$\operatorname{Argonne} \underset{\bigotimes}{\bigotimes}$

\title{
Investigation of Contaminant Sources at Navarre, Kansas
}

\author{
Environmental Science Division
}




\begin{abstract}
About Argonne National Laboratory
Argonne is a U.S. Department of Energy laboratory managed by UChicago Argonne, LLC under contract DE-AC02-06CH11357. The Laboratory's main facility is outside Chicago, at 9700 South Cass Avenue, Argonne, Illinois 60439. For information about Argonne, see www.anl.gov.
\end{abstract}

\title{
Availability of This Report
}

This report is available, at no cost, at http://www.osti.gov/bridge. It is also available on paper to the U.S. Department of Energy and its contractors, for a processing fee, from:

U.S. Department of Energy

Office of Scientific and Technical Information

P.O. Box 62

Oak Ridge, TN 37831-0062

phone (865) 576-8401

fax (865) 576-5728

reports@adonis.osti.gov

\section{Disclaimer}

This report was prepared as an account of work sponsored by an agency of the United States Government. Reference herein to any specific commercial product, process, or service by trade name, trademark, manufacturer, or otherwise, does not necessarily constitute or imply its endorsement, recommendation, or favoring by the United States Government or any agency thereof. The views and opinions of document authors expressed herein do not necessarily state or reflect those of the United States Government or any agency thereof, Argonne National Laboratory, or UChicago Argonne, LLC. 


\section{Investigation of Contaminant Sources at Navarre, Kansas}

by

Applied Geosciences and Environmental Management Section

Environmental Science Division, Argonne National Laboratory

July 2007

USDA 


\section{Contents}

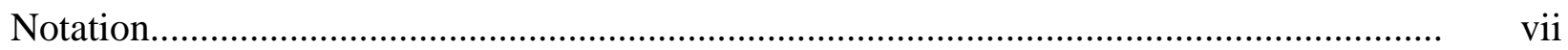

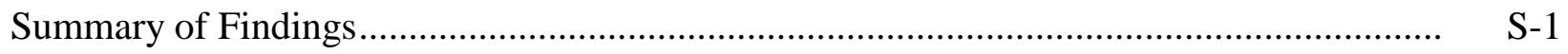

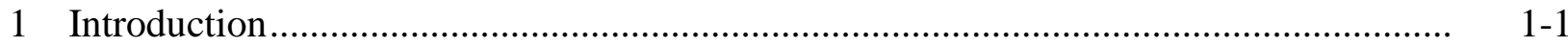

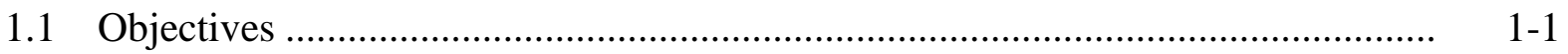

1.2 Background and Previous Investigations ................................................... $1-2$

2 Investigative Methods ................................................................................

2.1 Methods to Determine Groundwater Contaminant Levels and Groundwater Flow Direction in the General Study Area........................................................... 2-2

2.2 Methods to Identify Soil Source Areas Related to the Former CCC/USDA Property and Pathways for Contaminant Migration to Groundwater .................... 2-3

2.3 Methods to Investigate Specific Areas of Concern on the Co-op Property ............ 2-4

2.4 Methods to Investigate Potential Source Areas Identified on the Former CCC/USDA Property during the Investigation ........................................... $2-6$

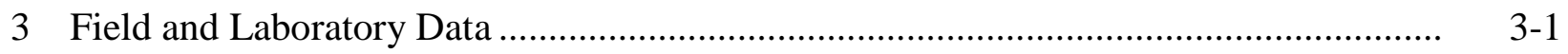

3.1 Cone Penetrometer Sensor Data .................................................................

3.2 Piezometer Construction ............................................................................ $3-2$

3.3 Coordinates Survey Data ................................................................................ 3-2

3.4 Analytical Data for Subsurface Soil Samples ...................................................... 3-2

3.4.1 Carbon Tetrachloride and Chloroform in Soils on and near the Former CCC/USDA Property.................................................................... 3-3

3.4.2 Carbon Tetrachloride and Chloroform in Soils on the Co-op Property...... 3-4

3.5 Groundwater Analytical Data ..................................................................... 3-5

3.5.1 Volatile Organic Compounds in Groundwater on and near the Former CCC/USDA Property....................................................................... 3 $3-6$

3.5.2 Volatile Organic Compounds in Groundwater on the Co-op Property....... 3-8

3.5.3 Volatile Organic Compounds in Groundwater in Areas Downgradient or Upgradient from the Target Areas ....................................................... 3-. 3

3.6 Groundwater Gradient Data .......................................................................... 3-10

3.7 Results of Quality Control Activities.............................................................. $3-11$

3.8 Waste Characterization, Handling, and Disposal .......................................... 3-14

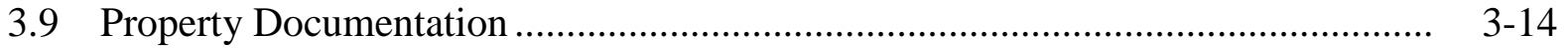

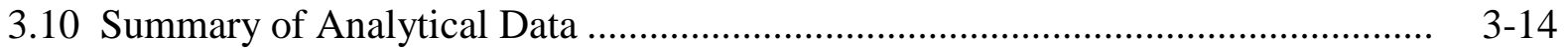

3.10.1 Former CCC/USDA Property …..................................................... $3-15$

3.10 .2 Co-op Property ........................................................................... $3-16$ 
4 Data Interpretation ............................................................................................... 4-1

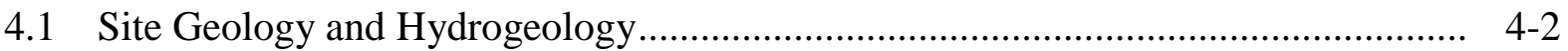

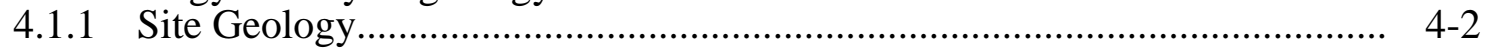

4.1.2 Site Hydrogeology ......................................................................... $4-4$

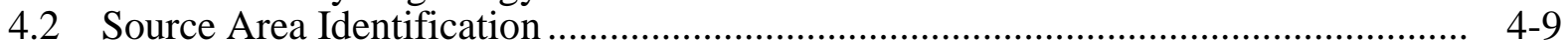

4.2.1 Evidence from Groundwater Data ...................................................... 4-10

4.2.2 Evidence from Soil Data ............................................................ 4-12

4.3 Contaminant Migration Pathways.......................................................................... 4-14

4.4 Contaminant Distribution throughout the General Investigation Area.................... 4-15

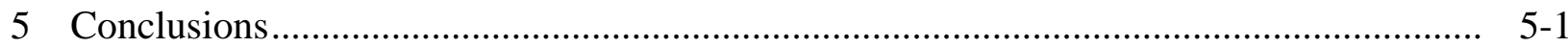

5.1 Former CCC/USDA Property ............................................................... $5-1$

5.2 Co-op Property Source Areas ….................................................................... $5-2$

5.3 Status of the Contaminant Plume in the General Investigation Area ....................... 5-5

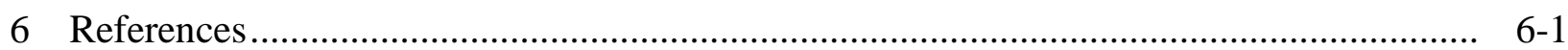

Appendix A: Electronic and Lithologic Logs....................................................... A

Appendix B: Well Construction Information for MW5 ............................................ B-1

Appendix C: Coordinates Survey Data ................................................................ C-1

Appendix D: Historical Data Summary for Monitoring Wells at Navarre........................ D-1

Supplement 1: Soil Sample Data...........................................................................(on CD)

Supplement 2: Water Sample Data .......................................................................(on CD)

Supplement 3: $\quad$ Water Level Data ..........................................................................(on CD)

Supplement 4: Quality Control for Sample Collection, Handling, and Analysis ..............(on CD)

Supplement 5: Chain-of-Custody Forms and Outside Laboratory Data ..........................(on CD)

Supplement 6: Wastewater Characterization Data.....................................................(on CD)

Supplement 7: Property Documentation .............................................................(on CD) 


\section{Tables}

2.1 Summary of activities during the 2006 investigation at Navarre, Kansas

3.1 Summary of organic analytical results for soil samples collected at the former CCC/USDA facility during the 2006 investigation at Navarre, Kansas....

3.2 Summary of organic analytical results for soil samples collected on the Co-op property during the 2006 investigation at Navarre, Kansas

3.3 Summary of results of organic analyses of vertical-profile groundwater samples collected with the cone penetrometer at the former CCC/USDA facility during the 2006 investigation at Navarre, Kansas

3.4 Summary of results of organic analyses of well samples and vertical-profile groundwater samples collected with the cone penetrometer on and near the Co-op property during the 2006 investigation at Navarre, Kansas.

3.5 Summary of results of organic analyses of well samples and vertical-profile groundwater samples collected with the cone penetrometer at locations downgradient or upgradient from the targeted areas during the 2006 investigation at Navarre,

Kansas .....

4.1 Monthly precipitation data for Enterprise, Kansas, approximately $7 \mathrm{mi}$ north of Navarre.

4.2 Daily precipitation data for Manhattan, Kansas, approximately 35 mi northeast of Navarre.

\section{Figures}

S.1 Locations of the former CCC/USDA facility, monitoring and private wells, and 2006 investigation activities at Navarre, with the approximate southern Co-op boundary in each year of the Co-op's southward expansion

S.2 Hydrogeologic cross section A-A', showing the vertical and lateral distribution of carbon tetrachloride in subsurface soil and groundwater

S.3 Hydrogeologic cross section B-B', showing the vertical and lateral distribution of carbon tetrachloride in subsurface soil and groundwater

S.4 Interpreted distribution of carbon tetrachloride in the groundwater plume, 2006, with groundwater elevations on November 28, 2006

S.5 Grain storage facilities at Navarre in 1965 and 1971 
1.1 Specific areas of concern on the properties formerly leased by the CCC/USDA and currently owned by the Co-op............................................................................. 1-4

1.2 Historical property ownership in the investigation area at Navarre ............................ 1-5

1.3 Location of Navarre, Kansas........................................................................................ 1-6

1.4 Results of soil gas analyses in the 2000 Papadopulos study....................................... 1-7

2.1 Investigated locations in the 2006 study at Navarre .................................................... 2-9

2.2 Groundwater level monitoring network …………............................................... 2-10

2.3 Groundwater sampling locations for chemical analyses............................................ 2-11

$2.4 \quad$ Vertical-profile soil sampling locations ............................................................... 2-12

3.1 Cone penetrometer electronic sensor logging locations ............................................ 3-29

3.2 Maximum carbon tetrachloride concentrations in soil samples................................. 3-30

3.3 Maximum chloroform concentrations in soil samples.............................................. 3-31

3.4 Maximum carbon tetrachloride concentrations in groundwater samples .................... 3-32

3.5 Maximum chloroform concentrations in groundwater samples ………….................... 3-33

3.6 Maximum tetrachloroethene concentrations in groundwater samples.......................... 3-34

3.7 Maximum carbon tetrachloride concentrations in soil and groundwater samples, with sample depths, in the area of greatest contamination

4.1 Locations of contaminant source areas at Navarre ................................................ 4-20

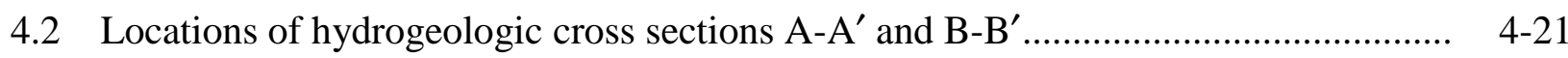

4.3 Hydrogeologic cross section A-A', showing the vertical and lateral distribution of carbon tetrachloride in subsurface soil and groundwater

4.4 Hydrogeologic cross section B-B', showing the vertical and lateral distribution of carbon tetrachloride in subsurface soil and groundwater

4.5 Hydrographs constructed from continuously monitored water levels in wells at Navarre, April 25, 2006, to November 28, 2006

4.6 Hydrographs for selected monitoring wells at Navarre, with precipitation data for Manhattan, Kansas, April 25, 2006, to November 28, 2006 
4.7 Potentiometric surface at Navarre, based on hand-measured water levels on April 24-25, 2006

4.8 Potentiometric surface at Navarre, based on water levels recorded by data loggers on August 11, 2006

4.9 Potentiometric surface at Navarre, based on water levels recorded by data loggers on August 31, 2006

4.10 Potentiometric surface at Navarre, based on water levels recorded by data loggers on September 23, 2006

4.11 Potentiometric surface at Navarre, based on hand-measured water levels on November 28, 2006

4.12 Interpreted distribution of carbon tetrachloride in the groundwater plume, 2006, with groundwater elevations on November 28, 2006

4.13 Locations of the former CCC/USDA facility, monitoring and private wells, and 2006 investigation activities at Navarre, with the approximate southern Co-op boundary in each year of the Co-op's southward expansion 


\section{Notation}

AGEM Applied Geosciences and Environmental Management

AMSL above mean sea level

AST aboveground storage tank

ASTM American Society for Testing and Materials

BGL below ground level

${ }^{\circ} \mathrm{C} \quad$ degree(s) Celsius

CAS Corrective Action Study

CCC Commodity Credit Corporation

CD compact disc

CLP Contract Laboratory Program

COC chain of custody

cpm count(s) per minute

CPT cone penetrometer

1,2-DCA 1,2-dichloroethane

DF dilution factor

EPA U.S. Environmental Protection Agency

ESC Expedited Site Characterization

$\mathrm{ft} \quad$ foot (feet)

g $\quad \operatorname{gram}(\mathrm{s})$

gal gallon(s)

GC-MS gas chromatograph-mass spectrometer

gpm gallon(s) per minute

$\mathrm{hr} \quad$ hour(s)

in. inch(es)

KDHE Kansas Department of Health and Environment

KGS Kansas Geological Survey

$\mu \mathrm{g} / \mathrm{kg} \quad$ microgram(s) per kilogram

$\mu \mathrm{g} / \mathrm{L} \quad$ microgram(s) per liter

$\mu \mathrm{m} \quad$ micrometer(s)

$\mu \mathrm{S} / \mathrm{cm} \quad$ microsiemen(s) per centimeter

MCL maximum contaminant level

$\mathrm{mg} / \mathrm{L} \quad$ milligram(s) per liter

mi mile(s)

min minute(s)

$\mathrm{mL} \quad$ milliliter(s) 
Investigation of Contaminant Sources at Navarre, Kansas

Version 00, 05/23/07

NAD North American Datum

NAVD North American Vertical Datum

PVC polyvinyl chloride

QA quality assurance

QC quality control

RBSL Risk Based Screening Level (Kansas Tier 2)

RPD relative percent difference

SDG sample delivery group

TOC top of casing

TU tritium unit(s)

USDA U.S. Department of Agriculture

VOC volatile organic compound 


\section{Investigation of Contaminant Sources at Navarre, Kansas}

\section{Summary of Findings}

The results of the 2006 investigation of contaminant sources at Navarre, Kansas, clearly demonstrate the following:

- Sources of carbon tetrachloride contamination were found on the Navarre Co-op property. These sources are the locations of the highest concentrations of carbon tetrachloride found in soil and groundwater at Navarre. The ongoing groundwater contamination at Navarre originates from these sources.

- The sources on the Co-op property are in locations where the Commodity Credit Corporation of the U.S. Department of Agriculture (CCC/USDA) never conducted grain storage operations.

- No definitive sources of carbon tetrachloride were identified on the portion of the current Co-op property formerly used by the CCC/USDA.

- The source areas on the Co-op property are consistent with the locations of the most intense Co-op operations, both historically and at present. The Co-op historically stored carbon tetrachloride for retail sale and used it as a grain fumigant in these locations.

- The distribution patterns of other contaminants (tetrachloroethene and nitrate) originating from sources on the Co-op property mimic the carbon tetrachloride plume. These other contaminants are not associated with CCC/USDA operations.

- The distribution of carbon tetrachloride at the Co-op source areas, particularly the absence of contamination in soils at depths less than $20 \mathrm{ft}$ below ground level, is consistent with vertical migration into the subsurface through a conduit (well Co-op 2), with subsequent lateral migration through the subsurface. 
- The groundwater flow direction, which is toward the west-northwest, is not consistent with migration of carbon tetrachloride in groundwater from the former CCC/USDA property to the source areas on the Co-op property.

- The absence of soil and groundwater contamination along surface drainage pathways on the former CCC/USDA property is not consistent with migration of carbon tetrachloride in surface water runoff from the former CCC/USDA property to the source areas on the Co-op property.

- The contamination detected in soil and groundwater samples collected along the northern boundary of the former CCC/USDA facility can be attributed to migration from the Co-op sources or to operations of the Co-op on the property after CCC/USDA operations ended.

- The southern boundary of the Co-op property has expanded over time, so that the Co-op has operated for a lengthy period in all areas previously leased by the CCC/USDA (Figure S.1). The Co-op began expanding onto the former CCC/USDA property in 1969 and has operated on that property longer than the CCC/USDA did. The use of carbon tetrachloride as a grain fumigant was standard industry practice until 1985, when the compound was banned by the U.S. Environmental Protection Agency.

- Petroleum-related contamination was detected on the southern part of the former CCC/USDA property. This contamination is associated with aboveground storage tanks that are owned and operated by the Co-op.

The major findings of the 2006 investigations are summarized in greater detail below. The 2006 investigation was implemented by the Environmental Science Division of Argonne National Laboratory on behalf of the CCC/USDA. 


\section{Findings Based on Technical Data}

\section{- Findings Related to the Co-op Property}

- Two clear source areas for carbon tetrachloride contamination in groundwater were identified on the Co-op property: one near the south door of the flat storage building, and the other near the former well Co-op 2 and the former Co-op chemical storage area. The CCC/USDA never leased or used this property. Source areas are generally defined as zones of highest contaminant concentrations in soil or groundwater (EPA 2007). The highest concentrations of carbon tetrachloride in soils and groundwater detected during the 2006 investigation occurred at the two locations identified as source areas. Carbon tetrachloride concentrations as high as $454 \mu \mathrm{g} / \mathrm{kg}$ in soil and $866 \mu \mathrm{g} / \mathrm{L}$ in groundwater were detected near the south door of the Co-op's flat storage building; concentrations as high as $1,094 \mu \mathrm{g} / \mathrm{kg}$ in soil and $3,104 \mu \mathrm{g} / \mathrm{L}$ in groundwater were detected in the vicinity of former well Co-op 2 and the former Co-op chemical storage area.

- The carbon tetrachloride contaminant distribution on the Co-op property indicated the presence of subsurface soil sources for the contamination found in groundwater at Navarre. The highest soil and groundwater concentrations were detected in the vicinity of former well Co-op 2, which could have served as a conduit for vertical contaminant migration. At boreholes in the source areas, high carbon tetrachloride concentrations were found in shallow groundwater samples and in some deeper vadose zone soil samples. In samples collected deeper in the boreholes, the concentrations generally decreased with depth. Figures S.2 and S.3 illustrate the vertical and lateral distribution of carbon tetrachloride in the subsurface at Navarre, along two hydrogeologic cross sections running from north to south and from west to east, respectively. Contamination concentrations detected east, west, north, and south of the source areas (TI-28 and TI-16) were significantly lower than in the source areas, confirming that the primary sources are (1) the area south of the flat 
storage building and (2) the area around well Co-op 2 that was formerly used for chemical storage.

- The absence of contamination in the shallow vadose zone throughout the investigation area is consistent with introduction of carbon tetrachloride into the subsurface through a conduit such as former well Co-op 2. The construction details of this well are not known; however, it was plugged in 1991 (KDHE 1992; KGS 2007).

- A total of 10 subsurface soil samples collected on the Co-op property contained carbon tetrachloride concentrations exceeding the Kansas Tier 2 Risk Based Screening Level (RBSL) of $200 \mu \mathrm{g} / \mathrm{kg}$ for the soil-togroundwater protection pathway; 55 groundwater samples collected on this property exhibited carbon tetrachloride concentrations exceeding the RBSL (and the maximum contaminant level [MCL]) of $5 \mu \mathrm{g} / \mathrm{L}$ for this compound in groundwater.

- Nitrate and tetrachloroethene were detected in groundwater samples collected on the Co-op property at the same source areas. The contaminant plumes of both compounds mimic the carbon tetrachloride plume. The maximum concentrations of nitrate $(344 \mathrm{mg} / \mathrm{L})$ and tetrachloroethene $(3.4 \mu \mathrm{g} / \mathrm{L})$ in the 1991 sampling event occurred at well Co-op 2 (KDHE 1992). These compounds are not associated with CCC/USDA operations.

- The Co-op's southern property boundary has expanded over time. This growth is illustrated in Figure S.1. The Co-op operations now encompass the entire area that the CCC/USDA formerly leased. Co-op operations have involved the storage, handling, mixing, and use of fertilizers and chemicals, including carbon tetrachloride. The Co-op has operated for years at the identified source areas and on the former CCC/USDA property, but the CCC/USDA never exclusively operated on or used any part of the Co-op property where the Co-op did not also operate. 


\section{- Findings Related to the Former CCC/USDA Property}

- No definitive source areas for carbon tetrachloride were found on the former CCC/USDA property. Contaminant patterns adjacent to and on the northern part of the former CCC/USDA property were consistent with migration through the subsurface from the source areas identified on the Co-op property.

- No soil samples collected on or adjacent to the former CCC/USDA property exhibited carbon tetrachloride concentrations above the RBSL of $200 \mu \mathrm{g} / \mathrm{kg}$ for the soil-to-groundwater protection pathway. Moreover, carbon tetrachloride was not detected above the method quantitation limit of $10 \mu \mathrm{g} / \mathrm{kg}$ in any vadose zone soil sample collected on or adjacent to the former CCC/USDA property, except at location TI-4, near the northern end of the property and also near the Co-op source areas.

- Sixteen groundwater samples collected on or adjacent to the former CCC/USDA property (near the source areas identified on the Co-op property) exhibited carbon tetrachloride concentrations exceeding the RBSL of $5 \mu \mathrm{g} / \mathrm{L}$, though the levels were significantly lower than in samples from the Co-op property. These 16 groundwater samples were all taken from locations on the northern third of the former CCC/USDA property, near the Co-op source areas. The carbon tetrachloride contamination in these samples can be attributed to migration through the subsurface from the Co-op source areas.

\section{- Findings Related to Surface Drainage}

- Soil and groundwater samples collected along surface drainage pathways on the former CCC/USDA property (locations TI-12, TI-8, TI-7, TI-2; Figure S.1) did not contain significant concentrations of carbon tetrachloride and did not exhibit contaminant distribution patterns that would demonstrate a source on the former CCC/USDA property in 2006 or earlier. 
- The absence of significant carbon tetrachloride contamination in vadose zone soils collected along surface drainage pathways on the former $\mathrm{CCC} / \mathrm{USDA}$ property is not consistent with an explanation asserting that the contamination detected at the identified source areas on the Co-op property originated on the former CCC/USDA property and migrated to the Co-op via surface drainage.

\section{- Findings Related to the Carbon Tetrachloride Plume}

- Figure S.4 illustrates the lateral distribution of maximum carbon tetrachloride concentrations in groundwater samples collected from monitoring wells and boreholes in 2006, with the groundwater gradient. The analytical data indicate that the carbon tetrachloride plume in groundwater extends a minimum of $1,500 \mathrm{ft}$ directly downgradient from the source areas identified on the Co-op property and is continuing to migrate toward the west-northwest. The concentration gradients identify the point of origin as the source areas identified on the Co-op property in the vicinity of (1) the south door of the flat storage building and (2) the former chemical storage area and well Co-op 2.

\section{- Findings Related to the Groundwater Flow Direction}

- The groundwater flow direction historically has been toward the westnorthwest (KDHE 1998a; Papadopulos 2001). Recent groundwater level measurements indicate similar flow patterns. The historical groundwater gradient is not consistent with migration from the former CCC/USDA property onto the Co-op property.

- The significantly lower concentrations of carbon tetrachloride detected in soil and groundwater on the former CCC/USDA property than at the identified source areas on the Co-op property are also inconsistent with migration from the former CCC/USDA property to the Co-op property. 


\section{Related Historical Information}

- In the standard practice of the time, the Co-op used carbon tetrachloride as a grain fumigant before the compound was banned in 1985. The Co-op also stored carbon tetrachloride for retail sale (Co-op 2000 [Exhibit 89]).

- The flat storage building on the Co-op property was used to store both grain and chemicals. Carbon tetrachloride was used by the Co-op until the mid 1980s and was used on several occasions in the 1970s at the flat storage building and other locations (iSi 2004; USDA 2007). (Carbon tetrachloride was banned by the EPA in December 1985.)

- Wheat was stored in the Co-op's flat storage building from 1974 to 1983. Milo was stored in the northern part of the building, and chemicals were stored in the southern part from 1990 to 1995 (Johnson 2000; Warders 2000). The earlier uses of the flat storage building, from its construction in 1958 to 1974, are not documented in the historical record.

- The area between the south door of the flat storage building and the dry fertilizer storage building has been and continues to be used intensely by the Co-op for storing, handling, and mixing chemicals. A 1971 aerial photograph (Figure S.5, right) documents the presence of the flat storage building (built in 1958), along with chemical storage containers. The former well (Co-op 2) adjacent to the former chemical storage area was used for chemical mixing and equipment washing (Warders 2000; Servi-Tech [1993] specified the location of well Co-op 2). With these adjacent operations, this well could have provided a direct conduit for contamination to enter the subsurface and migrate to groundwater.

- The Co-op used water from well Co-op 2 for mixing chemicals at and near the source areas (Warders 2000).

- In the 1991 sampling, the maximum carbon tetrachloride concentrations occurred at well Co-op $2(511 \mu \mathrm{g} / \mathrm{L})$ and well Co-op $3(535 \mu \mathrm{g} / \mathrm{L})(\mathrm{KDHE}$ 1992). (Well Co-op 3 lies approximately $450 \mathrm{ft}$ west-northwest 
(downgradient) from the location of former well Co-op 2.) Well Co-op 2 was still in use at the time of the 1991 sampling (approximately 25 years after CCC/USDA operations ended at Navarre), but it was plugged later that year (KGS 2007).

- The Co-op filled grain cars on the railroad tracks (Voit 2000) and fumigated the grain with carbon tetrachloride (iSi 2004).

- Grain storage by the CCC/USDA was limited in time. The CCC/USDA operation began in 1954, and removal of its bins began in 1963. By 1965 (Figure S.5, left), only five CCC/USDA bins remained. The use of carbon tetrachloride by other parties continued for another 20 years. For comparison, the Co-op began its operations in about 1919 and was reaching its full capacity by 1965 (Figure S.5, left). The Co-op's grain storage capacity has remained at approximately the same level to the present, though its operation has expanded southward to encompass the entire former CCC/USDA facility (Figure S.1).

- The grain storage capacity of the CCC/USDA operation was limited to a maximum of approximately 97,500 bushels. This capacity was calculated for 30 bins, as shown in the 1957 aerial photograph (Figure S.1, left), and an average capacity of 3,250 bushels per bin. For comparison, the Co-op's current capacity is approximately 895,000 bushels (BNSF Railway 2007). The Co-op's capacity was similar in 1965 (Figure S.5, left). The grain storage capacity ratio for the two facilities is approximately 1:9 (CCC/USDA capacity to Co-op capacity). The ratio of grain fumigant used during each year of operation could reasonably be similar. The CCC/USDA used carbon tetrachloride as a grain fumigant during its lease period (1954-1966). This was the standard industry practice at the time. The CCC/USDA did not store or mix fumigants on its property (PRC 1992). The Co-op reportedly used the pesticide Phostoxin in addition to the 80:20 mixture of carbon tetrachloride:carbon disulfide (Stroda 2000). 
- Carbon tetrachloride was brought to the CCC/USDA facility in a fumigant mixture ready for application (PRC 1992). No excess fumigant was left on the property to be stored or discarded.

- Nitrate contamination in groundwater at Navarre has historically been widespread. The maximum nitrate concentration detected was $344 \mathrm{mg} / \mathrm{L}$, at well Co-op 2, in 1991 (KDHE 1992). This well was plugged on September 5, 1991 (KGS 2007); its construction details are not known. A 1997 investigation by the Kansas Department of Health and Environment (KDHE) concluded that an ongoing nitrate source exists in the subsurface soils in the former chemical storage area, where liquid and dry fertilizers were stored and periodically released through spills and leakage. The KDHE linked the 1991 results for nitrate in groundwater from the Co-op 2 well $(344 \mathrm{mg} / \mathrm{L})$ to releases at the fertilizer storage area. Well KDHE-2 also contained a high concentration of nitrate $(330 \mathrm{mg} / \mathrm{L})$ in 1991 ; the KDHE interpreted this to be the result of migration from well Co-op 2 along the eastern drainage ditch on the Co-op property (KDHE 1998a). The nitrate contamination at Navarre is directly related to Co-op operations and is unique to the Co-op.

- Tetrachloroethene (also called tetrachloroethylene) was found in well Co-op 2 in 1991, at $3.4 \mu \mathrm{g} / \mathrm{L}$ (KDHE 1992). This contaminant is unique to the Co-op at Navarre.

- Additional documentation of spillage on the Co-op property includes stained soil around the former bulk liquid storage tanks just north of the dry fertilizer building (Servi-Tech 1993, Photo 9); removal of 3 tons of soil contaminated with oil (Linn 1990); and evidence of runoff and damaged vegetation in a drainage ditch adjacent to bulk liquid fertilizer tanks, as observed by KDHE staff on June 6, 1991 (KDHE 2004). 

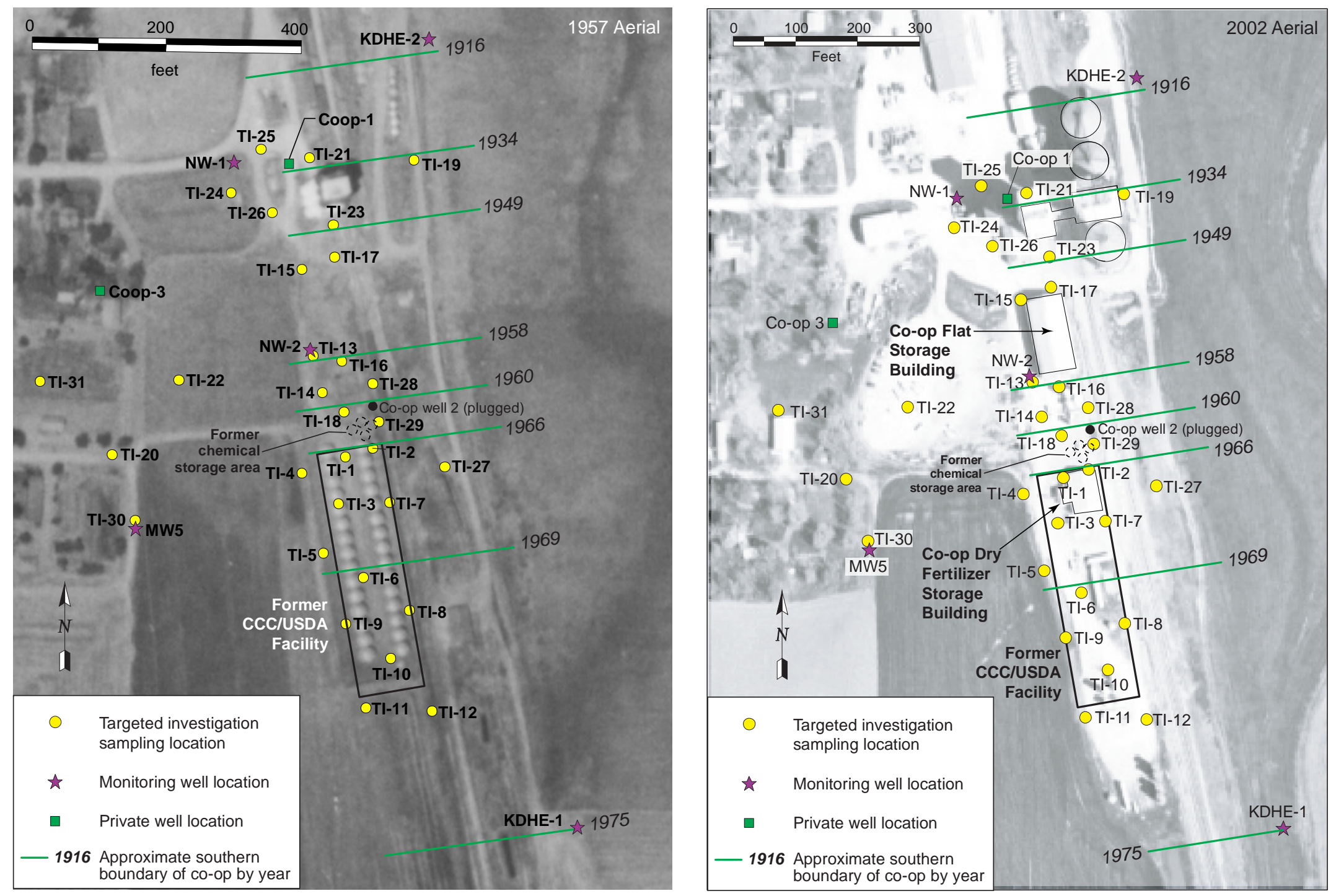

FIGURE S.1 Locations of the former CCC/USDA facility, monitoring and private wells, and 2006 investigation activities at Navarre, with the approximate southern Co-op boundary in each year of the Co-op's southward expansion. Source of photographs: USDA (1957); NAIP (2002). 


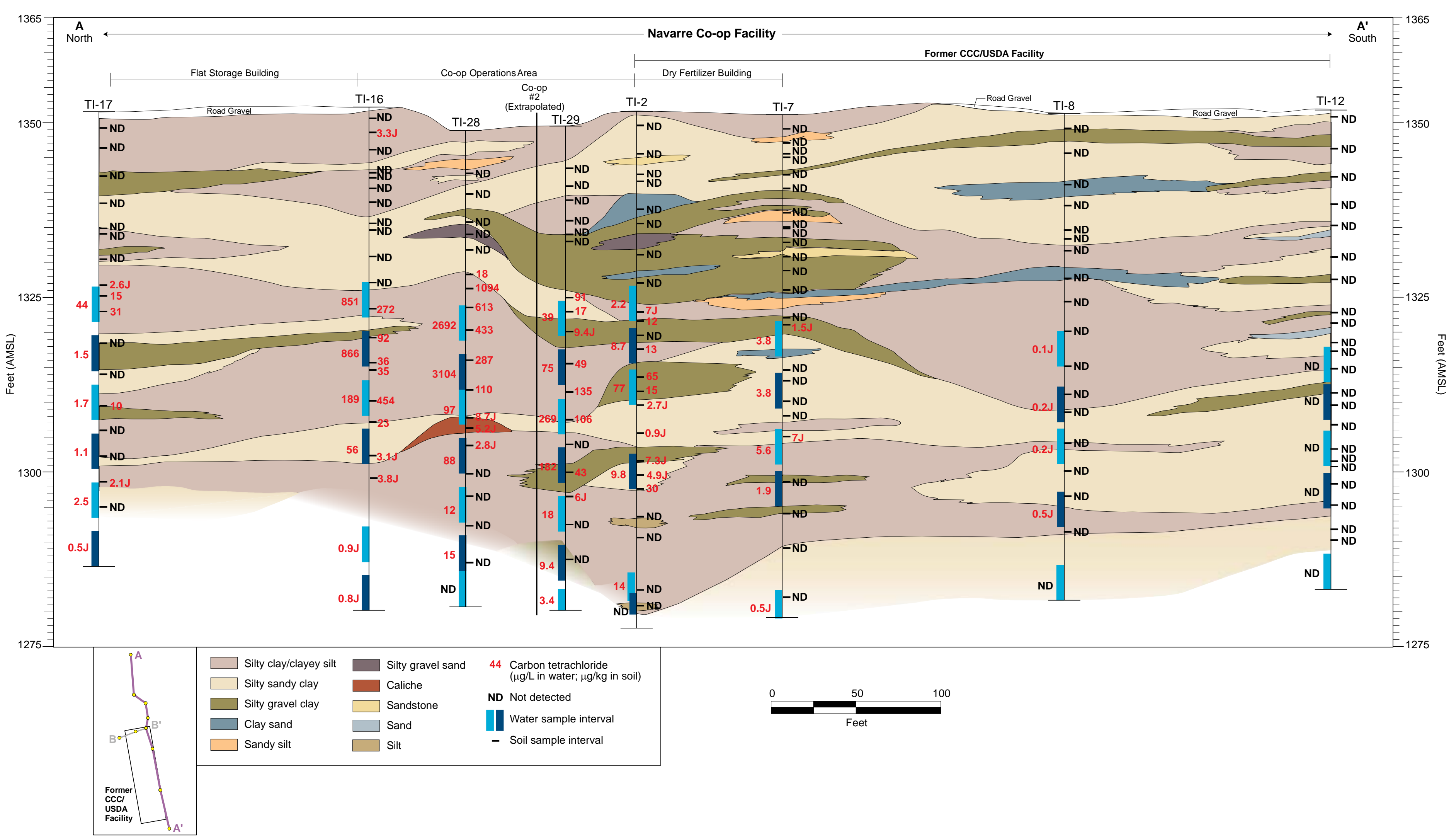

FIGURE S.2 Hydrogeologic cross section A-A' (vertically exaggerated), showing the vertical and lateral distribution of carbon tetrachloride in subsurface soil and groundwater. 


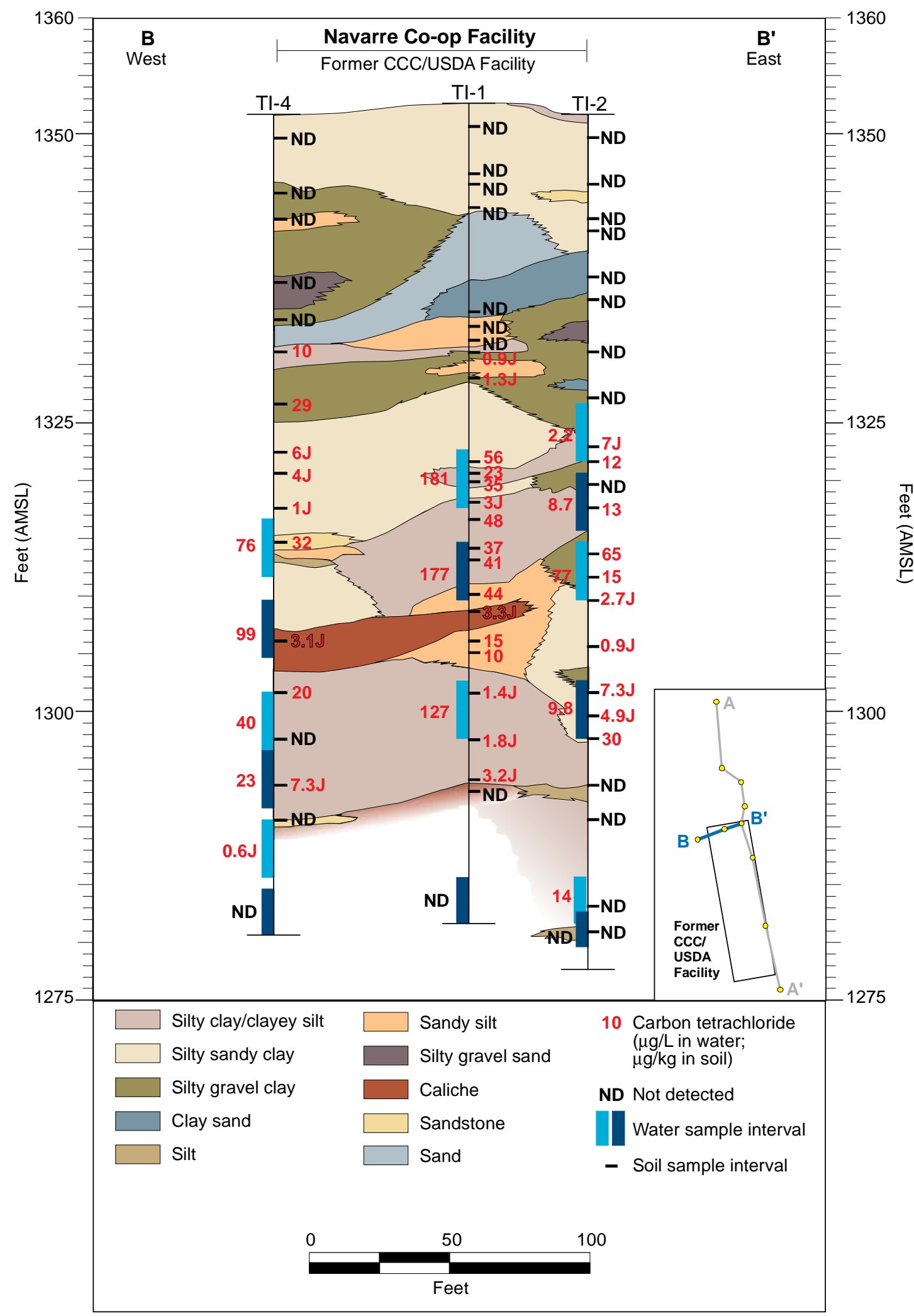

FIGURE S.3 Hydrogeologic cross section B-B' (vertically exaggerated), showing the vertical and lateral distribution of carbon tetrachloride in subsurface soil and groundwater. 


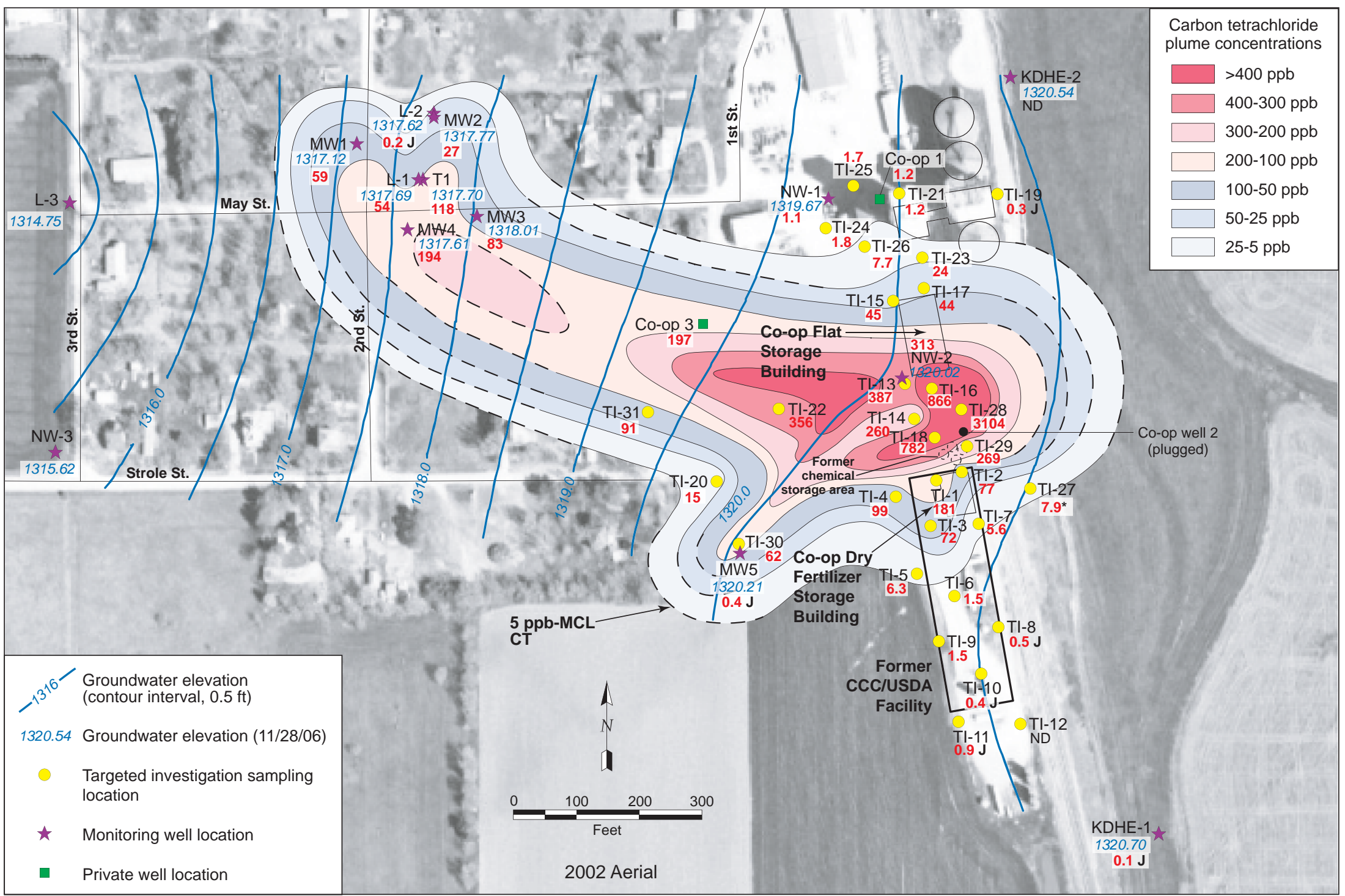

FIGURE S.4 Interpreted distribution of carbon tetrachloride in the groundwater plume, 2006, with groundwater elevations on November 28 , 2006. Source of photograph: NAIP (2002). 

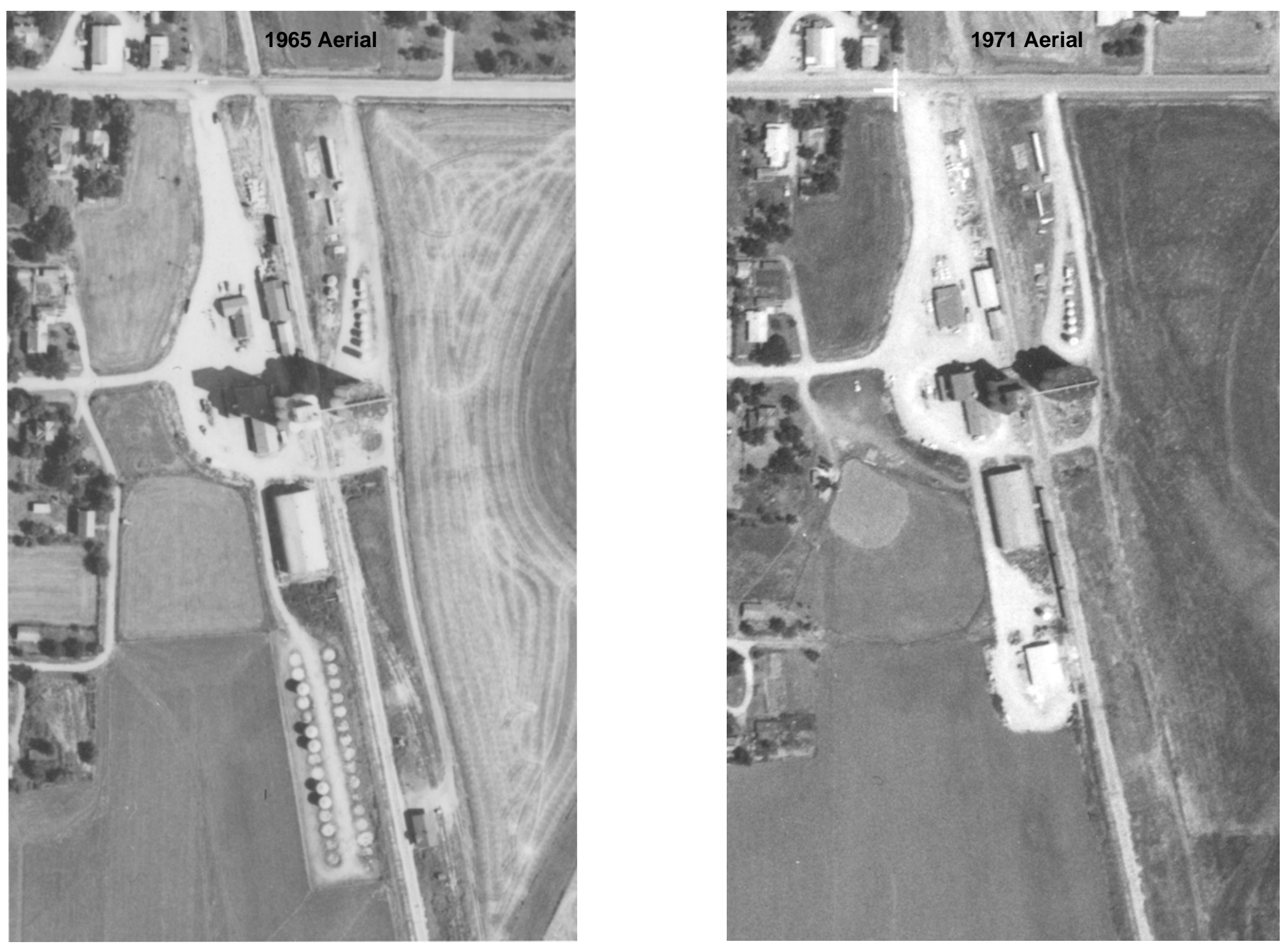

FIGURE S.5 Grain storage facilities at Navarre in 1965 (left) and 1971 (right). Bins are distinguished from foundations in the 1965 photograph (left) by shadows. Source of photographs: USDA $(1965,1971)$. 


\section{Introduction}

The Environmental Science Division of Argonne National Laboratory implemented the 2006 investigation at Navarre on behalf of the Commodity Credit Corporation of the U.S. Department of Agriculture (CCC/USDA). This investigation was designed to (1) investigate specific areas of concern for potential sources and potential source areas that might be associated with the past use of carbon tetrachloride and (2) evaluate migration pathways for previously identified carbon tetrachloride contamination in groundwater at Navarre. The investigation focused on the properties formerly leased by the CCC/USDA and currently owned by the Co-op. The specific areas of concern are shown in Figure 1.1.

A source is the place or object where the contaminant of concern is released. Source areas are defined as zones of highest contaminant concentrations in the soil or groundwater, or both (EPA 2007).

\subsection{Objectives}

Three objectives were proposed in the work plan (Argonne 2006). The work plan was approved by the Kansas Department of Health and Environment (KDHE) on February 20, 2006 (Carey 2006). The three objectives were as follows:

1. Determine groundwater contaminant levels and groundwater flow direction in the general study area.

2. Investigate the former CCC/USDA property for soil source areas and pathways for contaminant migration to groundwater.

3. Investigate specific areas of concern (flat storage building, former waste pit area adjacent to former well Co-op 2, and feed mill building) on the Co-op property that may be associated with the past use of carbon tetrachloride.

The investigation locations on the former CCC/USDA property and in the specific areas of concern were chosen on the basis of previously collected analytical data that indicated the presence of carbon tetrachloride in the subsurface, along with information from Co-op 
employees that grain and chemical storage and handling occurred at these locations. (Refer to Summary of Findings for a discussion of related historical information.) This report details and interprets the data collected during the 2006 investigation at Navarre. The investigation met the objectives defined in the work plan.

\subsection{Background and Previous Investigations}

The CCC/USDA operated a grain storage facility at Navarre, Kansas, from 1954 to approximately 1966, on property adjacent to the Navarre Co-op's operation. Over the years, the Co-op has expanded across the area formerly occupied by the CCC/USDA (Figure 1.2).

The unincorporated town of Navarre, Kansas, is located in Logan Township of Dickinson, County, in the eastern part of the state (Figure 1.3). The town lies about 95 mi west of Topeka, Kansas. The Dickinson County Clerk's Office reported that the 2006 population of Logan Township was approximately 208.

In 1991, the KDHE conducted a preliminary assessment of groundwater contamination detected in samples collected in 1990-1991 from drinking water wells in Navarre (KDHE 1992). In the preliminary assessment, the KDHE (1992) indicated that the carbon tetrachloride and nitrate contamination in groundwater resulted from activities conducted on the former CCC/USDA property and at the active Navarre Farmer's Union Co-op (the Co-op). At that time, the Co-op was working with the KDHE to resolve the problem by assisting the KDHE in development of a plan to provide a new drinking water supply to the residents of Navarre.

Site characterization studies conducted for the CCC/USDA by Argonne in the early 1990s (Argonne 1992, 1993, 1995) indicated that two aquifers (upper and lower) were present in Navarre. Separate aquifers could not be validated during the 2006 investigation. The investigations conducted in the 1990s had limited scopes of work and limited investigative techniques. In contrast, new technologies, coupled with the greater detail achieved in the 2006 investigation, enabled a more thorough study of the lithologic and hydrologic properties at Navarre.

In addition to groundwater samples, Argonne analyzed subsurface soil samples collected in 1993 at several locations on the former CCC/USDA property. Elevated carbon tetrachloride 
levels were detected in subsurface soil near the north end of the former CCC/USDA facility (Argonne 1993). During previous investigations, Argonne was unable to gain access to the Co-op area north of the former CCC/USDA facility for further investigation of soil sources of carbon tetrachloride (Argonne 2006).

In 2000, S.S. Papadopulos conducted a study on the Co-op and former CCC/USDA properties for the U.S. Department of Justice (Figure 1.4; Papadopulos 2000). The investigation included a soil gas survey at depths of $10 \mathrm{ft}$ and $17.5-26.5 \mathrm{ft}$ below ground level (BGL) and groundwater sampling at depths of 35.5-50 ft BGL. The soil gas survey was conducted in the vicinity of the feed mill, the flat storage building, former well Co-op 2 and chemical storage areas, and the dry fertilizer building. The groundwater survey was conducted in the vicinity of the flat storage building, former well Co-op 2, and the former chemical storage areas on the Co-op property. The soil gas survey and groundwater analyses showed elevated levels of carbon tetrachloride at locations throughout the investigation target area (Figure 1.4).

Because of concerns about potential health hazards, the CCC/USDA funded construction of a connection with the Dickinson County Rural Water District \#2 supply line to provide all households and businesses in Navarre with access to safe drinking water (KDHE 1998b). This project was completed on August 6, 2001 (KDHE 2001). 


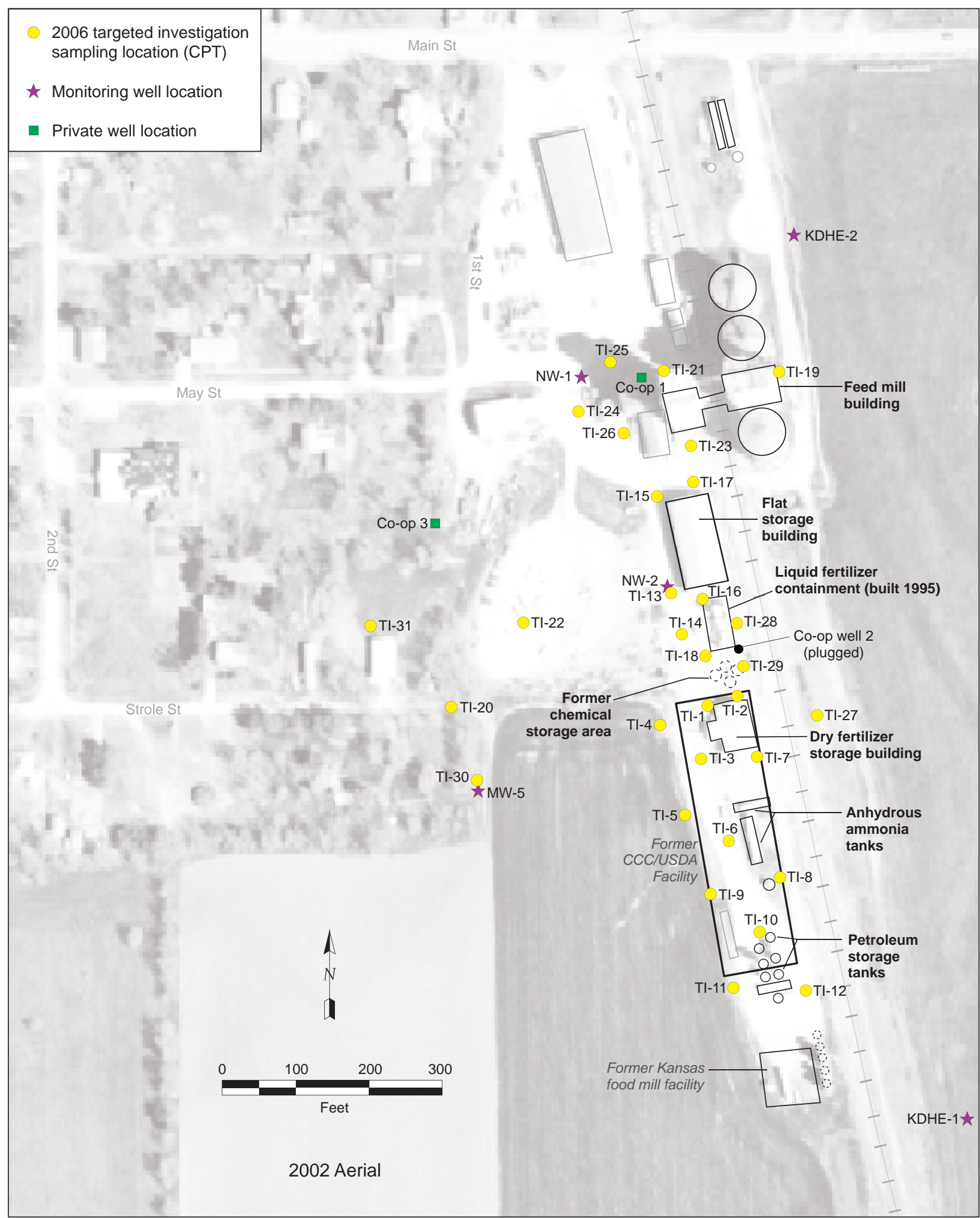

FIGURE 1.1 Specific areas of concern on the properties formerly leased by the CCC/USDA and currently owned by the Co-op. Source of photograph: NAIP (2002). 


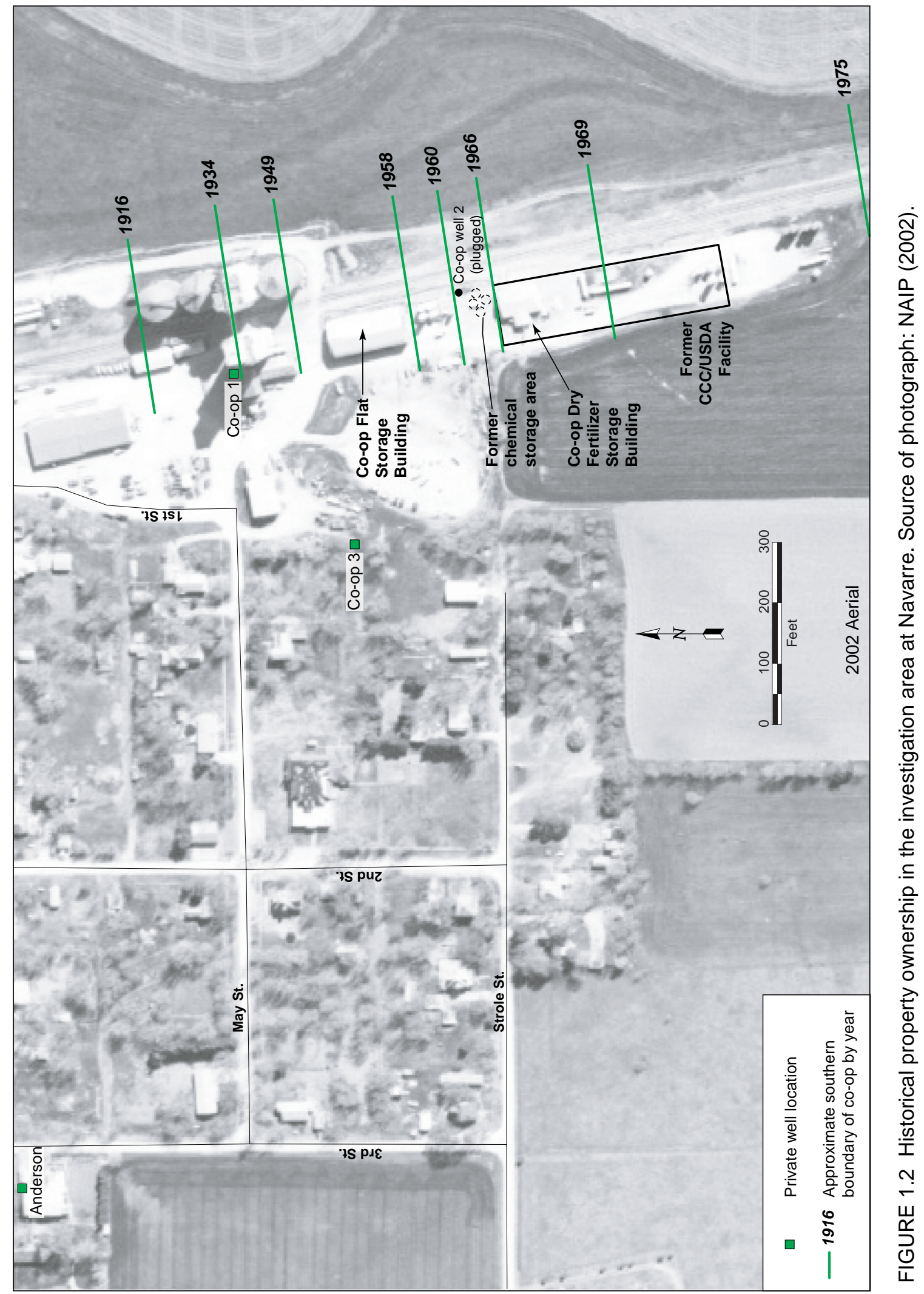




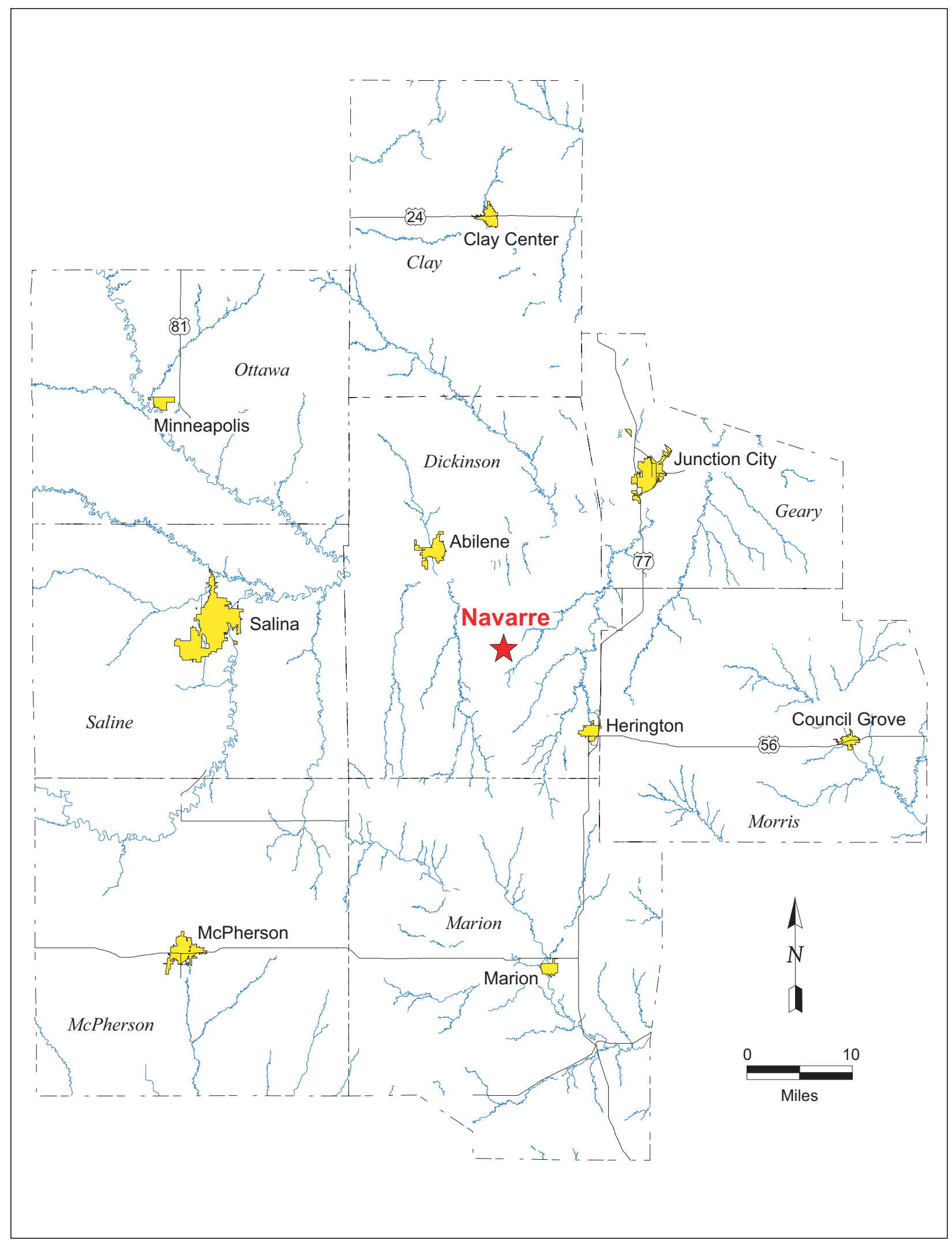

FIGURE 1.3 Location of Navarre, Kansas. 


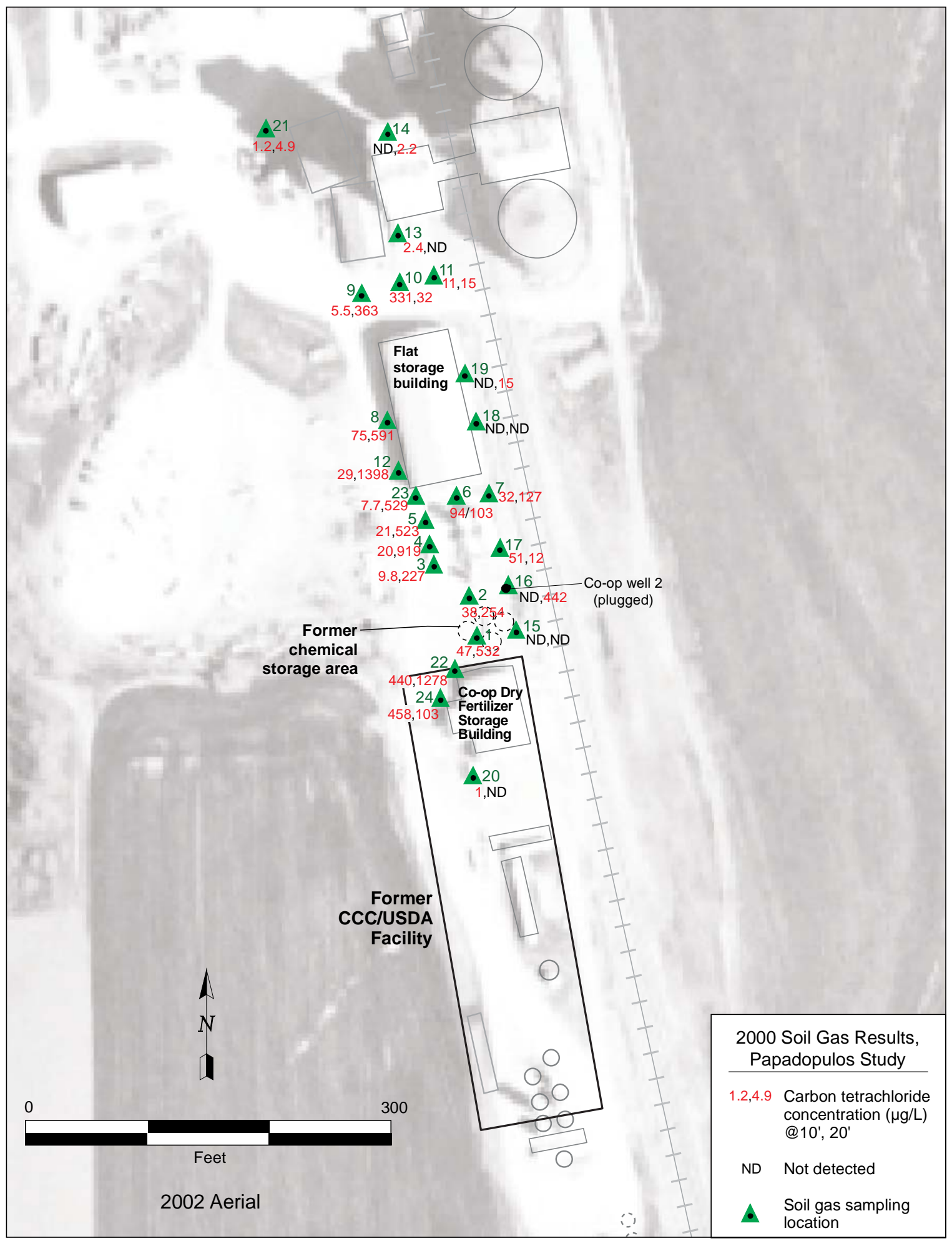

FIGURE 1.4 Results of soil gas analyses in the 2000 Papadopulos study. Source of data: Papadopulos (2000). Source of photograph: NAIP (2002). 


\section{Investigative Methods}

The 2006 investigation at Navarre was performed by using an iterative process of data collection, evaluation, and interpretation during field activities. This methodology ensured that the data necessary to achieve the specific technical objectives listed in Section 1 were obtained. Throughout data evaluation and interpretation, the CCC/USDA and KDHE project managers were kept informed of the analytical results as they were received, and modifications to the work plan (Argonne 2006) were made with their input and approval.

Throughout the field program, a comprehensive quality assurance/quality control (QA/QC) program was implemented to confirm the reliability of all information as it accumulated. Procedures for the individual techniques employed by Argonne at this site are in the Master Work Plan (Argonne 2002). This section provides a brief overview of the methods used to implement this investigation, and it identifies modifications made to the site-specific work plan (Argonne 2006) in response to data acquired during the field work.

The primary data collected in the 2006 investigation at the Navarre site included electronic logs and soil and groundwater samples. Electronic logs were used to evaluate the site lithology and determine target depths for groundwater samples. These data were collected by using the electronic capabilities of Argonne's cone penetrometer (CPT). Potential contaminant source areas were investigated at numerous locations by collecting soil and groundwater samples with the CPT.

All locations investigated in 2006 are shown in Figure 2.1. The activities at each location are summarized in Table 2.1.

The initial task consisted of attempts to collect electronic profiles from ground surface to bedrock at 17 CPT locations, in accordance with the procedures detailed in the Master Work Plan (Argonne 2002). The electronic logs are in Appendix A.

Soil samples were collected at $20 \mathrm{CPT}$ locations at 4-ft intervals, from the ground surface to the top of the saturated zone (at most locations) or to bedrock (at some locations). Because of the slow recharge rate at many locations, the depth to the saturated zone was estimated on the basis of groundwater levels in nearby wells and CPT locations. Soil samples were collected by using a 4-ft-long modified Macro-Core ${ }^{\circledR}$ soil sampler that allowed for discrete or continuous 
collection of soil samples at specified depths. Lithologic descriptions of soil cores are in Appendix A.

Groundwater samples were collected at $31 \mathrm{CPT}$ locations, at 5-ft intervals from the static water level to bedrock at most locations. Targeted sampling depths were determined from the electronic logs and lithologic descriptions. After targeted sampling intervals were identified, the CPT rods were pushed to the specified depth. The lead CPT rod was fitted with a 5-ft polyvinyl chloride (PVC) screen inserted inside the rod and attached to a disposable tip. After the target depth was reached, PVC riser pipe was inserted into the rods and threaded to the screen. The rods were then withdrawn $5 \mathrm{ft}$ to expose the screened area. If groundwater was not initially present, the CPT rods were withdrawn completely, and the temporary well was left in place until enough groundwater accumulated for sampling. Temporary wells were set at each location to the extent necessary to accomplish the investigational objectives. All temporary wells were subsequently plugged in accordance with KDHE regulations.

Soil and groundwater samples were collected in laboratory-approved containers, sealed, placed on ice (dry ice for soils), and transported to the Applied Geosciences and Environmental Management (AGEM) Laboratory at Argonne National Laboratory for preparation and analysis for volatile organic compounds (VOCs), including carbon tetrachloride, chloroform, methylene chloride, tetrachloroethene, and 1,2-dichloroethane (1,2-DCA). Selected groundwater samples were analyzed for tritium. In addition, some soil samples were submitted for grain size analysis.

\subsection{Methods to Determine Groundwater Contaminant Levels and Groundwater Flow Direction in the General Study Area}

The groundwater flow direction was determined from water level measurements collected in 14 monitoring wells (Figure 2.2).

Before groundwater samples were collected for VOCs analyses, each well was purged in accordance with procedures in the Master Work Plan (Argonne 2002). To determine contaminant concentrations in the general study area, groundwater samples were collected from 14 existing and new monitoring wells and from 3 private wells (Anderson, Co-op 1, and Co-op 3; Figure 2.3). 
The work plan (Argonne 2006) proposed sampling 12 monitoring wells and 2 private wells. The sample locations added during field activities included newly installed monitoring well MW5, existing monitoring well NW-3, and the Anderson private well. The additions were made for the following reasons:

- New monitoring well MW5 was installed during the 2006 investigation at the TI-30 location; it became the 14th monitoring well sampling location. The well was installed because the CPT achieved a depth of $88.5 \mathrm{ft}$ BGL (the maximum reached in the investigation) at this location, offering the opportunity to monitor the deepest accessible portion of the aquifer.

- Monitoring well NW-3 was added after it was located by using a metal detector. Previous attempts to locate that well had been unsuccessful. Several attempts to locate monitoring well NW-4 with a metal detector during the 2006 investigation were unsuccessful.

- The Anderson private well is located approximately 1 block north of monitoring well L-3. The data logger installed at well L-3 showed periodic drawdown with an unknown cause. A search of the area showed one known private well (Anderson well) near monitoring well L-3. To gauge its possible effects on the water level at well L-3, the Anderson well was pumped for a 24-hr period. Three groundwater samples were collected from this well - one at the start of pumping, one after several hours of pumping, and one at the end of the pumping period - to monitor the contaminant concentrations over time.

\subsection{Methods to Identify Soil Source Areas Related to the Former CCC/USDA Property and Pathways for Contaminant Migration to Groundwater}

The potential soil source areas and contaminant migration pathways were thoroughly investigated by collecting soil samples (Figure 2.4) and groundwater samples (Figure 2.3) in vertical profiles at 12 CPT locations (TI-1 through TI-12) on and near the former CCC/USDA property. For this technical objective, the work plan (Argonne 2006) proposed collecting soil and groundwater samples in vertical profiles at $12 \mathrm{CPT}$ locations on or adjacent to the former $\mathrm{CCC} / \mathrm{USDA}$ property, as well as installation of monitoring wells through use of conventional 
drilling methods in any borehole drilled into the deeper saturated zone (previously differentiated as the lower aquifer). The KDHE approved the 12 sample locations specified in the work plan (Carey 2006). These included locations along surface drainage pathways and near former grain bins.

Vertical-profile soil and groundwater samples were collected at the 12 proposed CPT (TI-1-TI-12) locations on and adjacent to the former CCC/USDA property. Four of the locations (TI-1, TI-2, TI-3, and TI-7) were positioned on the perimeter of the dry fertilizer building.

Monitoring wells were not installed on the former CCC/USDA property, because the presence of two separate aquifers could not be validated, and analytical data did not indicate a definitive source area to be present on the former CCC/USDA property. The lithology showed no distinctive continuous consolidated layers or bedrock to be present in the upper part of the lithologic section. Furthermore, analytical data for soil and groundwater showed no carbon tetrachloride contamination above the AGEM method quantitation limits $(10 \mu \mathrm{g} / \mathrm{kg}$ for soil and $1.0 \mu \mathrm{g} / \mathrm{L}$ for water) in samples collected on the former CCC/USDA property at depths from $70 \mathrm{ft}$ to $77.8 \mathrm{ft}$ BGL.

\subsection{Methods to Investigate Specific Areas of Concern on the Co-op Property (Flat Storage Building, Former Waste Pit Area Adjacent to Former Well Co-op 2, and Feed Mill Building)}

The investigation of specific areas of concern on the Co-op property included collection of soil samples from 8 CPT locations and groundwater samples from 19 CPT locations. Of these target locations, 13 were adjacent to storage buildings and an area that had previously been identified as the location of former well Co-op 2 and chemical storage area. Another 6 locations were upgradient or downgradient from the specific areas of concern on the Co-op property. The following samples were collected specifically to investigate the areas of concern:

- Vertical-profile soil samples at 8 locations adjacent to the flat storage building, former well Co-op 2, and the former chemical storage area: TI-13, TI-14, TI-15, TI-16, TI-17, TI-18, TI-28, and TI-29 (Figure 2.4).

- Vertical-profile groundwater samples at 13 locations adjacent to the feed mill, flat storage building, former well Co-op 2, and the former chemical storage 
area: TI-13, TI-14, TI-15, TI-16, TI-17, TI-18, TI-21, TI-23, TI-24, TI-25, TI-26, TI-28, and TI-29 (Figure 2.3).

- Vertical-profile groundwater samples at 6 locations upgradient and downgradient of the specific areas of concern: TI-19, TI-20, TI-22, TI-27, TI-30, and TI-31 (Figure 2.3).

The original scope of work (Argonne 2006) proposed the collection of soil and groundwater samples at up to 22 locations in the specific areas of concern on the Co-op property and at up to 13 additional locations upgradient and downgradient from these areas. In addition, installation of monitoring wells was proposed. The scope of work was modified as analytical data received in the field were discussed with the CCC/USDA and KDHE project managers. All modifications were approved by the CCC/USDA and KDHE project managers. The modifications were as follows:

- No soil and groundwater samples were collected at 7 locations proposed in the KDHE-approved work plan (Argonne 2006), at or near the specific areas of concern. Three of these locations were in an area that was not physically accessible. The decision to eliminate the other 4 locations - along the west side of the flat storage building and former well Co-op 2- was made after discussions with the KDHE and CCC/USDA project managers. Analyses of samples from nearby locations indicated that adequate data had been obtained to confirm a source area in the vicinity of the south door of the flat storage building, former well Co-op 2, and the former chemical storage area.

- No groundwater samples were collected at 4 proposed locations north and west of the feed mill building or at 5 locations south, west, and east of the former CCC/USDA property, because minimal contaminant concentrations had been detected at locations adjacent to these specific areas of concern. 


\subsection{Methods to Investigate Potential Source Areas Identified on the Former CCC/USDA Property during the Investigation}

The original scope of work (Argonne 2006) specified proposing additional work (in consultation with the CCC/USDA and KDHE project managers) if a source area of contamination was detected on the former CCC/USDA facility.

The investigation results did not indicate that a definitive source area was present on the former CCC/USDA property, and therefore additional work was not required. 
TABLE 2.1 Summary of activities during the 2006 investigation at Navarre, Kansas.

\begin{tabular}{|c|c|c|c|c|c|c|c|c|c|c|}
\hline Location & Type $^{a}$ & $\begin{array}{l}\text { Number of } \\
\text { Water } \\
\text { Samples }\end{array}$ & $\begin{array}{l}\text { Water } \\
\text { Sampling } \\
\text { Interval } \\
\text { (ft BGL) }\end{array}$ & $\begin{array}{l}\text { Number of } \\
\text { Soil } \\
\text { Samples }\end{array}$ & $\begin{array}{c}\text { Soil } \\
\text { Sampling } \\
\text { Interval } \\
\text { (ft BGL) }\end{array}$ & $\begin{array}{l}\text { Number of } \\
\text { Grain Size } \\
\text { Samples }\end{array}$ & $\begin{array}{c}\text { Grain Size } \\
\text { Sampling } \\
\text { Interval }\end{array}$ & $\begin{array}{l}\text { Lithology } \\
\text { Log }\end{array}$ & $\begin{array}{l}\text { Cone } \\
\text { Penetrometer } \\
\text { Sensor } \\
\text { Log }\end{array}$ & $\begin{array}{l}\text { Water } \\
\text { Level } \\
\text { Location }\end{array}$ \\
\hline
\end{tabular}

\section{Existing wells}

Co-op 1
Co-op 3
KDHE-1
KDHE-2
T1
MW1
MW2
MW3
MW4
NW-1
NW-2
NW-3
L-1
L-2
L-3

$\begin{array}{ccc}\text { DW } & 1 & \text { Unknown } \\ \text { DW } & 1 & \text { Unknown } \\ \text { MW } & 1 & 35-55 \\ \text { MW } & 1 & 25-45 \\ \text { MW } & 1 & 40-60 \\ \text { MW } & 1 & 43-58 \\ \text { MW } & 1 & 42.8-57.8 \\ \text { MW } & 1 & 44-59 \\ \text { MW } & 1 & 45-60 \\ \text { MW } & 1 & 40-50 \\ \text { MW } & 1 & 35.5-45.5 \\ \text { MW } & 1 & 38-48 \\ \text { MW } & 1 & 75-95 \\ \text { MW } & 1 & 80-90 \\ \text { MW } & 1 & 80-90 \\ \text { DW } & 3 & 68^{b}\end{array}$

New 2006 locations associated with former CCC/USDA facility

\begin{tabular}{|c|c|c|c|c|c|c|c|c|c|c|}
\hline TI-1 & CPT & 4 & $30-71$ & 24 & $2-59.5$ & - & - & Yes & Yes & - \\
\hline TI-2 & CPT & 6 & $25-72$ & 23 & $2-70.7$ & 15 & $13.2-71.5$ & Yes & Yes & - \\
\hline TI-3 & СРT & 3 & $32.2-56$ & 16 & $6-62$ & 4 & 21-42.5 & Yes & Yes & - \\
\hline $\mathrm{TI}-4$ & СРT & 6 & $35-71$ & 16 & $2-61$ & 3 & $16-31.5$ & Yes & - & - \\
\hline TI-5 & СРT & 4 & $28-77.8$ & 16 & $2-61$ & - & - & - & Yes & - \\
\hline TI-6 & CPT & 4 & $38-73$ & 15 & $2-61$ & - & - & Yes & Yes & - \\
\hline TI-7 & СРT & 5 & $29.5-72$ & 24 & $2-69$ & 4 & $33-68.6$ & Yes & Yes & - \\
\hline TI-8 & CPT & 5 & $31-69.5$ & 17 & $2-59.7$ & - & - & - & Yes & - \\
\hline TI-9 & СРT & 4 & $40-64$ & 21 & $4-69$ & - & - & Yes & Yes & - \\
\hline TI-10 & СРT & 5 & $40-71$ & 20 & $4.5-65.5$ & - & - & - & Yes & - \\
\hline TI-11 & СРT & 6 & $26-70.9$ & 17 & $2-60.5$ & - & - & - & Yes & - \\
\hline TI-12 & СРT & 5 & $34-68.7$ & 22 & $1-61.5$ & - & - & Yes & Yes & - \\
\hline
\end{tabular}


TABLE 2.1 (Cont.)

\begin{tabular}{|c|c|c|c|c|c|c|c|c|c|c|}
\hline Location & Type & $\begin{array}{c}\text { Number of } \\
\text { Water } \\
\text { Samples }\end{array}$ & $\begin{array}{l}\text { Water } \\
\text { Sampling } \\
\text { Interval } \\
\text { (ft BGL) }\end{array}$ & $\begin{array}{l}\text { Number of } \\
\text { Soil } \\
\text { Samples }\end{array}$ & $\begin{array}{c}\text { Soil } \\
\text { Sampling } \\
\text { Interval } \\
\text { (ft BGL) }\end{array}$ & $\begin{array}{l}\text { Number of } \\
\text { Grain Size } \\
\text { Samples }\end{array}$ & $\begin{array}{c}\text { Grain Size } \\
\text { Sampling } \\
\text { Interval }\end{array}$ & $\begin{array}{l}\text { Lithology } \\
\text { Log }\end{array}$ & $\begin{array}{l}\text { Cone } \\
\text { Penetrometer } \\
\text { Sensor } \\
\text { Log }\end{array}$ & $\begin{array}{l}\text { Water } \\
\text { Level } \\
\text { Location }\end{array}$ \\
\hline \multicolumn{11}{|c|}{ New 2006 locations on and downgradient from the Co-op property } \\
\hline TI-13 & СРT & 6 & $25-71.8$ & 23 & $2-58.9$ & - & - & - & Yes & - \\
\hline TI-14 & СPT & 6 & $26-72.4$ & 24 & $2-61.25$ & - & - & Yes & Yes & - \\
\hline TI-15 & СРТ & 6 & $15-72.1$ & 20 & $1-60.75$ & - & - & - & - & - \\
\hline TI-16 & СРТ & 6 & 25-72 & 19 & $1.5-53$ & - & - & Yes & - & - \\
\hline $\mathrm{TI}-17$ & СРТ & 6 & $25-65$ & 17 & $2.25-56.5$ & - & - & Yes & - & - \\
\hline TI-18 & СРТ & 7 & $25-71.5$ & 13 & 5-61 & - & - & - & - & - \\
\hline TI-19 & СРТ & 7 & $25-69.5$ & 0 & - & - & - & - & - & - \\
\hline TI-20 & СРT & 4 & $35-77$ & 0 & - & - & - & - & - & - \\
\hline TI-21 & СРТ & 6 & $25-65$ & 0 & - & - & - & - & - & - \\
\hline TI-22 & СРТ & 6 & 32-73.2 & 0 & - & - & - & - & Yes & - \\
\hline TI-23 & СРТ & 6 & $32-71.8$ & 0 & - & - & - & - & Yes & - \\
\hline TI-24 & СРТ & 7 & $23-74.4$ & 0 & - & - & - & - & - & - \\
\hline TI-25 & СРТ & 7 & $32-77.2$ & 0 & - & - & - & - & - & - \\
\hline TI-26 & СРТ & 8 & $25-76.8$ & 0 & - & - & - & - & - & - \\
\hline TI-27 & СРТ & 7 & $25-71.2$ & 0 & - & - & - & - & - & - \\
\hline TI-28 & СРT & 7 & $25-68$ & 18 & $6-61.75$ & - & - & Yes & Yes & - \\
\hline TI-29 & СРТ & 7 & $25-69.3$ & 17 & $6-62$ & - & - & Yes & - & - \\
\hline TI-30/MW5 & CPT, $\mathrm{P}$ & 8 & $32-88.5$ & 0 & - & - & - & - & Yes & X (MW5) \\
\hline TI-31 & СРT & 4 & $42-75.7$ & 0 & - & - & - & - & - & \\
\hline
\end{tabular}

a Types: CPT, cone penetrometer; DW, domestic well; MW, monitoring well; P, piezometer.

b Total depth.

c Well installed at this location during 2006 investigation. 


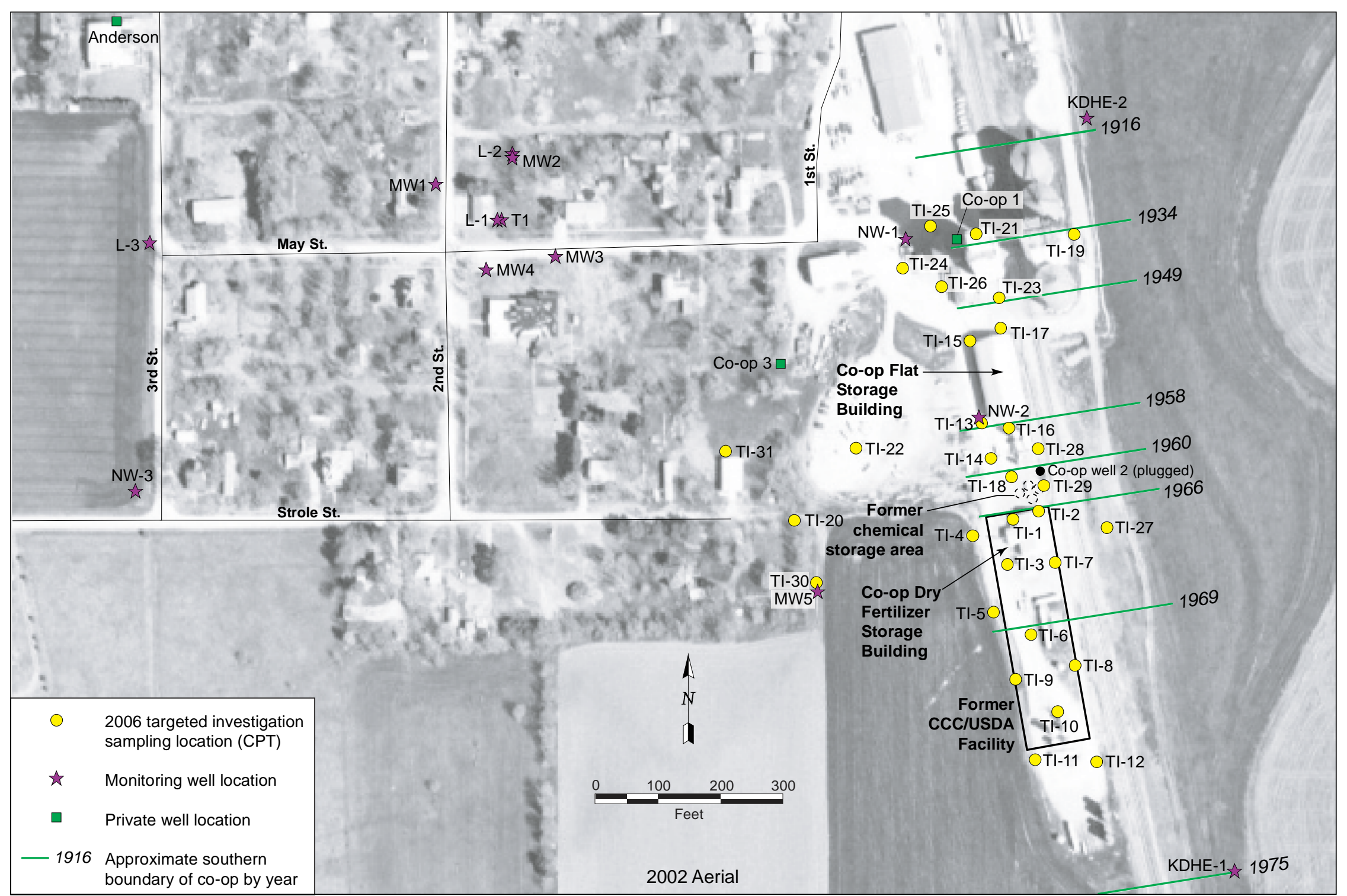




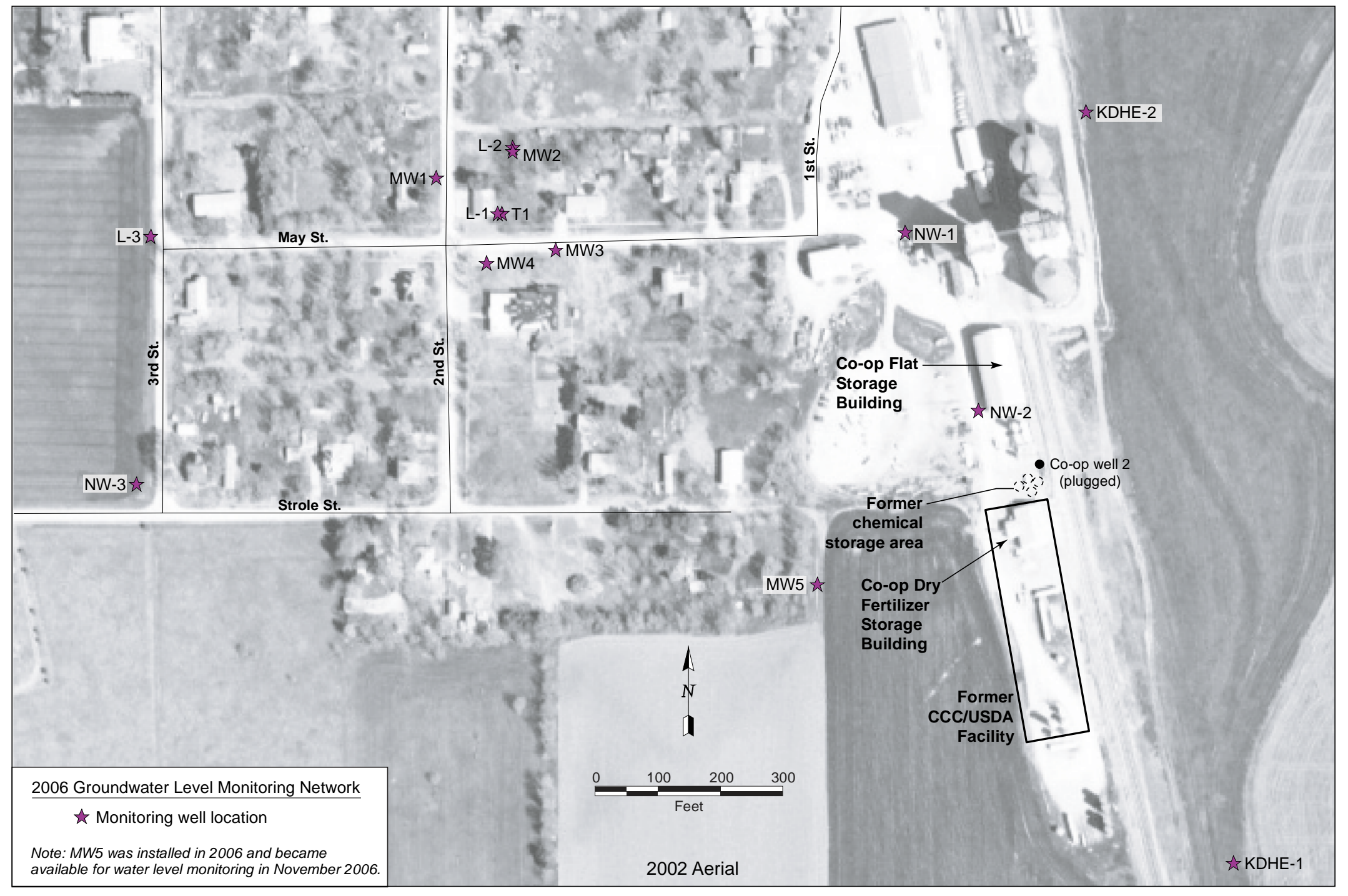

FIGURE 2.2 Groundwater level monitoring network. Source of photograph: NAIP (2002). 


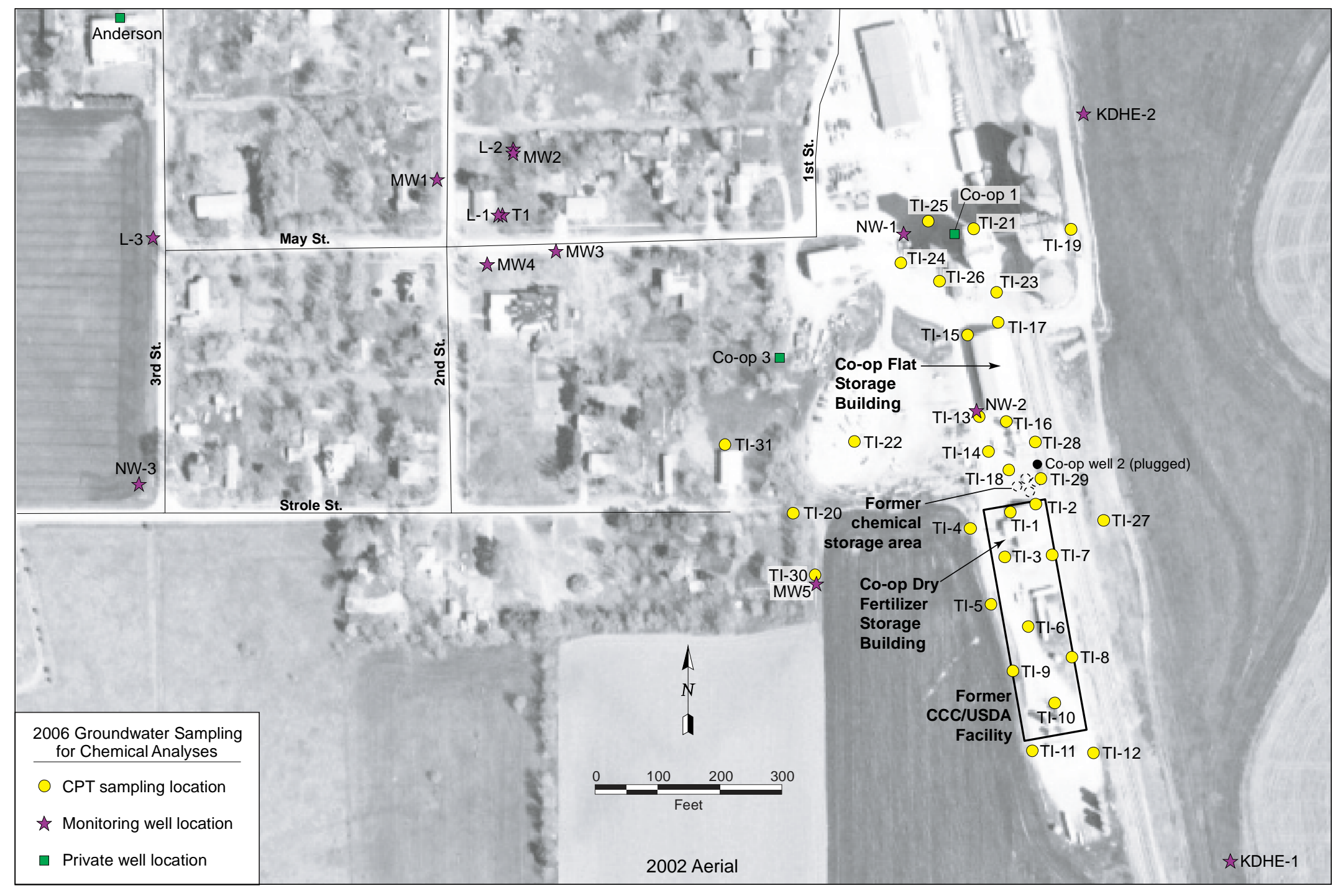

FIGURE 2.3 Groundwater sampling locations for chemical analyses. Source of photograph: NAIP (2002). 


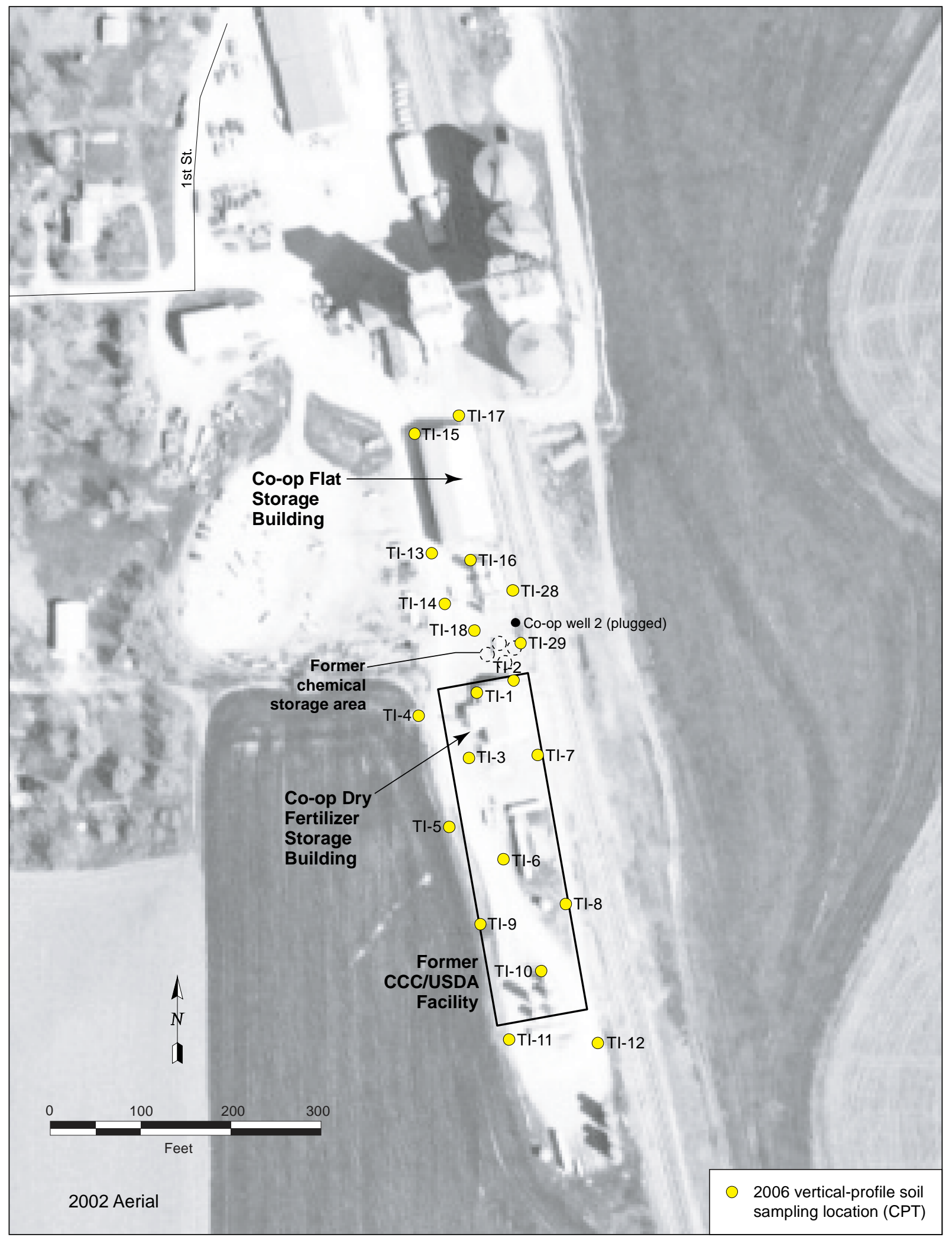

FIGURE 2.4 Vertical-profile soil sampling locations. Source of photograph: NAIP (2002). 


\section{Field and Laboratory Data}

This section presents the field and laboratory data generated during the Navarre investigation. The methods and procedures followed in collecting the data are described in detail in Section 2 of this document and in the Master Work Plan (Argonne 2002). A detailed interpretation of the data is in Section 4 of this document.

\subsection{Cone Penetrometer Sensor Data}

The CPT was used in an attempt to collect tip, sleeve, and (in some instances) conductance electronic sensor data from 17 boreholes (Figure 3.1). Electronic logs from some locations provided only limited data because of the type of lithology encountered. The electronic logs are in Appendix A. The investigated locations were distributed as follows:

- Eleven locations on and near the former CCC/USDA property (TI-1, TI-2, TI-3, TI-5, TI-6, TI-7, TI-8, TI-9, TI-10, TI-11, and TI-12).

- Four locations on or near the specific areas of concern on the Co-op property (TI-13, TI-14, TI-23, and TI-28).

- Two locations downgradient from the specific areas of concern (TI-22 and TI-30).

Measurements of tip and sleeve stresses were used to identify target zones for groundwater sampling and to estimate depth to bedrock. Tip stress is a measure of the resistance of the soil on the tip of the cone during penetration, and sleeve stress is a measure of the drag created along the sidewall of the cone. The ratio of tip stress to sleeve stress, called the friction ratio, is a tool for evaluating subsurface lithology. Because conductance data indicate the presence of water, these data are used to identify optimal intervals for water sampling.

The electronic sensor data collected during the Navarre investigation were limited by the type of lithology encountered. Zones of discontinuous consolidated material were encountered at various depths. This material limited cone penetration in several boreholes. Data from 4 locations (TI-2, TI-3, TI-6, and TI-9) were collected to depths ranging from approximately $51 \mathrm{ft}$ 
to $72.5 \mathrm{ft}$ BGL, and data from 7 locations (TI-1, TI-7, TI-8, TI-13, TI-22, TI-28, and TI-30) were collected to depths ranging from approximately $13.5 \mathrm{ft}$ to $24 \mathrm{ft}$ BGL. At 5 locations (TI-10, TI-11, TI-12, TI-14, and TI-23), pilot holes were used to obtain data at depths ranging from approximately $49.5 \mathrm{ft}$ to $72 \mathrm{ft}$ BGL.

The electronic sensor data did not indicate significant or distinct continuous zones of sand or gravel. This observation was supported by the soil cores collected from selected boreholes. Bedrock depths were determined by pushing Macro-Core ${ }^{\circledR}$ samplers until refusal was met. Depth to bedrock ranged from approximately $64 \mathrm{ft} \mathrm{BGL}$ in TI-3 to $88.5 \mathrm{ft}$ BGL in TI-30. To ensure that refusal was indicative of bedrock, confirmatory soil cores were collected to total depth from some boreholes.

\subsection{Piezometer Construction}

The CPT was used to install a permanent piezometer (sand point well), MW5 (Figure 2.2), at the TI-30 location, in accordance with Kansas Article 30 regulations. Piezometer MW5 was installed flush to the ground, to a depth of $88 \mathrm{ft}$ BGL. This location was chosen because of the depth achieved by the CPT. This is the deepest borehole completed during the 2006 investigation at Navarre. The construction diagram and the state well database registration for this piezometer are in Appendix B.

\subsection{Coordinates Survey Data}

The subsurface soil and groundwater sampling locations were surveyed by SchwabEaton, PA, of Manhattan, Kansas, to provide horizontal and vertical control for stratigraphic correlation and water level monitoring. Coordinates survey data are in Appendix C, Table C.1. A metal pin driven into the ground at each soil boring location was surveyed, and the top of the casing of each monitoring well was surveyed.

\subsection{Analytical Data for Subsurface Soil Samples}

A total of 382 subsurface soil samples were collected for VOCs analyses at $20 \mathrm{CPT}$ locations throughout the investigation area. The results for carbon tetrachloride and chloroform 
(maximum concentration at each location) are depicted in Figures 3.2 and 3.3. Results of all analyses for VOCs are in Supplement 1, Table S1.1. All supplements are on a compact disc (CD) inside the back cover of this report.

Discrete subsurface soil samples were collected from each borehole at approximately 4-ft intervals. The shallowest sample was collected at a depth of $2 \mathrm{ft}$ BGL, and the deepest was at $70.7 \mathrm{ft}$ BGL. The primary purpose was to investigate specific areas of concern for potential sources and migration pathways for carbon tetrachloride. Soil boring locations were chosen on the basis of analytical data from past investigations, as well as a review of areas where grain handling or chemical storage was thought to have occurred on the former CCC/USDA property or the Co-op property. Typically, grain- and chemical-handling activities are associated with doorways, equipment such as auger systems that transport grain from or to storage, and areas where maintenance or storage of equipment and chemicals occurred.

In addition to VOCs, selected soil samples from TI-2, TI-3, TI4, TI-6, TI-7, TI-12, TI-14, TI-16, and TI-29 were analyzed for particle size distribution and composition (Supplement 1, Table S1.2).

\subsubsection{Carbon Tetrachloride and Chloroform in Soils on and near the Former CCC/USDA Property}

On and near the former CCC/USDA property, 231 soil samples were collected from boreholes TI-1 through TI-12 (Figure 2.4). The samples were prepared and analyzed for VOCs by using U.S. Environmental Protection Agency (EPA) Methods 5030B and 8260B. The complete results of soil analyses for VOCs are in Supplement 1, Table S1.1. The results for these soil samples are summarized in Table 3.1 and in Figure 3.2 (carbon tetrachloride) and Figure 3.3 (chloroform). Table 3.1 and the other Section 3 data summary tables are grouped at the end of the section's text, before the figures.

Carbon tetrachloride was detected at or above the AGEM Laboratory method quantitation limit of $10 \mu \mathrm{g} / \mathrm{kg}$ in 20 soil samples from boreholes TI-1, TI-2, TI-3, TI-4, and TI-5. Trace levels (below the quantitation limit) were detected in 26 samples from TI-1, TI-2, TI-3, TI-4, TI-6, TI-7, and TI-9. All other soil samples collected at borehole locations on and near the southern boundary of the former CCC/USDA property, including all samples from boreholes TI-8, TI-10, 
TI-11, and TI-12, showed no detectable concentration of carbon tetrachloride (Figure 3.2 and Table 3.1).

Chloroform was detected at or above the AGEM Laboratory detection limit of $1.0 \mu \mathrm{g} / \mathrm{kg}$ in soil samples from boreholes TI-1, TI-2, TI-3, TI-4, TI-10, and TI-12 (Figure 3.3 and Table 3.1). Chloroform concentrations at or above the AGEM Laboratory method quantitation limit of $10 \mu \mathrm{g} / \mathrm{kg}$ were detected in 4 samples from TI-1 and 1 sample from TI-10.

No 1,2-DCA was detected above the AGEM Laboratory method quantitation limit of $10 \mu \mathrm{g} / \mathrm{kg}$ in soil samples from any boreholes on or near the former CCC/USDA property.

The highest carbon tetrachloride concentrations on or adjacent to the former CCC/USDA property occurred in the soil samples collected at locations TI-1 and TI-2 (Figure 3.2 and Table 3.1). These boreholes are along the northern boundary of the former CCC/USDA property, near the Co-op's dry fertilizer storage building. Nine of the 24 samples at TI-1 (collected at 31-47 ft BGL) contained carbon tetrachloride above the AGEM method quantitation limit of $10 \mu \mathrm{g} / \mathrm{kg}$, at concentrations ranging from $10 \mu \mathrm{g} / \mathrm{kg}$ at $47.5 \mathrm{ft}$ BGL to $56 \mu \mathrm{g} / \mathrm{kg}$ at $31 \mathrm{ft} \mathrm{BGL}$. At TI-2, 5 samples contained carbon tetrachloride at or above the AGEM method quantitation limit, at concentrations ranging from $12 \mu \mathrm{g} / \mathrm{kg}$ at $30 \mathrm{ft}$ BGL to $65 \mu \mathrm{g} / \mathrm{kg}$ at $38 \mathrm{ft}$ BGL. All of these concentrations are well below the Risk-Based Screening Level (RBSL) of $200 \mu \mathrm{g} / \mathrm{kg}$ for the soilto-groundwater protection pathway.

Chloroform was detected above the method quantitation limit in 4 soil samples collected from borehole TI-1 (at concentrations ranging from $10 \mu \mathrm{g} / \mathrm{kg}$ to $12 \mu \mathrm{g} / \mathrm{kg}$ ) and in 1 soil sample from borehole TI-10 (at $30 \mu \mathrm{g} / \mathrm{kg}$; Figure 3.3). Trace levels of carbon tetrachloride and chloroform were found in additional samples from both boreholes (Table 3.1).

\subsubsection{Carbon Tetrachloride and Chloroform in Soils on the Co-op Property}

On the Co-op property, 151 soil samples were collected from boreholes TI-13 through TI-18, TI-28, and TI-29 (Figure 2.4). These locations were at or near the specific areas of concern. The samples were prepared and analyzed for VOCs by using EPA Methods 5030B and 8260B. The complete results of analyses for VOCs in soil samples are in Supplement 1, 
Table S1.1. The results for the soil samples collected on the Co-op property are summarized in Table 3.2 and in Figure 3.2 (carbon tetrachloride) and Figure 3.3 (chloroform).

Carbon tetrachloride was detected at or above the AGEM Laboratory method quantitation limit of $10 \mu \mathrm{g} / \mathrm{kg}$ in 51 soil samples from boreholes TI-13 through TI-18, TI-28, and TI-29 (Figure 3.2 and Table 3.2). Ten of the samples from TI-13, TI-14, TI-16, TI-18, and TI-28 contained carbon tetrachloride concentrations above the RBSL of $200 \mu \mathrm{g} / \mathrm{kg}$. The concentrations above the RBSL ranged from $224 \mu \mathrm{g} / \mathrm{kg}$ to $1,094 \mu \mathrm{g} / \mathrm{kg}$ at depths of $22.5 \mathrm{ft}$ to $42 \mathrm{ft}$ BGL. Trace levels of carbon tetrachloride (below the method quantitation limit of $10 \mu \mathrm{g} / \mathrm{kg}$ ) were detected in 18 samples from the boreholes on the Co-op property. These 18 samples were distributed as follows: 3 samples from each of boreholes TI-13, TI-16, and TI-28; 2 samples from each of boreholes TI-14, TI-27, TI-18, and TI-29; and 1 sample from borehole TI-15. The other 82 soil samples from these boreholes contained no detectable concentrations of carbon tetrachloride.

Chloroform was found at trace to low concentrations (up to $107 \mu \mathrm{g} / \mathrm{kg}$ ) across the Co-op property, at TI-13 through TI-18, TI-28, and TI-29 (Figure 3.3). No 1,2-DCA was detected above the AGEM Laboratory method quantitation limit of $10 \mu \mathrm{g} / \mathrm{kg}$ in soil samples from any boreholes on the Co-op property.

The highest carbon tetrachloride concentration found in soil on the Co-op property (Table 3.2) occurred in a sample collected from the unsaturated zone at TI-28 $(1,094 \mu \mathrm{g} / \mathrm{kg}$ at $22.5 \mathrm{ft}$ BGL). Carbon tetrachloride was also detected above the method quantitation limit of $10 \mu \mathrm{g} / \mathrm{kg}$ in 5 additional samples from TI-28, at concentrations ranging from $18 \mu \mathrm{g} / \mathrm{kg}(20.5 \mathrm{ft}$ BGL) to $613 \mu \mathrm{g} / \mathrm{kg}$ (25.2 ft BGL). Trace levels of carbon tetrachloride and chloroform also occurred at this location.

Soil samples from boring TI-16 on the Co-op property had the second highest levels of carbon tetrachloride (concentrations of $23-454 \mu \mathrm{g} / \mathrm{kg}$, at depths of $28.8-45 \mathrm{ft} \mathrm{BGL}$ ).

\subsection{Groundwater Analytical Data}

A total of 196 groundwater samples were collected during the field activities. Of these, 18 samples were from 13 existing monitoring wells and 3 private wells. Historical data regarding the permanent monitoring points are summarized in Table D.1, Appendix D. The remaining 178 
groundwater samples were collected at $31 \mathrm{CPT}$ investigation locations, at discrete depths between $15 \mathrm{ft}$ and $88.5 \mathrm{ft}$ BGL.

Groundwater sampling locations are shown in Figure 2.3. Sample descriptions are on CD in Supplement 2, Table S2.1. Results of field parameter measurements on the samples are in Table S2.2. Results for VOCs are in Table S2.3, and tritium values are in Table S2.4.

Samples were collected from wells to estimate the extent of carbon tetrachloride contamination in groundwater in the general investigation area. Groundwater profiling at CPT locations was conducted to identify source areas and migration pathways for the carbon tetrachloride contamination. Locations for groundwater sampling with the CPT were selected on the basis of analytical data from past investigations and a review of areas where grain handling or chemical storage was thought to have occurred on the former CCC/USDA property and the Co-op property. In addition, several groundwater samples were collected at CPT locations elsewhere in the general investigation area to evaluate the extent of the contamination.

\subsubsection{Volatile Organic Compounds in Groundwater on and near the Former CCC/USDA Property}

Fifty-seven groundwater samples were collected at boreholes TI-1 through TI-12 on and near the former CCC/USDA property (Figure 2.3), at depths from $25 \mathrm{ft}$ to $77.8 \mathrm{ft}$ BGL. All samples were analyzed for VOCs by using EPA Method 524.2. Complete results for VOCs are in Table S2.3 in Supplement 2. Table 3.3 summarizes the VOCs results for groundwater samples collected on and adjacent to the former CCC/USDA property. Figure 3.4 illustrates the maximum carbon tetrachloride concentration detected in groundwater at all locations. Figures 3.5 and 3.6 show chloroform and tetrachloroethene concentrations in groundwater, respectively.

Carbon tetrachloride was detected at or above the AGEM Laboratory method quantitation limit of $1.0 \mu \mathrm{g} / \mathrm{L}$ in 26 groundwater samples from locations TI-1 through TI-7 and TI-9 (Figure 3.4 and Table 3.3). In addition, trace levels of carbon tetrachloride (below the AGEM Laboratory method quantitation limit) were detected in 15 groundwater samples from locations TI-4 and TI-6 through TI-11. No carbon tetrachloride was detected in the 5 samples collected at location TI-12. Carbon tetrachloride levels at TI-1, TI-2, TI-3, TI-4, TI-5, and TI-7 exceeded the RBSL and maximum contaminant level (MCL) of $5.0 \mu \mathrm{g} / \mathrm{L}$. 
The highest carbon tetrachloride concentrations found in groundwater on and adjacent to the former CCC/USDA property occurred in samples collected at TI-1, TI-2, TI-3, and TI-4. Three samples collected at TI-1 contained concentrations of $127-181 \mu \mathrm{g} / \mathrm{L}$ at $30-55 \mathrm{ft}$ BGL. Five samples from TI-2 contained concentrations of 2.2-77 $\mu \mathrm{g} / \mathrm{L}$ at 25-70 ft BGL. Three samples from TI-3 contained concentrations of $7.6-72 \mu \mathrm{g} / \mathrm{L}$ at $32.2-56 \mathrm{ft}$ BGL. Four samples from TI-4 contained quantifiable concentrations of $23-99 \mu \mathrm{g} / \mathrm{L}$ at $35-60 \mathrm{ft} \mathrm{BGL}$, and a trace concentration was found in a fifth sample collected at 61-66 ft BGL.

Sample locations TI-1 and TI-2 are along the northern boundary of the former CCC/USDA property (Figure 3.4). Location TI-3 is on the southwest corner of the Co-op's dry fertilizer building. Location TI-4 is near the northwest corner of the former CCC/USDA property, in a low area that collects drainage from the Co-op property to the north, the former $\mathrm{CCC} / \mathrm{USDA}$ property to the south, and an agricultural field to the southwest.

Chloroform was detected at or above the method quantitation limit of $1.0 \mu \mathrm{g} / \mathrm{L}$ in 23 groundwater samples from locations TI-1 through TI-7, as well as at trace levels in 5 samples from TI-5, TI-6, TI-7, and TI-11. All of the chloroform concentrations were below the RBSL and MCL of $80 \mu \mathrm{g} / \mathrm{L}$ for this compound (Figure 3.5 and Table 3.3).

All five groundwater samples from location TI-12, just south of the former CCC/USDA property, contained no detectable concentrations of carbon tetrachloride or chloroform. 1,2-Dichloroethane was not found in any groundwater sample collected on or near the former $\mathrm{CCC} / \mathrm{USDA}$ property.

The only other VOCs detected on or near the former CCC/USDA property were methylene chloride, tetrachloroethene, benzene, and toluene. Methylene chloride was detected in samples collected at TI-1, TI-4, TI-10, and TI-11, at concentrations ranging from a trace (below the method quantitation limit of $1.0 \mu \mathrm{g} / \mathrm{L}$ ) to $36 \mu \mathrm{g} / \mathrm{L}$ (Table 3.3). The MCL and RBSL for methylene chloride are both $5.0 \mu \mathrm{g} / \mathrm{L}$. Tetrachloroethene was detected at trace levels in 2 samples from TI-1, 1 sample from TI-2, and 3 samples from TI-4 (Figure 3.6). These CPT locations are along or near the northern border between the former CCC/USDA and Co-op properties. 
Benzene and toluene were detected in 4 samples from TI-10 and 1 sample from TI-11 (Table S2.3, Supplement 3). Both of these locations are adjacent to active aboveground storage tanks (ASTs) that are owned and operated by the Co-op.

\subsubsection{Volatile Organic Compounds in Groundwater on the Co-op Property}

On the Co-op property, 105 groundwater samples were collected at boreholes TI-13 through TI-19 and TI-21 through TI-29 (Figure 2.3). Sampling at these locations occurred at discrete depths between $15 \mathrm{ft}$ and $77.2 \mathrm{ft}$ BGL. Groundwater samples were also collected from 5 wells on the Co-op property: private wells Co-op 1 and Co-op 3 and existing monitoring wells KDHE-2, NW-1, and NW-2 (Figure 2.3). All samples were analyzed for VOCs by using EPA Method 524.2. Complete analytical results are in Supplement 2, Table S2.3. Table 3.4 summarizes the results of VOCs analyses on groundwater samples collected on the Co-op property. Figure 3.4 shows the maximum carbon tetrachloride concentration detected in groundwater at each location. Figures 3.5 and 3.6 show maximum chloroform and tetrachloroethene concentrations in groundwater, respectively.

Carbon tetrachloride was detected at or above the AGEM Laboratory method quantitation limit of $1.0 \mu \mathrm{g} / \mathrm{L}$ in the 4 groundwater samples from wells Co-op-1, Co-op-3, NW-1, and NW-2, as well as in 70 groundwater samples from boreholes TI-13 through TI-18 and TI-21 through TI-29 (Table 3.4 and Figure 3.4). Trace levels (below the method quantitation limit) were detected in 11 additional samples from boreholes TI-13, TI-16, TI-17, TI-19, TI-21, and TI-25. Carbon tetrachloride at or above the MCL (and the RBSL) of $5.0 \mu \mathrm{g} / \mathrm{L}$ was detected in the 2 samples from wells Co-op-3 and NW-2 and in 47 samples from boreholes TI-13 through TI-18, TI-22, TI-23, and TI-26 through TI-29.

Chloroform was detected at or above the AGEM Laboratory method quantitation limit of $1.0 \mu \mathrm{g} / \mathrm{L}$ in 63 samples from all CPT locations on the Co-op property, except for TI-19 and TI-21 (Table 3.4 and Figure 3.5). Trace concentrations of chloroform (below the quantitation limit) were found in 21 additional samples from well Co-op-1 and NW-1 and from boreholes TI-13, TI-16, TI-17, TI-18, TI-21, TI-23, TI-24, TI-25, TI-26, and TI-27. Chloroform at concentrations above the RBSL of $80 \mu \mathrm{g} / \mathrm{L}$ was detected in 13 samples from boreholes TI-13, TI-14, TI-16, TI-18, TI-28, and TI-29. 
The highest maximum carbon tetrachloride concentrations in groundwater samples from the Co-op property occurred at locations TI-13, TI-14, TI-16, TI-18, TI-22, TI-28, and TI-29, most often at depths of approximately $40 \mathrm{ft}$ BGL (except for TI-22, where the maximum concentration in groundwater occurred approximately $15 \mathrm{ft}$ deeper). The highest concentrations of carbon tetrachloride in soil also occurred at TI-13, TI-14, TI-16, TI-18, TI-28, and TI-29. No soil samples were collected at TI-22. These correlations are shown in Figure 3.7.

The only other VOCs detected in groundwater samples collected on the Co-op property were tetrachloroethene and methylene chloride (Table 3.4 and Figure 3.6). Tetrachloroethene was detected at trace concentrations (below the method quantitation limit of $1.0 \mu \mathrm{g} / \mathrm{L}$ ) in samples from 2 monitoring wells (Co-op 3 and NW-2). Tetrachloroethene was also detected at 6 CPT locations on the Co-op property (TI-13, TI-14, TI-16, TI-18, TI-22, and TI-28). Concentrations were at the trace level except at TI-28, where the highest concentration was $3.1 \mu \mathrm{g} / \mathrm{L}$. Methylene chloride was detected in samples collected from the same two monitoring wells (Co-op 3 and NW-2) and 8 at CPT locations on the Co-op property (TI-13, TI-14, TI-16, TI-18, TI-22, TI-27, TI-28, and TI-29). The highest concentration (12.6 $\mu \mathrm{g} / \mathrm{L})$ was detected at TI-14. The MCL and RBSL values for tetrachloroethene and methylene chloride are $5.0 \mu \mathrm{g} / \mathrm{L}$.

\subsubsection{Volatile Organic Compounds in Groundwater in Areas Downgradient or Upgradient from the Target Areas}

The CPT was used to collect 16 groundwater samples downgradient from the former CCC/USDA property and the Co-op property, at locations TI-20, TI-30/MW5, and TI-31. In addition, 14 groundwater samples were collected from 11 monitoring wells and 1 privately owned water well (Anderson) outside the former CCC/USDA property and the Co-op property. Table 3.5 summarizes the distribution of VOCs in these samples. The results provide additional information concerning the extent and concentrations of VOCs contamination in the groundwater plume in the general investigation area. Carbon tetrachloride concentrations at these locations are displayed in Figure 3.4, chloroform in Figure 3.5, and tetrachloroethene in Figure 3.6. Methylene chloride was not detected in any of the downgradient or upgradient samples.

Groundwater samples from the 3 CPT boreholes downgradient from (west of) the Co-op and CCC/USDA properties contained carbon tetrachloride at levels above the AGEM Laboratory method quantitation limit of $1.0 \mu \mathrm{g} / \mathrm{L}$. The highest contaminant levels were in boreholes TI-30 and TI-31, with concentrations in 2 samples from TI-30 and 3 samples from TI-31 exceeding the 
RBSL of $5 \mu \mathrm{g} / \mathrm{L}$ for carbon tetrachloride. In addition, 3 samples from TI-20 exceeded the RBSL of $5 \mu \mathrm{g} / \mathrm{L}$ for carbon tetrachloride, though the concentrations were lower.

The downgradient private well (Anderson) and 10 monitoring wells (T1, MW1, MW2, MW3, MW4, MW5, NW-3, L-1, L-2, and L-3) discussed here are located downgradient (west) of the Co-op property and northwest of the former CCC/USDA property; the upgradient monitoring well (KDHE-1) is southeast of the former CCC/USDA property (Figure 2.3).

Carbon tetrachloride was detected at concentrations above the RBSL of $5.0 \mu \mathrm{g} / \mathrm{L}$ in 7 of the off-site monitoring wells (T1, MW1, MW2, MW3, MW4, NW-3, and L-1) and in the private well. The other four monitoring wells (MW5, KDHE-1, L-2, and L-3) exhibited trace to nondetectable levels of carbon tetrachloride (Figure 3.4) and no detectable chloroform (Figure 3.5).

\subsection{Groundwater Gradient Data}

The direction of groundwater flow in the general investigation area was historically interpreted to be toward the northwest. The groundwater flow direction and contaminant migration (both currently and in the past) may be influenced, however, by the use of private wells to the west and northwest of the former CCC/USDA property and the Co-op property.

During the 2006 investigation, groundwater levels in monitoring wells and piezometers were initially measured by hand on April 24-25, 2006. Manual measurements were also made on May 11, 2006, November 3, 2006 (partial set), and November 28, 2006. The levels were measured (with an electronic meter) to the nearest $0.01 \mathrm{ft}$ from a surveyed reference point (the top of the casing in each well). Results are in Supplement 3, Table S3.1.

In April and May 2006, Argonne placed downhole pressure sensors with automatic data loggers in monitoring wells KDHE-1, KDHE-2, T1, MW1, MW2, MW3, MW4, NW-1, NW-2, NW-3, L-1, L-2, and L-3. The loggers record water levels at 4-hr intervals. The complete set of water levels recorded by the data loggers in April to November 2006 is in Supplement 3, Table S3.2. 
The data show that the hydraulic gradient did not vary significantly over the period of record, and indicate a predominant direction of groundwater flow toward the west-northwest. The addition of a (hand measured) groundwater depth measurement from MW5 for the first time on November 28, 2006, resulted in a reduced hydraulic gradient and an apparent flow direction that is slightly more toward the northwest in the area directly west of the former CCC/USDA property. The overall results are consistent with the previously determined northwesterly flow direction. Hydrographs developed from the data logger records indicate periodic drawdown of the water levels at well L-3 and in several additional monitoring wells. The cause of this drawdown is unknown. A discussion of the hydrogeology, along with groundwater flow maps and hydrographs, is in Section 4.1.2.

\subsection{Results of Quality Control Activities}

The QA/QC procedures for sample collection, handling, and analysis during the Navarre investigation are described in detail in the Master Work Plan (Argonne 2002) and the sitespecific work plan (Argonne 2006). A detailed QA/QC report addressing activities related to sample collection, handling, and analysis during the investigation is in Supplement 4 (on CD).

Results of QA/QC activities are summarized as follows:

- Sample integrity was maintained successfully throughout the collection, shipping, and analysis activities by documentation of samples as they were collected and the use of custody seals and chain-of-custody records. Chain-ofcustody records are in Supplement 5 (on CD).

- All samples were received with custody seals intact and at appropriate preservation conditions. With the exception of one trip blank that was broken during shipment, all samples were analyzed within required holding times. Carbon tetrachloride and chloroform were not detected in laboratory method blanks. Methylene chloride was present at trace concentrations in the methanol used for extraction of the soil samples. Detection of methylene chloride at similar concentrations in the soil samples is not reported. 
- As an indicator of cross-contamination of samples during shipment, 58 trip blanks were prepared and packed with soil or water samples shipped for organic analysis. Analytical results indicate that, overall, sample handling procedures were followed during the 2006 investigation; however, one trip blank broke during shipment. Because the trip blank was broken and not analyzed, three groundwater samples from location TI-27 with low levels of carbon tetrachloride reported $(1.1-7.9 \mu \mathrm{g} / \mathrm{L})$ are qualified to reflect the potential for cross-contamination during shipment.

- One field blank was collected to represent water used during equipment decontamination. Carbon tetrachloride and chloroform, the contaminants of primary concern in the investigation, were not detected in the field blank.

- To monitor decontamination procedures for reusable sampling equipment, 28 equipment rinsates were collected. Neither carbon tetrachloride nor chloroform was detected in the rinsate samples at concentrations above the method quantitation limit, indicating that cross-contamination of groundwater samples did not occur during sample collection. Trace concentrations of carbon tetrachloride $(0.1-0.8 \mu \mathrm{g} / \mathrm{L})$ were detected in three rinsate samples collected from the decontaminated sampling equipment following collection of groundwater samples with high levels $(118-260 \mu \mathrm{g} / \mathrm{L})$ of the contaminant present. These trace-level detections indicate that the decontamination procedures during the 2006 field investigation were good.

- One groundwater sample was rejected as non-representative of site conditions and was re-collected. During initial sampling at location TI-20 from a depth interval of $42-47 \mathrm{ft}$ BGL, very limited water was available for collection. After an overnight wait, sufficient water was available to collect the needed aliquots. The initial sample was rejected and is not included in the investigation data generated.

- Soil and groundwater samples were analyzed for VOCs at the AGEM Laboratory by using the purge-and-trap method. Dual analyses of samples were conducted as a measure of consistency in the sampling and analytical methodologies. The dual analyses were accomplished through analysis of 
replicate samples submitted to the laboratory or duplicate analyses of samples selected by the laboratory. Consistency in both the sampling and analytical methodologies is indicated by the average relative percent difference (RPD) values of $14.3 \%$ for carbon tetrachloride, $11.3 \%$ for chloroform, and $17.9 \%$ for methylene chloride in the dual analyses with the contaminants present. The data from the AGEM Laboratory are acceptable for quantitative determination of contaminant distribution.

- The analyses of water samples at the AGEM Laboratory by EPA Method 524.2 were verified at a second laboratory using EPA-defined Contract Laboratory Program (CLP) methodology. Of the 196 groundwater samples analyzed at the AGEM Laboratory, 21 samples (10.7\%) were also analyzed according to CLP methodology by Envirosystems, Inc., in Columbia, Maryland. Agreement was good over the range of contaminant concentrations detected. Samples analyzed at the AGEM Laboratory with no detection of contamination were analyzed by the CLP laboratory with similar results. Because of the higher quantitation limit for the CLP analysis of $5.0 \mu \mathrm{g} / \mathrm{L}$, very low concentrations detected by purge-and-trap analysis were sometimes not detected by the CLP analysis. For samples with contaminant concentrations above the purge-and-trap quantitation limit of $1.0 \mu \mathrm{g} / \mathrm{L}$, the average RPD values between the two laboratories were $20.5 \%$ for carbon tetrachloride and $16.3 \%$ for chloroform. Significant concentrations of methylene chloride (indicative of biodegradation of carbon tetrachloride to chloroform and then to methylene chloride) were confirmed by the CLP analysis. The Envirosystems data are in Supplement 5 (on CD).

- The analyses of soil samples at the AGEM Laboratory with EPA Method $8260 \mathrm{~B}$ were verified at a second laboratory using the same analytical method. Of the 382 vertical-profile soil samples analyzed at the AGEM Laboratory, 34 samples $(8.9 \%)$ were also analyzed by Severn-Trent Laboratories, Inc., in Colchester, Vermont. Agreement was good over the range of contaminant concentrations detected. Soil samples analyzed at the AGEM Laboratory with no detection of contamination were analyzed by Severn-Trent with similar results. For samples in which contamination was detected, the average RPD 
values between the two laboratories were $56.2 \%$ for carbon tetrachloride $28.3 \%$ for chloroform. The Severn-Trent data are in Supplement 5 (on CD).

- For the tritium analyses of groundwater samples at the University of Miami Tritium Laboratory in Miami, Florida, the instrument was calibrated with a standard, and dual analyses of samples gave comparable results. The data are acceptable for age dating of groundwaters.

- Soil samples selected for particle size analysis at the HWS Laboratory in Lincoln, Nebraska, were analyzed in accordance with ASTM D422-63 (2002), "Standard Test Method for Particle-Size Analysis of Soils" (ASTM International, http://www.astm.org). The distribution of particle sizes larger than $75 \mu \mathrm{m}$ was determined by sieving, while the distribution of smaller particle sizes was determined by a sedimentation process using a hydrometer. The data are suitable for use in evaluation of site lithology.

\subsection{Waste Characterization, Handling, and Disposal}

Wastewater generated by field activities was stored in polyurethane containers and aerated. Neither carbon tetrachloride nor chloroform was not detected above the method detection limit of $1.0 \mu \mathrm{g} / \mathrm{L}$ (Supplement 6 [on CD]). The water was discharged on-site, with the approval of the KDHE.

\subsection{Property Documentation}

Property documentation for the grain storage areas at Navarre is in Supplement 7 (on CD).

\subsection{Summary of Analytical Data}

The data collected during this investigation address the objectives detailed in the KDHEapproved site-specific work plan (Argonne 2006). Key results of the investigation are summarized below. 


\subsubsection{Former CCC/USDA Property}

\subsubsection{Soil Data for the Former CCC/USDA Property}

- Carbon tetrachloride was detected - at or above the AGEM Laboratory method quantitation limit of $10 \mu \mathrm{g} / \mathrm{kg}$ — in 20 of the 231 soil samples collected (at 5 of the 12 locations sampled) on or near the former CCC/USDA property. The highest carbon tetrachloride concentrations in these soil samples occurred at location TI-1 $(56 \mu \mathrm{g} / \mathrm{kg}$ at $31 \mathrm{ft}$ BGL, just above the saturated zone), along the northern border between the former CCC/USDA property and the Co-op property. Other soil samples that contained carbon tetrachloride above the method quantitation limit were at depths just above or within the saturated zone. The only exception was at TI-4, where groundwater was encountered at about $35 \mathrm{ft}$ BGL, and carbon tetrachloride was detected in soil samples from depths of $20.5 \mathrm{ft} \mathrm{BGL}$ and $25.0 \mathrm{ft}$ BGL.

- Contaminant levels in all of the soil samples collected on or near the former CCC/USDA property were below the RBSL values for the soil-togroundwater protection pathway of $200 \mu \mathrm{g} / \mathrm{kg}$ for carbon tetrachloride and $960 \mu \mathrm{g} / \mathrm{kg}$ for chloroform.

\subsubsection{Groundwater Data for the Former CCC/USDA Property}

- Carbon tetrachloride was detected at or above the RBSL of $5.0 \mu \mathrm{g} / \mathrm{L}$ in 16 of the 57 groundwater samples collected (at 6 of 12 locations sampled) on or near the former CCC/USDA property. The highest concentration $(181 \mu \mathrm{g} / \mathrm{L}$ at 30-35 ft BGL) was detected at location TI-1.

- Chloroform was detected at or above the method quantitation limit of $1.0 \mu \mathrm{g} / \mathrm{L}$ in 23 groundwater samples from locations TI-1 through TI-7; however, all of the chloroform concentrations were below the RBSL of $80 \mu \mathrm{g} / \mathrm{L}$ for this compound. 
- Tetrachloroethene was detected at trace levels in groundwater samples collected at TI-1, TI-2, and TI-4. None of the samples exceeded the RBSL of $5.0 \mu \mathrm{g} / \mathrm{L}$ for this compound in groundwater.

- Methylene chloride was detected at concentrations above the RBSL of $5.0 \mu \mathrm{g} / \mathrm{L}$ in 7 groundwater samples from locations TI-1, TI-4, TI-10, and TI-11. The highest concentration, $36 \mu \mathrm{g} / \mathrm{L}$, occurred in the sample collected at 40-45 ft BGL at location TI-10.

- Benzene was detected above the RBSL of $5 \mu \mathrm{g} / \mathrm{L}$ in 3 groundwater samples collected at locations TI-10 and TI-11. The highest concentration, $41 \mu \mathrm{g} / \mathrm{L}$, occurred in the sample collected at 33-38 ft BGL at location TI-11. Benzene is often found near petroleum storage facilities. The Co-op has active ASTs near TI-10 and TI-11. This location exhibited high concentrations of several VOCs that are typically associated with petroleum products.

- Toluene was detected in 4 groundwater samples from TI-10 and TI-11. The highest concentration was $27 \mu \mathrm{g} / \mathrm{L}$ in the sample collected at $40-45 \mathrm{ft}$ BGL from TI-10. None of the samples had concentrations exceeding the RBSL. Toluene is often found near petroleum storage facilities. The Co-op has active ASTs near TI-10 and TI-11.

\subsubsection{Co-op Property}

\subsubsection{Soil Data for the Co-op Property}

- Carbon tetrachloride was detected at or above the AGEM Laboratory method quantitation limit of $10 \mu \mathrm{g} / \mathrm{kg}$ in 51 of the 151 soil samples collected at all 8 locations investigated on the Co-op property. The maximum concentration was $1,094 \mu \mathrm{g} / \mathrm{kg}$ at $22.5 \mathrm{ft}$ BGL at location TI-28.

- Carbon tetrachloride was detected above the RBSL of $200 \mu \mathrm{g} / \mathrm{kg}$ in 10 of the 151 soil samples collected on the Co-op property (in 5 of the 8 locations investigated). The highest concentrations were detected near the south door of 
the flat storage building, the former well Co-op 2, and the former chemical storage area.

- Chloroform was found in soil at trace to low concentrations across the Co-op property, at all 8 locations investigated. None of the concentrations exceeded the RBSL value of $960 \mu \mathrm{g} / \mathrm{kg}$ for the soil-to-groundwater protection pathway.

\subsubsection{Groundwater Data for the Co-op Property}

- Carbon tetrachloride was detected at or above the RBSL of $5.0 \mu \mathrm{g} / \mathrm{L}$ in 47 of the 105 groundwater samples collected with the CPT on the Co-op property (at 12 of 16 locations investigated) and in 2 of the 5 wells sampled on the Co-op property $(197 \mu \mathrm{g} / \mathrm{L}$ in well Co-op 3 and $313 \mu \mathrm{g} / \mathrm{L}$ in well NW-2). The highest concentrations in groundwater occurred near the south door of the flat storage building, the former well Co-op 2, and the former chemical storage area.

- Chloroform was detected above the RBSL of $80 \mu \mathrm{g} / \mathrm{L}$ in 13 groundwater samples from boreholes TI-13, TI-14, TI-16, TI-18, TI-28, and TI-29. (There is no MCL for chloroform.) The highest concentrations occurred in samples collected near the south door of the flat storage building, former well Co-op 2, and the former chemical storage area: $535 \mu \mathrm{g} / \mathrm{L}$ at TI-16 (25-37 ft BGL) and $646 \mu \mathrm{g} / \mathrm{L}$ at TI-28 (32-37 ft BGL).

- Tetrachloroethene was detected at trace to low levels in samples collected from wells Co-op 3 and NW-2, as well as at CPT locations TI-13, TI-14, TI-16, TI-18, TI-22, and TI-28. None of the concentrations exceeded the RBSL of $5.0 \mu \mathrm{g} / \mathrm{L}$ for groundwater. The highest concentration was detected at TI-28 $(3.1 \mu \mathrm{g} / \mathrm{L}$ at 32-37 ft BGL), which is near the former well Co-op 2 and the former chemical storage area.

- Methylene chloride was detected above the RBSL of $5.0 \mu \mathrm{g} / \mathrm{L}$ in 6 groundwater samples from TI-14, TI-16, TI-18, and TI-28 on the Co-op property. The highest concentration was $12.6 \mu \mathrm{g} / \mathrm{L}$ in the sample collected at 
67.4-72.4 ft BGL at location TI-14, which is near the former chemical storage area. 
TABLE 3.1 Summary of organic analytical results for soil samples collected the former CCC/USDA facility during the 2006 investigation at Navarre, Kansas.

\begin{tabular}{|c|c|c|c|c|c|c|}
\hline \multirow[b]{2}{*}{ Location } & \multirow[b]{2}{*}{$\begin{array}{c}\text { Sample } \\
\text { Date }\end{array}$} & \multirow[b]{2}{*}{$\begin{array}{l}\text { Depth } \\
\text { (ft BGL) }\end{array}$} & \multirow[b]{2}{*}{$\begin{array}{c}\text { Number of } \\
\text { Samples }\end{array}$} & \multicolumn{3}{|c|}{ Concentration $(\mu \mathrm{g} / \mathrm{kg})$} \\
\hline & & & & $\begin{array}{c}\text { Carbon } \\
\text { Tetra- } \\
\text { chloride }\end{array}$ & $\begin{array}{l}\text { Chloro- } \\
\text { form }\end{array}$ & $\begin{array}{l}\text { Methylene } \\
\text { Chloride }\end{array}$ \\
\hline $\mathrm{Tl}-1$ & $4 / 7 / 06$ & $2.0-9.0$ & 4 & $\mathrm{ND}^{\mathrm{a}}$ & ND & ND \\
\hline $\mathrm{TI}-1$ & $4 / 7 / 06$ & 18.0 & 1 & ND & $1.0 \mathrm{~J}^{\mathrm{b}}$ & ND \\
\hline $\mathrm{TI}-1$ & $4 / 8 / 06-4 / 11 / 06$ & $19.2-20.5$ & 2 & ND & ND & ND \\
\hline $\mathrm{TI}-1$ & $4 / 11 / 06$ & 21.5 & 1 & $0.9 \mathrm{~J}$ & ND & ND \\
\hline $\mathrm{Tl}-1$ & $4 / 11 / 06$ & 23.8 & 1 & $1.3 \mathrm{~J}$ & ND & ND \\
\hline $\mathrm{TI}-1$ & $4 / 8 / 06$ & 31.0 & 1 & 56 & $3.7 \mathrm{~J}$ & ND \\
\hline $\mathrm{TI}-1$ & $4 / 13 / 06$ & 32.0 & 1 & 23 & $1.6 \mathrm{~J}$ & ND \\
\hline TI-1 & $4 / 13 / 06$ & 32.7 & 1 & 35 & 12 & ND \\
\hline $\mathrm{TI}-1$ & $4 / 13 / 06$ & 34.5 & 1 & $3.0 \mathrm{~J}$ & 11 & ND \\
\hline $\mathrm{TI}-1$ & $4 / 13 / 06$ & 36.0 & 1 & 48 & 10 & ND \\
\hline $\mathrm{TI}-1$ & $4 / 13 / 06$ & 38.5 & 1 & 37 & $3.8 \mathrm{~J}$ & ND \\
\hline $\mathrm{TI}-1$ & $4 / 13 / 06$ & 39.5 & 1 & 41 & $3.5 \mathrm{~J}$ & ND \\
\hline $\mathrm{TI}-1$ & $4 / 13 / 06$ & 42.5 & 1 & 44 & 10 & ND \\
\hline $\mathrm{TI}-1$ & $4 / 13 / 06$ & 44.0 & 1 & $3.3 \mathrm{~J}$ & $2.6 \mathrm{~J}$ & ND \\
\hline TI-1 & $4 / 13 / 06$ & 46.5 & 1 & 15 & $4.1 \mathrm{~J}$ & ND \\
\hline $\mathrm{TI}-1$ & $4 / 13 / 06$ & 47.5 & 1 & 10 & $2.3 \mathrm{~J}$ & ND \\
\hline $\mathrm{TI}-1$ & $4 / 13 / 06$ & 51.0 & 1 & $1.4 \mathrm{~J}$ & ND & ND \\
\hline TI-1 & $4 / 13 / 06$ & 55.0 & 1 & $1.8 \mathrm{~J}$ & ND & ND \\
\hline TI-1 & $4 / 13 / 06$ & 58.5 & 1 & $3.2 \mathrm{~J}$ & $2.2 \mathrm{~J}$ & ND \\
\hline TI-1 & $4 / 13 / 06$ & 59.5 & 1 & ND & ND & ND \\
\hline TI-2 & $4 / 5 / 06-4 / 6 / 06$ & $2.0-24.5$ & 8 & ND & ND & ND \\
\hline TI-2 & $4 / 6 / 06$ & 28.7 & 1 & $7.0 \mathrm{~J}$ & $1.5 \mathrm{~J}$ & ND \\
\hline TI-2 & $4 / 6 / 06$ & 30.0 & 1 & 12 & $6.8 \mathrm{~J}$ & ND \\
\hline TI-2 & $4 / 6 / 06$ & 32.0 & 1 & ND & $2.2 \mathrm{~J}$ & ND \\
\hline TI-2 & $4 / 6 / 06$ & 34.0 & 1 & 13 & $6.1 \mathrm{~J}$ & ND \\
\hline TI-2 & $4 / 6 / 06$ & 38.0 & 1 & 65 & $3.9 \mathrm{~J}$ & ND \\
\hline TI-2 & $4 / 6 / 06$ & 40.0 & 1 & 15 & $4.4 \mathrm{~J}$ & ND \\
\hline TI-2 & $4 / 6 / 06$ & 42.0 & 1 & $2.7 \mathrm{~J}$ & ND & ND \\
\hline TI-2 & $4 / 6 / 06$ & 46.0 & 1 & $0.9 \mathrm{~J}$ & $1.9 \mathrm{~J}$ & ND \\
\hline TI-2 & $4 / 6 / 06$ & 50.0 & 1 & $7.3 \mathrm{~J}$ & $1.3 \mathrm{~J}$ & ND \\
\hline TI-2 & $4 / 6 / 06$ & 52.0 & 1 & $4.9 \mathrm{~J}$ & $3 \mathrm{~J}$ & ND \\
\hline TI-2 & $4 / 6 / 06$ & 54.0 & 1 & 30 & $5.1 \mathrm{~J}$ & ND \\
\hline TI-2 & $4 / 6 / 06$ & $58.0-70.7$ & 4 & ND & ND & ND \\
\hline TI-3 & $4 / 4 / 06$ & $6.0-10.0$ & 2 & ND & ND & ND \\
\hline TI-3 & $4 / 6 / 06$ & 22.0 & 1 & ND & $1.0 \mathrm{~J}$ & ND \\
\hline TI-3 & $4 / 5 / 06-4 / 7 / 06$ & $24.5-32.0$ & 3 & ND & ND & ND \\
\hline TI-3 & $4 / 6 / 06$ & 36.0 & 1 & $2.7 \mathrm{~J}$ & $1.6 \mathrm{~J}$ & ND \\
\hline TI-3 & $4 / 6 / 06$ & 37.0 & 1 & ND & $1.1 \mathrm{~J}$ & ND \\
\hline TI-3 & $4 / 6 / 06$ & 40.0 & 1 & $1.4 \mathrm{~J}$ & $1.3 \mathrm{~J}$ & ND \\
\hline TI-3 & $4 / 6 / 06$ & 44.0 & 1 & $1.6 \mathrm{~J}$ & ND & ND \\
\hline TI-3 & $4 / 6 / 06$ & 48.0 & 1 & $2.6 \mathrm{~J}$ & $1.2 \mathrm{~J}$ & ND \\
\hline TI-3 & $4 / 13 / 06$ & 49.2 & 1 & ND & $1.9 \mathrm{~J}$ & ND \\
\hline TI-3 & $4 / 13 / 06$ & 52.8 & 1 & 21 & ND & ND \\
\hline TI-3 & $4 / 13 / 06$ & 56.5 & 1 & $1.3 \mathrm{~J}$ & ND & ND \\
\hline TI-3 & $4 / 13 / 06$ & $60.0-62.0$ & 2 & ND & ND & ND \\
\hline
\end{tabular}


TABLE 3.1 (Cont.)

\begin{tabular}{|c|c|c|c|c|c|c|}
\hline \multirow[b]{2}{*}{ Location } & \multirow[b]{2}{*}{$\begin{array}{l}\text { Sample } \\
\text { Date }\end{array}$} & \multirow[b]{2}{*}{$\begin{array}{l}\text { Depth } \\
\text { (ft BGL) }\end{array}$} & \multirow[b]{2}{*}{$\begin{array}{l}\text { Number of } \\
\text { Samples }\end{array}$} & \multicolumn{3}{|c|}{ Concentration $(\mu \mathrm{g} / \mathrm{kg})$} \\
\hline & & & & $\begin{array}{l}\text { Carbon } \\
\text { Tetra- } \\
\text { chloride }\end{array}$ & $\begin{array}{l}\text { Chloro- } \\
\text { form }\end{array}$ & $\begin{array}{c}\text { Methylene } \\
\text { Chloride }\end{array}$ \\
\hline TI-4 & $4 / 8 / 06$ & $2.0-17.7$ & 5 & ND & ND & ND \\
\hline TI-4 & $4 / 8 / 06$ & 20.5 & 1 & 10 & ND & ND \\
\hline TI-4 & $4 / 9 / 06$ & 25.0 & 1 & 29 & $1.3 \mathrm{~J}$ & ND \\
\hline TI-4 & $4 / 9 / 06$ & 29.2 & 1 & $6.0 \mathrm{~J}$ & $1.5 \mathrm{~J}$ & ND \\
\hline TI-4 & $4 / 9 / 06$ & 31.0 & 1 & $4.0 \mathrm{~J}$ & ND & ND \\
\hline TI-4 & $4 / 9 / 06$ & 34.0 & 1 & $1.0 \mathrm{~J}$ & ND & ND \\
\hline TI-4 & $4 / 9 / 06$ & 37.0 & 1 & 32 & $8.3 \mathrm{~J}$ & ND \\
\hline TI-4 & $4 / 9 / 06$ & 45.5 & 1 & $3.1 \mathrm{~J}$ & ND & ND \\
\hline TI-4 & $4 / 9 / 06$ & 50.0 & 1 & 20 & $1.8 \mathrm{~J}$ & ND \\
\hline TI-4 & $4 / 9 / 06$ & 54.0 & 1 & ND & ND & ND \\
\hline TI-4 & $4 / 9 / 06$ & 58.0 & 1 & $7.3 \mathrm{~J}$ & $0.9 \mathrm{~J}$ & ND \\
\hline TI-4 & $4 / 9 / 06$ & 61.0 & 1 & ND & ND & ND \\
\hline TI-5 & 4/10/06-4/11/06 & $2.0-52.0$ & 14 & ND & ND & ND \\
\hline TI-5 & $4 / 11 / 06$ & 53.0 & 1 & 15 & ND & ND \\
\hline TI-5 & $4 / 12 / 06$ & 61.0 & 1 & ND & ND & ND \\
\hline TI-6 & $4 / 20 / 06$ & $2.0-46.0$ & 12 & ND & ND & ND \\
\hline TI-6 & $4 / 20 / 06$ & 50.0 & 1 & $1.3 \mathrm{~J}$ & ND & ND \\
\hline TI-6 & $4 / 21 / 06$ & $53.4-61.0$ & 2 & ND & ND & ND \\
\hline TI-7 & $4 / 9 / 06$ & $2.0-29.0$ & 14 & ND & ND & ND \\
\hline TI-7 & $4 / 9 / 06$ & 30.0 & 1 & $1.5 \mathrm{~J}$ & ND & ND \\
\hline TI-7 & $4 / 9 / 06$ & $36.5-43.0$ & 4 & ND & ND & ND \\
\hline TI-7 & $4 / 9 / 06$ & 46.0 & 1 & $7.0 \mathrm{~J}$ & ND & ND \\
\hline TI-7 & $4 / 10 / 06$ & $52.5-69.0$ & 4 & ND & ND & ND \\
\hline TI-8 & 4/26/06-4/27/06 & $2.0-59.7$ & 17 & ND & ND & ND \\
\hline TI-9 & $4 / 22 / 06-4 / 23 / 06$ & $4.0-45.0$ & 15 & ND & ND & ND \\
\hline TI-9 & $4 / 23 / 06$ & 50.0 & 1 & $2.7 \mathrm{~J}$ & ND & ND \\
\hline TI-9 & $4 / 23 / 06$ & $53.0-69.0$ & 5 & ND & ND & ND \\
\hline TI-10 & $4 / 25 / 06$ & $4.5-13.5$ & 5 & ND & ND & ND \\
\hline TI-10 & $4 / 25 / 06$ & 17.0 & 1 & ND & $2.0 \mathrm{~J}$ & ND \\
\hline TI-10 & $4 / 25 / 06$ & 19.5 & 1 & ND & $8.0 \mathrm{~J}$ & ND \\
\hline TI-10 & $4 / 25 / 06$ & 20.8 & 1 & ND & 30 & 112 \\
\hline TI-10 & $4 / 26 / 06$ & 25.5 & 1 & ND & $1.2 \mathrm{~J}$ & ND \\
\hline $\mathrm{TI}-10$ & $4 / 26 / 06$ & $29.5-65.5$ & 11 & ND & ND & ND \\
\hline TI-11 & $4 / 22 / 06-4 / 25 / 06$ & $2.0-60.5$ & 17 & ND & ND & ND \\
\hline TI-12 & $5 / 3 / 06$ & $1.0-53.5$ & 19 & ND & ND & ND \\
\hline TI-12 & $5 / 3 / 06$ & 56.5 & 1 & ND & $1.0 \mathrm{~J}$ & ND \\
\hline TI-12 & $5 / 3 / 06$ & $60.0-61.5$ & 2 & ND & ND & ND \\
\hline
\end{tabular}

a ND, not detected at a method detection limit of $1.0 \mu \mathrm{g} / \mathrm{kg}$.

b Qualifier J indicates an estimated concentration below the quantitation limit of $10.0 \mu \mathrm{g} / \mathrm{kg}$. 
TABLE 3.2 Summary of organic analytical results for soil samples collected on the Co-op property during the 2006 investigation at Navarre, Kansas.

\begin{tabular}{|c|c|c|c|c|c|c|}
\hline \multirow[b]{2}{*}{ Location } & \multirow[b]{2}{*}{$\begin{array}{l}\text { Sample } \\
\text { Date }\end{array}$} & \multirow[b]{2}{*}{$\begin{array}{l}\text { Depth } \\
\text { (ft BGL) }\end{array}$} & \multirow[b]{2}{*}{$\begin{array}{c}\text { Number of } \\
\text { Samples }\end{array}$} & \multicolumn{3}{|c|}{ Concentration $(\mu \mathrm{g} / \mathrm{kg})$} \\
\hline & & & & $\begin{array}{c}\text { Carbon } \\
\text { Tetra- } \\
\text { chloride }\end{array}$ & $\begin{array}{l}\text { Chloro- } \\
\text { form }\end{array}$ & $\begin{array}{c}\text { Methylene } \\
\text { Chloride }\end{array}$ \\
\hline TI-13 & $5 / 2 / 06-5 / 3 / 06$ & $2.0-19.1$ & 9 & $\mathrm{ND}^{\mathrm{a}}$ & ND & ND \\
\hline TI-13 & $5 / 4 / 06$ & 22.8 & 1 & ND & $1.6 \mathrm{~J}^{\mathrm{b}}$ & ND \\
\hline TI-13 & $5 / 3 / 06$ & 27.0 & 1 & 176 & 33 & ND \\
\hline TI-13 & $5 / 3 / 06$ & 28.0 & 1 & 224 & 34 & ND \\
\hline TI-13 & $5 / 4 / 06$ & 30.5 & 1 & 37 & 14 & ND \\
\hline TI-13 & $5 / 3 / 06$ & 34.5 & 1 & 22 & $6.3 \mathrm{~J}$ & ND \\
\hline TI-13 & $5 / 3 / 06$ & 36.0 & 1 & 16 & $5.1 \mathrm{~J}$ & ND \\
\hline TI-13 & $5 / 3 / 06$ & 38.0 & 1 & 45 & 29 & ND \\
\hline TI-13 & $5 / 3 / 06$ & 40.0 & 1 & 18 & $8 \mathrm{~J}$ & ND \\
\hline TI-13 & $5 / 3 / 06$ & 42.9 & 1 & 30 & $5.6 \mathrm{~J}$ & ND \\
\hline TI-13 & $5 / 3 / 06$ & 47.0 & 1 & 11 & $3 \mathrm{~J}$ & ND \\
\hline TI-13 & $5 / 3 / 06$ & 50.0 & 1 & 20 & $2.4 \mathrm{~J}$ & ND \\
\hline TI-13 & $5 / 3 / 06$ & 51.5 & 1 & $3.1 \mathrm{~J}$ & $1.8 \mathrm{~J}$ & ND \\
\hline TI-13 & $5 / 3 / 06$ & 54.8 & 1 & $3.5 \mathrm{~J}$ & ND & ND \\
\hline TI-13 & $5 / 3 / 06$ & 58.9 & 1 & $1.9 \mathrm{~J}$ & $1.2 \mathrm{~J}$ & ND \\
\hline TI-14 & $5 / 5 / 06$ & $2.0-26.8$ & 12 & ND & ND & ND \\
\hline TI-14 & $5 / 5 / 06$ & 29.0 & 1 & 19 & $3.7 \mathrm{~J}$ & ND \\
\hline TI-14 & $5 / 5 / 06$ & 32.8 & 1 & 73 & $2.1 \mathrm{~J}$ & ND \\
\hline TI-14 & $5 / 5 / 06$ & 35.2 & 1 & 364 & 16 & ND \\
\hline TI-14 & $5 / 5 / 06$ & 38.0 & 1 & 194 & 15 & ND \\
\hline TI-14 & $5 / 5 / 06$ & 40.5 & 1 & 306 & 25 & ND \\
\hline TI-14 & $5 / 5 / 06$ & 44.5 & 1 & 114 & $2.3 \mathrm{~J}$ & ND \\
\hline TI-14 & $5 / 5 / 06$ & 46.2 & 1 & 160 & $2.6 \mathrm{~J}$ & ND \\
\hline TI-14 & $5 / 5 / 06$ & 49.0 & 1 & 66 & $5.5 \mathrm{~J}$ & ND \\
\hline TI-14 & $5 / 6 / 06$ & 52.8 & 1 & $2.6 \mathrm{~J}$ & $1.8 \mathrm{~J}$ & ND \\
\hline TI-14 & $5 / 6 / 06$ & 56.8 & 1 & $6.6 \mathrm{~J}$ & $3.6 \mathrm{~J}$ & ND \\
\hline TI-14 & $5 / 6 / 06$ & 57.8 & 1 & 50 & $4.0 \mathrm{~J}$ & ND \\
\hline TI-14 & $5 / 6 / 06$ & 61.2 & 1 & ND & ND & ND \\
\hline TI-15 & $5 / 5 / 06$ & $1.0-24.6$ & 9 & ND & ND & ND \\
\hline TI-15 & $5 / 5 / 06$ & 26.1 & 1 & 93 & $2.5 \mathrm{~J}$ & ND \\
\hline TI-15 & $5 / 5 / 06$ & 29.0 & 1 & 27 & ND & ND \\
\hline TI-15 & $5 / 5 / 06$ & 33.5 & 1 & 23 & ND & ND \\
\hline TI-15 & $5 / 5 / 06$ & 34.6 & 1 & 13 & ND & ND \\
\hline TI-15 & $5 / 5 / 06$ & 37.5 & 1 & 13 & $3.5 \mathrm{~J}$ & ND \\
\hline TI-15 & $5 / 5 / 06$ & 41.5 & 1 & 91 & $4.7 \mathrm{~J}$ & ND \\
\hline TI-15 & $5 / 5 / 06$ & 45.0 & 1 & $3.3 \mathrm{~J}$ & ND & ND \\
\hline TI-15 & 5/6/06 & $51.9-60.8$ & 4 & ND & ND & ND \\
\hline TI-16 & $5 / 7 / 06$ & 1.5 & 1 & ND & ND & ND \\
\hline TI-16 & $5 / 7 / 06$ & 3.5 & 1 & $3.3 \mathrm{~J}$ & ND & ND \\
\hline TI-16 & $5 / 7 / 06$ & $6.0-25.0$ & 9 & ND & ND & ND \\
\hline TI-16 & $5 / 7 / 06$ & 28.8 & 1 & 272 & 107 & ND \\
\hline TI-16 & $5 / 7 / 06$ & 32.9 & 1 & 92 & 33 & ND \\
\hline TI-16 & $5 / 7 / 06$ & 36.5 & 1 & 36 & $9.0 \mathrm{~J}$ & ND \\
\hline TI-16 & $5 / 7 / 06$ & 37.5 & 1 & 35 & $8.6 \mathrm{~J}$ & ND \\
\hline TI-16 & $5 / 7 / 06$ & 42.0 & 1 & 454 & 34 & ND \\
\hline TI-16 & $5 / 7 / 06$ & 45.0 & 1 & 23 & $8.8 \mathrm{~J}$ & ND \\
\hline TI-16 & $5 / 7 / 06$ & 49.8 & 1 & $3.1 \mathrm{~J}$ & ND & ND \\
\hline TI-16 & $5 / 7 / 06$ & 53.0 & 1 & $3.8 \mathrm{~J}$ & $1.7 \mathrm{~J}$ & ND \\
\hline
\end{tabular}


TABLE 3.2 (Cont.)

\begin{tabular}{|c|c|c|c|c|c|c|}
\hline \multirow[b]{2}{*}{ Location } & \multirow[b]{2}{*}{$\begin{array}{l}\text { Sample } \\
\text { Date }\end{array}$} & \multirow[b]{2}{*}{$\begin{array}{l}\text { Depth } \\
\text { (ft BGL) }\end{array}$} & \multirow[b]{2}{*}{$\begin{array}{c}\text { Number of } \\
\text { Samples }\end{array}$} & \multicolumn{3}{|c|}{ Concentration $(\mu \mathrm{g} / \mathrm{kg})$} \\
\hline & & & & $\begin{array}{l}\text { Carbon } \\
\text { Tetra- } \\
\text { chloride }\end{array}$ & $\begin{array}{l}\text { Chloro- } \\
\text { form }\end{array}$ & $\begin{array}{c}\text { Methylene } \\
\text { Chloride }\end{array}$ \\
\hline TI-17 & $5 / 7 / 06$ & $2.2-21.0$ & 7 & ND & ND & ND \\
\hline TI-17 & $5 / 7 / 06$ & 24.8 & 1 & $2.6 \mathrm{~J}$ & ND & ND \\
\hline $\mathrm{TI}-17$ & $5 / 7 / 06$ & 26.2 & 1 & 15 & $1.5 \mathrm{~J}$ & ND \\
\hline TI-17 & $5 / 7 / 06$ & 28.5 & 1 & 31 & ND & ND \\
\hline $\mathrm{Tl}-17$ & $5 / 7 / 06$ & 33.0 & 1 & ND & $7.9 \mathrm{~J}$ & ND \\
\hline $\mathrm{TI}-17$ & $5 / 7 / 06$ & 37.5 & 1 & ND & ND & ND \\
\hline $\mathrm{TI}-17$ & $5 / 7 / 06$ & 42.0 & 1 & 10 & ND & ND \\
\hline $\mathrm{TI}-17$ & $5 / 7 / 06$ & $45.5-49.2$ & 2 & ND & ND & ND \\
\hline $\mathrm{TI}-17$ & $5 / 7 / 06$ & 52.9 & 1 & $2.1 \mathrm{~J}$ & ND & ND \\
\hline $\mathrm{TI}-17$ & $5 / 7 / 06$ & 56.5 & 1 & ND & ND & ND \\
\hline TI-18 & $5 / 18 / 06$ & $5.0-20.8$ & 5 & ND & ND & ND \\
\hline TI-18 & $5 / 19 / 06$ & 29.0 & 1 & 10 & ND & ND \\
\hline TI-18 & $5 / 19 / 06$ & 33.5 & 1 & 56 & ND & ND \\
\hline TI-18 & $5 / 19 / 06$ & 37.0 & 1 & 109 & ND & ND \\
\hline TI-18 & $5 / 19 / 06$ & 41.0 & 1 & 400 & $9.2 \mathrm{~J}$ & ND \\
\hline TI-18 & $5 / 21 / 06$ & 45.0 & 1 & 46 & ND & ND \\
\hline TI-18 & $5 / 21 / 06$ & 53.0 & 1 & $4.0 \mathrm{~J}$ & $2.0 \mathrm{~J}$ & ND \\
\hline TI-18 & $5 / 21 / 06$ & 57.0 & 1 & ND & $1.4 \mathrm{~J}$ & ND \\
\hline TI-18 & $5 / 21 / 06$ & 61.0 & 1 & $2.9 \mathrm{~J}$ & $1.5 \mathrm{~J}$ & ND \\
\hline TI-28 & $5 / 18 / 06-5 / 19 / 06$ & $6.0-17.0$ & 5 & ND & ND & ND \\
\hline TI-28 & $5 / 18 / 06$ & 20.5 & 1 & 18 & $1.7 \mathrm{~J}$ & ND \\
\hline TI-28 & $5 / 18 / 06$ & 22.5 & 1 & 1094 & $4.4 \mathrm{~J}$ & ND \\
\hline TI-28 & $5 / 18 / 06$ & 25.2 & 1 & 613 & ND & ND \\
\hline TI-28 & $5 / 18 / 06$ & 28.5 & 1 & 433 & ND & ND \\
\hline TI-28 & $5 / 18 / 06$ & 32.8 & 1 & 287 & ND & ND \\
\hline TI-28 & $5 / 18 / 06$ & 37.0 & 1 & 110 & ND & ND \\
\hline TI-28 & $5 / 18 / 06$ & 41.0 & 1 & $8.7 \mathrm{~J}$ & ND & ND \\
\hline TI-28 & $5 / 18 / 06$ & 42.5 & 1 & $5.2 \mathrm{~J}$ & $1.9 \mathrm{~J}$ & ND \\
\hline TI-28 & $5 / 18 / 06$ & 45.0 & 1 & $2.8 \mathrm{~J}$ & ND & ND \\
\hline TI-28 & $5 / 19 / 06$ & $49.0-61.8$ & 4 & ND & ND & ND \\
\hline TI-29 & $5 / 19 / 06-5 / 21 / 06$ & $6.0-15.5$ & 5 & ND & ND & ND \\
\hline TI-29 & $5 / 19 / 06$ & 16.5 & 1 & ND & $0.7 \mathrm{~J}$ & ND \\
\hline TI-29 & $5 / 19 / 06$ & 24.5 & 1 & 91 & ND & ND \\
\hline TI-29 & $5 / 19 / 06$ & 26.5 & 1 & 17 & 10 & ND \\
\hline TI-29 & $5 / 19 / 06$ & 29.4 & 1 & $9.4 \mathrm{~J}$ & $7.8 \mathrm{~J}$ & ND \\
\hline TI-29 & $5 / 19 / 06$ & 34.0 & 1 & 49 & 16 & ND \\
\hline TI-29 & $5 / 19 / 06$ & 38.0 & 1 & 135 & $8 \mathrm{~J}$ & ND \\
\hline TI-29 & $5 / 19 / 06$ & 42.0 & 1 & 106 & $5.2 \mathrm{~J}$ & ND \\
\hline TI-29 & $5 / 19 / 06$ & 45.5 & 1 & ND & ND & ND \\
\hline TI-29 & $5 / 19 / 06$ & 49.5 & 1 & 43 & $5.9 \mathrm{~J}$ & ND \\
\hline TI-29 & $5 / 19 / 06$ & 53.0 & 1 & $6.0 \mathrm{~J}$ & $1.9 \mathrm{~J}$ & ND \\
\hline TI-29 & $5 / 21 / 06$ & $57.0-62.0$ & 2 & ND & ND & ND \\
\hline
\end{tabular}

a ND, not detected at a method detection limit of $1.0 \mu \mathrm{g} / \mathrm{kg}$.

b Qualifier J indicates an estimated concentration below the quantitation limit of $10.0 \mu \mathrm{g} / \mathrm{kg}$. 
TABLE 3.3 Summary of results of organic analyses of vertical-profile groundwater samples collected with the cone penetrometer at the former CCC/USDA facility during the 2006 investigation at Navarre, Kansas.

\begin{tabular}{|c|c|c|c|c|c|c|c|}
\hline \multirow[b]{2}{*}{ Location } & \multirow[b]{2}{*}{$\begin{array}{c}\text { Depth } \\
\text { (ft below TOC) }\end{array}$} & \multirow[b]{2}{*}{$\begin{array}{l}\text { Number of } \\
\text { Samples }\end{array}$} & \multirow[b]{2}{*}{$\begin{array}{l}\text { Sample } \\
\text { Date }\end{array}$} & \multicolumn{4}{|c|}{ Concentration $(\mu \mathrm{g} / \mathrm{L})$} \\
\hline & & & & $\begin{array}{c}\text { Carbon } \\
\text { Tetrachloride }\end{array}$ & Chloroform & $\begin{array}{l}\text { Methylene } \\
\text { Chloride }\end{array}$ & $\begin{array}{c}\text { Tetra- } \\
\text { chloroethene }\end{array}$ \\
\hline TI-1 & $30-35$ & 1 & 4/13/06 & 181 & 60 & 10 & $0.2 \mathrm{Ja}$ \\
\hline TI-1 & $38-43$ & 1 & 4/13/06 & 177 & 64 & 10 & $0.2 \mathrm{~J}$ \\
\hline TI-1 & $50-55$ & 1 & 4/13/06 & 127 & 56 & 8.2 & $N D^{b}$ \\
\hline TI-1 & $67-71$ & 1 & $4 / 14 / 06$ & ND & ND & ND & ND \\
\hline TI-2 & $25-30$ & 1 & $4 / 21 / 06$ & 2.2 & 2.0 & ND & ND \\
\hline TI-2 & $31-36$ & 1 & $4 / 21 / 06$ & 8.7 & 7.8 & ND & ND \\
\hline TI-2 & $37-42$ & 1 & $4 / 21 / 06$ & 77 & 9.2 & ND & $<0.1$ \\
\hline TI-2 & $49-54$ & 1 & $4 / 21 / 06$ & 9.8 & 2.7 & ND & ND \\
\hline TI-2 & $66-70$ & 1 & $4 / 21 / 06$ & 14 & 2.8 & ND & ND \\
\hline TI-2 & $69-72$ & 1 & $4 / 7 / 06$ & ND & ND & ND & ND \\
\hline TI-3 & $32.2-37.2$ & 1 & $4 / 7 / 06$ & 27 & 7.9 & ND & ND \\
\hline TI-3 & $43-48$ & 1 & 4/14/06 & 72 & 12 & ND & ND \\
\hline TI-3 & $51-56$ & 1 & $4 / 14 / 06$ & 7.6 & 1.6 & ND & ND \\
\hline TI-4 & $35-40$ & 1 & 4/10/06 & 76 & 55 & 3.6 & $0.2 \mathrm{~J}$ \\
\hline TI-4 & $42-47$ & 1 & 4/14/06 & 99 & 75 & 6.1 & ND \\
\hline TI-4 & $50-55$ & 1 & $4 / 11 / 06$ & 40 & 58 & $0.8 \mathrm{~J}$ & $0.2 \mathrm{~J}$ \\
\hline TI-4 & $55-60$ & 1 & $4 / 11 / 06$ & 23 & 7.0 & ND & $<0.1$ \\
\hline TI-4 & $61-66$ & 1 & 4/12/06 & $0.6 \mathrm{~J}$ & 3.6 & ND & ND \\
\hline $\mathrm{TI}-4$ & $67-71$ & 1 & 4/12/06 & ND & ND & ND & ND \\
\hline TI-5 & 28-33 & 1 & 4/13/06 & 1.1 & 1.2 & ND & ND \\
\hline TI-5 & $36-41$ & 1 & $4 / 12 / 06$ & 1.1 & 2.0 & ND & ND \\
\hline TI-5 & $49-54$ & 1 & 4/13/06 & 6.3 & $0.4 \mathrm{~J}$ & ND & ND \\
\hline TI-5 & $72.8-77.8$ & 1 & $4 / 20 / 06$ & ND & ND & ND & ND \\
\hline TI-6 & $38-43$ & 1 & 4/22/06 & 1.3 & 1.0 & ND & ND \\
\hline TI-6 & $49-54$ & 1 & $4 / 21 / 06$ & $0.4 \mathrm{~J}$ & ND & ND & ND \\
\hline TI-6 & $58-63$ & 1 & 4/22/06 & 1.5 & $0.1 \mathrm{~J}$ & ND & ND \\
\hline TI-6 & $68-73$ & 1 & $4 / 22 / 06$ & ND & ND & ND & ND \\
\hline TI-7 & $29.5-34.5$ & 1 & 4/20/06 & 3.8 & 1.7 & ND & ND \\
\hline TI-7 & $37-42$ & 1 & $4 / 20 / 06$ & 3.8 & 1.9 & ND & ND \\
\hline TI-7 & $45-50$ & 1 & $4 / 20 / 06$ & 5.6 & 2.7 & ND & ND \\
\hline TI-7 & $51-56$ & 1 & $4 / 21 / 06$ & 1.9 & 2.0 & ND & ND \\
\hline TI-7 & $68-72$ & 1 & $4 / 21 / 06$ & $0.5 \mathrm{~J}$ & $0.3 \mathrm{~J}$ & ND & ND \\
\hline TI-8 & $31-36$ & 1 & 4/28/06 & $0.1 \mathrm{~J}$ & ND & ND & ND \\
\hline TI-8 & $39-44$ & 1 & 4/28/06 & $0.2 \mathrm{~J}$ & ND & ND & ND \\
\hline TI-8 & $45-50$ & 1 & $4 / 28 / 06$ & $0.2 \mathrm{~J}$ & ND & ND & ND \\
\hline TI-8 & 54-59 & 1 & $5 / 2 / 06$ & $0.5 \mathrm{~J}$ & ND & ND & ND \\
\hline TI-8 & $64.5-69.5$ & 1 & $5 / 2 / 06$ & ND & ND & ND & ND \\
\hline TI-9 & $40-45$ & 1 & 4/24/06 & ND & ND & ND & ND \\
\hline TI-9 & $46-51$ & 1 & $4 / 21 / 06$ & 1.2 & ND & ND & ND \\
\hline TI-9 & $54-59$ & 1 & $4 / 23 / 06$ & $0.8 \mathrm{~J}$ & ND & ND & ND \\
\hline TI-9 & 59-64 & 1 & $4 / 22 / 06$ & 1.5 & ND & ND & ND \\
\hline
\end{tabular}


TABLE 3.3 (Cont.)

\begin{tabular}{|c|c|c|c|c|c|c|c|}
\hline \multirow[b]{2}{*}{ Location } & \multirow[b]{2}{*}{$\begin{array}{c}\text { Depth } \\
\text { (ft below TOC) }\end{array}$} & \multirow[b]{2}{*}{$\begin{array}{c}\text { Number of } \\
\text { Samples }\end{array}$} & \multirow[b]{2}{*}{$\begin{array}{l}\text { Sample } \\
\text { Date }\end{array}$} & \multicolumn{4}{|c|}{ Concentration ( $\mu \mathrm{g} / \mathrm{L})$} \\
\hline & & & & $\begin{array}{c}\text { Carbon } \\
\text { Tetrachloride }\end{array}$ & Chloroform & $\begin{array}{l}\text { Methylene } \\
\text { Chloride }\end{array}$ & $\begin{array}{c}\text { Tetra- } \\
\text { chloroethene }\end{array}$ \\
\hline TI-10 & $40-45$ & 1 & $4 / 26 / 06$ & $0.4 \mathrm{~J}$ & ND & 36 & ND \\
\hline TI-10 & $46-51$ & 1 & $4 / 27 / 06$ & $0.4 \mathrm{~J}$ & ND & 11 & ND \\
\hline TI-10 & $54-59$ & 1 & $4 / 27 / 06$ & $0.4 \mathrm{~J}$ & ND & ND & ND \\
\hline $\mathrm{TI}-10$ & $59-64$ & 1 & $4 / 27 / 06$ & $0.3 \mathrm{~J}$ & ND & ND & ND \\
\hline $\mathrm{TI}-10$ & $66-71$ & 1 & $4 / 25 / 06$ & ND & ND & ND & ND \\
\hline TI-11 & $26-31$ & 1 & $4 / 26 / 06$ & ND & ND & ND & ND \\
\hline TI-11 & $33-38$ & 1 & $4 / 27 / 06$ & ND & $0.2 \mathrm{~J}$ & 9.5 & ND \\
\hline $\mathrm{TI}-11$ & $40-45$ & 1 & $4 / 25 / 06$ & ND & ND & ND & ND \\
\hline TI-11 & $46.3-51.3$ & 1 & $4 / 23 / 06$ & $0.9 \mathrm{~J}$ & $0.1 \mathrm{~J}$ & ND & ND \\
\hline Tl-11 & $52.6-57.6$ & 1 & $4 / 27 / 06$ & $0.6 \mathrm{~J}$ & ND & ND & ND \\
\hline TI-11 & $65.9-70.9$ & 1 & $4 / 27 / 06$ & $0.4 \mathrm{~J}$ & ND & ND & ND \\
\hline TI-12 & $34-68.7$ & 5 & $4 / 28 / 06-5 / 4 / 06$ & ND & ND & ND & ND \\
\hline
\end{tabular}

a Qualifier J indicates an estimated concentration below the quantitation limit of $1.0 \mu \mathrm{g} / \mathrm{L}$.

b ND, not detected at a method detection limit of $0.1 \mu \mathrm{g} / \mathrm{L}$. 
TABLE 3.4 Summary of results of organic analyses of well samples and vertical-profile groundwater samples collected with the cone penetrometer on and near the Co-op property during the 2006 investigation at Navarre, Kansas.

\begin{tabular}{|c|c|c|c|c|c|c|}
\hline \multirow[b]{2}{*}{ Location } & \multirow[b]{2}{*}{$\begin{array}{c}\text { Depth } \\
\text { (ft below TOC) }\end{array}$} & \multirow[b]{2}{*}{$\begin{array}{l}\text { Sample } \\
\text { Date }\end{array}$} & \multicolumn{4}{|c|}{ Concentration $(\mu \mathrm{g} / \mathrm{L})$} \\
\hline & & & $\begin{array}{c}\text { Carbon } \\
\text { Tetrachloride }\end{array}$ & Chloroform & $\begin{array}{l}\text { Methylene } \\
\text { Chloride }\end{array}$ & $\begin{array}{c}\text { Tetra- } \\
\text { chloroethene }\end{array}$ \\
\hline \multicolumn{7}{|c|}{ Domestic wells } \\
\hline Co-op 1 & Unknown & $4 / 8 / 06$ & 1.2 & $0.8 \mathrm{Ja}$ & $N D^{b}$ & ND \\
\hline Co-op 3 & Unknown & $4 / 8 / 06$ & 197 & 44 & 3.6 & $0.2 \mathrm{~J}$ \\
\hline \multicolumn{7}{|c|}{ Monitoring wells } \\
\hline KDHE-2 & $25-45$ & $4 / 7 / 06$ & ND & ND & ND & ND \\
\hline NW-1 & $40-50$ & $4 / 8 / 06$ & 1.1 & $0.3 \mathrm{~J}$ & ND & ND \\
\hline NW-2 & $35.5-45.5$ & $4 / 9 / 06$ & 313 & 74 & 3.2 & $0.2 \mathrm{~J}$ \\
\hline \multicolumn{7}{|c|}{ Cone penetrometer samples } \\
\hline TI-13 & $25-30$ & $5 / 9 / 06$ & 27 & 16 & ND & ND \\
\hline TI-13 & $35-40$ & $5 / 5 / 06$ & 387 & 197 & $0.6 \mathrm{~J}$ & ND \\
\hline TI-13 & $42-47$ & $5 / 5 / 06$ & 116 & 60 & 1.2 & $0.1 \mathrm{~J}$ \\
\hline TI-13 & $48-53$ & $5 / 4 / 06$ & 22 & 5.4 & ND & ND \\
\hline TI-13 & $54-59$ & $5 / 5 / 06$ & 22 & 5.8 & ND & ND \\
\hline TI-13 & $66.8-71.8$ & $5 / 4 / 06$ & $0.6 \mathrm{~J}$ & $0.3 \mathrm{~J}$ & ND & ND \\
\hline TI-14 & $26-31$ & $5 / 6 / 06$ & 148 & 36 & 1.9 & $0.2 \mathrm{~J}$ \\
\hline TI-14 & $32-37$ & $5 / 6 / 06$ & 198 & 71 & ND & $0.3 \mathrm{~J}$ \\
\hline TI-14 & $38-43$ & $5 / 6 / 06$ & 260 & 126 & 8.0 & $0.5 \mathrm{~J}$ \\
\hline TI-14 & $47-52$ & $5 / 6 / 06$ & 229 & 90 & 5.2 & $0.4 \mathrm{~J}$ \\
\hline TI-14 & $54-59$ & $5 / 6 / 06$ & 72 & 48 & 2.8 & $0.2 \mathrm{~J}$ \\
\hline TI-14 & $67.4-72.4$ & $5 / 5 / 06$ & 6.0 & 280 & 12.6 & ND \\
\hline TI-15 & $15-20$ & $5 / 8 / 06$ & ND & ND & ND & ND \\
\hline TI-15 & $25-30$ & $5 / 9 / 06$ & 45 & 9.2 & ND & ND \\
\hline TI-15 & $35-40$ & $5 / 7 / 06$ & 21 & 6.0 & ND & ND \\
\hline TI-15 & $40-45$ & 5/6/06 & 3.4 & 1.7 & ND & ND \\
\hline TI-15 & $47-52$ & $5 / 6 / 06$ & 2.4 & 2.3 & ND & ND \\
\hline TI-15 & $67.1-72.1$ & 5/6/06 & ND & ND & ND & ND \\
\hline TI-16 & $25-30$ & $5 / 9 / 06$ & 851 & 535 & 2.6 & $0.1 \mathrm{~J}$ \\
\hline TI-16 & $32-37$ & $5 / 9 / 06$ & 866 & 535 & ND & $0.3 \mathrm{~J}$ \\
\hline TI-16 & $39-44$ & $5 / 8 / 06$ & 189 & 123 & 6.0 & $0.3 \mathrm{~J}$ \\
\hline TI-16 & $46-51$ & $5 / 9 / 06$ & 56 & 32 & ND & ND \\
\hline TI-16 & $60-65$ & $5 / 8 / 06$ & $0.9 \mathrm{~J}$ & $0.6 \mathrm{~J}$ & ND & ND \\
\hline TI-16 & $67-72$ & $5 / 8 / 06$ & $0.8 \mathrm{~J}$ & 2.4 & ND & ND \\
\hline $\mathrm{TI}-17$ & $25-30$ & $5 / 9 / 06$ & 44 & 3.2 & ND & ND \\
\hline TI-17 & $32-37$ & $5 / 8 / 06$ & 1.5 & $0.7 \mathrm{~J}$ & ND & ND \\
\hline TI-17 & $39-44$ & $5 / 9 / 06$ & 1.7 & 1.6 & ND & ND \\
\hline TI-17 & $46-51$ & $5 / 9 / 06$ & 1.1 & $0.6 \mathrm{~J}$ & ND & ND \\
\hline $\mathrm{TI}-17$ & $53-58$ & $5 / 9 / 06$ & 2.5 & 1.5 & ND & ND \\
\hline $\mathrm{TI}-17$ & $60-65$ & $5 / 9 / 06$ & $0.5 \mathrm{~J}$ & $0.9 \mathrm{~J}$ & ND & ND \\
\hline
\end{tabular}


TABLE 3.4 (Cont.)

\begin{tabular}{|c|c|c|c|c|c|c|}
\hline \multirow[b]{2}{*}{ Location } & \multirow[b]{2}{*}{$\begin{array}{c}\text { Depth } \\
\text { (ft below TOC) }\end{array}$} & \multirow[b]{2}{*}{$\begin{array}{l}\text { Sample } \\
\text { Date }\end{array}$} & \multicolumn{4}{|c|}{ Concentration $(\mu \mathrm{g} / \mathrm{L})$} \\
\hline & & & $\begin{array}{c}\text { Carbon } \\
\text { Tetrachloride }\end{array}$ & Chloroform & $\begin{array}{l}\text { Methylene } \\
\text { Chloride }\end{array}$ & $\begin{array}{c}\text { Tetra- } \\
\text { chloroethene }\end{array}$ \\
\hline \multicolumn{7}{|c|}{ Cone penetrometer samples (cont.) } \\
\hline TI-18 & $25-30$ & $5 / 23 / 06$ & 57 & 11 & ND & ND \\
\hline TI-18 & $30-35$ & $5 / 21 / 06$ & 266 & 27 & 1.1 & ND \\
\hline TI-18 & $35-40$ & $5 / 10 / 06$ & 782 & 47 & 2.7 & $0.2 \mathrm{~J}$ \\
\hline TI-18 & $42-47$ & $5 / 21 / 06$ & 218 & 48 & 7.3 & ND \\
\hline TI-18 & $49-54$ & $5 / 21 / 06$ & 325 & 92 & 2.8 & $0.4 \mathrm{~J}$ \\
\hline TI-18 & $56-61$ & $5 / 21 / 06$ & 70 & 35 & 1.5 & ND \\
\hline $\mathrm{TI}-18$ & $66.5-71.5$ & $5 / 22 / 06$ & ND & $0.2 \mathrm{~J}$ & ND & ND \\
\hline TI-19 & 25-30 & $5 / 11 / 06$ & ND & ND & ND & ND \\
\hline TI-19 & $32-37$ & $5 / 11 / 06$ & ND & ND & ND & ND \\
\hline TI-19 & $39-44$ & $5 / 11 / 06$ & $0.3 \mathrm{~J}$ & ND & ND & ND \\
\hline TI-19 & $46-51$ & $5 / 11 / 06$ & $0.3 \mathrm{~J}$ & ND & ND & ND \\
\hline TI-19 & $53-58$ & $5 / 11 / 06$ & $0.3 \mathrm{~J}$ & ND & ND & ND \\
\hline TI-19 & $60-65$ & $5 / 11 / 06$ & ND & ND & ND & ND \\
\hline TI-19 & $65.5-69.5$ & $5 / 11 / 06$ & ND & ND & ND & ND \\
\hline TI-21 & $25-30$ & $5 / 11 / 06$ & ND & ND & ND & ND \\
\hline TI-21 & $32-37$ & $5 / 11 / 06$ & $0.9 \mathrm{~J}$ & $0.9 \mathrm{~J}$ & ND & ND \\
\hline TI-21 & $39-44$ & $5 / 11 / 06$ & $0.9 \mathrm{~J}$ & $0.8 \mathrm{~J}$ & ND & ND \\
\hline TI-21 & $46-51$ & $5 / 11 / 06$ & 1.2 & $0.8 \mathrm{~J}$ & ND & ND \\
\hline TI-21 & $53-58$ & $5 / 11 / 06$ & $0.7 \mathrm{~J}$ & $0.3 \mathrm{~J}$ & ND & ND \\
\hline TI-21 & $60-65$ & $5 / 11 / 06$ & ND & $0.4 \mathrm{~J}$ & ND & ND \\
\hline TI-22 & $32-37$ & $5 / 10 / 06$ & 120 & 17 & ND & $0.2 \mathrm{~J}$ \\
\hline TI-22 & $39-44$ & $5 / 10 / 06$ & 26 & 12 & ND & ND \\
\hline TI-22 & $46-51$ & $5 / 9 / 06$ & 119 & 31 & 1.3 & $0.1 \mathrm{~J}$ \\
\hline TI-22 & $53-58$ & $5 / 10 / 06$ & 356 & 29 & 1.1 & ND \\
\hline TI-22 & $60-65$ & $5 / 10 / 06$ & 116 & 21 & 1.1 & ND \\
\hline TI-22 & $68.2-73.2$ & $5 / 10 / 06$ & ND & ND & ND & ND \\
\hline TI-23 & $32-37$ & $5 / 10 / 06$ & 24 & 2.6 & ND & ND \\
\hline TI-23 & $39-44$ & $5 / 11 / 06$ & 11 & 6.5 & ND & ND \\
\hline TI-23 & $46-51$ & $5 / 11 / 06$ & 5.1 & 2.0 & ND & ND \\
\hline TI-23 & $53-58$ & $5 / 11 / 06$ & 1.5 & $0.5 \mathrm{~J}$ & ND & ND \\
\hline TI-23 & $60-65$ & $5 / 10 / 06$ & 1.0 & ND & ND & ND \\
\hline TI-23 & $66.8-71.8$ & $5 / 10 / 06$ & ND & ND & ND & ND \\
\hline TI-24 & $23-30$ & $5 / 11 / 06$ & ND & ND & ND & ND \\
\hline TI-24 & $32-37$ & $5 / 10 / 06$ & ND & ND & ND & ND \\
\hline TI-24 & $39-44$ & $5 / 10 / 06$ & 1.5 & 1.9 & ND & ND \\
\hline TI-24 & $46-51$ & $5 / 10 / 06$ & 1.8 & 2.4 & ND & ND \\
\hline TI-24 & $53-58$ & $5 / 10 / 06$ & 1.4 & 1.7 & ND & ND \\
\hline TI-24 & $60-65$ & $5 / 10 / 06$ & 1.0 & $0.6 \mathrm{~J}$ & ND & ND \\
\hline TI-24 & $69.4-74.4$ & $5 / 10 / 06$ & ND & ND & ND & ND \\
\hline TI-25 & $32-37$ & $5 / 12 / 06$ & ND & ND & ND & ND \\
\hline TI-25 & $39-44$ & $5 / 11 / 06$ & ND & $0.2 \mathrm{~J}$ & ND & ND \\
\hline TI-25 & $46-51$ & $5 / 11 / 06$ & 1.7 & 1.4 & ND & ND \\
\hline TI-25 & $53-58$ & $5 / 11 / 06$ & $0.8 \mathrm{~J}$ & $0.2 \mathrm{~J}$ & ND & ND \\
\hline
\end{tabular}


TABLE 3.4 (Cont.)

\begin{tabular}{|c|c|c|c|c|c|c|}
\hline \multirow[b]{2}{*}{ Location } & \multirow[b]{2}{*}{$\begin{array}{c}\text { Depth } \\
\text { (ft below TOC) }\end{array}$} & \multirow[b]{2}{*}{$\begin{array}{l}\text { Sample } \\
\text { Date }\end{array}$} & \multicolumn{4}{|c|}{ Concentration $(\mu \mathrm{g} / \mathrm{L})$} \\
\hline & & & $\begin{array}{c}\text { Carbon } \\
\text { Tetrachloride }\end{array}$ & Chloroform & $\begin{array}{l}\text { Methylene } \\
\text { Chloride }\end{array}$ & $\begin{array}{c}\text { Tetra- } \\
\text { chloroethene }\end{array}$ \\
\hline \multicolumn{7}{|c|}{ Cone penetrometer samples (cont.) } \\
\hline TI-25 & $60-65$ & $5 / 12 / 06$ & ND & ND & ND & ND \\
\hline TI-25 & $66-71$ & $5 / 12 / 06$ & ND & ND & ND & ND \\
\hline TI-25 & $72.2-77.2$ & $5 / 12 / 06$ & ND & ND & ND & ND \\
\hline TI-26 & $25-30$ & $5 / 12 / 06$ & 1.1 & $0.7 \mathrm{~J}$ & ND & ND \\
\hline TI-26 & $32-37$ & $5 / 11 / 06$ & 2.7 & 3.7 & ND & ND \\
\hline TI-26 & $39-44$ & $5 / 11 / 06$ & 1.0 & 3.5 & ND & ND \\
\hline TI-26 & $46-51$ & $5 / 12 / 06$ & 5.0 & 4.3 & ND & ND \\
\hline TI-26 & $53-58$ & $5 / 11 / 06$ & 4.8 & 3.1 & ND & ND \\
\hline TI-26 & $60-65$ & $5 / 12 / 06$ & 7.7 & 11 & ND & ND \\
\hline TI-26 & $66-71$ & $5 / 12 / 06$ & 3.2 & 8.1 & ND & ND \\
\hline TI-26 & $71.8-76.8$ & $5 / 12 / 06$ & ND & ND & ND & ND \\
\hline TI-27 & $25-30$ & $5 / 19 / 06$ & 1.0 & $0.7 \mathrm{~J}$ & ND & ND \\
\hline TI-27 & $30-35$ & $5 / 20 / 06$ & $1.1^{\mathrm{c}}$ & $2.3^{c}$ & $0.2 \mathrm{Jc}^{\mathrm{c}}$ & ND \\
\hline TI-27 & $35-40$ & $5 / 19 / 06$ & ND & 1.6 & ND & ND \\
\hline TI-27 & $42-47$ & $5 / 20 / 06$ & $7.9^{\mathrm{c}}$ & $3.5^{\mathrm{c}}$ & ND & ND \\
\hline TI-27 & $49-54$ & $5 / 20 / 06$ & $2.0^{c}$ & $0.7 \mathrm{Jc}^{\mathrm{c}}$ & ND & ND \\
\hline TI-27 & $56-61$ & $5 / 21 / 06$ & ND & $0.6 \mathrm{~J}$ & ND & ND \\
\hline TI-27 & $66.2-71.2$ & $5 / 20 / 06$ & ND & ND & ND & ND \\
\hline TI-28 & $25-30$ & $5 / 21 / 06$ & 2692 & 238 & 1.3 & 1.3 \\
\hline TI-28 & $32-37$ & $5 / 20 / 06$ & 3104 & 646 & 6.3 & 3.1 \\
\hline TI-28 & $37-42$ & $5 / 20 / 06$ & 97 & 91 & 1.0 & ND \\
\hline TI-28 & $44-49$ & $5 / 20 / 06$ & 88 & 14 & ND & $0.1 \mathrm{~J}$ \\
\hline TI-28 & $51-56$ & $5 / 19 / 06$ & 12 & 2.7 & ND & ND \\
\hline TI-28 & $58-63$ & $5 / 19 / 06$ & 15 & 8.9 & ND & ND \\
\hline TI-28 & $63-68$ & $5 / 19 / 06$ & ND & ND & ND & ND \\
\hline TI-29 & $25-30$ & $5 / 23 / 06$ & 39 & 39 & 2.6 & ND \\
\hline TI-29 & $32-37$ & $5 / 22 / 06$ & 75 & 55 & 4.2 & ND \\
\hline TI-29 & $39-44$ & $5 / 21 / 06$ & 269 & 87 & 3.9 & ND \\
\hline TI-29 & $46-51$ & $5 / 21 / 06$ & 182 & 86 & 3.3 & ND \\
\hline TI-29 & $53-58$ & $5 / 21 / 06$ & 18 & 31 & 1.5 & ND \\
\hline TI-29 & $60-65$ & $5 / 21 / 06$ & 9.4 & 21 & $0.9 \mathrm{~J}$ & ND \\
\hline TI-29 & $66.3-69.3$ & $5 / 21 / 06$ & 3.4 & 4.3 & ND & ND \\
\hline
\end{tabular}

a Qualifier J indicates an estimated concentration below the quantitation limit of $1.0 \mu \mathrm{g} / \mathrm{L}$.

b ND, not detected at a method detection limit of $0.1 \mu \mathrm{g} / \mathrm{L}$.

c Cross-contamination possible. See discussion in Supplement 4, Section S4.1.3. 
TABLE 3.5 Summary of results of organic analyses of well samples and vertical-profile groundwater samples collected with the cone penetrometer at locations downgradient or upgradient from the targeted areas during the 2006 investigation at Navarre, Kansas.

\begin{tabular}{|c|c|c|c|c|c|c|}
\hline \multirow[b]{2}{*}{ Location } & \multirow[b]{2}{*}{$\begin{array}{c}\text { Depth } \\
\text { (ft below TOC) }\end{array}$} & \multirow[b]{2}{*}{$\begin{array}{l}\text { Sample } \\
\text { Date }\end{array}$} & \multicolumn{4}{|c|}{ Concentration $(\mu \mathrm{g} / \mathrm{L})$} \\
\hline & & & $\begin{array}{c}\text { Carbon } \\
\text { Tetrachloride }\end{array}$ & Chloroform & $\begin{array}{l}\text { Methylene } \\
\text { Chloride }\end{array}$ & $\begin{array}{c}\text { Tetra- } \\
\text { chloroethene }\end{array}$ \\
\hline \multicolumn{7}{|c|}{ Monitoring wells } \\
\hline KDHE-1 & $35-55$ & $4 / 7 / 06$ & $0.1 \mathrm{Ja}$ & $N D^{b}$ & ND & ND \\
\hline MW1 & $43-58$ & $4 / 5 / 06$ & 59 & 12 & ND & ND \\
\hline MW2 & $42.8-57.8$ & $4 / 6 / 06$ & 27 & 7.7 & ND & ND \\
\hline MW3 & $44-59$ & $4 / 6 / 06$ & 83 & 20 & ND & $0.1 \mathrm{~J}$ \\
\hline MW4 & $45-60$ & $4 / 6 / 06$ & 194 & 21 & ND & ND \\
\hline MW5 & $78-88$ & $5 / 25 / 06$ & $0.4 \mathrm{~J}$ & ND & ND & ND \\
\hline NW-3 & $38-48$ & $5 / 25 / 06$ & 34 & 2.0 & ND & ND \\
\hline $\mathrm{L}-1$ & $75-95$ & $4 / 8 / 06$ & 54 & 11 & ND & ND \\
\hline L-2 & $80-90$ & 4/6/06 & $0.2 \mathrm{~J}$ & ND & ND & ND \\
\hline L-3 & $80-90$ & $4 / 7 / 06$ & ND & ND & ND & ND \\
\hline $\mathrm{T} 1$ & $40-60$ & $4 / 7 / 06$ & 118 & 20 & ND & ND \\
\hline \multicolumn{7}{|c|}{ Domestic well } \\
\hline Anderson & $68^{d}$ & $5 / 23 / 06$ & 36 & 6.0 & ND & ND \\
\hline Anderson & $68^{d}$ & $5 / 24 / 06$ & 20 & 3.5 & ND & ND \\
\hline Anderson & $68^{d}$ & $5 / 25 / 06$ & 17 & 2.7 & ND & ND \\
\hline \multicolumn{7}{|c|}{ Cone penetrometer locations } \\
\hline TI-20 & $35-40$ & $5 / 22 / 06$ & 15 & 5.4 & ND & ND \\
\hline TI-20 & $42-47$ & $5 / 24 / 06$ & 13 & 3.4 & ND & ND \\
\hline TI-20 & $56-61$ & $5 / 23 / 06$ & 7.8 & 7.4 & ND & ND \\
\hline TI-20 & $72-77$ & $5 / 23 / 06$ & ND & ND & ND & ND \\
\hline TI-30 & $32-37$ & $5 / 25 / 06$ & $0.4 \mathrm{~J}$ & $0.5 \mathrm{~J}$ & ND & ND \\
\hline TI-30 & $39-44$ & $5 / 23 / 06$ & 1.1 & $0.4 \mathrm{~J}$ & ND & ND \\
\hline TI-30 & $43.8-48.8$ & $5 / 23 / 06$ & 2.5 & $0.9 \mathrm{~J}$ & ND & ND \\
\hline TI-30 & $53-58$ & 5/23/06 & 62 & 6.9 & ND & ND \\
\hline TI-30 & $60-65$ & $5 / 23 / 06$ & 43 & 5.5 & ND & ND \\
\hline TI-30 & $75-80$ & $5 / 23 / 06$ & ND & ND & ND & ND \\
\hline TI-30 & $83.5-88.5$ & $5 / 24 / 06$ & ND & ND & ND & ND \\
\hline TI-31 & $42-47$ & $5 / 25 / 06$ & 30 & 4.3 & ND & ND \\
\hline TI-31 & $53-58$ & $5 / 25 / 06$ & 91 & 14 & ND & ND \\
\hline TI-31 & $60-65$ & $5 / 25 / 06$ & 28 & 3.4 & ND & ND \\
\hline TI-31 & $70.7-75.7$ & $5 / 24 / 06$ & ND & ND & ND & ND \\
\hline
\end{tabular}

a Qualifier J indicates an estimated concentration below the quantitation limit of $1.0 \mu \mathrm{g} / \mathrm{L}$.

b ND, not detected at a method detection limit of $0.1 \mu \mathrm{g} / \mathrm{L}$.

c Sample from 1-in. piezometer installed in 2006 at the TI-30 location.

d Total depth. 


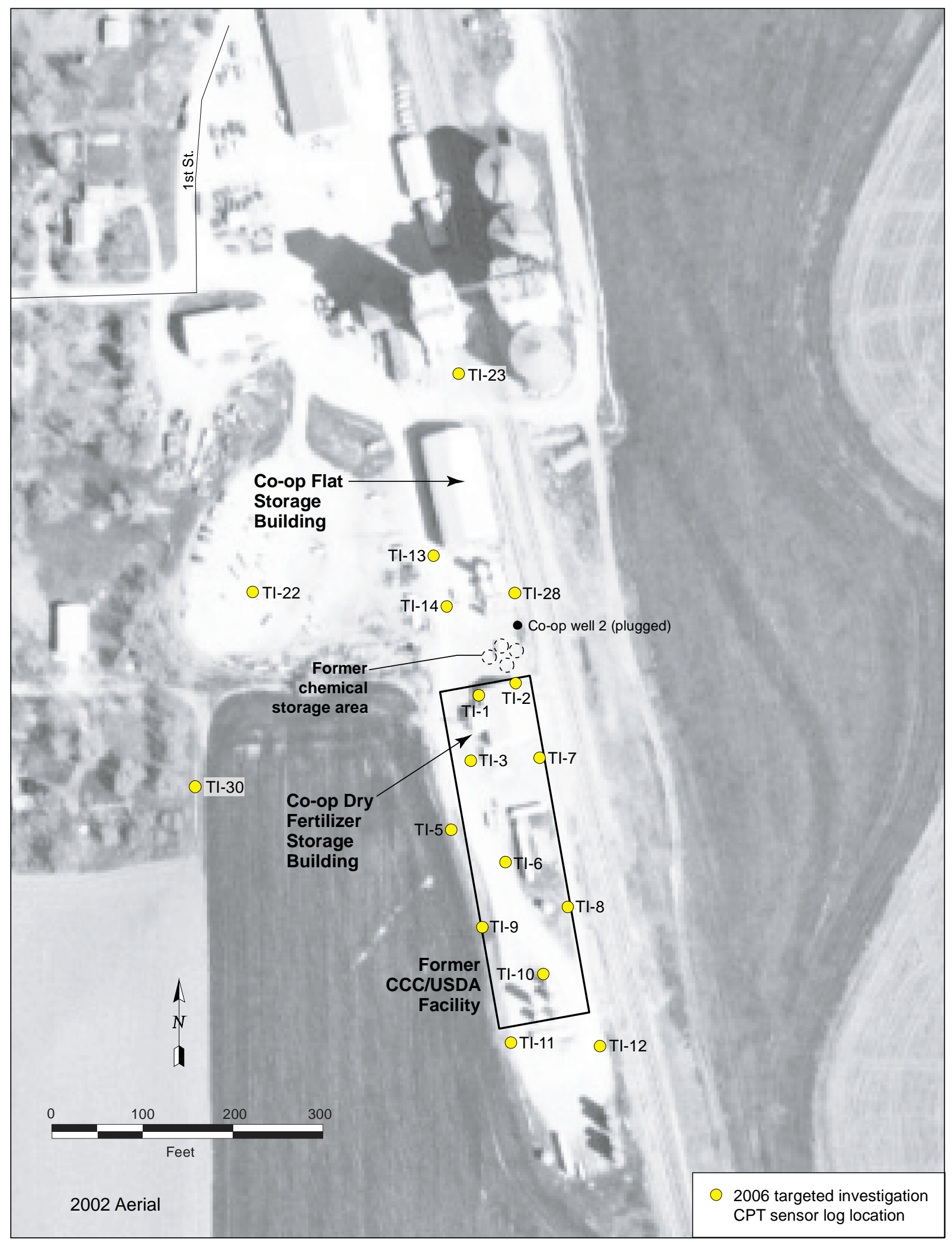

FIGURE 3.1 Cone penetrometer electronic sensor logging locations. Source of photograph: NAIP (2002). 


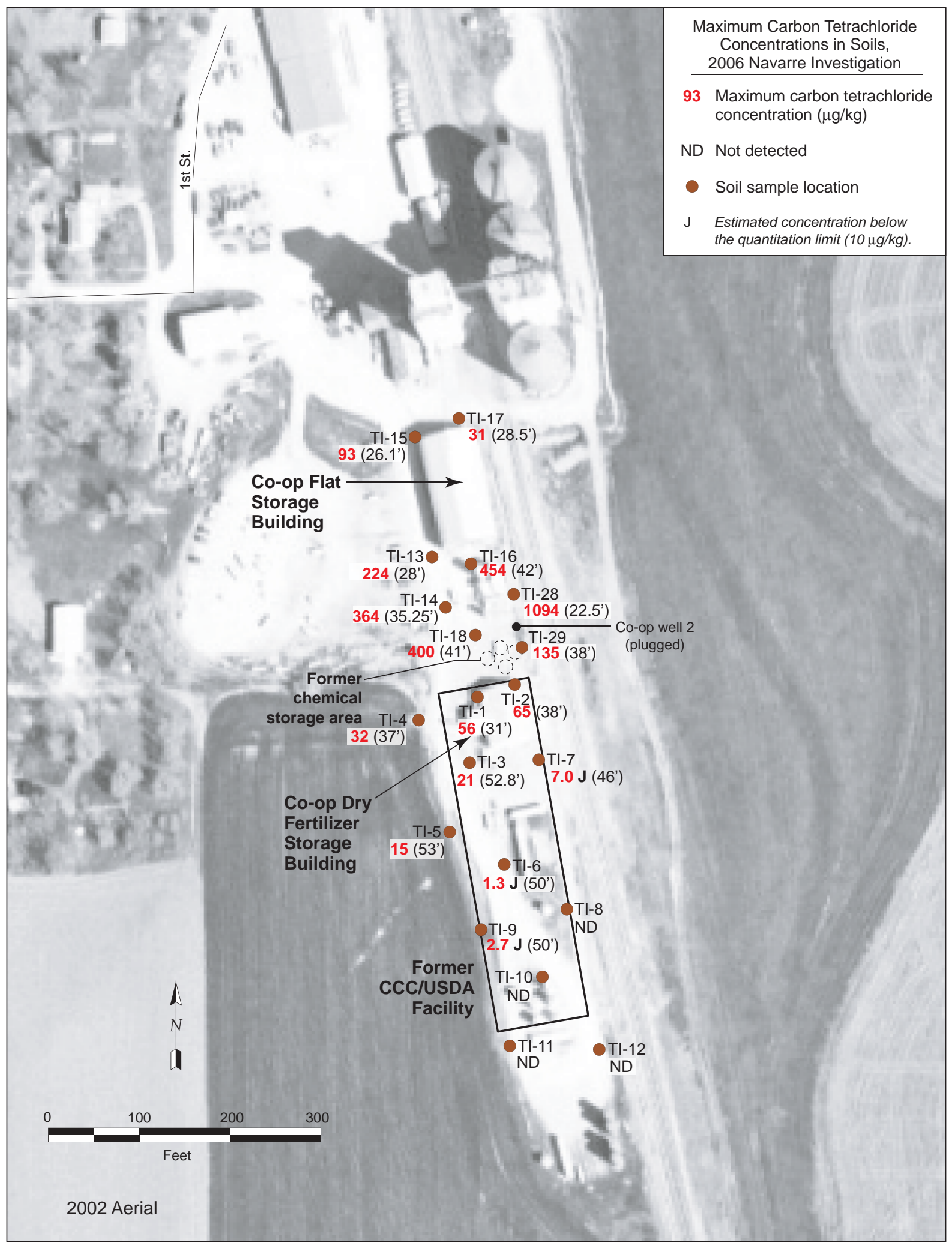

FIGURE 3.2 Maximum carbon tetrachloride concentrations in soil samples. Source of photograph: NAIP (2002). 


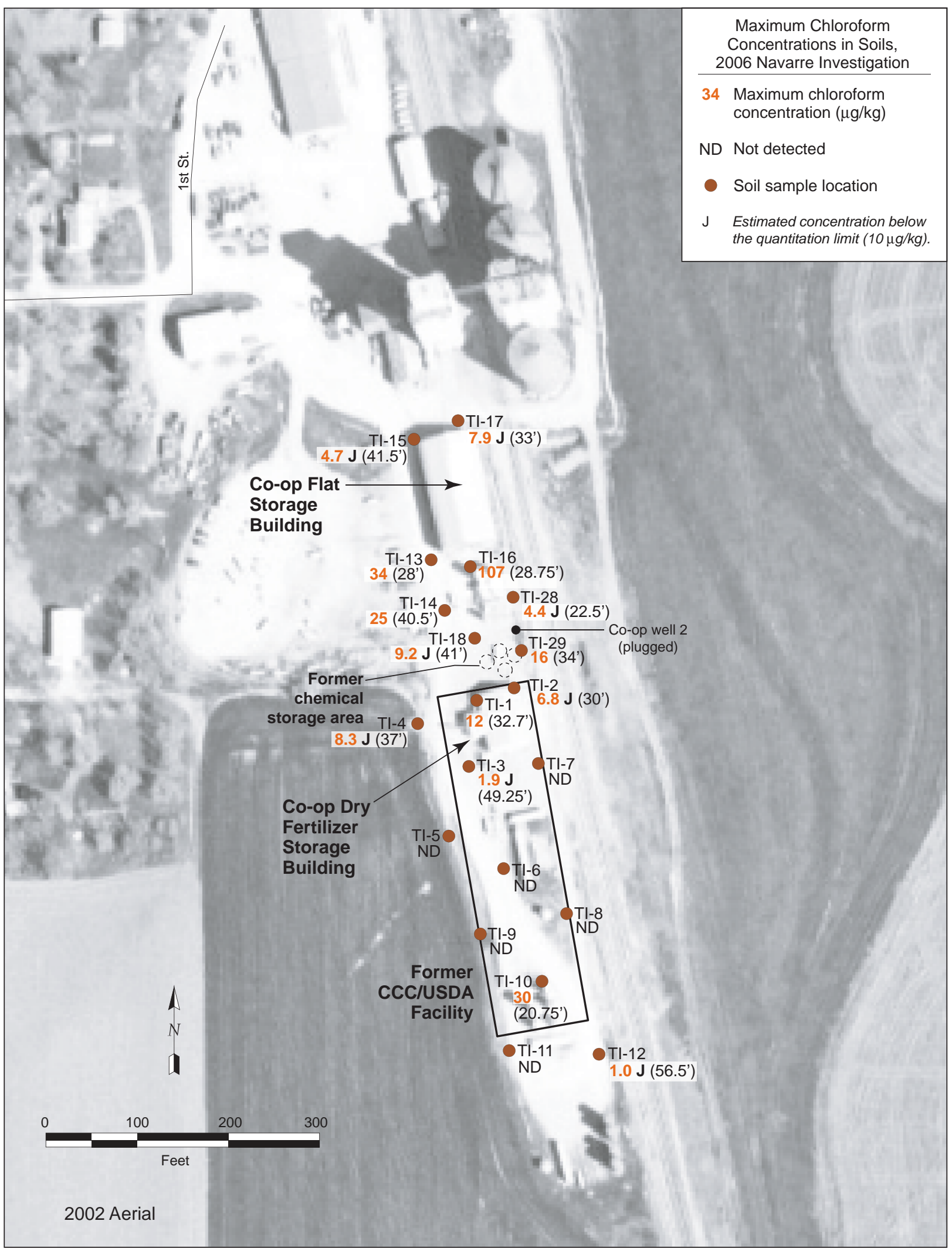

FIGURE 3.3 Maximum chloroform concentrations in soil samples. Source of photograph: NAIP (2002). 


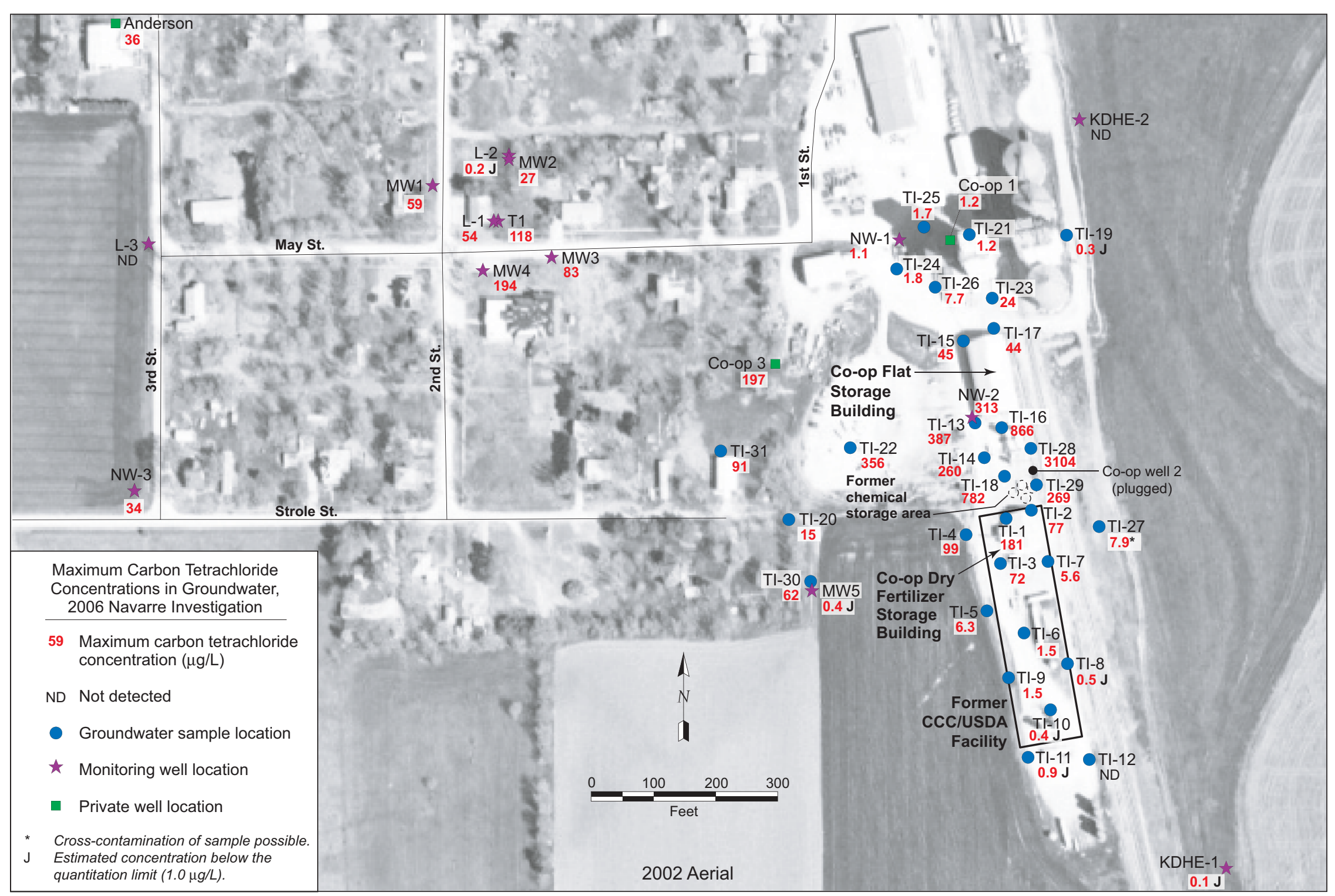

FIGURE 3.4 Maximum carbon tetrachloride concentrations in groundwater samples. Source of photograph: NAIP (2002). 


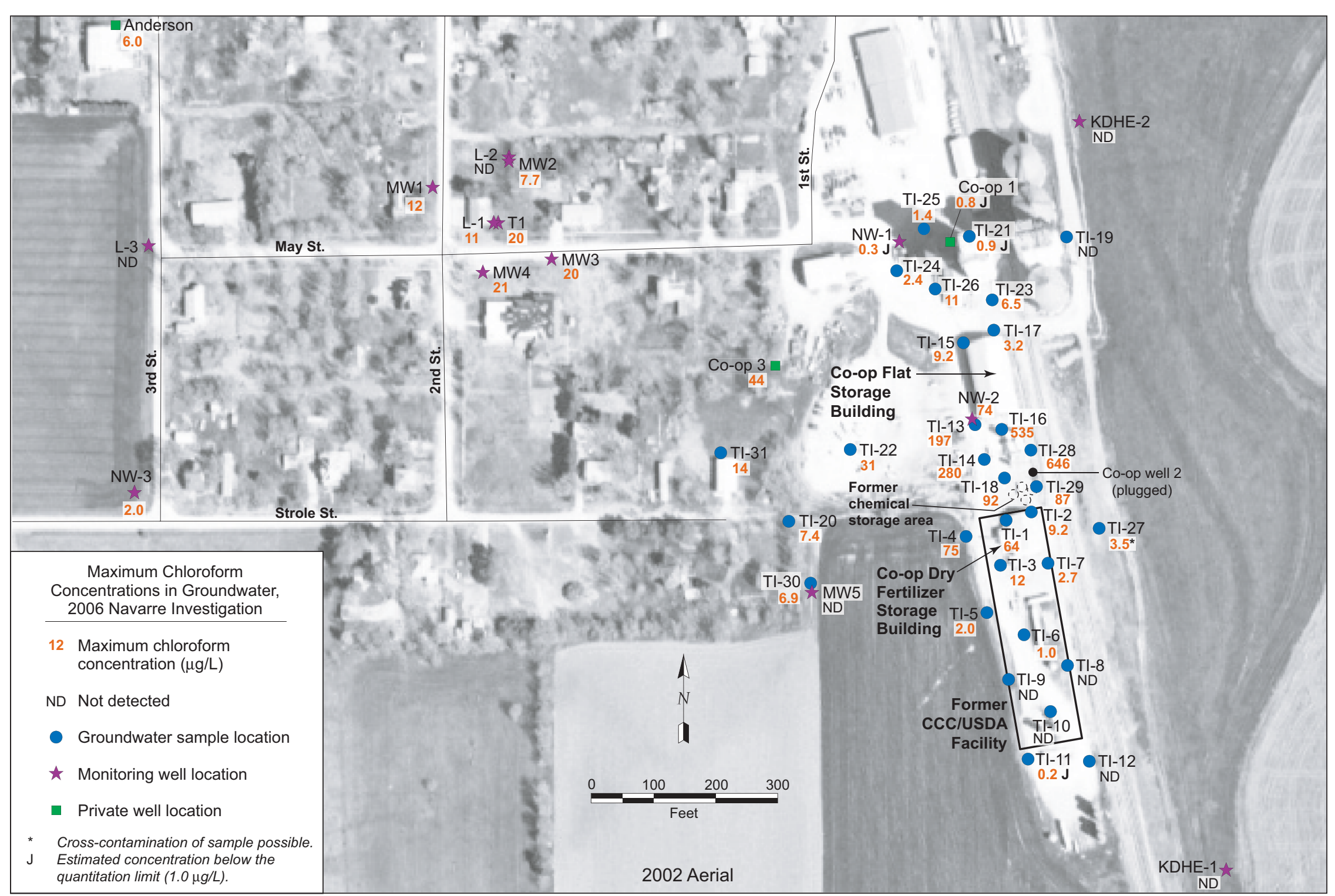

FIGURE 3.5 Maximum chloroform concentrations in groundwater samples. Source of photograph: NAIP (2002). 


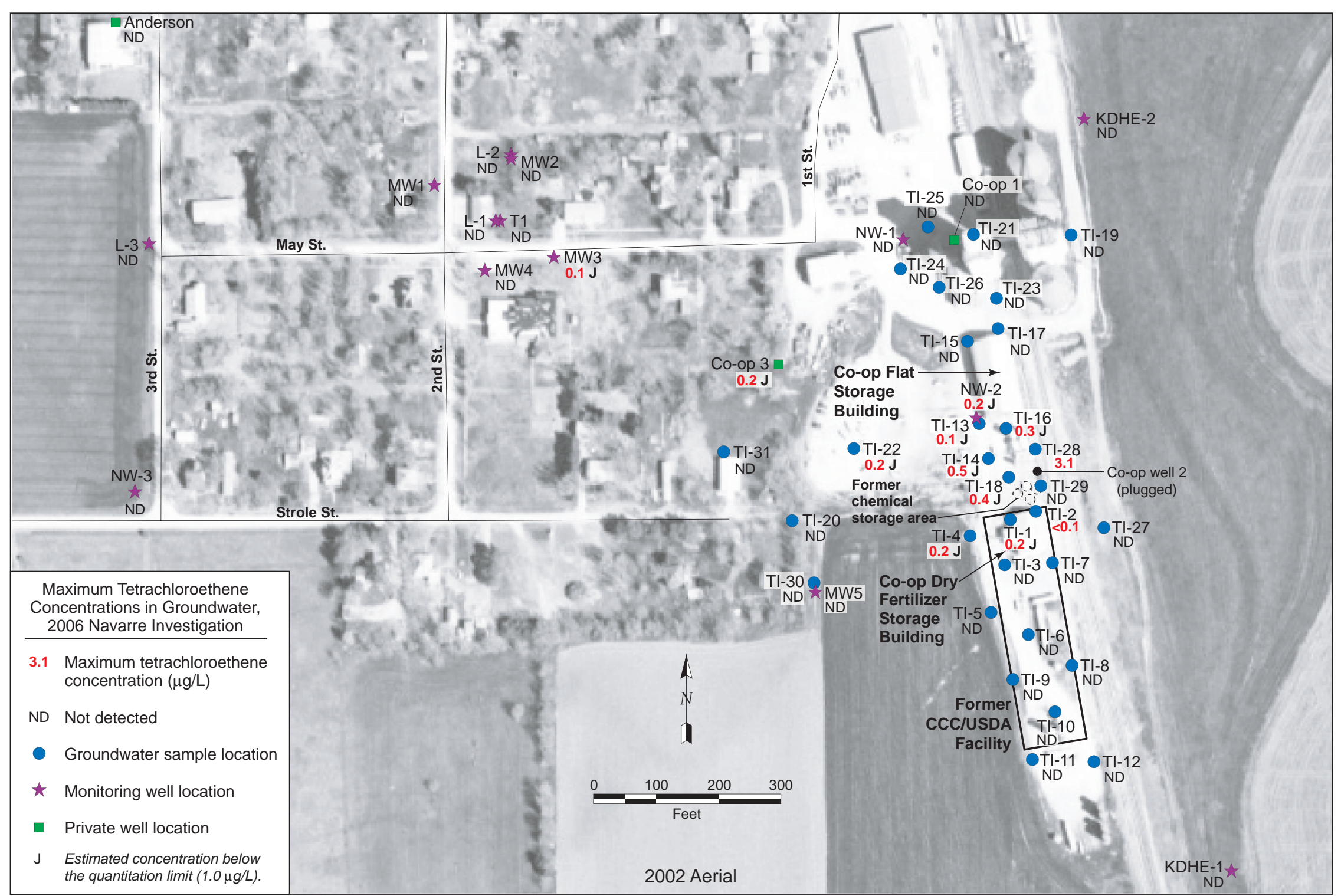

FIGURE 3.6 Maximum tetrachloroethene concentrations in groundwater samples. Source of photograph: NAIP (2002). 


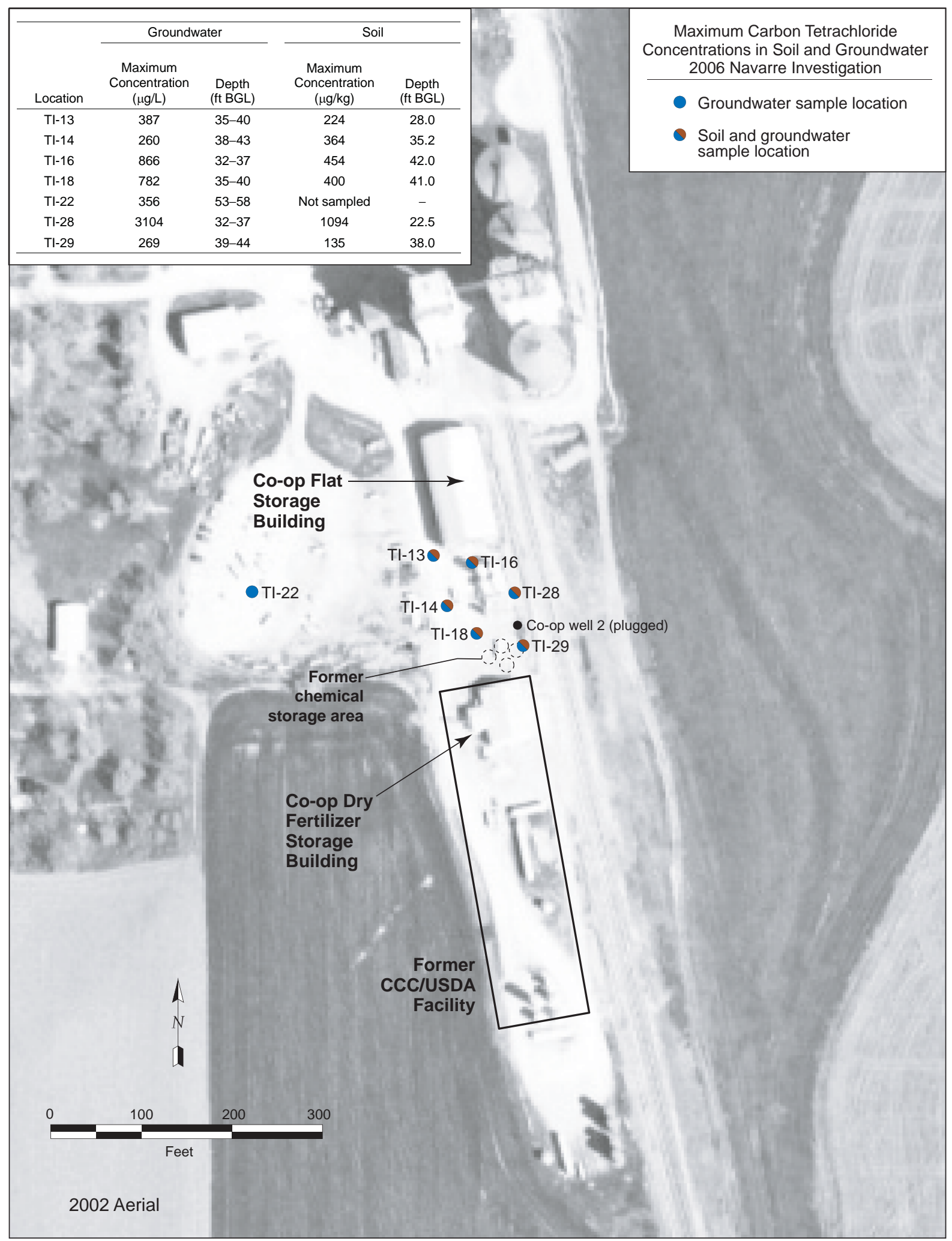

FIGURE 3.7 Maximum carbon tetrachloride concentrations in soil and groundwater samples, with sample depths, in the area of greatest contamination. Source of photograph: NAIP (2002). 


\section{Data Interpretation}

The 2006 investigation at Navarre was designed to accomplish two primary goals: (1) investigate specific areas of concern for potential sources that may be associated with the past use of carbon tetrachloride and (2) evaluate migration pathways for previously identified carbon tetrachloride contamination in groundwater. The task of accomplishing these goals included meeting the three technical objectives detailed in the KDHE-approved work plan (Section 1.1) and conducting further investigation at several locations on the former CCC/USDA property and on the Co-op property. The primary goals and technical objectives were met.

A source is the place or object where the contaminant of concern is released, and source areas are zones of highest contaminant concentrations in the soil or groundwater, or both (EPA 2007). Soil and groundwater analytical data collected during the Navarre investigation identified two primary source areas, both on the Co-op property. The primary source areas identified are as follows (Figure 4.1):

- The vicinity of the south door of the Co-op's flat storage building.

- The former well Co-op 2 and the area around the well that was formerly used for chemical storage.

The flat storage building was constructed in 1958. The identification of a location near this building as a source area is consistent with information in the Co-op's August 31, 2004, work plan (iSi 2004), which indicates that carbon tetrachloride was used by the Co-op until the mid 1980s and was used on several occasions at the flat storage building in the 1970s.

Former well Co-op 2 was used for chemical mixing and equipment washing (Warders 2000) until it was plugged on September 5, 1991 (KGS 2007). The date of this well's construction is not known; however, it may have been installed by the railroad prior to the Co-op's use of the area in which it was located (Stroda 2007). The former chemical storage area was used to store fertilizers.

Interpretations of the analytical data supporting these findings are presented in Section 4.2. 


\subsection{Site Geology and Hydrogeology}

\subsubsection{Site Geology}

The site lithology and potential migration pathways were evaluated by identifying soil types in samples collected during probing activities, evaluating CPT electronic sensor data, and conducting grain size analyses on selected soil samples. The soil samples and the electronic sensor data were evaluated by Kansas licensed geologists, and grain size analyses were conducted by a laboratory certified for geotechnical testing. Selected lithologic logs and complete electronic sensor data are in Appendix A. The grain size analysis results are in Table S1.2 in Supplement 1. The lithologic data were further compiled and are depicted on two generalized geologic cross sections constructed for the locations shown in Figure 4.2. The cross sections are shown in Figure 4.3 (north-south; A-A') and Figure 4.4 (west-east; B-B').

The predominant lithology observed in all boreholes consisted of unconsolidated zones of silty clay and silty sandy clay with intermixed layers of sandy silt, silty sand, clayey sand, silty gravelly sand, sand, and silt. Also observed in most boreholes were calcite crystals with vuggy porosity, intermixed throughout the lithologic column. Bedrock consisting of sandstone and limestone fragments was encountered in three boreholes near the dry fertilizer building.

The northern and southern portions of the site show thicker and more persistent zones of silty clay and silty sandy clay with minor zones of silty gravel clay and clayey sand and sand. This pattern is evident at TI-17, TI-16, and TI-28 on the Co-op property and at TI-12 and TI-8 on and adjacent to the former CCC/USDA property (Figure 4.3). The silty clay and clayey silt layers that predominate at TI-17, TI-16, TI-28, and TI-8 would likely slow contaminant migration. The highest carbon tetrachloride concentrations detected in the soil and groundwater were within the silty clay zones at TI-16 and TI-28.

In the central portion of the site, near former well Co-op 2 and the dry fertilizer building, lithology was more variable, with thicker zones of silty sandy clay with gravel or limestone fragments, clayey sand, and thin sandstone layers. This type of lithology, which is evident at TI-29, TI-2, and TI-7 (Figure 4.3) and at TI-1 (Figure 4.4) typically exhibits higher permeability that could facilitate contaminant migration. This central portion of the site with more variable lithology is also near the identified sources at former well Co-op 2 and the former chemical storage area. 
Thin layers of consolidated and semi-consolidated material were observed in four boreholes near the dry fertilizer building. These layers consisted of sandstone and caliche (zones of white to lighter colored very fine grained hard calcium carbonate deposits). A thin layer of poorly cemented, fragmented sandstone with occasional limestone fragments was encountered along the border between the former CCC/USDA property and the Co-op property (TI-2 and TI-4; Figure 4.4) and on the south side of the dry storage building at TI-3. Sandstone was encountered at 11-11.2 ft BGL at TI-2, at 25.5-26 ft BGL at TI-3, and at 34.8-35.2 ft BGL and 61-61.2 ft BGL at TI-4. These thin layers do not appear to be continuous throughout the area and are above the saturated zone at all locations except TI-4. Caliche was observed in three boreholes at depths below the water table: $59.8 \mathrm{ft}$ BGL at TI-1, at 44.8-46 ft BGL at TI-3, and at 44.5-46.5 ft BGL and 61.2-62 ft BGL at TI-4. These sandstone and caliche layers typically have lower permeability that could slow contaminant migration.

Limited comparable lithologic units continue across the area of the investigation. The most prevalent units are the silty clay, clayey silts, and the silty sandy clay layers that are evident at varying depths in all boreholes. No definitive lithologic data generated in the 2006 investigation would indicate the presence of a continuous confining layer separating the waterbearing zone into two distinct units. The data indicated that only one aquifer is present throughout the area investigated. This interpretation is evident from soil core data, which showed only limited thin layers of consolidated material, as well as the fact that groundwater samples were collected continuously throughout the water column to CPT refusal.

On the former CCC/USDA property, the shallowest depth to refusal was $56 \mathrm{ft}$ BGL at TI-3, and the deepest was $77.8 \mathrm{ft}$ BGL at TI-5. On the Co-op property, refusal occurred between $65 \mathrm{ft}$ BGL (at TI-17 and TI-21) and $77.2 \mathrm{ft}$ BGL (at TI-25). At borehole locations downgradient from the former CCC/USDA and Co-op properties, the deepest groundwater sample was collected at TI-30, at a depth of $88.5 \mathrm{ft} \mathrm{BGL}$.

The vertical and horizontal distribution of carbon tetrachloride in soil and groundwater at Navarre is displayed on generalized geologic cross sections A-A' (north-south; Figure 4.3) and B-B' (west-east; Figure 4.4). The cross sections show that the highest concentrations of carbon tetrachloride were detected in the silty clay and clayey silt layers at the areas near (1) former well Co-op 2 and the former chemical storage area (CPT location TI-28) and (2) the south door of the flat storage building (CPT location TI-16). These areas have been used intensely by the Co-op 
for storing, handling, and mixing of chemicals. Lower concentrations of carbon tetrachloride were also observed in silty gravel clay layers and to a lesser extent in silty sandy clay layers.

The depths of the highest carbon tetrachloride concentrations in groundwater were correlated with the depths of the highest soil concentrations. The highest soil concentrations were detected in the capillary fringe and at the groundwater interface in boring TI-28. The highest groundwater concentrations were detected in the upper 10-12 ft of the saturated column encountered in borings TI-16 and TI-28, at roughly 25-40 ft BGL.

The high concentrations detected in the deeper vadose zone soil samples and in the upper part of the saturated column (at roughly 25-40 ft BGL) at locations TI-16 and TI-28 indicate the presence of an ongoing soil source. An additional significant finding is nearly complete absence of carbon tetrachloride in soil samples collected in the shallow vadose zone (shallower than a depth of approximately $20 \mathrm{ft}$ BGL), except for a trace amount at $3.5 \mathrm{ft}$ BGL at TI-16. These findings are consistent with a migration pathway involving introduction of carbon tetrachloride into the subsurface through a conduit such as former well Co-op 2, with subsequent lateral movement of the contaminant to groundwater.

\subsubsection{Site Hydrogeology}

\subsubsection{Regional and Local Geologic and Hydrogeologic Background}

Navarre is located on a local surface drainage divide trending approximately north-south, at an average elevation of about 1,350 ft above mean sea level (AMSL). Drainage east of the town is toward Carry Creek (trending north-south) and several small, incised feeder streams to Carry Creek (trending east-west). Drainage to the west is toward Turkey Creek (trending northsouth, approximately $4 \mathrm{mi}$ west of the town), again along with several small feeder streams trending east-west.

Navarre lies within the Flint Hills physiographic province. The Permian bedrock units that underlie this area dip to the west, but they have been regionally eroded to form an eastwardsloping paleosurface. This paleosurface is now undergoing dissection by modern streams. Regional stratigraphic studies (Lee 1956; Moore et al. 1951) suggest that individual units consisting predominantly of shale and limestone within the Lower Permian bedrock have 
average thicknesses of 10-30 ft in the Navarre area. The water-bearing unit of concern at Navarre consists of variably to highly weathered sediments of the Permian Wellington Formation.

Estimates of regional dip ranging from $15 \mathrm{ft} / \mathrm{mi}$ to $35 \mathrm{ft} / \mathrm{mi}$ (due east to west) have been reported for the greater Flint Hills (Moore et al. 1951; Myers and Bigsby 1989). On this basis, local topographic relationships suggest that the groundwater-bearing unit identified at Navarre (or its lateral stratigraphic equivalents) is likely to have been penetrated by erosion along Turkey Creek, Carry Creek, and several of their associated feeder streams, thus forming natural hydraulic boundaries marking the potential maximum extent of groundwater migration to the east, west, or north from the vicinity of the town. Numerous springs identified at the headwaters of several of the streams trending east-west and feeding into Carry Creek are consistent with inferred groundwater discharge to the surface, within approximately $1 \mathrm{mi}$ east of the town.

Groundwater in the Flint Hills region of Dickinson County in general is obtained from more permeable horizons within the Permian bedrock units. The production capacity of registered wells in the vicinity of Navarre that are completed in these units is variable but generally low (5-25 gpm). Testing performed by Argonne (1993) suggested a potential long-term yield for well $\mathrm{T} 1$, installed near the center of the town, of roughly 4.5-9 gpm. The well was pumped for the Argonne test at $5.7 \mathrm{gpm}$.

At least 40 private wells have reportedly been drilled within the town of Navarre. Records for these wells are largely unavailable; however, information obtained by the KDHE for a limited number of wells (KDHE 1998a) suggests that private well depths may range from about $30 \mathrm{ft}$ to $85 \mathrm{ft}$. This range is generally comparable to the depth range represented by the screened intervals in the existing monitoring well network at the site (including wells MW5, L-1, L-2, and L-3). The KDHE findings indicate that most of the private wells and the monitoring wells are screened at depths shallower than $80 \mathrm{ft}$ BGL.

Several previous investigations have been performed to estimate hydraulic parameters for the water-bearing unit at Navarre. These include attempted pumping tests by Argonne in both the shallower and deeper portions of the saturated zone (Argonne 1993, 1995), slug testing of the existing monitoring wells and interpretation of the resulting data by Papadopulos (2001), and subsequent reinterpretation of the latter slug test data by Argonne. The quantitative results of these efforts have yielded estimated hydraulic conductivities ranging from $<1 \mathrm{ft} / \mathrm{day}$ to approximately $18 \mathrm{ft} /$ day for the shallower portion of the water-bearing unit $(\leq 65 \mathrm{ft} \mathrm{BGL})$, and 
approximately $36 \mathrm{ft} /$ day for the deeper interval screened at L-3 (79-89 ft BGL). The results for the monitoring wells grouped near the center of town (MW1-MW4, T1) showed considerable variation; the results of the analyses together suggest that the permeability distribution at Navarre is probably heterogeneous over relatively short distances, both laterally and vertically.

\subsubsection{Results of the Groundwater Level Monitoring}

To obtain detailed information on the patterns of groundwater level variations and flow at Navarre, groundwater levels were measured continuously in a suite of 12 monitoring wells (L-1, L-2, L-3, MW1, MW2, MW3, MW4, NW-1, NW-2, NW-3, KDHE-1, and KDHE-2) by using downhole pressure sensors with automatic data loggers. The loggers were programmed to collect one measurement every $4 \mathrm{hr}$. Periodically, water levels were also measured by hand in all of the monitoring wells at the site, by using an electronic drop line.

The results of the continuous water level monitoring are summarized in Figure 4.5. Complete data from the water level measurement activities in April to November 2006 are in Supplement 3 (on CD).

Figure 4.5 presents the hydrographs generated from the data logger records. With the exception of well L-3 (see below), the groundwater level traces for all of the monitored wells at Navarre are very similar in general morphology, showing a relatively slow, steady decline in levels throughout the spring and early summer of 2006, followed by a brief rebound in late August. The groundwater levels at the site subsequently remained fairly stable, declining only slightly during the fall and early winter of 2006. A maximum fluctuation in static water levels of approximately $3 \mathrm{ft}$ to $4 \mathrm{ft}$ was observed at a majority of the wells during the monitoring period.

Monthly rainfall totals for Enterprise, Kansas (approximately $7 \mathrm{mi}$ north of Navarre), indicate that relatively average precipitation was received in the Navarre area during the period of declining water levels prior to August 2006 (Table 4.1). More detailed precipitation records for Enterprise are not available; however, daily precipitation data for Manhattan, Kansas (approximately $35 \mathrm{mi}$ northeast of Navarre), are available (Table 4.2). The Manhattan precipitation data are compared in Figure 4.6 to the water level traces for selected monitoring wells at Navarre. Rainfall in April-July 2006 was received in numerous relatively small events, each yielding less than 1.5 in. of rain. In contrast, Figure 4.6 shows that the water level rebounds 
(by up to $2 \mathrm{ft}$ or more) observed at Navarre in August 2006 occurred in response to several heavy rains over a period of approximately 3 weeks. The available data for Enterprise and Manhattan suggest that Navarre received from 9 in. to 11 in. of rain during August 2006.

Figure 4.6 suggests that the apparent groundwater level responses to rainfall events are the greatest at wells NW-1, NW-2, and KDHE-2, on and near the northern portion of the Co-op property. This observation is consistent with a hypothesis that the greater responses reflect, in part, more effective vertical infiltration of precipitation in the northern portion of the Co-op property than in the remainder of the study area - due to the relatively large, flat areas of coarse surface gravel and the absence of vegetation in this portion of the property.

Argonne has previously identified static water levels that were both significantly lower (approximately 1,310 ft AMSL at MW1-MW4, in August 1992), and higher (approximately 1,325 $\mathrm{ft}$ at MW1-MW4, in May 1993) at Navarre than those observed during the current monitoring period. This pattern suggests that the recent data do not reflect the full range of groundwater levels that might have historically existed during (and following) the CCC/USDA grain storage activities at this site. Argonne water level monitoring also documented a rise of approximately $8 \mathrm{ft}$ in groundwater levels at MW1-MW4 in response to approximately $8.5 \mathrm{in}$. of rain over a 30-day period in April and May 1993, demonstrating that water level fluctuations can be greater and can occur more rapidly than those observed during the recent monitoring (Argonne 1995).

The hydrograph for monitoring well L-3 (Figure 4.5), located at the western edge of Navarre, depicts numerous large, downward "spikes" that appear to be superimposed on the common "background" pattern of water level variations observed at the other monitoring locations. Coincident spikes (of much smaller magnitude) are also apparent in the water level traces for almost all of the other wells, with the possible exceptions of KDHE-1 and KDHE-2. Additional small spikes in the traces for MW1, MW2, MW3, MW4, NW-1, and NW-2 are coincident with each other but seem to be unrelated to those detected at L-3. These observations strongly suggest the following:

1. Both vertical and lateral hydraulic communication exists throughout much, if not all, of the groundwater-bearing unit at Navarre. This communication is attributable to natural permeable hydraulic pathways, further facilitated by 
artificial pathways created by the continuous gravel packs or dual screens installed in some of the 40 or more private wells reported in Navarre.

2. Groundwater levels across much of the investigation site were influenced during the monitoring period by drawdown associated with the pumping of one (or more) nearby wells. As of this report, the well(s) responsible for the observed pumping effects have not been identified; however, there are no known large-capacity irrigation wells or other wells in the vicinity of the town.

Many of the prominent drawdown events identified at well L-3 began either in the early morning or mid to late afternoon and lasted from $8 \mathrm{hr}$ to $24 \mathrm{hr}$ or longer, although no regular pattern or time frame for water usage can be identified. Water level recovery to near-static levels appeared to require from 3-4 days at well L-3 following many of the individual drawdown events. These events occurred most frequently during the spring and early summer months of 2006, coinciding with the general decline in water levels observed across the site. Frequent pumping apparently ended following the heavy rains in late August 2006, because the groundwater levels briefly rebounded then remained fairly stable thereafter. These observations suggest that the frequent pumping might have contributed to the widespread decline in groundwater levels observed at Navarre during the spring and early summer of 2006. Nevertheless, a clear cause-and-effect relationship between the pumping and the groundwater level decline cannot be conclusively identified from the existing data.

Groundwater level measurements made by hand on April 24-25 and November 28, 2006, and values extracted from the data logger records for August 11 and September 23, 2006, were used to estimate the patterns of groundwater flow at Murdock under spring, summer, fall, and winter conditions. An additional data set for August 31, 2006, was also selected to illustrate the groundwater response to the late August rainfall/recharge event described above. The data were mechanically contoured to generate the potentiometric surface diagrams in Figure 4.7 (April 24-25), Figure 4.8 (August 11), Figure 4.9 (August 31), Figure 4.10 (September 23), and Figure 4.11 (November 28).

Figures 4.7-4.11 indicate that relatively little apparent change occurred in the direction of groundwater flow throughout the monitoring period, despite the fluctuations in groundwater elevations shown in Figure 4.5. Groundwater movement beneath the town to the west of the 
former CCC/USDA facility and the Co-op is predominantly toward the west or slightly northwest. The figures suggest that the hydraulic gradient driving groundwater movement increases slightly to the west under all of the observed flow conditions.

Figures 4.9 (August 31) and 4.10 (September 23) depict the groundwater conditions at the site, respectively, at the peak of the late August 2006 rainfall/recharge event and shortly after a smaller rainfall event in late September 2006. The slight "bowing" of the groundwater levels toward the west (suggested in each of these diagrams in the vicinity of the Co-op property) is qualitatively consistent with the hypothesis of preferential recharge to the saturated zone described above. The hydrographs in Figure 4.5 indicate that such effects are expected to be transient and relatively short lived.

A manual groundwater level measurement was available at monitoring well MW5 for the November 28, 2006, data set only. The resulting potentiometric surface (Figure 4.11) suggests a region of reduced hydraulic gradient and a slightly more northwestward component of groundwater flow in the vicinity of the former CCC/USDA facility.

\subsection{Source Area Identification}

The 2006 investigation at Navarre targeted several locations on the Co-op property and the former CCC/USDA property. The goal was to identify potential source areas and contaminant migration pathways. Activities to accomplish this goal included collection of soil samples from 20 CPT boreholes (TI-1 through TI-18, TI-28, and TI-29) and groundwater samples at 26 CPT locations (TI-1 through TI-19, TI-21, TI-23 through TI-26, TI-28, and TI-29). All investigated locations were near previously identified potential source areas (Figure 4.1).

Two source areas were identified at CPT boreholes TI-16 and TI-28 (Figure 4.12). Borehole TI-16 is at the door on the south side of the flat storage building. Borehole TI-28 is adjacent to the former location of well Co-op 2 and chemical storage areas on the Co-op property. Analytical data from CPT boreholes TI-13, TI-14, and TI-18 further substantiate the presence of a source in this area. Contaminant concentrations in both soil and groundwater decrease significantly at locations north, south, and west of the TI-16 and TI-28, consistent with origination of the contamination at and near these locations. Both TI-16 and TI-28 are near the 
former well Co-op 2, which could have functioned as a conduit for vertical contaminant migration.

Analytical data for soil and groundwater show that the area between the south end of the flat storage building (location TI-16 in cross section A-A'; Figure 4.3) and the north end of the dry fertilizer building (location TI-2 in Figure 4.3) exhibited the highest concentrations of carbon tetrachloride detected during the 2006 investigation. The groundwater data, coupled with the soil data, further indicate that the contamination detected in this area could have been introduced into the subsurface through a conduit such as former well Co-op 2.

The area between the flat storage building and the dry fertilizer building is owned by the Co-op and has been intensely used for chemical storage, handling, and mixing throughout the history of Co-op operation in this area. The CCC/USDA has never operated on or leased this area of the Co-op. Furthermore, the Co-op's southern property boundary has expanded over time to include all of the former CCC/USDA property (Figure 4.13). By 1969 the Co-op owned the entire northern half of the former CCC/USDA property, and by 1975 the Co-op owned the entire former CCC/USDA property. The Co-op used carbon tetrachloride as a grain fumigant at its facility until the mid 1980s (iSi 2004).

\subsubsection{Evidence from Groundwater Data}

Groundwater samples collected at TI-28 showed carbon tetrachloride concentrations of $2,692 \mu \mathrm{g} / \mathrm{L}$ at a depth of 25-30 ft BGL and 3,014 $\mu \mathrm{g} / \mathrm{L}$ at 32-37 ft BGL. These results represent the highest contaminant levels detected at all groundwater sampling locations during the 2006 investigation. As s Figure 4.3 shows, the concentrations at location TI-28 decreased significantly with depth (Table 3.4). The following pattern of high concentrations in the shallower zones and decreasing concentrations with depth at TI-28 indicates the presence of a carbon tetrachloride source in the immediate vicinity of this location:

- $2,692 \mu \mathrm{g} / \mathrm{L}$ at $25-30 \mathrm{ft}$ BGL

- $\quad 3,104 \mu \mathrm{g} / \mathrm{L}$ at $32-37 \mathrm{ft}$ BGL

- $\quad 97 \mu \mathrm{g} / \mathrm{L}$ at $37-42 \mathrm{ft}$ BGL 
- $\quad 88 \mu \mathrm{g} / \mathrm{L}$ at $44-49 \mathrm{ft}$ BGL

- $\quad 12 \mu \mathrm{g} / \mathrm{L}$ at $51-56 \mathrm{ft}$ BGL

- $\quad 15 \mu \mathrm{g} / \mathrm{L}$ at $58-63 \mathrm{ft}$ BGL

- Not detected at 63-68 ft BGL

A similar trend at location TI-16 (Table 3.4 and Figure 4.3) indicates the presence of another source area at this location. Results for carbon tetrachloride in groundwater at TI-16 were as follows:

- $\quad 851 \mu \mathrm{g} / \mathrm{L}$ at $25-30 \mathrm{ft}$ BGL

- $\quad 866 \mu \mathrm{g} / \mathrm{L}$ at $32-37 \mathrm{ft} \mathrm{BG}$

- $\quad 189 \mu \mathrm{g} / \mathrm{L}$ at $39-44 \mathrm{ft}$ BGL

- $\quad 56 \mu \mathrm{g} / \mathrm{L}$ at $46-51 \mathrm{ft} \mathrm{BGL}$

- Trace levels at 60-65 ft BGL and at 67-72 ft BGL

At downgradient and cross-gradient locations (TI-1, TI-2, TI-3, TI-4, TI-5, TI-7, TI-13, TI-14, TI-15, TI-18, and TI-29; Tables 3.3 and 3.4), lower carbon tetrachloride concentrations and contaminant distribution throughout the saturated column indicate that a source is nearby. At all of these locations except TI-1, the observation of maximum carbon tetrachloride concentrations at intermediate depths, with lower concentrations above and below, indicates lateral migration from a nearby source area. At TI-1, the concentrations were significantly lower than at the source areas. The highest concentration at TI-1 was detected in the shallower sample $(181 \mu \mathrm{g} / \mathrm{L}$ at $30-35 \mathrm{ft}$ BGL), and concentrations decreased with depth. Location TI-1 is adjacent to the former chemical storage area on the Co-op property.

Groundwater samples from the southern part of the former CCC/USDA property showed only trace to low concentrations of carbon tetrachloride. None of the concentrations indicated 
that a source area for carbon tetrachloride was present. Other VOCs detected in the southern part of the former CCC/USDA property were benzene, toluene, and methylene chloride at TI-10 and TI-11. The active ASTs owned and operated by the Co-op in this area are the source of these compounds.

Chloroform concentrations in groundwater samples at the source areas (TI-16 and TI-28) showed trends similar to the carbon tetrachloride distribution. Chloroform is a degradation product of carbon tetrachloride. The presence of chloroform at concentrations and with concentration trends that are comparable to those for carbon tetrachloride indicates that natural degradation is occurring. Other VOCs detected at the source areas and adjacent locations were tetrachloroethene and methylene chloride. Methylene chloride can also be a degradation product of carbon tetrachloride.

\subsubsection{Evidence from Soil Data}

Soil analytical data from locations TI-16 and TI-28 confirm that an ongoing source for carbon tetrachloride is present at these locations, as indicated by the concentrations detected and the contaminant distribution throughout the soil column (Table 3.2). Figure 4.3 shows the vertical distribution of carbon tetrachloride at both locations. The nearly complete absence of contamination in the upper part of the vadose zone at all sample soil locations during this investigation is further evidence that contamination may have migrated through a conduit such as former well Co-op 2.

At TI-28, the highest concentration of carbon tetrachloride $(1,094 \mu \mathrm{g} / \mathrm{kg})$ was detected in a soil sample just above the water table. This concentration far exceeds the RBSL of $200 \mu \mathrm{g} / \mathrm{kg}$ for the soil-to-groundwater protection pathway. Below this sample, the concentrations at TI-28 gradually decreased with depth.

Similar results were observed at TI-16 (Table 3.2). The primary differences between soil analytical data from TI-16 and TI-28 are that at TI-16, the following trends were observed:

1. The carbon tetrachloride concentrations were lower. 
2. No carbon tetrachloride (above the AGEM method quantitation limit of $10 \mu \mathrm{g} / \mathrm{kg}$ ) was detected above the water table.

3. One sample collected in an intermediate zone ( $42 \mathrm{ft} \mathrm{BGL}, 454 \mu \mathrm{g} / \mathrm{kg}$ ) showed a higher concentration than any other sample at the location.

Soil samples collected at adjacent locations (TI-1, TI-2, TI-14, and TI-18) generally showed higher carbon tetrachloride concentrations in the intermediate zone, with lower concentrations above and below. The only exception was the samples from location TI-13; here the highest carbon tetrachloride concentrations were detected in the upper zone, at the surface of the water table. This location is at the southwest corner of the flat storage building, where grain was loaded onto trucks.

The highest concentration detected in soil at or adjacent to the former CCC/USDA property was $65 \mu \mathrm{g} / \mathrm{kg}$ at $38 \mathrm{ft}$ BGL at TI-2 (Table 3.1). This concentration does not exceed the RBSL of $200 \mu \mathrm{g} / \mathrm{kg}$ for the soil-to-groundwater protection pathway. Lower concentrations were detected in soil samples at CPT locations TI-1, TI-3, and TI-4, as well as in one sample from location TI-5. The concentrations detected and their distribution throughout the soil column indicate that a source is nearby and that the contamination is likely associated with migration through groundwater.

The only other location on or adjacent to the former CCC/USDA property that contained soil contamination above the AGEM method quantitation limit of $10 \mu \mathrm{g} / \mathrm{kg}$ was TI-10 (Table 3.1). Carbon tetrachloride was not detected at any depth at this location; however, several other VOCs, including chloroform, were detected at depths from 17-25.5 ft BGL. This location is adjacent to ASTs that are owned by the Co-op and are actively being used to store and dispense diesel fuel and gasoline. The compounds detected in soil samples at this location include chloroform, methylene chloride, benzene, 1,1,2,2-tetrachloroethane, 1,1,2-trichloroethane, 1,1-dichloroethane, 1,2-dichloropropane, chlorobenzene, chlorodibromomethane, and toluene. Most of these compounds are typically associated with petroleum fuels or are breakdown products of fuels. The concentrations detected in the soil exceeded the RBSL for methylene chloride, benzene, 1,1,2,2-tetrachloroethane, and 1,1,2-trichloroethane. Petroleum-related contamination detected at this location is not associated with past activities of the CCC/USDA. 


\subsection{Contaminant Migration Pathways}

Potential horizontal and vertical migration pathways explored during this investigation included unsaturated and saturated flow, the influence of pumping wells, surface spillage, surface runoff, and potential migration through a conduit such as former well Co-op 2.

Soil and groundwater samples collected at locations approved by the KDHE along surface drainage pathways and near former locations of grain bins on the former CCC/USDA property did not contain significant concentrations of carbon tetrachloride and did not exhibit contaminant distribution patterns that would demonstrate a source from surface drainage or spillage on the former CCC/USDA property. This is evident from the absence of any detectable level of carbon tetrachloride or chloroform in shallow soil samples and the low concentrations (below the RBSL of $200 \mu \mathrm{g} / \mathrm{kg}$ for the soil-to-groundwater protection pathway) detected in soils at all locations on the former CCC/USDA property.

The absence of soil contamination (at concentrations above the AGEM method detection limit) in the upper part of the vadose zone (at depths shallower than approximately $20 \mathrm{ft} \mathrm{BGL)} \mathrm{at}$ all soil sampling locations indicates that contamination may have been introduced through a conduit such as former well Co-op 2. As Figure 4.13 illustrates, this well was located near TI-28 and was intensely used for chemical mixing and equipment washing before it was plugged on September 5, 1991 (KGS 2007). The date and type of construction for this well are not known.

The predominance of soil contamination in the saturated zone at locations downgradient and cross-gradient from the source areas at TI-16 and TI-28 indicates that contamination is likely associated with horizontal migration through the saturated zone.

The residual contamination detected at the cross-gradient locations on the former CCC/USDA property is likely associated with documented liquid grain fumigant handling and application activities on the Co-op property, at and near the south door of the flat storage building, former well Co-op 2, and the former chemical storage area.

The trace to low residual contaminant concentrations detected in groundwater on the southern portion of the former CCC/USDA facility are likely associated with the normal use of grain fumigants on the property by the CCC/USDA or the Co-op. The CCC/USDA operated in this area from 1954 to approximately 1965. The Co-op has owned and operated in the area 
formerly occupied by the CCC/USDA from approximately 1969 to the present. The Co-op continued to use carbon tetrachloride until the mid 1980s (iSi 2004).

\subsection{Contaminant Distribution throughout the General Investigation Area}

To identify the contaminant plume in the general investigation area, groundwater samples were collected from 13 monitoring wells, 3 private wells, and 3 CPT locations (Table 2.1 and Figure 3.4). The private wells sampled are not used as potable water sources; however, one well (Co-op 3) was historically used by the Co-op to wash equipment and mix bulk chemicals at the active chemical storage area, which is located within the area of highest contamination and is adjacent to TI-14, TI-16, TI-18, and TI-28. Water from well Co-op 3 drains into a concrete basin and also is discharged onto the gravel surrounding the active chemical storage tanks. These activities were observed by Argonne personnel during the investigation.

The analytical data for groundwater from the wells sampled in the general investigation area indicate that the carbon tetrachloride contamination is generally widespread and that the plume is migrating in a generally west to northwesterly direction. Figure 4.12 shows the known extent of the contaminant plume with concentration gradients. Groundwater level data collected during the investigation confirmed a west to northwestern flow direction.

The carbon tetrachloride concentrations detected in groundwater from monitoring wells downgradient from the source areas on the Co-op property have generally decreased, as compared to previous sampling events (Table D.1 in Appendix D). This trend is illustrated as follows:

- $\quad \mathrm{MW} 1: 59 \mu \mathrm{g} / \mathrm{L}$ in 2006; $157 \mu \mathrm{g} / \mathrm{L}$ in 1993

- $\quad \mathrm{MW} 2: 27 \mu \mathrm{g} / \mathrm{L}$ in 2006; $99 \mu \mathrm{g} / \mathrm{L}$ in 1993

- $M W 3: 83 \mu \mathrm{g} / \mathrm{L}$ in 2006; $395 \mu \mathrm{g} / \mathrm{L}$ in 1993

- MW4: $194 \mu \mathrm{g} / \mathrm{L}$ in 2006; $198 \mu \mathrm{g} / \mathrm{L}$ in 1993

- Co-op 3: $197 \mu \mathrm{g} / \mathrm{L}$ in 2006; $440 \mu \mathrm{g} / \mathrm{L}$ in 1992 
- $\quad \mathrm{T} 1: 118 \mu \mathrm{g} / \mathrm{L}$ in $2006 ; 249 \mu \mathrm{g} / \mathrm{L}$ in 1993

- $\quad \mathrm{L}-1: 54 \mu \mathrm{g} / \mathrm{L}$ in $2006 ; 100 \mu \mathrm{g} / \mathrm{L}$ in 1994

Well data from one downgradient location (NW-3) showed no significant change in carbon tetrachloride levels through time $(30.8 \mu \mathrm{g} / \mathrm{L}$ in 2000 and $34 \mu \mathrm{g} / \mathrm{L}$ in 2006$)$.

The 2006 carbon tetrachloride concentrations in most monitoring wells were the lowest since the wells were initially sampled in the early 1990s. The only exceptions are the slight increases in samples collected from NW-3 and the concentration fluctuations that have been detected periodically in L-1. The chloroform concentrations at some locations showed a trend indicating that natural degradation may be occurring. These results indicate that the contaminant plume continues to migrate and that the compounds are being degraded and diluted over time.

Results from existing monitoring well NW-2 on the Co-op property showed a slight increase from $2000(243 \mu \mathrm{g} / \mathrm{L})$ to the most recent sampling event in $2006(313 \mu \mathrm{g} / \mathrm{L})$. Well NW-2 is near the source areas identified on the Co-op property.

Other monitoring wells sampled during the 2006 investigation (KDHE-1, KDHE-2, L-2, L-3, and MW5) contained no carbon tetrachloride above the method quantitation limit of $1.0 \mu \mathrm{g} / \mathrm{L}$.

Samples from the Anderson private well contained carbon tetrachloride at levels ranging from $17 \mu \mathrm{g} / \mathrm{L}$ to $36 \mu \mathrm{g} / \mathrm{L}$. Three samples were collected from this well over a two-day period in an effort to gauge the potential impact of drawdown on a nearby monitoring well (L-3). Contaminant concentrations decreased after the initial sampling event on May 23, 2006, and drawdown was observed in well L-3.

The three downgradient CPT locations (TI-20, TI-30, and TI-31) showed carbon tetrachloride concentrations that exceeded the RBSL of $5.0 \mu \mathrm{g} / \mathrm{L}$. These locations are west of the Co-op property and the former CCC/USDA property.

Other VOCs detected in the general study area were chloroform, methylene chloride, and tetrachloroethene. Chloroform and methylene chloride are degradation products of carbon 
tetrachloride. Tetrachloroethene was detected at trace levels in groundwater samples collected from existing monitoring wells Co-op 3, MW-3, and NW-2.

Tetrachloroethene was historically detected in well Co-op 2, which was located near the identified source area on the Co-op property. This well was plugged in 1991. Construction details for Co-op 2 are unknown; however, it was sampled in 1991 with analytical results of $511 \mu \mathrm{g} / \mathrm{L}$ for carbon tetrachloride, $62.3 \mu \mathrm{g} / \mathrm{L}$ for chloroform, $6.6 \mu \mathrm{g} / \mathrm{L}$ for methylene chloride, $3.4 \mu \mathrm{g} / \mathrm{L}$ for tetrachloroethene, and $0.9 \mu \mathrm{g} / \mathrm{L}$ for 1,2 -DCA. 
TABLE 4.1 Monthly precipitation data for Enterprise, Kansas, approximately $7 \mathrm{mi}$ north of Navarre. Source of data: Kansas State University, K-State Research and Extension, Weather Data Library, http://www.oznet.ksu.edu/wdl/.

Precipitation (in.) in Month

Year Jan Feb Mar Apr May Jun Jul Aug Sep Oct Nov Dec Total

\begin{tabular}{lllllllllllllll}
\hline 2000 & 0.55 & 2.90 & 3.42 & 2.28 & 2.25 & 5.70 & 3.13 & 0.34 & 0.87 & 2.65 & 0.76 & 1.20 & 26.05 \\
2001 & 2.26 & 2.75 & 1.78 & 3.03 & 3.82 & 3.45 & 2.92 & 3.22 & 1.72 & 1.48 & 0.79 & 0.42 & 27.64 \\
2002 & 0.01 & 0.27 & 0.39 & 3.36 & 3.43 & 1.69 & 1.03 & 2.20 & 1.01 & 6.30 & 0.44 & 0.24 & 20.37 \\
2003 & 0.28 & 1.17 & 1.91 & 3.53 & 3.40 & 2.95 & 0.68 & 4.87 & 2.09 & 1.17 & 0.75 & 1.02 & 23.82 \\
2004 & 0.77 & 1.18 & 4.54 & 1.62 & 3.49 & 5.41 & 9.81 & 1.26 & 1.57 & 1.92 & 1.81 & 0.30 & 33.68 \\
2005 & 1.41 & 2.00 & 1.91 & 2.56 & 4.01 & 12.15 & 2.13 & 6.61 & 1.30 & 1.79 & 1.24 & 0.66 & 37.77 \\
2006 & 0.31 & 0.00 & $M^{a}$ & 2.83 & 2.91 & 2.88 & 1.33 & 9.04 & 1.67 & 2.72 & 0.05 & 1.81 & 25.55 \\
& & & & & & & & & & & & & &
\end{tabular}

a Data missing. 
TABLE 4.2 Daily precipitation data for Manhattan, Kansas, approximately 35 mi northeast of Navarre. Source of data: Kansas State University, K-State Research and Extension, Weather Data Library, http://www.oznet.ksu.edu/wdl/.

\begin{tabular}{lcccccccccc}
\hline & & & & & & & & & \\
& & & & \multicolumn{7}{c}{ Month in 2006} \\
Day & Apr & May & Jun & Jul & Aug & Sep & Oct & Nov & Dec \\
& & & & & & & & & \\
\hline & & & & & & & & & \\
1 & - a & - & 0.11 & - & - & - & - & - & - \\
2 & - & 0.58 & - & - & 0.67 & 0.14 & - & - & - \\
3 & - & 0.20 & - & 1.30 & - & - & - & - & - \\
4 & - & 0.18 & - & 0.01 & - & - & - & - & - \\
5 & - & - & 0.01 & - & - & - & - & - & - \\
6 & - & - & - & - & - & - & - & - & - \\
7 & - & - & - & - & - & - & - & - & - \\
8 & - & 0.67 & - & - & - & 0.01 & - & - & - \\
9 & - & 0.01 & - & 1.27 & - & 0.82 & 0.04 & - & - \\
10 & - & - & 0.07 & 0.34 & 0.49 & 0.04 & 0.40 & - & - \\
11 & - & - & 0.01 & 0.02 & 0.01 & - & - & - & - \\
12 & - & - & - & - & - & - & - & - & - \\
13 & - & - & - & - & 1.11 & - & - & - & - \\
14 & - & - & - & - & 1.02 & - & - & - & - \\
15 & - & - & - & - & 0.01 & 0.02 & 0.12 & - & - \\
16 & - & - & 0.28 & - & 0.01 & - & 0.06 & - & - \\
17 & - & - & 0.48 & - & 0.60 & 0.55 & 0.02 & - & - \\
18 & - & - & - & - & 3.42 & - & - & - & - \\
19 & - & - & - & - & 0.20 & - & - & - & - \\
20 & - & - & 0.03 & - & - & - & - & - & 0.42 \\
21 & - & - & 0.30 & 0.72 & - & 0.37 & 0.11 & - & 0.01 \\
22 & - & - & 0.01 & - & - & 0.03 & - & - & - \\
23 & - & - & - & - & - & 0.01 & - & - & - \\
24 & 0.19 & - & - & - & - & - & - & - & - \\
25 & 0.02 & - & 0.07 & - & 1.43 & - & 1.51 & - & - \\
26 & - & 0.31 & - & - & 1.83 & - & 0.13 & - & - \\
27 & - & - & - & 0.05 & 0.30 & - & 0.12 & 0.07 & - \\
28 & 1.28 & - & 0.04 & - & 0.04 & - & - & - & - \\
29 & 0.13 & 0.20 & 0.04 & - & - & - & - & - & - \\
30 & - & 0.70 & - & - & - & - & - & - & - \\
31 & & 0.03 & & - & - & & - & & - \\
Total & 1.62 & 2.88 & 1.45 & 3.71 & 11.14 & 1.99 & 2.51 & 0.07 & 0.43 \\
& & & & & & & & & \\
\hline & & & & & & & & &
\end{tabular}

a No recorded precipitation. 


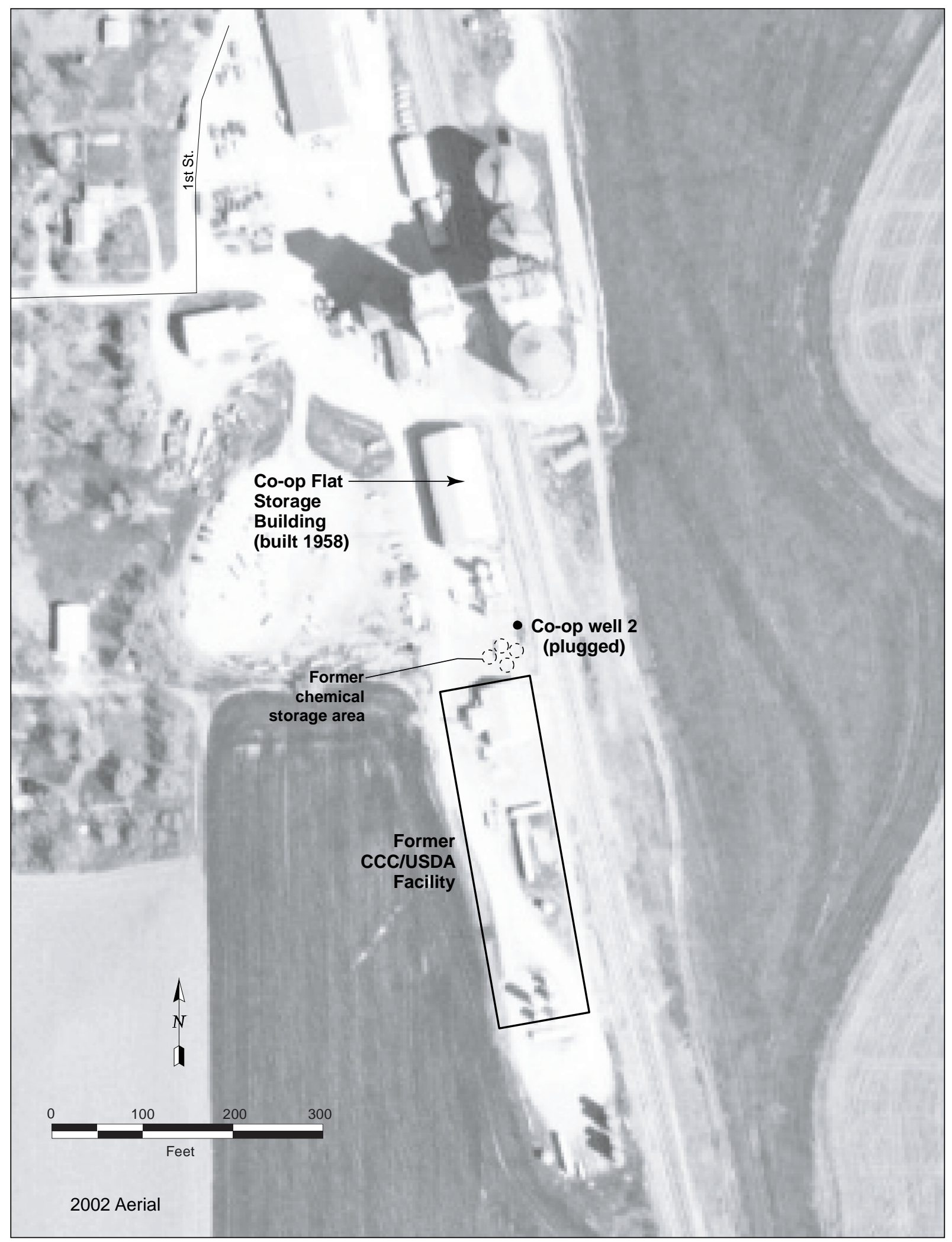

FIGURE 4.1 Locations of contaminant source areas at Navarre. Source of photograph: NAIP (2002). 


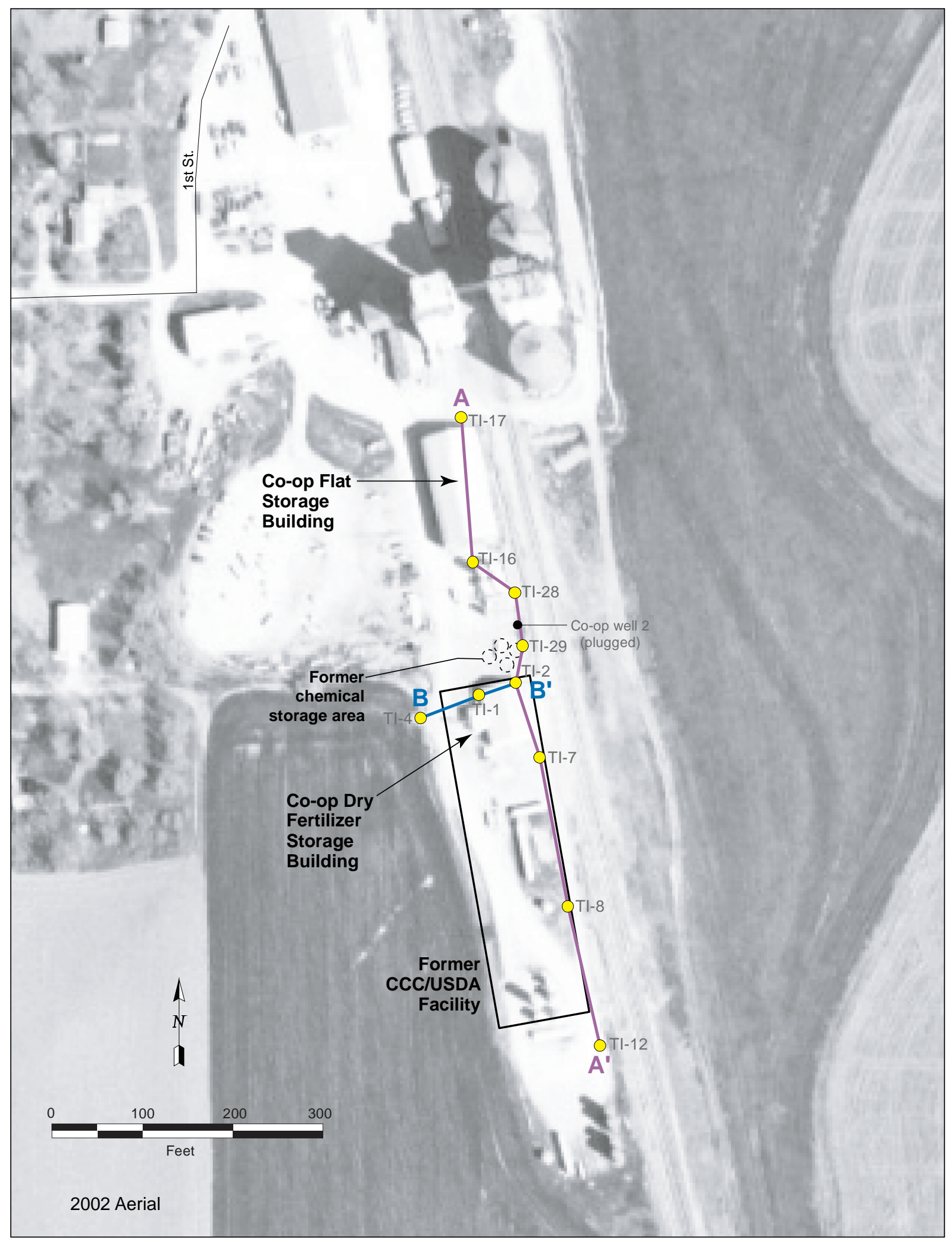

FIGURE 4.2 Locations of hydrogeologic cross sections A-A' and B-B'. Source of photograph: NAIP (2002). 


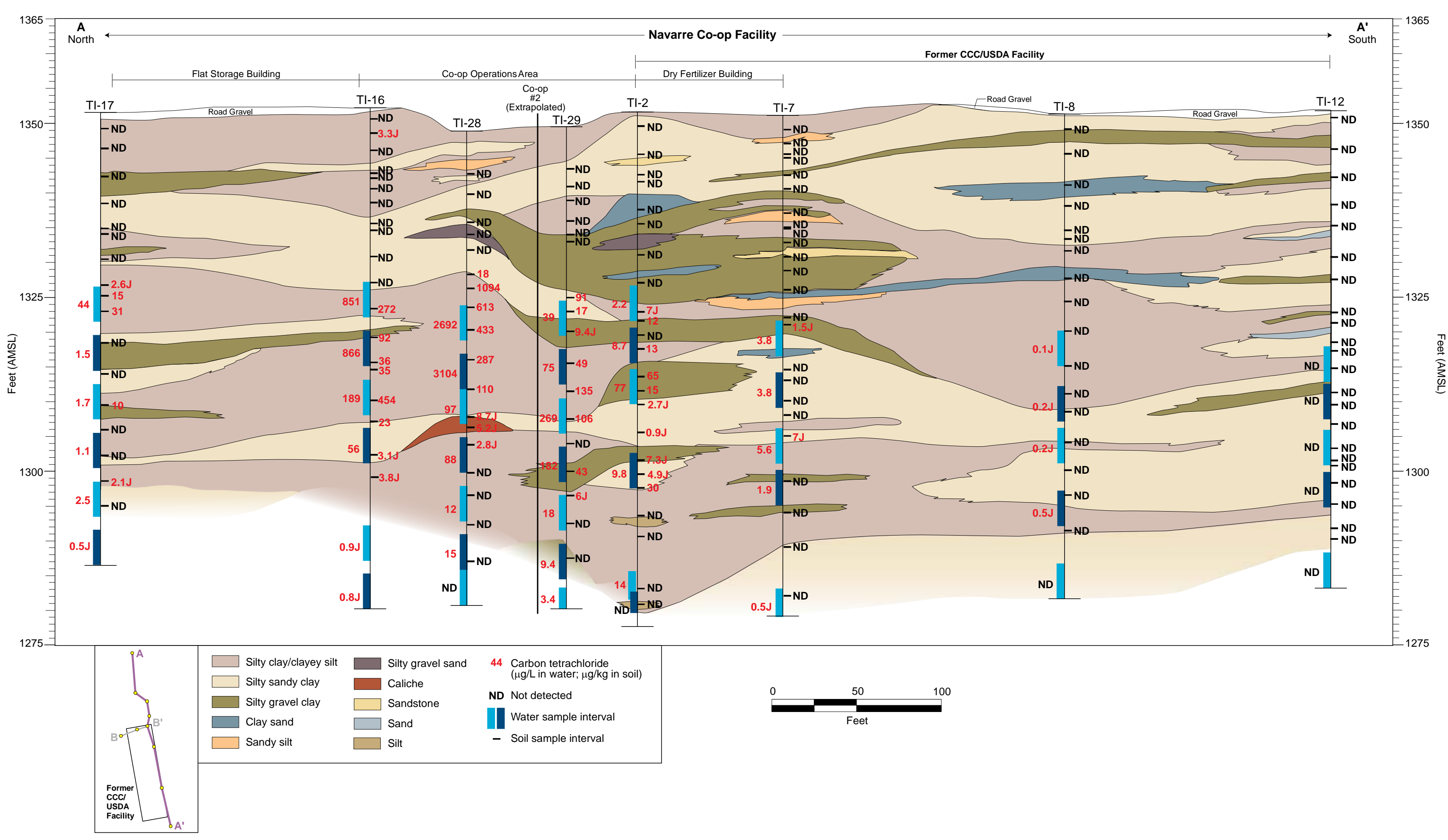

FIGURE 4.3 Hydrogeologic cross section A-A' (vertically exaggerated), showing the vertical and lateral distribution of carbon tetrachloride in subsurface soil and groundwater. 


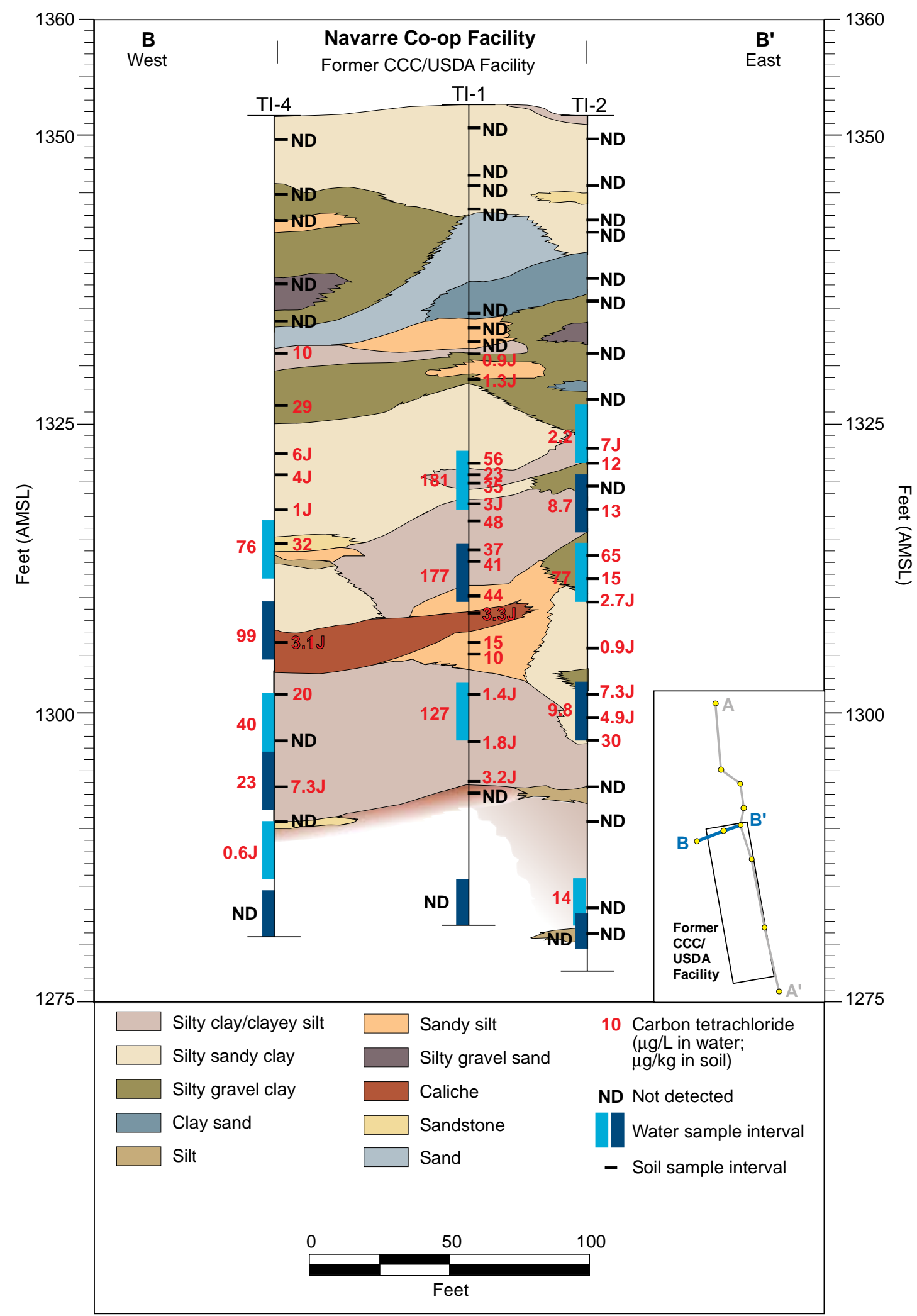

FIGURE 4.4 Hydrogeologic cross section B-B' (vertically exaggerated), showing the vertical and lateral distribution of carbon tetrachloride in subsurface soil and groundwater. 


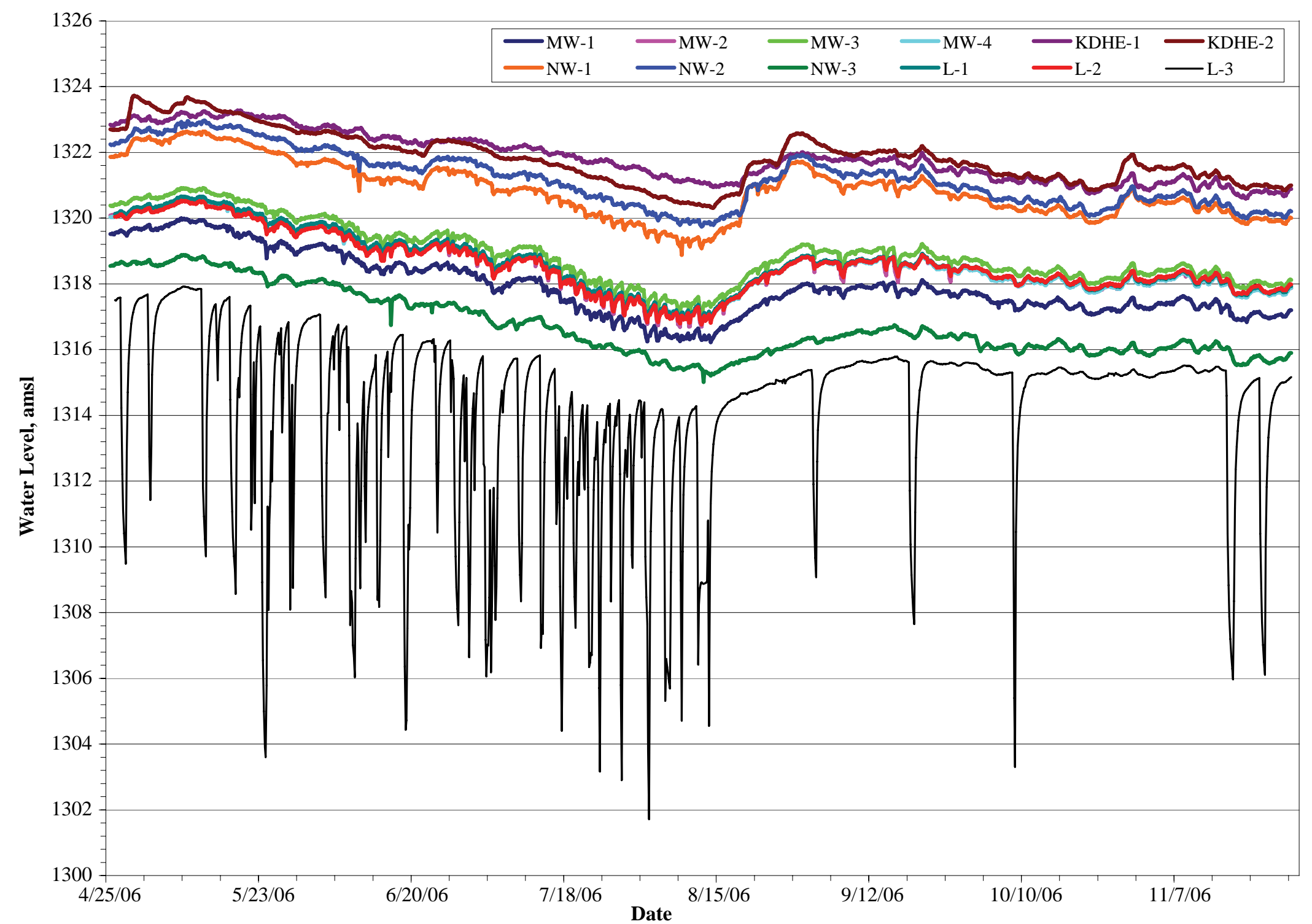

FIGURE 4.5 Hydrographs constructed from continuously monitored water levels in wells at Navarre, April 25, 2006, to November 28, 2006. 


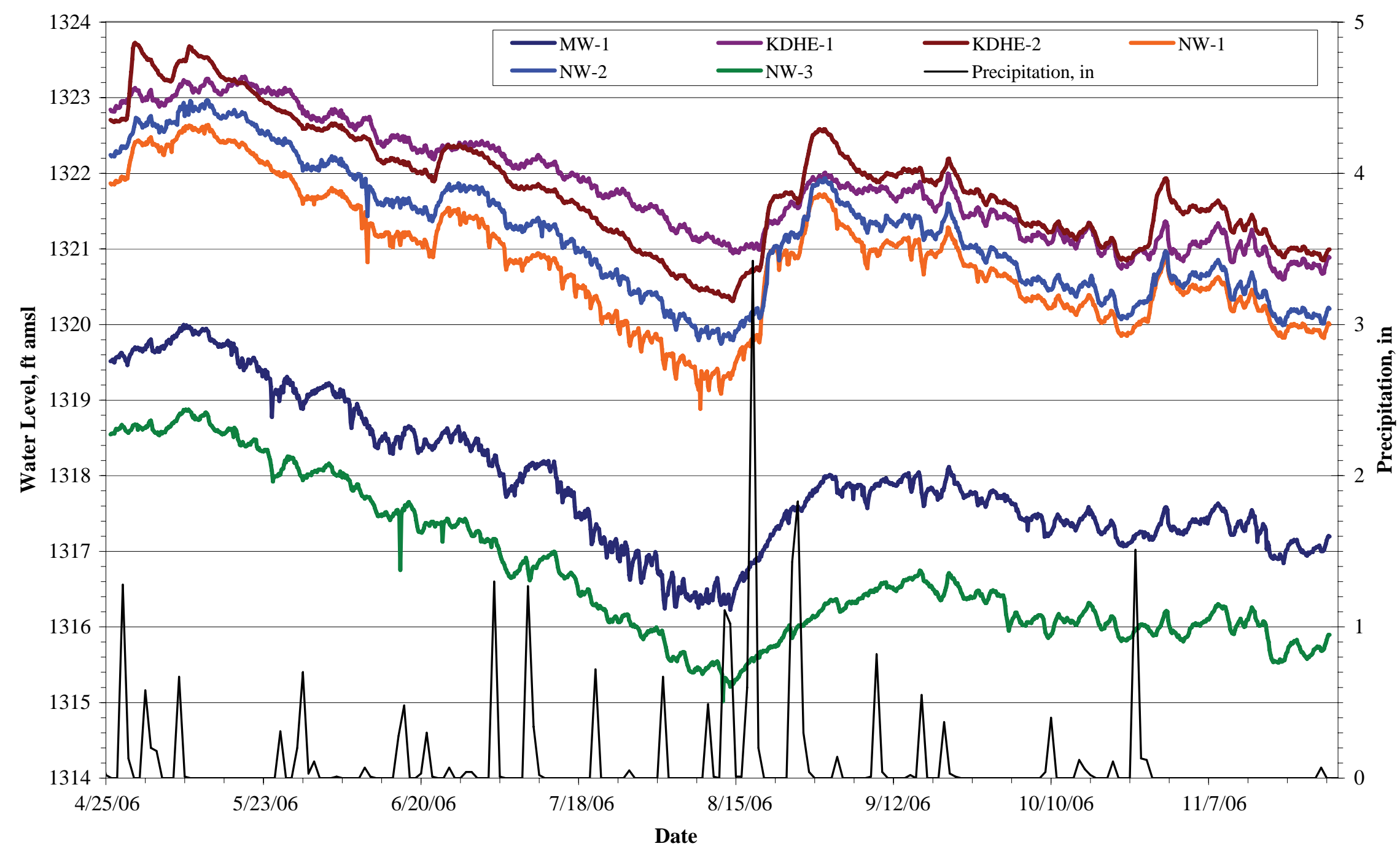

FIGURE 4.6 Hydrographs for selected monitoring wells at Navarre, with precipitation data for Manhattan, Kansas, April 25, 2006, to November 28, 2006. 


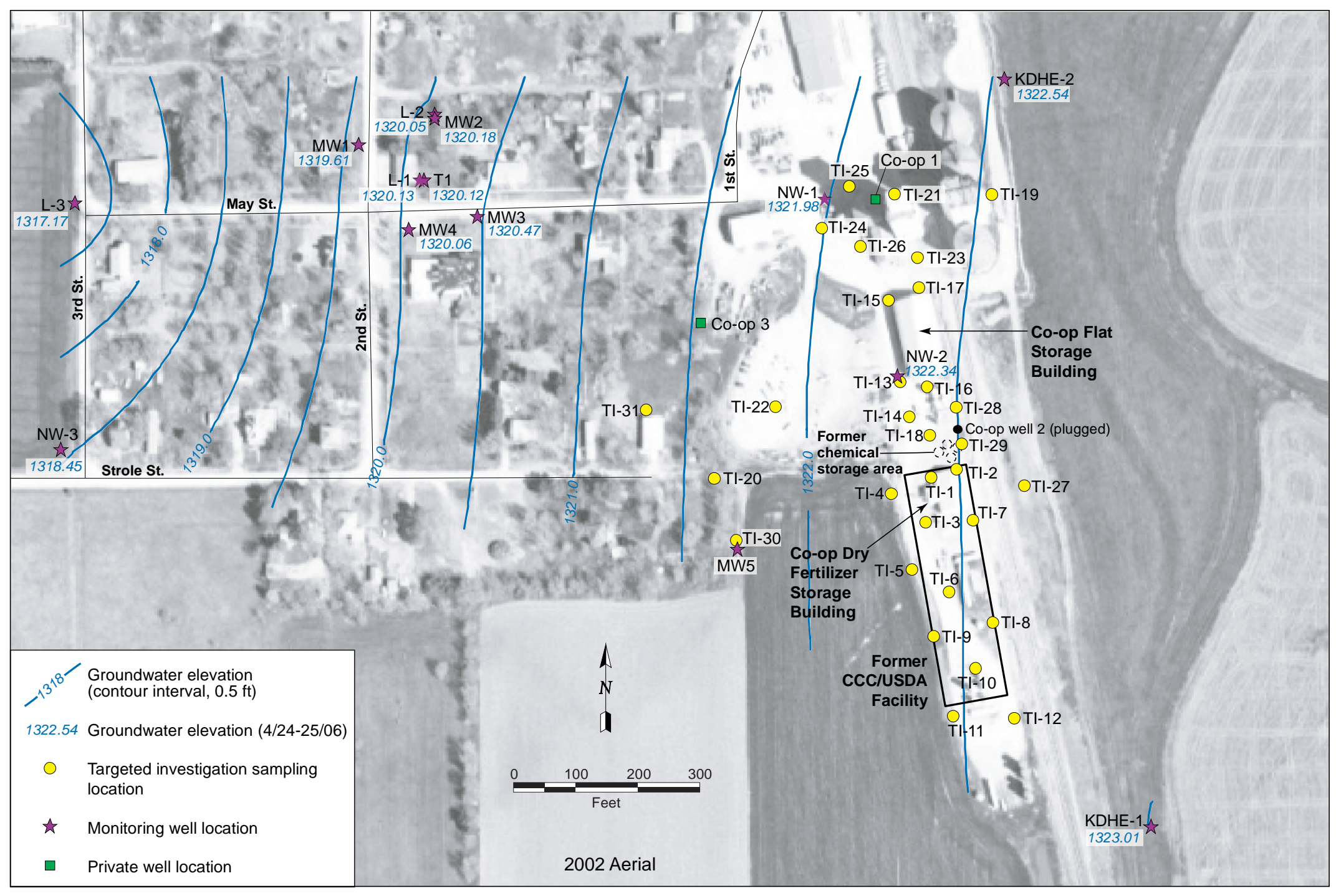

FIGURE 4.7 Potentiometric surface at Navarre, based on hand-measured water levels on April 24-25, 2006. Source of photograph: NAIP (2002). 


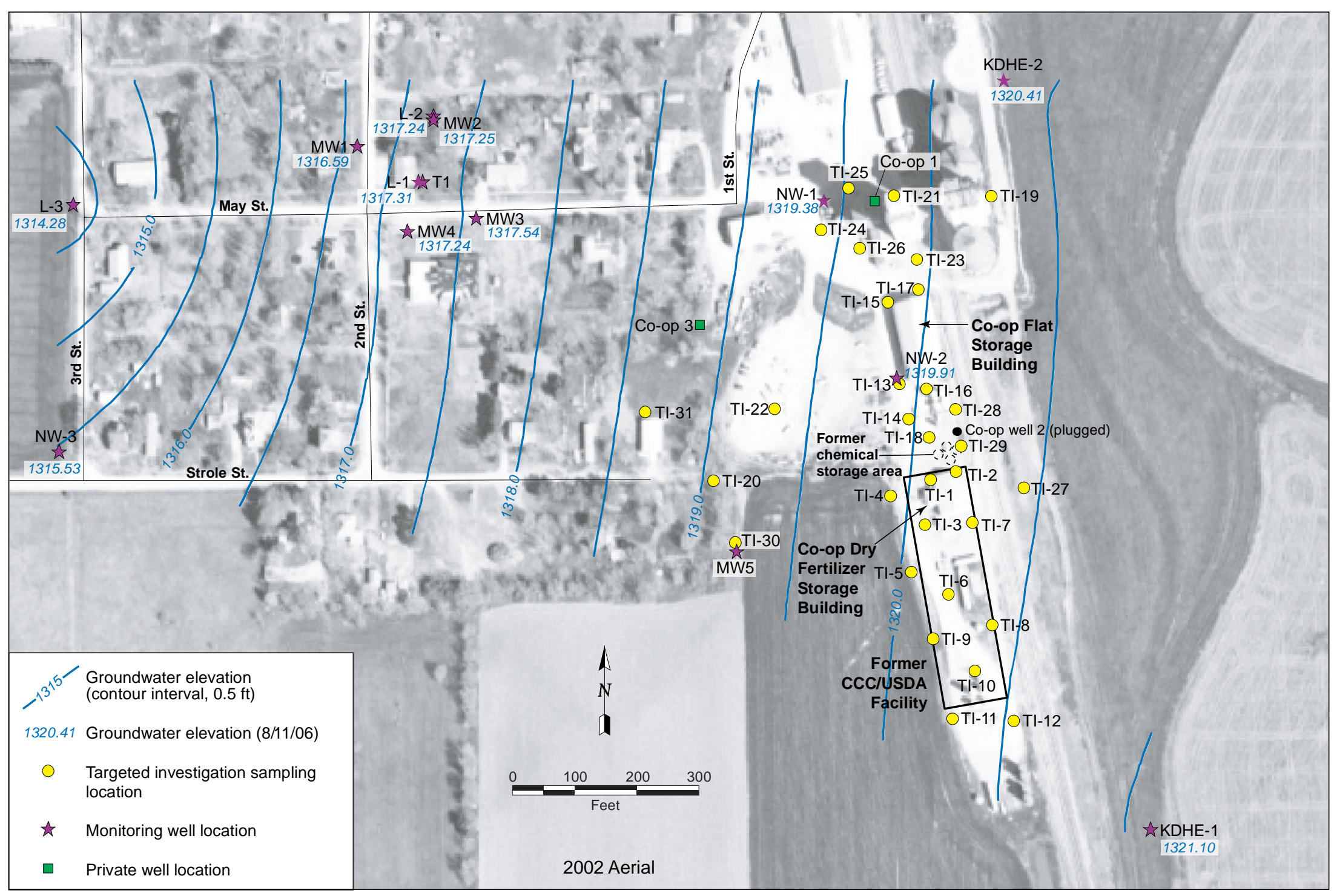

FIGURE 4.8 Potentiometric surface at Navarre, based on water levels recorded by data loggers on August 11, 2006. Source of photograph: NAIP (2002). 


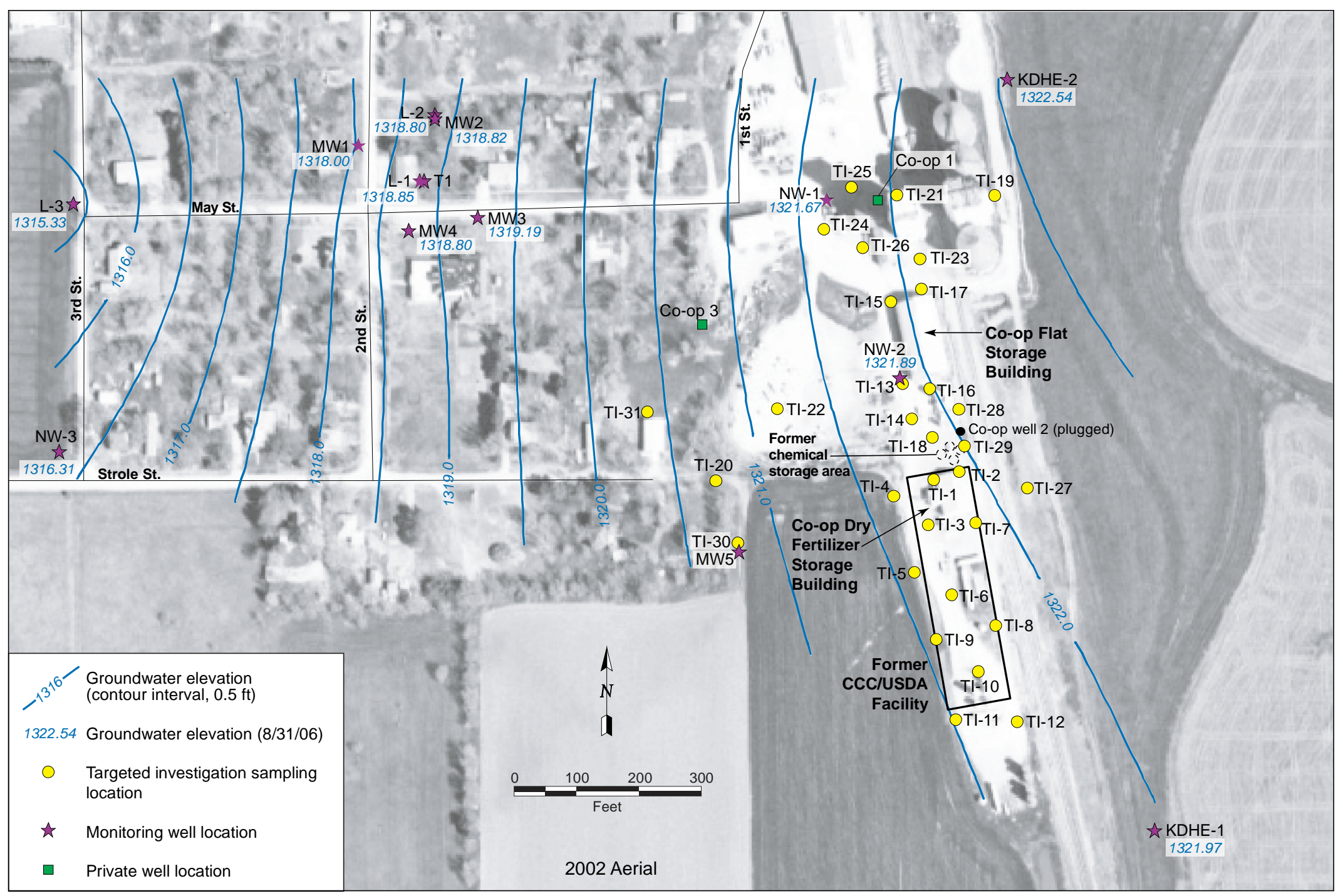

FIGURE 4.9 Potentiometric surface at Navarre, based on water levels recorded by data loggers on August 31, 2006. Source of photograph: NAIP (2002). 


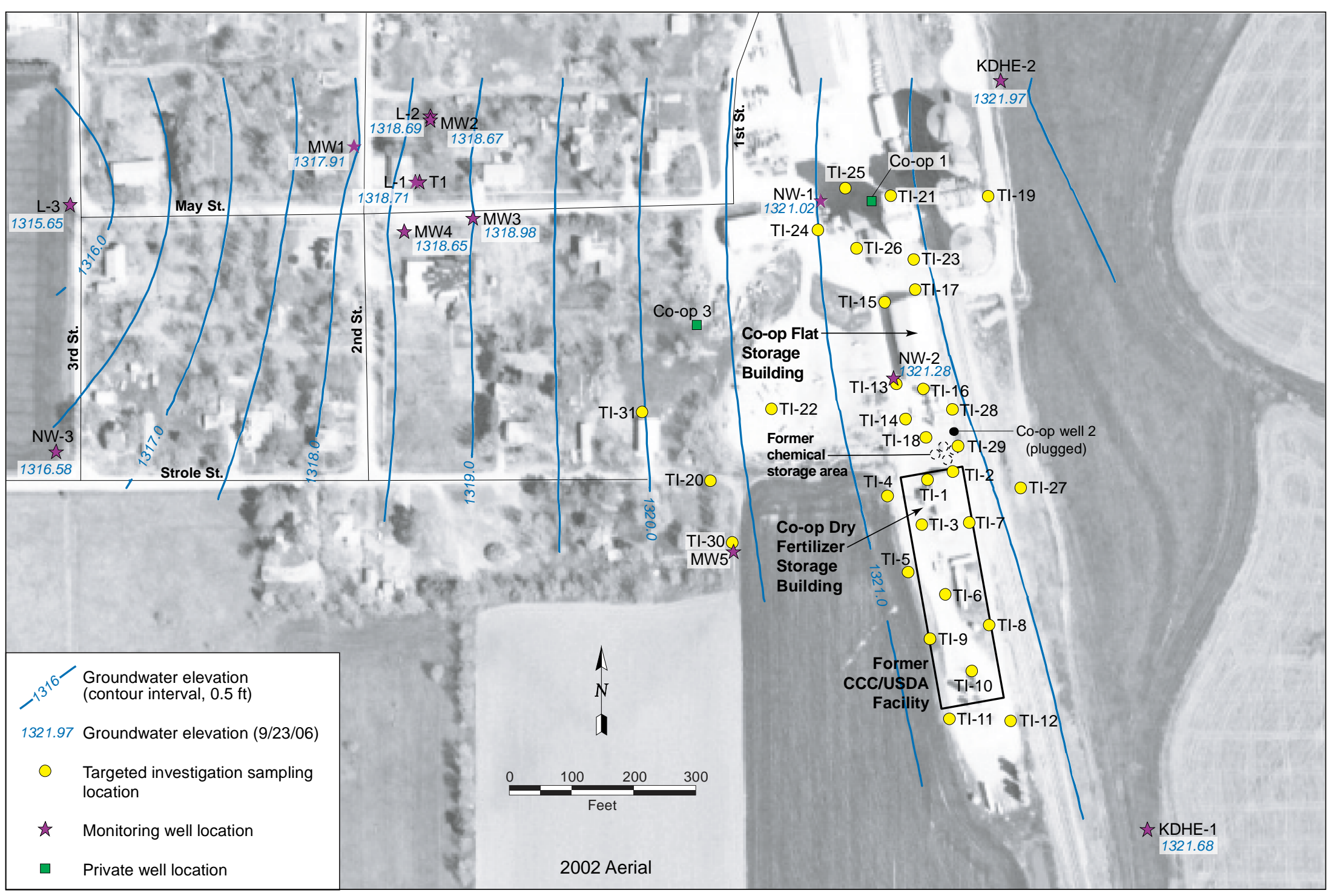

FIGURE 4.10 Potentiometric surface at Navarre, based on water levels recorded by data loggers on September 23, 2006. Source of photograph: NAIP (2002). 


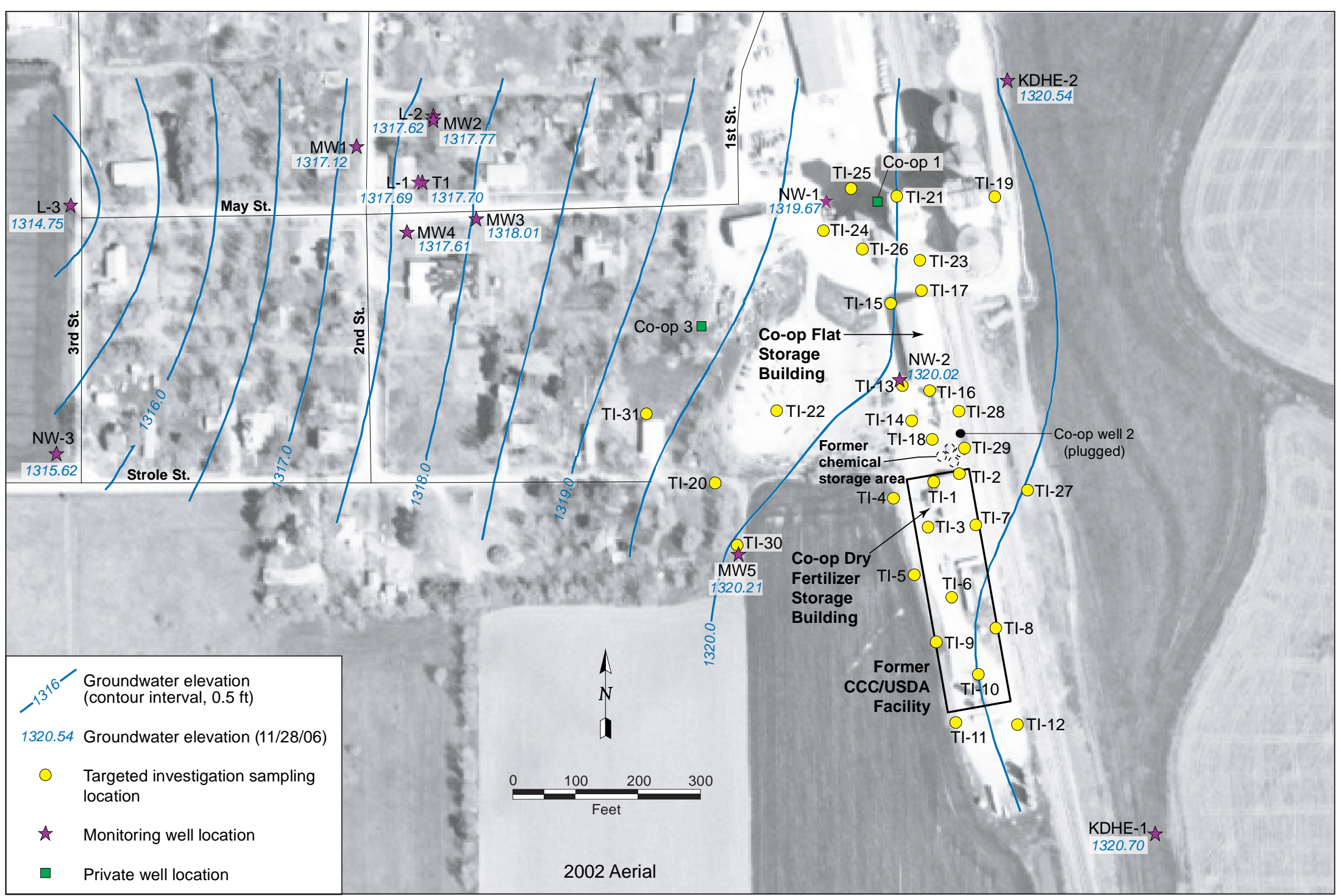

FIGURE 4.11 Potentiometric surface at Navarre, based on hand-measured water levels on November 28, 2006. Source of photograph: NAIP (2002). 


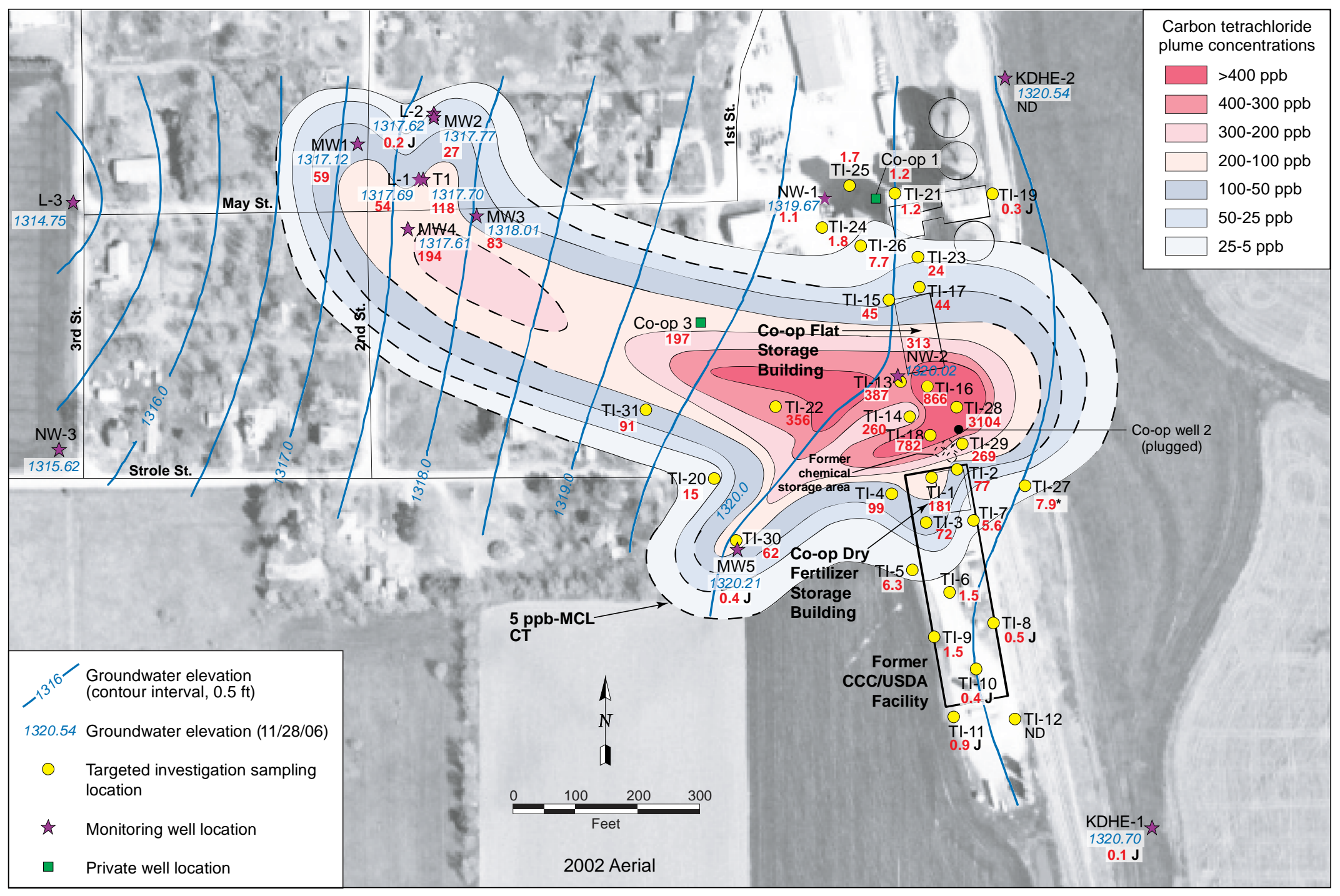

FIGURE 4.12 Interpreted distribution of carbon tetrachloride in the groundwater plume, 2006, with groundwater elevations on November 28, 2006. Source of photograph: NAIP (2002). 

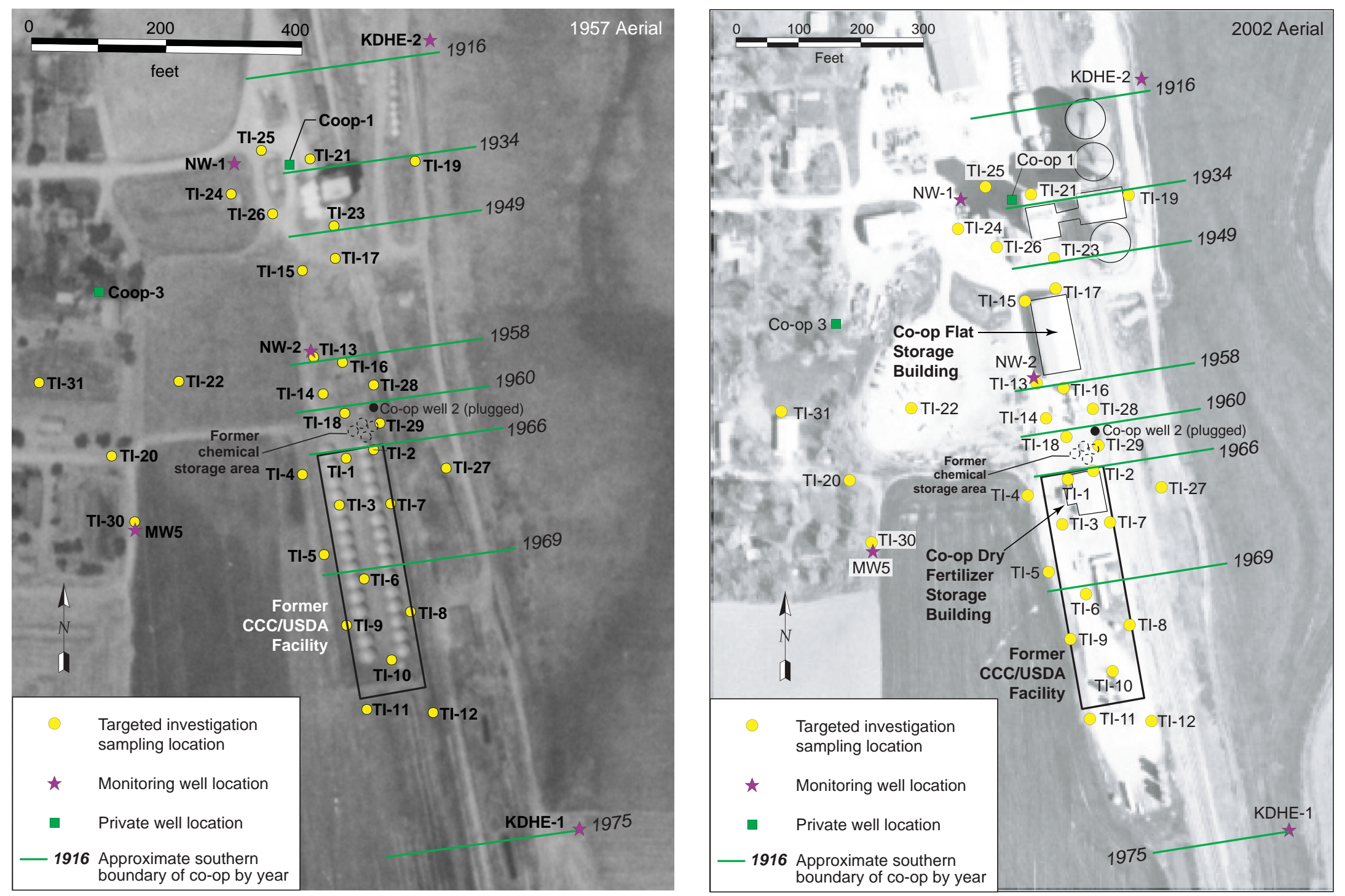

FIGURE 4.13 Locations of the former CCC/USDA facility, monitoring and private wells, and 2006 investigation activities at Navarre, with the approximate southern Co-op boundary in each year of the Co-op's southward expansion. Source of photographs: USDA (1957); NAIP (2002). 


\section{Conclusions}

\subsection{Former CCC/USDA Property}

Conclusions related to the former CCC/USDA property are as follows:

- No source areas for carbon tetrachloride were identified on the former CCC/USDA property. Residual carbon tetrachloride contamination detected at and near the dry fertilizer building is associated with the high concentrations detected in the area of TI-28, which is near former well Co-op 2 and the former chemical storage areas on the Co-op property. Only traces to low concentrations were detected on the southern part of the former CCC/USDA property. None of the concentrations detected in the southern part of the former CCC/USDA property would indicate a nearby source.

- No pathway for contaminant migration from surface soil to groundwater was identified at any location on the former CCC/USDA property. This conclusion is evident from the limited soil contamination detected, the significantly lower contaminant concentrations (as compared to the Co-op property), and the contaminant distribution throughout the soil and groundwater column. This finding further indicates that the CCC/USDA is not a responsible party for the contamination detected at the source areas identified on the Co-op property.

- One aquifer was identified during investigation activities. Data collected during the 2006 investigation showed that groundwater was encountered continuously throughout the stratigraphic column on the former CCC/USDA property, at depths from $25 \mathrm{ft}$ to $77.8 \mathrm{ft}$ BGL. The only potential confining layers that could indicate separate aquifers were consolidated materials of very thin, discontinuous sandstone layers with some limestone fragments. These materials occurred at depths of $10 \mathrm{ft} \mathrm{BGL}$ at location TI-2, $25.5 \mathrm{ft}$ BGL at TI-3, $18 \mathrm{ft}$ and $37 \mathrm{ft}$ BGL at TI-4, and $22 \mathrm{ft}$ BGL at TI-7. The 2006 investigation's finding of one aquifer is contrary to previous reports, which indicated that two aquifers were present. The information in previous reports was supported by data gathered during investigations that had limited scopes of work and limited investigative techniques. New technologies, coupled with 
the greater detail achieved in the 2006 investigation, enabled a more thorough study of the lithologic and hydrologic properties at Navarre.

- The distribution of groundwater contamination on the former CCC/USDA property is predominantly limited to the upper part of the water column. All but one groundwater sample collected from the deepest groundwater zones showed no carbon tetrachloride above the AGEM Laboratory method detection limit of $1.0 \mu \mathrm{g} / \mathrm{L}$. The only sample that contained carbon tetrachloride above this limit (at $14 \mu \mathrm{g} / \mathrm{L}$ ) was from location TI-2, at 66-70 ft BGL. This location is approximately $100 \mathrm{ft}$ south from (cross-gradient of) the source area at TI-28, and the contamination detected can be attributed to migration from this source area. The observed limited vertical migration of the contamination indicates that the lower part of the aquifer on the former CCC/USDA property has not been adversely impacted, and investigation into zones that may be present at deeper depths is not warranted.

- An active source for petroleum-related contamination exists on the southern end of the former CCC/USDA property. Benzene was detected in groundwater samples from TI-10 and TI-11 at concentrations above the RBSL of $5.0 \mu \mathrm{g} / \mathrm{L}$. Other petroleum-related compounds were also detected at these locations. These two sample locations are adjacent to active ASTs that are owned and operated by the Co-op.

- The groundwater flow direction was determined to be west to northwest. This finding is consistent with historical data. This flow direction, coupled with the low contaminant concentrations detected on the former CCC/USDA property (compared to concentrations detected at the source areas on the Co-op property), is not consistent with an explanation asserting that grain fumigant handling and use on the CCC/USDA property are related to or caused the contamination detected at the sources areas identified on the Co-op property.

\subsection{Co-op Property Source Areas}

Conclusions related to the Co-op property are as follows: 
- Two source areas were identified on the Co-op property. One source area is associated with the former well Co-op 2 and the former chemical storage activity at and adjacent to locations TI-14, TI-18, and TI-28. The other source area is at the south door of the flat storage building, at and near locations TI-16 and TI-13. These two source areas showed the highest concentrations of carbon tetrachloride in groundwater during the 2006 investigation, far exceeding the RBSL of $5.0 \mu \mathrm{g} / \mathrm{L}$ for this compound. Analytical data for soil samples from TI-28 showed a high carbon tetrachloride concentration just above the water table and decreasing concentrations at depth. The contaminant distribution trends indicate that sources are present at and adjacent to TI-16 and TI-28. The presence of sources in these areas is further supported by information in the Co-op's August 31, 2004, work plan (iSi 2004), indicating that carbon tetrachloride was used on the property until the mid 1980s and was applied to grain at the flat storage building on several occasions in the 1970s.

- Former Well Co-op 2. This well's proximity to former chemical storage bins and chemical mixing activities makes it a likely route for contaminant migration. The well's construction date and construction details are not known; however, it was plugged on September 5, 1991 (KGS 2007). This well may have been installed by the railroad prior to the Co-op's use (Stroda 2007). Analytical data for samples taken from this well in 1991 showed a high concentration of carbon tetrachloride. Other compounds detected in these samples included chloroform, methylene chloride, tetrachloroethene, and 1,2-DCA.

- Chloroform was detected in soil and groundwater samples on the Co-op property. The chloroform concentrations detected in 13 groundwater samples from the Co-op property exceeded the RBSL of $80 \mu \mathrm{g} / \mathrm{L}$ for this compound. The concentrations detected showed trends similar to the carbon tetrachloride contaminant distribution. The high ratio of chloroform to carbon tetrachloride indicates that natural degradation is occurring.

- Methylene chloride was detected on the Co-op property at concentrations above the RBSL of $5.0 \mu \mathrm{g} / \mathrm{L}$ for this compound in groundwater. Methylene 
chloride was detected at TI-13, TI-14, TI-16, TI-18, TI-22, TI-28, and TI-29. This compound is a degradation product of carbon tetrachloride and is also used as a solvent. The presence of methylene chloride indicates that natural degradation of carbon tetrachloride (and chloroform) is occurring.

- Tetrachloroethene was detected in groundwater samples collected at eight locations on the Co-op property. Trace to low levels of tetrachloroethene were detected at the source areas and at downgradient locations on the Co-op property. The highest concentration $(3.1 \mu \mathrm{g} / \mathrm{L})$ was detected at the source area identified at TI-28. The detection of tetrachloroethene at TI-28, with lower concentrations at downgradient and cross-gradient locations, further indicates a source at location TI-28. The tetrachloroethene contaminant plume mimics the carbon tetrachloride plume. This compound is not associated with CCC/USDA operations.

- Water from private well Co-op 3, which is contaminated with carbon tetrachloride at a concentration above the MCL and RBSL, was observed both being used for bulk chemical mixing and being discharged onto the ground surface near the source areas. The Co-op has, throughout its history, washed equipment and mixed bulk chemicals at the active chemical storage area adjacent to the source areas at TI-16 and TI-28 and former well Co-op 2. During the 2006 investigation, water from well Co-op 3 was observed to be draining into a concrete basin and onto the gravel surrounding the chemical storage tanks. Discharging water contaminated with carbon tetrachloride and other VOCs at concentrations above the regulatory limits onto the ground surface exacerbates the contamination problem and is a violation of Kansas discharge law.

- Only trace to low levels of carbon tetrachloride were detected at the feed mill. The carbon tetrachloride detected at the feed mill is not a source for the contamination detected at the source areas identified near the south door of the flat storage building and adjacent to and near former well Co-op 2 and the former chemical storage area. 


\subsection{Status of the Contaminant Plume in the General Investigation Area}

Conclusions related to the status of the contaminant plume are as follows:

- The carbon tetrachloride groundwater contaminant plume is generally widespread, as indicated by groundwater analytical data for sampled wells in the general investigation area. The concentrations detected in 2006 were generally lower than those reported for previous sampling events. This decline indicates that the plume continues to become degraded and diluted over time.

- Points of origin for the contamination detected in the general investigation area were found on the Co-op property. The points of origin on the Co-op property are the area at and near the southern door of the flat storage building, former well Co-op 2, and the former and active chemical storage areas. The analytical data collected at TI-16 and TI-28 and at adjacent locations indicate that the TI-16 and TI-28 areas are likely at or adjacent to the points of origin for the contamination detected in the general investigation area.

- The contaminant plume appears to be migrating west to northwest, as evidenced by groundwater flow and contaminant concentration data collected during the 2006 investigation.

- One aquifer is present throughout the general investigation area. Lithologic data and water level data collected during the investigation showed the effects of a nearby pumping well. Drawdown during pumping was observed in both shallow and deep wells. These findings indicate that only one aquifer is present, contrary to previous reports. 


\section{References}

Argonne, 1992, Final Work Plan: Expanded Site Investigation, Navarre, Kansas, prepared for the Commodity Credit Corporation, U.S. Department of Agriculture, Washington, D.C., by Argonne National Laboratory, Argonne, Illinois, September.

Argonne, 1993, Final Report: Expedited Site Characterization, Navarre, Kansas, prepared for the Commodity Credit Corporation, U.S. Department of Agriculture, Washington, D.C., by Argonne National Laboratory, Argonne, Illinois, December.

Argonne, 1995, Final Feasibility Study for Remedial Action at Navarre, Kansas, prepared for the Commodity Credit Corporation, U.S. Department of Agriculture, Washington, D.C., by Argonne National Laboratory, Argonne, Illinois, February.

Argonne, 2002, Final Master Work Plan: Environmental Investigations at Former CCC/USDA Facilities in Kansas, 2002 Revision, ANL/ER/TR-02/004, prepared for the Commodity Credit Corporation, U.S. Department of Agriculture, Washington, D.C., by Argonne National Laboratory, Argonne, Illinois, December.

Argonne, 2006, Revised Plan: Recommended Investigation for Navarre, Kansas, ANL/EVS/AGEM/TR-05-05, prepared for the Commodity Credit Corporation, U.S. Department of Agriculture, Washington, D.C., by Argonne National Laboratory, Argonne, Illinois, January.

BNSF Railway, 2007, BNSF Grain Elevator Directory, North Central Kansas Coop - Navarre, $K S$, http://www.bnsf.com/markets/agricultural/elevator/bin3/ele1883.html, March 27.

Carey, C., 2006, letter from Carey (Bureau of Environmental Remediation, Kansas Department of Health and Environment, Topeka, Kansas) to C. Roe (Commodity Credit Corporation, U.S. Department of Agriculture, Washington, D.C.), regarding Revised Plan: Recommended Investigation at Navarre, Kansas, February 20.

Co-op, 2000, Answers and Objections to the United States of America's First Set of Requests for Admissions, and Second set of Interrogatories and Request for Production of Documents to Defendants Navarre Farmers Union Cooperative Association and North Central Kansas 
Cooperative Association, Case No. 99-1100-JTM in the U.S. District Court for the District of Kansas, June 30.

EPA, 1994a, USEPA Contract Laboratory Program National Functional Guidelines for Organic Data Review, EPA 540/R-94/012, Office of Emergency and Remedial Response, U.S. Environmental Protection Agency, Washington, D.C., February.

EPA, 1994b, USEPA Contract Laboratory Program National Functional Guidelines for Inorganic Data Review, EPA 540/R-94/013, Office of Emergency and Remedial Response, U.S. Environmental Protection Agency, Washington, D.C., February.

EPA, 1995, Method 524.2: Measurement of Purgeable Organic Compounds in Water by Capillary Column Gas Chromatography/Mass Spectrometry, Revision 4.1, edited by J.W. Munch, National Exposure Research Laboratory, Office of Research and Development. U.S. Environmental Protection Agency, Cincinnati, Ohio (http://www.accustandard.com/asi/ pdfs/epa_methods/524_2.pdf).

EPA, 2007, Terms of Environment: Glossary, Abbreviations, and Acronyms, Office of Communications, Education, and Media Relations, U.S. Environmental Protection Agency, Washington, D.C. (originally EPA 175-B-97-001, revised December 1997), http://www.epa.gov/OCEPAterms, accessed April 6.

iSi, 2004, Carbon Tetrachloride Comprehensive Investigation Work Plan (Revision 1) for the North Central Kansas Cooperative, Navarre Branch, Navarre, Kansas, Integrated Solutions, Inc., Wichita, Kansas, August 31.

Johnson, J.E., 2000, Volume I of the Deposition of James Edward Johnson, Case No. 99-1100JTM in the U.S. District Court for the District of Kansas, November 29.

KDHE, 1992, Preliminary Assessment: Navarre Groundwater Site, Navarre, Kansas, Bureau of Environmental Remediation, Kansas Department of Health and Environment, Topeka, Kansas, January. 
KDHE, 1998a, Comprehensive Investigation of Navarre Site - Navarre, Dickinson County, Kansas, Kansas Department of Health and Environment, Bureau of Environmental Remediation, Topeka, Kansas, May.

KDHE, 1998b, "KDHE/USDA Water Supply Project in Navarre," in Kansas Environmental News - October 1998, Division of Environment, Kansas Department of Health and Environment, Topeka, Kansas, http://www.kdheks.gov/sbcs/download/ken9810.html\#navarre, accessed May 9, 2007.

KDHE, 2001, Quarterly Report Number 12 for Settlement Agreement between USDA/CCC and KDHE for Connection of Navarre Area Residences to Dickinson County Rural Water District \#2 in Kansas, Agreement number 04-XG-98-04-USDA-CCC, project period July 1, 2001 September 30, 2001, submitted to U.S. Department of Agriculture, Commodity Credit Corporation, by Kansas Department of Health and Environment, Topeka, Kansas, October 31.

KDHE, 2004, Consent Order, Case No. 03 E 0199, regarding the groundwater contamination at North Central Kansas Cooperative, Navarre Branch, Navarre, Kansas, March 18.

KGS, 2007, Water Well Completion Records (WWC5) Database, Kansas Geological Survey, Lawrence, Kansas, http://www.kgs.ku.edu/Magellan/WaterWell/index.html, April 6.

Lee, W., 1956, Stratigraphy and Structural Development of the Salina Basin Area, Kansas State Geological Survey Bulletin 121, 3-167.

Linn, C.H., 1990, letter from Linn (Solid Waste Section, Bureau of Air and Waste Management, Kansas Department of Health and Environment, Topeka, Kansas) to Navarre Farmers Union Coop Association (Navarre, Kansas), regarding Industrial Solid Waste Disposal Authorization Number 6048, November 8 (included in Exhibit 124 in Case No. 99-1100-JTM in the U.S. District Court for the District of Kansas).

Moore, R., J. Frye, W. Lee., and H. O'Connor, 1951, The Kansas Rock Column, Kansas State Geological Survey Bulletin 89, 31-52. 
Myers, N., and P. Bigsby, 1989, Hydrogeology and Ground-Water Quality Conditions at the Geary County Landfill, Northeast Kansas, 1988, Water Resources Research Investigations Report 89-4114, U.S. Geological Survey.

NAIP, 2002, aerial photograph of Navarre, Kansas, National Agricultural Imagery Program, U.S. Department of Agriculture, http://www.apfo.usda.gov/NAIP.html.

Papadopulos, 2000, (draft) Geoprobe ${ }^{\circledR}$ Assessment Report, Coop Facility, Navarre, Kansas, Terracon Project No. 01007102, prepared for S.S. Papadopulos and Associates, Bethesda, Maryland, and for the U.S. Department of Justice, by Terracon, Lexena, Kansas, November 17; transmitted to Argonne National Laboratory December 4, 2000.

Papadopulos, 2001, Supplemental Site Characterization, Navarre Superfund Site, Navarre, Kansas, draft report prepared for the Commodity Credit Corporation, U.S. Department of Agriculture, Washington, D.C., by S.S. Papadopulos \& Associates, Inc., January.

PRC, 1992, Navarre Groundwater Contamination Site, submitted to the U.S. Environmental Protection Agency, Region 7, Kansas City, Kansas, by PRC Environmental Management, Inc., Kansas City, Kansas, March 26.

Servi-Tech, 1993, Environmental Property Assessment: The Navarre Farmers Union Cooperative Association, Navarre, Kansas, submitted to the Navarre Farmers Union Cooperative Association, Navarre, Kansas, by Servi-Tech, Inc., Dodge City, Kansas, November 26.

Stroda, G.J., 2000, Deposition of Gregory J. Stroda, Case No. 99-1100-JTM in the U.S. District Court for the District of Kansas, August 30.

Stroda, G., 2007, personal communication from Stroda (Manager, Navarre Co-op, Navarre, Kansas) to L. Larsen (Larsen \& Associates, Inc., Lawrence, Kansas), stating that he believed that well Co-op 2 existed before the Co-op operated in that area and may have been installed by the railroad, April 27.

USDA, 1957, aerial photograph AYH-2T-127, U.S. Department of Agriculture, July 13.

USDA, 1965, aerial photograph AYH-4FF-101, U.S. Department of Agriculture, August 12. 
USDA, 1971, aerial photograph AYH-3MM-147, U.S. Department of Agriculture, June 28.

USDA, 2007, unpublished information from the Commodity Credit Corporation, U.S. Department of Agriculture, Washington, D.C., regarding previous communications with two former Navarre Co-op employees (P. Homman and R.M. Griffis) and a former Co-op fumigant contractor (L. Rakowski, formerly employed by Eco-Lab in Manhattan, Kansas) in 2000. These individuals indicated that the Co-op had regularly used the "80-20" fumigant mixture containing $20 \%$ carbon tetrachloride to treat grain at the Co-op flat storage building and at least one other building ("grain elevator") on the Co-op property.

Voit, K.M, 2000, Deposition of Karl Michael Voit, Case No. 99-1100-JTM in the U.S. District Court for the District of Kansas, December 5.

Warders, J.J., 2000, Deposition of Jeffrey J. Warders, Case No. 99-1100-JTM in the U.S. District Court for the District of Kansas, November 8. 


\section{Appendix A:}

\section{Electronic and Lithologic Logs}




\section{Argonne National Laboratory}

Project: Navarre

Geologist: Lorraine LaFreniere/Lisa Larsen
Elevation: $1352.62 \mathrm{ft}$.

Depth: $71 \mathrm{ft}$. BGL

\section{Boring ID: NATI-1}

Log Date: 4/5/2006

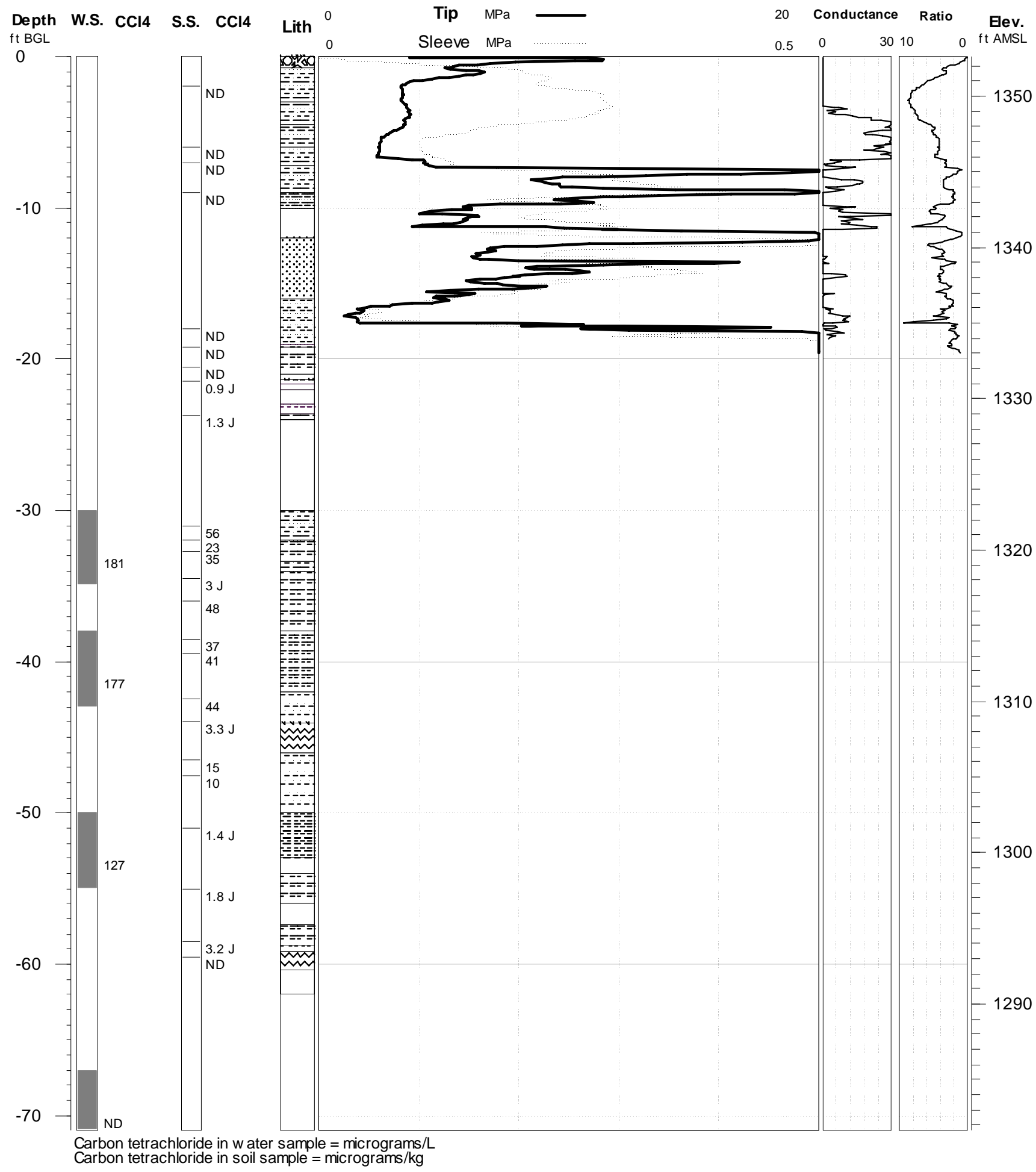




\section{Argonne National Laboratory}

Project: Navarre

Geologist: Lorraine LaFreniere/Lisa Larsen

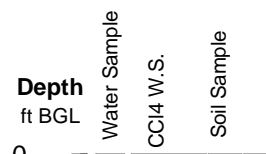

0

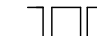

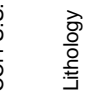

LITHOLOGY DESCRIPTION

Elev

$\mathrm{ft}$ AMSL

GRAVEL: Road, intermixed with silty clay.

CLAY, SILTY WITH SAND: Trace sand, brown (7.5YR), evidence of roots, dense, stiff.

$=-2$

D. ND

$-10$

ND

$-15$

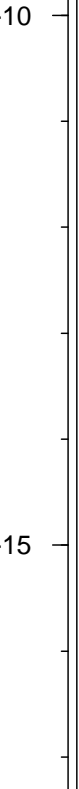

CLAY, SILTY WITH SAND: Abundant silt, yellow-brown (7.5YR), trace limestone fragment,

subrounded, some sand, white calcareous inclusions.

SILT, CLAYEY WITH SAND: Brown-yellow (7.5YR), increase in sand with depth, fine with coarse grains, scattered, oxidized.

SAND, SILTY WITH CLAY: Yellow-brown (7.5YR), coarse silt, medium sand, some clay matrix, oxidation evident, slightly calcareous, occasional calcite crystals.

NO RECOVERY

SAND: Slightly cemented, pale yellow (5Y), trace roots, oxidized, trace calcite from 14-16 ft., platy. 
NATI-1 Pg. 2

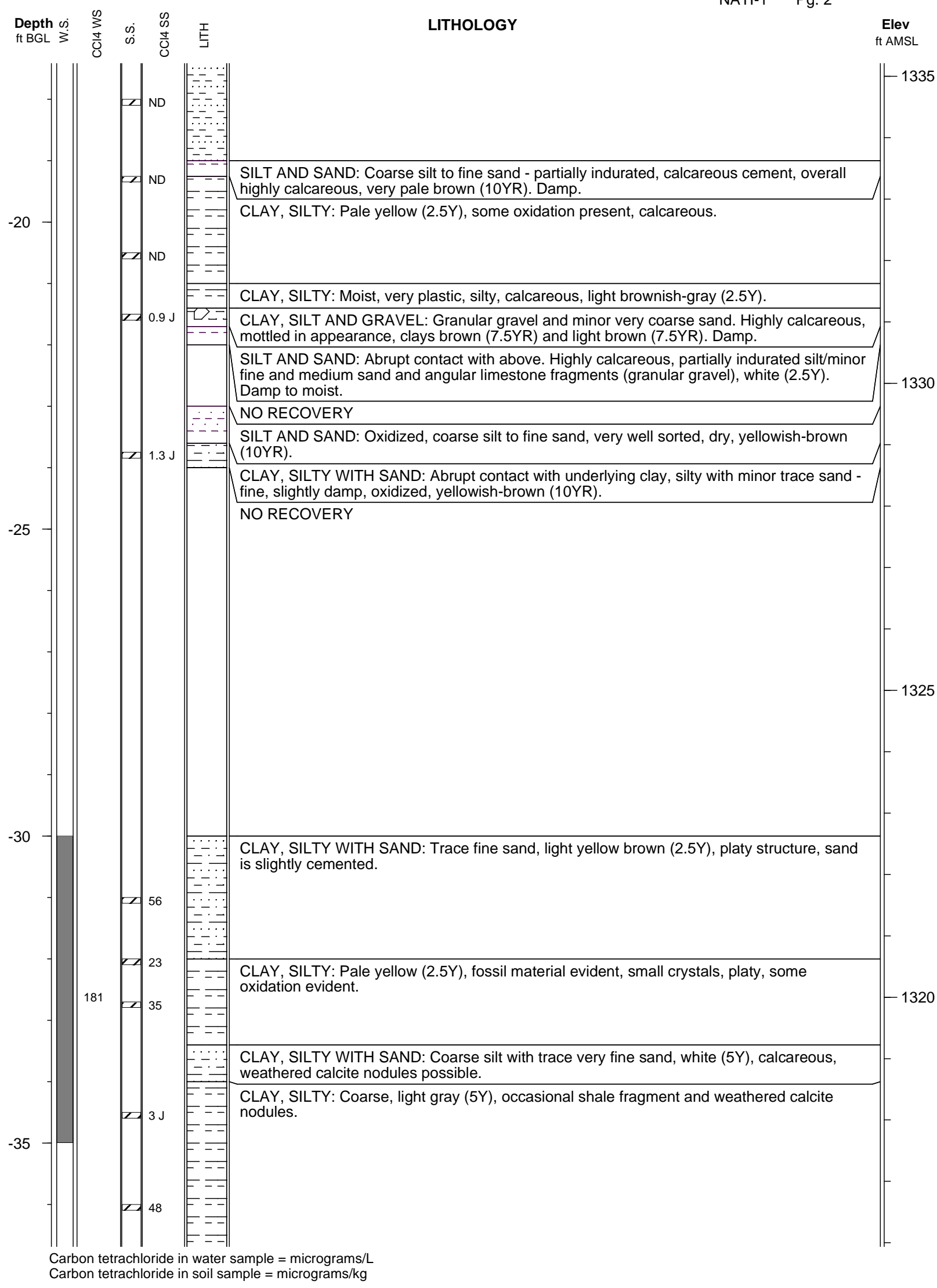




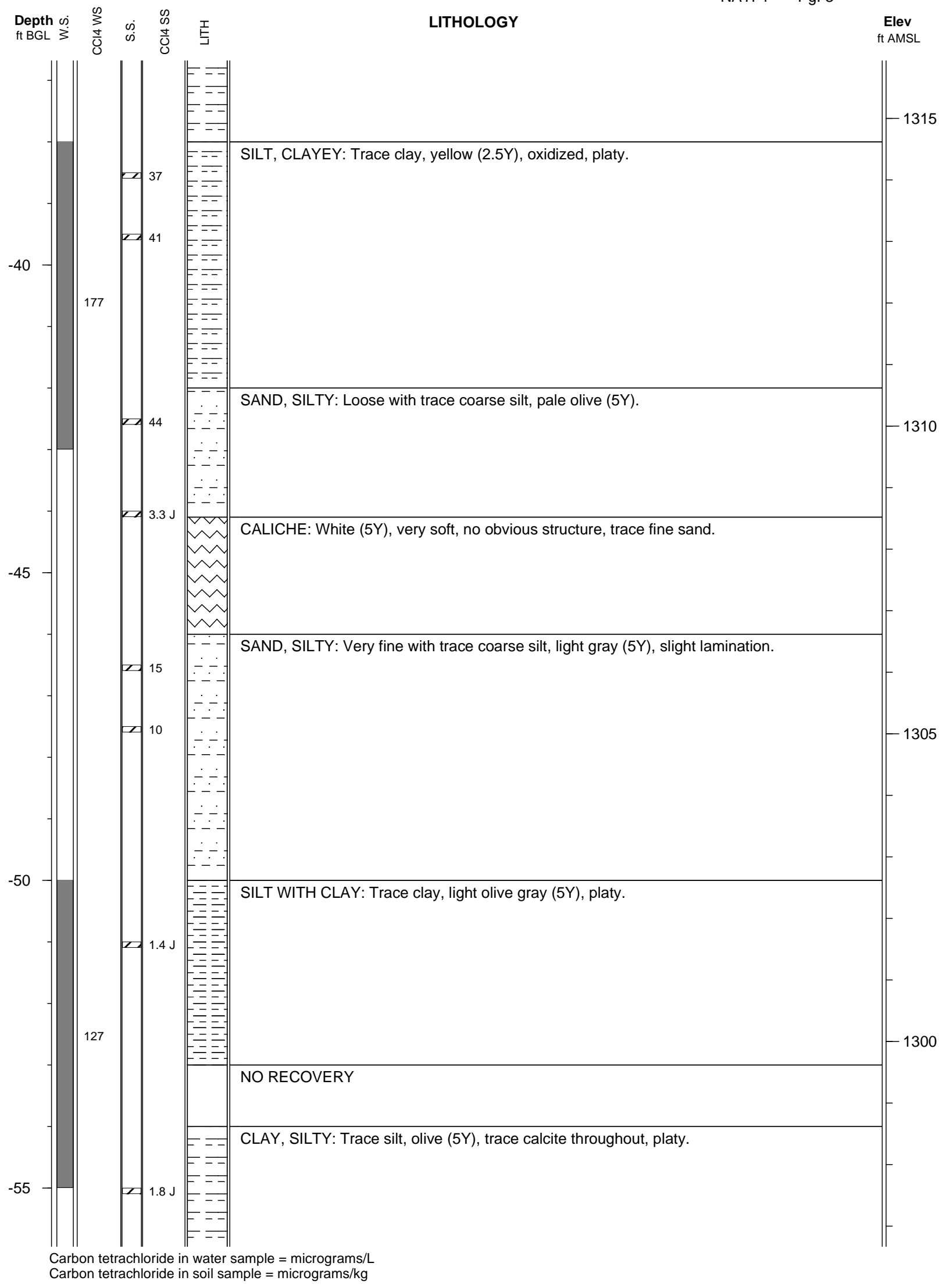


NATI-1 Pg. 4

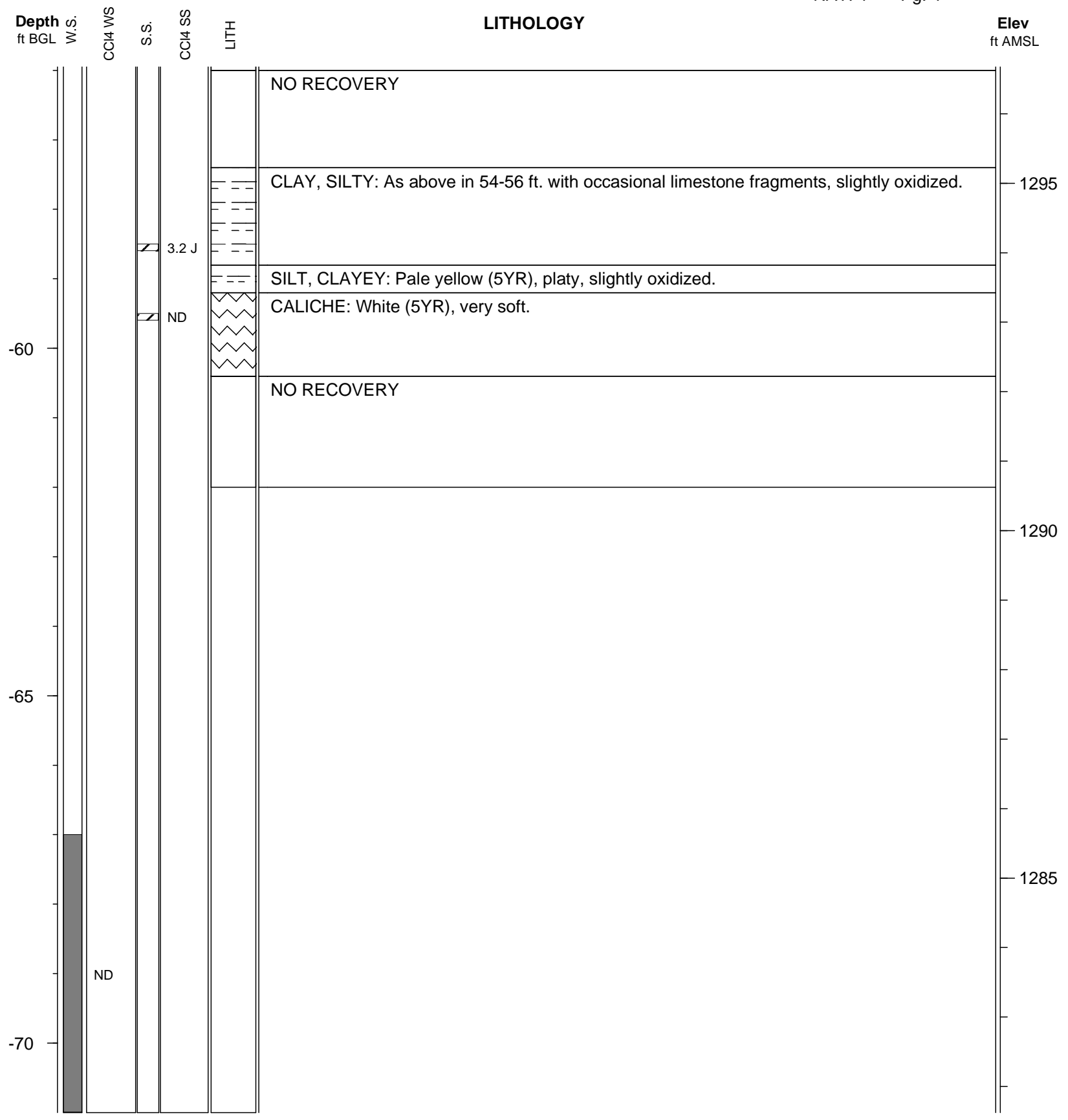

Carbon tetrachloride in water sample $=$ micrograms $/ \mathrm{L}$ Carbon tetrachloride in soil sample $=$ micrograms $/ \mathrm{kg}$ 


\section{Argonne National Laboratory} Project: Navarre

Geologist: Lorraine LaFreniere
Elevation: $1351.64 \mathrm{ft}$

Depth: $74 \mathrm{ft} . \mathrm{BGL}$

\section{Boring ID: NATI-2}

Log Date: 4/5/2006

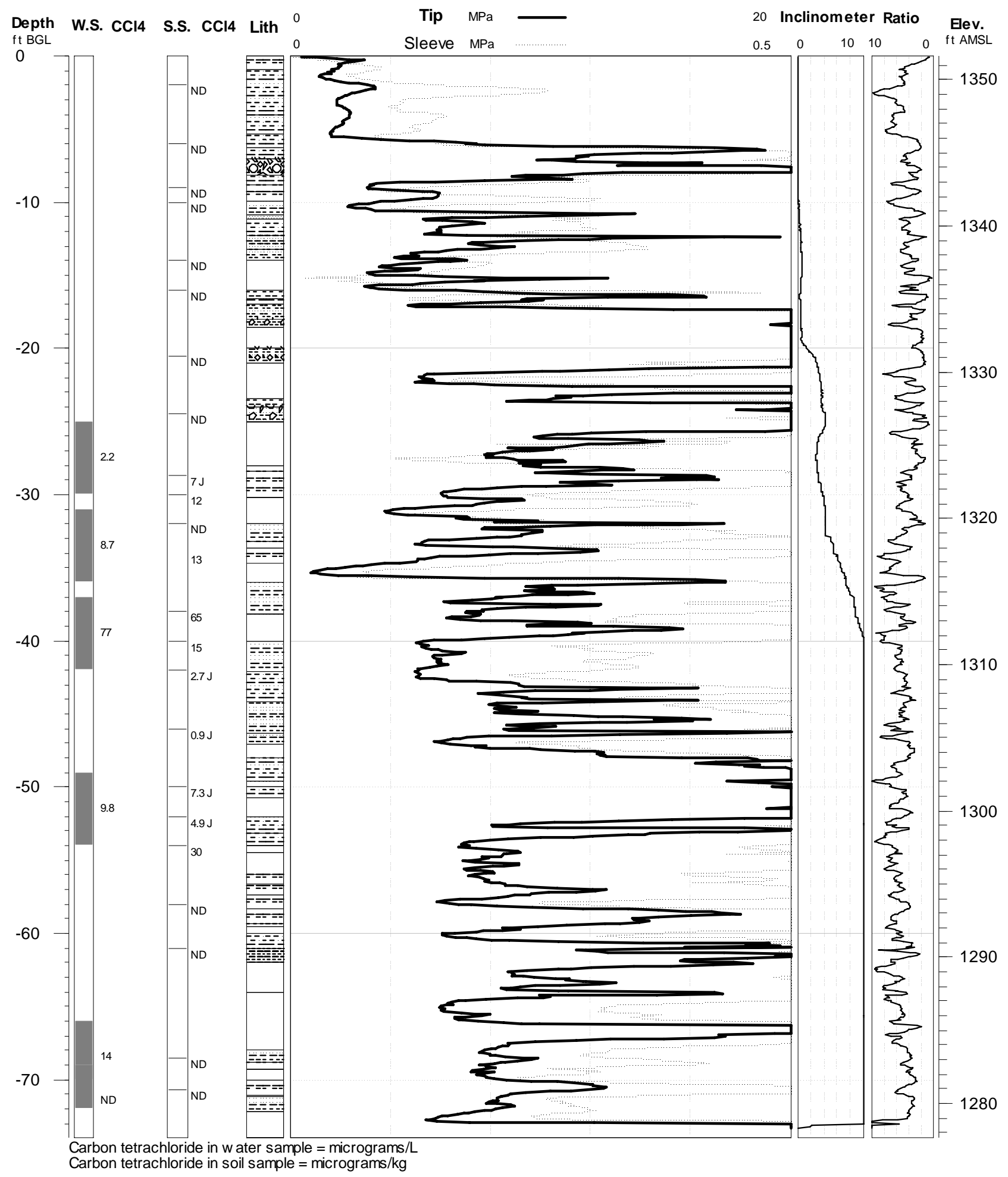




\section{Argonne National Laboratory}

\section{Project: Navarre}

Geologist: Lorraine LaFreniere

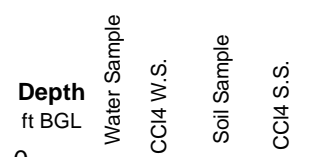

Elevation: $1351.64 \mathrm{ft}$

Boring ID: NATI-2

Depth: $74 \mathrm{ft}$. BGL

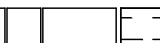

SILTY CLAY: Disturbed silty clay and road gravel.

CLAY, SILTY WITH SAND: Very silty with minor medium grained sand scattered

throughout. Damp. Brown (7.5YR). (7.5YR), very silty clay, becoming increasingly silty with depth, interspersed with poorly defined zone of sandy silt in a clay matrix. Sand very fine to very occasionally medium grained. Damp. Limestone cobble - rounded (1.2 cm length) at 5.8'. Matrix clays are non-calcareous. Sandy silt zones highly calcareous. Limestone cobbles throughout sequence increasing in frequency with depth.

SILT, CLAYEY WITH SAND: Very silty clay matrix with sand. Brownish-yellow (7.5YR). Clay - plastic, silty, fairly stiff, brownish-yellow (10YR) with fine grained sandy partings.

GRAVEL: "Rubble zone" with cobbles (calcareous cemented sandstone) throughout increasing in frequency with depth. Highly calcareous, rounded to sub-rounded to 2.5 " in length. Dry to slightly damp, vuggular solution porosity.

SILT, CLAYEY WITH SAND: Sand fine to very coarse grained, poorly sorted angular to sub-angular, mixed lithology occurring in lenses throughout. Sandy lenses highly calcareous, clay - non-calcareous. Damp. Brownish-yellow (10YR). CLAY, SILTY: Damp, yellowish-brown (10YR), slightly calcareous.

SILTY CLAY: Faintly laminated, silty clays, calcareous, alternating thin yellowish-brown (10YR) and light brownish-gray (10YR), non-calcareous. Damp.

SILT, CLAY, AND SAND: Fairly stiff with lenses and partings of fine sand, manganese staining, highly calcareous, yellowish-brown (10YR). Damp.

SANDSTONE: Sandy rubble zone - sandstone - calcium carbonate cemented yellowish-brown (10YR), damp. Sand fine to coarse grained, angular to sub-angular, highly calcareous.

SILT, CLAYEY WITH SAND: Dense, hard, stiff, silty clay with manganese partings, minor sand lenses, light yellowish-brown (2.5Y), calcareous.

SAND AND CLAY: Platy texture, sand in lenses and partings, slightly calcareous, light yellowish-brown (2.5Y), fine sand.

CLAYEY SAND: Light yellowish-brown (2.5Y), calcareous, poorly sorted fine to very coarse sand, sub-angular. Damp to dry.

Carbon tetrachloride in water sample $=$ micrograms $/ \mathrm{L}$

Carbon tetrachloride in soil sample $=$ micrograms $/ \mathrm{kg}$ 


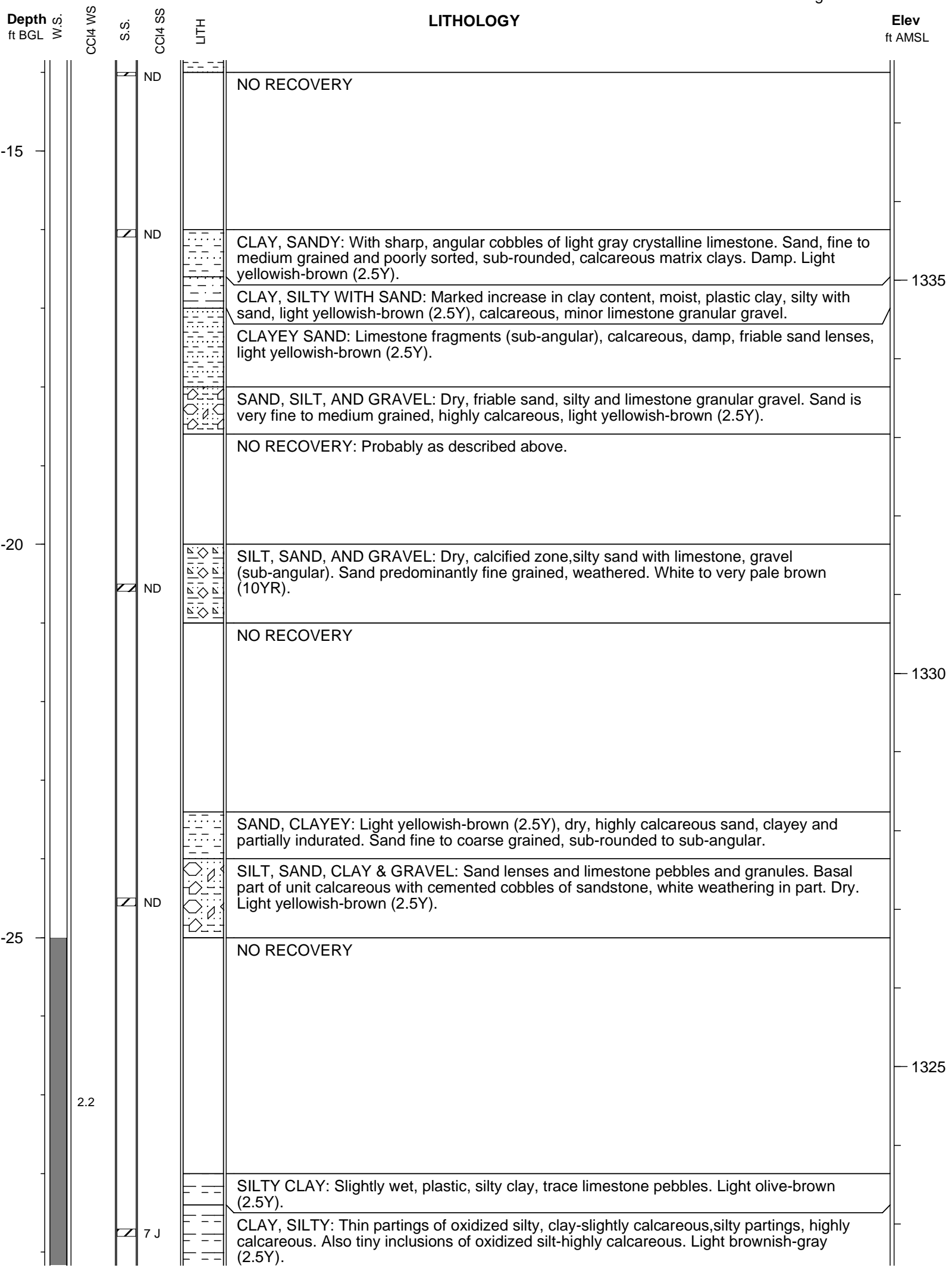




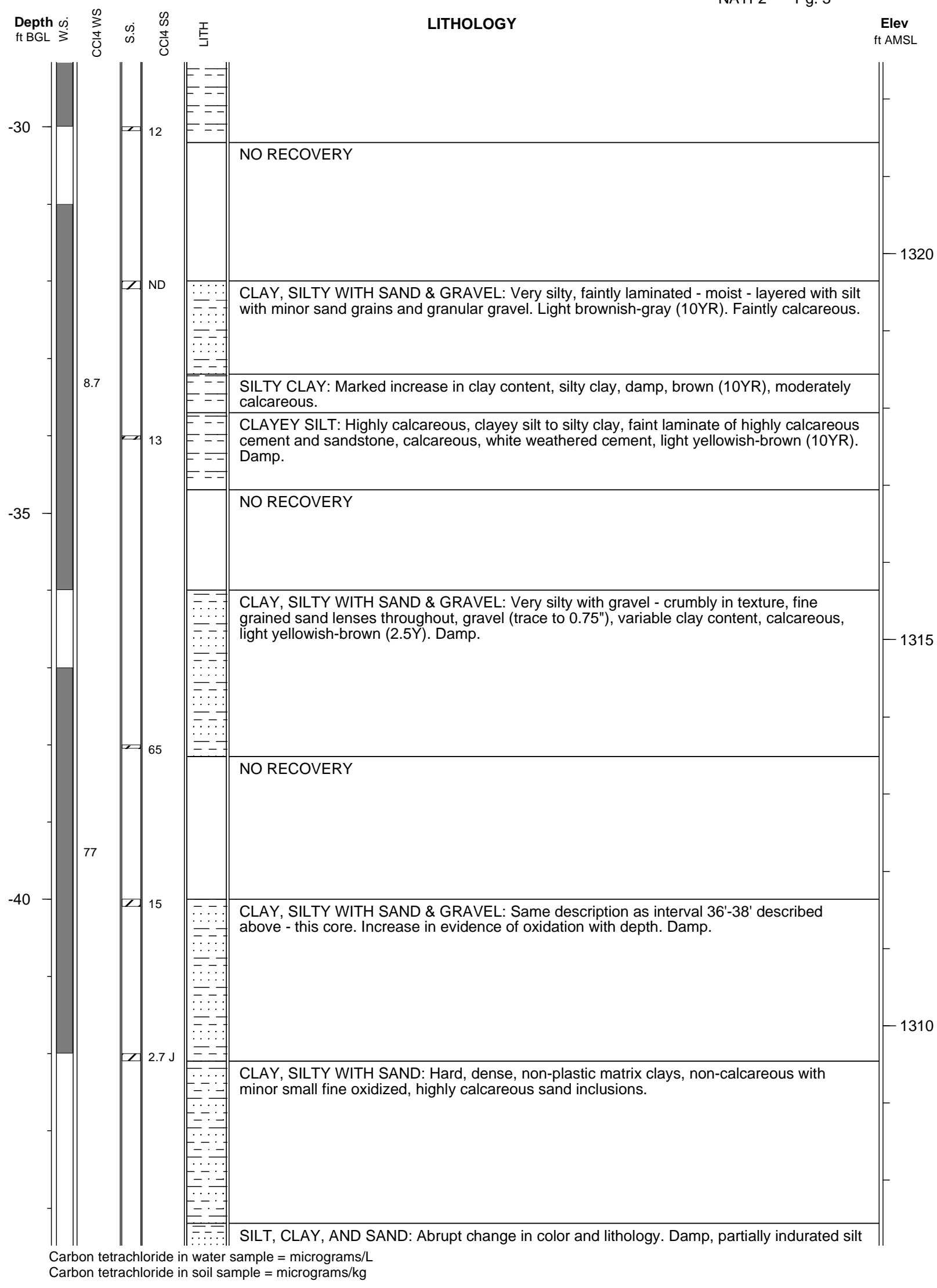


NATI-2 Pg. 4

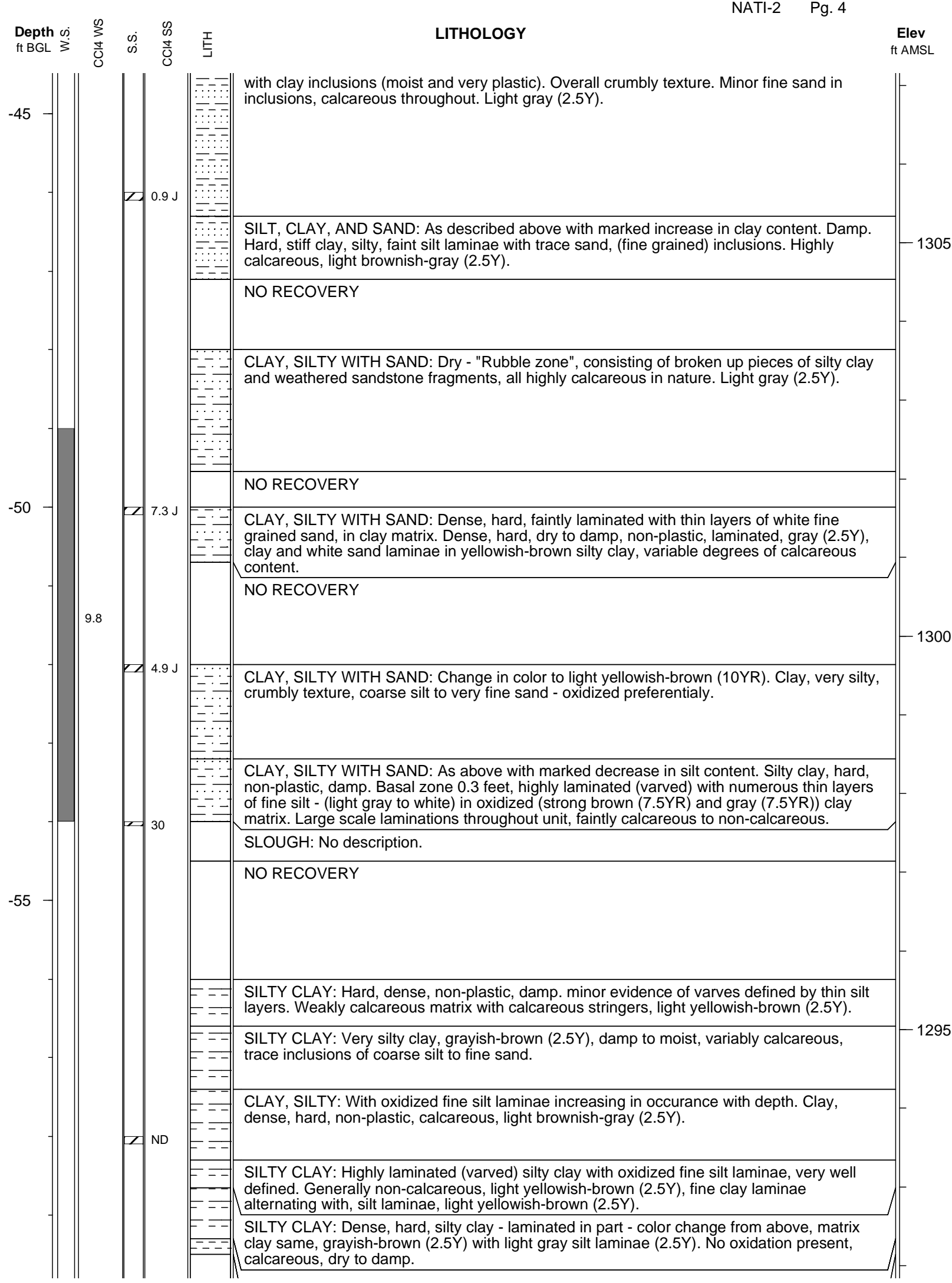




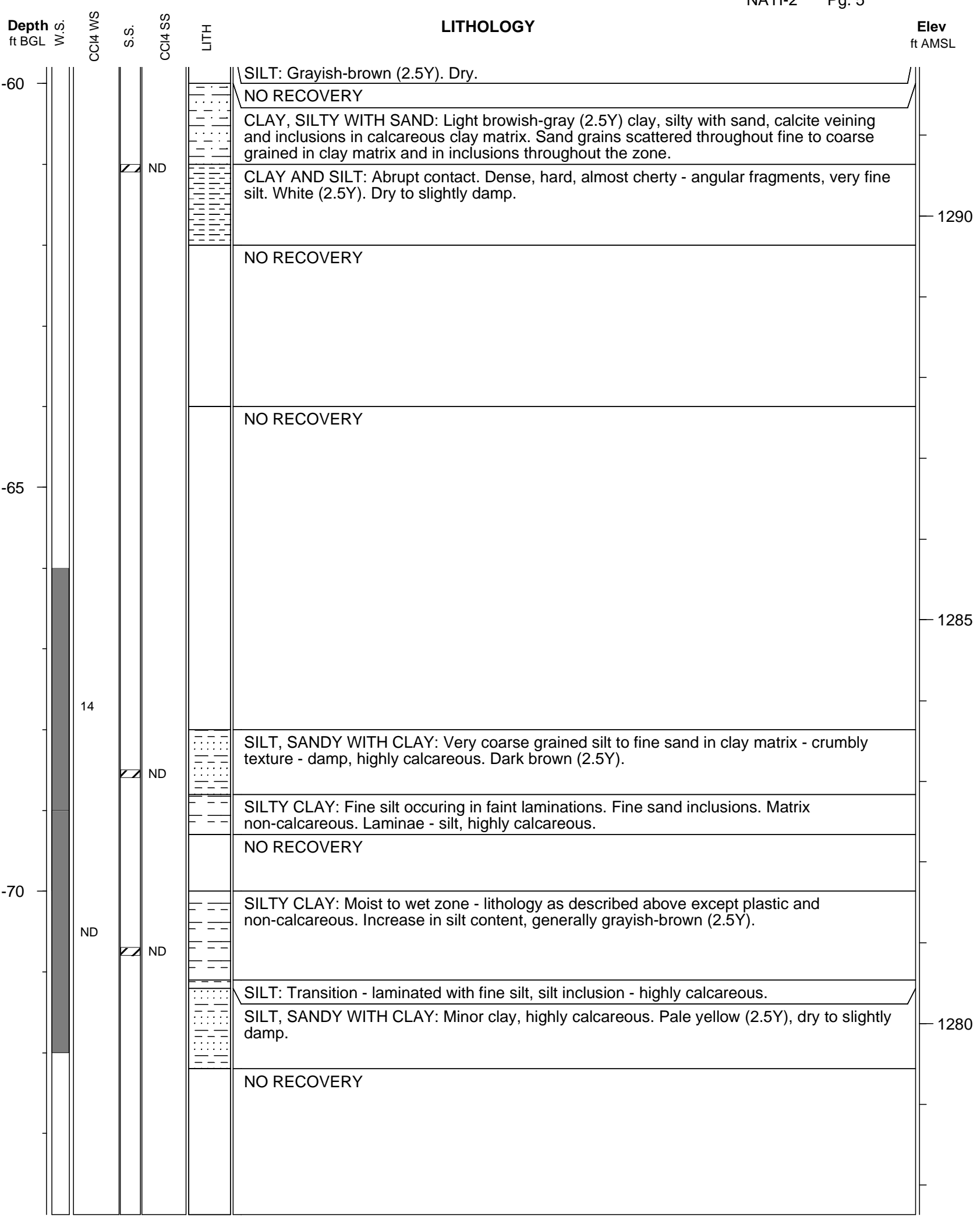




\section{Argonne National Laboratory}

Project: Navarre

Geologist: Lisa Larsen
Elevation: $1352.92 \mathrm{ft}$.

Depth: $64 \mathrm{ft} . \mathrm{BGL}$

\section{Boring ID: NATI-3}

Log Date: 4/5/2006

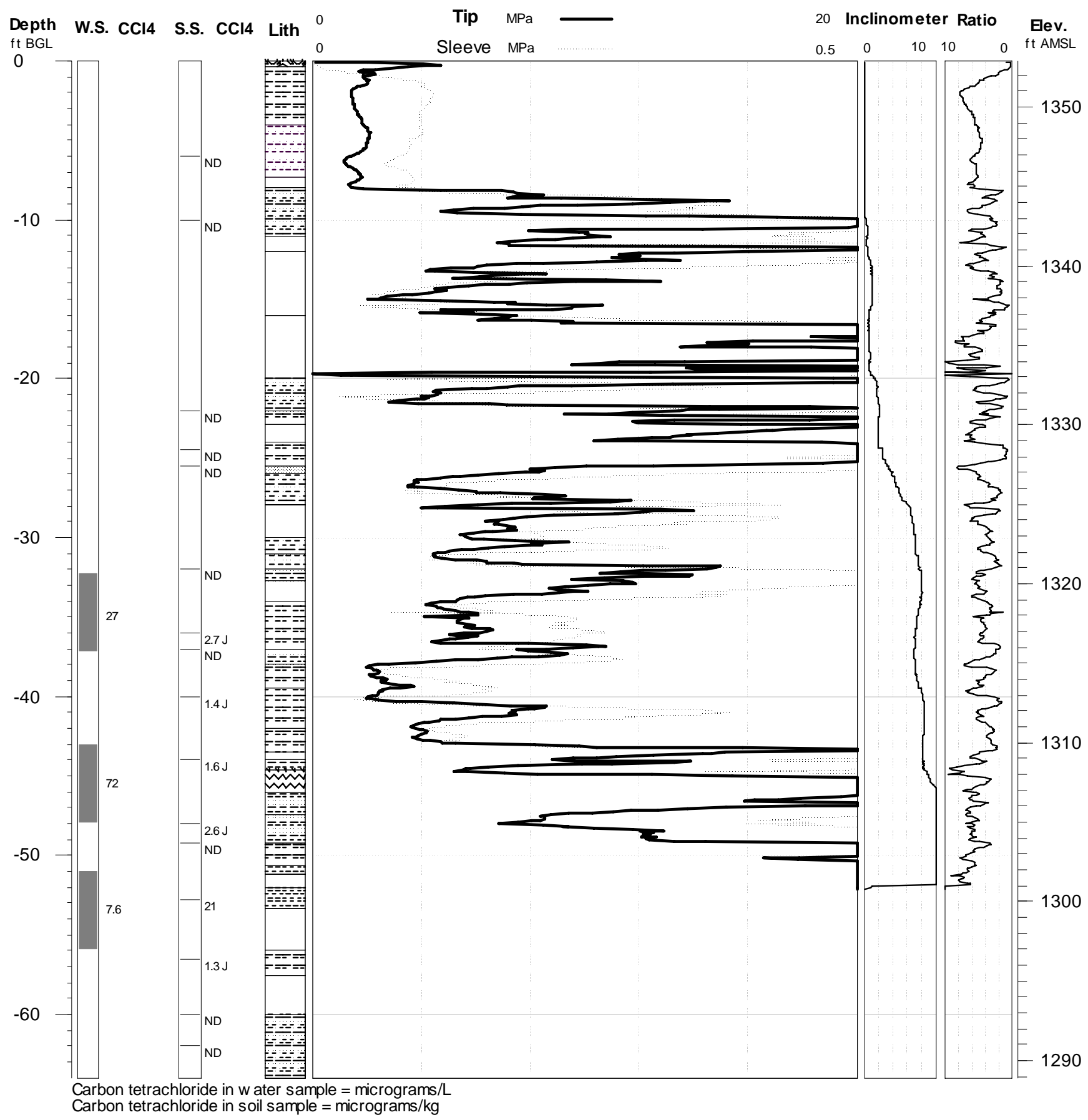




\section{Argonne National Laboratory}

Project: Navarre

Geologist: Lisa Larsen
Elevation: $1352.92 \mathrm{ft}$.

\section{Boring ID: NATI-3}

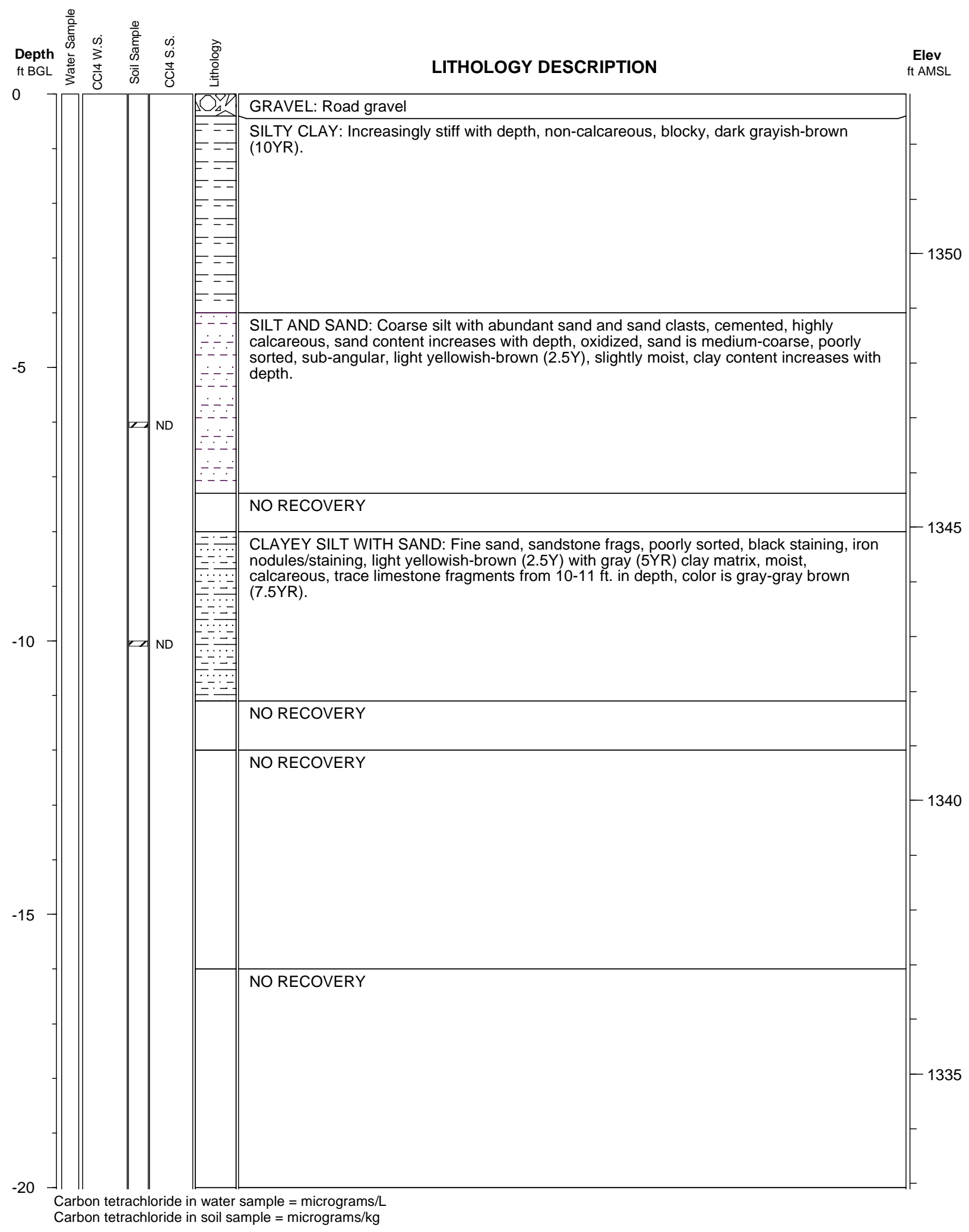


NATI-3 Pg. 2

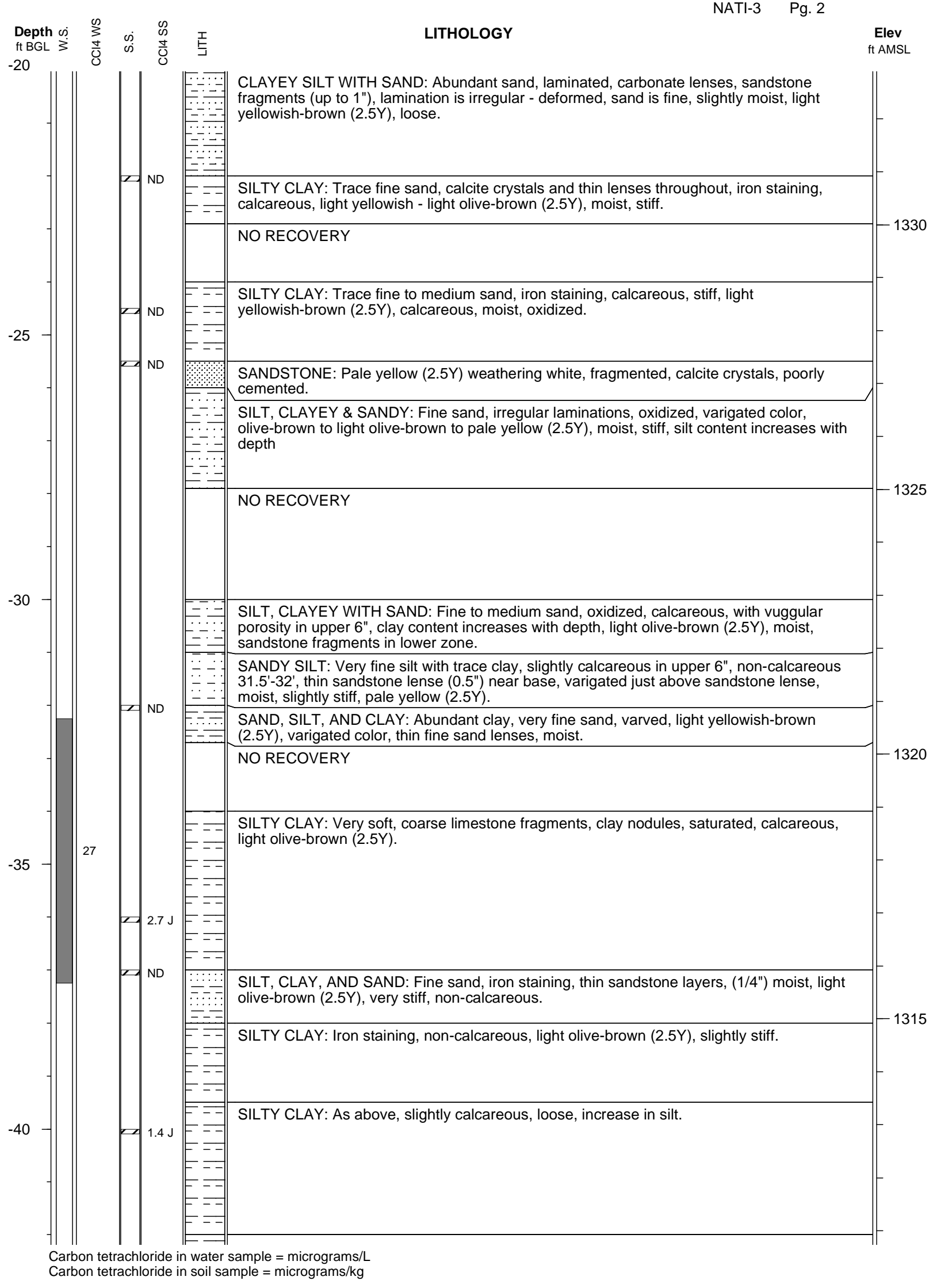


NATI-3 Pg. 3

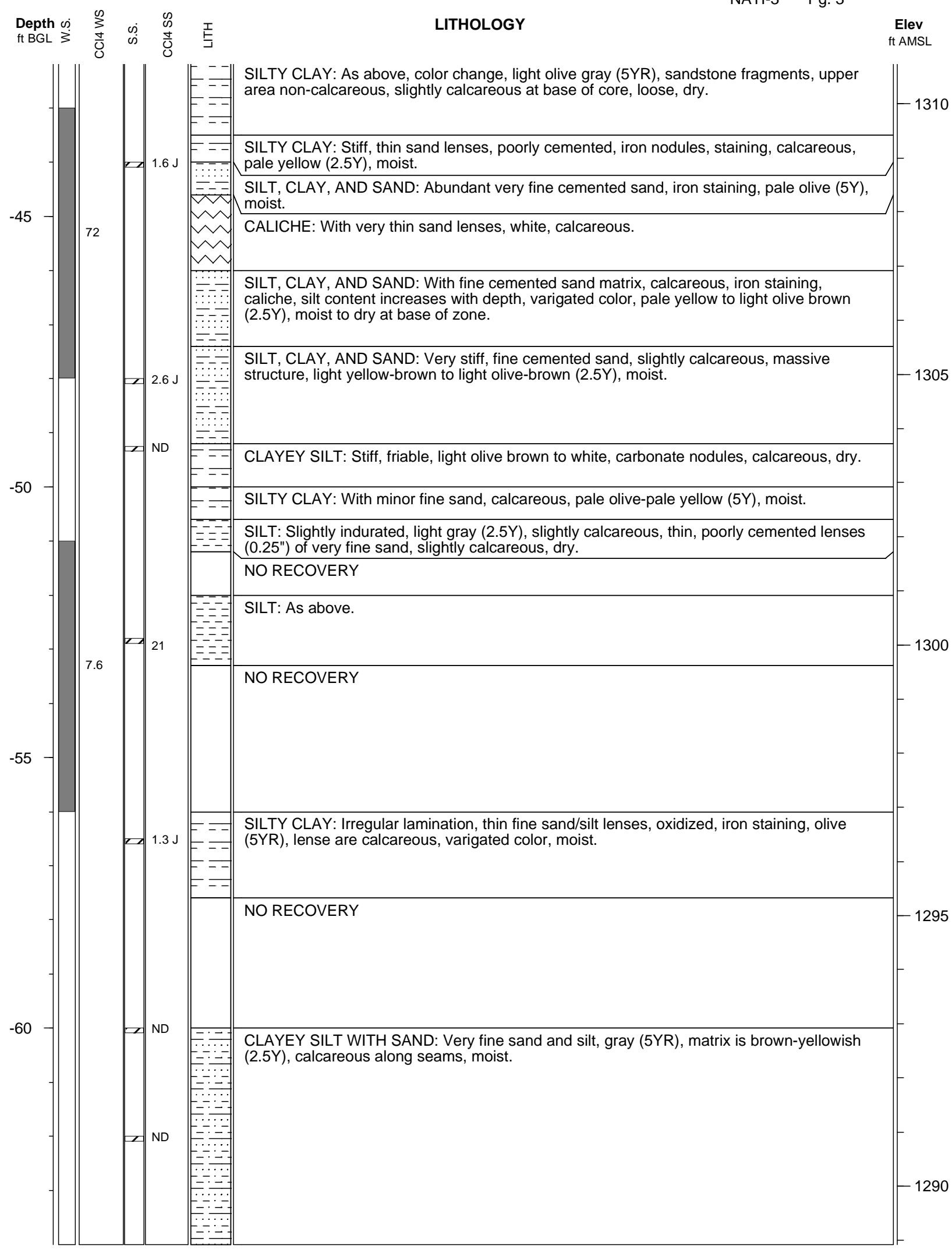

Carbon tetrachloride in water sample $=$ micrograms $/ \mathrm{L}$ Carbon tetrachloride in soil sample $=$ micrograms $/ \mathrm{kg}$ 


\section{Argonne National Laboratory}

Project: Navarre

Geologist: Lisa Larsen

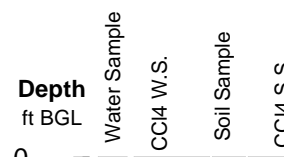

\section{ஸे}

Elevation: $1351.63 \mathrm{ft}$

\section{Boring ID: NATI-4}

Depth: $71 \mathrm{ft}$. BGL

SILT, CLAY, AND SAND: Trace sand, silt content increases with depth, few roots, iron staining at base of core, blocky structure, very dark gray (10YR) to dark gray (10YR) with depth, moist, stiff, non-calcareous.

CLAYEY SILT WITH SAND: Abrupt lithology change and sharp color change. Clayey silt, very fine sand, highly oxidized, vuggy porosity, irregular carbonate rich lenses throughout, sandstone pebbles and cobbles (up to $0.5 \mathrm{ft}$. diameter), angular to sub-rounded, dry, highly calcareous, except clay, small, black nodules, yellowish-brown (10YR).

SANDY SILT: Very fine, non-calcareous, poorly cemented with carbonate, varigated coloring, pale olive (2.5Y) with white carbonate viens.

NO RECOVERY

SILT, CLAY, AND SAND: As above, abundant silt, dark brown (7.5YR) to brown (10YR),

SILT, CLAY, AND SAND: Iron stained sand grains (sub-rounded, coarse), silt content increases with depth, dark gray (10YR) to dark brown (7.5YR), moist, stiff, non-calcareous.

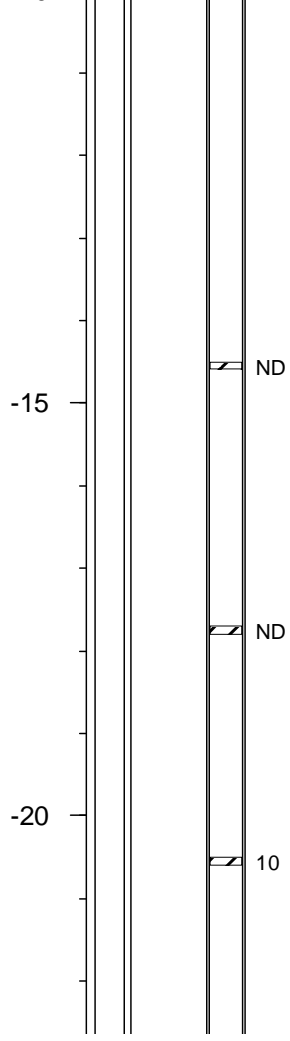

ND

SAND, SILT, AND GRAVEL: As above, decrease in clay, increase in sandstone pebbles, cobbles and sand, sub-rounded to angular.

SANDY SILT: Very fine, poorly cemented (carbonate), sandstone lenses (1-2" thick) irregular lamination, light olive-brown to light yellowish-brown (2.5Y), slightly moist, trace clay matrix, light gray-white coating, calcareous (except clay), clay content increases with depth, slightly moist-dry.

CLAYEY SILT WITH SAND: Sharp lithology change. Clayey silt with abundant fine sand, sandstone fragments, angular and platy, poorly cemented, calcareous cement, very stiff, iron staining, moist, light yellowish-brown (2.5Y).

SAND: Sharp lithology change. Sand poorly sorted, calcareous cement, fine to medium sand, pale yellow with white coating $(2.5 \mathrm{Y})$, dry. NO RECOVERY

CLAYEY SILT: Abundant clay, irregular vuggular porosity, calcite and carbonate (caliche?) lenses, carbonate nodules, light olive-brown to light yellowish-brown (2.5Y) with depth, varigated.

\section{NO RECOVERY}




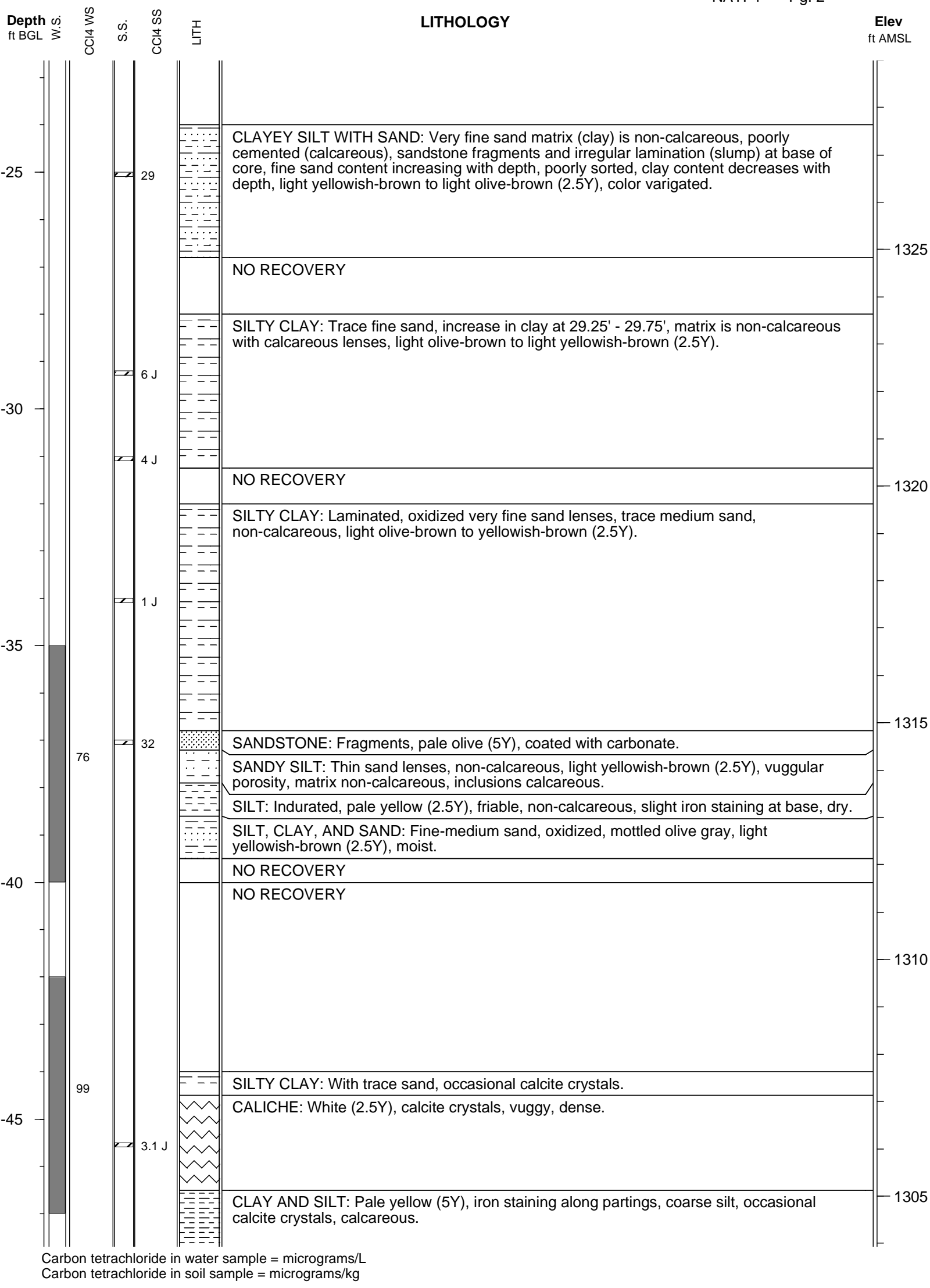


NATI-4 Pg. 3

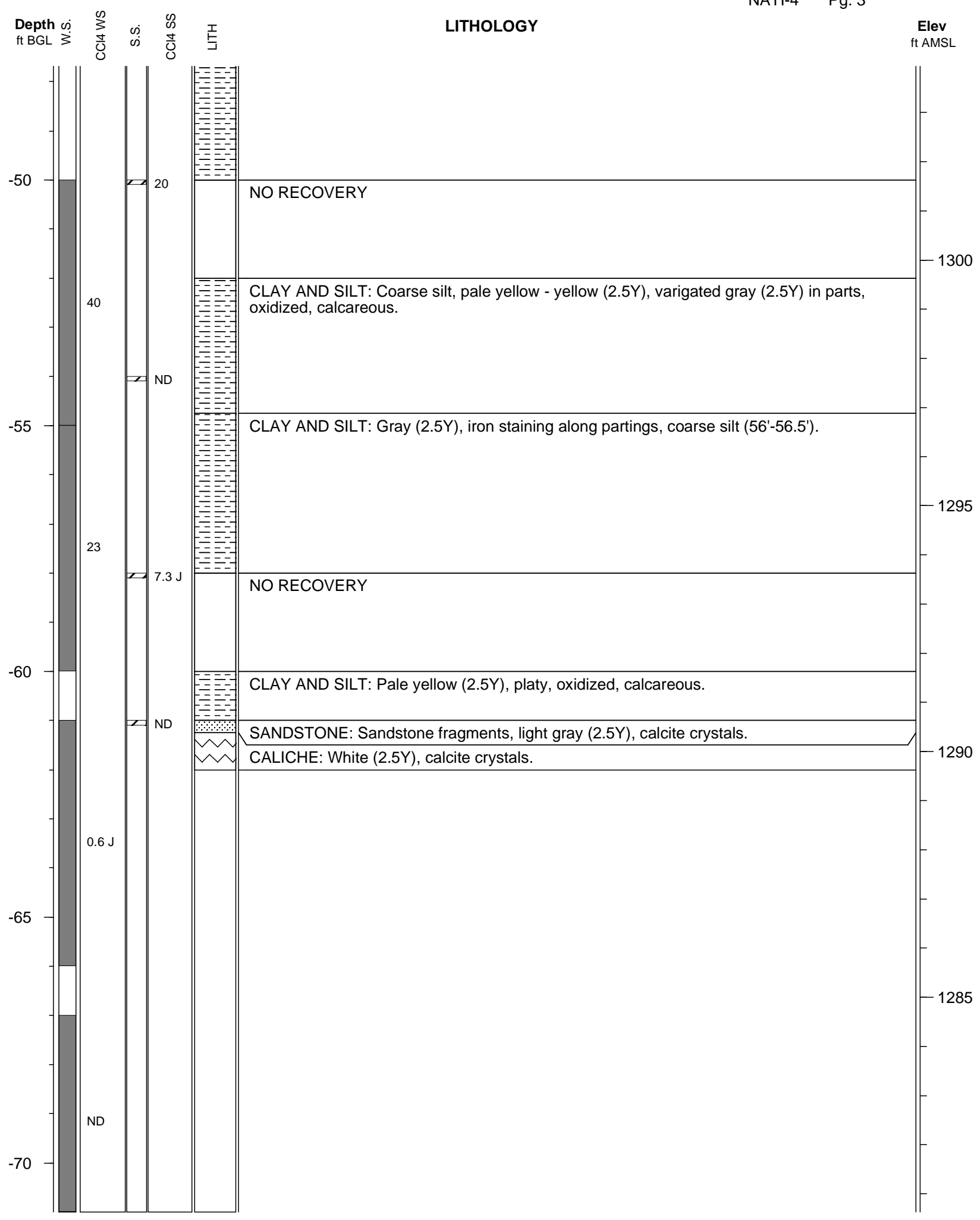

Carbon tetrachloride in water sample $=$ micrograms $/ \mathrm{L}$ Carbon tetrachloride in soil sample $=$ micrograms $/ \mathrm{kg}$ 


\section{Argonne National Laboratory}

\section{Project: Navarre}

Geologist: Lorraine LaFreniere
Elevation: $1351.96 \mathrm{ft}$.

Depth: $77.8 \mathrm{ft} . \mathrm{BGL}$

\section{Boring ID: NATI-5}

Log Date: 4/25/06

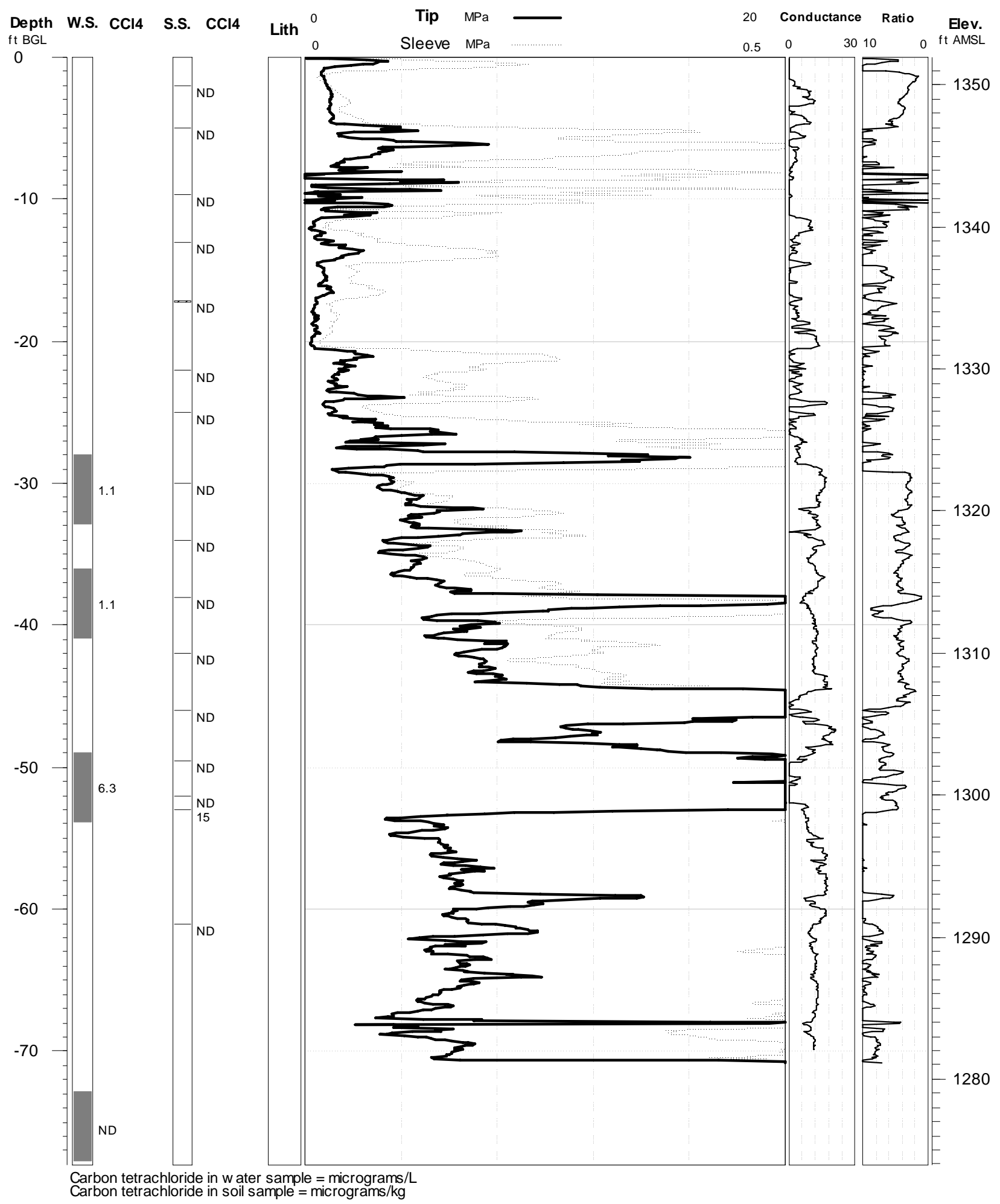




\section{Argonne National Laboratory}

Project: Navarre

Geologist: Lorraine LaFreniere/Lisa Larsen
Elevation: $1352.73 \mathrm{ft}$

Depth: $73 \mathrm{ft}$. BGL

\section{Boring ID: NATI-6}

Log Date: 4/24/2006

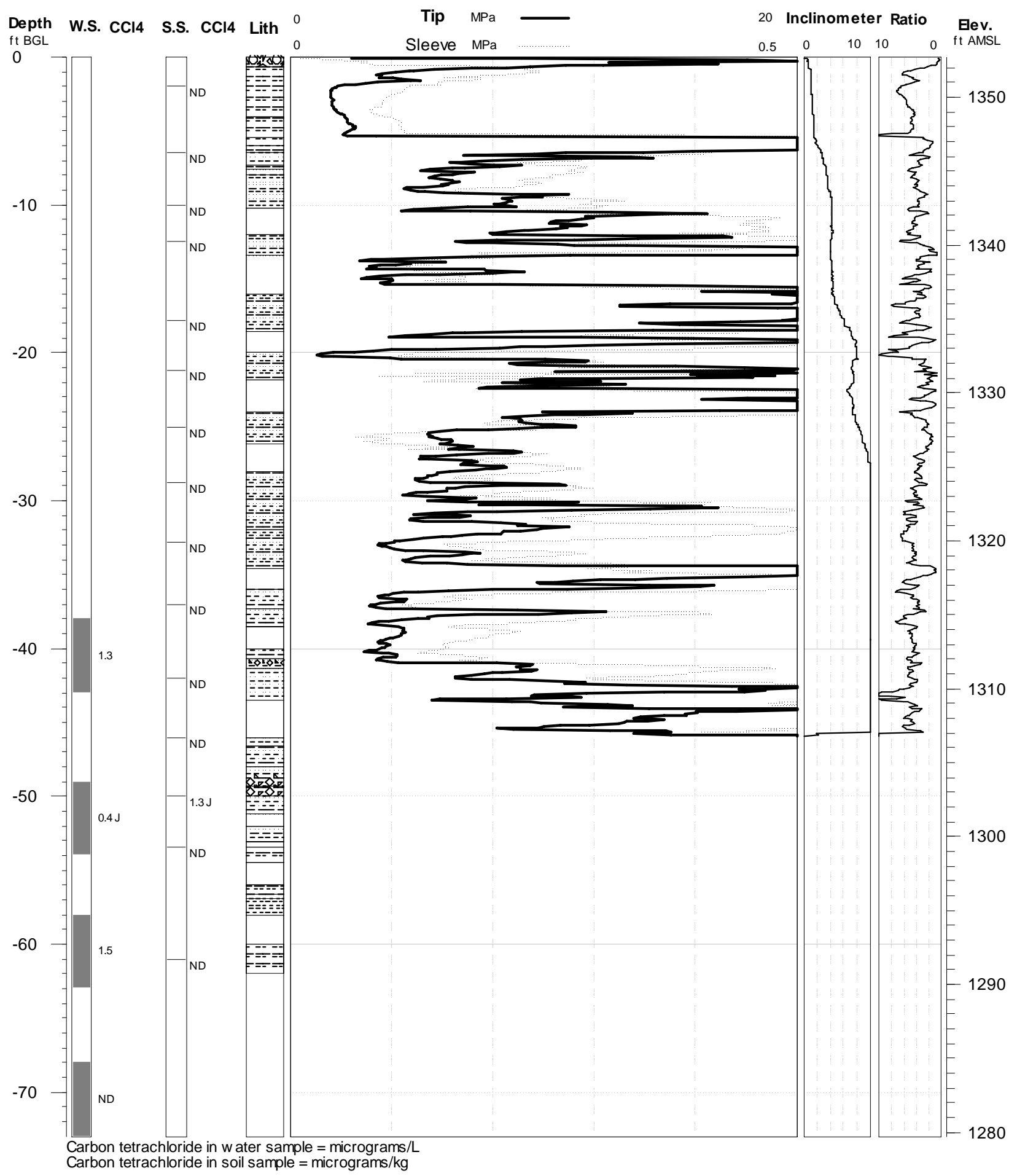




\section{Argonne National Laboratory}

Project: Navarre

Geologist: Lorraine LaFreniere/Lisa Larsen

\section{Boring ID: NATI-6}

Elevation: $1352.73 \mathrm{ft}$.

Depth: $73 \mathrm{ft}$. BGL

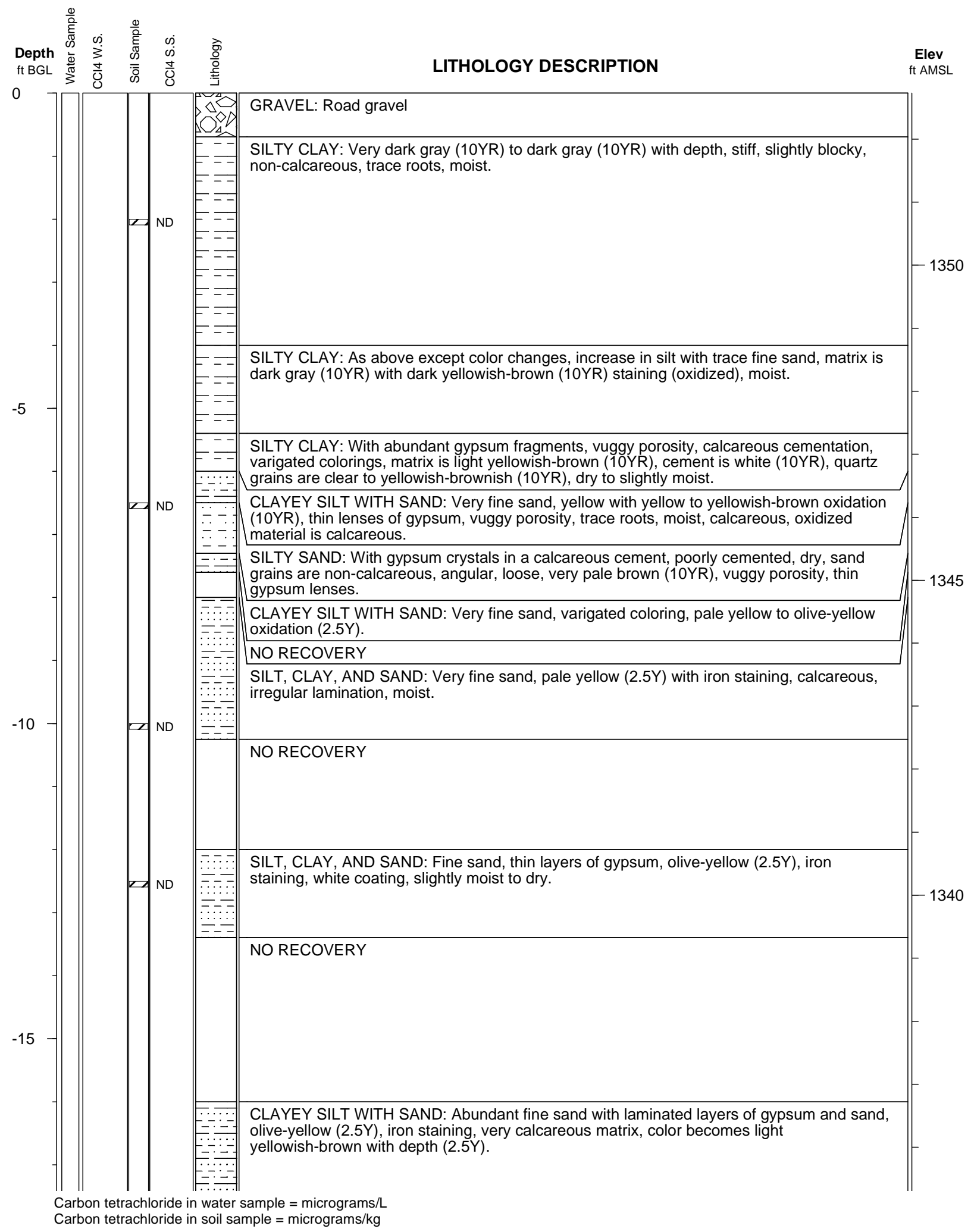


NATI-6 Pg. 2

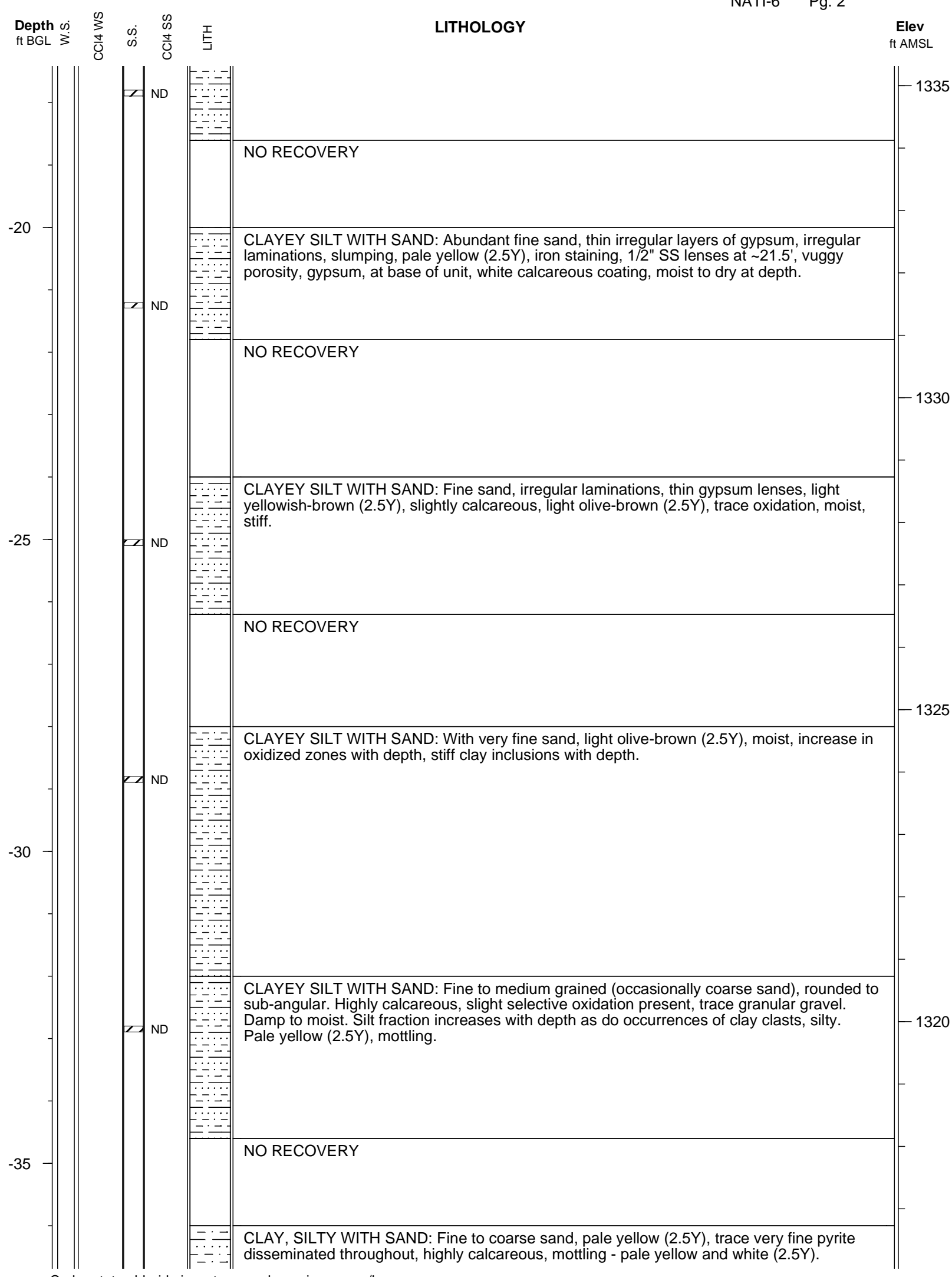




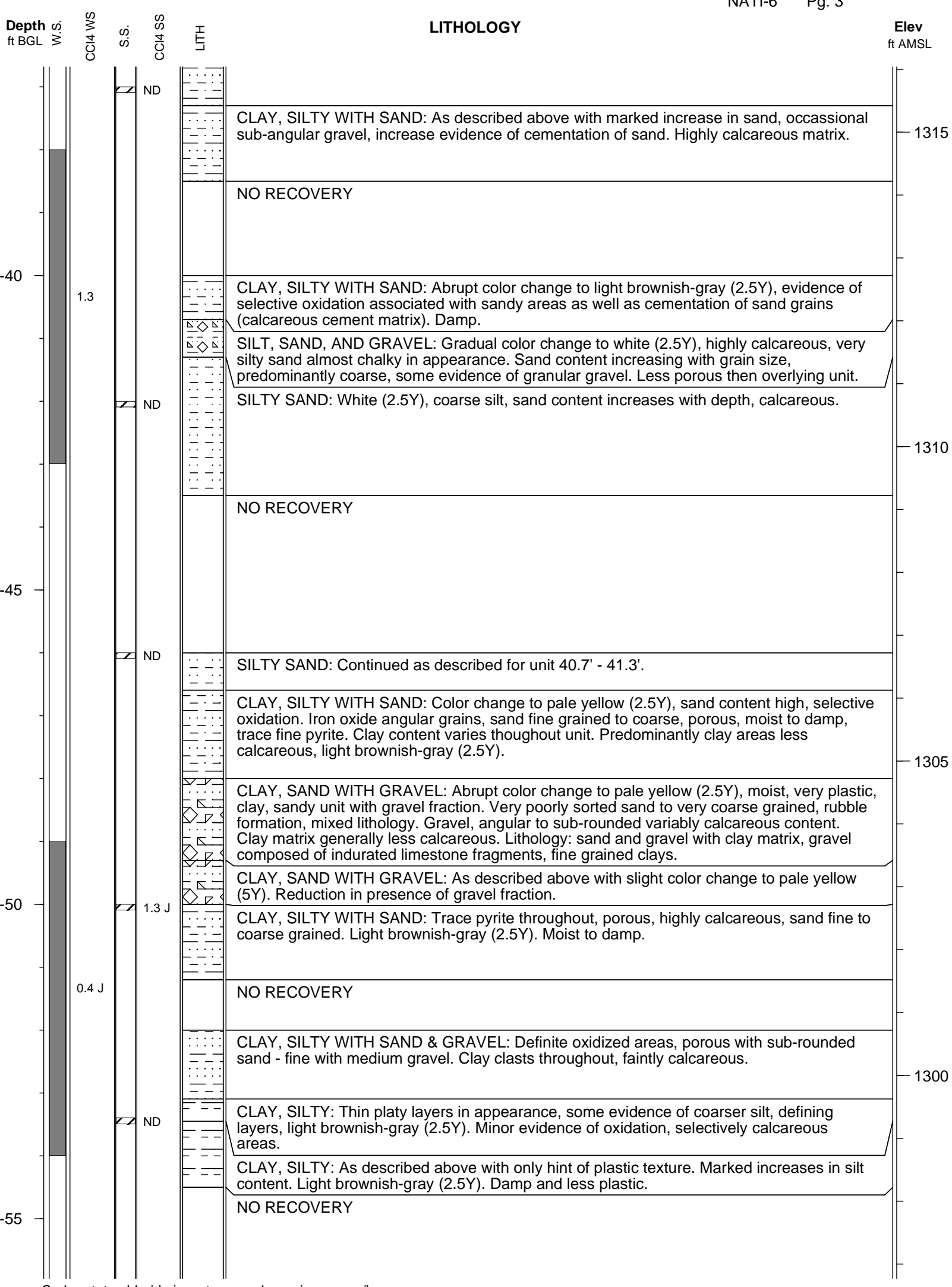


NATI-6

Pg. 4

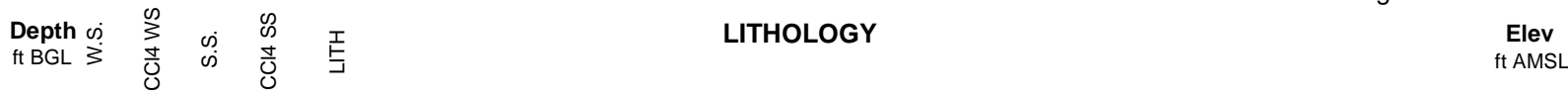

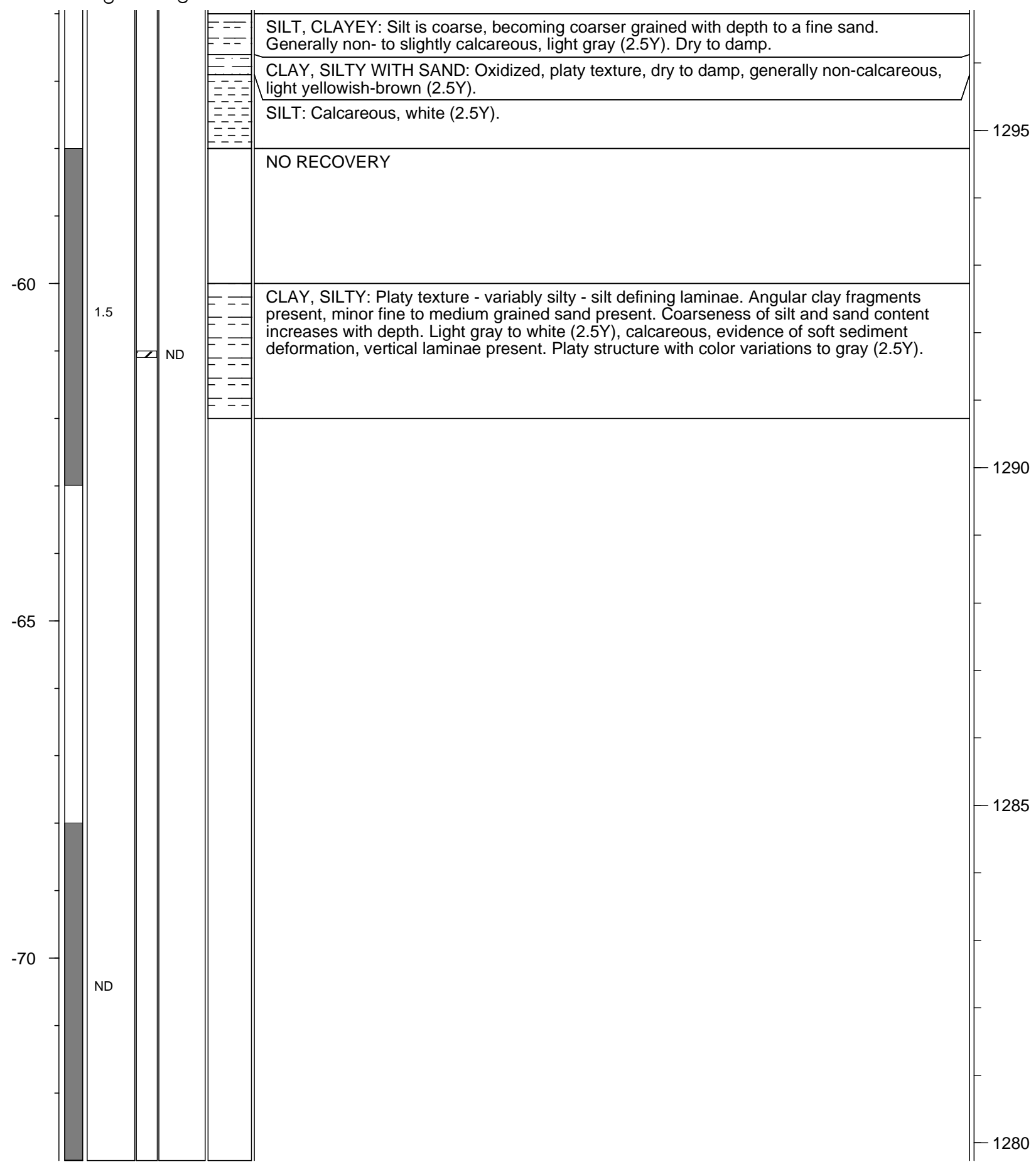

Carbon tetrachloride in water sample $=$ micrograms $/ \mathrm{L}$ Carbon tetrachloride in soil sample $=$ micrograms $/ \mathrm{kg}$ 


\section{Argonne National Laboratory}

\section{Project: Navarre}

Geologist: Lorraine LaFreniere
Elevation: $1351.14 \mathrm{ft}$.

Depth: 72 ft. BGL

\section{Boring ID: NATI-7}

Log Date: 4/8/2006

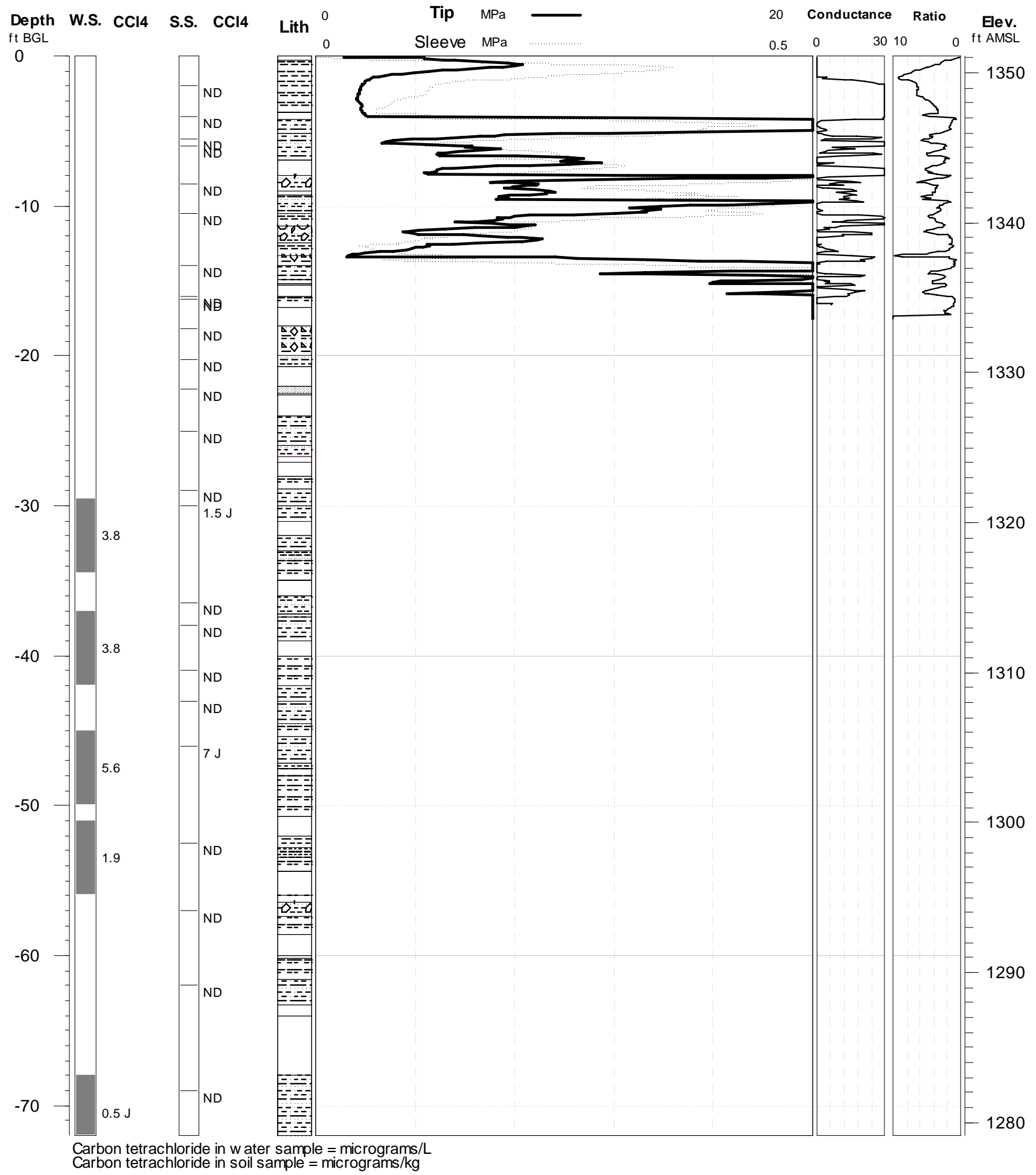




\section{Argonne National Laboratory}

\section{Project: Navarre}

Geologist: Lorraine LaFreniere
Elevation: $1351.14 \mathrm{ft}$

Depth: 72 ft. BGL

\section{Boring ID: NATI-7}

Elev CLAY: Fill CLAY: Dark grayish-brown (10YR), non-calcareous, damp, plastic.

SAND, SILTY: Dry, friable, sub-angular to sub-rounded quartz, light yellowish-brown (2.5Y) Highly calcareous, partially cemented.

CLAY, SILTY WITH SAND: With inclusions and lenses of highly calcareous oxidized silty sand, well sorted fine sand to coarse silt. Sand is light yellowish-brown (2.5Y) clay matrix brown (10YR) calcareous clay.

SILT, CLAYEY WITH SAND: Dry, very difficult zone to penetrate - silt, clayey with sand,

light yellowish-brown (2.5Y). Dry, medium sand in lenses, sub-rounded grains, calcareous.

NO RECOVERY

CLAY, SILT AND GRAVEL: Lenses of silt and trace granular gravel. Damp, siltier areas calcareous, damp to dry. Light brownish-gray (10YR).

CLAY, SILTY: As above - no gravel - more highly oxidized, light yellowish-brown (2.5Y), damp to dry, highly calcareous.

CLAYEY SILT WITH SAND: Color change - contact gradational, clayey silt with occassional sand grains (sub-rounded quartz). Highly calcareous in matrix, lenses of coarse silt to fine sand, very highly calcareous. Partly indurated lenses, light olive gray (5Y).

CLAY: Gradational contact into clay with tiny inclusions and very thin sandy partings. Light olive brown (2.5Y), highly calcareous.

SILT, SAND, CLAY \& GRAVEL: Fine grained silty, sand with clay inclusions, angular sandstone gravel (granular to pebble size), calcium carbonated cemented - faintly laminated, oxidized light yellowish-brown (2.5Y).

CLAY: Moist clay, plastic, silty, light yellowish-brown (2.5Y), calcareous.

CLAY,SILT,SAND \& GRAVEL: Wet, clay, silty with gravel and sand. Fossil fragments (pelecypod fragments) Gravel - sandstone (calcareous cementation), sand to very coarse grained "hash zone".

CLAY, SILTY WITH SAND: Dry clay, very silty with very fine sand, calcareous yellowish-brown (10YR), abundant evidence of iron oxide in laminae.

SANDY SILT: Dry, very sandy, silt. Sand very fine grained to coarse silt, occasional sub-angular quartz grains increasing to very coarse grained with depth.

SAND AND GRAVEL: Dry sand and gravel. Gravel (sandstone) with thin layers of calcification (white) with vuggular porosity calcite lined, dry, light yellowish-brown (10YR). NO RECOVERY

CLAYEY SILT: Limited recovery - dry, friable. Clayey silt. Light yellowish-brown (10YR), calcareous. 


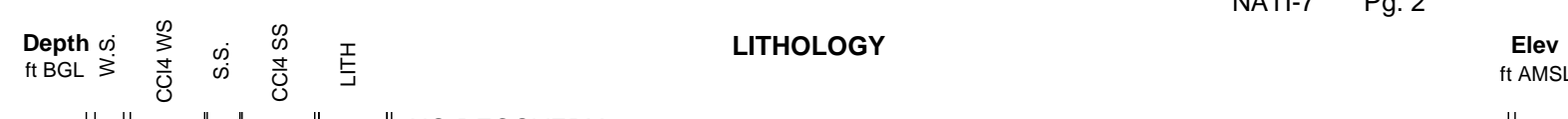

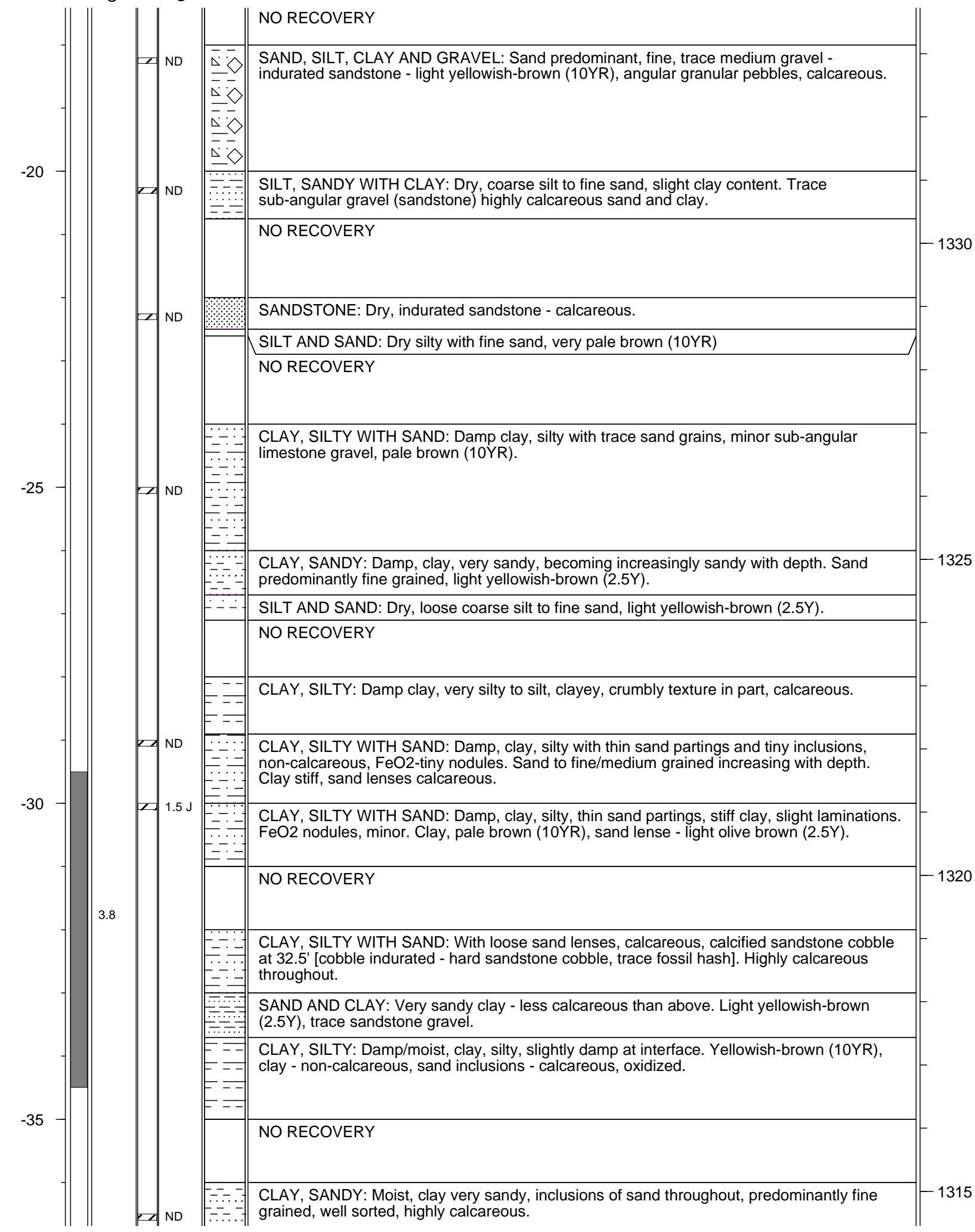


NATI-7 Pg. 3

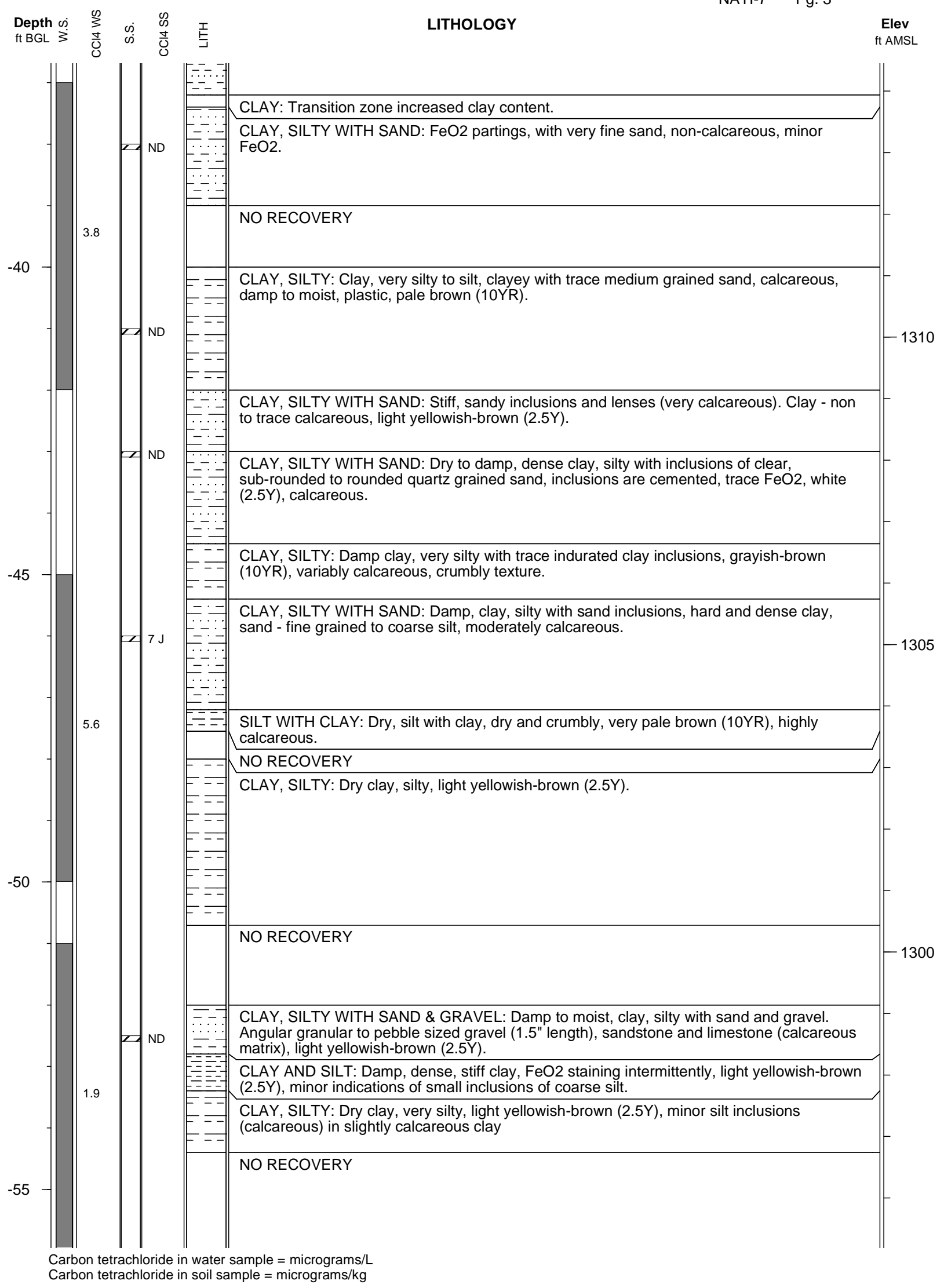


NATI-7 Pg. 4

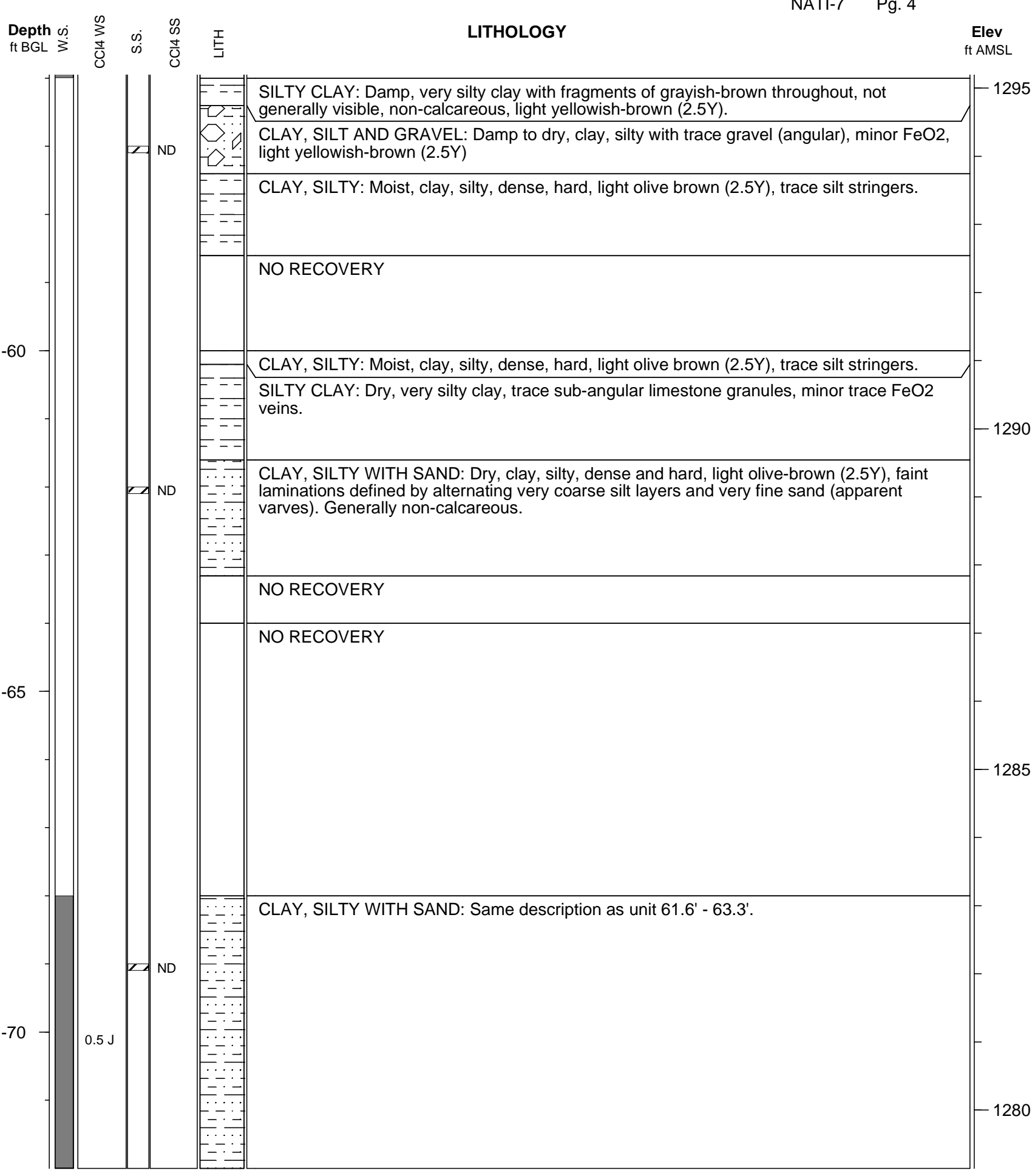




\section{Argonne National Laboratory}

Project: Navarre

Geologist: Lisa Larsen
Elevation: $1351.21 \mathrm{ft}$.

Depth: $69.5 \mathrm{ft} . \mathrm{BGL}$

\section{Boring ID: NATI-8}

Log Date: 4/10/2006

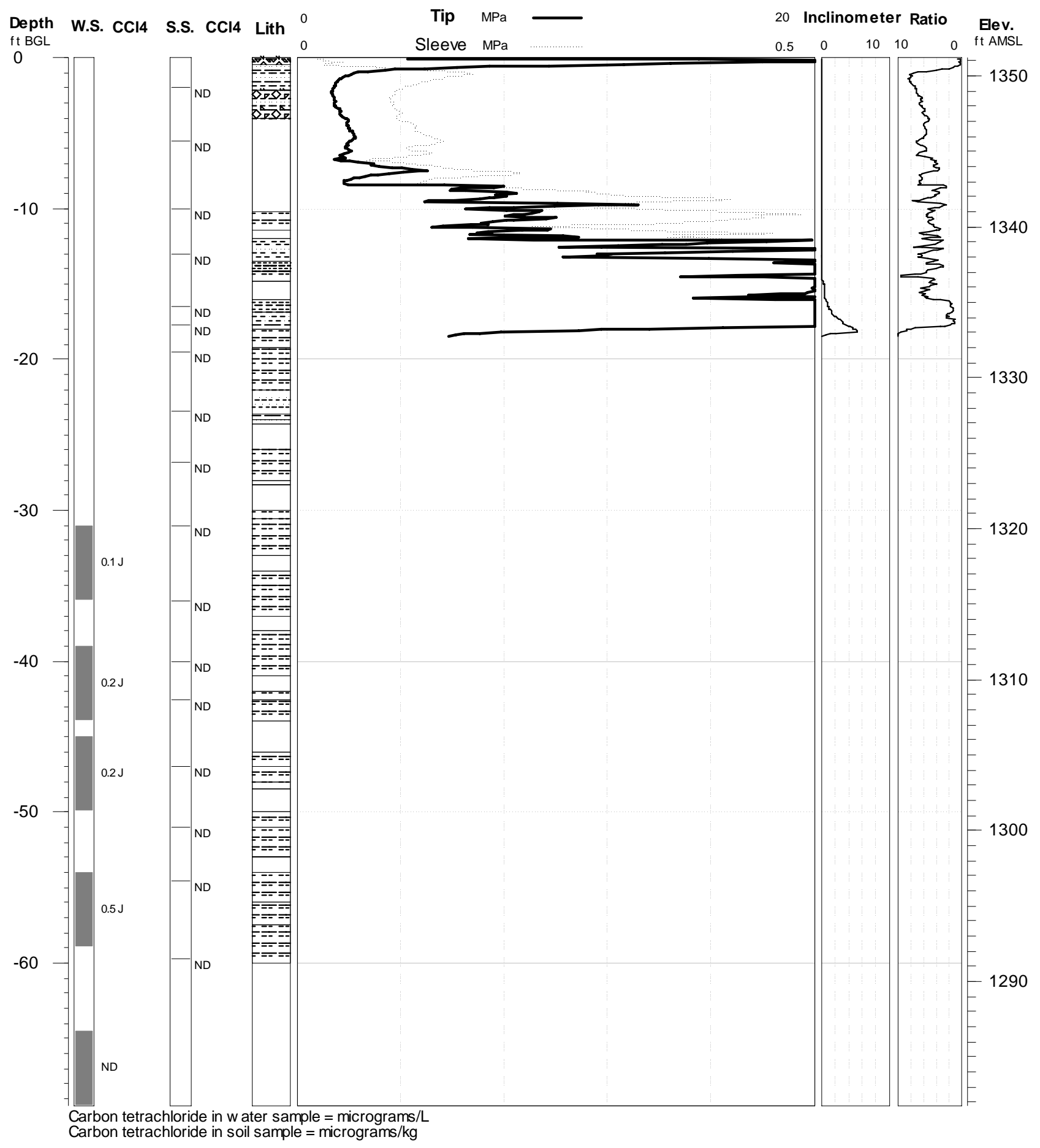




\section{Argonne National Laboratory}

Geologist: Lisa Larsen
Project: Navarre
Boring ID: NATI-8
Elevation: $1351.21 \mathrm{ft}$.

Depth: $69.5 \mathrm{ft}$. BGL

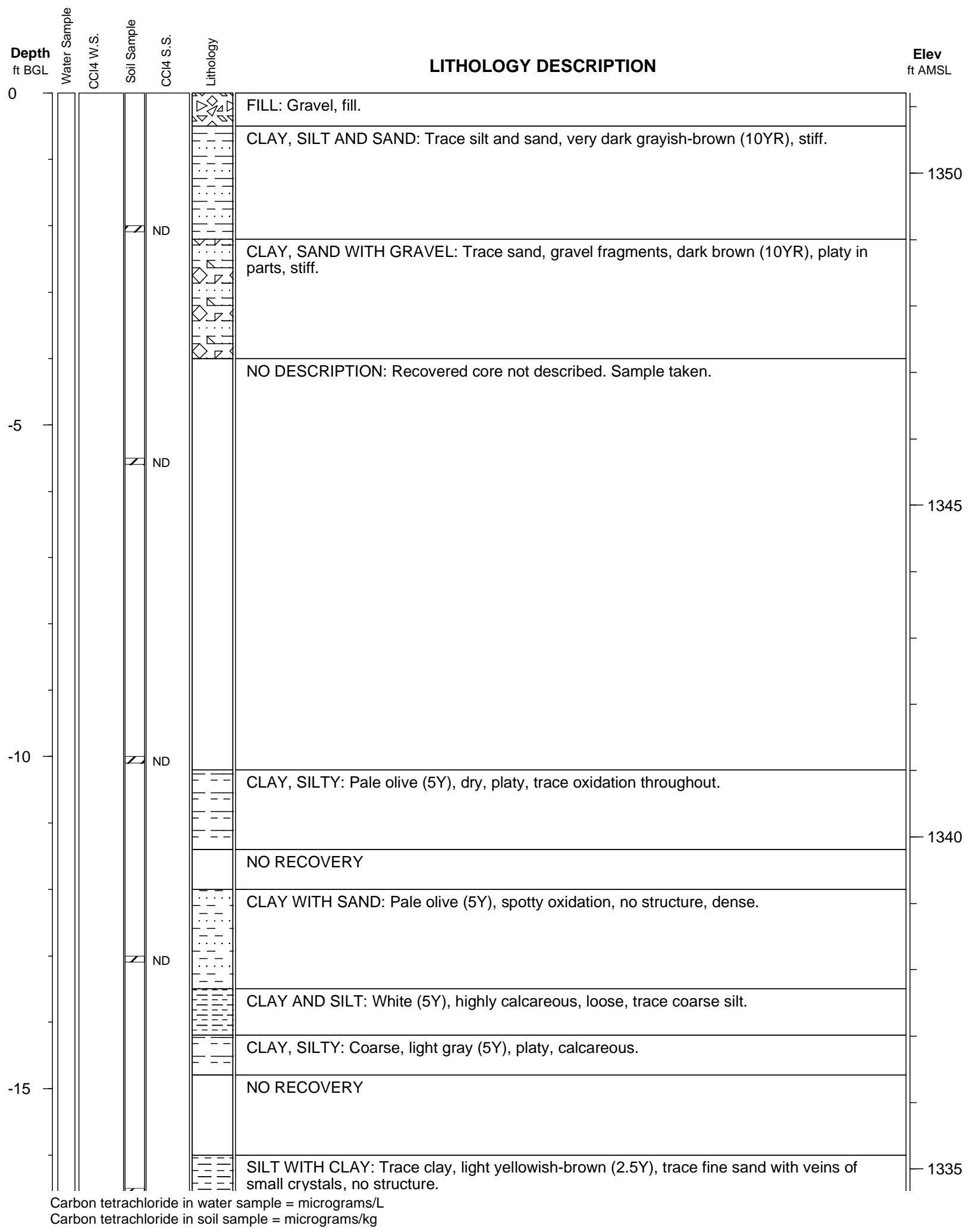


NATI-8 Pg. 2

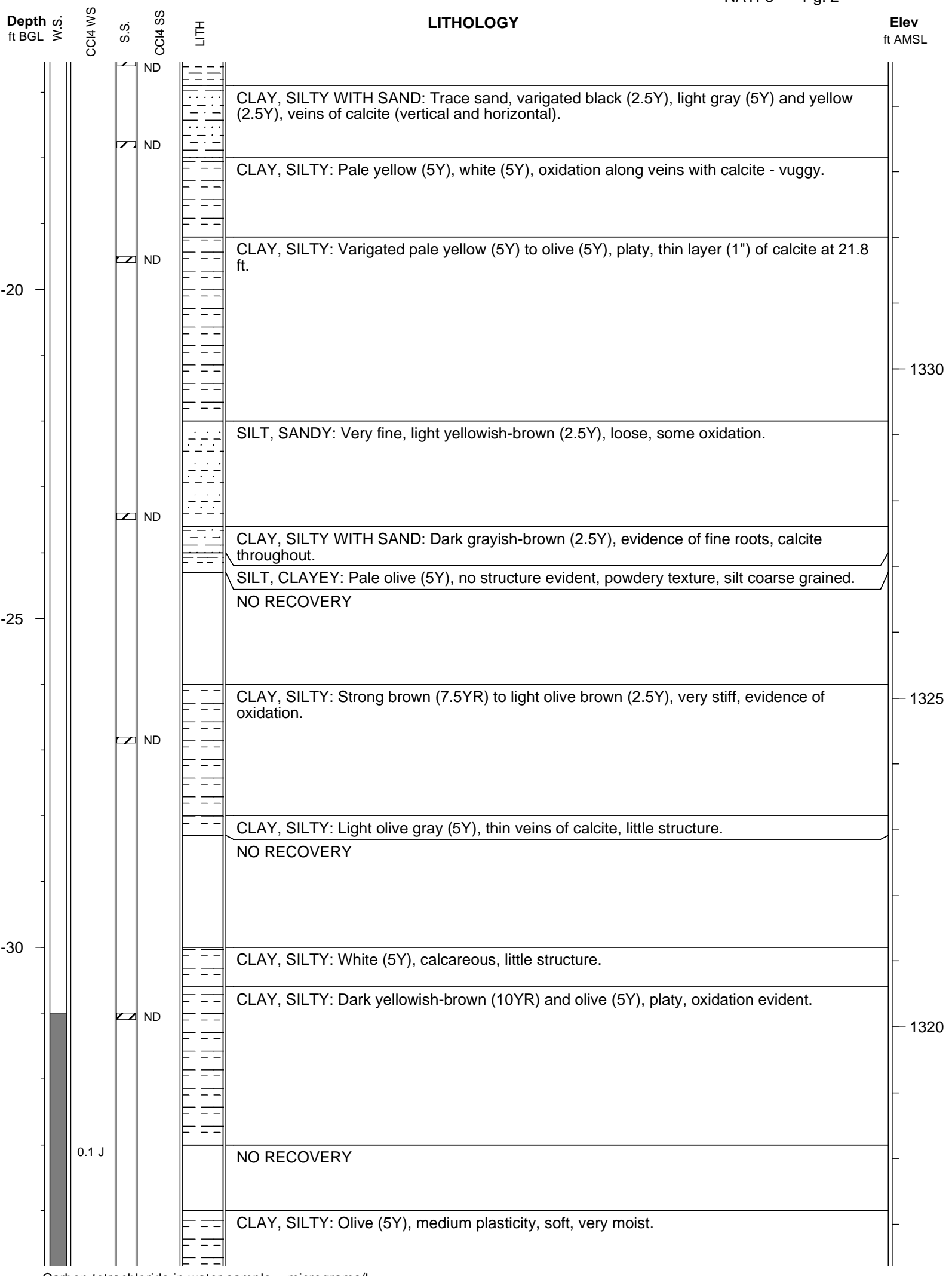


NATI-8 Pg. 3

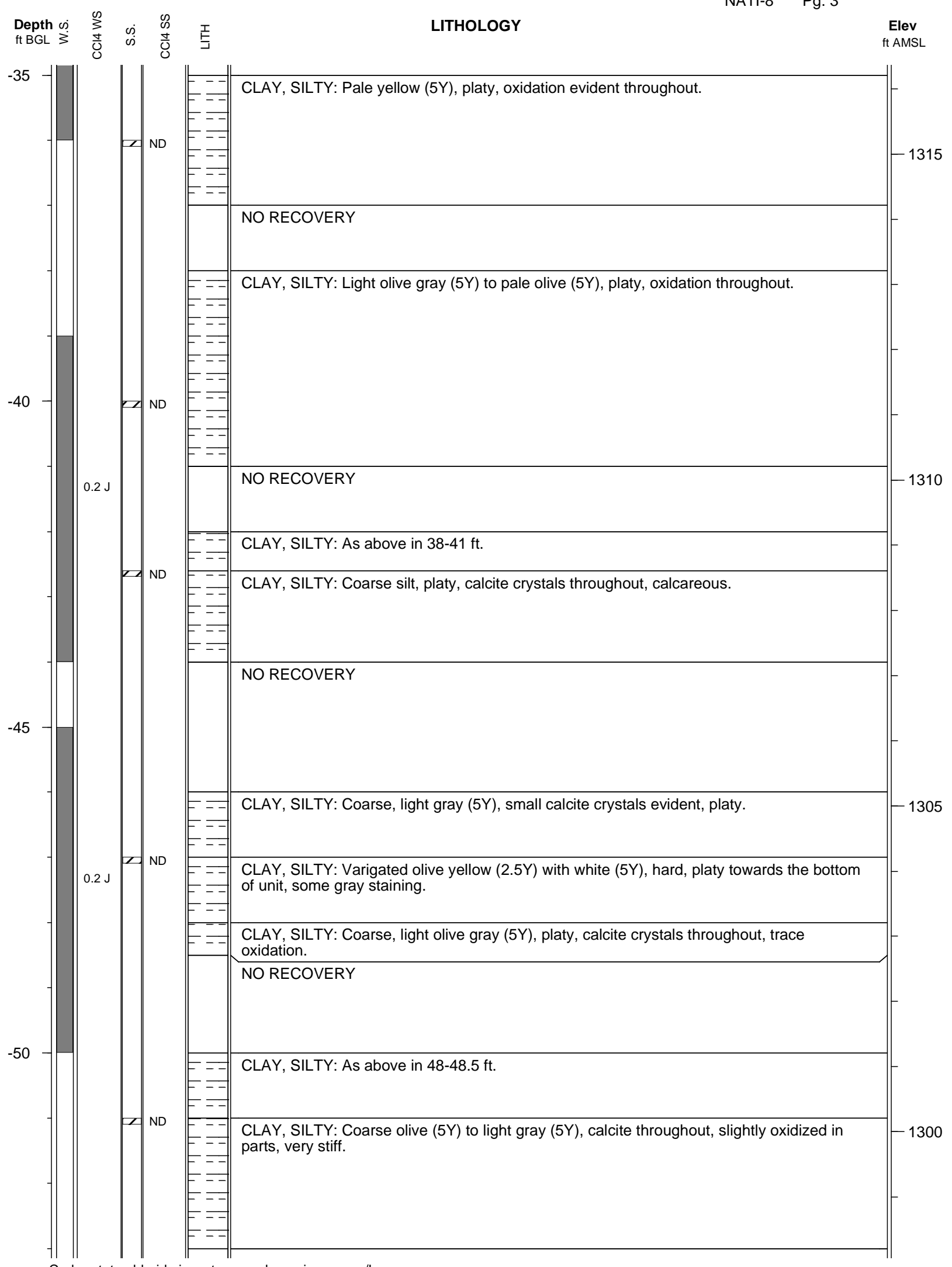


NATI-8 Pg. 4

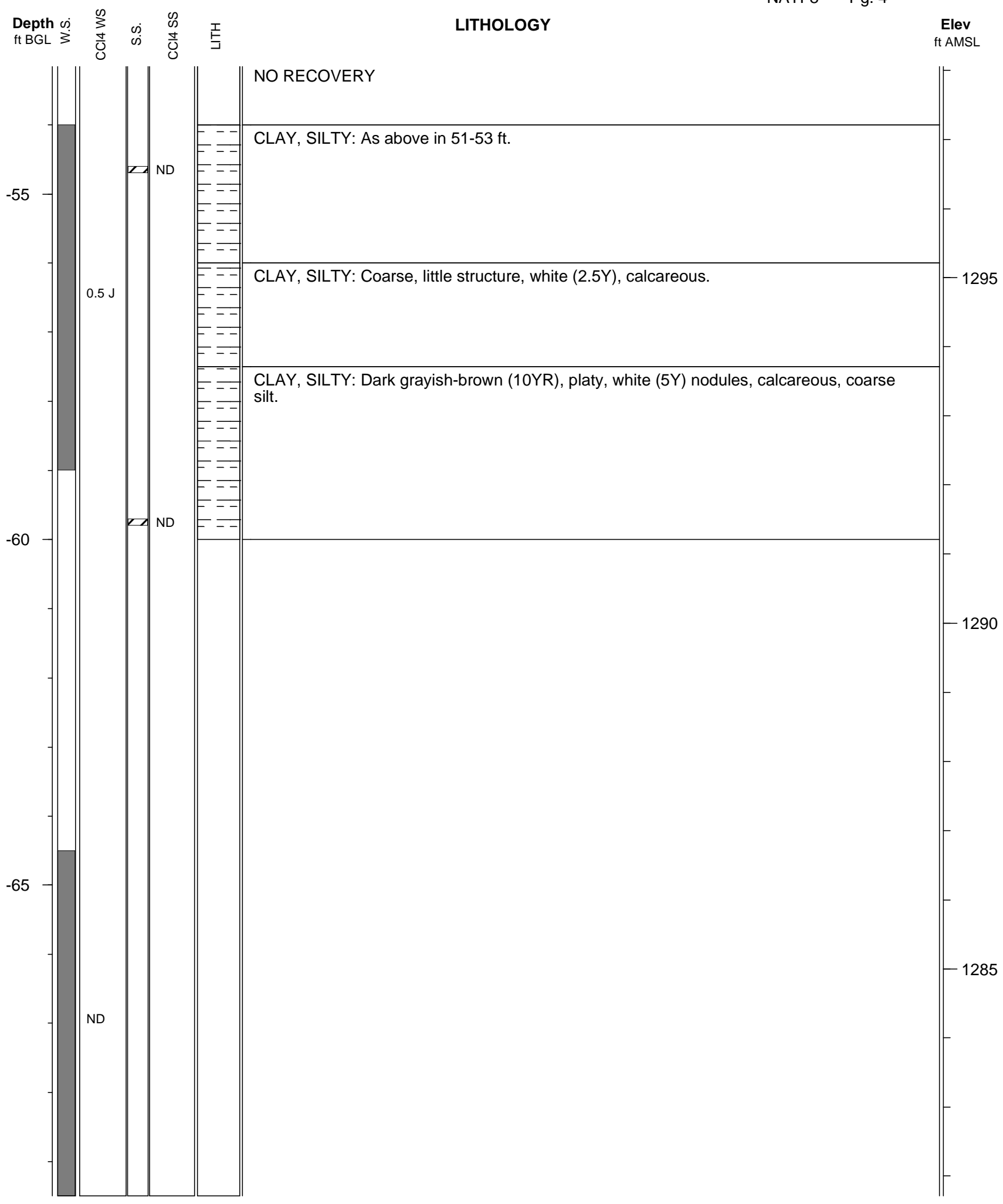




\section{Argonne National Laboratory}

\section{Project: Navarre}

Geologist: Lorraine LaFreniere
Elevation: $1351.55 \mathrm{ft}$.

Depth: $71 \mathrm{ft}$. BGL

\section{Boring ID: NATI-9}

Log Date: 4/21/2006

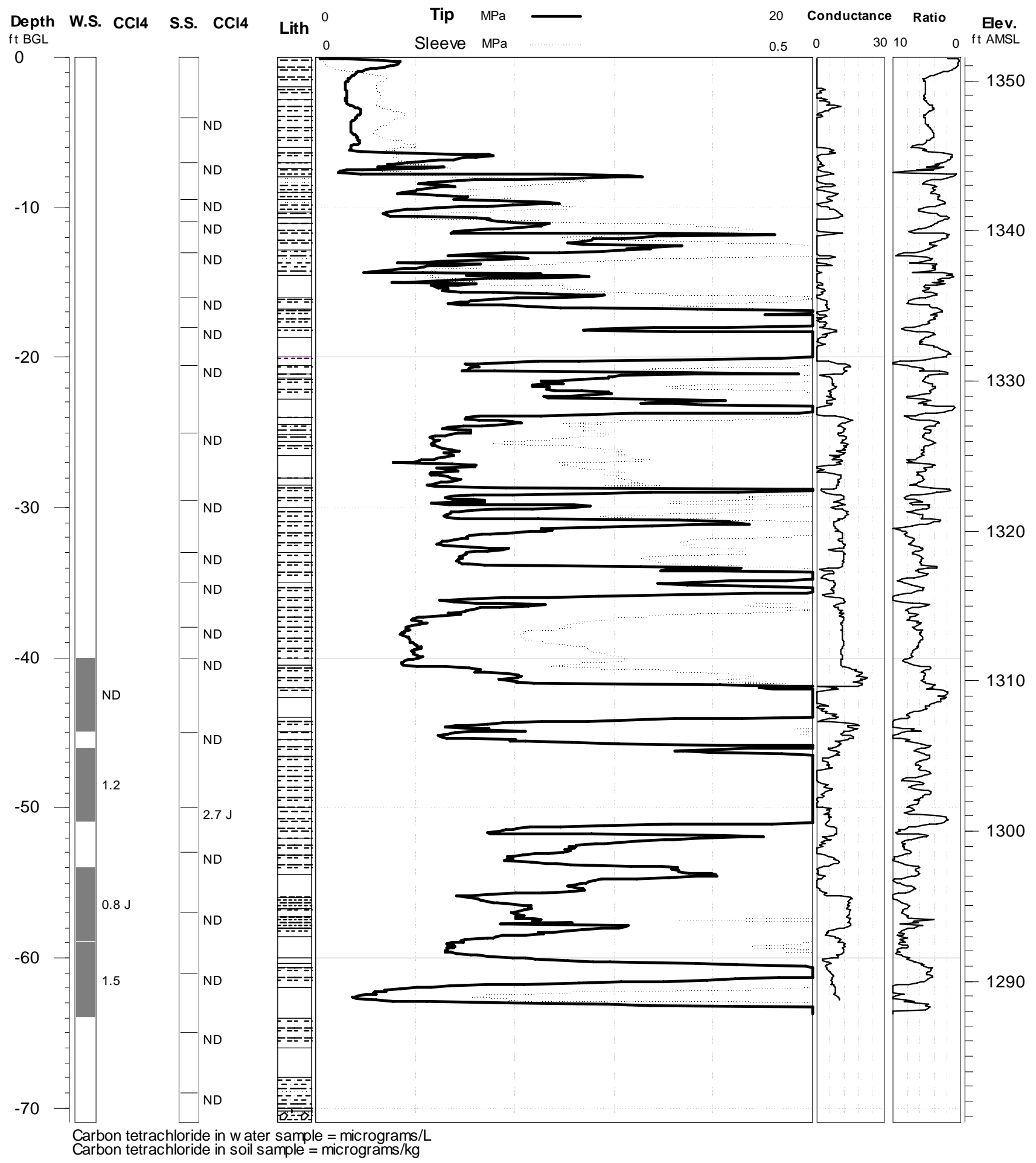




\section{Argonne National Laboratory}

\section{Project: Navarre}

Geologist: Lorraine LaFreniere

\section{Boring ID: NATI-9}

Elevation: $1351.55 \mathrm{ft}$.

Depth: $71 \mathrm{ft}$. BGL

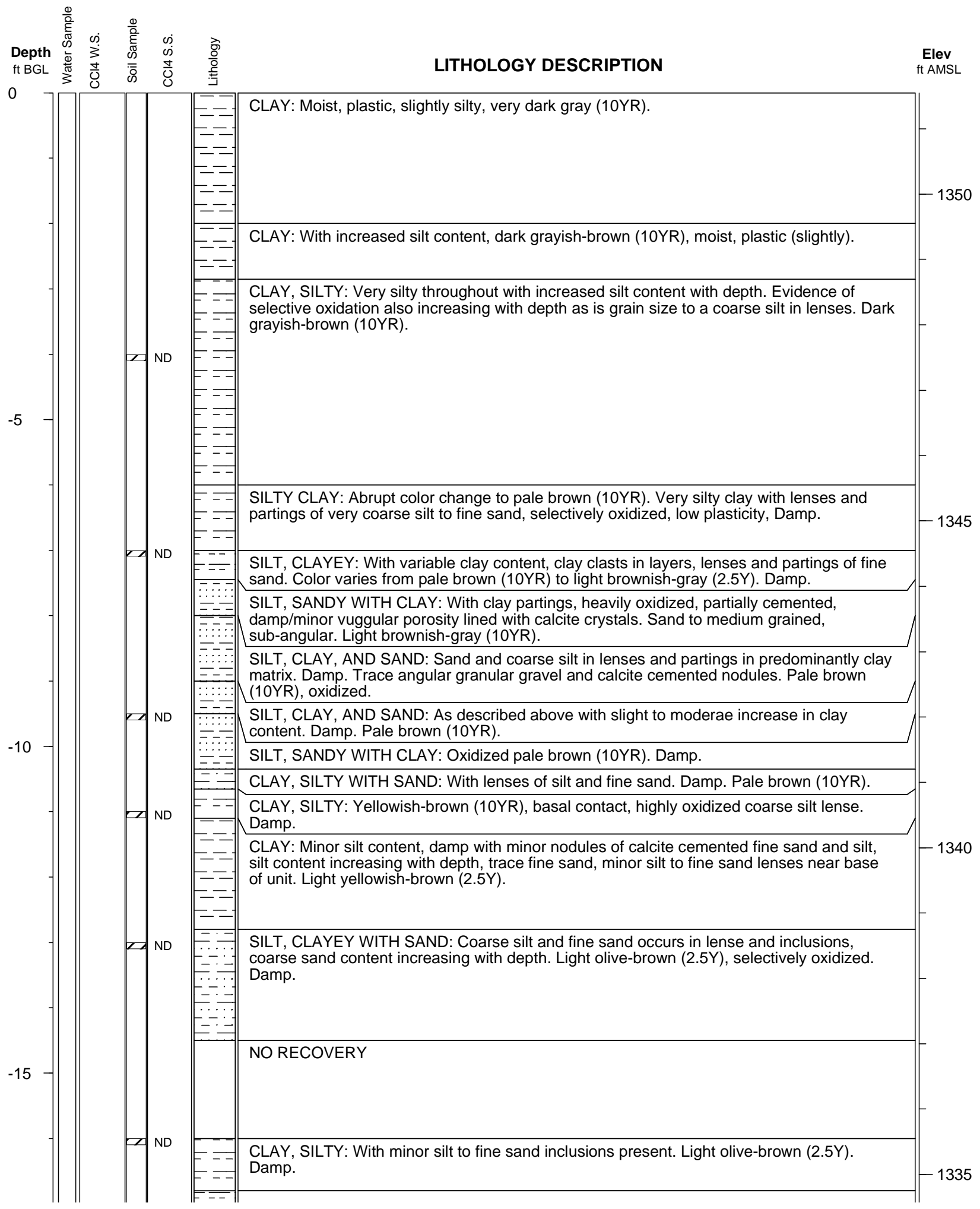

Carbon tetrachloride in water sample $=$ micrograms $/ \mathrm{L}$

Carbon tetrachloride in soil sample $=$ micrograms $/ \mathrm{kg}$ 


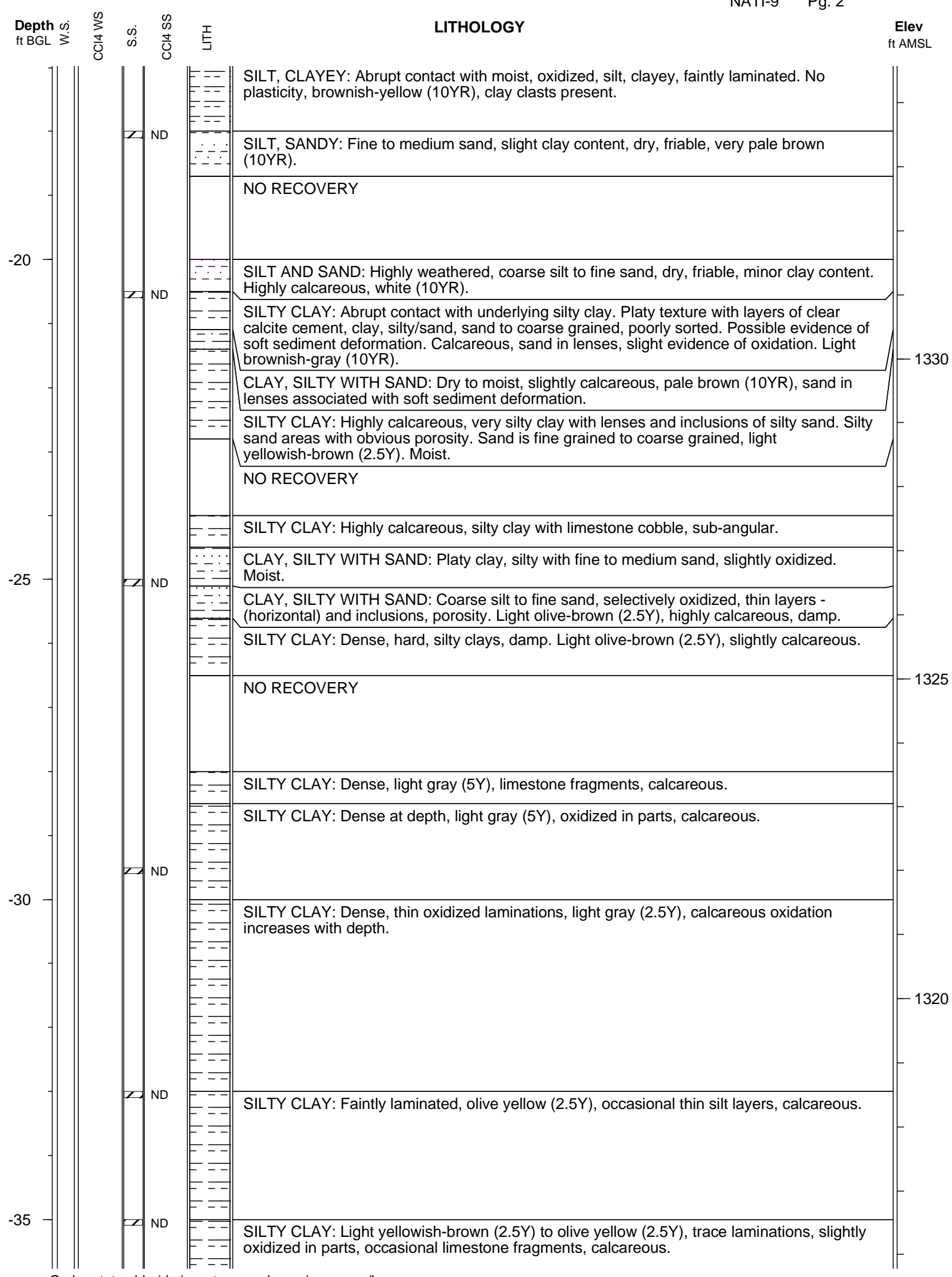




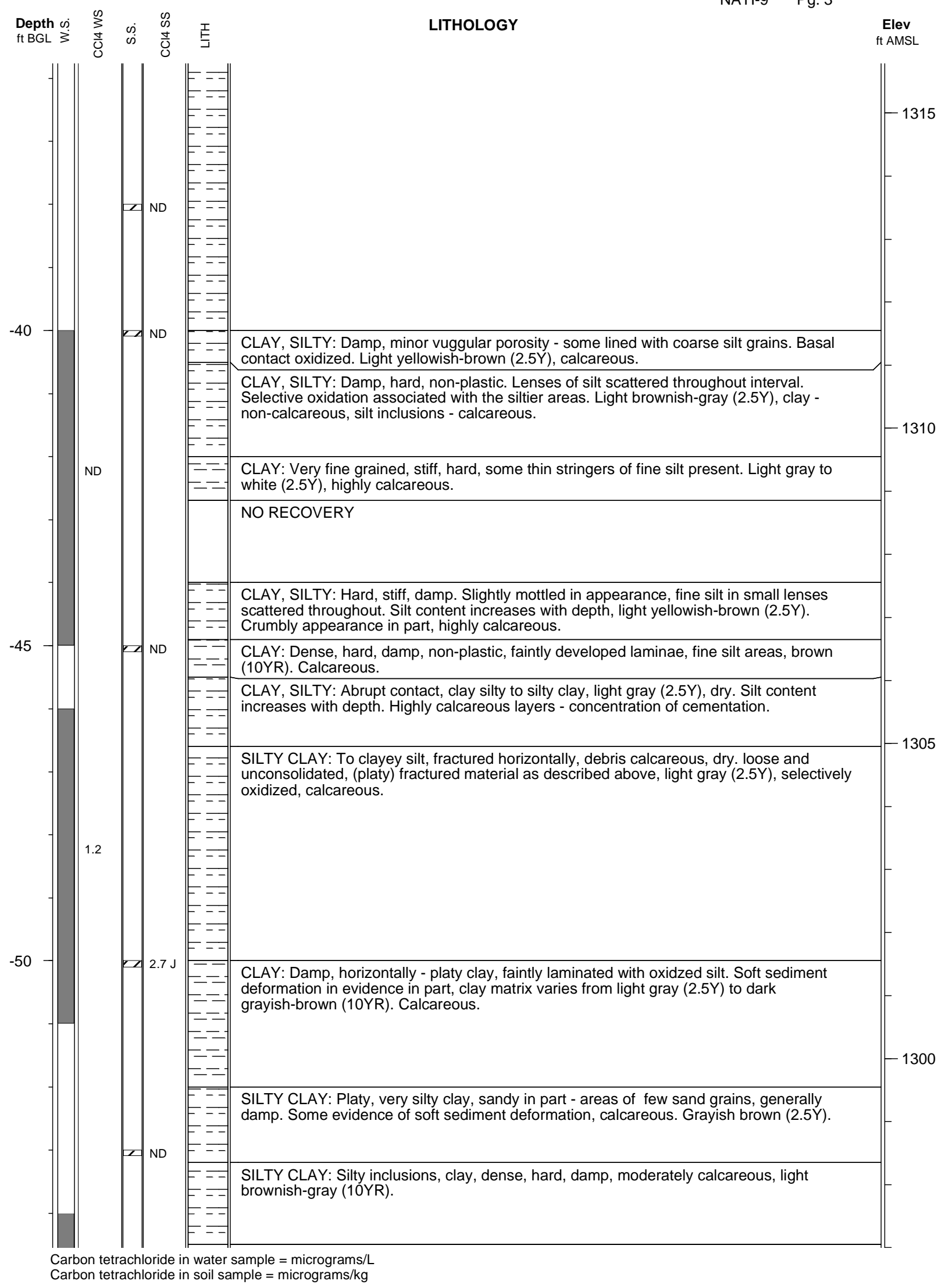




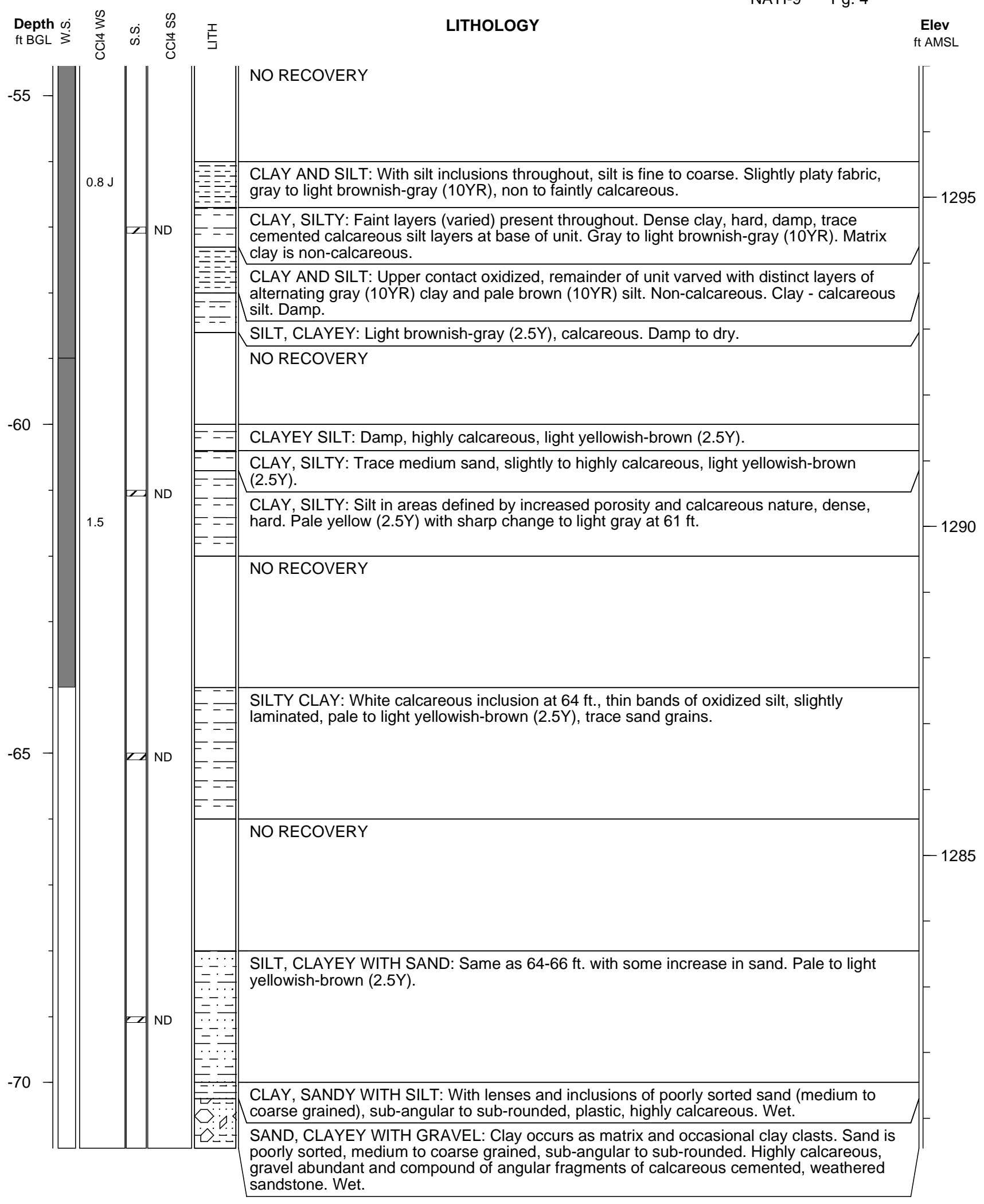




\section{Argonne National Laboratory}

Project: Navarre

Geologist: Lorraine LaFreniere
Elevation: $1353.21 \mathrm{ft}$

Depth: $71 \mathrm{ft} . \mathrm{BGL}$

\section{Boring ID: NATI-10}

Log Date: 4/25/2006

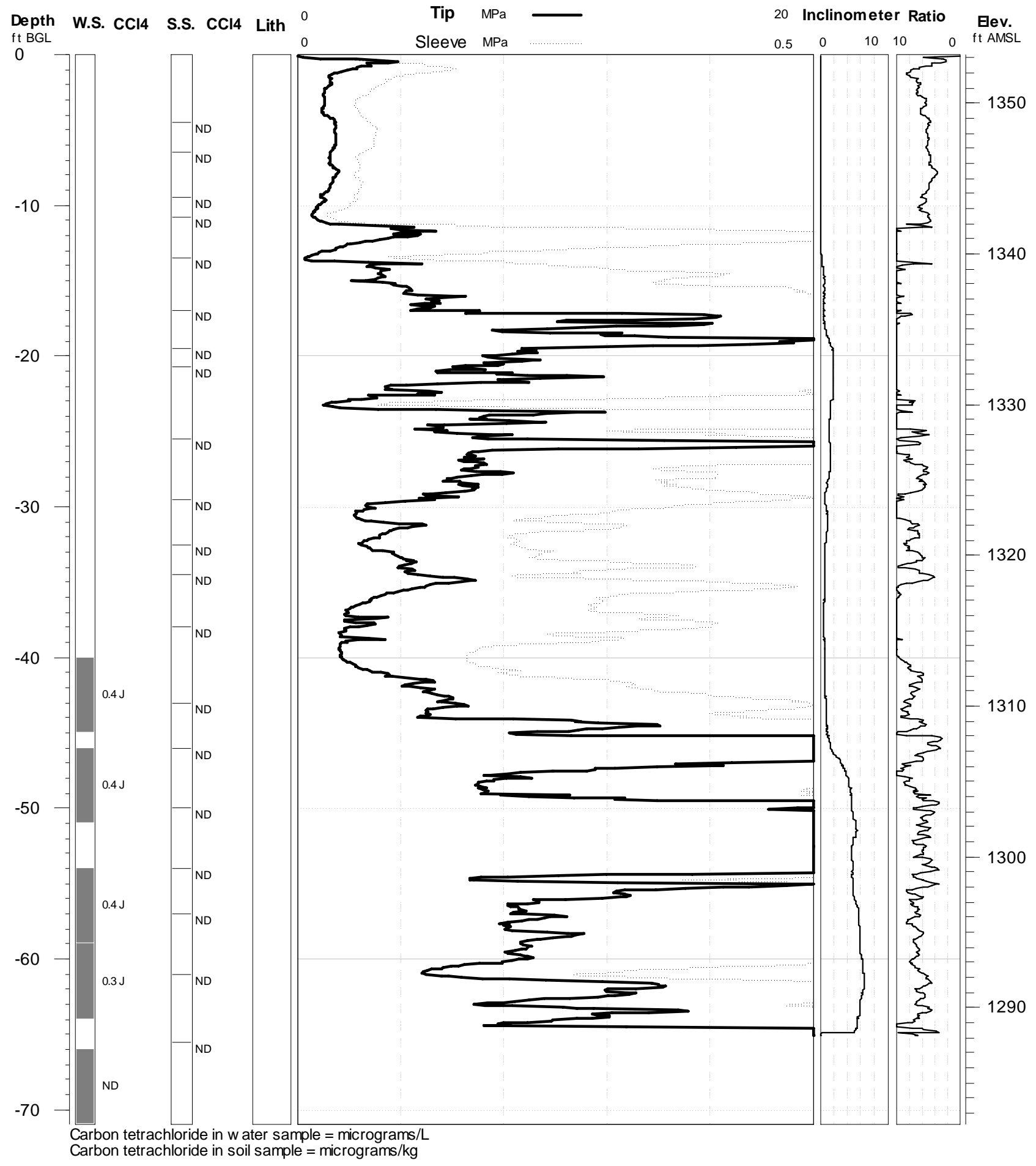




\section{Argonne National Laboratory}

Project: Navarre

Geologist: Lorraine LaFreniere
Elevation: $1352.07 \mathrm{ft}$.

Depth: 70.9 ft. BGL

\section{Boring ID: NATI-11}

Log Date: 4/27/2006

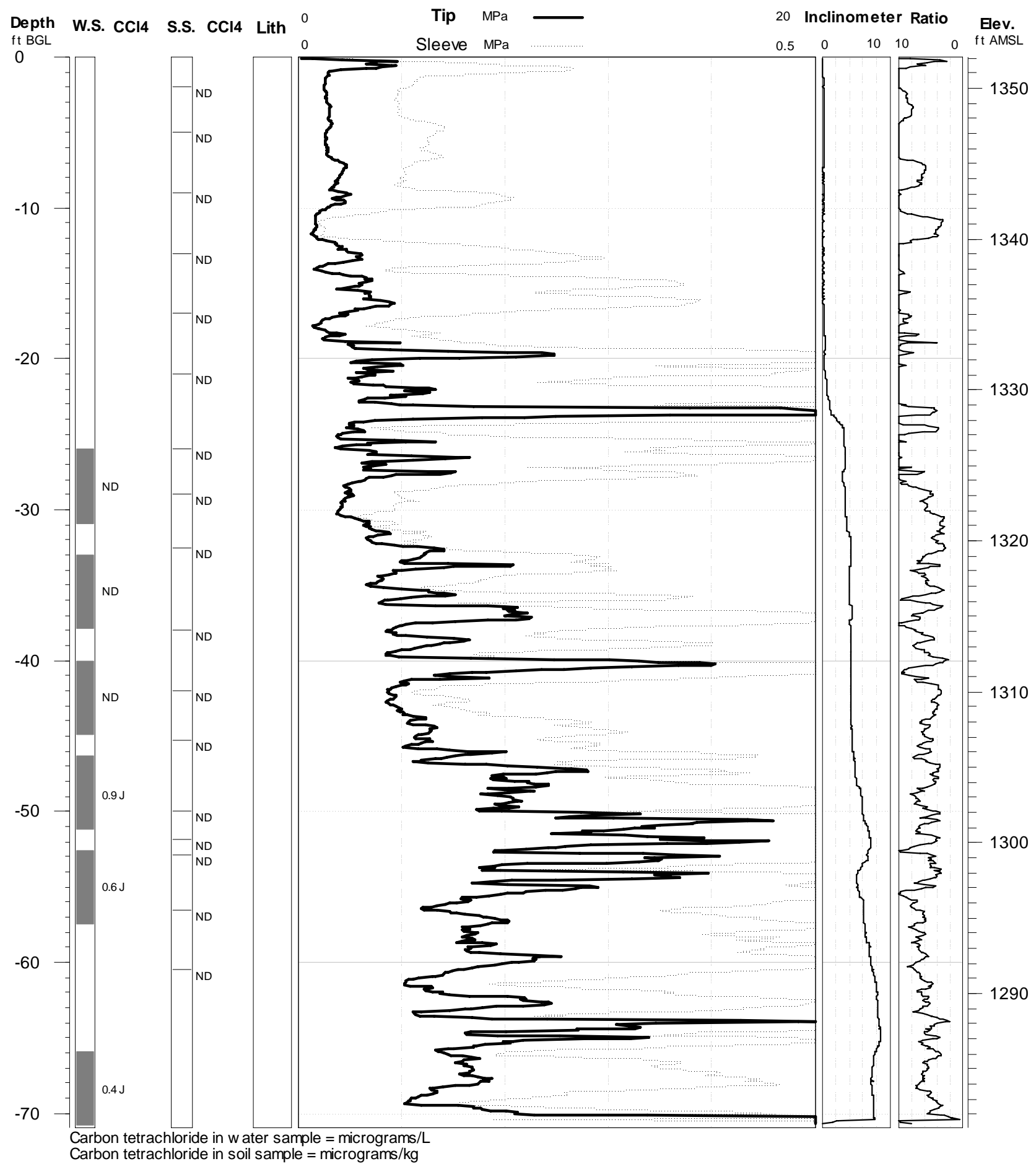




\section{Argonne National Laboratory}

Project: Navarre

Geologist: Lisa Larsen
Elevation: $1351.87 \mathrm{ft}$.

Depth: $68.68 \mathrm{ft}$. BGL

\section{Boring ID: NATI-12}

Log Date: 4/28/2006

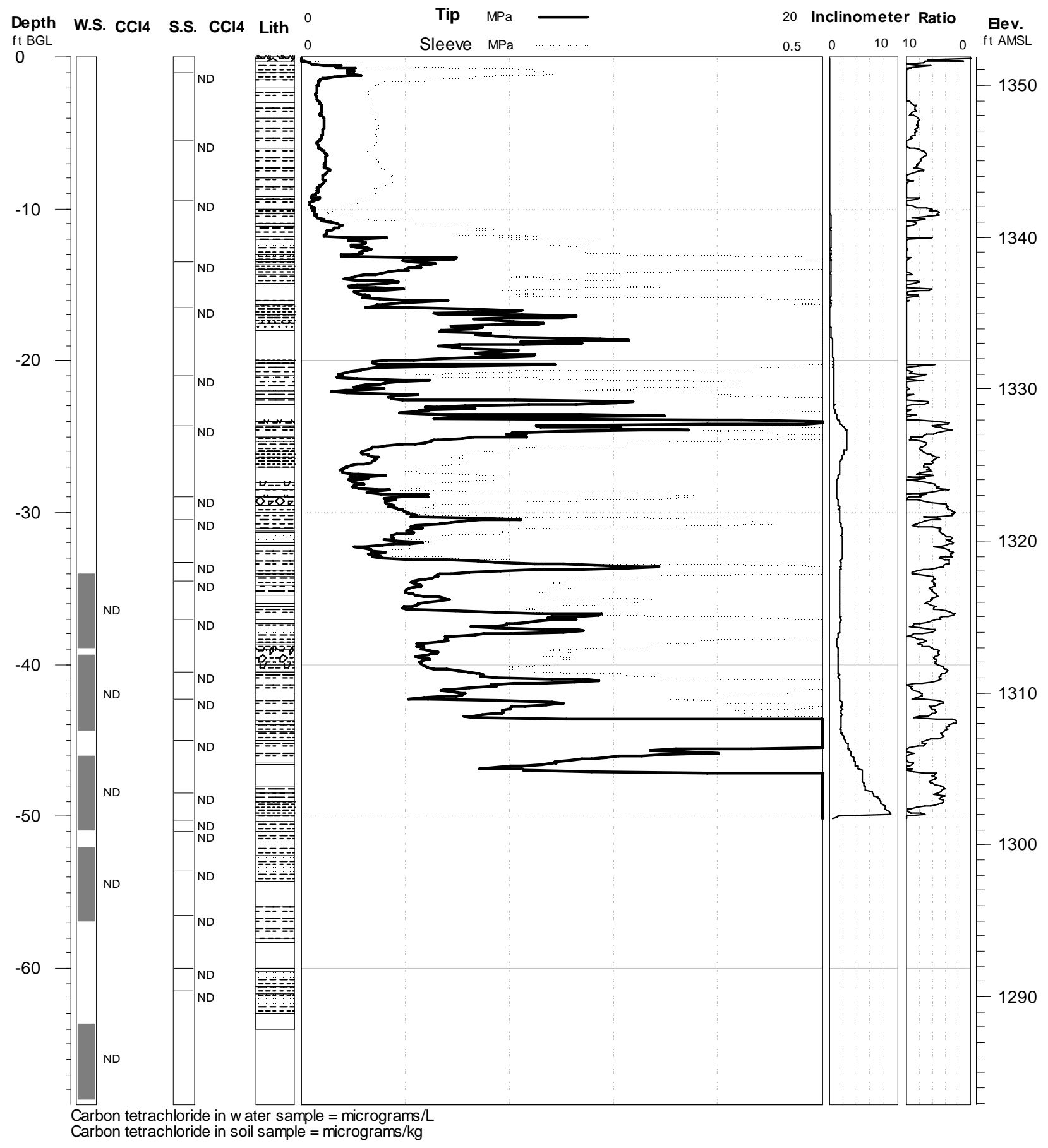




\section{Argonne National Laboratory}

Project: Navarre

Geologist: Lisa Larsen
Elevation: $1351.87 \mathrm{ft}$.

Depth: $68.68 \mathrm{ft}$. BGL

\section{Boring ID: NATI-12}

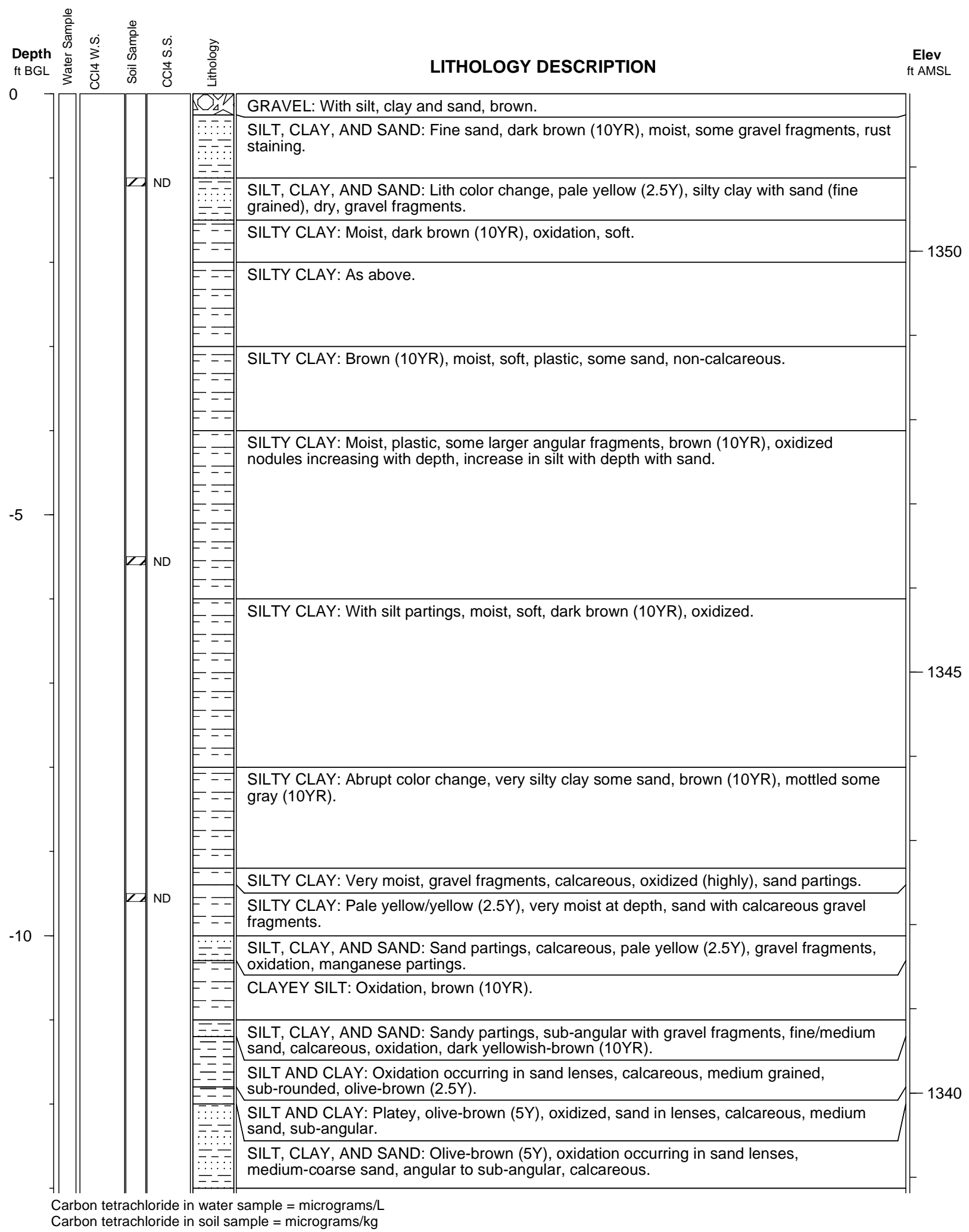


NATI-12 Pg. 2

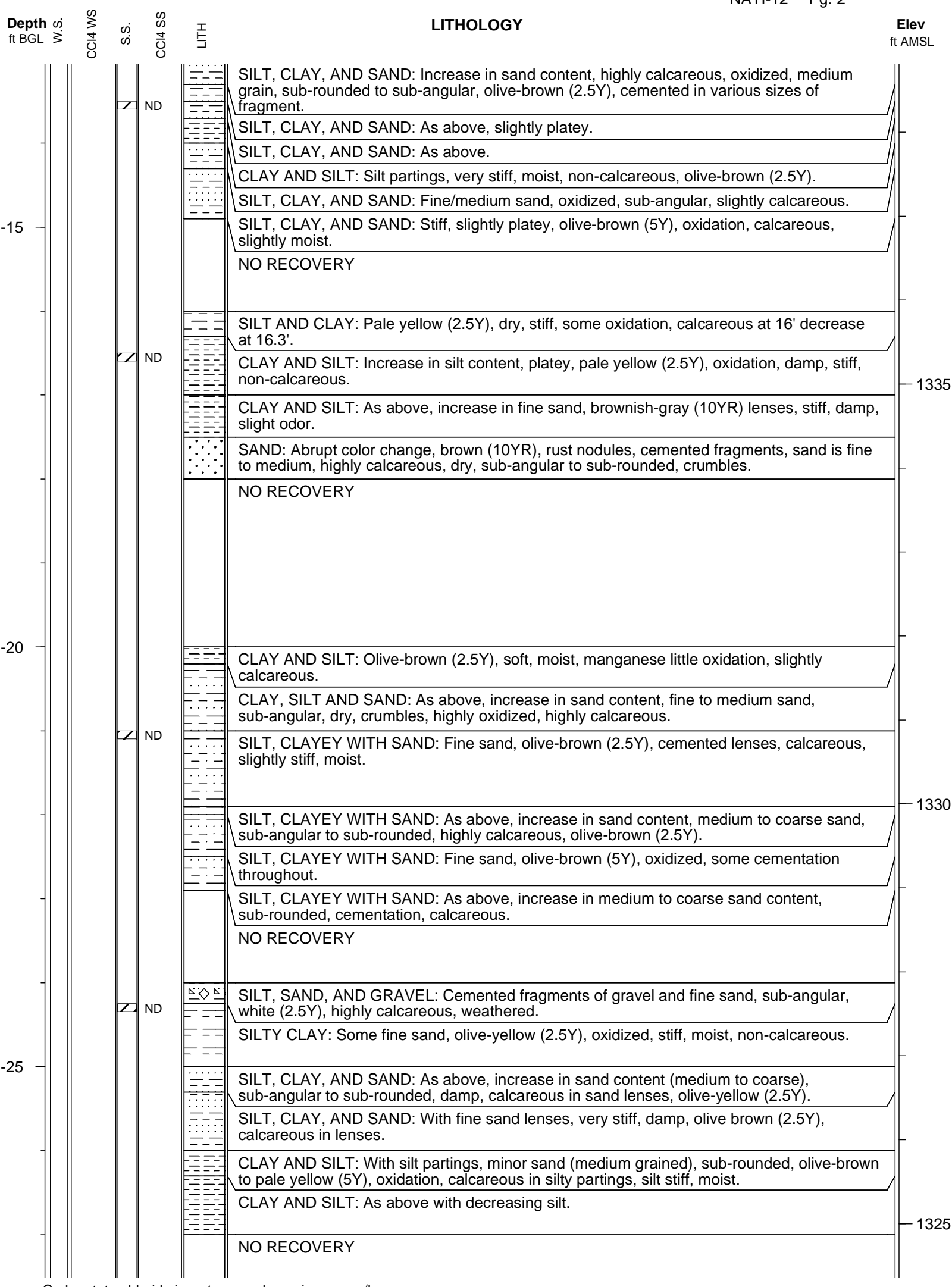




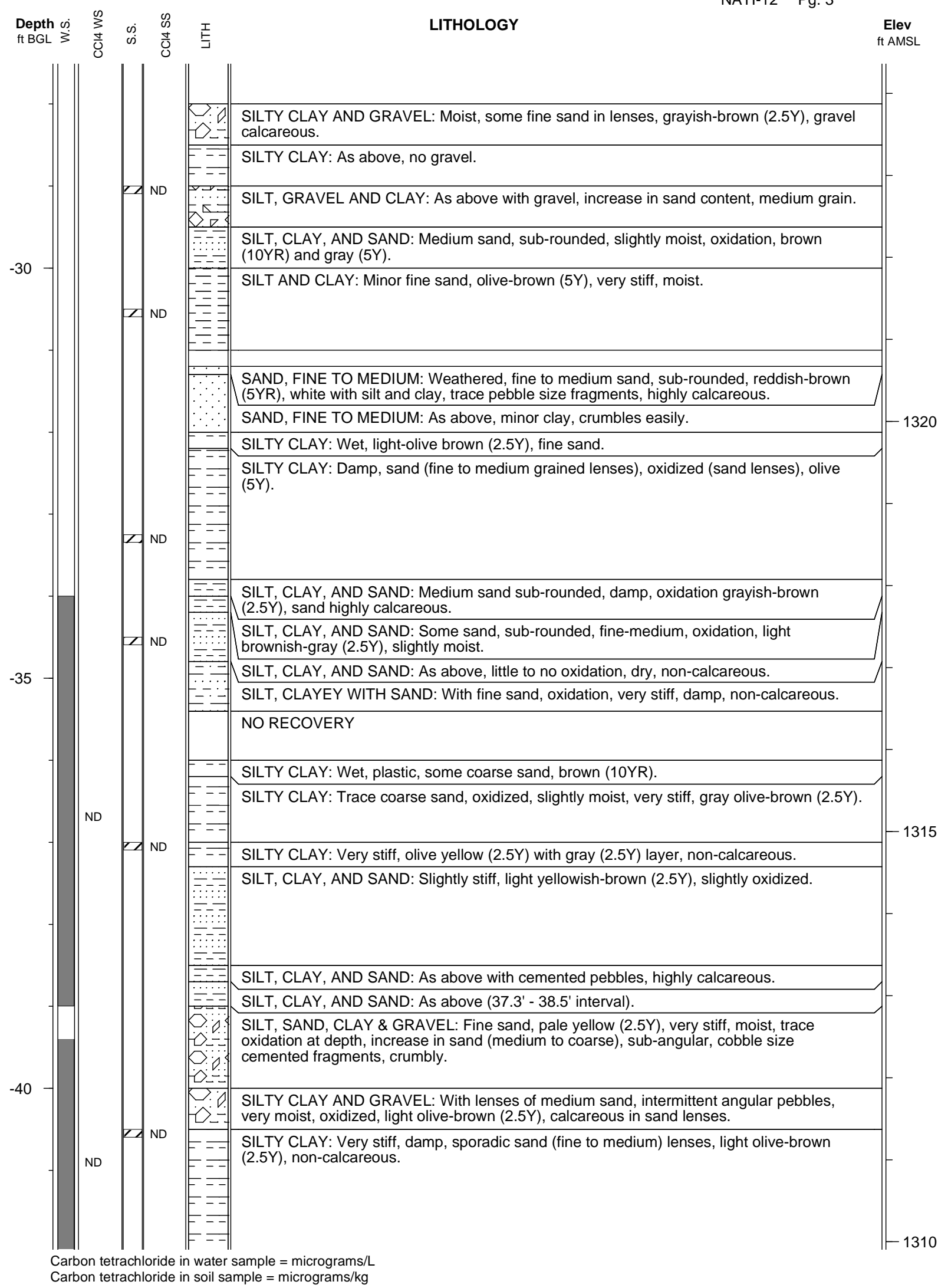


NATI-12 Pg. 4

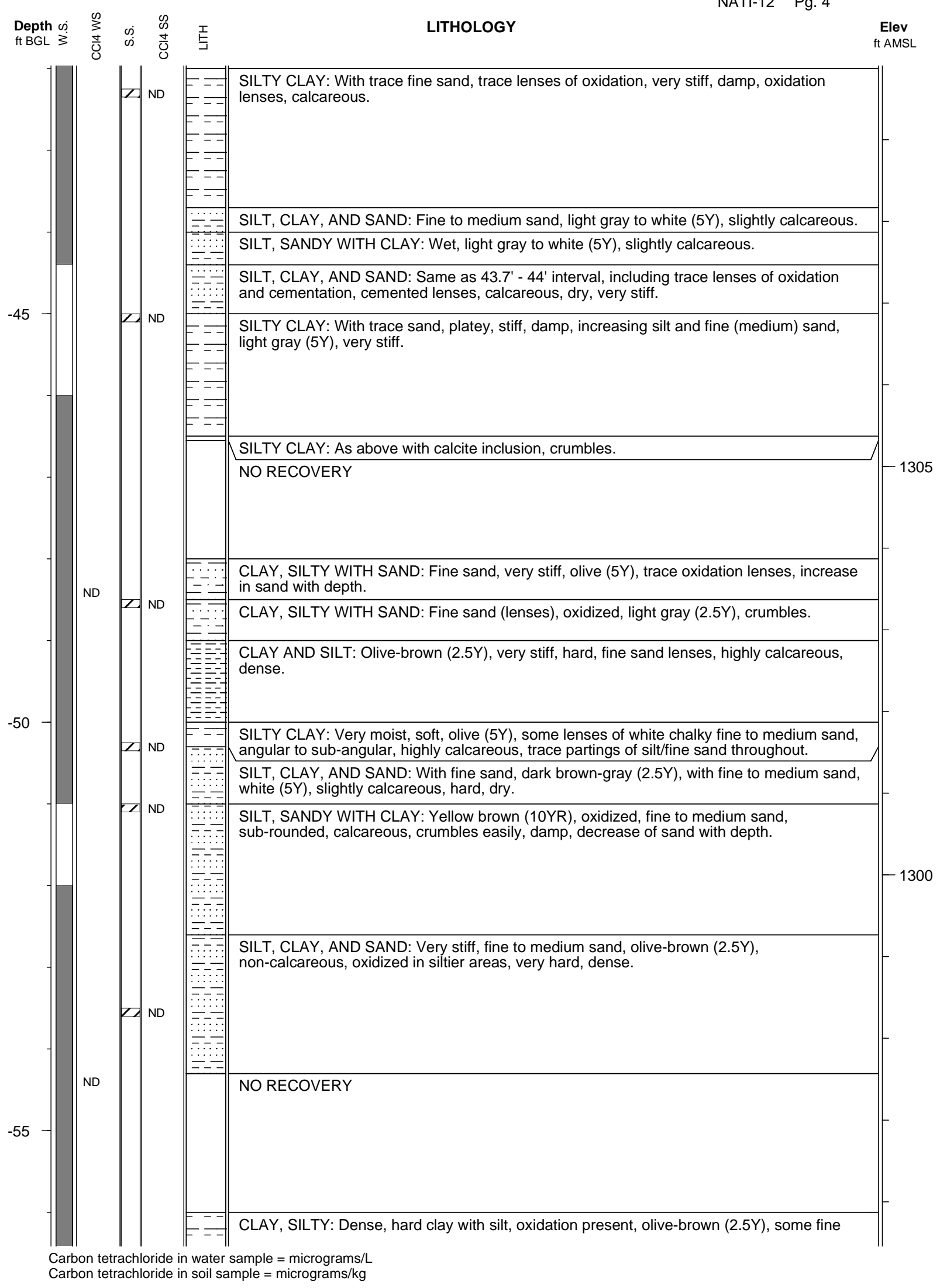


NATI-12 Pg. 5

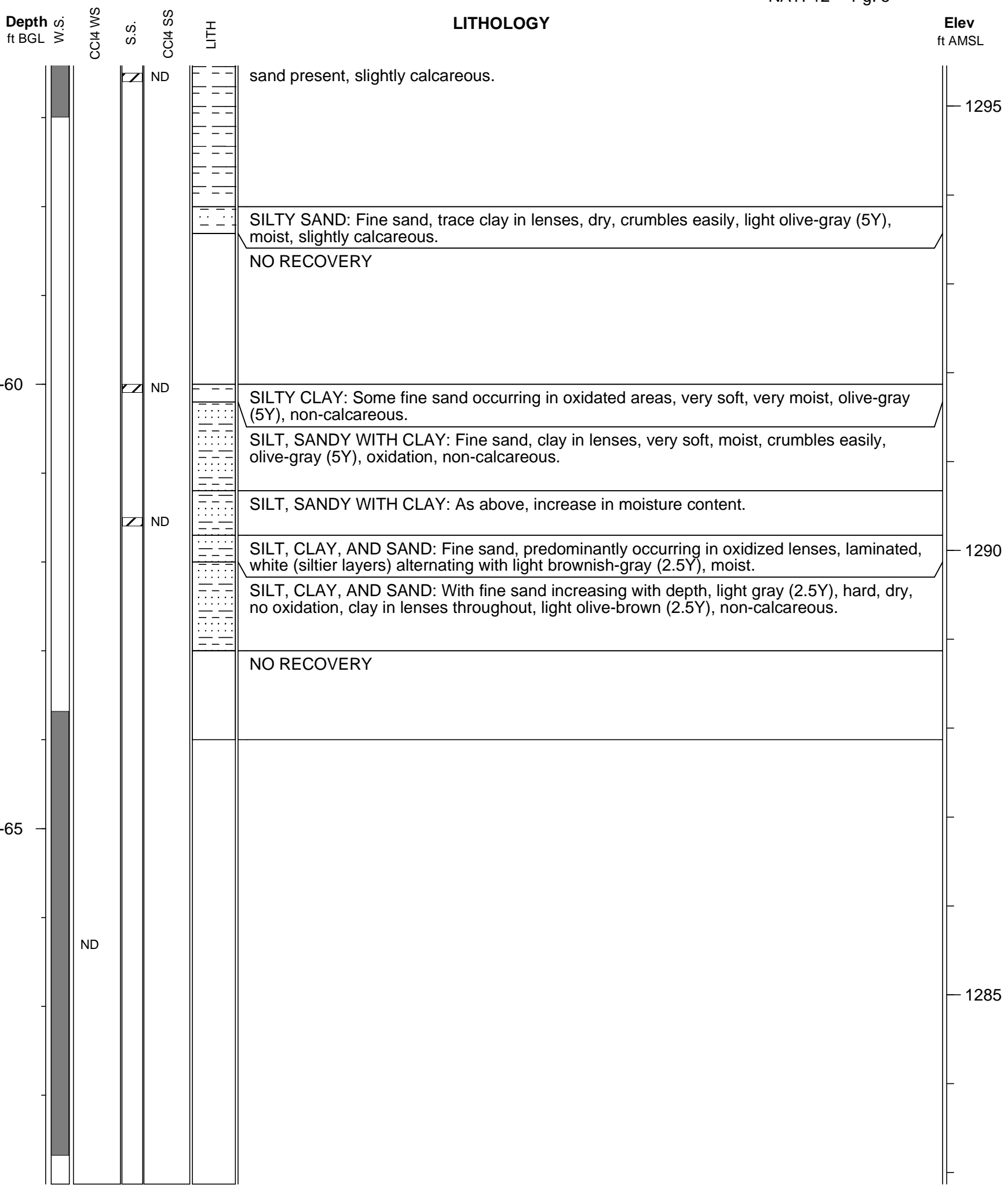




\section{Argonne National Laboratory}

Project: Navarre

Geologist: Lorraine LaFreniere
Elevation: $1351.73 \mathrm{ft}$.

Depth: $71.82 \mathrm{ft} . \mathrm{BGL}$

\section{Boring ID: NATI-13}

Log Date: 5/7/2006

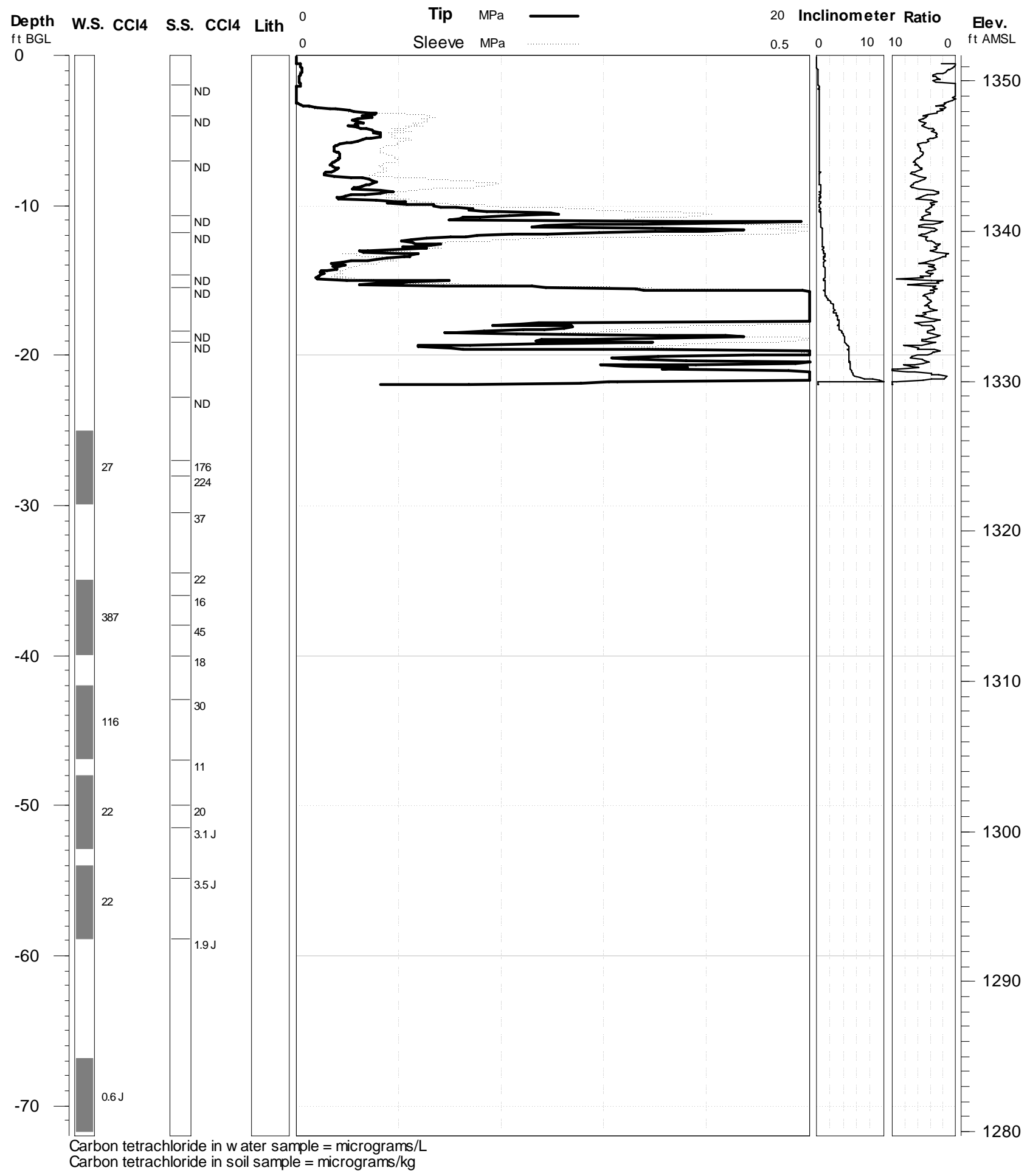




\section{Argonne National Laboratory}

Project: Navarre

Geologist: Lorraine LaFreniere
Elevation: $1352.68 \mathrm{ft}$

Depth: 72.4 ft. BGL

\section{Boring ID: NATI-14}

Log Date: 5/5/2006

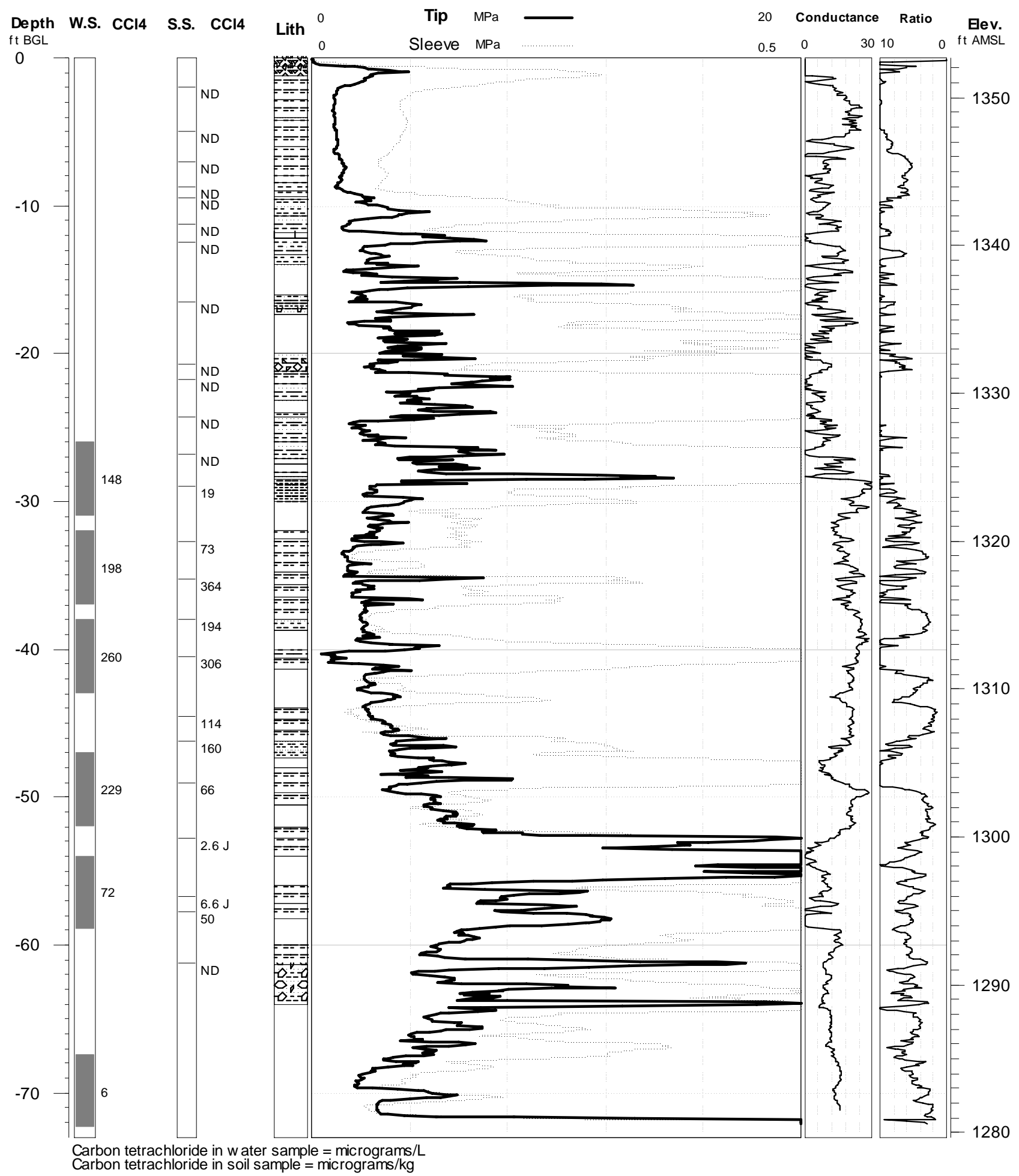


Argonne National Laboratory

Project: Navarre

Geologist: Lorraine LaFreniere

\section{Boring ID: NATI-14}

Elevation: $1352.68 \mathrm{ft}$.

Depth: $72.4 \mathrm{ft}$. BGL

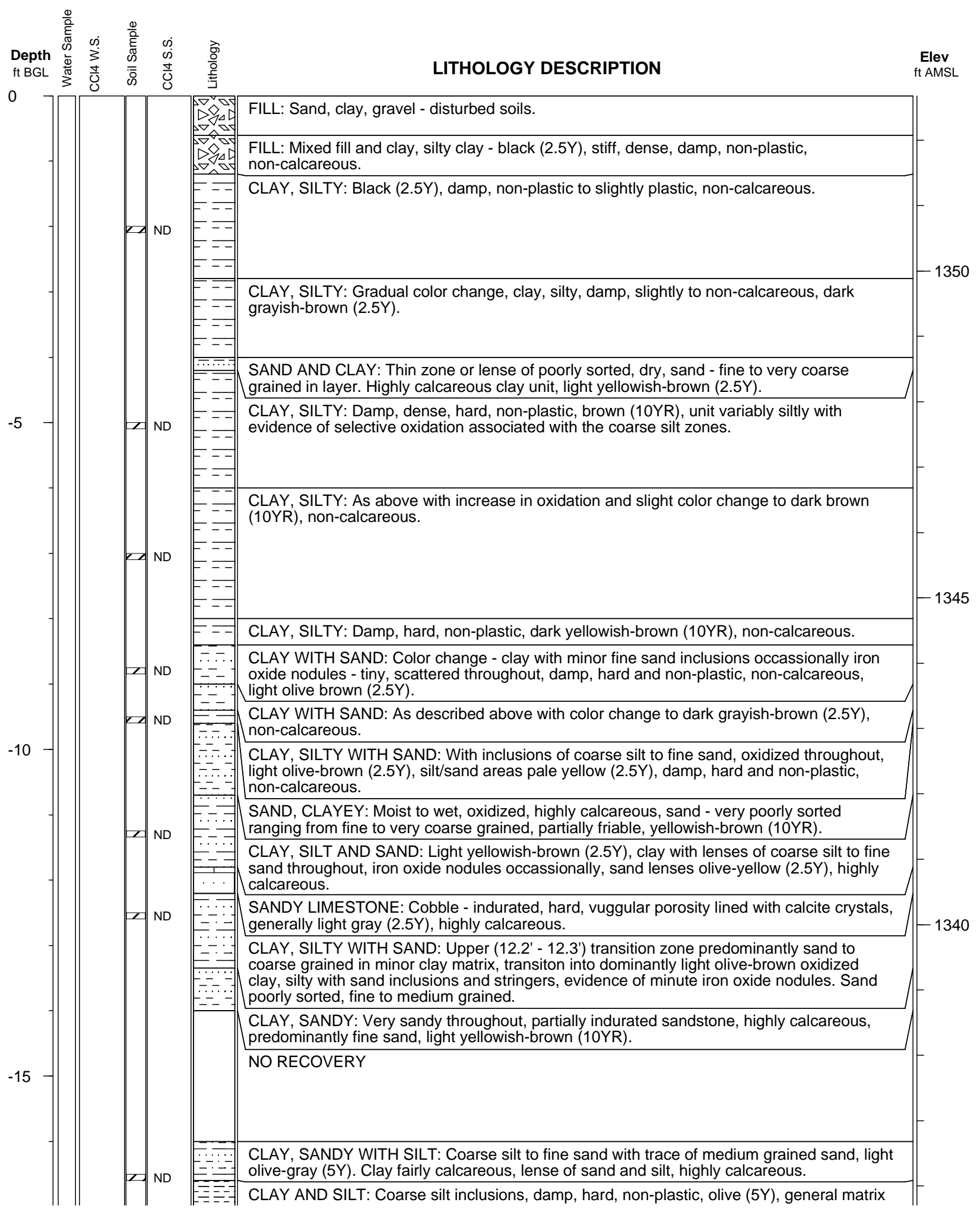


NATI-14 Pg. 2

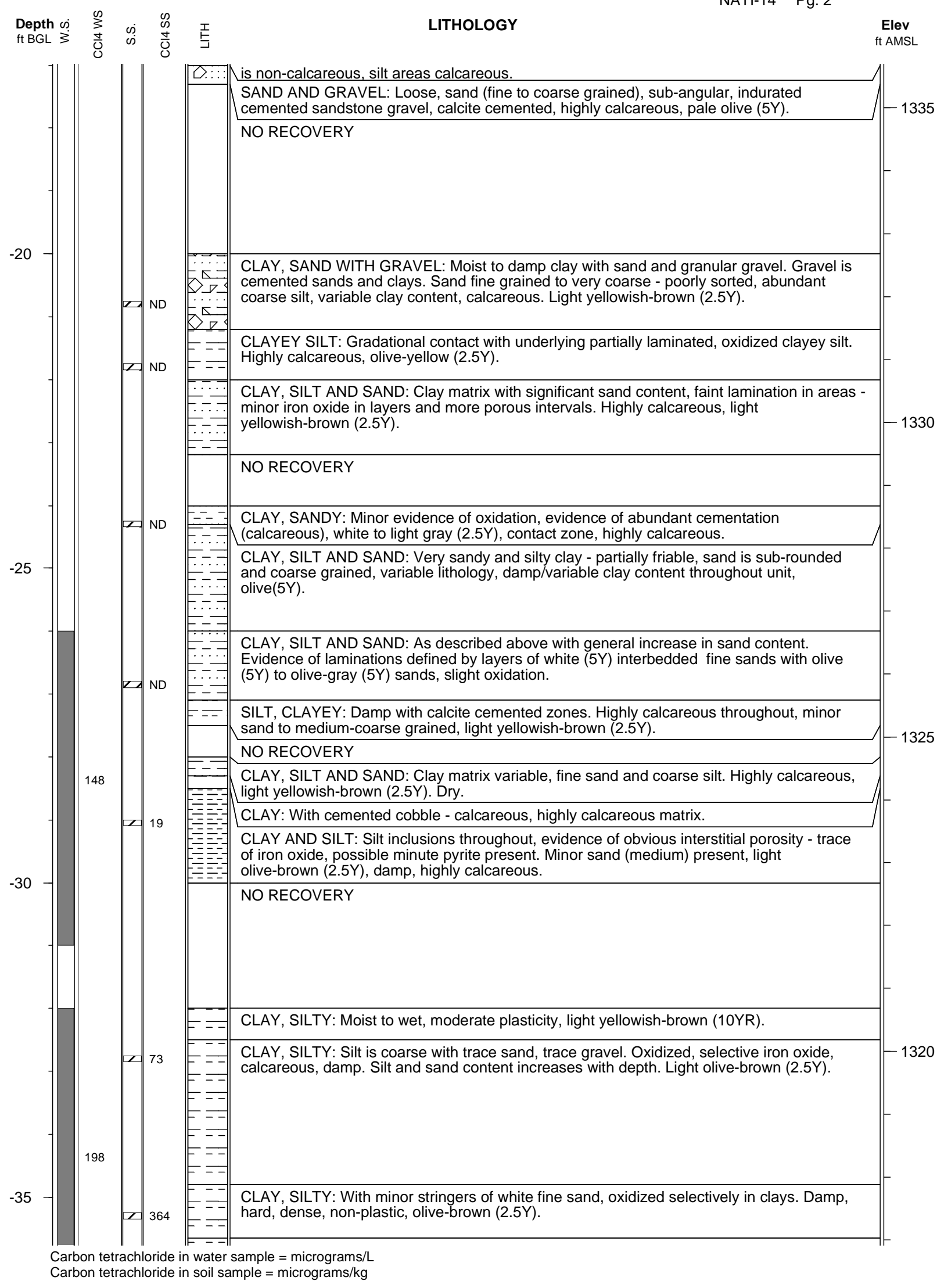


NATI-14 Pg. 3

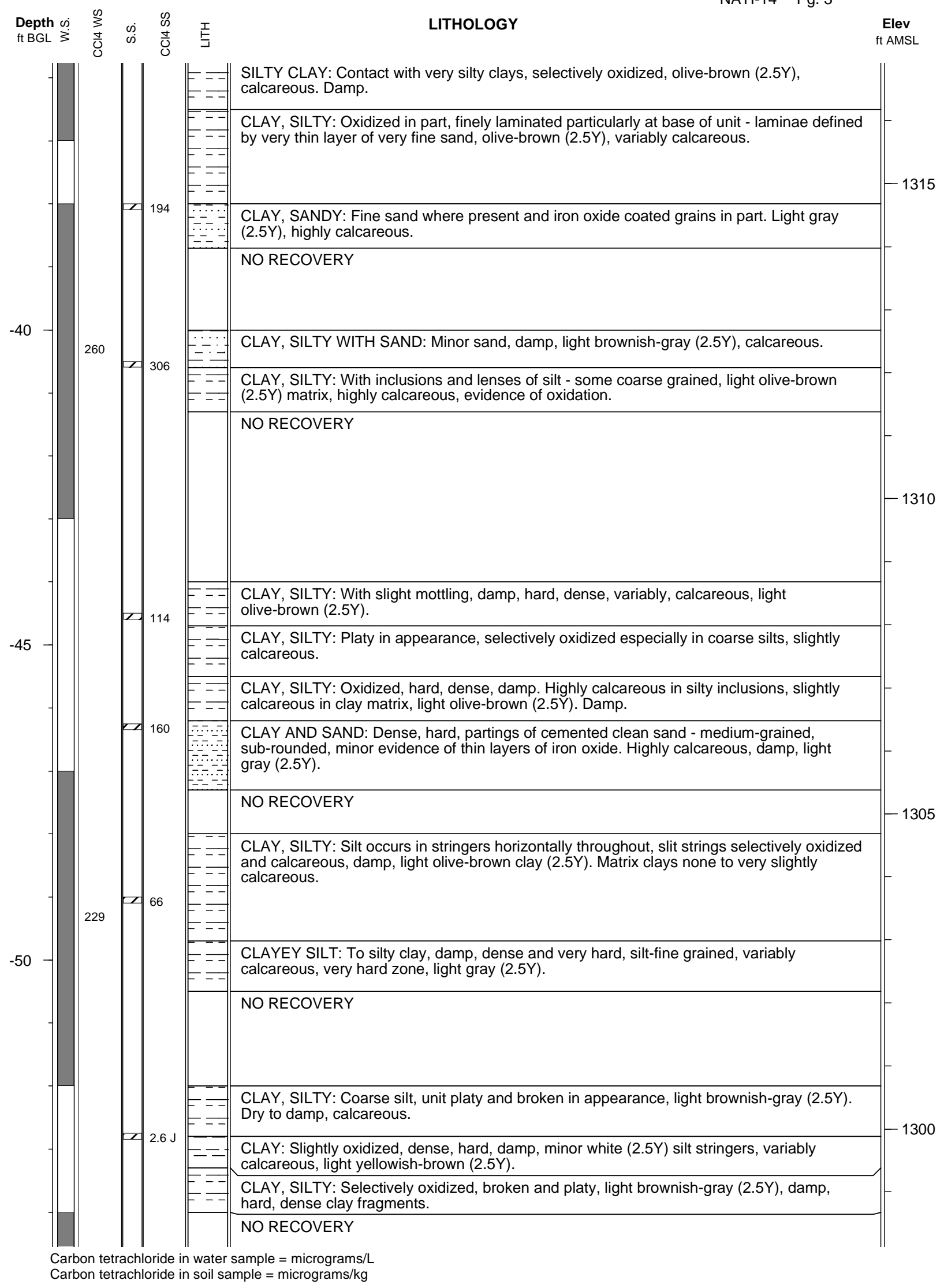


NATI-14 Pg. 4

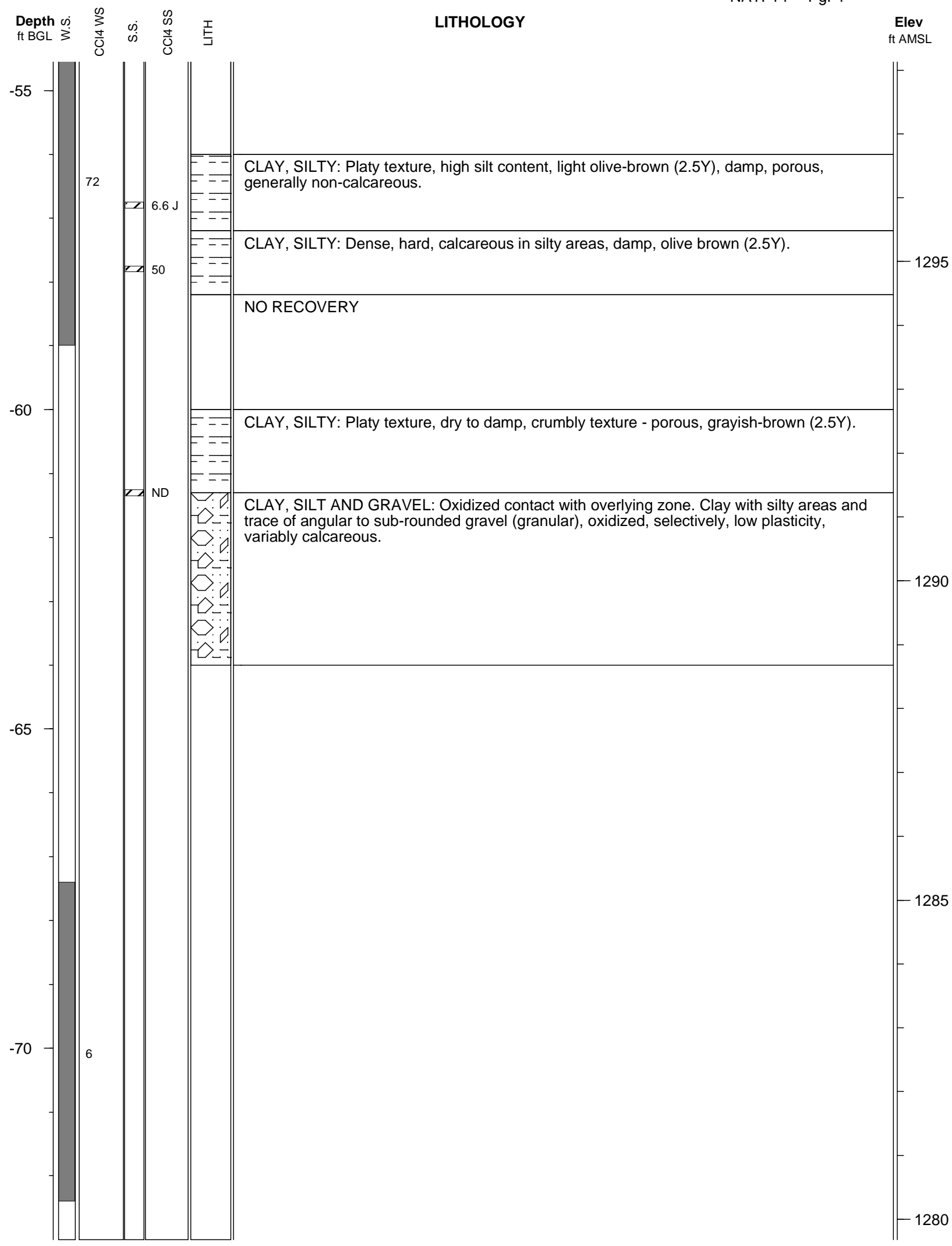

Carbon tetrachloride in water sample $=$ micrograms $/ \mathrm{L}$ Carbon tetrachloride in soil sample $=$ micrograms $/ \mathrm{kg}$ 
Argonne National Laboratory

\section{Project: Navarre}

Geologist: Lisa Larsen
Elevation: $1352.19 \mathrm{ft}$

Depth: $71.96 \mathrm{ft}$. BGL

\section{Boring ID: NATI-16}

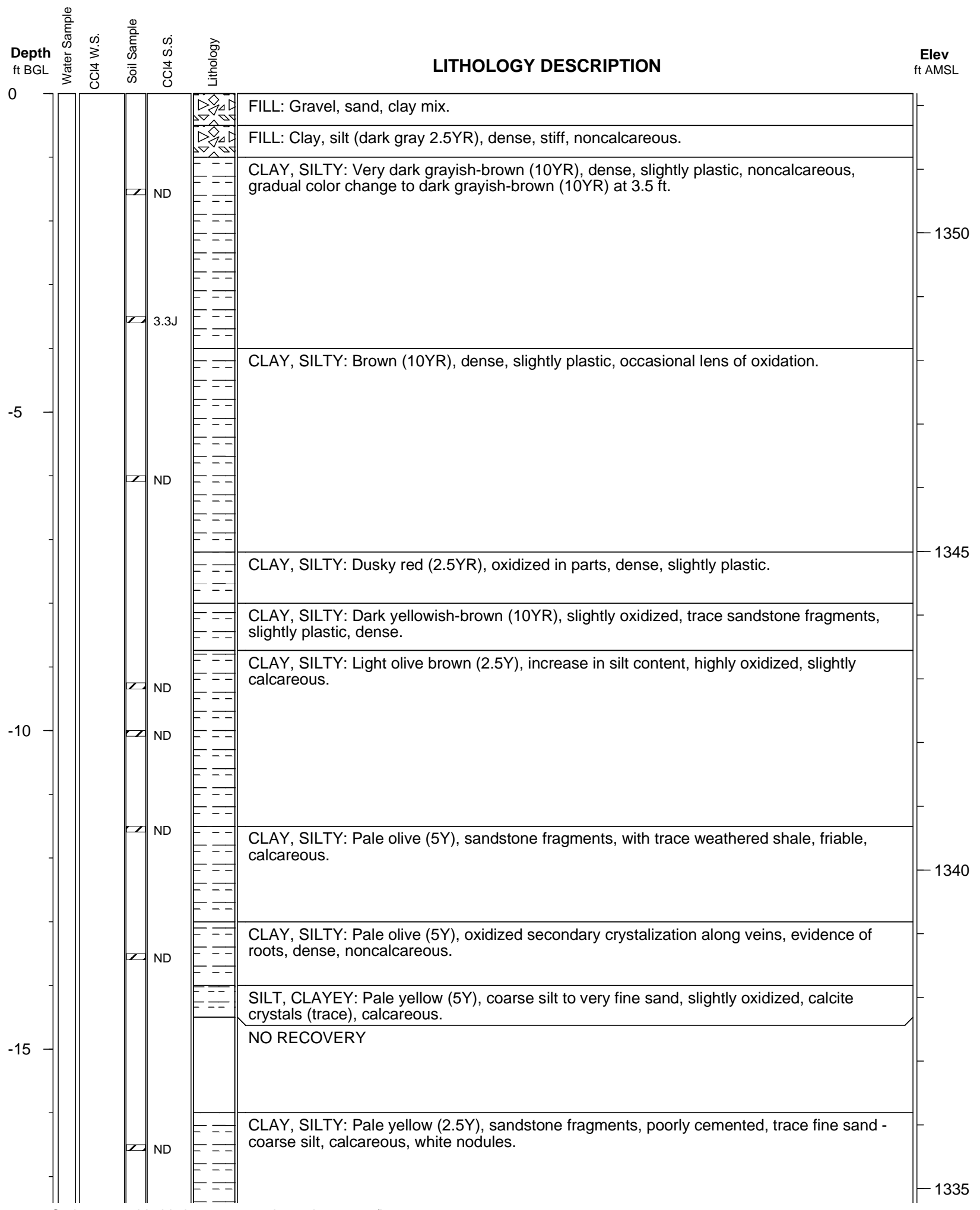

Carbon tetrachloride in water sample $=$ micrograms $/ L$

Carbon tetrachloride in soil sample $=$ micrograms $/ \mathrm{kg}$ 
NATI-16 Pg. 2

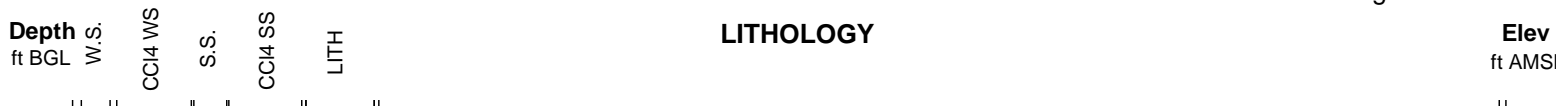

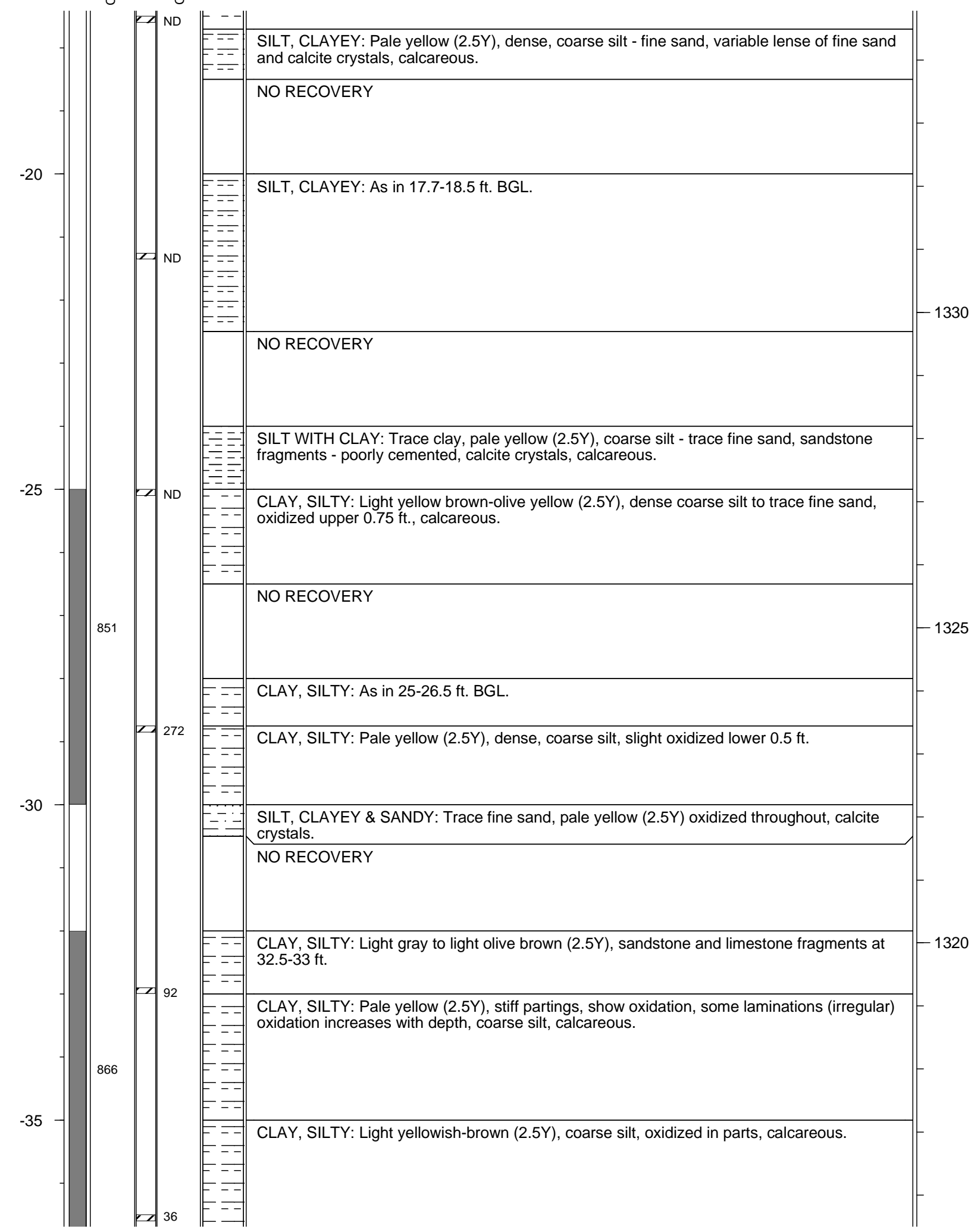


NATI-16 Pg. 3

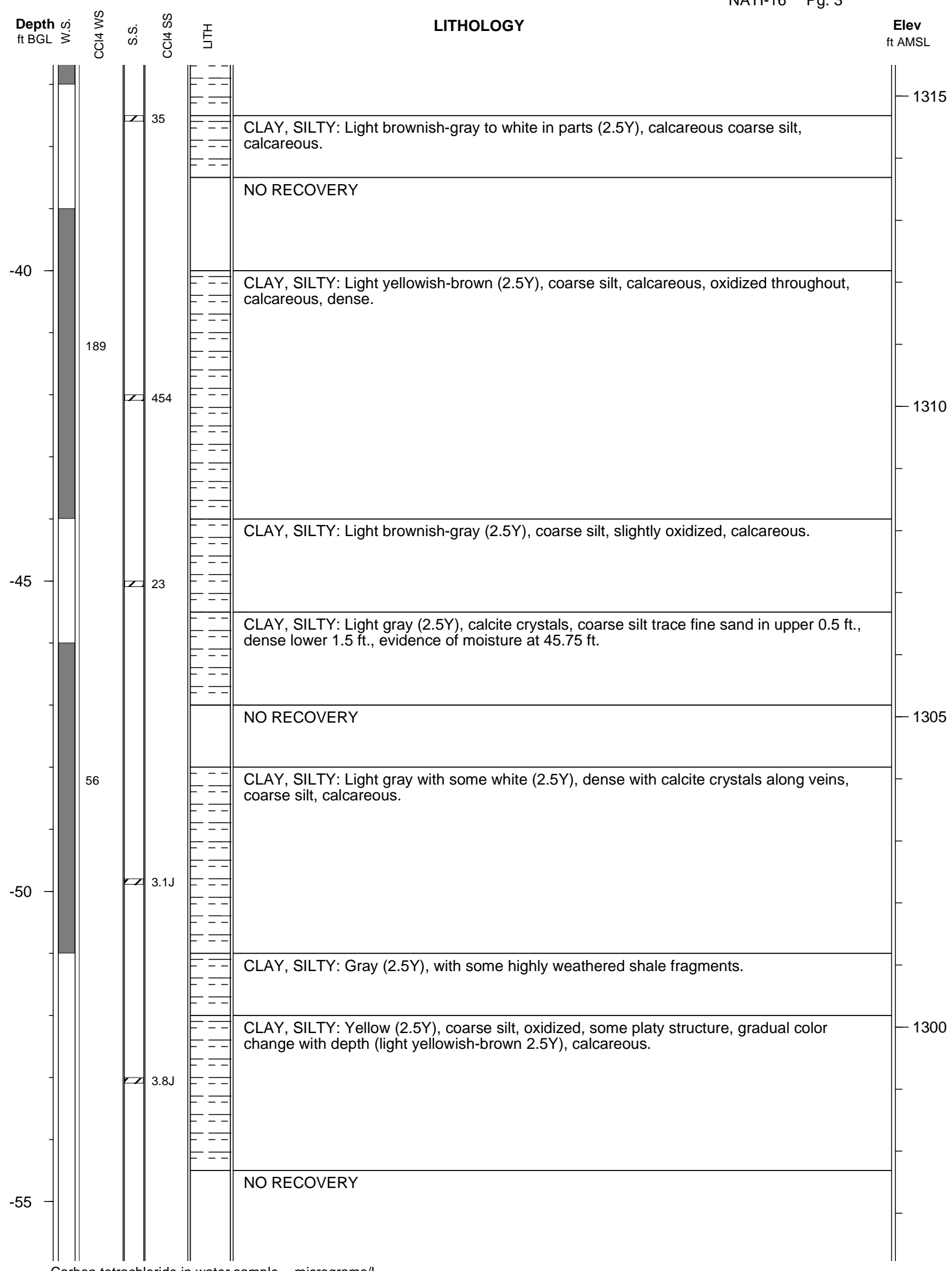

Carbon tetrachloride in water sample $=$ micrograms $/ \mathrm{L}$ Carbon tetrachloride in soil sample $=$ micrograms $/ \mathrm{kg}$ 
NATI-16 Pg. 4

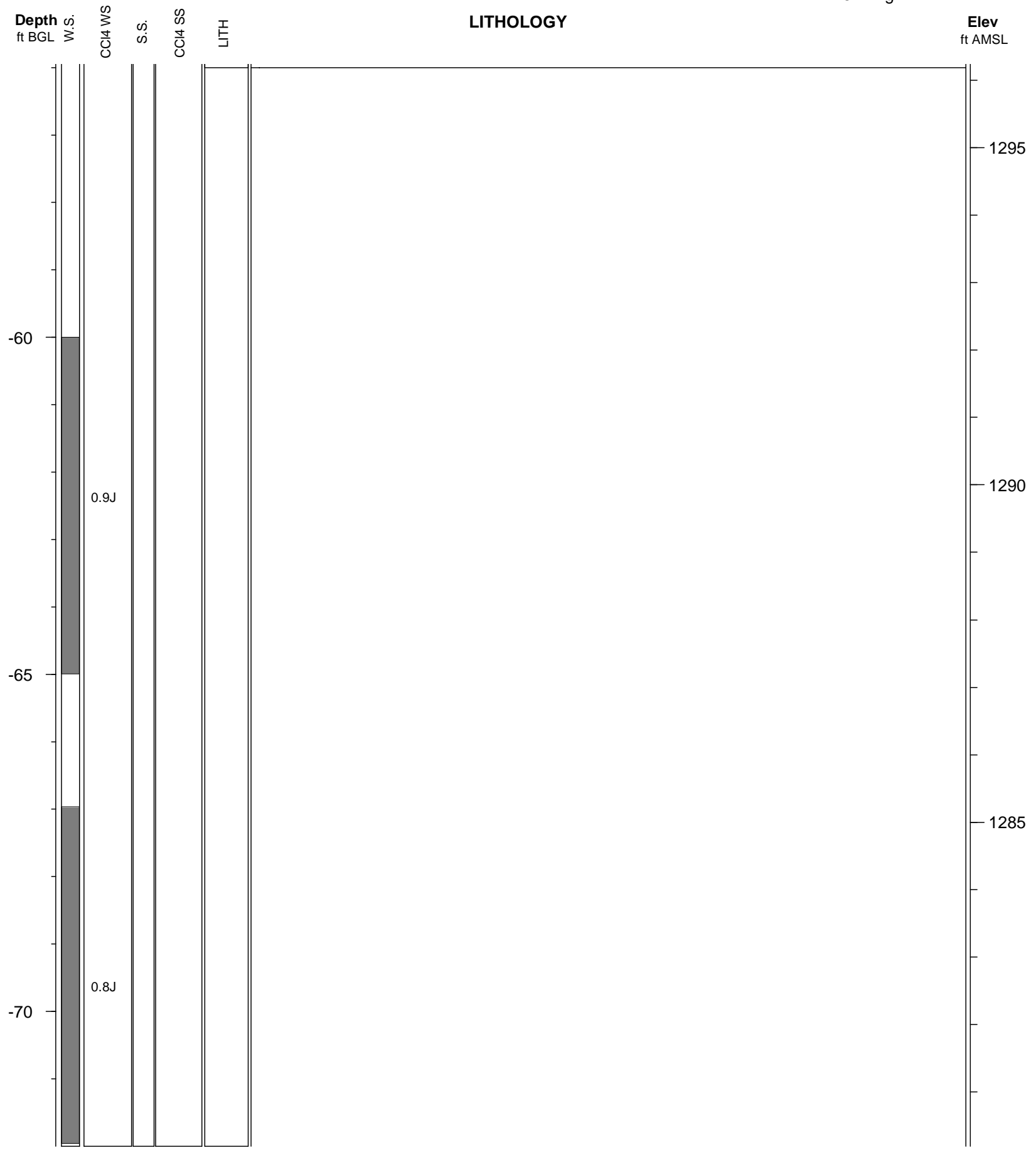

Carbon tetrachloride in water sample $=$ micrograms $/ \mathrm{L}$ Carbon tetrachloride in soil sample $=$ micrograms $/ \mathrm{kg}$ 


\section{Argonne National Laboratory}

Project: Navarre

Geologist: Lorraine LaFreniere
Elevation: $1351.54 \mathrm{ft}$

Depth: $65 \mathrm{ft}$. BGL

Boring ID: NATI-17

Geologist: Lorraine LaFreniere

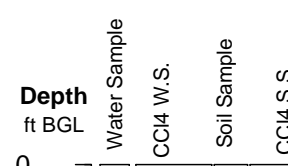

0

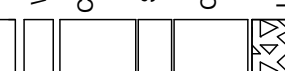

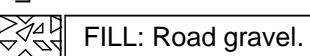

LITHOLOGY DESCRIPTION

Elev

CLAY, SILT AND GRAVEL: Mixed clay and road gravel - probably brought to site and

CLAY, SILT AND GRAVEL: Mixed clay and road gravel - pro
disturbed.
CLAY, SILTY: Dense, hard, black (10YR), non-calcareous.

\begin{tabular}{|l} 
CLAY, SILTY: Increasing silt content with depth, damp, dense, hard, dark grayish-brown \\
(10YR), non-calcareous, non-plastic.
\end{tabular}

\begin{tabular}{|l} 
CLAY, SILTY: Increasing silt content with depth, damp, dense, hard, dark grayish-brown \\
(10YR), non-calcareous, non-plastic.
\end{tabular}

CLAY, SILTY: Marked increase in silt content from overlying unit. Selectively oxidized in areas of concentrated coarse silt, damp, non-calcareous.

$+$

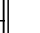

$E=-$

$=--$

$E=--$

NO RECOVERY

CLAY, SILTY: Dense, hard, damp, non-plastic, areas of selective oxidation - almost mottled in appearance, very dark grayish-brown (10YR).

CLAY, SILTY: As above with marked increase in silt content, slight oxidation, non-calcareous. 
NATI-17 Pg. 2

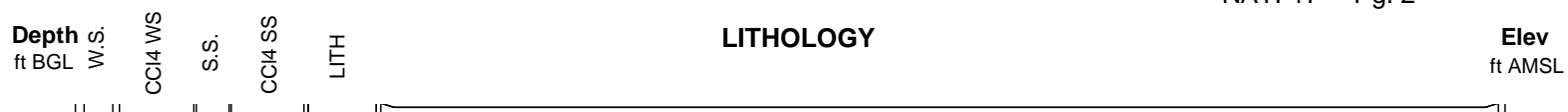

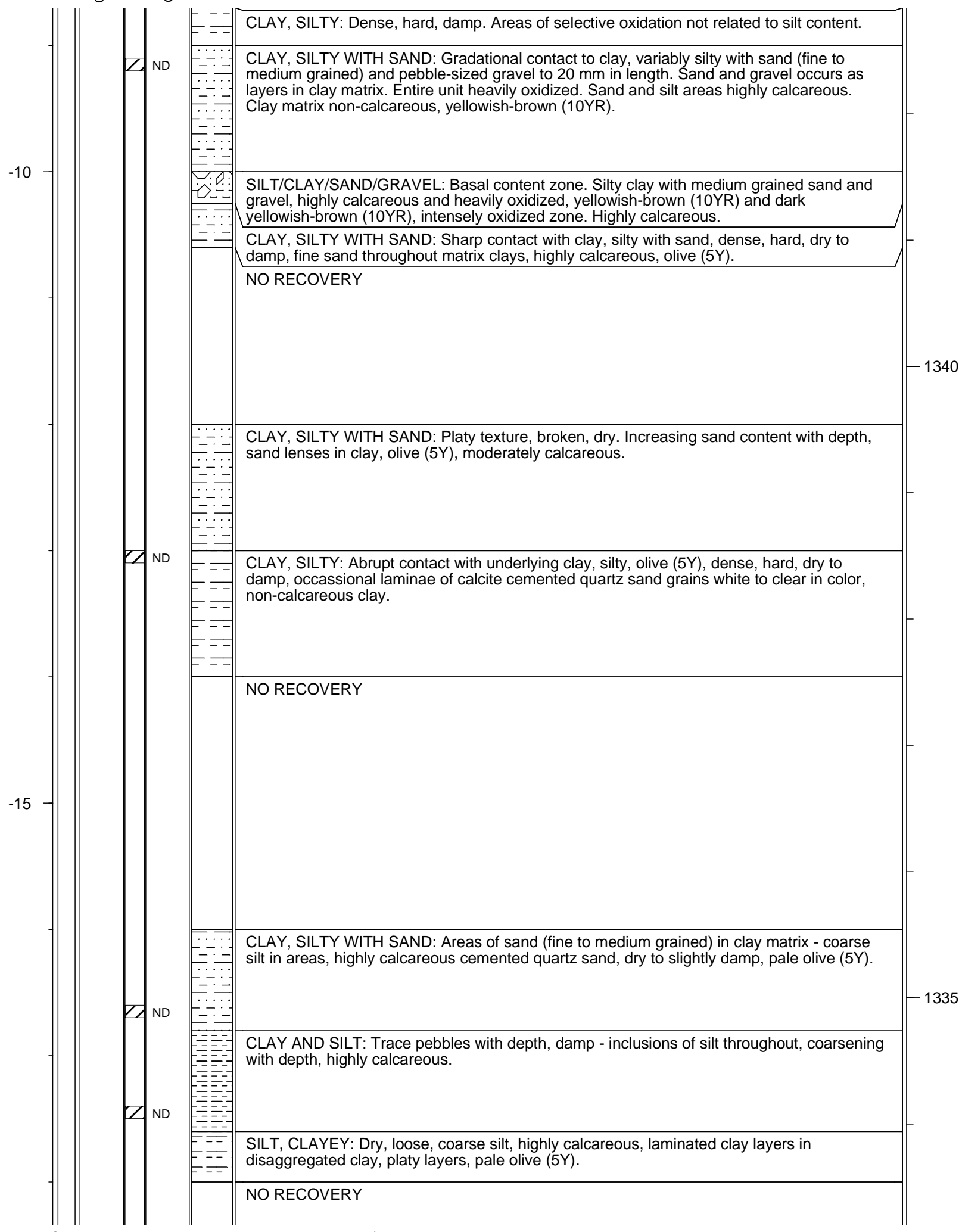


NATI-17 Pg. 3

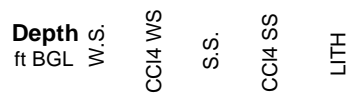

LITHOLOGY

Elev

ठ<smiles>C1=C[V]=CCC1</smiles>

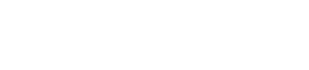

ft AMSL

CLAY,SILT,SAND \& GRAVEL: "Rubble zone", coarse silt to fine sand. Sand, angular to sub-angular, medium to coarse grained, granular to pebble-sized gravel. Damp to moist, grayish-brown (2.5Y), clay, highly calcareous, clear silt grains, basal contact - thin layers of

$E=-$ fine sand.

$-20$

4 ND

- - CLAY, SILTY: With lenses or inclusions of silt-some coarse grained. Clay generally non-calcareous, silt areas calcareous, light olive-brown (2.5Y).

E $=-$

CLAY, SILTY: As described above with marked increase in silt content. Clay, iron oxide

$E=-$ layers with associated fine grained, clear quartz sand, calcareous.

CLAY, SILTY WITH SAND: With faint laminations of silt and fine sand - oxidized. Highly

calcareous, light yellowish-brown (2.5Y), damp.

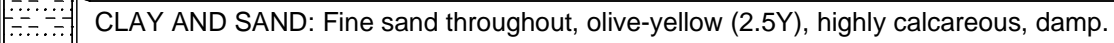

NO RECOVERY

CLAY, SILTY: Silt inclusions thro
$(2.5 Y)$, crumbly texture in part.

CLAY, SILTY: With occasional inclusions of coarse silt, clay non-calcareous, silt areas -

calcareous. Light olive-brown (2.5Y), clay, damp, dense, hard.

CLAY, SILTY: Silt inclusions throughout, calcareous, light olive-brown (2.5Y), minor evidence of iron oxide.

NO RECOVERY 
NATI-17 Pg. 4

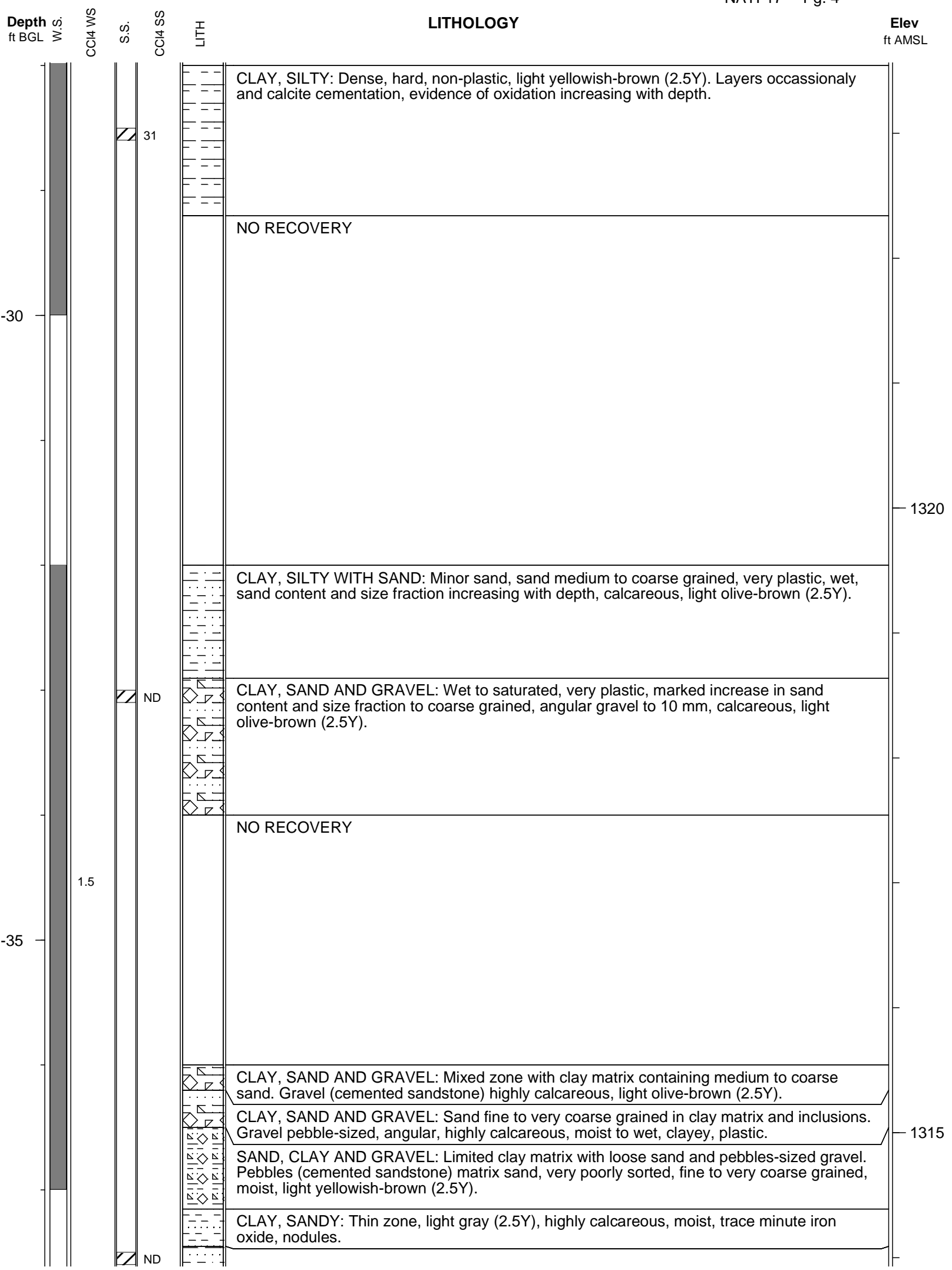

Carbon tetrachloride in water sample $=$ micrograms $/ \mathrm{L}$

Carbon tetrachloride in soil sample $=$ micrograms $/ \mathrm{kg}$ 
NATI-17 Pg. 5

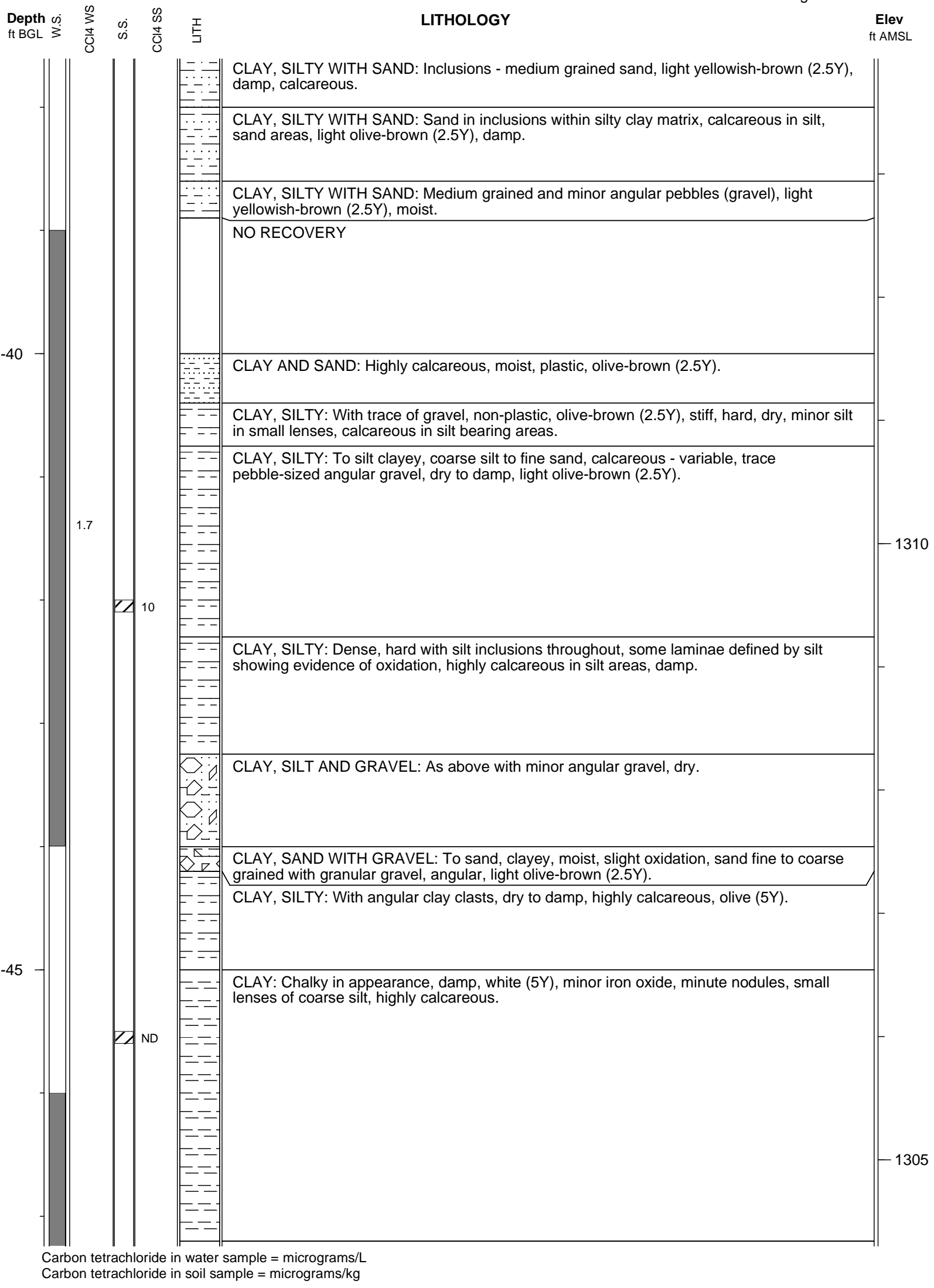


NATI-17 Pg. 6

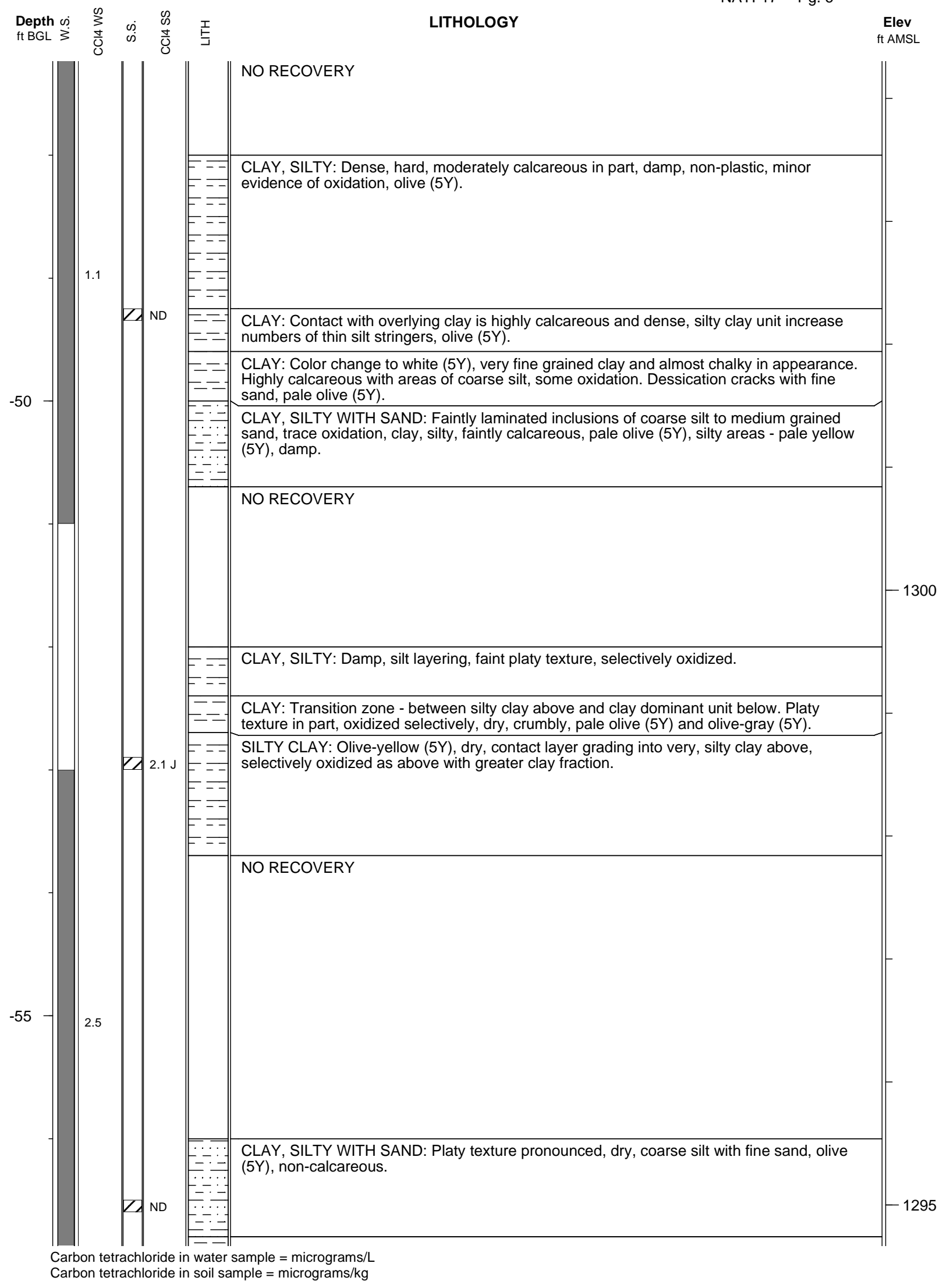


NATI-17 Pg. 7

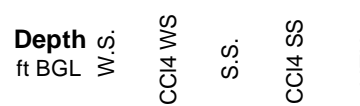

LITHOLOGY

Elev

10 CLAY: Marked reduction in silt content, dense, hard, non-plastic, olive (5Y), damp, non to slightly calcareous.

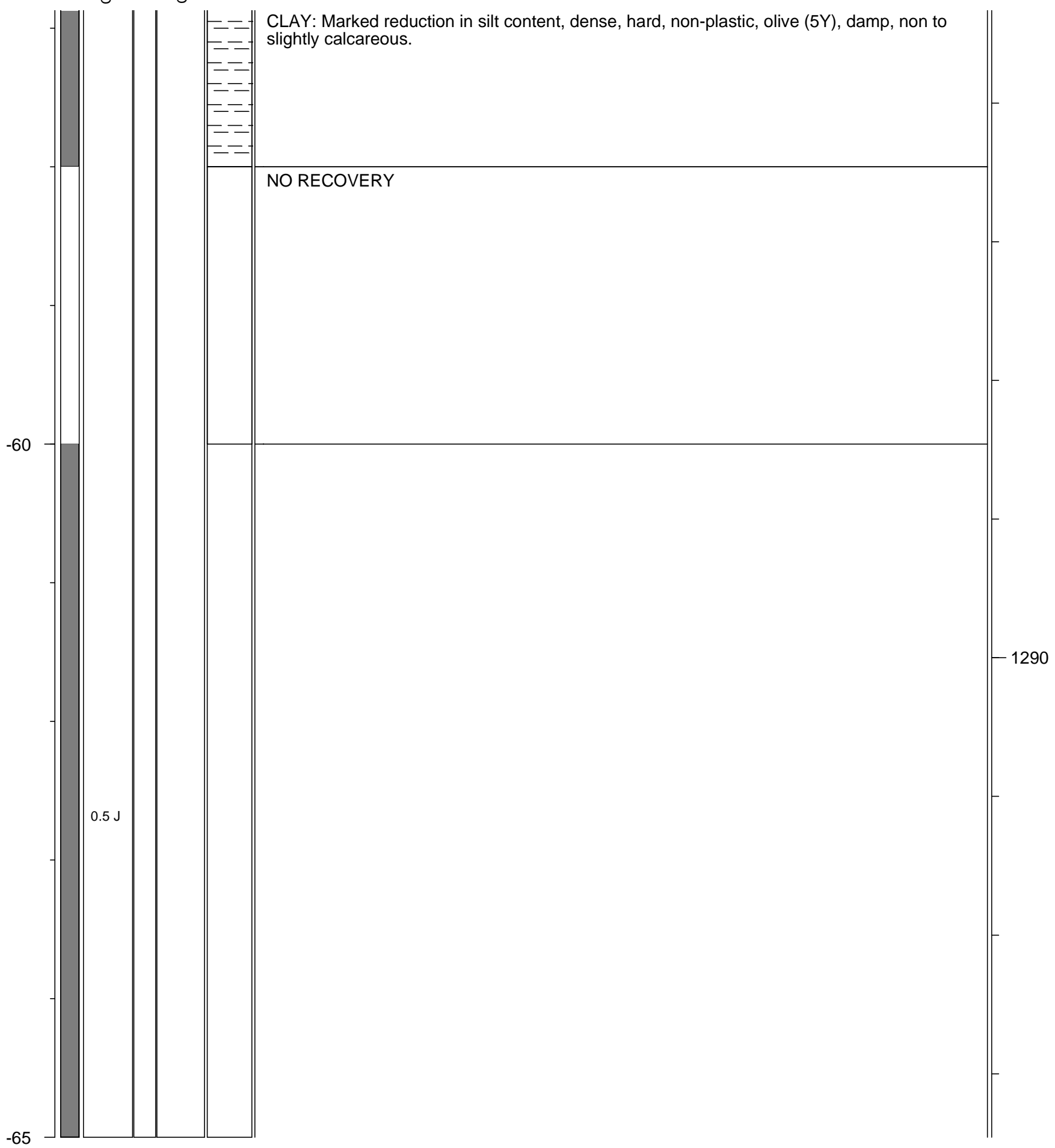

Carbon tetrachloride in water sample $=$ micrograms $/ \mathrm{L}$ Carbon tetrachloride in soil sample $=$ micrograms $/ \mathrm{kg}$ 
Version 00, 05/23/07

\section{Argonne National Laboratory}

Project: Navarre

Geologist: Lorraine LaFreniere
Elevation: $1352.68 \mathrm{ft}$.

Depth: 73.25 ft. BGL

\section{Boring ID: NATI-22}

Log Date: 5/9/2006

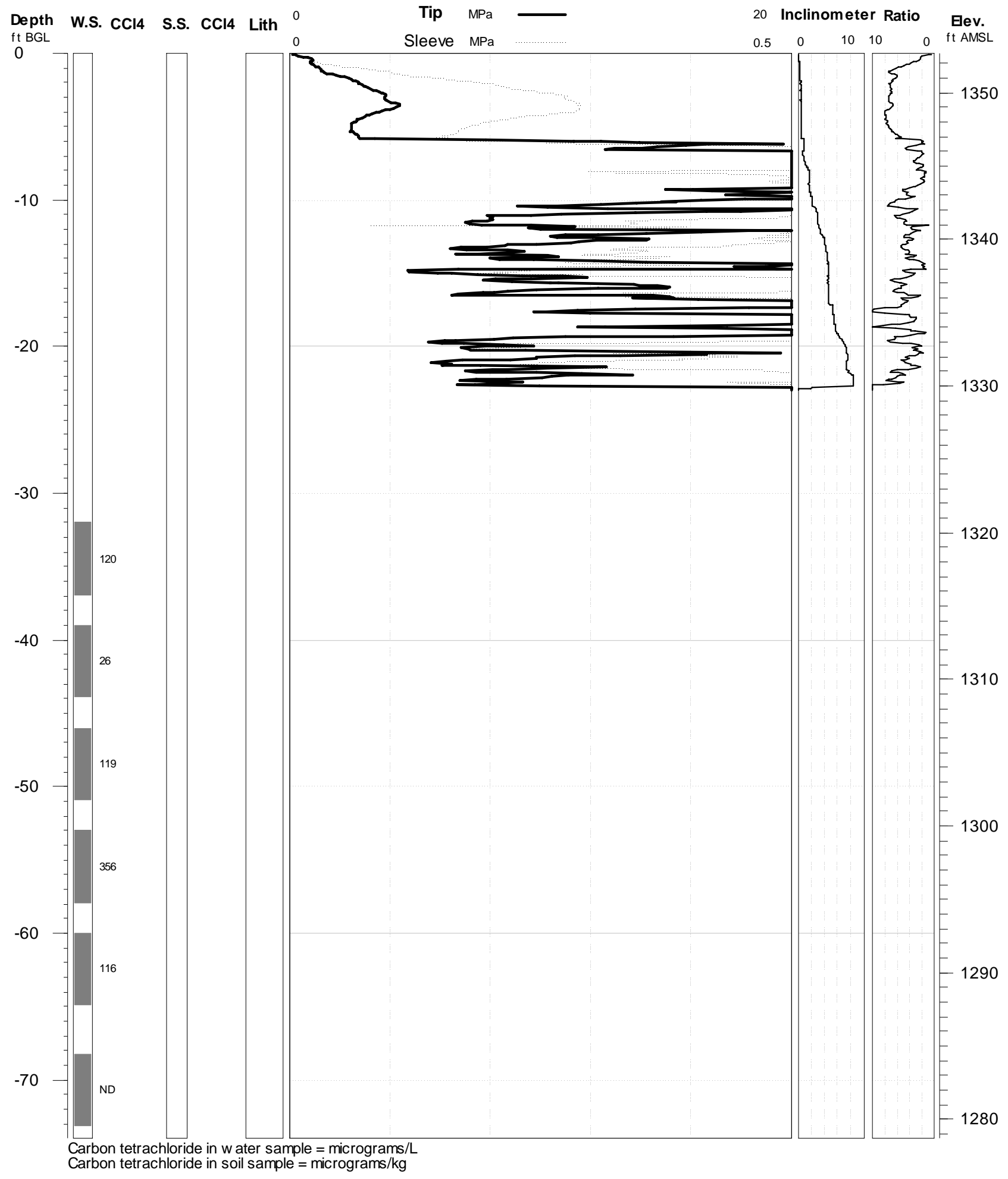




\section{Argonne National Laboratory}

\section{Project: Navarre}

Geologist: Lorraine LaFreniere
Elevation: $1351.79 \mathrm{ft}$.

Depth: $71.8 \mathrm{ft}$. BGL

\section{Boring ID: NATI-23}

Log Date: 5/10/2006

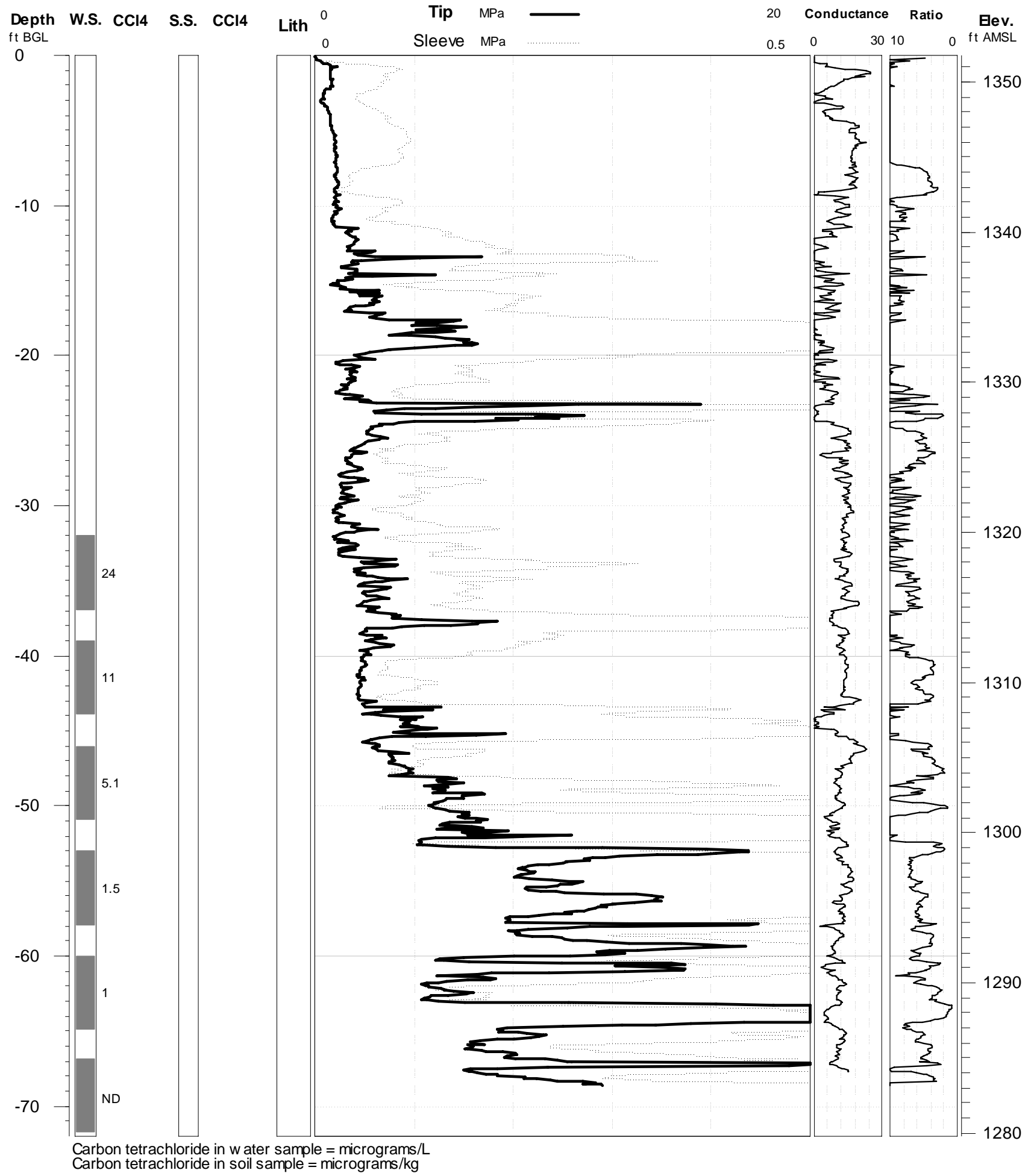




\section{Argonne National Laboratory}

Project: Navarre

Geologist: Lorraine LaFreniere/Lisa Larsen
Elevation: $1348.85 \mathrm{ft}$.

Depth: $68.03 \mathrm{ft} . \mathrm{BGL}$

\section{Boring ID: NATI-28}

Log Date: 5/18/2006

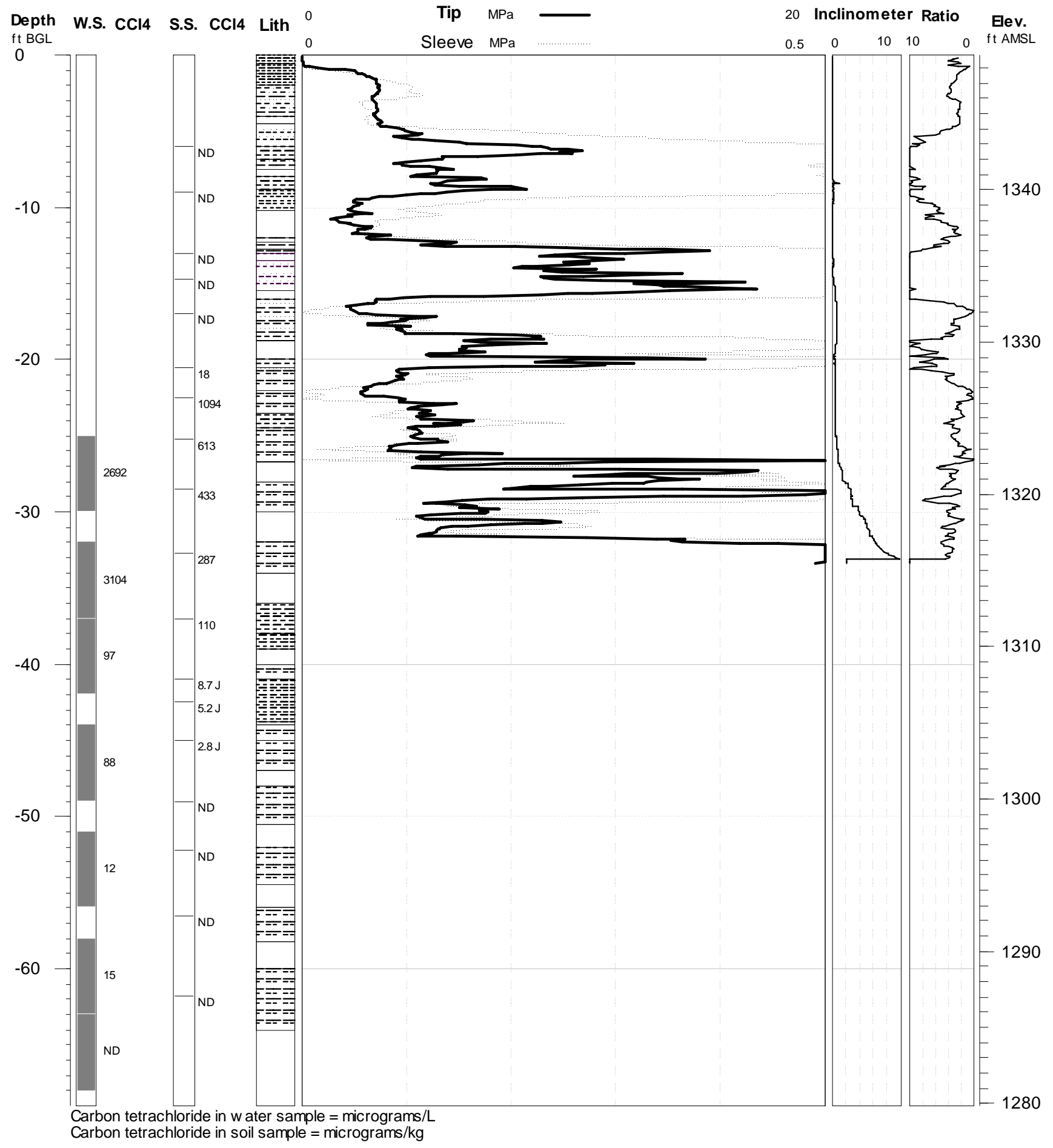




\section{Argonne National Laboratory}

Project: Navarre

Geologist: Lorraine LaFreniere/Lisa Larsen

\section{Boring ID: NATI-28}

Elevation: $1348.85 \mathrm{ft}$.

Depth: $68.03 \mathrm{ft}$. BGL

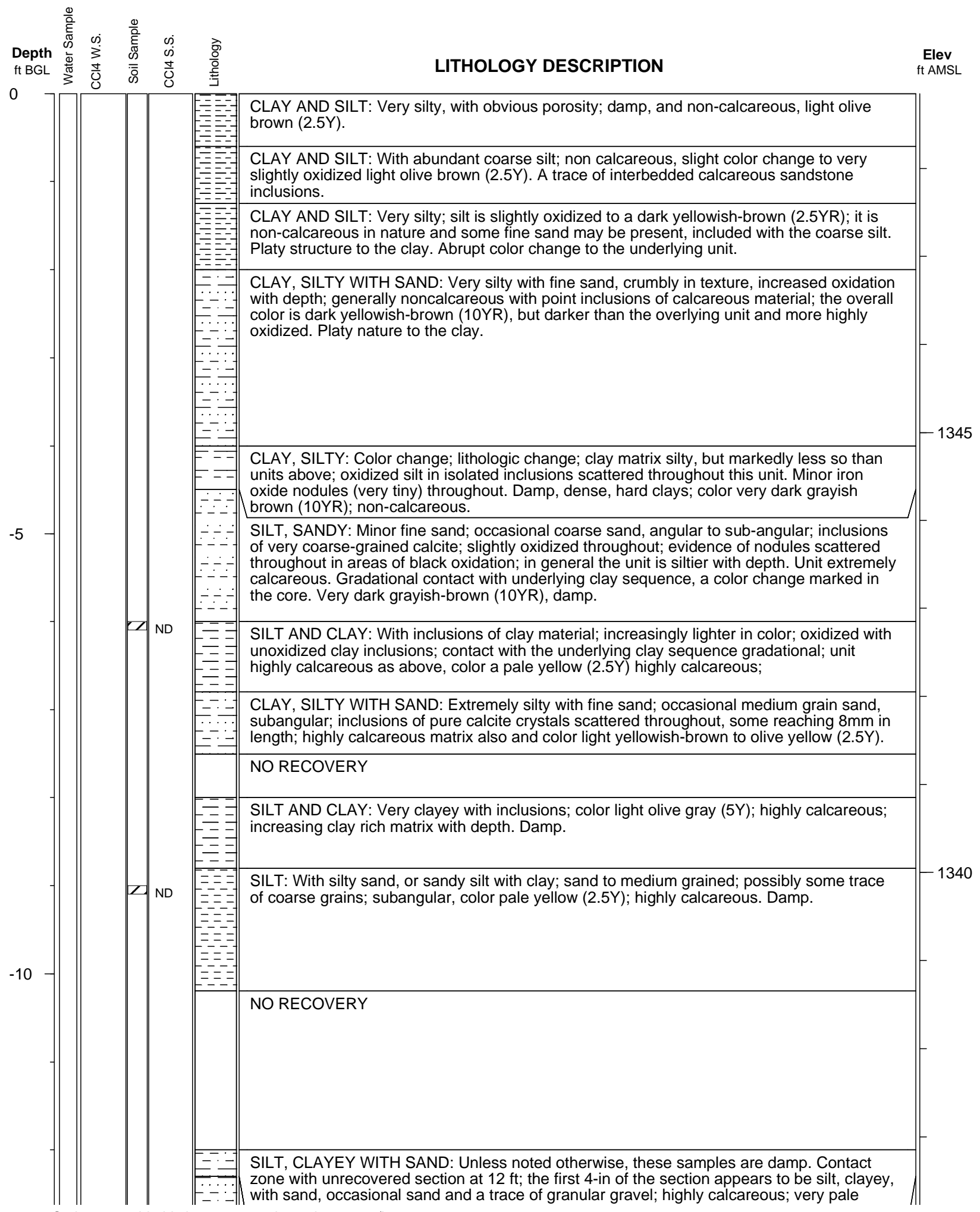


NATI-28 Pg. 2

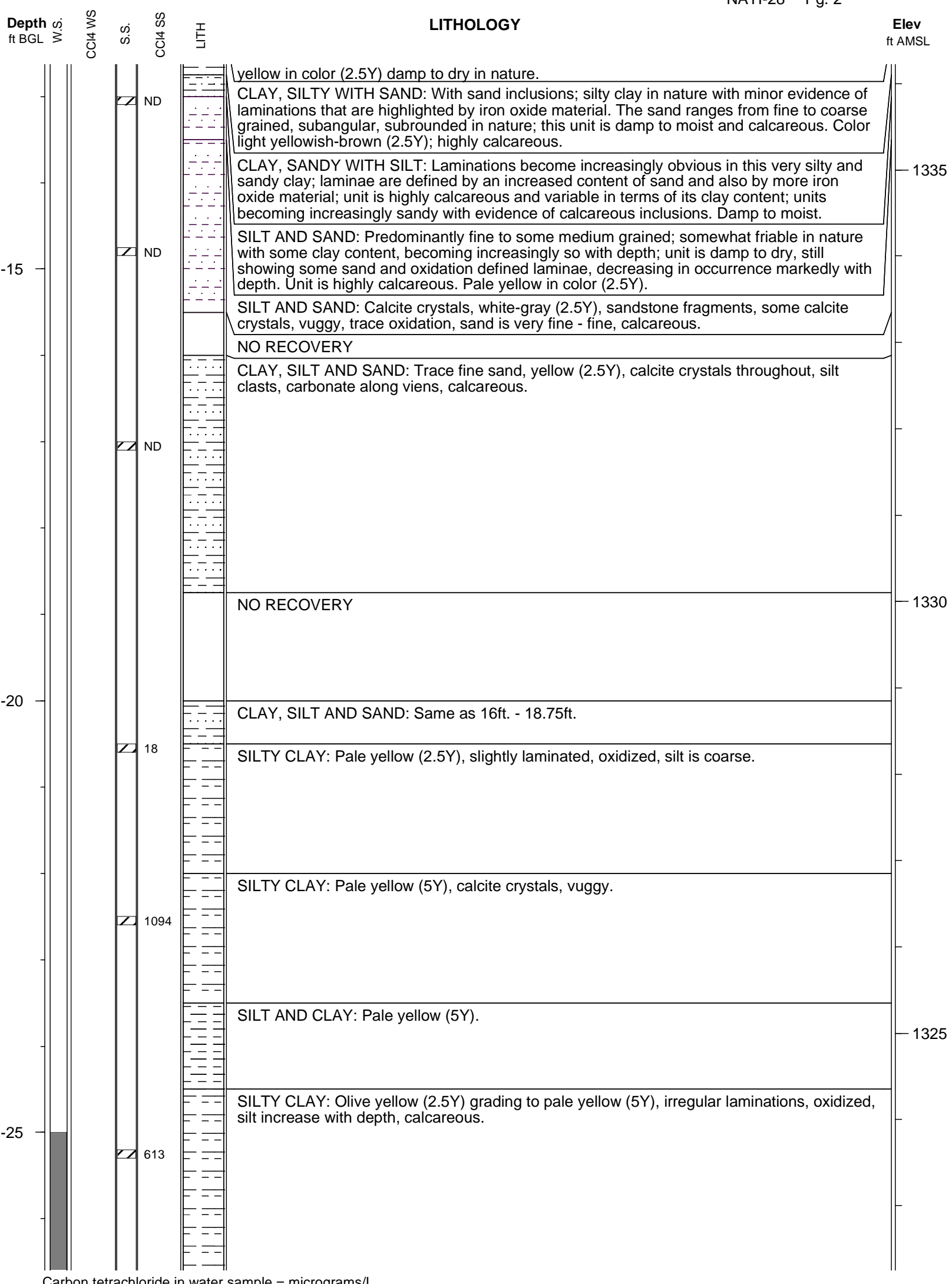

Carbon tetrachloride in water sample $=$ micrograms $/ \mathrm{L}$

Carbon tetrachloride in soil samble $=$ microarams $/ \mathrm{ka}$ 
NATI-28 Pg. 3

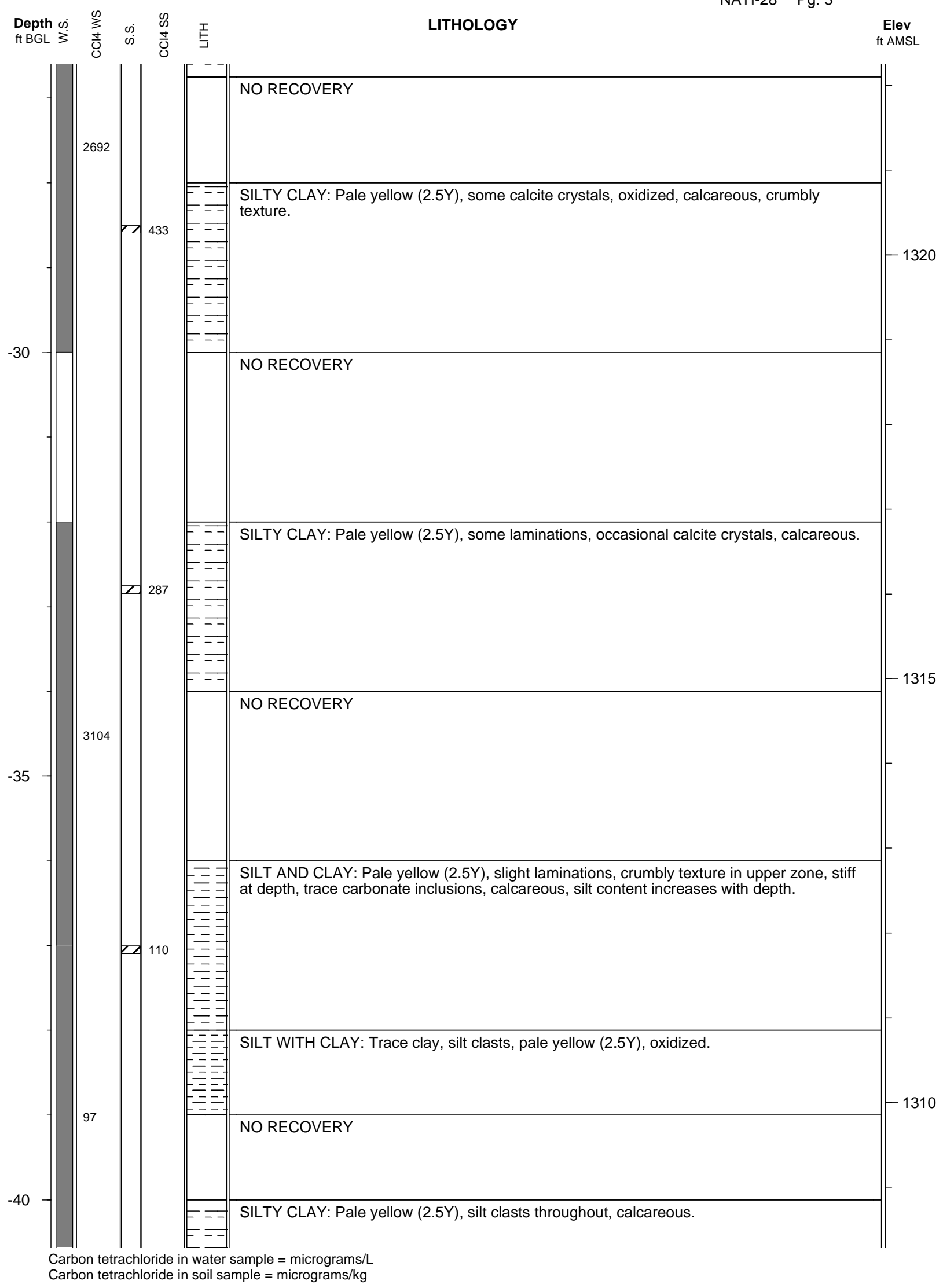


NATI-28 Pg. 4

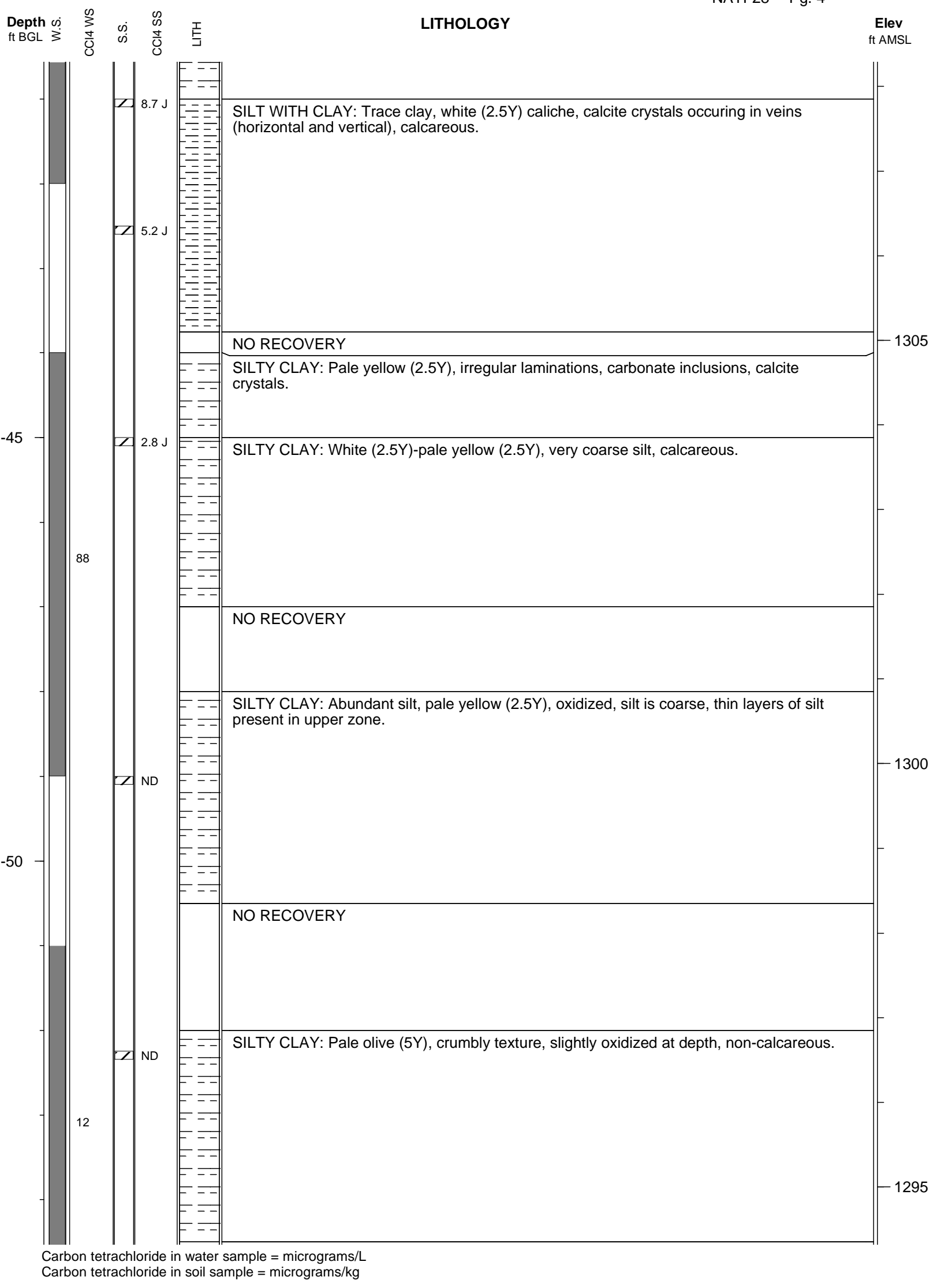


NATI-28 Pg. 5

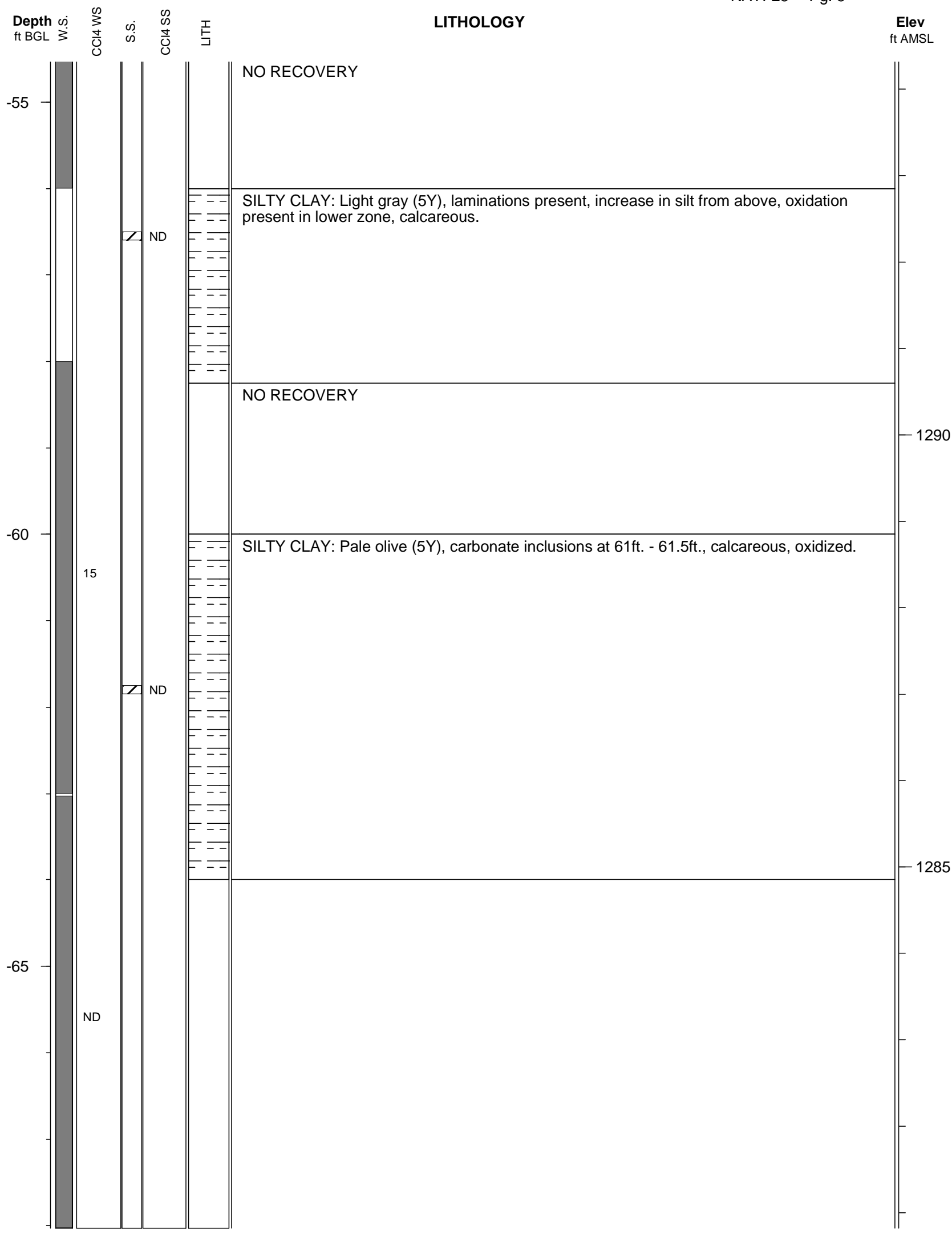

Carbon tetrachloride in water sample $=$ micrograms $/ \mathrm{L}$ Carbon tetrachloride in soil sample $=$ micrograms $/ \mathrm{kg}$ 


\section{Argonne National Laboratory}

Project: Navarre

Geologist: Lisa Larsen
Elevation: $1349.53 \mathrm{ft}$.

Depth: $69.3 \mathrm{ft} . \mathrm{BGL}$
Boring ID: NATI-29

\section{Boring ID: NATI-29}

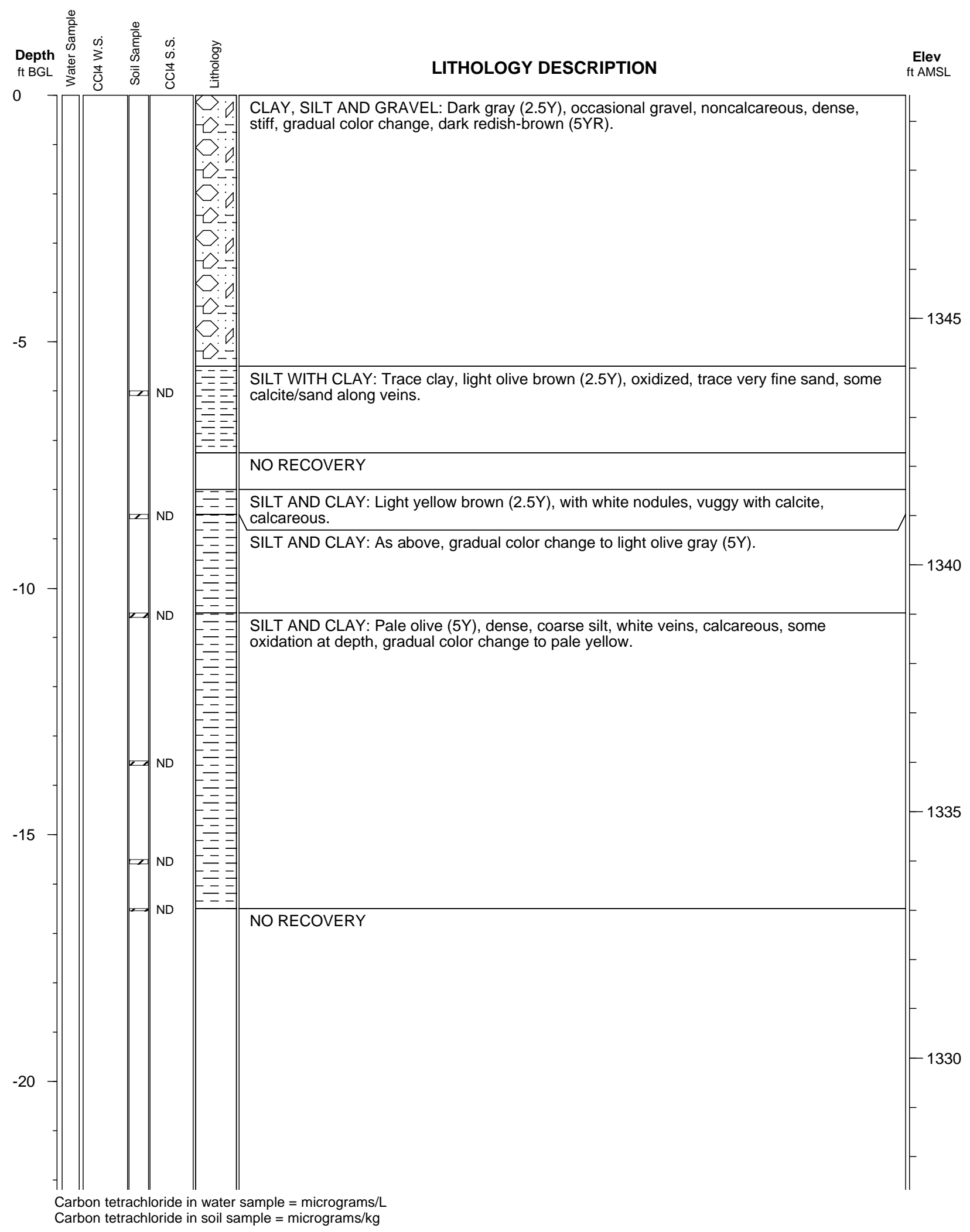


NATI-29 Pg. 2

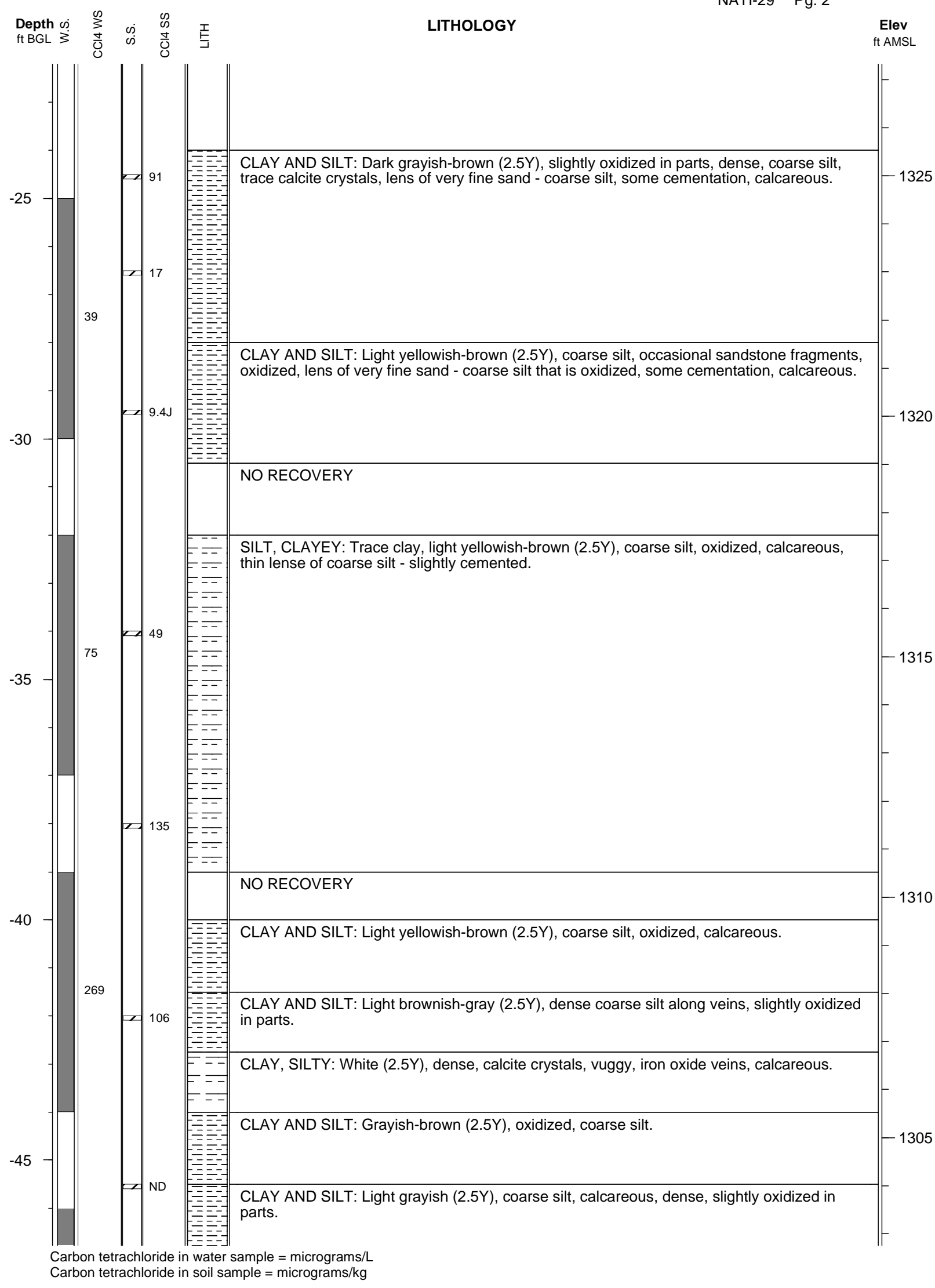


NATI-29 Pg. 3

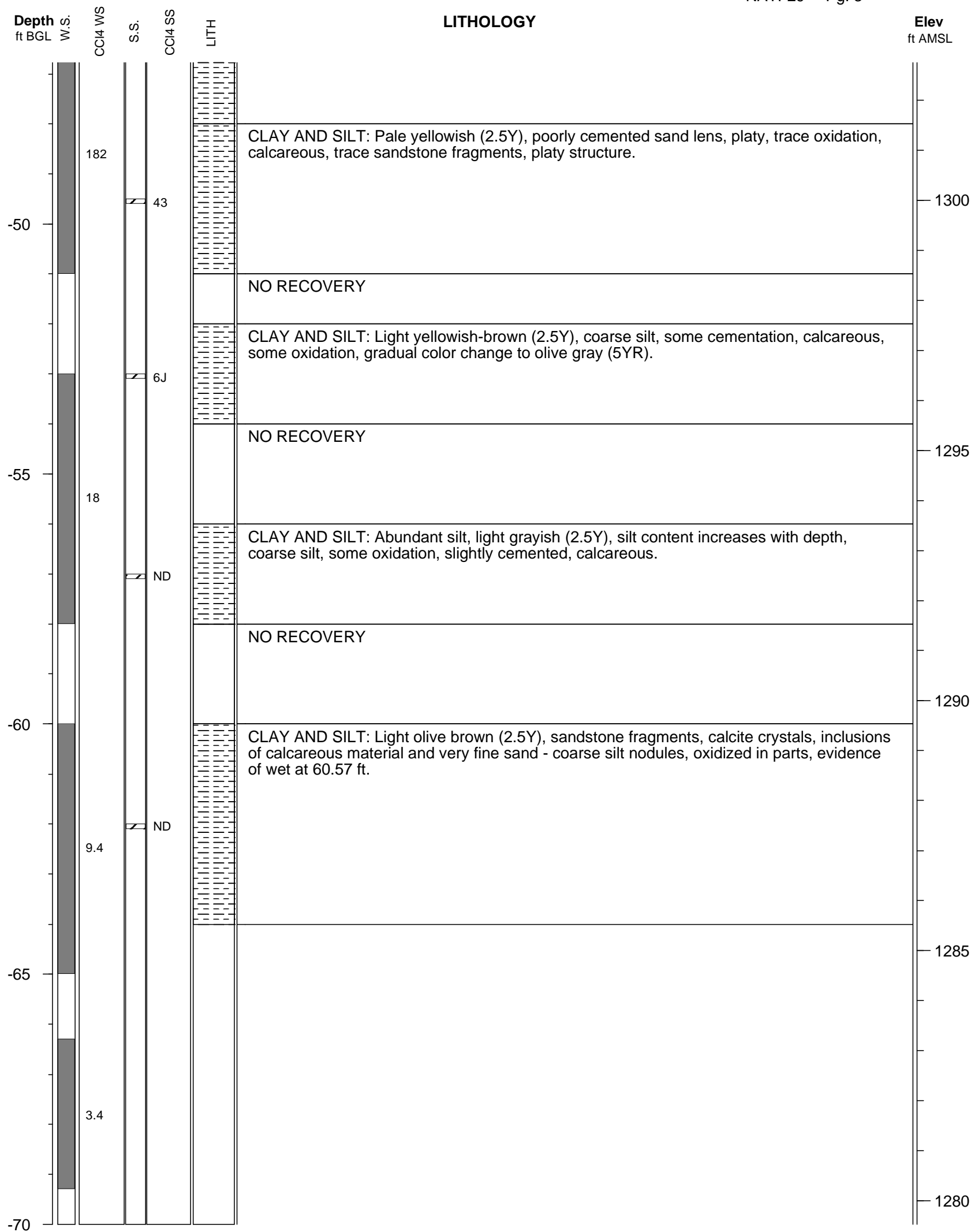

Carbon tetrachloride in water sample $=$ micrograms $/ \mathrm{L}$ Carbon tetrachloride in soil sample $=$ micrograms $/ \mathrm{kg}$ 


\section{Argonne National Laboratory}

Project: Navarre

Geologist: Lorraine LaFreniere
Elevation: $1353.77 \mathrm{ft}$

Depth: $88.5 \mathrm{ft} . \mathrm{BGL}$

\section{Boring ID: MW5}

Log Date: 5/22/2006

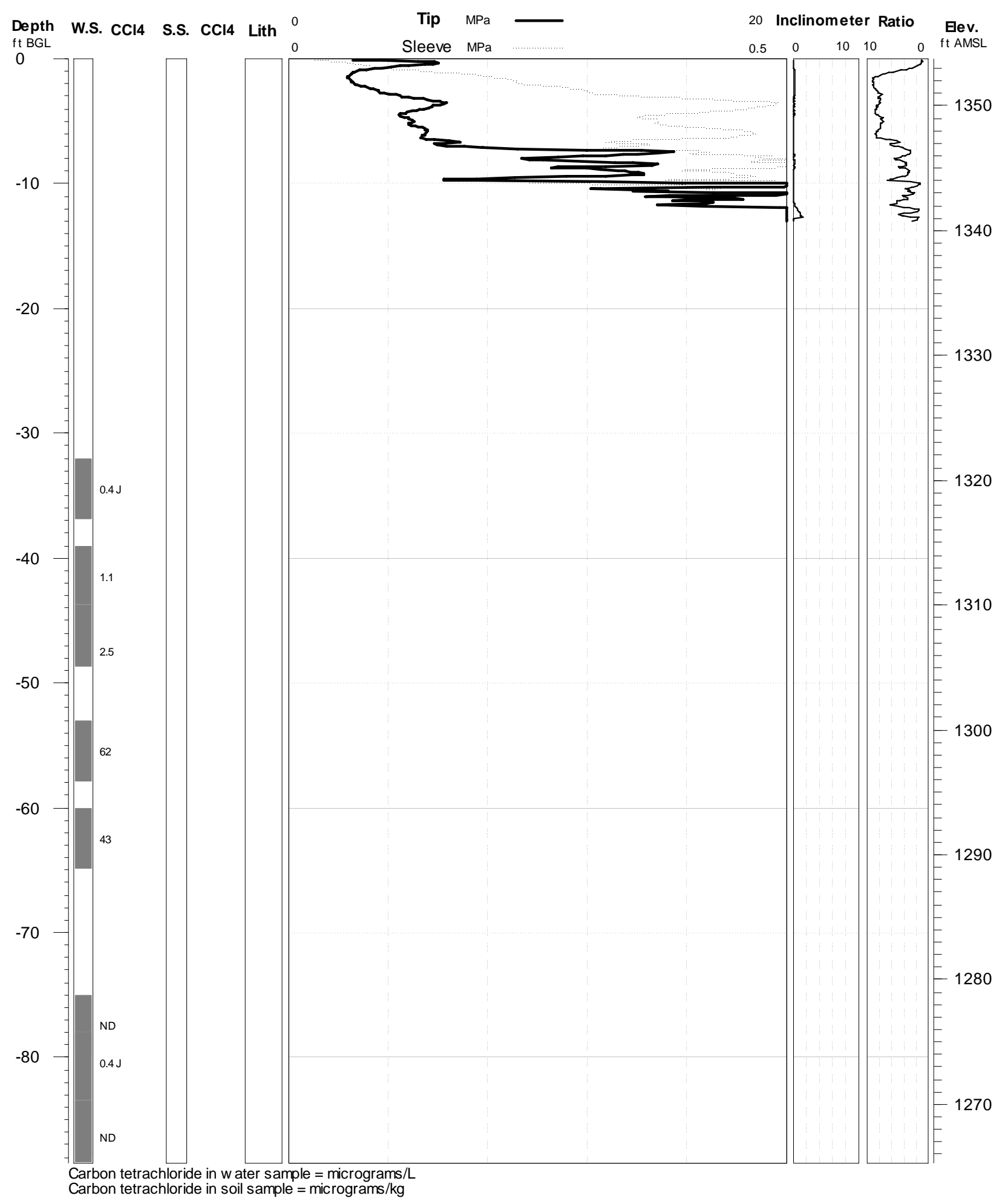




\section{Appendix B:}

\section{Well Construction Information for MW5}




\section{Piezometer (Sand Point Well) MW5: Navarre, KS}

SE $1 / 4$ of NE $1 / 4$ of NW 1/4 of Section 33, Twp. 14 South, Rge. 3 East

Dickinson County, State of Kansas

Date: $7 / 25 / 2006$

\section{WELL HEAD PROTECTION}

12" Morrison Brothers, Co. Model 418XA flush mount cover. Top of casing fitted with a (J-Plug) Morrison Brothers, Co. Model

678XA and a screened vent with a locking pipe plug and padlock.

\section{CONCRETE PAD}

Must be a minimum of 8 " thick and extend at least 8 " larger than the flush mount (28" minimum). Sloped to prevent pooling of water and vegetation around well and to allow for placement of a surveyor pin.

\section{IMPERVIOUS GROUT}

The well must be grouted with impervious grout and must be tremied in the hole, with clean fresh water, to have a minimum density of $9.4 \mathrm{lbs}$. per gallon. Grout must extend from the top of the bentonite chips to 3' BGL.

\section{WELL CASING}

Well casing shall terminate as high as possible inside the flush mount and be capped with a (J-Plug) Morrison Brothers, Co. Model 678XA locking plug and padlock.

1" PVC Schedule 40 threaded casing and Mill Slot (0.010") well screen.

\section{HOLE SIZE}

The hole must be at least 4.25" in diameter for the top 21' and grouted to the base of the flush mount

\section{GRAVEL / SAND PACK}

Gravel/sand pack screen size and gradation shall be determined based upon the grain size and gradation of portion or portions of the aquifer to be screened. Gravel pack shall be designed to stabilize the aquifer material and permit the fine fraction to move into the well during development. Gravel/sand pack shall extend to at least 2 ' above screen.

\section{CONTRACTOR LICENSING}

All wells must be constructed under the direction of a licensed water well contractor as specified under the Kansas Department of Health and Environment.

\section{REGISTRATION}

All wells must be registered with the Kansas Department of Health and Environment on form WWC-5 provided by that Department.

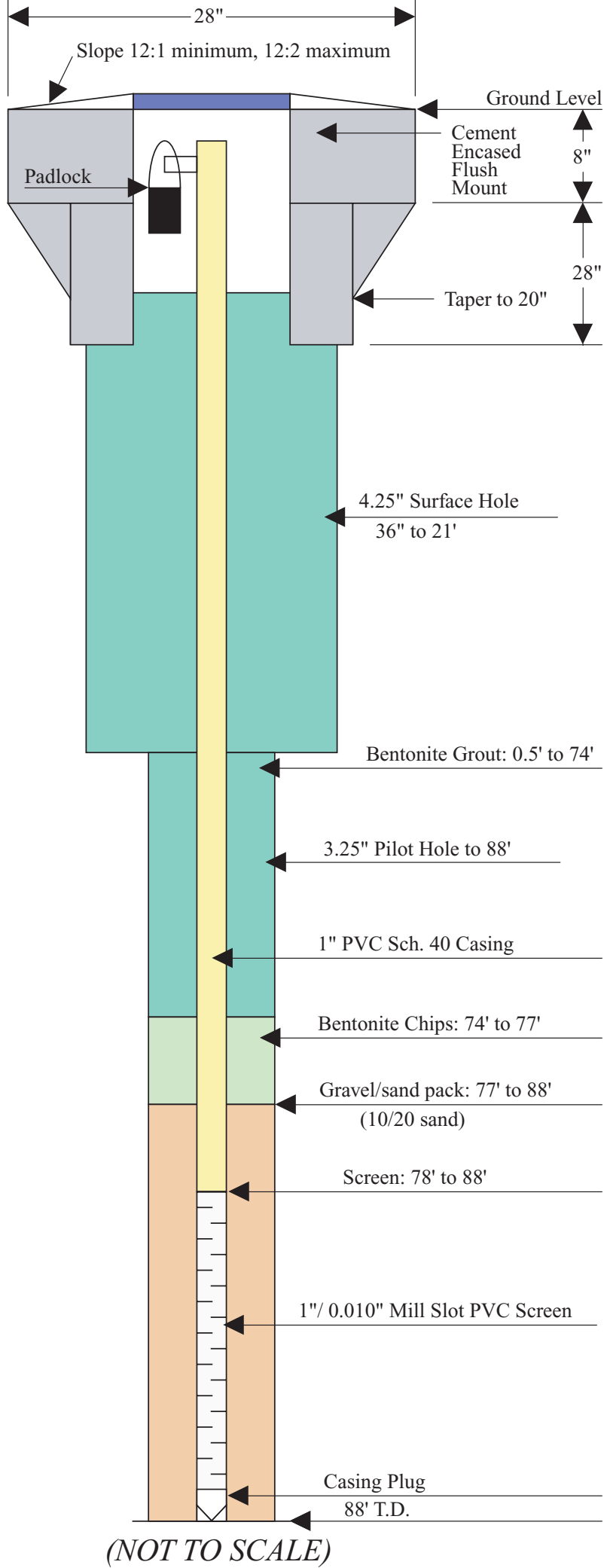




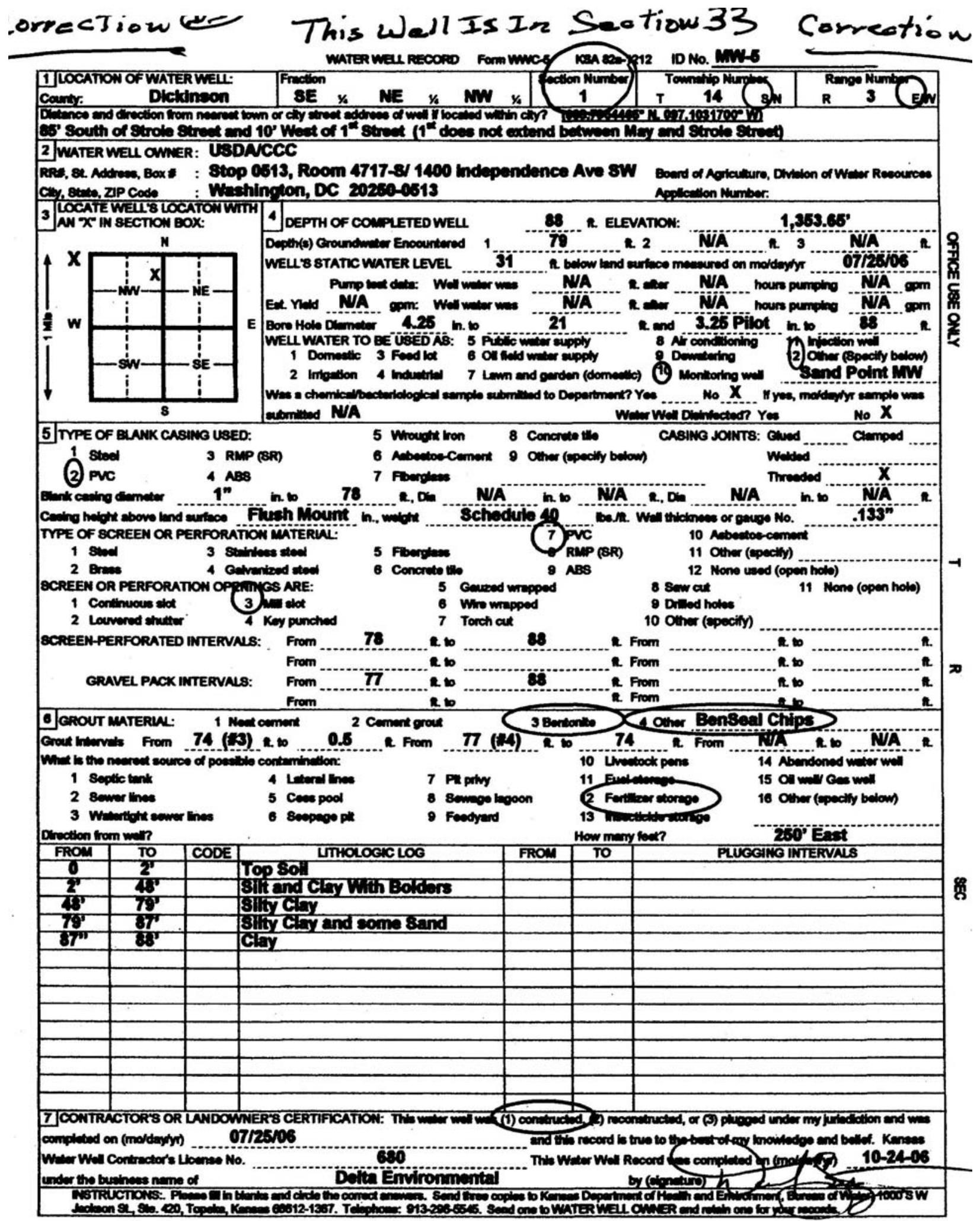




\section{Appendix C:}

Coordinates Survey Data 
TABLE C.1 Coordinates survey data for the 2006 investigation at Navarre.

\begin{tabular}{|c|c|c|c|}
\hline \multirow[b]{2}{*}{ Location } & \multicolumn{2}{|c|}{ Horizontal Location ${ }^{\mathrm{a}}$ (ft) } & \multirow{2}{*}{$\begin{array}{l}\text { Ground } \\
\text { Elevation } \\
\text { (ft AMSL) }\end{array}$} \\
\hline & Northing & Easting & \\
\hline
\end{tabular}

Cone penetrometer sampling locations

\begin{tabular}{llll} 
TI-1 & 169680.825 & 1568253.983 & 1352.62 \\
TI-2 & 169693.739 & 1568294.251 & 1351.64 \\
TI-3 & 169609.492 & 1568244.016 & 1352.92 \\
TI-4 & 169656.098 & 1568189.406 & 1351.63 \\
TI-5 & 169534.213 & 1568221.318 & 1351.96 \\
TI-6 & 169498.115 & 1568280.322 & 1352.73 \\
TI-7 & 169612.025 & 1568320.129 & 1351.14 \\
TI-8 & 169448.449 & 1568348.940 & 1351.21 \\
TI-9 & 169427.001 & 1568254.976 & 1351.55 \\
TI-10 & 169375.023 & 1568321.065 & 1353.21 \\
TI-11 & 169299.401 & 1568284.386 & 1352.07 \\
TI-12 & 169295.189 & 1568382.529 & 1351.87 \\
TI-13 & 169834.283 & 1568205.747 & 1351.73 \\
TI-14 & 169778.675 & 1568219.992 & 1352.68 \\
TI-15 & 169965.491 & 1568188.481 & 1351.48 \\
TI-16 & 169826.265 & 1568248.293 & 1352.19 \\
TI-17 & 169984.611 & 1568237.475 & 1351.54 \\
TI-18 & 169748.891 & 1568251.468 & 1353.02 \\
TI-19 & 170132.606 & 1568355.171 & 1349.12 \\
TI-20 & 169682.829 & 1567907.391 & 1353.45 \\
TI-21 & 170135.159 & 1568200.213 & 1352.16 \\
TI-22 & 169796.837 & 1568006.400 & 1352.68 \\
TI-23 & 170033.385 & 1568235.733 & 1351.79 \\
TI-24 & 170081.286 & 1568083.370 & 1352.64 \\
TI-25 & 170147.822 & 1568127.860 & 1352.37 \\
TI-26 & 170051.693 & 1568144.665 & 1352.67 \\
TI-27 & 169666.384 & 1568401.793 & 1350.43 \\
TI-28 & 169792.658 & 1568294.183 & 1348.85 \\
TI-29 & 169734.532 & 1568303.312 & 1349.53 \\
TI-30 & 169583.697 & 1567941.394 & 1353.77 \\
TI-31 & 169793.896 & 1567799.797 & 1353.68 \\
& & & \\
\hline
\end{tabular}

Well installed during 2006 investigation

$\begin{array}{llll}\text { MW-5 } & 169570.588 & 1567942.246 & 1353.65^{c}\end{array}$

Co-op flat storage building

NE COR BLDG $\quad 169961.942 \quad 1568249.252 \quad 1351.65$ SW COR BLDG $\quad 169831.725 \quad 1568214.414 \quad 1351.71$

Co-op liquid fertilizer tank concrete berm

$\begin{array}{llll}\text { NW COR CONC } & 169810.451 & 1568243.282 & 1354.10\end{array}$

$\begin{array}{llll}\text { SE COR CONC } \quad 169782.763 & 1568285.590 & 1354.07\end{array}$ 
TABLE C.1 (Cont.)

\begin{tabular}{llr}
\hline Location & Horizontal Location ${ }^{\mathrm{a}}(\mathrm{ft})$ & $\begin{array}{c}\text { Ground } \\
\text { Elevation }\end{array}$ \\
\cline { 2 - 3 } & Easting & $\begin{array}{c}\text { (ft AMSL) } \\
\text { L }\end{array}$ \\
\hline
\end{tabular}

Co-op dry fertilizer storage building

$\begin{array}{llll}\text { NE COR BLDG } & 169682.042 & 1568294.320 & 1352.02 \\ \text { SW COR BLDG } & 169614.770 & 1568264.631 & 1352.98\end{array}$

a Coordinates are in the State Plane, Kansas northern zone. Horizontal datum is North American Datum (NAD) 83.

b Vertical datum is North American Vertical Datum (NAVD) 29.

c Top of casing elevation, rather than ground elevation. MW5 was installed at the TI-30 location. 


\section{Appendix D:}

\section{Historical Analytical Data Summary}

for Monitoring Wells at Navarre, Kansas 
TABLE D.1 Historical analytical data summary for monitoring wells at Navarre, Kansas.

\begin{tabular}{|c|c|c|c|c|c|c|c|c|c|c|c|}
\hline \multirow[b]{2}{*}{ Well } & \multirow[b]{2}{*}{$\begin{array}{l}\text { Screen } \\
\text { Interval } \\
\text { (ft BGL) }\end{array}$} & \multirow[b]{2}{*}{$\begin{array}{c}\text { Sample } \\
\text { Date }\end{array}$} & \multirow{2}{*}{$\begin{array}{c}\text { Depth to } \\
\text { Water } \\
\text { (ft below } \\
\text { TOC)a }\end{array}$} & \multirow[b]{2}{*}{$\begin{array}{l}\text { Nitrate as } \\
N(\mathrm{mg} / \mathrm{L})\end{array}$} & \multicolumn{7}{|c|}{ Concentration $(\mu \mathrm{g} / \mathrm{L})$} \\
\hline & & & & & $\begin{array}{c}\text { Carbon } \\
\text { Tetrachloride }\end{array}$ & Chloroform & $\begin{array}{l}\text { Methylene } \\
\text { Chloride }\end{array}$ & $\begin{array}{l}\text { Tetrachloro- } \\
\text { ethylene }\end{array}$ & $\begin{array}{c}1,2- \\
\text { Dichloroethane }\end{array}$ & $\begin{array}{c}1,1,1- \\
\text { Trichloroethane } \\
\end{array}$ & $\begin{array}{l}\text { Chloro- } \\
\text { methane }\end{array}$ \\
\hline \multirow{5}{*}{ Co-op 1} & Unknown & \begin{tabular}{|l|}
$02 / 22 / 91$ \\
\end{tabular} & Unknown & 3.16 & $0.7 U^{b}$ & $0.5 \mathrm{U}$ & $0.9 \mathrm{U}$ & $1.1 \mathrm{U}$ & $0.6 \mathrm{U}$ & $0.7 \mathrm{U}$ & $5 \mathrm{U}$ \\
\hline & & \begin{tabular}{|l|}
$08 / 20 / 92$ \\
\end{tabular} & Unknown & 62 & $5 \mathrm{U}$ & $5 \mathrm{U}$ & $5 \mathrm{U}$ & $5 \mathrm{U}$ & $5 \mathrm{U}$ & $5 \mathrm{U}$ & $5 \mathrm{U}$ \\
\hline & & \begin{tabular}{|l|}
$12 / 04 / 97$ \\
\end{tabular} & Unknown & 80.1 & $N A^{C}$ & $\mathrm{NA}$ & $\mathrm{NA}$ & NA & NA & $\mathrm{NA}$ & $\mathrm{NA}$ \\
\hline & & \begin{tabular}{|l|}
$03 / 21 / 05$ \\
\end{tabular} & Unknown & $\mathrm{NR}^{\mathrm{d}}$ & $0.5 \mathrm{U}$ & $0.5 \mathrm{U}$ & NR & $\mathrm{NR}$ & NR & NR & NR \\
\hline & & \begin{tabular}{|l|}
$04 / 08 / 06$ \\
\end{tabular} & Unknown & $\mathrm{NA}$ & 1.2 & $0.8 \mathrm{~J}^{\mathrm{e}}$ & $0.1 \mathrm{U}$ & $0.1 \mathrm{U}$ & $0.1 \mathrm{U}$ & $0.1 \mathrm{U}$ & $0.1 \mathrm{U}$ \\
\hline Co-op 2 & $70(T D)^{f}$ & 02/22/91 & Unknown & 344 & 511 & 62.3 & 6.6 & 3.4 & 0.9 & $0.7 \mathrm{U}$ & $5 \mathrm{U}$ \\
\hline \multirow[t]{5}{*}{ Co-op 3} & Unknown & 02/22/91 & Unknown & 134 & 535 & 54.4 & 1.9 & $1.1 \mathrm{U}$ & $0.6 \mathrm{U}$ & $0.7 \mathrm{U}$ & $5 \mathrm{U}$ \\
\hline & & \begin{tabular}{|l|}
$08 / 19 / 92$ \\
\end{tabular} & Unknown & 95 & 440 & 40 & 5.7 & $5 \mathrm{U}$ & $5 \mathrm{U}$ & $5 \mathrm{U}$ & $5 U$ \\
\hline & & $12 / 04 / 97$ & Unknown & 137 & NA & NA & NA & NA & NA & NA & NA \\
\hline & & \begin{tabular}{|l|}
$03 / 21 / 05$ \\
\end{tabular} & Unknown & NR & 152 & 36 & NR & NR & NR & NR & NR \\
\hline & & \begin{tabular}{|l|}
$04 / 08 / 06$ \\
\end{tabular} & Unknown & NA & 197 & 44 & 3.6 & $0.2 \mathrm{~J}$ & $0.1 \mathrm{U}$ & $0.1 \mathrm{U}$ & $0.1 \mathrm{U}$ \\
\hline \multirow[t]{2}{*}{ Co-op 4} & Unknown & $02 / 22 / 91$ & Unknown & 22.4 & $0.7 \mathrm{U}$ & $0.5 \mathrm{U}$ & $0.9 \mathrm{U}$ & $1.1 \mathrm{U}$ & $0.6 \mathrm{U}$ & $0.7 \mathrm{U}$ & $5 \mathrm{U}$ \\
\hline & & $08 / 20 / 92$ & Unknown & 21 & $5 \mathrm{U}$ & $5 \mathrm{U}$ & $5 \mathrm{U}$ & $5 \mathrm{U}$ & $5 \mathrm{U}$ & $5 \mathrm{U}$ & $5 \mathrm{U}$ \\
\hline \multirow[t]{4}{*}{ KDHE-1 } & $35-55$ & \begin{tabular}{|l|}
$07 / 18 / 91$ \\
\end{tabular} & NR & 3.86 & $0.7 \mathrm{U}$ & $0.5 \mathrm{U}$ & $0.9 \mathrm{U}$ & $1.1 \mathrm{U}$ & $0.6 \mathrm{U}$ & $0.7 \mathrm{U}$ & $5 \mathrm{U}$ \\
\hline & & $08 / 21 / 92$ & 41.40 & 3.5 & $5 \mathrm{U}$ & $5 \mathrm{U}$ & $5 \mathrm{U}$ & $5 \mathrm{U}$ & $5 \mathrm{U}$ & $5 \mathrm{U}$ & $5 \mathrm{U}$ \\
\hline & & $12 / 05 / 97$ & 24.24 & 3.41 & NA & NA & NA & NA & NA & NA & NA \\
\hline & & $04 / 07 / 06$ & 27.04 & NA & $0.1 \mathrm{~J}$ & $0.1 \mathrm{U}$ & $0.1 \mathrm{U}$ & $0.1 \mathrm{U}$ & $0.1 \mathrm{U}$ & $0.1 \mathrm{U}$ & $0.1 \mathrm{U}$ \\
\hline \multirow[t]{4}{*}{ KDHE-2 } & $25-45$ & $07 / 18 / 91$ & NR & 330 & $0.7 \mathrm{U}$ & $0.5 \mathrm{U}$ & $0.9 \mathrm{U}$ & $1.1 \mathrm{U}$ & $0.6 \mathrm{U}$ & $0.7 \mathrm{U}$ & $5 \mathrm{U}$ \\
\hline & & \begin{tabular}{|l|l|}
$08 / 21 / 92$ \\
\end{tabular} & 33.01 & 315 & $5 \mathrm{U}$ & $5 \mathrm{U}$ & $5 \mathrm{U}$ & $5 \mathrm{U}$ & $5 \mathrm{U}$ & $5 \mathrm{U}$ & $5 \mathrm{U}$ \\
\hline & & $12 / 05 / 97$ & 22.52 & 175 & NA & $\mathrm{NA}$ & $\mathrm{NA}$ & $\mathrm{NA}$ & NA & $\mathrm{NA}$ & $\mathrm{NA}$ \\
\hline & & $04 / 07 / 06$ & 24.32 & NA & $0.1 \mathrm{U}$ & $0.1 \mathrm{U}$ & $0.1 \mathrm{U}$ & $0.1 \mathrm{U}$ & $0.1 \mathrm{U}$ & $0.1 \mathrm{U}$ & $0.1 \mathrm{U}$ \\
\hline \multirow[t]{3}{*}{$\mathrm{T} 1$} & $40-60$ & $04 / 06 / 93^{9}$ & 31.70 & 61 & 249 & 25 & NA & $\mathrm{NA}$ & $\mathrm{NA}$ & NA & NA \\
\hline & & \begin{tabular}{|l|}
$04 / 07 / 06$ \\
\end{tabular} & 30.81 & NA & 118 & 20 & $0.1 \mathrm{U}$ & $0.1 \mathrm{U}$ & $0.1 \mathrm{U}$ & $0.1 \mathrm{U}$ & $0.1 \mathrm{U}$ \\
\hline & & & & & & & & & & & \\
\hline
\end{tabular}


TABLE D.1 (Cont.)

\begin{tabular}{|c|c|c|c|c|c|c|c|c|c|c|c|}
\hline \multirow[b]{2}{*}{ Well } & \multirow{2}{*}{$\begin{array}{l}\text { Screen } \\
\text { Interval } \\
\text { (ft BGL) }\end{array}$} & \multirow[b]{2}{*}{$\begin{array}{l}\text { Sample } \\
\text { Date }\end{array}$} & \multirow{2}{*}{$\begin{array}{l}\text { Depth to } \\
\text { Water } \\
\text { (ft below } \\
\text { TOC) }\end{array}$} & \multirow[b]{2}{*}{$\begin{array}{c}\text { Nitrate as } \\
\mathrm{N}(\mathrm{mg} / \mathrm{L})\end{array}$} & \multicolumn{7}{|c|}{ Concentration $(\mu \mathrm{g} / \mathrm{L})$} \\
\hline & & & & & $\begin{array}{c}\text { Carbon } \\
\text { Tetrachloride }\end{array}$ & Chloroform & $\begin{array}{l}\text { Methylene } \\
\text { Chloride }\end{array}$ & $\begin{array}{c}\text { Tetrachloro- } \\
\text { ethylene }\end{array}$ & $\begin{array}{c}1,2- \\
\text { Dichloroethane }\end{array}$ & $\begin{array}{c}1,1,1- \\
\text { Trichloroethane }\end{array}$ & $\begin{array}{l}\text { Chloro- } \\
\text { methane }\end{array}$ \\
\hline \multirow[t]{5}{*}{$\mathrm{L}-1$} & $75-95$ & 03/02/949 & NR & NA & 100 & 17 & NA & NA & NA & NA & NA \\
\hline & & 03/14/949 & 26.86 & NA & 36 & 8 & NA & NA & NA & NA & NA \\
\hline & & $03 / 14 / 949$ & NR & NA & $84-94$ & 14-16 & NA & NA & NA & NA & NA \\
\hline & & $12 / 08 / 97$ & 26.70 & 31 & 27 & 5 & $2.5 \mathrm{U}$ & $0.5 \mathrm{U}$ & $0.5 \mathrm{U}$ & $0.5 \mathrm{U}$ & $0.8 \mathrm{U}$ \\
\hline & & 04/08/06 & 29.34 & NA & 54 & 11 & $0.1 \mathrm{U}$ & $0.1 \mathrm{U}$ & $0.1 \mathrm{U}$ & $0.1 \mathrm{U}$ & $0.1 \mathrm{U}$ \\
\hline \multirow[t]{3}{*}{$\mathrm{L}-2$} & $80-90$ & $03 / 14 / 949$ & 26.11 & NA & $5 U$ & $5 U$ & NA & NA & NA & NA & NA \\
\hline & & $02 / 05 / 98$ & NR & 0.6 & $1.2 \mathrm{U}$ & $0.5 \mathrm{U}$ & $2.5 U$ & $0.5 \mathrm{U}$ & $0.5 \mathrm{U}$ & $0.5 \mathrm{U}$ & $0.8 \mathrm{U}$ \\
\hline & & $04 / 06 / 06$ & 29.49 & NA & $0.2 \mathrm{~J}$ & $0.1 \mathrm{U}$ & $0.1 \mathrm{U}$ & $0.1 \mathrm{U}$ & $0.1 \mathrm{U}$ & $0.1 \mathrm{U}$ & $0.1 \mathrm{U}$ \\
\hline \multirow[t]{4}{*}{ L-3 } & $80-90$ & 03/14/949 & 28.05 & NA & $5 U$ & $5 \mathrm{U}$ & NA & $\mathrm{NA}$ & NA & NA & NA \\
\hline & & $02 / 05 / 98$ & NR & 4.42 & $1.2 \mathrm{U}$ & 1.1 & $2.5 \mathrm{U}$ & $0.5 \mathrm{U}$ & $0.5 \mathrm{U}$ & $0.5 \mathrm{U}$ & 1.9 \\
\hline & & $03 / 21 / 05$ & NR & NR & $0.5 \mathrm{U}$ & $0.5 \mathrm{U}$ & NR & $\mathrm{NR}$ & NR & NR & NR \\
\hline & & 04/07/06 & 29.75 & NA & $0.1 \mathrm{U}$ & $0.1 \mathrm{U}$ & $0.1 \mathrm{U}$ & $0.1 \mathrm{U}$ & $0.1 \mathrm{U}$ & $0.1 \mathrm{U}$ & $0.1 \mathrm{U}$ \\
\hline \multirow[t]{3}{*}{ MW1 } & $43-58$ & $04 / 06 / 93^{9}$ & 31.40 & NA & 157 & 20 & NA & NA & NA & NA & NA \\
\hline & & $12 / 08 / 97$ & 27.80 & 67.4 & 94 & 13 & $2.5 \mathrm{U}$ & 0.72 & $0.5 \mathrm{U}$ & $0.5 \mathrm{U}$ & $0.8 \mathrm{U}$ \\
\hline & & 04/05/06 & 31.11 & NA & 59 & 12 & $0.1 \mathrm{U}$ & $0.1 \mathrm{U}$ & $0.1 \mathrm{U}$ & $0.1 \mathrm{U}$ & $0.1 \mathrm{U}$ \\
\hline \multirow[t]{3}{*}{ MW2 } & $42.8-57.8$ & $04 / 06 / 93^{g}$ & 31.60 & 51 & 99 & 12 & NA & NA & NA & NA & NA \\
\hline & & $12 / 07 / 97$ & 26.40 & $N S^{h}$ & NS & NS & NS & NS & NS & NS & NS \\
\hline & & 04/06/06 & 31.42 & NA & 27 & 7.7 & $0.1 \mathrm{U}$ & $0.1 \mathrm{U}$ & $0.1 \mathrm{U}$ & $0.1 \mathrm{U}$ & $0.1 \mathrm{U}$ \\
\hline \multirow[t]{3}{*}{ MW3 } & $44-59$ & 04/06/939 & 32.00 & NA & 198 & 20 & NA & NA & NA & NA & NA \\
\hline & & $12 / 07 / 97$ & 28.75 & 100 & 100 & 24 & $2.5 \mathrm{U}$ & 0.72 & $0.5 \mathrm{U}$ & $0.5 \mathrm{U}$ & $0.8 \mathrm{U}$ \\
\hline & & 04/06/06 & 31.58 & NA & 83 & 20 & ND & $0.1 \mathrm{~J}$ & $0.1 \mathrm{U}$ & $0.1 \mathrm{U}$ & $0.1 \mathrm{U}$ \\
\hline \multirow[t]{3}{*}{ MW4 } & $45-60$ & $04 / 06 / 93^{g}$ & 32.40 & 62 & 395 & 25 & NA & NA & NA & NA & NA \\
\hline & & $12 / 08 / 97$ & NR & 52.2 & 220 & 19 & $2.5 \mathrm{U}$ & $0.5 \mathrm{U}$ & $0.5 \mathrm{U}$ & 1.5 & 1.2 \\
\hline & & $04 / 06 / 06$ & 32.21 & NA & 194 & 21 & $0.1 \mathrm{U}$ & $0.1 \mathrm{U}$ & $0.1 \mathrm{U}$ & $0.1 \mathrm{U}$ & $0.1 \mathrm{U}$ \\
\hline
\end{tabular}


TABLE D.1 (Cont.)

\begin{tabular}{|c|c|c|c|c|c|c|c|c|c|c|c|}
\hline \multirow[b]{2}{*}{ Well } & \multirow{2}{*}{$\begin{array}{l}\text { Screen } \\
\text { Interval } \\
\text { (ft BGL) }\end{array}$} & \multirow[b]{2}{*}{$\begin{array}{c}\text { Sample } \\
\text { Date }\end{array}$} & \multirow{2}{*}{$\begin{array}{l}\text { Depth to } \\
\text { Water } \\
\text { (ft below } \\
\text { TOC) }\end{array}$} & \multirow[b]{2}{*}{$\begin{array}{l}\text { Nitrate as } \\
\mathrm{N}(\mathrm{mg} / \mathrm{L})\end{array}$} & \multicolumn{7}{|c|}{ Concentration $(\mu \mathrm{g} / \mathrm{L})$} \\
\hline & & & & & $\begin{array}{c}\text { Carbon } \\
\text { Tetrachloride }\end{array}$ & Chloroform & $\begin{array}{l}\text { Methylene } \\
\text { Chloride }\end{array}$ & $\begin{array}{l}\text { Tetrachloro- } \\
\text { ethylene }\end{array}$ & $\begin{array}{c}1,2- \\
\text { Dichloroethane }\end{array}$ & $\begin{array}{c}1,1,1- \\
\text { Trichloroethane }\end{array}$ & $\begin{array}{l}\text { Chloro- } \\
\text { methane }\end{array}$ \\
\hline MW5 & $78-88$ & $05 / 25 / 06$ & 31 & NA & $0.4 \mathrm{~J}$ & $0.1 \mathrm{U}$ & $0.1 \mathrm{U}$ & $0.1 \mathrm{U}$ & $0.1 \mathrm{U}$ & $0.1 \mathrm{U}$ & $0.1 \mathrm{U}$ \\
\hline NW-1 & $40-50$ & $10 / 28 / 00$ & 31.30 & NA & $10 \mathrm{U}$ & $10 \mathrm{U}$ & $10 \mathrm{U}$ & $10 \mathrm{U}$ & $10 \mathrm{U}$ & $10 \mathrm{U}$ & $10 \mathrm{U}$ \\
\hline & & $04 / 08 / 06$ & 29.34 & NA & 1.1 & $0.3 \mathrm{~J}$ & $0.1 \mathrm{U}$ & $0.1 \mathrm{U}$ & $0.1 \mathrm{U}$ & $0.1 \mathrm{U}$ & $0.1 \mathrm{U}$ \\
\hline NW-2 & $35.5-45.5$ & $10 / 28 / 00$ & 29.85 & 97.2 & 243 & 39 & $10 U$ & $10 U$ & $10 U$ & $10 U$ & $10 \mathrm{U}$ \\
\hline & & $04 / 09 / 06$ & 28.09 & NA & 313 & 74 & 3.2 & $0.24 \mathrm{~J}$ & $0.1 \mathrm{U}$ & $0.1 \mathrm{U}$ & $0.1 \mathrm{U}$ \\
\hline NW-3 & $38-48$ & $05 / 25 / 06$ & 29.60 & NA & 34 & 2 & $0.1 \mathrm{U}$ & $0.1 \mathrm{U}$ & $0.1 \mathrm{U}$ & $0.1 \mathrm{U}$ & $0.1 \mathrm{U}$ \\
\hline NW-4 & $33.5-43.5$ & $05 / 25 / 06$ & Unk & NS & NS & NS & NS & NS & NS & NS & NS \\
\hline
\end{tabular}

a TOC, top of casing.

b Qualifier $U$ indicates that the contaminant was not detected at the indicated reporting limit.

c NA, not analyzed for this constituent

d NR, not reported in documentation on file at Argonne.

e Qualifier J indicates an estimated concentration below the method quantitation limit of $1.0 \mu \mathrm{g} / \mathrm{L}$.

f TD, total depth.

g Rapid-turnaround analysis for carbon tetrachloride and chloroform.

h NS, well could not be found and was not sampled in this event. 


\section{Supplementary Material for Investigation of Contaminant Sources at Navarre, Kansas}

Applied Geosciences and Environmental Management Section, Environmental Science Division,

Argonne National Laboratory, 9700 South Cass Avenue, Argonne, Illinois 60439

\section{Contents:}

S1_soilsamples.pdf Supplement 1:

Soil Sample Data

S2_watersamples.pdf Supplement 2:

Water Sample Data

S3_waterlevels.pdf Supplement 3:

Water Level Data

S4_qualitycontrol.pdf Supplement 4:

Quality Control for Sample Collection, Handling, and Analysis

S5_COC-analytics.pdf Supplement 5:

Chain-of-Custody Forms and Outside Laboratory Data

S6_wastewater.pdf Supplement 6:

Wastewater Characterization Data

S7_property.pdf $\quad$ Supplement 7:

Property Documentation

May 2007

Work sponsored by Commodity Credit Corporation, United States Department of Agriculture 


\section{Supplement 1:}

\section{Soil Sample Data}


TABLE S1.1 Organic results for vertical-profile soil samples collected during the 2006 investigation at Navarre, Kansas.

\begin{tabular}{|c|c|c|c|c|c|c|c|c|c|c|c|c|c|c|}
\hline \multirow[b]{2}{*}{ Location } & \multirow[b]{2}{*}{ Sample } & \multirow[b]{2}{*}{$\begin{array}{c}\text { Sample } \\
\text { Date }\end{array}$} & \multirow[b]{2}{*}{$\begin{array}{c}\text { Depth } \\
\text { (ft BGL) }\end{array}$} & \multicolumn{11}{|c|}{ Concentration $(\mu \mathrm{g} / \mathrm{kg})$} \\
\hline & & & & $\begin{array}{l}\text { Carbon } \\
\text { Tetra- } \\
\text { chloride }\end{array}$ & $\begin{array}{l}\text { Chloro- } \\
\text { form }\end{array}$ & $\begin{array}{l}\text { Methylene } \\
\text { Chloride }\end{array}$ & Benzene & $\begin{array}{c}1,1,2,2- \\
\text { Tetrachloro- } \\
\text { ethane }\end{array}$ & $\begin{array}{c}1,1,2- \\
\text { Trichloro- } \\
\text { ethane }\end{array}$ & $\begin{array}{c}1,1- \\
\text { Dichloro- } \\
\text { ethane }\end{array}$ & $\begin{array}{c}1,2- \\
\text { Dichloro- } \\
\text { propane }\end{array}$ & $\begin{array}{l}\text { Chloro- } \\
\text { benzene }\end{array}$ & $\begin{array}{l}\text { Chloro- } \\
\text { dibromo- } \\
\text { methane }\end{array}$ & Toluene \\
\hline
\end{tabular}

Former CCC/USDA grain storage facility sampling locations

$\begin{array}{lcccccc}\text { TI-1 } & \text { NATI1-S-20300 } & 4 / 7 / 06 & 2.0 & \text { ND } & \text { ND } & \text { ND } \\ \text { TI-1 } & \text { NATI1-S-20301 } & 4 / 7 / 06 & 6.0 & \text { ND } & \text { ND } & \text { ND } \\ \text { TI-1 } & \text { NATI1-S-20302 } & 4 / 7 / 06 & 7.0 & \text { ND } & \text { ND } & \text { ND } \\ \text { TI-1 } & \text { NATI1-S-20303 } & 4 / 7 / 06 & 9.0 & \text { ND } & \text { ND } & \text { ND } \\ \text { TI-1 } & \text { NATI1-S-20304 } & 4 / 7 / 06 & 18.0 & \text { ND } & 1.0 \mathrm{~J}^{\mathrm{b}} & \text { ND } \\ \text { TI-1 } & \text { NATI1-S-20464 } & 4 / 11 / 06 & 19.2 & \text { ND } & \text { ND } & \text { ND } \\ \text { TI-1 } & \text { NATI1-S-20305 } & 4 / 8 / 06 & 20.5 & \text { ND } & \text { ND } & \text { ND } \\ \text { TI-1 } & \text { NATI1-S-20465 } & 4 / 11 / 06 & 21.5 & 0.9 \mathrm{~J} & \text { ND } & \text { ND } \\ \text { TI-1 } & \text { NATI1-S-20467 } & 4 / 11 / 06 & 23.8 & 1.3 \mathrm{~J} & \text { ND } & \text { ND } \\ \text { TI-1 } & \text { NATI1-S-20306 } & 4 / 8 / 06 & 31.0 & 56 & 3.7 \mathrm{~J} & \text { ND } \\ \text { TI-1 } & \text { NATI1-S-20475 } & 4 / 13 / 06 & 32.0 & 23 & 1.6 \mathrm{~J} & \text { ND } \\ \text { TI-1 } & \text { NATI1-S-20476 } & 4 / 13 / 06 & 32.7 & 35 & 12 & \text { ND } \\ \text { TI-1 } & \text { NATI1-S-20477 } & 4 / 13 / 06 & 34.5 & 3.0 \mathrm{~J} & 11 & \text { ND } \\ \text { TI-1 } & \text { NATI1-S-20478 } & 4 / 13 / 06 & 36.0 & 48 & 10 & \text { ND } \\ \text { TI-1 } & \text { NATI1-S-20479 } & 4 / 13 / 06 & 38.5 & 37 & 3.8 \mathrm{~J} & \text { ND } \\ \text { TI-1 } & \text { NATI1-S-20480 } & 4 / 13 / 06 & 39.5 & 41 & 3.5 \mathrm{~J} & \text { ND } \\ \text { TI-1 } & \text { NATI1-S-20481 } & 4 / 13 / 06 & 42.5 & 44 & 10 & \text { ND } \\ \text { TI-1 } & \text { NATI1-S-20482 } & 4 / 13 / 06 & 44.0 & 3.3 \mathrm{~J} & 2.6 \mathrm{~J} & \text { ND } \\ \text { TI-1 } & \text { NATI1-S-20484 } & 4 / 13 / 06 & 46.5 & 15 & 4.1 \mathrm{~J} & \text { ND } \\ \text { TI-1 } & \text { NATI1-S-20485 } & 4 / 13 / 06 & 47.5 & 10 & 2.3 \mathrm{~J} & \text { ND } \\ \text { TI-1 } & \text { NATI1-S-20336 } & 4 / 13 / 06 & 51.0 & 1.4 \mathrm{~J} & \text { ND } & \text { ND } \\ \text { TI-1 } & \text { NATI1-S-20337 } & 4 / 13 / 06 & 55.0 & 1.8 \mathrm{~J} & \text { ND } & \text { ND } \\ \text { TI-1 } & \text { NATI1-S-20339 } & 4 / 13 / 06 & 58.5 & 3.2 \mathrm{~J} & 2.2 \mathrm{~J} & \text { ND } \\ \text { TI-1 } & \text { NATI1-S-20340 } & 4 / 13 / 06 & 59.5 & \text { ND } & \text { ND } & \text { ND } \\ & & & & & & \\ \text { TI-2 } & \text { NATI2-S-20406 } & 4 / 5 / 06 & 2.0 & \text { ND } & \text { ND } & \text { ND } \\ \text { TI-2 } & \text { NATI2-S-20407 } & 4 / 5 / 06 & 6.0 & \text { ND } & \text { ND } & \text { ND } \\ \text { TI-2 } & \text { NATI2-S-20408 } & 4 / 5 / 06 & 9.0 & \text { ND } & \text { ND } & \text { ND } \\ \text { TI-2 } & \text { NATI2-S-20409 } & 4 / 5 / 06 & 10.0 & \text { ND } & \text { ND } & \text { ND }\end{array}$


TABLE S1.1 (Cont.)

Concentration $(\mu \mathrm{g} / \mathrm{kg})$

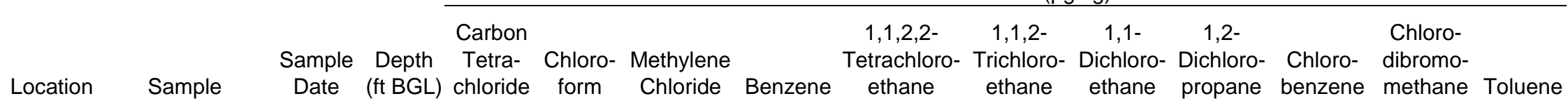

Former CCC/USDA grain storage facility sampling locations (cont.)

$\begin{array}{lcccccc}\text { TI-2 } & \text { NATI2-S-20410 } & 4 / 5 / 06 & 14.0 & \text { ND } & \text { ND } & \text { ND } \\ \text { TI-2 } & \text { NATI2-S-20411 } & 4 / 5 / 06 & 16.0 & \text { ND } & \text { ND } & \text { ND } \\ \text { TI-2 } & \text { NATI2-S-20413 } & 4 / 5 / 06 & 20.5 & \text { ND } & \text { ND } & \text { ND } \\ \text { TI-2 } & \text { NATI2-S-20414 } & 4 / 6 / 06 & 24.5 & \text { ND } & \text { ND } & \text { ND } \\ \text { TI-2 } & \text { NATI2-S-20415 } & 4 / 6 / 06 & 28.7 & 7.0 \mathrm{~J} & 1.5 \mathrm{~J} & \text { ND } \\ \text { TI-2 } & \text { NATI2-S-20416 } & 4 / 6 / 06 & 30.0 & 12 & 6.8 \mathrm{~J} & \text { ND } \\ \text { TI-2 } & \text { NATI2-S-20417 } & 4 / 6 / 06 & 32.0 & \text { ND } & 2.2 \mathrm{~J} & \text { ND } \\ \text { TI-2 } & \text { NATI2-S-20418 } & 4 / 6 / 06 & 34.0 & 13 & 6.1 \mathrm{~J} & \text { ND } \\ \text { TI-2 } & \text { NATI2-S-20419 } & 4 / 6 / 06 & 38.0 & 65 & 3.9 \mathrm{~J} & \text { ND } \\ \text { TI-2 } & \text { NATI2-S-20420 } & 4 / 6 / 06 & 40.0 & 15 & 4.4 \mathrm{~J} & \text { ND } \\ \text { TI-2 } & \text { NATI2-S-20421 } & 4 / 6 / 06 & 42.0 & 2.7 \mathrm{~J} & \text { ND } & \text { ND } \\ \text { TI-2 } & \text { NATI2-S-20422 } & 4 / 6 / 06 & 46.0 & 0.9 \mathrm{~J} & 1.9 \mathrm{~J} & \text { ND } \\ \text { TI-2 } & \text { NATI2-S-20423 } & 4 / 6 / 06 & 50.0 & 7.3 \mathrm{~J} & 1.3 \mathrm{~J} & \text { ND } \\ \text { TI-2 } & \text { NATI2-S-20424 } & 4 / 6 / 06 & 52.0 & 4.9 \mathrm{~J} & 3 \mathrm{~J} & \text { ND } \\ \text { TI-2 } & \text { NATI2-S-20425 } & 4 / 6 / 06 & 54.0 & 30 & 5.1 \mathrm{~J} & \text { ND } \\ \text { TI-2 } & \text { NATI2-S-20426 } & 4 / 6 / 06 & 58.0 & \text { ND } & \text { ND } & \text { ND } \\ \text { TI-2 } & \text { NATI2-S-20427 } & 4 / 6 / 06 & 61.0 & \text { ND } & \text { ND } & \text { ND } \\ \text { TI-2 } & \text { NATI2-S-20428 } & 4 / 7 / 06 & 68.5 & \text { ND } & \text { ND } & \text { ND } \\ \text { TI-2 } & \text { NATI2-S-20429 } & 4 / 7 / 06 & 70.7 & \text { ND } & \text { ND } & \text { ND } \\ & & & & & & \\ \text { TI-3 } & \text { NATI3-S-20286 } & 4 / 4 / 06 & 6.0 & \text { ND } & \text { ND } & \text { ND } \\ \text { TI-3 } & \text { NATI3-S-20287 } & 4 / 4 / 06 & 10.0 & \text { ND } & \text { ND } & \text { ND } \\ \text { TI-3 } & \text { NATI3-S-20295 } & 4 / 6 / 06 & 22.0 & \text { ND } & 1.0 \mathrm{~J} & \text { ND } \\ \text { TI-3 } & \text { NATI3-S-20296 } & 4 / 6 / 06 & 24.5 & \text { ND } & \text { ND } & \text { ND } \\ \text { TI-3 } & \text { NATI3-S-20297 } & 4 / 7 / 06 & 25.5 & \text { ND } & \text { ND } & \text { ND } \\ \text { TI-3 } & \text { NATI3-S-20289 } & 4 / 5 / 06 & 32.0 & \text { ND } & \text { ND } & \text { ND } \\ \text { TI-3 } & \text { NATI3-S-20290 } & 4 / 6 / 06 & 36.0 & 2.7 \mathrm{~J} & 1.6 \mathrm{~J} & \text { ND } \\ \text { TI-3 } & \text { NATI3-S-20291 } & 4 / 6 / 06 & 37.0 & \text { ND } & 1.1 \mathrm{~J} & \text { ND } \\ \text { TI-3 } & \text { NATI3-S-20292 } & 4 / 6 / 06 & 40.0 & 1.4 \mathrm{~J} & 1.3 \mathrm{~J} & \text { ND } \\ \text { TI-3 } & \text { NATI3-S-20293 } & 4 / 6 / 06 & 44.0 & 1.6 \mathrm{~J} & \text { ND } & \text { ND } \\ \text { TI-3 } & \text { NATI3-S-20294 } & 4 / 6 / 06 & 48.0 & 2.6 \mathrm{~J} & 1.2 \mathrm{~J} & \text { ND }\end{array}$


TABLE S1.1 (Cont.)

Concentration $(\mu \mathrm{g} / \mathrm{kg})$

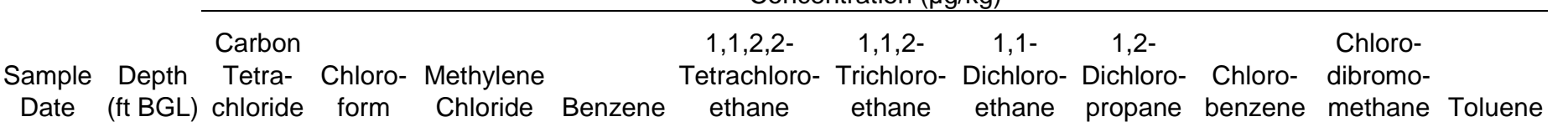

Former CCC/USDA grain storage facility sampling locations (cont.)

$\begin{array}{lcccccc}\text { TI-3 } & \text { NATI3-S-20375 } & 4 / 13 / 06 & 49.2 & \text { ND } & 1.9 \mathrm{~J} & \text { ND } \\ \text { TI-3 } & \text { NATI3-S-20376 } & 4 / 13 / 06 & 52.8 & 21 & \text { ND } & \text { ND } \\ \text { TI-3 } & \text { NATI3-S-20379 } & 4 / 13 / 06 & 56.5 & 1.3 \mathrm{~J} & \text { ND } & \text { ND } \\ \text { TI-3 } & \text { NATI3-S-20381 } & 4 / 13 / 06 & 60.0 & \text { ND } & \text { ND } & \text { ND } \\ \text { TI-3 } & \text { NATI3-S-20382 } & 4 / 13 / 06 & 62.0 & \text { ND } & \text { ND } & \text { ND } \\ & & & & & & \\ \text { TI-4 } & \text { NATI4-S-20308 } & 4 / 8 / 06 & 2.0 & \text { ND } & \text { ND } & \text { ND } \\ \text { TI-4 } & \text { NATI4-S-20309 } & 4 / 8 / 06 & 6.8 & \text { ND } & \text { ND } & \text { ND } \\ \text { TI-4 } & \text { NATI4-S-20310 } & 4 / 8 / 06 & 9.0 & \text { ND } & \text { ND } & \text { ND } \\ \text { TI-4 } & \text { NATI4-S-20311 } & 4 / 8 / 06 & 14.5 & \text { ND } & \text { ND } & \text { ND } \\ \text { TI-4 } & \text { NATI4-S-20312 } & 4 / 8 / 06 & 17.7 & \text { ND } & \text { ND } & \text { ND } \\ \text { TI-4 } & \text { NATI4-S-20313 } & 4 / 8 / 06 & 20.5 & 10 & \text { ND } & \text { ND } \\ \text { TI-4 } & \text { NATI4-S-20314 } & 4 / 9 / 06 & 25.0 & 29 & 1.3 \mathrm{~J} & \text { ND } \\ \text { TI-4 } & \text { NATI4-S-20316 } & 4 / 9 / 06 & 29.2 & 6.0 \mathrm{~J} & 1.5 \mathrm{~J} & \text { ND } \\ \text { TI-4 } & \text { NATI4-S-20315 } & 4 / 9 / 06 & 31.0 & 4.0 \mathrm{~J} & \text { ND } & \text { ND } \\ \text { TI-4 } & \text { NATI4-S-20317 } & 4 / 9 / 06 & 34.0 & 1.0 \mathrm{~J} & \text { ND } & \text { ND } \\ \text { TI-4 } & \text { NATI4-S-20319 } & 4 / 9 / 06 & 37.0 & 32 & 8.3 \mathrm{~J} & \text { ND } \\ \text { TI-4 } & \text { NATI4-S-20320 } & 4 / 9 / 06 & 45.5 & 3.1 \mathrm{~J} & \text { ND } & \text { ND } \\ \text { TI-4 } & \text { NATI4-S-20321 } & 4 / 9 / 06 & 50.0 & 20 & 1.8 \mathrm{~J} & \text { ND } \\ \text { TI-4 } & \text { NATI4-S-20322 } & 4 / 9 / 06 & 54.0 & \text { ND } & \text { ND } & \text { ND } \\ \text { TI-4 } & \text { NATI4-S-20323 } & 4 / 9 / 06 & 58.0 & 7.3 \mathrm{~J} & 0.9 \mathrm{~J} & \text { ND } \\ \text { TI-4 } & \text { NATI4-S-20324 } & 4 / 9 / 06 & 61.0 & \text { ND } & \text { ND } & \text { ND } \\ & & & & & & \\ \text { TI-5 } & \text { NATI5-S-20326 } & 4 / 10 / 06 & 2.0 & \text { ND } & \text { ND } & \text { ND } \\ \text { TI-5 } & \text { NATI5-S-20327 } & 4 / 10 / 06 & 5.0 & \text { ND } & \text { ND } & \text { ND } \\ \text { TI-5 } & \text { NATI5-S-20332 } & 4 / 11 / 06 & 9.7 & \text { ND } & \text { ND } & \text { ND } \\ \text { TI-5 } & \text { NATI5-S-20333 } & 4 / 11 / 06 & 13.0 & \text { ND } & \text { ND } & \text { ND } \\ \text { TI-5 } & \text { NATI5-S-20334 } & 4 / 11 / 06 & 17.2 & \text { ND } & \text { ND } & \text { ND } \\ \text { TI-5 } & \text { NATI5-S-20335 } & 4 / 11 / 06 & 22.0 & \text { ND } & \text { ND } & \text { ND } \\ \text { TI-5 } & \text { NATI5-S-20356 } & 4 / 11 / 06 & 25.0 & \text { ND } & \text { ND } & \text { ND } \\ \text { TI-5 } & \text { NATI5-S-20358 } & 4 / 11 / 06 & 30.0 & \text { ND } & \text { ND } & \text { ND }\end{array}$


TABLE S1.1 (Cont.)

Concentration $(\mu \mathrm{g} / \mathrm{kg})$

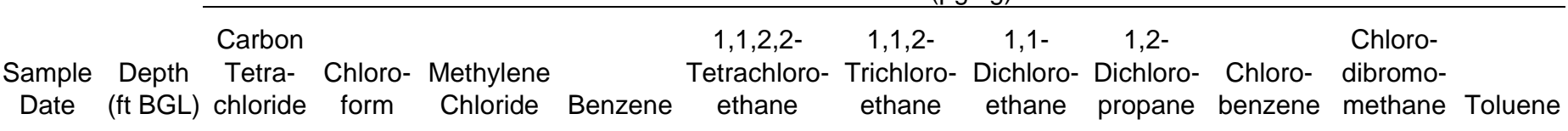

Location Sample Date (ft BGL) chloride form Chloride Benzene ethane ethane ethane propane benzene methane Toluene

Former CCC/USDA grain storage facility sampling locations (cont.)

$\begin{array}{lrrrrrl}\text { TI-5 } & \text { NATI5-S-20359 } & 4 / 11 / 06 & 34.0 & \text { ND } & \text { ND } & \text { ND } \\ \text { TI-5 } & \text { NATI5-S-20360 } & 4 / 11 / 06 & 38.0 & \text { ND } & \text { ND } & \text { ND } \\ \text { TI-5 } & \text { NATI5-S-20361 } & 4 / 11 / 06 & 42.0 & \text { ND } & \text { ND } & \text { ND } \\ \text { TI-5 } & \text { NATI5-S-20363 } & 4 / 11 / 06 & 46.0 & \text { ND } & \text { ND } & \text { ND } \\ \text { TI-5 } & \text { NATI5-S-20364 } & 4 / 11 / 06 & 49.5 & \text { ND } & \text { ND } & \text { ND } \\ \text { TI-5 } & \text { NATI5-S-20366 } & 4 / 11 / 06 & 52.0 & \text { ND } & \text { ND } & \text { ND } \\ \text { TI-5 } & \text { NATI5-S-20365 } & 4 / 11 / 06 & 53.0 & 15 & \text { ND } & \text { ND } \\ \text { TI-5 } & \text { NATI5-S-20367 } & 4 / 12 / 06 & 61.0 & \text { ND } & \text { ND } & \text { ND } \\ & & & & & & \\ \text { TI-6 } & \text { NATI6-S-20390 } & 4 / 20 / 06 & 2.0 & \text { ND } & \text { ND } & \text { ND } \\ \text { TI-6 } & \text { NATI6-S-20391 } & 4 / 20 / 06 & 6.5 & \text { ND } & \text { ND } & \text { ND } \\ \text { TI-6 } & \text { NATI6-S-20394 } & 4 / 20 / 06 & 10.0 & \text { ND } & \text { ND } & \text { ND } \\ \text { TI-6 } & \text { NATI6-S-20392 } & 4 / 20 / 06 & 12.5 & \text { ND } & \text { ND } & \text { ND } \\ \text { TI-6 } & \text { NATI6-S-20393 } & 4 / 20 / 06 & 17.8 & \text { ND } & \text { ND } & \text { ND } \\ \text { TI-6 } & \text { NATI6-S-20395 } & 4 / 20 / 06 & 21.2 & \text { ND } & \text { ND } & \text { ND } \\ \text { TI-6 } & \text { NATI6-S-20396 } & 4 / 20 / 06 & 25.0 & \text { ND } & \text { ND } & \text { ND } \\ \text { TI-6 } & \text { NATI6-S-20397 } & 4 / 20 / 06 & 28.8 & \text { ND } & \text { ND } & \text { ND } \\ \text { TI-6 } & \text { NATI6-S-20398 } & 4 / 20 / 06 & 32.8 & \text { ND } & \text { ND } & \text { ND } \\ \text { TI-6 } & \text { NATI6-S-20399 } & 4 / 20 / 06 & 37.0 & \text { ND } & \text { ND } & \text { ND } \\ \text { TI-6 } & \text { NATI6-S-20400 } & 4 / 20 / 06 & 42.0 & \text { ND } & \text { ND } & \text { ND } \\ \text { TI-6 } & \text { NATI6-S-20401 } & 4 / 20 / 06 & 46.0 & \text { ND } & \text { ND } & \text { ND } \\ \text { TI-6 } & \text { NATI6-S-20402 } & 4 / 20 / 06 & 50.0 & 1.3 ~ J & \text { ND } & \text { ND } \\ \text { TI-6 } & \text { NATI6-S-20404 } & 4 / 21 / 06 & 53.4 & \text { ND } & \text { ND } & \text { ND } \\ \text { TI-6 } & \text { NATI6-S-20405 } & 4 / 21 / 06 & 61.0 & \text { ND } & \text { ND } & \text { ND } \\ & & & & & & \\ \text { TI-7 } & \text { NATI7-S-20436 } & 4 / 9 / 06 & 2.0 & \text { ND } & \text { ND } & \text { ND } \\ \text { TI-7 } & \text { NATI7-S-20437 } & 4 / 9 / 06 & 4.0 & \text { ND } & \text { ND } & \text { ND } \\ \text { TI-7 } & \text { NATI7-S-20438 } & 4 / 9 / 06 & 5.5 & \text { ND } & \text { ND } & \text { ND } \\ \text { TI-7 } & \text { NATI7-S-20439 } & 4 / 9 / 06 & 6.0 & \text { ND } & \text { ND } & \text { ND } \\ \text { TI-7 } & \text { NATI7-S-20440 } & 4 / 9 / 06 & 8.5 & \text { ND } & \text { ND } & \text { ND } \\ \text { TI-7 } & \text { NATI7-S-20441 } & 4 / 9 / 06 & 10.5 & \text { ND } & \text { ND } & \text { ND }\end{array}$


TABLE S1.1 (Cont.)

Concentration $(\mu \mathrm{g} / \mathrm{kg})$

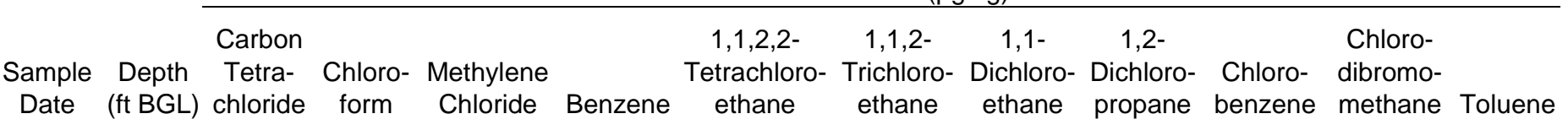

Location Sample

rage facility sampling locations (cont.)

Former CCC/USDA grain storage facility sampling locations (cont.)

$\begin{array}{lllllll} & & & \\ \text { TI-7 } & \text { NATI7-S-20442 } & 4 / 9 / 06 & 14.0 & \text { ND } & \text { ND } & \text { ND } \\ \text { TI-7 } & \text { NATI7-S-20444 } & 4 / 9 / 06 & 16.0 & \text { ND } & \text { ND } & \text { ND } \\ \text { TI-7 } & \text { NATI7-S-20443 } & 4 / 9 / 06 & 16.2 & \text { ND } & \text { ND } & \text { ND } \\ \text { TI-7 } & \text { NATI7-S-20445 } & 4 / 9 / 06 & 18.2 & \text { ND } & \text { ND } & \text { ND } \\ \text { TI-7 } & \text { NATI7-S-20446 } & 4 / 9 / 06 & 20.2 & \text { ND } & \text { ND } & \text { ND } \\ \text { TI-7 } & \text { NATI7-S-20447 } & 4 / 9 / 06 & 22.2 & \text { ND } & \text { ND } & \text { ND } \\ \text { TI-7 } & \text { NATI7-S-20448 } & 4 / 9 / 06 & 25.0 & \text { ND } & \text { ND } & \text { ND } \\ \text { TI-7 } & \text { NATI7-S-20449 } & 4 / 9 / 06 & 29.0 & \text { ND } & \text { ND } & \text { ND } \\ \text { TI-7 } & \text { NATI7-S-20450 } & 4 / 9 / 06 & 30.0 & 1.5 \text { J } & \text { ND } & \text { ND } \\ \text { TI-7 } & \text { NATI7-S-20451 } & 4 / 9 / 06 & 36.5 & \text { ND } & \text { ND } & \text { ND } \\ \text { TI-7 } & \text { NATI7-S-20452 } & 4 / 9 / 06 & 38.0 & \text { ND } & \text { ND } & \text { ND } \\ \text { TI-7 } & \text { NATI7-S-20453 } & 4 / 9 / 06 & 41.0 & \text { ND } & \text { ND } & \text { ND } \\ \text { TI-7 } & \text { NATI7-S-20454 } & 4 / 9 / 06 & 43.0 & \text { ND } & \text { ND } & \text { ND } \\ \text { TI-7 } & \text { NATI7-S-20455 } & 4 / 9 / 06 & 46.0 & 7.0 ~ J ~ & \text { ND } & \text { ND } \\ \text { TI-7 } & \text { NATI7-S-20456 } & 4 / 10 / 06 & 52.5 & \text { ND } & \text { ND } & \text { ND } \\ \text { TI-7 } & \text { NATI7-S-20457 } & 4 / 10 / 06 & 57.0 & \text { ND } & \text { ND } & \text { ND } \\ \text { TI-7 } & \text { NATI7-S-20458 } & 4 / 10 / 06 & 62.0 & \text { ND } & \text { ND } & \text { ND } \\ \text { TI-7 } & \text { NATI7-S-20459 } & 4 / 10 / 06 & 69.0 & \text { ND } & \text { ND } & \text { ND } \\ & & & & & & \\ \text { TI-8 } & \text { NATI8-S-20507 } & 4 / 26 / 06 & 2.0 & \text { ND } & \text { ND } & \text { ND } \\ \text { TI-8 } & \text { NATI8-S-20508 } & 4 / 26 / 06 & 5.5 & \text { ND } & \text { ND } & \text { ND } \\ \text { TI-8 } & \text { NATI8-S-20509 } & 4 / 26 / 06 & 10.0 & \text { ND } & \text { ND } & \text { ND } \\ \text { TI-8 } & \text { NATI8-S-20510 } & 4 / 26 / 06 & 13.0 & \text { ND } & \text { ND } & \text { ND } \\ \text { TI-8 } & \text { NATI8-S-20511 } & 4 / 26 / 06 & 16.5 & \text { ND } & \text { ND } & \text { ND } \\ \text { TI-8 } & \text { NATI8-S-20512 } & 4 / 26 / 06 & 17.8 & \text { ND } & \text { ND } & \text { ND } \\ \text { TI-8 } & \text { NATI8-S-20513 } & 4 / 26 / 06 & 19.5 & \text { ND } & \text { ND } & \text { ND } \\ \text { TI-8 } & \text { NATI8-S-20514 } & 4 / 26 / 06 & 23.4 & \text { ND } & \text { ND } & \text { ND } \\ \text { TI-8 } & \text { NATI8-S-20515 } & 4 / 26 / 06 & 26.8 & \text { ND } & \text { ND } & \text { ND } \\ \text { TI-8 } & \text { NATI8-S-20516 } & 4 / 26 / 06 & 31.0 & \text { ND } & \text { ND } & \text { ND } \\ \text { TI-8 } & \text { NATI8-S-20520 } & 4 / 26 / 06 & 36.0 & \text { ND } & \text { ND } & \text { ND } \\ \text { TI-8 } & \text { NATI8-S-20521 } & 4 / 26 / 06 & 40.0 & \text { ND } & \text { ND } & \text { ND }\end{array}$


TABLE S1.1 (Cont.)

Concentration $(\mu \mathrm{g} / \mathrm{kg})$

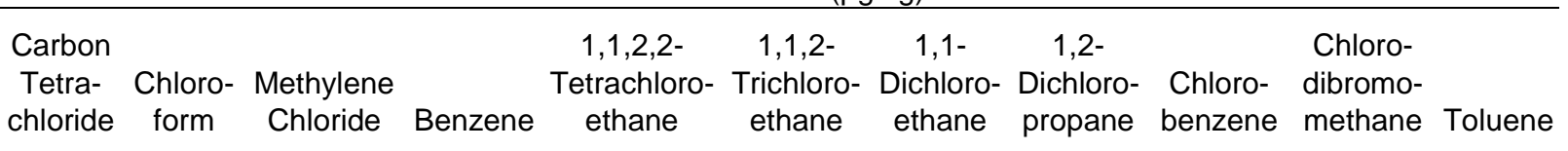

Date (ft BGL) chloride form Chloride Benzene ethane ethane ethane propane benzene methane Toluene

Location Sample $\begin{gathered}\text { Date (ft BGL) chloride form Cher CCC/USDA grain storage facility sampling locations (cont.) } \\ \text { Former }\end{gathered}$

$\begin{array}{lllllll}\text { TI-8 } & \text { NATI8-S-20522 } & 4 / 27 / 06 & 42.6 & \text { ND } & \text { ND } & \text { ND } \\ \text { TI-8 } & \text { NATI8-S-20524 } & 4 / 27 / 06 & 47.0 & \text { ND } & \text { ND } & \text { ND } \\ \text { TI-8 } & \text { NATI8-S-20525 } & 4 / 27 / 06 & 51.0 & \text { ND } & \text { ND } & \text { ND } \\ \text { TI-8 } & \text { NATI8-S-20526 } & 4 / 27 / 06 & 54.6 & \text { ND } & \text { ND } & \text { ND } \\ \text { TI-8 } & \text { NATI8-S-20528 } & 4 / 27 / 06 & 59.7 & \text { ND } & \text { ND } & \text { ND } \\ & & & & & & \\ \text { TI-9 } & \text { NATI9-S-20283 } & 4 / 22 / 06 & 4.0 & \text { ND } & \text { ND } & \text { ND } \\ \text { TI-9 } & \text { NATI9-S-20284 } & 4 / 22 / 06 & 7.0 & \text { ND } & \text { ND } & \text { ND } \\ \text { TI-9 } & \text { NATI9-S-20285 } & 4 / 22 / 06 & 9.5 & \text { ND } & \text { ND } & \text { ND } \\ \text { TI-9 } & \text { NATI9-S-20590 } & 4 / 22 / 06 & 11.0 & \text { ND } & \text { ND } & \text { ND } \\ \text { TI-9 } & \text { NATI9-S-20591 } & 4 / 22 / 06 & 13.0 & \text { ND } & \text { ND } & \text { ND } \\ \text { TI-9 } & \text { NATI9-S-20592 } & 4 / 22 / 06 & 16.0 & \text { ND } & \text { ND } & \text { ND } \\ \text { TI-9 } & \text { NATI9-S-20593 } & 4 / 22 / 06 & 18.0 & \text { ND } & \text { ND } & \text { ND } \\ \text { TI-9 } & \text { NATI9-S-20594 } & 4 / 22 / 06 & 20.5 & \text { ND } & \text { ND } & \text { ND } \\ \text { TI-9 } & \text { NATI9-S-20595 } & 4 / 22 / 06 & 25.0 & \text { ND } & \text { ND } & \text { ND } \\ \text { TI-9 } & \text { NATI9-S-20597 } & 4 / 22 / 06 & 29.5 & \text { ND } & \text { ND } & \text { ND } \\ \text { TI-9 } & \text { NATI9-S-20598 } & 4 / 22 / 06 & 33.0 & \text { ND } & \text { ND } & \text { ND } \\ \text { TI-9 } & \text { NATI9-S-20599 } & 4 / 22 / 06 & 35.0 & \text { ND } & \text { ND } & \text { ND } \\ \text { TI-9 } & \text { NATI9-S-20600 } & 4 / 22 / 06 & 38.0 & \text { ND } & \text { ND } & \text { ND } \\ \text { TI-9 } & \text { NATI9-S-20601 } & 4 / 22 / 06 & 40.0 & \text { ND } & \text { ND } & \text { ND } \\ \text { TI-9 } & \text { NATI9-S-20602 } & 4 / 23 / 06 & 45.0 & \text { ND } & \text { ND } & \text { ND } \\ \text { TI-9 } & \text { NATI9-S-20603 } & 4 / 23 / 06 & 50.0 & 2.7 ~ J & \text { ND } & \text { ND } \\ \text { TI-9 } & \text { NATI9-S-20604 } & 4 / 23 / 06 & 53.0 & \text { ND } & \text { ND } & \text { ND } \\ \text { TI-9 } & \text { NATI9-S-20605 } & 4 / 23 / 06 & 57.0 & \text { ND } & \text { ND } & \text { ND } \\ \text { TI-9 } & \text { NATI9-S-20606 } & 4 / 23 / 06 & 61.0 & \text { ND } & \text { ND } & \text { ND } \\ \text { TI-9 } & \text { NATI9-S-20607 } & 4 / 23 / 06 & 65.0 & \text { ND } & \text { ND } & \text { ND } \\ \text { TI-9 } & \text { NATI9-S-20608 } & 4 / 23 / 06 & 69.0 & \text { ND } & \text { ND } & \text { ND } \\ & & & & & & \\ \text { TI-10 } & \text { NATI10-S-20611 } & 4 / 25 / 06 & 4.5 & \text { ND } & \text { ND } & \text { ND } \\ \text { TI-10 } & \text { NATI10-S-20612 } & 4 / 25 / 06 & 6.5 & \text { ND } & \text { ND } & \text { ND } \\ \text { TI-10 } & \text { NATI10-S-20613 } & 4 / 25 / 06 & 9.5 & \text { ND } & \text { ND } & \text { ND }\end{array}$


TABLE S1.1 (Cont.)

Concentration $(\mu \mathrm{g} / \mathrm{kg})$

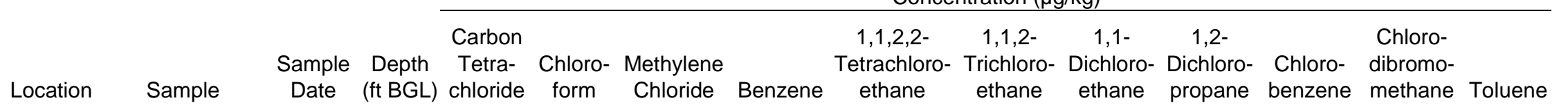

Former CCC/USDA grain storage facility sampling locations (cont.)

\begin{tabular}{|c|c|c|c|c|c|}
\hline TI-10 & NATI10-S-20614 & $4 / 25 / 06$ & 10.8 & ND & ND \\
\hline TI-10 & NATI10-S-20615 & $4 / 25 / 06$ & 13.5 & ND & ND \\
\hline TI-10 & NATI10-S-20616 & $4 / 25 / 06$ & 17.0 & ND & $2.0 \mathrm{~J}$ \\
\hline TI-10 & NATI10-S-20617 & $4 / 25 / 06$ & 19.5 & ND & $8.0 \mathrm{~J}$ \\
\hline TI-10 & NATI10-S-20618 & $4 / 25 / 06$ & 20.8 & ND & 30 \\
\hline TI-10 & NATI10-S-20619 & $4 / 26 / 06$ & 25.5 & ND & $1.2 \mathrm{~J}$ \\
\hline TI-10 & NATI10-S-20620 & $4 / 26 / 06$ & 29.5 & ND & ND \\
\hline TI-10 & NATI10-S-20621 & $4 / 26 / 06$ & 32.5 & ND & ND \\
\hline TI-10 & NATI10-S-20622 & $4 / 26 / 06$ & 34.5 & ND & ND \\
\hline TI-10 & NATI10-S-20623 & 4/26/06 & 38.0 & ND & ND \\
\hline TI-10 & NATI10-S-20624 & $4 / 26 / 06$ & 43.0 & ND & ND \\
\hline TI-10 & NATI10-S-20626 & 4/26/06 & 46.0 & ND & ND \\
\hline TI-10 & NATI10-S-20627 & $4 / 26 / 06$ & 50.0 & ND & ND \\
\hline TI-10 & NATI10-S-20636 & $4 / 26 / 06$ & 54.0 & ND & ND \\
\hline TI-10 & NATI10-S-20628 & $4 / 26 / 06$ & 57.0 & ND & ND \\
\hline TI-10 & NATI10-S-20629 & $4 / 26 / 06$ & 61.0 & ND & ND \\
\hline TI-10 & NATI10-S-20630 & $4 / 26 / 06$ & 65.5 & ND & ND \\
\hline TI-11 & NATI11-S-20354 & $4 / 22 / 06$ & 2.0 & ND & ND \\
\hline TI-11 & NATI11-S-20355 & $4 / 22 / 06$ & 5.0 & ND & ND \\
\hline TI-11 & NATI11-S-20488 & $4 / 22 / 06$ & 9.0 & ND & ND \\
\hline TI-11 & NATI11-S-20489 & $4 / 22 / 06$ & 13.0 & ND & ND \\
\hline TI-11 & NATI11-S-20491 & $4 / 22 / 06$ & 17.0 & ND & ND \\
\hline TI-11 & NATI11-S-20490 & $4 / 22 / 06$ & 21.0 & ND & ND \\
\hline TI-11 & NATI11-S-20492 & $4 / 23 / 06$ & 26.0 & ND & ND \\
\hline TI-11 & NATI11-S-20493 & $4 / 23 / 06$ & 29.0 & ND & ND \\
\hline TI-11 & NATI11-S-20494 & $4 / 23 / 06$ & 32.5 & ND & ND \\
\hline TI-11 & NATI11-S-20495 & $4 / 23 / 06$ & 38.0 & ND & ND \\
\hline TI-11 & NATI11-S-20496 & $4 / 23 / 06$ & 42.0 & ND & ND \\
\hline TI-11 & NATI11-S-20497 & $4 / 23 / 06$ & 45.3 & ND & ND \\
\hline TI-11 & NATI11-S-20498 & $4 / 23 / 06$ & 50.0 & ND & ND \\
\hline
\end{tabular}

\begin{tabular}{|c|c|c|c|c|c|c|c|}
\hline 464 & ND & 43 & ND & ND & ND & ND & 21 \\
\hline 243 & 338 & 357 & ND & 28 & 20 & 16 & 34 \\
\hline 1048 & 561 & 798 & 19 & 35 & 31 & 20 & 692 \\
\hline
\end{tabular}

ND 
TABLE S1.1 (Cont.)

Concentration $(\mu \mathrm{g} / \mathrm{kg})$

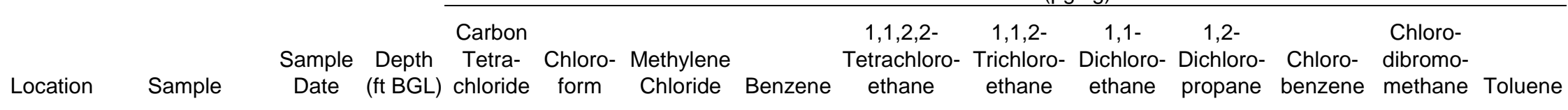

Former CCC/USDA grain storage facility sampling locations (cont.)

\begin{tabular}{|c|c|c|c|c|c|c|}
\hline TI-11 & NATI11-S-20499 & $4 / 24 / 06$ & 51.8 & ND & ND & ND \\
\hline TI-11 & NATI11-S-20502 & $4 / 24 / 06$ & 52.9 & ND & ND & ND \\
\hline TI-11 & NATI11-S-20503 & $4 / 25 / 06$ & 56.5 & ND & ND & ND \\
\hline TI-11 & NATI11-S-20505 & $4 / 25 / 06$ & 60.5 & ND & ND & ND \\
\hline TI-12 & NATI12-S-20734 & $5 / 3 / 06$ & 1.0 & ND & ND & ND \\
\hline TI-12 & NATI12-S-20735 & $5 / 3 / 06$ & 5.5 & ND & ND & ND \\
\hline TI-12 & NATI12-S-20736 & $5 / 3 / 06$ & 9.5 & ND & ND & ND \\
\hline TI-12 & NATI12-S-20737 & $5 / 3 / 06$ & 13.5 & ND & ND & ND \\
\hline TI-12 & NATI12-S-20738 & $5 / 3 / 06$ & 16.5 & ND & ND & ND \\
\hline TI-12 & NATI12-S-20739 & $5 / 3 / 06$ & 21.0 & ND & ND & ND \\
\hline TI-12 & NATI12-S-20741 & $5 / 3 / 06$ & 24.2 & ND & ND & ND \\
\hline TI-12 & NATI12-S-20742 & $5 / 3 / 06$ & 29.0 & ND & ND & ND \\
\hline TI-12 & NATI12-S-20743 & $5 / 3 / 06$ & 30.5 & ND & ND & ND \\
\hline TI-12 & NATI12-S-20744 & $5 / 3 / 06$ & 33.2 & ND & ND & ND \\
\hline TI-12 & NATI12-S-20745 & $5 / 3 / 06$ & 34.5 & ND & ND & ND \\
\hline $\mathrm{Tl}-12$ & NATI12-S-20746 & $5 / 3 / 06$ & 37.0 & ND & ND & ND \\
\hline TI-12 & NATI12-S-20747 & $5 / 3 / 06$ & 40.5 & ND & ND & ND \\
\hline $\mathrm{TI}-12$ & NATI12-S-20748 & $5 / 3 / 06$ & 42.2 & ND & ND & ND \\
\hline TI-12 & NATI12-S-20749 & $5 / 3 / 06$ & 45.0 & ND & ND & ND \\
\hline $\mathrm{TI}-12$ & NATI12-S-20750 & $5 / 3 / 06$ & 48.5 & ND & ND & ND \\
\hline $\mathrm{TI}-12$ & NATI12-S-20751 & $5 / 3 / 06$ & 50.2 & ND & ND & ND \\
\hline TI-12 & NATI12-S-20752 & $5 / 3 / 06$ & 51.0 & ND & ND & ND \\
\hline TI-12 & NATI12-S-20753 & $5 / 3 / 06$ & 53.5 & ND & ND & ND \\
\hline TI-12 & NATI12-S-20754 & $5 / 3 / 06$ & 56.5 & ND & $1.0 \mathrm{~J}$ & ND \\
\hline TI-12 & NATI12-S-20755 & $5 / 3 / 06$ & 60.0 & ND & ND & ND \\
\hline TI-12 & NATI12-S-20756 & $5 / 3 / 06$ & 61.5 & ND & ND & ND \\
\hline
\end{tabular}


TABLE S1.1 (Cont.)

Concentration $(\mu \mathrm{g} / \mathrm{kg})$

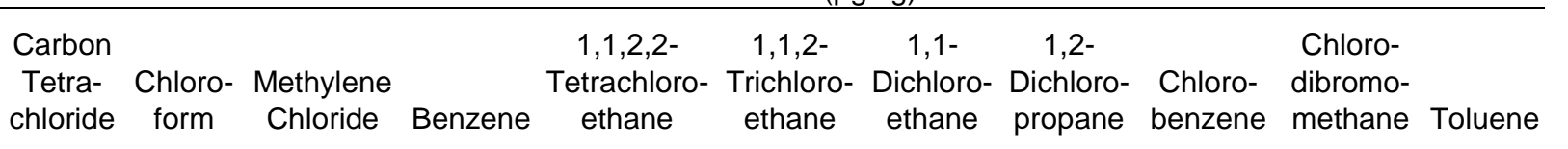

Location Sample Date (ft BGL) chloride form Chloride Benzene ethane ethane ethane propane benzene methane Toluene

\section{Co-op property sampling locations}

$\begin{array}{lcccccc}\text { TI-13 } & \text { NATI13-S-20532 } & 5 / 2 / 06 & 2.0 & \text { ND } & \text { ND } & \text { ND } \\ \text { TI-13 } & \text { NATI13-S-20533 } & 5 / 2 / 06 & 4.0 & \text { ND } & \text { ND } & \text { ND } \\ \text { TI-13 } & \text { NATI13-S-20534 } & 5 / 2 / 06 & 7.0 & \text { ND } & \text { ND } & \text { ND } \\ \text { TI-13 } & \text { NATI13-S-20535 } & 5 / 2 / 06 & 10.7 & \text { ND } & \text { ND } & \text { ND } \\ \text { TI-13 } & \text { NATI13-S-20536 } & 5 / 2 / 06 & 11.8 & \text { ND } & \text { ND } & \text { ND } \\ \text { TI-13 } & \text { NATI13-S-20537 } & 5 / 2 / 06 & 14.6 & \text { ND } & \text { ND } & \text { ND } \\ \text { TI-13 } & \text { NATI13-S-20538 } & 5 / 2 / 06 & 15.5 & \text { ND } & \text { ND } & \text { ND } \\ \text { TI-13 } & \text { NATI13-S-20539 } & 5 / 3 / 06 & 18.4 & \text { ND } & \text { ND } & \text { ND } \\ \text { TI-13 } & \text { NATI13-S-20540 } & 5 / 3 / 06 & 19.1 & \text { ND } & \text { ND } & \text { ND } \\ \text { TI-13 } & \text { NATI13-S-20557 } & 5 / 4 / 06 & 22.8 & \text { ND } & 1.6 \mathrm{~J} & \text { ND } \\ \text { TI-13 } & \text { NATI13-S-20541 } & 5 / 3 / 06 & 27.0 & 176 & 33 & \text { ND } \\ \text { TI-13 } & \text { NATI13-S-20542 } & 5 / 3 / 06 & 28.0 & 224 & 34 & \text { ND } \\ \text { TI-13 } & \text { NATI13-S-20558 } & 5 / 4 / 06 & 30.5 & 37 & 14 & \text { ND } \\ \text { TI-13 } & \text { NATI13-S-20543 } & 5 / 3 / 06 & 34.5 & 22 & 6.3 \mathrm{~J} & \text { ND } \\ \text { TI-13 } & \text { NATI13-S-20544 } & 5 / 3 / 06 & 36.0 & 16 & 5.1 \mathrm{~J} & \text { ND } \\ \text { TI-13 } & \text { NATI13-S-20545 } & 5 / 3 / 06 & 38.0 & 45 & 29 & \text { ND } \\ \text { TI-13 } & \text { NATI13-S-20546 } & 5 / 3 / 06 & 40.0 & 18 & 8 \mathrm{~J} & \text { ND } \\ \text { TI-13 } & \text { NATI13-S-20547 } & 5 / 3 / 06 & 42.9 & 30 & 5.6 \mathrm{~J} & \text { ND } \\ \text { TI-13 } & \text { NATI13-S-20548 } & 5 / 3 / 06 & 47.0 & 11 & 3 \mathrm{~J} & \text { ND } \\ \text { TI-13 } & \text { NATI13-S-20549 } & 5 / 3 / 06 & 50.0 & 20 & 2.4 \mathrm{~J} & \text { ND } \\ \text { TI-13 } & \text { NATI13-S-20550 } & 5 / 3 / 06 & 51.5 & 3.1 \mathrm{~J} & 1.8 \mathrm{~J} & \text { ND } \\ \text { TI-13 } & \text { NATI13-S-20553 } & 5 / 3 / 06 & 54.8 & 3.5 \mathrm{~J} & \text { ND } & \text { ND } \\ \text { TI-13 } & \text { NATI13-S-20554 } & 5 / 3 / 06 & 58.9 & 1.9 \mathrm{~J} & 1.2 \mathrm{~J} & \text { ND } \\ & & & & & & \\ \text { TI-14 } & \text { NATI14-S-20656 } & 5 / 5 / 06 & 2.0 & \text { ND } & \text { ND } & \text { ND } \\ \text { TI-14 } & \text { NATI14-S-20657 } & 5 / 5 / 06 & 5.0 & \text { ND } & \text { ND } & \text { ND } \\ \text { TI-14 } & \text { NATI14-S-20658 } & 5 / 5 / 06 & 7.0 & \text { ND } & \text { ND } & \text { ND } \\ \text { TI-14 } & \text { NATI14-S-20659 } & 5 / 5 / 06 & 8.8 & \text { ND } & \text { ND } & \text { ND } \\ \text { TI-14 } & \text { NATI14-S-20660 } & 5 / 5 / 06 & 9.5 & \text { ND } & \text { ND } & \text { ND } \\ \text { TI-14 } & \text { NATI14-S-20661 } & 5 / 5 / 06 & 11.2 & \text { ND } & \text { ND } & \text { ND } \\ \text { TI-14 } & \text { NATI14-S-20662 } & 5 / 5 / 06 & 12.5 & \text { ND } & \text { ND } & \text { ND }\end{array}$


TABLE S1.1 (Cont.)

Concentration $(\mu \mathrm{g} / \mathrm{kg})$

Carbon $\quad 1,1,2,2-\quad 1,1,2-\quad 1,1-\quad 1,2-\quad$ Chloro-

Sample Depth Tetra- Chloro- Methylene Tetrachloro- Trichloro- Dichloro- Dichloro- Chloro- dibromo-

Location Sample Date (ft BGL) chloride form Chloride Benzene ethane ethane ethane propane benzene methane Toluene

Co-op property sampling locations (cont.)

\begin{tabular}{|c|c|c|c|c|c|c|}
\hline TI-14 & NATI14-S-20663 & $5 / 5 / 06$ & 16.5 & ND & ND & ND \\
\hline TI-14 & NATI14-S-20664 & $5 / 5 / 06$ & 20.8 & ND & ND & ND \\
\hline TI-14 & NATI14-S-20665 & $5 / 5 / 06$ & 21.8 & ND & ND & ND \\
\hline TI-14 & NATI14-S-20667 & $5 / 5 / 06$ & 24.2 & ND & ND & ND \\
\hline TI-14 & NATI14-S-20668 & $5 / 5 / 06$ & 26.8 & ND & ND & ND \\
\hline $\mid-14$ & NATI14-S-20669 & $5 / 5 / 06$ & 29.0 & 19 & $3.7 \mathrm{~J}$ & תו \\
\hline TI-14 & NATI14-S-20670 & $5 / 5 / 06$ & 32.8 & 73 & $2.1 \mathrm{~J}$ & ND \\
\hline TI-14 & NATI14-S-20671 & $5 / 5 / 06$ & 35.2 & 364 & 16 & ND \\
\hline TI-14 & NATI14-S-20672 & $5 / 5 / 06$ & 38.0 & 194 & 15 & ND \\
\hline TI-14 & NATI14-S-20673 & $5 / 5 / 06$ & 40.5 & 306 & 25 & ND \\
\hline $\mid-14$ & NATI14-S-20674 & $5 / 5 / 06$ & 44.5 & 114 & $2.3 \mathrm{~J}$ & ND \\
\hline TI-14 & NATI14-S-20675 & $5 / 5 / 06$ & 46.2 & 160 & $2.6 \mathrm{~J}$ & ND \\
\hline TI-14 & NATI14-S-20686 & $5 / 5 / 06$ & 49.0 & 66 & $5.5 \mathrm{~J}$ & ND \\
\hline TI-14 & NATI14-S-20687 & $5 / 6 / 06$ & 52.8 & $2.6 \mathrm{~J}$ & $1.8 \mathrm{~J}$ & ND \\
\hline TI-14 & NATI14-S-20688 & $5 / 6 / 06$ & 56.8 & $6.6 \mathrm{~J}$ & $3.6 \mathrm{~J}$ & ND \\
\hline TI-14 & NATI14-S-20689 & $5 / 6 / 06$ & 57.8 & 50 & $4.0 \mathrm{~J}$ & ND \\
\hline $\mathrm{I}-14$ & NATI14-S-20690 & $5 / 6 / 06$ & 61.2 & ND & ND & ND \\
\hline $\mid-15$ & 66 & $5 / 5 / 06$ & 1. & ND & ND & ND \\
\hline TI-15 & NATI15-S-20567 & $5 / 5 / 06$ & 5.0 & ND & ND & ND \\
\hline Tl-15 & NATI15-S-20568 & $5 / 5 / 06$ & 8.5 & ND & ND & ND \\
\hline TI-15 & NATI15-S-20569 & $5 / 5 / 06$ & 12.4 & ND & ND & ND \\
\hline TI-15 & NATI15-S-20570 & $5 / 5 / 06$ & 13.0 & ND & ND & ND \\
\hline $\mathrm{Tl}-15$ & NATI15-S-20571 & $5 / 5 / 06$ & 17.7 & ND & ND & ND \\
\hline TI-15 & NATI15-S-20572 & $5 / 5 / 06$ & 19.0 & ND & ND & ND \\
\hline TI-15 & NATI15-S-20573 & $5 / 5 / 06$ & 21.0 & ND & ND & ND \\
\hline TI-15 & NATI15-S-20574 & $5 / 5 / 06$ & 24.6 & ND & ND & ND \\
\hline TI-15 & NATI15-S-20575 & $5 / 5 / 06$ & 26.1 & 93 & $2.5 \mathrm{~J}$ & ND \\
\hline TI-15 & NATI15-S-20576 & $5 / 5 / 06$ & 29.0 & 27 & ND & \\
\hline & & & & & & \\
\hline
\end{tabular}


TABLE S1.1 (Cont.)

Concentration $(\mu \mathrm{g} / \mathrm{kg})$

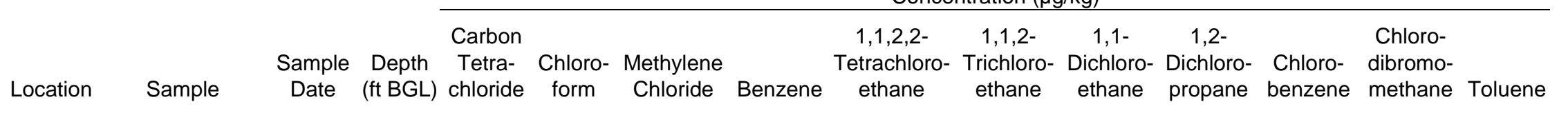

Co-op property sampling locations (cont.)

$\begin{array}{lcccccc}\text { TI-15 } & \text { NATI15-S-20578 } & 5 / 5 / 06 & 34.6 & 13 & \text { ND } & \text { ND } \\ \text { TI-15 } & \text { NATI15-S-20579 } & 5 / 5 / 06 & 37.5 & 13 & 3.5 \mathrm{~J} & \text { ND } \\ \text { TI-15 } & \text { NATI15-S-20580 } & 5 / 5 / 06 & 41.5 & 91 & 4.7 \mathrm{~J} & \text { ND } \\ \text { TI-15 } & \text { NATI15-S-20581 } & 5 / 5 / 06 & 45.0 & 3.3 \mathrm{~J} & \text { ND } & \text { ND } \\ \text { TI-15 } & \text { NATI15-S-20585 } & 5 / 6 / 06 & 51.9 & \text { ND } & \text { ND } & \text { ND } \\ \text { TI-15 } & \text { NATI15-S-20564 } & 5 / 6 / 06 & 52.5 & \text { ND } & \text { ND } & \text { ND } \\ \text { TI-15 } & \text { NATI15-S-20565 } & 5 / 6 / 06 & 57.1 & \text { ND } & \text { ND } & \text { ND } \\ \text { TI-15 } & \text { NATI15-S-20766 } & 5 / 6 / 06 & 60.8 & \text { ND } & \text { ND } & \text { ND } \\ & & & & & & \\ \text { TI-16 } & \text { NATI16-S-20770 } & 5 / 7 / 06 & 1.5 & \text { ND } & \text { ND } & \text { ND } \\ \text { TI-16 } & \text { NATI16-S-20771 } & 5 / 7 / 06 & 3.5 & 3.3 \mathrm{~J} & \text { ND } & \text { ND } \\ \text { TI-16 } & \text { NATI16-S-20772 } & 5 / 7 / 06 & 6.0 & \text { ND } & \text { ND } & \text { ND } \\ \text { TI-16 } & \text { NATI16-S-20773 } & 5 / 7 / 06 & 9.2 & \text { ND } & \text { ND } & \text { ND } \\ \text { TI-16 } & \text { NATI16-S-20774 } & 5 / 7 / 06 & 10.0 & \text { ND } & \text { ND } & \text { ND } \\ \text { TI-16 } & \text { NATI16-S-20775 } & 5 / 7 / 06 & 11.5 & \text { ND } & \text { ND } & \text { ND } \\ \text { TI-16 } & \text { NATI16-S-20776 } & 5 / 7 / 06 & 13.5 & \text { ND } & \text { ND } & \text { ND } \\ \text { TI-16 } & \text { NATI16-S-20777 } & 5 / 7 / 06 & 16.5 & \text { ND } & \text { ND } & \text { ND } \\ \text { TI-16 } & \text { NATI16-S-20778 } & 5 / 7 / 06 & 17.5 & \text { ND } & \text { ND } & \text { ND } \\ \text { TI-16 } & \text { NATI16-S-20779 } & 5 / 7 / 06 & 21.2 & \text { ND } & \text { ND } & \text { ND } \\ \text { TI-16 } & \text { NATI16-S-20780 } & 5 / 7 / 06 & 25.0 & \text { ND } & \text { ND } & \text { ND } \\ \text { TI-16 } & \text { NATI16-S-20781 } & 5 / 7 / 06 & 28.8 & 272 & 107 & \text { ND } \\ \text { TI-16 } & \text { NATI16-S-20782 } & 5 / 7 / 06 & 32.9 & 92 & 33 & \text { ND } \\ \text { TI-16 } & \text { NATI16-S-20783 } & 5 / 7 / 06 & 36.5 & 36 & 9.0 \mathrm{~J} & \text { ND } \\ \text { TI-16 } & \text { NATI16-S-20784 } & 5 / 7 / 06 & 37.5 & 35 & 8.6 \mathrm{~J} & \text { ND } \\ \text { TI-16 } & \text { NATI16-S-20785 } & 5 / 7 / 06 & 42.0 & 454 & 34 & \text { ND } \\ \text { TI-16 } & \text { NATI16-S-20786 } & 5 / 7 / 06 & 45.0 & 23 & 8.8 \mathrm{~J} & \text { ND } \\ \text { TI-16 } & \text { NATI16-S-20787 } & 5 / 7 / 06 & 49.8 & 3.1 \mathrm{~J} & \text { ND } & \text { ND } \\ \text { TI-16 } & \text { NATI16-S-20788 } & 5 / 7 / 06 & 53.0 & 3.8 \mathrm{~J} & 1.7 \mathrm{~J} & \text { ND } \\ & & & & & & \\ \text { TI-17 } & \text { NATI17-S-20796 } & 5 / 7 / 06 & 2.2 & \text { ND } & \text { ND } & \text { ND } \\ \text { TI-17 } & \text { NATI17-S-20797 } & 5 / 7 / 06 & 5.1 & \text { ND } & \text { ND } & \text { ND }\end{array}$


TABLE S1.1 (Cont.)

Concentration $(\mu \mathrm{g} / \mathrm{kg})$

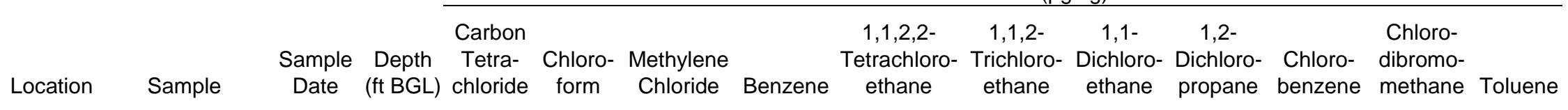

Co-op property sampling locations (cont.)

\begin{tabular}{|c|c|c|c|c|c|c|}
\hline $\mathrm{TI}-17$ & NATI17-S-20798 & $5 / 7 / 06$ & 9.1 & ND & ND & ND \\
\hline TI-17 & NATI17-S-20799 & $5 / 7 / 06$ & 13.0 & ND & ND & ND \\
\hline TI-17 & NATI17-S-20800 & $5 / 7 / 06$ & 16.6 & ND & ND & ND \\
\hline TI-17 & NATI17-S-20801 & $5 / 7 / 06$ & 17.4 & ND & ND & ND \\
\hline TI-17 & NATI17-S-20802 & $5 / 7 / 06$ & 21.0 & ND & ND & ND \\
\hline $\mathrm{TI}-17$ & NATI17-S-20803 & $5 / 7 / 06$ & 24.8 & $2.6 \mathrm{~J}$ & ND & ND \\
\hline $\mathrm{TI}-17$ & NATI17-S-20804 & $5 / 7 / 06$ & 26.2 & 15 & $1.5 \mathrm{~J}$ & ND \\
\hline $\mathrm{TI}-17$ & NATI17-S-20805 & $5 / 7 / 06$ & 28.5 & 31 & ND & ND \\
\hline $\mathrm{TI}-17$ & NATI17-S-20806 & $5 / 7 / 06$ & 33.0 & ND & $7.9 \mathrm{~J}$ & ND \\
\hline TI-17 & NATI17-S-20807 & $5 / 7 / 06$ & 37.5 & ND & ND & ND \\
\hline TI-17 & NATI17-S-20808 & $5 / 7 / 06$ & 42.0 & 10 & ND & ND \\
\hline TI-17 & NATI17-S-20809 & $5 / 7 / 06$ & 45.5 & ND & ND & ND \\
\hline TI-17 & NATI17-S-20810 & $5 / 7 / 06$ & 49.2 & ND & ND & ND \\
\hline $\mathrm{TI}-17$ & NATI17-S-20811 & $5 / 7 / 06$ & 52.9 & $2.1 \mathrm{~J}$ & ND & ND \\
\hline TI-17 & NATI17-S-20812 & $5 / 7 / 06$ & 56.5 & ND & ND & ND \\
\hline TI-18 & NATI18-S-20256 & $5 / 18 / 06$ & 5.0 & ND & ND & ND \\
\hline TI-18 & NATI18-S-20257 & $5 / 18 / 06$ & 9.0 & ND & ND & ND \\
\hline TI-18 & NATI18-S-20258 & $5 / 18 / 06$ & 13.0 & ND & ND & ND \\
\hline TI-18 & NATI18-S-20259 & $5 / 18 / 06$ & 16.5 & ND & ND & ND \\
\hline TI-18 & NATI18-S-20260 & $5 / 18 / 06$ & 20.8 & ND & ND & ND \\
\hline TI-18 & NATI18-S-20265 & $5 / 19 / 06$ & 29.0 & 10 & ND & ND \\
\hline TI-18 & NATI18-S-20676 & $5 / 19 / 06$ & 33.5 & 56 & ND & ND \\
\hline TI-18 & NATI18-S-20677 & $5 / 19 / 06$ & 37.0 & 109 & ND & ND \\
\hline TI-18 & NATI18-S-20678 & $5 / 19 / 06$ & 41.0 & 400 & $9.2 \mathrm{~J}$ & ND \\
\hline TI-18 & NATI18-S-20651 & $5 / 21 / 06$ & 45.0 & 46 & ND & ND \\
\hline TI-18 & NATI18-S-20652 & $5 / 21 / 06$ & 53.0 & $4.0 \mathrm{~J}$ & $2.0 \mathrm{~J}$ & ND \\
\hline TI-18 & NATI18-S-20653 & $5 / 21 / 06$ & 57.0 & ND & $1.4 \mathrm{~J}$ & ND \\
\hline TI-18 & NATI18-S-20654 & $5 / 21 / 06$ & 61.0 & $2.9 \mathrm{~J}$ & $1.5 \mathrm{~J}$ & ND \\
\hline
\end{tabular}


TABLE S1.1 (Cont.)

Concentration $(\mu \mathrm{g} / \mathrm{kg})$

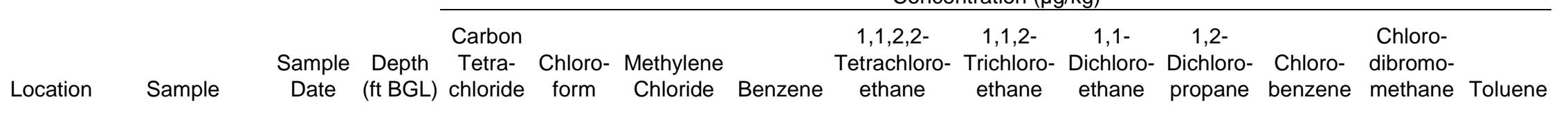

Co-op property sampling locations (cont.)

\begin{tabular}{|c|c|c|c|c|c|c|}
\hline TI-28 & NATI28-S-20696 & $5 / 19 / 06$ & 6.0 & ND & ND & ND \\
\hline TI-28 & NATI28-S-20236 & $5 / 18 / 06$ & 9.0 & ND & ND & ND \\
\hline TI-28 & NATI28-S-20237 & $5 / 18 / 06$ & 13.0 & ND & ND & ND \\
\hline TI-28 & NATI28-S-20238 & $5 / 18 / 06$ & 14.8 & ND & ND & ND \\
\hline TI-28 & NATI28-S-20239 & $5 / 18 / 06$ & 17.0 & ND & ND & ND \\
\hline TI-28 & NATI28-S-20240 & $5 / 18 / 06$ & 20.5 & 18 & $1.7 \mathrm{~J}$ & ND \\
\hline TI-28 & NATI28-S-20241 & $5 / 18 / 06$ & 22.5 & 1094 & $4.4 \mathrm{~J}$ & ND \\
\hline TI-28 & NATI28-S-20242 & $5 / 18 / 06$ & 25.2 & 613 & ND & ND \\
\hline TI-28 & NATI28-S-20243 & $5 / 18 / 06$ & 28.5 & 433 & ND & ND \\
\hline TI-28 & NATI28-S-20244 & $5 / 18 / 06$ & 32.8 & 287 & ND & ND \\
\hline TI-28 & NATI28-S-20246 & $5 / 18 / 06$ & 37.0 & 110 & ND & ND \\
\hline TI-28 & NATI28-S-20247 & $5 / 18 / 06$ & 41.0 & $8.7 \mathrm{~J}$ & ND & ND \\
\hline TI-28 & NATI28-S-20248 & $5 / 18 / 06$ & 42.5 & $5.2 \mathrm{~J}$ & $1.9 \mathrm{~J}$ & ND \\
\hline TI-28 & NATI28-S-20249 & $5 / 18 / 06$ & 45.0 & $2.8 \mathrm{~J}$ & ND & ND \\
\hline TI-28 & NATI28-S-20250 & $5 / 19 / 06$ & 49.0 & ND & ND & ND \\
\hline TI-28 & NATI28-S-20251 & $5 / 19 / 06$ & 52.2 & ND & ND & ND \\
\hline TI-28 & NATI28-S-20252 & $5 / 19 / 06$ & 56.5 & ND & ND & ND \\
\hline TI-28 & NATI28-S-20253 & $5 / 19 / 06$ & 61.8 & ND & ND & ND \\
\hline TI-29 & 98 & $5 / 1$ & 6.0 & ND & ND & ND \\
\hline TI-29 & NATI29-S-20855 & $5 / 19 / 06$ & 8.5 & ND & ND & ND \\
\hline TI-29 & NATI29-S-20697 & $5 / 19 / 06$ & 10.5 & ND & ND & ND \\
\hline TI-29 & NATI29-S-20703 & $5 / 19 / 06$ & 13.5 & ND & ND & ND \\
\hline TI-29 & NATI29-S-20956 & $5 / 21 / 06$ & 15.5 & ND & ND & ND \\
\hline TI-29 & NATI29-S-20705 & $5 / 19 / 06$ & 16.5 & ND & $0.7 \mathrm{~J}$ & ND \\
\hline TI-29 & NATI29-S-20886 & $5 / 19 / 06$ & 24.5 & 91 & ND & ND \\
\hline TI-29 & NATI29-S-20887 & $5 / 19 / 06$ & 26.5 & 17 & 10 & ND \\
\hline TI-29 & NATI29-S-20888 & $5 / 19 / 06$ & 29.4 & $9.4 \mathrm{~J}$ & $7.8 \mathrm{~J}$ & ND \\
\hline TI-29 & NATI29-S-20889 & $5 / 19 / 06$ & 34.0 & 49 & 16 & ND \\
\hline TI-29 & NATI29-S-20890 & $5 / 19 / 06$ & 38.0 & 135 & $8 \mathrm{~J}$ & ND \\
\hline TI-29 & NATI29-S-20891 & $5 / 19 / 06$ & 42.0 & 106 & $5.2 \mathrm{~J}$ & ND \\
\hline
\end{tabular}


TABLE S1.1 (Cont.)

Concentration $(\mu \mathrm{g} / \mathrm{kg})$

\begin{tabular}{|c|c|c|c|c|c|c|c|c|c|c|c|}
\hline \multirow[b]{2}{*}{ Location } & \multirow[b]{2}{*}{ Sample } & \multirow[b]{2}{*}{$\begin{array}{c}\text { Sample } \\
\text { Date }\end{array}$} & \multirow[b]{2}{*}{$\begin{array}{l}\text { Depth } \\
\text { (ft BGL) }\end{array}$} & \multicolumn{8}{|c|}{ Concentration $(\mu \mathrm{g} / \mathrm{kg})$} \\
\hline & & & & $\begin{array}{l}\text { Carbon } \\
\text { Tetra- Chloro- } \\
\text { chloride form }\end{array}$ & $\begin{array}{l}\text { Methylene } \\
\text { Chloride Benzene }\end{array}$ & $\begin{array}{l}1,1,2,2- \\
\text { Tetrachloro- } \\
\text { ethane }\end{array}$ & $\begin{array}{c}1,1,2- \\
\text { Trichloro- } \\
\text { ethane }\end{array}$ & $\begin{array}{l}1,1- \\
\text { Dichloro- } \\
\text { ethane }\end{array}$ & $\begin{array}{l}1,2- \\
\text { Dichloro- } \\
\text { propane }\end{array}$ & $\begin{array}{l}\text { Chloro- } \\
\text { benzene }\end{array}$ & $\begin{array}{l}\text { Chloro- } \\
\text { dibromo- } \\
\text { methane Toluene }\end{array}$ \\
\hline
\end{tabular}

Co-op property sampling locations (cont.)

$\begin{array}{lllllll}\text { TI-29 } & \text { NATI29-S-20892 } & 5 / 19 / 06 & 45.5 & \text { ND } & \text { ND } & \text { ND } \\ \text { TI-29 } & \text { NATI29-S-20893 } & 5 / 19 / 06 & 49.5 & 43 & 5.9 \mathrm{~J} & \text { ND } \\ \text { TI-29 } & \text { NATI29-S-20894 } & 5 / 19 / 06 & 53.0 & 6.0 \mathrm{~J} & 1.9 \mathrm{~J} & \text { ND } \\ \text { TI-29 } & \text { NATI29-S-20896 } & 5 / 21 / 06 & 57.0 & \text { ND } & \text { ND } & \text { ND } \\ \text { TI-29 } & \text { NATI29-S-20897 } & 5 / 21 / 06 & 62.0 & \text { ND } & \text { ND } & \text { ND }\end{array}$

a ND, not detected at a method detection limit of $1.0 \mu \mathrm{g} / \mathrm{kg}$.

b Qualifier J indicates an estimated concentration below the quantitation limit of $10.0 \mu \mathrm{g} / \mathrm{kg}$. 
TABLE S1.2 Particle size testing results for subsurface soil samples collected during the 2006 investigation at Navarre.

\begin{tabular}{|c|c|c|c|c|c|c|c|c|c|c|c|c|c|c|c|c|}
\hline \multirow[b]{2}{*}{ Location } & \multirow{2}{*}{$\begin{array}{l}\text { Depth } \\
\text { (ft BGL) }\end{array}$} & \multicolumn{15}{|c|}{ Particles Passing through Sieve Size (\%) } \\
\hline & & $3 / 4 "$ & 1/2" & 3/8" & $\# 4$ & $\# 10$ & \#18 & \#35 & $\# 40$ & \#60 & \#100 & \#120 & $\# 140$ & \#200 & \#230 & \#270 \\
\hline \multirow[t]{15}{*}{ TI-2 } & $13.2-13.4$ & 100 & 100 & 100 & 100 & 97.0 & 93.0 & 87.8 & 86.1 & 81.9 & 79.3 & 78.8 & 78.2 & 77.4 & 77.1 & 76.8 \\
\hline & $17-17.3$ & 100 & 100 & 100 & 97.3 & 89.1 & 76.9 & 67.2 & 65.2 & 60.1 & 56.4 & 55.5 & 54.6 & 52.6 & 51.4 & 50.6 \\
\hline & 24 & 100 & 100 & 97.6 & 90.6 & 83.0 & 73.7 & 66.5 & 64.8 & 59.1 & 46.2 & 42.5 & 38.7 & 33.1 & 30.9 & 29.7 \\
\hline & 28 & 100 & 94.0 & 88.3 & 76.6 & 69.3 & 65.4 & 63.0 & 62.5 & 60.9 & 58.9 & 58.3 & 57.5 & 56.2 & 55.8 & 55.5 \\
\hline & $29-29.2$ & 100 & 100 & 100 & 100 & 100 & 99.0 & 98.3 & 98.1 & 97.5 & 97.0 & 96.9 & 96.7 & 96.2 & 96.0 & 95.7 \\
\hline & $33.6-34$ & 100 & 100 & 97.5 & 94.1 & 90.2 & 87.4 & 85.0 & 84.3 & 82.2 & 80.1 & 79.6 & 78.8 & 77.5 & 77.0 & 76.6 \\
\hline & $37-38$ & 100 & 100 & 94.4 & 94.0 & 93.9 & 91.7 & 89.7 & 89.2 & 87.5 & 85.4 & 84.9 & 84.2 & 82.9 & 82.3 & 82.0 \\
\hline & 40 & 100 & 100 & 100 & 100 & 100 & 97.0 & 94.4 & 93.8 & 92.0 & 90.3 & 89.9 & 89.4 & 88.5 & 88.1 & 87.6 \\
\hline & 45 & 100 & 90.4 & 90.4 & 86.7 & 81.7 & 79.3 & 77.8 & 77.4 & 76.4 & 75.2 & 74.9 & 74.5 & 73.4 & 73.0 & 72.6 \\
\hline & $48-49$ & 100 & 100 & 100 & 98.4 & 94.5 & 92.6 & 90.8 & 90.4 & 89.5 & 88.6 & 88.4 & 88.1 & 87.3 & 86.7 & 86.1 \\
\hline & 50.5 & 100 & 100 & 100 & 99.8 & 97.9 & 93.4 & 91.0 & 90.5 & 89.3 & 88.1 & 87.7 & 87.0 & 85.4 & 84.5 & 83.6 \\
\hline & 53 & 100 & 100 & 100 & 100 & 100 & 97.7 & 94.7 & 94.0 & 92.0 & 90.1 & 89.6 & 88.9 & 87.3 & 86.2 & 85.3 \\
\hline & 58 & 100 & 100 & 100 & 100 & 100 & 99.7 & 98.9 & 98.6 & 97.8 & 97.0 & 96.8 & 96.6 & 95.9 & 95.4 & 94.6 \\
\hline & $61-61.5$ & 100 & 100 & 97.9 & 90.6 & 78.5 & 74.6 & 70.8 & 69.8 & 67.2 & 65.0 & 64.5 & 63.9 & 62.9 & 62.4 & 62.1 \\
\hline & $71-71.5$ & 100 & 100 & 100 & 100 & 100 & 99.7 & 99.3 & 99.3 & 98.9 & 98.6 & 98.4 & 98.2 & 97.5 & 97.0 & 96.4 \\
\hline \multirow[t]{4}{*}{ TI-3 } & $21-22$ & 100 & 96.3 & 87.7 & 79.0 & 71.0 & 66.0 & 61.8 & 61.0 & 58.2 & 53.7 & 52.2 & 50.1 & 45.4 & 42.8 & 42.0 \\
\hline & $34.5-35.5$ & 100 & 100 & 100 & 99.5 & 97.3 & 93.3 & 90.6 & 89.9 & 87.9 & 85.6 & 84.6 & 83.5 & 80.7 & 79.4 & 78.6 \\
\hline & $35.5-36$ & 100 & 100 & 100 & 99.3 & 97.5 & 95.2 & 93.4 & 92.9 & 91.5 & 89.7 & 89.2 & 88.5 & 86.9 & 86.1 & 85.6 \\
\hline & $42-42.5$ & 100 & 100 & 100 & 100 & 100 & 96.2 & 94.4 & 94.0 & 93.0 & 92.2 & 92.0 & 91.7 & 90.9 & 90.4 & 89.9 \\
\hline \multirow[t]{3}{*}{ TI-4 } & $16-16.5$ & 100 & 100 & 100 & 95.7 & 89.3 & 84.0 & 77.4 & 75.5 & 69.8 & 65.7 & 64.9 & 64.0 & 62.3 & 61.4 & 60.5 \\
\hline & $24.5-25$ & 100 & 100 & 100 & 100 & 100 & 99.1 & 98.3 & 98.1 & 97.0 & 95.8 & 95.4 & 95.0 & 93.9 & 93.5 & 93.3 \\
\hline & $31-31.5$ & 100 & 100 & 100 & 97.6 & 93.0 & 91.5 & 89.6 & 89.0 & 87.2 & 85.6 & 85.2 & 84.7 & 83.8 & 83.2 & 82.7 \\
\hline \multirow[t]{2}{*}{ TI-6 } & $41-42$ & 100 & 100 & 100 & 99.4 & 95.1 & 89.5 & 85.8 & 85.1 & 83.3 & 81.6 & 81.1 & 80.6 & 79.3 & 78.7 & 78.1 \\
\hline & $48.5-49$ & 100 & 96.6 & 93.8 & 89.5 & 84.0 & 80.1 & 77.8 & 77.3 & 76.3 & 75.4 & 75.1 & 74.8 & 73.8 & 73.2 & 72.5 \\
\hline \multirow[t]{4}{*}{ TI-7 } & $33-33.5$ & 100 & 100 & 100 & 96.7 & 88.5 & 84.4 & 81.0 & 80.2 & 78.0 & 75.5 & 74.8 & 73.8 & 71.5 & 70.7 & 70.1 \\
\hline & $36-36.5$ & 100 & 100 & 100 & 99.6 & 98.8 & 96.2 & 93.7 & 93.0 & 91.0 & 88.0 & 86.8 & 85.1 & 81.4 & 79.2 & 78.0 \\
\hline & $42.5-43$ & 100 & 100 & 100 & 99.0 & 95.7 & 94.3 & 92.0 & 91.2 & 89.1 & 86.9 & 86.3 & 85.4 & 83.0 & 81.9 & 81.4 \\
\hline & $68-68.6$ & 100 & 100 & 100 & 100 & 100 & 99.6 & 98.9 & 98.7 & 98.2 & 97.5 & 97.4 & 97.1 & 96.3 & 95.7 & 95.0 \\
\hline
\end{tabular}


TABLE S1.2 (Cont.)

\begin{tabular}{|c|c|c|c|c|c|c|c|c|c|c|c|c|c|c|c|c|}
\hline \multirow[b]{2}{*}{ Location } & \multirow{2}{*}{$\begin{array}{c}\text { Depth } \\
\text { (ft BGL) }\end{array}$} & \multicolumn{15}{|c|}{ Particles Passing through Sieve Size (\%) } \\
\hline & & $3 / 4 "$ & $1 / 2 "$ & $3 / 8 "$ & $\# 4$ & $\# 10$ & $\# 18$ & \#35 & $\# 40$ & $\# 60$ & $\# 100$ & \#120 & $\# 140$ & \#200 & \#230 & \#270 \\
\hline \multirow[t]{2}{*}{ TI-12 } & $29-30$ & 100 & 100 & 100 & 100 & 100 & 94.0 & 89.6 & 88.7 & 85.6 & 81.5 & 79.9 & 78.0 & 73.6 & 71.4 & 69.8 \\
\hline & $39-39.4$ & 100 & 100 & 100 & 100 & 100 & 99.5 & 98.5 & 98.2 & 97.5 & 96.8 & 96.6 & 96.4 & 95.9 & 95.6 & 95.4 \\
\hline \multirow[t]{2}{*}{ TI-14 } & $10-10.5$ & 100 & 100 & 100 & 99.4 & 98.4 & 95.4 & 93.3 & 92.8 & 90.3 & 80.7 & 76.5 & 72.2 & 64.7 & 61.7 & 59.5 \\
\hline & $34.6-35.3$ & 100 & 100 & 100 & 100 & 100 & 97.6 & 95.2 & 94.7 & 93.2 & 91.7 & 91.2 & 90.4 & 88.6 & 87.7 & 87.0 \\
\hline \multirow[t]{5}{*}{$\mathrm{TI}-16$} & $5.5-5.7$ & 100 & 100 & 100 & 100 & 100 & 100 & 99.8 & 99.7 & 99.5 & 99.3 & 99.2 & 99.1 & 98.8 & 98.4 & 97.7 \\
\hline & $14-14.5$ & 100 & 100 & 100 & 100 & 100 & 94.1 & 82.4 & 79.6 & 72.6 & 67.7 & 66.6 & 65.6 & 63.7 & 62.8 & 62.1 \\
\hline & $30-30.5$ & 100 & 100 & 100 & 100 & 100 & 97.4 & 91.8 & 90.6 & 87.4 & 81.4 & 78.8 & 75.6 & 70.1 & 68.5 & 67.2 \\
\hline & $38-38.5$ & 100 & 100 & 100 & 100 & 99.8 & 95.7 & 87.3 & 85.2 & 79.9 & 73.4 & 71.3 & 68.6 & 63.6 & 61.4 & 60.1 \\
\hline & $45.5-46$ & 82.7 & 59.1 & 47.7 & 36.8 & 34.9 & 32.3 & 27.7 & 26.8 & 24.1 & 20.9 & 20 & 19.1 & 17.2 & 16.4 & 15.8 \\
\hline \multirow[t]{5}{*}{ TI-29 } & $5-5.5$ & 100 & 100 & 100 & 100 & 100 & 100 & 98.4 & 98.1 & 95.2 & 90.7 & 89.4 & 88 & 85.7 & 84.9 & 84 \\
\hline & $28.5-29$ & 100 & 100 & 100 & 100 & 100 & 99.9 & 97.3 & 96.6 & 94.6 & 92.7 & 92.2 & 91.6 & 89.9 & 89 & 88 \\
\hline & $35-35.5$ & 100 & 100 & 100 & 100 & 100 & 97.8 & 95.6 & 95.2 & 94.2 & 93.3 & 93.1 & 92.8 & 91.9 & 91.5 & 90.9 \\
\hline & $48.5-49$ & 100 & 100 & 100 & 100 & 99.9 & 98.3 & 94.8 & 94 & 92.8 & 91.8 & 91.5 & 91 & 89.6 & 88.7 & 87.6 \\
\hline & $60-60.5$ & 100 & 100 & 100 & 100 & 100 & 99.9 & 97.3 & 96.8 & 95.4 & 94.1 & 93.8 & 93.2 & 92.1 & 91.5 & 90.8 \\
\hline
\end{tabular}


TABLE S1.3 Composition of subsurface soil samples collected during the 2006 investigation at Navarre.

\begin{tabular}{|c|c|c|c|c|c|}
\hline \multirow[b]{2}{*}{ Location } & \multirow{2}{*}{$\begin{array}{l}\text { Depth } \\
\text { (ft BGL) }\end{array}$} & \multicolumn{4}{|c|}{ Composition (\%) } \\
\hline & & Gravel & Sand & Silt & Clay \\
\hline \multirow[t]{15}{*}{ TI-2 } & $13.2-13.4$ & 0.0 & 22.6 & 37.2 & 40.2 \\
\hline & $17-17.3$ & 2.7 & 44.7 & 38.4 & 14.2 \\
\hline & 24 & 9.4 & 57.5 & 21.8 & 11.3 \\
\hline & 28 & 23.4 & 20.4 & 28.4 & 27.8 \\
\hline & $29-29.2$ & 0.0 & 3.8 & 52.8 & 43.4 \\
\hline & $33.6-34$ & 5.9 & 16.6 & 44.4 & 33.1 \\
\hline & $37-38$ & 6.0 & 11.1 & 49.0 & 33.9 \\
\hline & 40 & 0.0 & 11.5 & 52.6 & 35.9 \\
\hline & 45 & 13.3 & 13.3 & 31.1 & 42.3 \\
\hline & $48-49$ & 1.6 & 11.1 & 49.0 & 38.3 \\
\hline & 50.5 & 0.2 & 14.4 & 47.3 & 38.1 \\
\hline & 53 & 0.0 & 12.7 & 56.0 & 31.3 \\
\hline & 58 & 0.0 & 4.1 & 53.0 & 42.9 \\
\hline & $61-61.5$ & 9.4 & 27.7 & 21.7 & 41.2 \\
\hline & $71-71.5$ & 0.0 & 2.5 & 62.5 & 35.0 \\
\hline \multirow{4}{*}{ TI-3 } & $21-22$ & 21.0 & 33.6 & 28.5 & 16.9 \\
\hline & $34.5-35.5$ & 0.5 & 18.8 & 47.6 & 33.1 \\
\hline & $35.5-36$ & 0.7 & 12.4 & 50.6 & 36.3 \\
\hline & $42-42.5$ & 0.0 & 9.1 & 59.2 & 31.7 \\
\hline \multirow[t]{3}{*}{ TI-4 } & $16-16.5$ & 4.3 & 33.4 & 43.9 & 18.4 \\
\hline & $24.5-25$ & 0.0 & 6.1 & 60.0 & 33.9 \\
\hline & $31-31.5$ & 2.4 & 13.8 & 50.6 & 33.2 \\
\hline \multirow[t]{2}{*}{ TI-6 } & $41-42$ & 0.6 & 20.1 & 49.6 & 29.7 \\
\hline & $48.5-49$ & 10.5 & 15.7 & 54.4 & 19.4 \\
\hline \multirow[t]{4}{*}{ TI-7 } & $33-33.5$ & 3.3 & 25.2 & 42.1 & 29.4 \\
\hline & $36-36.5$ & 0.4 & 18.2 & 48.1 & 33.3 \\
\hline & $42.5-43$ & 1.0 & 16.0 & 42.0 & 41.0 \\
\hline & $68-68.6$ & 0.0 & 3.7 & 55.3 & 41.0 \\
\hline \multirow[t]{2}{*}{ TI-12 } & $29-30$ & 0.0 & 26.4 & 46.7 & 26.9 \\
\hline & 39-39.4 & 0.0 & 4.1 & 77.9 & 18.0 \\
\hline \multirow[t]{2}{*}{ TI-14 } & $10-10.5$ & 0.6 & 34.7 & 44.2 & 20.5 \\
\hline & $34.6-35.3$ & 0.0 & 11.4 & 65.5 & 23.1 \\
\hline \multirow[t]{5}{*}{ TI-16 } & $5.5-5.7$ & 0 & 1.2 & 50.4 & 48.4 \\
\hline & $14-14.5$ & 0 & 36.3 & 24.4 & 39.3 \\
\hline & $30-30.5$ & 0 & 29.9 & 41.5 & 28.6 \\
\hline & $38-38.5$ & 0 & 36.4 & 43.2 & 20.4 \\
\hline & $45.5-46$ & 63.2 & 19.6 & 11 & 6.2 \\
\hline
\end{tabular}


TABLE S1.3 (Cont.)

\begin{tabular}{|c|c|c|c|c|c|}
\hline \multirow[b]{2}{*}{ Location } & \multirow{2}{*}{$\begin{array}{c}\text { Depth } \\
\text { (ft BGL) }\end{array}$} & \multicolumn{4}{|c|}{ Composition (\%) } \\
\hline & & Gravel & Sand & Silt & Clay \\
\hline \multirow[t]{5}{*}{ TI-29 } & $5-5.5$ & 0 & 14.3 & 52.9 & 32.8 \\
\hline & $28.5-29$ & 0 & 10.1 & 56.4 & 33.5 \\
\hline & $35-35.5$ & 0 & 8.1 & 55.1 & 36.8 \\
\hline & $48.5-49$ & 0 & 10.4 & 51.6 & 38 \\
\hline & $60-60.5$ & 0 & 7.9 & 28.7 & 63.4 \\
\hline
\end{tabular}




\section{Supplement 2:}

\section{Water Sample Data}


TABLE S2.1 Groundwater samples collected during the 2006 investigation at Navarre.

\begin{tabular}{lcccc} 
Location & Sample & $\begin{array}{c}\text { Depth } \\
\text { (ft BGL) }\end{array}$ & $\begin{array}{c}\text { Sampling } \\
\text { Date }\end{array}$ & $\begin{array}{c}\text { Sampling } \\
\text { Time }\end{array}$ \\
\hline
\end{tabular}

\section{Existing well sampling}

\begin{tabular}{|c|c|c|c|c|c|}
\hline Co-op 1 & NACOOP1-W-20217 & Unknown & $4 / 8 / 06$ & $14: 45$ & DW \\
\hline Co-op 3 & NACOOP3-W-20218 & Unknown & $4 / 8 / 06$ & $14: 05$ & DW \\
\hline KDHE-1 & NAKDHE1-W-20203 & $35-55$ & $4 / 7 / 06$ & $11: 23$ & MW \\
\hline KDHE-2 & NAKDHE2-W-20205 & $25-45$ & $4 / 7 / 06$ & $13: 50$ & MW \\
\hline MW1 & NAMW01-W-20186 & $43-58$ & $4 / 5 / 06$ & $17: 07$ & MW \\
\hline MW2 & NAMW02-W-20188 & $42.8-57.8$ & $4 / 6 / 06$ & 9:00 & MW \\
\hline MW3 & NAMW03-W-20192 & $44-59$ & $4 / 6 / 06$ & $11: 48$ & MW \\
\hline MW4 & NAMW04-W-20194 & $45-60$ & $4 / 6 / 06$ & $14: 04$ & MW \\
\hline $\mathrm{T} 1$ & NAT1-W-20201 & $40-60$ & $4 / 7 / 06$ & $9: 45$ & MW \\
\hline $\mathrm{L}-1$ & NAL1-W-20211 & $75-95$ & $4 / 8 / 06$ & $9: 50$ & MW \\
\hline $\mathrm{L}-2$ & NAL2-W-20190 & $80-90$ & $4 / 6 / 06$ & $10: 15$ & MW \\
\hline L-3 & NAL3-W-20209 & $80-90$ & $4 / 7 / 06$ & $15: 50$ & MW \\
\hline
\end{tabular}

Sample collected from Co-op well \#1 after flushing line for $10 \mathrm{~min}$. Depth could not be measured because of pump.

Sample collected from Co-op well \#3 after flushing line for $10 \mathrm{~min}$. Depth could not be measured because of pump. Water used by Co-op to mix fertilizer.

Depth to water from top of casing $(T O C)=27.04 \mathrm{ft} \mathrm{BGL}$. Depth of well $=60 \mathrm{ft} \mathrm{BGL}$. Sample collected after purging of $21 \mathrm{gal}$.

Depth to water from TOC $=24.32 \mathrm{ft} \mathrm{BGL}$. Depth of well $=$ $44.93 \mathrm{ft} \mathrm{BGL}$. Sample collected after purging of 30 gal.

Depth to water from TOC $=31.11 \mathrm{ft} \mathrm{BGL}$. Depth of well = $59.84 \mathrm{ft} \mathrm{BGL}$. Sample collected after purging of 20 gal.

Depth to water from TOC $=31.42 \mathrm{ft}$ BGL. Depth of well = $57.1 \mathrm{ft} \mathrm{BGL}$. Sample collected after purging of $40 \mathrm{gal}$.

Depth to water from TOC $=31.58 \mathrm{ft} \mathrm{BGL}$. Depth of well $=$ $58 \mathrm{ft} \mathrm{BGL}$. Sample collected after purging of approximately $14 \mathrm{gal}$.

Depth to water from TOC $=32.21 \mathrm{ft} \mathrm{BGL}$. Depth of well $=$ $61.8 \mathrm{ft} \mathrm{BGL}$. Sample collected after purging of $20 \mathrm{gal}$. Depth to water from TOC $=30.81 \mathrm{ft} \mathrm{BGL}$. Depth of well $=$ $61 \mathrm{ft} \mathrm{BGL}$. Sample collected after purging of $105 \mathrm{gal}$.

Depth to water from TOC $=29.34 \mathrm{ft}$ BGL. Depth of well $=$ $95.35 \mathrm{ft} \mathrm{BGL}$. Sample collected after purging of 130 gal.

Depth to water from TOC $=29.49 \mathrm{ft} \mathrm{BGL}$. Depth of well = $90.91 \mathrm{ft} \mathrm{BGL}$. Sample collected after purging of 31 gal.

Depth to water from TOC $=29.75 \mathrm{ft} \mathrm{BGL}$. Depth of well $=$ $90 \mathrm{ft} \mathrm{BGL}$. Sample collected after purging of $46 \mathrm{gal}$. 
TABLE S2.1 (Cont.)

\begin{tabular}{|c|c|c|c|c|c|c|}
\hline Location & Sample & $\begin{array}{l}\text { Depth } \\
\text { (ft BGL) }\end{array}$ & $\begin{array}{c}\text { Sampling } \\
\text { Date }\end{array}$ & $\begin{array}{l}\text { Sampling } \\
\text { Time }\end{array}$ & Type $^{a}$ & Sample Description \\
\hline
\end{tabular}

\section{Existing well sampling (cont.)}

NANW1-W-20215

$40-50$

$4 / 8 / 06$

$11: 48$

NW-2

NANW2-W-20219

$35.5-45.5$

$4 / 9 / 06$

$13: 40$

NW-3

NANW3-W-20945

$38-48$

$5 / 25 / 06$

13:00

Anderson

NAANDER1-W-20941

$68^{b}$

$5 / 23 / 06$

$11: 50$

\section{Anderson}

NAANDER2-W-20942

NAANDER3-W-20943

$68^{b}$

$68^{b}$

$5 / 24 / 06$

$5 / 25 / 06$

$15: 30$

$11: 45$

Vertical-profile groundwater sampling at the former CCC/USDA grain storage facility

$\begin{array}{lccccc}\text { TI-1 } & \text { NATI1-W-20341 } & 30-35 & 4 / 13 / 06 & 18: 04 & \text { CPT } \\ \text { TI-1 } & \text { NATI1-W-20342 } & 38-43 & 4 / 13 / 06 & 19: 20 & \text { CPT } \\ & & & & \\ \text { TI-1 } & \text { NATI1-W-20344 } & 50-55 & 4 / 13 / 06 & 20: 07 & \text { CPT } \\ \text { TI-1 } & \text { NATI1-W-20266 } & 67-71 & 4 / 14 / 06 & 9: 46 & \text { CPT }\end{array}$

MW

MW

DW

Depth to water from TOC $=29.34 \mathrm{ft} \mathrm{BGL}$. Depth of well = $50.21 \mathrm{ft} \mathrm{BGL}$. Sample collected after purging of 11 gal.

Depth to water from TOC $=28.09 \mathrm{ft} \mathrm{BGL}$. Depth of well = $44.24 \mathrm{ft} \mathrm{BGL}$. Sample collected after purging of 20 gal.

Depth to water from TOC $=29.60 \mathrm{ft} \mathrm{BGL}$. Depth of well $=$ $41.8 \mathrm{ft} \mathrm{BGL}$. Collected after purging of $6 \mathrm{gal}$. Measured well depth during sampling is inconsistent with reported or registered screen intervals.

Anderson private well at 1524 Main Street. Northeast corner of shed located southwest of intersection of Main and Third Street. First of three samples collected over a three-day period when the well was continuously pumped. This is believed to be the same well sampled by Argonne on 8/22/92 with a location identifier of DW16. It was owned by Oswald at that time.

DW Second sample.

DW Third sample.

Good water recovery, immediately clear, low sediment content.

Good water recovery, immeditely oxidized, heavy sediment load.

Intermediate zone.

Deep zone. Abundant water immediately. Low turbidity. $40 \mathrm{ft}$ BGL of water in hole. 
TABLE S2.1 (Cont.)

\begin{tabular}{|c|c|c|c|c|c|c|}
\hline Location & Sample & $\begin{array}{l}\text { Depth } \\
\text { (ft BGL) }\end{array}$ & $\begin{array}{l}\text { Sampling } \\
\text { Date }\end{array}$ & $\begin{array}{l}\text { Sampling } \\
\text { Time }\end{array}$ & Type ${ }^{a}$ & Sample Description \\
\hline
\end{tabular}

Vertical-profile groundwater sampling at the former CCC/USDA grain storage facility (cont.)

\begin{tabular}{|c|c|c|c|c|c|c|}
\hline TI-2 & NATI2-W-20276 & $25-30$ & $4 / 21 / 06$ & $11: 15$ & CPT & High turbidity. Silt abundant. Water recovery immediately. \\
\hline TI-2 & NATI2-W-20277 & $31-36$ & $4 / 21 / 06$ & $11: 48$ & CPT & $\begin{array}{l}4.4 \mathrm{ft} \text { of water upon exposure of screen to formation. } \\
\text { Highly turbid with heavy sediment content; silty. Slow } \\
\text { initial recovery, with rapid response after } 1 \mathrm{hr} \text {. }\end{array}$ \\
\hline TI-2 & NATI2-W-20279 & $37-42$ & $4 / 21 / 06$ & $13: 45$ & CPT & $6 \mathrm{ft}$ of water upon exposure of screen to formation. \\
\hline TI-2 & NATI2-W-20281 & $49-54$ & $4 / 21 / 06$ & $14: 40$ & CPT & $\begin{array}{l}6 \mathrm{ft} \text { of water when screen was opened to the formation. } \\
\text { Relatively clear water, low turbidity. }\end{array}$ \\
\hline TI-2 & NATI2-W-20282 & $66-70$ & $4 / 21 / 06$ & $15: 30$ & CPT & $\begin{array}{l}\text { Could not penetrate below } 70 \mathrm{ft} \text { BGL. Heavily sedimented, } \\
\text { high turbidity. }\end{array}$ \\
\hline TI-2 & NATI2-W-20432 & $69-72$ & 4/7/06 & $15: 25$ & CPT & Water at $47 \mathrm{ft} \mathrm{BGL}$ in riser pipe. High turbidity. \\
\hline TI-3 & NATI3-W-20298 & $32.2-37.2$ & $4 / 7 / 06$ & $10: 22$ & CPT & $\begin{array}{l}\text { First aliquot for VOCs analysis clear, second aliquot } \\
\text { turbid. }\end{array}$ \\
\hline TI-3 & NATI3-W-20370 & 33-38 & $4 / 6 / 06$ & $8: 20$ & CPT & Sample collected for tritium analysis. Water turbid. \\
\hline TI-3 & NATI3-W-20383 & $43-48$ & $4 / 14 / 06$ & $7: 50$ & CPT & SW corner of dry fertilizer building. Turbid. \\
\hline TI-3 & NATI3-W-20385 & $51-56$ & $4 / 14 / 06$ & $9: 50$ & CPT & SW corner of dry fertilizer building. Turbid. \\
\hline $\mathrm{TI}-4$ & NATI4-W-20325 & $35-40$ & $4 / 10 / 06$ & $9: 35$ & CPT & West of NW corner of dry fertilizer building. Very turbid. \\
\hline $\mathrm{TI}-4$ & NATI4-W-20374 & $35-40$ & $4 / 13 / 06$ & $9: 45$ & CPT & $\begin{array}{l}\text { Sample collected for tritium analysis. Limited water, } \\
\text { turbid. } 500 \mathrm{~mL} \text { collected. }\end{array}$ \\
\hline $\mathrm{TI}-4$ & NATI4-W-20388 & $42-47$ & $4 / 14 / 06$ & $13: 40$ & CPT & West of NW corner of dry fertilizer building. Turbid. \\
\hline TI-4 & NATI4-W-20469 & $50-55$ & $4 / 11 / 06$ & $18: 30$ & CPT & $\begin{array}{l}6 \mathrm{ft} \text { of water upon opening core. Water carrying } \\
\text { tremendous amount of silt. Sampling could not be } \\
\text { done via bailer; collected by using Waterra pump. }\end{array}$ \\
\hline $\mathrm{Tl}-4$ & NATI4-W-20470 & $55-60$ & $4 / 11 / 06$ & $20: 01$ & CPT & Clear water recovered; little sediment fraction. \\
\hline TI-4 & NATI4-W-20472 & $61-66$ & $4 / 12 / 06$ & $10: 10$ & CPT & No description recorded. \\
\hline TI-4 & NATI4-W-20474 & $67-71$ & $4 / 12 / 06$ & $12: 00$ & CPT & $\begin{array}{l}\text { Reached refusal at } 71 \mathrm{ft} \mathrm{BGL.} 20 \mathrm{ft} \text { of water immediately } \\
\text { in this borehole. }\end{array}$ \\
\hline
\end{tabular}




\begin{tabular}{|c|c|c|c|c|c|c|}
\hline Location & Sample & $\begin{array}{l}\text { Depth } \\
\text { (ft BGL) }\end{array}$ & $\begin{array}{l}\text { Sampling } \\
\text { Date }\end{array}$ & $\begin{array}{l}\text { Sampling } \\
\text { Time }\end{array}$ & Type ${ }^{a}$ & Sample Description \\
\hline
\end{tabular}

Vertical-profile groundwater sampling at the former CCC/USDA grain storage facility (cont.)

\begin{tabular}{|c|c|c|c|c|c|c|}
\hline TI-5 & NATI5-W-20373 & $28-33$ & $4 / 13 / 06$ & $10: 12$ & CPT & $\begin{array}{l}\text { SW of dry fertilizer building, west edge of former } \\
\text { CCC/USDA facility. Very turbid. Limited water } \\
\text { available }(\sim 6 \mathrm{ft}) \text { after waiting overnight. Sample } \\
\text { collected without purge. KDHE collected a split } \\
\text { sample for VOCs and for nitrate, with nitrate field test } \\
\text { at } 56 \mathrm{ppm} \text {. }\end{array}$ \\
\hline TI-5 & NATI5-W-20368 & $36-41$ & $4 / 12 / 06$ & $11: 40$ & CPT & $\begin{array}{l}\text { SW of dry fertilizer building, west edge of former } \\
\text { CCC/USDA facility. Turbid and silty. }\end{array}$ \\
\hline TI-5 & NATI5-W-20371 & $49-54$ & 4/13/06 & $8: 10$ & CPT & $\begin{array}{l}\text { SW of dry fertilizer building. Turbid. KDHE collected a split } \\
\text { sample for VOCs and for nitrate, with field test for } \\
\text { nitrate at } 56 \mathrm{ppm} \text {. }\end{array}$ \\
\hline TI-5 & NATI5-W-20267 & $72.8-77.8$ & $4 / 20 / 06$ & $13: 13$ & CPT & Immediately $6 \mathrm{ft}$ of water in hole. \\
\hline TI-6 & NATI6-W-20353 & $38-43$ & $4 / 22 / 06$ & 15:04 & CPT & $\begin{array}{l}\text { South of SW corner of dry fertilizer building. Slightly turbid } \\
\text { water. }\end{array}$ \\
\hline TI-6 & NATI6-W-20348 & $49-54$ & $4 / 21 / 06$ & $19: 58$ & CPT & South of dry fertilizer building. Turbid. \\
\hline TI-6 & NATI6-W-20349 & $58-63$ & $4 / 22 / 06$ & $9: 18$ & CPT & South of dry fertilizer building. Slightly turbid. \\
\hline TI-6 & NATI6-W-20350 & $68-73$ & $4 / 22 / 06$ & 13:20 & CPT & South of dry fertilizer building. Brown, turbid water. \\
\hline TI-7 & NATI7-W-20269 & $29.5-34.5$ & $4 / 20 / 06$ & $14: 45$ & CPT & $\begin{array}{l}9 \mathrm{ft} \text { of water upon exposure of screen to the formation. } \\
\text { High turbidity. Heavy load of fine silt in water. }\end{array}$ \\
\hline $\mathrm{TI}-7$ & NATI7-W-20270 & $37-42$ & $4 / 20 / 06$ & $15: 59$ & CPT & $\begin{array}{l}18 \mathrm{ft} \text { of water immediately upon exposure of screen } \\
\text { interval. }\end{array}$ \\
\hline $\mathrm{TI}-7$ & NATI7-W-20272 & $45-50$ & $4 / 20 / 06$ & $17: 10$ & CPT & $\begin{array}{l}8 \mathrm{ft} \text { of water immediately. Very high turbidity. High silt } \\
\text { content. }\end{array}$ \\
\hline $\mathrm{TI}-7$ & NATI7-W-20273 & $51-56$ & $4 / 21 / 06$ & $7: 50$ & CPT & $\begin{array}{l}4 \mathrm{ft} \text { of water upon exposure of screen. Slow recovery. } \\
\text { High level of turbidity. }\end{array}$ \\
\hline TI-7 & NATI7-W-20274 & $68-72$ & $4 / 21 / 06$ & $8: 59$ & CPT & $\begin{array}{l}\text { Very slow water recovery. Could not sample with bailer. } \\
\text { Recovered only } 50 \mathrm{~mL} \text { of water with Waterra pump. }\end{array}$ \\
\hline $\mathrm{TI}-8$ & NATI8-W-20530 & $31-36$ & $4 / 28 / 06$ & $9: 20$ & CPT & $\begin{array}{l}\text { Southeast of SE corner of dry fertilizer building. Slightly } \\
\text { turbid water. Nitrate sample collected for Co-op. }\end{array}$ \\
\hline TI-8 & NATI8-W-20531 & 39-44 & $4 / 28 / 06$ & $10: 10$ & CPT & Slightly turbid. Nitrate sample collected for Co-op. \\
\hline
\end{tabular}


TABLE S2.1 (Cont.)

\begin{tabular}{|c|c|c|c|c|c|c|}
\hline Location & Sample & $\begin{array}{l}\text { Depth } \\
\text { (ft BGL) }\end{array}$ & $\begin{array}{l}\text { Sampling } \\
\text { Date }\end{array}$ & $\begin{array}{l}\text { Sampling } \\
\text { Time }\end{array}$ & Type ${ }^{a}$ & Sample Description \\
\hline
\end{tabular}

Vertical-profile groundwater sampling at the former CCC/USDA grain storage facility (cont.)

\begin{tabular}{|c|c|c|c|c|c|c|}
\hline TI-8 & NATI8-W-20641 & $45-50$ & $4 / 28 / 06$ & $17: 15$ & СРТ & Slow water recovery. \\
\hline TI-8 & NATI8-W-20732 & $54-59$ & $5 / 2 / 06$ & 18:00 & СРТ & $\begin{array}{l}\text { Along eastern edge of former CCC/USDA property. Very } \\
\text { silty. Nitrate sample collected for Co-op. }\end{array}$ \\
\hline TI-8 & NATI8-W-20731 & $64.5-69.5$ & $5 / 2 / 06$ & 17:05 & СРТ & $\begin{array}{l}\text { Along eastern edge of former CCC/USDA property. Very } \\
\text { silty. Nitrate sample collected for Co-op. }\end{array}$ \\
\hline TI-9 & NATI9-W-20609 & $40-45$ & $4 / 24 / 06$ & $14: 45$ & $\mathrm{CPT}$ & $\begin{array}{l}\text { Very slow water recovery. Waited } 24 \mathrm{hr} \text { to obtain } \\
\text { approximately } 17 \mathrm{ft} \text { of water in hole for sampling. }\end{array}$ \\
\hline TI-9 & NATI9-W-20586 & $46-51$ & $4 / 21 / 06$ & 19:35 & CPT & No description recorded. \\
\hline TI-9 & NATI9-W-20589 & $54-59$ & $4 / 23 / 06$ & 18:22 & СРT & $\begin{array}{l}\text { Water level } 28.5 \mathrm{ft} \text { BGL. Waited } 2 \text { days prior to sampling } \\
\text { because of lack of water. }\end{array}$ \\
\hline TI-9 & NATI9-W-20587 & $59-64$ & $4 / 22 / 06$ & $10: 10$ & $\mathrm{CPT}$ & Very slow water recovery. Clear water with little turbidity. \\
\hline $\mathrm{TI}-10$ & NATI10-W-20631 & $40-45$ & $4 / 26 / 06$ & $8: 10$ & СРТ & $\begin{array}{l}\text { Slow water recovery. Waited overnight prior to sampling. } \\
\text { Hole dry after collection of sample aliquots. }\end{array}$ \\
\hline $\mathrm{TI}-10$ & NATI10-W-20632 & $46-51$ & $4 / 27 / 06$ & $8: 59$ & СРT & Abundant immediate water recovery. \\
\hline $\mathrm{TI}-10$ & NATI10-W-20635 & $54-59$ & $4 / 27 / 06$ & $10: 46$ & СРТ & $\begin{array}{l}16 \mathrm{ft} \text { of water in hole upon opening of screen to formation. } \\
\text { Immediate recovery, moderate to low turbidity. }\end{array}$ \\
\hline $\mathrm{TI}-10$ & NATI10-W-20637 & $59-64$ & $4 / 27 / 06$ & $13: 10$ & СРТ & Slow water recovery initially, but $30 \mathrm{ft}$ of water after $1 \mathrm{hr}$. \\
\hline $\mathrm{TI}-10$ & NATI10-W-20610 & $66-71$ & $4 / 25 / 06$ & 14:20 & CPT & $\begin{array}{l}\text { Water slow entering borehole, with ample water after a } \\
\text { few minutes. }\end{array}$ \\
\hline $\mathrm{TI}-11$ & NATI11-W-20517 & $26-31$ & $4 / 26 / 06$ & $9: 12$ & $\mathrm{CPT}$ & $\begin{array}{l}\text { SW corner of former CCC/USDA property. Not enough } \\
\text { water for field parameters. Very turbid, silty water. }\end{array}$ \\
\hline $\mathrm{TI}-11$ & NATI11-W-20523 & $33-38$ & $4 / 27 / 06$ & $9: 15$ & СРТ & $\begin{array}{l}\text { SW corner of former CCC/USDA property. Not enough } \\
\text { water for field parameters. Very turbid, silty water. }\end{array}$ \\
\hline $\mathrm{TI}-11$ & NATI11-W-20506 & $40-45$ & $4 / 25 / 06$ & $17: 58$ & СРT & $\begin{array}{l}\text { SW corner of former CCC/USDA property. Very silty } \\
\text { water, thick. }\end{array}$ \\
\hline TI-11 & NATI11-W-20500 & $46.3-51.3$ & $4 / 23 / 06$ & $17: 42$ & СРТ & SW corner of former CCC/USDA property. Turbid. \\
\hline $\mathrm{TI}-11$ & NATI11-W-20638 & $52.6-57.6$ & $4 / 27 / 06$ & $16: 15$ & $\mathrm{CPT}$ & Slow water recovery. \\
\hline TI-11 & NATI11-W-20639 & $65.9-70.9$ & $4 / 27 / 06$ & $17: 13$ & СРТ & Abundant water present. \\
\hline
\end{tabular}




\begin{tabular}{|c|c|c|c|c|c|c|}
\hline Location & Sample & $\begin{array}{l}\text { Depth } \\
\text { (ft BGL) }\end{array}$ & $\begin{array}{l}\text { Sampling } \\
\text { Date }\end{array}$ & $\begin{array}{l}\text { Sampling } \\
\text { Time }\end{array}$ & Type ${ }^{a}$ & Sample Description \\
\hline
\end{tabular}

Vertical-profile groundwater sampling at the former CCC/USDA grain storage facility (cont.)

\begin{tabular}{|c|c|c|c|c|c|c|}
\hline $\mathrm{TI}-12$ & NATI12-W-20727 & $34-39$ & $5 / 2 / 06$ & $15: 30$ & CPT & $\begin{array}{l}\text { Southeast corner of former CCC/USDA property. Slightly } \\
\text { turbid. Nitrate sample collected for Co-op. }\end{array}$ \\
\hline TI-12 & NATI12-W-20728 & $39.4-44.4$ & $5 / 2 / 06$ & $15: 50$ & CPT & Silty. \\
\hline $\mathrm{Tl}-12$ & NATI12-W-20640 & $46-51$ & $4 / 28 / 06$ & $16: 30$ & CPT & Slow water recovery. \\
\hline $\mathrm{TI}-12$ & NATI12-W-20757 & $52-57$ & $5 / 4 / 06$ & $8: 28$ & CPT & $\begin{array}{l}\text { Southeast corner of former CCC/USDA property. Very } \\
\text { silty water. Duplicate sample collected for Co-op. }\end{array}$ \\
\hline $\mathrm{TI}-12$ & NATI12-W-20758 & $63.7-68.7$ & $5 / 4 / 06$ & $10: 03$ & CPT & Very silty water. Duplicate collected for Co-op. \\
\hline
\end{tabular}

Vertical-profile groundwater sampling on and downgradient from the Co-op property

\begin{tabular}{|c|c|c|c|c|c|c|}
\hline TI-13 & NATI13-W-20819 & $25-30$ & $5 / 9 / 06$ & $8: 58$ & CPT & Southwest corner of flat storage building. Very silty. \\
\hline $\mathrm{TI}-13$ & NATI13-W-20563 & $35-40$ & $5 / 5 / 06$ & $10: 00$ & CPT & Very turbid water. \\
\hline TI-13 & NATI13-W-20562 & $42-47$ & $5 / 5 / 06$ & $9: 30$ & CPT & Southwest corner of dry fertilizer building. Turbid water. \\
\hline $\mathrm{TI}-13$ & NATI13-W-20560 & $48-53$ & $5 / 4 / 06$ & $17: 20$ & CPT & No description recorded \\
\hline TI-13 & NATI13-W-20561 & $54-59$ & $5 / 5 / 06$ & $8: 26$ & CPT & Slightly turbid. \\
\hline TI-13 & NATI13-W-20555 & $66.8-71.8$ & $5 / 4 / 06$ & $8: 40$ & CPT & Southwest corner of flat storage building. Slightly turbid. \\
\hline TI-14 & NATI14-W-20693 & $26-31$ & $5 / 6 / 06$ & $14: 17$ & CPT & Turbid, silty with fine sand. \\
\hline TI-14 & NATI14-W-20695 & $32-37$ & $5 / 6 / 06$ & $15: 44$ & CPT & No description recorded. \\
\hline $\mathrm{Tl}-14$ & NATI14-W-20691 & $47-52$ & $5 / 6 / 06$ & $10: 53$ & CPT & West side of liquid fertilizer containment. Turbid. \\
\hline TI-14 & NATI14-W-20692 & $54-59$ & $5 / 6 / 06$ & 13:06 & CPT & Turbid water, silty. \\
\hline TI-14 & NATI14-W-20666 & $67.4-72.4$ & $5 / 5 / 06$ & 9:30 & CPT & West side of liquid fertilizer containment. Slightly turbid. \\
\hline TI-15 & NATI15-W-20644 & $15-20$ & $5 / 8 / 06$ & 8:05 & CPT & Slightly turbid. \\
\hline TI-15 & NATI15-W-20822 & $25-30$ & $5 / 9 / 06$ & 9:49 & CPT & Very silty. \\
\hline $\mathrm{Tl}-15$ & NATI15-W-20765 & $35-40$ & $5 / 7 / 06$ & $7: 40$ & CPT & Very silty, turbid. \\
\hline TI-15 & NATI15-W-20764 & $40-45$ & $5 / 6 / 06$ & $17: 50$ & CPT & Silty, turbid. \\
\hline $\mathrm{Tl}-15$ & NATI15-W-20763 & $47-52$ & $5 / 6 / 06$ & $16: 32$ & CPT & Very silty. Turbid, gray brown. \\
\hline TI-16 & NATI16-W-20818 & $25-30$ & $5 / 9 / 06$ & $8: 47$ & CPT & Very silty sample. \\
\hline TI-16 & NATI16-W-20817 & $32-37$ & $5 / 9 / 06$ & $8: 28$ & CPT & Very silty sample. \\
\hline
\end{tabular}




\begin{tabular}{|c|c|c|c|c|c|c|}
\hline Location & Sample & $\begin{array}{l}\text { Depth } \\
\text { (ft BGL) }\end{array}$ & $\begin{array}{l}\text { Sampling } \\
\text { Date }\end{array}$ & $\begin{array}{l}\text { Sampling } \\
\text { Time }\end{array}$ & Type ${ }^{a}$ & Sample Description \\
\hline
\end{tabular}

Vertical-profile groundwater sampling on and downgradient from the Co-op property (cont.)

\begin{tabular}{|c|c|c|c|c|c|c|}
\hline $\mathrm{TI}-16$ & NATI16-W-20791 & $39-44$ & $5 / 8 / 06$ & $16: 50$ & $\mathrm{CPT}$ & Very turbid water. \\
\hline TI-16 & NATI16-W-20816 & $46-51$ & $5 / 9 / 06$ & $7: 42$ & $\mathrm{CPT}$ & South of flat storage building. Silty. \\
\hline $\mathrm{TI}-16$ & NATI16-W-20789 & $60-65$ & $5 / 8 / 06$ & 13:05 & $\mathrm{CPT}$ & South door of flat storage building. Slightly turbid. \\
\hline $\mathrm{TI}-16$ & NATI16-W-20790 & $67-72$ & $5 / 8 / 06$ & $14: 40$ & $\mathrm{CPT}$ & Silty, turbid. \\
\hline $\mathrm{TI}-17$ & NATI17-W-20825 & $25-30$ & $5 / 9 / 06$ & $11: 43$ & $\mathrm{CPT}$ & Northeast corner of flat storage building. Silty. \\
\hline $\mathrm{TI}-17$ & NATI17-W-20793 & $32-37$ & $5 / 8 / 06$ & $15: 23$ & $\mathrm{CPT}$ & Turbid, silty. \\
\hline $\mathrm{TI}-17$ & NATI17-W-20826 & $39-44$ & $5 / 9 / 06$ & $11: 55$ & $\mathrm{CPT}$ & Silty. \\
\hline $\mathrm{TI}-17$ & NATI17-W-20814 & $46-51$ & $5 / 9 / 06$ & $7: 35$ & $\mathrm{CPT}$ & Northeast corner of flat storage building. Silty water. \\
\hline $\mathrm{TI}-17$ & NATI17-W-20829 & $53-58$ & $5 / 9 / 06$ & $13: 13$ & СРТ & Very silty. \\
\hline $\mathrm{TI}-17$ & NATI17-W-20828 & $60-65$ & $5 / 9 / 06$ & $13: 30$ & $\mathrm{CPT}$ & Very silty. \\
\hline TI-18 & NATI18-W-20921 & 25-30 & $5 / 23 / 06$ & $15: 40$ & $\mathrm{CPT}$ & $\begin{array}{l}\text { South side of liquid fertilizer concrete basin. Very silty. } \\
\text { Insufficient water for field parameters. }\end{array}$ \\
\hline TI-18 & NATI18-W-20655 & 30-35 & $5 / 21 / 06$ & 15:05 & CPT & Slightly turbid. Conductivity not recorded. \\
\hline TI-18 & NATI18-W-20831 & $35-40$ & $5 / 10 / 06$ & $7: 50$ & $\mathrm{CPT}$ & South of liquid fertilizer containment. Silty. \\
\hline $\mathrm{TI}-18$ & NATI18-W-20706 & $42-47$ & $5 / 21 / 06$ & 16:05 & $\mathrm{CPT}$ & $\begin{array}{l}\text { South side of liquid fertilizer containment. Very silty, } \\
\text { turbid. }\end{array}$ \\
\hline $\mathrm{TI}-18$ & NATI18-W-20707 & $49-54$ & $5 / 21 / 06$ & $17: 10$ & $\mathrm{CPT}$ & Very silty. \\
\hline TI-18 & NATI18-W-20709 & $56-61$ & $5 / 21 / 06$ & $18: 30$ & $\mathrm{CPT}$ & Very silty, turbid. \\
\hline $\mathrm{TI}-18$ & NATI18-W-20711 & $66.5-71.5$ & $5 / 22 / 06$ & $8: 40$ & $\mathrm{CPT}$ & Slightly turbid. \\
\hline TI-19 & NATI19-W-20231 & $25-30$ & $5 / 11 / 06$ & 23:55 & $\mathrm{CPT}$ & Far east of feed mill building, east of railroad tracks. Silty. \\
\hline TI-19 & NATI19-W-20863 & $32-37$ & $5 / 11 / 06$ & $12: 03$ & CPT & Far east of feed mill building, east of railroad tracks. Silty. \\
\hline TI-19 & NATI19-W-20864 & $39-44$ & $5 / 11 / 06$ & $13: 29$ & $\mathrm{CPT}$ & Silty. \\
\hline TI-19 & NATI19-W-20870 & $46-51$ & $5 / 11 / 06$ & $14: 05$ & $\mathrm{CPT}$ & Slightly silty. \\
\hline $\mathrm{TI}-19$ & NATI19-W-20868 & $53-58$ & $5 / 11 / 06$ & $15: 14$ & $\mathrm{CPT}$ & Fairly silty. \\
\hline TI-19 & NATI19-W-20869 & $60-65$ & $5 / 11 / 06$ & $15: 49$ & $\mathrm{CPT}$ & Slightly cloudy. \\
\hline TI-19 & NATI19-W-20872 & $65.5-69.5$ & $5 / 11 / 06$ & $17: 10$ & СРТ & Very silty. \\
\hline TI-20 & NATI20-W-20913 & $35-40$ & $5 / 22 / 06$ & $7: 30$ & CPT & Slightly turbid. \\
\hline TI-20 & NATI20-W-20713 & $42-47$ & $5 / 24 / 06$ & $13: 30$ & $\mathrm{CPT}$ & Very silty. Insufficient water for field parameters. \\
\hline
\end{tabular}


TABLE S2.1 (Cont.)

\begin{tabular}{|c|c|c|c|c|c|c|}
\hline Location & Sample & $\begin{array}{l}\text { Depth } \\
\text { (ft BGL) }\end{array}$ & $\begin{array}{c}\text { Sampling } \\
\text { Date }\end{array}$ & $\begin{array}{l}\text { Sampling } \\
\text { Time }\end{array}$ & Type $^{a}$ & Sample Description \\
\hline
\end{tabular}

Vertical-profile groundwater sampling on and downgradient from the Co-op property (cont.)

\begin{tabular}{|c|c|c|c|c|c|c|}
\hline TI-20 & NATI20-W-20914 & $56-61$ & $5 / 23 / 06$ & $14: 00$ & CPT & No description recorded. \\
\hline TI-20 & NATI20-W-20922 & $72-77$ & $5 / 23 / 06$ & $16: 00$ & CPT & $\begin{array}{l}\text { Northwest corner of J. Rock wheat field at east end of } \\
\text { Strole Street. Water slightly turbid. }\end{array}$ \\
\hline TI-21 & NATI21-W-20228 & $25-30$ & $5 / 11 / 06$ & 23:07 & CPT & Very little water, silty. No field parameters measured. \\
\hline TI-21 & NATI21-W-20885 & $32-37$ & $5 / 11 / 06$ & 22:07 & СРT & Very little water, silty. No field parameters measured. \\
\hline TI-21 & NATI21-W-20882 & 39-44 & $5 / 11 / 06$ & $21: 45$ & СРT & Very silty. \\
\hline TI-21 & NATI21-W-20881 & $46-51$ & $5 / 11 / 06$ & $21: 27$ & СPT & Silty. \\
\hline $\mathrm{TI}-21$ & NATI21-W-20867 & $53-58$ & $5 / 11 / 06$ & $14: 50$ & CPT & Northeast of feed mill building. Abundant, silty water. \\
\hline TI-22 & NATI22-W-20833 & $32-37$ & $5 / 10 / 06$ & 8:00 & СРT & Very silty water. \\
\hline TI-22 & NATI22-W-20834 & 39-44 & $5 / 10 / 06$ & $8: 10$ & CPT & Very silty water. \\
\hline TI-22 & NATI22-W-20830 & $46-51$ & $5 / 9 / 06$ & $17: 40$ & СРТ & Former ballfield. Slightly turbid to clear. \\
\hline TI-22 & NATI22-W-20836 & $53-58$ & $5 / 10 / 06$ & $9: 45$ & CPT & Very silty water. \\
\hline TI-22 & NATI22-W-20840 & $60-65$ & $5 / 10 / 06$ & $11: 58$ & CPT & Abundant water, fairly clear to slightly cloudy. \\
\hline TI-22 & NATI22-W-20846 & $68.2-73.2$ & $5 / 10 / 06$ & $11: 27$ & CPT & No description recorded. \\
\hline TI-23 & NATI23-W-20861 & $39-44$ & $5 / 11 / 06$ & $12: 45$ & СРТ & $\begin{array}{l}\text { Southeast corner of feed mill building. Fairly silty water, } \\
\text { abundant. }\end{array}$ \\
\hline TI-23 & NATI23-W-20862 & $46-51$ & $5 / 11 / 06$ & $12: 15$ & CPT & Little water, slightly silty. No field measurements done. \\
\hline TI-23 & NATI23-W-20865 & $53-58$ & $5 / 11 / 06$ & $14: 07$ & CPT & Silty sample, cloudy. \\
\hline TI-23 & NATI23-W-20856 & $60-65$ & $5 / 10 / 06$ & $17: 49$ & СPT & Southeast corner of feed mill building. Silty. \\
\hline TI-23 & NATI23-W-20859 & $66.8-71.8$ & $5 / 10 / 06$ & 18:35 & СРT & Silty. \\
\hline TI-24 & NATI24-W-20860 & 23-30 & $5 / 11 / 06$ & $10: 30$ & СРT & Very little water present. No field measurements done. \\
\hline TI-24 & NATI24-W-20858 & $32-37$ & $5 / 10 / 06$ & $18: 00$ & CPT & Silty. \\
\hline TI-24 & NATI24-W-20838 & $39-44$ & $5 / 10 / 06$ & $11: 25$ & СРТ & $\begin{array}{l}\text { Far west of feed mill building. Fairly clear water, only } \\
\text { slightly cloudy. }\end{array}$ \\
\hline TI-24 & NATI24-W-20841 & $46-51$ & $5 / 10 / 06$ & $13: 20$ & СРТ & Fairly silty water. \\
\hline TI-24 & NATI24-W-20842 & $53-58$ & $5 / 10 / 06$ & $15: 00$ & CPT & Slightly cloudy water. \\
\hline
\end{tabular}


TABLE S2.1 (Cont.)

\begin{tabular}{|c|c|c|c|c|c|c|}
\hline Location & Sample & $\begin{array}{l}\text { Depth } \\
\text { (ft BGL) }\end{array}$ & $\begin{array}{l}\text { Sampling } \\
\text { Date }\end{array}$ & $\begin{array}{l}\text { Sampling } \\
\text { Time }\end{array}$ & Type ${ }^{a}$ & Sample Description \\
\hline
\end{tabular}

Vertical-profile groundwater sampling on and downgradient from the Co-op property (cont.)

\begin{tabular}{|c|c|c|c|c|c|c|}
\hline TI-24 & NATI24-W-20843 & $60-65$ & $5 / 10 / 06$ & $16: 10$ & СPT & Abundant water, very silty. \\
\hline TI-24 & NATI24-W-20845 & $69.4-74.4$ & $5 / 10 / 06$ & $17: 20$ & СРТ & Silty water, abundant. \\
\hline TI-25 & NATI25-W-20722 & 32-37 & $5 / 12 / 06$ & $10: 10$ & СРТ & Very silty abundant water. \\
\hline TI-25 & NATI25-W-20875 & 39-44 & $5 / 11 / 06$ & 18:00 & СРТ & $\begin{array}{l}\text { Northwest corner of feed mill building. Slightly cloudy, } \\
\text { silty, milky. }\end{array}$ \\
\hline TI-25 & NATI25-W-20878 & $46-51$ & $5 / 11 / 06$ & 21:00 & CPT & Silty turbid water. \\
\hline TI-25 & NATI25-W-20884 & $53-58$ & $5 / 11 / 06$ & $21: 50$ & СРT & Very silty. \\
\hline TI-25 & NATI25-W-20232 & $60-65$ & $5 / 12 / 06$ & $0: 00$ & CPT & Very silty. \\
\hline TI-25 & NATI25-W-20720 & $66-71$ & $5 / 12 / 06$ & 2:00 & СРТ & Very silty, abundant water. \\
\hline TI-25 & NATI25-W-20235 & $72.2-77.2$ & $5 / 12 / 06$ & $0: 50$ & CPT & $\begin{array}{l}\text { Northwest corner of feed mill building. Very turbid/milky } \\
\text { sample. }\end{array}$ \\
\hline TI-26 & NATI26-W-20721 & $25-30$ & $5 / 12 / 06$ & $2: 20$ & СPT & Very little, very silty water. No field parameters measured. \\
\hline TI-26 & NATI26-W-20877 & $32-37$ & $5 / 11 / 06$ & 19:25 & СРT & $\begin{array}{l}\text { Southwest of feed mill building. Very turbid/silty/cloudy } \\
\text { sample. }\end{array}$ \\
\hline TI-26 & NATI26-W-20883 & $39-44$ & $5 / 11 / 06$ & $21: 20$ & СРТ & Southwest of feed mill building. Very silty water. \\
\hline TI-26 & NATI26-W-20723 & $46-51$ & $5 / 12 / 06$ & $10: 25$ & СРТ & Very silty, abundant water. \\
\hline TI-26 & NATI26-W-20229 & $53-58$ & $5 / 11 / 06$ & $22: 55$ & СРТ & Southwest corner of feed mill building. Fairly silty. \\
\hline TI-26 & NATI26-W-20233 & $60-65$ & $5 / 12 / 06$ & $0: 07$ & СРT & Very silty. \\
\hline TI-26 & NATI26-W-20719 & $66-71$ & $5 / 12 / 06$ & $1: 39$ & СРT & Cloudy sample. \\
\hline TI-26 & NATI26-W-20718 & $71.8-76.8$ & $5 / 12 / 06$ & $0: 47$ & CPT & Southwest corner of feed mill building. Turbid/silty water. \\
\hline TI-27 & NATI27-W-20680 & $25-30$ & $5 / 19 / 06$ & $13: 30$ & СРT & $\begin{array}{l}\text { Presumed upgradient location - west of railroad tracks } \\
\text { and dry fertilizer building. Very little turbidity. Very little } \\
\text { water in well (approximately } 2 \mathrm{ft} \text { ). Unable to collect } \\
\text { water for field measurements. }\end{array}$ \\
\hline TI-27 & NATI27-W-20704 & 30-35 & $5 / 20 / 06$ & $10: 15$ & CPT & Field measurements not recorded. \\
\hline TI-27 & NATI27-W-20684 & $35-40$ & $5 / 19 / 06$ & $16: 25$ & СРT & Turbid. \\
\hline TI-27 & NATI27-W-20699 & $42-47$ & $5 / 20 / 06$ & $8: 10$ & СРТ & Slightly turbid. \\
\hline TI-27 & NATI27-W-20700 & $49-54$ & $5 / 20 / 06$ & $9: 20$ & СРТ & Very turbid. \\
\hline
\end{tabular}


TABLE S2.1 (Cont.)

\begin{tabular}{|c|c|c|c|c|c|c|}
\hline Location & Sample & $\begin{array}{l}\text { Depth } \\
\text { (ft BGL) }\end{array}$ & $\begin{array}{c}\text { Sampling } \\
\text { Date }\end{array}$ & $\begin{array}{l}\text { Sampling } \\
\text { Time }\end{array}$ & Type $^{a}$ & Sample Description \\
\hline
\end{tabular}

Vertical-profile groundwater sampling on and downgradient from the Co-op property (cont.)

\begin{tabular}{|c|c|c|c|c|c|c|}
\hline TI-27 & NATI27-W-20905 & $56-61$ & $5 / 21 / 06$ & $11: 20$ & СРТ & Field measurements not recorded. \\
\hline TI-27 & NATI27-W-20649 & $66.2-71.2$ & $5 / 20 / 06$ & $16: 26$ & $\mathrm{CPT}$ & Very silty, turbid. \\
\hline TI-28 & NATI28-W-20904 & $25-30$ & $5 / 21 / 06$ & $12: 30$ & $\mathrm{CPT}$ & In ditch east of liquid fertilizer containment. Slightly turbid. \\
\hline TI-28 & NATI28-W-20851 & $32-37$ & $5 / 20 / 06$ & $8: 40$ & $\mathrm{CPT}$ & Very turbid. \\
\hline TI-28 & NATI28-W-20849 & $37-42$ & $5 / 20 / 06$ & $7: 48$ & СРT & Slightly turbid. \\
\hline TI-28 & NATI28-W-20850 & $44-49$ & $5 / 20 / 06$ & $8: 15$ & $\mathrm{CPT}$ & Very turbid. \\
\hline TI-28 & NATI28-W-20683 & $51-56$ & $5 / 19 / 06$ & $16: 21$ & $\mathrm{CPT}$ & Slightly turbid. \\
\hline TI-28 & NATI28-W-20254 & $58-63$ & $5 / 19 / 06$ & $14: 10$ & СРТ & $\begin{array}{l}\text { In ditch directly east of liquid fertilizer containment. } \\
\text { Slightly turbid water. }\end{array}$ \\
\hline TI-28 & NATI28-W-20647 & $63-68$ & $5 / 19 / 06$ & 15:08 & $\mathrm{CPT}$ & In ditch directly east of liquid fertilizer containment. Turbid. \\
\hline TI-29 & NATI29-W-20916 & $25-30$ & $5 / 23 / 06$ & $14: 50$ & CPT & Very silty. \\
\hline TI-29 & NATI29-W-20909 & $32-37$ & $5 / 22 / 06$ & $7: 35$ & СРТ & In ditch - southern location. \\
\hline TI-29 & NATI29-W-20900 & $39-44$ & $5 / 21 / 06$ & $9: 15$ & $\mathrm{CPT}$ & In ditch - southern location. Very silty, turbid. \\
\hline TI-29 & NATI29-W-20902 & $46-51$ & $5 / 21 / 06$ & $10: 20$ & СРТ & Very silty, turbid. \\
\hline TI-29 & NATI29-W-20903 & $53-58$ & $5 / 21 / 06$ & 13:00 & СРТ & $\begin{array}{l}\text { Very silty, turbid. Insufficient water for field } \\
\text { measurements. }\end{array}$ \\
\hline TI-29 & NATI29-W-20906 & $60-65$ & $5 / 21 / 06$ & $14: 29$ & СРТ & Very silty, turbid. \\
\hline TI-29 & NATI29-W-20907 & $66.3-69.3$ & $5 / 21 / 06$ & $16: 04$ & $\mathrm{CPT}$ & No description recorded. \\
\hline TI-30 & NATI30-W-20939 & $32-37$ & $5 / 25 / 06$ & $10: 20$ & CPT & Silty. Insufficient water for field parameters. \\
\hline TI-30 & NATI30-W-20920 & $39-44$ & $5 / 23 / 06$ & $12: 30$ & $\mathrm{CPT}$ & $\begin{array}{l}\text { Northwest corner of J. Rock wheat field, directly west of } \\
\text { dry fertilizer building. Water turbid. Field parameters } \\
\text { not measured. }\end{array}$ \\
\hline TI-30 & NATI30-W-20915 & $43.8-48.8$ & $5 / 23 / 06$ & $13: 50$ & $\mathrm{CPT}$ & $\begin{array}{l}\text { Directly west of dry fertilizer building and anhydrous } \\
\text { ammonia tanks. }\end{array}$ \\
\hline TI-30 & NATI30-W-20911 & $53-58$ & $5 / 23 / 06$ & $7: 30$ & СРТ & $\begin{array}{l}\text { West edge of wheat field - west of former CCC/USDA } \\
\text { property. Slightly turbid. }\end{array}$ \\
\hline TI-30 & NATI30-W-20918 & $60-65$ & $5 / 23 / 06$ & 10:05 & СРТ & Very silty, turbid. \\
\hline TI-30 & NATI30-W-20917 & $75-80$ & $5 / 23 / 06$ & $11: 05$ & СРТ & Slightly turbid. \\
\hline TI-30 & NATI30-W-20712 & $83.5-88.5$ & $5 / 24 / 06$ & $7: 15$ & $\mathrm{CPT}$ & No description recorded. \\
\hline
\end{tabular}


TABLE S2.1 (Cont.)

\begin{tabular}{|c|c|c|c|c|c|c|}
\hline Location & Sample & $\begin{array}{l}\text { Depth } \\
\text { (ft BGL) }\end{array}$ & $\begin{array}{c}\text { Sampling } \\
\text { Date }\end{array}$ & $\begin{array}{l}\text { Sampling } \\
\text { Time }\end{array}$ & Type ${ }^{a}$ & Sample Description \\
\hline
\end{tabular}

Vertical-profile groundwater sampling on and downgradient from the Co-op property (cont.)

$\begin{array}{lccccll}\text { TI-31 } & \text { NATI31-W-20947 } & 42-47 & 5 / 25 / 06 & 15: 02 & \text { CPT } & \text { No description recorded. } \\ \text { TI-31 } & \text { NATI31-W-20938 } & 53-58 & 5 / 25 / 06 & 10: 59 & \text { CPT } & \text { Slightly silty. } \\ \text { TI-31 } & \text { NATI31-W-20937 } & 60-65 & 5 / 25 / 06 & 9: 45 & \text { CPT } & \text { Very silty. } \\ \text { TI-31 } & \text { NATI31-W-20715 } & 70.7-75.7 & 5 / 24 / 06 & 18: 40 & \text { CPT } & \text { North side of shed, Beem property. Very turbid, silty. }\end{array}$

$\begin{array}{llll}\text { TI-31 NATI31-W-20715 } & \text { 50.7-75.7 } & \text { 5/24/06 } & \text { CPT }\end{array}$ North side of shed, Beem property. Very turbid, silty.

Sampling of monitoring well installed at the TI-30 location during the 2006 investigation

\begin{tabular}{|c|c|c|c|}
\hline MW5 & NATI30-W-20946 & $78-88$ & $5 / 25 / 06$ \\
\hline
\end{tabular}

a Types: CPT, cone penetrometer; DW, domestic well; MW, monitoring well; $\mathrm{P}$, piezometer.

b Total depth. 
TABLE S2.2 Results of field measurements made during groundwater sampling in the 2006 investigation at Navarre.

\begin{tabular}{|c|c|c|c|c|c|c|c|}
\hline Location & Sample & $\begin{array}{l}\text { Depth } \\
\text { (ft BGL) }\end{array}$ & $\begin{array}{l}\text { Sample } \\
\text { Date }\end{array}$ & Type $^{a}$ & $\begin{array}{l}\text { Temperature } \\
\left({ }^{\circ} \mathrm{C}\right)\end{array}$ & $\mathrm{pH}$ & $\begin{array}{l}\text { Conductivity } \\
\qquad(\mu \mathrm{S} / \mathrm{cm})\end{array}$ \\
\hline \multicolumn{8}{|c|}{ Existing well sampling } \\
\hline Co-op 1 & NACOOP1-W-20217 & Unknown & $4 / 8 / 06$ & DW & 11.4 & 7.42 & 1408 \\
\hline Co-op 3 & NACOOP3-W-20218 & Unknown & $4 / 8 / 06$ & DW & 11.4 & 7.34 & 1739 \\
\hline KDHE-1 & NAKDHE1-W-20203 & $35-55$ & $4 / 7 / 06$ & MW & 14.3 & 7.33 & 974 \\
\hline KDHE-2 & NAKDHE2-W-20205 & $25-45$ & $4 / 7 / 06$ & MW & 13.1 & 7.10 & 1450 \\
\hline MW1 & NAMW01-W-20186 & $43-58$ & $4 / 5 / 06$ & MW & 20.3 & 6.78 & 1498 \\
\hline MW2 & NAMW02-W-20188 & $42.8-57.8$ & $4 / 6 / 06$ & MW & 15.4 & 7.10 & 1420 \\
\hline MW3 & NAMW03-W-20192 & $44-59$ & $4 / 6 / 06$ & MW & 16.6 & 7.10 & 1559 \\
\hline MW4 & NAMW04-W-20194 & $45-60$ & $4 / 6 / 06$ & MW & 16.2 & 7.16 & 1445 \\
\hline $\mathrm{T} 1$ & NAT1-W-20201 & $40-60$ & $4 / 7 / 06$ & MW & 14.3 & 7.14 & 1558 \\
\hline L-1 & NAL1-W-20211 & $75-95$ & $4 / 8 / 06$ & MW & 13.8 & 7.36 & 1405 \\
\hline L-2 & NAL2-W-20190 & $80-90$ & $4 / 6 / 06$ & MW & 15.5 & 7.40 & 2790 \\
\hline L-3 & NAL3-W-20209 & $80-90$ & $4 / 7 / 06$ & MW & 14.2 & $N R^{b}$ & 2840 \\
\hline NW-1 & NANW1-W-20215 & $40-50$ & $4 / 8 / 06$ & MW & 13.8 & 7.25 & 1377 \\
\hline NW-2 & NANW2-W-20219 & $35.5-45.5$ & $4 / 9 / 06$ & MW & 17.6 & 7.25 & 1505 \\
\hline NW-3 & NANW3-W-20945 & $38-48$ & $5 / 25 / 06$ & MW & 21.9 & 7.50 & 886 \\
\hline Anderson & NAANDER1-W-20941 & $68^{c}$ & $5 / 23 / 06$ & DW & NR & NR & NR \\
\hline Anderson & NAANDER2-W-20942 & $68^{c}$ & $5 / 24 / 06$ & DW & NR & NR & NR \\
\hline Anderson & NAANDER3-W-20943 & $68^{c}$ & $5 / 25 / 06$ & DW & NR & NR & NR \\
\hline
\end{tabular}

Vertical-profile groundwater sampling at the former CCC/USDA grain storage facility

$\begin{array}{lrrrrrrr}\text { TI-1 } & \text { NATI1-W-20341 } & 30-35 & 4 / 13 / 06 & \text { CPT } & 20.9 & 7.18 & 4440 \\ \text { TI-1 } & \text { NATI1-W-20342 } & 38-43 & 4 / 13 / 06 & \text { CPT } & 20.1 & 7.13 & 4720 \\ \text { TI-1 } & \text { NATI1-W-20344 } & 50-55 & 4 / 13 / 06 & \text { CPT } & 20.1 & 7.17 & 4650 \\ \text { TI-1 } & \text { NATI1-W-20266 } & 67-71 & 4 / 14 / 06 & \text { CPT } & 18.9 & 7.57 & 1523 \\ & & & & & & & \\ \text { TI-2 } & \text { NATI2-W-20276 } & 25-30 & 4 / 21 / 06 & \text { CPT } & 19.6 & 7.18 & 5040 \\ \text { TI-2 } & \text { NATI2-W-20277 } & 31-36 & 4 / 21 / 06 & \text { CPT } & 19.6 & 7.06 & 4850 \\ \text { TI-2 } & \text { NATI2-W-20279 } & 37-42 & 4 / 21 / 06 & \text { CPT } & 19.5 & 7.24 & 2980 \\ \text { TI-2 } & \text { NATI2-W-20281 } & 49-54 & 4 / 21 / 06 & \text { CPT } & 19.6 & 7.47 & 1205 \\ \text { TI-2 } & \text { NATI2-W-20282 } & 66-70 & 4 / 21 / 06 & \text { CPT } & 21.6 & 7.66 & 1454 \\ \text { TI-2 } & \text { NATI2-W-20432 } & 69-72 & 4 / 7 / 06 & \text { CPT } & 16.3 & 7.62 & 1130 \\ & & & & & & & \\ \text { TI-3 } & \text { NATI3-W-20298 } & 32.2-37.2 & 4 / 7 / 06 & \text { CPT } & 15.6 & 7.25 & 3410 \\ \text { TI-3 } & \text { NATI3-W-20383 } & 43-48 & 4 / 14 / 06 & \text { CPT } & 16.2 & 7.49 & 1491 \\ \text { TI-3 } & \text { NATI3-W-20385 } & 51-56 & 4 / 14 / 06 & \text { CPT } & 16.7 & 7.59 & 800 \\ & & & & & & & 3450 \\ \text { TI-4 } & \text { NATI4-W-20325 } & 35-40 & 4 / 10 / 06 & \text { CPT } & 14.9 & 6.87 & 3450 \\ \text { TI-4 } & \text { NATI4-W-20388 } & 42-47 & 4 / 14 / 06 & \text { CPT } & 17.2 & 6.99 & 2640 \\ \text { TI-4 } & \text { NATI4-W-20469 } & 50-55 & 4 / 11 / 06 & \text { CPT } & 24.3 & 7.20 & 2410 \\ \text { TI-4 } & \text { NATI4-W-20470 } & 55-60 & 4 / 11 / 06 & \text { CPT } & 21.4 & 7.55 & 1254 \\ \text { TI-4 } & \text { NATI4-W-20472 } & 61-66 & 4 / 12 / 06 & \text { CPT } & 16.9 & 7.91 & 1349 \\ \text { TI-4 } & \text { NATI4-W-20474 } & 67-71 & 4 / 12 / 06 & \text { CPT } & 20.8 & 7.64 & 1508 \\ & & & & & & & \\ \text { TI-5 } & \text { NATI5-W-20373 } & 28-33 & 4 / 13 / 06 & \text { CPT } & \text { NR } & \text { NR } & \text { NR } \\ \text { TI-5 } & \text { NATI5-W-20368 } & 36-41 & 4 / 12 / 06 & \text { CPT } & 15.4 & 7.00 & 2190 \\ \text { TI-5 } & \text { NATI5-W-20371 } & 49-54 & 4 / 13 / 06 & \text { CPT } & 16.2 & 7.50 & 1057 \\ \text { TI-5 } & \text { NATI5-W-20267 } & 72.8-77.8 & 4 / 20 / 06 & \text { CPT } & 18.8 & 7.70 & 1694\end{array}$


TABLE S2.2 (Cont.)

\begin{tabular}{|c|c|c|c|c|c|c|c|}
\hline Location & Sample & $\begin{array}{l}\text { Depth } \\
\text { (ft BGL) }\end{array}$ & $\begin{array}{l}\text { Sample } \\
\text { Date }\end{array}$ & Type ${ }^{a}$ & $\begin{array}{c}\text { Temperature } \\
\left({ }^{\circ} \mathrm{C}\right)\end{array}$ & $\mathrm{pH}$ & $\begin{array}{c}\text { Conductivity } \\
(\mu \mathrm{S} / \mathrm{cm})\end{array}$ \\
\hline
\end{tabular}

Vertical-profile groundwater sampling at the former CCC/USDA grain storage facility (cont.)

\begin{tabular}{|c|c|c|c|c|c|c|c|}
\hline TI-6 & NATI6-W-20353 & $38-43$ & $4 / 22 / 06$ & CPT & NR & 7.58 & 1288 \\
\hline TI-6 & NATI6-W-20348 & $49-54$ & $4 / 21 / 06$ & CPT & 16.9 & 7.66 & 984 \\
\hline TI-6 & NATI6-W-20349 & $58-63$ & $4 / 22 / 06$ & CPT & 16.6 & 7.64 & 1374 \\
\hline TI-6 & NATI6-W-20350 & $68-73$ & $4 / 22 / 06$ & CPT & 17.6 & 7.35 & 1716 \\
\hline TI-7 & NATI7-W-20269 & 29.5-34.5 & $4 / 20 / 06$ & CPT & 20.9 & 7.18 & 4860 \\
\hline TI-7 & NATI7-W-20270 & $37-42$ & $4 / 20 / 06$ & CPT & 17.9 & 7.12 & 4740 \\
\hline TI-7 & NATI7-W-20272 & $45-50$ & $4 / 20 / 06$ & CPT & 19.1 & 7.13 & 4260 \\
\hline TI-7 & NATI7-W-20273 & $51-56$ & $4 / 21 / 06$ & CPT & 15.9 & 7.42 & 4980 \\
\hline TI-7 & NATI7-W-20274 & $68-72$ & $4 / 21 / 06$ & CPT & 19.8 & 7.77 & 1426 \\
\hline TI-8 & NATI8-W-20530 & $31-36$ & $4 / 28 / 06$ & CPT & 15.2 & 7.35 & 1058 \\
\hline TI-8 & NATI8-W-20531 & $39-44$ & $4 / 28 / 06$ & CPT & 14.9 & 7.47 & 86 \\
\hline TI-8 & NATI8-W-20641 & $45-50$ & $4 / 28 / 06$ & CPT & 16.3 & 7.80 & 5160 \\
\hline TI-8 & NATI8-W-20732 & $54-59$ & $5 / 2 / 06$ & CPT & 20.4 & 7.55 & 1147 \\
\hline TI-8 & NATI8-W-20731 & $64.5-69.5$ & $5 / 2 / 06$ & CPT & 19.8 & 7.55 & 1648 \\
\hline TI-9 & NATI9-W-20609 & $40-45$ & $4 / 24 / 06$ & CPT & 22.0 & 7.75 & 887 \\
\hline TI-9 & NATI9-W-20586 & $46-51$ & $4 / 21 / 06$ & CPT & 19.9 & 7.66 & 932 \\
\hline TI-9 & NATI9-W-20589 & $54-59$ & $4 / 23 / 06$ & CPT & 23.9 & 7.76 & 916 \\
\hline TI-9 & NATI9-W-20587 & $59-64$ & $4 / 22 / 06$ & CPT & 19.6 & 7.52 & 1160 \\
\hline TI-10 & NATI10-W-20631 & $40-45$ & $4 / 26 / 06$ & CPT & 15.4 & 7.64 & 880 \\
\hline TI-10 & NATI10-W-20632 & $46-51$ & $4 / 27 / 06$ & CPT & 16.4 & 7.59 & 85 \\
\hline TI-10 & NATI10-W-20635 & $54-59$ & $4 / 27 / 06$ & CPT & 17.1 & 7.67 & 866 \\
\hline TI-10 & NATI10-W-20637 & $59-64$ & $4 / 27 / 06$ & CPT & 18.3 & 7.51 & 991 \\
\hline TI-10 & NATI10-W-20610 & $66-71$ & $4 / 25 / 06$ & CPT & 17.3 & 7.69 & 1568 \\
\hline TI-11 & NATI11-W-20517 & $26-31$ & $4 / 26 / 06$ & CPT & NR & NR & NR \\
\hline TI-11 & NATI11-W-20523 & 33-38 & $4 / 27 / 06$ & CPT & NR & NR & $\mathrm{Nl}$ \\
\hline TI-11 & NATI11-W-20506 & $40-45$ & $4 / 25 / 06$ & CPT & NR & NR & $\mathrm{N}$ \\
\hline TI-11 & NATI11-W-20500 & $46.3-51.3$ & $4 / 23 / 06$ & CPT & 17.8 & 7.53 & 87 \\
\hline TI-11 & NATI11-W-20638 & $52.6-57.6$ & $4 / 27 / 06$ & CPT & 20.9 & 7.56 & 901 \\
\hline TI-11 & NATI11-W-20639 & $65.9-70.9$ & $4 / 27 / 06$ & СРT & 19.8 & 7.48 & 1215 \\
\hline TI-12 & NATI12-W-20727 & $34-39$ & $5 / 2 / 06$ & CPT & 20.5 & 7.42 & 96 \\
\hline TI-12 & NATI12-W-20728 & $39.4-44.4$ & $5 / 2 / 06$ & CPT & 21.2 & 7.70 & 911 \\
\hline TI-12 & NATI12-W-20640 & $46-51$ & $4 / 28 / 06$ & CPT & 16.7 & NR & 111 \\
\hline TI-12 & NATI12-W-20757 & $52-57$ & $5 / 4 / 06$ & CPT & 16.3 & 7.41 & 85 \\
\hline TI-12 & NATI12-W-20758 & $63.7-68.7$ & $5 / 4 / 06$ & CPT & 16.6 & 7.89 & 916 \\
\hline
\end{tabular}

Vertical-profile groundwater sampling on and downgradient from the Co-op property

$\begin{array}{llcllllr}\text { TI-13 } & \text { NATI13-W-20819 } & 25-30 & 5 / 9 / 06 & \text { CPT } & 15.7 & 7.54 & 1577 \\ \text { TI-13 } & \text { NATI13-W-20563 } & 35-40 & 5 / 5 / 06 & \text { CPT } & 13.2 & 7.51 & 1007 \\ \text { TI-13 } & \text { NATI13-W-20562 } & 42-47 & 5 / 5 / 06 & \text { CPT } & 17.6 & 7.31 & 1055 \\ \text { TI-13 } & \text { NATI13-W-20560 } & 48-53 & 5 / 4 / 06 & \text { CPT } & 18.1 & 7.25 & 974 \\ \text { TI-13 } & \text { NATI13-W-20561 } & 54-59 & 5 / 5 / 06 & \text { CPT } & 15.4 & 7.36 & 1165 \\ \text { TI-13 } & \text { NATI13-W-20555 } & 66.8-71.8 & 5 / 4 / 06 & \text { CPT } & 14.8 & 7.49 & 3910\end{array}$


TABLE S2.2 (Cont.)

\begin{tabular}{|c|c|c|c|c|c|c|c|}
\hline Location & Sample & $\begin{array}{l}\text { Depth } \\
\text { (ft BGL) }\end{array}$ & $\begin{array}{c}\text { Sample } \\
\text { Date }\end{array}$ & Type $^{a}$ & $\begin{array}{c}\text { Temperature } \\
\left({ }^{\circ} \mathrm{C}\right)\end{array}$ & $\mathrm{pH}$ & $\begin{array}{l}\text { Conductivity } \\
(\mu \mathrm{S} / \mathrm{cm})\end{array}$ \\
\hline
\end{tabular}

Vertical-profile groundwater sampling on and downgradient from the Co-op property (cont.)

\begin{tabular}{|c|c|c|c|c|c|c|c|}
\hline TI-14 & NATI14-W-20693 & $26-31$ & $5 / 6 / 06$ & CPT & 16.1 & 7.06 & 1436 \\
\hline TI-14 & NATI14-W-20695 & $32-37$ & $5 / 6 / 06$ & CPT & 16.5 & 6.74 & 9720 \\
\hline TI-14 & NATI14-W-20768 & $38-43$ & $5 / 6 / 06$ & CPT & 16.8 & 7.04 & 1192 \\
\hline TI-14 & NATI14-W-20691 & $47-52$ & $5 / 6 / 06$ & CPT & 16.2 & 7.05 & 7610 \\
\hline TI-14 & NATI14-W-20692 & $54-59$ & $5 / 6 / 06$ & CPT & 15.9 & 7.09 & 3860 \\
\hline TI-14 & NATI14-W-20666 & $67.4-72.4$ & $5 / 5 / 06$ & CPT & 17.7 & 7.11 & 4980 \\
\hline TI-15 & NATI15-W-20644 & $15-20$ & $5 / 8 / 06$ & CPT & 15.2 & 7.10 & 1277 \\
\hline TI-15 & NATI15-W-20822 & $25-30$ & $5 / 9 / 06$ & CPT & 18.2 & 7.42 & 787 \\
\hline TI-15 & NATI15-W-20765 & $35-40$ & $5 / 7 / 06$ & CPT & 13.4 & 7.5 & 1010 \\
\hline TI-15 & NATI15-W-20764 & $40-45$ & $5 / 6 / 06$ & CPT & 14.9 & 7.45 & 320 \\
\hline TI-15 & NATI15-W-20763 & $47-52$ & $5 / 6 / 06$ & CPT & 15.4 & 7.28 & 1123 \\
\hline TI-15 & NATI15-W-20761 & $67.1-72.1$ & $5 / 6 / 06$ & CPT & 15.9 & 7.09 & 3860 \\
\hline TI-16 & NATI16-W-20818 & $25-30$ & $5 / 9 / 06$ & CPT & 16.9 & 7.35 & 3650 \\
\hline TI-16 & NATI16-W-20817 & $32-37$ & $5 / 9 / 06$ & CPT & 16.5 & 7.22 & 3680 \\
\hline TI-16 & NATI16-W-20791 & $39-44$ & $5 / 8 / 06$ & CPT & 17.3 & 7.10 & 6720 \\
\hline TI-16 & NATI16-W-20816 & $46-51$ & $5 / 9 / 06$ & CPT & 16.0 & 7.37 & 1229 \\
\hline TI-16 & NATI16-W-20789 & $60-65$ & $5 / 8 / 06$ & CPT & 16.9 & 7.85 & 1853 \\
\hline TI-16 & NATI16-W-20790 & $67-72$ & $5 / 8 / 06$ & CPT & 17.7 & 7.69 & 1958 \\
\hline TI-17 & NATI17-W-20825 & $25-30$ & $5 / 9 / 06$ & CPT & 19.3 & 7.47 & 993 \\
\hline TI-17 & NATI17-W-20793 & $32-37$ & $5 / 8 / 06$ & CPT & NR & NR & NR \\
\hline TI-17 & NATI17-W-20826 & $39-44$ & $5 / 9 / 06$ & CPT & 18.9 & 7.37 & 1397 \\
\hline TI-17 & NATI17-W-20814 & $46-51$ & $5 / 9 / 06$ & CPT & 15.1 & 7.53 & 1266 \\
\hline TI-17 & NATI17-W-20829 & $53-58$ & $5 / 9 / 06$ & CPT & 20.1 & 7.38 & 1398 \\
\hline TI-17 & NATI17-W-20828 & $60-65$ & $5 / 9 / 06$ & CPT & 19.5 & 7.33 & 1397 \\
\hline TI-18 & NATI18-W-20921 & $25-30$ & $5 / 23 / 06$ & CPT & NR & NR & NR \\
\hline TI-18 & NATI18-W-20655 & $30-35$ & $5 / 21 / 06$ & CPT & 19.0 & 7.62 & NR \\
\hline TI-18 & NATI18-W-20831 & $35-40$ & $5 / 10 / 06$ & CPT & 11.6 & 6.74 & 13550 \\
\hline TI-18 & NATI18-W-20706 & $42-47$ & $5 / 21 / 06$ & CPT & 18.6 & 6.77 & 1363 \\
\hline TI-18 & NATI18-W-20707 & $49-54$ & $5 / 21 / 06$ & CPT & 19.6 & 6.79 & 1445 \\
\hline TI-18 & NATI18-W-20709 & $56-61$ & $5 / 21 / 06$ & CPT & 19.3 & 6.94 & 6810 \\
\hline TI-18 & NATI18-W-20711 & $66.5-71.5$ & $5 / 22 / 06$ & CPT & 17.6 & 7.41 & 1681 \\
\hline TI-19 & NATI19-W-20231 & $25-30$ & $5 / 11 / 06$ & CPT & 13.1 & 4.70 & 5160 \\
\hline TI-19 & NATI19-W-20863 & $32-37$ & $5 / 11 / 06$ & CPT & 14.7 & 7.52 & 1662 \\
\hline TI-19 & NATI19-W-20864 & 39-44 & $5 / 11 / 06$ & CPT & 15.2 & 7.45 & 1250 \\
\hline TI-19 & NATI19-W-20870 & $46-51$ & $5 / 11 / 06$ & CPT & 15.3 & 7.41 & 1703 \\
\hline TI-19 & NATI19-W-20868 & $53-58$ & $5 / 11 / 06$ & CPT & 15.9 & 7.61 & 1837 \\
\hline TI-19 & NATI19-W-20869 & $60-65$ & $5 / 11 / 06$ & CPT & 15.3 & 7.63 & 691 \\
\hline TI-19 & NATI19-W-20872 & $65.5-69.5$ & $5 / 11 / 06$ & CPT & 14.8 & 7.66 & 1237 \\
\hline TI-20 & NATI20-W-20913 & $35-40$ & $5 / 22 / 06$ & CPT & 19.1 & 7.31 & 1328 \\
\hline TI-20 & NATI20-W-20713 & $42-47$ & $5 / 24 / 06$ & CPT & NR & NR & NR \\
\hline TI-20 & NATI20-W-20914 & $56-61$ & $5 / 23 / 06$ & CPT & 19.6 & 7.95 & 1674 \\
\hline TI-20 & NATI20-W-20922 & $72-77$ & $5 / 23 / 06$ & CPT & 19.2 & 7.20 & 2460 \\
\hline
\end{tabular}


TABLE S2.2 (Cont.)

\begin{tabular}{|c|c|c|c|c|c|c|c|}
\hline Location & Sample & $\begin{array}{l}\text { Depth } \\
\text { (ft BGL) }\end{array}$ & $\begin{array}{l}\text { Sample } \\
\text { Date }\end{array}$ & Type $^{a}$ & $\begin{array}{c}\text { Temperature } \\
\left({ }^{\circ} \mathrm{C}\right)\end{array}$ & $\mathrm{pH}$ & $\begin{array}{l}\text { Conductivity } \\
(\mu \mathrm{S} / \mathrm{cm})\end{array}$ \\
\hline
\end{tabular}

Vertical-profile groundwater sampling on and downgradient from the Co-op property (cont.)

\begin{tabular}{|c|c|c|c|c|c|c|c|}
\hline TI-21 & NATI21-W-20228 & $25-30$ & $5 / 11 / 06$ & CPT & NR & NR & $\mathrm{NF}$ \\
\hline TI-21 & NATI21-W-20885 & $32-37$ & $5 / 11 / 06$ & CPT & NR & NR & \\
\hline TI-21 & NATI21-W-20882 & $39-44$ & $5 / 11 / 06$ & CPT & 14.6 & 7.40 & 132 \\
\hline TI-21 & NATI21-W-20881 & $46-51$ & $5 / 11 / 06$ & CPT & 15.3 & 7.44 & 12 \\
\hline TI-21 & NATI21-W-20867 & $53-58$ & $5 / 11 / 06$ & CPT & 17.4 & 7.35 & \\
\hline TI-21 & NATI21-W-20880 & $60-65$ & $5 / 11 / 06$ & CPT & 16.6 & 7.58 & 12 \\
\hline TI-22 & NATI22-W-20833 & $32-37$ & $5 / 10 / 06$ & CPT & 11.8 & 7.27 & \\
\hline TI-22 & NATI22-W-20834 & $39-44$ & $5 / 10 / 06$ & CPT & 12.1 & 7.62 & \\
\hline TI-22 & NATI22-W-20830 & $46-51$ & $5 / 9 / 06$ & CPT & 20.0 & 7.20 & $17 a$ \\
\hline TI-22 & NATI22-W-20836 & $53-58$ & $5 / 10 / 06$ & CPT & 15.4 & 7.58 & \\
\hline TI-22 & NATI22-W-20840 & $60-65$ & $5 / 10 / 06$ & CPT & 12.4 & 7.81 & \\
\hline TI-22 & NATI22-W-20846 & $68.2-73.2$ & $5 / 10 / 06$ & CPT & 15.3 & 7.68 & 25 \\
\hline TI-23 & NATI23-W-20844 & $32-37$ & $5 / 10 / 06$ & CPT & 15.9 & 7.89 & \\
\hline TI-23 & NATI23-W-20861 & $39-44$ & $5 / 11 / 06$ & CPT & 16.7 & 7.37 & 16 \\
\hline TI-23 & NATI23-W-20862 & $46-51$ & $5 / 11 / 06$ & CPT & NR & NR & \\
\hline TI-23 & NATI23-W-20865 & $53-58$ & $5 / 11 / 06$ & CPT & 15.3 & 7.41 & 170 \\
\hline TI-23 & NATI23-W-20856 & $60-65$ & $5 / 10 / 06$ & CPT & 15.4 & 7.92 & 105 \\
\hline TI-23 & NATI23-W-20859 & $66.8-71.8$ & $5 / 10 / 06$ & CPT & 16.0 & 7.72 & 15 \\
\hline TI-24 & NATI24-W-20860 & $23-30$ & $5 / 11 / 06$ & CPT & NR & NR & \\
\hline TI-24 & NATI24-W-20858 & $32-37$ & $5 / 10 / 06$ & CPT & 18.5 & 7.50 & 126 \\
\hline TI-24 & NATI24-W-20838 & 39-44 & $5 / 10 / 06$ & CPT & 17.3 & 7.29 & 142 \\
\hline TI-24 & NATI24-W-20841 & $46-51$ & $5 / 10 / 06$ & CPT & 15.8 & 7.52 & 12 \\
\hline TI-24 & NATI24-W-20842 & $53-58$ & $5 / 10 / 06$ & CPT & 17.9 & 7.38 & 129 \\
\hline TI-24 & NATI24-W-20843 & $60-65$ & $5 / 10 / 06$ & CPT & 16.5 & 7.45 & $15 \varepsilon$ \\
\hline TI-24 & NATI24-W-20845 & $69.4-74.4$ & $5 / 10 / 06$ & CPT & 16.2 & 7.61 & 170 \\
\hline TI-25 & NATI25-W-20722 & $32-37$ & $5 / 12 / 06$ & CPT & 16.3 & 7.22 & 122 \\
\hline TI-25 & NATI25-W-20875 & $39-44$ & $5 / 11 / 06$ & CPT & 17.6 & 7.85 & 145 \\
\hline TI-25 & NATI25-W-20878 & $46-51$ & $5 / 11 / 06$ & CPT & 15.9 & 7.30 & 131 \\
\hline TI-25 & NATI25-W-20884 & $53-58$ & $5 / 11 / 06$ & CPT & 15.9 & 7.48 & 1301 \\
\hline TI-25 & NATI25-W-20232 & $60-65$ & $5 / 12 / 06$ & CPT & 13.7 & 7.76 & 119 \\
\hline TI-25 & NATI25-W-20720 & $66-71$ & $5 / 12 / 06$ & CPT & NR & NR & \\
\hline TI-25 & NATI25-W-20235 & $72.2-77.2$ & $5 / 12 / 06$ & CPT & 15.0 & 7.55 & 118 \\
\hline TI-26 & NATI26-W-20721 & $25-30$ & $5 / 12 / 06$ & CPT & NR & NR & \\
\hline TI-26 & NATI26-W-20877 & $32-37$ & $5 / 11 / 06$ & CPT & 15.5 & 7.58 & 25 \\
\hline TI-26 & NATI26-W-20883 & $39-44$ & $5 / 11 / 06$ & CPT & 16.8 & 7.74 & 336 \\
\hline TI-26 & NATI26-W-20723 & $46-51$ & $5 / 12 / 06$ & CPT & 15.2 & 7.69 & 150 \\
\hline TI-26 & NATI26-W-20229 & $53-58$ & $5 / 11 / 06$ & CPT & 14.9 & 7.69 & 170 \\
\hline TI-26 & NATI26-W-20233 & $60-65$ & $5 / 12 / 06$ & CPT & 14.3 & 7.72 & 153 \\
\hline TI-26 & NATI26-W-20719 & $66-71$ & $5 / 12 / 06$ & CPT & 14.8 & 7.68 & 165 \\
\hline TI-26 & NATI26-W-20718 & $71.8-76.8$ & $5 / 12 / 06$ & CPT & 15.6 & 7.74 & 192 \\
\hline TI-27 & NATI27-W-20680 & $25-30$ & $5 / 19 / 06$ & CPT & NR & NR & \\
\hline TI-27 & NATI27-W-20704 & $30-35$ & $5 / 20 / 06$ & CPT & NR & NR & \\
\hline TI-27 & NATI27-W-20684 & $35-40$ & $5 / 19 / 06$ & CPT & 20.5 & 7.01 & $3<6$ \\
\hline TI-27 & NATI27-W-20699 & $42-47$ & $5 / 20 / 06$ & CPT & 16.3 & 7.63 & \\
\hline
\end{tabular}


TABLE S2.2 (Cont.)

\begin{tabular}{lccccc}
\hline Location & Sample & $\begin{array}{c}\text { Depth } \\
(\mathrm{ft} \mathrm{BGL})\end{array}$ & $\begin{array}{c}\text { Sample } \\
\text { Date }\end{array}$ & $\operatorname{Type}^{\mathrm{a}}$ & $\begin{array}{c}\text { Temperature } \\
\left({ }^{\circ} \mathrm{C}\right)\end{array}$ \\
$\mathrm{pH}$ & $\begin{array}{c}\text { Conductivity } \\
(\mu \mathrm{S} / \mathrm{cm})\end{array}$ \\
\hline
\end{tabular}

Vertical-profile groundwater sampling on and downgradient from the Co-op property (cont.)

\begin{tabular}{|c|c|c|c|c|c|c|c|}
\hline TI-27 & NATI27-W-20700 & $49-54$ & $5 / 20 / 06$ & CPT & 18.7 & 7.53 & 955 \\
\hline TI-27 & NATI27-W-20905 & $56-61$ & $5 / 21 / 06$ & CPT & 21.6 & 7.58 & 2150 \\
\hline TI-27 & NATI27-W-20649 & $66.2-71.2$ & $5 / 20 / 06$ & CPT & 18.2 & 7.39 & 1324 \\
\hline TI-28 & NATI28-W-20904 & $25-30$ & $5 / 21 / 06$ & CPT & 21.2 & 7.26 & 5720 \\
\hline TI-28 & NATI28-W-20851 & $32-37$ & $5 / 20 / 06$ & CPT & 15.2 & 7.23 & 4490 \\
\hline TI-28 & NATI28-W-20849 & $37-42$ & $5 / 20 / 06$ & CPT & 16.3 & 7.16 & 1675 \\
\hline TI-28 & NATI28-W-20850 & $44-49$ & $5 / 20 / 06$ & CPT & 15.0 & 7.92 & 2100 \\
\hline TI-28 & NATI28-W-20683 & $51-56$ & $5 / 19 / 06$ & CPT & 17.8 & 7.86 & 1313 \\
\hline TI-28 & NATI28-W-20254 & $58-63$ & $5 / 19 / 06$ & CPT & 17.2 & 7.10 & 1644 \\
\hline TI-28 & NATI28-W-20647 & $63-68$ & $5 / 19 / 06$ & CPT & 17.1 & 7.20 & 2140 \\
\hline TI-29 & NATI29-W-20916 & $25-30$ & $5 / 23 / 06$ & CPT & 18.6 & 6.93 & 1628 \\
\hline TI-29 & NATI29-W-20909 & $32-37$ & $5 / 22 / 06$ & CPT & 19.3 & 8.41 & 1284 \\
\hline TI-29 & NATI29-W-20900 & $39-44$ & $5 / 21 / 06$ & CPT & 18.2 & 7.76 & 7540 \\
\hline TI-29 & NATI29-W-20902 & $46-51$ & $5 / 21 / 06$ & CPT & 18.6 & 7.16 & 7700 \\
\hline TI-29 & NATI29-W-20903 & $53-58$ & $5 / 21 / 06$ & CPT & NR & NR & NR \\
\hline TI-29 & NATI29-W-20906 & $60-65$ & $5 / 21 / 06$ & CPT & 20.0 & 7.61 & 3720 \\
\hline TI-29 & NATI29-W-20907 & $66.3-69.3$ & $5 / 21 / 06$ & CPT & 16.8 & 7.57 & 2350 \\
\hline TI-30 & NATI30-W-20939 & $32-37$ & $5 / 25 / 06$ & CPT & NR & NR & NR \\
\hline TI-30 & NATI30-W-20920 & $39-44$ & $5 / 23 / 06$ & CPT & NR & NR & NR \\
\hline TI-30 & NATI30-W-20915 & $43.8-48.8$ & $5 / 23 / 06$ & CPT & 18.6 & 7.57 & 1036 \\
\hline TI-30 & NATI30-W-20911 & $53-58$ & $5 / 23 / 06$ & CPT & 14.9 & 7.85 & 978 \\
\hline TI-30 & NATI30-W-20918 & $60-65$ & $5 / 23 / 06$ & CPT & 19.4 & 7.70 & 1230 \\
\hline TI-30 & NATI30-W-20917 & $75-80$ & $5 / 23 / 06$ & CPT & 15.8 & 7.69 & 1882 \\
\hline TI-30 & NATI30-W-20712 & $83.5-88.5$ & $5 / 24 / 06$ & CPT & 16.6 & 7.57 & 3740 \\
\hline TI-31 & NATI31-W-20947 & $42-47$ & $5 / 25 / 06$ & CPT & 16.2 & 7.62 & 1374 \\
\hline TI-31 & NATI31-W-20938 & $53-58$ & $5 / 25 / 06$ & CPT & 15.4 & 7.56 & 767 \\
\hline TI-31 & NATI31-W-20937 & $60-65$ & $5 / 25 / 06$ & CPT & 15.6 & 7.57 & 2750 \\
\hline TI-31 & NATI31-W-20715 & $70.7-75.7$ & $5 / 24 / 06$ & CPT & 19.8 & 7.47 & 3730 \\
\hline
\end{tabular}

Sampling of monitoring well installed at the TI-30 location during the 2006 investigation

$\begin{array}{llllllll}\text { MW5 } & \text { NATI30-W-20946 } & 78-88 & 5 / 25 / 06 & \text { CPT/P } & 19.1 & 7.81 & 2360\end{array}$

a Types: CPT, cone penetrometer; DW, domestic well; MW, monitoring well; P, piezometer.

b NR, not recorded.

c Total depth. 
TABLE S2.3 Organic results for water samples collected during the 2006 investigation at Navarre, Kansas.

Concentration $(\mu \mathrm{g} / \mathrm{L})$

$\begin{array}{ccccc} & \text { Depth } & \text { Sample Sample } & \text { Carbon } & \text { Methylene } \\ \text { Location } & \text { Sample } & \text { (ft below TOC) } & \text { Date } & \text { Type } \\ & & & \text { Tetrachloride Chloroform Chloride chloroethene Benzene Toluene }\end{array}$

\section{Existing well sampling}

$\begin{array}{ll}\text { Co-op 1 } & \text { NACOOP1-W-20217 } \\ \text { Co-op 3 } & \text { NACOOP3-W-20218 } \\ \text { KDHE-1 } & \text { NAKDHE1-W-20203 } \\ \text { KDHE-2 } & \text { NAKDHE2-W-20205 } \\ \text { MW1 } & \text { NAMW01-W-20186 } \\ \text { MW2 } & \text { NAMW02-W-20188 } \\ \text { MW3 } & \text { NAMW03-W-20192 } \\ \text { MW4 } & \text { NAMW04-W-20194 } \\ \text { T1 } & \text { NAT1-W-20201 } \\ \text { L-1 } & \text { NAL1-W-20211 } \\ \text { L-2 } & \text { NAL2-W-20190 } \\ \text { L-3 } & \text { NAL3-W-20209 } \\ \text { NW-1 } & \text { NANW1-W-20215 } \\ \text { NW-2 } & \text { NANW2-W-20219 } \\ \text { NW-3 } & \text { NANW3-W-20945 } \\ \text { Anderson } & \text { NAANDER1-W-20941 } \\ \text { Anderson } & \text { NAANDER2-W-20942 } \\ \text { Anderson } & \text { NAANDER3-W-20943 }\end{array}$

$\begin{array}{ccccccc}\text { Unknown } & 4 / 8 / 06 & \text { DW } & 1.2 & 0.8 \mathrm{~J}^{\mathrm{b}} & \mathrm{ND}^{\mathrm{c}} & \mathrm{ND} \\ \text { Unknown } & 4 / 8 / 06 & \text { DW } & 197 & 44 & 3.6 & 0.2 \mathrm{~J} \\ 35-55 & 4 / 7 / 06 & \text { MW } & 0.1 \mathrm{~J} & \text { ND } & \text { ND } & \text { ND } \\ 25-45 & 4 / 7 / 06 & \text { MW } & \text { ND } & \text { ND } & \text { ND } & \text { ND } \\ 43-58 & 4 / 5 / 06 & \text { MW } & 59 & 12 & \text { ND } & \text { ND } \\ 42.8-57.8 & 4 / 6 / 06 & \text { MW } & 27 & 7.7 & \text { ND } & \text { ND } \\ 44-59 & 4 / 6 / 06 & \text { MW } & 83 & 20 & \text { ND } & 0.1 \mathrm{~J} \\ 45-60 & 4 / 6 / 06 & \text { MW } & 194 & 21 & \text { ND } & \text { ND } \\ 40-60 & 4 / 7 / 06 & \text { MW } & 118 & 20 & \text { ND } & \text { ND } \\ 75-95 & 4 / 8 / 06 & \text { MW } & 54 & 11 & \text { ND } & \text { ND } \\ 80-90 & 4 / 6 / 06 & \text { MW } & 0.2 \mathrm{~J} & \text { ND } & \text { ND } & \text { ND } \\ 80-90 & 4 / 7 / 06 & \text { MW } & \text { ND } & \text { ND } & \text { ND } & \text { ND } \\ 40-50 & 4 / 8 / 06 & \text { MW } & 1.1 & 0.3 \mathrm{~J} & \text { ND } & \text { ND } \\ 35.5-45.5 & 4 / 9 / 06 & \text { MW } & 313 & 74 & 3.2 & 0.2 \mathrm{~J} \\ 38-48 & 5 / 25 / 06 & \text { MW } & 34 & 2.0 & \text { ND } & \text { ND } \\ 68^{\mathrm{d}} & 5 / 23 / 06 & \text { DW } & 36 & 6.0 & \text { ND } & \text { ND } \\ 68^{\mathrm{d}} & 5 / 24 / 06 & \text { DW } & 20 & 3.5 & \text { ND } & \text { ND } \\ 68^{\mathrm{d}} & 5 / 25 / 06 & \text { DW } & 17 & 2.7 & \text { ND } & \text { ND }\end{array}$

Vertical-profile groundwater sampling at the former CCC/USDA facility

$\begin{array}{lrrrrrrrr}\text { TI-1 } & \text { NATI1-W-20341 } & 30-35 & 4 / 13 / 06 & \text { CPT } & 181 & 60 & 10 & 0.2 \mathrm{~J} \\ \text { TI-1 } & \text { NATI1-W-20342 } & 38-43 & 4 / 13 / 06 & \text { CPT } & 177 & 64 & 0.2 \mathrm{~J} \\ \text { TI-1 } & \text { NATI1-W-20344 } & 50-55 & 4 / 13 / 06 & \text { CPT } & 127 & 56 & 8.2 & \text { ND } \\ \text { TI-1 } & \text { NATI1-W-20266 } & 67-71 & 4 / 14 / 06 & \text { CPT } & \text { ND } & \text { ND } & \text { ND } & \text { ND } \\ & & & & & & & \text { ND } & \text { ND } \\ \text { TI-2 } & \text { NATI2-W-20276 } & 25-30 & 4 / 21 / 06 & \text { CPT } & 2.2 & 2.0 & \text { ND } & \text { ND } \\ \text { TI-2 } & \text { NATI2-W-20277 } & 31-36 & 4 / 21 / 06 & \text { CPT } & 8.7 & 7.8 & \end{array}$


TABLE S2.3 (Cont.)

Concentration $(\mu \mathrm{g} / \mathrm{L})$

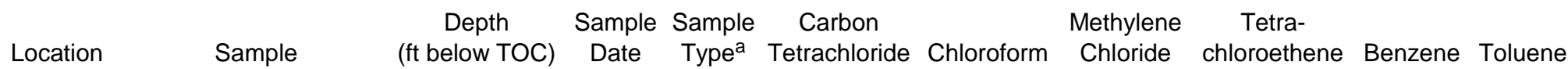

Vertical-profile groundwater sampling at the former CCC/USDA facility (cont.)

\begin{tabular}{|c|c|c|c|c|c|c|c|c|}
\hline TI-2 & NATI2-W-20279 & $37-42$ & $4 / 21 / 06$ & CPT & 77 & 9.2 & ND & $<0.1$ \\
\hline TI-2 & NATI2-W-20281 & $49-54$ & $4 / 21 / 06$ & CPT & 9.8 & 2.7 & ND & ND \\
\hline TI-2 & NATI2-W-20282 & $66-70$ & $4 / 21 / 06$ & CPT & 14 & 2.8 & ND & ND \\
\hline TI-2 & NATI2-W-20432 & $69-72$ & $4 / 7 / 06$ & СРТ & ND & ND & ND & ND \\
\hline TI-3 & NATI3-W-20298 & $32.2-37.2$ & $4 / 7 / 06$ & CPT & 27 & 7.9 & ND & ND \\
\hline TI-3 & NATI3-W-20383 & $43-48$ & $4 / 14 / 06$ & CPT & 72 & 12 & ND & ND \\
\hline TI-3 & NATI3-W-20385 & $51-56$ & $4 / 14 / 06$ & CPT & 7.6 & 1.6 & ND & ND \\
\hline TI-4 & NATI4-W-20325 & $35-40$ & $4 / 10 / 06$ & CPT & 76 & 55 & 3.6 & $0.2 \mathrm{~J}$ \\
\hline $\mathrm{TI}-4$ & NATI4-W-20388 & $42-47$ & $4 / 14 / 06$ & CPT & 99 & 75 & 6.1 & ND \\
\hline TI-4 & NATI4-W-20469 & $50-55$ & 4/11/06 & CPT & 40 & 58 & $0.8 \mathrm{~J}$ & $0.2 \mathrm{~J}$ \\
\hline TI-4 & NATI4-W-20470 & $55-60$ & 4/11/06 & CPT & 23 & 7.0 & ND & $<0.1$ \\
\hline TI-4 & NATI4-W-20472 & $61-66$ & $4 / 12 / 06$ & CPT & $0.6 \mathrm{~J}$ & 3.6 & ND & ND \\
\hline TI-4 & NATI4-W-20474 & $67-71$ & $4 / 12 / 06$ & СРT & ND & ND & ND & ND \\
\hline TI-5 & NATI5-W-20373 & $28-33$ & $4 / 13 / 06$ & СPT & 1.1 & 1.2 & ND & ND \\
\hline TI-5 & NATI5-W-20368 & $36-41$ & $4 / 12 / 06$ & CPT & 1.1 & 2.0 & ND & ND \\
\hline TI-5 & NATI5-W-20371 & $49-54$ & 4/13/06 & СPT & 6.3 & $0.4 \mathrm{~J}$ & ND & ND \\
\hline TI-5 & NATI5-W-20267 & $72.8-77.8$ & $4 / 20 / 06$ & CPT & ND & ND & ND & ND \\
\hline TI-6 & NATI6-W-20353 & $38-43$ & $4 / 22 / 06$ & CPT & 1.3 & 1.0 & ND & ND \\
\hline TI-6 & NATI6-W-20348 & $49-54$ & $4 / 21 / 06$ & CPT & $0.4 \mathrm{~J}$ & ND & ND & ND \\
\hline TI-6 & NATI6-W-20349 & $58-63$ & $4 / 22 / 06$ & CPT & 1.5 & $0.1 \mathrm{~J}$ & ND & ND \\
\hline TI-6 & NATI6-W-20350 & $68-73$ & $4 / 22 / 06$ & CPT & ND & ND & ND & ND \\
\hline TI-7 & NATI7-W-20269 & $29.5-34.5$ & $4 / 20 / 06$ & CPT & 3.8 & 1.7 & ND & ND \\
\hline TI-7 & NATI7-W-20270 & $37-42$ & $4 / 20 / 06$ & CPT & 3.8 & 1.9 & ND & ND \\
\hline TI-7 & NATI7-W-20272 & $45-50$ & $4 / 20 / 06$ & СPT & 5.6 & 2.7 & ND & ND \\
\hline TI-7 & NATI7-W-20273 & $51-56$ & $4 / 21 / 06$ & CPT & 1.9 & 2.0 & ND & ND \\
\hline TI-7 & NATI7-W-20274 & $68-72$ & $4 / 21 / 06$ & CPT & $0.5 \mathrm{~J}$ & $0.3 \mathrm{~J}$ & ND & ND \\
\hline
\end{tabular}


TABLE S2.3 (Cont.)

Concentration $(\mu \mathrm{g} / \mathrm{L})$

\begin{tabular}{|c|c|c|c|c|c|c|c|}
\hline Location & Sample & $\begin{array}{c}\text { Depth } \\
\text { (ft below TOC) }\end{array}$ & $\begin{array}{cc}\text { Sample } & \text { Sample } \\
\text { Date } & \text { Type }^{a}\end{array}$ & $\begin{array}{l}\text { Carbon } \\
\text { Tetrachloride }\end{array}$ & Chloroform & $\begin{array}{l}\text { Methylene } \\
\text { Chloride }\end{array}$ & $\begin{array}{l}\text { Tetra- } \\
\text { chloroethene Benzene Toluene }\end{array}$ \\
\hline
\end{tabular}

Vertical-profile groundwater sampling at the former CCC/USDA facility (cont.)

\begin{tabular}{|c|c|c|c|c|c|c|c|c|c|c|}
\hline TI-8 & NATI8-W-20530 & $31-36$ & 4/28/06 & CPT & $0.1 \mathrm{~J}$ & ND & ND & ND & & \\
\hline TI-8 & NATI8-W-20531 & $39-44$ & 4/28/06 & CPT & $0.2 \mathrm{~J}$ & ND & ND & ND & & \\
\hline TI-8 & NATI8-W-20641 & $45-50$ & $4 / 28 / 06$ & CPT & $0.2 \mathrm{~J}$ & ND & ND & ND & & \\
\hline TI-8 & NATI8-W-20732 & $54-59$ & $5 / 2 / 06$ & CPT & $0.5 \mathrm{~J}$ & ND & ND & ND & & \\
\hline TI-8 & NATI8-W-20731 & $64.5-69.5$ & $5 / 2 / 06$ & CPT & ND & ND & ND & ND & & \\
\hline TI-9 & NATI9-W-20609 & $40-45$ & $4 / 24 / 06$ & CPT & ND & ND & ND & ND & & \\
\hline TI-9 & NATI9-W-20586 & $46-51$ & $4 / 21 / 06$ & CPT & 1.2 & ND & ND & ND & & \\
\hline TI-9 & NATI9-W-20589 & $54-59$ & $4 / 23 / 06$ & CPT & $0.8 \mathrm{~J}$ & ND & ND & ND & & \\
\hline TI-9 & NATI9-W-20587 & $59-64$ & $4 / 22 / 06$ & CPT & 1.5 & ND & ND & ND & & \\
\hline TI-10 & NATI10-W-20631 & $40-45$ & $4 / 26 / 06$ & CPT & $0.4 \mathrm{~J}$ & ND & 36 & ND & 41 & 27 \\
\hline TI-10 & NATI10-W-20632 & $46-51$ & $4 / 27 / 06$ & CPT & $0.4 \mathrm{~J}$ & ND & 11 & ND & 25 & 11 \\
\hline $\mathrm{TI}-10$ & NATI10-W-20635 & $54-59$ & $4 / 27 / 06$ & CPT & $0.4 \mathrm{~J}$ & ND & ND & ND & 2.4 & 0 \\
\hline TI-10 & NATI10-W-20637 & $59-64$ & $4 / 27 / 06$ & CPT & $0.3 \mathrm{~J}$ & ND & ND & ND & 1.9 & 1.1 \\
\hline TI-10 & NATI10-W-20610 & $66-71$ & $4 / 25 / 06$ & CPT & ND & ND & ND & ND & & \\
\hline $\mathrm{TI}-11$ & NATI11-W-20517 & $26-31$ & 4/26/06 & CPT & ND & ND & ND & ND & & \\
\hline $\mathrm{TI}-11$ & NATI11-W-20523 & $33-38$ & $4 / 27 / 06$ & CPT & ND & $0.2 \mathrm{~J}$ & 9.5 & ND & 60 & 1 \\
\hline TI-11 & NATI11-W-20506 & $40-45$ & $4 / 25 / 06$ & CPT & ND & ND & ND & ND & & \\
\hline TI-11 & NATI11-W-20500 & $46.3-51.3$ & 4/23/06 & CPT & $0.9 \mathrm{~J}$ & $0.1 \mathrm{~J}$ & ND & ND & & \\
\hline TI-11 & NATI11-W-20638 & $52.6-57.6$ & $4 / 27 / 06$ & CPT & $0.6 \mathrm{~J}$ & ND & ND & ND & & \\
\hline TI-11 & NATI11-W-20639 & $65.9-70.9$ & $4 / 27 / 06$ & CPT & $0.4 \mathrm{~J}$ & ND & ND & ND & & \\
\hline TI-12 & NATI12-W-20727 & $34-39$ & $5 / 2 / 06$ & CPT & ND & ND & ND & ND & & \\
\hline TI-12 & NATI12-W-20728 & $39.4-44.4$ & $5 / 2 / 06$ & CPT & ND & ND & ND & ND & & \\
\hline TI-12 & NATI12-W-20640 & $46-51$ & 4/28/06 & CPT & ND & ND & ND & ND & & \\
\hline TI-12 & NATI12-W-20757 & $52-57$ & $5 / 4 / 06$ & CPT & ND & ND & ND & ND & & \\
\hline TI-12 & NATI12-W-20758 & $63.7-68.7$ & $5 / 4 / 06$ & CPT & ND & ND & ND & ND & & \\
\hline
\end{tabular}


TABLE S2.3 (Cont.)

Concentration $(\mu \mathrm{g} / \mathrm{L})$

$\begin{array}{ccccc} & \text { Depth } & \text { Sample Sample Carbon } & \text { Methylene } & \text { Tetra- } \\ \text { Location } & \text { Sample } & \text { (ft below TOC) } & \text { Date } & \text { Type } \\ \text { Tetrachloride Chloroform Chloride chloroethene Benzene Toluene }\end{array}$

Vertical-profile groundwater sampling on and downgradient from the Co-op property

\begin{tabular}{|c|c|c|c|c|c|c|c|c|}
\hline TI-13 & NATI13-W-20819 & $25-30$ & $5 / 9 / 06$ & CPT & 27 & 16 & ND & ND \\
\hline TI-13 & NATI13-W-20563 & $35-40$ & $5 / 5 / 06$ & CPT & 387 & 197 & $0.6 \mathrm{~J}$ & ND \\
\hline TI-13 & NATI13-W-20562 & $42-47$ & $5 / 5 / 06$ & CPT & 116 & 60 & 1.2 & $0.1 \mathrm{~J}$ \\
\hline TI-13 & NATI13-W-20560 & $48-53$ & $5 / 4 / 06$ & CPT & 22 & 5.4 & ND & ND \\
\hline TI-13 & NATI13-W-20561 & $54-59$ & $5 / 5 / 06$ & CPT & 22 & 5.8 & ND & ND \\
\hline TI-13 & NATI13-W-20555 & $66.8-71.8$ & $5 / 4 / 06$ & CPT & $0.6 \mathrm{~J}$ & $0.3 \mathrm{~J}$ & ND & ND \\
\hline $\mathrm{TI}-14$ & NATI14-W-20693 & $26-31$ & $5 / 6 / 06$ & CPT & 148 & 36 & 1.9 & $0.2 \mathrm{~J}$ \\
\hline TI-14 & NATI14-W-20695 & $32-37$ & $5 / 6 / 06$ & CPT & 198 & 71 & ND & $0.3 \mathrm{~J}$ \\
\hline $\mathrm{Tl}-14$ & NATI14-W-20768 & $38-43$ & $5 / 6 / 06$ & CPT & 260 & 126 & 8.0 & $0.5 \mathrm{~J}$ \\
\hline TI-14 & NATI14-W-20691 & $47-52$ & $5 / 6 / 06$ & CPT & 229 & 90 & 5.2 & $0.4 \mathrm{~J}$ \\
\hline $\mathrm{TI}-14$ & NATI14-W-20692 & $54-59$ & $5 / 6 / 06$ & CPT & 72 & 48 & 2.8 & $0.2 \mathrm{~J}$ \\
\hline TI-14 & NATI14-W-20666 & $67.4-72.4$ & $5 / 5 / 06$ & CPT & 6.0 & 280 & 12.6 & ND \\
\hline TI-15 & NATI15-W-20644 & $15-20$ & $5 / 8 / 06$ & СPT & ND & ND & ND & ND \\
\hline TI-15 & NATI15-W-20822 & $25-30$ & $5 / 9 / 06$ & CPT & 45 & 9.2 & ND & ND \\
\hline TI-15 & NATI15-W-20765 & $35-40$ & $5 / 7 / 06$ & CPT & 21 & 6.0 & ND & ND \\
\hline TI-15 & NATI15-W-20764 & $40-45$ & $5 / 6 / 06$ & CPT & 3.4 & 1.7 & ND & ND \\
\hline TI-15 & NATI15-W-20763 & $47-52$ & $5 / 6 / 06$ & CPT & 2.4 & 2.3 & ND & ND \\
\hline TI-15 & NATI15-W-20761 & $67.1-72.1$ & $5 / 6 / 06$ & СРT & ND & ND & ND & ND \\
\hline TI-16 & NATI16-W-20818 & $25-30$ & $5 / 9 / 06$ & CPT & 851 & 535 & 2.6 & $0.1 \mathrm{~J}$ \\
\hline TI-16 & NATI16-W-20817 & $32-37$ & $5 / 9 / 06$ & CPT & 866 & 535 & ND & $0.3 \mathrm{~J}$ \\
\hline TI-16 & NATI16-W-20791 & $39-44$ & $5 / 8 / 06$ & CPT & 189 & 123 & 6.0 & $0.3 \mathrm{~J}$ \\
\hline TI-16 & NATI16-W-20816 & $46-51$ & $5 / 9 / 06$ & CPT & 56 & 32 & ND & ND \\
\hline TI-16 & NATI16-W-20789 & $60-65$ & $5 / 8 / 06$ & CPT & $0.9 \mathrm{~J}$ & $0.6 \mathrm{~J}$ & ND & ND \\
\hline TI-16 & NATI16-W-20790 & $67-72$ & $5 / 8 / 06$ & CPT & $0.8 \mathrm{~J}$ & 2.4 & ND & ND \\
\hline $\mathrm{TI}-17$ & NATI17-W-20825 & $25-30$ & $5 / 9 / 06$ & CPT & 44 & 3.2 & ND & ND \\
\hline $\mathrm{TI}-17$ & NATI17-W-20793 & $32-37$ & $5 / 8 / 06$ & CPT & 1.5 & $0.7 \mathrm{~J}$ & ND & ND \\
\hline TI-17 & NATI17-W-20826 & $39-44$ & $5 / 9 / 06$ & CPT & 1.7 & 1.6 & ND & ND \\
\hline
\end{tabular}


TABLE S2.3 (Cont.)

Concentration $(\mu \mathrm{g} / \mathrm{L})$

$\begin{array}{ccccc} & \text { Depth } & \text { Sample Sample Carbon } & \text { Methylene } & \text { Tetra- } \\ \text { Location } & \text { Sample } & \text { (ft below TOC) } & \text { Date } & \text { Type } \\ \text { Tetrachloride Chloroform Chloride chloroethene Benzene Toluene }\end{array}$

Vertical-profile groundwater sampling on and downgradient from the Co-op property (cont.)

\begin{tabular}{|c|c|c|c|c|c|c|c|c|}
\hline TI-17 & NATI17-W-20814 & $46-51$ & $5 / 9 / 06$ & CPT & 1.1 & $0.6 \mathrm{~J}$ & ND & ND \\
\hline TI-17 & NATI17-W-20829 & $53-58$ & $5 / 9 / 06$ & $\mathrm{CPT}$ & 2.5 & 1.5 & ND & ND \\
\hline TI-17 & NATI17-W-20828 & $60-65$ & $5 / 9 / 06$ & СРТ & $0.5 \mathrm{~J}$ & $0.9 \mathrm{~J}$ & ND & ND \\
\hline TI-18 & NATI18-W-20921 & $25-30$ & $5 / 23 / 06$ & СРТ & 57 & 11 & ND & ND \\
\hline TI-18 & NATI18-W-20655 & 30-35 & $5 / 21 / 06$ & $\mathrm{CPT}$ & 266 & 27 & 1.1 & ND \\
\hline TI-18 & NATI18-W-20831 & $35-40$ & $5 / 10 / 06$ & СРТ & 782 & 47 & 2.7 & $0.2 \mathrm{~J}$ \\
\hline TI-18 & NATI18-W-20706 & $42-47$ & $5 / 21 / 06$ & $\mathrm{CPT}$ & 218 & 48 & 7.3 & ND \\
\hline TI-18 & NATI18-W-20707 & $49-54$ & $5 / 21 / 06$ & CPT & 325 & 92 & 2.8 & $0.4 \mathrm{~J}$ \\
\hline $\mathrm{Tl}-18$ & NATI18-W-20709 & $56-61$ & $5 / 21 / 06$ & СРT & 70 & 35 & 1.5 & ND \\
\hline TI-18 & NATI18-W-20711 & $66.5-71.5$ & $5 / 22 / 06$ & CPT & ND & $0.2 \mathrm{~J}$ & ND & ND \\
\hline TI-19 & NATI19-W-20231 & $25-30$ & $5 / 11 / 06$ & CPT & ND & ND & ND & ND \\
\hline TI-19 & NATI19-W-20863 & $32-37$ & $5 / 11 / 06$ & СPT & ND & ND & ND & ND \\
\hline TI-19 & NATI19-W-20864 & $39-44$ & $5 / 11 / 06$ & CPT & $0.3 \mathrm{~J}$ & ND & ND & ND \\
\hline TI-19 & NATI19-W-20870 & $46-51$ & $5 / 11 / 06$ & CPT & $0.3 \mathrm{~J}$ & ND & ND & ND \\
\hline TI-19 & NATI19-W-20868 & $53-58$ & $5 / 11 / 06$ & CPT & $0.3 \mathrm{~J}$ & ND & ND & ND \\
\hline TI-19 & NATI19-W-20869 & $60-65$ & $5 / 11 / 06$ & CPT & ND & ND & ND & ND \\
\hline TI-19 & NATI19-W-20872 & $65.5-69.5$ & $5 / 11 / 06$ & СPT & ND & ND & ND & ND \\
\hline TI-20 & NATI20-W-20913 & $35-40$ & $5 / 22 / 06$ & СРТ & 15 & 5.4 & ND & ND \\
\hline TI-20 & NATI20-W-20713 & $42-47$ & $5 / 24 / 06$ & СРТ & 13 & 3.4 & ND & ND \\
\hline TI-20 & NATI20-W-20914 & $56-61$ & $5 / 23 / 06$ & СРТ & 7.8 & 7.4 & ND & ND \\
\hline TI-20 & NATI20-W-20922 & $72-77$ & $5 / 23 / 06$ & СРТ & ND & ND & ND & ND \\
\hline TI-21 & NATI21-W-20228 & $25-30$ & $5 / 11 / 06$ & СРT & ND & ND & ND & ND \\
\hline TI-21 & NATI21-W-20885 & $32-37$ & $5 / 11 / 06$ & CPT & $0.9 \mathrm{~J}$ & $0.9 \mathrm{~J}$ & ND & ND \\
\hline TI-21 & NATI21-W-20882 & $39-44$ & $5 / 11 / 06$ & CPT & $0.9 \mathrm{~J}$ & $0.8 \mathrm{~J}$ & ND & ND \\
\hline TI-21 & NATI $21-W-20881$ & $46-51$ & $5 / 11 / 06$ & CPT & 1.2 & $0.8 \mathrm{~J}$ & ND & ND \\
\hline TI-21 & NATI21-W-20867 & $53-58$ & $5 / 11 / 06$ & CPT & $0.7 \mathrm{~J}$ & $0.3 \mathrm{~J}$ & ND & ND \\
\hline TI-21 & NATI21-W-20880 & $60-65$ & $5 / 11 / 06$ & CPT & ND & $0.4 \mathrm{~J}$ & ND & ND \\
\hline
\end{tabular}


TABLE S2.3 (Cont.)

Concentration $(\mu \mathrm{g} / \mathrm{L})$

$\begin{array}{ccccc} & \text { Depth } & \text { Sample Sample Carbon } & \text { Methylene } & \text { Tetra- } \\ \text { Location } & \text { Sample } & \text { (ft below TOC) } & \text { Date } & \text { Type } \\ & & & \text { Tetrachloride Chloroform Chloride chloroethene Benzene Toluene }\end{array}$

Vertical-profile groundwater sampling on and downgradient from the Co-op property (cont.)

\begin{tabular}{|c|c|c|c|c|c|c|c|c|}
\hline TI-22 & NATI22-W-20833 & $32-37$ & $5 / 10 / 06$ & CPT & 120 & 17 & ND & $0.2 \mathrm{~J}$ \\
\hline TI-22 & NATI22-W-20834 & $39-44$ & $5 / 10 / 06$ & CPT & 26 & 12 & ND & ND \\
\hline TI-22 & NATI22-W-20830 & $46-51$ & $5 / 9 / 06$ & СРT & 119 & 31 & 1.3 & $0.1 \mathrm{~J}$ \\
\hline TI-22 & NATI22-W-20836 & $53-58$ & $5 / 10 / 06$ & CPT & 356 & 29 & 1.1 & ND \\
\hline TI-22 & NATI22-W-20840 & $60-65$ & $5 / 10 / 06$ & CPT & 116 & 21 & 1.1 & ND \\
\hline TI-22 & NATI22-W-20846 & $68.2-73.2$ & $5 / 10 / 06$ & CPT & ND & ND & ND & ND \\
\hline TI-23 & NATI23-W-20844 & $32-37$ & $5 / 10 / 06$ & CPT & 24 & 2.6 & ND & ND \\
\hline TI-23 & NATI23-W-20861 & 39-44 & $5 / 11 / 06$ & CPT & 11 & 6.5 & ND & ND \\
\hline TI-23 & NATI23-W-20862 & $46-51$ & $5 / 11 / 06$ & CPT & 5.1 & 2.0 & ND & ND \\
\hline TI-23 & NATI23-W-20865 & $53-58$ & $5 / 11 / 06$ & CPT & 1.5 & $0.5 \mathrm{~J}$ & ND & ND \\
\hline TI-23 & NATI23-W-20856 & $60-65$ & $5 / 10 / 06$ & CPT & 1.0 & ND & ND & ND \\
\hline TI-23 & NATI23-W-20859 & $66.8-71.8$ & $5 / 10 / 06$ & СРT & ND & ND & ND & ND \\
\hline TI-24 & NATI24-W-20860 & $23-30$ & $5 / 11 / 06$ & СPT & ND & ND & ND & ND \\
\hline TI-24 & NATI24-W-20858 & $32-37$ & $5 / 10 / 06$ & СРT & ND & ND & ND & ND \\
\hline TI-24 & NATI24-W-20838 & $39-44$ & $5 / 10 / 06$ & СPT & 1.5 & 1.9 & ND & ND \\
\hline TI-24 & NATI24-W-20841 & $46-51$ & $5 / 10 / 06$ & CPT & 1.8 & 2.4 & ND & ND \\
\hline TI-24 & NATI24-W-20842 & $53-58$ & $5 / 10 / 06$ & СPT & 1.4 & 1.7 & ND & ND \\
\hline TI-24 & NATI24-W-20843 & $60-65$ & $5 / 10 / 06$ & СРT & 1.0 & $0.6 \mathrm{~J}$ & ND & ND \\
\hline TI-24 & NATI24-W-20845 & $69.4-74.4$ & $5 / 10 / 06$ & CPT & ND & ND & ND & ND \\
\hline TI-25 & NATI25-W-20722 & $32-37$ & $5 / 12 / 06$ & CPT & ND & ND & ND & ND \\
\hline TI-25 & NATI25-W-20875 & $39-44$ & $5 / 11 / 06$ & СРT & ND & $0.2 \mathrm{~J}$ & ND & ND \\
\hline TI-25 & NATI25-W-20878 & $46-51$ & $5 / 11 / 06$ & CPT & 1.7 & 1.4 & ND & ND \\
\hline TI-25 & NATI25-W-20884 & $53-58$ & $5 / 11 / 06$ & CPT & $0.8 \mathrm{~J}$ & $0.2 \mathrm{~J}$ & ND & ND \\
\hline TI-25 & NATI25-W-20232 & $60-65$ & $5 / 12 / 06$ & CPT & ND & ND & ND & ND \\
\hline TI-25 & NATI25-W-20720 & $66-71$ & $5 / 12 / 06$ & CPT & ND & ND & ND & ND \\
\hline TI-25 & NATI25-W-20235 & $72.2-77.2$ & $5 / 12 / 06$ & CPT & ND & ND & ND & ND \\
\hline
\end{tabular}


TABLE S2.3 (Cont.)

Concentration $(\mu \mathrm{g} / \mathrm{L})$

$\begin{array}{ccccc} & \text { Depth } & \text { Sample Sample Carbon } & \text { Methylene } & \text { Tetra- } \\ \text { Location } & \text { Sample } & \text { (ft below TOC) } & \text { Date } & \text { Type } \\ \text { Tetrachloride Chloroform Chloride chloroethene Benzene Toluene }\end{array}$

Vertical-profile groundwater sampling on and downgradient from the Co-op property (cont.)

\begin{tabular}{|c|c|c|c|c|c|c|c|c|}
\hline TI-26 & NATI26-W-20721 & $25-30$ & $5 / 12 / 06$ & СРТ & 1.1 & $0.7 \mathrm{~J}$ & ND & ND \\
\hline TI-26 & NATI26-W-20877 & $32-37$ & $5 / 11 / 06$ & СРТ & 2.7 & 3.7 & ND & ND \\
\hline TI-26 & NATI26-W-20883 & $39-44$ & $5 / 11 / 06$ & СРТ & 1.0 & 3.5 & ND & ND \\
\hline TI-26 & NATI26-W-20723 & $46-51$ & $5 / 12 / 06$ & СРТ & 5.0 & 4.3 & ND & ND \\
\hline TI-26 & NATI26-W-20229 & $53-58$ & $5 / 11 / 06$ & СРТ & 4.8 & 3.1 & ND & ND \\
\hline TI-26 & NATI26-W-20233 & $60-65$ & $5 / 12 / 06$ & СРТ & 7.7 & 11 & ND & ND \\
\hline TI-26 & NATI26-W-20719 & $66-71$ & $5 / 12 / 06$ & СРТ & 3.2 & 8.1 & ND & ND \\
\hline TI-26 & NATI26-W-20718 & $71.8-76.8$ & $5 / 12 / 06$ & СРТ & ND & ND & ND & ND \\
\hline TI-27 & NATI27-W-20680 & $25-30$ & $5 / 19 / 06$ & СРТ & 1.0 & $0.7 \mathrm{~J}$ & ND & ND \\
\hline TI-27 & NATI27-W-20704 & $30-35$ & $5 / 20 / 06$ & СРТ & $1.1^{\mathrm{e}}$ & $2.3^{e}$ & $0.2 \mathrm{Je}^{\mathrm{e}}$ & ND \\
\hline TI-27 & NATI27-W-20684 & $35-40$ & $5 / 19 / 06$ & СРT & ND & 1.6 & ND & ND \\
\hline TI-27 & NATI27-W-20699 & $42-47$ & $5 / 20 / 06$ & СРТ & $7.9^{\mathrm{e}}$ & $3.5^{\mathrm{e}}$ & ND & ND \\
\hline TI-27 & NATI27-W-20700 & $49-54$ & $5 / 20 / 06$ & СРT & $2.0^{\mathrm{e}}$ & $0.7 \mathrm{Je}$ & ND & ND \\
\hline TI-27 & NATI27-W-20905 & $56-61$ & $5 / 21 / 06$ & СРТ & ND & $0.6 \mathrm{~J}$ & ND & ND \\
\hline TI-27 & NATI27-W-20649 & $66.2-71.2$ & $5 / 20 / 06$ & СРТ & ND & ND & ND & ND \\
\hline TI-28 & NATI28-W-20904 & $25-30$ & $5 / 21 / 06$ & СРТ & 2692 & 238 & 1.3 & 1.3 \\
\hline TI-28 & NATI28-W-20851 & $32-37$ & $5 / 20 / 06$ & СРТ & 3104 & 646 & 6.3 & 3.1 \\
\hline TI-28 & NATI28-W-20849 & $37-42$ & $5 / 20 / 06$ & СРT & 97 & 91 & 1.0 & ND \\
\hline TI-28 & NATI28-W-20850 & $44-49$ & $5 / 20 / 06$ & СРT & 88 & 14 & ND & 0.1 \\
\hline TI-28 & NATI28-W-20683 & $51-56$ & $5 / 19 / 06$ & СРТ & 12 & 2.7 & ND & ND \\
\hline TI-28 & NATI28-W-20254 & $58-63$ & $5 / 19 / 06$ & СРТ & 15 & 8.9 & ND & ND \\
\hline TI-28 & NATI28-W-20647 & $63-68$ & $5 / 19 / 06$ & СРТ & ND & ND & ND & ND \\
\hline TI-29 & NATI29-W-20916 & $25-30$ & $5 / 23 / 06$ & СРТ & 39 & 39 & 2.6 & ND \\
\hline TI-29 & NATI29-W-20909 & $32-37$ & $5 / 22 / 06$ & СРТ & 75 & 55 & 4.2 & ND \\
\hline TI-29 & NATI29-W-20900 & $39-44$ & $5 / 21 / 06$ & СРТ & 269 & 87 & 3.9 & ND \\
\hline TI-29 & NATI29-W-20902 & $46-51$ & $5 / 21 / 06$ & CPT & 182 & 86 & 3.3 & ND \\
\hline TI-29 & NATI29-W-20903 & $53-58$ & $5 / 21 / 06$ & СРT & 18 & 31 & 1.5 & ND \\
\hline
\end{tabular}


TABLE S2.3 (Cont.)

Concentration $(\mu \mathrm{g} / \mathrm{L})$

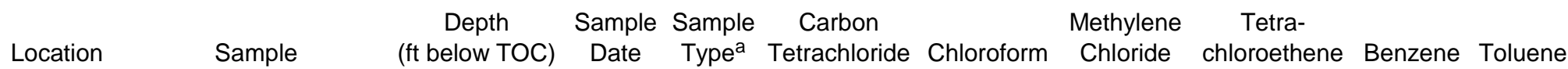

Vertical-profile groundwater sampling on and downgradient from the Co-op property (cont.)

\begin{tabular}{|c|c|c|c|c|c|c|c|}
\hline TI-29 & NATI29-W-20906 & $60-65$ & $5 / 21 / 06$ & CPT & 9.4 & 21 & $0.9 \mathrm{~J}$ \\
\hline TI-29 & NATI29-W-20907 & $66.3-69.3$ & $5 / 21 / 06$ & CPT & 3.4 & 4.3 & ND \\
\hline TI-30 & NATI30-W-20939 & $32-37$ & $5 / 25 / 06$ & CPT & $0.4 \mathrm{~J}$ & $0.5 \mathrm{~J}$ & ND \\
\hline TI-30 & NATI30-W-20920 & $39-44$ & $5 / 23 / 06$ & CPT & 1.1 & $0.4 \mathrm{~J}$ & ND \\
\hline TI-30 & NATI30-W-20915 & $43.8-48.8$ & $5 / 23 / 06$ & CPT & 2.5 & $0.9 \mathrm{~J}$ & ND \\
\hline TI-30 & NATI30-W-20911 & $53-58$ & $5 / 23 / 06$ & CPT & 62 & 6.9 & ND \\
\hline TI-30 & NATI30-W-20918 & $60-65$ & $5 / 23 / 06$ & CPT & 43 & 5.5 & ND \\
\hline TI-30 & NATI30-W-20917 & $75-80$ & $5 / 23 / 06$ & CPT & ND & ND & ND \\
\hline TI-30 & NATI30-W-20712 & $83.5-88.5$ & $5 / 24 / 06$ & CPT & ND & ND & ND \\
\hline TI-31 & NATI31-W-20947 & $42-47$ & $5 / 25 / 06$ & CPT & 30 & 4.3 & ND \\
\hline TI-31 & NATI31-W-20938 & $53-58$ & $5 / 25 / 06$ & CPT & 91 & 14 & ND \\
\hline TI-31 & NATI31-W-20937 & $60-65$ & $5 / 25 / 06$ & CPT & 28 & 3.4 & ND \\
\hline TI-31 & NATI31-W-20715 & $70.7-75.7$ & $5 / 24 / 06$ & CPT & ND & ND & ND \\
\hline
\end{tabular}

Sampling of monitoring well installed at the TI-30 location during the 2006 investigation

$\begin{array}{lllllllll}\text { MW5 NATI30-W-20946 } & 78-88 & 5 / 25 / 06 & \text { CPT/P } & 0.4 \mathrm{~J} & \text { ND } & \text { ND }\end{array}$

a Sample types: CPT, cone penetrometer; DW, domestic well; MW, monitoring well; P, piezometer.

b Qualifier J indicates an estimated concentration below the quantitation limit of $1.0 \mu \mathrm{g} / \mathrm{L}$.

c ND, not detected at a method detection limit of $0.1 \mu \mathrm{g} / \mathrm{L}$.

d Total depth.

e Cross-contamination possible. See discussion in Supplement 4, Section S4.1.3. 
TABLE S2.4 Tritium results from 2006 investigation at Navarre.

\begin{tabular}{lcccccc}
\hline Location & \multicolumn{1}{c}{ Sample } & $\begin{array}{c}\text { Depth } \\
\text { (ft BGL) }\end{array}$ & $\begin{array}{c}\text { Sampling } \\
\text { Date }\end{array}$ & Type $^{\mathrm{a}}$ & $\begin{array}{c}\text { Analysis } \\
\text { Date }\end{array}$ & $\begin{array}{c}\text { Tritium } \\
(\text { TU) }\end{array}$ \\
\hline Existing well sampling & & & & & & \\
Co-op 1 & NACOOP1-W-20217 & Unknown & $4 / 8 / 06$ & DW & $6 / 13 / 06$ & $5.22 \pm 0.17$ \\
Co-op 3 & NACOOP3-W-20218 & Unknown & $4 / 8 / 06$ & DW & $6 / 13 / 06$ & $3.82 \pm 0.13$ \\
KDHE-1 & NAKDHE1-W-20203 & $35-55$ & $4 / 7 / 06$ & MW & $6 / 13 / 06$ & $0.42 \pm 0.09$ \\
KDHE-2 & NAKDHE2-W-20205 & $25-45$ & $4 / 7 / 06$ & MW & $6 / 13 / 06$ & $4.46 \pm 0.15$ \\
T1 & NAT1-W-20201 & $40-60$ & $4 / 7 / 06$ & MW & $6 / 13 / 06$ & $4.24 \pm 0.14$ \\
L-1 & NAL1-W-20211 & $75-95$ & $4 / 8 / 06$ & MW & $6 / 13 / 06$ & $4.70 \pm 0.15$ \\
L-2 & NAL2-W-20190 & $80-90$ & $4 / 6 / 06$ & MW & $6 / 13 / 06$ & $1.06 \pm 0.09$ \\
L-3 & NAL3-W-20209 & $80-90$ & $4 / 7 / 06$ & MW & $6 / 13 / 06$ & $0.71 \pm 0.09$ \\
NW-1 & NANW1-W-20215 & $40-50$ & $4 / 8 / 06$ & MW & $6 / 13 / 06$ & $4.90 \pm 0.16$ \\
NW-2 & NANW2-W-20219 & $35.5-45.5$ & $4 / 9 / 06$ & MW & $6 / 13 / 06$ & $4.67 \pm 0.15$ \\
NW-3 & NANW3-W-20945 & $38-48$ & $5 / 25 / 06$ & MW & $8 / 4 / 06$ & $1.59 \pm 0.09$
\end{tabular}

Cone penetrometer sampling at targeted investigation locations

$\begin{array}{lllllll}\text { TI-2 } & \text { NATI2-W-20433 } & 69-72 & 4 / 7 / 06 & \text { CPT } & 6 / 13 / 06 & 0.03 \pm 0.09 \\ \text { TI-3 } & \text { NATI3-W-20370 } & 33-38 & 4 / 6 / 06 & \text { CPT } & 6 / 13 / 06 & 4.45 \pm 0.15 \\ & & & & & & \\ \text { TI-4 } & \text { NATI4-W-20374 } & 35-40 & 4 / 13 / 06 & \text { CPT } & 6 / 13 / 06 & 4.95 \pm 0.20 \\ \text { TI-4 } & \text { NATI4-W-20469 } & 50-55 & 4 / 11 / 06 & \text { CPT } & 6 / 13 / 06 & 4.07 \pm 0.20 \\ \text { TI-4 } & \text { NATI4-W-20470 } & 55-60 & 4 / 11 / 06 & \text { CPT } & 6 / 13 / 06 & 2.64 \pm 0.09 \\ \text { TI-4 } & \text { NATI4-W-20472 } & 61-66 & 4 / 12 / 06 & \text { CPT } & 6 / 13 / 06 & 0.45 \pm 0.09 \\ \text { TI-4 } & \text { NATI4-W-20474 } & 67-71 & 4 / 12 / 06 & \text { CPT } & 6 / 13 / 06 & 0.13 \pm 0.09 \\ & & & & & & \\ \text { TI-5 } & \text { NATI5-W-20368 } & 36-41 & 4 / 12 / 06 & \text { CPT } & 6 / 13 / 06 & 5.83 \pm 0.19 \\ \text { TI-5 } & \text { NATI5-W-20371 } & 49-54 & 4 / 13 / 06 & \text { CPT } & 6 / 13 / 06 & 1.40 \pm 0.09\end{array}$

Sampling of monitoring well installed at the TI-30 location during the 2006 targeted investigation

$\begin{array}{lllllll}\text { MW5 NATI30-W-20946 } & 78-88 & 5 / 25 / 06 & \text { CPT/P } & 8 / 4 / 06 & 0.03 \pm 0.09\end{array}$

a Types: CPT, penetrometer; DW, domestic well; MW, monitoring well; P, piezometer. 


\section{Supplement 3:}

\section{Water Level Data}


TABLE S3.1 Hand-measured water levels at Navarre.

\begin{tabular}{|c|c|c|c|c|c|c|c|c|c|c|c|c|c|c|c|}
\hline \multirow[b]{2}{*}{ Well } & \multirow[b]{2}{*}{$\begin{array}{l}\text { Screen } \\
\text { Interval } \\
\text { Depth } \\
\text { (ft BGL) }\end{array}$} & \multirow[b]{2}{*}{$\begin{array}{c}\text { Top of } \\
\text { Casing } \\
\text { Elevation } \\
\text { (ft AMSL) }\end{array}$} & \multirow[b]{2}{*}{$\begin{array}{l}\text { Depth of } \\
\text { Bottom } \\
\text { of Hole } \\
\text { (ft TOC) }\end{array}$} & \multicolumn{3}{|c|}{ April 24-25, 2006} & \multicolumn{3}{|c|}{ May 11,2006} & \multicolumn{3}{|c|}{ November $3,2006^{c}$} & \multicolumn{3}{|c|}{ November 28, 2006} \\
\hline & & & & Time & $\begin{array}{l}\text { Depth to } \\
\text { Water } \\
\text { (ft TOC) }\end{array}$ & $\begin{array}{l}\text { Groundwater } \\
\text { Elevation } \\
\text { (ft AMSL) }\end{array}$ & Time & $\begin{array}{l}\text { Depth to } \\
\text { Water } \\
\text { (ft TOC) }\end{array}$ & $\begin{array}{l}\text { Groundwater } \\
\text { Elevation } \\
\text { (ft AMSL) }\end{array}$ & Time & $\begin{array}{l}\text { Depth to } \\
\text { Water } \\
\text { (ft TOC) }\end{array}$ & $\begin{array}{l}\text { Groundwater } \\
\text { Elevation } \\
\text { (ft AMSL) }\end{array}$ & Time & $\begin{array}{l}\text { Depth to } \\
\text { Water } \\
\text { (ft TOC) }\end{array}$ & $\begin{array}{l}\text { Groundwater } \\
\text { Elevation } \\
\text { (ft AMSL) }\end{array}$ \\
\hline MW1 & 43-58 & 1351.36 & 59.85 & 18:10 & 31.75 & 1319.61 & 18:36 & 31.53 & 1319.83 & 13:16 & 34.1 & 1317.26 & $15: 36$ & 34.24 & 1317.12 \\
\hline MW2 & $42.8-57.8$ & 1352.31 & 56.89 & $17: 59$ & 32.13 & 1320.18 & 18:07 & 31.82 & 1320.49 & 12:55 & 34.36 & 1317.95 & $15: 25$ & 34.54 & 1317.77 \\
\hline MW3 & $44-59$ & 1352.88 & 58.30 & $17: 24$ & 32.41 & 1320.47 & 17:51 & 32.10 & 1320.78 & 13:30 & 34.64 & 1318.24 & 15:06 & 34.87 & 1318.01 \\
\hline MW4 & $45-60$ & 1352.94 & 61.95 & 17:17 & 32.88 & 1320.06 & $17: 45$ & 32.58 & 1320.36 & & & & $14: 58$ & 35.33 & 1317.61 \\
\hline$M W 5^{d}$ & 78-88 & $1353.65^{\mathrm{e}}$ & 88.00 & & & & & & & 11:50 & 32.23 & 1321.42 & $14: 27$ & 33.44 & 1320.21 \\
\hline KDHE-1 & $39-59^{f}$ & 1350.56 & 59.90 & $16: 37$ & 27.55 & 1323.01 & $16: 23$ & 27.56 & 1323.00 & $10: 44$ & 29.8 & 1320.76 & $13: 34$ & 29.86 & 1320.70 \\
\hline KDHE-29 & $25-45$ & 1347.86 & 54.95 & $16: 18$ & 25.32 & 1322.54 & 17:07 & 24.67 & 1323.19 & $11: 38$ & 26.81 & 1321.05 & $14: 12$ & 27.32 & 1320.54 \\
\hline$N W-1$ & $40-50$ & 1351.93 & 50.25 & $16: 02$ & 29.95 & 1321.98 & $16: 56$ & 29.45 & 1322.48 & & & & $13: 54$ & 32.26 & 1319.67 \\
\hline NW-2 & $35.5-45.5$ & 1350.93 & 44.75 & $15: 46$ & 28.59 & 1322.34 & $16: 40$ & 28.21 & 1322.72 & & & & $13: 20$ & 30.91 & 1320.02 \\
\hline$N W-3^{9}$ & $38-48$ & 1347.14 & 41.60 & 17:02 & $27.6^{\mathrm{h}}$ & 1319.54 & $17: 31$ & 28.62 & 1318.52 & $12: 21$ & 31.56 & 1315.58 & $14: 47$ & 31.52 & 1315.62 \\
\hline L-1 & $75-95$ & 1350.03 & 95.35 & 17:47 & 29.90 & 1320.13 & 18:00 & 29.61 & 1320.42 & $12: 40$ & 32.17 & 1317.86 & $15: 16$ & 32.34 & 1317.69 \\
\hline $\mathrm{L}-2$ & 80-90 & 1350.19 & 90.90 & 18:03 & 30.14 & 1320.05 & $18: 15$ & 29.87 & 1320.32 & 13:06 & 32.38 & 1317.81 & $15: 28$ & 32.57 & 1317.62 \\
\hline L-3 & 80-90 & 1347.55 & 90.06 & 18:16 & 30.38 & 1317.17 & $17: 22$ & 29.82 & 1317.73 & $12: 05$ & 32.76 & 1314.79 & $14: 35$ & 32.8 & 1314.75 \\
\hline $\mathrm{T} 1$ & $51-61^{\dagger}$ & 1351.72 & 61.30 & $17: 34$ & 31.60 & 1320.12 & $17: 55$ & 31.29 & 1320.43 & $12: 45$ & 33.86 & 1317.86 & $15: 10$ & 34.02 & 1317.70 \\
\hline
\end{tabular}

a Measured in Papadopulos (2000) investigation, except as noted.

b Measured by Argonne, April 24, 2006. Depths are in feet below the top of the casing.

c Incomplete set, repeated November 28, 2006.

d One-inch piezometer installed at the edge of field west of the Co-op during the 2006 investigation, at the TI-30 location. Not available for water level measurement until November 2006.

e Surveyed by Schwab-Eaton for Argonne 2006 investigation.

f Screen interval from video mapping by Argonne; different from previously reported interval.

9 Note discrepancy between bottom of hole and screen interval for wells KDHE-2 and NW-3.

hLevels falling slowly at the time of the measurement. Measurement is an approximation. 
TABLE S3.2 Automated water level measurements at Navarre, April 25, 2006, to November 28, 2006.

Depth to Water (ft TOC) in Well Indicated

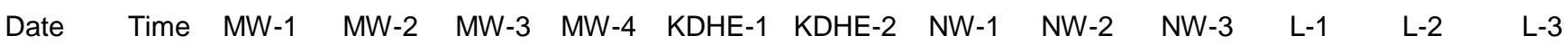

\begin{tabular}{|c|c|c|c|c|c|c|c|c|c|c|}
\hline 4/25/2006 & $20: 00$ & 31.84532 .224 & 32.49732 .909 & 27.718 & 25.154 & 30.063 & 28.687 & 28.642 & & \\
\hline 4/26/2006 & 0:00 & 31.84132 .229 & 32.50932 .913 & 27.737 & 25.165 & 30.075 & 28.704 & 28.635 & & \\
\hline 4/26/2006 & 4:00 & 31.82632 .213 & 32.49732 .901 & 27.733 & 25.170 & 30.068 & 28.698 & 28.635 & & \\
\hline /26/2006 & 8:00 & 31.85332 .224 & 32.50732 .913 & 27.743 & 25.177 & 30.073 & 28.689 & 28.630 & & \\
\hline $4 / 26 / 2006$ & $12: 00$ & 31.86132 .214 & 32.49032 .903 & 27.745 & 25.181 & 30.075 & 28.706 & 28.632 & & \\
\hline 4/26/2006 & $16: 00$ & 31.81232 .182 & 32.45932 .864 & 27.691 & 25.165 & 30.035 & 28.651 & 28.594 & 29.90430 .153 & 30.041 \\
\hline 4/26/2006 & $20: 00$ & 31.79632 .190 & 32.46532 .862 & 27.672 & 25.163 & 30.028 & 28.636 & 28.569 & 29.89530 .146 & 30.056 \\
\hline 4/27/2006 & $0: 00$ & 31.79632 .193 & 32.47332 .870 & 27.699 & 25.172 & 30.048 & 28.662 & 28.582 & 29.89730 .149 & 30.019 \\
\hline 4/27/2006 & 4:00 & 31.78132 .176 & 32.45932 .856 & 27.693 & 25.174 & 30.044 & 28.653 & 28.579 & 29.87830 .131 & 29.994 \\
\hline 4/27/2006 & 8:00 & 31.79432 .174 & 32.45732 .852 & 27.695 & 25.174 & 30.039 & 28.656 & 28.567 & 29.87330 .129 & 29.981 \\
\hline 4/27/2006 & $12: 00$ & 31.75932 .163 & 32.44832 .838 & 27.680 & 25.168 & 30.033 & 28.645 & 28.567 & 29.86030 .116 & 29.976 \\
\hline 4/27/2006 & $16: 00$ & 31.73632 .117 & 32.39832 .795 & 27.616 & 25.145 & 29.984 & 28.590 & 28.536 & 930.078 & 972 \\
\hline 4/27/2006 & 20:00 & 31.73632 .119 & 32.39732 .791 & 27.605 & 25.140 & 29.975 & 28.572 & 28.519 & 29.81630 .073 & 33.318 \\
\hline 4/28/2006 & $0: 00$ & 31.77132 .146 & 32.42132 .817 & 27.626 & 25.145 & 29.995 & 28.594 & 28.539 & 29.85330 .115 & 35.342 \\
\hline 4/28/2006 & $4: 00$ & 31.77932 .136 & 32.41032 .809 & 27.601 & 25.138 & 29.980 & 28.576 & 28.544 & 124 & 441 \\
\hline $4 / 28 / 2006$ & 8:00 & 31.79332 .154 & 32.42532 .828 & 27.609 & 25.142 & 30.013 & 28.590 & 28.564 & 47 & 141 \\
\hline 4/28/2006 & $12: 00$ & 31.83732 .188 & 32.45532 .862 & 27.626 & 25.153 & 30.011 & 28.596 & 28.594 & 29.91230 .198 & 37.663 \\
\hline 4/28/2006 & $16: 00$ & 31.84132 .182 & 32.44832 .858 & 27.605 & 25.142 & 29.986 & 28.581 & 28.604 & .209 & 38.060 \\
\hline 4/28/2006 & 20:00 & 31.89632 .182 & 32.44632 .862 & 27.584 & 25.091 & 30.000 & 28.559 & 28.614 & .217 & 676 \\
\hline 4/29/2006 & 0:00 & 31.82632 .173 & 32.43332 .848 & 27.576 & 24.976 & 29.889 & 28.521 & 28.622 & 0430.202 & 32.847 \\
\hline 4/29/2006 & 4:00 & 31.78732 .133 & 32.39132 .807 & 27.544 & 24.805 & 29.820 & 28.468 & 28.599 & 29.86030 .155 & 31.965 \\
\hline 4/29/2006 & 8:00 & 31.75732 .108 & 32.37032 .785 & 27.528 & 24.650 & 29.763 & 28.440 & 28.594 & 29.83130 .120 & 31.412 \\
\hline 4/29/2006 & $12: 00$ & 31.74432 .095 & 32.36032 .774 & 27.517 & 24.516 & 29.714 & 28.420 & 28.582 & 29.80930 .091 & 31.039 \\
\hline 4/29/2006 & $16: 00$ & 31.70932 .066 & 32.33332 .746 & 27.482 & 24.350 & 29.657 & 28.411 & 28.554 & 29.77730 .058 & 30.772 \\
\hline 4/29/2006 & $20: 00$ & 31.69132 .038 & 32.30332 .721 & 27.452 & 24.206 & 29.595 & 28.385 & 28.526 & 29.74830 .023 & 30.563 \\
\hline 4/30/2006 & 0:00 & 31.68332 .030 & 32.29432 .709 & 27.452 & 24.148 & 29.559 & 28.318 & 28.521 & 29.73530 .011 & 30.415 \\
\hline 4/30/2006 & $4: 00$ & 31.66432 .004 & 32.26732 .684 & 27.431 & 24.135 & 29.519 & 28.263 & 28.511 & 29.70629 .982 & 30.293 \\
\hline 4/30/2006 & 8:00 & 31.68732 .013 & 32.27732 .691 & 27.457 & 24.146 & 29.517 & 28.195 & 28.519 & 29.71129 .983 & 30.207 \\
\hline 4/30/2006 & $12: 00$ & 31.67432 .009 & 32.27132 .687 & 27.465 & 24.158 & 29.508 & 28.204 & 28.514 & 29.70529 .980 & 30.145 \\
\hline 4/30/2006 & $16: 00$ & 31.66631 .996 & 32.25932 .672 & 27.459 & 24.162 & 29.497 & 28.219 & 28.582 & 29.69129 .965 & 30.092 \\
\hline 4/30/2006 & $20: 00$ & 31.67432 .007 & 32.26732 .674 & 27.463 & 24.169 & 29.497 & 28.219 & 28.532 & 29.69429 .965 & 30.054 \\
\hline $5 / 1 / 2006$ & 0:00 & 31.69932 .030 & 32.28832 .697 & 27.513 & 24.193 & 29.519 & 28.259 & 28.549 & 29.71329 .985 & 30.016 \\
\hline $5 / 1 / 2006$ & 4:00 & 31.67932 .017 & 32.27832 .691 & 27.528 & 24.213 & 29.522 & 28.268 & 28.551 & 29.70829 .980 & 29.996 \\
\hline $5 / 1 / 2006$ & 8:00 & 31.69732 .030 & 32.29032 .703 & 27.554 & 24.238 & 29.530 & 28.283 & 28.561 & 29.71829 .989 & 29.976 \\
\hline
\end{tabular}


Depth to Water (ft TOC) in Well Indicated

$\begin{array}{llllllllllllll}\text { Date } & \text { Time } & \text { MW-1 } & \text { MW-2 } & \text { MW-3 } & \text { MW-4 } & \text { KDHE-1 } & \text { KDHE-2 } & \text { NW-1 } & \text { NW-2 } & \text { NW-3 } & \text { L-1 } & \text { L-2 } & \text { L-3 }\end{array}$

\begin{tabular}{|c|c|c|c|c|c|c|c|c|c|c|}
\hline $5 / 1 / 2006$ & $12: 00$ & 31.71832 .049 & 32.31532 .725 & 27.601 & 24.269 & 29.559 & 28.325 & 28.580 & 29.74030 .009 & 29.974 \\
\hline $1 / 2006$ & $16: 00$ & 31.67832 .019 & 32.28232 .691 & 27.575 & 24.282 & 29.539 & 28.292 & 28.561 & 29.70829 .982 & 29.961 \\
\hline $5 / 1 / 2006$ & $20: 00$ & 31.67632 .019 & 32.27932 .687 & 27.578 & 24.301 & 29.544 & 28.290 & 28.559 & 29.70129 .976 & .943 \\
\hline $5 / 2 / 2006$ & $0: 00$ & 31.68932 .021 & 32.28232 .687 & 27.592 & 24.322 & 29.553 & 28.303 & 28.559 & 29.70529 .978 & 29.930 \\
\hline $5 / 2 / 2006$ & 4:00 & 31.63331 .973 & 32.23732 .644 & 27.552 & 24.327 & 29.519 & 28.262 & 28.534 & 29.66229 .936 & 29.910 \\
\hline $5 / 2 / 2006$ & 8:00 & 31.65231 .994 & 32.25632 .664 & 27.588 & 24.354 & 29.541 & 28.290 & 28.546 & 29.67429 .945 & 902 \\
\hline $5 / 2 / 2006$ & $12: 00$ & 31.61331 .956 & 32.22332 .629 & 27.547 & 24.361 & 29.513 & 28.251 & 28.526 & 29.64429 .918 & 29.891 \\
\hline $5 / 2 / 2006$ & $16: 00$ & 31.58231 .920 & 32.18332 .584 & 27.494 & 24.352 & 29.475 & 28.198 & 28.486 & 29.60729 .885 & 29.872 \\
\hline /2/2006 & $20: 00$ & 31.57631 .926 & 32.18132 .580 & 27.475 & 24.356 & 29.473 & 28.187 & 28.469 & 29.60529 .885 & 31.996 \\
\hline 5/3/2006 & $0: 00$ & 31.55931 .901 & 32.15832 .562 & 27.458 & 24.356 & 29.453 & 28.173 & 28.461 & 29.59329 & 806 \\
\hline $5 / 3 / 2006$ & 4:00 & 31.66031 .973 & 32.22932 .638 & 27.550 & 24.396 & 29.513 & 28.255 & 28.526 & 29.66929 .956 & 36.122 \\
\hline 5/3/2006 & 8:00 & 31.71532 .017 & 32.27332 .684 & 27.596 & 24.426 & 29.550 & 28.297 & 28.582 & 29.72030 .011 & 34.488 \\
\hline 2006 & $12: 00$ & 31.70732 .009 & 32.26732 .682 & 27.582 & 24.438 & 29.546 & 28.292 & 28.597 & 29.72130 & 320 \\
\hline $5 / 3 / 2006$ & $16: 00$ & 31.72832 .004 & 32.27132 .685 & 27.596 & 24.456 & 29.546 & 28.279 & 28.617 & 29.7183 & 511 \\
\hline 5/3/2006 & 20:00 & 31.71532 .004 & 32.27132 .682 & 27.590 & 24.474 & 29.555 & 28.301 & 28.622 & 29.71130 .007 & 31.057 \\
\hline 5/4/2006 & $0: 00$ & 31.70332 .004 & 32.27932 .689 & 27.617 & 24.493 & 29.573 & 28.319 & 28.627 & 29.70830 .003 & 30.761 \\
\hline 5/4/2006 & 4:00 & 31.67031 .971 & 32.24832 .658 & 27.592 & 24.502 & 29.557 & 28.295 & 28.617 & 29.67429 & 46 \\
\hline $5 / 4 / 2006$ & 8:00 & 31.72032 .008 & 32.29232 .698 & 27.655 & 24.537 & 99 & 28.321 & 28.647 & 29.7032 & 30.408 \\
\hline 5/4/2006 & $12: 00$ & 31.72032 .021 & 32.30132 .705 & 27.676 & 24.558 & 29.639 & 28.389 & 28.655 & 29.71029 & 30.315 \\
\hline /4/2006 & $16: 00$ & 931.971 & 32.25932 .664 & 27.640 & 24.560 & 29.597 & 28.370 & 28.632 & 29.67129 & 30.222 \\
\hline 5/4/2006 & 20:00 & 631.960 & 32.24432 .650 & 27.623 & 24.569 & 29 & 28.319 & 28. & 29.65429 & 56 \\
\hline 5/5/2006 & $0: 00$ & 31.66431 .979 & 32.26732 .666 & 27.655 & 24.592 & 29.610 & 28.350 & 28.630 & 29.66929 & 30.114 \\
\hline 5/5/2006 & 4:00 & 31.64431 .958 & 32.24232 .643 & 27.640 & 24.599 & 29.683 & 28.363 & 28.614 & 29.64729 .930 & 30.061 \\
\hline $5 / 5 / 2006$ & 8:00 & 31.67831 .975 & 32.25832 .658 & 27.663 & 24.617 & 29.688 & 28.383 & 28.614 & 29.66229 & 30.032 \\
\hline $5 / 5 / 2006$ & $12: 00$ & 31.64831 .967 & 32.25432 .652 & 27.659 & 24.627 & 29.624 & 28.354 & 28.617 & 29.65429 .938 & 30.010 \\
\hline 5/5/2006 & $16: 00$ & 31.63131 .933 & 32.21932 .615 & 27.619 & 24.624 & 29.586 & 28.325 & 28.587 & 29.62029 .907 & 29.981 \\
\hline 5/5/2006 & $20: 00$ & 31.58631 .907 & 32.18932 .586 & 27.586 & 24.622 & 29.551 & 28.242 & 28.561 & 29.59329 .879 & 29.950 \\
\hline 5/6/2006 & 0:00 & 31.60031 .916 & 32.19832 .596 & 27.603 & 24.632 & 29.566 & 28.273 & 28.562 & 29.59829 .883 & 29.932 \\
\hline $5 / 6 / 2006$ & $4: 00$ & 31.56131 .892 & 32.17432 .568 & 27.575 & 24.627 & 29.553 & 28.257 & 28.542 & 29.57329 .859 & 29.908 \\
\hline 5/6/2006 & 8:00 & 31.57831 .903 & 32.18932 .580 & 27.594 & 24.638 & 29.568 & 28.235 & 28.544 & 29.58529 .868 & 29.897 \\
\hline 5/6/2006 & $12: 00$ & 31.61131 .901 & 32.18332 .576 & 27.592 & 24.645 & 29.575 & 28.259 & 28.534 & 29.57829 .859 & 29.866 \\
\hline 5/6/2006 & $16: 00$ & 31.55531 .871 & 32.15332 .547 & 27.554 & 24.634 & 29.646 & 28.264 & 28.508 & 29.55629 .839 & 29.846 \\
\hline $5 / 6 / 2006$ & 20:00 & 31.52531 .855 & 32.13832 .529 & 27.538 & 24.613 & 29.509 & 28.262 & 28.493 & 29.53629 .821 & 29.833 \\
\hline $5 / 7 / 2006$ & $0: 00$ & 31.54331 .861 & 32.14132 .531 & 27.546 & 24.567 & 29.515 & 28.255 & 28.494 & 29.53929 .823 & 29.824 \\
\hline
\end{tabular}


Depth to Water (ft TOC) in Well Indicated

$\begin{array}{llllllllllllll}\text { Date } & \text { Time } & \text { MW-1 } & \text { MW-2 } & \text { MW-3 } & \text { MW-4 } & \text { KDHE-1 } & \text { KDHE-2 } & \text { NW-1 } & \text { NW-2 } & \text { NW-3 } & \text { L-1 } & \text { L-2 } & \text { L-3 }\end{array}$

\begin{tabular}{|c|c|c|c|c|c|c|c|c|c|c|}
\hline 5/7/2006 & $4: 00$ & 31.50631 .842 & 32.12232 .512 & 27.525 & 24.507 & 29.493 & 28.259 & 28.476 & 29.51929 .803 & 29.808 \\
\hline 17/2006 & 8:00 & 31.50631 .846 & 32.12232 .513 & 27.532 & 24.467 & 29.491 & 28.251 & 28.468 & 29.52229 .807 & 29.799 \\
\hline /7/2006 & $12: 00$ & 31.52031 .848 & 32.12032 .510 & 27.523 & 24.438 & 29.482 & 28.275 & 28.469 & 29.51929 .803 & .790 \\
\hline 5/7/2006 & $16: 00$ & 31.47531 .802 & 32.07732 .469 & 27.471 & 24.409 & 29.444 & 28.288 & 28.433 & 29.48229 .768 & 29.773 \\
\hline $5 / 7 / 2006$ & 20:00 & 31.47531 .795 & 32.07132 .464 & 27.450 & 24.398 & 29.431 & 28.123 & 28.411 & 29.47829 .765 & 29.755 \\
\hline 5/8/2006 & $0: 00$ & 31.44231 .761 & 32.03332 .423 & 27.412 & 24.377 & 29.394 & 28.103 & 28.387 & 4129.730 & .737 \\
\hline 5/8/2006 & 4:00 & 31.40831 .730 & 32.00232 .394 & 27.381 & 24.370 & 29.374 & 28.052 & 28.365 & 29.40929 .693 & 29.711 \\
\hline $5 / 8 / 2006$ & 8:00 & 31.44731 .759 & 32.02932 .425 & 27.413 & 24.379 & 29.389 & 28.081 & 28.375 & 29.43829 .724 & 29.709 \\
\hline //8/2006 & $12: 00$ & 31.43431 .761 & 32.02932 .407 & 27.392 & 24.377 & 29.391 & 28.079 & 28.365 & 29.42629 .715 & 29.700 \\
\hline 5/8/2006 & $16: 00$ & 31.38131 .711 & 31.98332 .373 & 27.356 & 66 & 29.349 & 28.030 & 28.347 & 872 & 684 \\
\hline 5/8/2006 & $20: 00$ & 31.36431 .696 & 31.96232 .353 & 27.327 & 24.359 & 29.329 & 28.001 & 28.317 & 29.36729 .659 & 29.666 \\
\hline 5/9/2006 & $0: 00$ & 31.40831 .730 & 31.99332 .380 & 27.356 & 24.375 & 29.369 & 28.173 & 28.329 & 29.39729 .683 & 29.651 \\
\hline 5/9/2006 & 4:00 & 31.38531 .711 & 31.97632 .367 & 27.348 & 24.373 & 29.307 & 28.092 & 28.314 & 29.3772 & 31 \\
\hline $5 / 9 / 2006$ & 8:00 & 31.37331 .707 & 31.97432 .365 & 27.350 & 36 & 14 & 28.048 & 28.317 & 752 & 27 \\
\hline 5/9/2006 & $12: 00$ & 31.40531 .728 & 31.99532 .388 & 27.375 & 24.276 & 29.334 & 28.061 & 28.332 & 29.40229 .686 & 29.640 \\
\hline 5/9/2006 & $16: 00$ & 31.40331 .709 & 31.97432 .366 & 27.356 & 24.208 & 29.303 & 28.154 & 28.314 & 29.38229 .670 & 29.638 \\
\hline 5/9/2006 & 20:00 & 31.38931 .720 & 31.98132 .373 & 27.369 & 24.183 & 29.296 & 28.046 & 28.329 & 29.38929 & 49 \\
\hline 5/10/2006 & 0:00 & 31.4143 & 9832.388 & 27.396 & 88 & 07 & 27.973 & 28.342 & 29 & 29.651 \\
\hline $5 / 10 / 2006$ & 4:00 & 31.41231 .739 & 31.99832 .392 & 27.408 & 24.195 & 29.307 & 27.988 & 28.357 & 29.4062 & 29.653 \\
\hline 006 & 8:00 & 131.783 & 32.04032 .431 & 27.463 & 24.225 & 29.345 & 28.076 & 28. & 462 & 573 \\
\hline $5 / 10 / 2006$ & $12: 00$ & 31.4 & 32.06332 .453 & 27.490 & 24.248 & 58 & 28.108 & 28. & 702 & 95 \\
\hline 5/10/2006 & $16: 00$ & 31.45331 .773 & 32.02332 .418 & 27.442 & 24.243 & 32 & 28.053 & 28.397 & 29.4332 & 29.693 \\
\hline $5 / 10 / 2006$ & 20:00 & 31.44431 .773 & 32.02132 .418 & 27.438 & 24.255 & 29.332 & 28.050 & 28.445 & 29.43329 .723 & 29.695 \\
\hline $5 / 11 / 2006$ & $0: 00$ & 31.47331 .794 & 32.04632 .439 & 27.465 & 24.271 & 29.349 & 28.077 & 28.428 & 29.4582 & 29.709 \\
\hline $5 / 11 / 2006$ & 4:00 & 31.47531 .794 & 32.04632 .439 & 27.471 & 24.285 & 29.358 & 28.086 & 28.438 & 29.45529 .745 & 29.713 \\
\hline $5 / 11 / 2006$ & 8:00 & 31.47131 .798 & 32.04632 .441 & 27.475 & 24.297 & 29.360 & 28.088 & 28.446 & 29.45829 .748 & 29.722 \\
\hline $5 / 11 / 2006$ & $12: 00$ & 31.49031 .806 & 32.06532 .455 & 27.490 & 24.312 & 29.378 & 28.103 & 28.450 & 29.47329 .763 & 29.733 \\
\hline $5 / 11 / 2006$ & $16: 00$ & 31.45931 .808 & 32.04032 .427 & 27.448 & 24.312 & 29.358 & 28.066 & 28.443 & 29.45329 .746 & 29.733 \\
\hline $5 / 11 / 2006$ & 20:00 & 31.45531 .762 & 32.01232 .398 & 27.412 & 24.308 & 29.363 & 28.033 & 28.405 & 29.39929 .699 & 29.711 \\
\hline $5 / 12 / 2006$ & $0: 00$ & 31.43631 .751 & 32.00032 .390 & 27.410 & 24.315 & 29.325 & 28.028 & 28.400 & 29.39229 .692 & 29.711 \\
\hline $5 / 12 / 2006$ & 4:00 & 31.42031 .734 & 31.98532 .375 & 27.396 & 24.319 & 29.316 & 28.015 & 28.390 & 29.37529 .673 & 29.702 \\
\hline $5 / 12 / 2006$ & 8:00 & 31.42431 .740 & 31.99332 .378 & 27.404 & 24.327 & 29.325 & 28.026 & 28.395 & 29.37929 .677 & 29.702 \\
\hline $5 / 12 / 2006$ & $12: 00$ & 31.43831 .785 & 32.03132 .398 & 27.389 & 24.333 & 29.402 & 28.070 & 28.387 & 29.41129 .714 & 29.706 \\
\hline $5 / 12 / 2006$ & $16: 00$ & 31.42431 .742 & 31.99132 .363 & 27.337 & 24.326 & 29.343 & 28.015 & 28.357 & 29.37529 .675 & 32.393 \\
\hline
\end{tabular}


Depth to Water (ft TOC) in Well Indicated

$\begin{array}{llllllllllllll}\text { Date } & \text { Time } & \text { MW-1 } & \text { MW-2 } & \text { MW-3 } & \text { MW-4 } & \text { KDHE-1 } & \text { KDHE-2 } & \text { NW-1 } & \text { NW-2 } & \text { NW-3 } & \text { L-1 } & \text { L-2 } & \text { L-3 }\end{array}$

\begin{tabular}{|c|c|c|c|c|c|c|c|c|c|c|}
\hline $5 / 12 / 2006$ & $20: 00$ & 31.41431 .723 & 31.96832 .355 & 27.316 & 24.324 & 29.296 & 27.971 & 28.355 & 29.37529 .675 & 35.752 \\
\hline$/ 13 / 2006$ & $0: 00$ & 31.43831 .730 & 31.97232 .365 & 27.308 & 24.326 & 29.294 & 27.964 & 28.372 & 29.39429 .704 & 36.627 \\
\hline$/ 13 / 2006$ & $4: 00$ & 31.45331 .738 & 31.97932 .378 & 27.310 & 24.334 & 29.292 & 27.969 & 28.408 & 29.41629 .734 & 7.311 \\
\hline $13 / 2006$ & $8: 00$ & 31.51431 .783 & 32.01932 .424 & 27.346 & 24.350 & 29.320 & 28.006 & 28.466 & 29.47029 .792 & 37.836 \\
\hline /13/2006 & $12: 00$ & 31.56431 .854 & 32.08832 .478 & 27.366 & 24.370 & 29.374 & 28.046 & 28.508 & 29.53429 .863 & .757 \\
\hline$/ 13 / 2006$ & $16: 00$ & 31.57631 .833 & 32.06932 .474 & 27.360 & 24.380 & 29.354 & 28.030 & 28.526 & 29.51429 .841 & 2.311 \\
\hline 5/13/2006 & $20: 00$ & 31.55731 .835 & 32.07332 .476 & 27.364 & 24.393 & 29.358 & 28.035 & 28.529 & 29.51229 .828 & 31.602 \\
\hline $5 / 14 / 2006$ & 0:00 & 31.57231 .856 & 32.09432 .498 & 27.400 & 24.416 & 29.387 & 28.072 & 28.551 & 29.52729 .834 & 35 \\
\hline 5/14/2006 & $4: 00$ & 31.57031 .861 & 32.10532 .504 & 27.419 & 24.435 & 29.407 & 28.094 & 28.569 & 29.52729 .832 & .825 \\
\hline $5 / 14 / 2006$ & $8: 00$ & 31.60331 .890 & 32.13632 .533 & 27.465 & 24.463 & 29.440 & 28.138 & 28.587 & 29.55129 .850 & 30.612 \\
\hline 5/14/2006 & $12: 00$ & 31.59831 .890 & 32.13832 .533 & 27.469 & 24.481 & 29.451 & 28.145 & 28.597 & 29.54929 .848 & 30.464 \\
\hline $5 / 14 / 2006$ & $16: 00$ & 31.61131 .888 & 32.13632 .531 & 27.467 & 97 & 29.454 & 28.147 & 28.589 & 4425 & 49 \\
\hline 5/14/2006 & $20: 00$ & 31.61931 .905 & 32.14532 .537 & 27.473 & 24.513 & 29.471 & 28.152 & 28.587 & 532 & .249 \\
\hline $5 / 15 / 2006$ & $0: 00$ & 31.65631 .933 & 32.17632 .568 & 27.504 & 24.536 & 29.498 & 28.185 & 28.609 & 29.58329 .876 & 30.200 \\
\hline $5 / 15 / 2006$ & $4: 00$ & 31.62131 .918 & 32.16632 .559 & 27.502 & 24.551 & 29.502 & 28.185 & 28.609 & 29.56829 .863 & 30.171 \\
\hline $5 / 15 / 2006$ & 8:00 & 31.63331 .937 & 32.18132 .570 & 27.517 & 24.571 & 29.518 & 28.202 & 28.617 & 802 & 243 \\
\hline $5 / 15 / 2006$ & $12: 00$ & 31.65431 .941 & 32.18732 .580 & 27.517 & 24.583 & 29.522 & 28.205 & 28.632 & 952 & 32.483 \\
\hline 5/15/2006 & $16: 00$ & 31.65031 .928 & 32.17832 .572 & 27.492 & 24.589 & 29.511 & 28.185 & 28.642 & 29.5882 & 31.177 \\
\hline $5 / 15 / 2006$ & $20: 00$ & 31.61931 .930 & 32.18932 .572 & 27.465 & 24.592 & 29.502 & 28.165 & 28.627 & 802 & 30.727 \\
\hline $5 / 16 / 2006$ & $0: 00$ & 31.63931 .941 & 32.20032 .586 & 27.479 & 24.604 & 29.524 & 28.189 & 28.637 & 952 & 486 \\
\hline 5/16/2006 & $4: 00$ & 31.61931 .920 & 32.17832 .566 & 27.473 & 24.613 & 29.518 & 28.185 & 28.627 & 732 & 30.331 \\
\hline $5 / 16 / 2006$ & 8:00 & 31.62131 .920 & 32.17932 .568 & 27.479 & 24.625 & 29.524 & 28.194 & 28.617 & 29.5762 & 30.227 \\
\hline $5 / 16 / 2006$ & $12: 00$ & 31.59431 .900 & 32.15732 .545 & 27.454 & 24.626 & 29.513 & 28.174 & 28.604 & 29.55329 .852 & 30.154 \\
\hline $5 / 16 / 2006$ & $16: 00$ & 31.57631 .873 & 32.12632 .512 & 27.415 & 24.618 & 29.489 & 28.136 & 28.577 & 29.5242 & 30.092 \\
\hline $5 / 16 / 2006$ & $20: 00$ & 31.63531 .913 & 32.15732 .555 & 27.391 & 24.615 & 29.489 & 28.121 & 28.566 & 29.56329 .865 & 30.054 \\
\hline $5 / 17 / 2006$ & $0: 00$ & 31.64231 .922 & 32.16332 .561 & 27.398 & 24.622 & 29.496 & 28.134 & 28.582 & 29.57329 .867 & 30.056 \\
\hline $5 / 17 / 2006$ & 4:00 & 31.60331 .903 & 32.14932 .539 & 27.390 & 24.625 & 29.498 & 28.134 & 28.569 & 29.55129 .843 & 30.019 \\
\hline $5 / 17 / 2006$ & 8:00 & 31.76131 .960 & 32.22932 .725 & 27.404 & 24.634 & 29.511 & 28.150 & 28.580 & 29.63229 .901 & 29.992 \\
\hline $5 / 17 / 2006$ & $12: 00$ & 31.67031 .932 & 32.19332 .613 & 27.385 & 24.634 & 29.505 & 28.143 & 28.599 & 29.60029 .887 & 29.974 \\
\hline $5 / 17 / 2006$ & $16: 00$ & 31.61331 .928 & 32.16832 .563 & 27.346 & 24.626 & 29.513 & 28.110 & 28.677 & 29.57329 .874 & 29.950 \\
\hline $5 / 17 / 2006$ & $20: 00$ & 31.70931 .979 & 32.21832 .655 & 27.312 & 24.618 & 29.496 & 28.090 & 28.554 & 29.61729 .903 & 33.070 \\
\hline $5 / 18 / 2006$ & $0: 00$ & 31.66831 .945 & 32.19332 .598 & 27.344 & 24.636 & 29.511 & 28.123 & 28.574 & 29.60529 .898 & 35.302 \\
\hline $5 / 18 / 2006$ & $4: 00$ & 31.68331 .958 & 32.20532 .610 & 27.374 & 24.650 & 29.533 & 28.158 & 28.597 & 29.62929 .932 & 36.485 \\
\hline $5 / 18 / 2006$ & 8:00 & 31.78532 .006 & 32.26032 .719 & 27.389 & 24.662 & 29.551 & 28.180 & 28.640 & 29.69829 .998 & 37.262 \\
\hline
\end{tabular}


Depth to Water (ft TOC) in Well Indicated

$\begin{array}{llllllllllllll}\text { Date } & \text { Time } & \text { MW-1 } & \text { MW-2 } & \text { MW-3 } & \text { MW-4 } & \text { KDHE-1 } & \text { KDHE-2 } & \text { NW-1 } & \text { NW-2 } & \text { NW-3 } & \text { L-1 } & \text { L-2 } & \text { L-3 }\end{array}$

\begin{tabular}{|c|c|c|c|c|c|c|c|c|c|c|}
\hline $5 / 18 / 2006$ & $12: 00$ & 31.88032 .057 & 1532.790 & 27.373 & 24.668 & 29.553 & 28.174 & 28 & 8130.087 & .834 \\
\hline $5 / 18 / 2006$ & $16: 00$ & 31.87132 .061 & 32.30532 .778 & 27.339 & 24.664 & 29.582 & 28.169 & 28.738 & 29.77730 .096 & .261 \\
\hline$/ 18 / 2006$ & $20: 00$ & 31.92332 .097 & 32.39732 .839 & 27.319 & 24.663 & 29.553 & 28.154 & 28.783 & 29.83130 .151 & .983 \\
\hline $19 / 2006$ & $0: 00$ & 31.88632 .088 & 32.33432 .776 & 27.318 & 24.666 & 29.549 & 28.152 & 28.788 & 29.81630 .140 & .151 \\
\hline$/ 19 / 2006$ & $4: 00$ & 31.80832 .034 & 32.27732 .713 & 27.283 & 24.659 & 29.524 & 28.123 & 28.764 & 29.75030 .073 & .762 \\
\hline 5/19/2006 & 8:00 & 31.83232 .063 & 32.29032 .735 & 27.287 & 24.662 & 29.531 & 28.134 & 28.746 & 29.75530 .078 & 32.413 \\
\hline /19/2006 & $12: 00$ & 31.96432 .118 & 32.37032 .868 & 27.295 & 24.669 & 29.553 & 28.145 & 28.783 & 29.83330 .133 & 32.995 \\
\hline $5 / 19 / 2006$ & $16: 00$ & 31.90032 .097 & 32.34532 .797 & 27.281 & 24.670 & 29.553 & 28.134 & 28.791 & 29.7943 & 817 \\
\hline 5/19/2006 & $20: 00$ & 31.93132 .108 & 32.36632 .829 & 27.298 & 24.680 & 29.567 & 28.163 & 28.804 & 29.81830 .118 & .336 \\
\hline $5 / 20 / 2006$ & $0: 00$ & 31.90032 .124 & 32.37032 .809 & 27.346 & 24.701 & 29.604 & 28.211 & 28.796 & 29.81130 .111 & 31.051 \\
\hline 5/20/2006 & $4: 00$ & 31.86932 .112 & 32.36032 .786 & 27.375 & 24.719 & 29.624 & 28.242 & 28.786 & 887 & 30.831 \\
\hline 5/20/2006 & $8: 00$ & 2232.082 & 32.33432 .752 & 27.369 & 728 & 24 & 28.240 & 28.768 & 53 & 63 \\
\hline $5 / 20 / 2006$ & $12: 00$ & 31.84132 .106 & 32.34932 .770 & 27.379 & 24.740 & 29.633 & 28.251 & 28.778 & 29.76730 & 539 \\
\hline $5 / 20 / 2006$ & $16: 00$ & 31.87232 .137 & 32.38932 .797 & 27.379 & 24.753 & 29.659 & 28.260 & 28.778 & 29.80130 .095 & 30.450 \\
\hline 06 & $20: 00$ & 532.128 & 32.36132 .764 & 27.377 & 761 & 29.657 & 28.257 & 28.746 & 29.7793 & 377 \\
\hline 06 & $0: 00$ & 032.118 & 32.36532 .762 & 27.404 & 777 & 29.677 & 28.284 & 28.738 & 29. & 322 \\
\hline $5 / 21 / 2006$ & $4: 00$ & 31.80132 .078 & 32.32832 .727 & 27.387 & 24.784 & 29.664 & 28.271 & 28.720 & 29.73230 .025 & 30.264 \\
\hline $5 / 21 / 2006$ & $8: 00$ & 31.80132 .085 & 32.37832 .762 & 27.400 & 24.795 & 29.682 & 28.286 & 28.713 & 29.74030 & 30.227 \\
\hline 006 & $12: 00$ & 132.152 & 32.38732 .766 & 27.418 & 307 & 29.706 & 28.308 & 28.718 & 29. & 213 \\
\hline $5 / 21 / 2006$ & $16: 00$ & 31.85732 .143 & 32.36632 .756 & 27.396 & 24.809 & 29.693 & 28.288 & 28.715 & 29.7863 & 37.028 \\
\hline $5 / 21 / 2006$ & $20: 00$ & 31.91032 .242 & 32.42732 .801 & 27.413 & 24.823 & 29.730 & 28.317 & 28.804 & 29.86030 .175 & 35.962 \\
\hline $5 / 22 / 2006$ & $0: 00$ & 31.97632 .228 & 32.44732 .844 & 27.458 & 24.844 & 29.752 & 28.361 & 28.836 & 29.89230 .204 & 32.913 \\
\hline $5 / 22 / 2006$ & $4: 00$ & 31.92232 .200 & 32.42932 .829 & 27.458 & 53 & 29.752 & 28.363 & 28.824 & 29.86730 & 31.939 \\
\hline $5 / 22 / 2006$ & $8: 00$ & 31.94132 .223 & 32.45832 .854 & 27.506 & 24.878 & 29.786 & 28.403 & 28.852 & 29. & 36.224 \\
\hline $5 / 22 / 2006$ & $12: 00$ & 31.97832 .228 & 32.46432 .862 & 27.498 & 24.888 & 29.790 & 28.407 & 28.857 & 29.89730 .206 & 32.960 \\
\hline $5 / 22 / 2006$ & $16: 00$ & 31.96332 .234 & 32.45832 .848 & 27.462 & 24.883 & 29.770 & 28.366 & 28.864 & 29.8 & 31.914 \\
\hline $5 / 22 / 2006$ & $20: 00$ & 32.04432 .295 & 32.50432 .929 & 27.456 & 24.892 & 29.779 & 28.379 & 28.864 & 29. & 31.400 \\
\hline $5 / 23 / 2006$ & $0: 00$ & 32.01732 .264 & 32.49232 .897 & 27.477 & 24.906 & 29.799 & 28.403 & 28.869 & 29.92930 .229 & 31.093 \\
\hline $5 / 23 / 2006$ & $4: 00$ & 31.97432 .242 & 32.47332 .870 & 27.475 & 24.915 & 29.799 & 28.403 & 28.849 & 29.89230 .191 & 30.871 \\
\hline $5 / 23 / 2006$ & 8:00 & 31.96232 .230 & 32.47532 .872 & 27.496 & 24.929 & 29.814 & 28.425 & 28.846 & 29.88930 .189 & 30.842 \\
\hline $5 / 23 / 2006$ & $12: 00$ & 32.07532 .264 & 32.52332 .985 & 27.473 & 24.932 & 29.803 & 28.407 & 28.859 & 29.95130 .233 & 32.463 \\
\hline $5 / 23 / 2006$ & $16: 00$ & 31.98632 .219 & 32.46632 .882 & 27.439 & 24.925 & 29.779 & 28.377 & 28.846 & 29.90230 .200 & 38.626 \\
\hline $5 / 23 / 2006$ & $20: 00$ & 32.06632 .289 & 32.52332 .946 & 27.425 & 24.927 & 29.799 & 28.377 & 28.884 & 29.98230 .291 & 41.007 \\
\hline $5 / 24 / 2006$ & $0: 00$ & 32.07332 .297 & 32.53432 .952 & 27.465 & 24.941 & 29.821 & 28.416 & 28.937 & 30.00230 .328 & 42.553 \\
\hline
\end{tabular}


Depth to Water (ft TOC) in Well Indicated

$\begin{array}{llllllllllllll}\text { Date } & \text { Time } & \text { MW-1 } & \text { MW-2 } & \text { MW-3 } & \text { MW-4 } & \text { KDHE-1 } & \text { KDHE-2 } & \text { NW-1 } & \text { NW-2 } & \text { NW-3 } & \text { L-1 } & \text { L-2 } & \text { L-3 }\end{array}$

\begin{tabular}{|c|c|c|c|c|c|c|c|c|c|c|}
\hline $5 / 24 / 2006$ & $4: 00$ & 32.07932 .301 & 32.53232 .954 & 27.462 & 24.948 & 828 & 28.418 & 28.980 & 30.02230 .368 & 649 \\
\hline $5 / 24 / 2006$ & 8:00 & 32.18132 .413 & 32.60733 .025 & 27.500 & 24.964 & 29.865 & 28.458 & 29.046 & 30.12530 .492 & 43.948 \\
\hline /24/2006 & $12: 00$ & 32.58532 .529 & 32.73233 .246 & 27.515 & 24.976 & 29.894 & 28.485 & 29.129 & 30.31830 .678 & 41.765 \\
\hline 24/2006 & $16: 00$ & 32.30332 .500 & 32.69433 .136 & 27.491 & 24.980 & 29.899 & 28.474 & 29.265 & 30.23030 .598 & 36.328 \\
\hline $5 / 24 / 2006$ & $20: 00$ & 32.27232 .494 & 32.70233 .135 & 27.475 & 24.983 & 29.899 & 28.469 & 29.202 & 30.21530 .576 & .466 \\
\hline $5 / 25 / 2006$ & $0: 00$ & 32.27432 .485 & 32.70033 .132 & 27.498 & 24.996 & 29.916 & 28.491 & 29.205 & 30.21030 .569 & 36.828 \\
\hline$/ 25 / 2006$ & $4: 00$ & 32.22432 .453 & 32.67733 .107 & 27.496 & 25.003 & 29.917 & 28.496 & 29.195 & 30.17430 .523 & 36.018 \\
\hline 5/25/2006 & $8: 00$ & 32.27232 .475 & 32.70433 .146 & 27.521 & 25.017 & 29.934 & 28.518 & 29.195 & 30.1913 & 027 \\
\hline $5 / 25 / 2006$ & $12: 00$ & 32.28432 .496 & 32.71733 .148 & 27.527 & 25.026 & 29.945 & 28.529 & 29.195 & 30.2033 & 35.553 \\
\hline $5 / 25 / 2006$ & $16: 00$ & 32.22732 .439 & 32.67133 .125 & 27.477 & 25.017 & 29.914 & 28.476 & 29.168 & 30.14430 .476 & 33.777 \\
\hline $5 / 25 / 2006$ & $20: 00$ & 32.37432 .568 & 32.86433 .317 & 27.467 & 25.022 & 29.952 & 28.500 & 29.183 & 90 & 32.627 \\
\hline 2006 & 0:00 & 32.27232 .519 & 32.76733 .195 & 27.498 & 35 & 63 & 28. & 29.175 & 28 & 52 \\
\hline $5 / 26 / 2006$ & $4: 00$ & 32.18532 .439 & 32.68933 .109 & 27.475 & 25.033 & 29.941 & 28.507 & 29.115 & 30.1273 & 31.675 \\
\hline $5 / 26 / 2006$ & 8:00 & 32.17932 .434 & 32.69233 .099 & 27.512 & 25.052 & 29.963 & 28.537 & 29.099 & 30.11030 & 31.405 \\
\hline 06 & $12: 00$ & 32.26032 .481 & 32.77233 .227 & 27.506 & 50 & 29.976 & 28.555 & 98 & 863 & 214 \\
\hline $5 / 26 / 2006$ & $16: 00$ & 32.11332 .390 & 32.65033 .062 & 27.443 & 40 & 29.918 & 28. & 37 & 74 & D39 \\
\hline $5 / 26 / 2006$ & 20:00 & 32.07932 .378 & 32.64133 .033 & 27.431 & 25.040 & 29.921 & 28.471 & 28.989 & 30.03930 .341 & 31.697 \\
\hline $5 / 27 / 2006$ & $0: 00$ & 32.09732 .407 & 32.66233 .052 & 27.481 & 25.063 & 29.967 & 28.524 & 29.011 & 30.0583 & 106 \\
\hline 006 & $4: 00$ & 32.05232 .361 & 32.62033 .009 & 27.473 & 25.061 & 29.954 & 28. & 28. & 22 & 398 \\
\hline $5 / 27 / 2006$ & 8:00 & 32.13632 .401 & 32.65633 .103 & 27.460 & 25.063 & 29.943 & 28.509 & 28.935 & 30.056 & 34.067 \\
\hline $5 / 27 / 2006$ & $12: 00$ & 32.08732 .378 & 32.64133 .036 & 27.473 & 25.073 & 29.954 & 28.526 & 28.980 & 30.0513 & 33.024 \\
\hline $5 / 27 / 2006$ & $16: 00$ & 32.09732 .435 & 32.64333 .027 & 27.454 & 25.072 & 29.952 & 28.513 & 28.940 & 30.07130 .383 & 31.788 \\
\hline $5 / 27 / 2006$ & $20: 00$ & 32.15932 .449 & 32.68933 .099 & 27.466 & 25.080 & 29.972 & 28.529 & 28.952 & 30.11530 .415 & 31.312 \\
\hline $5 / 28 / 2006$ & $0: 00$ & 32.14832 .460 & 32.69433 .086 & 27.510 & 25.095 & 30.005 & 28. & 28.962 & 118 & 31.057 \\
\hline $5 / 28 / 2006$ & $4: 00$ & 32.13432 .443 & 32.68133 .066 & 27.517 & 25.109 & 30.011 & 28.584 & 28.952 & 30.09330 & 30.887 \\
\hline $5 / 28 / 2006$ & 8:00 & 32.24932 .494 & 32.76133 .203 & 27.546 & 25.125 & 30.036 & 28.612 & 28.962 & 30.15230 & 30.776 \\
\hline $5 / 28 / 2006$ & $12: 00$ & 32.26532 .536 & 32.78633 .207 & 27.563 & 25.132 & 30.056 & 28.632 & 29.020 & 30.20630 & 30.716 \\
\hline $5 / 28 / 2006$ & $16: 00$ & 32.21232 .536 & 32.76733 .154 & 27.562 & 25.142 & 30.073 & 28.637 & 29.007 & 30.18130 .483 & 34.027 \\
\hline $5 / 28 / 2006$ & $20: 00$ & 32.26032 .578 & 32.79933 .176 & 27.575 & 25.147 & 30.102 & 28.656 & 29.027 & 30.21330 .525 & 39.459 \\
\hline 5/29/2006 & $0: 00$ & 32.33132 .633 & 32.85233 .238 & 27.606 & 25.165 & 30.135 & 28.694 & 29.078 & 30.29430 .618 & 34.031 \\
\hline $5 / 29 / 2006$ & $4: 00$ & 32.29832 .603 & 32.83333 .221 & 27.619 & 25.174 & 30.140 & 28.705 & 29.095 & 30.27230 .593 & 32.623 \\
\hline $5 / 29 / 2006$ & 8:00 & 32.33332 .637 & 32.86433 .254 & 27.667 & 25.197 & 30.175 & 28.749 & 29.123 & 30.29630 .616 & 38.804 \\
\hline 006 & $12: 00$ & 32.39932 .715 & 32.94233 .327 & 27.696 & 25.214 & 30.222 & 28.786 & 29.179 & 30.38230 .717 & 35.938 \\
\hline /29/2006 & $16: 00$ & 32.46932 .749 & 32.98233 .383 & 27.688 & 25.220 & 30.237 & 28.786 & 29.196 & 30.42130 .755 & 33.591 \\
\hline
\end{tabular}


Depth to Water (ft TOC) in Well Indicated

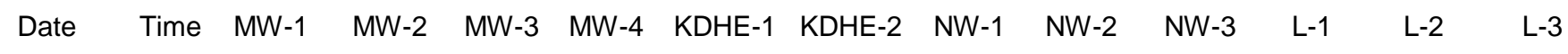

\begin{tabular}{|c|c|c|c|c|c|c|c|c|c|c|}
\hline $5 / 29 / 2006$ & $20: 00$ & 32.45432 .795 & 33.00333 .389 & 27.677 & 25.227 & 30.259 & 28.791 & 29.206 & 30.44330 .771 & 32.612 \\
\hline /30/2006 & $0: 00$ & 32.47532 .796 & 33.03033 .413 & 27.769 & 25.271 & 30.332 & 28.892 & 29.251 & 30.45130 .764 & 32.049 \\
\hline $5 / 30 / 2006$ & $4: 00$ & 32.38232 .705 & 32.95033 .336 & 27.728 & 25.266 & 30.264 & 28.835 & 29.196 & 30.36730 .678 & 1.653 \\
\hline /30/2006 & 8:00 & 32.39932 .700 & 32.95333 .332 & 27.769 & 25.271 & 30.279 & 28.859 & 29.211 & 30.35530 .660 & .403 \\
\hline /30/2006 & $12: 00$ & 32.38232 .688 & 32.94833 .327 & 27.790 & 25.267 & 30.275 & 28.868 & 29.214 & 30.34330 .645 & 31.230 \\
\hline /30/2006 & $16: 00$ & 32.34932 .673 & 32.93433 .311 & 27.803 & 25.255 & 30.277 & 28.875 & 29.216 & 30.32630 .629 & 31.097 \\
\hline $5 / 30 / 2006$ & $20: 00$ & 32.29032 .622 & 32.87933 .254 & 27.751 & 25.218 & 30.233 & 28.811 & 29.171 & 30.26930 .569 & 30.971 \\
\hline 5/31/2006 & $0: 00$ & 32.31532 .643 & 32.90633 .280 & 27.807 & 25.236 & 30.266 & 28.859 & 29.183 & 3930.582 & 30.896 \\
\hline 5/31/2006 & $4: 00$ & 32.28632 .624 & 32.88933 .260 & 27.811 & 25.236 & 30.261 & 28.861 & 29.176 & 30.26930 .562 & 30.827 \\
\hline $5 / 31 / 2006$ & $8: 00$ & 32.28032 .620 & 32.88933 .258 & 27.830 & 25.246 & 30.264 & 28.872 & 29.176 & 30.26230 .554 & 30.778 \\
\hline $5 / 31 / 2006$ & $12: 00$ & 32.28632 .625 & 32.89833 .266 & 27.849 & 25.259 & 30.277 & 28.89 & 29.183 & 30.26930 .559 & 30.743 \\
\hline $5 / 31 / 2006$ & $16: 00$ & 32.24532 .584 & 32.85233 .221 & 27.801 & 25.239 & 30.246 & 28.842 & 29.140 & 30 & 30.714 \\
\hline 5/31/2006 & $20: 00$ & 32.24132 .595 & 32.86833 .221 & 27.803 & 25.241 & 30.253 & 28.842 & 29.145 & 30. & 30.674 \\
\hline $6 / 1 / 2006$ & $0: 00$ & 32.27532 .603 & 32.87333 .240 & 27.832 & 25.264 & 30.341 & 28.868 & 29.138 & 30.24730 .534 & 30.634 \\
\hline $6 / 1 / 2006$ & $4: 00$ & 32.22832 .572 & 32.84533 .209 & 27.824 & 25.264 & 30.250 & 28.855 & 29.125 & 530.505 & 30.614 \\
\hline 06 & 8:00 & 32.26132 .578 & 32.85433 .217 & 27.845 & 25.278 & 30.261 & 28.879 & 29.135 & 30. & 597 \\
\hline $6 / 1 / 2006$ & $12: 00$ & 32.23732 .586 & 32.86433 .225 & 27.867 & 25.288 & 30.268 & 28.881 & 29.142 & 30. & 30.603 \\
\hline $6 / 1 / 2006$ & $16: 00$ & 32.20632 .553 & 32.82833 .188 & 27.830 & 25.269 & 30.237 & 28.842 & 29.117 & 330.485 & 30.579 \\
\hline $6 / 1 / 2006$ & $20: 00$ & 32.22232 .578 & 32.84733 .193 & 27.830 & 25.269 & 30.248 & 28.894 & 29.107 & 30. & 557 \\
\hline $6 / 2 / 2006$ & $0: 00$ & 32.21232 .568 & 32.84933 .203 & 27.859 & 25.287 & 30.255 & 28.846 & 29.107 & 30. & 546 \\
\hline $6 / 2 / 2006$ & $4: 00$ & 32.19832 .555 & 32.83533 .193 & 27.859 & 25.287 & 30.250 & 28.809 & 29.110 & 83 & 30.535 \\
\hline $6 / 2 / 2006$ & 8:00 & 32.20232 .559 & 32.84133 .197 & 27.878 & 25.296 & 30.257 & 28.756 & 29.112 & 30. & 30.530 \\
\hline 6/2/2006 & $12: 00$ & 32.20032 .551 & 32.83333 .189 & 27.876 & 25.297 & 30.250 & 28.784 & 29.115 & 130.480 & 30.535 \\
\hline 6/2/2006 & $16: 00$ & 32.17732 .555 & 32.82433 .170 & 27.842 & 25.278 & 30.244 & 28.837 & 29.120 & 630.483 & 30.521 \\
\hline $6 / 2 / 2006$ & $20: 00$ & 32.17332 .555 & 32.81833 .164 & 27.820 & 25.266 & 30.233 & 28.817 & 29.079 & 30.18430 .478 & 30.506 \\
\hline $6 / 3 / 2006$ & $0: 00$ & 32.18232 .551 & 32.82433 .172 & 27.838 & 25.276 & 30.237 & 28.833 & 29.079 & 30.18430 .474 & 30.495 \\
\hline 6/3/2006 & 4:00 & 32.14832 .515 & 32.79033 .142 & 27.815 & 25.264 & 30.211 & 28.806 & 29.052 & 30.15230 .443 & 30.481 \\
\hline $6 / 3 / 2006$ & 8:00 & 32.14632 .511 & 32.78833 .139 & 27.820 & 25.267 & 30.211 & 28.811 & 29.054 & 30.14730 .436 & 30.490 \\
\hline $6 / 3 / 2006$ & $12: 00$ & 32.16532 .506 & 32.79333 .139 & 27.809 & 25.262 & 30.202 & 28.8 & 29.049 & 30.14730 .438 & 33.604 \\
\hline $6 / 3 / 2006$ & $16: 00$ & 32.13832 .485 & 32.76733 .119 & 27.771 & 25.237 & 30.169 & 28.76 & 29.031 & 30.13730 .432 & 36.002 \\
\hline $6 / 3 / 2006$ & $20: 00$ & 32.15932 .502 & 32.77233 .129 & 27.749 & 25.223 & 30.160 & 28.74 & 29.047 & 30.15930 .465 & 37.242 \\
\hline $6 / 4 / 2006$ & 0:00 & 32.16532 .491 & 32.75733 .127 & 27.742 & 25.218 & 30.146 & 28.734 & 29.067 & 30.16630 .481 & 38.051 \\
\hline 6/4/2006 & $4: 00$ & 32.17332 .481 & 32.74433 .119 & 27.715 & 25.202 & 30.122 & 28.705 & 29.074 & 30.16930 .494 & 38.626 \\
\hline $6 / 4 / 2006$ & $8: 00$ & 32.24932 .549 & 32.83133 .205 & 27.774 & 25.230 & 30.171 & 28.762 & 29.140 & 30.24530 .571 & 39.083 \\
\hline
\end{tabular}


Depth to Water (ft TOC) in Well Indicated

$\begin{array}{lllllllllllllll}\text { Date } & \text { Time } & \text { MW-1 } & \text { MW-2 } & \text { MW-3 } & \text { MW-4 } & \text { KDHE-1 } & \text { KDHE-2 } & \text { NW-1 } & \text { NW-2 } & \text { NW-3 } & \text { L-1 } & \text { L-2 } & \text { L-3 }\end{array}$

\begin{tabular}{|c|c|c|c|c|c|c|c|c|c|c|}
\hline 6/4/2006 & $12: 00$ & 32.29032 .576 & 32.81633 .197 & 27.732 & 25.213 & 30.155 & 28.731 & 29.153 & 30.26730 .607 & 35.006 \\
\hline 6/4/2006 & $16: 00$ & 32.25332 .580 & 32.82033 .195 & 27.711 & 25.199 & 30.155 & 28.716 & 29.173 & 30.26230 .596 & 3.489 \\
\hline 6/4/2006 & $20: 00$ & 32.32132 .624 & 32.85633 .236 & 27.723 & 25.204 & 30.160 & 28.727 & 29.175 & 30.28930 .622 & 3.476 \\
\hline $6 / 5 / 2006$ & $0: 00$ & 32.32332 .633 & 32.88333 .258 & 27.759 & 25.227 & 30.188 & 28.764 & 29.190 & 30.30630 .622 & 32.280 \\
\hline 6/5/2006 & 4:00 & 32.28232 .595 & 32.85433 .233 & 27.757 & 25.229 & 30.177 & 28.762 & 29.183 & 30.26730 .578 & 31.826 \\
\hline $6 / 5 / 2006$ & $8: 00$ & 32.27532 .606 & 32.87133 .240 & 27.759 & 25.230 & 30.182 & 28.767 & 29.175 & 30.26430 .569 & 31.536 \\
\hline $6 / 5 / 2006$ & $12: 00$ & 32.31932 .624 & 32.88933 .266 & 27.801 & 25.259 & 30.213 & 28.809 & 29.190 & 30.27930 .578 & 31.327 \\
\hline 6/5/2006 & $16: 00$ & 32.21832 .540 & 32.81033 .184 & 27.734 & 25.229 & 30.166 & 28.742 & 29.135 & 30.20330 & 144 \\
\hline $6 / 5 / 2006$ & $20: 00$ & 32.23432 .559 & 32.84733 .215 & 27.728 & 25.225 & 30.166 & 28.738 & 29.160 & 30.21330 .509 & 32.156 \\
\hline $6 / 6 / 2006$ & $0: 00$ & 32.31032 .639 & 32.90433 .268 & 27.822 & 25.280 & 30.239 & 28.828 & 29.211 & 30.28230 .574 & 31.245 \\
\hline $6 / 6 / 2006$ & 4:00 & 32.23432 .570 & 32.83733 .203 & 27.774 & 25.257 & 30.200 & 28.784 & 29.158 & 2233 & 26 \\
\hline 6/6/2006 & 8:00 & 32.31432 .637 & 32.89633 .280 & 27.809 & 25.280 & 30.228 & 28.822 & 29.172 & 282 & 918 \\
\hline $6 / 6 / 2006$ & $12: 00$ & 32.37832 .675 & 32.95333 .346 & 27.834 & 25.297 & 30.257 & 28.846 & 29.215 & 30.33530 .616 & 30.851 \\
\hline $6 / 6 / 2006$ & $16: 00$ & 32.36032 .722 & 32.93233 .297 & 27.819 & 25.296 & 30.244 & 28.828 & 29. & 340 & 96 \\
\hline 006 & $20: 00$ & 32.34932 .683 & 32.99033 .338 & 27.832 & 25.304 & 66 & 28. & 29. & 3283 & \\
\hline 6/7/2006 & $0: 00$ & 32.37632 .707 & 32.99133 .356 & 27.878 & 25.329 & 30.297 & 28.899 & 29.238 & 30.362 & 10 \\
\hline 6/7/2006 & $4: 00$ & 32.35632 .687 & 32.96533 .330 & 27.897 & 25.341 & 30.304 & 28.908 & 29.241 & 30.34330 & 31.369 \\
\hline 6/7/2006 & 8:00 & 32.42132 .747 & 33.00533 .368 & 27.932 & 25.364 & 26 & 28. & 29.258 & 3943 & 48 \\
\hline 6/7/2006 & $12: 00$ & 32.65532 .853 & 33.13233 .605 & 27.939 & 25.375 & 30.359 & 28.952 & 29.316 & 30.5273 & 37 \\
\hline $6 / 7 / 2006$ & $16: 00$ & 32.72932 .910 & 33.19733 .712 & 27.914 & 25.371 & 30.370 & 28.945 & 29.369 & 30.60530 & 30.958 \\
\hline $6 / 7 / 2006$ & $20: 00$ & 32.62032 .893 & 33.22433 .622 & 27.909 & 25.371 & 30.374 & 28.947 & 29.384 & 30.57130 & 30.911 \\
\hline 6/8/2006 & 0:00 & 32.57132 .865 & 33.16733 .554 & 27.957 & 25.400 & 396 & 28. & 29.384 & 5393 & 398 \\
\hline $6 / 8 / 2006$ & $4: 00$ & 32.47532 .791 & 33.08133 .460 & 27.932 & 25.393 & 30.370 & 28.965 & 29.341 & 30.45830 .742 & 30.842 \\
\hline 6/8/2006 & 8:00 & 32.48132 .804 & 33.09833 .471 & 27.985 & 25.422 & 30.405 & 29.011 & 29.356 & 30.46530 .751 & 33.159 \\
\hline $6 / 8 / 2006$ & $12: 00$ & 32.46232 .789 & 33.07333 .440 & 27.957 & 25.417 & 30.401 & 28.994 & 29.341 & 30.44830 .738 & 31.476 \\
\hline $6 / 8 / 2006$ & $16: 00$ & 32.41132 .734 & 33.01433 .383 & 27.916 & 25.400 & 30.357 & 28.95 & 29.303 & 30.39730 & 34.166 \\
\hline $6 / 8 / 2006$ & $20: 00$ & 32.45432 .766 & 33.07333 .436 & 27.901 & 25.393 & 30.352 & 28.936 & 29.306 & 30.42930 .726 & 39.933 \\
\hline 6/9/2006 & $0: 00$ & 32.54432 .834 & 33.10033 .477 & 27.926 & 25.410 & 30.381 & 28.969 & 29.364 & 30.51730 .831 & 38.897 \\
\hline 6/9/2006 & 4:00 & 32.50532 .795 & 33.06033 .442 & 27.901 & 25.400 & 30.361 & 28.945 & 29.382 & 30.49230 & 39.249 \\
\hline 6/9/2006 & 8:00 & 32.53232 .812 & 33.07533 .464 & 27.913 & 25.410 & 30.366 & 28.958 & 29.422 & 30.51730 .850 & 40.542 \\
\hline 6/9/2006 & $12: 00$ & 32.59632 .882 & 33.16533 .507 & 27.891 & 25.396 & 30.658 & 29.108 & 29.455 & 30.56830 .901 & 40.883 \\
\hline $6 / 9 / 2006$ & $16: 00$ & 32.60632 .931 & 33.21833 .558 & 27.857 & 25.378 & 30.536 & 29.108 & 29.462 & 30.63730 .981 & 41.519 \\
\hline 6/9/2006 & $20: 00$ & 32.64932 .998 & 33.26433 .607 & 27.830 & 25.370 & 30.494 & 29.02 & 29.470 & 30.68431 .032 & 37.905 \\
\hline $6 / 10 / 2006$ & $0: 00$ & 32.67832 .977 & 33.24933 .614 & 27.845 & 25.385 & 30.512 & 29.068 & 29.495 & 30.69131 .034 & 35.072 \\
\hline
\end{tabular}


Depth to Water (ft TOC) in Well Indicated

$\begin{array}{llllllllllllll}\text { Date } & \text { Time } & \text { MW-1 } & \text { MW-2 } & \text { MW-3 } & \text { MW-4 } & \text { KDHE-1 } & \text { KDHE-2 } & \text { NW-1 } & \text { NW-2 } & \text { NW-3 } & \text { L-1 } & \text { L-2 } & \text { L-3 }\end{array}$

\begin{tabular}{|c|c|c|c|c|c|c|c|c|c|c|}
\hline $6 / 10 / 2006$ & $4: 00$ & 32.60832 .903 & 933.548 & 27.826 & 25.385 & 30.441 & 29.003 & 29.483 & 30.60830 .946 & 797 \\
\hline $6 / 10 / 2006$ & 8:00 & 32.63733 .017 & 33.29133 .593 & 27.838 & 25.394 & 30.797 & 29.21 & 29.467 & 30.64730 .974 & 4.377 \\
\hline b/10/2006 & $12: 00$ & 32.72533 .177 & 33.54633 .769 & 27.845 & 25.412 & 31.105 & 29.496 & 29.478 & 30.79931 .107 & 7.238 \\
\hline$/ 10 / 2006$ & $16: 00$ & 32.70333 .089 & 33.37433 .693 & 27.834 & 25.412 & 30.671 & 29.203 & 29.475 & 30.75731 .090 & 8.812 \\
\hline b/10/2006 & $20: 00$ & 32.70333 .097 & 33.32333 .659 & 27.813 & 25.410 & 30.596 & 29.104 & 29.472 & 30.75231 .099 & 155 \\
\hline $6 / 11 / 2006$ & $0: 00$ & 32.81133 .123 & 33.34833 .700 & 27.853 & 25.440 & 30.593 & 29.112 & 29.505 & 30.78431 .125 & 4.018 \\
\hline $6 / 11 / 2006$ & $4: 00$ & 32.74433 .083 & 33.33133 .697 & 27.909 & 25.475 & 30.593 & 29.143 & 29.523 & 30.76031 .090 & .090 \\
\hline $6 / 11 / 2006$ & 8:00 & 32.76233 .112 & 33.35433 .712 & 27.943 & 25.504 & 30.598 & 29.148 & 29.538 & 30.7603 & 537 \\
\hline $6 / 11 / 2006$ & $12: 00$ & 32.83433 .222 & 33.41133 .763 & 27.982 & 25.535 & 30.649 & 29.185 & 29.566 & 30.84331 .169 & .703 \\
\hline $6 / 11 / 2006$ & $16: 00$ & 32.87133 .257 & 33.44533 .794 & 27.989 & 25.548 & 30.682 & 29.198 & 29.578 & 30.88731 .222 & 37.406 \\
\hline $6 / 11 / 2006$ & $20: 00$ & 32.97433 .382 & 33.60333 .938 & 28.028 & 25.576 & 30.742 & 29.253 & 29.621 & 0731 & 671 \\
\hline $6 / 12 / 2006$ & 0:00 & 32.98233 .351 & 9933.924 & 28.070 & 25.608 & 758 & 29.286 & 29.664 & 03 & 325 \\
\hline $6 / 12 / 2006$ & $4: 00$ & 32.95333 .287 & 33.52533 .892 & 28.095 & 25.632 & 30.746 & 29.295 & 29.687 & 5131.267 & 296 \\
\hline $6 / 12 / 2006$ & 8:00 & 32.99833 .302 & 33.55233 .947 & 28.158 & 25.671 & 30.768 & 29.339 & 29.725 & 6131.267 & 32.960 \\
\hline 006 & $12: 00$ & 32.93033 .247 & 633.886 & 28.158 & 25.682 & 30.766 & 29.333 & 29.725 & 16 & 738 \\
\hline $6 / 12 / 2006$ & $16: 00$ & 32.89833 .232 & 3833.888 & 28.150 & 25.687 & 30.759 & 29.324 & 29.717 & 89 & 530 \\
\hline $6 / 12 / 2006$ & 20:00 & 32.87733 .219 & 33.51033 .867 & 28.141 & 25.683 & 30.744 & 29.315 & 29.707 & 30.88231 .180 & 32.366 \\
\hline $6 / 13 / 2006$ & $0: 00$ & 32.89833 .211 & 33.49333 .859 & 28.189 & 25.712 & 30.768 & 29.35 & 29.725 & 30.87731 & 32.251 \\
\hline $6 / 13 / 2006$ & $4: 00$ & 32.84033 .161 & 33.44733 .814 & 28.175 & 25.710 & 30.748 & 29.331 & 29.700 & 283 & 162 \\
\hline $6 / 13 / 2006$ & 8:00 & 32.84233 .162 & 33.45533 .820 & 28.208 & 25.729 & 30.764 & 29.353 & 29.715 & 30.82831 .119 & 32.089 \\
\hline $6 / 13 / 2006$ & $12: 00$ & 32.86533 .148 & 33.43533 .811 & 28.195 & 25.726 & 30.751 & 29.337 & 29.700 & 30.81831 & 31.711 \\
\hline $6 / 13 / 2006$ & $16: 00$ & 32.80333 .112 & 33.39233 .761 & 28.148 & 25.698 & 30.717 & 29.291 & 29.677 & 30.77731 .070 & 32.975 \\
\hline $6 / 13 / 2006$ & $20: 00$ & 32.87133 .194 & 33.46633 .828 & 28.129 & 25.680 & 30.709 & 29.269 & 29.672 & 30.84331 .136 & 162 \\
\hline $6 / 14 / 2006$ & $0: 00$ & 32.90033 .202 & 33.46033 .838 & 28.158 & 25.699 & 30.728 & 29.302 & 29.715 & 30. & 863 \\
\hline $6 / 14 / 2006$ & $4: 00$ & 32.87133 .158 & 33.42233 .803 & 28.131 & 25.689 & 30.709 & 29.275 & 29.720 & 30.85531 .176 & 39.379 \\
\hline $6 / 14 / 2006$ & 8:00 & 32.93733 .245 & 33.47933 .861 & 28.139 & 25.694 & 30.724 & 29.289 & 29.750 & 30.92631 .258 & 37.369 \\
\hline $6 / 14 / 2006$ & $12: 00$ & 33.05833 .562 & 33.75234 .026 & 28.120 & 25.687 & 30.888 & 29.351 & 29.778 & 31.14431 .457 & 34.600 \\
\hline $6 / 14 / 2006$ & $16: 00$ & 33.05133 .555 & 33.77534 .042 & 28.078 & 25.657 & 30.883 & 29.349 & 29.750 & 31.16931 .486 & 33.493 \\
\hline $6 / 14 / 2006$ & $20: 00$ & 32.96933 .382 & 33.63233 .953 & 28.060 & 25.657 & 30.775 & 29.284 & 29.730 & 31.00231 .329 & 32.836 \\
\hline $6 / 15 / 2006$ & $0: 00$ & 33.07233 .555 & 33.78534 .069 & 28.087 & 25.678 & 30.910 & 29.377 & 29.722 & 31.17431 .488 & 32.417 \\
\hline $6 / 15 / 2006$ & $4: 00$ & 32.93533 .291 & 33.55733 .910 & 28.060 & 25.664 & 30.782 & 29.302 & 29.689 & 30.95831 .267 & 32.109 \\
\hline $6 / 15 / 2006$ & 8:00 & 32.94133 .245 & 33.52933 .896 & 28.087 & 25.694 & 30.773 & 29.315 & 29.692 & 30.91931 .214 & 31.892 \\
\hline $6 / 15$ & $12: 00$ & 32.88333 .194 & 33.47733 .845 & 28.085 & 25.699 & 30.748 & 29.295 & 29.679 & 30.87231 & 31.788 \\
\hline $6 / 15 / 2006$ & $16: 00$ & 32.85033 .152 & 33.43433 .795 & 28.062 & 25.682 & 30.735 & 29.282 & 29.649 & 30.81831 .116 & 31.615 \\
\hline
\end{tabular}


Depth to Water (ft TOC) in Well Indicated

$\begin{array}{llllllllllllll}\text { Date } & \text { Time } & \text { MW-1 } & \text { MW-2 } & \text { MW-3 } & \text { MW-4 } & \text { KDHE-1 } & \text { KDHE-2 } & \text { NW-1 } & \text { NW-2 } & \text { NW-3 } & \text { L-1 } & \text { L-2 } & \text { L-3 }\end{array}$

\begin{tabular}{|c|c|c|c|c|c|c|c|c|c|c|}
\hline $6 / 15 / 2006$ & 20:00 & 32.84633 .158 & 33.46833 .836 & 28.054 & 25.671 & 30.704 & 29.256 & 29.642 & 30.82131 .107 & 34.815 \\
\hline /16/2006 & 0:00 & 32.85233 .160 & 33.45133 .818 & 28.085 & 25.694 & 30.720 & 29.278 & 29.702 & 30.83131 .121 & 33.119 \\
\hline 6/16/2006 & 4:00 & 32.85433 .308 & 33.54433 .842 & 28.114 & 25.715 & 30.808 & 29.32 & 29.689 & 30.88031 .156 & 32.264 \\
\hline 6/16/2006 & 8:00 & 32.99433 .437 & 33.67033 .969 & 28.120 & 25.726 & 30.879 & 29.384 & 30.439 & 31.05631 .358 & 32.844 \\
\hline 6/16/2006 & $12: 00$ & 32.89633 .249 & 33.51733 .865 & 28.102 & 25.724 & 30.782 & 29.333 & 29.720 & 30.90931 .205 & 31.925 \\
\hline o/16/2006 & $16: 00$ & 32.87533 .156 & 33.43933 .795 & 28.074 & 25.705 & 30.728 & 29.284 & 29.654 & 30.82631 .110 & .664 \\
\hline 6/16/2006 & 20:00 & 32.79733 .131 & 33.41633 .781 & 28.070 & 25.698 & 30.711 & 29.267 & 29.626 & 30.79431 .076 & 31.505 \\
\hline $6 / 17 / 2006$ & $0: 00$ & 32.82033 .148 & 33.43433 .793 & 28.141 & 25.744 & 30.773 & 29.337 & 29.644 & 30.80431 .079 & 31.389 \\
\hline $6 / 17 / 2006$ & 4:00 & 32.72533 .063 & 33.35033 .707 & 28.078 & 25.708 & 30.709 & 29.271 & 29.581 & 30.72331 .001 & 301 \\
\hline $6 / 17 / 2006$ & 8:00 & 32.74433 .083 & 33.37133 .722 & 28.133 & 25.744 & 30.742 & 29.311 & 29.601 & 30.73531 .008 & 31.250 \\
\hline $6 / 17 / 2006$ & $12: 00$ & 32.73533 .069 & 33.35733 .711 & 28.143 & 25.752 & 30.746 & 29.322 & 29.596 & 30.72030 .994 & 31.208 \\
\hline $6 / 17 / 2006$ & $16: 00$ & 32.72333 .078 & 33.34033 .687 & 28.089 & 25.719 & 30.711 & 29.271 & 29.553 & 30.7233 & 170 \\
\hline $6 / 17 / 2006$ & $20: 00$ & 32.70533 .045 & 33.32133 .669 & 28.091 & 25.717 & 30.709 & 29.26 & 29.536 & 893 & 137 \\
\hline $6 / 18 / 2006$ & 0:00 & 32.71933 .063 & 33.34633 .695 & 28.150 & 25.761 & 30.744 & 29.313 & 29.566 & 30.70830 .979 & 31.117 \\
\hline $6 / 18 / 2006$ & 4:00 & 32.71533 .059 & 33.34833 .695 & 28.175 & 25.775 & 30.759 & 29.335 & 29.573 & 30.70630 .977 & 31.106 \\
\hline $6 / 18 / 2006$ & 8:00 & 32.74633 .086 & 33.37833 .724 & 28.225 & 25.805 & 30.793 & 29.379 & 29.601 & 303 & 04 \\
\hline $6 / 18 / 2006$ & $12: 00$ & 32.75833 .107 & 33.39233 .736 & 28.250 & 25.824 & 30.811 & 29.401 & 29.636 & 30.7503 & 102 \\
\hline $6 / 18 / 2006$ & $16: 00$ & 32.75233 .093 & 33.37633 .720 & 28.223 & 25.814 & 30.797 & 29.375 & 29.636 & 30.74231 .016 & 37.404 \\
\hline $6 / 18 / 2006$ & $20: 00$ & 32.79333 .114 & 33.42233 .769 & 28.221 & 25.814 & 30.795 & 29.372 & 29.664 & 30.78231 .065 & 41.195 \\
\hline $6 / 19 / 2006$ & 0:00 & 32.84833 .143 & 33.42433 .789 & 28.235 & 25.824 & 30.804 & 29.383 & 29.717 & 383 & 118 \\
\hline $6 / 19 / 2006$ & $4: 00$ & 32.89833 .179 & 33.44933 .824 & 28.250 & 25.833 & 30.819 & 29.399 & 29.780 & 30.89031 & 42.834 \\
\hline 6/19/2006 & 8:00 & 33.00833 .278 & 33.59233 .957 & 28.289 & 25.856 & 30.859 & 29.443 & 29.858 & 30.99231 & 40.028 \\
\hline $6 / 19 / 2006$ & $12: 00$ & 33.05833 .283 & 33.57833 .959 & 28.279 & 25.854 & 30.855 & 29.45 & 29.919 & 31.01531 .336 & 36.879 \\
\hline $6 / 19 / 2006$ & $16: 00$ & 33.00033 .260 & 33.53833 .926 & 28.262 & 25.844 & 30.848 & 29.43 & 29.932 & 30.98531 & 37.628 \\
\hline $6 / 19 / 2006$ & $20: 00$ & 33.01333 .274 & 33.58033 .957 & 28.246 & 25.839 & 30.841 & 29.414 & 29.932 & 30.99231 & 36.365 \\
\hline $6 / 20 / 2006$ & 0:00 & 33.03133 .297 & 33.56533 .952 & 28.254 & 25.842 & 30.850 & 29.421 & 29.936 & 31.00531 .313 & 34.240 \\
\hline $6 / 20 / 2006$ & 4:00 & 33.00233 .287 & 33.56133 .942 & 28.283 & 25.863 & 30.871 & 29.445 & 29.944 & 30.98031 .282 & 33.391 \\
\hline $6 / 20 / 2006$ & 8:00 & 32.99233 .340 & 33.62433 .969 & 28.275 & 25.861 & 30.912 & 29.465 & 29.919 & 31.00231 .291 & 32.851 \\
\hline 6/20/2006 & $12: 00$ & 32.94933 .241 & 33.53333 .903 & 28.246 & 25.847 & 30.851 & 29.43 & 29.891 & 30.93131 .218 & 32.486 \\
\hline $6 / 20 / 2006$ & $16: 00$ & 32.88133 .179 & 33.46633 .834 & 28.196 & 25.814 & 30.806 & 29.379 & 29.841 & 30.86531 .150 & 32.207 \\
\hline $6 / 20 / 2006$ & $20: 00$ & 32.88333 .232 & 33.52533 .867 & 28.193 & 25.814 & 30.828 & 29.377 & 29.815 & 30.88731 .180 & 31.996 \\
\hline $6 / 21 / 2006$ & 0:00 & 32.91833 .264 & 33.53833 .883 & 28.235 & 25.839 & 30.868 & 29.421 & 29.818 & 30.91631 .202 & 31.843 \\
\hline $6 / 21 / 2006$ & 4:00 & 32.94333 .306 & 33.56733 .910 & 28.279 & 25.874 & 30.917 & 29.467 & 29.830 & 30.94831 .233 & 31.733 \\
\hline $6 / 21 / 2006$ & 8:00 & 32.95533 .331 & 33.58833 .930 & 28.300 & 25.890 & 30.941 & 29.491 & 29.828 & 30.96831 .253 & 31.651 \\
\hline
\end{tabular}


Depth to Water (ft TOC) in Well Indicated

$\begin{array}{llllllllllllll}\text { Date } & \text { Time } & \text { MW-1 } & \text { MW-2 } & \text { MW-3 } & \text { MW-4 } & \text { KDHE-1 } & \text { KDHE-2 } & \text { NW-1 } & \text { NW-2 } & \text { NW-3 } & \text { L-1 } & \text { L-2 } & \text { L-3 }\end{array}$

\begin{tabular}{|c|c|c|c|c|c|c|c|c|c|c|}
\hline $6 / 21 / 2006$ & $12: 00$ & 32.98233 .378 & 33.63233 .959 & 28.335 & 25.913 & 31.030 & 29.547 & 29.853 & 31.00031 .278 & 31.589 \\
\hline /21/2006 & $16: 00$ & 32.96533 .363 & 33.61133 .942 & 28.312 & 25.911 & 30.966 & 29.516 & 29.833 & 30.98531 .271 & 31.529 \\
\hline 6/21/2006 & 20:00 & 32.96933 .392 & 33.64133 .959 & 28.275 & 25.895 & 30.968 & 29.491 & 29.800 & 31.00431 .293 & .474 \\
\hline 6/22/2006 & $0: 00$ & 33.01733 .384 & 33.64333 .991 & 28.363 & 25.960 & 31.030 & 29.564 & 29.818 & 31.01631 .284 & 1.376 \\
\hline /22/2006 & 4:00 & 32.96533 .323 & 33.58633 .934 & 28.340 & 25.955 & 31.036 & 29.527 & 29.797 & 30.96631 .234 & 31.336 \\
\hline o/22/2006 & 8:00 & 32.99633 .331 & 33.59933 .953 & 28.373 & 25.967 & 30.934 & 29.502 & 29.828 & 30.97331 .236 & .330 \\
\hline 6/22/2006 & $12: 00$ & 32.96933 .302 & 33.57633 .932 & 28.336 & 25.937 & 30.846 & 29.476 & 29.861 & 30.95131 .220 & .334 \\
\hline 6/22/2006 & $16: 00$ & 32.92833 .259 & 33.53633 .891 & 28.279 & 25.881 & 30.766 & 29.408 & 29.825 & 30.90931 .178 & .319 \\
\hline $6 / 22 / 2006$ & $20: 00$ & 32.90833 .224 & 33.50233 .865 & 28.250 & 25.819 & 30.697 & 29.359 & 29.815 & 8031.149 & 303 \\
\hline $6 / 23 / 2006$ & 0:00 & 32.91433 .224 & 33.50233 .867 & 28.265 & 25.780 & 30.667 & 29.357 & 29.828 & 30.88031 .147 & .301 \\
\hline $6 / 23 / 2006$ & 4:00 & 32.88533 .186 & 33.46633 .836 & 28.242 & 25.726 & 30.616 & 29.317 & 29.823 & 4831.114 & 31.292 \\
\hline $6 / 23 / 2006$ & $8: 00$ & 32.87933 .179 & 33.46033 .832 & 28.258 & 25.690 & 93 & 29.309 & 29.830 & 4331.107 & .290 \\
\hline $6 / 23 / 2006$ & $12: 00$ & 32.85933 .162 & 33.44533 .816 & 28.254 & 25.657 & 63 & 29.284 & 29.825 & 283 & 285 \\
\hline 6/23/2006 & $16: 00$ & 32.82433 .116 & 33.39733 .771 & 28.218 & 25.611 & 30.510 & 29.229 & 29.805 & 30.78431 .050 & 31.290 \\
\hline $6 / 23 / 2006$ & 20:00 & 32.79533 .089 & 33.36133 .736 & 28.193 & 25.572 & 74 & 29.19 & 30.062 & 5231.021 & .350 \\
\hline $6 / 24 / 2006$ & 0:00 & 32.79533 .089 & 33.36533 .740 & 28.208 & 25.558 & 68 & 29.187 & 29. & 573 & 252 \\
\hline $6 / 24 / 2006$ & 4:00 & 32.77833 .069 & 33.34833 .722 & 28.218 & 25.548 & 30.456 & 29.179 & 29.803 & 383 & 234 \\
\hline $6 / 24 / 2006$ & 8:00 & 32.76433 .048 & 33.32733 .703 & 28.208 & 25.534 & 30.441 & 29.155 & 29.790 & 30.71330 .979 & 31.930 \\
\hline $6 / 24 / 2006$ & $12: 00$ & 32.79133 .046 & 33.32333 .701 & 28.221 & 25.523 & 30.434 & 29.155 & 29.787 & 30.72030 .986 & 434 \\
\hline 006 & $16: 00$ & 32.73133 .012 & 33.28933 .664 & 28.187 & 25.497 & 03 & 29.113 & 29.757 & 863 & 026 \\
\hline $6 / 24 / 2006$ & $20: 00$ & 32.77033 .021 & 33.28133 .666 & 28.179 & 25.488 & 30.388 & 29.093 & 29.767 & 70330.983 & 37.114 \\
\hline $6 / 25 / 2006$ & 0:00 & 32.84433 .091 & 33.34233 .732 & 28.212 & 25.507 & 30.392 & 29.073 & 29.815 & 30.77931 .063 & 34.981 \\
\hline $6 / 25 / 2006$ & $4: 00$ & 32.86133 .255 & 33.45933 .779 & 28.218 & 25.502 & 30.494 & 29.139 & 29.840 & 30.85031 .121 & 33.289 \\
\hline $6 / 25 / 2006$ & 8:00 & 32.92033 .203 & 33.44533 .818 & 28.237 & 25.514 & 30.477 & 29.159 & 29.856 & 8031.167 & 32.603 \\
\hline $6 / 25 / 2006$ & $12: 00$ & 32.88933 .139 & 33.39733 .781 & 28.252 & 25.519 & 30.459 & 29.152 & 29.871 & 30.82331 & 32.220 \\
\hline $6 / 25 / 2006$ & $16: 00$ & 32.81933 .089 & 33.35433 .734 & 28.227 & 25.504 & 30.428 & 29.119 & 29.868 & 30.77231 .052 & 31.987 \\
\hline $6 / 25 / 2006$ & $20: 00$ & 32.80533 .080 & 33.35433 .732 & 28.216 & 25.498 & 30.421 & 29.106 & 29.853 & 30.76031 .034 & 31.786 \\
\hline 6/26/2006 & 0:00 & 32.80533 .074 & 33.34433 .726 & 28.244 & 25.516 & 30.430 & 29.122 & 29.866 & 30.75531 .027 & 31.662 \\
\hline 6/26/2006 & 4:00 & 32.77633 .044 & 33.31433 .697 & 28.235 & 25.512 & 30.417 & 29.106 & 29.853 & 30.72330 .992 & 31.562 \\
\hline 6/26/2006 & 8:00 & 32.75633 .032 & 33.30233 .683 & 28.235 & 25.513 & 30.417 & 29.101 & 29.835 & 30.70830 .975 & 31.485 \\
\hline $6 / 26 / 2006$ & $12: 00$ & 32.75233 .031 & 33.30433 .681 & 28.221 & 25.514 & 30.468 & 29.117 & 29.825 & 30.70630 .975 & 31.427 \\
\hline $6 / 26 / 2006$ & $16: 00$ & 32.70732 .981 & 33.25633 .636 & 28.187 & 25.495 & 30.401 & 29.064 & 29.795 & 30.65930 .928 & 31.371 \\
\hline 6/26/2006 & 20:00 & 32.77833 .249 & 33.49533 .765 & 28.156 & 25.479 & 30.488 & 29.073 & 29.767 & 30.80331 .061 & 31.319 \\
\hline $6 / 27 / 2006$ & 0:00 & 32.90233 .433 & 33.64033 .887 & 28.185 & 25.498 & 30.609 & 29.163 & 29.775 & 30.97331 .231 & 31.294 \\
\hline
\end{tabular}


Depth to Water (ft TOC) in Well Indicated

$\begin{array}{llllllllllllll}\text { Date } & \text { Time } & \text { MW-1 } & \text { MW-2 } & \text { MW-3 } & \text { MW-4 } & \text { KDHE-1 } & \text { KDHE-2 } & \text { NW-1 } & \text { NW-2 } & \text { NW-3 } & \text { L-1 } & \text { L-2 } & \text { L-3 }\end{array}$

\begin{tabular}{|c|c|c|c|c|c|c|c|c|c|c|}
\hline $6 / 27 / 2006$ & 4:00 & 32.88333 .274 & 33.53333 .856 & 28.181 & 25.502 & 30.550 & 29.156 & 29.775 & 30.91631 .191 & 31.274 \\
\hline$/ 27 / 2006$ & $8: 00$ & 32.87133 .202 & 33.46033 .816 & 28.196 & 25.518 & 30.503 & 29.141 & 29.787 & 30.85531 .130 & 33.449 \\
\hline $6 / 27 / 2006$ & $12: 00$ & 32.83633 .135 & 33.40533 .773 & 28.208 & 25.530 & 30.492 & 29.137 & 29.797 & 30.80631 .076 & 1.870 \\
\hline /27/2006 & $16: 00$ & 32.80133 .070 & 33.33833 .713 & 28.173 & 25.511 & 30.454 & 29.09 & 29.780 & 30.74431 .016 & .100 \\
\hline /27/2006 & $20: 00$ & 32.83833 .335 & 33.56233 .824 & 28.150 & 25.504 & 30.574 & 29.119 & 29.762 & 30.88531 .141 & 32.439 \\
\hline$/ 28 / 2006$ & $0: 00$ & 32.94133 .316 & 33.55633 .887 & 28.187 & 25.532 & 30.585 & 29.172 & 29.785 & 30.96031 .242 & 6.264 \\
\hline $6 / 28 / 2006$ & $4: 00$ & 32.88333 .179 & 33.43433 .805 & 28.177 & 25.535 & 30.516 & 29.13 & 29.812 & 30.86231 .145 & 37.842 \\
\hline $6 / 28 / 2006$ & 8:00 & 32.91233 .179 & 33.44933 .832 & 28.198 & 25.553 & 30.519 & 29.143 & 29.858 & 931.167 & .779 \\
\hline 5/28/2006 & $12: 00$ & 32.94933 .167 & 33.42633 .824 & 28.195 & 25.555 & 30.508 & 29.132 & 29.898 & 30.88531 .181 & .439 \\
\hline $6 / 28 / 2006$ & $16: 00$ & 32.97633 .188 & 33.42433 .834 & 28.162 & 25.541 & 30.496 & 29.102 & 29.916 & 30.91131 .218 & 39.929 \\
\hline $6 / 28 / 2006$ & $20: 00$ & 33.03333 .297 & 33.50233 .895 & 28.145 & 25.537 & 30.519 & 29.108 & 29.946 & 30.99731 .313 & 36.802 \\
\hline $6 / 29 / 2006$ & 0:00 & 33.01733 .243 & 33.47433 .881 & 28.172 & 25.560 & 19 & 29.117 & 29. & 64 & 647 \\
\hline $6 / 29 / 2006$ & $4: 00$ & 32.97033 .205 & 33.44733 .854 & 28.176 & 25.567 & 30.516 & 29.121 & 29.987 & 216 & 646 \\
\hline $6 / 29 / 2006$ & 8:00 & 32.95333 .196 & 33.45133 .852 & 28.203 & 25.586 & 30.532 & 29.145 & 29.989 & 30.90131 .189 & 33.035 \\
\hline $6 / 29 / 2006$ & $12: 00$ & 32.92833 .173 & 33.42433 .824 & 28.193 & 25.586 & 30.521 & 29.139 & 29.981 & 30.87231 .160 & 32.627 \\
\hline $6 / 29 / 2006$ & $16: 00$ & 32.90033 .156 & 33.39733 .791 & 28.170 & 25.572 & 05 & 29.115 & 29.954 & 36 & 333 \\
\hline $6 / 29 / 2006$ & $20: 00$ & 32.86733 .120 & 33.37833 .769 & 28.150 & 25.567 & 30.494 & 29.099 & 29.929 & 990 & 939 \\
\hline 6/30/2006 & $0: 00$ & 32.92633 .154 & 33.40133 .795 & 28.178 & 25.594 & 30.521 & 29.126 & 29.931 & 331.120 & 33.026 \\
\hline 6/30/2006 & $4: 00$ & 32.87533 .205 & 33.42133 .781 & 28.172 & 25.593 & 543 & 29.123 & 29.919 & 831 & 32.417 \\
\hline $6 / 30 / 2006$ & 8:00 & 33.02133 .395 & 33.59633 .928 & 28.185 & 25.610 & 45 & 29.203 & 29. & 22 & 181 \\
\hline 6/30/2006 & $12: 00$ & 33.00633 .255 & 33.48933 .875 & 28.178 & 25.610 & 30.583 & 29.17 & 29.959 & 331.231 & 38.137 \\
\hline 6/30/2006 & $16: 00$ & 32.95933 .196 & 33.43933 .836 & 28.145 & 25.594 & 45 & 29.132 & 30.057 & 0731.202 & 40.909 \\
\hline 6/30/2006 & $20: 00$ & 33.04133 .196 & 33.46633 .863 & 28.133 & 25.590 & 30.534 & 29.117 & 30.027 & 831.218 & 38.686 \\
\hline 7/1/2006 & $0: 00$ & 33.08033 .264 & 33.50133 .906 & 28.160 & 25.617 & 30.576 & 29.152 & 30.059 & 8031.287 & 35.369 \\
\hline $7 / 1 / 2006$ & $4: 00$ & 33.02733 .291 & 33.51633 .910 & 28.173 & 25.631 & 30.609 & 29.174 & 30.062 & 30.99231 .298 & 34.082 \\
\hline 7/1/2006 & $8: 00$ & 33.08833 .337 & 33.56933 .955 & 28.193 & 25.650 & 30.638 & 29.203 & 30.070 & 31.02731 .326 & 33.344 \\
\hline $7 / 1 / 2006$ & $12: 00$ & 33.04533 .337 & 33.55833 .942 & 28.189 & 25.655 & 30.656 & 29.212 & 30.057 & 31.01731 .313 & 32.879 \\
\hline $7 / 1 / 2006$ & $16: 00$ & 33.03133 .321 & 33.54833 .936 & 28.166 & 25.650 & 30.650 & 29.194 & 30.034 & 30.99731 .291 & 35.827 \\
\hline $7 / 1 / 2006$ & $20: 00$ & 33.01933 .299 & 33.56933 .942 & 28.154 & 25.650 & 30.638 & 29.187 & 30.027 & 30.98031 .266 & 33.686 \\
\hline $7 / 2 / 2006$ & $0: 00$ & 33.16933 .669 & 33.86834 .139 & 28.195 & 25.680 & 30.838 & 29.306 & 30.052 & 31.23531 .501 & 32.897 \\
\hline $7 / 2 / 2006$ & 4:00 & 33.22433 .762 & 33.94334 .196 & 28.200 & 25.698 & 30.906 & 29.357 & 30.044 & 31.31131 .585 & 32.503 \\
\hline $7 / 2 / 2006$ & $8: 00$ & 33.20733 .546 & 33.79034 .141 & 28.233 & 25.724 & 30.795 & 29.339 & 30.054 & 31.20131 .490 & 32.257 \\
\hline $7 / 2 / 2006$ & $12: 00$ & 33.22433 .544 & 33.73334 .106 & 28.221 & 25.726 & 30.766 & 29.32 & 30.133 & 31.19331 .490 & 32.087 \\
\hline $7 / 2 / 2006$ & $16: 00$ & 33.23433 .536 & 33.70634 .100 & 28.200 & 25.717 & 30.747 & 29.295 & 30.062 & 31.17931 .479 & 31.965 \\
\hline
\end{tabular}


Depth to Water (ft TOC) in Well Indicated

$\begin{array}{llllllllllllll}\text { Date } & \text { Time } & \text { MW-1 } & \text { MW-2 } & \text { MW-3 } & \text { MW-4 } & \text { KDHE-1 } & \text { KDHE-2 } & \text { NW-1 } & \text { NW-2 } & \text { NW-3 } & \text { L-1 } & \text { L-2 } & \text { L-3 }\end{array}$

\begin{tabular}{|c|c|c|c|c|c|c|c|c|c|c|}
\hline 7/2/2006 & $20: 00$ & 33.14433 .428 & 33.64934 .036 & 28.193 & 25.721 & 30.729 & 29.278 & 30.029 & 31.09131 .371 & 31.865 \\
\hline 7/3/2006 & $0: 00$ & 33.14433 .426 & 33.68134 .059 & 28.233 & 25.750 & 30.762 & 29.324 & 30.037 & 31.10031 .368 & 31.808 \\
\hline 7/3/2006 & $4: 00$ & 33.09033 .365 & 33.62334 .004 & 28.237 & 25.759 & 30.753 & 29.317 & 30.027 & 31.03931 .308 & 1.746 \\
\hline 7/3/2006 & $8: 00$ & 33.09733 .399 & 33.68534 .047 & 28.262 & 25.777 & 30.775 & 29.346 & 30.039 & 31.05631 .319 & 5.034 \\
\hline 7/3/2006 & $12: 00$ & 33.21833 .490 & 33.69734 .081 & 28.254 & 25.780 & 30.789 & 29.346 & 30.112 & 31.13931 .415 & 35.127 \\
\hline 7/3/2006 & $16: 00$ & 33.23833 .544 & 33.71434 .092 & 28.229 & 25.772 & 30.793 & 29.328 & 30.117 & 31.18631 .475 & 40.380 \\
\hline 7/3/2006 & $20: 00$ & 33.28333 .561 & 33.79234 .165 & 28.222 & 25.772 & 30.797 & 29.331 & 30.158 & 3331.534 & 494 \\
\hline 7/4/2006 & $0: 00$ & 33.35933 .582 & 33.80234 .204 & 28.279 & 25.809 & 30.840 & 29.39 & 30.218 & 31.29131 .592 & 40.526 \\
\hline 7/4/2006 & $4: 00$ & 33.32533 .547 & 33.77334 .185 & 28.289 & 25.821 & 30.840 & 29.394 & 30.256 & 31.27231 .581 & 40.551 \\
\hline 7/4/2006 & 8:00 & 33.34733 .561 & 33.83834 .239 & 28.318 & 25.841 & 30.862 & 29.423 & 30.297 & 31.29431 .599 & .089 \\
\hline 7/4/2006 & $12: 00$ & 33.34733 .561 & 33.81334 .226 & 28.335 & 25.856 & 30.873 & 29.441 & 332 & 601 & 827 \\
\hline 7/4/2006 & $16: 00$ & 33.34333 .587 & 33.78834 .196 & 28.304 & 25.847 & 30.864 & 29.417 & 30.339 & 31.28931 .603 & 41.366 \\
\hline 7/4/2006 & $20: 00$ & 33.47433 .724 & 33.93334 .324 & 28.319 & 25.865 & 30.926 & 29.452 & 30.370 & 31.42431 .742 & 39.344 \\
\hline$/ 5 / 2006$ & $0: 00$ & 34.070 & 34.22334 .512 & 28.377 & 25.904 & 31.136 & 29.586 & 420 & 623 & 788 \\
\hline $7 / 5 / 2006$ & $4: 00$ & 34.148 & 34.30334 .576 & 28.377 & 25.920 & 31.203 & 29.633 & 30.435 & 7303 & 756 \\
\hline 7/5/2006 & $8: 00$ & 33.62033 .941 & 34.17934 .545 & 28.425 & 25.955 & 31.141 & 29.655 & 30.471 & 31.63731 .944 & 38.126 \\
\hline 7/5/2006 & $12: 00$ & 33.59333 .878 & 34.10534 .492 & 28.432 & 25.974 & 31.125 & 29.646 & 30.486 & 31.58131 .887 & 39.773 \\
\hline 7/5/2006 & $16: 00$ & 33.55233 .821 & 34.04234 .437 & 28.400 & 25.967 & 31.092 & 29.606 & 19 & 5293 & .761 \\
\hline 7/5/2006 & $20: 00$ & 33.53433 .772 & 34.00634 .410 & 28.406 & 25.976 & 31.070 & 29.599 & 29 & 933 & 36.073 \\
\hline 7/6/2006 & 0:00 & 33.55733 .802 & 34.03034 .435 & 28.452 & 26.003 & 31.094 & 29.637 & & 5123 & 618 \\
\hline $7 / 6 / 2006$ & $4: 00$ & 33.747 & 33.99034 .394 & 28.454 & 26.011 & 31.085 & 29.635 & 24 & 5631.745 & 365 \\
\hline 7/6/2006 & $8: 00$ & 33.53633 .802 & 34.03034 .425 & 28.490 & 26.034 & 31.105 & 29.666 & 30.529 & 8531.778 & 384 \\
\hline 7/6/2006 & $12: 00$ & 33.55433 .812 & 34.04034 .435 & 28.494 & 26.043 & 31.118 & 29.672 & 30.526 & 31.49531 .776 & 33.052 \\
\hline $7 / 6 / 2006$ & $16: 00$ & 33.62033 .745 & 33.98534 .390 & 28.465 & 26.031 & 31.092 & 29.641 & 30.483 & 31.44131 .718 & 32.795 \\
\hline $7 / 6 / 2006$ & $20: 00$ & 33.46233 .711 & 33.99234 .376 & 28.446 & 26.024 & 31.079 & 29.626 & 30.473 & 9731.669 & 33.464 \\
\hline 7/7/2006 & $0: 00$ & 33.48933 .760 & 34.01534 .400 & 28.485 & 26.052 & 31.112 & 29.666 & 30.481 & 31.43931 .709 & 32.904 \\
\hline 7/7/2006 & $4: 00$ & 33.43133 .699 & 33.96334 .351 & 28.483 & 26.057 & 31.101 & 29.661 & 30.456 & 31.38731 .656 & 32.627 \\
\hline 7/7/2006 & $8: 00$ & 33.43333 .692 & 33.98034 .372 & 28.500 & 26.066 & 31.114 & 29.677 & 30.453 & 31.38231 .643 & 32.461 \\
\hline 7/7/2006 & $12: 00$ & 33.40033 .680 & 33.95034 .327 & 28.492 & 26.066 & 31.110 & 29.666 & 30.435 & 31.35831 .621 & 32.350 \\
\hline 7/7/2006 & $16: 00$ & 33.33333 .606 & 33.88334 .261 & 28.450 & 26.045 & 31.067 & 29.628 & 30.387 & 31.28931 .554 & 32.235 \\
\hline 7/7/2006 & $20: 00$ & 33.35733 .772 & 34.01534 .308 & 28.431 & 26.032 & 31.141 & 29.63 & 30.357 & 31.34531 .594 & 32.146 \\
\hline $7 / 8 / 2006$ & $0: 00$ & 33.44633 .872 & 34.12034 .423 & 28.448 & 26.052 & 31.216 & 29.707 & 30.350 & 31.50431 .771 & 32.100 \\
\hline 7/8/2006 & $4: 00$ & 33.35933 .675 & 33.94834 .306 & 28.442 & 26.052 & 31.125 & 29.661 & 30.324 & 31.34531 .608 & 32.027 \\
\hline 7/8/2006 & $8: 00$ & 33.36633 .677 & 34.01534 .363 & 28.479 & 26.075 & 31.141 & 29.692 & 30.339 & 31.35031 .599 & 31.987 \\
\hline
\end{tabular}


Depth to Water (ft TOC) in Well Indicated

$\begin{array}{llllllllllllll}\text { Date } & \text { Time } & \text { MW-1 } & \text { MW-2 } & \text { MW-3 } & \text { MW-4 } & \text { KDHE-1 } & \text { KDHE-2 } & \text { NW-1 } & \text { NW-2 } & \text { NW-3 } & \text { L-1 } & \text { L-2 } & \text { L-3 }\end{array}$

\begin{tabular}{|c|c|c|c|c|c|c|c|c|c|c|}
\hline 7/8/2006 & $12: 00$ & 33.31233 .622 & 2334.280 & 28.448 & 26.061 & 114 & 29.663 & 30.317 & 963 & .945 \\
\hline 7/8/2006 & $16: 00$ & 33.25733 .578 & 33.86534 .220 & 28.415 & 26.043 & 31.085 & 29.626 & 30.276 & 31.24531 .503 & 31.890 \\
\hline 7/8/2006 & $20: 00$ & 33.24433 .570 & 33.82134 .179 & 28.391 & 26.031 & 31.052 & 29.593 & 30.327 & 31.21831 .488 & 31.845 \\
\hline /9/2006 & $0: 00$ & 33.28533 .601 & 33.86334 .214 & 28.423 & 26.054 & 31.090 & 29.628 & 30.425 & 31.25231 .510 & 31.832 \\
\hline $7 / 9 / 2006$ & $4: 00$ & 33.23233 .534 & 33.80934 .175 & 28.414 & 26.055 & 31.061 & 29.615 & 30.496 & 31.20031 .459 & .821 \\
\hline $7 / 9 / 2006$ & 8:00 & 33.21233 .515 & 33.79634 .159 & 28.417 & 26.057 & 31.059 & 29.61 & 30.572 & 31.18331 .437 & 31.823 \\
\hline $7 / 9 / 2006$ & $12: 00$ & 33.23033 .515 & 33.79634 .159 & 28.421 & 26.061 & 31.059 & 29.613 & 30.544 & 31.18331 .437 & 31.825 \\
\hline 7/9/2006 & $16: 00$ & 33.19933 .483 & 33.76234 .130 & 28.392 & 26.045 & 31.037 & 29.588 & 30.466 & 31.156 & 310 \\
\hline 7/9/2006 & $20: 00$ & 33.18933 .473 & 33.74434 .114 & 28.373 & 26.033 & 31.014 & 29.562 & 30.410 & 31.14931 .408 & .178 \\
\hline 7/10/2006 & $0: 00$ & 33.20133 .477 & 33.74834 .122 & 28.373 & 26.033 & 31.017 & 29.562 & 30.395 & 31.16731 .437 & 38.407 \\
\hline 7/10/2006 & $4: 00$ & 33.22433 .490 & 33.75634 .136 & 28.377 & 26.038 & 31.025 & 29.566 & 30.400 & 131.466 & .206 \\
\hline 2006 & $8: 00$ & 33.26533 .513 & 33.77334 .157 & 28.377 & 38 & 21 & 29. & 08 & 218 & 41 \\
\hline 7/10/2006 & $12: 00$ & 33.25533 .509 & 33.77534 .159 & 28.373 & 26.036 & 019 & 29.562 & 30.410 & 31.22031 .506 & 861 \\
\hline $7 / 10 / 2006$ & $16: 00$ & 33.20833 .473 & 33.73934 .122 & 28.333 & 26.015 & 30.992 & 29.531 & 30.374 & 31.18131 .463 & 33.854 \\
\hline 006 & $20: 00$ & 433.521 & 33.76034 .136 & 28.318 & 26.004 & 86 & 29.52 & 45 & 86 & 256 \\
\hline 7/11/2006 & $0: 00$ & 33.22433 .502 & 33.76434 .142 & 28.346 & 26.027 & 01 & 29.555 & 27 & 57 & 335 \\
\hline 7/11/2006 & 4:00 & 33.20733 .471 & 33.74134 .116 & 28.343 & 26.027 & 30.999 & 29.624 & 30.302 & 31.15731 .422 & 32.567 \\
\hline $7 / 11 / 2006$ & $8: 00$ & 33.19333 .479 & 33.75234 .126 & 28.375 & 26.047 & 31.045 & 29.65 & 30.299 & 31.15631 .421 & 32.370 \\
\hline $7 / 11 / 2006$ & $12: 00$ & 33.20333 .492 & 33.76434 .132 & 28.398 & 26.061 & 28 & 29.763 & 30. & 31.161 & 250 \\
\hline $7 / 11 / 2006$ & $16: 00$ & 33.17733 .470 & 33.74634 .111 & 28.392 & 26.054 & 025 & 29.584 & 30.278 & 31.14231 .401 & 32.140 \\
\hline 7/11/2006 & $20: 00$ & 33.16833 .456 & 33.73534 .102 & 28.394 & 26.052 & 31.012 & 29.575 & 30.264 & 31.12931 .384 & 32.040 \\
\hline $7 / 12 / 2006$ & $0: 00$ & 33.20133 .481 & 33.75834 .126 & 28.433 & 26.073 & 31.037 & 29.61 & 30.276 & 31.15131 .406 & 31.983 \\
\hline $7 / 12 / 2006$ & $4: 00$ & 33.17933 .475 & 33.75434 .118 & 28.444 & 26.080 & 31.043 & 29.615 & 30.271 & 31.14231 .395 & 927 \\
\hline 7/12/2006 & 8:00 & 33.21033 .496 & 33.77334 .134 & 28.469 & 26.096 & 56 & 29.639 & 30.276 & 31.15731 .406 & 887 \\
\hline 7/12/2006 & $12: 00$ & 33.20133 .498 & 33.77734 .136 & 28.473 & 26.100 & 31.070 & 29.646 & 30.273 & 31.16231 .413 & 31.859 \\
\hline 7/12/2006 & $16: 00$ & 33.16433 .462 & 33.74134 .103 & 28.442 & 26.082 & 31.041 & 29.615 & 30.248 & 31.12931 .384 & 31.830 \\
\hline 7/12/2006 & $20: 00$ & 33.25733 .770 & 33.97934 .232 & 28.452 & 26.091 & 31.194 & 29.679 & 30.243 & 31.30331 .546 & 31.799 \\
\hline 7/13/2006 & 0:00 & 33.30633 .663 & 33.88734 .230 & 28.452 & 26.098 & 31.118 & 29.666 & 30.233 & 31.29631 .566 & 31.777 \\
\hline $7 / 13 / 2006$ & $4: 00$ & 33.24233 .559 & 33.81934 .173 & 28.436 & 26.091 & 31.085 & 29.639 & 30.220 & 31.21631 .474 & 31.763 \\
\hline 7/13/2006 & 8:00 & 33.23233 .566 & 33.80434 .156 & 28.436 & 26.093 & 31.074 & 29.633 & 30.205 & 31.20631 .470 & 31.746 \\
\hline $7 / 13 / 2006$ & $12: 00$ & 33.23033 .530 & 33.79434 .150 & 28.444 & 26.096 & 31.074 & 29.633 & 30.223 & 31.18631 .443 & 31.739 \\
\hline 7/13/2006 & $16: 00$ & 33.17333 .483 & 33.75234 .107 & 28.413 & 26.079 & 31.052 & 29.608 & 30.193 & 31.14231 .399 & 31.726 \\
\hline 7/13/2006 & $20: 00$ & 33.25733 .597 & 33.84734 .185 & 28.404 & 26.073 & 31.061 & 29.604 & 30.203 & 31.22531 .488 & 40.624 \\
\hline 7/14/2006 & $0: 00$ & 33.38433 .686 & 33.91834 .279 & 28.456 & 26.108 & 31.114 & 29.659 & 30.266 & 31.35031 .627 & 39.926 \\
\hline
\end{tabular}


Depth to Water (ft TOC) in Well Indicated

$\begin{array}{llllllllllllll}\text { Date } & \text { Time } & \text { MW-1 } & \text { MW-2 } & \text { MW-3 } & \text { MW-4 } & \text { KDHE-1 } & \text { KDHE-2 } & \text { NW-1 } & \text { NW-2 } & \text { NW-3 } & \text { L-1 } & \text { L-2 } & \text { L-3 }\end{array}$

\begin{tabular}{|c|c|c|c|c|c|c|c|c|c|c|}
\hline 7/14/2006 & $4: 00$ & 33.39233 .667 & 33.90834 .283 & 28.467 & 26.116 & 31.118 & 29.672 & 30.316 & 31.36031 .639 & 40.201 \\
\hline 7/14/2006 & $8: 00$ & 33.57733 .774 & 00634.383 & 28.534 & 26.159 & 31.169 & 29.734 & 30.400 & 31.46331 .756 & 37.091 \\
\hline 7/14/2006 & $12: 00$ & 33.50333 .753 & 34.00634 .389 & 28.559 & 26.177 & 31.196 & 29.747 & 30.458 & 31.45831 .743 & 34.979 \\
\hline 7/14/2006 & $16: 00$ & 33.45433 .718 & 33.97534 .355 & 28.544 & 26.172 & 31.176 & 29.743 & 30.480 & 31.41931 .701 & 34.015 \\
\hline 7/14/2006 & $20: 00$ & 33.44633 .709 & 33.97934 .355 & 28.554 & 26.179 & 31.178 & 29.745 & 30.493 & 31.40231 .676 & 33.431 \\
\hline $7 / 15 / 2006$ & 0:00 & 33.57734 .078 & 34.28634 .547 & 28.586 & 26.200 & 31.369 & 29.853 & 30.508 & 64031.893 & 33.050 \\
\hline $7 / 15 / 2006$ & $4: 00$ & 33.71034 .207 & 0034.639 & 28.605 & 26.218 & 31.455 & 29.917 & & 008 & 789 \\
\hline 7/15/2006 & $8: 00$ & 33.64133 .992 & 34.27834 .604 & 28.636 & 26.242 & 31.373 & 29.912 & 30.521 & 31.64531 .907 & 32.600 \\
\hline $7 / 15 / 2006$ & $12: 00$ & 33.63233 .929 & 34.20834 .560 & 28.643 & 26.253 & 31.349 & 29.904 & 30.548 & 31.60031 .858 & 32.474 \\
\hline $7 / 15 / 2006$ & $16: 00$ & 33.55033 .855 & 4834.500 & 28.617 & 26.241 & 31.309 & 29.864 & 38 & 273 & 32.370 \\
\hline $7 / 15 / 2006$ & $20: 00$ & 33.56733 .870 & 6434.510 & 28.601 & 26.232 & 293 & 29.842 & & 273 & 32.297 \\
\hline 7/16/2006 & $0: 00$ & 33.61233 .893 & 34.16234 .526 & 28.615 & 26.244 & 31.304 & 29.857 & 30.533 & 31.56131 .814 & 32.246 \\
\hline $7 / 16 / 2006$ & $4: 00$ & 33.52233 .823 & 34.09334 .457 & 28.600 & 26.237 & 31.278 & 29.833 & 30.513 & 9031.747 & 32.188 \\
\hline 2006 & $8: 00$ & 3933.907 & 34.15834 .517 & 28.599 & 26.235 & 31.278 & 29 & 95 & 373 & 32.140 \\
\hline 7/16/2006 & $12: 00$ & 33.61633 .914 & 34.13934 .508 & 28.595 & 26.237 & 280 & 29 & 90 & 331 & 33.628 \\
\hline 7/16/2006 & $16: 00$ & 33.55033 .865 & 34.09734 .457 & 28.559 & 26.216 & 31.267 & 29.8 & 30.470 & 51931.787 & 36.857 \\
\hline 7/16/2006 & $20: 00$ & 33.62833 .903 & 34.16234 .518 & 28.552 & 26.213 & 31.278 & 29.796 & 88 & 643 & 197 \\
\hline 7/17/2006 & 0:00 & 33.62033 .916 & 34.16234 .530 & 28.565 & 26.232 & 31.295 & 29.818 & & 358 & 33.998 \\
\hline 7/17/2006 & $4: 00$ & 33.57933 .870 & 34.12234 .492 & 28.576 & 26.239 & 284 & 29.822 & & 316 & 33.276 \\
\hline 7/17/2006 & $8: 00$ & 33.77234 .327 & 34.52634 .759 & 28.590 & 26.255 & 92 & 29.926 & & 513 & 38.766 \\
\hline 2006 & $12: 00$ & 33.91234 .461 & 34.62134 .858 & 28.594 & 26.262 & 31 & 29 & & 0732.281 & 683 \\
\hline 7/17/2006 & $16: 00$ & 33.86634 .193 & 34.42334 .770 & 28.578 & 26.264 & 31.448 & 29.935 & 06 & 175 & 43.146 \\
\hline 7/17/2006 & $20: 00$ & 33.87534 .178 & 34.42734 .784 & 28.580 & 26.269 & 31.415 & 29.915 & 54 & 6832 & 40.099 \\
\hline 7/18/2006 & $0: 00$ & 33.90734 .174 & 34.41734 .802 & 28.649 & 26.308 & 31.444 & 29.968 & 53 & 31.88732 .183 & 36.365 \\
\hline 7/18/2006 & 4:00 & 33.85034 .114 & 34.36034 .747 & 28.655 & 26.320 & 31.433 & 29.961 & 63 & 2632.115 & 35.010 \\
\hline 7/18/2006 & $8: 00$ & 33.89334 .182 & 34.39634 .780 & 28.674 & 26.334 & 31.442 & 29.974 & 30.781 & 31.86832 .163 & 34.224 \\
\hline 7/18/2006 & $12: 00$ & 33.93634 .233 & 34.40234 .784 & 28.670 & 26.338 & 31.442 & 29.968 & 30.770 & 31.89032 .194 & 35.455 \\
\hline 7/18/2006 & $16: 00$ & 33.86834 .102 & 34.33034 .716 & 28.630 & 26.324 & 31.450 & 29.946 & 30.727 & 31.78932 .073 & 36.084 \\
\hline 7/18/2006 & $20: 00$ & 33.86434 .173 & 34.38134 .747 & 28.613 & 26.313 & 31.413 & 29.919 & 758 & 31.82532 .112 & 34.525 \\
\hline 7/19/2006 & $0: 00$ & 33.91634 .201 & 34.40834 .780 & 28.647 & 26.339 & 31.457 & 29.961 & 30.760 & 31.86332 .143 & 33.703 \\
\hline 7/19/2006 & $4: 00$ & 33.82734 .114 & 34.34334 .723 & 28.651 & 26.345 & 31.435 & 29.954 & 30.738 & 31.78932 .059 & 33.296 \\
\hline 7/19/2006 & $8: 00$ & 33.89934 .340 & 34.55134 .849 & 28.661 & 26.354 & 31.568 & 30.027 & 30.727 & 31.94632 .208 & 33.028 \\
\hline 7/19/2006 & $12: 00$ & 33.84834 .176 & 34.40434 .763 & 28.666 & 26.362 & 31.495 & 29.994 & 30.720 & 31.83032 .104 & 32.842 \\
\hline 7/19/2006 & $16: 00$ & 33.79934 .161 & 34.37134 .718 & 28.642 & 26.355 & 31.495 & 29.979 & 30.692 & 31.79932 .073 & 33.874 \\
\hline
\end{tabular}


Depth to Water (ft TOC) in Well Indicated

$\begin{array}{llllllllllllll}\text { Date } & \text { Time } & \text { MW-1 } & \text { MW-2 } & \text { MW-3 } & \text { MW-4 } & \text { KDHE-1 } & \text { KDHE-2 } & \text { NW-1 } & \text { NW-2 } & \text { NW-3 } & \text { L-1 } & \text { L-2 } & \text { L-3 }\end{array}$

\begin{tabular}{|c|c|c|c|c|c|c|c|c|c|c|}
\hline 7/19/2006 & $20: 00$ & 33.83234 .198 & 34.44434 .772 & 28.628 & 26.355 & 31.517 & 29.979 & 30.697 & 31.83332 .104 & 37.432 \\
\hline 7/20/2006 & 0:00 & 33.94434 .328 & 34.50334 .839 & 28.678 & 26.387 & 31.554 & 30.02 & 30.763 & 31.93132 .208 & 9.067 \\
\hline 7/20/2006 & $4: 00$ & 34.06834 .593 & 34.74534 .999 & 28.699 & 26.405 & 31.716 & 30.115 & 30.793 & 32.14532 .416 & .015 \\
\hline 7/20/2006 & $8: 00$ & 34.26534 .520 & 34.69735 .076 & 28.757 & 26.443 & 31.654 & 30.14 & 30.869 & 32.20132 .505 & 38.030 \\
\hline 7/20/2006 & $12: 00$ & 34.23234 .488 & 34.64435 .041 & 28.745 & 26.449 & 31.619 & 30.113 & 30.896 & 32.16932 .471 & 5.557 \\
\hline 7/20/2006 & $16: 00$ & 34.14834 .388 & 34.58534 .986 & 28.736 & 26.451 & 31.592 & 30.093 & 30.902 & 32.07932 .367 & 966 \\
\hline 7/20/2006 & $20: 00$ & 34.08634 .323 & 4134.937 & 28.720 & 26.445 & 31.561 & 30.065 & 30.899 & 0532.287 & 971 \\
\hline 7/21/2006 & $0: 00$ & 34.04534 .313 & 34.54534 .933 & 28.732 & 26.454 & 31.574 & 30.08 & 30.904 & 32.00532 .279 & 34.261 \\
\hline 7/21/2006 & $4: 00$ & 34.00234 .237 & 34.47634 .864 & 28.730 & 26.454 & 31.552 & 30.067 & 30.886 & 31.92432 .194 & 33.697 \\
\hline $7 / 21 / 2006$ & 8:00 & 34.04334 .308 & 4134.925 & 28.814 & 26.498 & 31.601 & 30.14 & .924 & 7732.247 & 442 \\
\hline $7 / 21 / 2006$ & $12: 00$ & 34.05134 .298 & 4334.923 & 28.860 & 26.528 & 31.630 & 30.175 & 944 & 6832.225 & 236 \\
\hline 7/21/2006 & $16: 00$ & 34.06634 .399 & 34.56234 .925 & 28.828 & 26.523 & 31.645 & 30.151 & 30.914 & 32.02732 .316 & 35.346 \\
\hline $7 / 21 / 2006$ & $20: 00$ & 34.20134 .534 & 34.66735 .021 & 28.829 & 26.533 & 31.703 & 30.179 & 30.914 & 32.14932 .445 & 35.800 \\
\hline$/ 22 / 2006$ & $0: 00$ & 34.25234 .781 & 34.91535 & 28.859 & 55 & 31.875 & 30.278 & 42 & 1832 & 111 \\
\hline $7 / 22 / 2006$ & $4: 00$ & 34.25834 .804 & 34.9603 & 28.859 & 26.565 & 915 & 30.307 & 42 & 4832.608 & 550 \\
\hline 7/22/2006 & $8: 00$ & 34.21134 .583 & 34.85735 .184 & 28.885 & 26.588 & 31.840 & 30.311 & 30.954 & 32.23832 .500 & 33.243 \\
\hline 7/22/2006 & $12: 00$ & 34.17634 .553 & 34.82935 .156 & 28.887 & 26.599 & 31.838 & 30.305 & 67 & 9832.460 & 35.745 \\
\hline 7/22/2006 & $16: 00$ & 34.19534 .572 & 34.77735 .117 & 28.849 & 26.588 & 31.829 & 30.272 & 57 & 0832.487 & 208 \\
\hline 7/22/2006 & $20: 00$ & 34.29734 .612 & 34.81235 .158 & 28.834 & 26.588 & 31.836 & 30.263 & 30.979 & 5732.549 & 41.073 \\
\hline 7/23/2006 & 0:00 & 34.44334 .699 & 34.87535 .264 & 28.872 & 26.613 & 31.840 & 30.298 & 43 & 8532.680 & 40.772 \\
\hline 7/23/2006 & $4: 00$ & 34.594 & 34.80035 .203 & 28.868 & 18 & 305 & 30.281 & 88 & 0432.597 & 345 \\
\hline 7/23/2006 & $8: 00$ & 34.30634 .545 & 34.80835 .199 & 28.879 & 26.627 & 31.794 & 30.281 & 123 & 6532.553 & 37.765 \\
\hline 7/23/2006 & $12: 00$ & 34.28134 .545 & 34.76435 .152 & 28.862 & 26.623 & 31.802 & 30.27 & 128 & 24532.542 & 35.820 \\
\hline 7/23/2006 & $16: 00$ & 34.24034 .585 & 34.76635 .127 & 28.820 & 26.606 & 31.829 & 30.245 & 31.093 & 32.24832 .546 & 34.839 \\
\hline 7/23/2006 & $20: 00$ & 34.37134 .796 & 34.94535 .272 & 28.811 & 26.607 & 398 & 30.267 & 98 & 10032.715 & 397 \\
\hline 7/24/2006 & $0: 00$ & 34.37134 .727 & 34.91135 .268 & 28.847 & 26.639 & 31.900 & 30.311 & 31.123 & 32.37332 .657 & 34.168 \\
\hline 7/24/2006 & $4: 00$ & 34.28934 .608 & 34.81035 .184 & 28.829 & 26.637 & 31.844 & 30.283 & 31.088 & 32.27032 .547 & 33.756 \\
\hline $7 / 24 / 2006$ & $8: 00$ & 34.24234 .524 & 34.75335 .140 & 28.841 & 26.644 & 31.811 & 30.274 & 31.075 & 32.19832 .465 & 35.284 \\
\hline $7 / 24 / 2006$ & $12: 00$ & 34.22234 .519 & 34.71835 .097 & 28.816 & 26.634 & 31.787 & 30.245 & 31.055 & 32.18432 .463 & 41.846 \\
\hline 7/24/2006 & $16: 00$ & 34.19934 .458 & 34.69435 .084 & 28.787 & 26.616 & 31.747 & 30.208 & 31.053 & 32.15732 .434 & 44.384 \\
\hline 7/24/2006 & $20: 00$ & 34.26134 .595 & 34.74135 .109 & 28.772 & 26.606 & 31.756 & 30.199 & 31.078 & 32.23832 .535 & 38.456 \\
\hline $7 / 25 / 2006$ & $0: 00$ & 34.45434 .950 & 35.06735 .348 & 28.813 & 26.636 & 31.955 & 30.318 & 31.126 & 32.52232 .806 & 36.210 \\
\hline 7/25/2006 & $4: 00$ & 34.45334 .963 & 35.11135 .383 & 28.813 & 26.648 & 32.004 & 30.356 & 31.128 & 32.53632 .812 & 35.107 \\
\hline 7/25/2006 & $8: 00$ & 34.49335 .036 & 35.18735 .442 & 28.830 & 26.662 & 32.052 & 30.402 & 31.126 & 32.59032 .861 & 34.436 \\
\hline
\end{tabular}


Depth to Water (ft TOC) in Well Indicated

$\begin{array}{llllllllllllll}\text { Date } & \text { Time } & \text { MW-1 } & \text { MW-2 } & \text { MW-3 } & \text { MW-4 } & \text { KDHE-1 } & \text { KDHE-2 } & \text { NW-1 } & \text { NW-2 } & \text { NW-3 } & \text { L-1 } & \text { L-2 } & \text { L-3 }\end{array}$

\begin{tabular}{|c|c|c|c|c|c|c|c|c|c|c|}
\hline 7/25/2006 & $12: 00$ & 34.36134 .678 & 34.90735 .270 & 28.820 & 26.666 & 31.904 & 30.338 & 31.113 & 32.35332 .624 & 33.991 \\
\hline 7/25/2006 & $16: 00$ & 34.28934 .652 & 34.84435 .195 & 28.786 & 26.650 & 31.898 & 30.296 & 31.075 & 32.29932 .584 & 4.374 \\
\hline 7/25/2006 & $20: 00$ & 34.33234 .735 & 34.91735 .238 & 28.765 & 26.646 & 31.942 & 30.292 & 31.045 & 32.34332 .628 & 3.730 \\
\hline 7/26/2006 & $0: 00$ & 34.40634 .745 & 34.92035 .268 & 28.790 & 26.678 & 31.935 & 30.318 & 31.048 & 32.37832 .655 & 33.491 \\
\hline 7/26/2006 & 4:00 & 34.29534 .604 & 34.81935 .180 & 28.789 & 26.685 & 31.889 & 30.303 & 31.030 & 32.25732 .520 & 33.265 \\
\hline $7 / 26 / 2006$ & 8:00 & 34.24234 .553 & 34.80235 .168 & 28.832 & 26.711 & 31.884 & 30.322 & 31.032 & 32.21532 .469 & .865 \\
\hline 7/26/2006 & $12: 00$ & 34.28534 .582 & 34.78535 .148 & 28.849 & 26.726 & 31.884 & 30.329 & 31.030 & 32.22832 .495 & 33.201 \\
\hline $7 / 26 / 2006$ & $16: 00$ & 34.33034 .699 & 34.82935 .184 & 28.833 & 26.726 & 31.902 & 30.322 & 31.025 & 32.30432 .582 & 39.213 \\
\hline 7/26/2006 & $20: 00$ & 34.46234 .958 & 35.09835 .362 & 28.828 & 26.729 & 32.028 & 30.367 & 31.035 & 32.49032 .754 & .258 \\
\hline 7/27/2006 & 0:00 & 34.67535 .190 & 35.29635 .552 & 28.889 & 26.766 & 32.168 & 30.486 & 31.106 & 32.72332 .993 & 35.109 \\
\hline 7/27/2006 & 4:00 & 34.73735 .222 & 35.34135 .605 & 28.899 & 26.785 & 32.210 & 30.523 & 31.123 & 32.76433 .029 & 34.330 \\
\hline 7/27/2006 & 8:00 & 34.63634 .986 & 35.19135 .540 & 28.937 & 26.819 & 32.130 & 30.532 & 66 & 32.63732 .903 & 885 \\
\hline 7/27/2006 & $12: 00$ & 34.55034 .855 & 35.07835 .446 & 28.968 & 26.840 & 088 & 21 & 31 & 32.52232 .777 & 588 \\
\hline 7/27/2006 & $16: 00$ & 34.49934 .811 & 35.02535 .389 & 28.989 & 26.860 & 32.077 & 30.523 & 31.181 & 32.46032 .717 & 33.867 \\
\hline 7/27/2006 & 20:00 & 34.45834 .749 & 34.98235 .356 & 28.968 & 26.856 & 32.033 & 30.488 & 31. & 32.40932 .664 & 33.364 \\
\hline 7/28/2006 & 0:00 & 34.44734 .749 & 34.97435 .344 & 29.002 & 26.877 & 059 & 12 & 31 & 32.40732 .659 & 203 \\
\hline 7/28/2006 & 4:00 & 34.37934 .673 & 34.91535 .287 & 29.012 & 26.886 & 32.033 & 30.503 & 31 & 32.33832 .582 & 33.088 \\
\hline 7/28/2006 & 8:00 & 34.35734 .652 & 34.91735 .281 & 29.031 & 26.898 & 32.030 & 30.51 & 31.186 & 32.31832 .557 & 35.339 \\
\hline $7 / 28 / 2006$ & $12: 00$ & 34.35334 .625 & 34.87935 .260 & 29.033 & 26.900 & 32.017 & 30.499 & 31.204 & 32.30732 .548 & 41.757 \\
\hline 2006 & $16: 00$ & 34.35734 .589 & 34.84235 .231 & 29.004 & 26.884 & 979 & 64 & 17 & 32.29632 .559 & 645 \\
\hline 7/28/2006 & $20: 00$ & 34.51334 .956 & 35.11135 .403 & 28.993 & 26.874 & 32.081 & 30.488 & 31.244 & 32.52232 .797 & 38.963 \\
\hline 7/29/2006 & 0:00 & 34.70635 .241 & 35.33835 .599 & 29.027 & 26.904 & 32.243 & 30.596 & 31.302 & 32.78733 .071 & 36.460 \\
\hline 7/29/2006 & $4: 00$ & 34.77235 .262 & 35.39335 .677 & 29.037 & 26.916 & 32.287 & 30.642 & 31.320 & 32.86533 .149 & 35.302 \\
\hline 7/29/2006 & 8:00 & 34.66534 .998 & 35.26635 .612 & 29.058 & 26.930 & 32.179 & 30.613 & 31.332 & 32.66432 .927 & 34.609 \\
\hline 7/29/2006 & $12: 00$ & 34.71234 .996 & 35.18635 .573 & 29.058 & 26.935 & 32.148 & 30.598 & 31.352 & 32.67232 .945 & 35.176 \\
\hline 7/29/2006 & $16: 00$ & 34.63834 .922 & 35.10535 .493 & 29.029 & 26.927 & 32.101 & 30.556 & 31.325 & 32.58832 .858 & 35.419 \\
\hline 7/29/2006 & $20: 00$ & 34.57534 .842 & 35.04835 .440 & 29.010 & 26.916 & 32.075 & 30.525 & 31.297 & 32.51932 .783 & 34.228 \\
\hline 7/30/2006 & 0:00 & 34.52734 .798 & 35.02035 .403 & 29.033 & 26.934 & 32.079 & 30.534 & 31.299 & 32.46832 .725 & 33.798 \\
\hline 7/30/2006 & 4:00 & 34.43334 .690 & 34.93835 .326 & 29.017 & 26.927 & 32.044 & 30.51 & 31.272 & 32.37732 .626 & 33.535 \\
\hline 7/30/2006 & 8:00 & 34.42334 .694 & 34.91135 .297 & 29.019 & 26.930 & 32.026 & 30.501 & 31.246 & 32.36232 .621 & 34.622 \\
\hline 7/30/2006 & $12: 00$ & 34.40834 .737 & 34.93835 .297 & 29.016 & 26.935 & 32.081 & 30.505 & 31.262 & 32.38332 .646 & 37.800 \\
\hline 7/30/2006 & $16: 00$ & 34.43734 .794 & 34.96635 .317 & 28.981 & 26.925 & 32.112 & 30.497 & 31.249 & 32.42932 .708 & 38.185 \\
\hline 7/30/2006 & 20:00 & 34.50334 .910 & 35.02435 .364 & 28.966 & 26.930 & 32.148 & 30.499 & 31.231 & 32.51932 .821 & 35.074 \\
\hline 7/31/2006 & 0:00 & 34.54434 .901 & 35.06435 .415 & 28.998 & 26.958 & 32.168 & 30.539 & 31.251 & 32.53632 .812 & 34.275 \\
\hline
\end{tabular}


Depth to Water (ft TOC) in Well Indicated

$\begin{array}{lllllllllllllll}\text { Date } \quad \text { Time } & \text { MW-1 } & \text { MW-2 } & \text { MW-3 } & \text { MW-4 } & \text { KDHE-1 } & \text { KDHE-2 } & \text { NW-1 } & \text { NW-2 } & \text { NW-3 } & \text { L-1 } & \text { L-2 } & \text { L-3 }\end{array}$

\begin{tabular}{|c|c|c|c|c|c|c|c|c|c|c|}
\hline 7/31/2006 & $4: 00$ & 34.46234 .781 & 34.98735 .352 & 28.989 & 26.962 & 32.119 & 30.521 & 31.229 & 3932.701 & 33.834 \\
\hline 7/31/2006 & $8: 00$ & 34.44334 .745 & 35.02035 .375 & 29.002 & 26.972 & 32.101 & 30.529 & 31.229 & 32.41232 .664 & 33.548 \\
\hline 7/31/2006 & $12: 00$ & 34.44734 .751 & 35.00335 .360 & 29.012 & 26.978 & 32.097 & 30.534 & 31.236 & 32.40932 .650 & 33.358 \\
\hline 7/31/2006 & $16: 00$ & 34.37134 .652 & 34.91335 .285 & 28.993 & 26.965 & 32.057 & 30.508 & 31.211 & 32.33332 .573 & 33.222 \\
\hline $7 / 31 / 2006$ & $20: 00$ & 34.41534 .716 & 34.98235 .328 & 28.993 & 26.964 & 32.057 & 30.505 & 31.196 & 32.36832 .621 & 3.101 \\
\hline $8 / 1 / 2006$ & $0: 00$ & 34.59734 .848 & 35.04835 .426 & 29.046 & 26.994 & 32.126 & 30.563 & 31.231 & 32.50032 .757 & 33.098 \\
\hline 3/1/2006 & $4: 00$ & 34.65134 .836 & 35.04135 .442 & 29.056 & 27.002 & 32.117 & 30.567 & 31.246 & 32.50732 .759 & 33.134 \\
\hline $8 / 1 / 2006$ & $8: 00$ & 34.66534 .905 & 9035.483 & 29.079 & 27.015 & 32.134 & 30.589 & 31.279 & 32.55732 .807 & .828 \\
\hline $8 / 1 / 2006$ & $12: 00$ & 34.62634 .893 & 35.07535 .460 & 29.090 & 27.025 & 32.145 & 30.598 & 31.284 & 32.54232 .794 & 33.592 \\
\hline $8 / 1 / 2006$ & $16: 00$ & 34.68534 .922 & 35.06435 .440 & 29.072 & 27.011 & 32.119 & 30.578 & 31.262 & 32.55432 .828 & 33.300 \\
\hline$/ 1 / 2006$ & $20: 00$ & 34.66935 .159 & 35.30635 .567 & 29.058 & 27.006 & 32.254 & 30.618 & 31.239 & 32.68632 .927 & 3.156 \\
\hline $8 / 2 / 2006$ & $0: 00$ & 34.8 & 7135.724 & 29. & 27.039 & 32.398 & 19 & 294 & 8733.140 & 353 \\
\hline $8 / 2 / 2006$ & $4: 00$ & 35.00835 .450 & 35.55935 .834 & 29.108 & 27.055 & 32.451 & 30.766 & 31.340 & 33.00033 .257 & 39.084 \\
\hline 8/2/2006 & $8: 00$ & 35.11435 .531 & 35.67735 .949 & 29.142 & 27.082 & 32.515 & 30.83 & 31.398 & 33.10333 .354 & 39.906 \\
\hline $8 / 2 / 2006$ & $12: 00$ & 35.222 & 35.44535 .816 & 29.167 & 27.106 & 32.402 & 07 & 458 & 193 & 757 \\
\hline $8 / 2 / 2006$ & $16: 00$ & 34.93635 .117 & 35.34935 .781 & 29.141 & 27.098 & 32.325 & 30.748 & 31.506 & 5633 & 841 \\
\hline 8/2/2006 & $20: 00$ & 34.93635 .115 & 35.34235 .749 & 29.202 & 27.135 & 32.345 & 30.792 & 31.582 & 32.85133 .137 & 39.530 \\
\hline $8 / 3 / 2006$ & $0: 00$ & 34.83535 .055 & 35.29235 .695 & 29.217 & 27.145 & 32.329 & 30.788 & 31.610 & 32.78233 .060 & 37.080 \\
\hline 8/3/2006 & $4: 00$ & 34.77234 .999 & 35.24835 .650 & 29.236 & 27.161 & 32.320 & 325 & 31.622 & 32.72332 .996 & 358 \\
\hline $8 / 3 / 2006$ & $8: 00$ & 34.74334 .981 & 35.24535 .636 & 29.284 & 27.184 & 32.338 & 30.745 & 31.635 & 32.69432 .951 & 35.105 \\
\hline 8/3/2006 & $12: 00$ & 34.71034 .956 & 35.22735 .610 & 29.309 & 27.205 & 32.351 & 30.838 & 31.640 & 32.66032 .909 & 34.598 \\
\hline $8 / 3 / 2006$ & $16: 00$ & 34.64234 .891 & 35.16335 .546 & 29.284 & 27.193 & 32.311 & 30.798 & 31.607 & 32.59132 .838 & 34.228 \\
\hline 8/3/2006 & $20: 00$ & 34.70434 .935 & 35.19535 .573 & 29.290 & 27.189 & 32.320 & 30.796 & 31.587 & 32.61832 .869 & .989 \\
\hline 8/4/2006 & 0:00 & 34.96035 .361 & 35.51135 .802 & 29.325 & 27.216 & 32.508 & 30.896 & 31.605 & 32.92433 .166 & 33.887 \\
\hline $8 / 4 / 2006$ & $4: 00$ & 35.09035 .501 & 35.64335 .926 & 29.317 & 27.223 & 32.577 & 30.935 & 31.607 & 33.05933 .302 & 33.814 \\
\hline $8 / 4 / 2006$ & $8: 00$ & 35.09235 .569 & 35.73436 .002 & 29.353 & 27.246 & 32.646 & 30.999 & 31.630 & 33.12033 .350 & 33.707 \\
\hline $8 / 4 / 2006$ & $12: 00$ & 34.99135 .298 & 35.48935 .851 & 29.344 & 27.251 & 32.502 & 30.939 & 31.643 & 32.94433 .202 & 33.581 \\
\hline 8/4/2006 & $16: 00$ & 34.94635 .254 & 35.41635 .791 & 29.299 & 27.230 & 32.438 & 30.88 & 31.597 & 32.88733 .151 & 33.468 \\
\hline 8/4/2006 & $20: 00$ & 34.83535 .142 & 35.37435 .736 & 29.277 & 27.210 & 32.424 & 30.854 & 31.577 & 32.79633 .040 & 33.377 \\
\hline $8 / 5 / 2006$ & 0:00 & 34.90935 .163 & 35.35535 .732 & 29.240 & 27.221 & 32.393 & 30.792 & 31.564 & 32.82133 .080 & 33.355 \\
\hline $8 / 5 / 2006$ & $4: 00$ & 34.91735 .098 & 35.30035 .702 & 29.257 & 27.207 & 32.360 & 30.812 & 31.534 & 32.77433 .024 & 33.382 \\
\hline $8 / 5 / 2006$ & $8: 00$ & 34.90335 .060 & 35.30235 .699 & 29.269 & 27.210 & 32.351 & 30.812 & 31.537 & 32.73632 .969 & 33.694 \\
\hline & $12: 00$ & 34.75334 .988 & 35.23335 .620 & 29.271 & 27.217 & 32.340 & 30.805 & 31.524 & 32.66432 .899 & 37.547 \\
\hline $8 / 5 / 2006$ & $16: 00$ & 34.72635 .007 & 35.22435 .591 & 29.244 & 27.209 & 32.354 & 30.785 & 31.521 & 32.66732 .920 & 42.236 \\
\hline
\end{tabular}


Depth to Water (ft TOC) in Well Indicated

$\begin{array}{llllllllllllll}\text { Date } & \text { Time } & \text { MW-1 } & \text { MW-2 } & \text { MW-3 } & \text { MW-4 } & \text { KDHE-1 } & \text { KDHE-2 } & \text { NW-1 } & \text { NW-2 } & \text { NW-3 } & \text { L-1 } & \text { L-2 } & \text { L-3 }\end{array}$

\begin{tabular}{|c|c|c|c|c|c|c|c|c|c|c|}
\hline 8/5/2006 & $20: 00$ & 34.84635 .246 & 35.35935 .693 & 29.232 & 27.212 & 32.433 & 30.803 & 31.529 & 32.84633 .138 & 40.960 \\
\hline /6/2006 & $0: 00$ & 34.93235 .264 & 35.40135 .765 & 29.280 & 27.253 & 32.458 & 30.854 & 31.597 & 32.91533 .206 & 41.208 \\
\hline 8/6/2006 & $4: 00$ & 34.97135 .226 & 35.38435 .765 & 29.290 & 27.263 & 32.438 & 30.858 & 31.630 & 32.90233 .193 & 41.423 \\
\hline 8/6/2006 & 8:00 & 34.92835 .197 & 35.41035 .789 & 29.327 & 27.281 & 32.449 & 30.882 & 31.690 & 32.88733 .166 & 41.658 \\
\hline $8 / 6 / 2006$ & $12: 00$ & 34.99535 .256 & 35.42235 .814 & 29.334 & 27.293 & 32.455 & 30.895 & 31.720 & 32.95133 .251 & 41.859 \\
\hline 8/6/2006 & $16: 00$ & 34.98135 .237 & 35.39735 .789 & 29.299 & 27.283 & 32.422 & 30.858 & 31.761 & 32.92933 .230 & 38.320 \\
\hline 8/6/2006 & $20: 00$ & 35.01035 .271 & 35.46035 .852 & 29.359 & 27.306 & 32.469 & 30.913 & 31.776 & 5933.246 & .460 \\
\hline 8/7/2006 & $0: 00$ & 34.98535 .233 & 35.44535 .838 & 29.380 & 27.325 & 32.486 & 30.933 & 31.784 & 32.93433 .206 & 35.519 \\
\hline $8 / 7 / 2006$ & $4: 00$ & 34.95035 .161 & 35.40335 .791 & 29.403 & 27.344 & 32.489 & 30.948 & 31.789 & 32.85833 .116 & 34.925 \\
\hline 06 & 8:00 & 34.96935 .163 & 35.40535 .804 & 29.441 & 27.360 & 32.495 & 55 & 31.791 & 32. & 564 \\
\hline $8 / 7 / 2006$ & $12: 00$ & 34.94835 .195 & 35.40935 .796 & 29.440 & 27.369 & 32.500 & 30.97 & 31.789 & 32. & 325 \\
\hline 8/7/2006 & $16: 00$ & 34.90335 .169 & 35.44935 .806 & 29.409 & 27.357 & 32.486 & 30.946 & 31.754 & 32.83333 .067 & 34.053 \\
\hline $8 / 7 / 2006$ & $20: 00$ & 34.90335 .374 & 35.55535 .828 & 29.401 & 27.348 & 32.595 & 30.981 & 31.741 & 32. & 851 \\
\hline 8/8/2006 & $0: 00$ & 35.03435 .545 & 35.72535 .975 & 29.451 & 27.381 & 32.710 & 76 & 63 & 23 & 18 \\
\hline $8 / 8 / 2006$ & 4:00 & 35.00635 .545 & 35.74035 .979 & 29.426 & 27.381 & 32.741 & 89 & 31.725 & 33. & 603 \\
\hline $8 / 8 / 2006$ & 8:00 & 35.10435 .621 & 35.81336 .055 & 29.461 & 27.399 & 32.794 & 38 & 31.733 & 33. & 538 \\
\hline 8/8/2006 & $12: 00$ & 35.01235 .332 & 35.59335 .934 & 29.463 & 27.410 & 32.668 & 98 & 41 & 733 & 73 \\
\hline 8/8/2006 & $16: 00$ & 34.93835 .260 & 35.50835 .843 & 29.424 & 27.392 & 33.046 & 36 & 31.761 & 32. & 336 \\
\hline 8/8/2006 & $20: 00$ & 34.96735 .222 & 35.47035 .828 & 29.409 & 27.381 & 32.546 & 99 & 31.771 & 32.90233 .175 & 37.693 \\
\hline $8 / 9 / 2006$ & $0: 00$ & 35.05135 .336 & 35.54835 .912 & 29.438 & 27.397 & 32.615 & 31.034 & 31.811 & 33.00833 .277 & 35.966 \\
\hline $8 / 9$ & $4: 00$ & 35.309 & 35.51735 .873 & 29.419 & 27.397 & 32.624 & 21 & 31.794 & 32. & 114 \\
\hline $8 / 9 / 2006$ & 8:00 & 34.98735 .355 & 35.53835 .879 & 29.411 & 27.399 & 32.644 & 31.023 & 31.771 & 32. & 34.589 \\
\hline 8/9/2006 & $12: 00$ & 35.01835 .347 & 35.53635 .889 & 29.415 & 27.404 & 32.624 & 31.025 & 31.763 & 32.99733 & 250 \\
\hline $8 / 9 / 2006$ & $16: 00$ & 34.92435 .210 & 35.44135 .800 & 29.369 & 27.385 & 32.573 & 30.975 & 31.736 & 32.88033 & 995 \\
\hline 8/9/2006 & $20: 00$ & 34.86235 .197 & 35.46835 .800 & 29.357 & 27.371 & 32.551 & 57 & 31.741 & 32.8 & 800 \\
\hline $8 / 10 / 2006$ & 0:00 & 35.03835 .611 & 35.75035 .981 & 29.388 & 27.394 & 32.750 & 31.067 & 31.730 & 33.12233 .366 & 33.670 \\
\hline $8 / 10 / 2006$ & 4:00 & 35.04135 .605 & 35.79236 .016 & 29.395 & 27.408 & 32.798 & 31.107 & 31.710 & 33.14033 .368 & 33.570 \\
\hline $8 / 10 / 2006$ & 8:00 & 34.95235 .306 & 35.55935 .892 & 29.399 & 27.417 & 32.652 & 31.065 & 31.700 & 32.95633 .195 & 33.488 \\
\hline $8 / 10 / 2006$ & $12: 00$ & 34.94635 .264 & 35.48335 .822 & 29.407 & 27.418 & 32.635 & 31.047 & 31.680 & 32.89933 .149 & 33.428 \\
\hline $8 / 10 / 2006$ & $16: 00$ & 34.92835 .254 & 35.46835 .814 & 29.417 & 27.420 & 32.604 & 31.028 & 31.665 & 32.89433 .144 & 33.375 \\
\hline $8 / 10 / 2006$ & $20: 00$ & 35.00835 .262 & 35.48735 .840 & 29.399 & 27.415 & 32.575 & 31.008 & 31.655 & 32.90233 .149 & 33.371 \\
\hline $8 / 11 / 2006$ & 0:00 & 34.89335 .186 & 35.43935 .796 & 29.451 & 27.438 & 32.584 & 31.039 & 31.695 & 32.83833 .067 & 33.340 \\
\hline $8 / 11 / 2006$ & $4: 00$ & 34.82135 .098 & 35.37235 .736 & 29.463 & 27.447 & 32.559 & 31.03 & 31.685 & 32.76732 .996 & 33.306 \\
\hline $8 / 11 / 2006$ & 8:00 & 34.77435 .057 & 35.34235 .701 & 29.464 & 27.447 & 32.548 & 31.021 & 31.662 & 32.72532 .949 & 33.267 \\
\hline
\end{tabular}


Depth to Water (ft TOC) in Well Indicated

$\begin{array}{llllllllllllll}\text { Date } & \text { Time } & \text { MW-1 } & \text { MW-2 } & \text { MW-3 } & \text { MW-4 } & \text { KDHE-1 } & \text { KDHE-2 } & \text { NW-1 } & \text { NW-2 } & \text { NW-3 } & \text { L-1 } & \text { L-2 } & \text { L-3 }\end{array}$

\begin{tabular}{|c|c|c|c|c|c|c|c|c|c|c|}
\hline $8 / 11 / 2006$ & $12: 00$ & 34.75935 .022 & 35.31335 .669 & 29.476 & 27.452 & 32.535 & 31.023 & 31.657 & 32.69932 .918 & 34.383 \\
\hline $3 / 11 / 2006$ & $16: 00$ & 34.71434 .996 & 35.27535 .630 & 29.457 & 27.436 & 32.513 & 30.992 & 31.647 & 32.66932 .894 & 41.133 \\
\hline $8 / 11 / 2006$ & $20: 00$ & 34.80435 .212 & 35.42835 .714 & 29.442 & 27.422 & 32.564 & 30.99 & 31.667 & 32.77933 .011 & 39.319 \\
\hline 3/12/2006 & $0: 00$ & 34.96135 .486 & 35.68535 .922 & 29.492 & 27.452 & 32.748 & 31.114 & 31.736 & 33.04633 .284 & 38.801 \\
\hline$/ 12 / 2006$ & $4: 00$ & 35.02035 .556 & 35.75935 .990 & 29.493 & 27.464 & 32.803 & 31.153 & 31.756 & 33.12333 .364 & 652 \\
\hline $3 / 12 / 2006$ & $8: 00$ & 35.06935 .611 & 35.82036 .047 & 29.499 & 27.475 & 32.845 & 31.186 & 31.783 & 33.18133 .423 & 3.630 \\
\hline $8 / 12 / 2006$ & $12: 00$ & 35.05135 .380 & 35.65835 .990 & 29.512 & 27.492 & 32.741 & 31.164 & 31.816 & 33.07333 .330 & 38.663 \\
\hline $8 / 12 / 2006$ & $16: 00$ & 34.97335 .250 & 35.54635 .902 & 29.486 & 27.484 & 32.661 & 31.107 & 32.175 & 6533.217 & 972 \\
\hline 3/12/2006 & $20: 00$ & 34.93235 .214 & 35.47335 .834 & 29.464 & 27.468 & 32.615 & 31.063 & 31.864 & 32.91033 .164 & 663 \\
\hline $8 / 13 / 2006$ & $0: 00$ & 35.05135 .363 & 35.53635 .896 & 29.491 & 27.484 & 32.630 & 31.08 & 31.864 & 33.02733 .312 & 38.655 \\
\hline 8/13/2006 & $4: 00$ & 35.05335 .319 & 35.52935 .904 & 29.494 & 27.485 & 32.619 & 31.072 & 31.869 & 33.01533 .282 & 38.635 \\
\hline 8/13/2006 & 8:00 & 35.00635 .245 & 35.51735 .892 & 29.516 & 27.496 & 32.613 & 83 & 89 & 633 & 99 \\
\hline 8/13/2006 & $12: 00$ & 34.98735 .205 & 35.47335 .863 & 29.512 & 27.494 & 32.597 & 31.076 & 31.912 & 32.92933 & 50 \\
\hline 8/13/2006 & $16: 00$ & 34.93235 .159 & 35.42635 .813 & 29.487 & 27.482 & 32.573 & 31.045 & 31.917 & 32.87733 .127 & 42.993 \\
\hline $8 / 13 / 2006$ & $20: 00$ & 35.01235 .277 & 35.48135 .863 & 29.520 & 27.496 & 32.590 & 31.065 & 31.942 & 32.97033 .250 & 37.964 \\
\hline 006 & 0:00 & 35.1333 & 35.57635 .957 & 29.583 & 27.528 & 32.648 & 27 & 31.980 & 363 & 34 \\
\hline $8 / 14 / 2006$ & $4: 00$ & 35.06135 .290 & 35.49235 .890 & 29.562 & 27.538 & 32.570 & 31.089 & 31.920 & 32.98033 & 255 \\
\hline $8 / 14 / 2006$ & 8:00 & 35.02235 .245 & 35.50435 .890 & 29.605 & 27.549 & 32.599 & 31.107 & 31.937 & 32.93933 .173 & 34.761 \\
\hline $8 / 14 / 2006$ & $12: 00$ & 34.98135 .214 & 35.49235 .867 & 29.618 & 27.545 & 32.542 & 38 & 45 & 0533 & 427 \\
\hline $8 / 14 / 2006$ & $16: 00$ & 34.92235 .155 & 35.44135 .818 & 29.603 & 27.505 & 32.515 & 91 & 22 & 1833 & 181 \\
\hline $8 / 14 / 2006$ & $20: 00$ & 34.85635 .096 & 35.38935 .760 & 29.566 & 27.447 & 32.442 & 31.019 & 31.889 & 32.78433 .011 & 984 \\
\hline $8 / 15 / 2006$ & $0: 00$ & 34.85635 .089 & 35.38835 .762 & 29.605 & 27.424 & 32.424 & 31.032 & 31.889 & 32.78233 .002 & 33.842 \\
\hline $8 / 15 / 2006$ & $4: 00$ & 34.81335 .053 & 35.35335 .724 & 29.599 & 27.390 & 32.389 & 31.012 & 31.879 & 32.74332 .962 & 33.729 \\
\hline $8 / 15 / 2006$ & $8: 00$ & 34.78235 .020 & 35.32535 .695 & 29.597 & 27.358 & 32.355 & 30.992 & 31.861 & 32.71332 .929 & 33.634 \\
\hline $8 / 15 / 2006$ & $12: 00$ & 34.76335 .007 & 35.31135 .677 & 29.610 & 27.341 & 32.342 & 30.988 & 31.852 & 32.69332 .907 & 33.561 \\
\hline $8 / 15 / 2006$ & $16: 00$ & 34.73334 .946 & 35.25035 .624 & 29.572 & 27.302 & 32.287 & 30.939 & 31.814 & 32.63932 .856 & 33.532 \\
\hline $8 / 15 / 2006$ & $20: 00$ & 34.68734 .941 & 35.22435 .589 & 29.549 & 27.265 & 32.254 & 30.902 & 31.783 & 32.61732 .839 & 33.450 \\
\hline $8 / 16 / 2006$ & $0: 00$ & 34.69034 .924 & 35.21835 .587 & 29.560 & 27.251 & 32.245 & 30.9 & 31.783 & 32.60832 .821 & 33.408 \\
\hline $8 / 16 / 2006$ & 4:00 & 34.64634 .895 & 35.18935 .554 & 29.551 & 27.233 & 32.230 & 30.882 & 31.763 & 32.57132 .785 & 33.360 \\
\hline $8 / 16 / 2006$ & $8: 00$ & 34.74935 .218 & 35.45435 .709 & 29.558 & 27.226 & 32.393 & 30.955 & 31.758 & 32.77932 .982 & 33.322 \\
\hline $8 / 16 / 2006$ & $12: 00$ & 34.73535 .026 & 35.31535 .658 & 29.562 & 27.226 & 32.305 & 30.933 & 31.753 & 32.69332 .909 & 33.298 \\
\hline $8 / 16 / 2006$ & $16: 00$ & 34.65534 .923 & 35.22235 .574 & 29.526 & 27.200 & 32.241 & 30.878 & 31.715 & 32.60332 .817 & 33.262 \\
\hline $8 / 16 / 2006$ & $20: 00$ & 34.70634 .904 & 35.21035 .564 & 29.497 & 27.177 & 32.214 & 30.84 & 31.695 & 32.58132 .792 & 33.227 \\
\hline $8 / 17 / 2006$ & $0: 00$ & 34.60934 .872 & 35.17035 .529 & 29.520 & 27.180 & 32.205 & 30.845 & 31.685 & 32.54632 .756 & 33.202 \\
\hline
\end{tabular}


Depth to Water (ft TOC) in Well Indicated

$\begin{array}{llllllllllllll}\text { Date } & \text { Time } & \text { MW-1 } & \text { MW-2 } & \text { MW-3 } & \text { MW-4 } & \text { KDHE-1 } & \text { KDHE-2 } & \text { NW-1 } & \text { NW-2 } & \text { NW-3 } & \text { L-1 } & \text { L-2 } & \text { L-3 }\end{array}$

\begin{tabular}{|c|c|c|c|c|c|c|c|c|c|c|}
\hline $8 / 17 / 2006$ & 4:00 & 34.60734 .836 & 35.13435 .493 & 29.520 & 27.179 & 32.183 & 30.834 & 31.667 & 32.50932 .719 & 33.158 \\
\hline $3 / 17 / 2006$ & $8: 00$ & 34.56834 .815 & 35.11535 .480 & 29.534 & 27.175 & 32.179 & 30.831 & 31.659 & 32.49532 .704 & 33.143 \\
\hline $8 / 17 / 2006$ & $12: 00$ & 34.56834 .823 & 35.12535 .470 & 29.522 & 27.166 & 32.216 & 30.873 & 31.642 & 32.48732 .695 & 33.125 \\
\hline 3/17/2006 & $16: 00$ & 34.51134 .768 & 35.06935 .425 & 29.495 & 27.142 & 32.139 & 30.781 & 31.617 & 32.44632 .657 & .105 \\
\hline$/ 17 / 2006$ & $20: 00$ & 34.51934 .775 & 35.06235 .419 & 29.492 & 27.129 & 32.121 & 30.761 & 31.601 & 32.44632 .663 & 33.083 \\
\hline $8 / 18 / 2006$ & $0: 00$ & 34.51334 .772 & 35.06735 .429 & 29.528 & 27.143 & 32.132 & 30.781 & 31.619 & 32.44632 .653 & 3.076 \\
\hline 8/18/2006 & $4: 00$ & 34.51534 .779 & 35.07535 .433 & 29.555 & 27.154 & 32.141 & 30.796 & 31.644 & 32.44632 .650 & 33.070 \\
\hline 8/18/2006 & 8:00 & 34.47634 .731 & 35.02935 .391 & 29.518 & 27.131 & 32.103 & 30.763 & 31.602 & 32.40932 .619 & 50 \\
\hline 8/18/2006 & $12: 00$ & 34.48234 .730 & 35.02535 .388 & 29.522 & 27.127 & 32.097 & 30.763 & 31.594 & 32.40632 .617 & 047 \\
\hline 8/18/2006 & $16: 00$ & 34.45334 .697 & 34.99335 .354 & 29.489 & 27.106 & 32.070 & 30.717 & 31.572 & 32.37532 .586 & 33.027 \\
\hline 8/18/2006 & $20: 00$ & 34.44934 .699 & 34.99735 .358 & 29.505 & 27.105 & 32.070 & 30.715 & 31.574 & 32.37532 .582 & 33.014 \\
\hline 8/19/2006 & 0:00 & 34.48234 .743 & 35.03935 .397 & 29.563 & 27.138 & 32.143 & 89 & 99 & 0932.608 & 92 \\
\hline 8/19/2006 & $4: 00$ & 34.47234 .732 & 35.02935 .388 & 29.572 & 27.145 & 32.165 & 30.842 & 31.592 & 32.40232 .602 & 32.976 \\
\hline 8/19/2006 & 8:00 & 34.38834 .642 & 34.88435 .260 & 29.514 & 27.103 & 32.061 & 30.834 & 31.518 & 32.30732 .509 & 32.932 \\
\hline $8 / 19 / 2006$ & $12: 00$ & 34.42734 .646 & 34.89635 .278 & 29.522 & 27.064 & 31.849 & 30.798 & 31.536 & 32.31632 .524 & 32.883 \\
\hline 006 & $16: 00$ & 6934.610 & 34.85435 .241 & 29.443 & 26.969 & 31.714 & 45 & 31.523 & 32.27932 & 32 \\
\hline $8 / 19 / 2006$ & $20: 00$ & 34.33934 .562 & 34.80135 .198 & 29.369 & 26.858 & 31.563 & 30.686 & 31.505 & 32.24032 .453 & 32.881 \\
\hline 8/20/2006 & $0: 00$ & 34.33234 .541 & 34.78535 .190 & 29.340 & 26.768 & 31.442 & 30.604 & 31.511 & 32.22632 .435 & 32.883 \\
\hline $8 / 20 / 2006$ & $4: 00$ & 34.29534 .486 & 34.73035 .147 & 29.299 & 26.669 & 31.313 & 16 & 31.508 & 32.17732 .387 & 32.879 \\
\hline $8 / 20 / 2006$ & 8:00 & 34.27534 .456 & 34.70135 .125 & 29.292 & 26.581 & 31.234 & 28 & 08 & 32.15232 .364 & 32 \\
\hline 8/20/2006 & $12: 00$ & 34.29334 .426 & 34.67135 .104 & 29.282 & 26.500 & 31.158 & 30.388 & 31.513 & 32.12832 .338 & 32.892 \\
\hline 8/20/2006 & $16: 00$ & 34.22834 .378 & 34.61835 .053 & 29.221 & 26.396 & 31.072 & 30.062 & 31.485 & 32.08332 .296 & 32.910 \\
\hline $8 / 20 / 2006$ & $20: 00$ & 34.20934 .333 & 34.57235 .008 & 29.186 & 26.317 & 31.008 & 30 & 31.460 & 32.03432 .247 & 32.864 \\
\hline $8 / 21 / 2006$ & $0: 00$ & 34.20134 .319 & 34.56435 .004 & 29.211 & 26.285 & 30.988 & 29.996 & 31.470 & 32.02532 .234 & 32.855 \\
\hline $8 / 21 / 2006$ & $4: 00$ & 34.13734 .279 & 34.52634 .970 & 29.194 & 26.242 & 30.953 & 29.961 & 31.445 & 31.99032 .199 & 32.839 \\
\hline $8 / 21 / 2006$ & 8:00 & 34.14234 .279 & 34.52434 .967 & 29.215 & 26.225 & 30.951 & 29.956 & 31.455 & 31.98032 .188 & 32.830 \\
\hline $8 / 21 / 2006$ & $12: 00$ & 34.14834 .289 & 34.53634 .974 & 29.229 & 26.214 & 30.979 & 29.98 & 31.458 & 31.99232 .205 & 32.833 \\
\hline $8 / 21 / 2006$ & $16: 00$ & 34.12534 .243 & 34.49234 .933 & 29.196 & 26.181 & 30.942 & 29.921 & 31.445 & 31.94832 .159 & 32.817 \\
\hline $8 / 21 / 2006$ & 20:00 & 34.08034 .224 & 34.46534 .905 & 29.184 & 26.161 & 30.926 & 29.894 & 31.440 & 31.92632 .136 & 32.802 \\
\hline $8 / 22 / 2006$ & $0: 00$ & 34.07634 .219 & 34.46734 .910 & 29.213 & 26.170 & 30.951 & 29.91 & 31.455 & 31.92632 .132 & 32.802 \\
\hline $8 / 22 / 2006$ & 4:00 & 34.04334 .184 & 34.43334 .873 & 29.200 & 26.160 & 30.946 & 29.888 & 31.430 & 31.89232 .099 & 32.790 \\
\hline $8 / 22 / 2006$ & 8:00 & 34.04534 .192 & 34.43734 .875 & 29.221 & 26.172 & 30.968 & 29.899 & 31.440 & 31.89232 .099 & 32.786 \\
\hline $8 / 22 / 2006$ & $12: 00$ & 34.02734 .177 & 34.42534 .857 & 29.219 & 26.172 & 31.015 & 29.901 & 31.432 & 31.87732 .090 & 32.788 \\
\hline $8 / 22 / 2006$ & $16: 00$ & 34.06334 .276 & 34.56434 .937 & 29.183 & 26.163 & 31.207 & 30.08 & 31.400 & 31.96032 .167 & 32.773 \\
\hline
\end{tabular}


Depth to Water (ft TOC) in Well Indicated

$\begin{array}{llllllllllllll}\text { Date } & \text { Time } & \text { MW-1 } & \text { MW-2 } & \text { MW-3 } & \text { MW-4 } & \text { KDHE-1 } & \text { KDHE-2 } & \text { NW-1 } & \text { NW-2 } & \text { NW-3 } & \text { L-1 } & \text { L-2 } & \text { L-3 }\end{array}$

\begin{tabular}{|c|c|c|c|c|c|c|c|c|c|c|}
\hline 8/22/2006 & $20: 00$ & 34.01834 .205 & 34.45934 .861 & 29.150 & 26.151 & 31.068 & 29.934 & 31.372 & 31.89432 .105 & 32.751 \\
\hline $3 / 23 / 2006$ & $0: 00$ & 34.01234 .182 & 34.43334 .847 & 29.167 & 26.170 & 31.050 & 29.914 & 31.372 & 31.87232 .081 & 32.746 \\
\hline 8/23/2006 & $4: 00$ & 33.95934 .127 & 34.37634 .792 & 29.131 & 26.154 & 31.015 & 29.861 & 31.339 & 31.82132 .034 & 32.731 \\
\hline 3/23/2006 & 8:00 & 33.94234 .103 & 34.34734 .769 & 29.123 & 26.154 & 31.008 & 29.837 & 31.331 & 31.79632 .006 & 32.715 \\
\hline /23/2006 & $12: 00$ & 33.91634 .081 & 34.32634 .743 & 29.112 & 26.153 & 31.008 & 29.822 & 31.306 & 31.77731 .988 & 32.706 \\
\hline $8 / 23 / 2006$ & $16: 00$ & 33.86234 .021 & 34.26734 .683 & 29.047 & 26.121 & 30.962 & 29.764 & 31.263 & 31.72131 .935 & 32.684 \\
\hline 8/23/2006 & $20: 00$ & 33.84834 .008 & 34.25634 .673 & 29.020 & 26.110 & 30.948 & 29.742 & 31.238 & 31.70831 .920 & 32.658 \\
\hline 8/24/2006 & 0:00 & 33.82734 .006 & 34.24034 .653 & 29.020 & 26.116 & 30.953 & 29.742 & 31.230 & 8831 & 32.644 \\
\hline 3/24/2006 & $4: 00$ & 33.94434 .342 & 34.51334 .816 & 29.018 & 26.124 & 134 & 29.82 & 31.208 & .90932 & 32.627 \\
\hline $8 / 24 / 2006$ & 8:00 & 33.98634 .290 & 34.50534 .851 & 29.004 & 26.131 & 31.132 & 29.837 & 31.193 & 31.94132 .159 & 32.611 \\
\hline 3/24/2006 & $12: 00$ & 33.90134 .110 & 34.34534 .739 & 28.993 & 26.135 & 31.048 & 29.786 & 31.170 & 31.79432 .010 & 32.607 \\
\hline 8/24/2006 & $16: 00$ & 33.82734 .017 & 34.25434 .657 & 28.951 & 26.114 & 95 & 29.729 & 218 & 0631 & 596 \\
\hline 8/24/2006 & $20: 00$ & 33.79533 .981 & 34.21434 .622 & 28.933 & 26.112 & 979 & 29.709 & 31.311 & 31.66731 & 32.585 \\
\hline $8 / 25 / 2006$ & 0:00 & 33.79133 .962 & 34.20634 .618 & 28.951 & 26.133 & 30.986 & 29.72 & 31.336 & 31.65731 .868 & 32.589 \\
\hline $8 / 25 / 2006$ & $4: 00$ & 33.77233 .948 & 34.18734 .598 & 28.951 & 26.142 & 30.990 & 29.72 & 31.299 & 31.63531 .846 & 32.589 \\
\hline 2006 & 8:00 & 333.960 & 34.19834 .616 & 28.983 & 26.174 & 15 & 29.749 & 31.281 & 493 & 32.587 \\
\hline $8 / 25 / 2006$ & $12: 00$ & 33.81933 .996 & 34.23134 .638 & 28.997 & 26.193 & 055 & 29.778 & 31.251 & 31.67831 & 32.593 \\
\hline $8 / 25 / 2006$ & $16: 00$ & 33.78833 .964 & 34.19834 .608 & 28.974 & 26.190 & 31.037 & 29.751 & 31.220 & 4731.857 & 32.593 \\
\hline $8 / 25 / 2006$ & $20: 00$ & 33.78033 .939 & 34.17734 .593 & 28.962 & 26.191 & 028 & 29.742 & 31.192 & 3431 & 32.578 \\
\hline $8 / 26 / 2006$ & $0: 00$ & 33.76633 .947 & 34.17034 .585 & 29.022 & 26.255 & 52 & 29.784 & 31.185 & 2031 & 32.465 \\
\hline 8/26/2006 & $4: 00$ & 33.78433 .958 & 34.19534 .610 & 29.010 & 26.244 & 021 & 29.733 & 31.170 & 4031 & 32.485 \\
\hline $8 / 26 / 2006$ & 8:00 & 33.80133 .972 & 34.20434 .624 & 28.979 & 26.216 & 975 & 29.718 & 31.183 & 31.65431 & 32.489 \\
\hline $8 / 26 / 2006$ & $12: 00$ & 33.81133 .958 & 34.18934 .626 & 28.916 & 26.158 & 30.900 & 29.735 & 31.180 & 4931 & 32.503 \\
\hline $8 / 26 / 2006$ & $16: 00$ & 33.82533 .972 & 34.18734 .614 & 28.872 & 26.087 & 30.842 & 29.696 & 31.180 & 31.65131 .857 & 32.511 \\
\hline $8 / 26 / 2006$ & 20:00 & 33.78833 .915 & 34.13534 .575 & 28.814 & 26.003 & 30.760 & 29.623 & 31.162 & 31.61031 .817 & 32.507 \\
\hline $8 / 27 / 2006$ & $0: 00$ & 33.77433 .913 & 34.13434 .577 & 28.814 & 25.936 & 30.725 & 29.544 & 31.177 & 31.61331 .819 & 32.507 \\
\hline 8/27/2006 & 4:00 & 33.76033 .867 & 34.08834 .530 & 28.778 & 25.849 & 30.725 & 29.504 & 31.149 & 31.56631 .771 & 32.567 \\
\hline $8 / 27 / 2006$ & 8:00 & 33.70433 .844 & 34.06134 .503 & 28.759 & 25.781 & 30.672 & 29.529 & 31.129 & 31.53931 .744 & 32.469 \\
\hline 8/27/2006 & $12: 00$ & 33.70033 .829 & 34.04834 .495 & 28.761 & 25.740 & 30.625 & 29.52 & 31.139 & 31.53131 .735 & 32.463 \\
\hline $8 / 27 / 2006$ & $16: 00$ & 33.65533 .772 & 33.98934 .438 & 28.709 & 25.670 & 30.508 & 29.544 & 31.104 & 31.48031 .689 & 32.576 \\
\hline 8/27/2006 & $20: 00$ & 33.66933 .751 & 33.96434 .414 & 28.684 & 25.606 & 30.455 & 29.495 & 31.099 & 31.45831 .665 & 32.494 \\
\hline $8 / 28 / 2006$ & 0:00 & 33.63433 .745 & 33.96034 .410 & 28.692 & 25.558 & 30.426 & 29.385 & 31.109 & 31.45631 .662 & 32.467 \\
\hline 8/28/2006 & $4: 00$ & 33.59633 .700 & 33.91434 .371 & 28.661 & 25.484 & 30.364 & 29.302 & 31.091 & 31.41431 .620 & 32.443 \\
\hline 8/28/2006 & $8: 00$ & 33.60433 .690 & 33.90134 .363 & 28.657 & 25.437 & 30.329 & 29.204 & 31.086 & 31.40431 .607 & 32.423 \\
\hline
\end{tabular}


Depth to Water (ft TOC) in Well Indicated

$\begin{array}{llllllllllllll}\text { Date } & \text { Time } & \text { MW-1 } & \text { MW-2 } & \text { MW-3 } & \text { MW-4 } & \text { KDHE-1 } & \text { KDHE-2 } & \text { NW-1 } & \text { NW-2 } & \text { NW-3 } & \text { L-1 } & \text { L-2 } & \text { L-3 }\end{array}$

\begin{tabular}{|c|c|c|c|c|c|c|c|c|c|c|}
\hline 8/28/2006 & $12: 00$ & 33.62633 .677 & 33.88434 .348 & 28.636 & 25.393 & 30.291 & 29.138 & 31.066 & 31.39531 .603 & 32.407 \\
\hline $3 / 28 / 2006$ & $16: 00$ & 33.55233 .652 & 33.85534 .313 & 28.607 & 25.352 & 30.260 & 29.087 & 31.043 & 31.36031 .567 & 32.396 \\
\hline 8/28/2006 & $20: 00$ & 33.58733 .666 & 33.86934 .334 & 28.634 & 25.349 & 30.260 & 29.092 & 31.056 & 31.37931 .583 & 32.383 \\
\hline 3/29/2006 & $0: 00$ & 33.57933 .656 & 33.86134 .320 & 28.650 & 25.340 & 30.260 & 29.096 & 31.064 & 31.36531 .570 & 32.381 \\
\hline /29/2006 & $4: 00$ & 33.53233 .633 & 33.83434 .295 & 28.644 & 25.320 & 30.242 & 29.078 & 31.053 & 31.33831 .542 & 32.374 \\
\hline $8 / 29 / 2006$ & $8: 00$ & 33.56333 .639 & 33.84234 .297 & 28.656 & 25.319 & 30.249 & 29.083 & 31.043 & 31.34331 .545 & 32.361 \\
\hline 8/29/2006 & $12: 00$ & 33.52633 .635 & 33.83634 .291 & 28.654 & 25.313 & 30.251 & 29.083 & 31.033 & 31.33831 .543 & 32.352 \\
\hline 8/29/2006 & $16: 00$ & 33.50133 .605 & 33.79634 .258 & 28.610 & 25.285 & 30.216 & 29.037 & 31.008 & 0431.514 & 32.341 \\
\hline 8/29/2006 & $20: 00$ & 33.49933 .578 & 33.77534 .238 & 28.592 & 25.276 & 30.209 & 29.021 & 30.985 & 31.27931 .487 & 32.323 \\
\hline 8/30/2006 & $0: 00$ & 33.46433 .571 & 33.77234 .226 & 28.610 & 25.287 & 30.225 & 29.039 & 30.980 & 31.27431 .480 & 32.312 \\
\hline 8/30/2006 & $4: 00$ & 33.44233 .546 & 33.75134 .207 & 28.611 & 25.290 & 30.227 & 29.037 & 30.973 & 31.25231 .458 & 32.303 \\
\hline 8/30/2006 & 8:00 & 33.45233 .544 & 33.74934 .203 & 28.619 & 25.301 & 30.238 & 29. & 30 & 473 & 32.288 \\
\hline 8/30/2006 & $12: 00$ & 33.44033 .533 & 33.74334 .209 & 28.615 & 25.305 & 30.242 & 29.05 & 45 & 2433 & 32.319 \\
\hline 8/30/2006 & $16: 00$ & 33.37633 .477 & 33.68634 .140 & 28.560 & 25.275 & 30.209 & 29.002 & 30.902 & 31.18831 .394 & 32.274 \\
\hline $8 / 30 / 2006$ & $20: 00$ & 33.36833 .477 & 33.68034 .130 & 28.552 & 25.278 & 30.216 & 28.995 & 30.882 & 31.18131 .388 & 32.246 \\
\hline 2006 & 0:00 & 33.38633 .481 & 33.69034 .142 & 28.583 & 25.305 & 30.238 & 29. & 89 & 88 & 32.235 \\
\hline $8 / 31 / 2006$ & $4: 00$ & 33.36833 .489 & 33.69534 .138 & 28.590 & 25.319 & 30.260 & 29.041 & 30.879 & 388 & 32.224 \\
\hline $8 / 31 / 2006$ & 8:00 & 33.37233 .485 & 33.70334 .148 & 28.604 & 25.336 & 30.277 & 29.056 & 30.877 & 8931.392 & 32.212 \\
\hline $8 / 31 / 2006$ & $12: 00$ & 33.36633 .485 & 33.70134 .142 & 28.611 & 25.349 & 30.295 & 29. & 30 & 363 & 32.204 \\
\hline $8 / 31 / 2006$ & $16: 00$ & 33.34733 .464 & 33.67634 .121 & 28.588 & 25.345 & 30.287 & 29. & 44 & 70 & 32.190 \\
\hline $8 / 31 / 2006$ & $20: 00$ & 33.35533 .479 & 33.68234 .121 & 28.587 & 25.356 & 30.300 & 29.056 & 30.831 & 7131.375 & 32.179 \\
\hline $9 / 1 / 2006$ & $0: 00$ & 33.36133 .485 & 33.69534 .136 & 28.611 & 25.382 & 30.331 & 29.087 & 30.839 & 31.18131 .383 & 32.173 \\
\hline $9 / 1 / 2006$ & 4:00 & 33.40333 .538 & 33.73934 .168 & 28.656 & 25.417 & 30.382 & 29.14 & 30.849 & 31.21131 .410 & 32.177 \\
\hline $9 / 1 / 2006$ & $8: 00$ & 33.40333 .578 & 33.78134 .197 & 28.657 & 25.432 & 30.417 & 29.162 & 30.842 & 31.24731 .449 & 32.173 \\
\hline $9 / 1 / 2006$ & $12: 00$ & 33.39233 .529 & 33.74734 .181 & 28.661 & 25.446 & 30.410 & 29.164 & 30.839 & 31.22231 .425 & 32.166 \\
\hline 9/1/2006 & $16: 00$ & 33.37433 .510 & 33.72834 .160 & 28.644 & 25.447 & 30.413 & 29.153 & 30.826 & 31.20331 .407 & 32.476 \\
\hline $9 / 1 / 2006$ & $20: 00$ & 33.44033 .747 & 33.92434 .268 & 28.644 & 25.461 & 30.537 & 29.202 & 30.824 & 31.33631 .523 & 35.643 \\
\hline $9 / 2 / 2006$ & $0: 00$ & 33.58733 .954 & 34.10134 .418 & 28.682 & 25.500 & 30.663 & 29.301 & 30.859 & 31.52931 .726 & 37.049 \\
\hline $9 / 2 / 2006$ & $4: 00$ & 33.54833 .698 & 33.90934 .334 & 28.692 & 25.523 & 30.559 & 29.277 & 30.894 & 31.40131 .618 & 37.891 \\
\hline $9 / 2 / 2006$ & $8: 00$ & 33.58533 .700 & 33.92234 .363 & 28.736 & 25.564 & 30.583 & 29.31 & 30.953 & 31.42131 .636 & 38.469 \\
\hline $9 / 2 / 2006$ & $12: 00$ & 33.60633 .717 & 33.92834 .371 & 28.757 & 25.592 & 30.610 & 29.336 & 30.988 & 31.43431 .653 & 34.958 \\
\hline $9 / 2 / 2006$ & $16: 00$ & 33.57333 .700 & 33.90934 .352 & 28.736 & 25.595 & 30.605 & 29.325 & 30.983 & 31.41431 .634 & 33.997 \\
\hline 9/2/2006 & $20: 00$ & 33.63533 .677 & 33.89034 .338 & 28.723 & 25.601 & 30.603 & 29.317 & 30.970 & 31.39631 .614 & 33.477 \\
\hline 9/3/2006 & $0: 00$ & 33.54233 .668 & 33.88834 .330 & 28.740 & 25.622 & 30.621 & 29.336 & 30.968 & 31.37731 .593 & 33.145 \\
\hline
\end{tabular}


Depth to Water (ft TOC) in Well Indicated

$\begin{array}{llllllllllllll}\text { Date } & \text { Time } & \text { MW-1 } & \text { MW-2 } & \text { MW-3 } & \text { MW-4 } & \text { KDHE-1 } & \text { KDHE-2 } & \text { NW-1 } & \text { NW-2 } & \text { NW-3 } & \text { L-1 } & \text { L-2 } & \text { L-3 }\end{array}$

\begin{tabular}{|c|c|c|c|c|c|c|c|c|c|c|}
\hline $9 / 3 / 2006$ & $4: 00$ & 33.51733 .652 & 33.87334 .311 & 28.730 & 25.629 & 30.623 & 29.332 & 30.950 & 31.35831 .569 & 32.910 \\
\hline /3/2006 & 8:00 & 33.52033 .650 & 33.87434 .315 & 28.750 & 25.648 & 30.641 & 29.354 & 30.947 & 31.35731 .567 & 32.744 \\
\hline 9/3/2006 & $12: 00$ & 33.51533 .647 & 33.87334 .311 & 28.744 & 25.655 & 30.652 & 29.358 & 30.935 & 31.35231 .563 & 32.622 \\
\hline $9 / 3 / 2006$ & $16: 00$ & 33.47833 .647 & 33.85734 .279 & 28.700 & 25.640 & 30.650 & 29.325 & 30.887 & 31.33331 .549 & 32.511 \\
\hline $9 / 3 / 2006$ & $20: 00$ & 33.46233 .624 & 33.83834 .264 & 28.688 & 25.641 & 30.643 & 29.321 & 30.872 & 31.31331 .522 & 32.429 \\
\hline 9/4/2006 & 0:00 & 33.47433 .631 & 33.85234 .279 & 28.717 & 25.671 & 30.665 & 29.354 & 30.867 & 31.32031 .527 & 32.374 \\
\hline 9/4/2006 & $4: 00$ & 33.46033 .614 & 33.84034 .268 & 28.715 & 25.682 & 30.672 & 29.358 & 30.859 & 31.30831 .512 & 32.321 \\
\hline 9/4/2006 & 8:00 & 33.50733 .637 & 33.86534 .293 & 28.753 & 25.714 & 30.705 & 29.398 & 30.869 & 31.32831 .527 & 32.292 \\
\hline $9 / 4 / 2006$ & $12: 00$ & 33.49933 .662 & 33.89034 .311 & 28.776 & 25.736 & 30.734 & 29.429 & 30.879 & 31.35031 .549 & 32.277 \\
\hline /4/2006 & $16: 00$ & 33.49133 .643 & 33.87334 .299 & 28.751 & 25.736 & 30.727 & 29.414 & 30.854 & 3531.536 & 32.248 \\
\hline $9 / 4 / 2006$ & $20: 00$ & 33.67133 .671 & 33.91134 .336 & 28.774 & 25.759 & 30.754 & 29.442 & 30.866 & 563 & 32.224 \\
\hline 9/5/2006 & $0: 00$ & 33.51133 .683 & 33.91634 .332 & 28.801 & 25.784 & 30.782 & 29.473 & 30.872 & 31.36731 .562 & 32.215 \\
\hline $9 / 5 / 2006$ & $4: 00$ & 33.50133 .673 & 33.90934 .322 & 28.803 & 25.796 & 30.791 & 29.477 & 30.859 & 31.35531 .554 & 32.197 \\
\hline $9 / 5 / 2006$ & 8:00 & 33.51533 .696 & 33.92834 .340 & 28.828 & 25.818 & 30.818 & 29.508 & 76 & 569 & 32.192 \\
\hline $9 / 5 / 2006$ & $12: 00$ & 33.52433 .687 & 33.92634 & 28.830 & 25.832 & 30.824 & 29.517 & 69 & 573 & 32.190 \\
\hline $9 / 5 / 2006$ & $16: 00$ & 33.48133 .654 & 33.89334 .305 & 28.786 & 25.814 & 30.809 & 29.48 & 30.828 & 31.34031 .542 & 32.168 \\
\hline $9 / 5 / 2006$ & 20:00 & 33.48333 .656 & 33.88834 .299 & 28.774 & 25.814 & 30.807 & 29.475 & 30 & 131 & 32.150 \\
\hline 9/6/2006 & 0:00 & 33.48733 .673 & 33.90934 .316 & 28.805 & 25.841 & 30.835 & 29.508 & 16 & 51 & 32.144 \\
\hline $9 / 6 / 2006$ & $4: 00$ & 33.48533 .671 & 33.90934 .315 & 28.805 & 25.849 & 30.844 & 29.515 & 30 & 543 & 32.131 \\
\hline 9/6/2006 & 8:00 & 33.49933 .677 & 33.91434 .320 & 28.811 & 25.862 & 30.844 & 29.526 & & 51 & 32.124 \\
\hline 9/6/2006 & $12: 00$ & 33.61833 .863 & 34.08834 .448 & 28.821 & 25.876 & 66 & 29.594 & 30 & 731.720 & 32.126 \\
\hline $9 / 6 / 2006$ & $16: 00$ & 33.719 & 33.95634 .354 & 28.771 & 25.853 & 30.884 & 29.53 & 30.770 & 931 & 32.111 \\
\hline $9 / 6 / 2006$ & 20:00 & 33.66334 .038 & 34.22934 .514 & 28.755 & 25.851 & 31.041 & 29.59 & 30.760 & 9731.784 & 32.093 \\
\hline 9/7/2006 & $0: 00$ & 33.70334 .155 & 34.33034 .600 & 28.776 & 25.878 & 31.119 & 29.654 & 30.763 & 31.70831 .899 & 32.095 \\
\hline 9/7/2006 & 4:00 & 33.73134 .190 & 34.36434 .634 & 28.765 & 25.883 & 31.147 & 29.674 & 30 & 4231 & 32.090 \\
\hline 9/7/2006 & $8: 00$ & 33.78834 .245 & 34.41934 .690 & 28.784 & 25.906 & 31.187 & 29.716 & 30.765 & 31.79931 .990 & 32.088 \\
\hline 9/7/2006 & $12: 00$ & 33.69633 .941 & 34.18334 .561 & 28.793 & 25.922 & 31.061 & 29.674 & 30.773 & 31.61831 .821 & 32.101 \\
\hline 9/7/2006 & $16: 00$ & 33.62033 .837 & 34.07334 .465 & 28.742 & 25.895 & 30.990 & 29.603 & 30.733 & 31.51731 .722 & 32.086 \\
\hline 9/7/2006 & $20: 00$ & 33.56533 .761 & 34.00434 .405 & 28.722 & 25.890 & 30.946 & 29.566 & 30.712 & 31.44531 .649 & 32.066 \\
\hline 9/8/2006 & $0: 00$ & 33.55033 .749 & 33.99334 .395 & 28.753 & 25.915 & 30.955 & 29.583 & 30.728 & 31.43131 .629 & 32.068 \\
\hline 9/8/2006 & $4: 00$ & 33.52033 .713 & 33.96234 .365 & 28.751 & 25.920 & 30.941 & 29.574 & 30.710 & 31.39931 .596 & 32.057 \\
\hline $9 / 8 / 2006$ & 8:00 & 33.52833 .715 & 33.96434 .369 & 28.778 & 25.941 & 30.955 & 29.594 & 30.717 & 31.39931 .593 & 32.049 \\
\hline 9/8/2006 & $12: 00$ & 33.52833 .722 & 33.96834 .365 & 28.761 & 25.953 & 30.937 & 29.585 & 30.730 & 31.39931 .594 & 32.053 \\
\hline 9/8/2006 & $16: 00$ & 33.48533 .673 & 33.92234 .328 & 28.765 & 25.941 & 30.939 & 29.579 & 30.700 & 31.36031 .556 & 32.038 \\
\hline
\end{tabular}


Depth to Water (ft TOC) in Well Indicated

$\begin{array}{llllllllllllll}\text { Date } & \text { Time } & \text { MW-1 } & \text { MW-2 } & \text { MW-3 } & \text { MW-4 } & \text { KDHE-1 } & \text { KDHE-2 } & \text { NW-1 } & \text { NW-2 } & \text { NW-3 } & \text { L-1 } & \text { L-2 } & \text { L-3 }\end{array}$

\begin{tabular}{|c|c|c|c|c|c|c|c|c|c|c|}
\hline 9/8/2006 & $20: 00$ & 33.47233 .658 & 33.90934 .313 & 28.763 & 25.945 & 30.935 & 29.572 & 30.685 & 31.34031 .534 & 32.022 \\
\hline /9/2006 & 0:00 & 33.49133 .679 & 33.93334 .334 & 28.809 & 25.975 & 30.990 & 29.614 & 30.712 & 31.36331 .556 & 32.022 \\
\hline /9/2006 & 4:00 & 33.46833 .656 & 33.91134 .313 & 28.778 & 25.969 & 31.025 & 29.594 & 30.687 & 31.34031 .532 & 32.009 \\
\hline /9/2006 & $8: 00$ & 33.47433 .709 & 33.94934 .328 & 28.786 & 25.973 & 30.982 & 29.605 & 30.675 & 31.36531 .553 & .978 \\
\hline /9/2006 & $12: 00$ & 33.49533 .744 & 33.97934 .354 & 28.790 & 25.975 & 31.006 & 29.625 & 30.672 & 31.39631 .587 & 1.975 \\
\hline /9/2006 & $16: 00$ & 33.45033 .656 & 33.90734 .299 & 28.740 & 25.945 & 30.946 & 29.691 & 30.634 & 31.33531 .532 & 31.949 \\
\hline /9/2006 & 20:00 & 33.42333 .620 & 33.87434 .269 & 28.730 & 25.930 & 30.908 & 29.691 & 30.612 & 0131.492 & 918 \\
\hline 9/10/2006 & 0:00 & 33.42933 .622 & 33.88034 .277 & 28.759 & 25.936 & 30.904 & 29.667 & 30.624 & 31.30631 .496 & 1.922 \\
\hline /10/2006 & 4:00 & 33.40933 .603 & 33.85734 .256 & 28.743 & 25.911 & 30.871 & 29.636 & 30.612 & 31.28431 .474 & 31.918 \\
\hline $9 / 10 / 2006$ & 8:00 & 33.40733 .597 & 33.85034 .250 & 28.753 & 25.900 & 30.857 & 29.608 & 30.6 & 31.27631 .465 & .904 \\
\hline /2006 & $12: 00$ & 33.43933 .607 & 33.85734 .264 & 28.765 & 892 & 348 & 29.596 & 30 & 33 & 906 \\
\hline 9/10/2006 & $16: 00$ & 33.39433 .599 & 33.84434 .238 & 28.745 & 25.863 & 30.831 & 29.488 & 30.599 & 31.27131 .461 & 31.902 \\
\hline 9/10/2006 & $20: 00$ & 33.42733 .593 & 33.83834 .242 & 28.757 & 25.855 & 30.817 & 29.493 & 30.667 & 31.27231 .460 & 31.895 \\
\hline 006 & 0:00 & 33.44833 .635 & 33.87434 .279 & 28.799 & 25.870 & 42 & 28 & 30. & 33 & 911 \\
\hline $9 / 11 / 2006$ & 4:00 & 33.44633 .631 & 33.87634 .281 & 28.813 & 25.869 & 342 & 29.537 & 30. & 501 & 920 \\
\hline 9/11/2006 & 8:00 & 33.46633 .639 & 33.88234 .295 & 28.826 & 25.869 & 30.846 & 29.546 & 30.657 & 31.31831 .509 & 31.915 \\
\hline 9/11/2006 & $12: 00$ & 33.47033 .652 & 33.89934 .305 & 28.859 & 25.881 & 30.866 & 29.574 & 30.672 & 31.33031 & 922 \\
\hline 9/11/2006 & $16: 00$ & 33.45633 .641 & 33.89034 .293 & 28.853 & 25.870 & 55 & 29.566 & 30.670 & 203 & 40 \\
\hline 9/11/2006 & 20:00 & 33.46633 .654 & 33.89934 .301 & 28.867 & 25.874 & 366 & 29.577 & 30.667 & 30 & 31.929 \\
\hline 9/12/2006 & 0:00 & 33.48333 .671 & 33.92034 .322 & 28.897 & 25.892 & 30.886 & 29.605 & 30.690 & 503 & 31.937 \\
\hline 9/12/2006 & 4:00 & 33.47633 .660 & 33.91134 .313 & 28.899 & 25.888 & 30.884 & 29.603 & 30.699 & 31.34031 .529 & 31.944 \\
\hline 9/12/2006 & 8:00 & 33.49133 .671 & 33.92234 .324 & 28.918 & 25.897 & 30.893 & 29.612 & 30.707 & 31.35031 .536 & 944 \\
\hline 9/12/2006 & $12: 00$ & 33.49333 .679 & 33.93034 .330 & 28.933 & 25.908 & 08 & 29.632 & 30.717 & 31.35831 & 31.949 \\
\hline 9/12/2006 & $16: 00$ & 33.47933 .652 & 33.90134 .297 & 28.887 & 25.874 & 30.890 & 29.605 & 30.682 & 3131.522 & 942 \\
\hline 9/12/2006 & $20: 00$ & 33.43933 .629 & 33.88034 .275 & 28.868 & 25.860 & 59 & 29.57 & 30. & 93 & 931 \\
\hline 9/13/2006 & $0: 00$ & 33.43933 .633 & 33.88234 .279 & 28.874 & 25.862 & 64 & 29.574 & 30.672 & 1431 & 31.929 \\
\hline 9/13/2006 & 4:00 & 33.41533 .607 & 33.85734 .254 & 28.861 & 25.851 & 30.846 & 29.557 & 30.654 & 31.28631 .476 & 31.924 \\
\hline 9/13/2006 & 8:00 & 33.44233 .618 & 33.86634 .268 & 28.872 & 25.858 & 30.849 & 29.566 & 30.662 & 31.30131 .487 & 31.918 \\
\hline 9/13/2006 & $12: 00$ & 33.40233 .593 & 33.84634 .238 & 28.847 & 25.844 & 30.837 & 29.544 & 30.641 & 31.27131 .461 & 31.906 \\
\hline 9/13/2006 & $16: 00$ & 33.37233 .559 & 33.80434 .193 & 28.788 & 25.807 & 30.804 & 29.502 & 30.596 & 31.24031 .438 & 31.889 \\
\hline 9/13/2006 & 20:00 & 33.35533 .540 & 33.78634 .179 & 28.773 & 25.798 & 30.789 & 29.48 & 30.571 & 31.21831 .408 & 31.873 \\
\hline $9 / 14 / 2006$ & $0: 00$ & 33.35533 .548 & 33.79234 .187 & 28.790 & 25.811 & 30.795 & 29.495 & 30.578 & 31.22531 .414 & 31.869 \\
\hline 9/14/2006 & 4:00 & 33.34333 .538 & 33.78734 .179 & 28.790 & 25.812 & 30.795 & 29.495 & 30.568 & 31.21831 .405 & 31.862 \\
\hline 9/14/2006 & 8:00 & 33.35333 .546 & 33.79634 .189 & 28.807 & 25.825 & 30.804 & 29.508 & 30.571 & 31.22531 .407 & 31.858 \\
\hline
\end{tabular}


Depth to Water (ft TOC) in Well Indicated

$\begin{array}{llllllllllllll}\text { Date } & \text { Time } & \text { MW-1 } & \text { MW-2 } & \text { MW-3 } & \text { MW-4 } & \text { KDHE-1 } & \text { KDHE-2 } & \text { NW-1 } & \text { NW-2 } & \text { NW-3 } & \text { L-1 } & \text { L-2 } & \text { L-3 }\end{array}$

\begin{tabular}{|c|c|c|c|c|c|c|c|c|c|c|}
\hline $9 / 14 / 2006$ & $12: 00$ & 33.37233 .553 & 0734.213 & 28.809 & 25.832 & 30.820 & 29.522 & 30.566 & 3231.414 & .853 \\
\hline 9/14/2006 & $16: 00$ & 33.32533 .521 & 33.77134 .162 & 28.768 & 25.805 & 30.782 & 29.481 & 30.548 & 31.20031 .387 & 31.842 \\
\hline$/ 14 / 2006$ & $20: 00$ & 33.40733 .781 & 33.96634 .268 & 28.770 & 25.809 & 30.901 & 29.526 & 30.543 & 31.34331 .520 & 31.833 \\
\hline$/ 15 / 2006$ & $0: 00$ & 33.51333 .960 & 34.13434 .403 & 28.791 & 25.828 & 31.014 & 29.605 & 30.548 & 31.50431 .684 & 31.836 \\
\hline $9 / 15 / 2006$ & $4: 00$ & 33.57134 .047 & 34.21934 .473 & 28.790 & 25.837 & 31.070 & 29.645 & 30.543 & 31.58531 .768 & .836 \\
\hline /15/2006 & 8:00 & 33.52633 .761 & 34.00234 .375 & 28.809 & 25.853 & 30.935 & 29.609 & 30.563 & 31.42831 .620 & 31.844 \\
\hline$/ 15 / 2006$ & $12: 00$ & 33.46033 .679 & 33.92734 .309 & 28.791 & 25.848 & 30.899 & 29.581 & 30.551 & 31.35731 .543 & 31.844 \\
\hline $9 / 15 / 2006$ & $16: 00$ & 33.39233 .607 & 33.85934 .238 & 28.740 & 25.814 & 30.873 & 29.53 & 30.535 & 31.28431 .469 & .827 \\
\hline $9 / 15 / 2006$ & $20: 00$ & 33.37033 .567 & 33.81734 .205 & 28.713 & 25.805 & 30.813 & 29.488 & 30.518 & 31.24931 .438 & .825 \\
\hline $9 / 16 / 2006$ & $0: 00$ & 33.36333 .569 & 33.81934 .207 & 28.745 & 25.819 & 30.831 & 29.515 & 30.518 & 31.24431 .430 & 31.818 \\
\hline /16/2006 & $4: 00$ & 33.35533 .557 & 33.81334 .199 & 28.755 & 25.832 & 30.840 & 29.523 & 18 & 31.235 & 311 \\
\hline 006 & $8: 00$ & 933.533 & 33.78834 .176 & 28.742 & 25.823 & 13 & 29. & & 31.213 & \\
\hline 9/16/2006 & $12: 00$ & 33.31633 .517 & 33.77534 .158 & 28.728 & 25.816 & 30.804 & 29.501 & 30.493 & 31.19831 & 791 \\
\hline 2006 & $16: 00$ & 33.36333 .780 & 33.97034 .248 & 28.673 & 25.786 & 30.921 & 29.513 & 30.445 & 31.33831 .514 & 31.774 \\
\hline 006 & $20: 00$ & 633.946 & 34.11634 .371 & 28.692 & 25.796 & 014 & 29. & 52 & 4803 & 67 \\
\hline 2006 & $0: 00$ & 33.58534 .044 & 34.21634 .473 & 28.738 & 25.833 & 096 & 29.638 & 30.475 & 31.5713 & 776 \\
\hline 9/17/2006 & 4:00 & 33.64534 .135 & 34.31334 .561 & 28.788 & 25.886 & 31.163 & 29.735 & 30.498 & 31.67231 .846 & 31.758 \\
\hline $9 / 17 / 2006$ & $8: 00$ & 33.76234 .257 & 34.43734 .683 & 28.895 & 25.960 & 31.269 & 29.81 & 30.576 & 31.78931 .965 & 31.805 \\
\hline 006 & $12: 00$ & 33.74534 .034 & 34.28034 .618 & 28.889 & 25.966 & 72 & 29.797 & 88 & 31.686 & 340 \\
\hline 006 & $16: 00$ & 33.63033 .871 & 34.12634 .499 & 28.866 & 25.948 & 31.065 & 29.755 & 30.591 & 31.546 & 31.847 \\
\hline 9/17/2006 & $20: 00$ & 33.58333 .795 & 34.05334 .442 & 28.872 & 25.945 & 31.019 & 29.709 & 30.591 & 31.47531 .660 & 31.856 \\
\hline $9 / 18 / 2006$ & $0: 00$ & 33.56133 .761 & 34.02334 .416 & 28.895 & 25.952 & 31.001 & 29.709 & 30.614 & 31.44531 .627 & 31.867 \\
\hline $9 / 18 / 2006$ & $4: 00$ & 33.53433 .728 & 33.99034 .387 & 28.905 & 25.950 & 30.981 & 29.7 & 30.614 & 31.41231 .593 & 867 \\
\hline 9/18/2006 & 8:00 & 33.54633 .734 & 33.99834 .397 & 28.949 & 25.973 & 92 & 29.731 & 30.649 & 98 & 902 \\
\hline 9/18/2006 & $12: 00$ & 33.53433 .723 & 33.98534 .385 & 28.960 & 25.973 & 30.988 & 29.726 & 30.659 & 31.40631 .585 & 898 \\
\hline 006 & $16: 00$ & 33.51733 .687 & 33.95334 .348 & 28.930 & 25.946 & 30.961 & 29.695 & 30.644 & 31.37231 & 887 \\
\hline 9/18/2006 & $20: 00$ & 33.52433 .715 & 33.97534 .371 & 28.964 & 25.964 & 79 & 29.72 & 30.664 & 31.39731 & 893 \\
\hline 9/19/2006 & $0: 00$ & 33.54833 .738 & 33.99834 .395 & 29.004 & 25.987 & 31.001 & 29.753 & 30.692 & 31.41931 .594 & 31.909 \\
\hline $9 / 19 / 2006$ & $4: 00$ & 33.56933 .749 & 34.00234 .399 & 29.010 & 25.987 & 31.001 & 29.755 & 30.699 & 31.42631 .604 & 31.918 \\
\hline 9/19/2006 & 8:00 & 33.58933 .770 & 34.03034 .430 & 29.054 & 26.008 & 31.030 & 29.79 & 30.725 & 31.45031 .625 & 31.937 \\
\hline $9 / 19 / 2006$ & $12: 00$ & 33.60233 .780 & 34.04234 .440 & 29.064 & 26.015 & 31.039 & 29.801 & 30.750 & 31.46531 .644 & 35.840 \\
\hline 9/19/2006 & $16: 00$ & 33.57333 .734 & 33.99234 .395 & 29.006 & 25.973 & 30.990 & 29.735 & 30.730 & 31.43131 .616 & 37.476 \\
\hline 06 & $20: 00$ & 33.67833 .749 & 33.99834 .407 & 28.989 & 25.959 & 30.981 & 29.724 & 30.755 & 31.45331 & 38.404 \\
\hline $9 / 20 / 2006$ & $0: 00$ & 33.60633 .753 & 34.00434 .418 & 28.987 & 25.957 & 30.975 & 29.72 & 30.793 & 31.47131 .671 & 39.086 \\
\hline
\end{tabular}


Depth to Water (ft TOC) in Well Indicated

$\begin{array}{llllllllllllll}\text { Date } & \text { Time } & \text { MW-1 } & \text { MW-2 } & \text { MW-3 } & \text { MW-4 } & \text { KDHE-1 } & \text { KDHE-2 } & \text { NW-1 } & \text { NW-2 } & \text { NW-3 } & \text { L-1 } & \text { L-2 } & \text { L-3 }\end{array}$

\begin{tabular}{|c|c|c|c|c|c|c|c|c|c|c|}
\hline 9/20/2006 & $4: 00$ & 33.61633 .755 & 34.00234 .420 & 28.974 & 25.946 & 30.963 & 29.709 & 30.818 & 31.48031 .686 & 39.544 \\
\hline $9 / 20 / 2006$ & $8: 00$ & 33.64933 .770 & 34.01734 .442 & 28.966 & 25.941 & 30.959 & 29.7 & 30.838 & 31.51031 .720 & 39.901 \\
\hline 9/20/2006 & $12: 00$ & 33.64133 .740 & 33.98734 .412 & 28.918 & 25.906 & 30.939 & 29.658 & 30.835 & 31.48531 .700 & .497 \\
\hline$/ 20 / 2006$ & $16: 00$ & 33.54633 .690 & 33.93134 .346 & 28.826 & 25.849 & 30.868 & 29.576 & 30.793 & 31.42431 .638 & .841 \\
\hline /20/2006 & $20: 00$ & 33.55633 .684 & 33.92634 .338 & 28.820 & 25.837 & 30.857 & 29.567 & 30.782 & 31.40231 .611 & .990 \\
\hline $9 / 21 / 2006$ & $0: 00$ & 33.51833 .645 & 33.89534 .309 & 28.795 & 25.821 & 30.835 & 29.545 & 30.760 & 31.36831 .573 & 3.457 \\
\hline 9/21/2006 & $4: 00$ & 33.44633 .601 & 33.85534 .268 & 28.765 & 25.796 & 30.804 & 29.512 & 30.709 & 31.31931 .520 & 33.080 \\
\hline $9 / 21 / 2006$ & $8: 00$ & 33.40733 .567 & 33.81934 .228 & 28.732 & 25.777 & 30.760 & 29.481 & 30.664 & 31.27631 .474 & 32.792 \\
\hline $9 / 21 / 2006$ & $12: 00$ & 33.35933 .523 & 33.77534 .177 & 28.682 & 25.738 & 30.753 & 29.44 & 30.614 & 3231.429 & 32.635 \\
\hline $9 / 21 / 2006$ & $16: 00$ & 33.27333 .426 & 33.67434 .082 & 28.565 & 25.671 & 30.645 & 29.329 & 30.505 & 31.13531 .336 & 32.380 \\
\hline /21/2006 & $20: 00$ & 33.24433 .424 & 33.67234 .070 & 28.575 & 25.664 & 30.658 & 29.336 & 30.479 & 31.11531 .306 & 32.234 \\
\hline $9 / 22 / 2006$ & $0: 00$ & 33.28833 .470 & 33.72034 .117 & 28.646 & 25.708 & 30.707 & 29.402 & 30. & 573 & 148 \\
\hline $9 / 22 / 2006$ & $4: 00$ & 33.31033 .489 & 33.74434 .140 & 28.690 & 25.740 & 30.735 & 29.446 & 30.507 & 783 & 32.073 \\
\hline 9/22/2006 & 8:00 & 33.35733 .525 & 33.77934 .176 & 28.740 & 25.775 & 30.775 & 29.49 & 30.538 & 31.20631 .390 & 32.019 \\
\hline $9 / 22 / 2006$ & $12: 00$ & 33.35733 .544 & 33.80034 .193 & 28.770 & 25.798 & 30.804 & 29.526 & 30.553 & 2831.408 & 31.988 \\
\hline 9/22/2006 & $16: 00$ & 33.35533 .542 & 33.79834 .189 & 28.770 & 25.805 & 304 & 29.528 & 30. & 233 & 953 \\
\hline $9 / 22 / 2006$ & $20: 00$ & 33.46833 .681 & 33.90534 .287 & 28.813 & 25.833 & 30.857 & 29.576 & 30.575 & 403 & 31.931 \\
\hline 9/23/2006 & $0: 00$ & 33.46433 .664 & 33.90834 .297 & 28.799 & 25.865 & 30.828 & 29.572 & 30.608 & 511 & 31.929 \\
\hline $9 / 23 / 2006$ & $4: 00$ & 33.46433 .658 & 33.91034 .301 & 28.885 & 25.888 & 30.908 & 29.649 & 30. & 363 & 915 \\
\hline 9/23/2006 & 8:00 & 33.45433 .645 & 33.90334 .291 & 28.884 & 25.895 & 13 & 29.649 & 11 & 00 & 900 \\
\hline 2006 & $12: 00$ & 33.48133 .675 & 33.93734 .322 & 28.926 & 25.927 & 30.948 & 29.691 & 30.646 & 533 & 31.906 \\
\hline 9/23/2006 & $16: 00$ & 33.48933 .687 & 33.95034 .334 & 28.947 & 25.943 & 30.968 & 29.713 & 30.661 & 31.36331 & 31.909 \\
\hline 9/23/2006 & $20: 00$ & 33.53633 .747 & 34.00634 .383 & 28.999 & 25.983 & 31.025 & 29.768 & 30.694 & 31.41231 .582 & 31.911 \\
\hline 9/24/2006 & $0: 00$ & 33.56533 .764 & 34.03234 .416 & 29.054 & 26.024 & 31.056 & 29.817 & 30.734 & 31.43931 .613 & 31.933 \\
\hline 9/24/2006 & $4: 00$ & 33.58333 .782 & 34.04934 .434 & 29.079 & 26.049 & 31.081 & 29.845 & 30.754 & 31.45531 .629 & 31.944 \\
\hline 9/24/2006 & $8: 00$ & 33.61233 .818 & 34.08934 .471 & 29.127 & 26.086 & 31.121 & 29.891 & 30.790 & 31.48731 .658 & 31.955 \\
\hline 9/24/2006 & $12: 00$ & 33.65333 .848 & 34.12034 .501 & 29.154 & 26.108 & 31.152 & 29.922 & 30.818 & 31.52131 .695 & 31.977 \\
\hline $9 / 24 / 2006$ & $16: 00$ & 33.62033 .829 & 34.09734 .473 & 29.121 & 26.096 & 31.136 & 29.894 & 30.810 & 31.49931 .675 & 31.982 \\
\hline 9/24/2006 & $20: 00$ & 33.63533 .846 & 34.11634 .493 & 29.129 & 26.107 & 31.149 & 29.907 & 30.815 & 31.52031 .693 & 31.984 \\
\hline $9 / 25 / 2006$ & $0: 00$ & 33.62833 .842 & 34.11234 .489 & 29.135 & 26.116 & 31.156 & 29.916 & 30.823 & 31.51431 .690 & 31.991 \\
\hline $9 / 25 / 2006$ & 4:00 & 33.61433 .823 & 34.09934 .477 & 29.127 & 26.115 & 31.152 & 29.907 & 30.807 & 31.50231 .677 & 31.993 \\
\hline $9 / 25 / 2006$ & $8: 00$ & 33.61833 .827 & 34.10134 .481 & 29.139 & 26.122 & 31.158 & 29.92 & 30.812 & 31.50231 .677 & 31.988 \\
\hline 9/25/2006 & $12: 00$ & 33.60633 .818 & 34.09134 .471 & 29.127 & 26.119 & 31.158 & 29.914 & 30.812 & 31.49531 .670 & 32.004 \\
\hline $9 / 25 / 2006$ & $16: 00$ & 33.57133 .783 & 34.05534 .436 & 29.085 & 26.092 & 31.132 & 29.878 & 30.787 & 31.46131 .638 & 31.988 \\
\hline
\end{tabular}




\begin{tabular}{|c|c|c|c|c|c|c|c|c|c|c|}
\hline $9 / 25 / 2006$ & $20: 00$ & 33.59633 .816 & 34.12234 .491 & 29.096 & 26.101 & 31.149 & 29.889 & 30.800 & 31.49231 .660 & 31.982 \\
\hline 26/2006 & 0:00 & 33.60433 .818 & 34.09934 .479 & 29.108 & 26.114 & 31.156 & 29.907 & 30.807 & 31.49731 .669 & 31.988 \\
\hline $9 / 26 / 2006$ & $4: 00$ & 33.58933 .804 & 34.08634 .463 & 29.102 & 26.112 & 31.156 & 29.9 & 30.797 & 31.48231 .656 & .986 \\
\hline /26/2006 & 8:00 & 33.59833 .818 & 34.09334 .469 & 29.114 & 26.126 & 31.165 & 29.916 & 30.800 & 31.49231 .660 & .984 \\
\hline $26 / 2006$ & $12: 00$ & 33.57933 .799 & 34.07834 .454 & 29.098 & 26.117 & 31.160 & 29.905 & 30.780 & 31.47531 .649 & .984 \\
\hline $9 / 26 / 2006$ & $16: 00$ & 33.58933 .949 & 34.16834 .465 & 29.033 & 26.075 & 31.198 & 29.876 & 30.734 & 31.52031 .684 & .971 \\
\hline $9 / 26 / 2006$ & 20:00 & 33.70234 .152 & 34.35234 .604 & 29.018 & 26.070 & 31.309 & 29.929 & 30.714 & 31.69631 .864 & 31.955 \\
\hline$/ 27 / 2006$ & 0:00 & 33.75134 .241 & 34.43834 .681 & 29.010 & 26.075 & 31.362 & 29.966 & 30.712 & 31.78431 .954 & .953 \\
\hline /27/2006 & 4:00 & 33.67133 .939 & 34.20934 .561 & 29.025 & 26.089 & 31.231 & 29.922 & 30.712 & 31.60531 .782 & .955 \\
\hline //27/2006 & 8:00 & 33.68633 .930 & 34.20634 .569 & 29.085 & 26.138 & 31.231 & 29.966 & 30.754 & 59731.769 & 962 \\
\hline $9 / 27 / 2006$ & $12: 00$ & 33.72533 .939 & 34.21334 .587 & 29.118 & 26.165 & 31.264 & 29.991 & 30.777 & 31.61231 .784 & 31.975 \\
\hline /27/2006 & $16: 00$ & 33.66133 .896 & 34.17334 .540 & 29.093 & 26.154 & 31.242 & 29.966 & 30.757 & 31.56831 .742 & 31.977 \\
\hline 2006 & $20: 00$ & 33.71733 .922 & 34.20034 .565 & 29.139 & 26.186 & 31.273 & 30.008 & 30.795 & 883 & 984 \\
\hline $9 / 28 / 2006$ & $0: 00$ & 33.71933 .954 & 34.23634 .602 & 29.198 & 26.226 & 313 & 30.057 & 30.828 & 23 & .004 \\
\hline $9 / 28 / 2006$ & 4:00 & 33.72933 .962 & 34.24634 .614 & 29.221 & 26.248 & 31.331 & 30.079 & 30.853 & 31.63431 .799 & 32.024 \\
\hline /28/2006 & 8:00 & 33.77033 .983 & 34.26934 .636 & 29.254 & 26.272 & 31.355 & 30.11 & 30.876 & 31.65231 .817 & 32.033 \\
\hline 9/28/2006 & $12: 00$ & 33.74933 .973 & 34.26134 .626 & 29.252 & 26.280 & 351 & 30.108 & 30.876 & 31.64731 & 042 \\
\hline 9/28/2006 & $16: 00$ & 33.68233 .920 & 34.20634 .569 & 29.185 & 26.239 & 31.309 & 30.046 & 30.840 & 9331.766 & 32.035 \\
\hline $9 / 28 / 2006$ & $20: 00$ & 33.66533 .899 & 34.17934 .544 & 29.154 & 26.221 & 31.284 & 30.019 & 30.820 & 7131.742 & 32.024 \\
\hline 006 & 0:00 & 33.63033 .861 & 34.14334 .508 & 29.111 & 26.196 & 251 & 29.982 & 30.780 & 393 & 008 \\
\hline 9/29/2006 & $4: 00$ & 33.57733 .816 & 34.09534 .459 & 29.062 & 26.163 & 31.211 & 29.933 & 30.744 & 1923 & 991 \\
\hline 9/29/2006 & 8:00 & 33.56533 .791 & 34.07234 .438 & 29.037 & 26.145 & 31.185 & 29.907 & 30.714 & 6831.638 & 31.968 \\
\hline 9/29/2006 & $12: 00$ & 33.57733 .808 & 34.09034 .456 & 29.064 & 26.161 & 31.205 & 29.933 & 30.724 & 31.48031 .647 & 31.962 \\
\hline /2006 & $16: 00$ & 33.55633 .793 & 34.07634 .438 & 29.041 & 26.151 & 89 & 29.916 & 30.704 & 663 & 951 \\
\hline 9/29/2006 & $20: 00$ & 33.57333 .829 & 34.10834 .463 & 29.052 & 26.161 & 31.209 & 29.931 & 30.722 & 31.48331 .651 & 31.946 \\
\hline 9/30/2006 & 0:00 & 33.57333 .812 & 34.09934 .459 & 29.062 & 26.170 & 31.211 & 29.94 & 30.724 & 31.48231 .649 & 31.944 \\
\hline 9/30/2006 & 4:00 & 33.58133 .816 & 34.10134 .463 & 29.073 & 26.181 & 31.220 & 29.949 & 30.719 & 31.48831 .655 & 31.944 \\
\hline 9/30/2006 & 8:00 & 33.61033 .852 & 34.13534 .497 & 29.116 & 26.212 & 31.251 & 29.991 & 30.749 & 31.51731 .680 & 31.951 \\
\hline 9/30/2006 & $12: 00$ & 33.65733 .882 & 34.16934 .532 & 29.156 & 26.244 & 31.287 & 30.033 & 30.775 & 31.54831 .713 & 31.962 \\
\hline 9/30/2006 & $16: 00$ & 33.62633 .865 & 34.15234 .512 & 29.131 & 26.233 & 31.273 & 30.013 & 30.760 & 31.53431 .702 & 31.962 \\
\hline 9/30/2006 & $20: 00$ & 33.63433 .876 & 34.16034 .520 & 29.131 & 26.237 & 31.280 & 30.015 & 30.777 & 31.54431 .708 & 31.964 \\
\hline 10/1/2006 & 0:00 & 33.65333 .884 & 34.16934 .532 & 29.144 & 26.248 & 31.287 & 30.03 & 30.782 & 31.55331 .720 & 31.966 \\
\hline $10 / 1 / 2006$ & 4:00 & 33.62933 .869 & 34.15834 .518 & 29.141 & 26.248 & 31.284 & 30.026 & 30.777 & 31.54131 .707 & 31.968 \\
\hline 10/1/2006 & 8:00 & 33.65533 .870 & 34.15834 .522 & 29.142 & 26.249 & 31.284 & 30.028 & 30.774 & 31.54131 .707 & 31.971 \\
\hline
\end{tabular}


Depth to Water (ft TOC) in Well Indicated

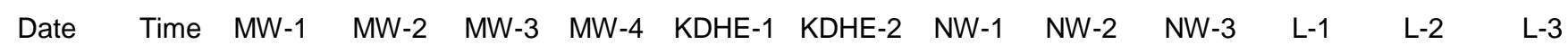

\begin{tabular}{|c|c|c|c|c|c|c|c|c|c|c|}
\hline 10/1/2006 & $12: 00$ & 33.63533 .873 & 34.16234 .522 & 29.140 & 26.255 & 31.289 & 30.03 & 30.772 & 31.54431 .710 & 31.977 \\
\hline $10 / 1 / 2006$ & $16: 00$ & 33.60833 .838 & 34.12834 .487 & 29.094 & 26.221 & 31.256 & 29.995 & 30.820 & 31.50731 .675 & 31.971 \\
\hline 10/1/2006 & $20: 00$ & 33.69433 .850 & 34.14134 .504 & 29.102 & 26.228 & 31.260 & 29.997 & 30.913 & 31.52431 .686 & .986 \\
\hline $10 / 2 / 2006$ & $0: 00$ & 33.63933 .875 & 34.16634 .526 & 29.131 & 26.253 & 31.286 & 30.028 & 31.024 & 31.54631 .708 & .008 \\
\hline 0/2/2006 & $4: 00$ & 33.63033 .867 & 34.15834 .518 & 29.123 & 26.251 & 31.282 & 30.026 & 31.092 & 31.54131 .702 & 32.030 \\
\hline $10 / 2 / 2006$ & $8: 00$ & 33.67833 .896 & 34.18534 .551 & 29.154 & 26.270 & 31.307 & 30.052 & 31.180 & 31.57131 .728 & 2.064 \\
\hline 10/2/2006 & $12: 00$ & 33.68233 .911 & 34.20434 .569 & 29.169 & 26.281 & 31.324 & 30.072 & 31.241 & 31.58531 .746 & 32.101 \\
\hline 10/2/2006 & $16: 00$ & 33.68433 .908 & 34.19634 .563 & 29.138 & 26.265 & 31.306 & 30.048 & 31.178 & 31.58331 .746 & 115 \\
\hline 10/2/2006 & $20: 00$ & 33.71533 .958 & 34.29734 .643 & 29.158 & 26.280 & 31.340 & 30.077 & 31.148 & 31.62731 .780 & 135 \\
\hline 10/3/2006 & $0: 00$ & 33.73533 .960 & 34.26134 .628 & 29.179 & 26.297 & 31.348 & 30.096 & 31.115 & 31.63731 .793 & 32.152 \\
\hline 10/3/2006 & $4: 00$ & 33.72333 .951 & 34.24834 .616 & 29.177 & 26.297 & 31.349 & 30.096 & 31.080 & 31.62931 .786 & 32.157 \\
\hline 10/3/2006 & 8:00 & 33.73933 .966 & 34.26534 .630 & 29.194 & 26.313 & 64 & 16 & 64 & 543 & 167 \\
\hline 10/3/2006 & $12: 00$ & 33.74533 .981 & 34.27434 .634 & 29.207 & 26.323 & 31.375 & 30.127 & 31.047 & 31.6513 & 174 \\
\hline 10/3/2006 & $16: 00$ & 33.73833 .975 & 34.26134 .620 & 29.171 & 26.304 & 31.357 & 30.105 & 30.999 & 31.64431 .803 & 32.163 \\
\hline $10 / 3 / 2006$ & $20: 00$ & 33.82233 .996 & 34.31234 .673 & 29.194 & 26.323 & 31.377 & 30.125 & 30.999 & 31.67331 .828 & 32.163 \\
\hline 006 & 0:00 & 33.80334 .038 & 34.33934 .700 & 29.259 & 26.366 & 428 & 87 & 31.034 & 1031 & 181 \\
\hline 2006 & $4: 00$ & 33.84234 .068 & 34.36834 .730 & 29.307 & 26.403 & 31.466 & 30.233 & 31.054 & 31.74031 & 197 \\
\hline 10/4/2006 & 8:00 & 33.90534 .116 & 34.41734 .781 & 29.367 & 26.445 & 31.512 & 30.29 & 31.090 & 31.78631 & 32.221 \\
\hline $10 / 4 / 2006$ & $12: 00$ & 33.92634 .162 & 34.46534 .826 & 29.428 & 26.489 & 31.561 & 30.348 & 31.132 & 31.8303 & 32.256 \\
\hline 10/4/2006 & $16: 00$ & 33.91834 .160 & 34.45534 .816 & 29.415 & 26.493 & 567 & 41 & 31.120 & 283 & 32.265 \\
\hline 10/4/2006 & $20: 00$ & 33.93834 .175 & 34.47634 .837 & 29.441 & 26.512 & 31.583 & 30.368 & 31.160 & 001 & 32.281 \\
\hline 10/5/2006 & $0: 00$ & 33.94234 .181 & 34.48234 .841 & 29.447 & 26.523 & 31.592 & 30.374 & 31.170 & 31.85532 .007 & 32.298 \\
\hline 10/5/2006 & $4: 00$ & 33.93834 .176 & 34.48034 .839 & 29.447 & 26.530 & 31.598 & 30.376 & 31.165 & 31.85032 .003 & 32.303 \\
\hline 10/5/2006 & $8: 00$ & 33.95334 .190 & 34.49334 .853 & 29.461 & 26.542 & 31.612 & 30.385 & 31.165 & 31.86532 .018 & 32.312 \\
\hline 10/5/2006 & $12: 00$ & 33.95734 .203 & 34.50334 .857 & 29.459 & 26.553 & 31.625 & 30.401 & 31.175 & 31.87032 .025 & 32.325 \\
\hline 10/5/2006 & $16: 00$ & 33.91834 .158 & 34.45734 .814 & 29.415 & 26.523 & 31.583 & 30.35 & 31.130 & 31.83031 .987 & 32.311 \\
\hline 10/5/2006 & $20: 00$ & 34.08434 .173 & 34.46534 .830 & 29.413 & 26.523 & 31.585 & 30.35 & 31.127 & 31.85032 .005 & 32.305 \\
\hline $10 / 6 / 2006$ & $0: 00$ & 33.93334 .175 & 34.47634 .833 & 29.434 & 26.538 & 31.603 & 30.37 & 31.130 & 31.85032 .003 & 32.311 \\
\hline 10/6/2006 & 4:00 & 33.93234 .179 & 34.48034 .835 & 29.440 & 26.547 & 31.609 & 30.376 & 31.132 & 31.84832 .001 & 32.310 \\
\hline $10 / 6 / 2006$ & $8: 00$ & 33.93734 .179 & 34.48434 .839 & 29.445 & 26.554 & 31.614 & 30.385 & 31.127 & 31.85532 .009 & 32.305 \\
\hline 10/6/2006 & $12: 00$ & 33.94534 .190 & 34.49534 .851 & 29.455 & 26.565 & 31.620 & 30.399 & 31.140 & 31.86532 .018 & 32.316 \\
\hline 10/6/2006 & $16: 00$ & 33.90034 .142 & 34.44234 .800 & 29.378 & 26.517 & 31.559 & 30.328 & 31.100 & 31.81831 .972 & 32.296 \\
\hline 10/6/2006 & $20: 00$ & 33.88934 .139 & 34.43534 .788 & 29.372 & 26.510 & 31.565 & 30.319 & 31.085 & 31.80631 .959 & 32.283 \\
\hline 10/7/2006 & $0: 00$ & 33.89834 .143 & 34.44634 .802 & 29.392 & 26.526 & 31.578 & 30.339 & 31.087 & 31.81931 .972 & 32.285 \\
\hline
\end{tabular}


Depth to Water (ft TOC) in Well Indicated

$\begin{array}{llllllllllllll}\text { Date } & \text { Time } & \text { MW-1 } & \text { MW-2 } & \text { MW-3 } & \text { MW-4 } & \text { KDHE-1 } & \text { KDHE-2 } & \text { NW-1 } & \text { NW-2 } & \text { NW-3 } & \text { L-1 } & \text { L-2 } & \text { L-3 }\end{array}$

\begin{tabular}{|c|c|c|c|c|c|c|c|c|c|c|}
\hline 10/7/2006 & 4:00 & 33.87734 .124 & 34.42734 .779 & 29.372 & 26.519 & 31.565 & 30.324 & 31.062 & 31.79731 .950 & 32.276 \\
\hline 0/7/2006 & 8:00 & 33.91034 .133 & 34.43634 .788 & 29.388 & 26.528 & 31.574 & 30.337 & 31.069 & 31.80431 .954 & 32.267 \\
\hline 10/7/2006 & $12: 00$ & 33.92234 .173 & 34.45534 .806 & 29.388 & 26.535 & 31.583 & 30.35 & 31.069 & 31.83531 .996 & 2.272 \\
\hline 10/7/2006 & $16: 00$ & 33.86534 .114 & 34.41334 .765 & 29.340 & 26.507 & 31.547 & 30.299 & 31.039 & 31.78731 .941 & 32.258 \\
\hline 10/7/2006 & 20:00 & 33.88534 .135 & 34.42734 .779 & 29.349 & 26.510 & 31.559 & 30.31 & 31.032 & 31.79931 .952 & 32.247 \\
\hline 10/8/2006 & $0: 00$ & 33.89434 .143 & 34.44034 .792 & 29.370 & 26.530 & 31.576 & 30.332 & 31.037 & 31.81131 .963 & 32.254 \\
\hline 10/8/2006 & $4: 00$ & 33.88334 .133 & 34.43434 .786 & 29.369 & .531 & 576 & 332 & 37 & 56 & 2.252 \\
\hline 10/8/2006 & 8:00 & 33.90834 .156 & 34.45934 .810 & 29.403 & 26.556 & 31.601 & 30.363 & 31.047 & 31.82531 .974 & 32.254 \\
\hline 10/8/2006 & $12: 00$ & 33.95734 .217 & 34.49034 .837 & 29.413 & 26.567 & 31.620 & 30.378 & 31.062 & 31.87032 .032 & 35.934 \\
\hline 10/8/2006 & $16: 00$ & 33.99434 .222 & 34.49734 .859 & 29.382 & 26.549 & 31.605 & 30.354 & 31.092 & 31.90232 & 42.148 \\
\hline 10/8/2006 & 20:00 & 34.16734 .272 & 34.54134 .922 & 29.401 & .567 & 627 & 376 & & 62 & 1.248 \\
\hline 10/9/2006 & 0:00 & 34.12134 .315 & 34.58934 .969 & 29.441 & 26.591 & 31.663 & 30.418 & 31.264 & 32.03232 .226 & 37.093 \\
\hline 10/9/2006 & $4: 00$ & 34.12334 .323 & 34.60434 .984 & 29.455 & 26.607 & 31.678 & 30.434 & 31.291 & 32.04132 .235 & 35.383 \\
\hline 2006 & 8:00 & 634.355 & 34.63835 & 29.482 & 26.627 & 02 & 46 & 22 & 47 & .486 \\
\hline 10/9/2006 & $12: 00$ & 34.15834 .367 & 34.65035 & 29.499 & 26.642 & 718 & 30.48 & 34 & 46 & 3.944 \\
\hline 10/9/2006 & $16: 00$ & 34.12534 .333 & 34.62735 .000 & 29.478 & 26.634 & 31.709 & 30.462 & 31 & 32.03432 .209 & 33.571 \\
\hline 10/9/2006 & $20: 00$ & 34.13234 .353 & 34.64835 .012 & 29.482 & 26.637 & 716 & 30.471 & 31 & 4432.213 & 33.303 \\
\hline $10 / 10 / 2006$ & $0: 00$ & 34.10734 .330 & 34.62934 .996 & 29.478 & 26.639 & 16 & 471 & 89 & 2732.196 & 3.111 \\
\hline 10/10/2006 & 4:00 & 34.08434 .310 & 34.61034 .976 & 29.468 & 26.634 & 707 & 30.462 & 31.279 & 69 & 32.960 \\
\hline 10/10/2006 & 8:00 & 34.05234 .283 & 34.58334 .947 & 29.441 & 26.618 & 31.687 & 36 & 38 & 7032.134 & 32.832 \\
\hline 10/10/2006 & $12: 00$ & 734.243 & 34.54134 & 29.388 & 88 & 56 & 92 & 91 & 92 & 32.723 \\
\hline $10 / 10 / 2006$ & $16: 00$ & 534.188 & 34.48634 .847 & 29.332 & 26.549 & 685 & 30.341 & 35 & 40 & 32.681 \\
\hline 10/10/2006 & $20: 00$ & 33.92834 .167 & 34.46534 .824 & 29.307 & 26.531 & 31.592 & 30.317 & 02 & 332.016 & 32.544 \\
\hline $10 / 11 / 2006$ & 0:00 & 33.91034 .159 & 34.45334 .808 & 29.298 & 26.524 & 31.583 & 30.31 & 31.079 & 3531.998 & 32.489 \\
\hline 10/11/2006 & 4:00 & 33.87334 .117 & 34.41534 .771 & 29.263 & 26.503 & 554 & 30.277 & 31 & 65 & 32.435 \\
\hline $10 / 11 / 2006$ & 8:00 & 33.89834 .114 & 34.41034 .769 & 29.263 & 26.498 & 31.547 & 30.273 & 31.021 & 31.79431 .954 & 32.384 \\
\hline 10/11/2006 & $12: 00$ & 33.90834 .156 & 34.45434 .806 & 29.321 & 26.535 & 31.590 & 30.321 & 31.052 & 31.82831 .983 & 32.358 \\
\hline $10 / 11 / 2006$ & $16: 00$ & 33.95134 .197 & 34.49434 .847 & 29.363 & 26.570 & 31.616 & 30.368 & 31.067 & 31.87032 .025 & 32.347 \\
\hline 10/11/2006 & $20: 00$ & 33.96334 .207 & 34.50534 .859 & 29.380 & 26.586 & 31.638 & 30.383 & 31.080 & 31.87732 .031 & 32.329 \\
\hline 10/12/2006 & $0: 00$ & 33.95934 .209 & 34.50934 .863 & 29.392 & 26.597 & 31.654 & 30.398 & 31.082 & 31.88432 .040 & 32.320 \\
\hline $10 / 12 / 2006$ & 4:00 & 33.99034 .239 & 34.54134 .892 & 29.434 & 26.625 & 31.685 & 30.436 & 31.109 & 31.91232 .062 & 32.316 \\
\hline 10/12/2006 & 8:00 & 34.01534 .266 & 34.56834 .920 & 29.470 & 26.653 & 31.711 & 30.471 & 31.127 & 31.93632 .085 & 32.316 \\
\hline $10 / 12 / 2006$ & $12: 00$ & 34.00234 .260 & 34.56234 .908 & 29.457 & 26.651 & 31.713 & 30.462 & 31.122 & 31.92632 .080 & 32.309 \\
\hline 10/12/2006 & $16: 00$ & 33.94734 .207 & 34.50934 .855 & 29.397 & 26.612 & 31.671 & 30.411 & 31.085 & 31.88032 .038 & 32.294 \\
\hline
\end{tabular}




\begin{tabular}{|c|c|c|c|c|c|c|c|c|c|c|}
\hline 0/12/2006 & $0: 00$ & 33.95134 .203 & 34.50734 .855 & 29.378 & 26.611 & 31.665 & 30.405 & 31.072 & 31.87732 .031 & 32.278 \\
\hline /13/2006 & $0: 00$ & 33.97434 .226 & 34.53234 .878 & 29.424 & 26.634 & 31.687 & 30.433 & 31.094 & 31.89932 .049 & 32.276 \\
\hline $10 / 13 / 2006$ & $4: 00$ & 33.99234 .241 & 34.54934 .898 & 29.451 & 26.657 & 31.707 & 30.46 & 31.107 & 31.91632 .067 & 2.278 \\
\hline 10/13/2006 & 8:00 & 34.00034 .251 & 34.55634 .906 & 29.464 & 26.667 & 31.722 & 30.473 & 31.107 & 31.92432 .074 & 32.278 \\
\hline 0/13/2006 & $12: 00$ & 34.02534 .287 & 34.59334 .934 & 29.497 & 26.688 & 31.744 & 30.507 & 31.135 & 31.95332 .100 & 2.285 \\
\hline 10/13/2006 & $16: 00$ & 33.98634 .241 & 34.54734 .895 & 29.447 & .658 & 31.709 & 30.46 & 31.109 & 432.067 & 274 \\
\hline 10/13/2006 & $20: 00$ & 33.99834 .256 & 34.56034 .906 & 29.464 & 26.671 & 31.722 & 30.473 & 31.115 & 31.92432 .074 & 32.272 \\
\hline $10 / 14 / 2006$ & 0:00 & 34.03934 .287 & 34.59334 .937 & 29.503 & 26.697 & 31.756 & 30.511 & 31.130 & 31.95332 .098 & 32.278 \\
\hline $10 / 14 / 2006$ & 4:00 & 34.03734 .294 & 34.60234 .949 & 29.518 & 26.711 & 31.767 & 30.528 & 31.147 & 31.96632 .116 & 2.285 \\
\hline $10 / 14 / 2006$ & 8:00 & 34.08434 .323 & 34.63134 .978 & 29.551 & .732 & 795 & 30.557 & 31.162 & 9732.144 & 2.296 \\
\hline $10 / 14 / 2006$ & $12: 00$ & 34.06034 .323 & 34.63434 .978 & 29.549 & 26.738 & 31.804 & 30.561 & 31.165 & 31.99532 .142 & 32.300 \\
\hline $10 / 14 / 2006$ & $16: 00$ & 34.02534 .281 & 34.59134 .937 & 29.491 & 26.701 & 31.758 & 30.506 & 31.140 & 31.95632 .107 & 32.289 \\
\hline 006 & 20:00 & 434.260 & 34.56634 .906 & 9.461 & 26.679 & 36 & 30.477 & 31.117 & 2932.080 & 32.276 \\
\hline $10 / 15 / 2006$ & $0: 00$ & 33.96334 .228 & 34.53534 .873 & 29.420 & 26.657 & 705 & 30.44 & 31.079 & 9932.049 & 32.263 \\
\hline $10 / 15 / 2006$ & $4: 00$ & 33.93034 .192 & 34.49734 .841 & 29.390 & 26.637 & 31.676 & 30.414 & 31.057 & 31.86532 .018 & 32.243 \\
\hline $10 / 15 / 2006$ & 8:00 & 33.93134 .194 & 34.49934 .841 & 29.399 & 26.637 & 31.680 & 30.418 & 31.044 & 86532.014 & 32.234 \\
\hline $10 / 15 / 2006$ & $12: 00$ & 33.93734 .201 & 34.50734 .849 & 29.401 & 26.641 & 85 & 30.427 & 31.034 & 37032.020 & 2.218 \\
\hline 10/15/2006 & $16: 00$ & 33.91234 .169 & 34.46934 .810 & 29.355 & 26.612 & 31.651 & 30.38 & 31.011 & 3331.985 & 32.201 \\
\hline 10/15/2006 & $20: 00$ & 33.88934 .154 & 34.45734 .799 & 29.341 & 26.600 & 31.638 & 30.372 & 30.986 & 31.82331 .974 & 32.183 \\
\hline /2006 & $0: 00$ & 134.152 & 34.45334 .794 & 29.338 & 26.602 & 634 & 63 & 81 & 831.969 & 32.174 \\
\hline 10/16/2006 & $4: 00$ & 33.86134 .127 & 34.43134 .773 & 29.318 & 26.588 & 31.618 & 30.389 & 30.961 & 31.79731 .950 & 32.165 \\
\hline 10/16/2006 & 8:00 & 33.84434 .106 & 34.41134 .751 & 29.295 & 26.570 & 31.601 & 30.389 & 30.940 & 31.77731 .930 & 32.147 \\
\hline 10/16/2006 & $12: 00$ & 33.82034 .087 & 34.38934 .728 & 29.267 & 26.554 & 31.576 & 30.405 & 30.915 & 31.75731 .910 & 32.134 \\
\hline 10/16/2006 & $16: 00$ & 33.77634 .041 & 34.34334 .683 & 29.213 & 26.521 & 31.541 & 30.42 & 30.875 & 031 & 32.105 \\
\hline 10/16/2006 & 20:00 & 33.86934 .074 & 34.36434 .712 & 29.225 & 26.519 & 31.545 & 30.385 & 30.877 & 31.74531 .897 & 32.094 \\
\hline 10/17/2006 & $0: 00$ & 33.81534 .085 & 34.37934 .720 & 29.253 & 26.535 & 31.565 & 30.323 & 30.892 & 31.74331 .894 & 32.094 \\
\hline 10/17/2006 & 4:00 & 33.84234 .108 & 34.40834 .749 & 29.299 & 26.565 & 31.594 & 30.286 & 30.913 & 31.77031 .916 & 32.094 \\
\hline 10/17/2006 & 8:00 & 33.86534 .125 & 34.42734 .769 & 29.315 & 26.581 & 31.616 & 30.323 & 30.923 & 31.78931 .938 & 32.099 \\
\hline 10/17/2006 & $12: 00$ & 33.91434 .181 & 34.48434 .824 & 29.378 & 26.625 & 31.671 & 30.409 & 30.966 & 31.84331 .985 & 32.123 \\
\hline 10/17/2006 & $16: 00$ & 33.90834 .178 & 34.48034 .818 & 29.370 & 26.627 & 31.667 & 30.405 & 30.971 & 31.83831 .985 & 32.123 \\
\hline 10/17/2006 & $20: 00$ & 33.95134 .218 & 34.51834 .859 & 29.416 & 26.660 & 31.700 & 30.444 & 30.998 & 31.87732 .021 & 32.136 \\
\hline $10 / 18 / 2006$ & $0: 00$ & 33.98434 .250 & 34.55634 .894 & 29.463 & 26.695 & 31.736 & 30.491 & 31.026 & 31.91132 .056 & 32.159 \\
\hline 10/18/2006 & 4:00 & 34.01734 .283 & 34.59134 .929 & 29.511 & 26.731 & 31.769 & 30.535 & 31.066 & 31.94332 .083 & 32.174 \\
\hline 10/18/2006 & 8:00 & 34.07034 .340 & 34.64834 .987 & 29.579 & 26.776 & 31.826 & 30.603 & 31.122 & 32.00032 .140 & 32.203 \\
\hline
\end{tabular}




\begin{tabular}{|c|c|c|c|c|c|c|c|c|c|c|}
\hline 10/18/2006 & $12: 00$ & 34.10334 .376 & 34.68635 .023 & 29.620 & 26.806 & 31.868 & 30.645 & 31.160 & 32.03632 .178 & 32.234 \\
\hline 10/18/2006 & $16: 00$ & 34.09934 .372 & 68435.021 & 29.612 & 26.813 & 31.871 & 30.641 & 31.167 & 32.03432 .176 & 32.247 \\
\hline 10/18/2006 & $20: 00$ & 34.13234 .410 & 34.71635 .051 & 29.651 & 26.838 & 31.902 & 30.671 & 31.208 & 32.06332 .204 & 2.269 \\
\hline 10/19/2006 & $0: 00$ & 34.13434 .410 & 34.72235 .057 & 29.651 & 26.847 & 31.908 & 30.678 & 31.223 & 32.07332 .215 & 2.287 \\
\hline 10/19/2006 & 4:00 & 34.11334 .384 & 34.69735 .033 & 29.627 & 26.840 & 31.893 & 30.66 & 31.213 & 32.05132 .195 & 32.289 \\
\hline 10/19/2006 & $8: 00$ & 34.10734 .376 & 34.69035 .025 & 29.620 & 26.836 & 31.888 & 30.649 & 31.208 & 32.04432 .185 & 32.291 \\
\hline 10/19/2006 & $12: 00$ & 34.11134 .385 & 34.69735 .029 & 29.616 & 26.836 & 31.891 & 30.652 & 31.208 & 32.04932 .195 & 32.303 \\
\hline 10/19/2006 & $16: 00$ & 34.07734 .334 & 34.64234 .970 & 29.539 & 26.791 & 31.835 & 30.583 & 31.150 & 31.99532 .142 & 32.283 \\
\hline 10/19/2006 & 20:00 & 34.05234 .327 & 34.63434 .969 & 29.535 & 26.780 & 31.833 & 30.574 & 31.142 & 31.99032 .133 & 32.274 \\
\hline 10/20/2006 & $0: 00$ & 34.05834 .340 & 34.64634 .976 & 29.549 & 26.792 & 31.842 & 30.59 & 31.150 & 31.99732 .144 & 32.278 \\
\hline$/ 2006$ & $4: 00$ & 34.03534 .308 & 34.61934 .953 & 29.522 & 26.778 & 31.822 & 30.568 & 31.125 & 31.97532 .118 & 32.267 \\
\hline 10/20/2006 & 8:00 & 34.02534 .300 & 34.61034 .943 & 29.514 & 26.771 & 813 & 30.557 & 31.119 & 1.96332 .109 & 32.258 \\
\hline 10/20/2006 & $12: 00$ & 34.01534 .281 & 34.59134 .923 & 29.483 & 26.754 & 31.795 & 30.537 & 31.094 & 31.94632 .094 & 32.256 \\
\hline $10 / 20 / 2006$ & $16: 00$ & 33.95334 .231 & 34.53934 .871 & 29.420 & 26.713 & 31.747 & 30.48 & 31.049 & 31.89732 .045 & 32.227 \\
\hline $10 / 20 / 2006$ & $20: 00$ & 434.252 & 34.56034 .888 & 29.443 & 26.718 & 756 & 91 & 051 & 31.91432 .058 & 32.221 \\
\hline $10 / 21 / 2006$ & $0: 00$ & 33.99234 .273 & 34.58134 .908 & 29.470 & 26.736 & 31.778 & 30.515 & 31.061 & 31.93132 .072 & 32.225 \\
\hline 10/21/2006 & $4: 00$ & 34.02134 .298 & 34.60634 .937 & 29.503 & 26.759 & 31.804 & 30.548 & 31.079 & 31.95832 .098 & 32.229 \\
\hline /2006 & 8:00 & 34.07434 .347 & 34.65934 .990 & 29.570 & 26.799 & 48 & 08 & 31.122 & 32.00232 .136 & 32.238 \\
\hline 10/21/2006 & $12: 00$ & 34.17734 .423 & 34.73535 .070 & 29.656 & 26.865 & 31.915 & 30.694 & 31.190 & 32.08032 .219 & 32.274 \\
\hline 10/21/2006 & $16: 00$ & 34.18534 .463 & 34.77535 .108 & 29.699 & 26.896 & 31.959 & 30.735 & 31.238 & 32.12032 .255 & 32.298 \\
\hline 10/21/2006 & $20: 00$ & 34.22634 .503 & 34.82135 .151 & 29.752 & 26.933 & 32.008 & 30.784 & 31.278 & 32.16232 .295 & 32.327 \\
\hline 2/2006 & 0:00 & 34.24934 .526 & 34.84435 .176 & 29.779 & 26.962 & 32.032 & 30.815 & 31.321 & 32.18832 .322 & 32.356 \\
\hline $10 / 22 / 2006$ & $4: 00$ & 34.26934 .545 & 34.86135 .190 & 29.796 & 26.979 & 32.050 & 30.832 & 31.341 & 32.20132 .335 & 32.376 \\
\hline 10/22/2006 & 8:00 & 34.27734 .549 & 34.86735 .200 & 29.808 & 26.992 & 32.061 & 30.843 & 31.357 & 32.21332 .344 & 32.387 \\
\hline $10 / 22 / 2006$ & $12: 00$ & 34.28434 .562 & 34.88435 .213 & 29.819 & 27.004 & 32.079 & 30.859 & 31.374 & 32.22532 .360 & 32.407 \\
\hline 10/22/2006 & $16: 00$ & 34.24734 .532 & 34.84835 .174 & 29.767 & 26.977 & 32.048 & 30.815 & 31.341 & 32.19332 .332 & 32.409 \\
\hline $10 / 22 / 2006$ & $20: 00$ & 34.29434 .560 & 34.86935 .198 & 29.777 & 26.984 & 32.059 & 30.826 & 31.352 & 32.21832 .351 & 32.413 \\
\hline 10/23/2006 & $0: 00$ & 34.26834 .545 & 34.86335 .194 & 29.783 & 26.990 & 32.059 & 30.828 & 31.362 & 32.21032 .348 & 32.427 \\
\hline 10/23/2006 & 4:00 & 34.28834 .545 & 34.86535 .196 & 29.790 & 27.000 & 32.063 & 30.835 & 31.362 & 32.20832 .344 & 32.433 \\
\hline 10/23/2006 & $8: 00$ & 34.29034 .553 & 34.87135 .207 & 29.796 & 27.007 & 32.068 & 30.841 & 31.369 & 32.22032 .357 & 32.433 \\
\hline 10/23/2006 & $12: 00$ & 34.28034 .558 & 34.87835 .207 & 29.798 & 27.011 & 32.079 & 30.848 & 31.367 & 32.22332 .359 & 32.444 \\
\hline $10 / 23 / 2006$ & $16: 00$ & 34.25734 .528 & 34.84235 .168 & 29.750 & 26.981 & 32.039 & 30.802 & 31.341 & 32.18632 .326 & 32.435 \\
\hline 10/23/2006 & $20: 00$ & 34.25334 .539 & 34.85235 .178 & 29.752 & 26.979 & 32.045 & 30.804 & 31.341 & 32.19632 .332 & 32.433 \\
\hline $10 / 24 / 2006$ & $0: 00$ & 34.24934 .532 & 34.85035 .178 & 29.758 & 26.986 & 32.048 & 30.808 & 31.336 & 32.19632 .333 & 32.440 \\
\hline
\end{tabular}


Depth to Water (ft TOC) in Well Indicated

$\begin{array}{lllllllllllllll}\text { Date } & \text { Time } & \text { MW-1 } & \text { MW-2 } & \text { MW-3 } & \text { MW-4 } & \text { KDHE-1 } & \text { KDHE-2 } & \text { NW-1 } & \text { NW-2 } & \text { NW-3 } & \text { L-1 } & \text { L-2 } & \text { L-3 }\end{array}$

\begin{tabular}{|c|c|c|c|c|c|c|c|c|c|c|}
\hline $10 / 24 / 2006$ & 4:00 & 34.23834 .523 & 34.83835 .166 & 29.750 & 26.984 & 32.043 & 30.802 & 31.329 & 32.18432 .320 & 32.435 \\
\hline 0/24/2006 & 8:00 & 34.23334 .511 & 34.82935 .157 & 29.733 & 26.976 & 32.032 & 30.788 & 31.321 & 32.17632 .311 & 32.429 \\
\hline 0/24/2006 & $12: 00$ & 34.20834 .488 & 34.81035 .133 & 29.710 & 26.962 & 32.019 & 30.766 & 31.304 & 32.15632 .295 & 2.420 \\
\hline $10 / 24 / 2006$ & $16: 00$ & 34.16134 .441 & 34.75635 .082 & 29.641 & 26.919 & 31.966 & 30.705 & 31.256 & 32.10832 .248 & 32.402 \\
\hline $10 / 24 / 2006$ & 20:00 & 34.19234 .481 & 34.77935 .096 & 29.639 & 26.910 & 31.970 & 30.702 & 31.248 & 32.12532 .260 & 32.391 \\
\hline $10 / 25 / 2006$ & $0: 00$ & 34.14834 .433 & 34.74535 .072 & 29.623 & 26.902 & 31.950 & 30.691 & 31.230 & 32.10132 .238 & 32.384 \\
\hline $10 / 25 / 2006$ & $4: 00$ & 34.13434 .414 & 34.72835 .057 & 29.612 & 893 & 1.937 & 76 & 215 & 7932.218 & 373 \\
\hline $10 / 25 / 2006$ & 8:00 & 34.13034 .406 & 34.71835 .047 & 29.598 & 26.886 & 31.922 & 30.665 & 31.203 & 32.07132 .209 & 32.360 \\
\hline $10 / 25 / 2006$ & $12: 00$ & 34.12634 .410 & 34.72035 .045 & 29.600 & 26.886 & 31.930 & 30.667 & 31.208 & 32.06832 .206 & 32.353 \\
\hline 2006 & $16: 00$ & 34.11234 .395 & 34.69535 .024 & 29.562 & 26.866 & 1.895 & 30.634 & 31.185 & 5632.202 & .342 \\
\hline $10 / 25 / 2006$ & 20:00 & 34.09934 .374 & 34.68435 .016 & 29.558 & 26.861 & 28 & 27 & 162 & 3432.253 & 2.358 \\
\hline 10/26/2006 & 0:00 & 34.10934 .393 & 34.70135 .027 & 29.576 & 26.872 & 31.895 & 30.638 & 31.160 & 32.05132 .185 & 32.272 \\
\hline 10/26/2006 & $4: 00$ & 34.10534 .382 & 34.69435 .021 & 29.577 & 26.865 & 31.877 & 30.63 & 31.157 & 32.04232 .177 & 32.278 \\
\hline 10/26/2006 & 8:00 & 34.1093 & 34.69735 .025 & 29.589 & 58 & 66 & 27 & 62 & 432.178 & 32.280 \\
\hline $10 / 26 / 2006$ & $12: 00$ & 34.17534 .399 & 34.71335 .043 & 29.610 & 26.859 & 875 & 30.647 & 31.177 & 132.195 & 32.287 \\
\hline 10/26/2006 & $16: 00$ & 34.10934 .387 & 34.69935 .027 & 29.589 & 26.835 & 31.855 & 30.619 & 31.167 & 32.04732 .180 & 32.287 \\
\hline $10 / 26 / 2006$ & $20: 00$ & 34.15334 .416 & 34.72635 .057 & 29.616 & 26.838 & 31.882 & 34 & 31.175 & 32.07632 .209 & 32.289 \\
\hline 10/27/2006 & $0: 00$ & 34.14634 .420 & 34.73035 .063 & 29.633 & 26.833 & 31.888 & 32 & 195 & 7932.228 & 32.342 \\
\hline 10/27/2006 & 4:00 & 34.13034 .395 & 34.69035 .031 & 29.643 & 26.829 & 31.893 & 30.612 & 31.185 & 5432.255 & 32.285 \\
\hline 10/27/2006 & 8:00 & 34.15934 .422 & 34.70135 .047 & 29.691 & 26.817 & 31.844 & 79 & 31.218 & 32.07632 .209 & 32.260 \\
\hline 10/27/2006 & $12: 00$ & 34.20634 .467 & 34.74335 .088 & 29 & .787 & 31.778 & 55 & 248 & 2032.253 & 32.289 \\
\hline 10/27/2006 & $16: 00$ & 34.17334 .422 & 34.70735 .059 & 29.627 & 26.704 & 31.678 & 30.588 & 31.246 & 32.08532 .222 & 32.300 \\
\hline 10/27/2006 & $20: 00$ & 34.16734 .429 & 34.71135 .059 & 29.606 & 26.621 & 31.614 & 30.493 & 31.263 & 32.08132 .215 & 32.311 \\
\hline 10/28/2006 & 0:00 & 34.20834 .465 & 34.75335 .100 & 29.618 & 26.554 & 31.596 & 30.4 & 31.289 & 32.12532 .261 & 32.329 \\
\hline 10/28/2006 & 4:00 & 34.19634 .435 & 34.72435 .086 & 29.614 & 26.484 & 31.545 & 321 & 304 & 0732.240 & 32.347 \\
\hline 10/28/2006 & 8:00 & 34.16934 .399 & 34.68835 .055 & 29.581 & 26.404 & 31.492 & 30.268 & 31.299 & 32.07632 .211 & 32.351 \\
\hline 10/28/2006 & $12: 00$ & 34.15034 .370 & 34.65735 .028 & 29.550 & 26.322 & 31.450 & 30.403 & 31.286 & 32.05232 .188 & 32.349 \\
\hline 10/28/2006 & $16: 00$ & 34.10534 .296 & 34.57934 .951 & 29.456 & 26.214 & 31.370 & 30.303 & 31.298 & 31.97732 .117 & 32.331 \\
\hline 10/28/2006 & $20: 00$ & 34.08034 .273 & 34.55634 .922 & 29.441 & 26.154 & 31.335 & 30.272 & 31.251 & 31.95132 .089 & 32.318 \\
\hline 10/29/2006 & $0: 00$ & 34.04134 .253 & 34.53534 .908 & 29.428 & 26.124 & 31.306 & 30.255 & 31.220 & 31.93432 .071 & 32.314 \\
\hline 10/29/2006 & 4:00 & 34.01534 .220 & 34.50534 .882 & 29.412 & 26.096 & 31.277 & 30.231 & 31.200 & 31.90732 .045 & 32.309 \\
\hline 10/29/2006 & 8:00 & 33.99534 .198 & 34.48234 .861 & 29.405 & 26.077 & 31.255 & 30.211 & 31.193 & 31.88432 .020 & 32.300 \\
\hline 10/29/2006 & $12: 00$ & 34.00534 .184 & 34.46934 .849 & 29.395 & 26.059 & 31.239 & 30.191 & 31.175 & 31.87332 .007 & 32.287 \\
\hline 10/29/2006 & $16: 00$ & 33.92734 .125 & 34.40434 .783 & 29.328 & 26.015 & 31.180 & 30.12 & 31.127 & 31.81131 .949 & 32.265 \\
\hline
\end{tabular}




\begin{tabular}{|c|c|c|c|c|c|c|c|c|c|c|}
\hline 0/29/2006 & $0: 00$ & 33.89634 .091 & 34.37134 .753 & 29.305 & 26.001 & 31.153 & 30.087 & 31.099 & 31.78231 .916 & 32.247 \\
\hline 0/30/2006 & $0: 00$ & 33.85334 .057 & 34.33534 .710 & 29.267 & 25.976 & 31.120 & 30.05 & 31.064 & 31.74331 .879 & 32.225 \\
\hline $10 / 30 / 2006$ & $4: 00$ & 33.79933 .996 & 34.27234 .651 & 29.207 & 25.936 & 31.069 & 29.986 & 31.008 & 31.68631 .821 & 2.194 \\
\hline 10/30/2006 & 8:00 & 33.77733 .975 & 34.25134 .630 & 29.197 & 5.927 & 31.051 & 29.964 & 30.988 & 31.66131 .797 & 32.172 \\
\hline 0/30/2006 & $12: 00$ & 33.77733 .979 & 34.25334 .628 & 29.207 & 25.927 & 31.054 & 29.97 & 30.980 & 31.66131 .796 & 2.156 \\
\hline 10/30/2006 & $16: 00$ & 33.80934 .010 & 34.28434 .659 & 29.253 & 5.958 & 31.073 & 30.001 & 30.990 & 8631.816 & .152 \\
\hline 10/30/2006 & $20: 00$ & 33.98434 .169 & 34.44634 .826 & 29.434 & 26.078 & 31.215 & 30.167 & 31.106 & 31.84331 .962 & 32.192 \\
\hline 10/31/2006 & 0:00 & 34.02134 .222 & 34.50734 .882 & 29.526 & 26.147 & 31.291 & 30.255 & 31.175 & 31.90032 .018 & 32.229 \\
\hline /2006 & 4:00 & 34.04534 .249 & 34.53334 .908 & 29.566 & 26.188 & 31.328 & 30.295 & 31.210 & 31.92632 .047 & 32.254 \\
\hline 10/31/2006 & 8:00 & 34.07534 .277 & 34.56034 .935 & 29.606 & 26.223 & 361 & 30.325 & 31.255 & 9533 & 2.285 \\
\hline 10/31/2006 & $12: 00$ & 34.08334 .293 & 34.57734 .949 & 29.624 & 26.246 & 31.388 & 30.343 & 31.270 & 31.96832 .089 & 32.307 \\
\hline 10/31/2006 & $16: 00$ & 34.03434 .233 & 34.52434 .898 & 29.564 & 26.216 & 31.344 & 30.286 & 31.240 & 31.91932 .045 & 32.300 \\
\hline 2006 & 20:00 & 34.03634 .241 & 34.52834 .902 & 29.570 & 26.226 & 59 & 30.292 & 31.256 & 243 & 32.309 \\
\hline 11/1/2006 & $0: 00$ & 34.05234 .249 & 34.53534 .908 & 29.580 & 26.241 & 373 & 30.299 & 31.260 & 9313 & 32.316 \\
\hline $11 / 1 / 2006$ & $4: 00$ & 34.07334 .264 & 34.54734 .922 & 29.599 & 26.260 & 31.390 & 30.319 & 31.273 & 31.94432 .067 & 32.320 \\
\hline $11 / 1 / 2006$ & 8:00 & 34.08934 .288 & 34.57334 .945 & 29.631 & 26.288 & 423 & 30.347 & 31.296 & 31.96832 & 32.338 \\
\hline $11 / 1 / 2006$ & $12: 00$ & 34.12134 .325 & 34.61334 .983 & 29.679 & 26.325 & 461 & 30.385 & 31.326 & 32.00332 & 2.358 \\
\hline $11 / 1 / 2006$ & $16: 00$ & 34.07934 .287 & 34.57534 .943 & 29.637 & 26.311 & 31.441 & 30.358 & 31.306 & 96632 & 32.356 \\
\hline $11 / 1 / 2006$ & $20: 00$ & 34.11534 .319 & 34.60434 .970 & 29.670 & 26.334 & 31.472 & 30.385 & 31.336 & 31.99832 & 32.367 \\
\hline $11 / 2 / 2006$ & $0: 00$ & 34.12634 .334 & 34.62334 .990 & 29.699 & 26.360 & 501 & 30.414 & 31 & 32.0143 & 32.382 \\
\hline $11 / 2 / 2006$ & $4: 00$ & 34.10934 .311 & 34.60434 .972 & 29.691 & 26.367 & 31.499 & 30.411 & 31.354 & 31.99532 .117 & 32.384 \\
\hline $11 / 2 / 2006$ & 8:00 & 34.11434 .317 & 34.61034 .978 & 29.702 & 26.382 & 14 & 30.42 & 31.356 & 32.00032 & 32.391 \\
\hline 11/2/2006 & $12: 00$ & 34.13834 .346 & 34.63435 .002 & 29.723 & 26.403 & 31.540 & 30.444 & 31.381 & 32.02532 .146 & 32.407 \\
\hline 11/2/2006 & $16: 00$ & 34.08534 .302 & 34.59134 .953 & 29.660 & 26.373 & 03 & 30.389 & 31 & 31.9773 & 32.393 \\
\hline 11/2/2006 & 20:00 & 34.08334 .293 & 34.58134 .945 & 29.662 & 26.369 & 31.505 & 30.409 & 31.336 & 31.97632 .098 & 32.388 \\
\hline 11/3/2006 & $0: 00$ & 34.10934 .298 & 34.58734 .949 & 29.652 & 26.376 & 31.510 & 30.387 & 31.328 & 31.97632 .098 & 32.391 \\
\hline 11/3/2006 & 4:00 & 34.07034 .285 & 34.57534 .936 & 29.637 & 26.374 & 31.505 & 30.38 & 31.321 & 31.96632 .086 & 32.377 \\
\hline $11 / 3 / 2006$ & 8:00 & 34.06234 .277 & 34.56834 .925 & 29.626 & 26.373 & 31.499 & 30.369 & 31.308 & 31.95632 .078 & 32.371 \\
\hline $11 / 3 / 2006$ & $12: 00$ & 34.03134 .249 & 34.53734 .896 & 29.581 & 26.353 & 31.472 & 30.336 & 31.273 & 31.93132 .056 & 32.362 \\
\hline $11 / 3 / 2006$ & $16: 00$ & 33.98634 .22 & 34.50334 .845 & 29.507 & 26.302 & 31.43 & 30.277 & 31.217 & 31.88732 .025 & 32.329 \\
\hline $11 / 3 / 2006$ & 20:00 & 33.98234 .193 & $34.48 \quad 34.831$ & 29.505 & 26.3 & 31.428 & 30.27 & 31.202 & 31.86532 & 32.315 \\
\hline $11 / 4 / 2006$ & $0: 00$ & 33.96734 .19 & 34.47434 .824 & 29.501 & 26.302 & 31.428 & 30.268 & 31.195 & $31.86 \quad 31.991$ & 32.309 \\
\hline $11 / 4 / 2006$ & 4:00 & 33.93734 .154 & 34.44234 .79 & 29.464 & 26.285 & 31.403 & 30.239 & 31.162 & 31.82631 .958 & 32.282 \\
\hline $11 / 4 / 2006$ & 8:00 & 33.94934 .169 & 34.45534 .804 & 29.487 & 26.302 & 31.421 & 30.259 & 31.157 & 31.83831 .967 & 32.272 \\
\hline
\end{tabular}


Depth to Water (ft TOC) in Well Indicated

$\begin{array}{llllllllllllll}\text { Date } & \text { Time } & \text { MW-1 } & \text { MW-2 } & \text { MW-3 } & \text { MW-4 } & \text { KDHE-1 } & \text { KDHE-2 } & \text { NW-1 } & \text { NW-2 } & \text { NW-3 } & \text { L-1 } & \text { L-2 } & \text { L-3 }\end{array}$

\begin{tabular}{|c|c|c|c|c|c|c|c|c|c|c|}
\hline $11 / 4 / 2006$ & $12: 00$ & 33.97234 .207 & 34.48234 .826 & 29.495 & 26.309 & 31.45 & 30.281 & 31.164 & 31.86531 .998 & 32.269 \\
\hline 1/4/2006 & $16: 00$ & 33.93734 .162 & 34.44634 .794 & 29.461 & 26.295 & 31.414 & 30.242 & 31.142 & 31.82831 .96 & .253 \\
\hline 11/4/2006 & $20: 00$ & 33.96734 .188 & 34.47434 .824 & 29.499 & 26.325 & 31.443 & 30.279 & 31.149 & 31.85531 .982 & 32.253 \\
\hline $11 / 5 / 2006$ & 0:00 & 33.97434 .195 & 34.48434 .833 & 29.518 & 26.345 & 31.463 & 30.299 & 31.162 & 31.86531 .993 & 32.26 \\
\hline 1/5/2006 & $4: 00$ & 33.96934 .192 & 34.47834 .828 & 29.512 & 26.348 & 31.463 & 30.299 & 31.152 & 31.85831 .985 & 32.251 \\
\hline 1/5/2006 & 8:00 & 33.97434 .201 & 34.48834 .833 & 29.52 & 26.357 & 31.476 & 30.306 & 31.154 & 31.86231 .987 & 32.245 \\
\hline 11/5/2006 & $12: 00$ & 33.99734 .22 & 34.50934 .855 & 29.532 & 26.369 & 31.492 & 30.325 & 31.177 & 013 & 32.251 \\
\hline $11 / 5 / 2006$ & $16: 00$ & 33.93334 .167 & $34.45 \quad 34.796$ & 29.464 & 26.33 & 31.445 & 30.261 & 31.144 & 31.82831 .956 & 32.233 \\
\hline 1/5/2006 & 20:00 & 33.95534 .169 & 34.45534 .804 & 29.472 & 26.336 & 31.452 & 30.27 & 31.139 & 31.83331 .96 & 32.225 \\
\hline $1 / 6 / 2006$ & 0:00 & 1534.175 & 34.808 & 29.482 & 26.348 & 31.463 & 30.283 & 31.132 & $31.84 \quad 31$ & 32.229 \\
\hline $11 / 6 / 2006$ & $4: 00$ & 33.93134 .155 & 1634.793 & 29.472 & 26.346 & 56 & 30.277 & 19 & 31 & 32.222 \\
\hline $11 / 6 / 2006$ & 8:00 & 33.9834 .167 & 34.45334 .81 & 29.476 & 26.353 & 31.461 & 30.281 & 31.122 & 31.84331 .969 & 32.214 \\
\hline $11 / 6 / 2006$ & $12: 00$ & 33.94534 .173 & 34.808 & 29.48 & 26.36 & 31.47 & 30.288 & 31 & 31 & 32.211 \\
\hline 006 & $16: 00$ & 434.144 & 134.777 & 29.438 & 26.334 & 43 & 25 & 91 & 34 & 32.198 \\
\hline $11 / 6 / 2006$ & 20:00 & 33.9934 .142 & 134.779 & 29.436 & 26.337 & 47 & 30.257 & 31 & 093 & 32.189 \\
\hline $11 / 7 / 2006$ & $0: 00$ & 33.93134 .157 & 1434.79 & 29.457 & 26.355 & 31.463 & 30.277 & 31.094 & 31.8263 & 32.196 \\
\hline $11 / 7 /$ & $4: 00$ & 33.89834 .127 & 734 & 29.432 & 26.341 & 443 & 53 & 74 & 43 & 32.185 \\
\hline $11 / 7 / 2006$ & $8: 00$ & 33.86734 .097 & 534.73 & 29.395 & 26.318 & 19 & 222 & 43 & 385 & 32.163 \\
\hline $11 / 7 / 2006$ & $12: 00$ & 33.84734 .087 & 34.36834 .712 & 29.372 & 26.307 & 31.405 & 30.206 & 31.028 & 31.74531 .876 & 32.149 \\
\hline $11 / 7 / 2006$ & $16: 00$ & 33.81334 .045 & 34.33334 .677 & 29.33 & 26.281 & 31.374 & 30.167 & 31 & 31.71331 .839 & 32.132 \\
\hline 006 & $20: 00$ & 33.82434 .045 & 34.32834 .673 & 29.34 & 26.277 & 92 & 184 & & 08 & 32.116 \\
\hline $11 / 8 / 2006$ & $0: 00$ & 33.834 .03 & 34.31634 .661 & 29.315 & 26.274 & 31.361 & 30.156 & 30.973 & 993 & 32.109 \\
\hline $11 / 8 / 2006$ & 4:00 & 33.76934 .003 & 34.28834 .632 & 29.284 & 26.256 & 31.337 & 30.127 & 30.937 & 31.66931 .797 & 32.094 \\
\hline $11 / 8 / 2006$ & 8:00 & 33.75633 .984 & $34.27 \quad 34.616$ & 29.263 & 26.246 & 31.322 & 30.109 & 30.912 & 31.64931 .777 & 32.072 \\
\hline $11 / 8 / 2006$ & $12: 00$ & 33.75633 .994 & 34.27634 .618 & 29.265 & 26.248 & 31.326 & 30.116 & 30 & 31.65231 .779 & 32.061 \\
\hline $11 / 8 / 2006$ & $16: 00$ & 33.72833 .973 & 34.25334 .589 & 29.221 & 26.219 & 31.302 & 30.076 & 30.887 & $31.63 \quad 31.757$ & 32.041 \\
\hline $11 / 8 / 2006$ & 20:00 & 33.74633 .982 & 34.26134 .6 & 29.25 & 26.237 & 31.311 & 30.098 & 30.897 & $31.64 \quad 31.763$ & 32.034 \\
\hline $11 / 9 / 2006$ & 0:00 & 33.76534 .012 & 34.28834 .628 & 29.276 & 26.26 & 31.337 & 30.129 & 30.907 & 31.66431 .785 & 32.036 \\
\hline $11 / 9 / 2006$ & $4: 00$ & 33.78334 .089 & 34.30734 .647 & 29.307 & 26.285 & 31.362 & 30.16 & 30.92 & 31.68131 .801 & 32.043 \\
\hline $11 / 9 / 2006$ & 8:00 & 33.80734 .047 & 34.33134 .673 & 29.34 & 26.311 & 31.388 & 30.191 & 30.932 & 31.70331 .821 & 32.047 \\
\hline $11 / 9 / 2006$ & $12: 00$ & 33.82634 .059 & $34.35 \quad 34.69$ & 29.353 & 26.323 & 31.401 & 30.209 & 30.947 & 31.72131 .841 & 32.05 \\
\hline $11 / 9 / 2006$ & $16: 00$ & 33.79534 .044 & 34.31834 .657 & 29.307 & 26.302 & 31.375 & 30.171 & 30.92 & 31.69631 .819 & 32.043 \\
\hline $11 / 9 / 2006$ & $20: 00$ & 33.83634 .048 & 34.34134 .687 & 29.328 & 26.315 & 31.393 & 30.189 & 30.925 & 31.72331 .845 & 32.047 \\
\hline $11 / 10 / 2006$ & 0:00 & 33.8334 .077 & 34.35234 .692 & 29.338 & 26.327 & 31.408 & 30.204 & 30.927 & 31.72531 .847 & 32.052 \\
\hline
\end{tabular}


Depth to Water (ft TOC) in Well Indicated

$\begin{array}{llllllllllllll}\text { Date } & \text { Time } & \text { MW-1 } & \text { MW-2 } & \text { MW-3 } & \text { MW-4 } & \text { KDHE-1 } & \text { KDHE-2 } & \text { NW-1 } & \text { NW-2 } & \text { NW-3 } & \text { L-1 } & \text { L-2 } & \text { L-3 }\end{array}$

\begin{tabular}{|c|c|c|c|c|c|c|c|c|c|c|}
\hline $11 / 10 / 2006$ & 4:00 & 33.88134 .127 & 34.40834 .747 & 29.411 & 26.371 & 31.459 & 30.272 & 30.983 & 31.77731 .89 & 32.07 \\
\hline 1/10/2006 & 8:00 & 33.93534 .174 & 34.45734 .797 & 29.474 & 26.417 & 31.512 & 30.334 & 31.021 & 31.82431 .936 & 32.087 \\
\hline $1 / 10 / 2006$ & $12: 00$ & 33.99234 .228 & 34.52234 .859 & 29.549 & 26.475 & 31.574 & 30.407 & 31.091 & $31.88 \quad 31.994$ & 2.118 \\
\hline $11 / 10 / 2006$ & $16: 00$ & 34.02934 .275 & $34.57 \quad 34.902$ & 29.597 & 26.515 & 31.627 & 30.462 & 31.134 & 31.92432 .034 & 32.143 \\
\hline $11 / 10 / 2006$ & 20:00 & 34.10934 .357 & 34.65234 .988 & 29.699 & 26.586 & 31.702 & 30.555 & 31.215 & 32.00532 .113 & 32.187 \\
\hline $11 / 11 / 2006$ & $0: 00$ & 34.1434 .384 & 34.68235 .017 & 29.729 & 26.618 & 31.736 & 30.59 & 31.248 & 3732 & 32.218 \\
\hline $11 / 11 / 2006$ & $4: 00$ & 34.1434 .384 & 34.68835 .023 & 29.737 & 0.632 & 31.749 & 30.601 & 31.273 & 157 & 245 \\
\hline $11 / 11 / 2006$ & 8:00 & 34.1434 .387 & 34.68635 .021 & 29.737 & 26.64 & 31.756 & 30.601 & 31.278 & 32.04232 .155 & 32.26 \\
\hline $11 / 11 / 2006$ & $12: 00$ & 34.13834 .383 & 34.68635 .022 & 29.725 & 26.642 & 31.753 & 30.599 & 31.283 & 32.04232 .158 & 32.269 \\
\hline $1 / 11 / 2006$ & $16: 00$ & 34.09934 .317 & 34.61334 .955 & 29.629 & 26.586 & 31.687 & 30.513 & 31.233 & 833 & 2.253 \\
\hline $11 / 11 / 2006$ & 20:00 & 34.04134 .28 & 34.57534 .914 & 29.591 & 26.558 & 654 & 30.471 & 97 & 062 & 2.242 \\
\hline $11 / 12 / 2006$ & 0:00 & 34.0434 .258 & 34.55434 .892 & 29.564 & 26.544 & 31.636 & 30.449 & 31.175 & 31.92232 .04 & 32.231 \\
\hline $11 / 12 / 2006$ & $4: 00$ & 33.9934 .231 & 34.53234 .869 & 29.535 & 26.528 & 31.614 & 30.422 & 31.152 & 31.8973 & 32.22 \\
\hline $11 / 12 / 2006$ & 8:00 & 33.96834 .214 & 34.51234 .849 & 29.511 & 26.512 & 96 & 03 & 29 & 773 & 32.207 \\
\hline $11 / 12 / 2006$ & $12: 00$ & 33.97434 & 34.50534 .842 & 29.491 & 26.501 & 85 & 94 & 09 & 723 & 32.191 \\
\hline $11 / 12 / 2006$ & $16: 00$ & 33.95834 .176 & 34.47634 .816 & 29.458 & 26.477 & 563 & 30.361 & 31.081 & 31.8463 & 32.174 \\
\hline $11 / 12 / 2006$ & $20: 00$ & 34.00234 .233 & 34.53334 .871 & 29.535 & 26.524 & 23 & 29 & 19 & 953 & 32.183 \\
\hline $11 / 13 / 2006$ & $0: 00$ & 34.04834 .26 & 34.55834 .896 & 29.562 & 26.553 & & 53 & 34 & 31 & 32.185 \\
\hline 11/13/2006 & 4:00 & 34.07234 .298 & 34.60234 .939 & 29.616 & 26.595 & 685 & 30.511 & 31.17 & 613 & 32.205 \\
\hline $11 / 13 / 2006$ & 8:00 & 34.08934 .313 & 34.61734 .957 & 29.637 & 26.611 & 31.707 & 30 & & 7632 & 32.216 \\
\hline 2006 & $12: 00$ & 534.301 & 34.60634 .945 & 29.62 & 11 & 98 & 22 & 82 & 683 & 32.218 \\
\hline $11 / 13 / 2006$ & $16: 00$ & 33.99734 .222 & 34.53234 .867 & 29.514 & 26.545 & 31 & 38 & 31 & 13 & 32.191 \\
\hline $11 / 13 / 2006$ & $20: 00$ & 33.97834 .22 & 34.52234 .853 & 29.501 & 26.533 & 31.629 & 30.425 & 96 & 8532.002 & 32.18 \\
\hline $11 / 14 / 2006$ & 0:00 & 33.98634 .212 & 34.51634 .851 & 29.504 & 26.535 & 31.625 & 30.422 & 31.096 & 31.87931 .994 & 32.174 \\
\hline $11 / 14 / 2006$ & 4:00 & 33.91734 .161 & 34.46534 .798 & 29.435 & 26.496 & 578 & 65 & 31 & 313 & 32.149 \\
\hline $11 / 14 / 2006$ & 8:00 & 33.91734 .134 & 34.43634 .775 & 29.401 & 26.473 & 31.545 & 30.33 & 31.013 & 31.80931 .927 & 32.127 \\
\hline $11 / 14 / 2006$ & $12: 00$ & 33.85734 .108 & 34.40434 .736 & 29.361 & 26.448 & 31.516 & 30.295 & 30.983 & $31.77 \quad 31.89$ & 32.109 \\
\hline $11 / 14 / 2006$ & $16: 00$ & 33.81634 .064 & 34.35834 .694 & 29.307 & 26.408 & & 30.242 & 30.932 & $31.73 \quad 31.849$ & 32.074 \\
\hline $11 / 14 / 2006$ & $20: 00$ & 33.84134 .097 & 34.39134 .718 & 29.349 & 26.429 & 31.503 & 30.277 & 30.95 & $31.75 \quad 31.865$ & 32.07 \\
\hline 11/15/2006 & 0:00 & 33.90434 .127 & 34.42734 .755 & 29.399 & 26.468 & 31.539 & 30.328 & 30.978 & 31.78131 .898 & 32.072 \\
\hline $11 / 15 / 2006$ & 4:00 & 33.89434 .142 & 34.44434 .775 & 29.432 & 26.489 & 31.563 & 30.358 & 30.985 & 31.79931 .912 & 32.072 \\
\hline $11 / 15 / 2006$ & 8:00 & 33.98834 .235 & 34.53934 .869 & 29.547 & 26.566 & 31.645 & 30.464 & 31.069 & 31.88931 .996 & 32.103 \\
\hline $11 / 15 / 2006$ & $12: 00$ & 34.03934 .286 & 34.59134 .922 & 29.593 & 26.618 & 31.678 & 30.519 & 31.106 & 31.94432 .053 & 32.132 \\
\hline 11/15/2006 & $16: 00$ & 34.23534 .347 & 34.63434 .99 & 29.6 & 26.627 & 31.711 & 30.53 & 31.122 & 32.02232 .133 & 32.143 \\
\hline
\end{tabular}




\begin{tabular}{|c|c|c|c|c|c|c|c|c|c|c|c|}
\hline /15/2006 & 20:00 & 34.10134 .357 & 34.65 & 34.978 & 29.637 & 26.648 & 31.744 & 30.566 & 31.164 & 32.111 & 32.165 \\
\hline 1/16/2006 & 0:00 & 34.1334 .342 & 34.646 & 34.982 & 29.644 & 26.658 & 31.749 & 30.574 & 31.175 & 32.00332 .115 & 32.178 \\
\hline $11 / 16 / 2006$ & $4: 00$ & 34.11234 .33 & 34.636 & 34.972 & 29.627 & 26.655 & 31.742 & 30.561 & 31.17 & 31.99532 .107 & 2.183 \\
\hline $11 / 16 / 2006$ & 8:00 & 34.08334 .322 & 34.633 & 34.965 & 29.627 & 26.656 & 31.747 & 30.561 & 31.17 & 31.98832 .1 & 32.187 \\
\hline 1/16/2006 & $12: 00$ & 34.06634 .319 & 34.629 & 34.957 & 29.625 & 26.658 & 31.749 & 30.566 & 31.164 & 31.97732. & 32.194 \\
\hline $11 / 16 / 2006$ & $16: 00$ & 33.99934 .262 & 34.57 & 34.892 & 29.545 & .611 & 31.698 & 30.495 & 31.117 & 2132.036 & 951 \\
\hline 11/16/2006 & 20:00 & 34.01934 .266 & 34.568 & 34.894 & 29.537 & 26.6 & 31.687 & 30.484 & 31.117 & 31.93132 .049 & 37.159 \\
\hline $11 / 17 / 2006$ & 0:00 & 34.03634 .271 & 34.568 & 34.902 & 29.529 & 26.598 & 31.68 & 30.482 & 31.132 & 31.94632 .073 & 38.722 \\
\hline 1/17/2006 & 4:00 & 34.07734 .3 & 34.594 & 34.933 & 29.545 & 26.607 & 31.694 & 30.493 & 31.167 & 31.98532 & 39.674 \\
\hline 11/17/2006 & 8:00 & 34.32934 .389 & 34.667 & 35.027 & 29.6 & 0.646 & 31.74 & 30.546 & 31.245 & 32.08532 & 354 \\
\hline 11/17/2006 & $12: 00$ & 34.23934 .439 & 34.728 & 35.082 & 29.656 & 26.686 & 31.789 & 30.605 & 31.336 & 32.13932 .288 & 40.868 \\
\hline $11 / 17 / 2006$ & $16: 00$ & 34.2834 .467 & 34.76 & 35.117 & 29.669 & 26.702 & 31.809 & 30.623 & 31.389 & 32.18432 .337 & 41.26 \\
\hline 11/17/2006 & 20:00 & 34.36634 .543 & 34.836 & 35.2 & 29.742 & 6.745 & 31.868 & 30.691 & 31.49 & 32.26732 & 86 \\
\hline $11 / 18 / 2006$ & $0: 00$ & 34.42134 .6 & 34.892 & 35.256 & 29.788 & 26.78 & 31.913 & 30.742 & 31.558 & 32.32632 & 203 \\
\hline $11 / 18 / 2006$ & $4: 00$ & 34.43834 .617 & 34.913 & 35.28 & 29.808 & 26.801 & 31.93 & 30.762 & 31.609 & 32.34332 .499 & 36.37 \\
\hline $11 / 18 / 2006$ & 8:00 & 34.44834 .634 & 34.939 & 35.301 & 29.834 & 6.824 & 31.961 & 30.791 & 31.634 & 5332 & 197 \\
\hline /2006 & $12: 00$ & 34.4634 .651 & 34.96 & 35.319 & 29.857 & 26.845 & 31.984 & 17 & 31.656 & 6532 & 01 \\
\hline $11 / 18 / 2006$ & $16: 00$ & 34.41734 .628 & 34.93 & 35.284 & 29.819 & 26.831 & 31.97 & 30.788 & 31.639 & 32.32632 & 34.029 \\
\hline $11 / 18 / 2006$ & $20: 00$ & 34.43834 .668 & 34.964 & 35.307 & 29.85 & 26.852 & 31.999 & 30.817 & 31.646 & 32.34532 & 33.702 \\
\hline /2006 & 0:00 & 34.44434 .67 & 34.977 & 35.323 & 29.882 & 26.878 & 32.023 & 30 & 51 & 32.35332 & 467 \\
\hline 11/19/2006 & $4: 00$ & 34.45434 .676 & 34.993 & 35.339 & 29.907 & 26.898 & 32.045 & 30.876 & 31.654 & $32.36 \quad 32.483$ & 33.283 \\
\hline $11 / 19 / 2006$ & 8:00 & 34.46334 .678 & 34.997 & 35.341 & 29.921 & 26.916 & 32.059 & 30.892 & 31.659 & 32.36332 .481 & 33.141 \\
\hline 11/19/2006 & $12: 00$ & 34.45834 .691 & 35.012 & 35.35 & 29.938 & 26.931 & 32.079 & 30.91 & 31.659 & $32.37 \quad 32.487$ & 33.042 \\
\hline $11 / 19 / 2006$ & $16: 00$ & 34.39734 .632 & 34.951 & 35.288 & 29.871 & 26.896 & 32.032 & 30.85 & 31.619 & 32.31332 & 32.944 \\
\hline 11/19/2006 & $20: 00$ & 34.41734 .648 & 34.968 & 35.308 & 29.896 & 26.914 & 32.048 & 30.872 & 31.629 & 32.32632 .443 & 32.873 \\
\hline $11 / 20 / 2006$ & $11 / 20 /$ & 34.45634 .687 & 35.006 & 35.343 & 29.946 & 26.949 & 32.09 & 30.921 & 31.649 & 32.35732 .472 & 32.836 \\
\hline $11 / 20 / 2006$ & 4:00 & 34.45434 .689 & 35.016 & 35.35 & 29.965 & 26.967 & 32.103 & 30.938 & 31.644 & 32.36532 .477 & 32.792 \\
\hline $11 / 20 / 2006$ & 8:00 & 34.51834 .687 & 35.004 & 35.352 & 29.94 & 26.961 & 32.088 & 30.921 & 31.629 & 32.37832 .492 & 32.752 \\
\hline $11 / 20 / 2006$ & $12: 00$ & 34.44434 .678 & 35.006 & 35.341 & 29.955 & 26.972 & 32.103 & 30.934 & 31.629 & 32.35832 .472 & 32.73 \\
\hline $11 / 20 / 2006$ & $16: 00$ & 34.3734 .615 & 34.941 & 35.268 & 29.869 & 26.924 & 32.048 & 30.859 & 31.571 & 32.29232 .406 & 32.683 \\
\hline $11 / 20 / 2006$ & $20: 00$ & 34.34334 .59 & 34.909 & 35.239 & 29.84 & 26.905 & 32.017 & 30.83 & 31.538 & 32.26232 .379 & 32.648 \\
\hline $11 / 21 / 2006$ & 0:00 & 34.33134 .589 & 34.907 & 35.229 & 29.834 & 26.9 & 32.015 & 30.826 & 31.52 & 32.25232 .368 & 32.625 \\
\hline $11 / 21 / 2006$ & 4:00 & 34.27234 .52 & 34.842 & 35.168 & 29.773 & 26.868 & 31.961 & 30.768 & 31.46 & 32.19632 .313 & 32.583 \\
\hline $11 / 21 / 2006$ & 8:00 & 34.27234 .514 & 34.833 & 35.162 & 29.767 & 26.861 & 31.959 & 30.762 & 31.447 & 32.18332 .297 & 32.548 \\
\hline
\end{tabular}


Depth to Water (ft TOC) in Well Indicated

$\begin{array}{llllllllllllll}\text { Date } & \text { Time } & \text { MW-1 } & \text { MW-2 } & \text { MW-3 } & \text { MW-4 } & \text { KDHE-1 } & \text { KDHE-2 } & \text { NW-1 } & \text { NW-2 } & \text { NW-3 } & \text { L-1 } & \text { L-2 } & \text { L-3 }\end{array}$

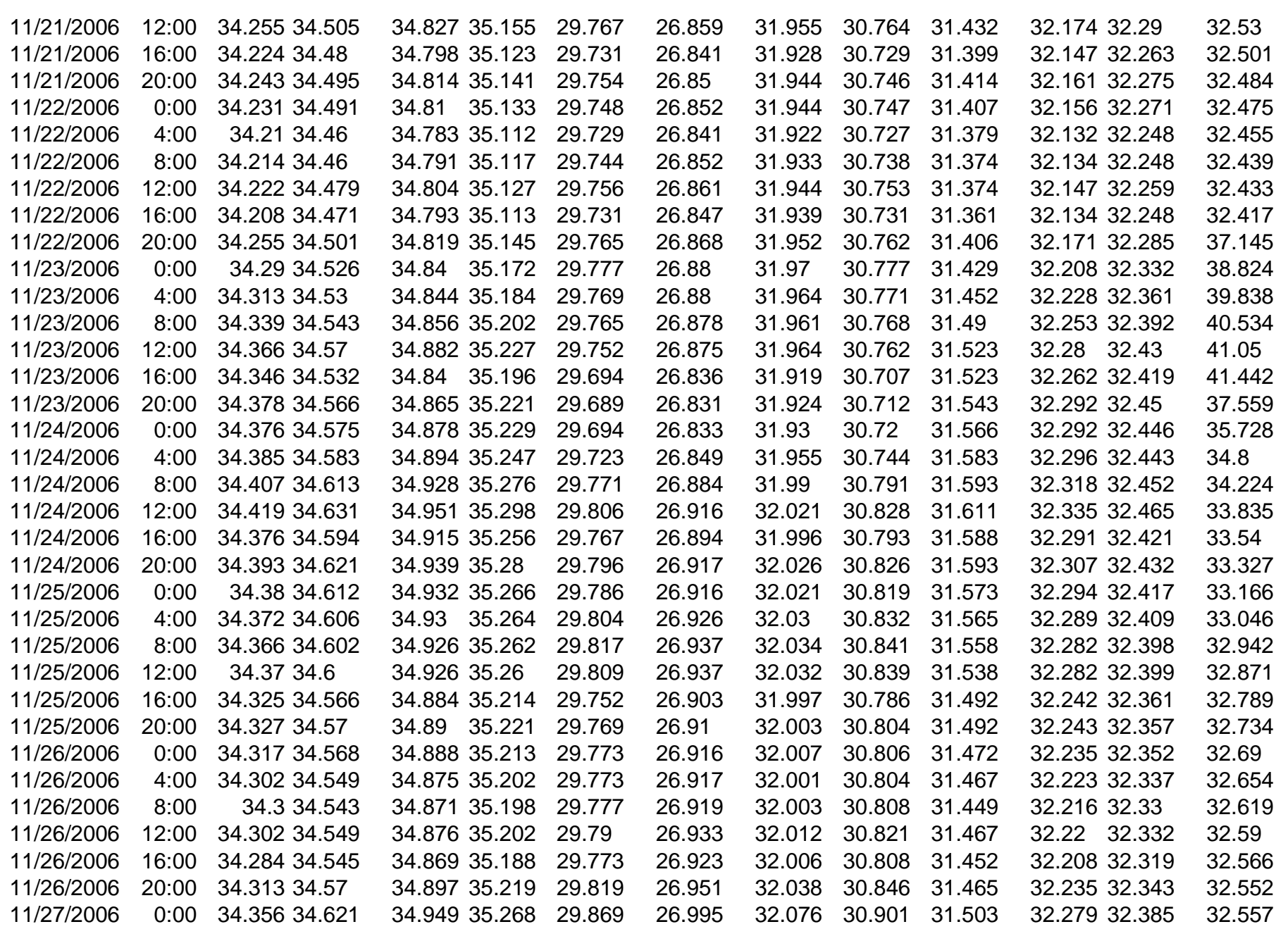


TABLE S3.2 (Cont.)

Depth to Water (ft TOC) in Well Indicated

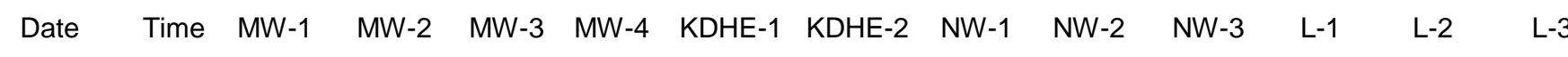

\begin{tabular}{|c|c|c|c|c|c|c|c|c|c|c|}
\hline $11 / 27 / 2006$ & $4: 00$ & 34.35634 .615 & 34.94735 .268 & 29.888 & 27.005 & 32.094 & 30.914 & 31.503 & 32.28232 .388 & 32.552 \\
\hline 11/27/2006 & 8:00 & 34.35834 .606 & 34.93735 .262 & 29.884 & 27.007 & 32.092 & 30.91 & 31.498 & 32.27732 .387 & 32.545 \\
\hline 11/27/2006 & $12: 00$ & 34.34534 .604 & 34.94135 .258 & 29.879 & 27.009 & 32.107 & 30.914 & 31.482 & 32.27532 .383 & 32.532 \\
\hline 11/27/2006 & $16: 00$ & 34.334 .547 & 34.87335 .192 & 29.796 & 26.96 & 32.034 & 30.837 & 31.432 & 32.21332 .326 & 32.508 \\
\hline 11/27/2006 & $20: 00$ & 34.26534 .528 & 34.85435 .17 & 29.777 & 26.944 & 32.019 & 30.815 & 31.407 & 32.19132 .306 & 32.486 \\
\hline $11 / 28 / 2006$ & $0: 00$ & 34.21234 .471 & 35.117 & 29.719 & 26.91 & 31.974 & 30.764 & 31.359 & 32.14232 .259 & 32.457 \\
\hline $11 / 28 / 2006$ & $4: 00$ & 34.17534 .438 & 34.76635 .079 & 29.683 & 26.882 & 31.932 & 30.722 & 31.313 & 32.10732 .222 & 32.428 \\
\hline $11 / 28 / 2006$ & 8:00 & 34.15934 .422 & 34.74335 .055 & 29.664 & 26.868 & 31.912 & 30.707 & 31.293 & 32.08632 .199 & 32.406 \\
\hline $11 / 28 / 2006$ & $12: 00$ & 34.16334 .438 & $34.76 \quad 35.07$ & 29.673 & 26.866 & 31.928 & 30.722 & 31.293 & 32.09532 .206 & 32.388 \\
\hline $11 / 28 / 2006$ & $16: 00$ & 34.415 & 34.73735 .039 & 29.641 & 26.849 & 32.231 & 30.707 & 31.265 & 32.07432 .184 & 32.364 \\
\hline $11 / 28 / 2006$ & $20: 00$ & $34.204 \quad 34.478$ & 34.80435 .11 & 29.735 & 26.898 & 32.289 & 30.779 & 31.321 & 32.14532 .241 & 32.373 \\
\hline
\end{tabular}




\section{Supplement 4:}

Quality Control for Sample Collection, Handling, and Analysis 


\section{Supplement 4:}

\section{Quality Control for Sample Collection, Handling, and Analysis}

Soil and groundwater samples were collected in April and May 2006 at Navarre, Kansas, to complete the approved scope of work for the investigation (Argonne 2006). The quality assurance/quality control (QA/QC) procedures followed for sample collection, handling, and analysis followed are described in detail in the Master Work Plan (Argonne 2002) and the sitespecific work plan (Argonne 2006).

The following sections discuss the quality of the analytical data generated during the Navarre investigation. Evaluation of the analytical data was consistent with U.S. Environmental Protection Agency guidelines (EPA 1994a,b).

\section{S4.1 Sampling to Monitor Sampling Collection, Handling, and Analysis Procedures}

Sample collection and handling activities were monitored by the documentation of samples as they were collected and the use of chain-of-custody (COC) forms and custody seals to ensure sample integrity during sample handling and shipment. The QA/QC samples collected included a field blank, equipment rinsates, and trip blanks. Field replicate samples were collected, and other samples were selected for duplicate analyses as a measure of analytical precision. The QA/QC samples are listed in Table S4.1. Analytical results for carbon tetrachloride and chloroform in QA/QC samples collected to monitor sample collection and handling activities are in Table S4.2. The COC forms are in Supplement 5.

\section{S4.1.1 Field Blanks}

One field blank was collected, representing water used during equipment decontamination. Carbon tetrachloride and chloroform, the contaminants of concern in the investigation, were not detected in the field blank. 


\section{S4.1.2 Equipment Rinsates}

Twenty-eight equipment rinsates were collected to monitor decontamination procedures for reusable sampling equipment. Neither carbon tetrachloride nor chloroform was detected above the method quantitation limit in the rinsate samples, indicating that cross-contamination of groundwater samples did not occur during sample collection. Three rinsate samples were collected from the decontaminated sampling equipment after collection of groundwater samples with high levels of carbon tetrachloride (118-260 $\mu \mathrm{g} / \mathrm{L}$; see results for locations MW4, T1, and TI-14 in Supplement 2, Table S2.3). The detection of only trace concentrations of carbon tetrachloride $(0.1-0.8 \mu \mathrm{g} / \mathrm{L})$ in these three rinsates (Table S4.2) indicates that decontamination procedures during the 2006 field investigation were satisfactory.

\section{S4.1.3 Trip Blanks}

As an indicator of cross-contamination of samples during shipment, 58 trip blanks were prepared and included with soil or water samples shipped off-site for organic analysis. Included in this total were 27 water trip blanks ( 1 of which was broken during shipment) and 23 soil trip blanks sent to the Applied Geosciences and Environmental Management (AGEM) Laboratory at Argonne National Laboratory; 4 water trip blanks sent to Envirosystems, Inc. (ENVSY), in Columbia, Maryland; and 4 soil trip blanks sent to Severn-Trent Laboratories (STL) in Colchester, Vermont. Analytical results, shown in Table S4.2, indicate that although sample handling procedures were followed overall during the 2006 investigation, cross-contamination during shipment might have occurred in the following instances:

- Samples from locations TI-27 and TI-28 were shipped together to the AGEM Laboratory on three different days (May 19, 20, and 22, 2006; see Table S4.3). Trip blanks accompanying the samples on May 19 and May 22 arrived intact and contained no detectable volatile organic compounds (VOCs), demonstrating that no cross-contamination occurred during shipment. However, the trip blank accompanying the samples on May 20 arrived broken and could not be analyzed. One of the samples from location TI-28 in that shipment contained a very high level of carbon tetrachloride $(3,104 \mu \mathrm{g} / \mathrm{L})$, and cross-contamination of other samples in that shipment cannot be ruled out. The high contaminant levels in samples from location TI-28 in the May 20 shipment are not questioned, because a similar concentration was detected in a sample from TI-28 shipped on May 22, 
2006, with an intact trip blank. However, the much lower contaminant levels in samples from location TI-27 in the May 20 shipment could have resulted from cross-contamination. Results for the affected samples from location TI-27 are qualified in the water sample data table (Supplement 2, Table S2.3).

- Trip blank NAQCTB-052306, shipped with seven groundwater samples to ENVSY on May 23, 2006 (COC 4029), for verification analysis, contained carbon tetrachloride at a concentration of $1.5 \mu \mathrm{g} / \mathrm{L}$. Also in that shipment (as above) was a sample from location TI-28 with a very high concentration of carbon tetrachloride and a sample from location TI-27 (NATI27-W-20700) in which carbon tetrachloride was detected by ENVSY at $1.6 \mu \mathrm{g} / \mathrm{L}$ (similar to the concentration in the trip blank). In analysis of sample NATI27-W-20700 at the AGEM Laboratory, no contamination was detected. The discrepancy between the AGEM Laboratory result and the ENVSY result is assumed to be the result of cross-contamination. Qualification of the AGEM Laboratory result is not warranted. See also Section S4.4.

\section{S4.1.4 Replicate Samples and Duplicate Analyses}

As an indicator of the consistency of the sampling methodology followed and to provide a measure of analytical precision, replicate soil and groundwater samples were collected. In addition, samples were selected by the AGEM Laboratory for duplicate organic analyses. Additional soil and groundwater samples were selected for verification organic analysis at a secondary laboratory. Replicate samples, samples selected for duplicate analyses, and samples selected for verification organic analysis are listed in Table S4.1.

\section{S4.1.5 Sample Representativeness}

One groundwater sample was rejected as non-representative of site conditions, and a replacement sample was collected. The original groundwater sample, NATI20-W-20912, was collected at location TI-20 from a depth interval of 42-47 ft BGL on May 23, 2006. Very limited water was available for collection. Carbon tetrachloride was detected at $1.2 \mu \mathrm{g} / \mathrm{L}$. Sample NATI20-W-20713 was collected at the same location and interval on the following day; carbon tetrachloride was detected at $13 \mu \mathrm{g} / \mathrm{L}$. Because the latter level of contamination is consistent with 
other vertical-profile samples at the TI-20 location, the initial sample was rejected and is not included in the investigation data (Supplement 2, Table S2.3).

\section{S4.2 Quality Control for Organic Analysis of Soil and Water Samples at the AGEM Laboratory}

Vertical-profile subsurface soil sampling was conducted at 20 locations to investigate site contamination. In this effort, 382 soil samples were collected. Four additional samples were collected at location CP-1, which was investigated to confirm historical lithology data. Fifteen replicate samples were collected for QC purposes. The subsurface soils were analyzed at the AGEM Laboratory for VOCs, including carbon tetrachloride and chloroform, by using a modification of EPA Method 8260B (purge-and-trap method), as referenced in the EPA's SW-846 (http://www.epa.gov/epaoswer/hazwaste/test/8_series.htm) to achieve a quantitation limit of $10.0 \mu \mathrm{g} / \mathrm{kg}$.

Soil samples were quick-frozen on dry ice as they were collected. At the laboratory, the VOCs in each soil sample were extracted with methanol from the sample matrix. For the purgeand-trap soil analyses, an aliquot of the methanol extract was purged, and the volatile species were transferred to a sorbent tube. After purging, the sorbent tube was heated and backflushed with an inert gas to desorb the components into a gas chromatograph-mass spectrometer (GC-MS) system.

Groundwater sampling was conducted at 47 locations, including 3 private wells, 13 monitoring wells (or piezometers) installed during prior investigations, and 31 newly established vertical-profile direct-push sampling locations. In total, 196 groundwater samples (and an additional 35 field replicate samples) were collected for organic analysis at the AGEM Laboratory with EPA Method 524.2 (EPA 1995) to achieve a quantitation limit of $1.0 \mu \mathrm{g} / \mathrm{L}$. (The sample discussed in Section S4.1.5 that was rejected as non-representative of site conditions is not included in the total of 196 samples.)

Water samples shipped to the AGEM Laboratory were analyzed by the purge-and-trap method with a GC-MS system. For the purge-and-trap analyses, VOCs present in the groundwater sample were extracted (purged) from the sample matrix by bubbling an inert gas through the sample. The purged components were trapped in a sorbent tube. After purging, the 
sorbent tube was heated and backflushed with an inert gas to desorb the components into the GC-MS system.

For both the soil and water analyses, the compounds eluting from the GC column were identified by retention time and by comparison with reference library spectra. The concentration of each component was calculated by comparison of the MS response for the quantitation ion to corresponding calibration curves, the responses for internal standards, or both. The internal standard recovery limits were $80-120 \%$. Calibration checks with each sample delivery group (SDG) were required to be within $\pm 20 \%$ of the standard.

Samples submitted to the AGEM Laboratory for organic analysis were analyzed in 58 SDGs, as shown in Table S4.4. The QA/QC procedures followed included analysis of instrument calibration check standards, analysis of laboratory blanks, monitoring of surrogate spike recovery, and duplicate laboratory analyses. Significant results include the following:

- Samples shipped to the AGEM Laboratory were received with custody seals intact and at the appropriate temperature. All samples were analyzed within required holding times (except for the broken trip blank discussed in Section S4.1.3).

- Carbon tetrachloride and chloroform, contaminants of concern in the investigation, were not detected in laboratory method blanks analyzed with the samples. Methylene chloride was present at trace concentrations in the methanol used for extraction of the soil samples. Detection of methylene chloride at similar concentrations in the soil samples is not reported.

- For each SDG, analytical instrument calibration was monitored by the analysis of calibration check standards. Table S4.4 shows the relative percent difference (RPD) values between the known and calculated concentrations of the standards. The concentrations of calibration check standards measured in all SDGs were within the acceptable range of $\pm 20 \%$.

- Several samples had carbon tetrachloride and/or chloroform concentrations above instrument calibration when they were analyzed undiluted (dilution 
factor $=1$ or DF1). The samples were reanalyzed at dilution as indicated in Table S4.4.

- Surrogate standard determinations were performed on samples and blanks by using surrogate spike compounds fluorobenzene, 1,4-dichlorobenzene- $\mathrm{d}_{4}$, and bromofluorobenzene. Table S4.4 shows the percent recovery of these systemmonitoring compounds for each analysis. With the following exceptions, the surrogate recoveries were within the specified range of $80-120 \%$ for all samples in either the initial analysis of the sample or a successful reanalysis of the sample:

- In the analysis of rinsate NAQCRI-W-20189 in SDG 06-4-7, the recovery of surrogate compound fluorobenzene was $78 \%$. Recovery of other surrogates was within control limits. The result for NAQCRI-W-20189 is accepted without qualification.

- In the analysis of rinsate NAQCRI-W-20187 in SDG 06-4-7, the recovery of surrogate compound fluorobenzene was $78 \%$. Recovery of other surrogates was within control limits. The result for NAQCRI-W-20187 is accepted without qualification.

- In the analysis of trip blank NAQCTB-S-20208 in SDG 06-4-11b, the recovery of surrogate compound dichlorobenzene- $\mathrm{d}_{4}$ was $79 \%$. Recovery of other surrogates was within control limits, and the result for NAQCTBS-20208 is accepted without qualification.

- In the analysis of rinsate NAQCRI-W-20386 in SDG 06-4-18a, the recoveries of surrogate compounds fluorobenzene and bromofluorobenzene were $131 \%$ and $125 \%$, respectively. High surrogate recovery would not inhibit detection of contamination, and the result for NAQCRI-W-20386 is accepted without qualification.

- In the analysis of trip blank NAQCTB-S-20346 in SDG 06-4-26b, the recovery of surrogate compound dichlorobenzene- $\mathrm{d}_{4}$ was $68 \%$. Recovery 
of other surrogates was within control limits. The result for NAQCTB-S20346 is accepted without qualification.

- In the analysis of groundwater sample NATI11-W-20506 in SDG 06-4$27 \mathrm{a}$, the recovery of surrogate compound fluorobenzene was $136 \%$. The high recovery would not inhibit detection of contamination. The result for NATI11-W-20506 is accepted without qualification.

- In the analysis of groundwater sample NATI10-W-20627 in SDG 06-5-2b, the recovery of surrogate compound dichlorobenzene- $\mathrm{d}_{4}$ was $79.2 \%$. Recovery of other surrogates was within control limits. The result for NATI10-W-20627 is accepted without qualification.

- In the analysis of soil sample NATI12-S-20754 in SDG 06-5-6, the recoveries of surrogate compounds fluorobenzene and dichlorobenzene- $\mathrm{d}_{4}$ were $126 \%$ and $132 \%$, respectively, while the recovery of surrogate compound bromofluorobenzene was $48 \%$. No contamination was indicated in any samples from the TI-12 sampling location. The result for NATI12-S-20754 is accepted without qualification.

- In the analysis of soil sample NATI12-S-20747 in SDG 06-5-6, the recovery of surrogate compound bromofluorobenzene was $197 \%$. The high recovery would not inhibit detection of contamination. The result for NATI12-S-20747 is accepted without qualification.

- In the analysis of soil sample NATI14-S-20665 in SDG 06-5-8b, the recovery of surrogate compound fluorobenzene was $79 \%$. Recovery of other surrogates was within control limits. The result for NATI14-S-20665 is accepted without qualification.

- In the analysis of trip blank NAQCTB-W-20694 in SDG 06-5-10a, the recoveries of surrogate compounds fluorobenzene, bromofluorobenzene, and dichlorobenzene- $\mathrm{d}_{4}$ were $70 \%, 67 \%$, and $75 \%$, respectively. Evaluation of the water samples associated with trip blank 
NAQCTB-W-20694 indicated that cross-contamination did not occur. The result is accepted without qualification.

- Dual analyses of soil and groundwater samples were conducted at the AGEM Laboratory as a measure of consistency in the sampling and analytical methodologies. The dual analyses were accomplished through the analysis of replicate samples submitted to the laboratory or duplicate analyses of samples selected by the laboratory. Table $\mathrm{S} 4.5$ summarizes the analytical results for carbon tetrachloride and chloroform in the primary samples and the associated replicate or duplicate analyses. Consistency in both the sampling and analytical methodologies is indicated by the average RPD values of $14.3 \%$ for carbon tetrachloride, $11.3 \%$ for chloroform, and $17.9 \%$ for methylene chloride, for those dual analyses in which the contaminants were present.

The analytical data from the AGEM Laboratory are acceptable for quantitative determination of contaminant distribution.

\section{S4.3 Quality Control for Verification Organic Analysis of Soil Samples by Severn-Trent Laboratories}

In accordance with the QA/QC procedures defined in the Master Work Plan (Argonne 2002), selected soil samples analyzed at the AGEM Laboratory for VOCs by using EPA Method $8260 \mathrm{~B}$ were subjected to verification analysis at a second laboratory with the same analytical procedure. Thirty-four of the 382 vertical-profile soil samples analyzed at the AGEM Laboratory ( $8.9 \%$ of the soil samples) were also analyzed by STL. The results were reported in 4 SDGs. The STL data packages are in Supplement 5.

The QA/QC procedures followed at STL included initial and continuing calibration of instruments, analysis of laboratory blanks, monitoring of surrogate spike recovery, and analyses of laboratory QC samples. Significant results include the following:

- Soil samples shipped to STL were received with custody seals intact and at the appropriate temperature. All samples were analyzed within required holding times. 
- Analytical instruments were properly tuned; initial and continuing calibration checks remained within the allowable range.

- Carbon tetrachloride and chloroform, the primary contaminants of concern in the investigation, were not detected in the associated blanks analyzed with the samples. Low levels of chloromethane, bromomethane, methylene chloride, butanone, and xylene were present in the methanol used for soil extraction and were detected in most of the samples, but these low concentrations are not reported.

- Surrogate standard determinations were performed on samples and blanks by using the surrogate spike compounds toluene- $\mathrm{d}_{8}, 1,2$-dichloroethane- $\mathrm{d}_{4}$, bromofluorobenzene, and 1,2-dichlorobenzene- $\mathrm{d}_{4}$. Table S4.6 shows the percent recovery of the each system-monitoring compound in each analysis. Recoveries of the surrogate compounds were within the target range for most analyses. The recovery of 1,2-dichlorobenzene- $\mathrm{d}_{4}$ trended low for most samples in SDG 113901, but other surrogate compounds were recovered well. Qualification of the data is not warranted.

- To evaluate the matrix effect of samples on the analytical methodology, laboratory QC samples containing a suite of spike compounds that included carbon tetrachloride and chloroform were analyzed with each SDG. Table S4.7 shows the percent recoveries of carbon tetrachloride and chloroform in the spiked analyses. Quality control limits were met for these analyses.

Analytical results for soil samples analyzed at the AGEM Laboratory with EPA Method $8260 \mathrm{~B}$ are supported by the results from STL obtained with the same analytical method. The verification organic results for the soil samples are summarized in Table S4.8. Agreement is good over the range of contaminant concentrations detected. Soil samples analyzed at the AGEM Laboratory with no detection of contamination were analyzed at STL with similar results. The inherent heterogeneity of soil samples is evident in the average RPD values of $56.2 \%$ for carbon tetrachloride and $28.3 \%$ for chloroform. 


\section{S4.4 Quality Control for Verification Organic Analysis of Groundwater Samples by Envirosystems, Inc.}

In accordance with the QA/QC procedures defined in the Master Work Plan (Argonne 2002), the analyses of water samples at the AGEM Laboratory with EPA Method 524.2 were verified at a second laboratory using EPA-defined Contract Laboratory Program (CLP) methodology. Twenty-one of the 196 groundwater samples analyzed at the AGEM Laboratory (10.7\% of the groundwater samples) were also analyzed according to CLP methodology by ENVSY. The results were reported in 4 SDGs. The ENVSY data packages are in Supplement 5.

The QA/QC procedures followed in the CLP analyses included initial and continuing calibration of instruments, analysis of laboratory blanks, and monitoring of surrogate spike recovery. Significant results include the following:

- Samples shipped to the CLP laboratory were received with custody seals intact and at the appropriate temperature. All samples were analyzed within required holding times.

- Analytical instruments were properly tuned; initial and continuing calibration checks remained within the allowable ranges for all contaminants of interest.

- Carbon tetrachloride and chloroform were not detected in the laboratory method blanks. Methylene chloride was present at low concentrations in laboratory blanks. Similar concentrations reported in most samples resulted in qualification of the methylene chloride results, except for sample NATI14-W20666, which had methylene chloride present at $12 \mu \mathrm{g} / \mathrm{L}$.

- As discussed in Section S4.1.3, cross-contamination of sample NATI27-W20905 during shipment to ENVSY is a possibility. Having been shipped with sample NATI28-W-20904 (in which carbon tetrachloride was detected at 3,104 $\mu \mathrm{g} / \mathrm{L}$ ), sample NATI27-W-20905 contained carbon tetrachloride at $1.6 \mu \mathrm{g} / \mathrm{L}$, similar to the concentration of $1.5 \mu \mathrm{g} / \mathrm{L}$ detected in the associated trip blank. 
- Surrogate standard determinations were performed on samples and blanks by using the surrogate spike compounds toluene- $\mathrm{d}_{8}$, bromofluorobenzene, and 1,2-dichloroethane- $\mathrm{d}_{4}$. Table S4.9 shows the percent recovery of each systemmonitoring compound for each CLP analysis. With few exceptions, the recoveries of the surrogate spikes were within the acceptable ranges (identified in Table S4.9) specific to the surrogates. High recovery of multiple surrogates for several samples in SDG 605051 would not inhibit contaminant detection and does not warrant qualification of the data. For several samples in SDG 606057, recovery of the surrogate bromofluorobenzene was marginally low, while recovery of the other surrogates was good. The data are accepted without qualification.

Analytical results for groundwater samples analyzed at the AGEM Laboratory with EPA Method 524.2 are supported by the analytical results from ENVSY, obtained by using EPA CLP methodology. The verification organic results for the groundwater samples are summarized in Table S4.10. Agreement is good over the range of contaminant concentrations detected. Samples analyzed at the AGEM Laboratory with no detection of contamination were analyzed at the CLP laboratory with similar results. Because of the higher quantitation limit of $5.0 \mu \mathrm{g} / \mathrm{L}$ for the CLP analysis, very low concentrations detected at the AGEM Laboratory by purge-and-trap analysis were sometimes not detected by the CLP analysis. For samples with contaminant concentrations above the purge-and-trap quantitation limit of $1.0 \mu \mathrm{g} / \mathrm{L}$, the RPD values for carbon tetrachloride ranged from $0 \%$ to $44.4 \%$, while those for chloroform ranged from $1.8 \%$ to $36.4 \%$. Significant concentrations of methylene chloride, indicative of biodegradation of carbon tetrachloride (via the intermediate compound chloroform), were confirmed by CLP analysis of sample NATI14-W20666.

\section{S4.5 Quality Control for Tritium Analyses of Groundwater Samples at the University of Miami Tritium Laboratory}

Groundwater samples were collected for tritium analysis at the University of Miami Tritium Laboratory in Miami, Florida, to aid in geochemical characterization of the waterbearing zone. Tritium concentrations were reported on the basis of the U.S. National Institute of Standards and Technology tritium water standard \#4926E, with a half-life of 12.32 years. Concentrations were reported in tritium units (TU), equivalent to 3.222 picocuries per kilogram of water. Because counting efficiency and background concentration are different for each 
instrument, the reported concentrations were corrected for cosmic intensity and gas pressure. Typical efficiencies are equivalent to 1 count per minute (cpm) per 2.4 TU. Background is about $0.3 \mathrm{cpm}$, known to $\pm 0.02 \mathrm{cpm}$. The tritium results for the groundwater samples are acceptable for evaluating the age of the groundwater.

\section{S4.6 Quality Control for Particle Size Analyses of Soil Samples at HWS Laboratory}

To aid in the evaluation of site lithology, soil samples were selected during coring activities for particle size analysis at the HWS Laboratory in Lincoln, Nebraska. The analysis was conducted in accordance with American Society for Testing and Materials method ASTM D42263 (2002). The distribution of particle sizes larger than $75 \mu \mathrm{m}$ was determined by sieving, while the distribution of smaller particle sizes was determined by a sedimentation process using a hydrometer. 
TABLE S4.1 Quality control samples collected to monitor sample collection, handling, and analysis activities at Navarre, Kansas.

\begin{tabular}{|c|c|c|c|c|c|c|c|c|c|}
\hline Location & Sample & $\begin{array}{c}\text { Depth } \\
\text { (ft BGL) }\end{array}$ & $\begin{array}{l}\text { Sample } \\
\text { Date }\end{array}$ & Time & $\operatorname{coc}^{a}$ & Medium & $\begin{array}{l}\text { Sample } \\
\text { Type }^{\text {b }}\end{array}$ & $\begin{array}{l}\text { Log; } \\
\text { Page }^{\mathrm{C}}\end{array}$ & Sample Description \\
\hline
\end{tabular}

\section{Field blank}

QC

NAQCFB-W-20199

$-$

4/6/06 16:35 $3711 \quad$ Water

\section{Equipment rinsates}

\begin{tabular}{|c|c|c|c|c|c|}
\hline NAQCRI-W-20187 & - & $4 / 5 / 06$ & $17: 15$ & 3711 & Water \\
\hline NAQCRI-W-20189 & - & $4 / 6 / 06$ & $9: 15$ & 3711 & Water \\
\hline NAQCRI-W-20191 & - & $4 / 6 / 06$ & $10: 30$ & 3711 & Water \\
\hline NAQCRI-W-20193 & - & $4 / 6 / 06$ & $12: 00$ & 3711 & Water \\
\hline NAQCRI-W-20196 & - & $4 / 6 / 06$ & $14: 15$ & 3711 & Water \\
\hline NAQCRI-W-20202 & - & $4 / 7 / 06$ & $9: 50$ & 3713 & Water \\
\hline NATI3-W-20299 & - & $4 / 7 / 06$ & 10:36 & 3713 & Water \\
\hline NAQCRI-W-20204 & - & $4 / 7 / 06$ & $11: 30$ & 3713 & Water \\
\hline NAQCRI-W-20206 & - & $4 / 7 / 06$ & $13: 55$ & 3713 & Water \\
\hline NAQCRI-W-20210 & - & $4 / 7 / 06$ & $15: 55$ & 3518 & Water \\
\hline NAQCRI-W-20212 & - & $4 / 8 / 06$ & $10: 10$ & 3518 & Water \\
\hline NAQCRI-W-20216 & - & $4 / 8 / 06$ & $12: 10$ & 3518 & Water \\
\hline
\end{tabular}

RI 06-BN; 10 Rinsate of decontaminated Redi-Flo tubing after collection of sample NAMW01-W-20186.

RI 06-BN; 14 Rinsate of decontaminated Redi-Flo tubing after collection of sample NAMW02-W-20188 and replicate NAQCDU-W-20200.

RI 06-BN; 18 Rinsate of decontaminated Redi-Flo tubing after collection of sample NAL2-W-20190.

RI 06-BN; 22 Rinsate of decontaminated Redi-Flo tubing after collection of sample NAMW03-W-20192.

RI 06-BN; 26 Rinsate of decontaminated Redi-Flo tubing after collection of sample NAMW04-W-20194.

RI 06-BN; 30 Rinsate of decontaminated Redi-Flo tubing after collection of sample NAT1-W-20201.

RI $\quad 06-3 ; 13 \quad$ Rinsate of decontaminated sampling bailer after collection of sample NATI3-W-20298.

RI 06-BN; 38 Rinsate of decontaminated Redi-Flo tubing after collection of sample NAKDHE1-W-20203.

RI 06-BN; 42 Rinsate of decontaminated Redi-Flo tubing after collection of sample NAKDHE2-W-20205.

RI 06-BN; 46 Rinsate of decontaminated Redi-Flo tubing after collection of sample NAL3-W-20209.

RI 06-BN; 50 Rinsate of decontaminated Redi-Flo tubing after collection of sample NAL1-W-20211 and replicate NAQCDU-W-20213.

RI $\quad 06-B N ; 54$ Rinsate of decontaminated Redi-Flo tubing after collection of sample NANW1-W-20215 and replicate NAQCDU-W-20225. 
TABLE S4.1 (Cont.)

\begin{tabular}{ccccccccc}
\hline Location & $\begin{array}{c}\text { Depth } \\
\text { (ft BGL) }\end{array}$ & $\begin{array}{c}\text { Sample } \\
\text { Date }\end{array}$ & Time & COC $^{a}$ & Medium & $\begin{array}{c}\text { Sample } \\
\text { Type }^{\mathrm{b}}\end{array}$ & $\begin{array}{c}\text { Log; } \\
\text { Page }^{\mathrm{c}}\end{array}$ & Sample Description \\
\hline
\end{tabular}

Equipment rinsates (cont.)

\begin{tabular}{|c|c|c|c|c|c|}
\hline QC & NATI4-W-20473 & - & $4 / 12 / 06$ & $10: 20$ & 3685 \\
\hline QC & NAQCRIN-W-20372 & - & $4 / 13 / 06$ & $8: 26$ & 3520 \\
\hline QC & NATI1-W-20345 & - & $4 / 13 / 06$ & $20: 07$ & 3698 \\
\hline QC & NAQCRIN-W-20386 & - & $4 / 14 / 06$ & $10: 00$ & 3698 \\
\hline QC & NATI5QC-W-20268 & - & $4 / 20 / 06$ & $13: 20$ & 4706 \\
\hline QC & NATI2-W-20280 & - & $4 / 21 / 06$ & $13: 50$ & 4710 \\
\hline QC & NATI6RIN-W-20352 & - & $4 / 22 / 06$ & $13: 38$ & 4704 \\
\hline QC & NATI10QC-W-20633 & - & $4 / 27 / 06$ & $9: 20$ & 4719 \\
\hline QC & NAQCRIN-W-20760 & - & $5 / 4 / 06$ & $10: 30$ & 4141 \\
\hline QC & NAQCRIN-W-20769 & - & $5 / 6 / 06$ & $17: 15$ & 4730 \\
\hline QC & NAQCRIN-W-20837 & - & $5 / 10 / 06$ & $9: 55$ & 3521 \\
\hline QC & NAQCRIN-W-20874 & - & $5 / 11 / 06$ & $17: 22$ & 4147 \\
\hline QC & NAQCRIN-W-20234 & - & $5 / 12 / 06$ & $0: 45$ & 4148 \\
\hline QC & NAQCRIN-W-20648 & - & $5 / 19 / 06$ & $14: 30$ & 4761 \\
\hline QC & NAQCRIN-W-20908 & - & $5 / 21 / 06$ & $16: 20$ & 4156 \\
\hline QC & NAQCRIN-W-20924 & - & $5 / 23 / 06$ & $13: 15$ & 4158 \\
\hline
\end{tabular}

Water $\quad \mathrm{RI} \quad 06-1 ; 45 \quad$ Rinsate of decontaminated sampling bailer after collection of sample NATI4-W-20472.

Rinsate of decontaminated sampling bailer after collection of sample NATI5-W-20371.
Rinsate of decontaminated sampling bailer after collection of sample NATI1-W-20344.
RI $\quad 06-1 ; 73$

$\mathrm{RI} \quad 06-3 ; 93$

Rinsate of decontaminated sampling bailer after collection of sample NATI3-W-20385.

RI 06-1;93 Rinsate of decontaminated sampling bailer after collection of sample NATI5-W-20267.

RI $\quad 06-1 ; 139$ Rinsate of decontaminated sampling bailer after collection of sample NATI2-W-20279.

RI 06-3; 149 Rinsate of decontaminated sampling bailer after collection of sample NATI6-W-20350 and replicate NATI6-W-20351.

RI $\quad 06-1 ; 197$ Rinsate of decontaminated sampling bailer after collection of sample NATI10-W-20632.

RI $\quad 06-5 ; 53 \quad$ Rinsate of decontaminated sampling bailer after collection of sample NATI12-W-20758.

RI 06-4; 125 Rinsate of decontaminated sampling bailer after collection of sample NATI14-W-20768.

RI 06-5; 153 Rinsate of decontaminated sampling bailer after collection of sample NATI22-W-20836.

RI 06-6; $57 \quad$ Rinsate of decontaminated sampling bailer after collection of sample NATI19-W-20872.

RI 06-6; 129 Rinsate of decontaminated sampling bailer after collection of sample NATI26-W-20233.

RI 06-7; $33 \quad$ Rinsate of decontaminated sampling bailer after collection of sample NATI28-W-20254 and replicate NATI28-W-20255.

RI 06-7; 139 Rinsate of decontaminated sampling bailer after collection of sample NATI29-W-20907.

RI 06-8; 25 Rinsate of decontaminated sampling bailer after collection of sample NATI30-W-20920. 
TABLE S4.1 (Cont.)

\begin{tabular}{|c|c|c|c|c|c|c|c|c|c|}
\hline Location & Sample & $\begin{array}{c}\text { Depth } \\
\text { (ft BGL) }\end{array}$ & $\begin{array}{l}\text { Sample } \\
\text { Date }\end{array}$ & Time & $\mathrm{COC}^{\mathrm{a}}$ & Medium & $\begin{array}{l}\text { Sample } \\
\text { Type }^{\text {b }}\end{array}$ & $\begin{array}{l}\text { Log; } \\
\text { Page }^{\mathrm{C}}\end{array}$ & Sample Description \\
\hline
\end{tabular}

Trip blanks

$\begin{array}{ll}\text { QC } & \text { NAQCTB-S-20197 } \\ \text { QC } & \text { NAQCTB-W-20198 } \\ \text { QC } & \text { NAQCTB-W-20207 } \\ \text { QC } & \text { NAQCTB-S-20208 } \\ \text { QC } & \text { NAQCTB-W-20214 } \\ \text { QC } & \text { NATripBlank-S-20226 } \\ \text { QC } & \text { NATripBlank-S-20227 } \\ \text { QC } & \text { NAQCTB-W-11APR06 } \\ \text { QC } & \text { NAQCTB-S-20362 } \\ \text { QC } & \text { NAQCTB-W-20369 } \\ \text { QC } & \text { NAQCTB-S-20377 } \\ \text { QC } & \text { NAQCTB-W-20378 } \\ \text { QC } & \text { NAQCTB-W-20389 } \\ \text { QC } & \text { NAQCTB-S-20387 } \\ \text { QC } & \text { NA-MEOHBLANK- } \\ & \text { 18APR06 }\end{array}$

\begin{tabular}{|c|c|c|c|c|c|c|}
\hline - & $4 / 6 / 06$ & $15: 10$ & 4546 & Soil & TB & $06-\mathrm{BN} ; 33$ \\
\hline - & $4 / 6 / 06$ & $15: 30$ & 3711 & Water & TB & 06-BN; 33 \\
\hline - & $4 / 7 / 06$ & $14: 20$ & 3713 & Water & TB & $06-\mathrm{BN} ; 45$ \\
\hline - & $4 / 7 / 06$ & $14: 40$ & 4113 & Soil & TB & 06-BN; 45 \\
\hline- & $4 / 8 / 06$ & $8: 45$ & 3518 & Water & TB & 06-BN; 53 \\
\hline - & $4 / 10 / 06$ & & 4108 & Soil & TB & COC 4108 \\
\hline - & $4 / 10 / 06$ & & 4110 & Soil & TB & COC 4110 \\
\hline- & $4 / 11 / 06$ & $15: 00$ & 2478 & Water & TB & $\mathrm{COC}$ \\
\hline - & $4 / 11 / 06$ & & 4107 & Soil & TB & $06-3 ; 28$ \\
\hline- & $4 / 12 / 06$ & $15: 50$ & 3685 & Water & TB & $06-3 ; 45$ \\
\hline - & $4 / 13 / 06$ & $15: 30$ & 3519 & Soil & TB & $06-3 ; 73$ \\
\hline - & $4 / 13 / 06$ & $15: 35$ & 3520 & Water & TB & $06-3 ; 77$ \\
\hline - & $4 / 14 / 06$ & $14: 00$ & 3698 & Water & TB & $06-3 ; 105$ \\
\hline - & $4 / 14 / 06$ & & 3714 & Soil & TB & $06-3 ; 97$ \\
\hline- & $4 / 18 / 06$ & $15: 00$ & 4023 & Soil & TB & $\mathrm{coc}$ \\
\hline
\end{tabular}

sent to the AGEM Laboratory with soil samples listed on COCs 4545, 4546, and 4102.

Trip blank sent to the AGEM Laboratory with water samples listed on COC 3711.

rip blank sent to the AGEM Laboratory with water samples listed on COC 3713.

kent to the AGEM Laboratory with soil samples listed on COCs 4113 and 4104.

Trip blank sent to the AGEM Laboratory with water samples listed on COC 3518. samples listed on COCs 4101 and 4108 . samples listed on COCs 4101 and 4108 . samples listed on COCs 4109 and 4110 .

Trip blank sent to ENVSY for verification organic analysis with water samples listed on $\mathrm{COC}$ 2478.

Trip blank sent to the AGEM Laboratory with soil samples listed on COCs 4105 and 4107.

Trip blank sent to the AGEM Laboratory with water samples listed on COC 3685.

Trip blank sent to the AGEM Laboratory with soil samples listed on COCs 3768 and 3519.

Trip blank sent to the AGEM Laboratory with water samples listed on COC 3520.

Trip blank sent to the AGEM Laboratory with water samples listed on COC 3698.

Trip blank sent to the AGEM Laboratory with soil samples listed on COC 3714.

Trip blank with sent to STL for verification organic analysis with soil samples listed on COC 4023. 
TABLE S4.1 (Cont.)

\begin{tabular}{|c|c|c|c|c|c|c|c|c|c|}
\hline Location & Sample & $\begin{array}{c}\text { Depth } \\
\text { (ft BGL) }\end{array}$ & $\begin{array}{l}\text { Sample } \\
\text { Date }\end{array}$ & Time & $\mathrm{coc}^{\mathrm{a}}$ & Medium & $\begin{array}{l}\text { Sample } \\
\text { Type }^{\mathrm{b}}\end{array}$ & $\begin{array}{c}\text { Log; } \\
\text { Page }^{\mathrm{C}}\end{array}$ & Sample Description \\
\hline
\end{tabular}

Trip blanks (cont.)

$\begin{array}{ll}\text { QC } & \text { NAQCTB-S-20486 } \\ \text { QC } & \text { NAQCTB-W-20487 } \\ \text { QC } & \text { NAQCTB-S-20346 } \\ \text { QC } & \text { NAQCTB-W-20347 } \\ \text { QC } & \text { NATI9QC-W-20588 } \\ \text { QC } & \text { NAQCTB-S-20716 } \\ \text { QC } & \text { NAQCTB-W-20717 } \\ \text { QC } & \text { NAQCTB-W-20518 } \\ \text { QC } & \text { NAQCTB-S-20519 } \\ \text { QC } & \text { NAQCTB-S-20529 } \\ \text { QC } & \text { NAQCTB-W-20726 } \\ \text { QC } & \text { NA-S-BLANK-02MAY06 } \\ \text { QC } & \text { NAQCTB-W-20730 } \\ \text { QC } & \text { NAQCTB-W-20733 } \\ \text { QC } & \text { NAQCTB-S-20551 }\end{array}$

\begin{tabular}{|c|c|c|c|c|c|c|c|}
\hline- & $4 / 20 / 06$ & $16: 00$ & 4709 & Soil & TB & $06-3 ; 113$ & $\begin{array}{l}\text { Trip blank sent to the AGEM Laboratory with soil } \\
\text { samples listed on COC } 4709 \text {. }\end{array}$ \\
\hline & $4 / 20 / 06$ & $16: 00$ & 4706 & Water & TB & $06-3 ; 117$ & $\begin{array}{l}\text { Trip blank sent to the AGEM Laboratory with } \\
\text { water samples listed on COC } 4706 \text {. }\end{array}$ \\
\hline & $4 / 21 / 06$ & $15: 00$ & 4705 & Soil & TB & $06-3 ; 121$ & $\begin{array}{l}\text { Trip blank sent to the AGEM Laboratory with soil } \\
\text { samples listed on COC } 4705 \text {. }\end{array}$ \\
\hline & $4 / 21 / 06$ & $16: 30$ & 4710 & Water & TB & $06-3 ; 135$ & $\begin{array}{l}\text { Trip blank sent to the AGEM Laboratory with } \\
\text { water samples listed on COC } 4710 \text {. }\end{array}$ \\
\hline & $4 / 22 / 06$ & $9: 22$ & 4711 & Water & TB & $06-1 ; 165$ & $\begin{array}{l}\text { Trip blank sent to the AGEM Laboratory with } \\
\text { water samples listed on COCs } 4701 \text { and } 4711 \text {. }\end{array}$ \\
\hline & $4 / 24 / 06$ & $16: 00$ & 4702 & Soil & TB & $06-3 ; 169$ & $\begin{array}{l}\text { Trip blank sent to the AGEM Laboratory with soil } \\
\text { samples listed on COCs } 4702,4712 \text {, and } \\
4713 \text {. }\end{array}$ \\
\hline & $4 / 24 / 06$ & $16: 10$ & 4714 & Water & TB & $06-3 ; 173$ & $\begin{array}{l}\text { Trip blank sent to the AGEM Laboratory with } \\
\text { water samples listed on COCs } 4704 \text { and } 4714 \text {. }\end{array}$ \\
\hline & $4 / 26 / 06$ & $16: 50$ & 4722 & Water & TB & $06-3 ; 185$ & $\begin{array}{l}\text { Trip blank sent to the AGEM Laboratory with } \\
\text { water samples listed on COC } 4722 \text {. }\end{array}$ \\
\hline & $4 / 26 / 06$ & $16: 50$ & 4114 & Soil & TB & $06-3 ; 189$ & $\begin{array}{l}\text { Trip blank sent to the AGEM Laboratory with soil } \\
\text { samples listed on COCs } 4716,4717 \text {, and } \\
4114 \text {. }\end{array}$ \\
\hline & $4 / 27 / 06$ & 15:01 & 4122 & Soil & TB & $06-3 ; 201$ & $\begin{array}{l}\text { Trip blank sent to the AGEM Laboratory with soil } \\
\text { samples listed on COC } 4122 \text {. }\end{array}$ \\
\hline & $4 / 27 / 06$ & $15: 30$ & 4719 & Water & TB & $06-4 ; 17$ & $\begin{array}{l}\text { Trip blank sent to the AGEM Laboratory with } \\
\text { water samples listed on COC } 4719 \text {. }\end{array}$ \\
\hline & $5 / 2 / 06$ & $11: 15$ & 4024 & Soil & TB & COC & $\begin{array}{l}\text { Trip blank sent to STL for verification organic } \\
\text { analysis with soil samples listed on COC } 4024\end{array}$ \\
\hline & $5 / 2 / 06$ & $15: 50$ & 4124 & Water & TB & $06-5 ; 17$ & $\begin{array}{l}\text { Trip blank sent to the AGEM Laboratory with } \\
\text { water samples listed on COC } 4124 \text {. }\end{array}$ \\
\hline & $5 / 3 / 06$ & $10: 08$ & 4126 & Water & TB & $06-5 ; 29$ & $\begin{array}{l}\text { Trip blank sent to the AGEM Laboratory with } \\
\text { water samples listed on COC } 4126 \text {. }\end{array}$ \\
\hline - & $5 / 3 / 06$ & $16: 30$ & 4129 & Soil & TB & $06-4 ; 25$ & $\begin{array}{l}\text { Trip blank sent to the AGEM Laboratory with soil } \\
\text { samples listed on COCs } 4118 \text { and } 4129 \text {. }\end{array}$ \\
\hline
\end{tabular}


TABLE S4.1 (Cont.)

\begin{tabular}{|c|c|c|c|c|c|c|c|c|c|}
\hline Location & Sample & $\begin{array}{c}\text { Depth } \\
\text { (ft BGL) }\end{array}$ & $\begin{array}{l}\text { Sample } \\
\text { Date }\end{array}$ & Time & $\mathrm{COC}^{\mathrm{a}}$ & Medium & $\begin{array}{l}\text { Sample } \\
\text { Type }^{\mathrm{b}}\end{array}$ & $\begin{array}{l}\text { Log; } \\
\text { Page }^{\mathrm{C}}\end{array}$ & Sample Description \\
\hline
\end{tabular}

Trip blanks (cont.)

\begin{tabular}{|c|c|c|c|c|c|c|c|c|c|}
\hline$Q C$ & NAQCTB-S-20552 & - & $5 / 3 / 06$ & $16: 30$ & 4137 & Soil & TB & $06-4 ; 29$ & $\begin{array}{l}\text { Trip blank sent to the AGEM Laboratory with soil } \\
\text { samples listed on COC } 4137 \text {. }\end{array}$ \\
\hline QC & NAQCTB-W-20759 & - & $5 / 4 / 06$ & $10: 19$ & 4141 & Water & TB & $06-5 ; 49$ & $\begin{array}{l}\text { Trip blank sent to the AGEM Laboratory with } \\
\text { water samples listed on COC } 4141 \text {. }\end{array}$ \\
\hline QC & NAQCTB-S-20559 & - & $5 / 4 / 06$ & $14: 26$ & 4138 & Soil & TB & $06-4 ; 41$ & $\begin{array}{l}\text { Trip blank sent to the AGEM Laboratory with soil } \\
\text { samples listed on COCs } 4130 \text { and } 4138 \text {. }\end{array}$ \\
\hline QC & NAQCTB-S-20582 & - & $5 / 5 / 06$ & $16: 00$ & 4133 & Soil & TB & $06-4 ; 73$ & $\begin{array}{l}\text { Trip blank sent to the AGEM Laboratory with soil } \\
\text { samples listed on COC } 4133 \text {. }\end{array}$ \\
\hline QC & NAQCTB-S-20583 & - & $5 / 5 / 06$ & $17: 00$ & 4134 & Soil & TB & $06-4 ; 77$ & $\begin{array}{l}\text { Trip blank sent to the AGEM Laboratory with soil } \\
\text { samples listed on COCs } 4134 \text { and } 4135 \text {. }\end{array}$ \\
\hline QC & NAQCTB-W-20584 & - & $5 / 5 / 06$ & $17: 00$ & 4131 & Water & TB & $06-4 ; 81$ & $\begin{array}{l}\text { Trip blank sent to the AGEM Laboratory with } \\
\text { water samples listed on COC } 4131 \text {. }\end{array}$ \\
\hline QC & NAQCTB-S-20767 & - & $5 / 6 / 06$ & $8: 00$ & 4731 & Soil & TB & $06-4 ; 113$ & $\begin{array}{l}\text { Trip blank sent to the AGEM Laboratory with soil } \\
\text { samples listed on COCs } 4132 \text { and } 4731 \text {. }\end{array}$ \\
\hline$Q C$ & NAQCTB-W-20694 & - & $5 / 6 / 06$ & $14: 00$ & 4730 & Water & TB & $06-4 ; 101$ & $\begin{array}{l}\text { Trip blank sent to the AGEM Laboratory with } \\
\text { water samples listed on COCs } 4730 \text { and } 4758 \text {. }\end{array}$ \\
\hline QC & $\begin{array}{l}\text { NA-MEOHBLANK- } \\
\text { 08MAY06 }\end{array}$ & - & $5 / 8 / 06$ & $12: 00$ & 4025 & Soil & TB & $\mathrm{COC}$ & $\begin{array}{l}\text { Trip blank sent to STL for verification organic } \\
\text { analysis with water samples listed on COC } \\
4025 \text {. }\end{array}$ \\
\hline QC & NAQCTB-S-20794 & - & $5 / 8 / 06$ & $17: 00$ & 3765 & Soil & TB & $06-4 ; 169$ & $\begin{array}{l}\text { Trip blank sent to the AGEM Laboratory with soil } \\
\text { samples listed on COCs } 4729 \text { and } 3765 \text {. }\end{array}$ \\
\hline QC & NAQCTB-S-20795 & - & $5 / 8 / 06$ & 17:00 & 4728 & Soil & TB & $06-4 ; 173$ & $\begin{array}{l}\text { Trip blank sent to the AGEM Laboratory with soil } \\
\text { samples listed on COCs } 3767 \text { and } 4728 \text {. }\end{array}$ \\
\hline QC & NAQCTB-050706 & - & $5 / 8 / 06$ & & 4026 & Water & TB & $\mathrm{COC}$ & $\begin{array}{l}\text { Trip blank sent to ENVSY for verification organic } \\
\text { analysis with water samples listed on COC } \\
4026 \text {. }\end{array}$ \\
\hline QC & NAQCTB-W-20824 & - & $5 / 9 / 06$ & $10: 39$ & 4143 & Water & TB & $06-5 ; 93$ & $\begin{array}{l}\text { Trip blank sent to the AGEM Laboratory with } \\
\text { water samples listed on COCs } 4143 \text { and } 4144 \text {. }\end{array}$ \\
\hline QC & NAQCTB-W-20866 & - & $5 / 11 / 06$ & $14: 30$ & 4146 & Water & TB & $06-6 ; 25$ & $\begin{array}{l}\text { Trip blank sent to the AGEM Laboratory with } \\
\text { water samples listed on COCs } 4146 \text { and } 4149 \text {. }\end{array}$ \\
\hline QC & NAQCTB-W-20873 & - & $5 / 11 / 06$ & $17: 15$ & 4147 & Water & TB & $06-6 ; 53$ & $\begin{array}{l}\text { Trip blank sent to the AGEM Laboratory with } \\
\text { water samples listed on COC } 4147 \text {. }\end{array}$ \\
\hline
\end{tabular}


TABLE S4.1 (Cont.)

\begin{tabular}{|c|c|c|c|c|c|c|c|c|c|}
\hline Location & Sample & $\begin{array}{c}\text { Depth } \\
\text { (ft BGL) }\end{array}$ & $\begin{array}{l}\text { Sample } \\
\text { Date }\end{array}$ & Time & $\mathrm{COC}^{\mathrm{a}}$ & Medium & $\begin{array}{l}\text { Sample } \\
\text { Type }^{\text {b }}\end{array}$ & $\begin{array}{l}\text { Log; } \\
\text { Page }^{\mathrm{C}}\end{array}$ & Sample Description \\
\hline
\end{tabular}

Trip blanks (cont.)

\begin{tabular}{|c|c|c|c|c|c|c|c|c|c|}
\hline QC & NAQCTB-W-20724 & - & $5 / 12 / 06$ & $11: 00$ & 4148 & Water & TB & $06-6 ; 165$ & $\begin{array}{l}\text { Trip blank sent to the AGEM Laboratory with } \\
\text { water samples listed on COC } 4148 \text {. }\end{array}$ \\
\hline QC & NAQCTB-S-20263 & - & $5 / 18 / 06$ & $16: 30$ & 4723 & Soil & TB & $06-7 ; 13$ & $\begin{array}{l}\text { Trip blank sent to the AGEM Laboratory with soil } \\
\text { samples listed on COC } 4723 \text {. }\end{array}$ \\
\hline QC & $\begin{array}{l}\text { NA-MEOHBLANK- } \\
\text { 19MAY06 }\end{array}$ & - & $5 / 19 / 06$ & 9:00 & 4027 & Soil & TB & $\mathrm{COC}$ & $\begin{array}{l}\text { Trip blank sent to the STL for verification organic } \\
\text { analysis with soil samples listed on COC } 4027 \text {. }\end{array}$ \\
\hline QC & NAQCTB-S-20681 & - & $5 / 19 / 06$ & $14: 00$ & 4725 & Soil & TB & $06-7 ; 37$ & $\begin{array}{l}\text { Trip blank sent to the AGEM Laboratory with soil } \\
\text { samples listed on COCs } 4724 \text { and } 4725 \text {. }\end{array}$ \\
\hline QC & NAQCTB-W-20682 & - & $5 / 19 / 06$ & 14:00 & 4761 & Water & TB & $06-7 ; 41$ & $\begin{array}{l}\text { Trip blank sent to the AGEM Laboratory with } \\
\text { water samples listed on COC } 4761 \text {. }\end{array}$ \\
\hline QC & NAQCTB-W-20702 & - & $5 / 20 / 06$ & $10: 00$ & 4154 & Water & TB & $06-7 ; 81$ & $\begin{array}{l}\text { Trip blank sent to the AGEM Laboratory with } \\
\text { water samples listed on COC } 4154 \text {. Trip blank } \\
\text { was broken during shipment and was not } \\
\text { analyzed. }\end{array}$ \\
\hline QC & NAQCTB-W-20650 & - & $5 / 20 / 06$ & $17: 00$ & 4156 & Water & TB & $06-7 ; 93$ & $\begin{array}{l}\text { Trip blank sent to the AGEM Laboratory with } \\
\text { water samples listed on COCs } 4156 \text { and } 4727 \text {. }\end{array}$ \\
\hline QC & NAQCTB-S-20899 & - & $5 / 21 / 06$ & 9:00 & 4155 & Soil & TB & $06-7 ; 97$ & $\begin{array}{l}\text { Trip blank sent to the AGEM Laboratory with soil } \\
\text { samples listed on COCs } 4157 \text { and } 4155 \text {. }\end{array}$ \\
\hline QC & NAQCTB-052306 & - & $5 / 23 / 06$ & 10:00 & 4029 & Water & TB & $\mathrm{COC}$ & $\begin{array}{l}\text { Trip blank sent to ENVSY for verification organic } \\
\text { analysis with water samples listed on COC } \\
4029 \text {. }\end{array}$ \\
\hline QC & NAQCTB-W-20925 & - & $5 / 23 / 06$ & $16: 45$ & 4158 & Water & TB & $06-8 ; 29$ & $\begin{array}{l}\text { Trip blank sent to the AGEM Laboratory with } \\
\text { water samples listed on COC } 4158 \text {. }\end{array}$ \\
\hline QC & NAQCTB-W-20714 & - & $5 / 24 / 06$ & $14: 00$ & 3766 & Water & TB & $06-8 ; 41$ & $\begin{array}{l}\text { Trip blank sent to the AGEM Laboratory with } \\
\text { water samples listed on COC } 3766 \text {. }\end{array}$ \\
\hline QC & NAQCTB-052506 & - & $5 / 25 / 06$ & $11: 30$ & 4030 & Water & TB & $\mathrm{cOC}$ & $\begin{array}{l}\text { Trip blank sent to ENVSY for verification organic } \\
\text { analysis with water samples listed on COC } \\
4030 \text {. }\end{array}$ \\
\hline QC & NAQCTB-W-20944 & - & $5 / 25 / 06$ & $14: 00$ & 4127 & Water & TB & $06-8 ; 69$ & $\begin{array}{l}\text { Trip blank sent to the AGEM Laboratory with } \\
\text { water samples listed on COC } 4127 \text {. }\end{array}$ \\
\hline
\end{tabular}


TABLE S4.1 (Cont.)

\begin{tabular}{|c|c|c|c|c|c|c|c|c|c|}
\hline Location & Sample & $\begin{array}{c}\text { Depth } \\
\text { (ft BGL) }\end{array}$ & $\begin{array}{c}\text { Sample } \\
\text { Date }\end{array}$ & Time & $\mathrm{COC}^{\mathrm{a}}$ & Medium & $\begin{array}{c}\text { Sample } \\
\text { Type }\end{array}$ & $\begin{array}{l}\text { Log; } \\
\text { Page }^{c}\end{array}$ & Sample Description \\
\hline
\end{tabular}

Replicate soil samples

$\begin{array}{llcclll}\text { TI-1 } & \text { NATI1-S-20307 } & 31 & 4 / 8 / 06 & 13: 55 & 4101 & \text { Soil } \\ \text { TI-1 } & \text { NATI1-S-20483 } & 44 & 4 / 13 / 06 & 15: 15 & 3768 & \text { Soil } \\ \text { TI-2 } & \text { NATI2-S-20412 } & 16 & 4 / 5 / 06 & 17: 40 & 4545 & \text { Soil } \\ \text { TI-3 } & \text { NATI3-S-20380 } & 56.5 & 4 / 13 / 06 & 16: 35 & 3714 & \text { Soil } \\ \text { TI-4 } & \text { NATI4-S-20318 } & 34 & 4 / 9 / 06 & 9: 40 & 4101 & \text { Soil } \\ \text { TI-5 } & \text { NATI5-S-20357 } & 25 & 4 / 11 / 06 & 10: 10 & 4105 & \text { Soil } \\ \text { TI-6 } & \text { NATI6-S-20403 } & 50 & 4 / 20 / 06 & 17: 52 & 4705 & \text { Soil } \\ \text { TI-8 } & \text { NATI8-S-20527 } & 54.6 & 4 / 27 / 06 & 11: 50 & 4122 & \text { Soil } \\ \text { TI-9 } & \text { NATI9-S-20596 } & 25 & 4 / 22 / 06 & 15: 50 & 4712 & \text { Soil } \\ \text { TI-10 } & \text { NATI10-S-20625 } & 43 & 4 / 26 / 06 & 11: 43 & 4716 & \text { Soil } \\ \text { TI-11 } & \text { NATI11-S-20504 } & 56.5 & 4 / 25 / 06 & 8: 08 & 4114 & \text { Soil } \\ \text { TI-12 } & \text { NATI12-S-20740 } & 21 & 5 / 3 / 06 & 13: 27 & 4137 & \text { Soil } \\ \text { TI-18 } & \text { NATI18-S-20679 } & 41 & 5 / 19 / 06 & 11: 05 & 4724 & \text { Soil } \\ \text { TI-28 } & \text { NATI28-S-20245 } & 32.8 & 5 / 18 / 06 & 16: 03 & 4724 & \text { Soil } \\ \text { TI-29 } & \text { NATI29-S-20898 } & 62 & 5 / 21 / 06 & 8: 20 & 4155 & \text { Soil }\end{array}$

Soil samples selected by the AGEM Laboratory for duplicate organic analyses

$\begin{array}{llcclll}\text { TI-1 } & \text { NATI1-S-20467DUP } & 23.8 & 4 / 11 / 06 & 10: 34 & 4107 & \text { Soil } \\ \text { TI-2 } & \text { NATI2-S-20414DUP } & 24.5 & 4 / 6 / 06 & 9: 00 & 4545 & \text { Soil } \\ \text { TI-2 } & \text { NATI2-S-20419DUP } & 38 & 4 / 6 / 06 & 10: 22 & 4545 & \text { Soil } \\ \text { TI-3 } & \text { NATI3-S-20297DUP } & 25.5 & 4 / 7 / 06 & 8: 26 & 4104 & \text { Soil } \\ \text { TI-4 } & \text { NATI4-S-20324DUP } & 61 & 4 / 9 / 06 & 17: 52 & 4108 & \text { Soil } \\ \text { TI-5 } & \text { NATI5-S-20334DUP } & 17.2 & 4 / 11 / 06 & 9: 03 & 4105 & \text { Soil } \\ \text { TI-5 } & \text { NATI5-S-20364DUP } & 49.5 & 4 / 11 / 06 & 16: 16 & 3768 & \text { Soil } \\ \text { TI-6 } & \text { NATI6-S-20390DUP } & 2 & 4 / 20 / 06 & 8: 34 & 4709 & \text { Soil } \\ \text { TI-7 } & \text { NATI7-S-20449DUP } & 29 & 4 / 9 / 06 & 15: 36 & 4109 & \text { Soil } \\ \text { TI-10 } & \text { NATI10-S-20625 } & 43 & 4 / 26 / 06 & 11: 43 & 4716 & \text { Soil } \\ \text { TI-10 } & \text { NATI10-S-20636DUP } & 54 & 4 / 26 / 06 & 14: 58 & 4717 & \text { Soil } \\ \text { TI-11 } & \text { NATI11-S-20495DUP } & 38 & 4 / 23 / 06 & 11: 50 & 4702 & \text { Soil } \\ \text { TI-11 } & \text { NATI11-S-20496DUP } & 42 & 4 / 23 / 06 & 13: 44 & 4702 & \text { Soil } \\ \text { TI-14 } & \text { NATI14-S-20671DUP } & 35.2 & 5 / 5 / 06 & 14: 57 & 4135 & \text { Soil } \\ \text { TI-14 } & \text { NATI14-S-20674DUP } & 44.5 & 5 / 5 / 06 & 16: 57 & 4132 & \text { Soil }\end{array}$

$\begin{array}{lll}\text { CPT } & 06-1 ; 24 & \text { Duplicate analysis. } \\ \text { CPT } & 06-1 ; 4 & \text { Duplicate analysis. } \\ \text { CPT } & 06-1 ; 4 & \text { Duplicate analysis. } \\ \text { CPT } & 06-3 ; 5 & \text { Duplicate analysis. } \\ \text { CPT } & 06-3 ; 20 & \text { Duplicate analysis. } \\ \text { CPT } & 06-3 ; 28 & \text { Duplicate analysis. } \\ \text { CPT } & 06-3 ; 28 & \text { Duplicate analysis. } \\ \text { CPT } & 06-3 ; 108 & \text { Duplicate analysis. } \\ \text { CPT } & 06-1 ; 20 & \text { Duplicate analysis. } \\ \text { CPT } & 06-1 ; 184 & \text { Replicate of sample NATI10-S-20624. } \\ \text { CPT } & 06-1 ; 184 & \text { Duplicate analysis. } \\ \text { CPT } & 06-3 ; 156 & \text { Duplicate analysis. } \\ \text { CPT } & 06-3 ; 156 & \text { Duplicate analysis. } \\ \text { CPT } & 06-4 ; 60 & \text { Duplicate analysis. } \\ \text { CPT } & 06-4 ; 60 & \text { Duplicate analysis. }\end{array}$

CPT 06-3; 16 Replicate of sample NATI1-S-20306.

CPT $06-1 ; 52$ Replicate of sample NATI1-S-20482.

CPT $06-1 ; 4 \quad$ Replicate of sample NATI2-S-20411.

CPT 06-3; 68 Replicate of sample NATI3-S-20379.

CPT 06-3; 20 Replicate of sample NATI4-S-20317.

CPT 06-3; 28 Replicate of sample NATI5-S-20356.

CPT 06-3; 108 Replicate of sample NATI6-S-20402.

CPT 06-3; 180 Replicate of sample NATI8-S-20526.

CPT 06-1;172 Replicate of sample NATI9-S-20595.

CPT 06-1; 184 Replicate of sample NATI10-S-20624.

CPT 06-3; 156 Replicate of sample NATI11-S-20503.

CPT 06-5; 32 Replicate of sample NATI12-S-20739.

CPT 06-7; 8 Replicate of sample NATI18-S-20678.

CPT 06-7; 4 Replicate of sample NATI28-S-20244.

CPT 06-7; 64 Replicate of sample NATI29-S-20897.

NATI14-S-20674DUP

СРT $06-4 ; 60$

Duplicate analysis. 
TABLE S4.1 (Cont.)

\begin{tabular}{|c|c|c|c|c|c|c|c|c|c|}
\hline Location & Sample & $\begin{array}{c}\text { Depth } \\
\text { (ft BGL) }\end{array}$ & $\begin{array}{c}\text { Sample } \\
\text { Date }\end{array}$ & Time & $\mathrm{cOC}^{\mathrm{a}}$ & Medium & $\begin{array}{l}\text { Sample } \\
\text { Type }^{b}\end{array}$ & $\begin{array}{l}\text { Log; } \\
\text { Page }^{\text {C }}\end{array}$ & Sample Description \\
\hline
\end{tabular}

Soil samples selected by the AGEM Laboratory for duplicate organic analyses (cont.)

\begin{tabular}{|c|c|c|c|c|c|c|c|c|c|}
\hline $\mathrm{TI}-15$ & NATI15-S-20766DUP & 60.8 & $5 / 6 / 06$ & $10: 40$ & 4731 & Soil & CPT & $06-4 ; 56$ & Duplicate analysis. \\
\hline $\mathrm{TI}-17$ & NATI17-S-20798DUP & 9.1 & $5 / 7 / 06$ & $10: 40$ & 4729 & Soil & СРТ & $06-4 ; 138$ & Duplicate analysis. \\
\hline $\mathrm{TI}-17$ & NATI17-S-20810DUP & 49.25 & $5 / 7 / 06$ & $16: 37$ & 4729 & Soil & СРТ & $06-4 ; 138$ & Duplicate analysis. \\
\hline TI-18 & NATI18-S-20677DUP & 37 & $5 / 19 / 06$ & $10: 27$ & 4724 & Soil & CPT & $06-7 ; 8$ & Duplicate analysis. \\
\hline TI-28 & NATI28-S-20244DUP & 32.8 & $5 / 18 / 06$ & $16: 03$ & 4724 & Soil & CPT & $06-7 ; 4$ & Duplicate analysis. \\
\hline TI-29 & NATI29-S-20703DUP & 13.5 & $5 / 19 / 06$ & $9: 50$ & 4157 & Soil & СРT & $06-7 ; 64$ & Duplicate analysis. \\
\hline TI-29 & NATI29-S-20894DUP & 53 & $5 / 19 / 06$ & $17: 47$ & 4157 & Soil & CPT & $06-7 ; 64$ & Duplicate analysis. \\
\hline $\mathrm{CP} 1$ & NACP1-S-20329DUP & 9.1 & $4 / 10 / 06$ & $11: 30$ & 4105 & Soil & СРT & $06-3 ; 32$ & Duplicate analysi \\
\hline
\end{tabular}

Replicate groundwater samples

$\begin{array}{ll}\text { TI-1 } & \text { NATI1-W-20343 } \\ \text { MW2 } & \text { NAQCDU-W-20200 } \\ \text { NW-1 } & \text { NAQCDU-W-20225 } \\ \text { L-1 } & \text { NAQCDU-W-20213 } \\ \text { TI-2 } & \text { NATI2-W-20278 } \\ \text { TI-2 } & \text { NATI2-W-20433 } \\ \text { TI-3 } & \text { NATI3-W-20384 } \\ \text { TI-4 } & \text { NATI4-W-20471 } \\ \text { TI-6 } & \text { NATI6-W-20351 } \\ \text { TI-7 } & \text { NATI7-W-20271 } \\ \text { TI-10 } & \text { NATI10-W-20634 } \\ \text { TI-11 } & \text { NATI11-W-20501 } \\ \text { TI-12 } & \text { NATI12-W-20729 } \\ \text { TI-13 } & \text { NATI13-W-20820 } \\ & \\ \text { TI-13 } & \text { NATI13-W-20556 } \\ \text { TI-15 } & \text { NATI15-W-20821 } \\ & \\ \text { TI-15 } & \text { NATI15-W-20823 } \\ \text { TI-15 } & \text { NATI15-W-20762 } \\ \text { TI-17 } & \text { NATI17-W-20827 } \\ \text { TI-18 } & \text { NATI18-W-20832 }\end{array}$

$\begin{array}{cclll}38-43 & 4 / 13 / 06 & 19: 20 & 3698 & \text { Water } \\ 42.8-57.8 & 4 / 6 / 06 & 9: 00 & 3711 & \text { Water } \\ 40-50 & 4 / 8 / 06 & 11: 48 & 3518 & \text { Water } \\ 75-95 & 4 / 8 / 06 & 9: 50 & 3518 & \text { Water } \\ 31-36 & 4 / 21 / 06 & 11: 48 & 4710 & \text { Water } \\ 69-72 & 4 / 7 / 06 & 16: 53 & 3518 & \text { Water } \\ 43-48 & 4 / 14 / 06 & 7: 50 & 3698 & \text { Water } \\ 55-60 & 4 / 11 / 06 & 20: 01 & 3685 & \text { Water } \\ 68-73 & 4 / 22 / 06 & 13: 20 & 4704 & \text { Water } \\ 37-42 & 4 / 20 / 06 & 16: 00 & 4706 & \text { Water } \\ 46-51 & 4 / 27 / 06 & 8: 59 & 4719 & \text { Water } \\ 46.3-51.3 & 4 / 23 / 06 & 17: 42 & 4704 & \text { Water } \\ 39.4-44.4 & 5 / 2 / 06 & 15: 50 & 4124 & \text { Water } \\ 42-47 & 5 / 9 / 06 & 9: 05 & 4143 & \text { Water } \\ & & & & \\ 66.8-71.8 & 5 / 4 / 06 & 8: 40 & 4141 & \text { Water } \\ 15-20 & 5 / 9 / 06 & 9: 26 & 4143 & \text { Water } \\ & & & & \\ 25-30 & 5 / 9 / 06 & 9: 49 & 4143 & \text { Water } \\ 67.1-72.1 & 5 / 6 / 06 & 13: 10 & 4730 & \text { Water } \\ 39-44 & 5 / 9 / 06 & 11: 55 & 4143 & \text { Water } \\ 35-40 & 5 / 10 / 06 & 7: 50 & 3521 & \text { Water }\end{array}$

CPT 06-1;61 Replicate of sample NATI1-W-20342.

MW 06-BN; 14 Replicate of sample NAMW02-W-20188.

MW 06-BN; 54 Replicate of sample NANW1-W-20215.

MW 06-BN; 50 Replicate of sample NAL1-W-20211.

CPT 06-1; 129 Replicate of sample NATI2-W-20277.

CPT $06-1 ; 13 \quad$ Replicate of sample NATI2-W-20432.

CPT $06-3 ; 85$ Replicate of sample NATI3-W-20383.

CPT $06-1 ; 37$ Replicate of sample NATI4-W-20470.

CPT $06-3 ; 145$ Replicate of sample NATI6-W-20350.

CPT 06-1; 105 Replicate of sample NATI7-W-20270.

CPT 06-1; 201 Replicate of sample NATI10-W-20632.

CPT $\quad 06-3 ; 165$ Replicate of sample NATI11-W-20500.

CPT $06-5 ; 13 \quad$ Replicate of sample NATI12-W-20728.

CPT $06-5 ; 77$ Replicate of sample NATI13-W-20562 collected on $5 / 5 / 06$.

CPT 06-4; 37 Replicate of sample NATI13-W-20555.

CPT 06-5; $81 \quad$ Replicate of sample NATI15-W-20644 collected on $5 / 8 / 06$.

CPT $06-5 ; 89$ Replicate of sample NATI15-W-20822.

CPT 06-4;89 Replicate of sample NATI15-W-20761.

CPT $06-5 ; 105$ Replicate of sample NATI17-W-20826.

CPT $06-5 ; 125$ Replicate of sample NATI18-W-20831. 
TABLE S4.1 (Cont.)

\begin{tabular}{|c|c|c|c|c|c|c|c|c|c|}
\hline Location & Sample & $\begin{array}{c}\text { Depth } \\
\text { (ft BGL) }\end{array}$ & $\begin{array}{c}\text { Sample } \\
\text { Date }\end{array}$ & Time & $\mathrm{COC}^{\mathrm{a}}$ & Medium & $\begin{array}{c}\text { Sample } \\
\text { Type }^{\text {b }}\end{array}$ & $\begin{array}{l}\text { Log; } \\
\text { Page }^{c}\end{array}$ & Sample Description \\
\hline
\end{tabular}

Replicate groundwater samples (cont.)

$\begin{array}{lccclll}\text { TI-18 } & \text { NATI18-W-20710 } & 56-61 & 5 / 21 / 06 & 18: 30 & 4156 & \text { Water } \\ \text { TI-19 } & \text { NATI19-W-20871 } & 46-51 & 5 / 11 / 06 & 14: 15 & 4149 & \text { Water } \\ \text { TI-20 } & \text { NATI20-W-20923 } & 72-77 & 5 / 23 / 06 & 16: 00 & 4158 & \text { Water } \\ \text { TI-21 } & \text { NATI21-W-20879 } & 60-65 & 5 / 11 / 06 & 21: 20 & 4147 & \text { Water } \\ \text { TI-22 } & \text { NATI22-W-20835 } & 39-44 & 5 / 10 / 06 & 8: 10 & 3521 & \text { Water } \\ \text { TI-22 } & \text { NATI22-W-20847 } & 68.2-73.2 & 5 / 10 / 06 & 11: 28 & 3521 & \text { Water } \\ \text { TI-23 } & \text { NATI23-W-20857 } & 60-65 & 5 / 10 / 06 & 17: 54 & 4146 & \text { Water } \\ \text { TI-24 } & \text { NATI24-W-20839 } & 39-44 & 5 / 10 / 06 & 11: 25 & 3521 & \text { Water } \\ \text { TI-25 } & \text { NATI25-W-20876 } & 39-44 & 5 / 11 / 06 & 18: 10 & 4147 & \text { Water } \\ \text { TI-26 } & \text { NATI26-W-20230 } & 53-58 & 5 / 11 / 06 & 22: 55 & 4148 & \text { Water } \\ \text { TI-27 } & \text { NATI27-W-20701 } & 49-54 & 5 / 20 / 06 & 9: 20 & 4154 & \text { Water } \\ \text { TI-28 } & \text { NATI28-W-20255 } & 58-63 & 5 / 19 / 06 & 14: 10 & 4761 & \text { Water } \\ \text { TI-29 } & \text { NATI29-W-20901 } & 39-44 & 5 / 21 / 06 & 9: 15 & 4156 & \text { Water } \\ \text { TI-30 } & \text { NATI30-W-20919 } & 75-80 & 5 / 23 / 06 & 11: 05 & 4158 & \text { Water } \\ \text { TI-31 } & \text { NATI31-W-20936 } & 70.7-75.7 & 5 / 24 / 06 & 18: 40 & 4127 & \text { Water }\end{array}$

Groundwater samples selected by the AGEM Laboratory for duplicate organic analyses

$\begin{array}{lllllll}\text { MW3 } & \text { NAMW03-W-20192 } & 44-59 & 4 / 6 / 06 & 11: 48 & 3711 & \text { Water } \\ & & & & & & \\ \text { L-1 } & \text { NAL1-W-20211 } & 75-95 & 4 / 8 / 06 & 9: 50 & 3518 & \text { Water } \\ & & & & & & \\ \text { TI-2 } & \text { NATI2-W-20282 } & 66-70 & 4 / 21 / 06 & 15: 30 & 4710 & \text { Water } \\ \text { TI-2 } & \text { NATI2-W-20432 } & 69-72 & 4 / 7 / 06 & 15: 25 & 3713 & \text { Water } \\ \text { TI-4 } & \text { NATI4-W-20472 } & 61-66 & 4 / 12 / 06 & 10: 10 & 3685 & \text { Water } \\ \text { TI-6 } & \text { NATI6-W-20353 } & 38-43 & 4 / 22 / 06 & 15: 04 & 4704 & \text { Water } \\ & & & & & & \\ \text { TI-7 } & \text { NATI7-W-20271 } & 37-42 & 4 / 20 / 06 & 16: 00 & 4706 & \text { Water } \\ \text { TI-10 } & \text { NATI10-W-20637 } & 59-64 & 4 / 27 / 06 & 13: 10 & 4719 & \text { Water }\end{array}$

06-BN; 06-BN; 22 Depth to water from TOC $=31.58 \mathrm{ft}$ BGL. Depth of well $=58 \mathrm{ft} \mathrm{BGL}$. Sample collected after purging of approximately 14 gal.

06-BN; 06-BN; 50 Depth to water from TOC $=29.34 \mathrm{ft} \mathrm{BGL}$. Depth 50

of well $=95.35 \mathrm{ft} \mathrm{BGL}$. Sample collected after purging of 130 gal.

06-1; 14906-1; 149 Could not penetrate below 70 ft BGL. Heavily sedimented, high turbidity.

$06-1 ; 9 \quad 06-1 ; 9 \quad$ Water at $47 \mathrm{ft} \mathrm{BGL}$ in riser pipe. High turbidity

06-1; 41 06-1; $41 \quad$ No description recorded.

06-3; 15306-3; 153 South of southwest corner of dry fertilizer building. Slightly turbid water.

06-1; 10506-1; 105 Replicate of sample NATI7-W-20270.

06-2; 9 06-2; $9 \quad$ Slow water recovery initially, but $30 \mathrm{ft}$ of water after $1 \mathrm{hr}$ 
TABLE S4.1 (Cont.)

\begin{tabular}{|c|c|c|c|c|c|c|c|c|c|}
\hline Location & Sample & $\begin{array}{c}\text { Depth } \\
\text { (ft BGL) }\end{array}$ & $\begin{array}{c}\text { Sample } \\
\text { Date }\end{array}$ & Time & $\mathrm{COC}^{\mathrm{a}}$ & Medium & $\begin{array}{c}\text { Sample } \\
\text { Type }^{b}\end{array}$ & $\begin{array}{c}\text { Log; } \\
\text { Page }^{\mathrm{C}}\end{array}$ & Sample Description \\
\hline
\end{tabular}

Groundwater samples selected by the AGEM Laboratory for duplicate organic analyses (cont.)

\begin{tabular}{|c|c|c|c|c|c|c|c|c|}
\hline TI-10 & NATI10-W-20610 & $66-71$ & $4 / 25 / 06$ & 14:20 & 4722 & Water & $06-1 ; 18106-1 ; 181$ & $\begin{array}{l}\text { Water slow entering borehole; ample water after } \\
\text { a few minutes. }\end{array}$ \\
\hline TI-11 & NATI11-W-20639 & $65.9-70.9$ & $4 / 27 / 06$ & $17: 13$ & 4719 & Water & $06-2 ; 1706-2 ; 17$ & Abundant water present. \\
\hline $\mathrm{TI}-12$ & NATI12-W-20727 & 34-39 & $5 / 2 / 06$ & $15: 30$ & 4124 & Water & $06-5 ; 5 \quad 06-5 ; 5$ & $\begin{array}{l}\text { Southeast corner of former CCC/USDA property. } \\
\text { Slightly turbid. Nitrate sample collected for } \\
\text { Co-op. }\end{array}$ \\
\hline TI-14 & NATI14-W-20666 & $67.4-72.4$ & $5 / 5 / 06$ & $9: 30$ & 4026 & Water & $06-4 ; 5306-4 ; 53$ & $\begin{array}{l}\text { West side of liquid fertilizer containment. Slightly } \\
\text { turbid. }\end{array}$ \\
\hline $\mathrm{TI}-16$ & NATI16-W-20791 & $39-44$ & $5 / 8 / 06$ & $16: 50$ & 4144 & Water & $06-4 ; 16106-4 ; 161$ & Very turbid water. \\
\hline $\mathrm{TI}-17$ & NATI17-W-20829 & $53-58$ & $5 / 9 / 06$ & $13: 13$ & 4143 & Water & $06-5 ; 11306-5 ; 113$ & Very silty. \\
\hline TI-18 & NATI18-W-20710 & $56-61$ & $5 / 21 / 06$ & $18: 30$ & 4156 & Water & $06-7 ; 15706-7 ; 157$ & Replicate of sample NATI18-W-20709. \\
\hline TI-19 & NATI19-W-20868 & $53-58$ & $5 / 11 / 06$ & $15: 14$ & 4146 & Water & $06-6 ; 3306-6 ; 33$ & Fairly silty. \\
\hline TI-21 & NATI21-W-20885 & $32-37$ & $5 / 11 / 06$ & $22: 07$ & 4147 & Water & $06-6 ; 10106-6 ; 101$ & $\begin{array}{l}\text { Very little water, silty. No field parameters } \\
\text { measured. }\end{array}$ \\
\hline TI-21 & NATI21-W-20880 & $60-65$ & $5 / 11 / 06$ & $21: 15$ & 4147 & Water & $06-6 ; 8106-6 ; 81$ & $\begin{array}{l}\text { Northeast corner of feed mill building. Very } \\
\text { silty/cloudy sample. Abundant water. }\end{array}$ \\
\hline TI-24 & NATI24-W-20843 & $60-65$ & $5 / 10 / 06$ & $16: 10$ & 4145 & Water & $06-5 ; 17706-5 ; 177$ & Abundant water, very silty. \\
\hline TI-24 & NATI24-W-20845 & $69.4-74.4$ & $5 / 10 / 06$ & $17: 20$ & 4146 & Water & $06-5 ; 18106-5 ; 181$ & Silty water, abundant. \\
\hline TI-26 & NATI26-W-20719 & $66-71$ & $5 / 12 / 06$ & $1: 39$ & 4148 & Water & $06-6 ; 14506-6 ; 145$ & Cloudy sample. \\
\hline TI-28 & NATI28-W-20849 & $37-42$ & $5 / 20 / 06$ & $7: 48$ & 4154 & Water & $06-7 ; 53$ 06-7; 53 & Slightly turbid. \\
\hline TI-28 & NATI28-W-20683 & $51-56$ & $5 / 19 / 06$ & $16: 21$ & 4761 & Water & $06-7 ; 4506-7 ; 45$ & Slightly turbid. \\
\hline TI-30 & NATI30-W-20917 & $75-80$ & $5 / 23 / 06$ & $11: 05$ & 4158 & Water & 06-7; 19306-7; 193 & Slightly turbid. \\
\hline TI-30 & NATI30-W-20712 & $83.5-88.5$ & $5 / 24 / 06$ & $7: 15$ & 4030 & Water & $06-8 ; 3306-8 ; 33$ & No description recorded. \\
\hline
\end{tabular}

Soil samples selected for verification organic analysis at Severn-Trent Laboratories

$\begin{array}{llcllll}\text { TI-1 } & \text { NATI1-S-20303 } & 9 & 4 / 7 / 06 & 14: 31 & 4023 & \text { Soil } \\ \text { TI-1 } & \text { NATI1-S-20476 } & 32.7 & 4 / 13 / 06 & 11: 50 & 4023 & \text { Soil } \\ \text { TI-1 } & \text { NATI1-S-20340 } & 59.5 & 4 / 13 / 06 & 18: 20 & 4023 & \text { Soil } \\ \text { TI-2 } & \text { NATI2-S-20408 } & 9 & 4 / 5 / 06 & 17: 10 & 4023 & \text { Soil } \\ \text { TI-2 } & \text { NATI2-S-20413 } & 20.5 & 4 / 5 / 06 & 18: 22 & 4023 & \text { Soil } \\ \text { TI-3 } & \text { NATI3-S-20295 } & 22 & 4 / 6 / 06 & 15: 55 & 4023 & \text { Soil }\end{array}$

$\begin{array}{lll}\text { CPT } & 06-3 ; 16 & \text { No description recorded. } \\ \text { CPT } & 06-1 ; 52 & \text { No description recorded. } \\ \text { CPT } & 06-1 ; 52 & \text { No description recorded. } \\ \text { CPT } & 06-1 ; 4 & \text { No description recorded. } \\ \text { CPT } & 06-1 ; 4 & \text { No description recorded. } \\ \text { CPT } & 06-3 ; 5 & \text { No description recorded. }\end{array}$


TABLE S4.1 (Cont.)

\begin{tabular}{|c|c|c|c|c|c|c|c|c|c|}
\hline Location & Sample & $\begin{array}{c}\text { Depth } \\
\text { (ft BGL) }\end{array}$ & $\begin{array}{c}\text { Sample } \\
\text { Date }\end{array}$ & Time & $\mathrm{COC}^{\mathrm{a}}$ & Medium & $\begin{array}{c}\text { Sample } \\
\text { Type }^{b}\end{array}$ & $\begin{array}{c}\text { Log; } \\
\text { Page }^{\mathrm{C}}\end{array}$ & Sample Description \\
\hline
\end{tabular}

Soil samples selected for verification organic analysis at Severn-Trent Laboratories (cont.)

\begin{tabular}{|c|c|c|c|c|c|c|c|c|c|}
\hline $\mathrm{TI}-4$ & NATI4-S-20308 & 2 & $4 / 8 / 06$ & $15: 30$ & 4023 & Soil & CPT & $06-3 ; 20$ & $\begin{array}{l}\text { Vertical soil profile at TI-4 location, west of the } \\
\text { dry fertilizer storage building along west side } \\
\text { of road. }\end{array}$ \\
\hline $\mathrm{TI}-4$ & NATI4-S-20311 & 14.5 & $4 / 8 / 06$ & $17: 03$ & 4023 & Soil & CPT & $06-3 ; 20$ & No description recorded. \\
\hline TI-5 & NATI5-S-20358 & 30 & $4 / 11 / 06$ & $10: 44$ & 4023 & Soil & CPT & $06-3 ; 28$ & No description recorded. \\
\hline TI-6 & NATI6-S-20390 & 2 & $4 / 20 / 06$ & $8: 34$ & 4024 & Soil & CPT & $06-3 ; 108$ & $\begin{array}{l}\text { Vertical soil profile at TI- } 6 \text { location south, of dry } \\
\text { fertilizer storage building. }\end{array}$ \\
\hline TI-7 & NATI7-S-20453 & 41 & $4 / 9 / 06$ & $17: 38$ & 4023 & Soil & CPT & $06-1 ; 20$ & No description recorded. \\
\hline TI-7 & NATI7-S-20454 & 43 & $4 / 9 / 06$ & $17: 38$ & 4023 & Soil & CPT & $06-1 ; 20$ & No description recorded. \\
\hline TI-8 & NATI8-S-20511 & 16.5 & $4 / 26 / 06$ & $13: 28$ & 4024 & Soil & CPT & $06-3 ; 180$ & No description recorded. \\
\hline TI-8 & NATI8-S-20527 & 54.6 & $4 / 27 / 06$ & $11: 50$ & 4024 & Soil & CPT & $06-3 ; 180$ & Replicate of sample NATI8-S-20526. \\
\hline TI-9 & NATI9-S-20603 & 50 & $4 / 23 / 06$ & $10: 15$ & 4024 & Soil & CPT & $06-1 ; 172$ & No description recorded. \\
\hline TI-9 & NATI9-S-20607 & 65 & $4 / 23 / 06$ & $16: 51$ & 4024 & Soil & CPT & $06-1 ; 172$ & No description recorded. \\
\hline $\mathrm{TI}-10$ & NATI10-S-20620 & 29.5 & $4 / 26 / 06$ & $10: 00$ & 4024 & Soil & CPT & $06-1 ; 184$ & No description recorded. \\
\hline TI-10 & NATI10-S-20636 & 54 & $4 / 26 / 06$ & $14: 58$ & 4025 & Soil & CPT & $06-1 ; 184$ & No description recorded. \\
\hline $\mathrm{TI}-11$ & NATI11-S-20496 & 42 & $4 / 23 / 06$ & $13: 44$ & 4024 & Soil & CPT & $06-3 ; 156$ & No description recorded. \\
\hline $\mathrm{TI}-12$ & NATI12-S-20735 & 5.5 & $5 / 3 / 06$ & $10: 50$ & 4025 & Soil & CPT & $06-5 ; 32$ & No description recorded. \\
\hline $\mathrm{TI}-12$ & NATI12-S-20750 & 48.5 & $5 / 3 / 06$ & $17: 59$ & 4025 & Soil & CPT & $06-5 ; 32$ & No description recorded. \\
\hline $\mathrm{TI}-13$ & NATI13-S-20538 & 15.5 & $5 / 2 / 06$ & $17: 50$ & 4025 & Soil & CPT & $06-4 ; 20$ & No description recorded. \\
\hline $\mathrm{TI}-13$ & NATI13-S-20541 & 27 & $5 / 3 / 06$ & $11: 40$ & 4025 & Soil & CPT & $06-4 ; 20$ & No description recorded. \\
\hline $\mathrm{TI}-13$ & NATI13-S-20543 & 34.5 & $5 / 3 / 06$ & $13: 42$ & 4025 & Soil & CPT & $06-4 ; 20$ & No description recorded. \\
\hline $\mathrm{TI}-13$ & NATI13-S-20545 & 38 & $5 / 3 / 06$ & $14: 17$ & 4025 & Soil & CPT & $06-4 ; 20$ & No description recorded. \\
\hline TI-14 & NATI14-S-20664 & 20.8 & $5 / 5 / 06$ & $13: 25$ & 4025 & Soil & CPT & $06-4 ; 60$ & No description recorded. \\
\hline $\mathrm{TI}-14$ & NATI14-S-20673 & 40.5 & $5 / 5 / 06$ & $16: 18$ & 4027 & Soil & CPT & $06-4 ; 60$ & No description recorded. \\
\hline $\mathrm{TI}-14$ & NATI14-S-20689 & 57.8 & $5 / 6 / 06$ & $8: 20$ & 4027 & Soil & CPT & $06-4 ; 60$ & No description recorded. \\
\hline $\mathrm{TI}-15$ & NATI15-S-20575 & 26.1 & $5 / 5 / 06$ & $14: 28$ & 4025 & Soil & CPT & $06-4 ; 56$ & No description recorded. \\
\hline TI-15 & NATI15-S-20578 & 34.6 & $5 / 5 / 06$ & $15: 45$ & 4027 & Soil & CPT & $06-4 ; 56$ & No description recorded. \\
\hline $\mathrm{TI}-16$ & NATI16-S-20773 & 9.25 & $5 / 7 / 06$ & $10: 02$ & 4027 & Soil & CPT & $06-4 ; 134$ & No description recorded. \\
\hline $\mathrm{TI}-16$ & NATI16-S-20783 & 36.5 & $5 / 7 / 06$ & $15: 28$ & 4027 & Soil & CPT & $06-4 ; 134$ & No description recorded. \\
\hline $\mathrm{TI}-17$ & NATI17-S-20808 & 42 & $5 / 7 / 06$ & $15: 20$ & 4027 & Soil & CPT & $06-4 ; 138$ & No description recorded. \\
\hline CP1 & NACP1-S-20330 & 14.8 & $4 / 10 / 06$ & $13: 30$ & 4023 & Soil & CPT & $06-3 ; 32$ & No description recorded. \\
\hline
\end{tabular}


TABLE S4.1 (Cont.)

\begin{tabular}{|c|c|c|c|c|c|c|c|c|c|}
\hline Location & Sample & $\begin{array}{c}\text { Depth } \\
\text { (ft BGL) }\end{array}$ & $\begin{array}{c}\text { Sample } \\
\text { Date }\end{array}$ & Time & $\mathrm{COC}^{\mathrm{a}}$ & Medium & $\begin{array}{c}\text { Sample } \\
\text { Type }^{b}\end{array}$ & $\begin{array}{c}\text { Log; } \\
\text { Page }^{c}\end{array}$ & Sample Description \\
\hline
\end{tabular}

Groundwater samples selected for verification organic analysis by Envirosystems, Inc.

\begin{tabular}{|c|c|c|c|c|c|c|c|c|c|}
\hline $\mathrm{L}-2$ & NAL2-W-20190 & $80-90$ & $4 / 6 / 06$ & $10: 15$ & 2478 & Water & MW & $06-\mathrm{BN} ; 18$ & $\begin{array}{l}\text { Depth to water from TOC }=29.49 \mathrm{ft} \text {. Depth of } \\
\text { well }=90.91 \mathrm{ft} \text { below TOC. Sample collected } \\
\text { after purging of } 31 \mathrm{gal} .\end{array}$ \\
\hline TI-2 & NATI2-W-20433 & 69-72 & $4 / 7 / 06$ & $16: 53$ & 2478 & Water & CPT & $06-1 ; 13$ & Replicate of sample NATI2-W-20432. \\
\hline TI-3 & NATI3-W-20298 & $32.2-37.2$ & $4 / 7 / 06$ & $10: 22$ & 2478 & Water & СPT & $06-3 ; 9$ & $\begin{array}{l}\text { First aliquot for volatile organic analyses clear; } \\
\text { second aliquot turbid. }\end{array}$ \\
\hline TI-4 & NATI4-W-20325 & $35-40$ & $4 / 10 / 06$ & $9: 35$ & 2478 & Water & СРТ & $06-3 ; 25$ & $\begin{array}{l}\text { West of northwest corner of dry fertilizer building. } \\
\text { Very turbid. }\end{array}$ \\
\hline $\mathrm{TI}-13$ & NATI13-W-20563 & $35-40$ & $5 / 5 / 06$ & $10: 00$ & 4026 & Water & CPT & $06-4 ; 69$ & Very turbid water. \\
\hline $\mathrm{TI}-13$ & NATI13-W-20562 & $42-47$ & $5 / 5 / 06$ & $9: 30$ & 4026 & Water & CPT & $06-4 ; 65$ & $\begin{array}{l}\text { Southwest corner of dry fertilizer building. Turbid } \\
\text { water. }\end{array}$ \\
\hline $\mathrm{TI}-13$ & NATI13-W-20560 & $48-53$ & $5 / 4 / 06$ & $17: 20$ & 4026 & Water & CPT & $06-4 ; 45$ & No description recorded. \\
\hline $\mathrm{TI}-13$ & NATI13-W-20561 & $54-59$ & $5 / 5 / 06$ & $8: 26$ & 4026 & Water & СРT & $06-4 ; 49$ & Slightly turbid. \\
\hline $\mathrm{TI}-14$ & NATI14-W-20666 & $67.4-72.4$ & $5 / 5 / 06$ & $9: 30$ & 4026 & Water & СРТ & $06-4 ; 53$ & $\begin{array}{l}\text { West side of liquid fertilizer containment. Slightly } \\
\text { turbid. }\end{array}$ \\
\hline $\mathrm{TI}-18$ & NATI18-W-20655 & 30-35 & $5 / 21 / 06$ & 15:05 & 4029 & Water & СPT & $06-7 ; 125$ & Slightly turbid. Conductivity not recorded. \\
\hline $\mathrm{TI}-18$ & NATI18-W-20706 & $42-47$ & $5 / 21 / 06$ & $16: 05$ & 4029 & Water & СPT & $06-7 ; 145$ & $\begin{array}{l}\text { South side of liquid fertilizer containment. Very } \\
\text { silty, turbid. }\end{array}$ \\
\hline $\mathrm{TI}-18$ & NATI18-W-20709 & $56-61$ & $5 / 21 / 06$ & $18: 30$ & 4029 & Water & CPT & $06-7 ; 153$ & Very silty, turbid. \\
\hline TI-20 & NATI20-W-20913 & $35-40$ & $5 / 22 / 06$ & $7: 30$ & 4030 & Water & CPT & $06-7 ; 177$ & Slightly turbid. \\
\hline TI-27 & NATI27-W-20700 & $49-54$ & $5 / 20 / 06$ & $9: 20$ & 4029 & Water & CPT & $06-7 ; 73$ & Very turbid. \\
\hline TI-27 & NATI27-W-20905 & $56-61$ & $5 / 21 / 06$ & $11: 20$ & 4029 & Water & CPT & $06-7 ; 121$ & Field measurements not recorded. \\
\hline TI-28 & NATI28-W-20904 & $25-30$ & $5 / 21 / 06$ & $12: 30$ & 4029 & Water & СРТ & $06-7 ; 117$ & $\begin{array}{l}\text { In ditch east of liquid fertilizer containment. } \\
\text { Slightly turbid. }\end{array}$ \\
\hline TI-29 & NATI29-W-20916 & $25-30$ & $5 / 23 / 06$ & $14: 50$ & 4030 & Water & CPT & 06-7;189 & Very silty. \\
\hline TI-29 & NATI29-W-20901 & $39-44$ & $5 / 21 / 06$ & $9: 15$ & 4029 & Water & CPT & $06-7 ; 105$ & Replicate of sample NATI29-W-20900. \\
\hline $\mathrm{TI}-30$ & NATI30-W-20920 & 39-44 & $5 / 23 / 06$ & $12: 30$ & 4030 & Water & CPT & $06-8 ; 9$ & $\begin{array}{l}\text { Northwest corner of J. Rock wheatfield, directly } \\
\text { west of dry fertilizer building. Water turbid. } \\
\text { Field parameters not measured. }\end{array}$ \\
\hline $\mathrm{TI}-30$ & NATI30-W-20919 & $75-80$ & $5 / 23 / 06$ & $11: 05$ & 4030 & Water & CPT & 06-7;201 & Replicate of sample NATI30-W-20917. \\
\hline TI-30 & NATI30-W-20712 & 83. $5-88.5$ & $5 / 24 / 06$ & $7: 15$ & 4030 & Water & СРT & $06-8 ; 33$ & No description recorded. \\
\hline
\end{tabular}


TABLE S4.1 (Cont.)

a Chain-of-custody form number.

b Sample types: CPT, cone penetrometer; MW, monitoring well; RI, rinsate; TB, trip blank.

c Location of record in logbook; on file at Argonne. 
TABLE S4.2 Results of carbon tetrachloride and chloroform analyses on quality control samples collected to monitor sample collection and handling activities during the 2006 investigation at Navarre, Kansas.

Concentration

$(\mu \mathrm{g} / \mathrm{L}$ in water; $\mu \mathrm{gkg}$ in soil)

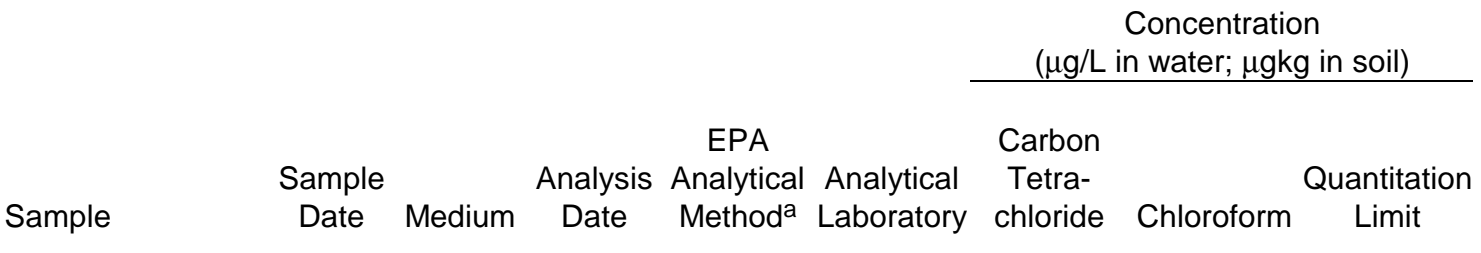

Field blank

NAQCFB-W-20199

Equipment rinsates

NAQCRI-W-20187

NAQCRI-W-20189

NAQCRI-W-20191

NAQCRI-W-20193

NAQCRI-W-20196

NAQCRI-W-20202

NAQCRI-W-20204

NAQCRI-W-20206

NAQCRI-W-20210

NATI3-W-20299

NAQCRI-W-20212

NAQCRI-W-20216

NATI4-W-20473

NAQCRIN-W-20372

NATI1-W-20345

NAQCRIN-W-20386

NATI5QC-W-20268

NATI2-W-20280

NATI6RIN-W-20352

NATI10QC-W-20633

NAQCRIN-W-20760

NAQCRIN-W-20769

NAQCRIN-W-20837

NAQCRIN-W-20874

NAQCRIN-W-20234

NAQCRIN-W-20648

NAQCRIN-W-20908

NAQCRIN-W-20924

Trip blanks

NAQCTB-W-20198

NAQCTB-W-20207

NAQCTB-W-20214

NAQCTB-W-20369

NAQCTB-W-20378

NAQCTB-W-20389

NAQCTB-W-20487

NAQCTB-W-20347

NATI9QC-W-20588 $\begin{array}{lllllll}\text { 4/6/06 Water 4/7/06 } & 524.2 & \text { AGEM } & \text { ND } & \text { ND } & 1.0\end{array}$

$\begin{array}{cccccccc}4 / 5 / 06 & \text { Water } & 4 / 7 / 06 & 524.2 & \text { AGEM } & \text { ND } & \text { ND } & 1.0 \\ 4 / 6 / 06 & \text { Water } & 4 / 7 / 06 & 524.2 & \text { AGEM } & \text { ND } & \text { ND } & 1.0 \\ 4 / 6 / 06 & \text { Water } & 4 / 7 / 06 & 524.2 & \text { AGEM } & \text { ND } & \text { ND } & 1.0 \\ 4 / 6 / 06 & \text { Water } & 4 / 7 / 06 & 524.2 & \text { AGEM } & \text { ND } & \text { ND } & 1.0 \\ 4 / 6 / 06 & \text { Water } & 4 / 7 / 06 & 524.2 & \text { AGEM } & 0.8 \text { Jc } & \text { ND } & 1.0 \\ 4 / 7 / 06 & \text { Water } & 4 / 8 / 06 & 524.2 & \text { AGEM } & 0.3 \mathrm{~J} & \text { ND } & 1.0 \\ 4 / 7 / 06 & \text { Water } & 4 / 8 / 06 & 524.2 & \text { AGEM } & \text { ND } & \text { ND } & 1.0 \\ 4 / 7 / 06 & \text { Water } & 4 / 8 / 06 & 524.2 & \text { AGEM } & \text { ND } & \text { ND } & 1.0 \\ 4 / 7 / 06 & \text { Water } & 4 / 11 / 06 & 524.2 & \text { AGEM } & \text { ND } & \text { ND } & 1.0 \\ 4 / 7 / 06 & \text { Water } & 4 / 8 / 06 & 524.2 & \text { AGEM } & \text { ND } & \text { ND } & 1.0 \\ 4 / 8 / 06 & \text { Water } & 4 / 11 / 06 & 524.2 & \text { AGEM } & \text { ND } & \text { ND } & 1.0 \\ 4 / 8 / 06 & \text { Water } & 4 / 11 / 06 & 524.2 & \text { AGEM } & \text { ND } & \text { ND } & 1.0 \\ 4 / 12 / 06 & \text { Water } & 4 / 13 / 06 & 524.2 & \text { AGEM } & \text { ND } & \text { ND } & 1.0 \\ 4 / 13 / 06 & \text { Water } & 4 / 14 / 06 & 524.2 & \text { AGEM } & \text { ND } & \text { ND } & 1.0 \\ 4 / 13 / 06 & \text { Water } & 4 / 18 / 06 & 524.2 & \text { AGEM } & \text { ND } & \text { ND } & 1.0 \\ 4 / 14 / 06 & \text { Water } & 4 / 18 / 06 & 524.2 & \text { AGEM } & \text { ND } & \text { ND } & 1.0 \\ 4 / 20 / 06 & \text { Water } & 4 / 21 / 06 & 524.2 & \text { AGEM } & \text { ND } & \text { ND } & 1.0 \\ 4 / 21 / 06 & \text { Water } & 4 / 25 / 06 & 524.2 & \text { AGEM } & \text { ND } & \text { ND } & 1.0 \\ 4 / 22 / 06 & \text { Water } & 4 / 25 / 06 & 524.2 & \text { AGEM } & \text { ND } & \text { ND } & 1.0 \\ 4 / 27 / 06 & \text { Water } & 4 / 28 / 06 & 524.2 & \text { AGEM } & \text { ND } & \text { ND } & 1.0 \\ 5 / 4 / 06 & \text { Water } & 5 / 8 / 06 & 524.2 & \text { AGEM } & \text { ND } & \text { ND } & 1.0 \\ 5 / 6 / 06 & \text { Water } & 5 / 12 / 06 & 524.2 & \text { AGEM } & 0.1 \mathrm{~J} & \text { ND } & 1.0 \\ 5 / 10 / 06 & \text { Water } & 5 / 12 / 06 & 524.2 & \text { AGEM } & \text { ND } & \text { ND } & 1.0 \\ 5 / 11 / 06 & \text { Water } & 5 / 13 / 06 & 524.2 & \text { AGEM } & \text { ND } & 0.1 \mathrm{~J} & 1.0 \\ 5 / 12 / 06 & \text { Water } & 5 / 13 / 06 & 524.2 & \text { AGEM } & \text { ND } & \text { ND } & 1.0 \\ 5 / 19 / 06 & \text { Water } & 5 / 20 / 06 & 524.2 & \text { AGEM } & \text { ND } & \text { ND } & 1.0 \\ 5 / 21 / 06 & \text { Water } & 5 / 23 / 06 & 524.2 & \text { AGEM } & \text { ND } & 0.2 \mathrm{~J} & 1.0 \\ 5 / 23 / 06 & \text { Water } & 5 / 24 / 06 & 524.2 & \text { AGEM } & \text { ND } & 0.2 \mathrm{~J} & 1.0\end{array}$

$\begin{array}{ccccc}4 / 6 / 06 & \text { Water } & 4 / 7 / 06 & 524.2 & \text { AGEM } \\ 4 / 7 / 06 & \text { Water } & 4 / 8 / 06 & 524.2 & \text { AGEM } \\ 4 / 8 / 06 & \text { Water } & 4 / 11 / 06 & 524.2 & \text { AGEM } \\ 4 / 12 / 06 & \text { Water } & 4 / 13 / 06 & 524.2 & \text { AGEM } \\ 4 / 13 / 06 & \text { Water } & 4 / 14 / 06 & 524.2 & \text { AGEM } \\ 4 / 14 / 06 & \text { Water } & 4 / 17 / 06 & 524.2 & \text { AGEM } \\ 4 / 20 / 06 & \text { Water } & 4 / 21 / 06 & 524.2 & \text { AGEM } \\ 4 / 21 / 06 & \text { Water } & 4 / 22 / 06 & 524.2 & \text { AGEM } \\ 4 / 22 / 06 & \text { Water } & 4 / 25 / 06 & 524.2 & \text { AGEM }\end{array}$

$\mathrm{ND}$

ND $\quad 1.0$

ND $\quad 1.0$

$\begin{array}{lll}\text { ND } & \text { ND } & 1.0 \\ \text { ND } & \text { ND } & 1.0\end{array}$

ND $\quad$ ND $\quad 1.0$

ND ND $\quad 1.0$

ND $\quad$ ND $\quad 1.0$

ND $\quad 0.1 \mathrm{~J} \quad 1.0$

ND ND $\quad 1.0$ 
TABLE S4.2 (Cont.)

Concentration

$(\mu \mathrm{g} / \mathrm{L}$ in water; $\mu \mathrm{gkg}$ in soil)

EPA Carbon

Sample Date Medium Date Method ${ }^{a}$ Laboratory chloride Chloroform Limit

Trip blanks (cont.)

NAQCTB-W-20717

NAQCTB-W-20518

NAQCTB-W-20726

NAQCTB-W-20730

NAQCTB-W-20733

NAQCTB-W-20759

NAQCTB-W-20584

NAQCTB-W-20694

NAQCTB-W-20824

NAQCTB-W-20866

NAQCTB-W-20873

NAQCTB-W-20724

NAQCTB-W-20682

NAQCTB-W-20702

NAQCTB-W-20650

NAQCTB-W-20925

NAQCTB-W-20714

NAQCTB-W-20944

NAQCTB-S-20197

NAQCTB-S-20208

NATripBlank-S-20226

NATripBlank-S-20227

NAQCTB-S-20362

NAQCTB-S-20377

NAQCTB-S-20387

NAQCTB-S-20486

NAQCTB-S-20346

NAQCTB-S-20716

NAQCTB-S-20519

NAQCTB-S-20529

NAQCTB-S-20551

NAQCTB-S-20552

NAQCTB-S-20559

NAQCTB-S-20582

NAQCTB-S-20583

NAQCTB-S-20767

NAQCTB-S-20794

NAQCTB-S-20795

NAQCTB-S-20263

NAQCTB-S-20681

NAQCTB-S-20899

NAQCTB-W-11APR06

NAQCTB-050706

\begin{tabular}{|c|c|c|c|c|c|c|c|}
\hline $4 / 24 / 06$ & Water & $4 / 25 / 06$ & 524.2 & AGEM & ND & ND & 1.0 \\
\hline $4 / 26 / 06$ & Water & $4 / 27 / 06$ & 524.2 & AGEM & ND & ND & 1.0 \\
\hline $4 / 27 / 06$ & Water & 4/28/06 & 524.2 & AGEM & ND & $0.2 \mathrm{~J}$ & 1.0 \\
\hline $5 / 2 / 06$ & Water & $5 / 3 / 06$ & 524.2 & AGEM & ND & ND & 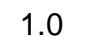 \\
\hline $5 / 3 / 06$ & Water & $5 / 4 / 06$ & 524.2 & AGEM & ND & ND & 1.0 \\
\hline $5 / 4 / 06$ & Water & $5 / 5 / 06$ & 524.2 & AGEM & ND & ND & .0 \\
\hline $5 / 5 / 06$ & Water & $5 / 5 / 06$ & 524.2 & AGEM & ND & $0.5 \mathrm{~J}$ & 4 \\
\hline $5 / 6 / 06$ & Water & $5 / 10 / 06$ & 524.2 & AGEM & ND & ND & \\
\hline $5 / 9 / 06$ & Water & $5 / 10 / 06$ & 524.2 & AGEM & ND & $0.1 \mathrm{~J}$ & \\
\hline $5 / 11 / 06$ & Water & $5 / 12 / 06$ & 524.2 & AGEM & ND & $0.1 \mathrm{~J}$ & 1.0 \\
\hline $5 / 11 / 06$ & Water & $5 / 13 / 06$ & 524.2 & AGEM & ND & ND & \\
\hline $5 / 12 / 06$ & Water & $5 / 15 / 06$ & 524.2 & AGEM & ND & ND & \\
\hline $5 / 19 / 06$ & Water & $5 / 20 / 06$ & 524.2 & AGEM & ND & ND & 1.0 \\
\hline $5 / 20 / 06$ & Waterd & - & - & - & - & - & \\
\hline $5 / 20 / 06$ & Water & $5 / 23 / 06$ & 524.2 & AGEM & ND & ND & 1. \\
\hline $5 / 23 / 06$ & Water & $5 / 24 / 06$ & 524.2 & AGEM & ND & $0.7 \mathrm{~J}$ & \\
\hline $5 / 24 / 06$ & Water & $5 / 25 / 06$ & 524.2 & AGEM & ND & ND & \\
\hline $5 / 25 / 06$ & Water & 5/26/06 & 524.2 & AGEM & ND & $0.3 \mathrm{~J}$ & 1.0 \\
\hline $4 / 6 / 06$ & Soil & $4 / 11 / 06$ & $8260 \mathrm{~B}$ & AGEM & ND & ND & 10 \\
\hline $4 / 7 / 06$ & Soil & $4 / 11 / 06$ & $8260 \mathrm{~B}$ & AGEM & ND & ND & \\
\hline 4/10/06 & Soil & 4/21/06 & $8260 \mathrm{~B}$ & AGEM & ND & ND & 10 \\
\hline 4/10/06 & Soil & 4/21/06 & $8260 \mathrm{~B}$ & AGEM & ND & ND & 10 \\
\hline $4 / 11 / 06$ & Soil & $4 / 21 / 06$ & $8260 \mathrm{~B}$ & AGEM & ND & ND & 10 \\
\hline $4 / 13 / 06$ & Soil & 4/21/06 & $8260 \mathrm{~B}$ & AGEM & ND & ND & 10 \\
\hline $4 / 14 / 06$ & Soil & 4/20/06 & $8260 \mathrm{~B}$ & AGEM & ND & ND & 10 \\
\hline $4 / 20 / 06$ & Soil & $4 / 26 / 06$ & $8260 B$ & AGEM & ND & ND & 10 \\
\hline $4 / 21 / 06$ & Soil & 4/26/06 & $8260 \mathrm{~B}$ & AGEM & ND & ND & 10 \\
\hline $4 / 24 / 06$ & Soil & $5 / 1 / 06$ & $8260 \mathrm{~B}$ & AGEM & $2.1 \mathrm{~J}$ & $2.7 \mathrm{~J}$ & 10 \\
\hline $4 / 26 / 06$ & Soil & $5 / 2 / 06$ & $8260 \mathrm{~B}$ & AGEM & $8.9 \mathrm{~J}$ & $4.2 \mathrm{~J}$ & 10 \\
\hline $4 / 27 / 06$ & Soil & $5 / 2 / 06$ & $8260 \mathrm{~B}$ & AGEM & ND & ND & 10 \\
\hline $5 / 3 / 06$ & Soil & $5 / 8 / 06$ & $8260 \mathrm{~B}$ & AGEM & $3.4 \mathrm{~J}$ & $2.3 \mathrm{~J}$ & 10 \\
\hline $5 / 3 / 06$ & Soil & $5 / 5 / 06$ & $8260 \mathrm{~B}$ & AGEM & ND & ND & 10 \\
\hline $5 / 4 / 06$ & Soil & $5 / 8 / 06$ & $8260 \mathrm{~B}$ & AGEM & ND & ND & 10 \\
\hline $5 / 5 / 06$ & Soil & $5 / 18 / 06$ & $8260 \mathrm{~B}$ & AGEM & ND & ND & 10 \\
\hline $5 / 5 / 06$ & Soil & $5 / 15 / 06$ & $8260 \mathrm{~B}$ & AGEM & $8 \mathrm{~J}$ & $4.9 \mathrm{~J}$ & 10 \\
\hline $5 / 6 / 06$ & Soil & $5 / 17 / 06$ & $8260 \mathrm{~B}$ & AGEM & ND & ND & 10 \\
\hline $5 / 8 / 06$ & Soil & $5 / 17 / 06$ & $8260 \mathrm{~B}$ & AGEM & ND & ND & 10 \\
\hline $5 / 8 / 06$ & Soil & $5 / 17 / 06$ & $8260 \mathrm{~B}$ & AGEM & ND & ND & 10 \\
\hline $5 / 18 / 06$ & Soil & $5 / 22 / 06$ & $8260 \mathrm{~B}$ & AGEM & ND & ND & 10 \\
\hline $5 / 19 / 06$ & Soil & $5 / 25 / 06$ & $8260 \mathrm{~B}$ & AGEM & ND & ND & 10 \\
\hline $5 / 21 / 06$ & Soil & 5/25/06 & $8260 \mathrm{~B}$ & AGEM & ND & ND & 10. \\
\hline $4 / 11 / 06$ & Water & $4 / 20 / 06$ & 8260 & ENVSY & ND & ND & 5. \\
\hline $5 / 8 / 06$ & Water & 05/13/06 & 8260 & ENVSY & ND & ND & 5. \\
\hline
\end{tabular}


TABLE S4.2 (Cont.)

\begin{tabular}{|c|c|c|c|c|c|c|c|c|}
\hline \multirow[b]{2}{*}{ Sample } & \multirow[b]{2}{*}{$\begin{array}{l}\text { Sample } \\
\text { Date }\end{array}$} & \multirow[b]{2}{*}{ Medium } & \multirow[b]{2}{*}{$\begin{array}{l}\text { Analysis } \\
\text { Date }\end{array}$} & \multirow[b]{2}{*}{$\begin{array}{c}\text { EPA } \\
\text { Analytical } \\
\text { Method }^{a}\end{array}$} & \multirow[b]{2}{*}{$\begin{array}{l}\text { Analytical } \\
\text { Laboratory }\end{array}$} & \multicolumn{3}{|c|}{$\begin{array}{c}\text { Concentration } \\
(\mu \mathrm{g} / \mathrm{L} \text { in water; } \mu \mathrm{gkg} \text { in soil })\end{array}$} \\
\hline & & & & & & $\begin{array}{c}\text { Carbon } \\
\text { Tetra- } \\
\text { chloride }\end{array}$ & Chloroform & $\begin{array}{l}\text { Quantitation } \\
\text { Limit }\end{array}$ \\
\hline \multicolumn{9}{|l|}{ Trip blanks (cont.) } \\
\hline NAQCTB-052306 & $5 / 23 / 06$ & Water & 05/24/06 & 8260 & ENVSY & $1.5 \mathrm{~J}$ & ND & 5.0 \\
\hline NAQCTB-052506 & $5 / 25 / 06$ & Water & $05 / 31 / 06$ & 8260 & ENVSY & ND & ND & 5.0 \\
\hline NA-MEOHBLANK-18APR06 & $4 / 18 / 06$ & Soil & $4 / 28 / 06$ & $8260 \mathrm{~B}$ & STL & ND & ND & 10.0 \\
\hline NA-S-BLANK-02MAY06 & $5 / 2 / 06$ & Soil & $5 / 10 / 06$ & $8260 B$ & STL & ND & ND & 10.0 \\
\hline NA-MEOHBLANK-08MAY06 & $5 / 8 / 06$ & Soil & $5 / 19 / 06$ & $8260 \mathrm{~B}$ & STL & ND & ND & 10.0 \\
\hline NA-MEOHBLANK-19MAY06 & $5 / 19 / 06$ & Soil & $5 / 24 / 06$ & $8260 B$ & STL & ND & $3.2 \mathrm{~J}$ & 10.0 \\
\hline
\end{tabular}

a Analytical methods: EPA Method 524.2 and EPA Method 8260B.

b Not detected at the method detection limit.

c Qualifier J indicates an estimated concentration below the indicated method quantitation limit.

d Vial was broken; sample not analyzed. 
TABLE S4.3 Shipment information showing potential for cross-contamination of some groundwater samples from location TI-27.

\begin{tabular}{|c|c|c|c|c|c|c|c|c|c|}
\hline \multirow[b]{2}{*}{ Location } & \multirow[b]{2}{*}{ Sample } & \multirow[b]{2}{*}{$\begin{array}{c}\text { Depth } \\
\text { (ft below TOC) }\end{array}$} & \multirow[b]{2}{*}{$\begin{array}{l}\text { Sample } \\
\text { Date }\end{array}$} & \multirow[b]{2}{*}{$\begin{array}{c}\text { COC } \\
\text { Number }\end{array}$} & \multirow[b]{2}{*}{$\begin{array}{l}\text { Shipment } \\
\text { Date }\end{array}$} & \multirow[b]{2}{*}{$\begin{array}{l}\text { Sample } \\
\text { Type }^{a}\end{array}$} & \multicolumn{3}{|c|}{ Concentration $(\mu \mathrm{g} / \mathrm{L})$} \\
\hline & & & & & & & $\begin{array}{c}\text { Carbon } \\
\text { Tetrachloride }\end{array}$ & Chloroform & $\begin{array}{c}\text { Methylene } \\
\text { Chloride }\end{array}$ \\
\hline \multicolumn{10}{|c|}{ Analysis of trip blank indicates no evidence of cross-contamination in shipment on May 19, 2006.} \\
\hline TI-27 & NATI27-W-20680 & $25-30$ & $5 / 19 / 06$ & 4761 & $5 / 19 / 06$ & СPT & 1.0 & $0.7 \mathrm{Jb}^{\mathrm{b}}$ & $N^{c}$ \\
\hline TI-27 & NATI27-W-20684 & $35-40$ & $5 / 19 / 06$ & 4761 & $5 / 19 / 06$ & CPT & ND & 1.6 & ND \\
\hline TI-28 & NATI28-W-20683 & $51-56$ & $5 / 19 / 06$ & 4761 & $5 / 19 / 06$ & СРT & 12 & 2.7 & ND \\
\hline TI-28 & NATI28-W-20254 & $58-63$ & $5 / 19 / 06$ & 4761 & $5 / 19 / 06$ & СPT & 15 & 8.9 & ND \\
\hline TI-28 & NATI28-W-20255 & $58-63$ & $5 / 19 / 06$ & 4761 & $5 / 19 / 06$ & СPT & 26 & 10 & ND \\
\hline TI-28 & NATI28-W-20647 & $63-68$ & $5 / 19 / 06$ & 4761 & $5 / 19 / 06$ & CPT & ND & ND & ND \\
\hline QC & NAQCTB-W-20682 & - & $5 / 19 / 06$ & 4761 & $5 / 19 / 06$ & TB & ND & ND & ND \\
\hline \multicolumn{10}{|c|}{ Trip blank was broken: potential for cross-contamination in shipment on May 20, 2006.} \\
\hline TI-27 & NATI27-W-20704 & 30-35 & $5 / 20 / 06$ & 4154 & $5 / 20 / 06$ & CPT & 1.1 & 2.3 & $0.2 \mathrm{~J}$ \\
\hline TI-27 & NATI27-W-20699 & $42-47$ & $5 / 20 / 06$ & 4154 & $5 / 20 / 06$ & СРТ & 7.9 & 3.5 & ND \\
\hline TI-27 & NATI27-W-20700 & $49-54$ & $5 / 20 / 06$ & 4154 & $5 / 20 / 06$ & СРТ & 2.0 & $0.7 \mathrm{~J}$ & ND \\
\hline TI-27 & NATI27-W-20701 & $49-54$ & $5 / 20 / 06$ & 4154 & $5 / 20 / 06$ & СРТ & 1.8 & $0.6 \mathrm{~J}$ & ND \\
\hline TI-28 & NATI28-W-20851 & $32-37$ & $5 / 20 / 06$ & 4154 & $5 / 20 / 06$ & CPT & 3,104 & 646 & 6.3 \\
\hline TI-28 & NATI28-W-20849 & $37-42$ & $5 / 20 / 06$ & 4154 & $5 / 20 / 06$ & СРТ & 97 & 91 & 1.0 \\
\hline TI-28 & NATI28-W-20850 & $44-49$ & $5 / 20 / 06$ & 4154 & $5 / 20 / 06$ & СРT & 88 & 14 & ND \\
\hline QC & NAQCTB-W-20702 & - & $5 / 20 / 06$ & 4154 & $5 / 20 / 06$ & $\mathrm{~TB}^{\mathrm{d}}$ & - & - & - \\
\hline \multicolumn{10}{|c|}{ Analysis of trip blank indicates no evidence of cross-contamination in shipment on May 22, 2006.} \\
\hline TI-27 & NATI27-W-20905 & $56-61$ & $5 / 21 / 06$ & 4156 & $5 / 22 / 06$ & СРТ & ND & $0.6 \mathrm{~J}$ & ND \\
\hline TI-27 & NATI27-W-20649 & $66.25-71.25$ & $5 / 20 / 06$ & 4156 & $5 / 22 / 06$ & СРT & ND & ND & ND \\
\hline TI-28 & NATI28-W-20904 & $25-30$ & $5 / 21 / 06$ & 4156 & $5 / 22 / 06$ & СРT & 2,692 & 238 & 1.3 \\
\hline QC & NAQCTB-W-20650 & - & $5 / 20 / 06$ & 4156 & $5 / 22 / 06$ & TB & ND & ND & ND \\
\hline
\end{tabular}

a Sample types: CPT, cone penetrometer; TB, trip blank.

b Qualifier J indicates an estimated concentration below the AGEM Laboratory method quantitation limit of $1.0 \mu \mathrm{g} / \mathrm{L}$.

c ND, not detected at a method detection limit of $0.1 \mu \mathrm{g} / \mathrm{L}$.

d Vial was broken during shipment; sample not analyzed. 
TABLE S4.4 Calibration and surrogate recovery data for organic analyses of soil and water samples at the AGEM Laboratory.

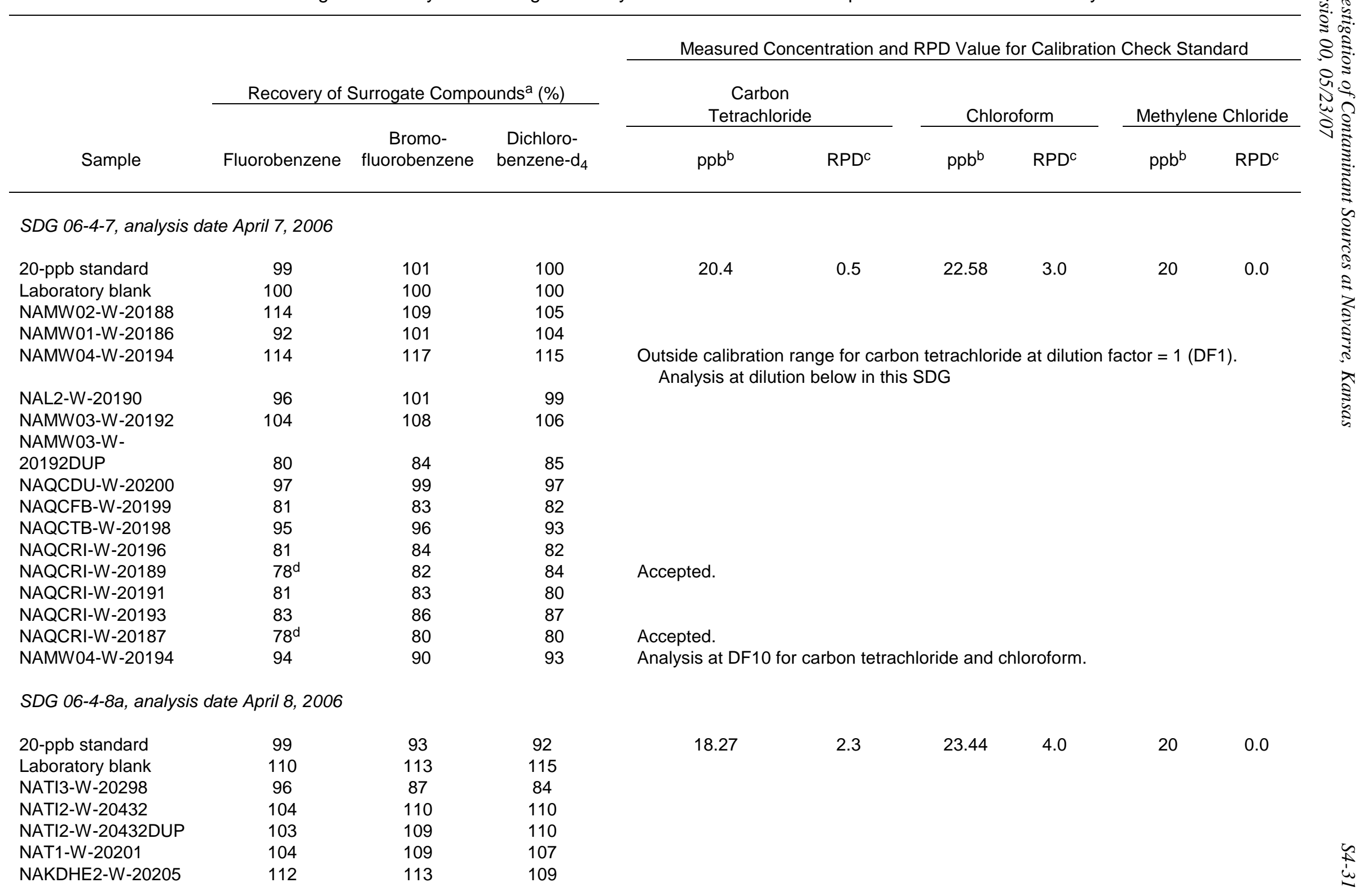


SDG 06-4-8a, analysis date April 8, 2006 (cont.)

$\begin{array}{lccc}\text { NAKDHE1-W-20203 } & 101 & 105 & 101 \\ \text { NATI3-W-20299 } & 116 & 114 & 108 \\ \text { NAQCTB-W-20207 } & 99 & 94 & 90 \\ \text { NAQCRI-W-20204 } & 108 & 106 & 104 \\ \text { NAQCRI-W-20206 } & 85 & 86 & 84 \\ \text { NAQCRI-W-20202 } & 107 & 103 & 98\end{array}$

SDG 06-4-8b, analysis date April 8, 2006

$\begin{array}{lccc}\text { 20-ppb standard } & 80 & 109 & 103 \\ \text { Laboratory blank } & 100 & 100 & 100 \\ \text { NATI2-S-20416 } & 112 & 106 & 111 \\ \text { NATI2-S-20417 } & 100 & 68^{\mathrm{d}} & 111 \\ \text { NATI2-S-20418 } & 96 & 58^{\mathrm{d}} & 112 \\ \text { NATI2-S-20419 } & 95 & 111 & 117 \\ \text { NATI2-S-20420 } & 92 & 111 & 109 \\ \text { NATI2-S-20421 } & 91 & 64^{\mathrm{d}} & 116 \\ \text { NATI2-S-20424 } & 90 & 110 & 109 \\ \text { NATI2-S-20419DUP } & 87 & 105 & 104 \\ \text { NATI3-S-20290 } & 85 & 99 & 99 \\ \text { NATI3-S-20291 } & 85 & 102 & 100 \\ \text { NATI3-S-20289 } & 34^{\mathrm{d}} & 37^{\mathrm{d}} & 40^{\mathrm{d}} \\ \text { NATI3-S-20293 } & 73^{\mathrm{d}} & 79^{\mathrm{d}} & 82 \\ \text { NATI2-S-20409 } & 84 & 93 & 95 \\ \text { NATI2-S-20425 } & 87 & 55^{\mathrm{d}} & 97\end{array}$

$\begin{array}{llllll}17.98 & 2.7 & 16.8 & 4.3 & 17.59 & 3.2\end{array}$

Reanalyzed in SDG 06-4-10a.

Reanalyzed in SDG 06-4-10a.

Reanalyzed in SDG 06-4-10a.

Reanalyzed in SDG 06-4-10b.

Reanalyzed in SDG 06-4-10b.

Reanalyzed in SDG 06-4-10b. 
SDG 06-4-10a, analysis date April 10, 2006

$\begin{array}{lccc}\text { 20-ppb standard } & 100 & 100 & 100 \\ \text { Laboratory blank } & 100 & 100 & 100 \\ \text { NATI2-S-20415 } & 104 & 109 & 109 \\ \text { NATI3-S-20286 } & 106 & 106 & 98 \\ \text { NATI2-S-20408 } & 94 & 85 & 87 \\ \text { NATI3-S-20294 } & 90 & 91 & 94 \\ \text { NATI2-S-20422 } & 87 & 92 & 93 \\ \text { NATI2-S-20412 } & 102 & 105 & 105 \\ \text { NATI2-S-20411 } & 95 & 97 & 98 \\ \text { NATI3-S-20292 } & 101 & 101 & 101 \\ \text { NATI3-S-20287 } & 94 & 100 & 100 \\ \text { NATI2-S-20414 } & 94 & 96 & 92 \\ \text { NATI2-S-20414DUP } & 115 & 112 & 102 \\ \text { Methanol blank } & 100 & 100 & 100 \\ \text { NATI2-S-20417 } & 108 & 113 & 112 \\ \text { NATI2-S-20418 } & 100 & 105 & 104 \\ \text { NATI2-S-20421 } & 117 & 116 & 115\end{array}$

Measured Concentration and RPD Value for Calibration Check Standard

\begin{tabular}{|c|c|c|c|c|c|c|c|c|c|}
\hline \multirow[b]{3}{*}{ Sample } & \multicolumn{3}{|c|}{ Recovery of Surrogate Compounds ${ }^{\mathrm{a}}(\%)$} & \multirow{2}{*}{\multicolumn{2}{|c|}{$\begin{array}{c}\text { Carbon } \\
\text { Tetrachloride }\end{array}$}} & \multirow{2}{*}{\multicolumn{2}{|c|}{ Chloroform }} & \multirow{2}{*}{\multicolumn{2}{|c|}{ Methylene Chloride }} \\
\hline & & & & & & & & & \\
\hline & Fluorobenzene & $\begin{array}{l}\text { Bromo- } \\
\text { fluorobenzene }\end{array}$ & $\begin{array}{c}\text { Dichloro- } \\
\text { benzene- } \mathrm{d}_{4}\end{array}$ & $\mathrm{ppb}^{\mathrm{b}}$ & $\mathrm{RPD}^{\mathrm{c}}$ & $\mathrm{ppb}^{\mathrm{b}}$ & $\mathrm{RPD}^{\mathrm{c}}$ & $\mathrm{ppb}^{\mathrm{b}}$ & $\mathrm{RPD}^{\mathrm{C}}$ \\
\hline
\end{tabular}

SDG 06-4-10b, analysis date April 10, 2006

$\begin{array}{lccc}\text { 20-ppb standard } & 100 & 100 & 100 \\ \text { Laboratory blank } & 100 & 100 & 100 \\ \text { NATI3-S-20289 } & 107 & 110 & 112 \\ \text { NATI3-S-20293 } & 111 & 111 & 105 \\ \text { NATI2-S-20425 } & 95 & 95 & 89 \\ \text { NATI2-S-20407 } & 97 & 98 & 94 \\ \text { NATI2-S-20423 } & 97 & 94 & 91 \\ \text { NATI3-S-20295 } & 103 & 100 & 89\end{array}$


SDG 06-4-10b, analysis date April 10, 2006 (cont.)

$\begin{array}{cccc}\text { NATI2-S-20406 } & 110 & 111 & 107 \\ \text { NATI2-S-20410 } & 111 & 102 & 96\end{array}$

SDG 06-4-11a, analysis date April 11, 2006

$\begin{array}{lccc}\text { 20-ppb standard } & 100 & 107 & 103 \\ \text { Laboratory blank } & 100 & 100 & 100 \\ \text { NATI2-W-20433 } & 99 & 138^{\mathrm{d}} & 127^{\mathrm{d}} \\ \text { NAL3-W-20209 } & 93 & 100 & 104 \\ \text { NACOOP3-W-20218 } & 100 & 107 & 100 \\ & & & \\ \text { NAL1-W-20211 } & 97 & 103 & 98 \\ \text { NAL1-W-20211DUP } & 98 & 104 & 103 \\ \text { NANW1-W-20215 } & 96 & 104 & 101 \\ \text { NANW2-W-20219 } & 100 & 107 & 99 \\ & & & \\ \text { NACOOP1-W-20217 } & 95 & 101 & 96 \\ \text { NATI4-W-20325 } & 101 & 122^{\mathrm{d}} & 115 \\ \text { NAQCDU-W-20213 } & 91 & 95 & 96 \\ \text { NAQCDU-W-20225 } & 89 & 98 & 93 \\ \text { NAQCRI-W-20212 } & 93 & 108 & 94 \\ \text { NAQCRI-W-20210 } & 92 & 104 & 99 \\ \text { NAQCTB-W-20214 } & 93 & 103 & 94 \\ \text { NAQCRI-W-20216 } & 93 & 108 & 94 \\ \text { Laboratory blank 2 } & 90 & 105 & 101 \\ \text { NACOOP3-W-20218 } & 90 & 109 & 103 \\ \text { NANW2-W-20219 } & 89 & 106 & 104 \\ \text { NANW2-W-20219DUP } & 92 & 113 & 107\end{array}$

\begin{tabular}{|c|c|c|c|c|c|}
\hline \multicolumn{2}{|c|}{$\begin{array}{c}\text { Carbon } \\
\text { Tetrachloride }\end{array}$} & \multicolumn{2}{|c|}{ Chloroform } & \multicolumn{2}{|c|}{ Methylene Chloride } \\
\hline $\mathrm{ppb}^{\mathrm{b}}$ & $\mathrm{RPD}^{\mathrm{c}}$ & $\mathrm{ppb}^{\mathrm{b}}$ & $\mathrm{RPD}^{\mathrm{c}}$ & $\mathrm{ppb}^{\mathrm{b}}$ & $\mathrm{RPD}^{\mathrm{c}}$ \\
\hline
\end{tabular}

\section{Bromo- Dichloro-}

Sample Fluorobenzene fluorobenzene benzene- $d_{4}$

96

\section{0-ppb standard}

Laboratory blank

$127^{\circ}$

104

Reanalyzed in SDG 06-4-12a.

Outside calibration range for carbon tetrachloride at DF1. Analysis at dilution below in this SDG. Methylene chloride and tetrachloroethylene reported.

Outside calibration range for carbon tetrachloride at zero dilution. Analysis at dilution below in this SDG. Methylene chloride and tetrachloroethylene reported.

Reanalyzed in SDG 06-4-12a.

Analysis at DF5 for carbon tetrachloride and chloroform.

Analysis at DF10 for carbon tetrachloride and chloroform.

Duplicate analysis at DF10. 
SDG 06-4-11b, analysis date April 11, 2006

20-ppb standard
Laboratory blank
NATI2-S-20428
NATI1-S-20303
NATI2-S-20413
NAQCTB-S-20197
NATI2-S-20426
NATI3-S-20297
NATI3-S-20297DUP
NATI1-S-20302
NATI1-S-20301
NATI2-S-20429
NATI2-S-20427
NATI3-S-20296
NAQCTB-S-20208
NATI1-S-20300

$\begin{array}{ccc}100 & 100 & 100 \\ 105 & 103 & 104 \\ 82 & 85 & 88 \\ 104 & 107 & 109 \\ 83 & 89 & 95 \\ 101 & 96 & 97 \\ 88 & 91 & 99 \\ 95 & 96 & 101 \\ 86 & 85 & 88 \\ 85 & 88 & 96 \\ 86 & 90 & 97 \\ 92 & 96 & 101 \\ 85 & 92 & 98 \\ 83 & 86 & 91 \\ 99 & 98 & 79 d \\ 86 & 90 & 97\end{array}$

Measured Concentration and RPD Value for Calibration Check Standard

\begin{tabular}{|c|c|c|c|c|c|c|c|c|c|}
\hline \multirow[b]{3}{*}{ Sample } & \multicolumn{3}{|c|}{ Recovery of Surrogate Compounds ${ }^{\mathrm{a}}(\%)$} & \multirow{2}{*}{\multicolumn{2}{|c|}{$\begin{array}{c}\text { Carbon } \\
\text { Tetrachloride }\end{array}$}} & \multirow{2}{*}{\multicolumn{2}{|c|}{ Chloroform }} & \multirow{2}{*}{\multicolumn{2}{|c|}{ Methylene Chloride }} \\
\hline & & & & & & & & & \\
\hline & Fluorobenzene & $\begin{array}{l}\text { Bromo- } \\
\text { fluorobenzene }\end{array}$ & $\begin{array}{c}\text { Dichloro- } \\
\text { benzene- } \mathrm{d}_{4}\end{array}$ & $\mathrm{ppb}^{\mathrm{b}}$ & $\mathrm{RPD}^{\mathrm{c}}$ & $\mathrm{ppb}^{\mathrm{b}}$ & $\mathrm{RPD}^{\mathrm{c}}$ & $\mathrm{ppb}^{\mathrm{b}}$ & $\mathrm{RPD}^{\mathrm{C}}$ \\
\hline
\end{tabular}

SDG 06-4-12a, analysis date April 12, 2006

$\begin{array}{lccc}\text { 20-ppb standard } & 89 & 117 & 86 \\ \text { Laboratory blank } & 100 & 100 & 100 \\ \text { NATI2-W-20433 } & 87 & 120 & 113 \\ \text { NATI4-W-20325 } & 100 & 116 & 112\end{array}$

NATI4-W-20325

100

SDG 06-4-12b, analysis date April 12, 2006

20-ppb standard

Methanol blank

NATI1-S-20306

$\begin{array}{cc}87 & 98 \\ 90^{d} & 91 \\ 171^{d} & 150^{d}\end{array}$

99
94

$130^{\mathrm{d}}$
21.58

1.9

23.89

4.4

19.83

0.2

Accepted.
0.3

0.1

Reanalyzed in SDG 06-4-14b.
24.63

5.2

0.0 
SDG 06-4-12b, analysis date April 12, 2006 (cont.)

$\begin{array}{lccc}\text { NATI1-S-20305 } & 98 & 79^{\mathrm{d}} & 70^{\mathrm{d}} \\ \text { NATI1-S-20307 } & 93 & 88 & 89 \\ \text { NATI1-S-20304 } & 87 & 86 & 88 \\ \text { NATI4-S-20321 } & 130^{\mathrm{d}} & 122^{\mathrm{d}} & 118 \\ \text { NATI4-S-20310 } & 108 & 101 & 96 \\ \text { Methanol blank } 2 & 110 & 109 & 106 \\ \text { NATI4-S-20318 } & 94 & 94 & 93 \\ \text { NATI4-S-20314 } & 105 & 101 & 98 \\ \text { NATI4-S-20317 } & 111 & 104 & 102 \\ \text { NATI4-S-20308 } & 115 & 106 & 102 \\ \text { NATI4-S-20322 } & 93 & 90 & 90 \\ \text { NATI4-S-20316 } & 116 & 105 & 103 \\ \text { NATI4-S-20312 } & 122^{\mathrm{d}} & 115 & 115\end{array}$

Measured Concentration and RPD Value for Calibration Check Standard

\begin{tabular}{|c|c|c|c|c|c|c|c|c|c|}
\hline \multirow[b]{3}{*}{ Sample } & \multicolumn{3}{|c|}{ Recovery of Surrogate Compounds ${ }^{a}(\%)$} & \multirow{2}{*}{\multicolumn{2}{|c|}{$\begin{array}{c}\text { Carbon } \\
\text { Tetrachloride }\end{array}$}} & \multirow{2}{*}{\multicolumn{2}{|c|}{ Chloroform }} & \multirow{2}{*}{\multicolumn{2}{|c|}{ Methylene Chloride }} \\
\hline & & & & & & & & & \\
\hline & Fluorobenzene & $\begin{array}{l}\text { Bromo- } \\
\text { fluorobenzene }\end{array}$ & $\begin{array}{c}\text { Dichloro- } \\
\text { benzene- } \mathrm{d}_{4}\end{array}$ & $\mathrm{ppb}^{\mathrm{b}}$ & $\mathrm{RPD}^{\mathrm{c}}$ & $\mathrm{ppb}^{\mathrm{b}}$ & $\mathrm{RPD}^{\mathrm{c}}$ & $\mathrm{ppb}^{\mathrm{b}}$ & $\mathrm{RPD}^{\mathrm{c}}$ \\
\hline
\end{tabular}

Reanalyzed in SDG 06-4-14b.

Reanalyzed in SDG 06-4-14b.

SDG 06-4-13, analysis date April 13, 2006

$\begin{array}{lccc}\text { 20-ppb standard } & 100 & 105 & 110 \\ \text { Laboratory blank } & 113 & 111 & 109 \\ \text { NATI4-W-20471 } & 88 & 91 & 93 \\ \text { NATI4-W-20474 } & 117 & 119 & 120 \\ \text { NATI4-W-20469 } & 100 & 105 & 106 \\ \text { NATI5-W-20368 } & 124^{\mathrm{d}} & 127^{\mathrm{d}} & 127^{\mathrm{d}} \\ \text { NATI4-W-20470 } & 89 & 94 & 96 \\ \text { NATI4-W-20472 } & 98 & 106 & 108 \\ \text { NATI4-W-20472DUP } & 91 & 94 & 93 \\ \text { NATI4-W-20473 } & 99 & 98 & 96 \\ \text { NAQCTB-W-20369 } & 89 & 85 & 83\end{array}$

Reanalyzed in SDG 06-4-14a.

17.21

3.7

17.91

2.8

20

0.0

Reanalyzed in SDG 06-4-17. 
SDG 06-4-14a, analysis date April 14, 2006

$\begin{array}{lccc}\text { 20-ppb standard } & 100 & 100 & 100 \\ \text { Laboratory blank } & 100 & 100 & 100 \\ \text { NATI4-S-20312 } & 111 & 100 & 98 \\ \text { NATI4-S-20323 } & 98 & 108 & 104 \\ \text { NATI4-S-20309 } & 100 & 100 & 100 \\ \text { NATI4-S-20313 } & 111 & 116 & 96 \\ \text { NATI4-S-20319 } & 95 & 102 & 101 \\ \text { NATI4-S-20324 } & 108 & 104 & 92 \\ \text { NATI4-S-20324DUP } & 96 & 83 & 92 \\ \text { NATI4-S-20315 } & 93 & 112 & 78 \\ \text { NATI4-S-20311 } & 90 & 95 & 105 \\ \text { NATI4-S-20320 } & 93 & 90 & 93 \\ \text { NATI7-S-20446 } & 88 & 87 & 89 \\ \text { NATI7-S-20451 } & 88 & 91 & 91 \\ \text { NATI7-S-20445 } & 102 & 100 & 85 \\ \text { NATI7-S-20448 } & 82 & 88 & 92 \\ \text { NATI7-S-20457 } & 85 & 88 & 87 \\ \text { Methanol blank2 } & 100 & 100 & 100 \\ \text { NATI7-S-20455 } & 98 & 103 & 102 \\ \text { NATI7-S-20456 } & 83 & 90 & 97 \\ \text { NATI7-S-20452 } & 99 & 98 & 89 \\ \text { NATI7-S-20454 } & 86 & 94 & 105 \\ \text { NATI7-S-20442 } & 83 & 90 & 97 \\ \text { NATI1-S-20464 } & 103 & 97 & 91 \\ \text { NATI1-S-20467 } & 81 & 89 & 90 \\ \text { NATI1-S-20467DUP } & 98 & 88 & 84\end{array}$

Measured Concentration and RPD Value for Calibration Check Standard

\begin{tabular}{|c|c|c|c|c|c|c|c|c|c|}
\hline \multirow[b]{3}{*}{ Sample } & \multicolumn{3}{|c|}{ Recovery of Surrogate Compounds ${ }^{\mathrm{a}}(\%)$} & \multirow{2}{*}{\multicolumn{2}{|c|}{$\begin{array}{c}\text { Carbon } \\
\text { Tetrachloride }\end{array}$}} & \multirow{2}{*}{\multicolumn{2}{|c|}{ Chloroform }} & \multirow{2}{*}{\multicolumn{2}{|c|}{ Methylene Chloride }} \\
\hline & & & & & & & & & \\
\hline & Fluorobenzene & $\begin{array}{l}\text { Bromo- } \\
\text { fluorobenzene }\end{array}$ & $\begin{array}{c}\text { Dichloro- } \\
\text { benzene- } \mathrm{d}_{4}\end{array}$ & $\mathrm{ppb}^{\mathrm{b}}$ & $\mathrm{RPD}^{\mathrm{c}}$ & $\mathrm{ppb}^{\mathrm{b}}$ & $\mathrm{RPD}^{\mathrm{c}}$ & $\mathrm{ppb}^{\mathrm{b}}$ & $\mathrm{RPD}^{\mathrm{C}}$ \\
\hline
\end{tabular}

04

100

101

92

$78^{d}$

105

93

89

91
85

92

102
97

89

105

91

90
84

Reanalyzed in SDG 06-4-18b. 
SDG 06-4-14b, analysis date April 14, 2006

$\begin{array}{lccc}\text { 20-ppb standard } & 114 & 110 & 106 \\ \text { Laboratory blank } & 93 & 94 & 98 \\ \text { NATI5-W-20373 } & 101 & 102 & 102 \\ \text { NATI5-W-20371 } & 102 & 103 & 99 \\ \text { NAQCRIN-W-20372 } & 106 & 103 & 98 \\ \text { NAQCTB-W-20378 } & 102 & 104 & 103 \\ \text { Laboratory blank } & 97 & 87 & 83 \\ \text { Methanol blank } & 100 & 100 & 100 \\ \text { NATI1-S-20306 } & 102 & 96 & 86 \\ \text { NATI1-S-20305 } & 108 & 107 & 93 \\ \text { NATI4-S-20321 } & 108 & 99 & 86\end{array}$

SDG 06-4-17, analysis date April 17, 2006

$\begin{array}{lccc}\text { 20-ppb standard } & 98 & 87 & 98 \\ \text { Laboratory blank } & 101 & 92 & 86 \\ \text { NATI5-W-20368 } & 104 & 103 & 97 \\ \text { NATI1-W-20344 } & 98 & 98 & 113 \\ \text { NATI1-W-20341 } & 97 & 99 & 105 \\ \text { NATI1-W-20343 } & 100 & 114 & 126^{d} \\ \text { NATI3-W-20383 } & 94 & 101 & 99 \\ \text { NATI1-W-20342 } & 99 & 108 & 114 \\ & & & \\ \text { NAQCTB-W-20389 } & 91 & 83 & 80 \\ \text { NATI1-W-20266 } & 92 & 118 & 101 \\ \text { NATI3-W-20385 } & 95 & 99 & 96\end{array}$

Measured Concentration and RPD Value for Calibration Check Standard

\begin{tabular}{|c|c|c|c|c|c|}
\hline \multicolumn{2}{|c|}{$\begin{array}{c}\text { Carbon } \\
\text { Tetrachloride }\end{array}$} & \multicolumn{2}{|c|}{ Chloroform } & \multicolumn{2}{|c|}{ Methylene Chloride } \\
\hline $\mathrm{ppb}^{\mathrm{b}}$ & $\mathrm{RPD}^{\mathrm{c}}$ & $\mathrm{ppb}^{\mathrm{b}}$ & $\mathrm{RPD}^{\mathrm{c}}$ & $\mathrm{ppb}^{\mathrm{b}}$ & $\mathrm{RPD}^{\mathrm{c}}$ \\
\hline
\end{tabular}

Bromo- Dichloro-

$\begin{array}{llllll}16.57 & 4.7 & 16.22 & 5.2 & 16.89 & 4.2\end{array}$

Outside calibration range for carbon tetrachloride at DF1. Analysis at DF5 in 06-418a. Methylene chloride reported.

Outside calibration range for carbon tetrachloride at DF1. Analysis at DF5 in 06-418a. Methylene chloride and tetrachloroethylene reported.

Outside calibration range for carbon tetrachloride at DF1. Analysis at DF5 in 06-418a. Methylene chloride reported.

Outside calibration range for carbon tetrachloride at DF1. Analysis at DF5 in 06-418a. Methylene chloride and tetrachloroethylene reported. 
aboratory blank

NAQCRIN-W-20386

NATI1-W-20345

NATI1-W-20344

NATI1-W-20341

NATI1-W-20343

NATI1-W-20342

95

100

$131^{\mathrm{d}}$

103

109

88

89

SDG 06-4-18b, analysis date April 18, 2006

20-ppb standard

Methanol blank

NATI4-S-20315

NATI1-S-20465

NATI8-S-20462

NATI7-S-20439

NATI7-S-20438

NATI7-S-20441

NATI7-S-20444

NATI7-S-20449

NATI7-S-20449DUP

Methanol blank 2

NATI7-S-20443
Measured Concentration and RPD Value for Calibration Check Standard

\begin{tabular}{|c|c|c|c|c|c|}
\hline \multicolumn{2}{|c|}{$\begin{array}{c}\text { Carbon } \\
\text { Tetrachloride }\end{array}$} & \multicolumn{2}{|c|}{ Chloroform } & \multicolumn{2}{|c|}{ Methylene Chloride } \\
\hline $\mathrm{ppb}^{\mathrm{b}}$ & $\mathrm{RPD}^{\mathrm{c}}$ & $\mathrm{ppb}^{\mathrm{b}}$ & $\mathrm{RPD}^{\mathrm{c}}$ & $\mathrm{ppb}^{\mathrm{b}}$ & $\mathrm{RPD}^{\mathrm{C}}$ \\
\hline
\end{tabular}

\section{0}

89

101

$\begin{array}{cc}96 & 90 \\ 100 & 100 \\ 125^{d} & 115 \\ 103 & 98 \\ 98 & 96 \\ 115 & 111 \\ 89 & 88 \\ 91 & 87\end{array}$

20.49

0.6

22.94

3.4

20

0.0

Accepted.

Analysis at DF5 for carbon tetrachloride and chloroform.

Analysis at DF5 for carbon tetrachloride and chloroform.

Analysis at DF5 for carbon tetrachloride and chloroform.

Analysis at DF5 for carbon tetrachloride and chloroform.

19.46

0.7

17.88

2.8

18.35

Reanalyzed in SDG 06-4-19. 
SDG 06-4-18b, analysis date April 18, 2006 (cont.)

$\begin{array}{lccc}\text { NATI7-S-20437 } & 92 & 96 & 97 \\ \text { NATI7-S-20447 } & 99 & 92 & 83 \\ \text { NATI7-S-20440 } & 94 & 79 d & 88 \\ \text { NATI5-S-20363 } & 94 & 93 & 112 \\ \text { NATI7-S-20459 } & 89 & 99 & 93 \\ \text { NATI7-S-20453 } & 93 & 108 & 95 \\ \text { NATI7-S-20436 } & 99 & 96 & 95 \\ \text { NATI7-S-20458 } & 88 & 96 & 91 \\ \text { NATI7-S-20450 } & 100 & 92 & 87\end{array}$

Measured Concentration and RPD Value for Calibration Check Standard

\begin{tabular}{|c|c|c|c|c|c|c|c|c|c|}
\hline \multirow[b]{3}{*}{ Sample } & \multicolumn{3}{|c|}{ Recovery of Surrogate Compounds ${ }^{a}(\%)$} & \multirow{2}{*}{\multicolumn{2}{|c|}{$\begin{array}{c}\text { Carbon } \\
\text { Tetrachloride }\end{array}$}} & \multirow{2}{*}{\multicolumn{2}{|c|}{ Chloroform }} & \multirow{2}{*}{\multicolumn{2}{|c|}{ Methylene Chloride }} \\
\hline & & & & & & & & & \\
\hline & Fluorobenzene & $\begin{array}{c}\text { Bromo- } \\
\text { fluorobenzene }\end{array}$ & $\begin{array}{c}\text { Dichloro- } \\
\text { benzene- } \mathrm{d}_{4}\end{array}$ & $\mathrm{ppb}^{\mathrm{b}}$ & $\mathrm{RPD}^{\mathrm{c}}$ & $\mathrm{ppb}^{\mathrm{b}}$ & $\mathrm{RPD}^{\mathrm{c}}$ & $\mathrm{ppb}^{\mathrm{b}}$ & $\mathrm{RPD}^{\mathrm{c}}$ \\
\hline
\end{tabular}

SDG 06-4-19, analysis date April 19, 2006

$\begin{array}{lccc}\text { 20-ppb standard } & 100 & 100 & 100 \\ \text { Laboratory blank } & 100 & 100 & 100 \\ \text { NATripBlank-S-20227 } & 103 & 90 & 145^{\mathrm{d}} \\ \text { NATripBlank-S-20226 } & 103 & 173^{\mathrm{d}} & 145^{\mathrm{d}} \\ \text { NATI5-S-20360 } & 100 & 118 & 114 \\ \text { NATI5-S-20361 } & 96 & 105 & 108 \\ \text { NATI5-S-20335 } & 103 & 105 & 108 \\ \text { NATI5-S-20357 } & 100 & 65^{\mathrm{d}} & 105 \\ \text { NATI5-S-20334 } & 97 & 100 & 97 \\ \text { NATI5-S-20334DUP } & 103 & 103 & 107 \\ \text { Methanol blank } & 100 & 100 & 100 \\ \text { NATI8-S-20462 } & 105 & 97 & 82 \\ \text { NATI7-S-20440 } & 93 & 91 & 83 \\ \text { NACP1-S-20331 } & 103 & 93 & 90 \\ \text { NATI5-S-20332 } & 97 & 101 & 99 \\ \text { NATI5-S-20359 } & 92 & 92 & 102 \\ \text { NATI5-S-20356 } & 98 & 91 & 107\end{array}$

Reanalyzed in SDG 06-4-19.

Reanalyzed in SDG 06-4-21b.

Reanalyzed in SDG 06-4-21b.

Reanalyzed in SDG 06-4-20a. 
SDG 06-4-20a, analysis date April 20, 2006

$\begin{array}{lccc}\text { 20-ppb standard } & 100 & 100 & 100 \\ \text { Laboratory blank } & 105 & 82 & 88 \\ \text { NATripBlank-S-20227 } & 113 & 167^{\mathrm{d}} & 154^{\mathrm{d}} \\ \text { NATripBlank-S-20226 } & 114 & 173^{\mathrm{d}} & 147^{\mathrm{d}} \\ \text { NATI5-S-20357 } & 113 & 100 & 120 \\ \text { NAQCTB-S-20362 } & 119 & 96 & 146^{\mathrm{d}} \\ \text { NATI5-S-20333 } & 124^{\mathrm{d}} & 123^{\mathrm{d}} & 102 \\ \text { NATI1-S-20479 } & 116 & 104 & 93 \\ \text { NATI1-S-20482 } & 106 & 100 & 101 \\ \text { NATI1-S-20481 } & 100 & 100 & 100 \\ \text { NATI5-S-20364 } & 108 & 89 & 96 \\ \text { NATI5-S-20364DUP } & 103 & 100 & 102 \\ \text { NATI5-S-20366 } & 117 & 99 & 88 \\ \text { NATI1-S-20485 } & 117 & 101 & 89 \\ \text { NATI1-S-20484 } & 104 & 98 & 95 \\ \text { NATI3-S-20376 } & 111 & 102 & 86 \\ \text { NATI1-S-20483 } & 111 & 120 & 91\end{array}$

20.89

1.1

20.01

0.0

18.5

1.9

Reanalyzed in SDG 06-4-21b.

Reanalyzed in SDG 06-4-21b.

Reanalyzed in SDG 06-4-21b.

Reanalyzed in SDG 06-4-21b. 
SDG 06-4-20b, analysis date April 20, 2006

$\begin{array}{lccc}\text { 20-ppb standard } & 98 & 98 & 94 \\ \text { Laboratory blank } & 101 & 90 & 80 \\ \text { NATI1-S-20476 } & 120 & 115 & 100 \\ \text { NATI1-S-20475 } & 107 & 106 & 100 \\ \text { NATI5-S-20367 } & 114 & 114 & 104 \\ \text { NATI1-S-20478 } & 109 & 109 & 106 \\ \text { NATI1-S-20480 } & 115 & 113 & 103 \\ \text { NATI1-S-20477 } & 99 & 101 & 98 \\ \text { NATI5-S-20365 } & 108 & 106 & 97 \\ \text { NAQCTB-S-20377 } & 94 & 88 & 78 \\ \text { NATI3-S-20375 } & 100 & 96 & 89 \\ \text { NATI3-S-20380 } & 106 & 109 & 103 \\ \text { NATI1-S-20339 } & 100 & 99 & 91 \\ \text { NATI3-S-20379 } & 116 & 114 & 108 \\ \text { NAQCTB-S-20387 } & 96 & 96 & 87 \\ \text { NATI1-S-20336 } & 114 & 109 & 98\end{array}$

SDG 06-4-21a, analysis date April 21, 2006

$\begin{array}{lccc}\text { 20-ppb standard } & 100 & 100 & 100 \\ \text { Laboratory blank } & 81 & 93 & 97 \\ \text { NATI5-W-20267 } & 100 & 100 & 100 \\ \text { NATI7-W-20269 } & 100 & 102 & 110 \\ \text { NATI7-W-20270 } & 97 & 103 & 117 \\ \text { NATI7-W-20271 } & 99 & 98 & 111 \\ \text { NATI7-W-20271DUP } & 96 & 97 & 114 \\ \text { NAQCTB-W-20487 } & 95 & 88 & 93 \\ \text { NATI5QC-W-20268 } & 96 & 99 & 97\end{array}$

Reanalyzed in SDG 06-4-21b.

$\begin{array}{llllll}18.92 & 1.4 & 19.8 & 0.3 & 21.74 & 2.1\end{array}$


SDG 06-4-21b, analysis date April 21, 2006

$\begin{array}{lccc}\text { 20-ppb standard } & 100 & 100 & 100 \\ \text { Laboratory blank } & 100 & 100 & 100 \\ \text { NATI3-S-20381 } & 107 & 115 & 113 \\ \text { NATI3-S-20382 } & 97 & 106 & 115 \\ \text { NATI1-S-20337 } & 58^{\mathrm{d}} & 66^{\mathrm{d}} & 73^{\mathrm{d}} \\ \text { NATI1-S-20340 } & 94 & 103 & 109 \\ \text { NAQCTB-S-20377 } & 109 & 112 & 99 \\ \text { NAQCTB-S-20362 } & 97 & 102 & 102 \\ \text { NATripBlank-S-20226 } & 100 & 105 & 106 \\ \text { NATripBlank-S-20227 } & 109 & 111 & 113 \\ \text { NATI5-S-20333 } & 99 & 106 & 114\end{array}$

SDG 06-4-22, analysis date April 22, 2006

20-ppb standard

Laboratory blank

NATI7-W-20272

NATI7-W-20273

NATI7-W-20274

NATI2-W-20276

NATI2-W-20277

NATI2-W-20279

NATI2-W-20282

NATI2-W-20282DUP

NATI2-W-20278

NAQCTB-W-20347

NATI2-W-20281
Measured Concentration and RPD Value for Calibration Check Standard

\begin{tabular}{|c|c|c|c|c|c|}
\hline \multicolumn{2}{|c|}{$\begin{array}{c}\text { Carbon } \\
\text { Tetrachloride }\end{array}$} & \multicolumn{2}{|c|}{ Chloroform } & \multicolumn{2}{|c|}{ Methylene Chloride } \\
\hline $\mathrm{ppb}^{\mathrm{b}}$ & $\mathrm{RPD}^{\mathrm{c}}$ & $\mathrm{ppb}^{\mathrm{b}}$ & $\mathrm{RPD}^{\mathrm{c}}$ & $\mathrm{ppb}^{\mathrm{b}}$ & $\mathrm{RPD}^{\mathrm{c}}$ \\
\hline
\end{tabular}

Reanalyzed in SDG 06-4-26b.

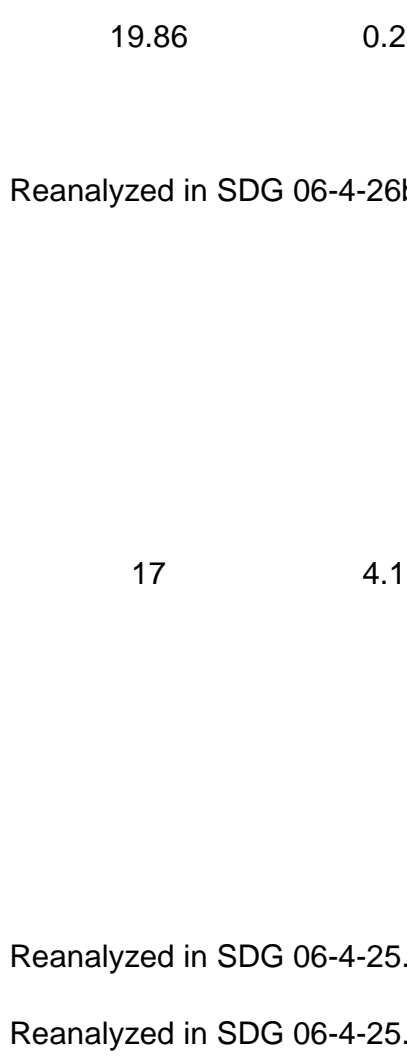

0.9

20

0.0

$\begin{array}{llll}4.1 & 19.77 & 0.3 & 20\end{array}$

20

0.0

$\begin{array}{cccc}108 & 116 & 113 & 17 \\ 84 & 89 & 91 & \\ 87 & 90 & 90 & \\ 84 & 89 & 91 & \\ 116 & 111 & 109 & \\ 82 & 87 & 88 & \\ 93 & 96 & 96 & \\ 94 & 94 & 92 & \\ 99 & 91 & 87 & \text { Reanalyzed in SDG 06-4-25. } \\ 91 & 94 & 93 & \text { Reanalyzed in SDG 06-4-25. } \\ 131^{\mathrm{d}} & 129^{\mathrm{d}} & 116 & \\ 86 & 87 & 86 & \end{array}$


SDG 06-4-25, analysis date April 25, 2006

$\begin{array}{lccc}\text { 20-ppb standard } & 100 & 100 & 100 \\ \text { Laboratory blank } & 92 & 94 & 95 \\ \text { NATI9-W-20609 } & 62^{\mathrm{d}} & 59^{\mathrm{d}} & 59^{\mathrm{d}} \\ \text { NATI9-W-20589 } & 123^{\mathrm{d}} & 125^{\mathrm{d}} & 126^{\mathrm{d}} \\ \text { NATI6-W-20350 } & 125^{\mathrm{d}} & 124^{\mathrm{d}} & 122^{\mathrm{d}} \\ & & & \\ \text { NATI6-W-20351 } & 102 & 104 & 108 \\ \text { NATI11-W-20500 } & 96 & 104 & 107 \\ \text { NATI6-W-20353 } & 100 & 105 & 111 \\ \text { NATI6-W-20353DUP } & 93 & 99 & 101 \\ \text { NATI9-W-20586 } & 115 & 119 & 120 \\ \text { NATI9-W-20587 } & 112 & 116 & 116 \\ \text { NATI6-W-20348 } & 122^{\mathrm{d}} & 122^{\mathrm{d}} & 117 \\ \text { NATI6-W-20349 } & 92 & 88 & 88 \\ \text { NATI2-W-20281 } & 97 & 95 & 94 \\ \text { NATI2-W-20278 } & 102 & 105 & 107 \\ \text { NATI6RIN-W-20352 } & 82 & 81 & 80 \\ \text { NAQCTB-W-20717 } & 95 & 97 & 98 \\ \text { NATI11-W-20501 } & 94 & 100 & 100 \\ \text { NATI2-W-20280 } & 102 & 98 & 95 \\ \text { NATI9QC-W-20588 } & 83 & 86 & 88\end{array}$

Measured Concentration and RPD Value for Calibration Check Standard

\begin{tabular}{|c|c|c|c|c|c|c|c|c|c|}
\hline \multirow[b]{3}{*}{ Sample } & \multicolumn{3}{|c|}{ Recovery of Surrogate Compounds ${ }^{\mathrm{a}}(\%)$} & \multirow{2}{*}{\multicolumn{2}{|c|}{$\begin{array}{c}\text { Carbon } \\
\text { Tetrachloride }\end{array}$}} & \multirow{2}{*}{\multicolumn{2}{|c|}{ Chloroform }} & \multirow{2}{*}{\multicolumn{2}{|c|}{ Methylene Chloride }} \\
\hline & & & & & & & & & \\
\hline & Fluorobenzene & $\begin{array}{l}\text { Bromo- } \\
\text { fluorobenzene }\end{array}$ & $\begin{array}{c}\text { Dichloro- } \\
\text { benzene- } \mathrm{d}_{4}\end{array}$ & $\mathrm{ppb}^{\mathrm{b}}$ & $\mathrm{RPD}^{\mathrm{c}}$ & $\mathrm{ppb}^{\mathrm{b}}$ & $\mathrm{RPD}^{\mathrm{c}}$ & $\mathrm{ppb}^{\mathrm{b}}$ & $\mathrm{RPD}^{\mathrm{C}}$ \\
\hline
\end{tabular}

NATI9QC-W-20588

SDG 06-4-26a, analysis date April 26, 2006

20-ppb standard

Laboratory blank

NATI9-W-20589

NATI9-W-20609

NATI6-W-20348

$\begin{array}{ccc}100 & 100 & 100 \\ 100 & 100 & 100 \\ 80 & 82 & 86 \\ 101 & 91 & 93 \\ 155^{\mathrm{d}} & 171^{\mathrm{d}} & 168^{\mathrm{d}}\end{array}$

17.76

3.0

18.89

1.4

28.6

8.8

Reanalyzed in SDG 06-4-26a.

Reanalyzed in SDG 06-4-26a

Reanalyzed in SDG 06-4-26a also with high recovery. Contaminants of interest were not detected in either analysis. Accept this analysis.

Reanalyzed in SDG 06-4-27a.

$\begin{array}{rrrrr}1.9 & 22.28 & 2.7 & 22.02 & 2.4 \\ & & & & \\ & & & & \\ & & & \end{array}$


SDG 06-4-26a, analysis date April 26, 2006 (cont.)

NATI6-W-20350
Methanol blank
NATI6-S-20402
NATI9-S-20592
NATI11-S-20355
NATI11-S-20490
NATI11-S-20488
NATI11-S-20493
NATI9-S-20600
Methanol blank 2
NATI9-S-20593
NATI9-S-20602
NATI11-S-20354
NATI11-S-20496
NATI11-S-20496DUP
NATI9-S-20605

$154^{d}$
105
95
87
88
98
85
85
85
94
94
87
81
84
84
86

$162^{d}$

102

93

89

$186^{\mathrm{d}}$

95

96

107

107
98

91

91

86

82

86

$165^{\mathrm{d}}$

SDG 06-4-26b, analysis date April 26, 2006

20-ppb standard

Methanol blank

NATI1-S-20337

NATI6-S-20398

NATI6-S-20392

NATI6-S-20395

NATI6-S-20396

NATI6-S-20397

NATI6-S-20394

$\begin{array}{ccc}100 & 100 & 100 \\ 85 & 84 & 90 \\ 115 & 116 & 97 \\ 96 & 102 & 107 \\ 113 & 128^{\mathrm{d}} & 140^{\mathrm{d}} \\ 98 & 105 & 111 \\ 99 & 108 & 114 \\ 96 & 96 & 99 \\ 102 & 104 & 105\end{array}$

Second analysis with high recovery. First analysis in SDG 06-4-25. Contaminants of interest were not detected in either analysis. Accept first analysis.

Reanalyzed in SDG 06-5-1.

Reanalyzed in SDG 06-5-1.

Reanalyzed in SDG 06-5-1.

20.68

0.8

22.87

3.3

20

Reanalyzed in SDG 06-5-1. 
SDG 06-4-26b, analysis date April 26, 2006 (cont.)

NATI6-S-20391

NATI6-S-20393

NATI6-S-20399

NATI6-S-20390

Reanalyzed in SDG 06-5-3.

NATI6-S-20390DUP

NATI6-S-20401

NATI6-S-20404

NATI6-S-20403

NAQCTB-S-20486

NATI6-S-20405

NAQCTB-S-20346

NATI6-S-20400

$\begin{array}{ccc}82 & 90 & 96 \\ 87 & 90 & 94 \\ 91 & 103 & 112 \\ 77^{d} & 89 & 100 \\ 89 & 101 & 109 \\ 83 & 93 & 94 \\ 95 & 107 & 114 \\ 88 & 96 & 99 \\ 97 & 104 & 88 \\ 81 & 88 & 92 \\ 81 & 86 & 68^{d} \\ 82 & 91 & 99\end{array}$

Measured Concentration and RPD Value for Calibration Check Standard

Recovery of Surrogate Compounds ${ }^{\mathrm{a}}(\%)$

Bromo-

Dichloro-

etrachloride

\begin{tabular}{|c|c|c|c|c|c|}
\hline \multicolumn{2}{|c|}{$\begin{array}{l}\text { Carbon } \\
\text { Tetrachloride }\end{array}$} & \multicolumn{2}{|c|}{ Chloroform } & \multicolumn{2}{|c|}{ Methylene Chloride } \\
\hline $\mathrm{ppb}^{\mathrm{b}}$ & $\mathrm{RPD}^{\mathrm{c}}$ & $\mathrm{ppb}^{\mathrm{b}}$ & $\mathrm{RPD}^{\mathrm{c}}$ & $\mathrm{ppb}^{\mathrm{b}}$ & $\mathrm{RPD}^{\mathrm{c}}$ \\
\hline
\end{tabular}

SDG 06-4-27a, analysis date April 27, 2006

\section{0-ppb standard}

Laboratory blank

NATI11-W-20517

NATI11-W-20506

NATI10-W-20610

NATI10-W-20610DUP

NAQCTB-W-20518

NATI6-W-20348

Methanol blank

NATI6-S-20392

NATI6-S-20402

NATI9-S-20605

NATI11-S-20494

NATI9-S-20604

$\begin{array}{ccc}100 & 100 & 100 \\ 92 & 80 & 87 \\ 109 & 88 & 90 \\ 136^{\mathrm{d}} & 117 & 120 \\ 101 & 102 & 114 \\ 98 & 92 & 100 \\ 96 & 87 & 87 \\ 108 & 120 & 113 \\ 100 & 100 & 100 \\ 91 & 83 & 69^{\mathrm{d}} \\ 96 & 76^{\mathrm{d}} & 81 \\ 88 & 72^{\mathrm{d}} & 74^{\mathrm{d}} \\ 99 & 49^{\mathrm{d}} & 110 \\ 112 & 125^{\mathrm{d}} & 153^{\mathrm{d}}\end{array}$

21

1.2

20.06

0.1

22.12

Accepted.

Reanalyzed in SDG 06-5-1.

Reanalyzed in SDG 06-5-1.

Reanalyzed in SDG 06-5-1.

Reanalyzed in SDG 06-5-1.

Reanalyzed in SDG 06-5-1. 


$\begin{array}{lccc}\text { 20-ppb standard } & 102 & 105 & 111 \\ \text { Methanol blank } & 110 & 106 & 109 \\ \text { NATI11-S-20490 } & 86 & 83 & 51^{d} \\ \text { NATI9-S-20590 } & 103 & 107 & 108 \\ \text { NATI11-S-20492 } & 107 & 103 & 95 \\ \text { NATI9-S-20595 } & 111 & 115 & 112 \\ \text { NATI9-S-20596 } & 83 & 87 & 88 \\ \text { NATI9-S-20597 } & 101 & 101 & 92 \\ \text { NATI9-S-20608 } & 97 & 100 & 93 \\ \text { NATI9-S-20598 } & 92 & 93 & 82 \\ \text { NATI11-S-20498 } & 88 & 102 & 100 \\ \text { NATI9-S-20607 } & 87 & 89 & 87 \\ \text { NATI11-S-20491 } & 98 & 105 & 98 \\ \text { NATI9-S-20285 } & 90 & 87 & 80\end{array}$

SDG 06-4-28a, analysis date April 28, 2006

$\begin{array}{lccc}\text { 20-ppb standard } & 91 & 83 & 89 \\ \text { Laboratory blank } & 95 & 88 & 91 \\ \text { NATI10-W-20631 } & 98 & 116 & 114 \\ \text { NATI10-W-20632 } & 105 & 112 & 109\end{array}$

Reanalyzed in SDG 06-5-1.

Reanalyzed in SDG 06-5-1.

Reanalyzed in SDG 06-4-27b.

Reanalyzed in SDG 06-5-1.

Reanalyzed in SDG 06-5-1.
18.52
1.9

19.7

0.4

20

0.0

Reanalyzed in SDG 06-5-1.

114

109
18.0

2.6

18.44

2.0

20

0.0 
SDG 06-4-28a, analysis date April 28, 2006 (cont.)

$\begin{array}{lccc}\text { NATI10-W-20635 } & 114 & 110 & 118 \\ \text { NATI10-W-20634 } & 103 & 111 & 108 \\ \text { NATI11-W-20523 } & 105 & 96 & 101 \\ \text { NATI11-W-20638 } & 110 & 105 & 108 \\ \text { NATI10-W-20637 } & 93 & 88 & 96 \\ \text { NATI10-W-20637DUP } & 101 & 95 & 99 \\ \text { NATI10QC-W-20633 } & 100 & 95 & 101 \\ \text { NAQCTB-W-20726 } & 99 & 89 & 89\end{array}$

SDG 06-4-28b, analysis date April 28, 2006

$\begin{array}{lccc}\text { 20-ppb standard } & 100 & 100 & 100 \\ \text { Methanol blank } & 100 & 100 & 100 \\ \text { NATI9-S-20603 } & 108 & 108 & 107 \\ \text { NATI9-S-20284 } & 109 & 195^{\mathrm{d}} & 151^{\mathrm{d}} \\ \text { NATI11-S-20497 } & 111 & 113 & 108 \\ \text { NAQCTB-S-20716 } & 133^{\mathrm{d}} & 215^{\mathrm{d}} & 159^{\mathrm{d}} \\ \text { NATI9-S-20606 } & 107 & 114 & 117 \\ \text { NATI9-S-20601 } & 109 & 107 & 104 \\ \text { NATI9-S-20599 } & 105 & 112 & 98 \\ \text { Methanol blank } 2 & 97 & 102 & 100 \\ \text { NATI8-S-20508 } & 92 & 86 & 93 \\ \text { NATI11-S-20502 } & 112 & 103 & 102 \\ \text { NATI8-S-20510 } & 109 & 113 & 113 \\ \text { NATI8-S-20515 } & 109 & 117 & 120 \\ \text { Methanol blank 3 } & 100 & 100 & 100 \\ \text { NATI8-S-20509 } & 98 & 94 & 90\end{array}$

$\begin{array}{lllll}18.76 & 1.6 & 18.55 & 1.9 & 19.6\end{array}$

Reanalyzed in SDG 06-5-1.

Reanalyzed in SDG 06-5-1. 
SDG 06-5-1, analysis date May 1, 2006

$\begin{array}{lccc}\text { 20-ppb standard } & 100 & 100 & 100 \\ \text { Methanol blank } & 100 & 100 & 100 \\ \text { NATI9-S-20284 } & 91 & 100 & 105 \\ \text { NAQCTB-S-20716 } & 99 & 102 & 90 \\ \text { NATI11-S-20490 } & 88 & 91 & 102 \\ \text { NATI6-S-20392 } & 98 & 103 & 116 \\ \text { NATI6-S-20402 } & 96 & 99 & 115 \\ \text { NATI9-S-20605 } & 86 & 91 & 102 \\ \text { NATI11-S-20489 } & 99 & 99 & 97 \\ \text { NATI9-S-20604 } & 103 & 104 & 105 \\ \text { NATI9-S-20283 } & 94 & 97 & 115 \\ \text { NATI9-S-20591 } & 109 & 107 & 105 \\ \text { NATI9-S-20594 } & 94 & 97 & 115 \\ \text { NATI11-S-20495 } & 107 & 106 & 94 \\ \text { NATI11-S-20495DUP } & 89 & 93 & 108 \\ \text { Methanol blank } 2 & 100 & 100 & 100 \\ \text { NATI11-S-20494 } & 83 & 81 & 80 \\ \text { NATI11-S-20504 } & 93 & 104 & 108 \\ \text { NATI11-S-20499 } & 87 & 97 & 114 \\ \text { NATI8-S-20514 } & 92 & 97 & 101 \\ \text { NATI8-S-20507 } & 100 & 107 & 112 \\ \text { NATI8-S-20511 } & 88 & 96 & 113 \\ \text { NATI8-S-20513 } & 97 & 106 & 108\end{array}$

Measured Concentration and RPD Value for Calibration Check Standard

\begin{tabular}{|c|c|c|c|c|c|c|c|c|c|}
\hline \multirow[b]{3}{*}{ Sample } & \multicolumn{3}{|c|}{ Recovery of Surrogate Compounds ${ }^{\mathrm{a}}(\%)$} & \multirow{2}{*}{\multicolumn{2}{|c|}{$\begin{array}{c}\text { Carbon } \\
\text { Tetrachloride }\end{array}$}} & \multirow{2}{*}{\multicolumn{2}{|c|}{ Chloroform }} & \multirow{2}{*}{\multicolumn{2}{|c|}{ Methylene Chloride }} \\
\hline & & & & & & & & & \\
\hline & Fluorobenzene & $\begin{array}{l}\text { Bromo- } \\
\text { fluorobenzene }\end{array}$ & $\begin{array}{c}\text { Dichloro- } \\
\text { benzene- } \mathrm{d}_{4}\end{array}$ & $\mathrm{ppb}^{\mathrm{b}}$ & $\mathrm{RPD}^{\mathrm{c}}$ & $\mathrm{ppb}^{\mathrm{b}}$ & $\mathrm{RPD}^{\mathrm{c}}$ & $\mathrm{ppb}^{\mathrm{b}}$ & $\mathrm{RPD}^{\mathrm{C}}$ \\
\hline
\end{tabular}

SDG 06-5-2a, analysis date May 2, 2006

$\begin{array}{lccc}\text { 20-ppb standard } & 100 & 100 & 100 \\ \text { Laboratory blank } & 100 & 100 & 100 \\ \text { NATI8-W-20531 } & 101 & 93 & 98\end{array}$


SDG 06-5-2a, analysis date May 2, 2006 (cont.)

NATI8-W-20641

NATI8-W-20530

NATI12-W-20640

NATI11-W-20639

NATI11-W-20639DUP

Carbo

Recovery of Surrogate Compounds ${ }^{\mathrm{a}}(\%)$

Bromo-

Dichloro-

Tetrachloride

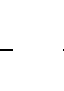

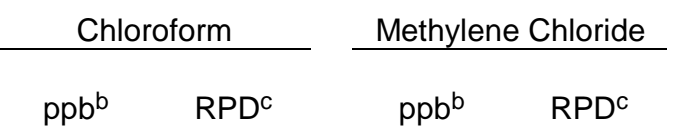

Methanol blank

NATI8-S-20512

NATI11-S-20505

NATI10-S-20611

NATI10-S-20612

NATI10-S-20618

NATI11-S-20503

NATI10-S-20615

Methanol blank 2

NATI10-S-20620

NATI10-S-20614

NATI10-S-20617

NATI10-S-20613

NATI10-S-20621

NATI10-S-20622

$\begin{array}{ccc}99 & 108 & 110 \\ 98 & 87 & 100 \\ 94 & 84 & 100 \\ 97 & 81 & 91 \\ 95 & 82 & 92 \\ 100 & 100 & 100 \\ 105 & 102 & 105 \\ 103 & 102 & 109 \\ 97 & 101 & 111 \\ 97 & 95 & 107 \\ 92 & 115 & 102 \\ 96 & 118 & 103 \\ 101 & 119 & 108 \\ 99 & 81 & 84 \\ 96 & 67^{d} & 76^{d} \\ 91 & 59^{d} & 69^{d} \\ 110 & 110 & 107 \\ 101 & 109 & 107 \\ 111 & 93 & 104 \\ 103 & 89 & 91\end{array}$

SDG 06-5-2b, analysis date May 2, 2006

$\begin{array}{lccc}\text { 20-ppb standard } & 85 & 101 & 119 \\ \text { Laboratory blank } & 100 & 100 & 100 \\ \text { NATI10-S-20627 } & 87 & 90 & 79.2^{\mathrm{d}} \\ \text { NATI10-S-20624 } & 84 & 89 & 96 \\ \text { NATI10-S-20616 } & 85 & 119 & 105 \\ \text { NATI10-S-20626 } & 85 & 90 & 96\end{array}$

Reanalyzed in SDG 06-5-3.

Reanalyzed in SDG 06-5-3.

TI10-S-20626

19

9.2

96 
SDG 06-5-2b, analysis date May 2, 2006 (cont.)

NAQCTB-S-20519

NATI10-S-20636

NATI10-S-20636DUP

NATI10-S-20619

NATI10-S-20623

NATI10-S-20628

NATI8-S-20522

NATI8-S-20521

NAQCTB-S-20529

NATI8-S-20516

SDG 06-5-3, analysis date May 3, 2006

20-ppb standard

Laboratory blank

NATI12-W-20728

NATI12-W-20729

NATI12-W-20727

NATI12-W-20727DUP

NAQCTB-W-20730

Methanol blank

NATI8-S-20528

NATI8-S-20526

NATI10-S-20625

NATI10-S-20625DUP

NATI8-S-20524

NATI8-S-20520

NATI8-S-20525

NATI8-S-20527

112

$\begin{array}{ccc}98 & 101 & 90 \\ 87 & 91 & 95 \\ 80 & 87 & 89 \\ 96 & 94 & 98 \\ 99 & 100 & 103 \\ 91 & 91 & 95 \\ 100 & 104 & 105 \\ 91 & 94 & 98 \\ 95 & 97 & 94 \\ 92 & 101 & 106\end{array}$

Measured Concentration and RPD Value for Calibration Check Standard

\begin{tabular}{|c|c|c|c|c|c|}
\hline \multicolumn{2}{|c|}{$\begin{array}{c}\text { Carbon } \\
\text { Tetrachloride }\end{array}$} & \multicolumn{2}{|c|}{ Chloroform } & \multicolumn{2}{|c|}{ Methylene Chloride } \\
\hline $\mathrm{ppb}^{\mathrm{b}}$ & $\mathrm{RPD}^{\mathrm{c}}$ & $\mathrm{ppb}^{\mathrm{b}}$ & $\mathrm{RPD}^{\mathrm{c}}$ & $\mathrm{ppb}^{\mathrm{b}}$ & $\mathrm{RPD}^{\mathrm{C}}$ \\
\hline
\end{tabular}

Bromo- Dichloro-

$\begin{array}{ccc}104 & 98 & 94 \\ 93 & 88 & 87 \\ 101 & 102 & 102 \\ 107 & 112 & 113 \\ 100 & 99 & 102 \\ 102 & 105 & 108 \\ 94 & 92 & 92 \\ 91 & 85 & 94 \\ 111 & 107 & 98 \\ 103 & 105 & 109 \\ 119 & 115 & 115 \\ 108 & 108 & 108 \\ 113 & 117 & 119 \\ 111 & 111 & 112 \\ 112 & 114 & 116 \\ 109 & 106 & 109\end{array}$

16.57

4.7

19.81

0.2

27.37

7.8 
102

Laboratory blank

NATI8-W-20731

NATI8-W-20732

85

NAQCTB-W-20733

SDG 06-5-5a, analysis date May 5, 2006

20-ppb standard

Laboratory blank

105

NATI13-W-20556

NATI13-W-20555

NATI12-W-20757

NATI12-W-20758

NAQCRIN-W-20760

NAQCTB-W-20759

Laboratory blank 2

Methanol blank

NATI12-S-20737

NATI12-S-20744

NATI12-S-20741

NATI12-S-20739

NATI12-S-20746

$\begin{array}{ccc}100 & 100 & 100 \\ 93 & 89 & 88 \\ 106 & 111 & 112 \\ 107 & 113 & 118 \\ 103 & 101 & 104 \\ 109 & 122^{\mathrm{d}} & 122^{\mathrm{d}} \\ 107 & 135^{\mathrm{d}} & 138^{\mathrm{d}} \\ 115 & 111 & 115 \\ 106 & 103 & 103 \\ 98 & 92 & 86 \\ 114 & 105 & 110 \\ 109 & 104 & 107 \\ 111 & 100 & 103 \\ 108 & 94 & 102 \\ 106 & 100 & 103\end{array}$

21.58

1.9

21.43

1.7

1.6
Reanalyzed in SDG 06-5-8a.

Reanalyzed in SDG 06-5-8a. 
SDG 06-5-5a, analysis date May 5, 2006 (cont.)

NATI12-S-20743
NATI12-S-20740
NATI12-S-20736
NATI12-S-20742
NATI12-S-20735
NATI12-S-20745
NATI12-S-20738
NATI12-S-20734

SDG 06-5-5b, analysis date May 5, 2006

$\begin{array}{lccc}\text { 20-ppb standard } & 99 & 98 & 95 \\ \text { Laboratory blank } & 108 & 108 & 109 \\ \text { NATI13-W-20560 } & 91 & 95 & 94 \\ \text { NATI13-W-20561 } & 103 & 107 & 104 \\ \text { NATI13-W-20562 } & 92 & 96 & 94 \\ \text { NATI13-W-20563 } & 96 & 97 & 95 \\ \text { NATI14-W-20666 } & & & \\ & 89 & 92 & 90 \\ \text { NATI14-W-20666DUP } & 89 & 92 & 90 \\ & & & \\ \text { NAQCTB-W-20584 } & 103 & 100 & 97 \\ \text { Laboratory blank 2 } & 100 & 100 & 100 \\ \text { Methanol blank } & 112 & 98 & 83 \\ \text { NATI13-S-20549 } & 103 & 92 & 84 \\ \text { NATI13-S-20557 } & 120 & 106 & 93 \\ \text { NATI12-S-20749 } & 105 & 95 & 88\end{array}$

Reanalyzed in SDG 06-5-8b.

$\begin{array}{llllll}20.11 & 0.1 & 19.95 & 0.1 & 21.57 & 1.9\end{array}$

Outside calibration for carbon tetrachloride at DF1. Analyzed at dilution in SDG 065-8a. Methylene chloride and tetrachloroethylene reported.

Outside calibration for carbon tetrachloride at DF1. Analyzed at dilution in SDG 065-8a. Methylene chloride reported.

Outside calibration for carbon tetrachloride at DF1. Analyzed at dilution in SDG 065-8a. Methylene chloride reported.

Outside calibration for carbon tetrachloride at DF1. Analyzed at dilution in SDG 065-8a. Methylene chloride reported. 
aboratory blank

NATI13-S-20541

NATI13-S-20542

NATI13-S-20543

NATI13-S-20544

NATI13-S-20545

NATI13-S-20545DUP

NATI13-S-20546

NATI13-S-20533

NATI13-S-20547

Methanol blank

NATI13-S-20532

NATI13-S-20540

NATI13-S-20535

NATI13-S-20538

NATI13-S-20534

NATI10-S-20630

NATI10-S-20629

NATI13-S-20548

NATI13-S-20539

NATI13-S-20537

NATI13-S-20536

NAQCTB-S-20552
Measured Concentration and RPD Value for Calibration Check Standard

\section{Recovery of Surrogate Compounds ${ }^{\mathrm{a}}(\%)$}

Bromo-

Dichloro-

Fluorobenzene fluorobenzene benzene- $d_{4}$

\section{Carbon}

Tetrachloride

$\mathrm{ppb}^{\mathrm{b}}$
Chloroform

$\mathrm{ppb}^{\mathrm{b}}$ $\mathrm{ppb}^{\mathrm{b}}$

Methylene Chloride

$\mathrm{RPD}^{\mathrm{c}}$

97

88

$\begin{array}{lcl}119 & 107 & 97 \\ 105 & 97 & 88\end{array}$

0.3

18.78

1.6

25.19

5.7 
SDG 06-5-6, analysis date May 6, 2006

20-ppb standard
Laboratory blank
NATI12-S-20754
NATI12-S-20755
NATI13-S-20558
NATI12-S-20750
NATI12-S-20756
NATI12-S-20752
NATI13-S-20553
Methanol blank
NATI13-S-20550
NATI12-S-20751
NATI12-S-20747
NATI13-S-20554

100
100
$126^{\mathrm{d}}$
110
100
93
86
93
91
100
90
87
85
87

100
100
$48^{d}$
114
105
97
99
111
$133^{d}$
100
94
101
$197^{d}$
97

Measured Concentration and RPD Value for Calibration Check Standard

100

114

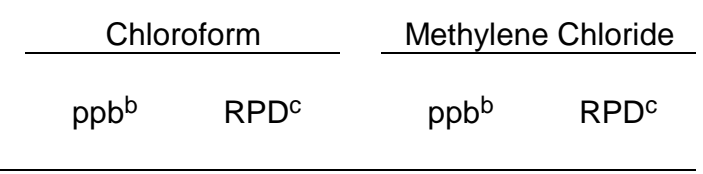

SDG 06-5-8a, analysis date May 8, 2006

$\begin{array}{lccc}\text { 20-ppb standard } & 84 & 97 & 83 \\ \text { Laboratory blank } & 100 & 100 & 100 \\ \text { NATI13-W-20562 } & 103 & 107 & 112 \\ \text { NATI13-W-20563 } & 102 & 90 & 89 \\ \text { NATI14-W-20666 } & 109 & 92 & 96 \\ \text { NATI14-W-20666DUP } & 91 & 81 & 86 \\ \text { NATI12-W-20758 } & 108 & 106 & 109 \\ \text { NAQCRIN-W-20760 } & 97 & 91 & 95\end{array}$

Accepted.

Accepted.

Accested

$\begin{array}{lllllll}83 & 17.64 & 3.1 & 19.87 & 0.2 & 19.37 & 0.8 \\ 100 & & & & & & \\ 12 & & & & & \\ 89 & & & & & \\ 96 & & & & & \\ 86 & & & & & \\ 09 & & & & \end{array}$


SDG 06-5-8b, analysis date May 8, 2006

$\begin{array}{lccc}\text { 20-ppb standard } & 88 & 95 & 111 \\ \text { Laboratory blank } & 100 & 100 & 100 \\ \text { NATI13-S-20553 } & 99 & 94 & 86 \\ \text { NAQCTB-S-20551 } & 88 & 83 & 82 \\ \text { NAQCTB-S-20559 } & 84 & 91 & 96 \\ \text { NATI14-S-20671 } & 82 & 83 & 84 \\ \text { Methanol blank } & 100 & 100 & 100 \\ \text { NATI14-S-20672 } & 91 & 95 & 102 \\ \text { NATI14-S-20659 } & 104 & 103 & 102 \\ \text { NATI14-S-20670 } & 102 & 102 & 100 \\ \text { NATI14-S-20660 } & 110 & 110 & 105 \\ \text { NATI14-S-20668 } & 120 & 113 & 103 \\ \text { NATI14-S-20665 } & 79 \mathrm{~d} & 80 & 80 \\ \text { NATI14-S-20658 } & 87 & 85 & 86 \\ \text { NATI14-S-20657 } & 96 & 95 & 92 \\ \text { NATI12-S-20734 } & 101 & 100 & 96 \\ \text { NATI14-S-20663 } & 110 & 116 & 118 \\ \text { NATI14-S-20664 } & 106 & 106 & 107 \\ \text { NATI14-S-20662 } & 107 & 102 & 101 \\ \text { NATI14-S-20669 } & 103 & 97 & 96 \\ \text { NATI14-S-20661 } & 99 & 95 & 98\end{array}$

Measured Concentration and RPD Value for Calibration Check Standard

\begin{tabular}{|c|c|c|c|c|c|c|c|c|c|}
\hline \multirow[b]{3}{*}{ Sample } & \multicolumn{3}{|c|}{ Recovery of Surrogate Compounds ${ }^{\mathrm{a}}(\%)$} & \multirow{2}{*}{\multicolumn{2}{|c|}{$\begin{array}{c}\text { Carbon } \\
\text { Tetrachloride }\end{array}$}} & \multirow{2}{*}{\multicolumn{2}{|c|}{ Chloroform }} & \multirow{2}{*}{\multicolumn{2}{|c|}{ Methylene Chloride }} \\
\hline & & & & & & & & & \\
\hline & Fluorobenzene & $\begin{array}{l}\text { Bromo- } \\
\text { fluorobenzene }\end{array}$ & $\begin{array}{c}\text { Dichloro- } \\
\text { benzene- } \mathrm{d}_{4}\end{array}$ & $\mathrm{ppb}^{\mathrm{b}}$ & $\mathrm{RPD}^{\mathrm{c}}$ & $\mathrm{ppb}^{\mathrm{b}}$ & $\mathrm{RPD}^{\mathrm{c}}$ & $\mathrm{ppb}^{\mathrm{b}}$ & $\mathrm{RPD}^{\mathrm{C}}$ \\
\hline
\end{tabular}

SDG 06-5-10a, analysis date May 10, 2006

$\begin{array}{lccc}\text { 20-ppb standard } & 100 & 100 & 100 \\ \text { Laboratory blank } & 100 & 100 & 100 \\ \text { NATI15-W-20761 } & 96 & 88 & 83 \\ \text { NATI14-W-20768 } & 91 & 93 & 93\end{array}$

Accepted.

NATI14-W-20768 
SDG 06-5-10a, analysis date May 10, 2006 (cont.)

$\begin{array}{lccc}\text { NATI15-W-20764 } & 106 & 100 & 96 \\ \text { NATI14-W-20695 } & 84 & 87 & 87 \\ \text { NATI16-W-20790 } & 101 & 102 & 103 \\ \text { NATI17-W-20793 } & 98 & 98 & 101 \\ \text { NATI15-W-20762 } & 95 & 93 & 93 \\ \text { NATI15-W-20822 } & 84 & 83 & 86 \\ \text { NATI16-W-20817 } & 92 & 90 & 89 \\ & & & \\ \text { NATI17-W-20827 } & 92 & 93 & 91 \\ \text { NATI17-W-20826 } & 95 & 91 & 87 \\ \text { NATI16-W-20791 } & 94 & 91 & 89 \\ & & & \\ \text { NATI17-W-20814 } & 90 & 87 & 86 \\ \text { NATI13-W-20819 } & 87 & 86 & 88 \\ \text { NATI15-W-20821 } & 98 & 96 & 95 \\ \text { NATI15-W-20823 } & 73^{\mathrm{d}} & 74^{\mathrm{d}} & 74^{\mathrm{d}} \\ \text { NATI17-W-20829 } & 77^{\mathrm{d}} & 80 & 82 \\ \text { NATI17-W-20829DUP } & 97 & 93 & 90 \\ \text { NAQCTB-W-20824 } & 99 & 94 & 100 \\ \text { NAQCTB-W-20694 } & 70^{\mathrm{d}} & 67^{\mathrm{d}} & 75^{\mathrm{d}} \\ \text { NAQCRIN-W-20769 } & 0^{\mathrm{d}} & 141^{\mathrm{d}} & 118^{\mathrm{d}}\end{array}$

Outside calibration for carbon tetrachloride at DF1. Analyzed at dilution in SDG 065-12b. Tetrachloroethylene reported.

Outside calibration for carbon tetrachloride and chloroform at DF1. Analyzed at dilution in SDG 06-5-12b. Tetrachloroethylene reported.

Outside calibration for carbon tetrachloride at DF1. Analyzed at dilution in SDG 065-12b. Tetrachloroethylene reported.

Reanalyzed in SDG 06-5-13a. Reanalyzed in SDG 06-5-12b.

Accepted.

Reanalyzed in SDG 06-5-12a.

SDG 06-5-10b, analysis date May 10, 2006

20-ppb standard

Laboratory blank

NATI16-W-20789

NATI14-W-20692

$\begin{array}{ccc}104 & 119 & 95 \\ 100 & 100 & 100 \\ 79^{d} & 77^{d} & 67^{d} \\ 101 & 115 & 110\end{array}$

20.85

1.0

21.69

2.0

21.23

1.5

Reanalyzed in SDG 06-5-13a. 
SDG 06-5-10b, analysis date May 10, 2006 (cont.)

NATI14-W-20693
NATI15-W-20644
NATI15-W-20765
NATI15-W-20763
NATI14-W-20691

NATI13-W-20820

NATI17-W-20828

NATI17-W-20825

NATI16-W-20818

NATI16-W-20816

SDG 06-5-12a, analysis date May 12, 2006

$\begin{array}{ccc}92 & 111 & 119 \\ 101 & 101 & 100 \\ 96 & 96 & 93 \\ 91 & 99 & 99 \\ 93 & 91 & 86 \\ & & \\ 106 & 96 & 91 \\ & & \\ 95 & 87 & 84 \\ 91 & 87 & 83 \\ 99 & 86 & 83 \\ 102 & 101 & 105\end{array}$

Outside calibration for carbon tetrachloride at DF1. Analyzed at dilution in SDG 065-12b. Methylene chloride and tetrachloroethylene reported.

Outside calibration for carbon tetrachloride at DF1. Analyzed at dilution in SDG 06$5-12 b$. Methylene chloride and tetrachloroethylene reported.

Outside calibration for carbon tetrachloride at DF1. Analyzed at dilution in SDG 065-12b. Methylene chloride reported.

Outside calibration for carbon tetrachloride and chloroform at zero dilution.

Analyzed at dilution in SDG 06-5-12a. Tetrachloroethylene reported.

$\begin{array}{lccc}\text { 20-ppb standard } & 92 & 106 & 82 \\ \text { Laboratory blank } & 100 & 100 & 100 \\ \text { NATI22-W-20830 } & 94 & 81 & 82 \\ \text { NATI18-W-20831 } & 118 & 111 & 118 \\ & & & \\ \text { NATI22-W-20833 } & 104 & 97 & 104 \\ \text { NATI19-W-20868 } & 87 & 89 & 94 \\ \text { NATI19-W-20868DUP } & 94 & 99 & 106 \\ \text { NATI23-W-20862 } & 99 & 108 & 116 \\ \text { NATI19-W-20871 } & 92 & 95 & 98 \\ \text { NATI24-W-20860 } & 92 & 92 & 94 \\ \text { NATI23-W-20856 } & 88 & 89 & 96\end{array}$


SDG 06-5-12a, analysis date May 12, 2006 (cont.)

NATI19-W-20870

NATI23-W-20861

NATI23-W-20859

NATI24-W-20858

Laboratory blank 2

NATI19-W-20864

NATI19-W-20869

NATI23-W-20865

NATI19-W-20863

NATI23-W-20857

NATI24-W-20845

NATI24-W-20845DUP

NAQCTB-W-20866

NAQCRIN-W-20837

NAQCRIN-W-20769

Laboratory blank 3

NATI16-W-20818

SDG 06-5-12b, analysis date May 12, 2006

20-ppb standard

Laboratory blank

NATI22-W-20834

NATI22-W-20836

$\begin{array}{cc}100 & 100 \\ 96 & 90 \\ 102 & 96 \\ 113 & 109 \\ & \\ 107 & 117 \\ 105 & 109 \\ 104 & 110\end{array}$

NATI24-W-20838

NATI22-W-20846

NATI22-W-20840
Measured Concentration and RPD Value for Calibration Check Standard

\begin{tabular}{|c|c|c|c|c|c|}
\hline \multicolumn{2}{|c|}{$\begin{array}{c}\text { Carbon } \\
\text { Tetrachloride }\end{array}$} & \multicolumn{2}{|c|}{ Chloroform } & \multicolumn{2}{|c|}{ Methylene Chloride } \\
\hline $\mathrm{ppb}^{\mathrm{b}}$ & $\mathrm{RPD}^{\mathrm{c}}$ & $\mathrm{ppb}^{\mathrm{b}}$ & $\mathrm{RPD}^{\mathrm{c}}$ & $\mathrm{ppb}^{\mathrm{b}}$ & $\mathrm{RPD}^{\mathrm{c}}$ \\
\hline
\end{tabular}

Reanalyzed in SDG 06-5-13a.

Analysis at DF20 for carbon tetrachloride, chloroform, and methylene chloride.

$\begin{array}{cc}95 & 94 \\ 94 & 101 \\ 96 & 96 \\ 84 & 86 \\ 87 & 89 \\ 78^{d} & 81 \\ 101 & 100 \\ 80 & 81 \\ 88 & 94 \\ 94 & 91 \\ 88 & 85 \\ 95 & 93 \\ 93 & 88 \\ 89 & 87 \\ 91 & 90 \\ 88 & 84\end{array}$

100

89

96

109

111

110

111
22.64

3.1

21.32

1.6

24.24

4.8

Outside calibration for carbon tetrachloride at DF1. Analyzed at dilution in SDG 065-13a. Methylene chloride reported.

Outside calibration for carbon tetrachloride at DF1. Analyzed at dilution in SDG 065-13a. Methylene chloride reported. 
SDG 06-5-12b, analysis date May 12, 2006 (cont.)

$\begin{array}{lccc}\text { NATI24-W-20841 } & 101 & 107 & 108 \\ \text { NATI24-W-20842 } & 99 & 100 & 96 \\ \text { NATI24-W-20843 } & 97 & 98 & 90 \\ \text { NATI24-W-20843DUP } & 99 & 104 & 97 \\ \text { NATI23-W-20844 } & 101 & 105 & 95 \\ \text { NATI18-W-20832 } & 92 & 101 & 94 \\ & & & \\ \text { NATI22-W-20835 } & 101 & 105 & 95 \\ \text { NATI24-W-20839 } & 86 & 84 & 91 \\ \text { NATI22-W-20847 } & 93 & 93 & 85 \\ \text { NATI21-W-20867 } & 92 & 92 & 85 \\ \text { NATI14-W-20768 } & 95 & 99 & 94 \\ \text { NATI13-W-20820 } & 100 & 111 & 97 \\ \text { NATI16-W-20817 } & 96 & 99 & 90 \\ \text { NATI16-W-20791 } & 92 & 95 & 85 \\ \text { NATI16-W-20791DUP } & 86 & 88 & 81 \\ \text { Laboratory blank } 2 & 100 & 100 & 100 \\ \text { NATI17-W-20829 } & 101 & 106 & 104 \\ \text { NATI14-W-20695 } & 98 & 97 & 94 \\ \text { NATI14-W-20693 } & 98 & 100 & 95 \\ \text { NATI14-W-20691 } & 97 & 97 & 93\end{array}$

Outside calibration for carbon tetrachloride at DF1. Analyzed at dilution in SDG 065-13a. Methylene chloride reported.

Analysis at DF5 for carbon tetrachloride, chloroform, and methylene chloride Analysis at DF5 for carbon tetrachloride and chloroform.

Analysis at DF20 for carbon tetrachloride and chloroform.

Analysis at DF5 for carbon tetrachloride, chloroform, and methylene chloride.

Analysis at DF5 for carbon tetrachloride, chloroform, and methylene chloride.

Analysis at DF5 for carbon tetrachloride and chloroform.

Analysis at DF5 for carbon tetrachloride and chloroform.

Analysis at DF5 for carbon tetrachloride and chloroform.

SDG 06-5-13a, analysis date May 13, 2006

$\begin{array}{lccc}\text { 20-ppb standard } & 99 & 97 & 98 \\ \text { Laboratory blank } & 104 & 110 & 105 \\ \text { NATI16-W-20789 } & 96 & 93 & 103 \\ \text { NATI15-W-20823 } & 104 & 99 & 109 \\ \text { NATI26-W-20233 } & 99 & 91 & 99\end{array}$

20.91

1.1

19.64

0.5

22.29 
SDG 06-5-13a, analysis date May 13, 2006 (cont.)

$\begin{array}{lccc}\text { NATI26-W-20230 } & 100 & 93 & 104 \\ \text { NATI26-W-20723 } & 99 & 109 & 102 \\ \text { NATI26-W-20719 } & 99 & 107 & 103 \\ \text { NATI26-W-20719DUP } & 95 & 89 & 95 \\ \text { NATI25-W-20720 } & 96 & 92 & 97 \\ \text { NATI25-W-20722 } & 97 & 95 & 102 \\ \text { NATI19-W-20231 } & 96 & 90 & 97 \\ \text { NATI26-W-20229 } & 87 & 83 & 88 \\ \text { NATI25-W-20232 } & 108 & 100 & 95 \\ \text { NATI25-W-20235 } & 90 & 84 & 89 \\ \text { NATI26-W-20718 } & 90 & 96 & 90 \\ \text { NATI26-W-20721 } & 94 & 95 & 105 \\ \text { Laboratory blank } 2 & 102 & 81 & 87 \\ \text { NATI19-W-20869 } & 107 & 92 & 98 \\ \text { NATI22-W-20836 } & 106 & 85 & 91 \\ \text { NATI22-W-20840 } & 108 & 90 & 95 \\ \text { NATI18-W-20832 } & 103 & 87 & 93 \\ \text { NATI18-W-20831 } & 104 & 86 & 91 \\ \text { NATI18-W-20831DUP } & 106 & 85 & 90\end{array}$

Analysis at DF10 for carbon tetrachloride and chloroform.

Analysis at DF5 for carbon tetrachloride and chloroform.

Analysis at DF20 for carbon tetrachloride and chloroform.

Analysis at DF20 for carbon tetrachloride and chloroform.

Analysis at DF20 for carbon tetrachloride and chloroform.

SDG 06-5-13b, analysis date May 13, 2006

$\begin{array}{lccc}\text { 20-ppb standard } & 100 & 100 & 100 \\ \text { Laboratory blank } & 100 & 100 & 100 \\ \text { NATI21-W-20228 } & 102 & 92 & 91 \\ \text { NATI19-W-20872 } & 104 & 106 & 101 \\ \text { NATI21-W-20881 } & 109 & 104 & 107 \\ \text { NATI21-W-20885 } & 103 & 101 & 103 \\ \text { NATI21-W-20885DUP } & 96 & 96 & 97\end{array}$

$\begin{array}{llllll}21.17 & 1.4 & 21.33 & 1.6 & 20.8\end{array}$

1.0 
SDG 06-5-13b, analysis date May 13, 2006 (cont.)

$\begin{array}{lccc}\text { NATI25-W-20884 } & 99 & 113 & 96 \\ \text { NATI26-W-20877 } & 102 & 112 & 110 \\ \text { NATI21-W-20879 } & 103 & 106 & 105 \\ \text { NATI26-W-20883 } & 93 & 117 & 114 \\ \text { NATI21-W-20882 } & 94 & 97 & 99 \\ \text { NATI25-W-20878 } & 87 & 109 & 99 \\ \text { NATI25-W-20875 } & 81 & 110 & 99 \\ \text { NATI25-W-20876 } & 0^{\mathrm{d}} & 0^{\mathrm{d}} & 0^{\mathrm{d}} \\ \text { NATI21-W-20880 } & 0^{\mathrm{d}} & 0^{\mathrm{d}} & 0^{\mathrm{d}} \\ \text { NAQCTB-W-20724 } & 82 & 71^{\mathrm{d}} & 68^{\mathrm{d}} \\ \text { NAQCTB-W-20873 } & 118 & 92 & 92 \\ \text { NAQCRIN-W-20234 } & 118 & 94 & 89 \\ \text { NAQCRIN-W-20874 } & 116 & 92 & 86\end{array}$

Reanalyzed in SDG 06-5-15.

Reanalyzed in SDG 06-5-15.

Reanalyzed in SDG 06-5-15.

Chloroform $\quad$ Methylene Chloride

$\begin{array}{llll}\mathrm{ppb}^{\mathrm{b}} & \mathrm{RPD} & \mathrm{ppb}^{\mathrm{b}} & \mathrm{RPD}^{\mathrm{c}}\end{array}$

SDG 06-5-15, analysis date May 15, 2006

$\begin{array}{lc}\text { 20-ppb standard } & 111 \\ \text { Laboratory blank } & 100 \\ \text { NATI25-W-20876 } & 101 \\ \text { NATI21-W-20880 } & 100 \\ \text { NATI21-W-20880DUP } & 97 \\ \text { NAQCTB-W-20724 } & 96 \\ \text { Methanol blank } & 100 \\ \text { NATI14-S-20671DUP } & 100 \\ \text { NATI14-S-20667 } & 102 \\ \text { NATI14-S-20656 } & 98 \\ \text { NAQCTB-S-20583 } & 95 \\ \text { NATI15-S-20568 } & 99 \\ \text { NATI15-S-20573 } & 98\end{array}$

$\begin{array}{cc}118 & 106 \\ 100 & 100 \\ 104 & 101 \\ 103 & 102 \\ 102 & 103 \\ 100 & 101 \\ 100 & 100 \\ 103 & 106 \\ 99 & 105 \\ 104 & 110 \\ 100 & 104 \\ 103 & 105 \\ 104 & 108\end{array}$

20.59

0.7

18.58

1.8

22.33

2.8

98


SDG 06-5-15, analysis date May 15, 2006 (cont.)

$\begin{array}{lccc}\text { NATI15-S-20570 } & 112 & 152^{\mathrm{d}} & 144^{\mathrm{d}} \\ \text { NATI15-S-20575 } & 105 & 108 & 89 \\ \text { NATI15-S-20571 } & 96 & 105 & 107 \\ \text { NATI15-S-20574 } & 106 & 111 & 116 \\ \text { NATI15-S-20576 } & 107 & 114 & 116 \\ \text { NATI15-S-20569 } & 102 & 106 & 106 \\ \text { NATI15-S-20572 } & 102 & 107 & 110 \\ \text { NATI15-S-20566 } & 105 & 109 & 93\end{array}$

Measured Concentration and RPD Value for Calibration Check Standard

\begin{tabular}{|c|c|c|c|c|c|c|c|c|c|}
\hline \multirow[b]{3}{*}{ Sample } & \multicolumn{3}{|c|}{ Recovery of Surrogate Compounds ${ }^{a}(\%)$} & \multirow{2}{*}{\multicolumn{2}{|c|}{$\begin{array}{c}\text { Carbon } \\
\text { Tetrachloride }\end{array}$}} & \multirow{2}{*}{\multicolumn{2}{|c|}{ Chloroform }} & \multirow{2}{*}{\multicolumn{2}{|c|}{ Methylene Chloride }} \\
\hline & & & & & & & & & \\
\hline & Fluorobenzene & $\begin{array}{l}\text { Bromo- } \\
\text { fluorobenzene }\end{array}$ & $\begin{array}{c}\text { Dichloro- } \\
\text { benzene- } \mathrm{d}_{4}\end{array}$ & $\mathrm{ppb}^{\mathrm{b}}$ & $\mathrm{RPD}^{\mathrm{c}}$ & $\mathrm{ppb}^{\mathrm{b}}$ & $\mathrm{RPD}^{\mathrm{c}}$ & $\mathrm{ppb}^{\mathrm{b}}$ & $\mathrm{RPD}^{\mathrm{c}}$ \\
\hline
\end{tabular}

SDG 06-5-16a, analysis date May 16, 2006

$\begin{array}{lccc}\text { 20-ppb standard } & 83 & 101 & 100 \\ \text { Methanol blank } & 95 & 90 & 91 \\ \text { NATI15-S-20567 } & 93 & 107 & 107 \\ \text { NAQCTB-S-20582 } & 107 & 194^{\mathrm{d}} & 153^{\mathrm{d}} \\ \text { NATI17-S-20809 } & 101 & 119 & 121^{\mathrm{d}} \\ \text { NATI17-S-20796 } & 98 & 118 & 117 \\ \text { NATI17-S-20804 } & 97 & 104 & 114 \\ \text { NATI16-S-20781 } & 93 & 107 & 111 \\ \text { NATI17-S-20802 } & 92 & 108 & 117 \\ \text { Methanol blank } 2 & 100 & 100 & 100 \\ \text { NATI17-S-20799 } & 90 & 101 & 101 \\ \text { NATI17-S-20798 } & 88 & 97 & 100 \\ \text { NATI17-S-20798DUP } & 88 & 97 & 101 \\ \text { NATI17-S-20803 } & 89 & 99 & 100 \\ \text { NATI17-S-20811 } & 91 & 105 & 105 \\ \text { NATI17-S-20808 } & 91 & 154^{\mathrm{d}} & 107 \\ \text { NATI17-S-20801 } & 88 & 105 & 103 \\ \text { NATI16-S-20774 } & 93 & 102 & 104\end{array}$

Reanalyzed in SDG 06-5-18.

107

106

93

.

00

91

$53^{d}$

$121^{d}$

117

114

111
117

100

101

100

101

105

107

104 
SDG 06-5-16b, analysis date May 16, 2006

$\begin{array}{lccc}\text { 20-ppb standard } & 100 & 100 & 100 \\ \text { Methanol blank } & 100 & 100 & 100 \\ \text { NATI17-S-20812 } & 98 & 90 & 104 \\ \text { NATI17-S-20797 } & 97 & 92 & 108 \\ \text { NATI17-S-20810 } & 99 & 94 & 110 \\ \text { NATI17-S-20810DUP } & 97 & 91 & 109 \\ \text { NATI17-S-20806 } & 97 & 90 & 104 \\ \text { NATI17-S-20800 } & 97 & 90 & 103 \\ \text { NATI17-S-20807 } & 97 & 101 & 104 \\ \text { Methanol blank2 } & 97 & 88 & 103 \\ \text { NATI17-S-20805 } & 96 & 92 & 104 \\ \text { NATI16-S-20780 } & 94 & 86 & 102 \\ \text { NATI16-S-20783 } & 94 & 89 & 103 \\ \text { NATI16-S-20787 } & 95 & 92 & 104 \\ \text { NATI16-S-20788 } & 96 & 99 & 108 \\ \text { NATI16-S-20776 } & 96 & 102 & 106 \\ \text { NATI16-S-20779 } & 97 & 91 & 106 \\ \text { NATI14-S-20673 } & 98 & 98 & 112 \\ \text { NATI16-S-20786 } & 98 & 103 & 109\end{array}$


SDG 06-5-16b, analysis date May 16, 2006 (cont.)

$\begin{array}{lccc}\text { NATI16-S-20777 } & 95 & 102 & 106 \\ \text { NATI15-S-20564 } & 96 & 95 & 109 \\ \text { NATI16-S-20778 } & 95 & 93 & 108 \\ \text { NATI15-S-20581 } & 96 & 94 & 106 \\ \text { NATI14-S-20687 } & 85 & 96 & 99 \\ \text { NATI14-S-20686 } & 91 & 102 & 103\end{array}$

SDG 06-5-17, analysis date May 17, 2006

$\begin{array}{lccc}\text { 20-ppb standard } & 100 & 100 & 100 \\ \text { Methanol blank } & 111 & 113 & 111 \\ \text { NATI14-S-20688 } & 93 & 89 & 100 \\ \text { NATI14-S-20689 } & 91 & 87 & 98 \\ \text { NATI15-S-20565 } & 93 & 89 & 98 \\ \text { NATI14-S-20690 } & 91 & 85 & 94 \\ \text { NATI16-S-20784 } & 88 & 84 & 94 \\ \text { NATI14-S-20675 } & 89 & 92 & 91 \\ \text { NATI15-S-20579 } & 70^{\text {d }} & 68^{d} & 78^{d} \\ \text { Methanol blank } & 89 & 87 & 89 \\ \text { NATI14-S-20674 } & 88 & 89 & 89 \\ \text { NATI14-S-20674DUP } & 88 & 80 & 90 \\ \text { NAQCTB-S-20794 } & 84 & 83 & 86 \\ \text { NAQCTB-S-20795 } & 91 & 94 & 98 \\ \text { NAQCTB-S-20767 } & 85 & 81 & 91 \\ \text { NATI15-S-20580 } & 96 & 91 & 102 \\ \text { NATI15-S-20577 } & 91 & 94 & 98\end{array}$

$\begin{array}{llllll}18.66 & 1.7 & 18.8 & 1.5 & 18.91 & 1.4\end{array}$

Reanalyzed in SDG 06-5-18. 
SDG 06-5-18, analysis date May 18, 2006

$\begin{array}{lccc}\text { 20-ppb standard } & 100 & 100 & 100 \\ \text { Laboratory blank } & 116 & 115 & 116 \\ \text { NATI15-S-20578 } & 91 & 96 & 96 \\ \text { NATI15-S-20766 } & 94 & 92 & 103 \\ \text { NATI15-S-20766DUP } & 87 & 92 & 90 \\ \text { NATI15-S-20570 } & 109 & 112 & 109 \\ \text { NATI15-S-20585 } & 87 & 92 & 92 \\ \text { NAQCTB-S-20582 } & 84 & 93 & 87 \\ \text { NATI17-S-20809 } & 83 & 80 & 87 \\ \text { NATI16-S-20770 } & 86 & 85 & 94 \\ \text { NATI17-S-20808 } & 85 & 82 & 90 \\ \text { NATI15-S-20579 } & 90 & 88 & 96\end{array}$

SDG 06-5-20, analysis date May 20, 2006

$\begin{array}{lccc}\text { 20-ppb standard } & 96 & 102 & 94 \\ \text { Laboratory blank } & 100 & 100 & 100 \\ \text { NATI28-W-20647 } & 97 & 88 & 90 \\ \text { NATI28-W-20254 } & 97 & 99 & 100 \\ \text { NATI28-W-20255 } & 95 & 99 & 102 \\ \text { NATI27-W-20680 } & 94 & 100 & 97 \\ \text { NATI27-W-20684 } & 89 & 95 & 91 \\ \text { NATI28-W-20683 } & 88 & 92 & 87 \\ \text { NATI28-W-20683DUP } & 84 & 85 & 81 \\ \text { NAQCTB-W-20682 } & 84 & 80 & 82 \\ \text { NAQCRIN-W-20648 } & 82 & 91 & 82\end{array}$

Measured Concentration and RPD Value for Calibration Check Standard

Carbon

$\mathrm{ppb}^{\mathrm{b}}$

$\mathrm{RPD}^{\mathrm{c}}$

Chloroform

$\mathrm{ppb}^{\mathrm{b}} \quad \mathrm{RPD}^{\mathrm{c}}$

Methylene Chloride

$\mathrm{ppb}^{\mathrm{b}}$

$\mathrm{RPD}^{\mathrm{C}}$

9.64

0.5

19.02

1.3

18.8

1.5

03

87

90
96

88

20.67

20.67

19.87

0.2

19.57

0.5 
SDG 06-5-22a, analysis date May 22, 2006

20-ppb standard
Laboratory blank
NATI28-W-20851
NATI28-W-20849
NATI28-W-20849DUP
NATI28-W-20850
NATI27-W-20701
NATI27-W-20704
NATI27-W-20699
NATI27-W-20700
Methanol blank
NATI28-S-20240
NATI18-S-20260
NATI18-S-20257
NATI18-S-20258
NATI28-S-20238
Methanol blank 2
NATI28-S-20249
NATI28-S-20251
NATI28-S-20248
NATI28-S-20245
NAQCTB-S-20681

115
85
104

111
101
103
111
97
95
95
109
111
113
111
112
113
102
103
106
106
105
118

101

80

96

108

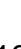

115

102

120

118
97

97
91

89

89
96

96
99

107

103

101

100

91
97

97
93

98

98
$159^{d}$

SDG 06-5-22b, analysis date May 22, 2006

$\begin{array}{lccc}\text { 20-ppb standard } & 107 & 118 & 118 \\ \text { Methanol blank } & 106 & 111 & 113 \\ \text { NATI28-S-20242 } & 98 & 104 & 107\end{array}$

113

116

115

111

110

105

92

106

101

100

96

90

101

95

102

$133^{\mathrm{d}}$

118

Measured Concentration and RPD Value for Calibration Check Standard

\begin{tabular}{|c|c|c|c|c|c|}
\hline \multicolumn{2}{|c|}{$\begin{array}{c}\text { Carbon } \\
\text { Tetrachloride }\end{array}$} & \multicolumn{2}{|c|}{ Chloroform } & \multicolumn{2}{|c|}{ Methylene Chloride } \\
\hline $\mathrm{ppb}^{\mathrm{b}}$ & $\mathrm{RPD}^{\mathrm{c}}$ & $\mathrm{ppb}^{\mathrm{b}}$ & $\mathrm{RPD}^{\mathrm{c}}$ & $\mathrm{ppb}^{\mathrm{b}}$ & $\mathrm{RPD}^{\mathrm{c}}$ \\
\hline
\end{tabular}

Reanalyzed in SDG 06-5-25a.

Outside calibration for carbon tetrachloride at DF1. Analyzed at DF100 in SDG 065-23. Methylene chloride and tetrachloroethylene reported.

20.72

0.9

19.23

1.0

18.81

1.5 
SDG 06-5-22b, analysis date May 22, 2006 (cont.)

$\begin{array}{lccc}\text { NATI28-S-20243 } & 106 & 119 & 120 \\ \text { NATI28-S-20241 } & 101 & 11 & 96 \\ \text { NATI28-S-20239 } & 102 & 105 & 99 \\ \text { NATI28-S-20244 } & 107 & 106 & 115 \\ \text { NATI28-S-20244DUP } & 101 & 109 & 108 \\ \text { NATI18-S-20679 } & 96 & 104 & 103 \\ \text { Methanol blank } 2 & 99 & 91 & 92 \\ \text { NATI28-S-20237 } & 94 & 95 & 89 \\ \text { NATI18-S-20256 } & 92 & 93 & 90 \\ \text { NATI18-S-20259 } & 88 & 82 & 85 \\ \text { NATI28-S-20236 } & 98 & 88 & 92 \\ \text { NAQCTB-S-20263 } & 84 & 84 & 85 \\ \text { NATI18-S-20265 } & 96 & 99 & 94 \\ \text { NATI18-S-20678 } & 91 & 97 & 92 \\ \text { Methanol blank 3 } & 95 & 98 & 95 \\ \text { NATI18-S-20676 } & 103 & 111 & 111 \\ \text { NATI28-S-20252 } & 97 & 91 & 93 \\ \text { NATI28-S-20247 } & 97 & 92 & 95 \\ \text { NATI28-S-20250 } & 95 & 90 & 94 \\ \text { NATI28-S-20253 } & 97 & 97 & 93 \\ \text { NATI28-S-20246 } & 96 & 89 & 92 \\ \text { NATI18-S-20677 } & 94 & 85 & 93 \\ \text { NATI18-S-20677DUP } & 95 & 87 & 90\end{array}$

SDG 06-5-23, analysis date May 23, 2006

$\begin{array}{llcccccccc}20-p p b \text { standard } & 92 & 106 & 98 & 24.12 & 4.7 & 22.73 & 3.2 & 22.55 & 3.0 \\ \text { Laboratory blank } & 88 & 89 & 90 & \end{array}$


SDG 06-5-23, analysis date May 23, 2006 (cont.)

$\begin{array}{lccc}\text { NATI18-W-20655 } & 89 & 113 & 105 \\ \text { NATI18-W-20706 } & 99 & 113 & 117 \\ \text { NATI18-W-20711 } & 97 & 113 & 109 \\ \text { NATI18-W-20709 } & 96 & 116 & 115 \\ \text { NATI18-W-20707 } & 92 & 111 & 110 \\ & & & \\ \text { NATI18-W-20710 } & 87 & 92 & 97 \\ \text { NATI18-W-20710DUP } & 89 & 105 & 102 \\ \text { NATI27-W-20905 } & 89 & 95 & 100 \\ \text { NATI27-W-20649 } & 87 & 100 & 96 \\ \text { NATI28-W-20904 } & 85 & 90 & 92 \\ \text { NATI29-W-20906 } & 75^{\mathrm{d}} & 84 & 88 \\ \text { NATI29-W-20907 } & 88 & 90 & 94 \\ \text { NATI29-W-20909 } & 70^{\mathrm{d}} & 80 & 79^{d} \\ & & & \\ \text { NATI29-W-20902 } & 85 & 96 & 100 \\ & & & \\ \text { NATI29-W-20903 } & 87 & 92 & 96 \\ \text { NATI29-W-20900 } & 74^{\mathrm{d}} & 81 & 79^{d} \\ & & & \\ \text { NATI29-W-20901 } & 105 & 120 & 117 \\ \text { NAQCRIN-W-20908 } & 112 & 111 & 110 \\ \text { NAQCTB-W-20650 } & 96 & 100 & 96 \\ \text { NATI18-W-20655 } & 99 & 107 & 107 \\ \text { NATI18-W-20706 } & 96 & 91 & 95\end{array}$

Outside calibration for carbon tetrachloride at DF1. Analysis at DF10 below in this SDG. Methylene chloride reported.

Outside calibration for carbon tetrachloride at DF1. Analysis at DF10 below in this SDG. Methylene chloride reported.

Outside calibration for carbon tetrachloride at DF1. Analysis at DF10 in SDG 06-524. Methylene chloride and tetrachloroethylene reported.

Outside calibration for carbon tetrachloride at DF1. Analysis at DF100 in SDG 065-24. Methylene chloride and tetrachloroethylene reported.

Reanalyzed in SDG 06-5-24.

Outside calibration for carbon tetrachloride at DF1. Analysis at DF5 in SDG 06-524. Methylene chloride is detected in the reanalysis.

Outside calibration for carbon tetrachloride at DF1. Analysis at DF5 in SDG 06-524. Methylene chloride reported.

Outside calibration for carbon tetrachloride at DF1. Analysis at DF10 in SDG 06-524. Methylene chloride reported.

Outside calibration for carbon tetrachloride at DF1. Analysis at DF10 in SDG 06-524. Methylene chloride and tetrachloroethylene reported.

Analysis at DF10 for carbon tetrachloride and chloroform. Analysis at DF10 for carbon tetrachloride and chloroform. 
SDG 06-5-23, analysis date May 23, 2006 (cont.)

NATI28-W-20851

NATI28-W-20851DUP
101

85
93

85

Analysis at DF100 for carbon tetrachloride and chloroform. Analysis at DF100 for carbon tetrachloride and chloroform.

22.32

2.7

21.51

1.8

21.21

1.5

Laboratory blank

NATI30-W-20911

NATI30-W-20915

NATI30-W-20917

NATI30-W-20917DUP

NATI30-W-20920

NATI30-W-20918

NATI30-W-20919

NATI20-W-20912

NATI20-W-20913

NATI20-W-20922

NATI20-W-20923

NATI20-W-20914

NATI29-W-20916

NATI18-W-20921

NAQCTB-W-20925

NAQCRIN-W-20924

NATI29-W-20906

NATI29-W-20901

NATI29-W-20902

NATI28-W-20904

NATI28-W-20904DUP

NATI18-W-20707

112
88
114
109
109
95
106
104
$127^{d}$
89
98
90
88
95
90
89
89
93
92
93
94
96
87
90

90

103

109

111

103

113

$135^{\mathrm{d}}$

91

100

91

91

97

98

92

90

90

93

92

96

82

85
Measured Concentration and RPD Value for Calibration Check Standard

Chloroform

Methylene Chloride

$\mathrm{ppb}^{\mathrm{b}} \quad \mathrm{RPD}^{\mathrm{c}}$

Reanalyzed in SDG 06-5-25a.

Analysis at DF10 for carbon tetrachloride and chloroform Analysis at DF5 for carbon tetrachloride and chloroform.

Analysis at DF100 for carbon tetrachloride and chloroform. Analysis at DF100 for carbon tetrachloride and chloroform. Analysis at DF10 for carbon tetrachloride and chloroform. 
aboratory blank

NATI30-W-20712

NATI30-W-20712DUP

NATI20-W-20713

NAQCTB-W-20714

NATI30-W-20919

Methanol blank

NATI29-S-20697

NATI29-S-20897

NATI29-S-20698

NATI29-S-20891

NATI29-S-20892

NATI29-S-20703

NATI29-S-20703DUP

NATI29-S-20887

NAQCTB-S-20899

NATI29-S-20898

NATI18-S-20651

NAQCTB-S-20681

NATI29-S-20956

\section{8}

88

88
Measured Concentration and RPD Value for Calibration Check Standard

\begin{tabular}{|c|c|c|c|c|c|}
\hline \multicolumn{2}{|c|}{$\begin{array}{c}\text { Carbon } \\
\text { Tetrachloride }\end{array}$} & \multicolumn{2}{|c|}{ Chloroform } & \multicolumn{2}{|c|}{ Methylene Chloride } \\
\hline $\mathrm{ppb}^{\mathrm{b}}$ & $\mathrm{RPD}^{\mathrm{c}}$ & $\mathrm{ppb}^{\mathrm{b}}$ & $\mathrm{RPD}^{\mathrm{c}}$ & $\mathrm{ppb}^{\mathrm{b}}$ & $\mathrm{RPD}^{\mathrm{c}}$ \\
\hline
\end{tabular}

Analysis at DF5 for carbon tetrachloride and chloroform.
84 Analysis at DF10 for carbon tetrachloride and chloroform. 
SDG 06-5-25b, analysis date May 25, 2006

$\begin{array}{lccc}\text { 20-ppb standard } & 94 & 99 & 97 \\ \text { Laboratory blank } & 117 & 116 & 116 \\ \text { NATI29-S-20893 } & 100 & 100 & 96 \\ \text { NATI18-S-20652 } & 107 & 112 & 103 \\ \text { NATI29-S-20896 } & 103 & 100 & 100 \\ \text { NATI18-S-20653 } & 100 & 103 & 100 \\ \text { NATI28-S-20696 } & 103 & 106 & 109 \\ \text { NATI29-S-20894 } & 100 & 102 & 103 \\ \text { NATI29-S-20894DUP } & 98 & 97 & 93 \\ \text { NATI29-S-20705 } & 96 & 93 & 94 \\ \text { NATI29-S-20855 } & 95 & 95 & 97 \\ \text { NATI29-S-20886 } & 93 & 97 & 95 \\ \text { NATI29-S-20888 } & 97 & 95 & 95 \\ \text { NATI29-S-20889 } & 95 & 93 & 93 \\ \text { NATI29-S-20890 } & 91 & 90 & 91 \\ \text { NATI18-S-20654 } & 90 & 90 & 88\end{array}$

\begin{tabular}{|c|c|c|c|c|c|}
\hline \multicolumn{2}{|c|}{$\begin{array}{c}\text { Carbon } \\
\text { Tetrachloride }\end{array}$} & \multicolumn{2}{|c|}{ Chloroform } & \multicolumn{2}{|c|}{ Methylene Chloride } \\
\hline $\mathrm{ppb}^{\mathrm{b}}$ & $\mathrm{RPD}^{\mathrm{c}}$ & $\mathrm{ppb}^{\mathrm{b}}$ & $\mathrm{RPD}^{\mathrm{c}}$ & $\mathrm{ppb}^{\mathrm{b}}$ & $\mathrm{RPD}^{\mathrm{C}}$ \\
\hline
\end{tabular}

Bromobenzene-d

6.34

5.0

22.04

2.4

19.65

0.4

SDG 06-5-26, analysis date May 26, 2006

$\begin{array}{lc}\text { 20-ppb standard } & 94 \\ \text { Laboratory blank } & 96 \\ \text { NATI31-W-20947 } & 104 \\ \text { NATI31-W-20715 } & 100 \\ \text { NATI31-W-20936 } & 99 \\ \text { NAANDER2-W-20942 } & 91 \\ \text { NATI30-W-20939 } & 91 \\ \text { NATI31-W-20938 } & 93 \\ \text { NAANDER3-W-20943 } & 89 \\ \text { NATI31-W-20937 } & 90\end{array}$

$\begin{array}{cc}103 & 93 \\ 91 & 94 \\ 109 & 106 \\ 116 & 114 \\ 109 & 107 \\ 103 & 98 \\ 98 & 94 \\ 95 & 95 \\ 93 & 90 \\ 97 & 95\end{array}$

18.4

17.61

3.2

17.14 
a Quality control range for recovery $=80-120 \%$.

b Concentration in parts per billion ( $\mu \mathrm{g} / \mathrm{L}$ in water or $\mu \mathrm{g} / \mathrm{kg}$ in soil).

c Quality control range for RPD $= \pm 20 \%$.

d Surrogate recovery outside quality control range. 
TABLE S4.5 Comparison of AGEM Laboratory results for primary and secondary organic analyses of samples collected during the 2006 investigation at Navarre, Kansas.

\begin{tabular}{|c|c|c|c|c|c|c|c|c|}
\hline \multirow[b]{2}{*}{ Location } & \multirow[b]{2}{*}{ Depth $^{a}$} & \multirow[b]{2}{*}{$\begin{array}{c}\text { Sample } \\
\text { Date }\end{array}$} & \multirow[b]{2}{*}{ Medium } & \multirow[b]{2}{*}{ Sample } & \multirow[b]{2}{*}{$\begin{array}{c}\text { Analysis } \\
\text { Type }\end{array}$} & \multicolumn{3}{|c|}{$\begin{array}{c}\text { Concentration } \\
(\mu \mathrm{g} / \mathrm{L} \text { in water; } \mu \mathrm{g} / \mathrm{kg} \text { in soil })\end{array}$} \\
\hline & & & & & & $\begin{array}{c}\text { Carbon } \\
\text { Tetrachloride }\end{array}$ & Chloroform & $\begin{array}{l}\text { Methylene } \\
\text { Chloride }\end{array}$ \\
\hline \multirow[t]{2}{*}{$\mathrm{CP} 1$} & 9.1 & $4 / 10 / 06$ & Soil & NACP1-S-20329 & Primary sample & $N D^{b}$ & ND & ND \\
\hline & & & & NACP1-S-20329DUP & Duplicate analysis & ND & ND & ND \\
\hline \multirow[t]{3}{*}{ L-1 } & $75-95$ & $4 / 8 / 06$ & Water & NAL1-W-20211 & Primary sample & 54 & 11 & ND \\
\hline & & & & NAL1-W-20211DUP & Duplicate analysis & 54 & 11 & ND \\
\hline & & & & NAQCDU-W-20213 & Replicate sample & 57 & 11 & ND \\
\hline \multirow[t]{2}{*}{ MW2 } & $42.8-57.8$ & $4 / 6 / 06$ & Water & NAMW02-W-20188 & Primary sample & 27 & 7.7 & ND \\
\hline & & & & NAQCDU-W-20200 & Replicate sample & 27 & 7.8 & ND \\
\hline \multirow[t]{2}{*}{ MW3 } & $44-59$ & $4 / 6 / 06$ & Water & NAMW03-W-20192 & Primary sample & 83 & 20 & ND \\
\hline & & & & NAMW03-W-20192DUP & Duplicate analysis & 83 & 20 & ND \\
\hline \multirow[t]{2}{*}{ NW-1 } & $40-50$ & $4 / 8 / 06$ & Water & NANW1-W-20215 & Primary sample & 1.1 & $0.3 \mathrm{~J}^{\mathrm{c}}$ & ND \\
\hline & & & & NAQCDU-W-20225 & Replicate sample & 1.2 & $0.4 \mathrm{~J}$ & ND \\
\hline \multirow[t]{2}{*}{ TI-1 } & 23.75 & $4 / 11 / 06$ & Soil & NATI1-S-20467 & Primary sample & $1.3 \mathrm{~J}$ & ND & ND \\
\hline & & & & NATI1-S-20467DUP & Duplicate analysis & $1.4 \mathrm{~J}$ & $0.7 \mathrm{~J}$ & ND \\
\hline \multirow[t]{2}{*}{ TI-1 } & 31 & $4 / 8 / 06$ & Soil & NATI1-S-20306 & Primary sample & 56 & $3.7 \mathrm{~J}$ & ND \\
\hline & & & & NATI1-S-20307 & Replicate sample & 68 & $1.8 \mathrm{~J}$ & ND \\
\hline \multirow[t]{2}{*}{ Tl-1 } & $38-43$ & $4 / 13 / 06$ & Water & NATI1-W-20342 & Primary sample & 177 & 64 & 10.2 \\
\hline & & & & NATI1-W-20343 & Replicate sample & 168 & 61 & 8.8 \\
\hline \multirow[t]{2}{*}{ TI-1 } & 44 & $4 / 13 / 06$ & Soil & NATI1-S-20482 & Primary sample & $3.3 \mathrm{~J}$ & $2.6 \mathrm{~J}$ & ND \\
\hline & & & & NATI1-S-20483 & Replicate sample & $1.9 \mathrm{~J}$ & $2.7 \mathrm{~J}$ & ND \\
\hline \multirow[t]{2}{*}{ TI-2 } & 16 & $4 / 5 / 06$ & Soil & NATI2-S-20411 & Primary sample & ND & ND & ND \\
\hline & & & & NATI2-S-20412 & Replicate sample & ND & ND & ND \\
\hline \multirow[t]{2}{*}{ TI-2 } & 24.5 & $4 / 6 / 06$ & Soil & NATI2-S-20414 & Primary sample & ND & ND & ND \\
\hline & & & & NATI2-S-20414DUP & Duplicate analysis & ND & ND & ND \\
\hline \multirow[t]{2}{*}{ TI-2 } & $31-36$ & $4 / 21 / 06$ & Water & NATI2-W-20277 & Primary sample & 8.7 & 7.8 & ND \\
\hline & & & & NATI2-W-20278 & Replicate sample & 7.8 & 7.8 & ND \\
\hline \multirow[t]{2}{*}{ TI-2 } & 38 & $4 / 6 / 06$ & Soil & NATI2-S-20419 & Primary sample & 65 & $3.9 \mathrm{~J}$ & ND \\
\hline & & & & NATI2-S-20419DUP & Duplicate analysis & 70 & $4.1 \mathrm{~J}$ & ND \\
\hline \multirow[t]{2}{*}{ TI-2 } & $66-70$ & $4 / 21 / 06$ & Water & NATI2-W-20282 & Primary sample & 14 & 2.8 & ND \\
\hline & & & & NATI2-W-20282DUP & Duplicate analysis & 14 & 2.8 & ND \\
\hline \multirow[t]{3}{*}{ TI-2 } & $69-72$ & $4 / 7 / 06$ & Water & NATI2-W-20432 & Primary sample & ND & ND & ND \\
\hline & & & & NATI2-W-20432DUP & Duplicate analysis & ND & ND & ND \\
\hline & & & & NATI2-W-20433 & Replicate sample & ND & ND & ND \\
\hline
\end{tabular}


TABLE S4.5 (Cont.)

\begin{tabular}{|c|c|c|c|c|c|c|c|c|}
\hline \multirow[b]{2}{*}{ Location } & \multirow[b]{2}{*}{ Depth $^{\mathrm{a}}$} & \multirow[b]{2}{*}{$\begin{array}{l}\text { Sample } \\
\text { Date }\end{array}$} & \multirow[b]{2}{*}{ Medium } & \multirow[b]{2}{*}{ Sample } & \multirow[b]{2}{*}{$\begin{array}{l}\text { Analysis } \\
\text { Type }\end{array}$} & \multicolumn{3}{|c|}{$\begin{array}{c}\text { Concentration } \\
(\mu \mathrm{g} / \mathrm{L} \text { in water; } \mu \mathrm{g} / \mathrm{kg} \text { in soil })\end{array}$} \\
\hline & & & & & & $\begin{array}{c}\text { Carbon } \\
\text { Tetrachloride }\end{array}$ & Chloroform & $\begin{array}{l}\text { Methylene } \\
\text { Chloride }\end{array}$ \\
\hline \multirow[t]{2}{*}{ TI-3 } & 25.5 & $4 / 7 / 06$ & Soil & NATI3-S-20297 & Primary sample & ND & ND & ND \\
\hline & & & & NATI3-S-20297DUP & Duplicate analysis & ND & ND & ND \\
\hline \multirow[t]{2}{*}{ TI-3 } & $43-48$ & $4 / 14 / 06$ & Water & NATI3-W-20383 & Primary sample & 72 & 12 & ND \\
\hline & & & & NATI3-W-20384 & Replicate sample & 57 & 11 & ND \\
\hline \multirow[t]{2}{*}{ TI-3 } & 56.5 & $4 / 13 / 06$ & Soil & NATI3-S-20379 & Primary sample & $1.3 \mathrm{~J}$ & ND & ND \\
\hline & & & & NATI3-S-20380 & Replicate sample & $2.1 \mathrm{~J}$ & $1 \mathrm{~J}$ & ND \\
\hline \multirow{2}{*}{ TI-4 } & 34 & $4 / 9 / 06$ & Soil & NATI4-S-20317 & Primary sample & $0.8 \mathrm{~J}$ & ND & ND \\
\hline & & & & NATI4-S-20318 & Replicate sample & $3.1 \mathrm{~J}$ & $2.8 \mathrm{~J}$ & ND \\
\hline \multirow[t]{2}{*}{ TI-4 } & $55-60$ & $4 / 11 / 06$ & Water & NATI4-W-20470 & Primary sample & 23 & 7 & ND \\
\hline & & & & NATI4-W-20471 & Replicate sample & 23 & 6.3 & ND \\
\hline \multirow[t]{2}{*}{ TI-4 } & 61 & $4 / 9 / 06$ & Soil & NATI4-S-20324 & Primary sample & ND & ND & ND \\
\hline & & & & NATI4-S-20324DUP & Duplicate analysis & ND & ND & ND \\
\hline \multirow[t]{2}{*}{ TI-4 } & $61-66$ & $4 / 12 / 06$ & Water & NATI4-W-20472 & Primary sample & $0.6 \mathrm{~J}$ & 3.6 & ND \\
\hline & & & & NATI4-W-20472DUP & Duplicate analysis & $0.6 \mathrm{~J}$ & 3.6 & ND \\
\hline \multirow[t]{2}{*}{ TI-5 } & 17.2 & $4 / 11 / 06$ & Soil & NATI5-S-20334 & Primary sample & ND & ND & ND \\
\hline & & & & NATI5-S-20334DUP & Duplicate analysis & ND & ND & ND \\
\hline \multirow[t]{2}{*}{ TI-5 } & $25-25$ & $4 / 11 / 06$ & Soil & NATI5-S-20356 & Primary sample & ND & ND & ND \\
\hline & & & & NATI5-S-20357 & Replicate sample & ND & ND & ND \\
\hline \multirow[t]{2}{*}{ TI-5 } & 49.5 & $4 / 11 / 06$ & Soil & NATI5-S-20364 & Primary sample & ND & ND & ND \\
\hline & & & & NATI5-S-20364DUP & Duplicate analysis & $2.9 \mathrm{~J}$ & ND & ND \\
\hline \multirow[t]{2}{*}{ TI-6 } & 2 & $4 / 20 / 06$ & Soil & NATI6-S-20390 & Primary sample & ND & ND & ND \\
\hline & & & & NATI6-S-20390DUP & Duplicate analysis & ND & ND & ND \\
\hline \multirow[t]{2}{*}{ TI-6 } & $38-43$ & $4 / 22 / 06$ & Water & NATI6-W-20353 & Primary sample & 1.3 & 1 & ND \\
\hline & & & & NATI6-W-20353DUP & Duplicate analysis & 1.3 & 1 & ND \\
\hline \multirow[t]{2}{*}{ TI-6 } & 50 & $4 / 20 / 06$ & Soil & NATI6-S-20402 & Primary sample & $1.3 \mathrm{~J}$ & ND & ND \\
\hline & & & & NATI6-S-20403 & Replicate sample & $1 \mathrm{~J}$ & ND & ND \\
\hline \multirow[t]{2}{*}{ TI-6 } & $68-73$ & $4 / 22 / 06$ & Water & NATI6-W-20350 & Primary sample & ND & ND & ND \\
\hline & & & & NATI6-W-20351 & Replicate sample & ND & ND & ND \\
\hline \multirow[t]{2}{*}{ TI-7 } & 29 & $4 / 9 / 06$ & Soil & NATI7-S-20449 & Primary sample & ND & ND & ND \\
\hline & & & & NATI7-S-20449DUP & Duplicate analysis & ND & ND & ND \\
\hline \multirow[t]{3}{*}{ TI-7 } & $37-42$ & $4 / 20 / 06$ & Water & NATI7-W-20270 & Primary sample & 3.8 & 1.9 & ND \\
\hline & & & & NATI7-W-20271 & Replicate sample & 3 & 1.7 & ND \\
\hline & & & & NATI7-W-20271DUP & Duplicate analysis & 2.8 & 1.5 & ND \\
\hline
\end{tabular}


TABLE S4.5 (Cont.)

\begin{tabular}{|c|c|c|c|c|c|c|c|c|}
\hline \multirow[b]{2}{*}{ Location } & \multirow[b]{2}{*}{ Depth $^{a}$} & \multirow[b]{2}{*}{$\begin{array}{c}\text { Sample } \\
\text { Date }\end{array}$} & \multirow[b]{2}{*}{ Medium } & \multirow[b]{2}{*}{ Sample } & \multirow[b]{2}{*}{$\begin{array}{c}\text { Analysis } \\
\text { Type }\end{array}$} & \multicolumn{3}{|c|}{$\begin{array}{c}\text { Concentration } \\
(\mu \mathrm{g} / \mathrm{L} \text { in water; } \mu \mathrm{g} / \mathrm{kg} \text { in soil) }\end{array}$} \\
\hline & & & & & & $\begin{array}{c}\text { Carbon } \\
\text { Tetrachloride }\end{array}$ & Chloroform & $\begin{array}{l}\text { Methylene } \\
\text { Chloride }\end{array}$ \\
\hline \multirow[t]{2}{*}{ TI-8 } & 54.6 & $4 / 27 / 06$ & Soil & NATI8-S-20526 & Primary sample & ND & ND & ND \\
\hline & & & & NATI8-S-20527 & Replicate sample & ND & ND & ND \\
\hline \multirow[t]{2}{*}{ TI-9 } & 25 & $4 / 22 / 06$ & Soil & NATI9-S-20595 & Primary sample & ND & ND & ND \\
\hline & & & & NATI9-S-20596 & Replicate sample & ND & ND & ND \\
\hline \multirow[t]{3}{*}{$\mathrm{Tl}-10$} & 43 & $4 / 26 / 06$ & Soil & NATI10-S-20624 & Primary sample & ND & ND & ND \\
\hline & & & & NATI10-S-20625 & Replicate sample & ND & ND & ND \\
\hline & & & & NATI10-S-20625DUP & Duplicate analysis & ND & ND & ND \\
\hline \multirow[t]{2}{*}{ TI-10 } & $46-51$ & $4 / 27 / 06$ & Water & NATI10-W-20632 & Primary sample & $0.4 \mathrm{~J}$ & ND & 11 \\
\hline & & & & NATI10-W-20634 & Replicate sample & $0.1 \mathrm{~J}$ & $0.1 \mathrm{~J}$ & 6.2 \\
\hline \multirow[t]{2}{*}{ TI-10 } & 54 & $4 / 26 / 06$ & Soil & NATI10-S-20636 & Primary sample & ND & ND & ND \\
\hline & & & & NATI10-S-20636DUP & Duplicate analysis & ND & ND & ND \\
\hline \multirow[t]{2}{*}{ TI-10 } & $59-64$ & $4 / 27 / 06$ & Water & NATI10-W-20637 & Primary sample & $0.3 \mathrm{~J}$ & ND & ND \\
\hline & & & & NATI10-W-20637DUP & Duplicate analysis & $0.4 \mathrm{~J}$ & ND & ND \\
\hline \multirow{2}{*}{ TI-10 } & $66-71$ & $4 / 25 / 06$ & Water & NATI10-W-20610 & Primary sample & ND & ND & ND \\
\hline & & & & NATI10-W-20610DUP & Duplicate analysis & ND & ND & ND \\
\hline \multirow[t]{2}{*}{ TI-11 } & 38 & $4 / 23 / 06$ & Soil & NATI11-S-20495 & Primary sample & ND & ND & ND \\
\hline & & & & NATI11-S-20495DUP & Duplicate analysis & ND & ND & ND \\
\hline \multirow[t]{2}{*}{ Tl-11 } & 42 & $4 / 23 / 06$ & Soil & NATI11-S-20496 & Primary sample & ND & ND & ND \\
\hline & & & & NATI11-S-20496DUP & Duplicate analysis & ND & ND & ND \\
\hline \multirow[t]{2}{*}{ TI-11 } & $46.3-51.3$ & $4 / 23 / 06$ & Water & NATI11-W-20500 & Primary sample & $0.9 \mathrm{~J}$ & $0.1 \mathrm{~J}$ & ND \\
\hline & & & & NATI11-W-20501 & Replicate sample & 1 & ND & ND \\
\hline \multirow[t]{2}{*}{ TI-11 } & 56.5 & $4 / 25 / 06$ & Soil & NATI11-S-20503 & Primary sample & ND & ND & ND \\
\hline & & & & NATI11-S-20504 & Replicate sample & ND & ND & ND \\
\hline \multirow[t]{2}{*}{ TI-11 } & $65.9-70.9$ & $4 / 27 / 06$ & Water & NATI111-W-20639 & Primary sample & $0.4 \mathrm{~J}$ & ND & ND \\
\hline & & & & NATI11-W-20639DUP & Duplicate analysis & $0.4 \mathrm{~J}$ & ND & ND \\
\hline \multirow[t]{2}{*}{ TI-12 } & 21 & $5 / 3 / 06$ & Soil & NATI12-S-20739 & Primary sample & ND & ND & ND \\
\hline & & & & NATI12-S-20740 & Replicate sample & ND & ND & ND \\
\hline \multirow[t]{2}{*}{ TI-12 } & $34-39$ & $5 / 2 / 06$ & Water & NATI12-W-20727 & Primary sample & ND & ND & ND \\
\hline & & & & NATI12-W-20727DUP & Duplicate analysis & ND & ND & ND \\
\hline \multirow[t]{2}{*}{ TI-12 } & $39.4-44.4$ & $5 / 2 / 06$ & Water & NATI12-W-20728 & Primary sample & ND & ND & ND \\
\hline & & & & NATI12-W-20729 & Replicate sample & ND & ND & ND \\
\hline \multirow[t]{2}{*}{$\mathrm{TI}-13$} & $42-47$ & $5 / 5 / 06$ & Water & NATI13-W-20562 & Primary sample & 116 & 60 & 1.2 \\
\hline & & & & NATI13-W-20820 & Replicate sample & 155 & 80 & 1.2 \\
\hline
\end{tabular}


TABLE S4.5 (Cont.)

\begin{tabular}{|c|c|c|c|c|c|c|c|c|}
\hline \multirow[b]{2}{*}{ Location } & \multirow[b]{2}{*}{ Depth $^{\mathrm{a}}$} & \multirow[b]{2}{*}{$\begin{array}{l}\text { Sample } \\
\text { Date }\end{array}$} & \multirow[b]{2}{*}{ Medium } & \multirow[b]{2}{*}{ Sample } & \multirow[b]{2}{*}{$\begin{array}{l}\text { Analysis } \\
\text { Type }\end{array}$} & \multicolumn{3}{|c|}{$\begin{array}{c}\text { Concentration } \\
(\mu \mathrm{g} / \mathrm{L} \text { in water; } \mu \mathrm{g} / \mathrm{kg} \text { in soil })\end{array}$} \\
\hline & & & & & & $\begin{array}{l}\text { Carbon } \\
\text { Tetrachloride }\end{array}$ & Chloroform & $\begin{array}{l}\text { Methylene } \\
\text { Chloride }\end{array}$ \\
\hline \multirow[t]{2}{*}{ TI-13 } & $66.8-71.8$ & $5 / 4 / 06$ & Water & NATI13-W-20555 & Primary sample & $0.6 \mathrm{~J}$ & $0.3 \mathrm{~J}$ & ND \\
\hline & & & & NATI13-W-20556 & Replicate sample & $0.5 \mathrm{~J}$ & $0.2 \mathrm{~J}$ & ND \\
\hline \multirow[t]{2}{*}{ TI-14 } & 35.25 & $5 / 5 / 06$ & Soil & NATI14-S-20671 & Primary sample & 364 & 16 & ND \\
\hline & & & & NATI14-S-20671DUP & Duplicate analysis & 387 & $6.1 \mathrm{~J}$ & ND \\
\hline \multirow[t]{2}{*}{ TI-14 } & 44.5 & $5 / 5 / 06$ & Soil & NATI14-S-20674 & Primary sample & 114 & $2.3 \mathrm{~J}$ & ND \\
\hline & & & & NATI14-S-20674DUP & Duplicate analysis & 139 & $2.9 \mathrm{~J}$ & ND \\
\hline \multirow[t]{2}{*}{ TI-14 } & $67.4-72.4$ & $5 / 5 / 06$ & Water & NATI14-W-20666 & Primary sample & 6 & 280 & 12.6 \\
\hline & & & & NATI14-W-20666DUP & Duplicate analysis & 6.6 & 319 & 12.1 \\
\hline \multirow[t]{2}{*}{ Tl-15 } & $15-20$ & $5 / 8 / 06$ & Water & NATI15-W-20644 & Primary sample & ND & ND & ND \\
\hline & & & & NATI15-W-20821 & Replicate sample & ND & ND & ND \\
\hline \multirow[t]{2}{*}{ TI-15 } & $25-30$ & $5 / 9 / 06$ & Water & NATI15-W-20822 & Primary sample & 45 & 9.2 & ND \\
\hline & & & & NATI15-W-20823 & Replicate sample & 35 & 9.5 & ND \\
\hline \multirow[t]{2}{*}{ TI-15 } & 60.75 & $5 / 6 / 06$ & Soil & NATI15-S-20766 & Primary sample & ND & ND & ND \\
\hline & & & & NATI15-S-20766DUP & Duplicate analysis & ND & ND & ND \\
\hline \multirow[t]{2}{*}{ TI-15 } & $67.1-72.1$ & $5 / 6 / 06$ & Water & NATI15-W-20761 & Primary sample & ND & ND & ND \\
\hline & & & & NATI15-W-20762 & Replicate sample & ND & ND & ND \\
\hline \multirow[t]{2}{*}{ TI-16 } & $39-44$ & $5 / 8 / 06$ & Water & NATI16-W-20791 & Primary sample & 189 & 123 & 6 \\
\hline & & & & NATI16-W-20791DUP & Duplicate analysis & 197 & 128 & 6 \\
\hline \multirow[t]{2}{*}{ TI-17 } & 9.1 & $5 / 7 / 06$ & Soil & NATI17-S-20798 & Primary sample & ND & ND & ND \\
\hline & & & & NATI17-S-20798DUP & Duplicate analysis & ND & ND & ND \\
\hline \multirow[t]{2}{*}{$\mathrm{TI}-17$} & $39-44$ & $5 / 9 / 06$ & Water & NATI17-W-20826 & Primary sample & 1.7 & 1.6 & ND \\
\hline & & & & NATI17-W-20827 & Replicate sample & 2.6 & 3.5 & ND \\
\hline \multirow[t]{2}{*}{ TI-17 } & 49.25 & $5 / 7 / 06$ & Soil & NATI17-S-20810 & Primary sample & ND & ND & ND \\
\hline & & & & NATI17-S-20810DUP & Duplicate analysis & ND & ND & ND \\
\hline \multirow[t]{2}{*}{ TI-17 } & $53-58$ & $5 / 9 / 06$ & Water & NATI17-W-20829 & Primary sample & 2.5 & 1.5 & ND \\
\hline & & & & NATI17-W-20829DUP & Duplicate analysis & 2.3 & 1.4 & ND \\
\hline \multirow[t]{2}{*}{ TI-18 } & 37 & $5 / 19 / 06$ & Soil & NATI18-S-20677 & Primary sample & 109 & ND & ND \\
\hline & & & & NATI18-S-20677DUP & Duplicate analysis & 107 & ND & ND \\
\hline \multirow[t]{2}{*}{ TI-18 } & $35-40$ & $5 / 10 / 06$ & Water & NATI18-W-20831 & Primary sample & 782 & 47 & 2.7 \\
\hline & & & & NATI18-W-20832 & Replicate sample & 809 & 41 & 1.1 \\
\hline \multirow[t]{2}{*}{ TI-18 } & 41 & $5 / 19 / 06$ & Soil & NATI18-S-20678 & Primary sample & 400 & $9.2 \mathrm{~J}$ & ND \\
\hline & & & & NATI18-S-20679 & Replicate sample & 348 & $9.6 \mathrm{~J}$ & ND \\
\hline
\end{tabular}


TABLE S4.5 (Cont.)

\begin{tabular}{|c|c|c|c|c|c|c|c|c|}
\hline \multirow[b]{2}{*}{ Location } & \multirow[b]{2}{*}{ Depth $^{a}$} & \multirow[b]{2}{*}{$\begin{array}{l}\text { Sample } \\
\text { Date }\end{array}$} & \multirow[b]{2}{*}{ Medium } & \multirow[b]{2}{*}{ Sample } & \multirow[b]{2}{*}{$\begin{array}{l}\text { Analysis } \\
\text { Type }\end{array}$} & \multicolumn{3}{|c|}{$\begin{array}{c}\text { Concentration } \\
(\mu \mathrm{g} / \mathrm{L} \text { in water; } \mu \mathrm{g} / \mathrm{kg} \text { in soil })\end{array}$} \\
\hline & & & & & & $\begin{array}{c}\text { Carbon } \\
\text { Tetrachloride }\end{array}$ & Chloroform & $\begin{array}{l}\text { Methylene } \\
\text { Chloride }\end{array}$ \\
\hline \multirow[t]{3}{*}{ TI-18 } & $56-61$ & $5 / 21 / 06$ & Water & NATI18-W-20709 & Primary sample & 70 & 35 & 1.5 \\
\hline & & & & NATI18-W-20710 & Replicate sample & 77 & 38 & 1.5 \\
\hline & & & & NATI18-W-20710DUP & Duplicate analysis & 68 & 34 & 1.4 \\
\hline \multirow[t]{2}{*}{ TI-19 } & $46-51$ & $5 / 11 / 06$ & Water & NATI19-W-20870 & Primary sample & $0.3 \mathrm{~J}$ & ND & ND \\
\hline & & & & NATI19-W-20871 & Replicate sample & $0.2 \mathrm{~J}$ & ND & ND \\
\hline \multirow[t]{2}{*}{ TI-19 } & $53-58$ & $5 / 11 / 06$ & Water & NATI19-W-20868 & Primary sample & $0.3 \mathrm{~J}$ & ND & ND \\
\hline & & & & NATI19-W-20868DUP & Duplicate analysis & $0.3 \mathrm{~J}$ & ND & ND \\
\hline \multirow[t]{2}{*}{ TI-20 } & $72-77$ & $5 / 23 / 06$ & Water & NATI20-W-20922 & Primary sample & ND & ND & ND \\
\hline & & & & NATI20-W-20923 & Replicate sample & ND & ND & ND \\
\hline \multirow[t]{2}{*}{ TI-21 } & $32-37$ & $5 / 11 / 06$ & Water & NATI21-W-20885 & Primary sample & $0.9 \mathrm{~J}$ & $0.9 \mathrm{~J}$ & ND \\
\hline & & & & NATI21-W-20885DUP & Duplicate analysis & $0.8 \mathrm{~J}$ & $0.9 \mathrm{~J}$ & ND \\
\hline \multirow[t]{3}{*}{ TI-21 } & $60-65$ & $5 / 11 / 06$ & Water & NATI21-W-20880 & Primary sample & ND & $0.4 \mathrm{~J}$ & ND \\
\hline & & & & NATI21-W-20880DUP & Duplicate analysis & ND & $0.4 \mathrm{~J}$ & ND \\
\hline & & & & NATI21-W-20879 & Replicate sample & $0.4 \mathrm{~J}$ & $0.7 \mathrm{~J}$ & ND \\
\hline \multirow[t]{2}{*}{ TI-22 } & $39-44$ & $5 / 10 / 06$ & Water & NATI22-W-20834 & Primary sample & 26 & 12 & ND \\
\hline & & & & NATI22-W-20835 & Replicate sample & 35 & 14 & ND \\
\hline \multirow[t]{2}{*}{ TI-22 } & $68.2-73.2$ & $5 / 10 / 06$ & Water & NATI22-W-20846 & Primary sample & ND & ND & ND \\
\hline & & & & NATI22-W-20847 & Replicate sample & ND & ND & ND \\
\hline \multirow[t]{2}{*}{ TI-23 } & $60-65$ & $5 / 10 / 06$ & Water & NATI23-W-20856 & Primary sample & 1 & ND & ND \\
\hline & & & & NATI23-W-20857 & Replicate sample & 1.9 & ND & ND \\
\hline \multirow[t]{2}{*}{ TI-24 } & $39-44$ & $5 / 10 / 06$ & Water & NATI24-W-20838 & Primary sample & 1.5 & 1.9 & ND \\
\hline & & & & NATI24-W-20839 & Replicate sample & 2.2 & 2.6 & ND \\
\hline \multirow[t]{2}{*}{ TI-24 } & $60-65$ & $5 / 10 / 06$ & Water & NATI24-W-20843 & Primary sample & 1 & $0.6 \mathrm{~J}$ & ND \\
\hline & & & & NATI24-W-20843DUP & Duplicate analysis & $0.8 \mathrm{~J}$ & $0.5 \mathrm{~J}$ & ND \\
\hline \multirow[t]{2}{*}{ TI-24 } & $69.4-74.4$ & $5 / 10 / 06$ & Water & NATI24-W-20845 & Primary sample & ND & ND & ND \\
\hline & & & & NATI24-W-20845DUP & Duplicate analysis & ND & ND & ND \\
\hline \multirow[t]{2}{*}{ TI-25 } & $39-44$ & $5 / 11 / 06$ & Water & NATI25-W-20875 & Primary sample & ND & $0.2 \mathrm{~J}$ & ND \\
\hline & & & & NATI25-W-20876 & Replicate sample & ND & ND & ND \\
\hline \multirow[t]{2}{*}{ TI-26 } & $53-58$ & $5 / 11 / 06$ & Water & NATI26-W-20229 & Primary sample & 4.8 & 3.1 & ND \\
\hline & & & & NATI26-W-20230 & Replicate sample & 3.6 & 2.7 & ND \\
\hline \multirow[t]{2}{*}{ TI-26 } & $66-71$ & $5 / 12 / 06$ & Water & NATI26-W-20719 & Primary sample & 3.2 & 8.1 & ND \\
\hline & & & & NATI26-W-20719DUP & Duplicate analysis & 3.2 & 8.2 & ND \\
\hline
\end{tabular}


TABLE S4.5 (Cont.)

\begin{tabular}{|c|c|c|c|c|c|c|c|c|}
\hline \multirow[b]{2}{*}{ Location } & \multirow[b]{2}{*}{ Depth $^{a}$} & \multirow[b]{2}{*}{$\begin{array}{l}\text { Sample } \\
\text { Date }\end{array}$} & \multirow[b]{2}{*}{ Medium } & \multirow[b]{2}{*}{ Sample } & \multirow[b]{2}{*}{$\begin{array}{l}\text { Analysis } \\
\text { Type }\end{array}$} & \multicolumn{3}{|c|}{$\begin{array}{c}\text { Concentration } \\
(\mu \mathrm{g} / \mathrm{L} \text { in water; } \mu \mathrm{g} / \mathrm{kg} \text { in soil })\end{array}$} \\
\hline & & & & & & $\begin{array}{c}\text { Carbon } \\
\text { Tetrachloride }\end{array}$ & Chloroform & $\begin{array}{l}\text { Methylene } \\
\text { Chloride }\end{array}$ \\
\hline \multirow[t]{2}{*}{ TI-27 } & $49-54$ & $5 / 20 / 06$ & Water & NATI27-W-20700 & Primary sample & 2 & $0.7 \mathrm{~J}$ & ND \\
\hline & & & & NATI27-W-20701 & Replicate sample & 1.8 & $0.6 \mathrm{~J}$ & ND \\
\hline \multirow[t]{3}{*}{ TI-28 } & 32.75 & $5 / 18 / 06$ & Soil & NATI28-S-20244 & Primary sample & 287 & ND & ND \\
\hline & & & & NATI28-S-20244DUP & Duplicate analysis & 290 & $3.3 \mathrm{~J}$ & ND \\
\hline & & & & NATI28-S-20245 & Replicate sample & 298 & $5 \mathrm{~J}$ & ND \\
\hline \multirow[t]{2}{*}{ TI-28 } & $37-42$ & $5 / 20 / 06$ & Water & NATI28-W-20849 & Primary sample & 97 & 91 & 1 \\
\hline & & & & NATI28-W-20849DUP & Duplicate analysis & 101 & 97 & 1 \\
\hline \multirow[t]{2}{*}{ TI-28 } & $51-56$ & $5 / 19 / 06$ & Water & NATI28-W-20683 & Primary sample & 12 & 2.7 & ND \\
\hline & & & & NATI28-W-20683DUP & Duplicate analysis & 13 & 2.6 & ND \\
\hline \multirow[t]{2}{*}{ TI-28 } & $58-63$ & $5 / 19 / 06$ & Water & NATI28-W-20254 & Primary sample & 15 & 8.9 & ND \\
\hline & & & & NATI28-W-20255 & Replicate sample & 26 & 10 & ND \\
\hline \multirow[t]{2}{*}{ TI-29 } & 13.5 & $5 / 19 / 06$ & Soil & NATI29-S-20703 & Primary sample & ND & ND & ND \\
\hline & & & & NATI29-S-20703DUP & Duplicate analysis & ND & ND & ND \\
\hline \multirow[t]{2}{*}{ TI-29 } & $39-44$ & $5 / 21 / 06$ & Water & NATI29-W-20900 & Primary sample & 269 & 87 & 3.9 \\
\hline & & & & NATI29-W-20901 & Replicate sample & 239 & 86 & 3.8 \\
\hline \multirow[t]{2}{*}{ TI-29 } & 53 & $5 / 19 / 06$ & Soil & NATI29-S-20894 & Primary sample & $6 \mathrm{~J}$ & $1.9 \mathrm{~J}$ & ND \\
\hline & & & & NATI29-S-20894DUP & Duplicate analysis & $6.1 \mathrm{~J}$ & $2 \mathrm{~J}$ & ND \\
\hline \multirow[t]{2}{*}{ TI-29 } & 62 & $5 / 21 / 06$ & Soil & NATI29-S-20897 & Primary sample & ND & ND & ND \\
\hline & & & & NATI29-S-20898 & Replicate sample & ND & ND & ND \\
\hline \multirow[t]{3}{*}{ TI-30 } & $75-80$ & $5 / 23 / 06$ & Water & NATI30-W-20917 & Primary sample & ND & ND & ND \\
\hline & & & & NATI30-W-20917DUP & Duplicate analysis & $0.7 \mathrm{~J}$ & $0.3 \mathrm{~J}$ & ND \\
\hline & & & & NATI30-W-20919 & Replicate sample & ND & ND & ND \\
\hline \multirow[t]{2}{*}{ TI-30 } & $83.5-88.5$ & $5 / 24 / 06$ & Water & NATI30-W-20712 & Primary sample & ND & ND & ND \\
\hline & & & & NATI30-W-20712DUP & Duplicate analysis & ND & ND & ND \\
\hline \multirow[t]{2}{*}{ TI-31 } & $70.7-75.7$ & $5 / 24 / 06$ & Water & NATI31-W-20715 & Primary sample & ND & ND & ND \\
\hline & & & & NATI31-W-20936 & Replicate sample & ND & ND & ND \\
\hline
\end{tabular}

a Depths for soil samples are in ft BGL; depths for water samples are in ft below TOC.

b ND, not detected at the AGEM Laboratory method detection limit of $0.1 \mu \mathrm{g} / \mathrm{L}$ for water samples or $1.0 \mu \mathrm{g} / \mathrm{kg}$ for soil samples.

c Qualifier $\mathrm{J}$ indicates an estimated concentration below the AGEM Laboratory quantitation limit of $0.1 \mu \mathrm{g} / \mathrm{L}$ for water samples and $10.0 \mu \mathrm{g} / \mathrm{kg}$ for soil samples. 
TABLE S4.6 Recovery of system-monitoring compounds in verification organic analyses of soil samples by STL with EPA Method 8260B.

\begin{tabular}{|c|c|c|c|c|c|c|}
\hline \multirow[b]{2}{*}{ Sample } & \multirow[b]{2}{*}{$\begin{array}{l}\text { Analysis } \\
\text { Date }\end{array}$} & \multirow[b]{2}{*}{ SDG } & \multicolumn{4}{|c|}{ Recoverya (\%) } \\
\hline & & & Toluene- $d_{8}$ & $\begin{array}{l}\text { 1,2-Dichloro- } \\
\text { ethane-d }\end{array}$ & $\begin{array}{l}\text { Bromofluoro- } \\
\text { benzene }\end{array}$ & $\begin{array}{l}\text { 1,2-Dichloro- } \\
\text { benzene- } d_{4}\end{array}$ \\
\hline NATI4-S-20308 & 4/28/06 & 113901 & 95 & 102 & 113 & $69^{b}$ \\
\hline NATI7-S-20454 & $4 / 28 / 06$ & 113901 & 95 & 98 & 105 & $76^{b}$ \\
\hline NATI7-S-20453 & $4 / 28 / 06$ & 113901 & 98 & 100 & 112 & $70^{\mathrm{b}}$ \\
\hline NATI2-S-20408 & $4 / 28 / 06$ & 113901 & 97 & 96 & $123^{b}$ & $63^{b}$ \\
\hline NATI1-S-20303 & $4 / 28 / 06$ & 113901 & 97 & 102 & $123^{b}$ & $69^{b}$ \\
\hline NATI4-S-20311 & $4 / 28 / 06$ & 113901 & 98 & 97 & 114 & $69^{b}$ \\
\hline NATI1-S-20476 & 4/28/06 & 113901 & 98 & 96 & 115 & $74^{b}$ \\
\hline NACP1-S-20330 & $4 / 28 / 06$ & 113901 & 98 & 100 & 109 & $78^{b}$ \\
\hline NATI2-S-20413 & $4 / 28 / 06$ & 113901 & 99 & 97 & 114 & $70^{\mathrm{b}}$ \\
\hline NATI3-S-20295 & $4 / 28 / 06$ & 113901 & 99 & 97 & $126^{b}$ & $62^{b}$ \\
\hline NA-MEOHBLANK-18APR06 & $4 / 28 / 06$ & 113901 & 97 & 100 & $149^{b}$ & $58^{b}$ \\
\hline NATI1-S-20340 & $4 / 28 / 06$ & 113901 & 96 & 98 & $122^{\mathrm{b}}$ & $66^{b}$ \\
\hline NATI5-S-20358 & $4 / 28 / 06$ & 113901 & 98 & 97 & 119 & $72^{b}$ \\
\hline LB042806LCS & $4 / 28 / 06$ & 113901 & 96 & 101 & 96 & 100 \\
\hline MBLK042806LB & $4 / 28 / 06$ & 113901 & 98 & 108 & 104 & 103 \\
\hline MEOHBLK & $5 / 10 / 06$ & 114211 & 96 & 107 & 96 & 88 \\
\hline MEOHLCS & $5 / 10 / 06$ & 114211 & 95 & 111 & 93 & 90 \\
\hline NATI8-S-20527 & $5 / 10 / 06$ & 114211 & 95 & 100 & 96 & 92 \\
\hline NATI8-S-20511 & $5 / 10 / 06$ & 114211 & 94 & 98 & 95 & 84 \\
\hline NATI9-S-20603 & $5 / 10 / 06$ & 114211 & 98 & 99 & 101 & 92 \\
\hline NATI10-S-20620 & $5 / 10 / 06$ & 114211 & 98 & 102 & 102 & 90 \\
\hline NATI6-S-20390 & $5 / 10 / 06$ & 114211 & 97 & 103 & 97 & 92 \\
\hline NATI11-S-20496 & $5 / 10 / 06$ & 114211 & 95 & 97 & 97 & 88 \\
\hline NATI9-S-20607 & $5 / 10 / 06$ & 114211 & 98 & 100 & 102 & 90 \\
\hline NA-S-BLANK-02MAY06 & $5 / 10 / 06$ & 114211 & 100 & 97 & 105 & 91 \\
\hline LA051006LCS & $5 / 10 / 06$ & 114211 & 101 & 100 & 100 & 102 \\
\hline MBLK051006LA & $5 / 10 / 06$ & 114211 & 97 & 105 & 98 & 98 \\
\hline MEOH LCS & $5 / 19 / 06$ & 114309 & 100 & 111 & 96 & 93 \\
\hline NA-MEOHBLANK-08MAY06 & $5 / 19 / 06$ & 114309 & 97 & 109 & 96 & 96 \\
\hline NATI10-S-20636 & $5 / 19 / 06$ & 114309 & 97 & 104 & 96 & 92 \\
\hline NATI12-S-20735 & $5 / 19 / 06$ & 114309 & 99 & 101 & 97 & 94 \\
\hline NATI13-S-20538 & $5 / 19 / 06$ & 114309 & 100 & 103 & 97 & 92 \\
\hline NATI12-S-20750 & $5 / 19 / 06$ & 114309 & 99 & 100 & 101 & 92 \\
\hline NATI13-S-20541 & $5 / 19 / 06$ & 114309 & 97 & 105 & 100 & 91 \\
\hline NATI15-S-20575 & $5 / 19 / 06$ & 114309 & 98 & 100 & 97 & 91 \\
\hline NATI13-S-20545 & $5 / 19 / 06$ & 114309 & 101 & 106 & 102 & 93 \\
\hline NATI14-S-20664 & $5 / 19 / 06$ & 114309 & 99 & 98 & 99 & 93 \\
\hline NATI13-S-20543 & $5 / 19 / 06$ & 114309 & 96 & 98 & 99 & 92 \\
\hline LB051806LCS & $5 / 19 / 06$ & 114309 & 99 & 102 & 97 & 97 \\
\hline MBLK051806LB & $5 / 19 / 06$ & 114309 & 101 & 106 & 103 & 100 \\
\hline MEOHLCS & $5 / 24 / 06$ & 114417 & 102 & 91 & 98 & 93 \\
\hline NA-MEOHBLANK-19MAY06 & $5 / 24 / 06$ & 114417 & $119^{b}$ & 108 & 119 & 103 \\
\hline NATI14-S-20673 & $5 / 24 / 06$ & 114417 & $138^{b}$ & 132 & $133^{b}$ & 119 \\
\hline NATI15-S-20578 & $5 / 24 / 06$ & 114417 & 110 & 108 & 108 & 104 \\
\hline NATI17-S-20808 & $5 / 24 / 06$ & 114417 & $118^{b}$ & 112 & 114 & 106 \\
\hline
\end{tabular}


TABLE S4.6 (Cont.)

\begin{tabular}{|c|c|c|c|c|c|c|}
\hline \multirow[b]{2}{*}{ Sample } & \multirow[b]{2}{*}{$\begin{array}{l}\text { Analysis } \\
\text { Date }\end{array}$} & \multirow[b]{2}{*}{ SDG } & \multicolumn{4}{|c|}{ Recoverya (\%) } \\
\hline & & & Toluene- $\mathrm{d}_{8}$ & $\begin{array}{l}\text { 1,2-Dichloro- } \\
\text { ethane-d }\end{array}$ & $\begin{array}{l}\text { Bromofluoro- } \\
\text { benzene }\end{array}$ & $\begin{array}{l}\text { 1,2-Dichloro- } \\
\text { benzene-d }_{4}\end{array}$ \\
\hline NATI16-S-20773 & $5 / 24 / 06$ & 114417 & 103 & 89 & 100 & 93 \\
\hline NATI16-S-20783 & $5 / 24 / 06$ & 114417 & 101 & 94 & 100 & 91 \\
\hline NATI14-S-20689 & $5 / 24 / 06$ & 114417 & 103 & 97 & 98 & 93 \\
\hline LA052406LCS & $5 / 24 / 06$ & 114417 & 104 & 100 & 97 & 100 \\
\hline MBLK052406LB & $5 / 24 / 06$ & 114417 & 96 & 94 & 98 & 97 \\
\hline
\end{tabular}

a Quality control ranges:

$\begin{array}{ll}\text { Compound } & \text { Range }(\%) \\ & \\ \text { Toluene-d } & 81-117 \\ \text { 1,2-Dichloroethane- } d_{4} & 80-120 \\ \text { Bromofluorobenzene } & 74-121 \\ \text { 1,2-Dichlorobenzene- } d_{4} & 80-120\end{array}$

b Result outside quality control range. 
TABLE S4.7 Recovery of contaminants of concern in laboratory quality control samples during verification organic analysis of soil samples by STL.

\begin{tabular}{|c|c|c|c|c|c|c|c|c|}
\hline \multirow[b]{2}{*}{ Sample } & \multirow[b]{2}{*}{$\begin{array}{c}\text { Analysis } \\
\text { Date }\end{array}$} & \multirow[b]{2}{*}{ SDG } & \multicolumn{3}{|c|}{ Carbon Tetrachloride } & \multicolumn{3}{|c|}{ Chloroform } \\
\hline & & & $\begin{array}{c}\text { Spiked } \\
\text { Concentration } \\
(\mu \mathrm{g} / \mathrm{L})\end{array}$ & $\begin{array}{c}\text { Detected } \\
\text { Concentration } \\
(\mu \mathrm{g} / \mathrm{L})\end{array}$ & $\begin{array}{c}\text { Recoverya } \\
(\%)\end{array}$ & $\begin{array}{c}\text { Spiked } \\
\text { Concentration } \\
(\mu \mathrm{g} / \mathrm{L})\end{array}$ & $\begin{array}{c}\text { Detected } \\
\text { Concentration } \\
(\mu \mathrm{g} / \mathrm{L})\end{array}$ & $\begin{array}{c}\text { Recovery } \\
\text { (\%) }\end{array}$ \\
\hline LC042806LCS & 4/28/06 & 113901 & 130 & 110 & 85 & 130 & 120 & 92 \\
\hline LB042806LCS & 4/28/06 & 113901 & 10 & 9.5 & 95 & 10 & 9.7 & 97 \\
\hline MEOHLCS & $5 / 10 / 06$ & 114211 & 100 & 87 & 87 & 100 & 89 & 89 \\
\hline LA051006LCS & 5/10/06 & 114211 & 10 & 9.8 & 98 & 10 & 9.7 & 97 \\
\hline MEOH LCS & 5/19/06 & 114309 & 100 & 92 & 92 & 100 & 97 & 97 \\
\hline LB051806LCS & 5/19/06 & 114309 & 10 & 9.3 & 93 & 10 & 9.4 & 94 \\
\hline MEOHLCS & $5 / 24 / 06$ & 114417 & 100 & 75 & 75 & 100 & 82 & 82 \\
\hline LA052406LCS & $5 / 24 / 06$ & 114417 & 10 & 8.6 & 86 & 10 & 9.0 & 90 \\
\hline
\end{tabular}

a Quality control range for carbon tetrachloride recovery $=75-120 \%$.

b Quality control range for chloroform recovery $=80-125 \%$. 
TABLE S4.8 Comparisons of organic results for verification analyses of soil samples collected during the 2006 investigation at Navarre, Kansas.

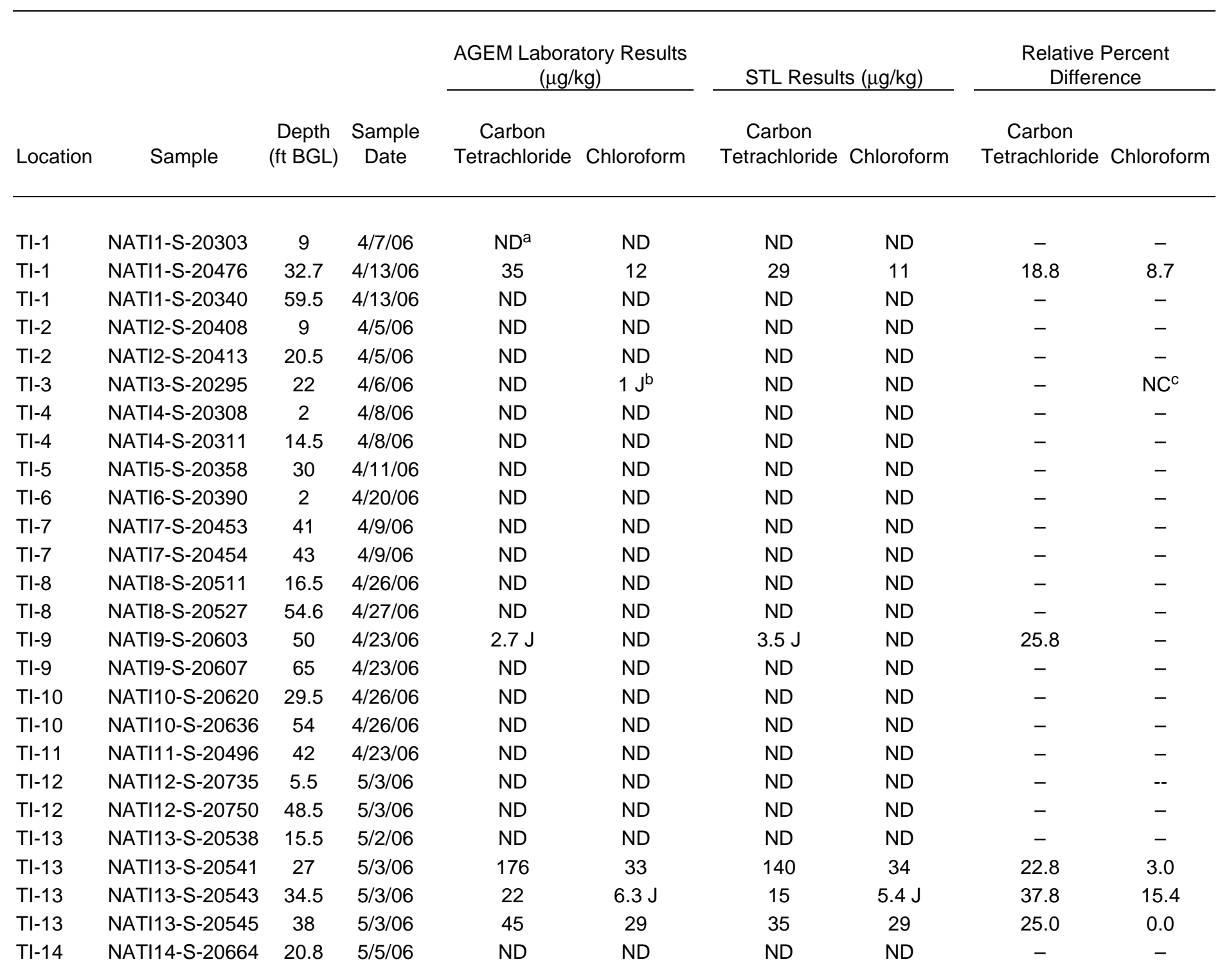


TABLE S4.8 (Cont.)

\begin{tabular}{|c|c|c|c|c|c|c|c|c|c|}
\hline \multirow[b]{2}{*}{ Location } & \multirow[b]{2}{*}{ Sample } & \multirow[b]{2}{*}{$\begin{array}{c}\text { Depth } \\
\text { (ft BGL) }\end{array}$} & \multirow[b]{2}{*}{$\begin{array}{l}\text { Sample } \\
\text { Date }\end{array}$} & \multicolumn{2}{|c|}{$\begin{array}{l}\text { AGEM Laboratory } \\
\text { Results }(\mu \mathrm{g} / \mathrm{kg})\end{array}$} & \multicolumn{2}{|c|}{ STL Results $(\mu \mathrm{g} / \mathrm{kg})$} & \multicolumn{2}{|c|}{$\begin{array}{c}\text { Relative Percent } \\
\text { Difference }\end{array}$} \\
\hline & & & & $\begin{array}{c}\text { Carbon } \\
\text { Tetrachloride }\end{array}$ & Chloroform & $\begin{array}{c}\text { Carbon } \\
\text { Tetrachloride }\end{array}$ & Chloroform & $\begin{array}{c}\text { Carbon } \\
\text { Tetrachloride }\end{array}$ & Chloroform \\
\hline $\mathrm{TI}-14$ & NATI14-S-20689 & 57.8 & $5 / 6 / 06$ & 50 & $4 \mathrm{~J}$ & 16 & ND & 103.0 & NC \\
\hline TI-15 & NATI15-S-20575 & 26.1 & $5 / 5 / 06$ & 93 & $2.5 \mathrm{~J}$ & 77 & $2.7 \mathrm{~J}$ & 18.8 & 7.7 \\
\hline $\mathrm{Tl}-15$ & NATI15-S-20578 & 34.6 & $5 / 5 / 06$ & 13 & ND & $5.9 \mathrm{~J}$ & ND & 75.1 & - \\
\hline $\mathrm{TI}-16$ & NATI16-S-20773 & 9.25 & $5 / 7 / 06$ & ND & ND & ND & ND & - & - \\
\hline $\mathrm{TI}-16$ & NATI16-S-20783 & 36.5 & $5 / 7 / 06$ & 36 & $9 \mathrm{~J}$ & 13 & $3.6 \mathrm{~J}$ & 93.9 & 85.7 \\
\hline
\end{tabular}

a ND, not detected at a method detection limit of $1.0 \mu \mathrm{g} / \mathrm{kg}$.

b Qualifier J indicates an estimated concentration below the method quantitation limit of $10.0 \mu \mathrm{g} / \mathrm{kg}$.

c NC, not calculated. 
TABLE S4.9 Recovery of system-monitoring compounds in verification organic analyses of water samples by ENVSY.

\begin{tabular}{|c|c|c|c|c|c|}
\hline \multirow[b]{2}{*}{ Sample } & \multirow[b]{2}{*}{$\begin{array}{l}\text { Analysis } \\
\text { Date }\end{array}$} & \multirow[b]{2}{*}{ SDG } & \multicolumn{3}{|c|}{ Recoverya (\%) } \\
\hline & & & Toluene-d 8 & $\begin{array}{l}\text { Bromofluoro- } \\
\text { benzene }\end{array}$ & $\begin{array}{l}\text { 1,2-Dichloro- } \\
\text { ethane-d }\end{array}$ \\
\hline VBLKHO & 4/20/06 & 605051 & 106 & 96 & 102 \\
\hline NATI3-W-20298 & $4 / 20 / 06$ & 605051 & $134^{b}$ & $120^{b}$ & 126 \\
\hline NATI4-W-20325 & $4 / 20 / 06$ & 605051 & 94 & $84^{b}$ & 92 \\
\hline NATI2-W-20433 & $4 / 20 / 06$ & 605051 & $132^{b}$ & $120^{b}$ & 130 \\
\hline NAL2-W-20190 & $4 / 20 / 06$ & 605051 & 106 & 94 & 102 \\
\hline NAQCTB-W-11APR06 & $4 / 20 / 06$ & 605051 & $118^{b}$ & 104 & 114 \\
\hline VHBLKHO & $4 / 20 / 06$ & 605051 & $122^{b}$ & 102 & 118 \\
\hline VBLKF2 & $5 / 13 / 06$ & 606057 & 104 & $78^{b}$ & 90 \\
\hline NATI13-W-20561 & $5 / 13 / 06$ & 606057 & 104 & $78^{b}$ & 94 \\
\hline NATI13-W-20560 & $5 / 13 / 06$ & 606057 & 104 & $78^{b}$ & 94 \\
\hline NATI14-W-20666 & $5 / 13 / 06$ & 606057 & 106 & $80^{b}$ & 98 \\
\hline NATI13-W-20563 & $5 / 13 / 06$ & 606057 & 106 & $78^{b}$ & 102 \\
\hline NATI13-W-20562 & $5 / 13 / 06$ & 606057 & 106 & $78^{b}$ & 94 \\
\hline NAQCTB-050706 & $5 / 13 / 06$ & 606057 & 104 & $76^{b}$ & 94 \\
\hline VHBLKFK & $5 / 13 / 06$ & 606057 & 104 & $82^{b}$ & 100 \\
\hline VBLKHP & $5 / 17 / 06$ & 606057 & 102 & 100 & 104 \\
\hline NATI14-W-20666DL & $5 / 17 / 06$ & 606057 & 102 & 98 & 102 \\
\hline NATI13-W-20563DL & $5 / 17 / 06$ & 606057 & 98 & 98 & 104 \\
\hline VBLKHW & $5 / 24 / 06$ & 605053 & 102 & 102 & 108 \\
\hline NATI18-W-20706 & $5 / 24 / 06$ & 605053 & 100 & 100 & 108 \\
\hline NATI29-W-20901 & $5 / 24 / 06$ & 605053 & 100 & 102 & 110 \\
\hline NATI18-W-20655 & $5 / 24 / 06$ & 605053 & 100 & 102 & 110 \\
\hline NATI27-W-20905 & $5 / 24 / 06$ & 605053 & 100 & 104 & 114 \\
\hline NATI18-W-20709 & $5 / 24 / 06$ & 605053 & 100 & 102 & 108 \\
\hline NATI27-W-20700 & $5 / 24 / 06$ & 605053 & 102 & 102 & 110 \\
\hline NATI28-W-20904 & $5 / 24 / 06$ & 605053 & 102 & 100 & 110 \\
\hline NAQCTB-052306 & $5 / 24 / 06$ & 605053 & 100 & 98 & 108 \\
\hline MBLKHD & $5 / 31 / 06$ & 605053 & 102 & 102 & 108 \\
\hline NATI18-W-20706RE & $5 / 31 / 06$ & 605053 & 104 & 100 & 96 \\
\hline NATI29-W-20901RE & $5 / 31 / 06$ & 605053 & 104 & 108 & 108 \\
\hline NATI18-W-20655RE & $5 / 31 / 06$ & 605053 & 102 & 109 & 110 \\
\hline MBLKHE & $6 / 1 / 06$ & 605053 & 106 & 96 & 102 \\
\hline NATI28-W-20904RE & $6 / 1 / 06$ & 605053 & 104 & 94 & 104 \\
\hline
\end{tabular}


TABLE S4.9 (Cont.)

\begin{tabular}{|c|c|c|c|c|c|}
\hline \multirow[b]{2}{*}{ Sample } & \multirow[b]{2}{*}{$\begin{array}{c}\text { Analysis } \\
\text { Date }\end{array}$} & \multirow[b]{2}{*}{ SDG } & \multicolumn{3}{|c|}{ Recoverya (\%) } \\
\hline & & & Toluene- $d_{8}$ & $\begin{array}{l}\text { Bromofluoro- } \\
\text { benzene }\end{array}$ & $\begin{array}{l}\text { 1,2-Dichloro- } \\
\text { ethane- } \mathrm{d}_{4}\end{array}$ \\
\hline VBLKHD & $5 / 31 / 06$ & 605054 & 100 & 108 & 110 \\
\hline NAQCTB-052506 & $5 / 31 / 06$ & 605054 & 100 & 108 & 110 \\
\hline NATI29-W-20916 & $5 / 31 / 06$ & 605054 & 100 & 106 & 110 \\
\hline NATI30-W-20920 & $5 / 31 / 06$ & 605054 & 98 & 106 & 110 \\
\hline NATI30-W-20712 & $5 / 31 / 06$ & 605054 & 98 & 108 & 110 \\
\hline NATI20-W-20913 & $5 / 31 / 06$ & 605054 & 98 & 110 & 112 \\
\hline NATI30-W-20919 & $5 / 31 / 06$ & 605054 & 98 & 110 & 110 \\
\hline
\end{tabular}

a Quality control ranges:

Compound

Toluene- $\mathrm{d}_{8}$

Bromofluorobenzene

1,2-Dichloroethane- $d_{4}$

b Result outside quality control range.
Range (\%)

88-110

86-115

76-114 
TABLE S4.10 Comparison of organic results for verification analyses of groundwater samples collected during the 2006 investigation at Navarre, Kansas.

\begin{tabular}{|c|c|c|c|c|c|c|c|c|c|c|c|c|}
\hline \multirow[b]{2}{*}{ Location } & \multirow[b]{2}{*}{ Sample } & \multirow[b]{2}{*}{$\begin{array}{c}\text { Depth } \\
\text { (ft below TOC) }\end{array}$} & \multirow[b]{2}{*}{$\begin{array}{l}\text { Sample } \\
\text { Date }\end{array}$} & \multicolumn{3}{|c|}{ AGEM Laboratory Results ( $\mu \mathrm{g} / \mathrm{L})$} & \multicolumn{3}{|c|}{ ENVSY Results $(\mu \mathrm{g} / \mathrm{L})$} & \multicolumn{3}{|c|}{ Relative Percent Difference } \\
\hline & & & & $\begin{array}{c}\text { Carbon } \\
\text { Tetrachloride }\end{array}$ & $\begin{array}{l}\text { Chloro- } \\
\text { form }\end{array}$ & $\begin{array}{l}\text { Methylene } \\
\text { Chloride }\end{array}$ & $\begin{array}{c}\text { Carbon } \\
\text { Tetrachloride }\end{array}$ & $\begin{array}{l}\text { Chloro- } \\
\text { form }\end{array}$ & $\begin{array}{l}\text { Methylene } \\
\text { Chloride }\end{array}$ & $\begin{array}{c}\text { Carbon } \\
\text { Tetrachloride }\end{array}$ & $\begin{array}{l}\text { Chloro- } \\
\text { form }\end{array}$ & $\begin{array}{c}\text { Methylene } \\
\text { Chloride }\end{array}$ \\
\hline L-2 & NAL2-W-20190 & $80-90$ & $4 / 6 / 06$ & $0.2 \mathrm{~J}^{\mathrm{a}}$ & $N D^{b}$ & ND & ND & ND & $1.3 \mathrm{JB}^{\mathrm{C}}$ & $\mathrm{NC}^{d}$ & - & - \\
\hline TI-2 & NATI2-W-20433 & $69-72$ & $4 / 7 / 06$ & ND & ND & ND & ND & ND & $2 \mathrm{JB}$ & - & - & - \\
\hline TI-3 & NATI3-W-20298 & $32.2-37.2$ & $4 / 7 / 06$ & 27 & 7.9 & ND & 23 & 11 & $1.6 \mathrm{JB}$ & 16.0 & 32.8 & - \\
\hline $\mathrm{TI}-4$ & NATI4-W-20325 & $35-40$ & $4 / 10 / 06$ & 76 & 55 & 3.6 & 95 & 78 & $4.7 \mathrm{JB}$ & 22.2 & 34.6 & $\mathrm{NC}$ \\
\hline TI-13 & NATI13-W-20563 & $35-40$ & $5 / 5 / 06$ & 387 & 197 & $0.6 \mathrm{~J}$ & $330 \mathrm{E}^{\mathrm{e}}$ & 180 & $2.5 \mathrm{JB}$ & 15.9 & 9.0 & \\
\hline TI-13 & NATI13-W-20562 & $42-47$ & $5 / 5 / 06$ & 116 & 60 & 1.2 & 130 & 73 & $2.9 \mathrm{JB}$ & 11.4 & 19.5 & \\
\hline TI-13 & NATI13-W-20560 & $48-53$ & $5 / 4 / 06$ & 22 & 5.4 & ND & 16 & 5.2 & $2 \mathrm{JB}$ & 31.6 & 3.8 & \\
\hline $\mathrm{Tl}-13$ & NATI13-W-20561 & $54-59$ & $5 / 5 / 06$ & 22 & 5.8 & ND & 18 & 5.6 & $2.2 \mathrm{JB}$ & 20.0 & 3.5 & \\
\hline TI-14 & NATI14-W-20666 & $67.4-72.4$ & $5 / 5 / 06$ & 6 & 280 & 12.6 & $4 \mathrm{~J}$ & 320 & 12 & 40.0 & 13.3 & 4.9 \\
\hline TI-18 & NATI18-W-20655 & $30-35$ & $5 / 21 / 06$ & 266 & 27 & 1.1 & 390 & 39 & $4.1 \mathrm{JB}$ & 37.8 & 36.4 & NC \\
\hline TI-18 & NATI18-W-20706 & $42-47$ & $5 / 21 / 06$ & 218 & 48 & 7.3 & 270 & 55 & $7.3 \mathrm{JB}$ & 21.3 & 13.6 & $\mathrm{NC}$ \\
\hline TI-18 & NATI18-W-20709 & $56-61$ & $5 / 21 / 06$ & 70 & 35 & 1.5 & 110 & 44 & $4 \mathrm{JB}$ & 44.4 & 22.8 & NC \\
\hline TI-20 & NATI20-W-20913 & $35-40$ & $5 / 22 / 06$ & 15 & 5.4 & ND & 15 & 5.5 & $3.7 \mathrm{JB}$ & 0.0 & 1.8 & - \\
\hline TI-27 & NATI27-W-20700 & $49-54$ & $5 / 20 / 06$ & 2 & $0.7 \mathrm{~J}$ & ND & $1.3 \mathrm{~J}$ & $0.6 \mathrm{~J}$ & $2.5 \mathrm{JB}$ & 42.4 & 15.3 & - \\
\hline TI-27 & NATI27-W-20905 & $56-61$ & $5 / 21 / 06$ & ND & $0.6 \mathrm{~J}$ & ND & $1.6 \mathrm{~J}$ & ND & $3 \mathrm{JB}$ & $\mathrm{NC}$ & $\mathrm{NC}$ & - \\
\hline TI-28 & NATI28-W-20904 & $25-30$ & $5 / 21 / 06$ & 2692 & 238 & 1.3 & 3100 & 200 & $4.2 \mathrm{JB}$ & 14.1 & 17.4 & $\mathrm{NC}$ \\
\hline TI-29 & NATI29-W-20916 & $25-30$ & $5 / 23 / 06$ & 39 & 39 & 2.6 & 38 & 35 & $6 \mathrm{~B}$ & 2.6 & 10.8 & $\mathrm{NC}$ \\
\hline TI-29 & NATI29-W-20901 & $39-44$ & $5 / 21 / 06$ & 239 & 86 & 3.8 & 220 & 77 & $5.8 \mathrm{~B}$ & 8.3 & 11.0 & $\mathrm{NC}$ \\
\hline TI-30 & NATI30-W-20920 & $39-44$ & $5 / 23 / 06$ & 1.1 & $0.4 \mathrm{~J}$ & ND & $1.1 \mathrm{~J}$ & ND & $3.3 \mathrm{JB}$ & 0 & NC & - \\
\hline TI-30 & NATI30-W-20919 & $75-80$ & $5 / 23 / 06$ & ND & ND & ND & ND & ND & $3.9 \mathrm{JB}$ & - & - & - \\
\hline TI-30 & NATI30-W-20712 & $83.5-88.5$ & $5 / 24 / 06$ & ND & ND & ND & ND & ND & $3.4 \mathrm{JB}$ & - & - & - \\
\hline
\end{tabular}

a Qualifier $\mathrm{J}$ indicates an estimated concentration below the quantitation limit of $1.0 \mu \mathrm{g} / \mathrm{L}$ for analyses at the AGEM Laboratory or $5.0 \mu \mathrm{g} / \mathrm{L}$ for analyses by ENVSY.

b ND, not detected at the method detection limit.

C Qualifier B indicates that the contaminant was present in the laboratory blank.

d NC, not calculated.

e Qualifier E indicates a result outside the calibration range at DF1.

f Cross-contamination of sample NATI27-W-20905 might have occurred during shipment to ENVSY. This sample was shipped with sample NATI28-W-20904, which contained carbon tetrachloride at $3,104 \mu \mathrm{g} / \mathrm{L}$ 


\section{Supplement 5:}

\section{Chain-of-Custody Forms and} Outside Laboratory Data 


\section{Supplement 5 Contents}

COC Forms AGEM Laboratory ................................................................................. 3 of 340

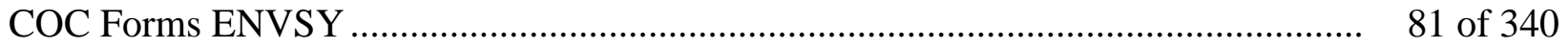

COC Forms STL ........................................................................................ 85 of 340

ENVSY Report 0605051 ................................................................................. 89 of 340

ENVSY Report 0606053 .................................................................................... 110 of 340

ENVSY Report 0606054................................................................................ 152 of 340

ENVSY Report 0606057................................................................................... 176 of 340

STL Report 113901 ..................................................................................................... 207 of 340

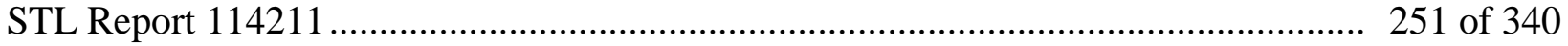

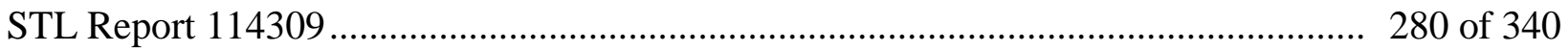

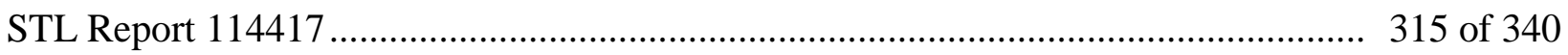




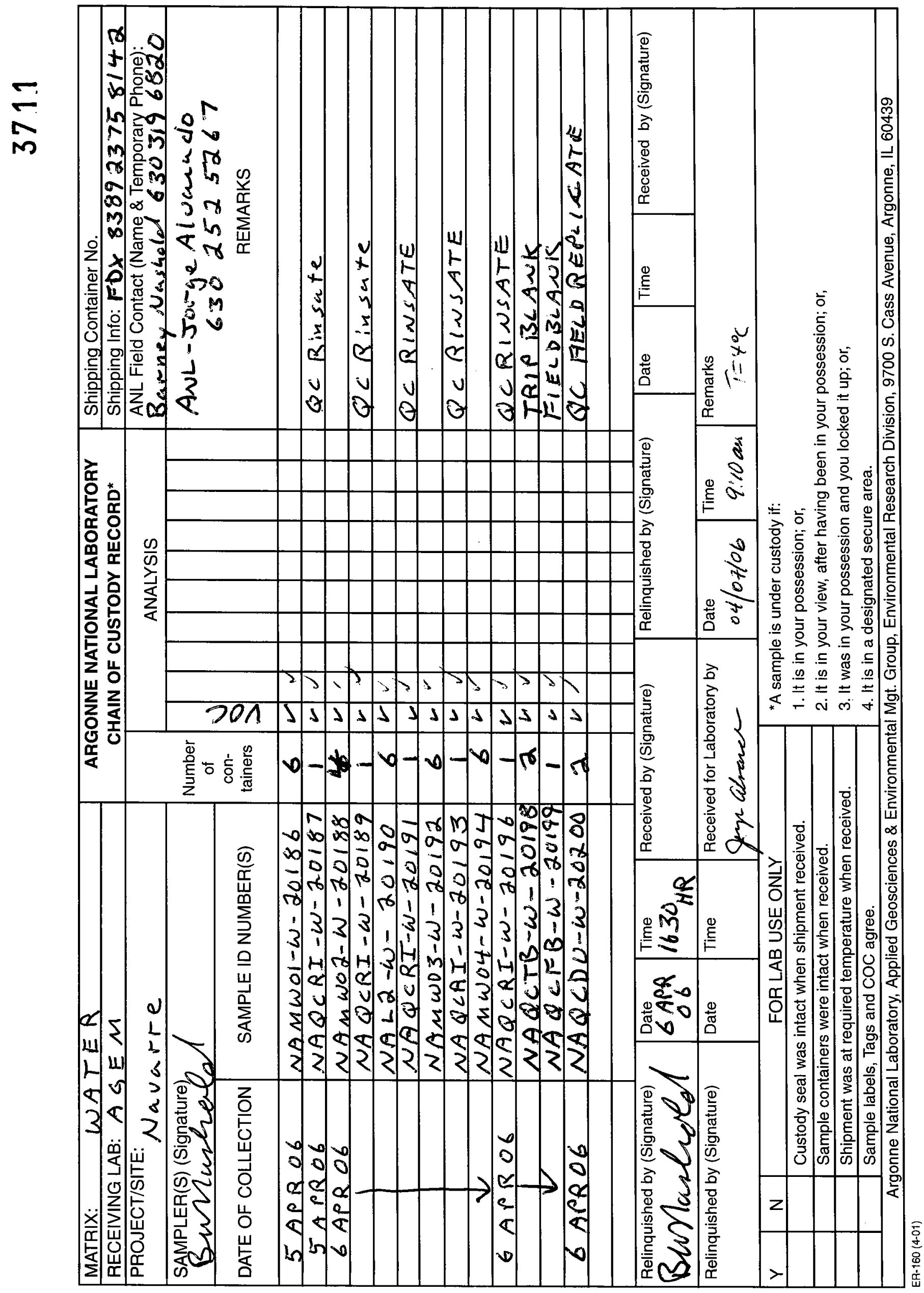




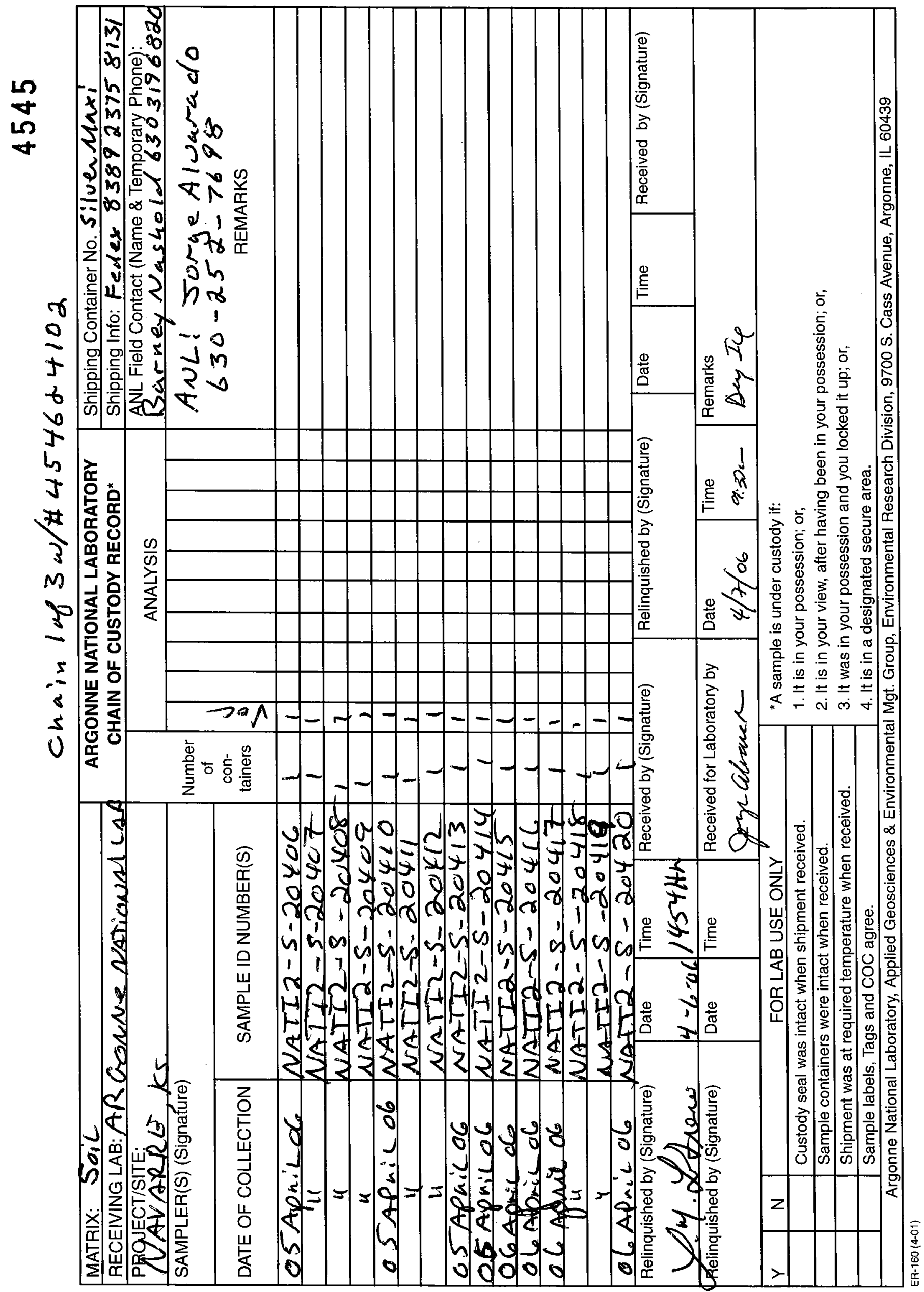




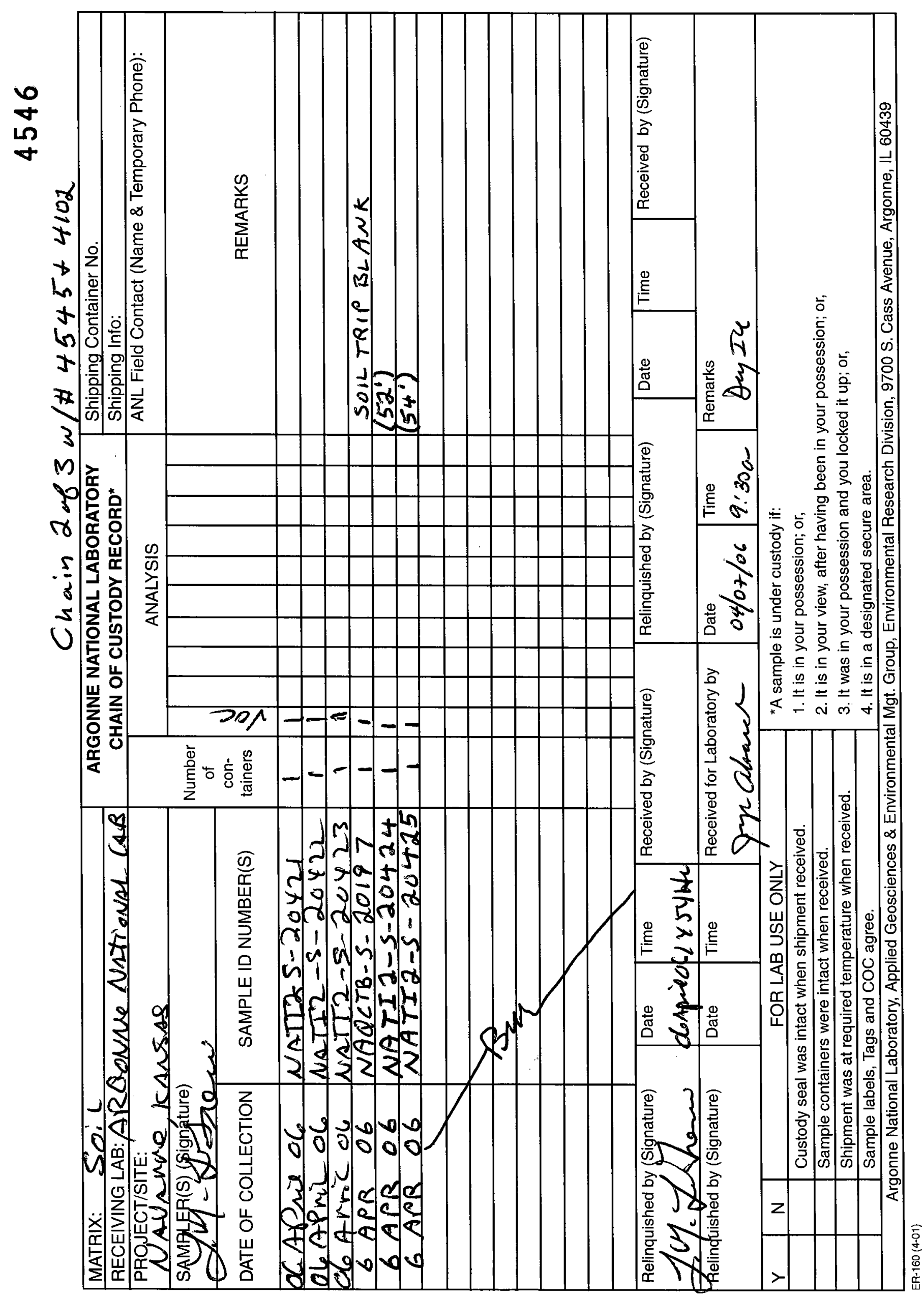




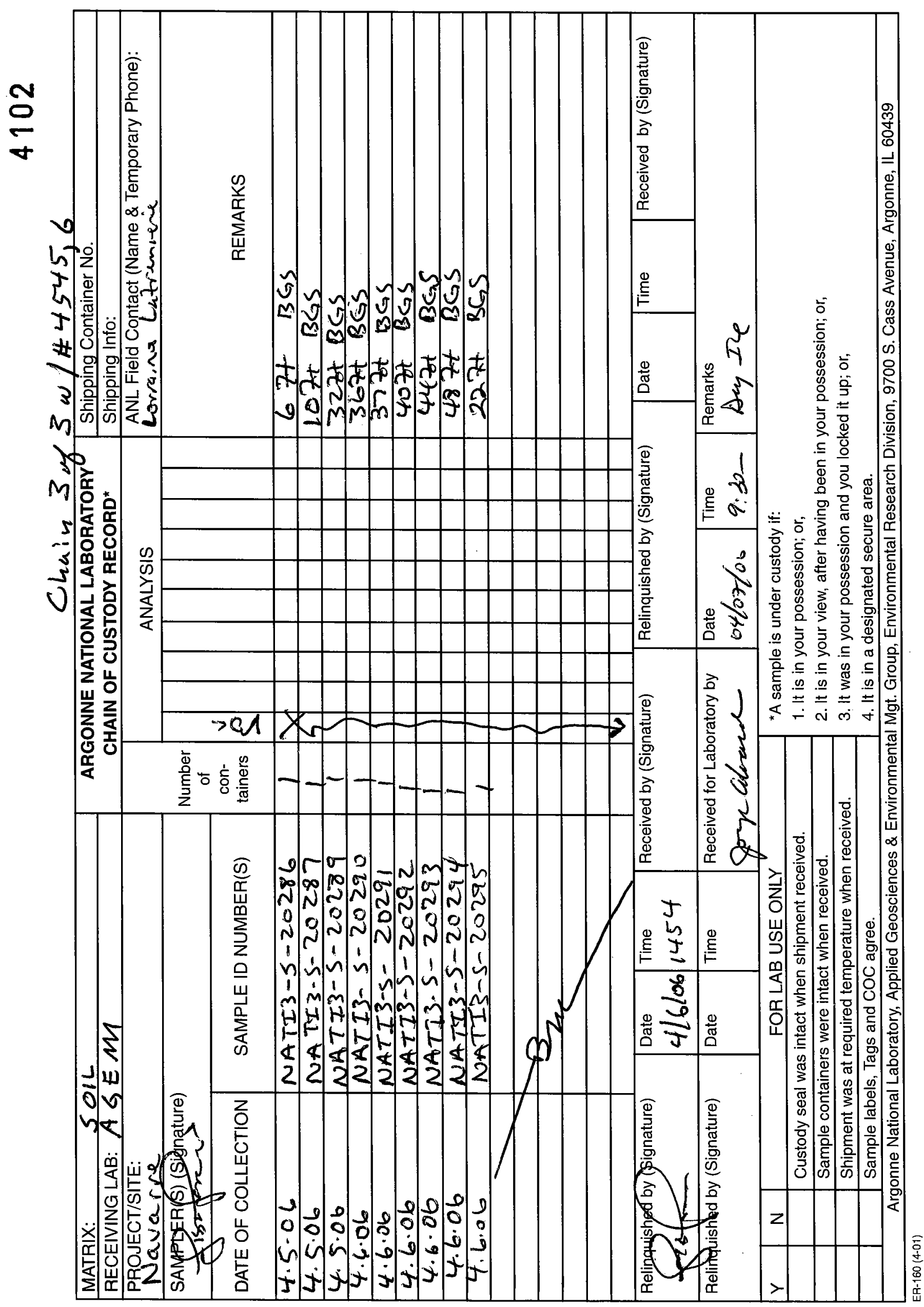




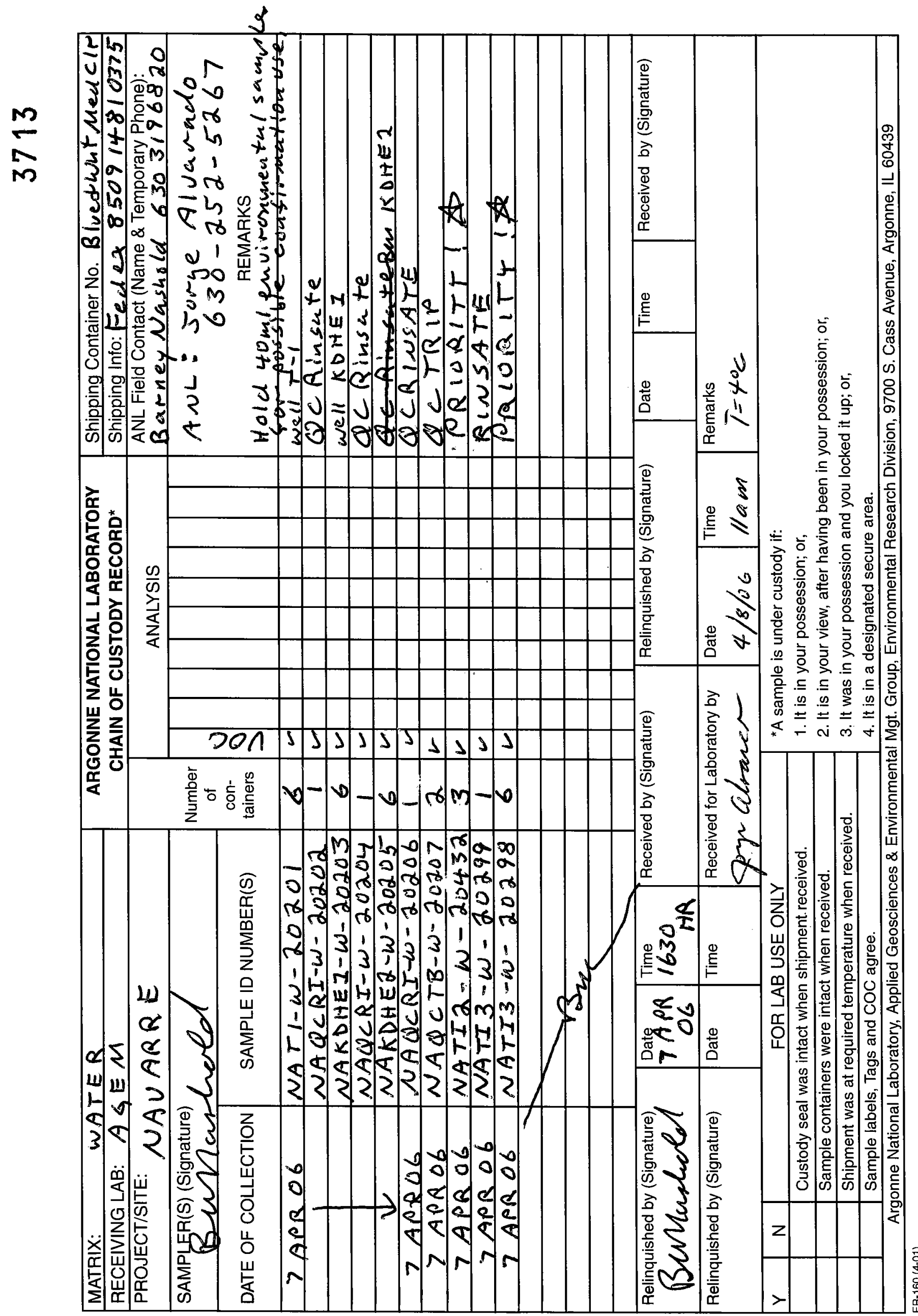




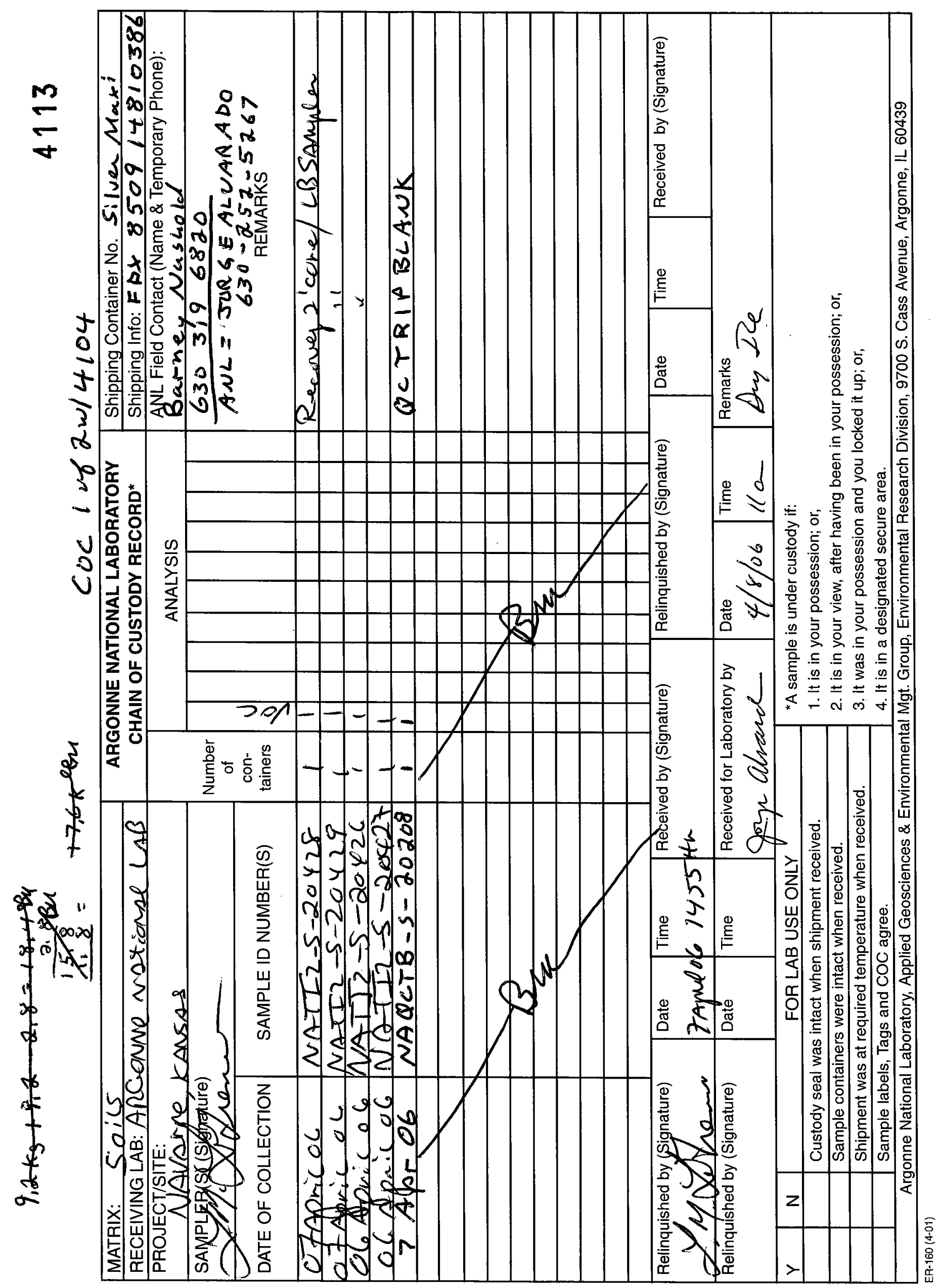




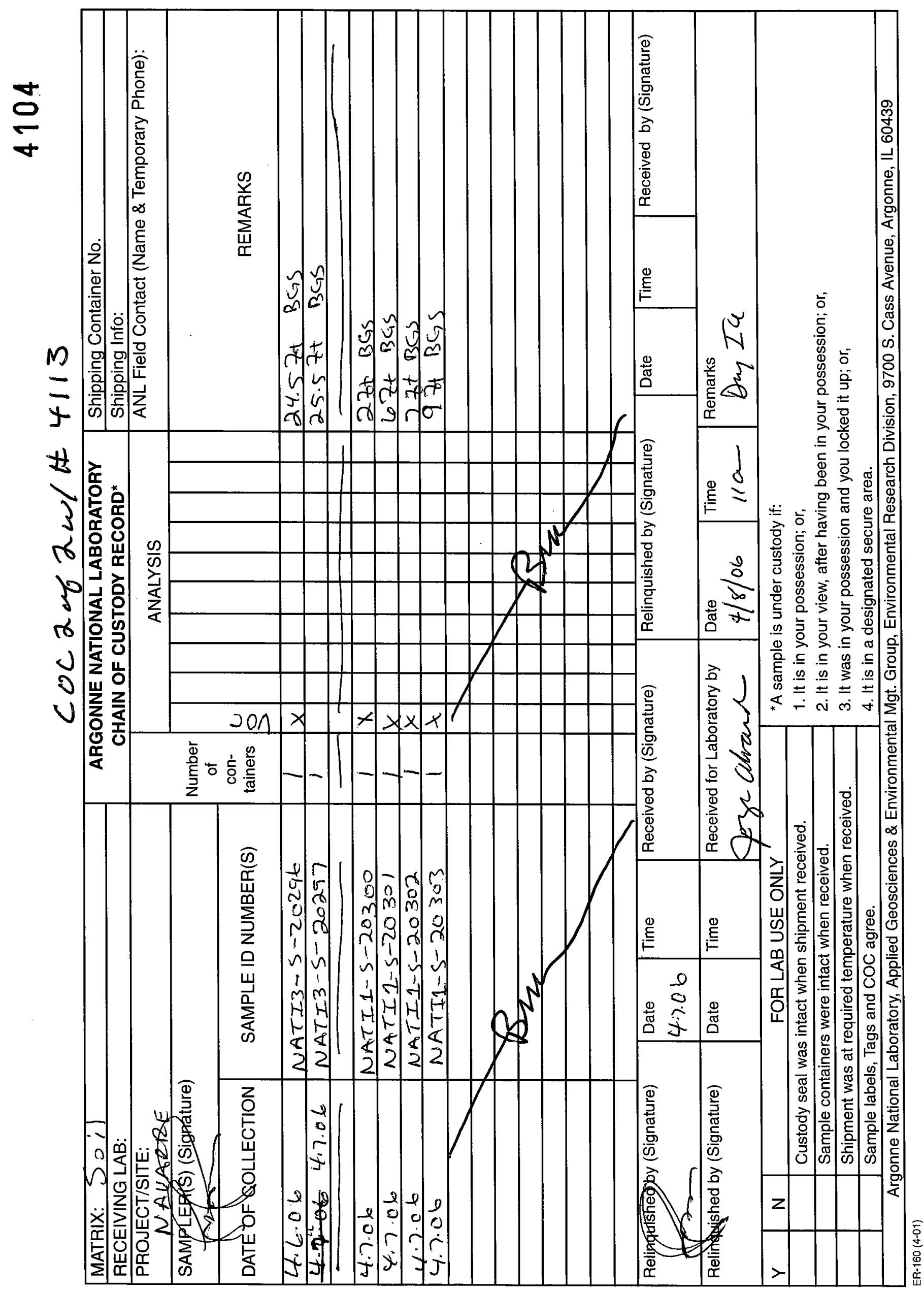




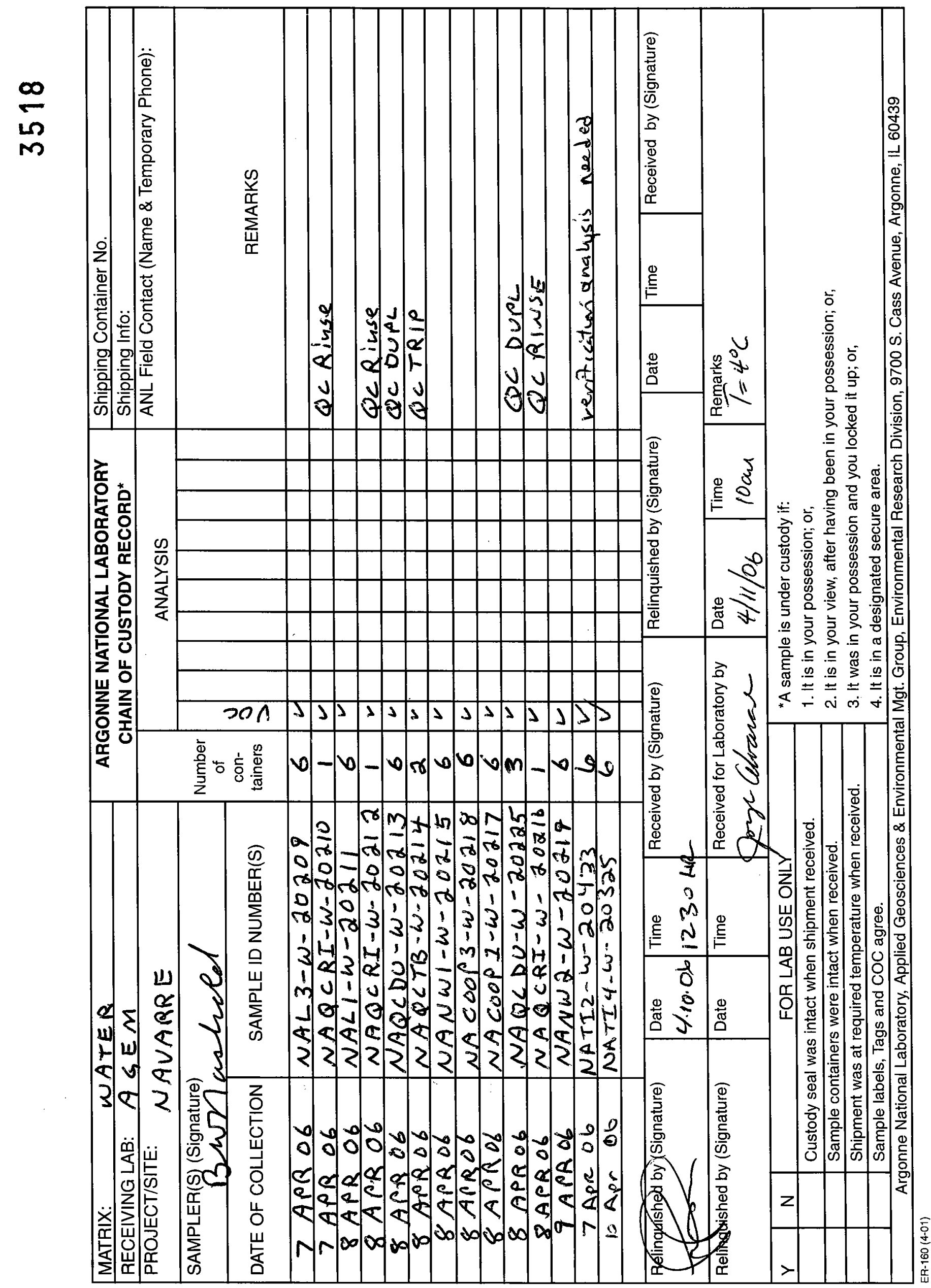




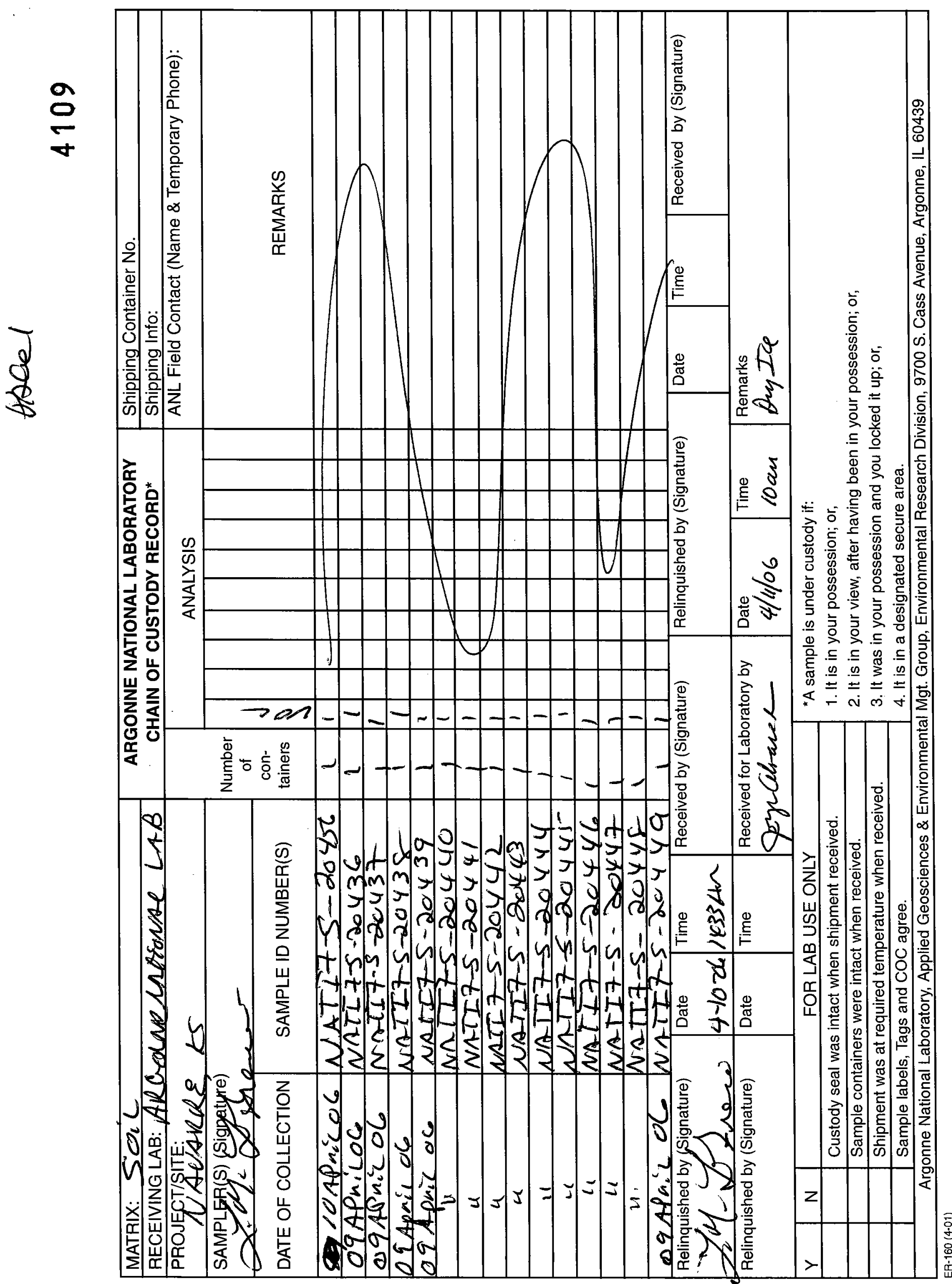




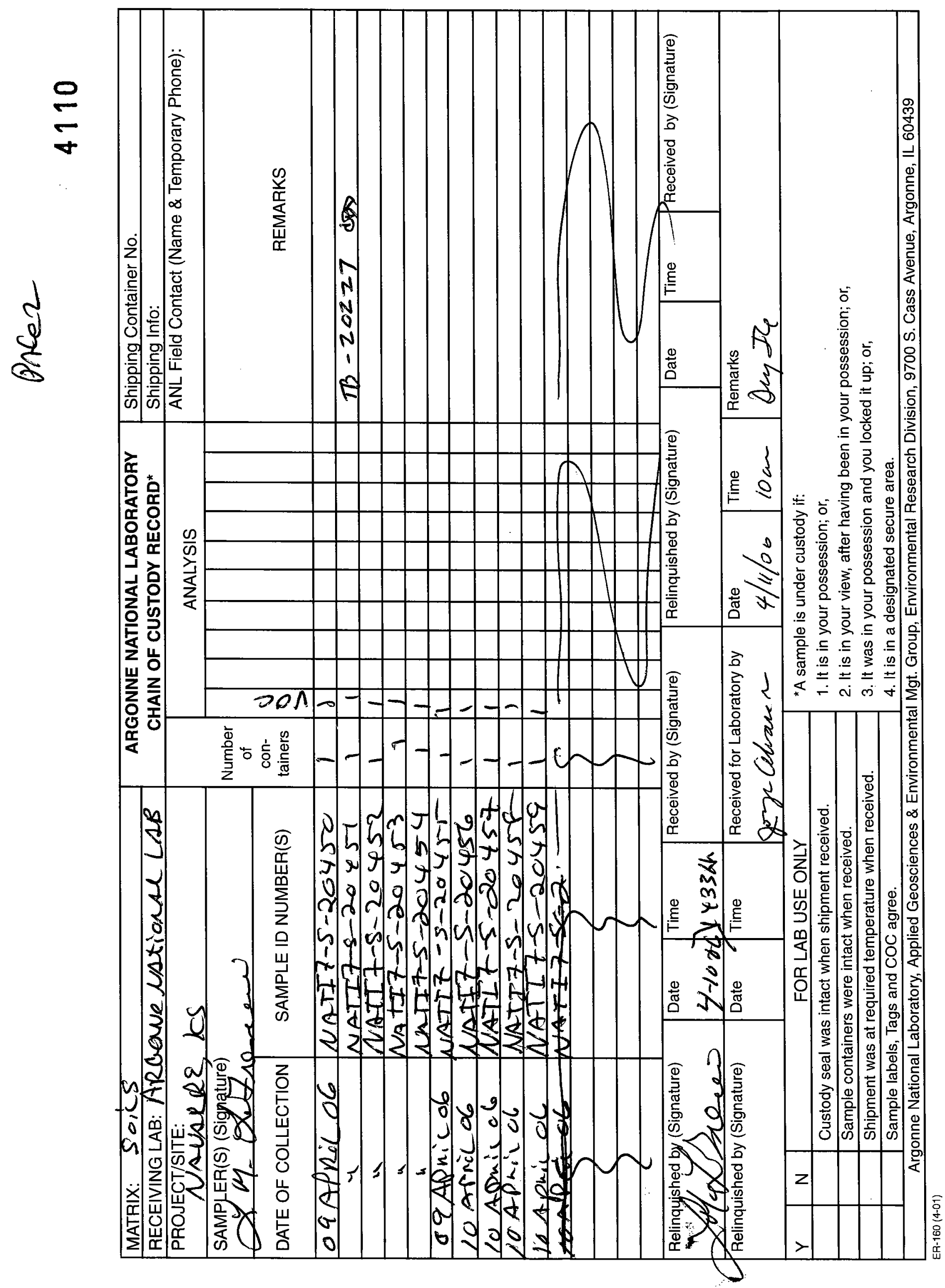




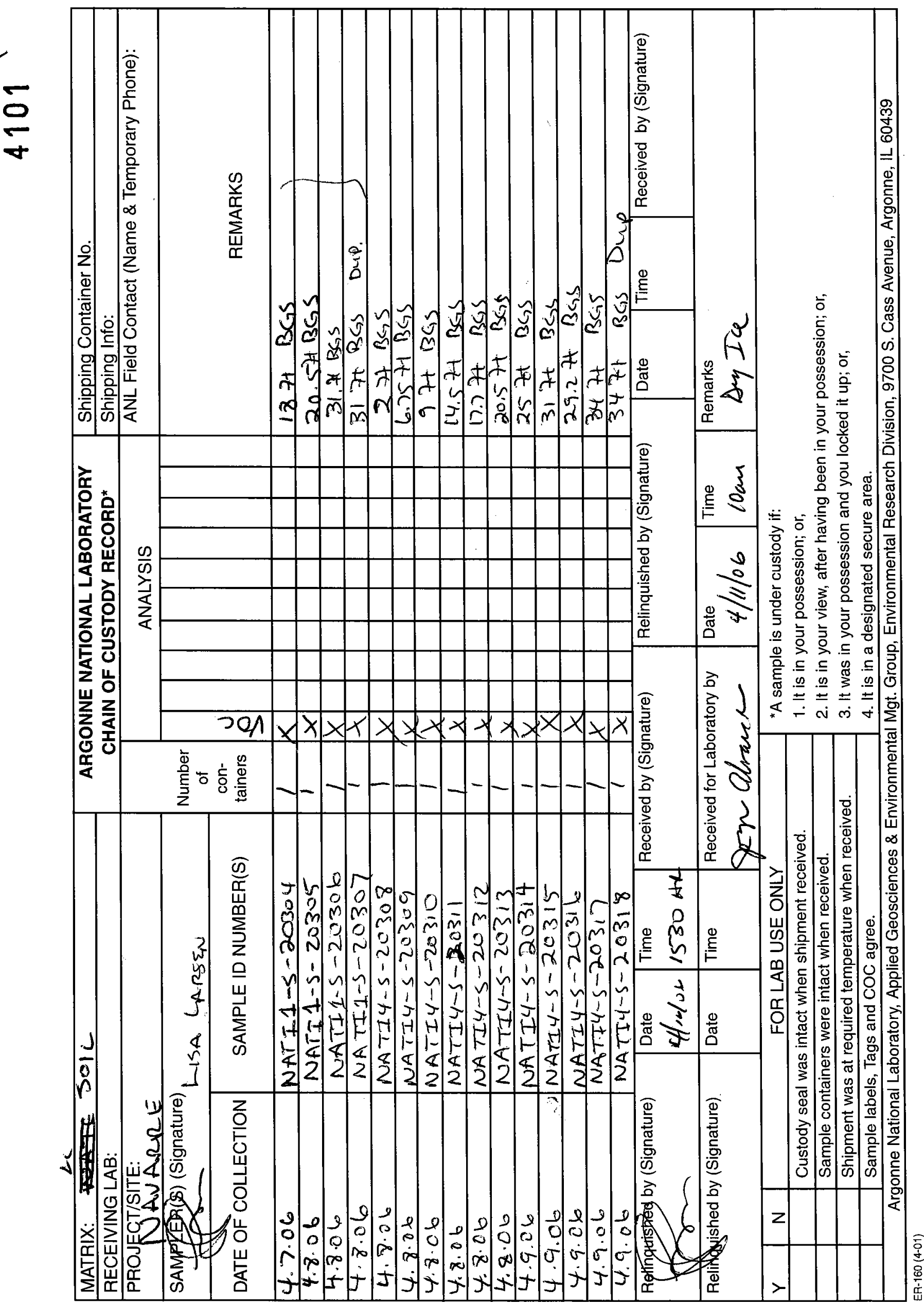




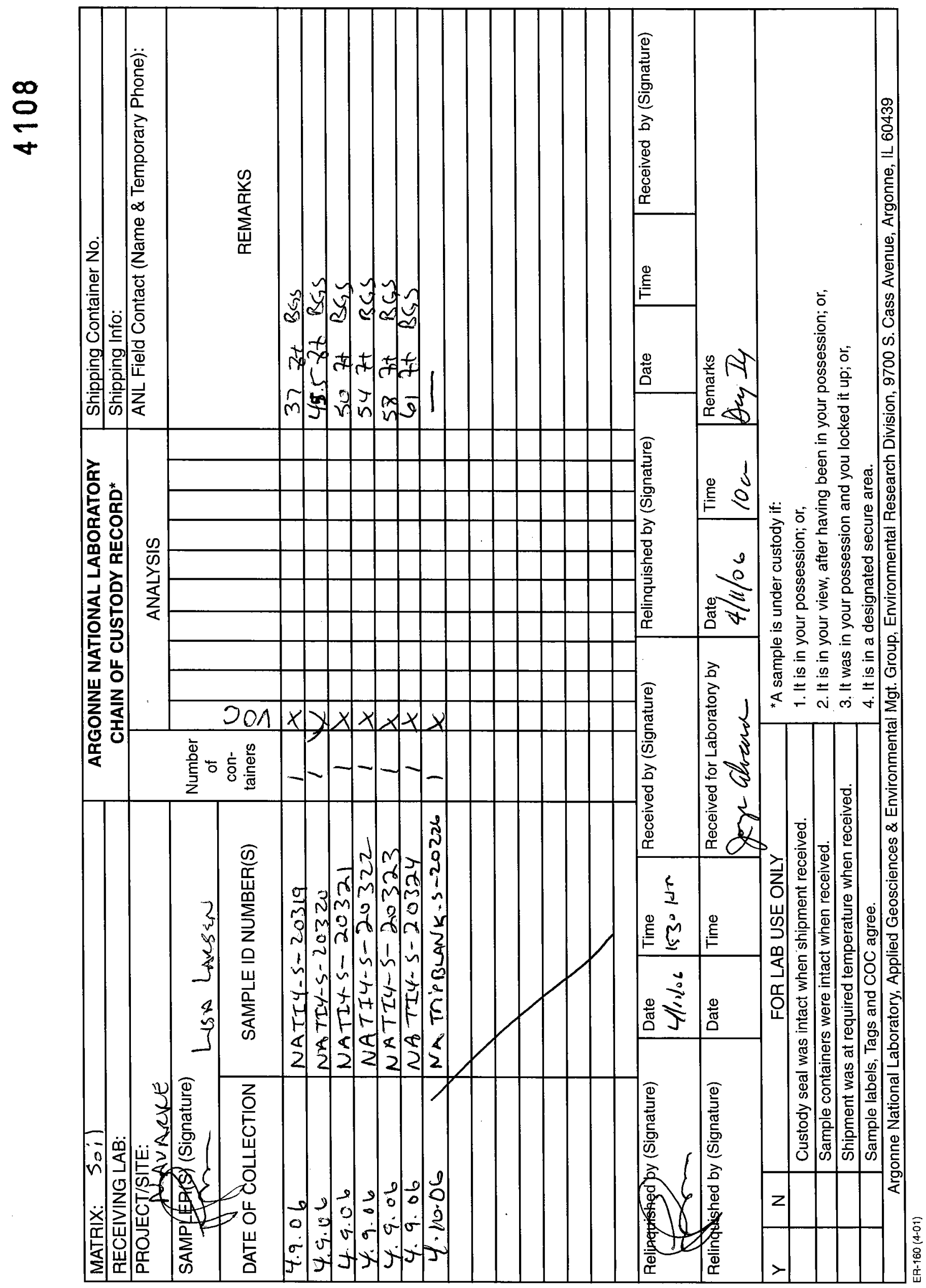




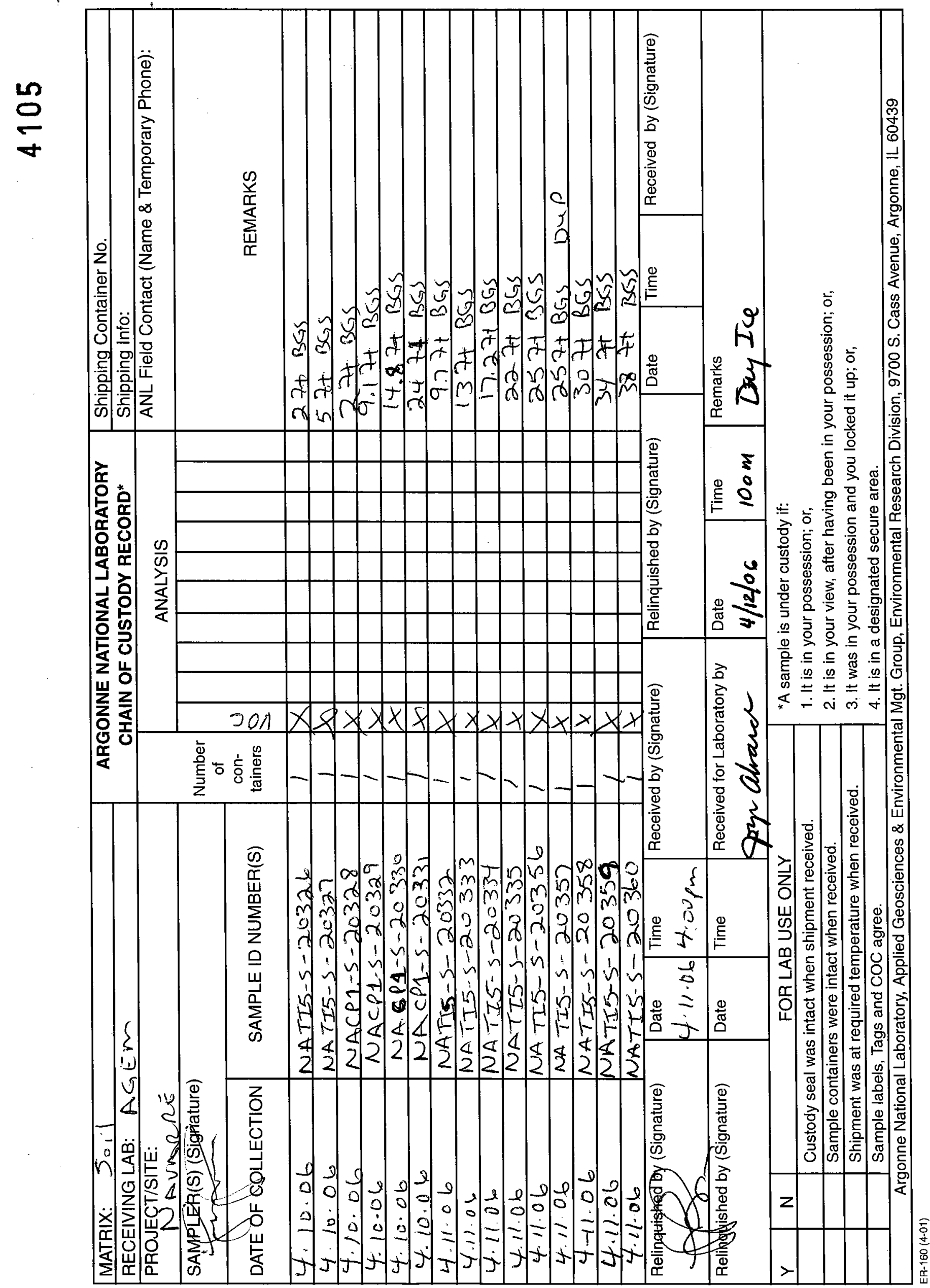




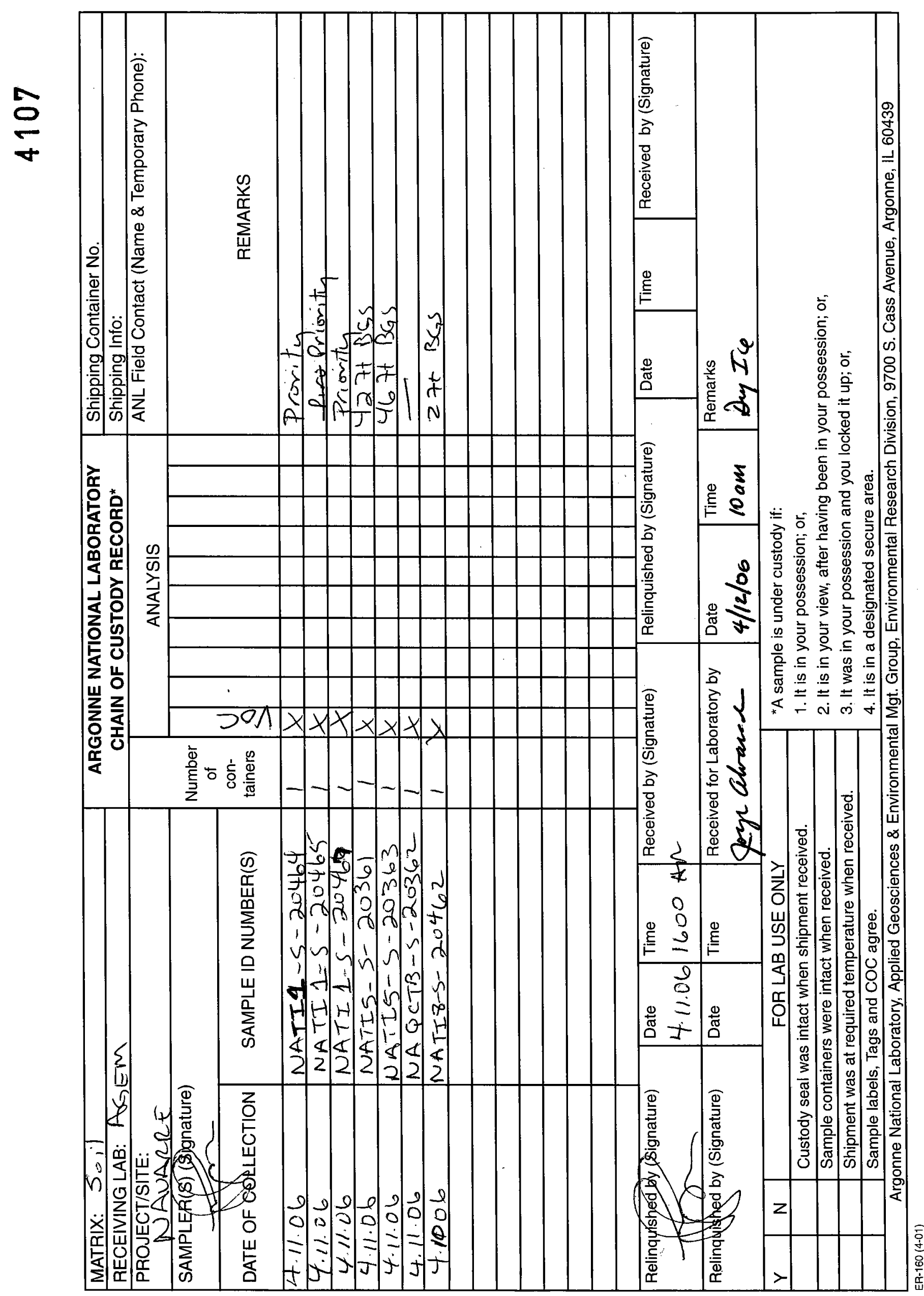




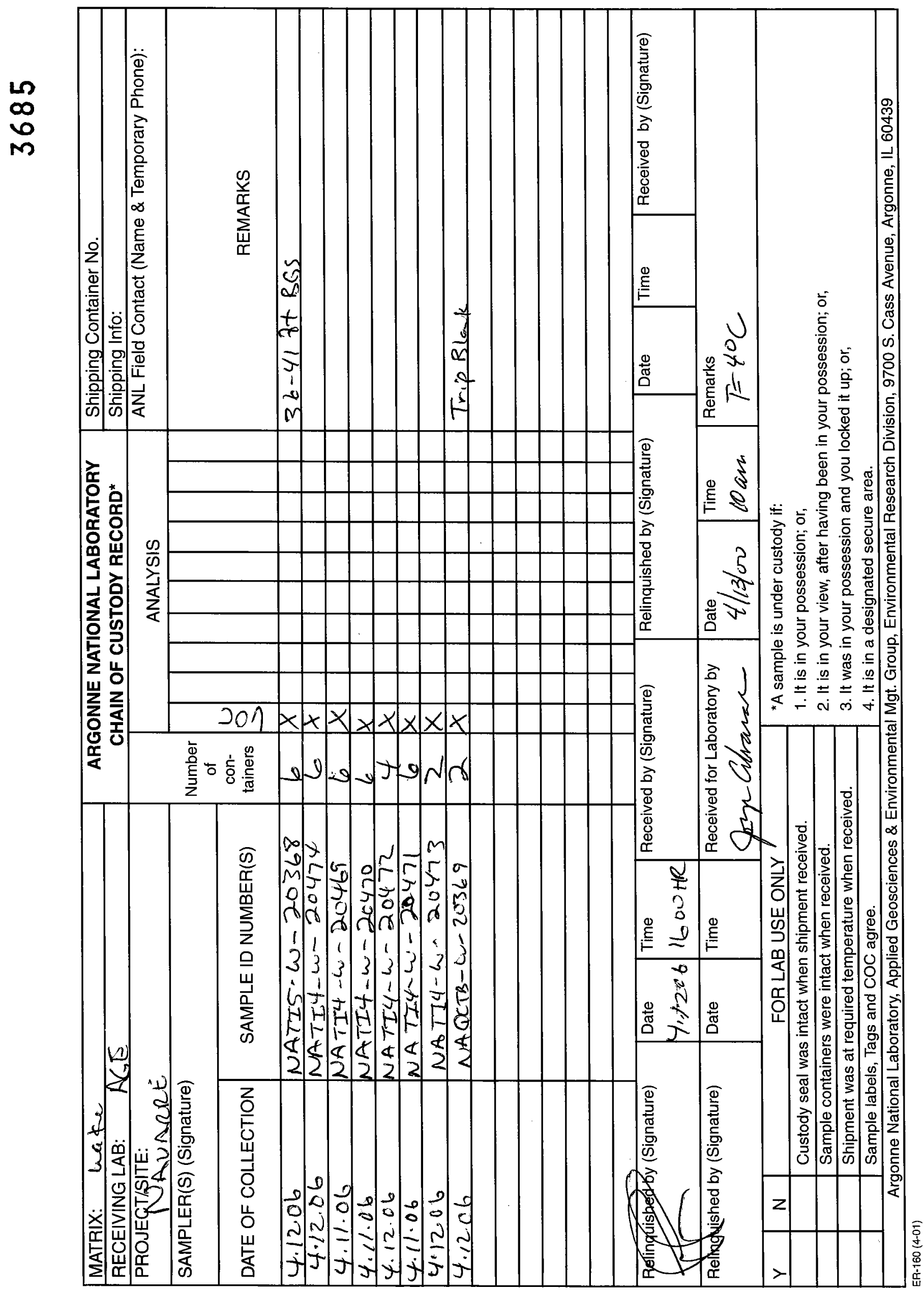




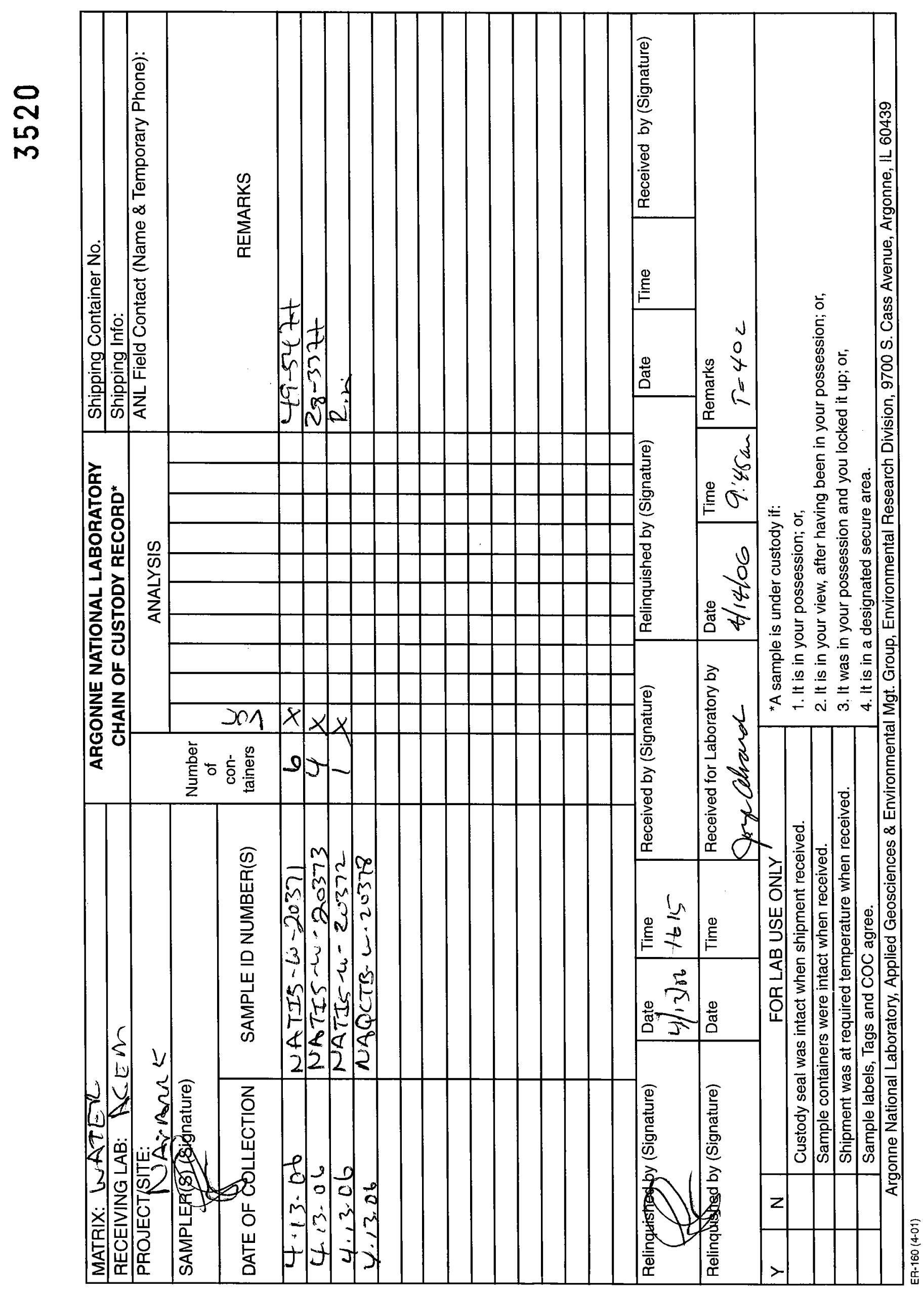




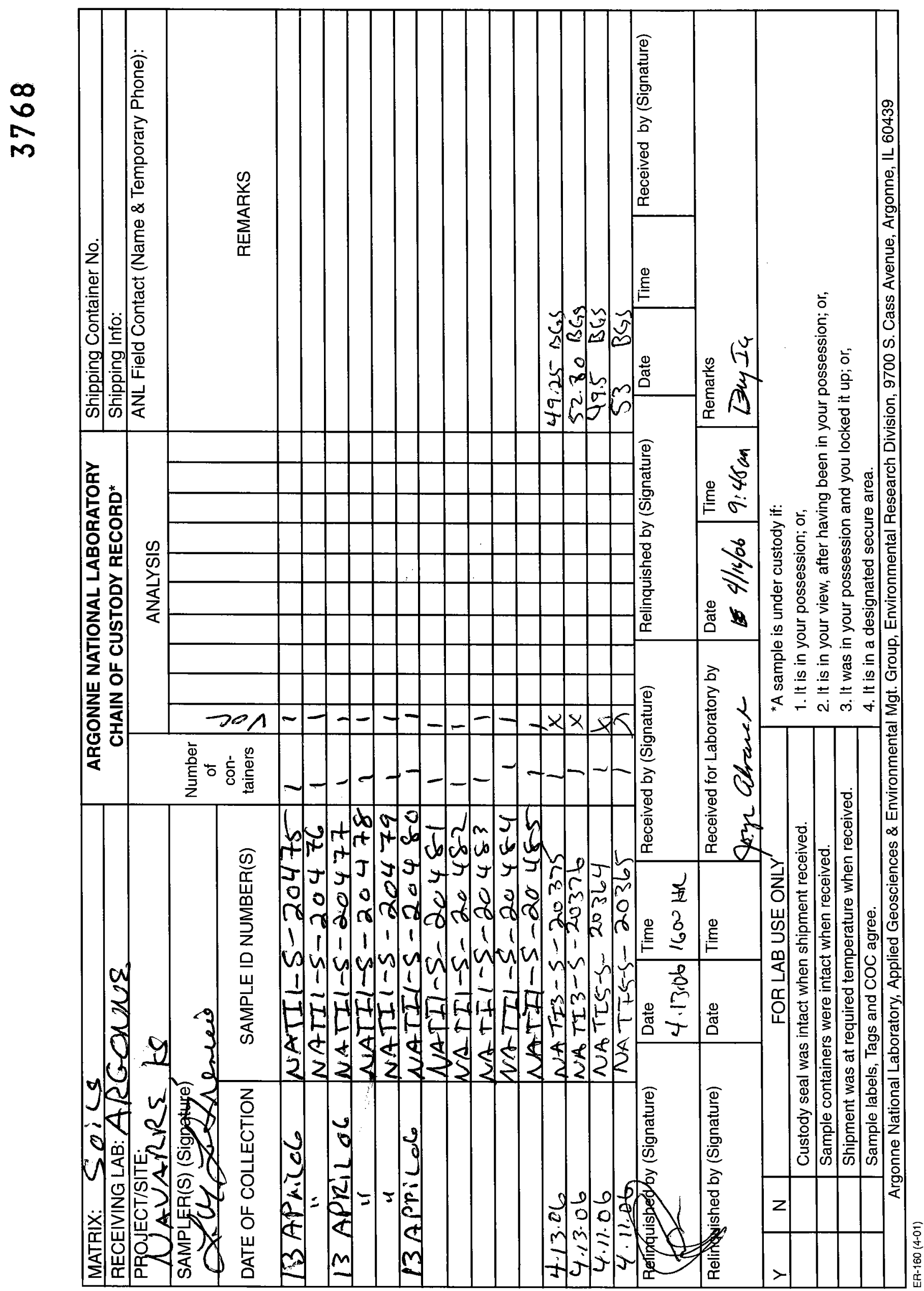




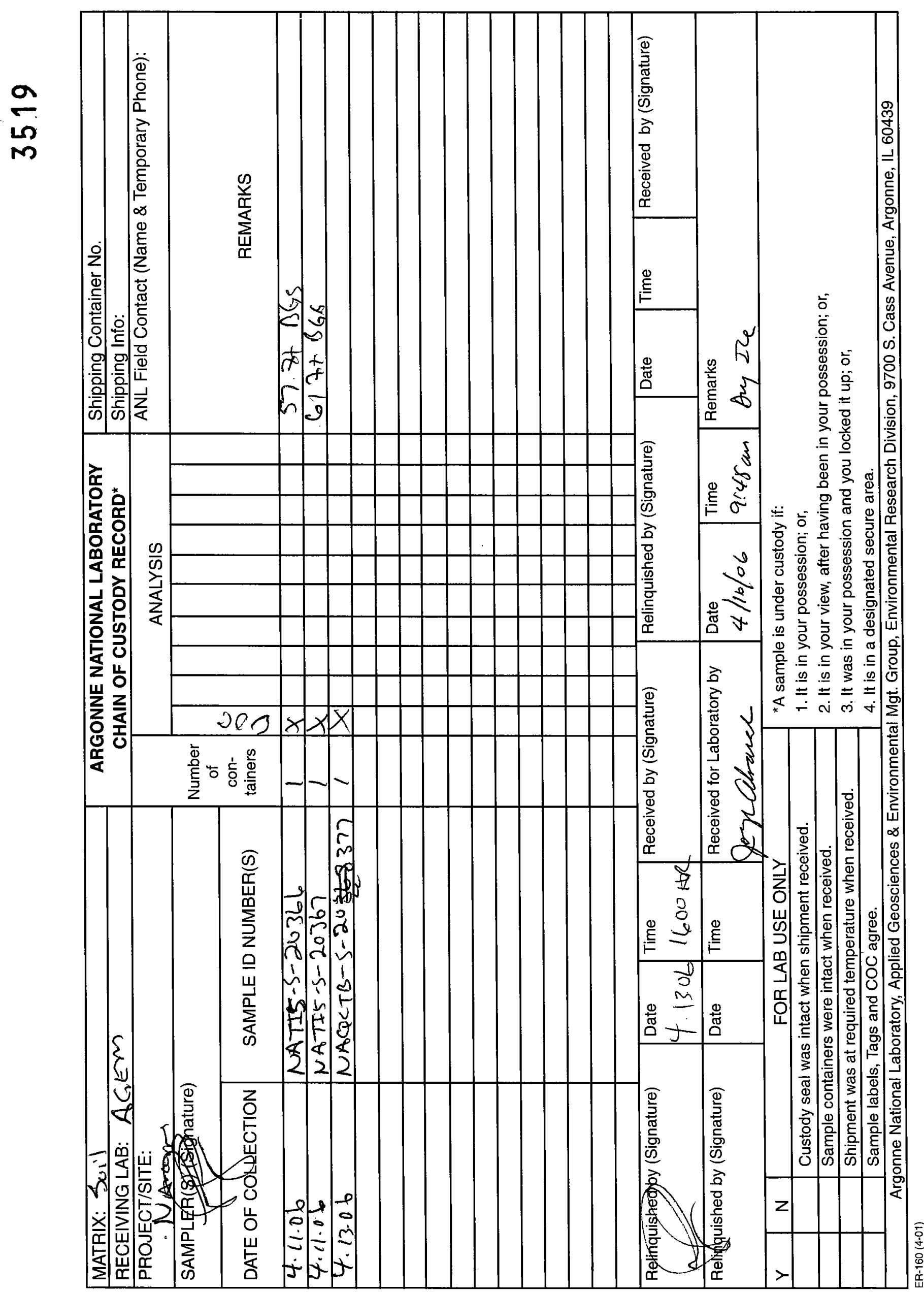




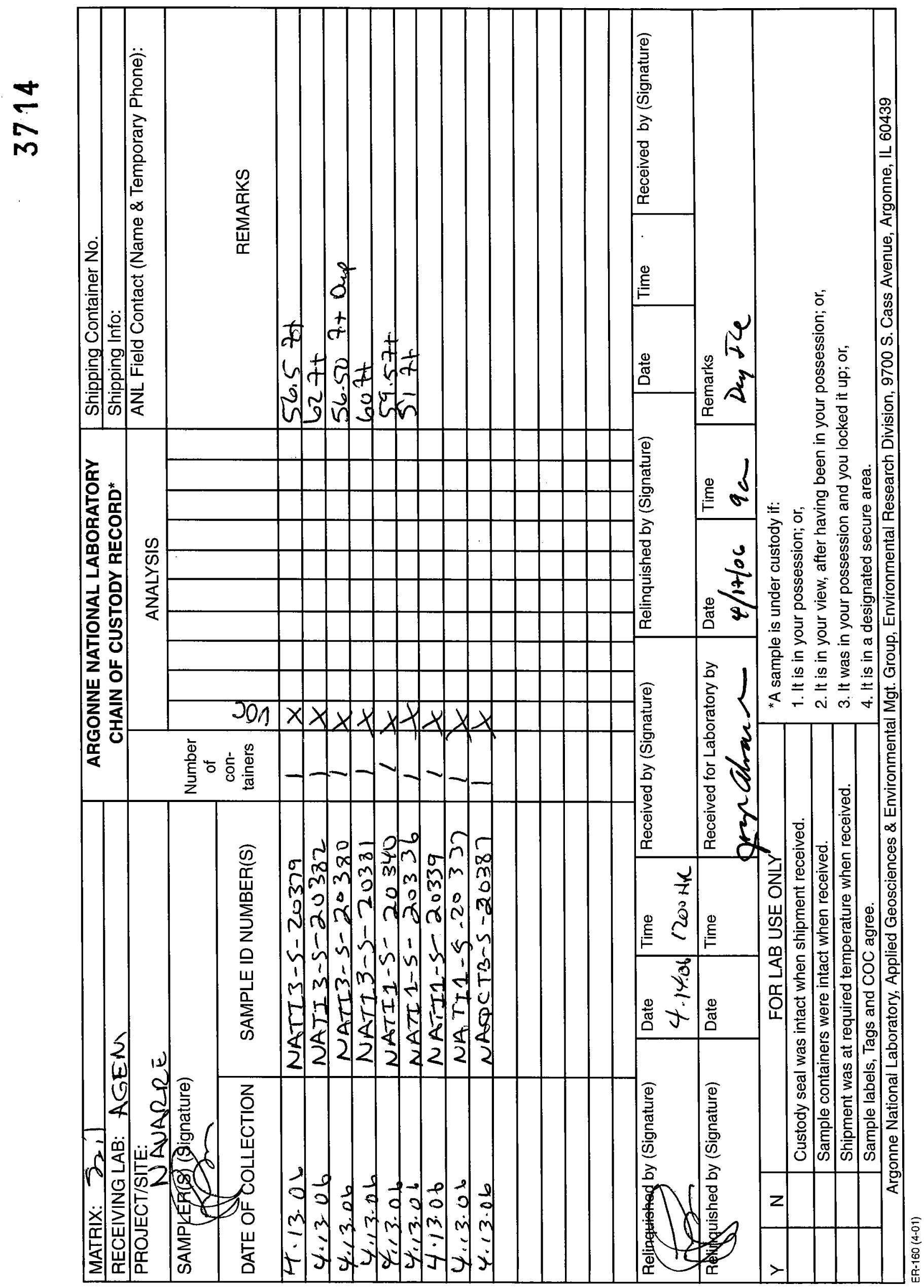




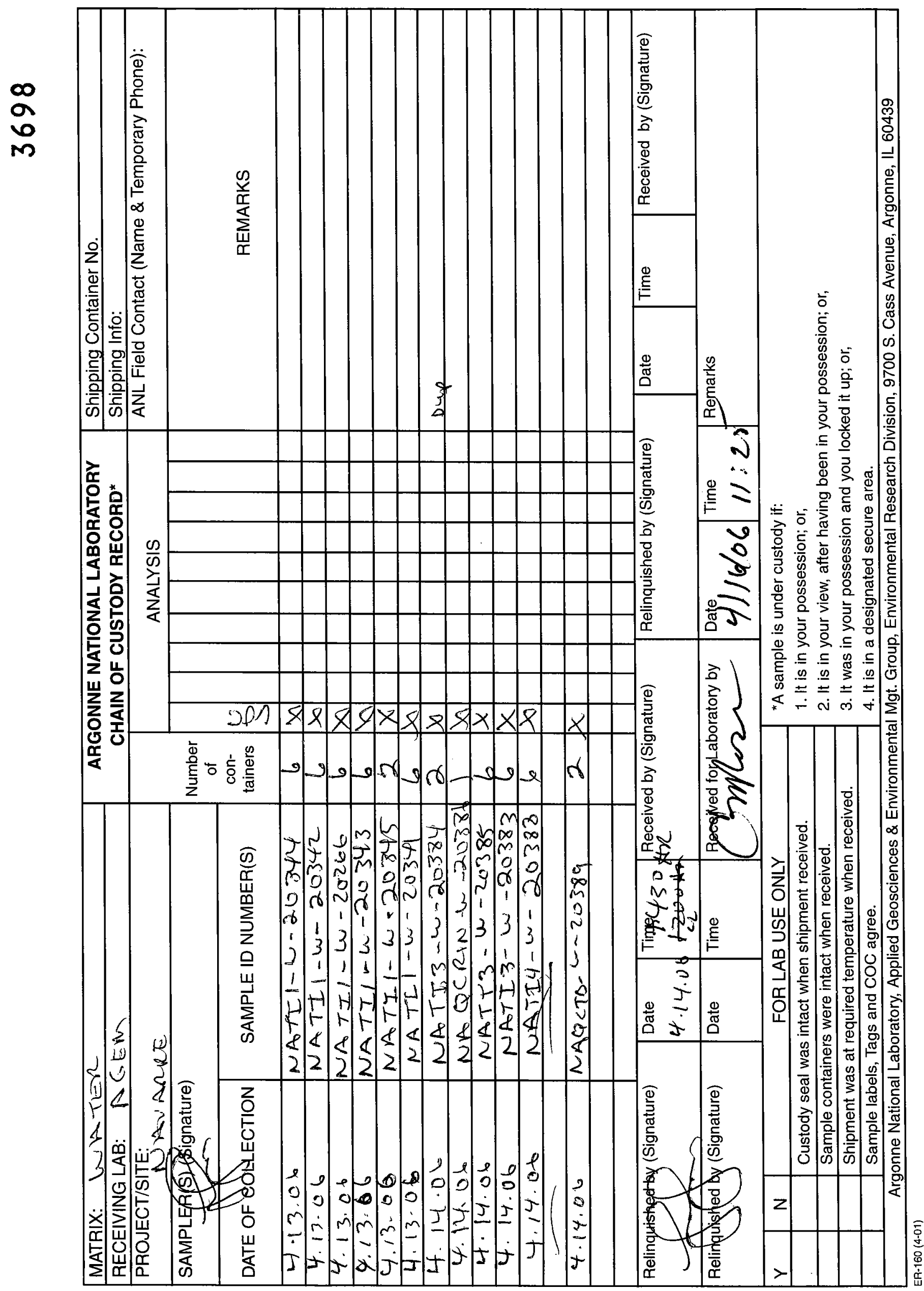




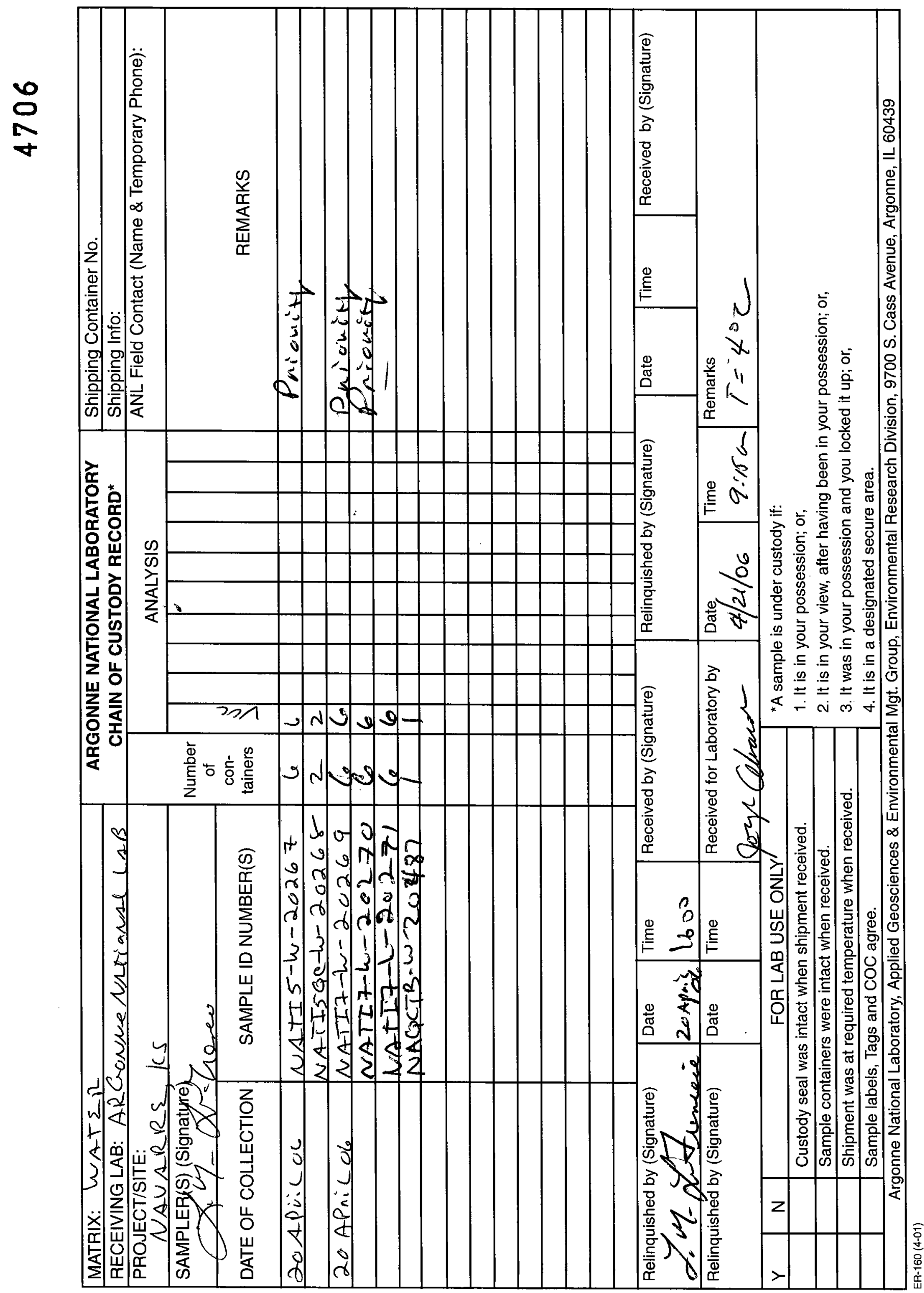




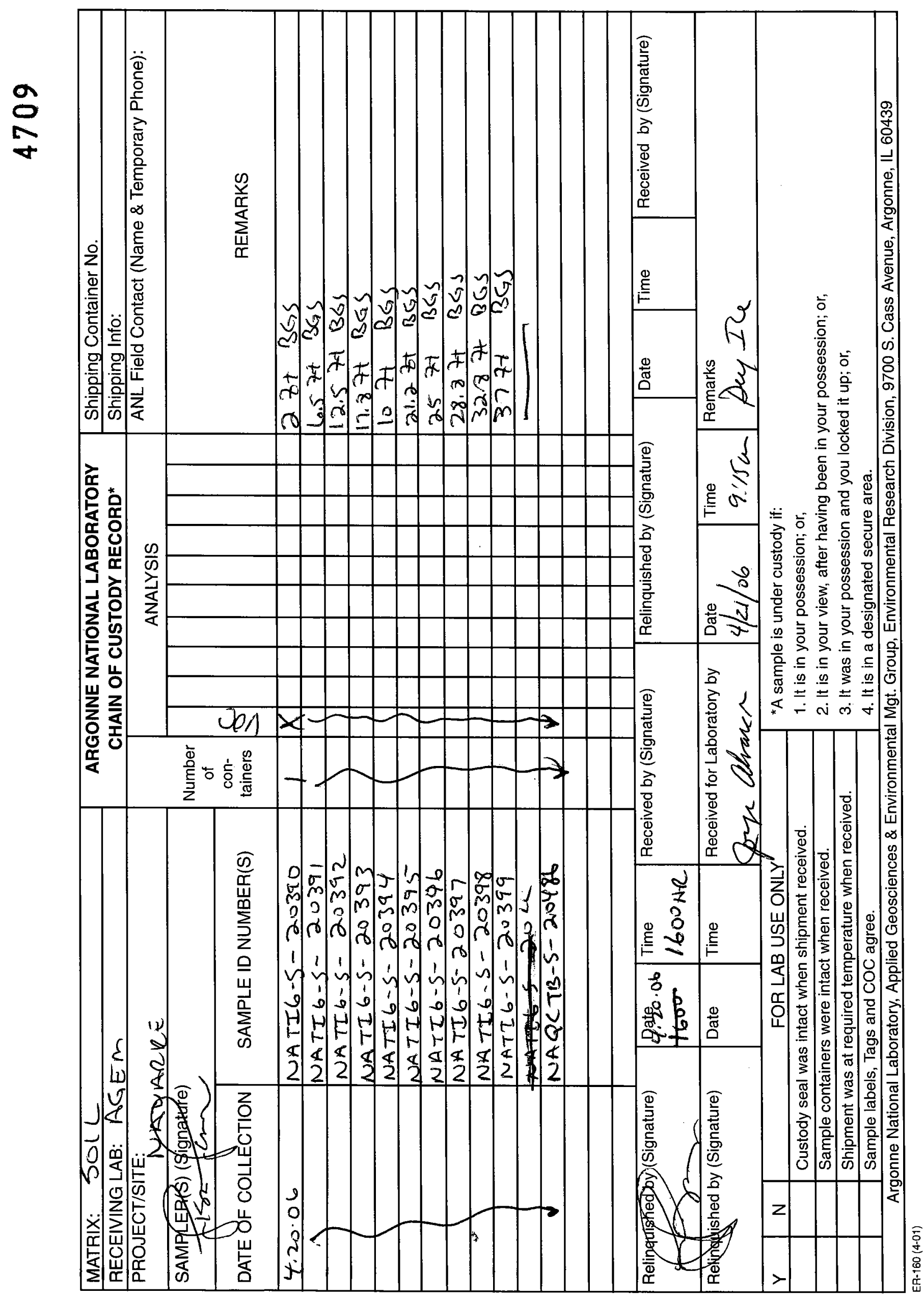




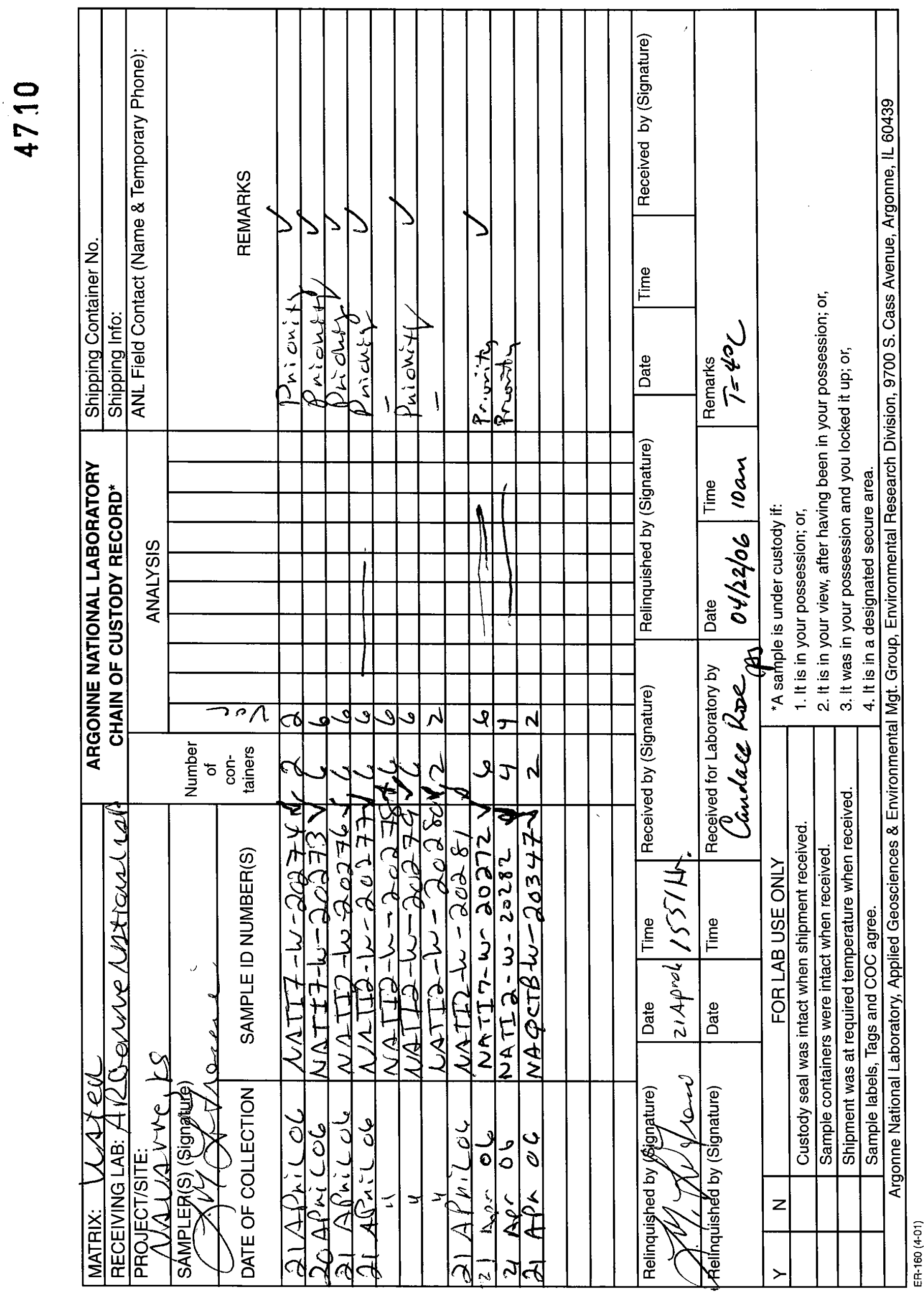




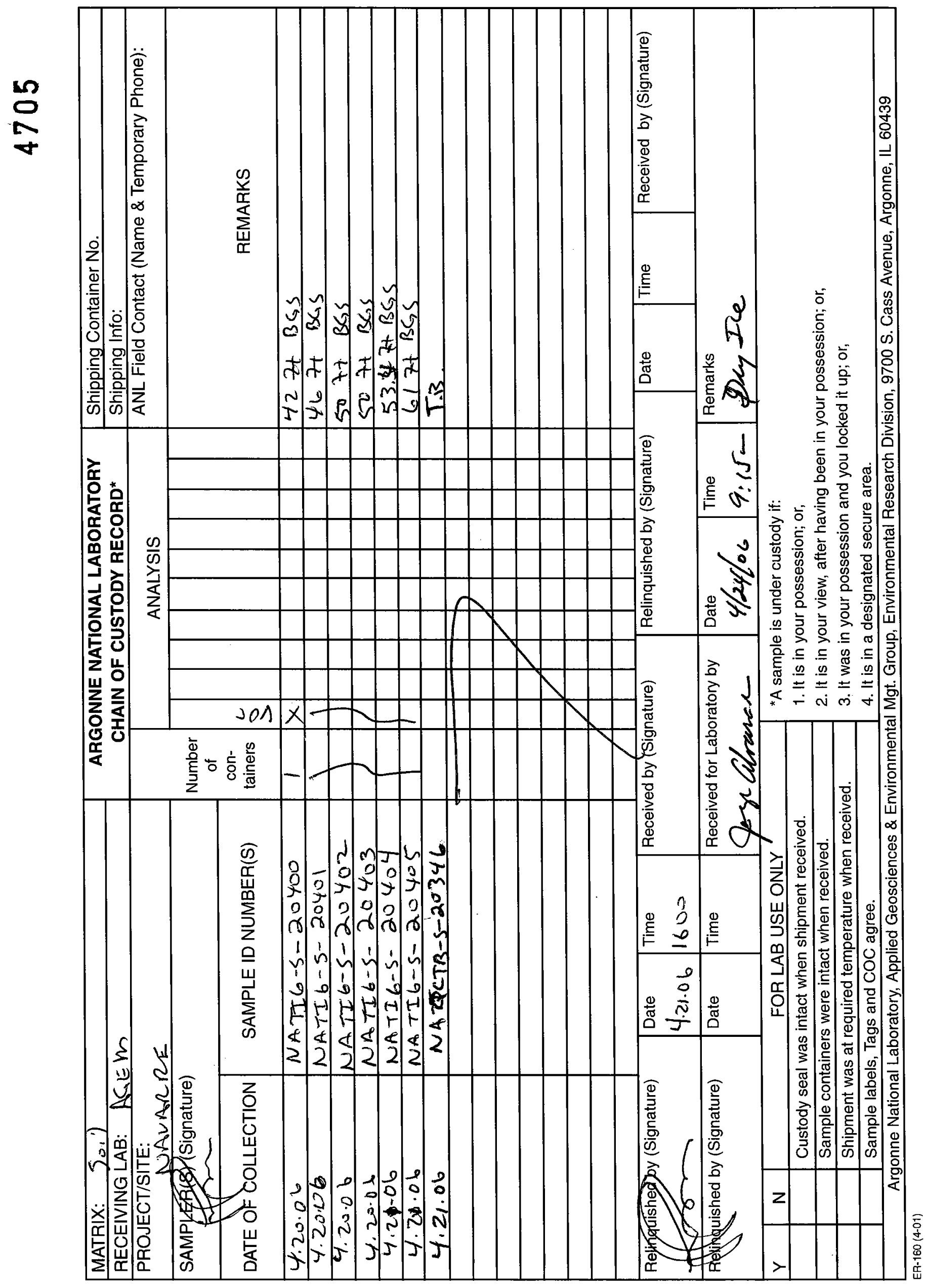




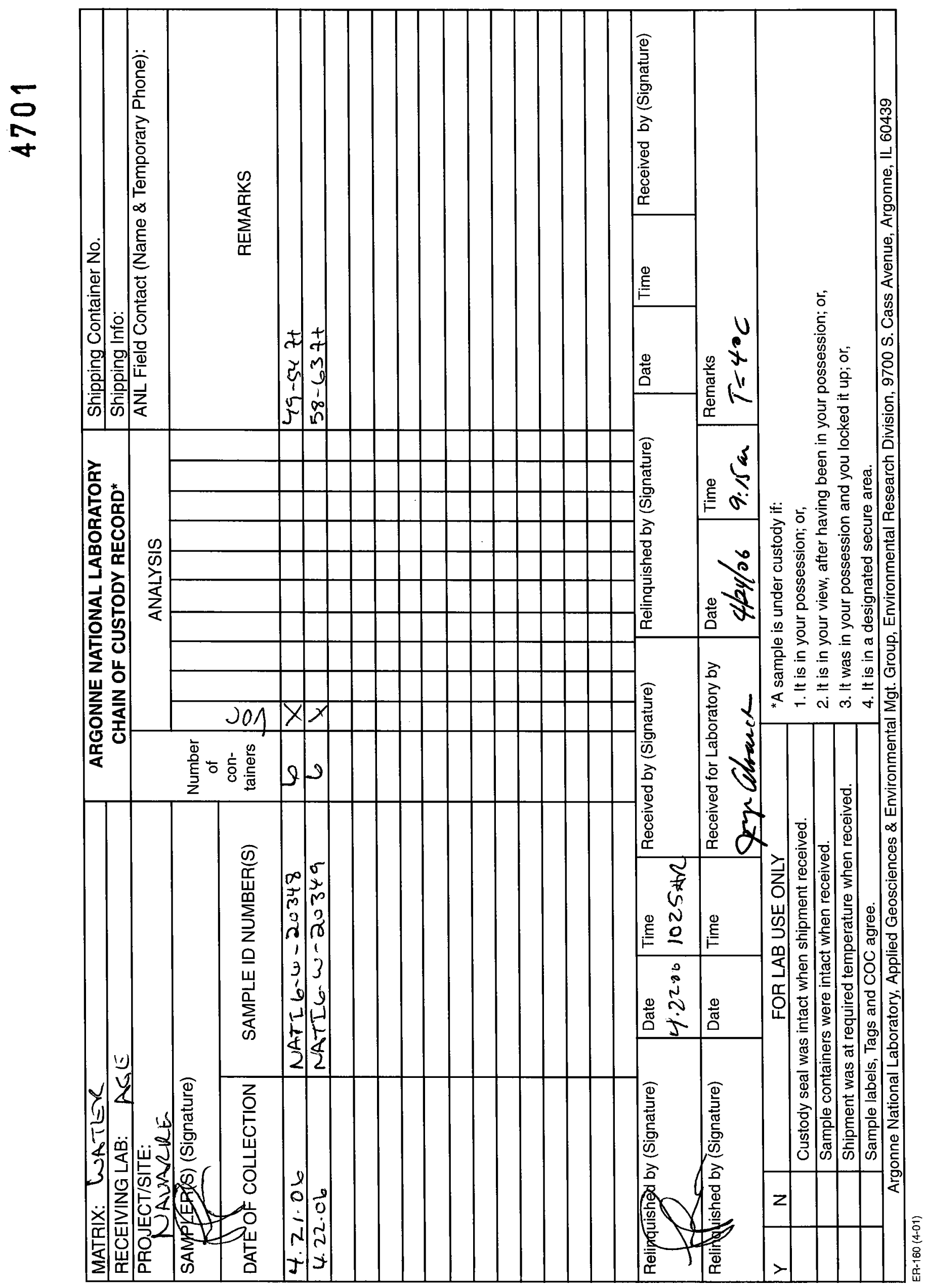




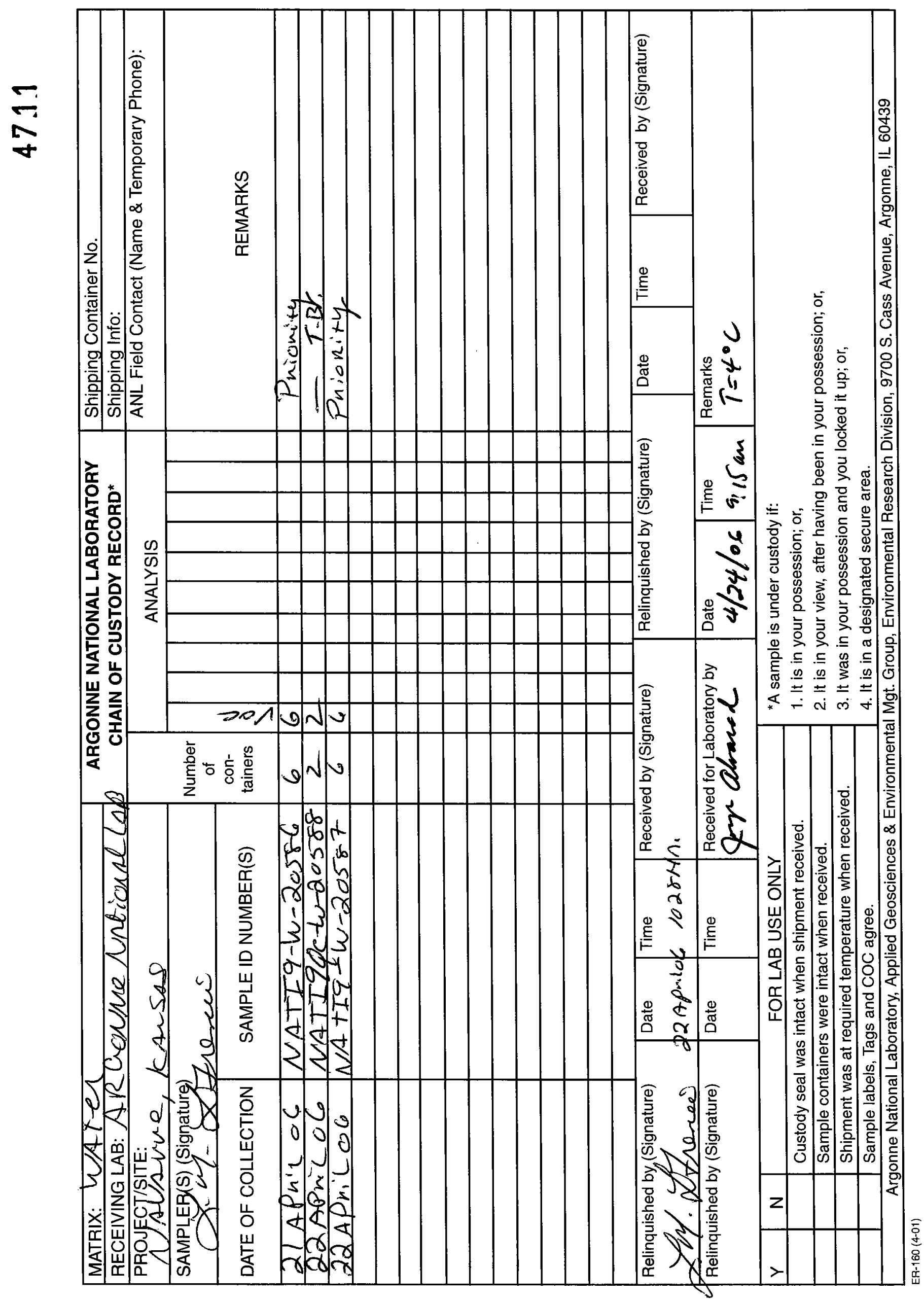




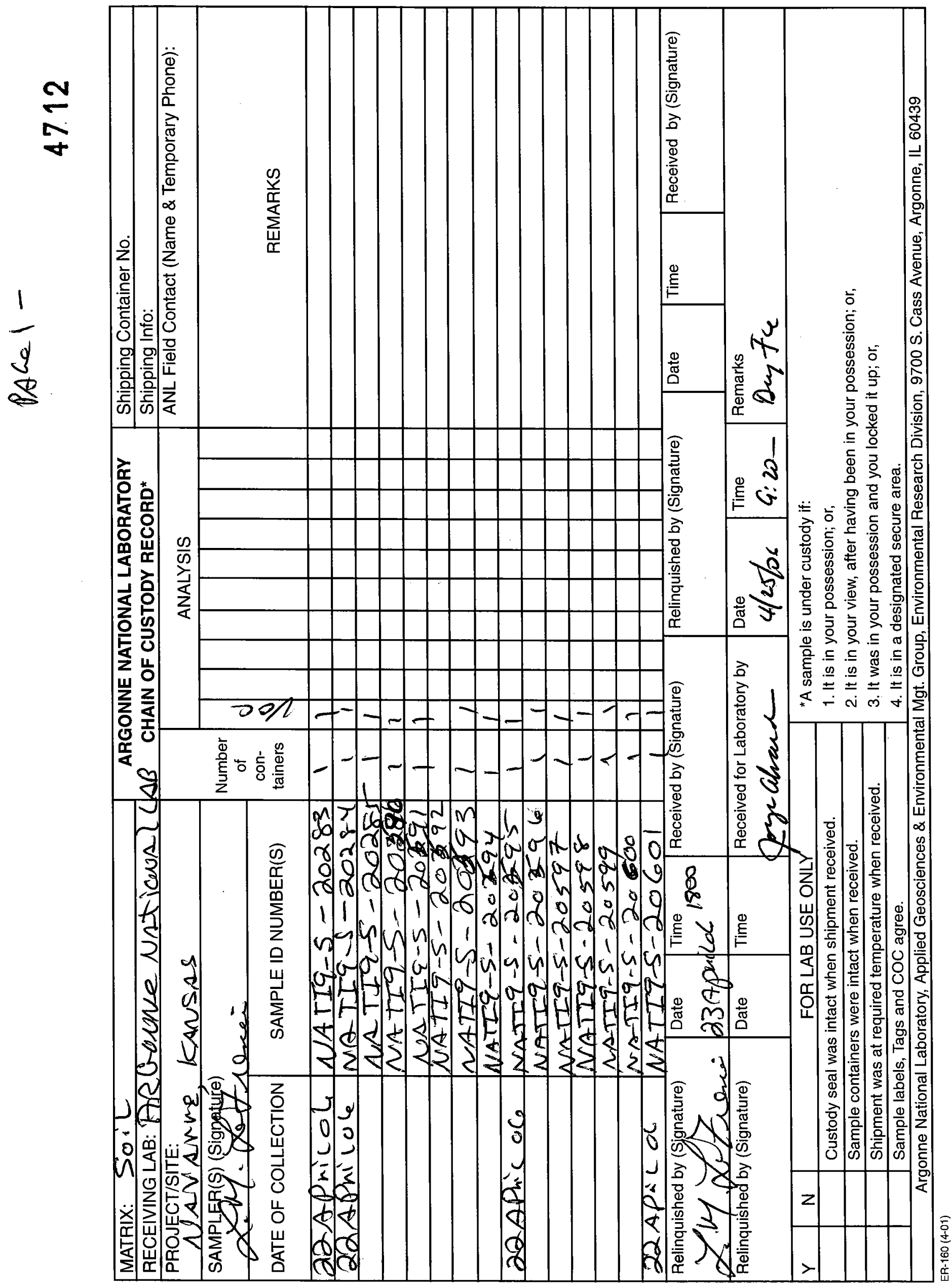




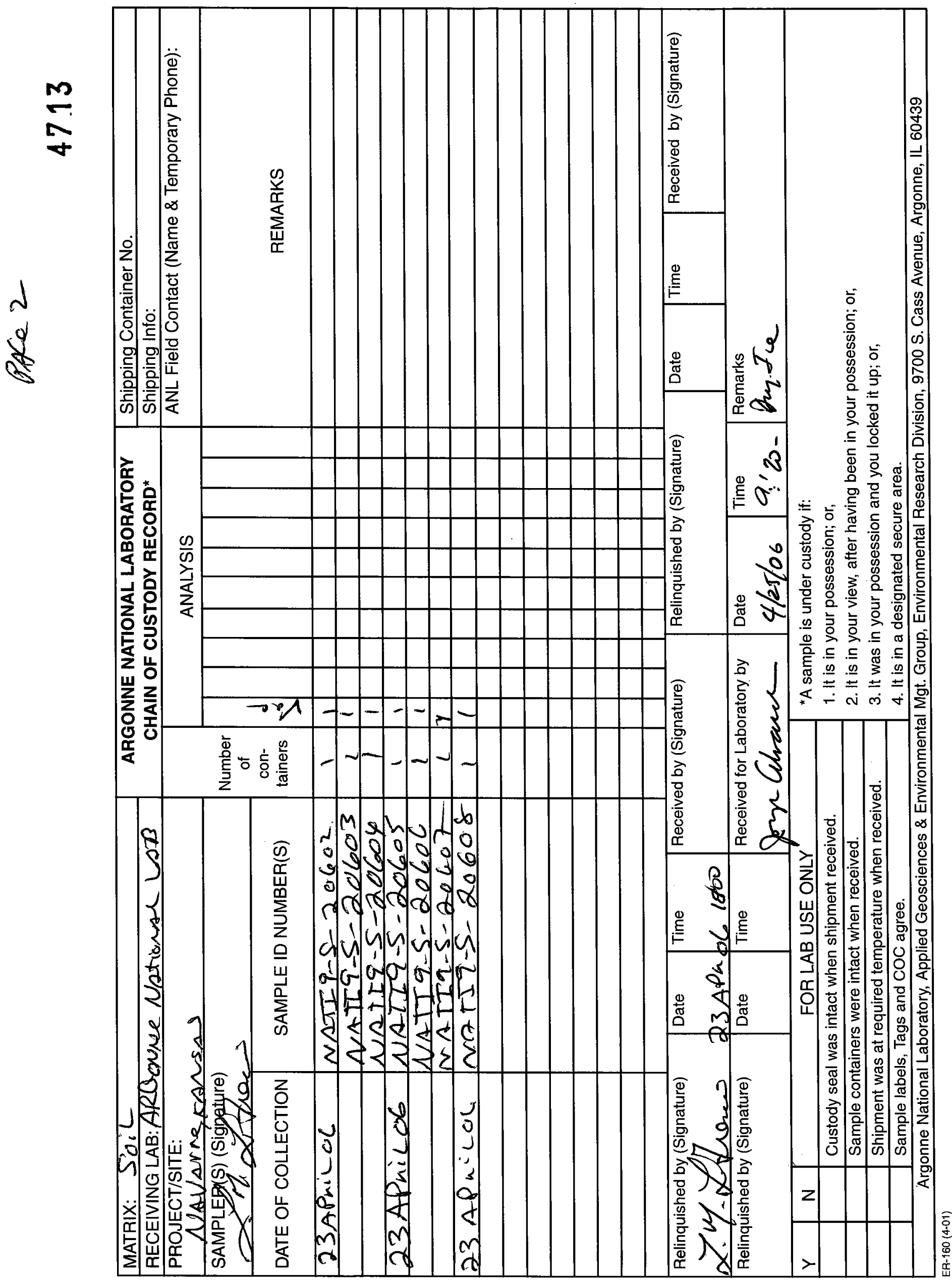




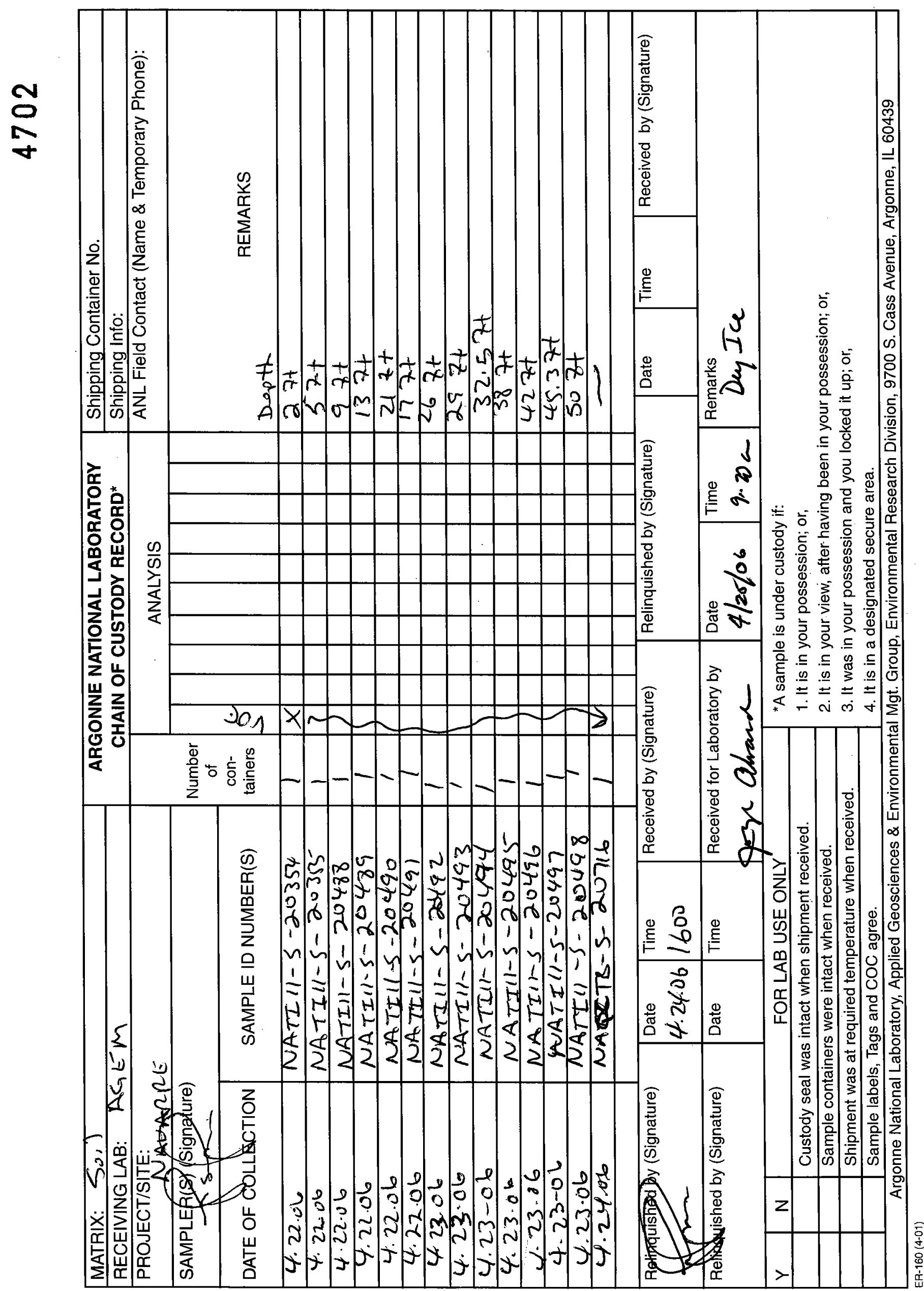




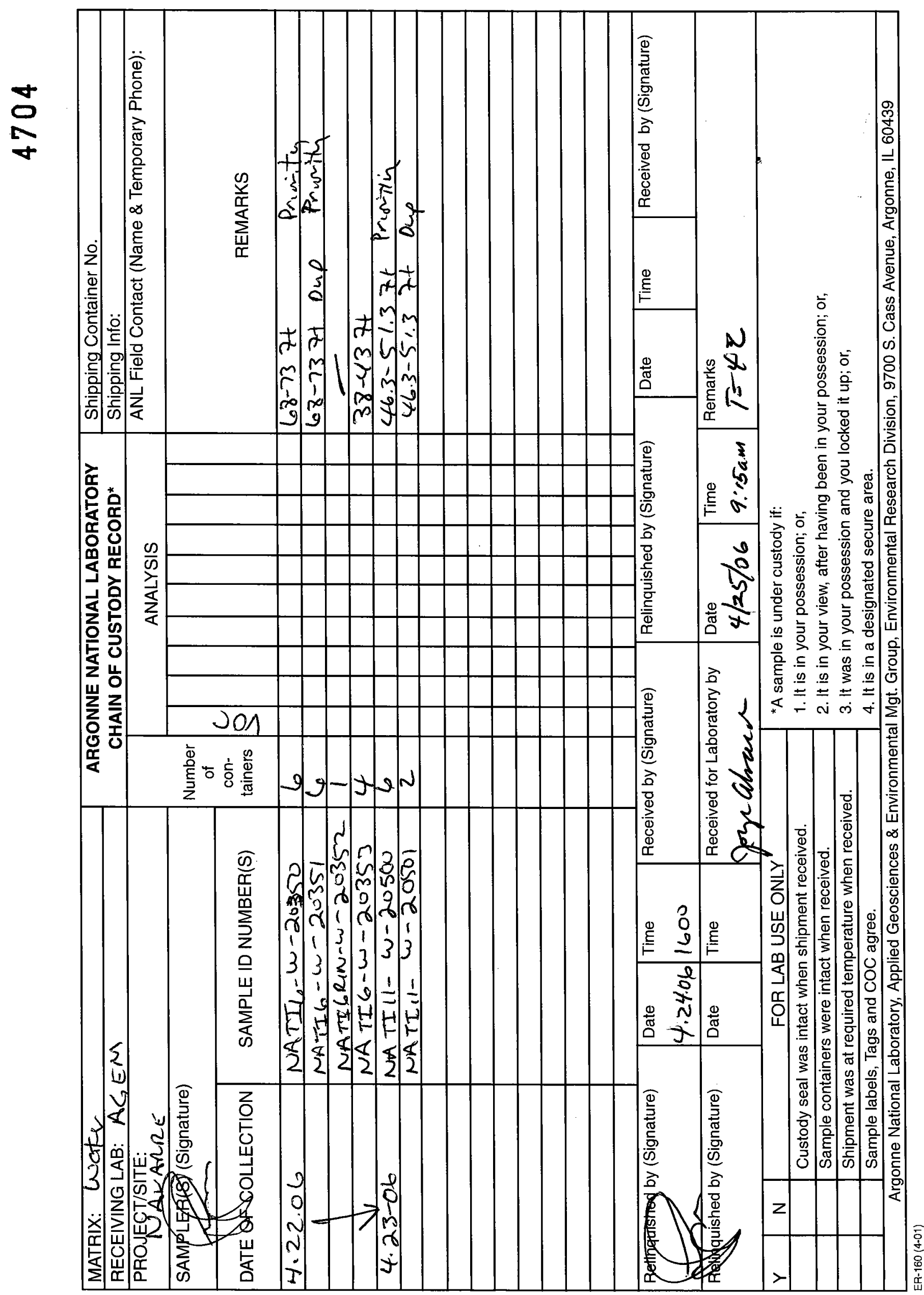




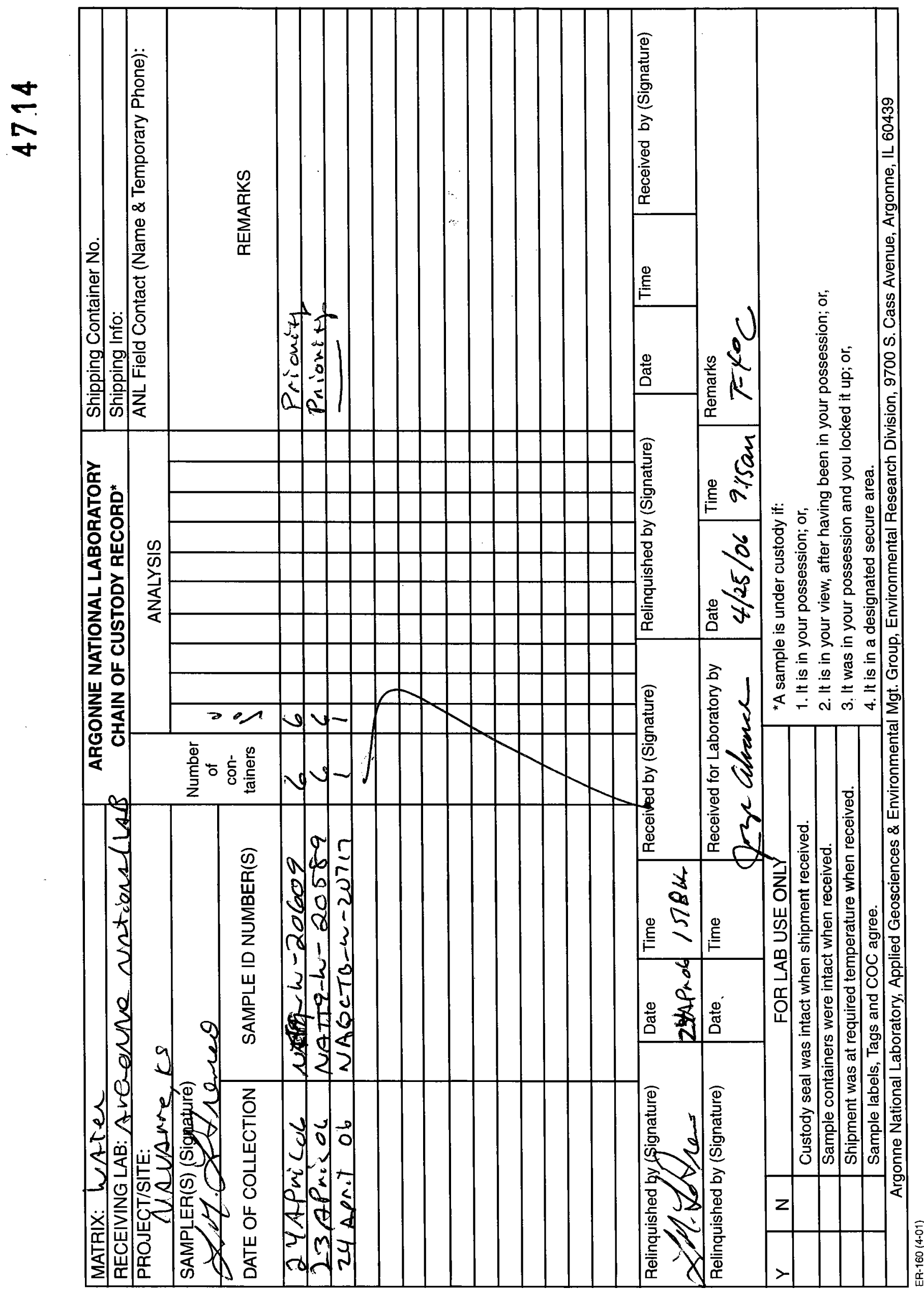




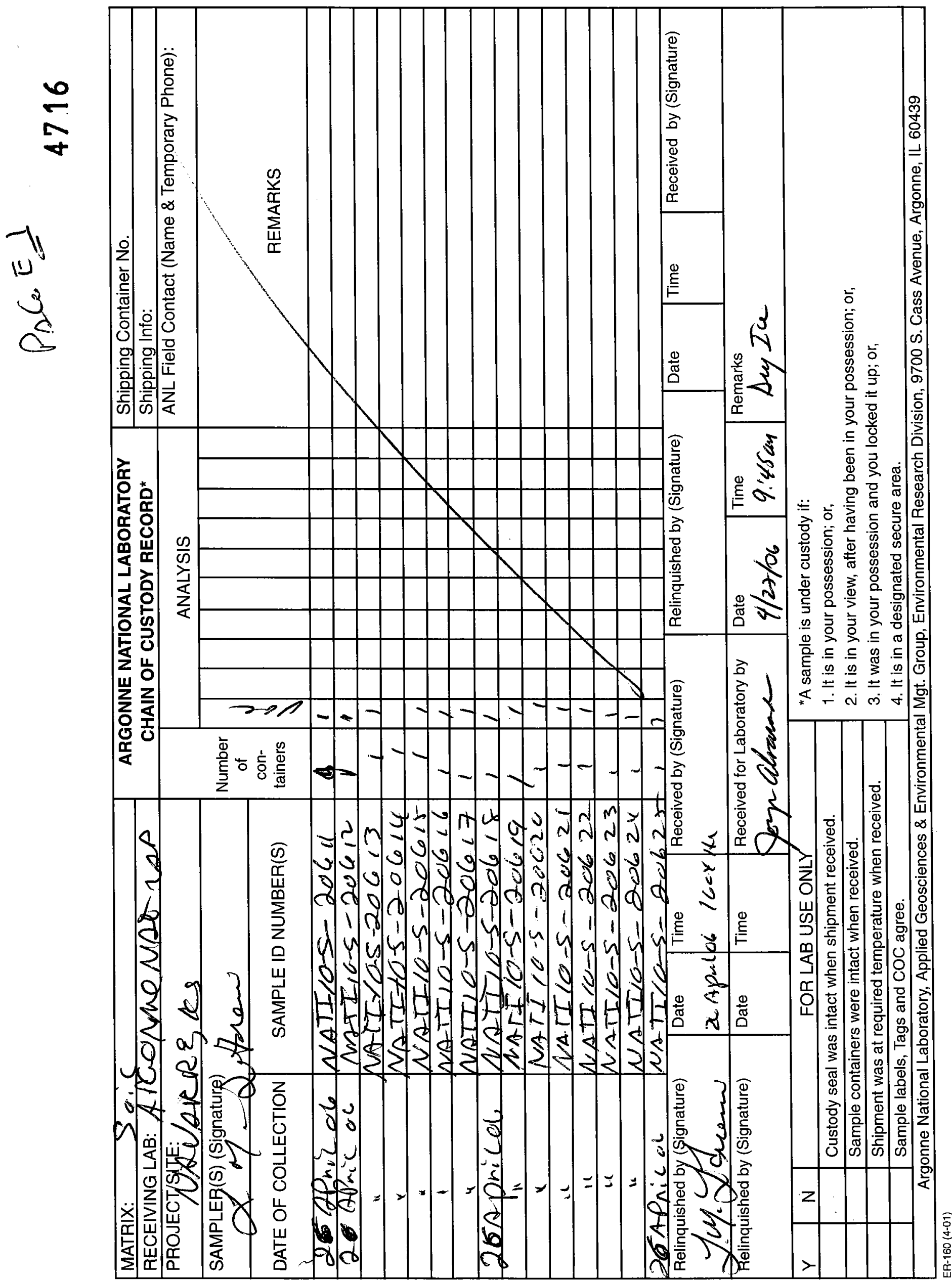




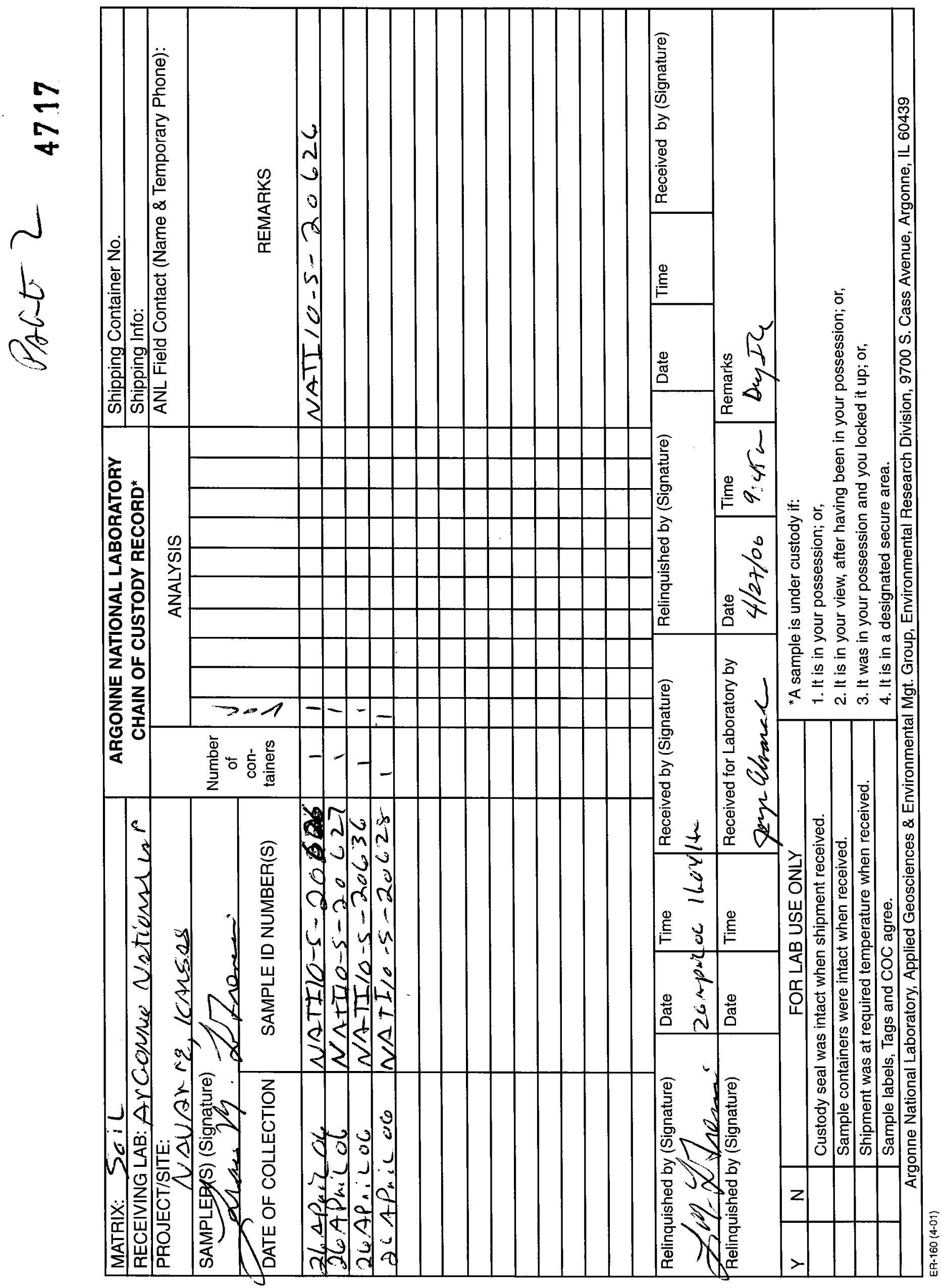




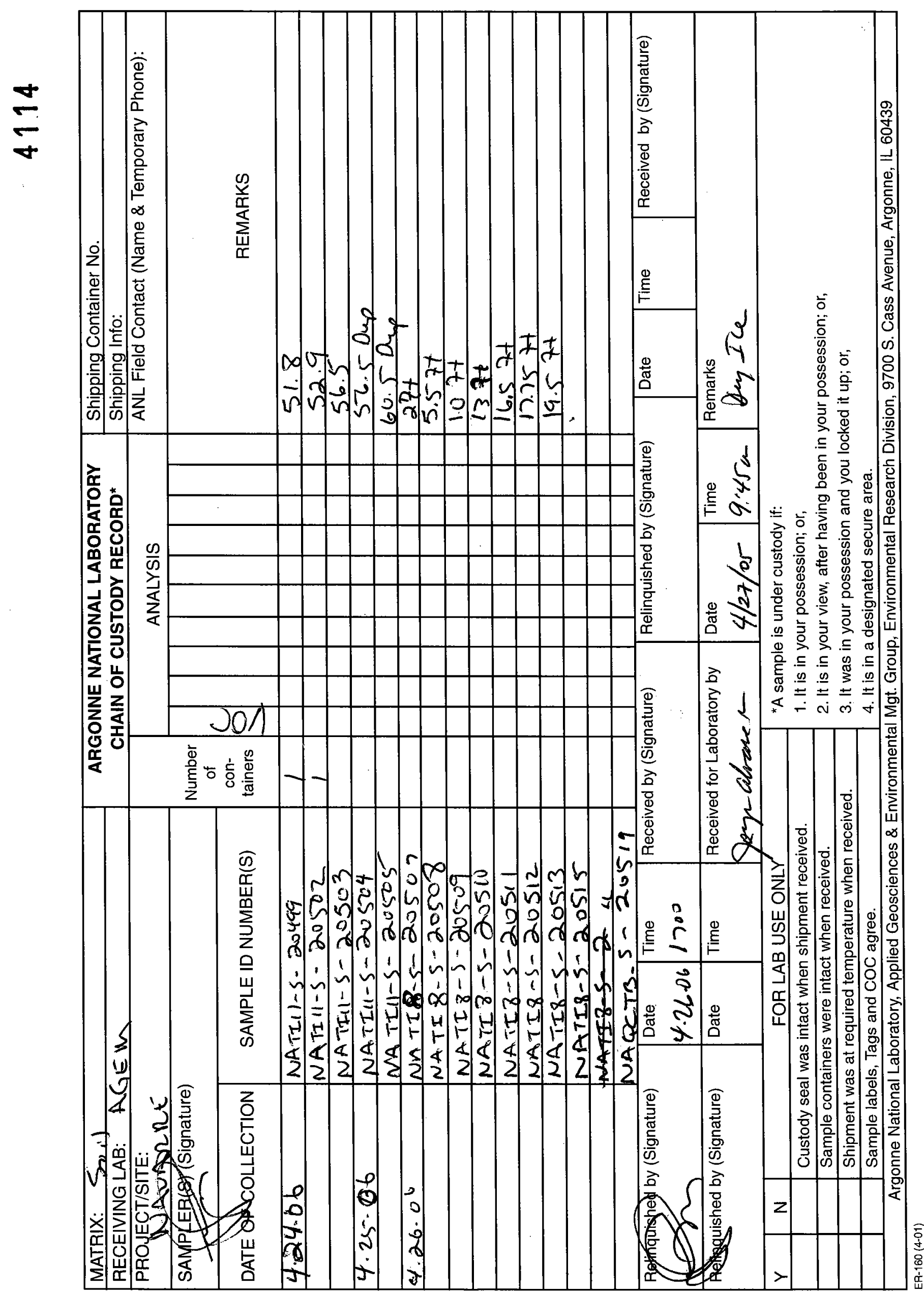




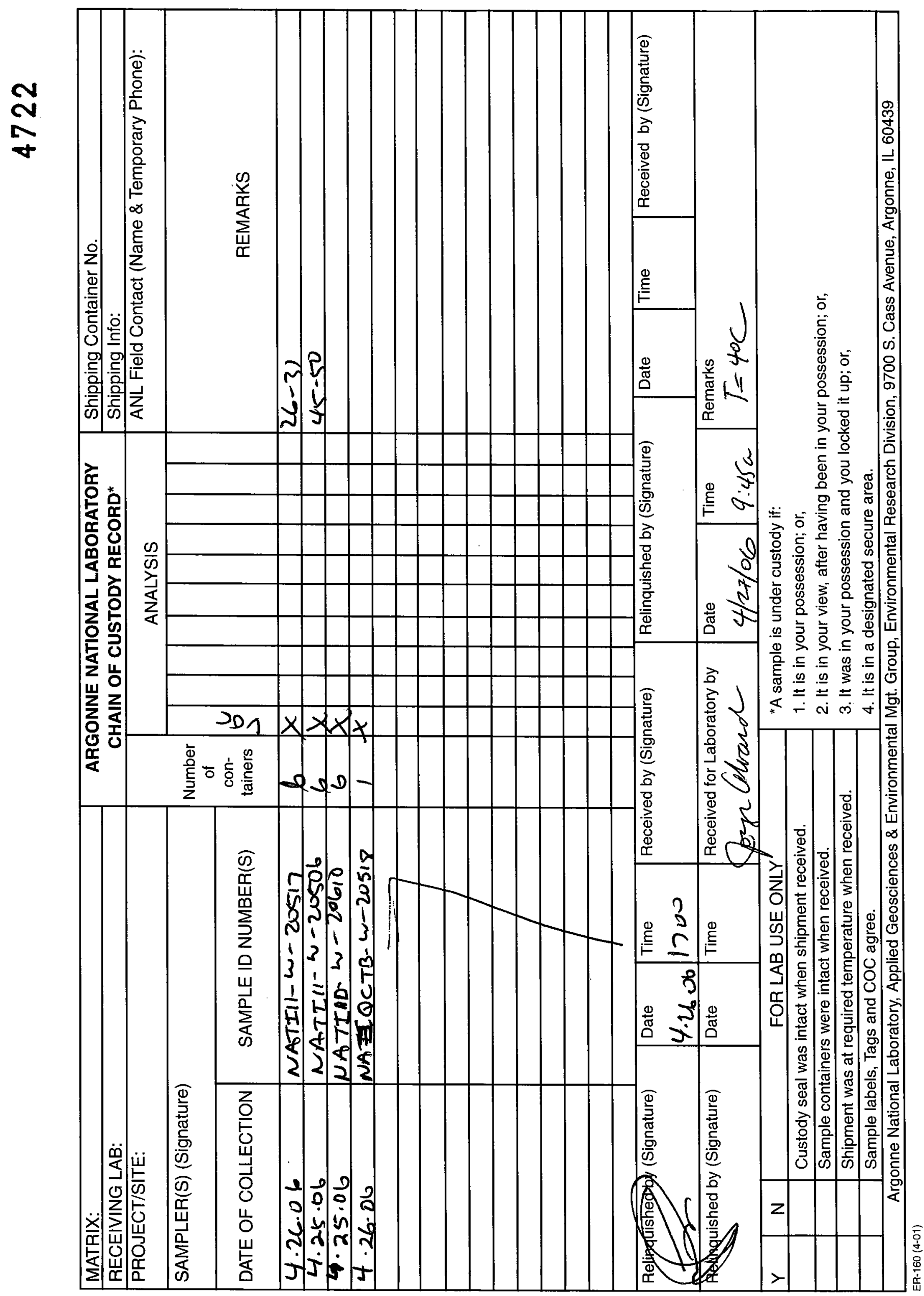




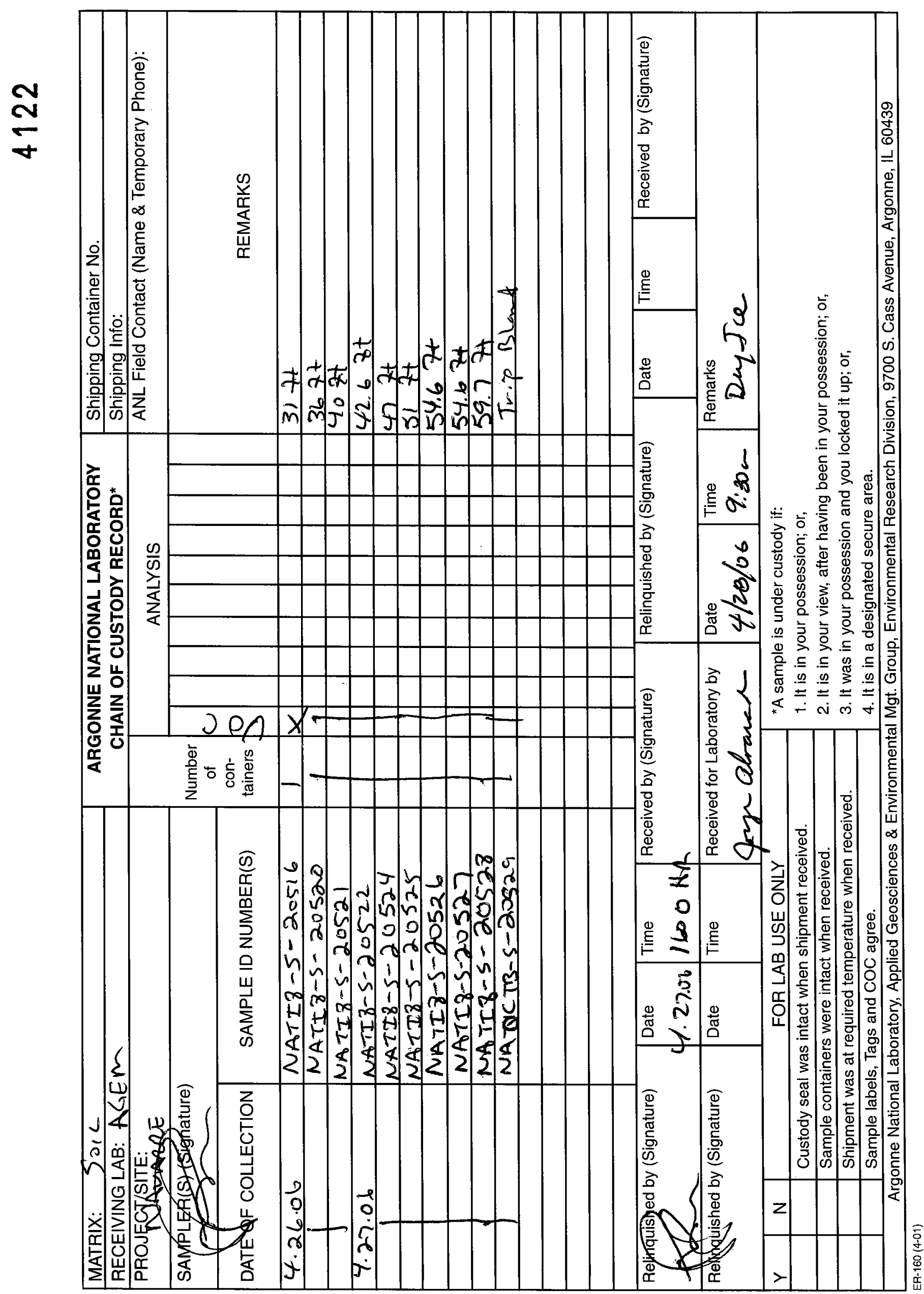




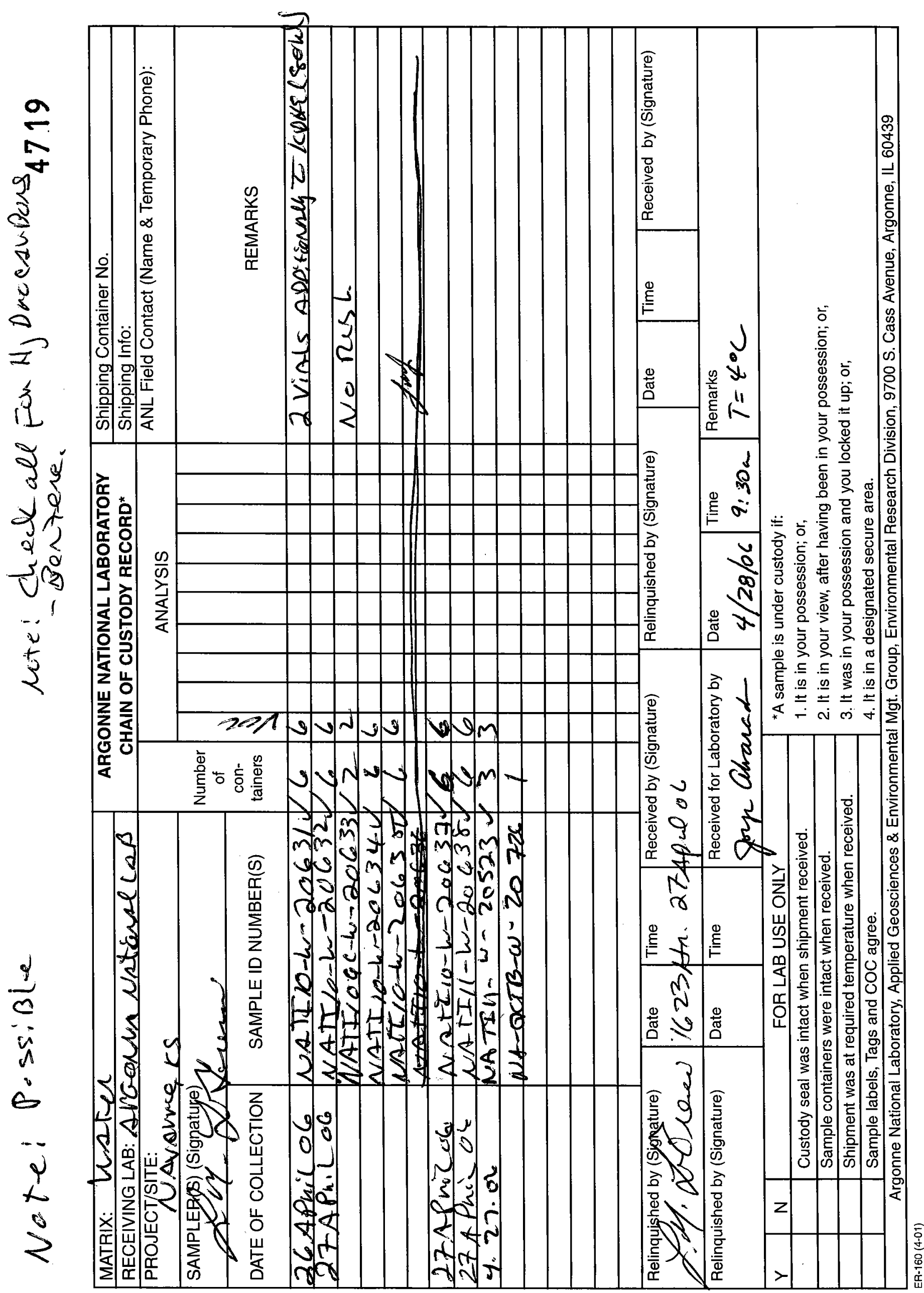




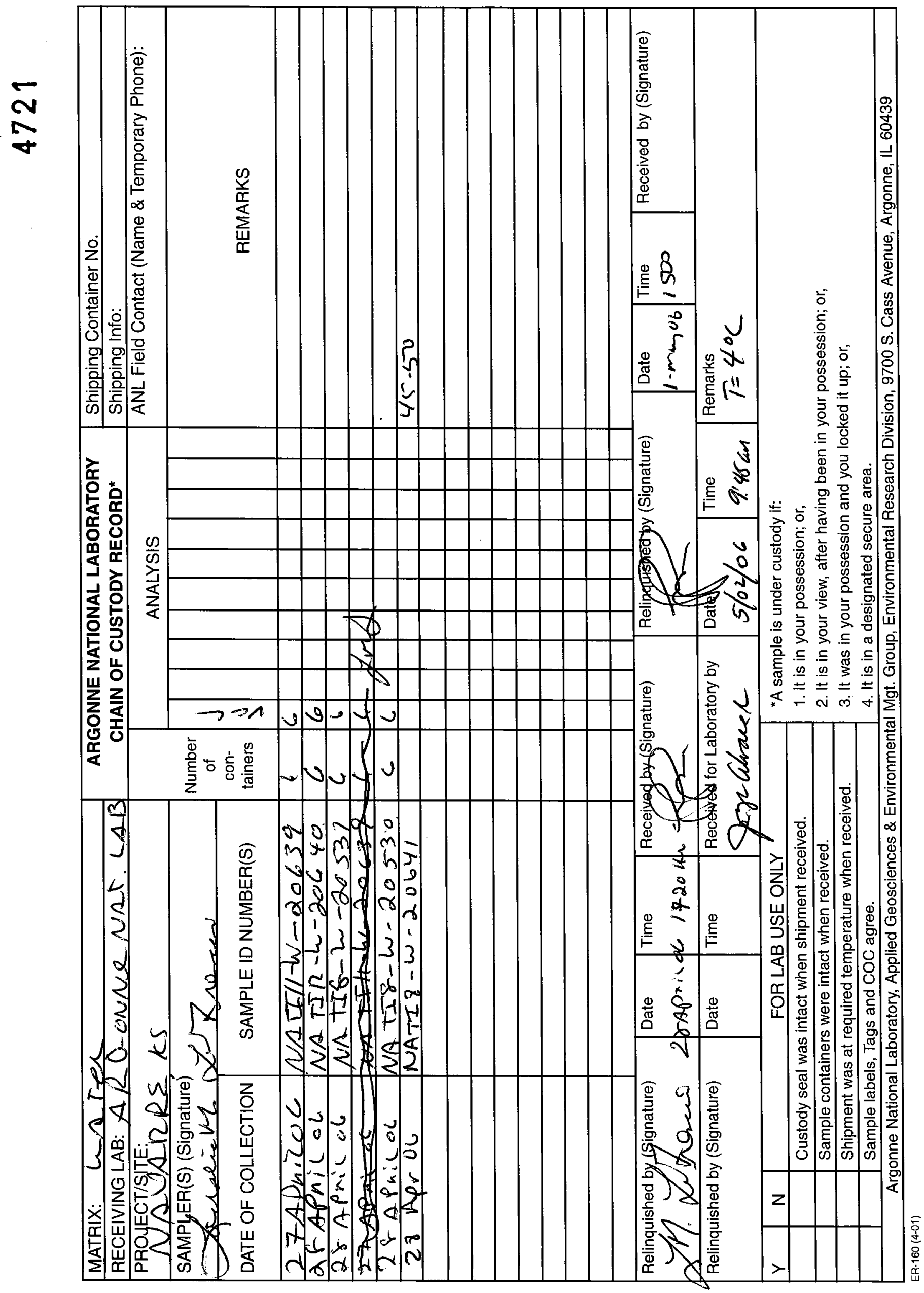




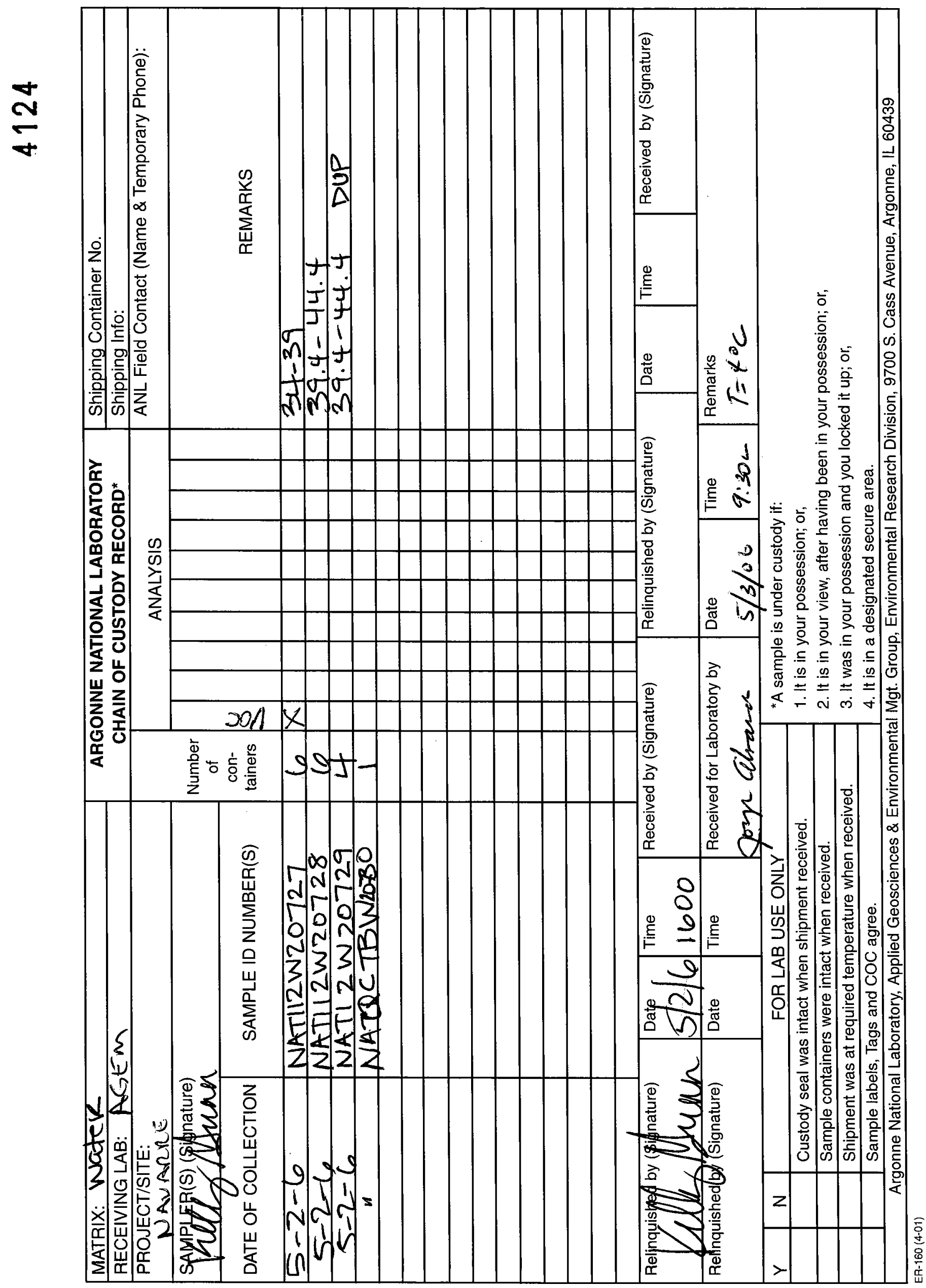




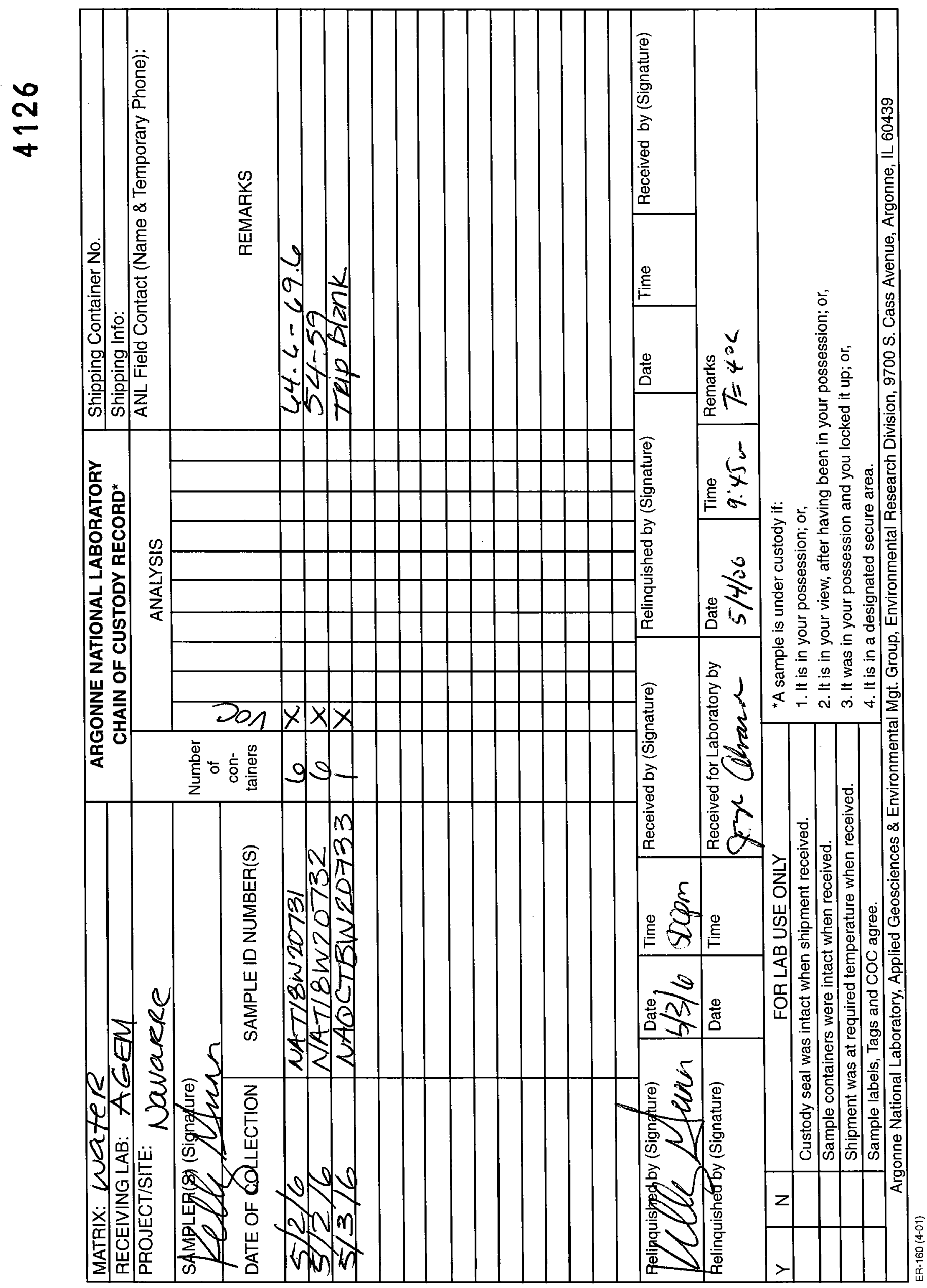




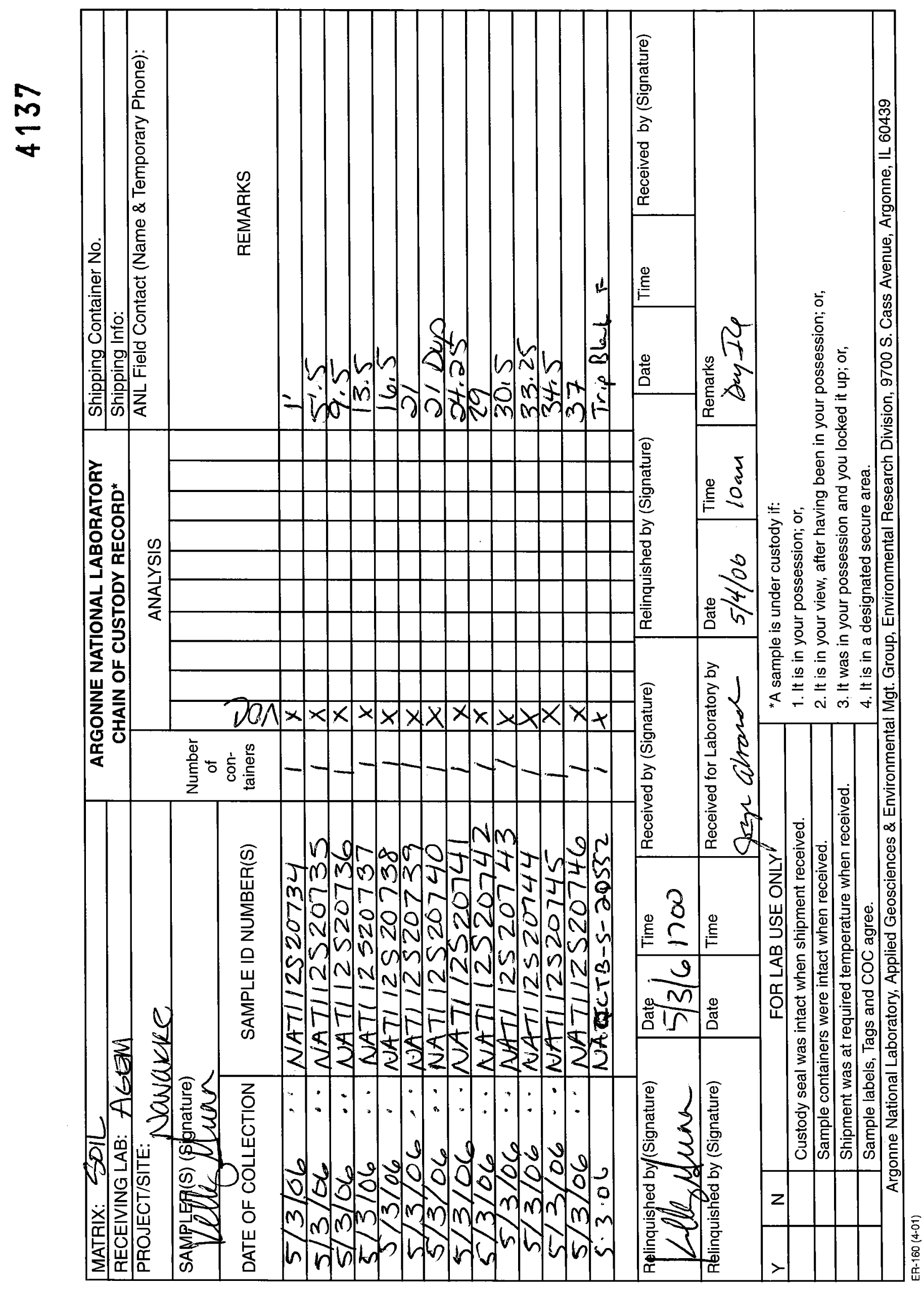




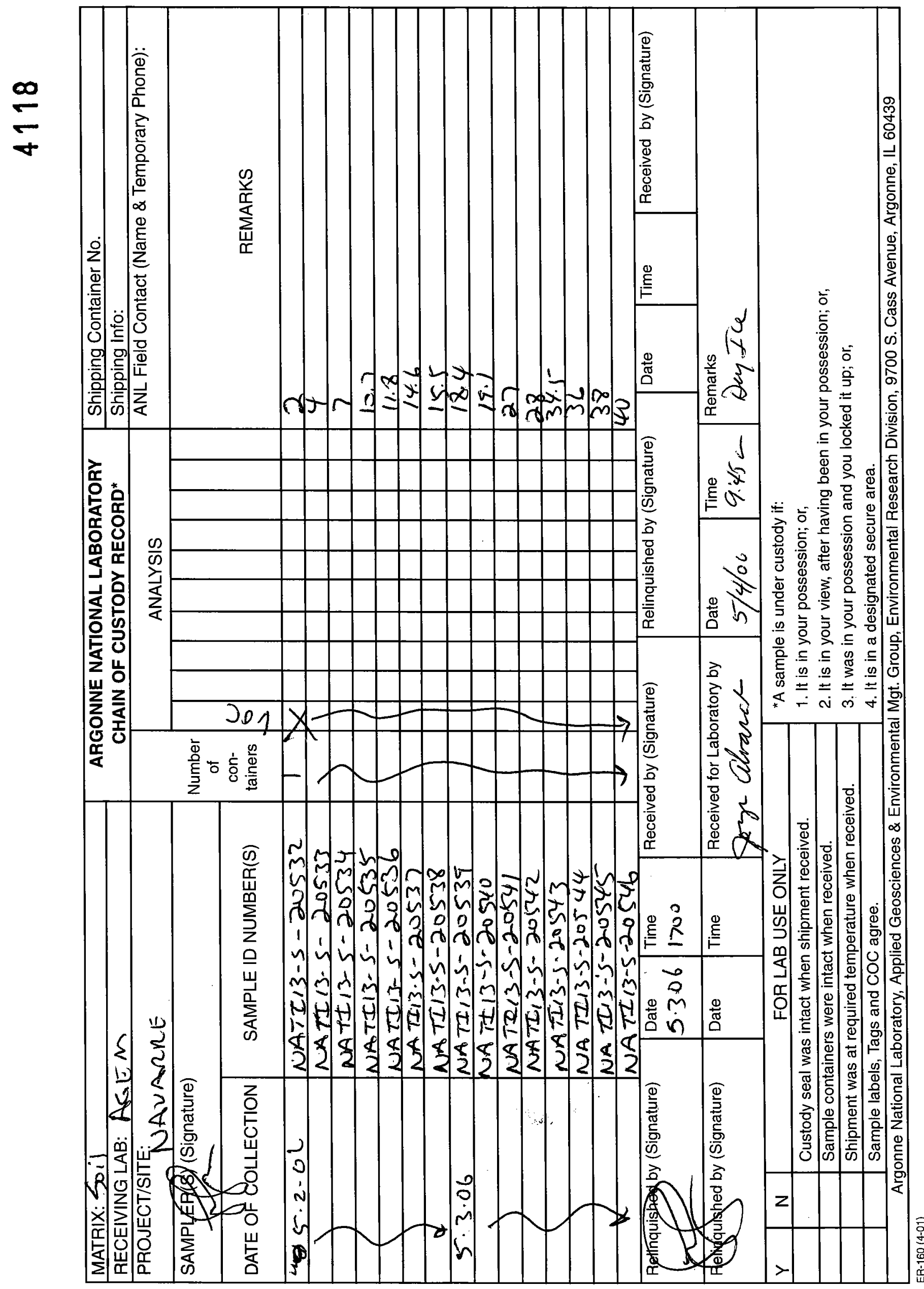




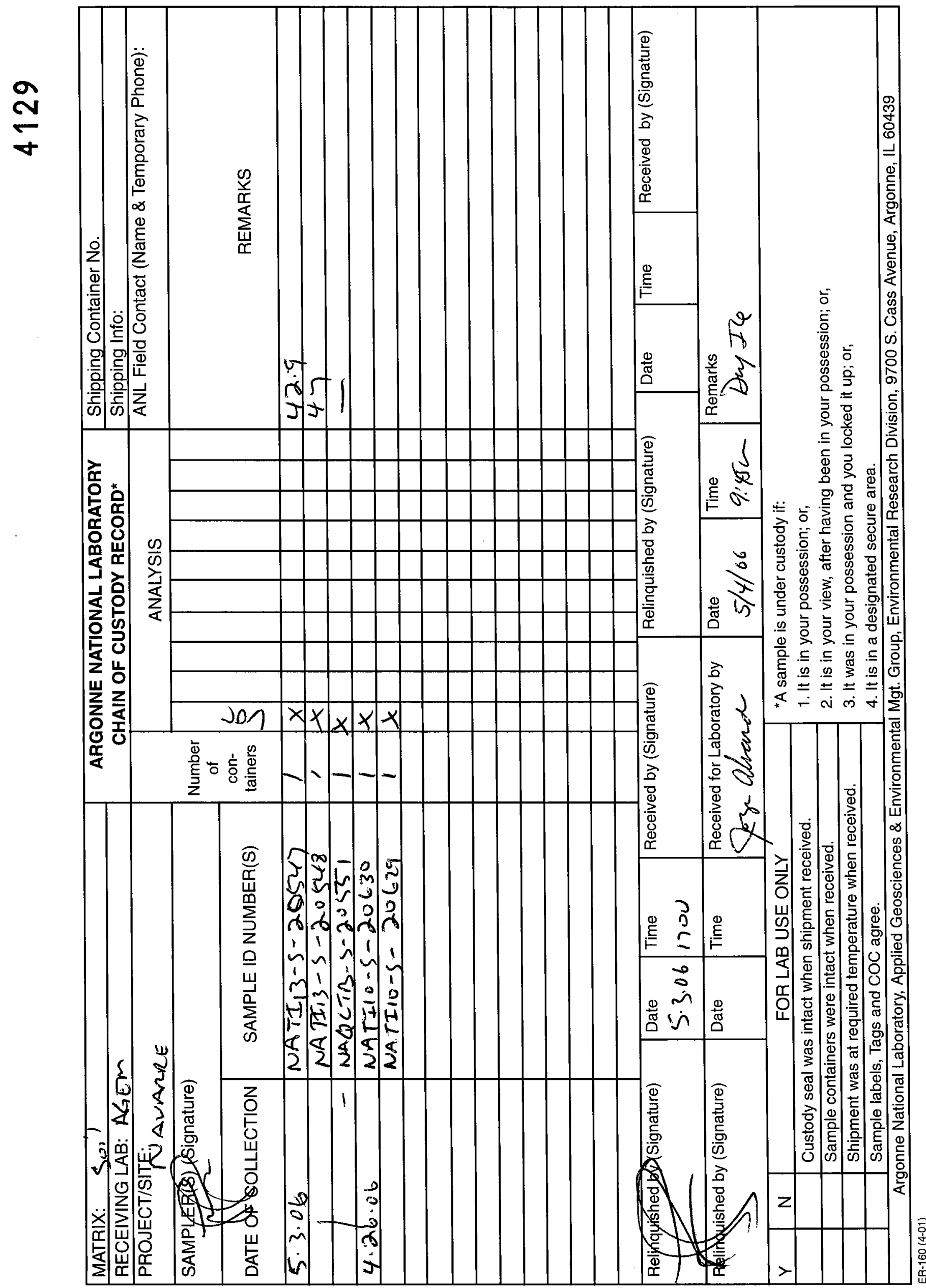




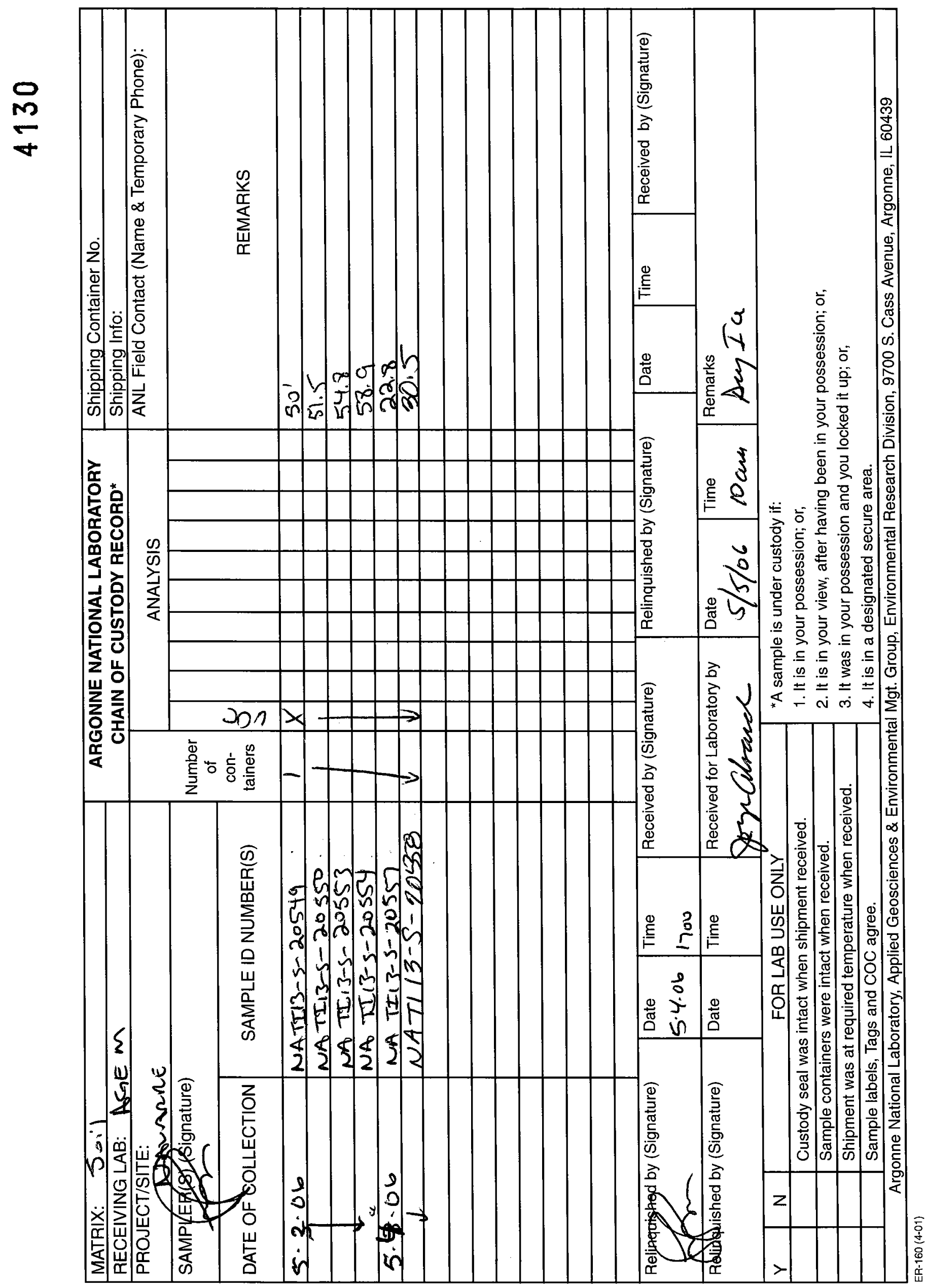




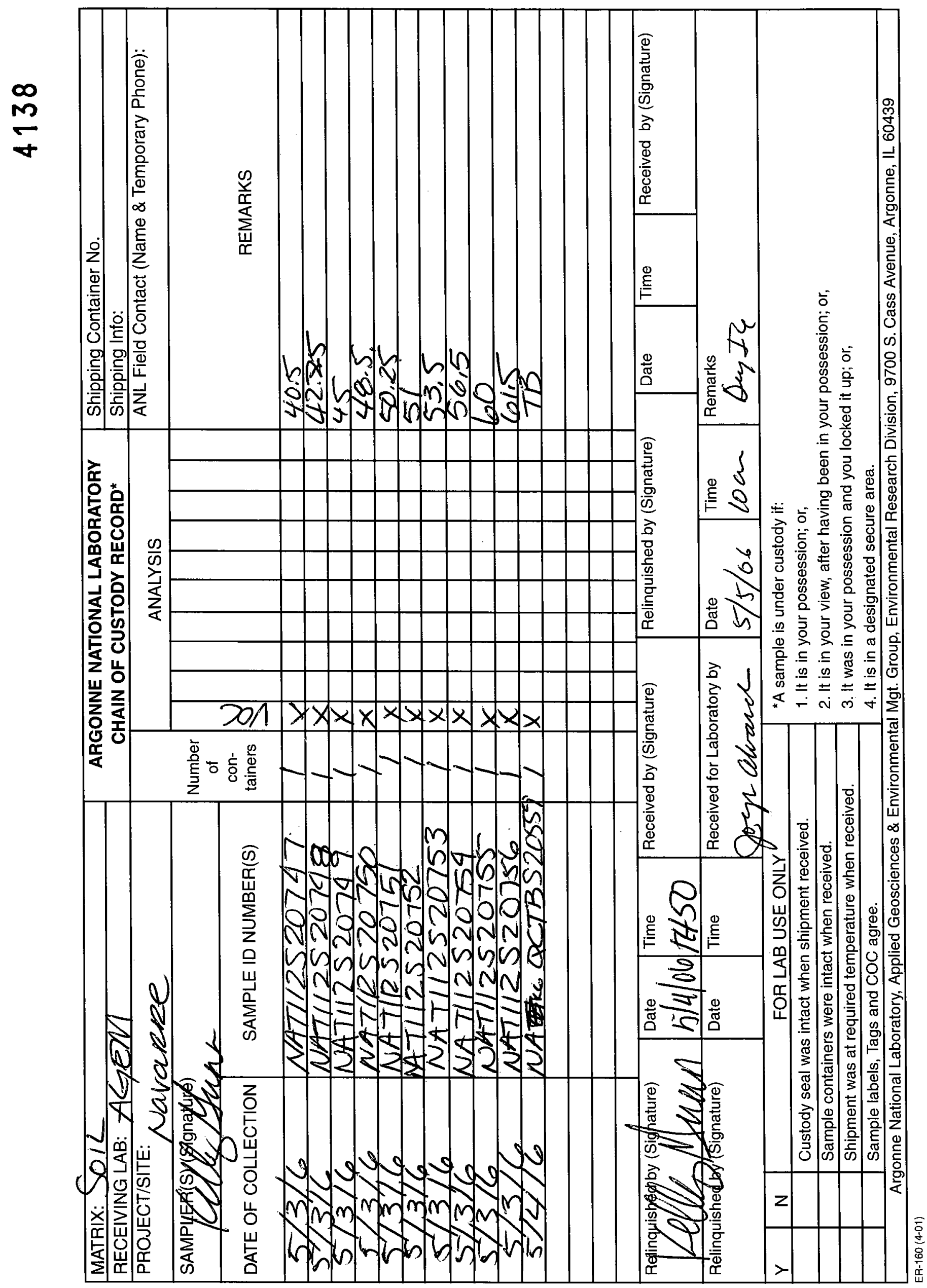




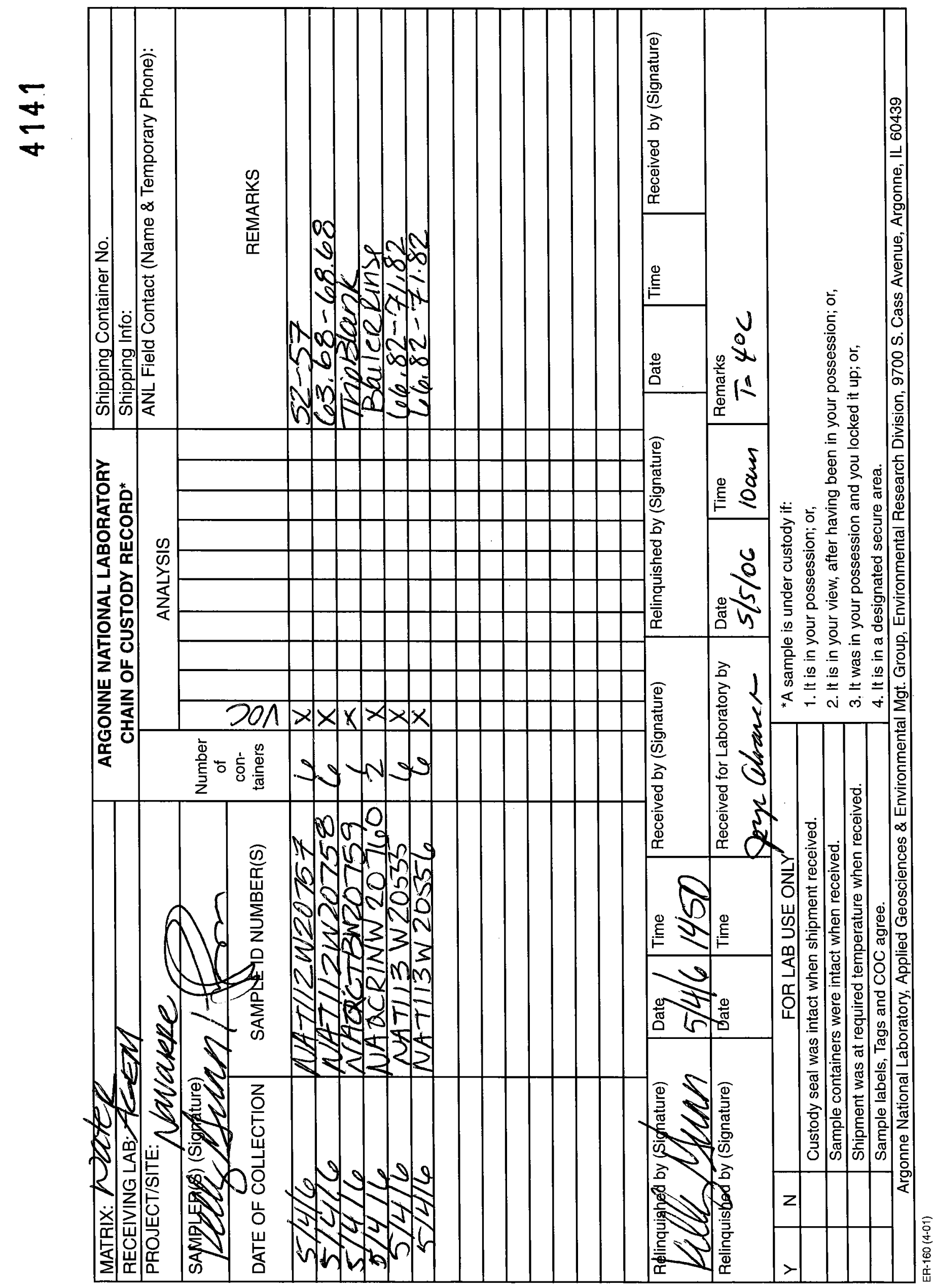




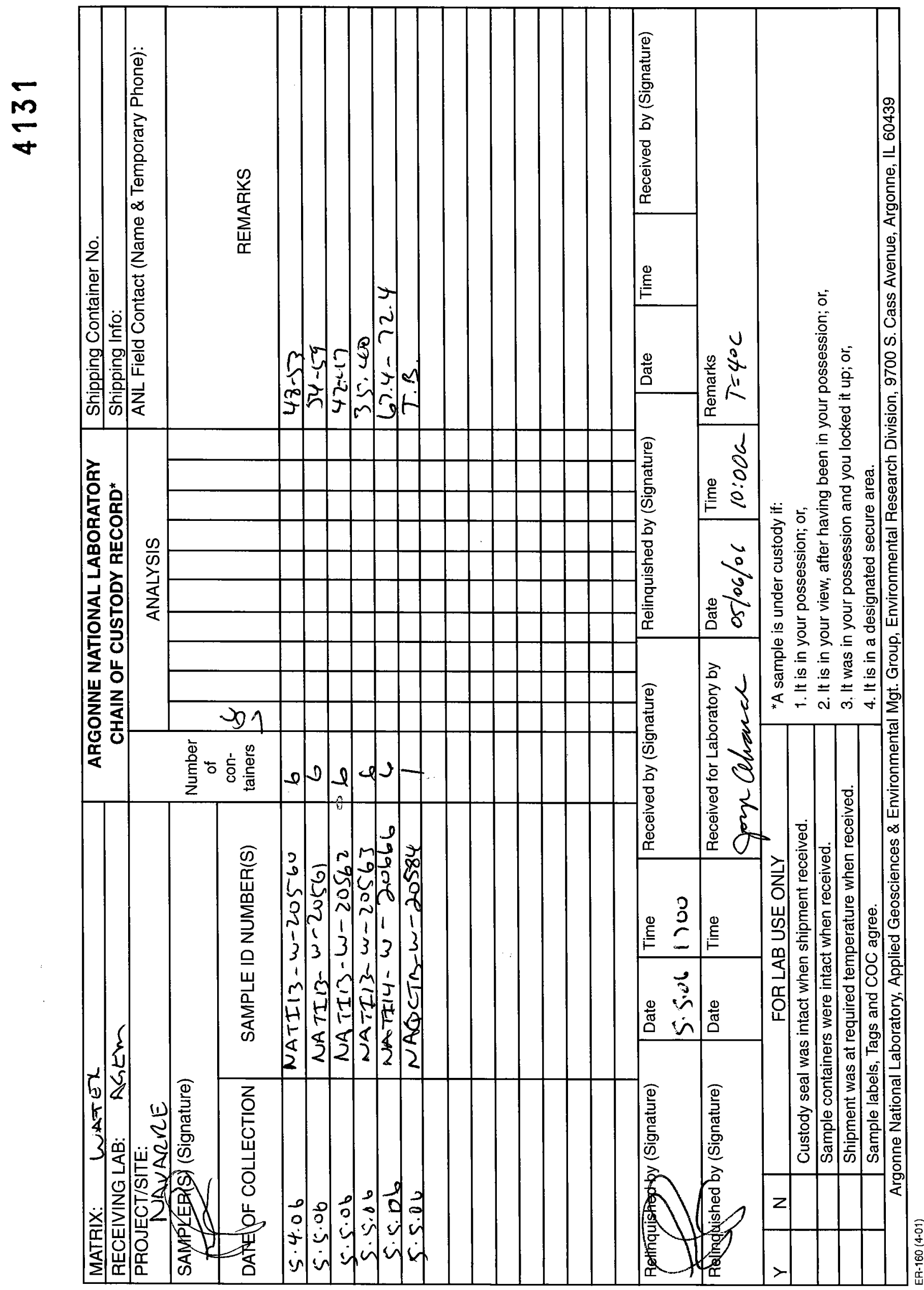




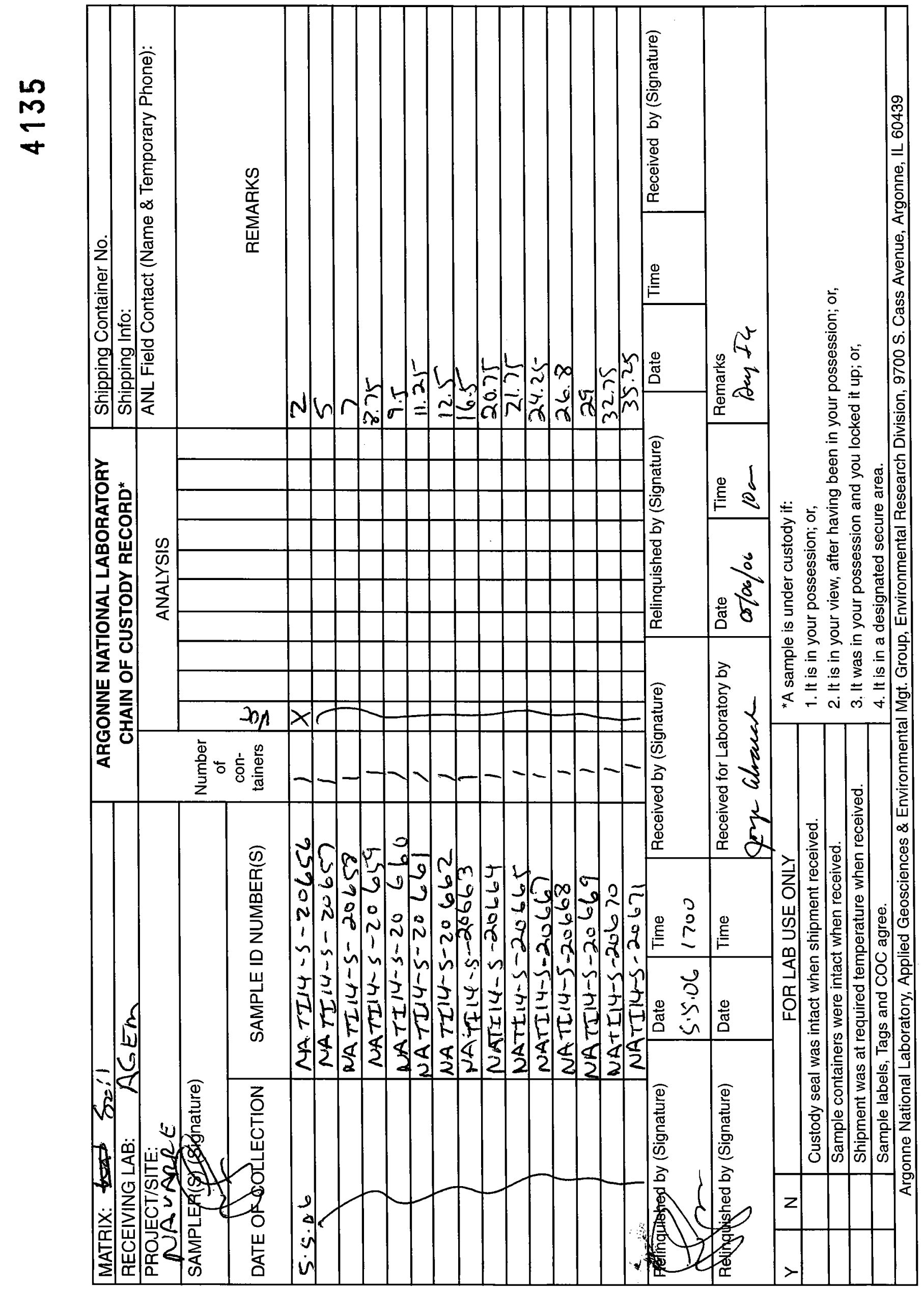




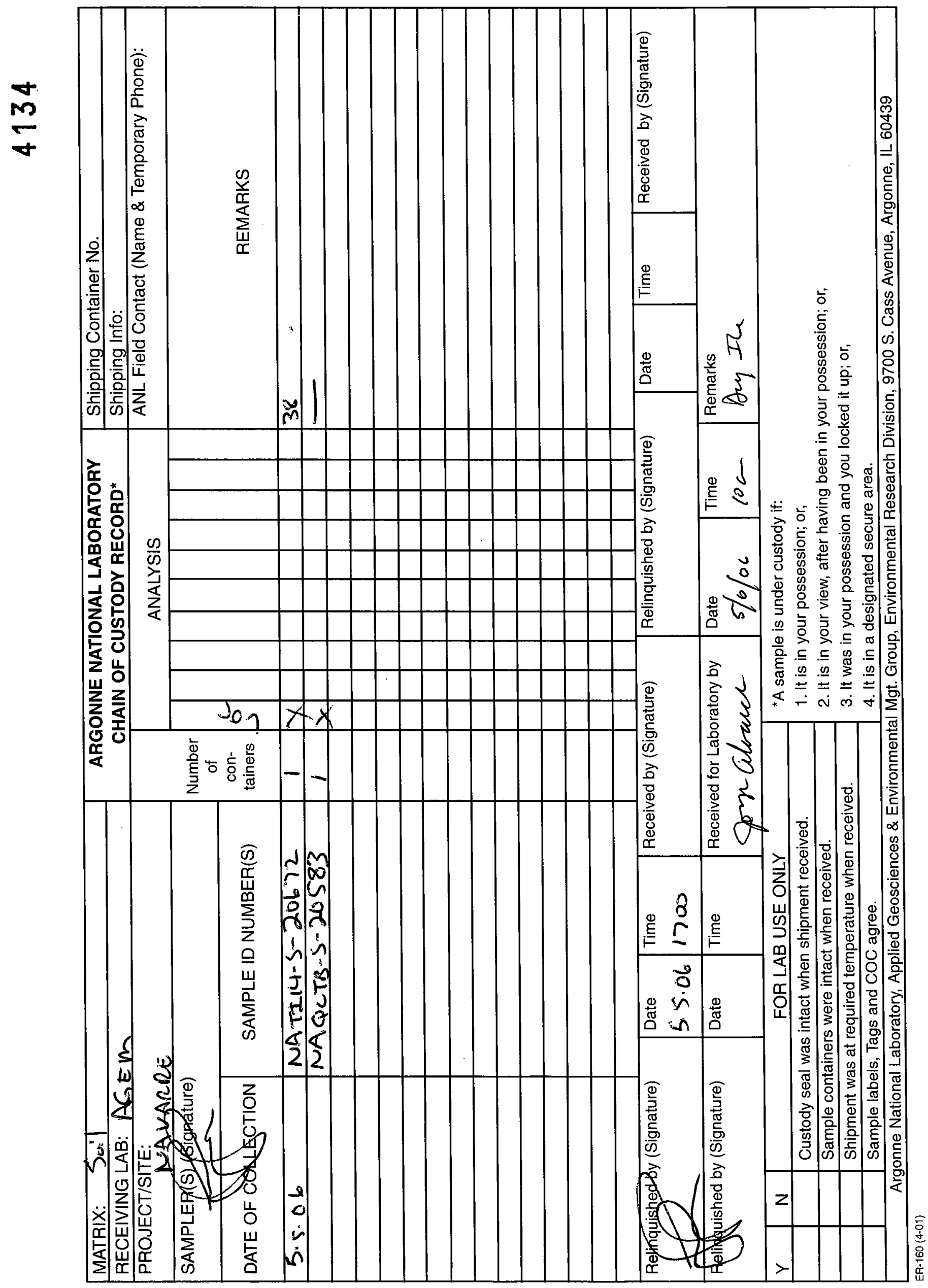




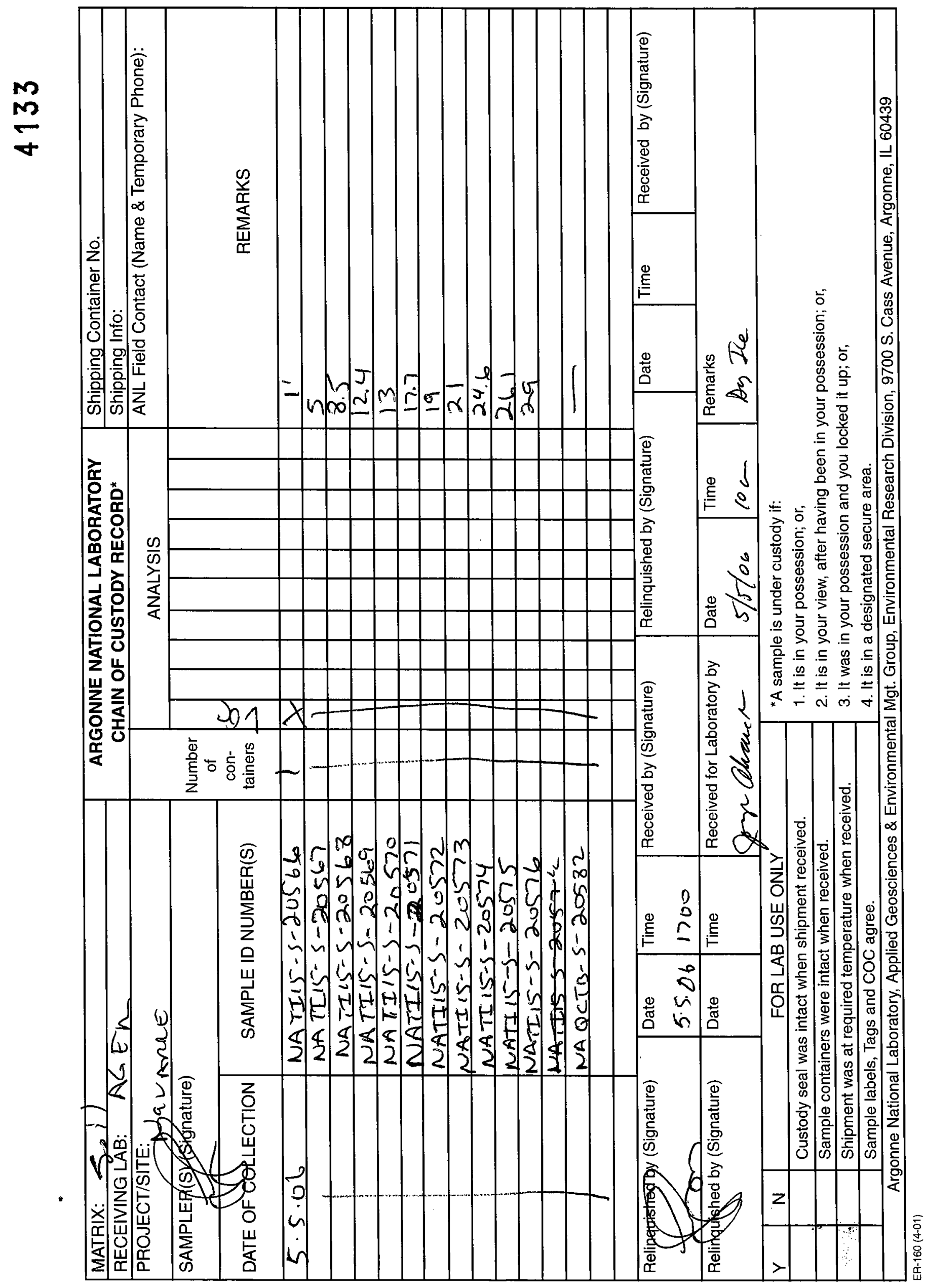




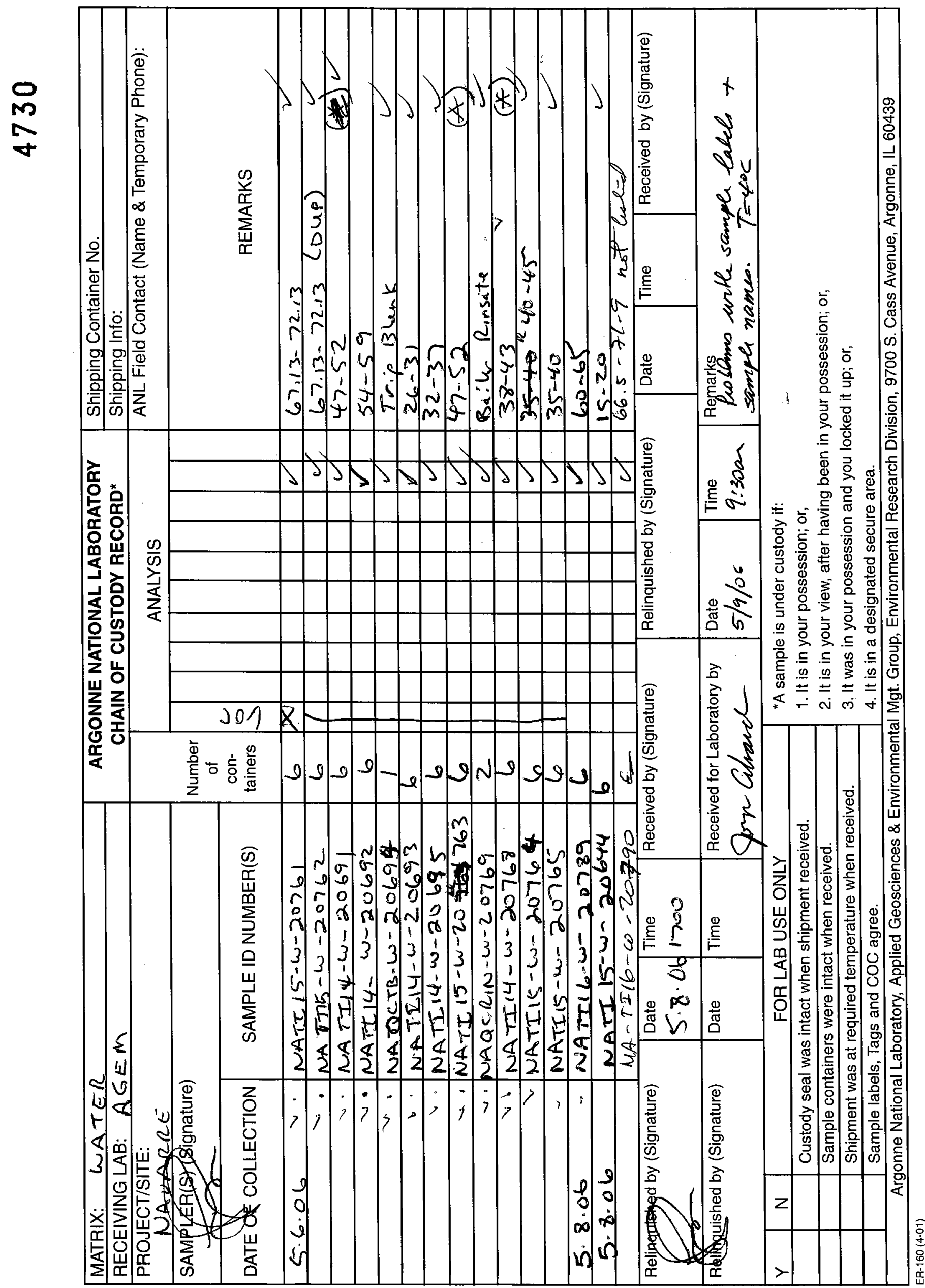




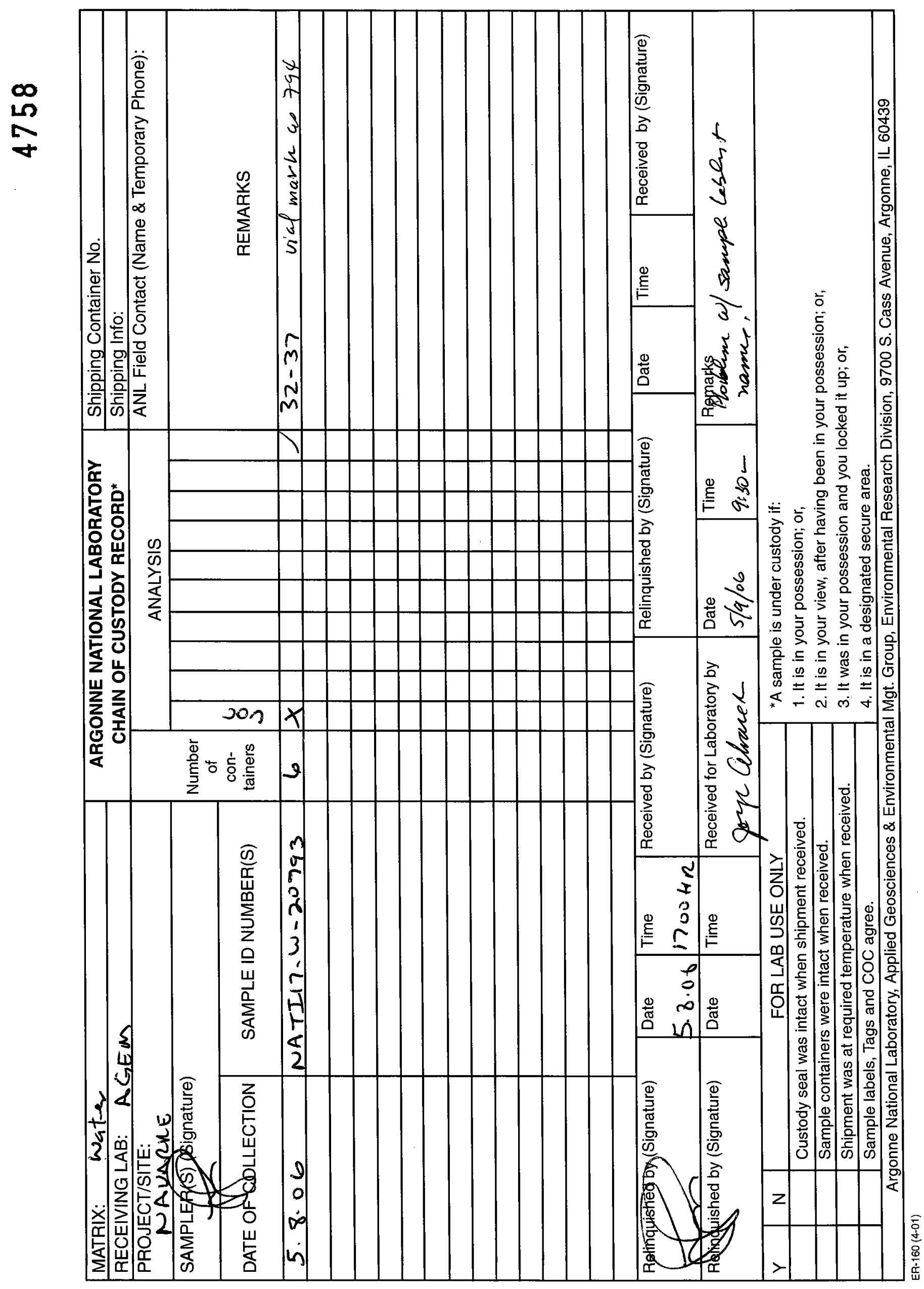




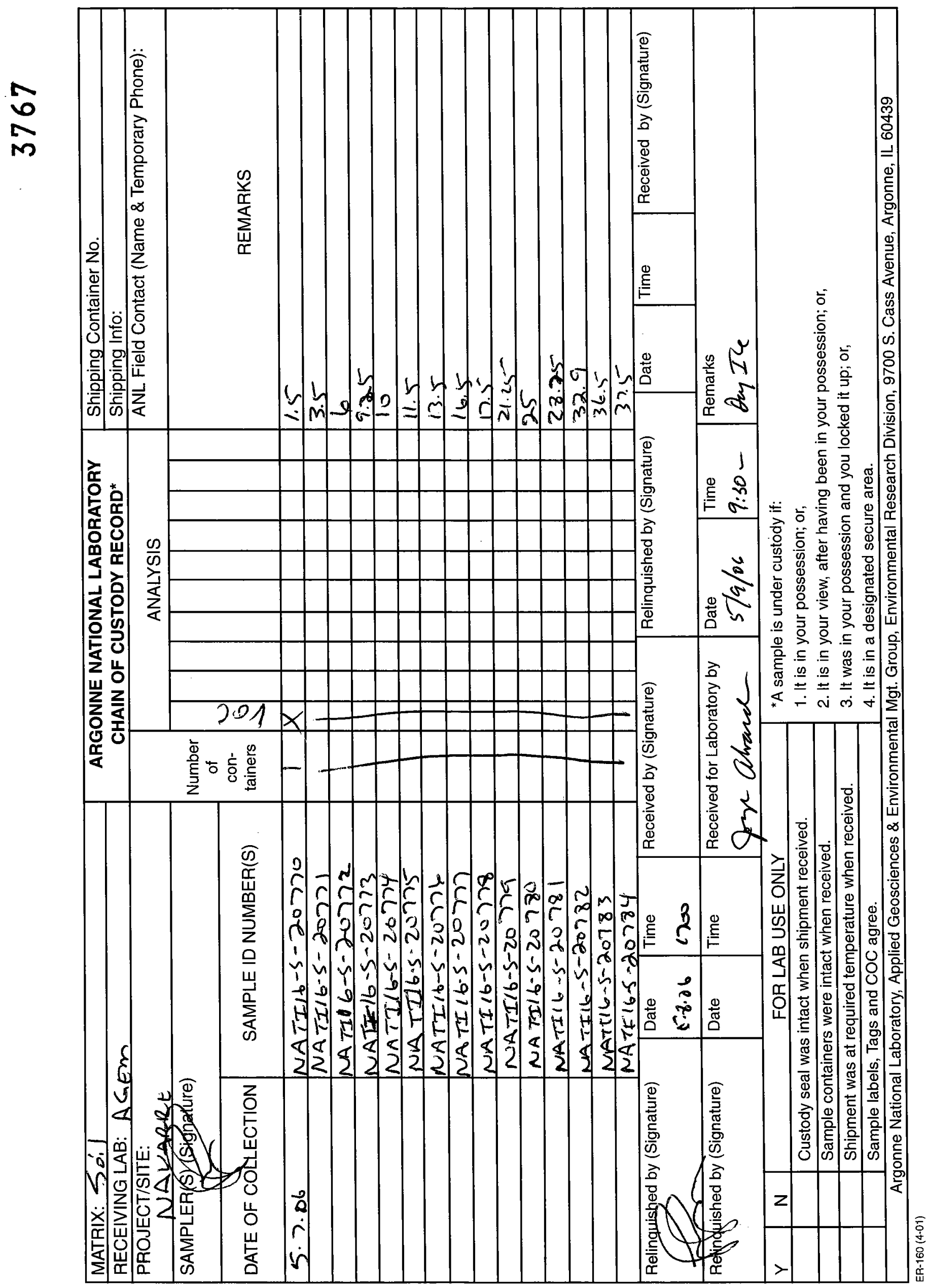




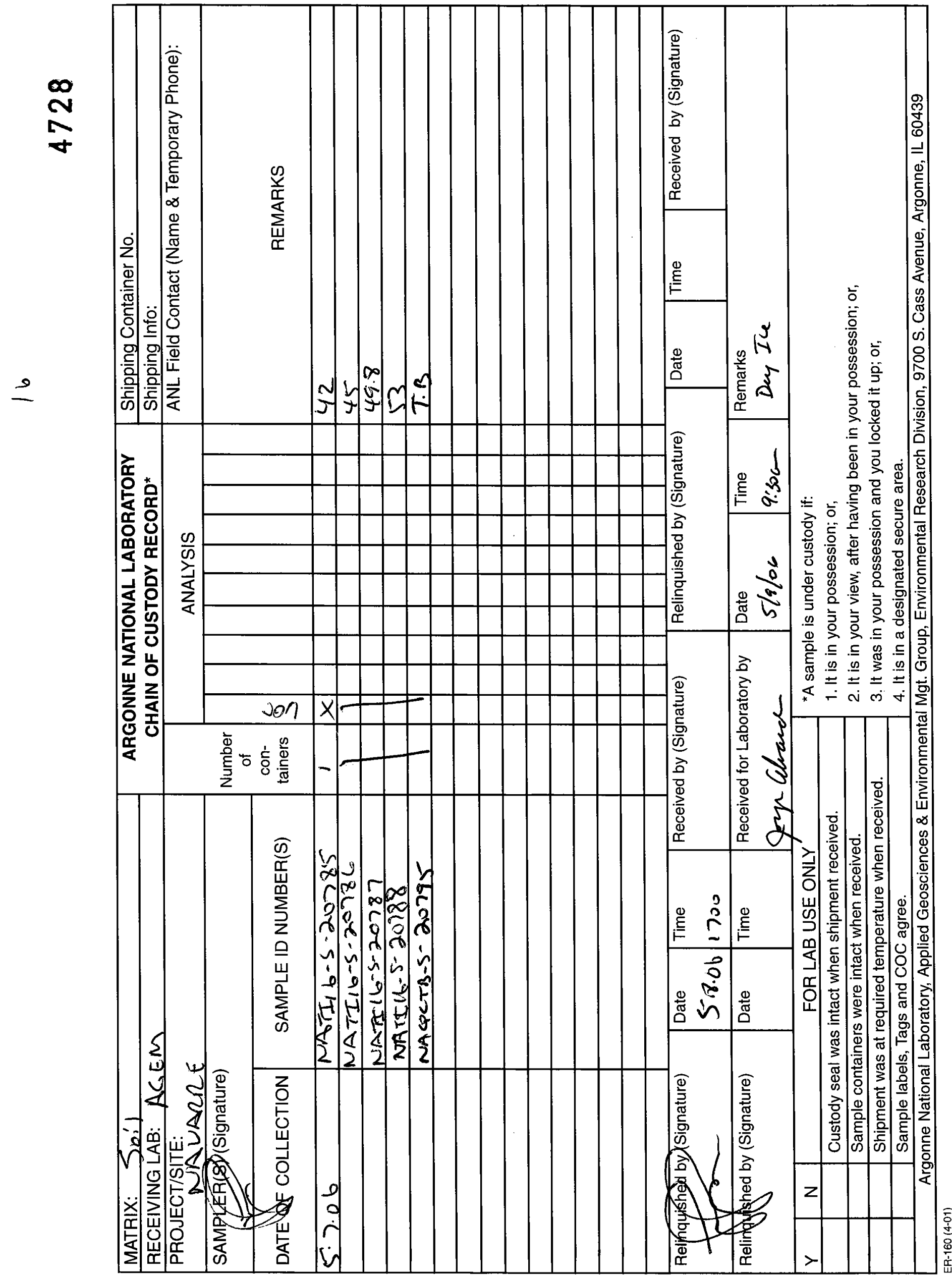




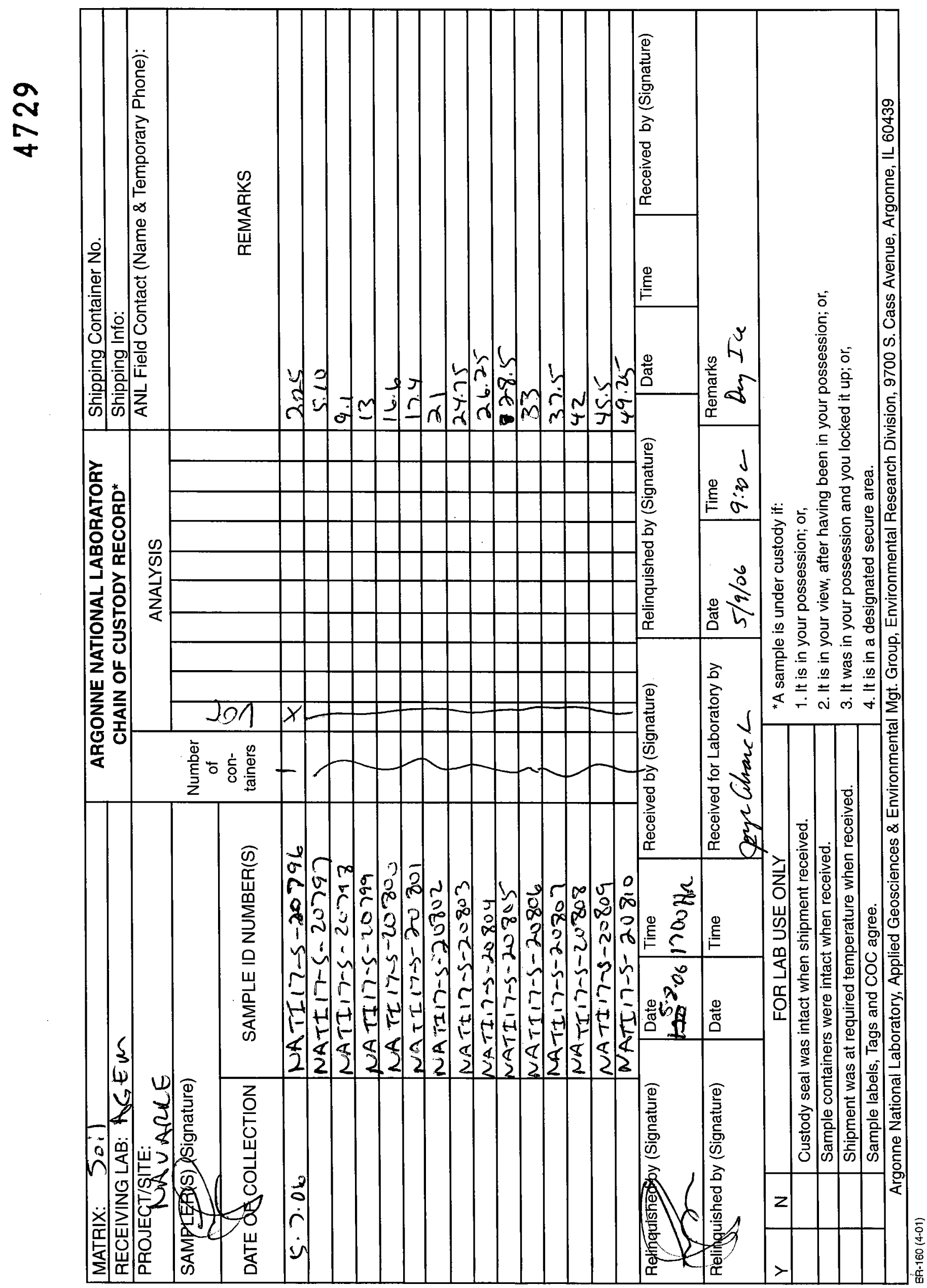




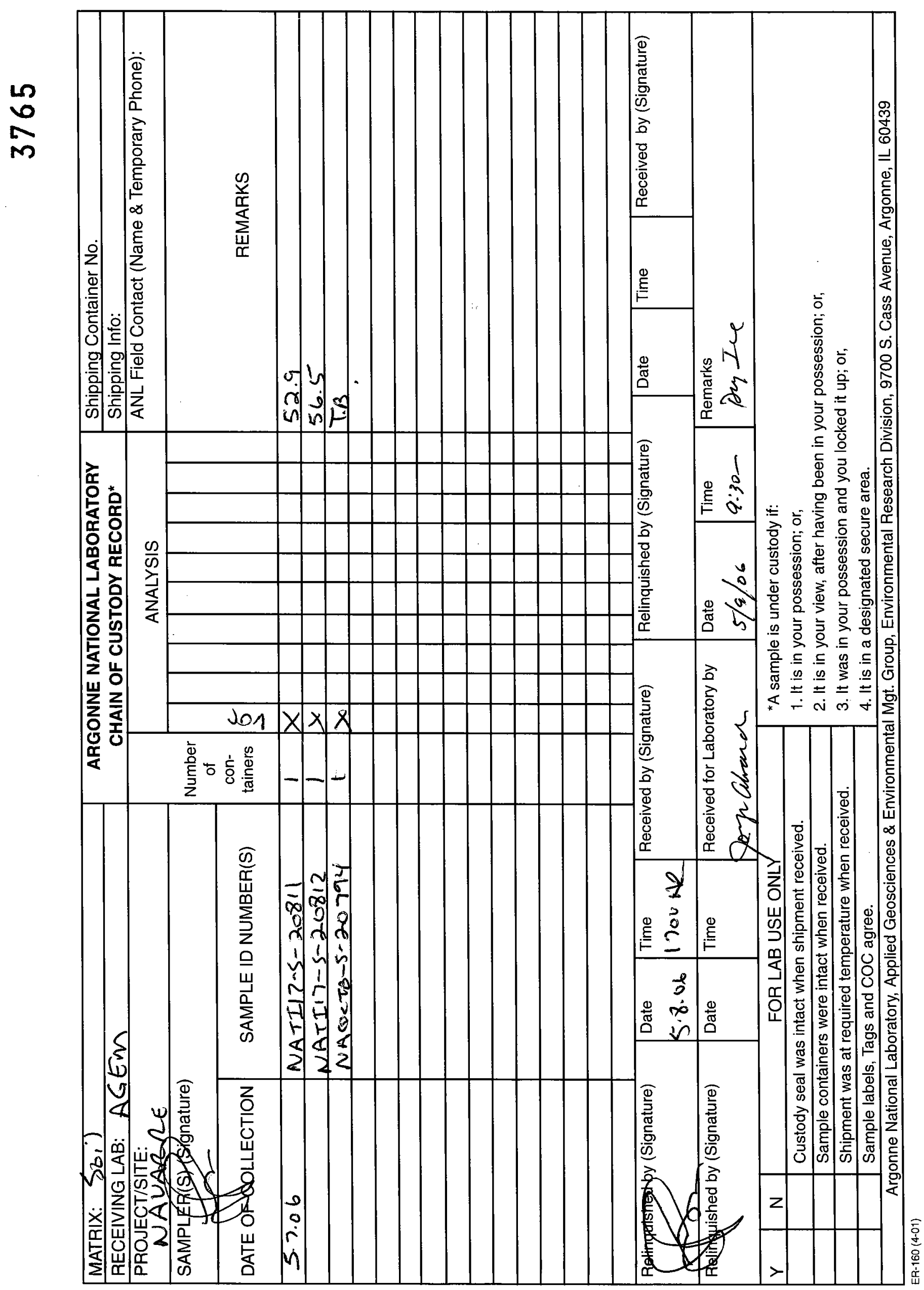




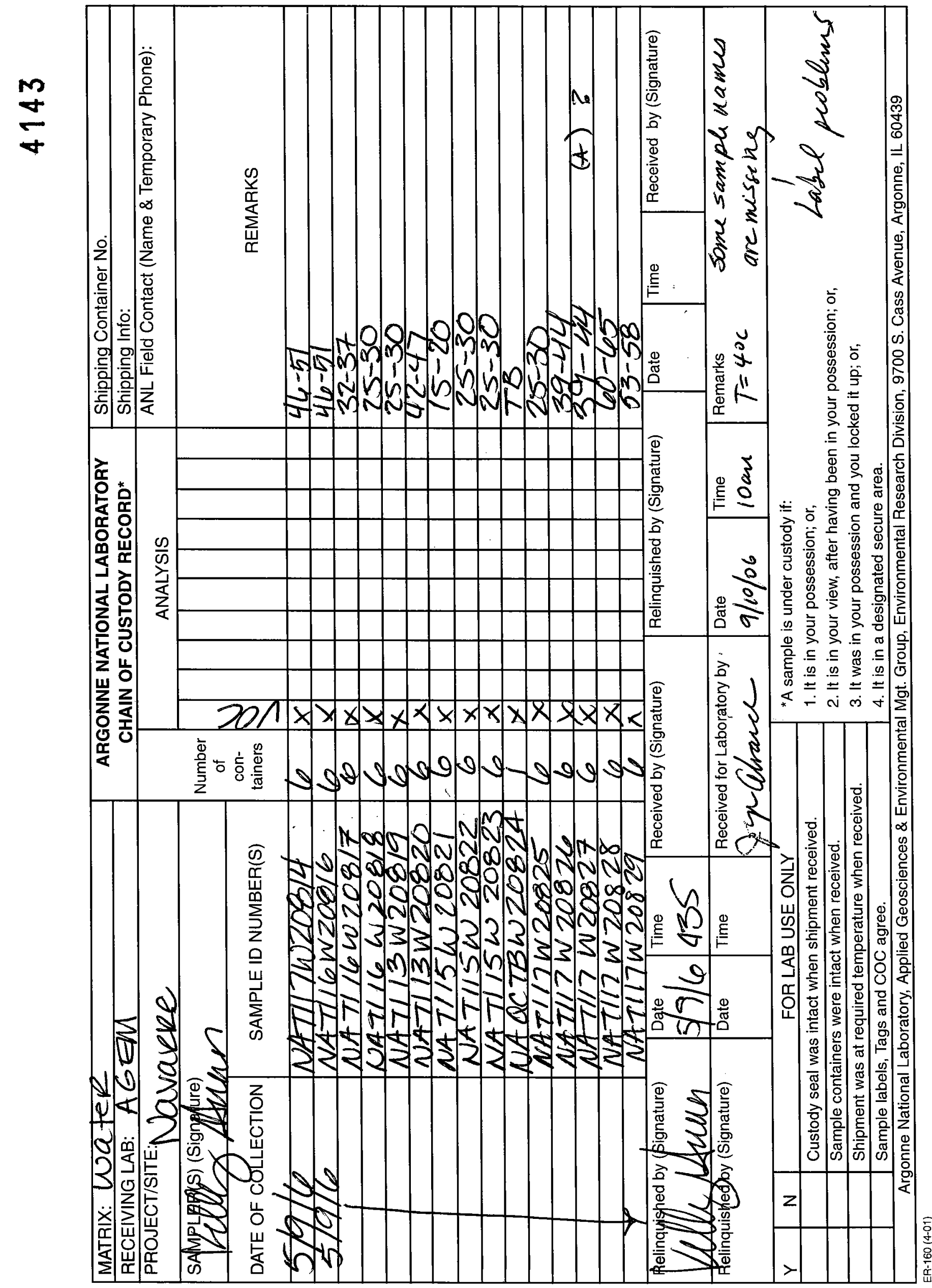




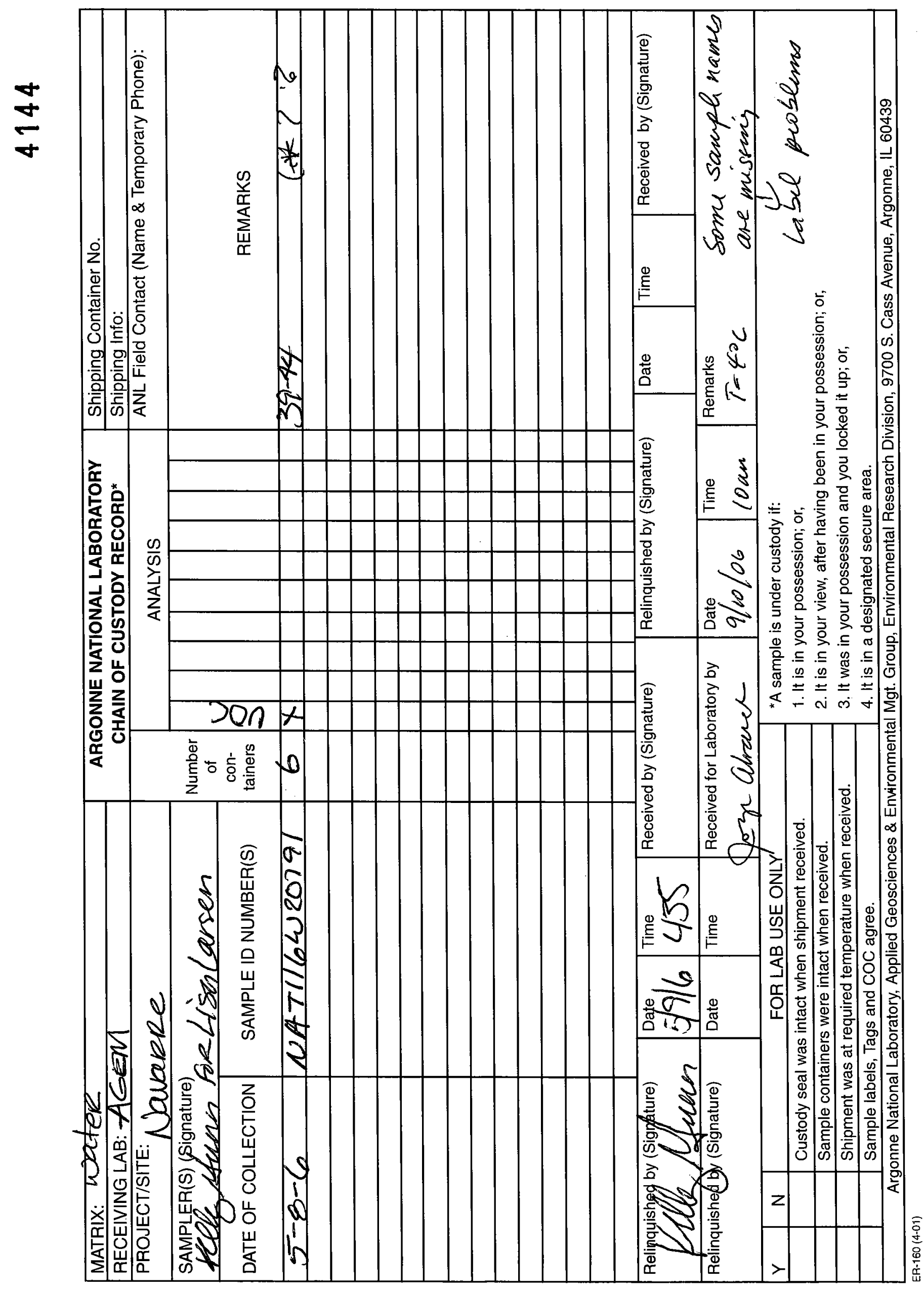




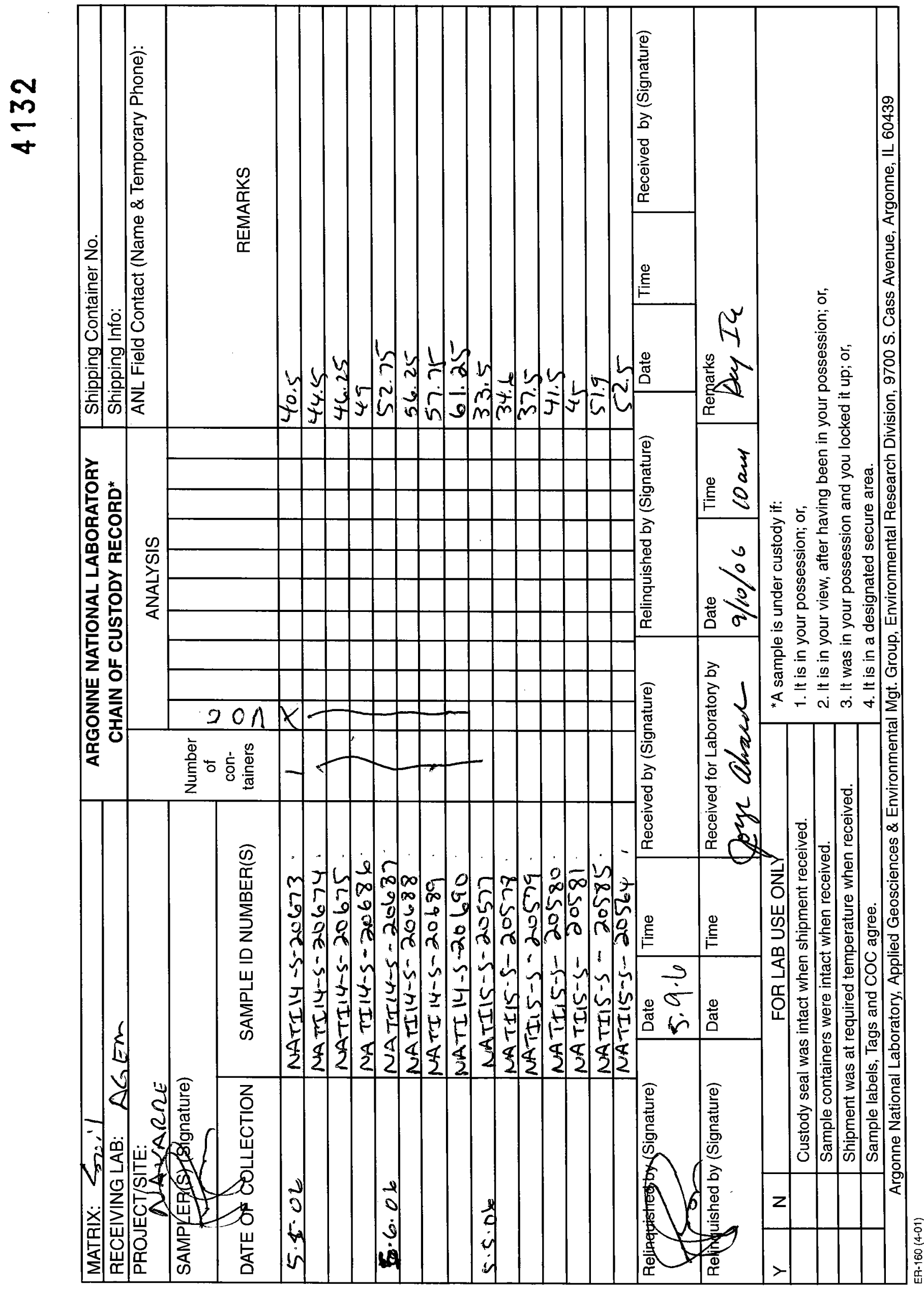




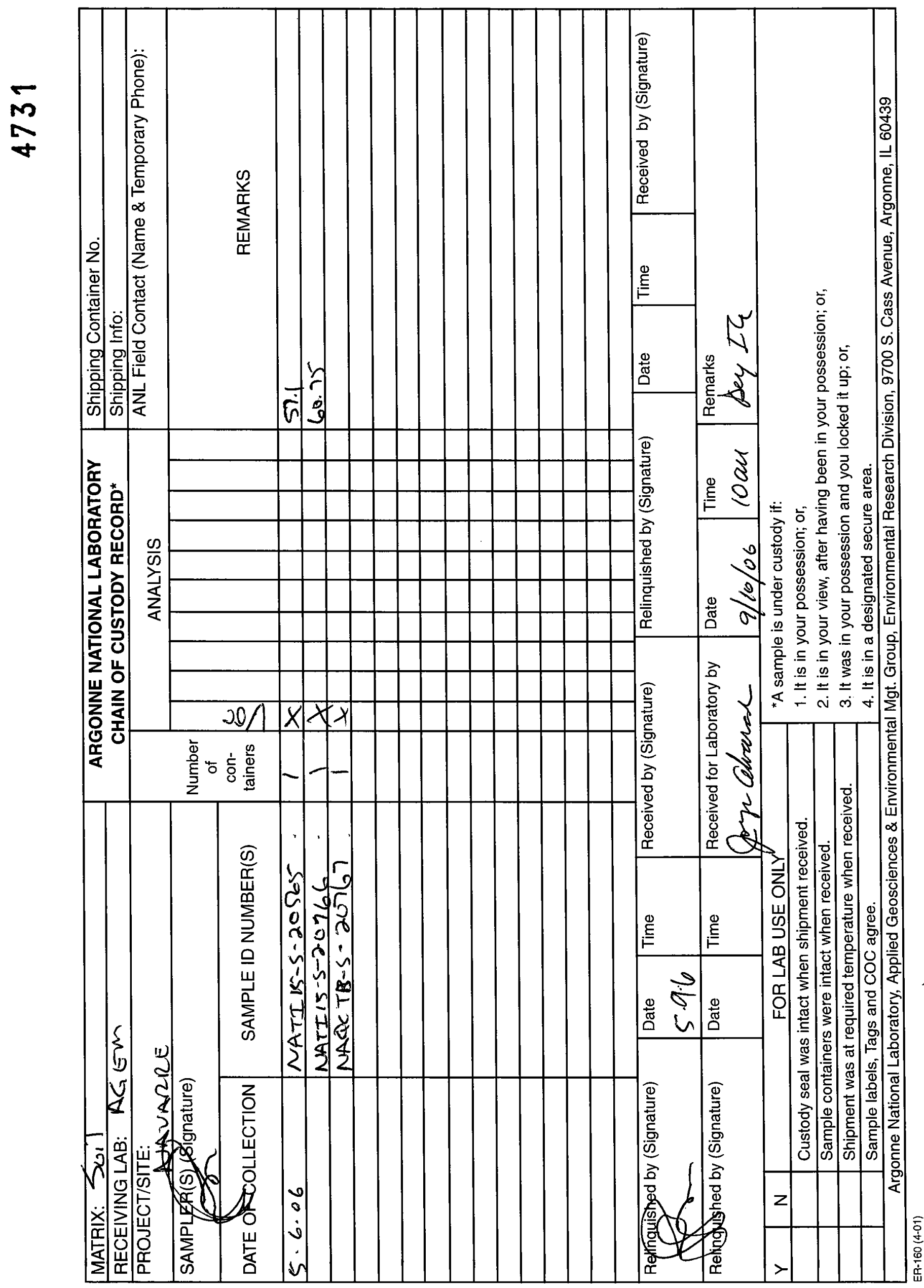




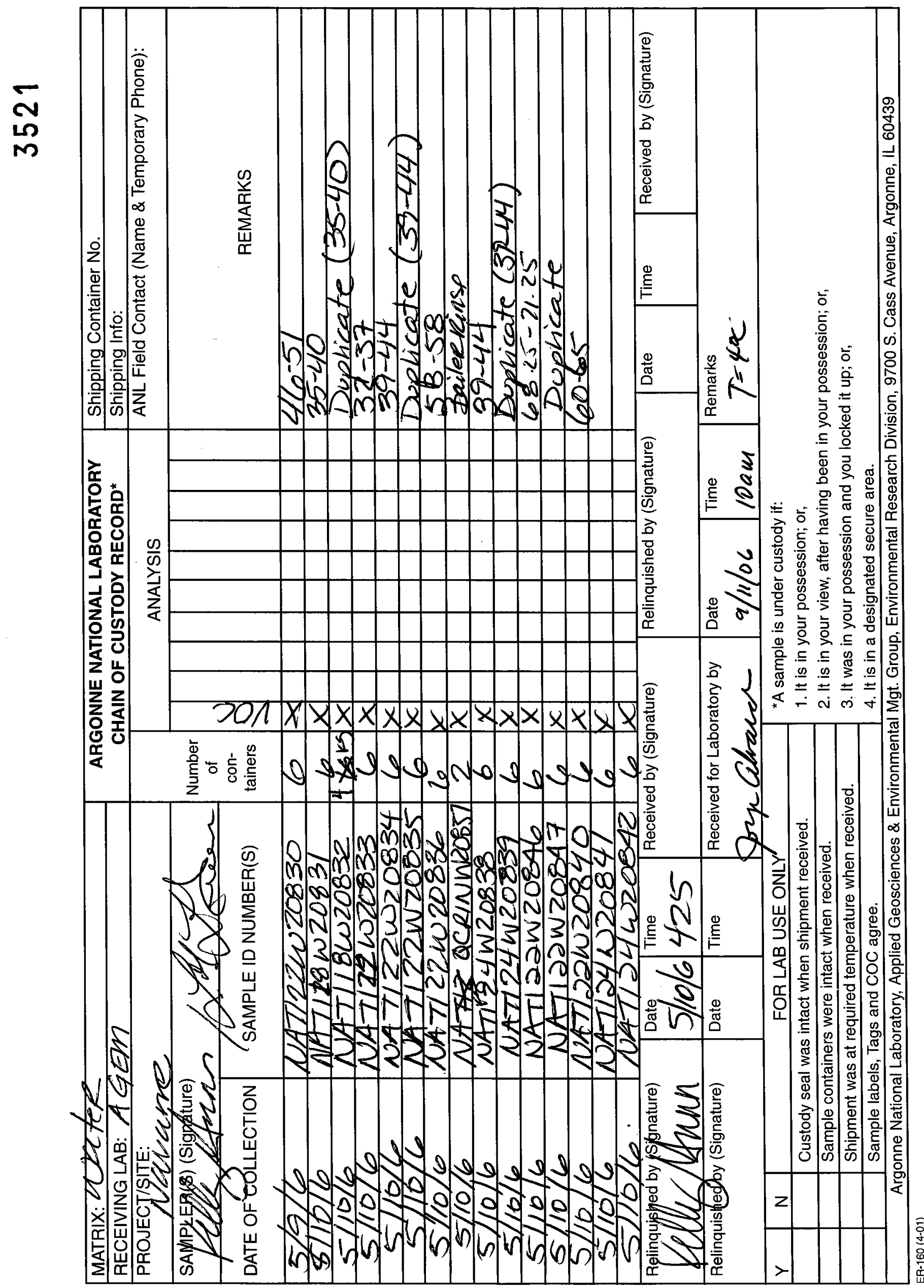




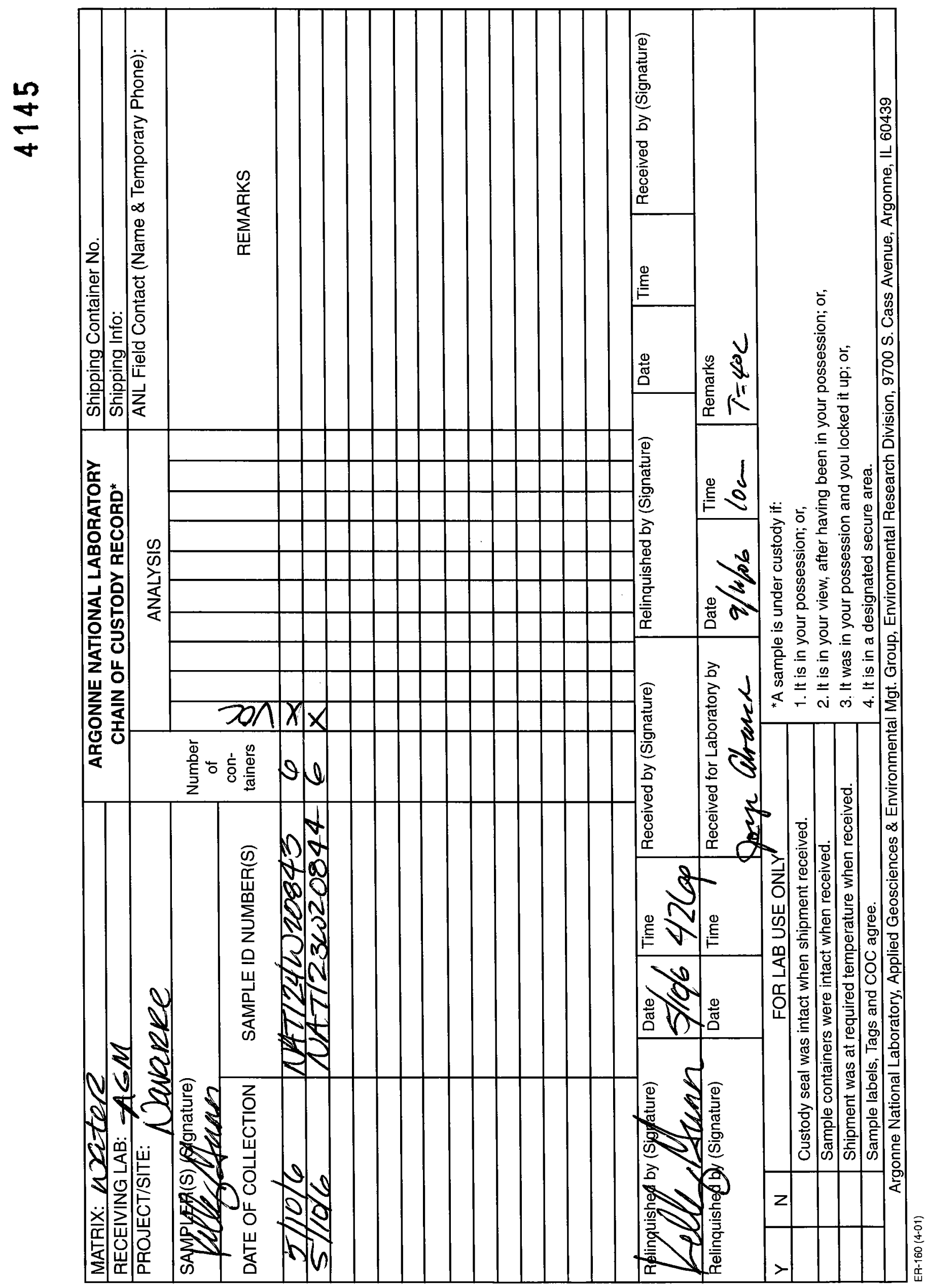




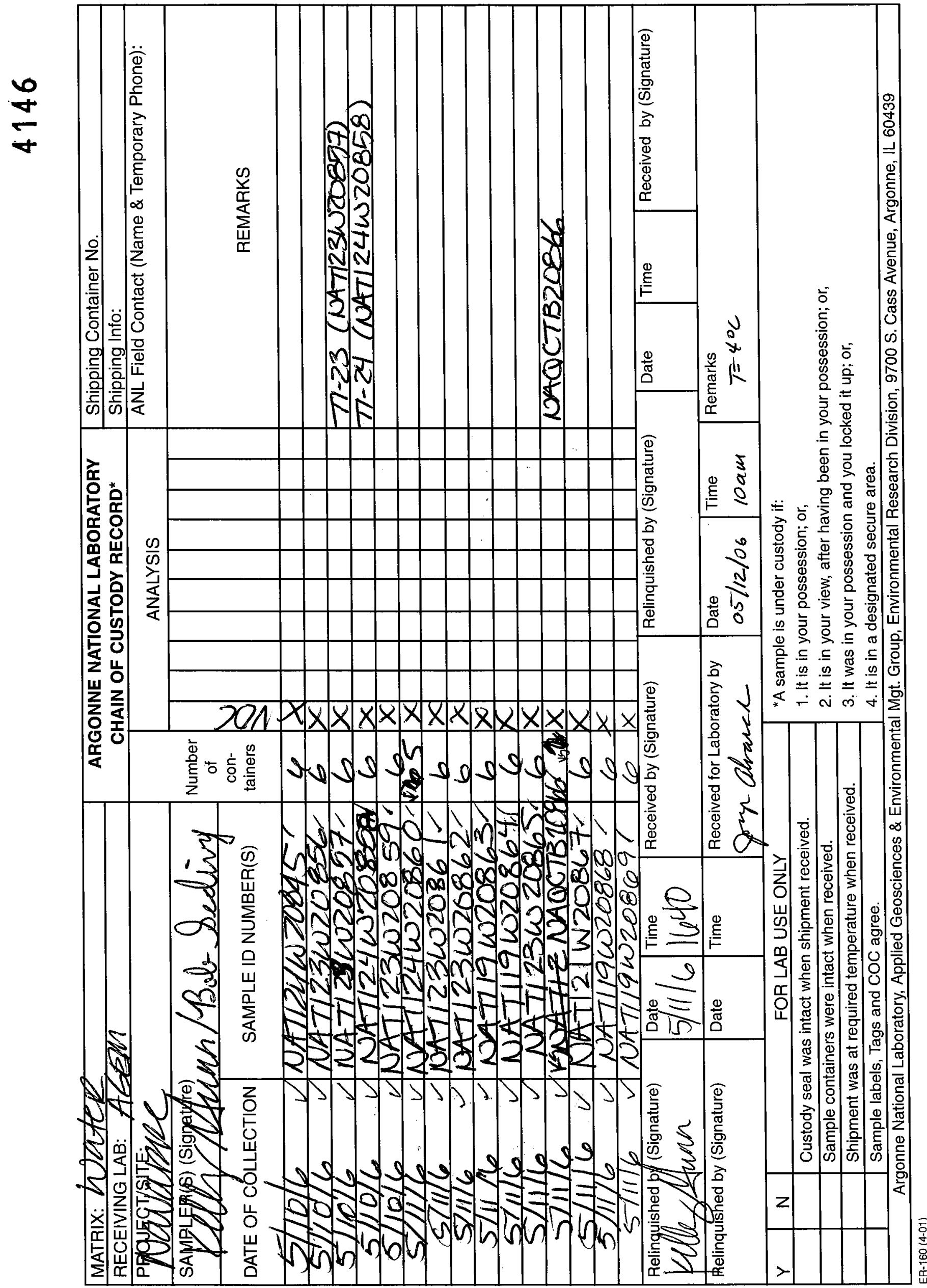




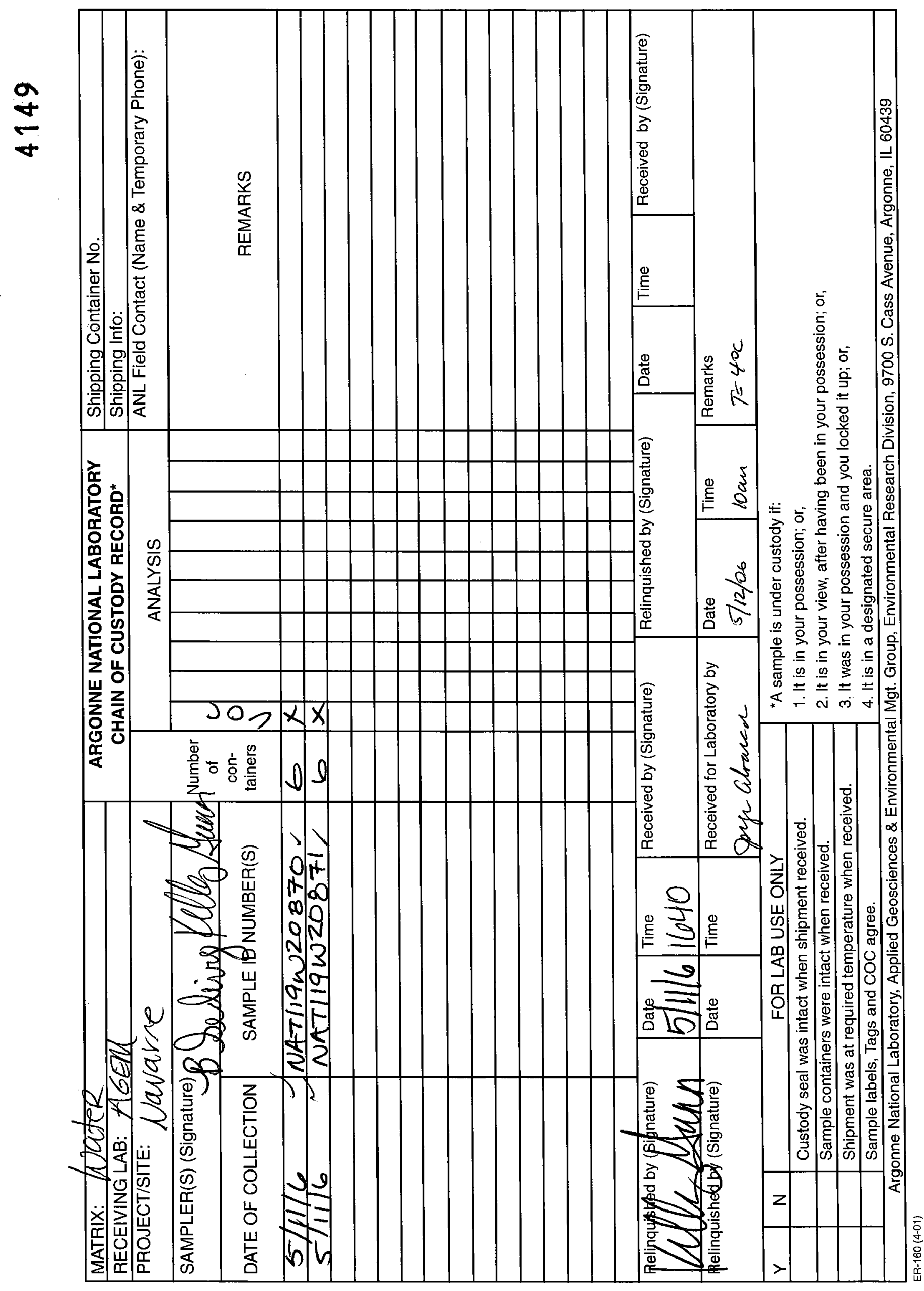




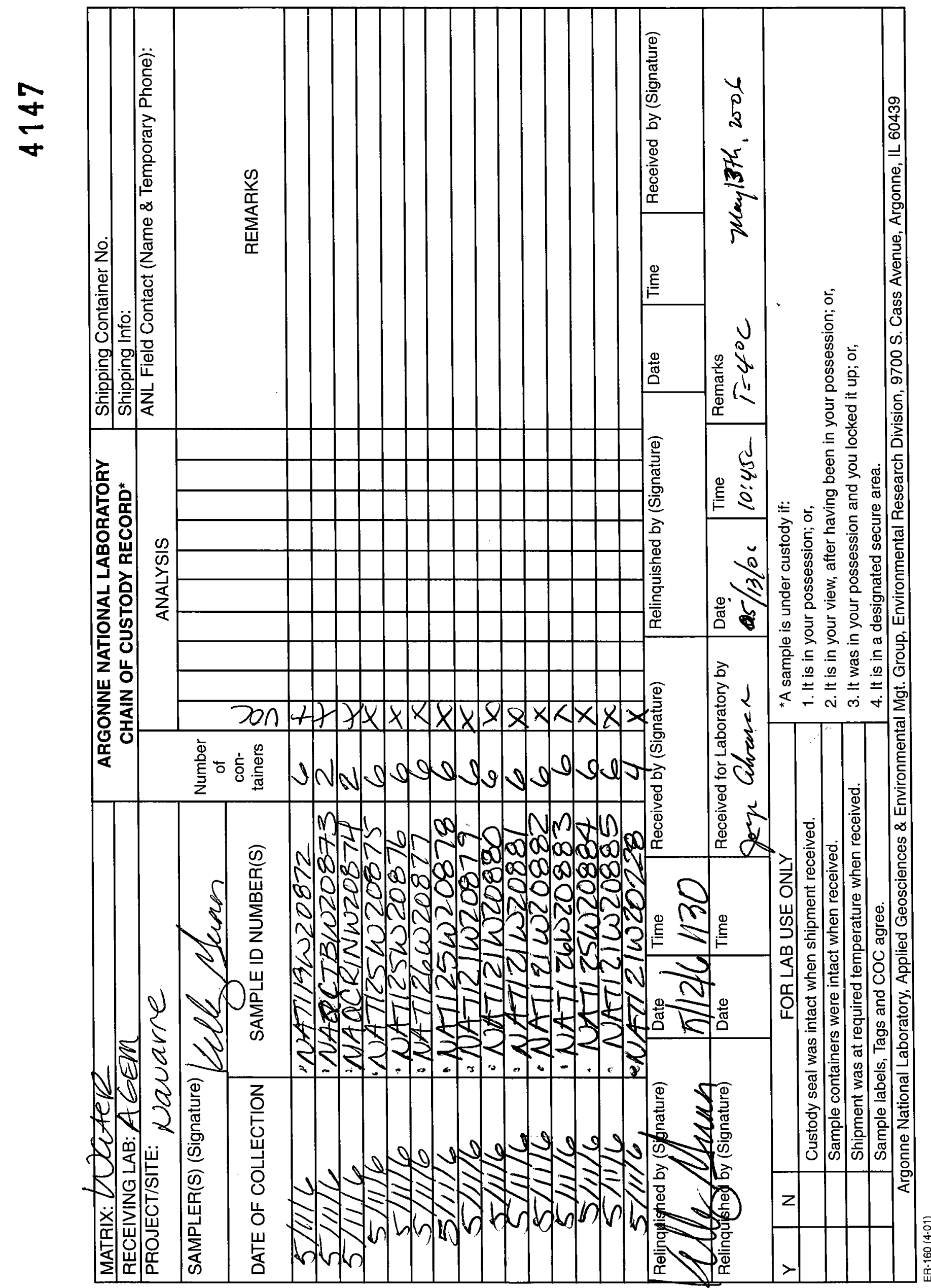




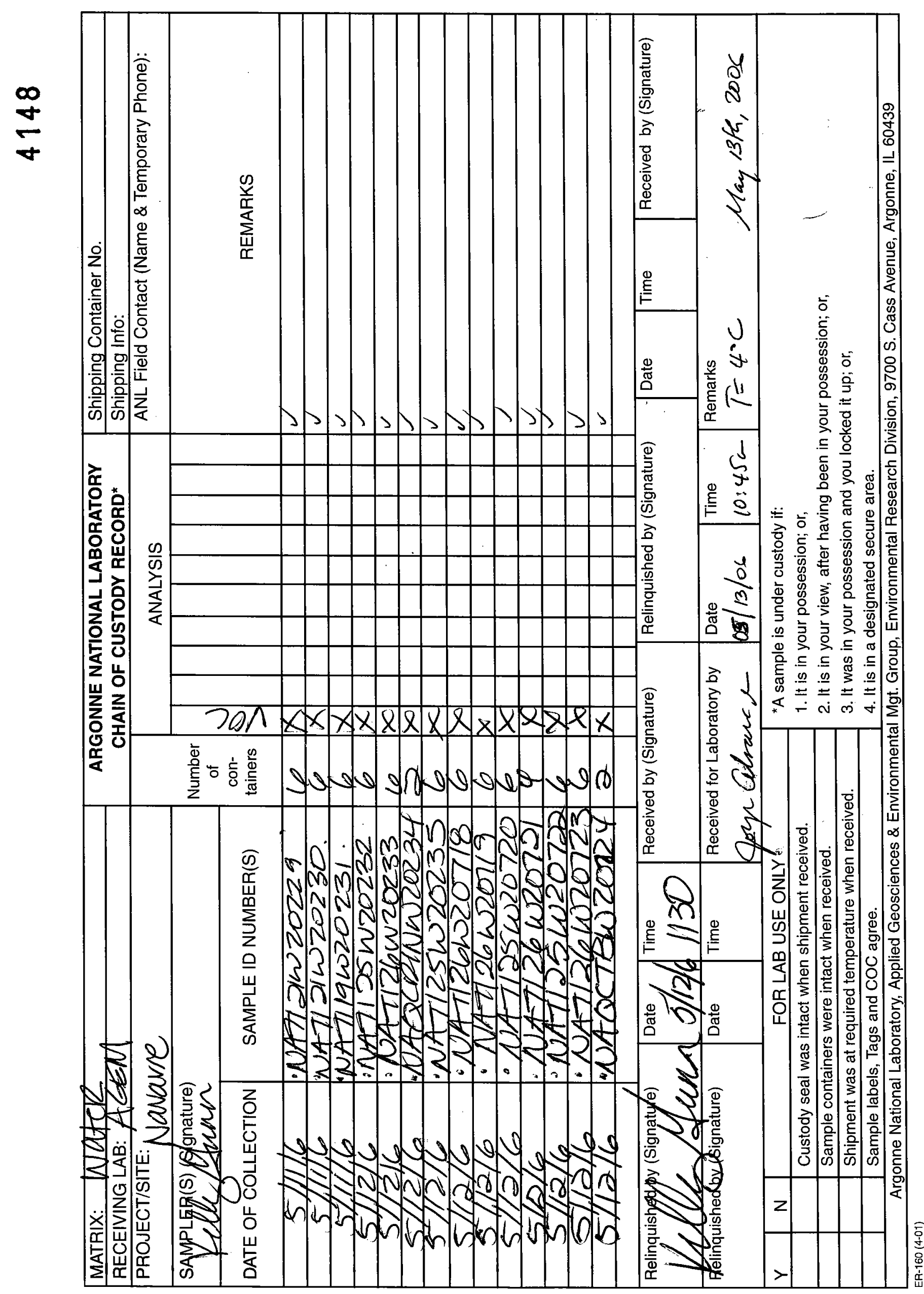




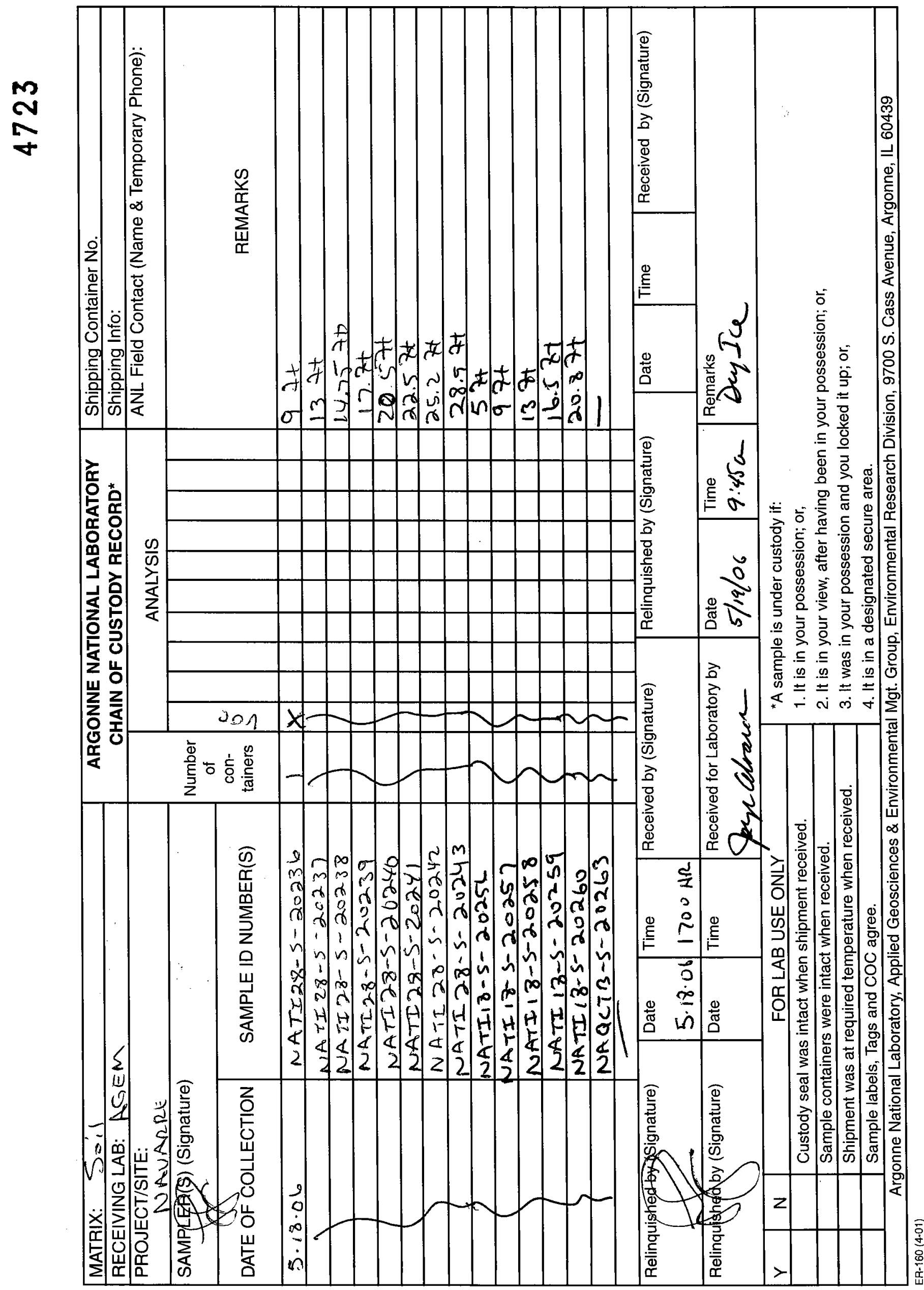




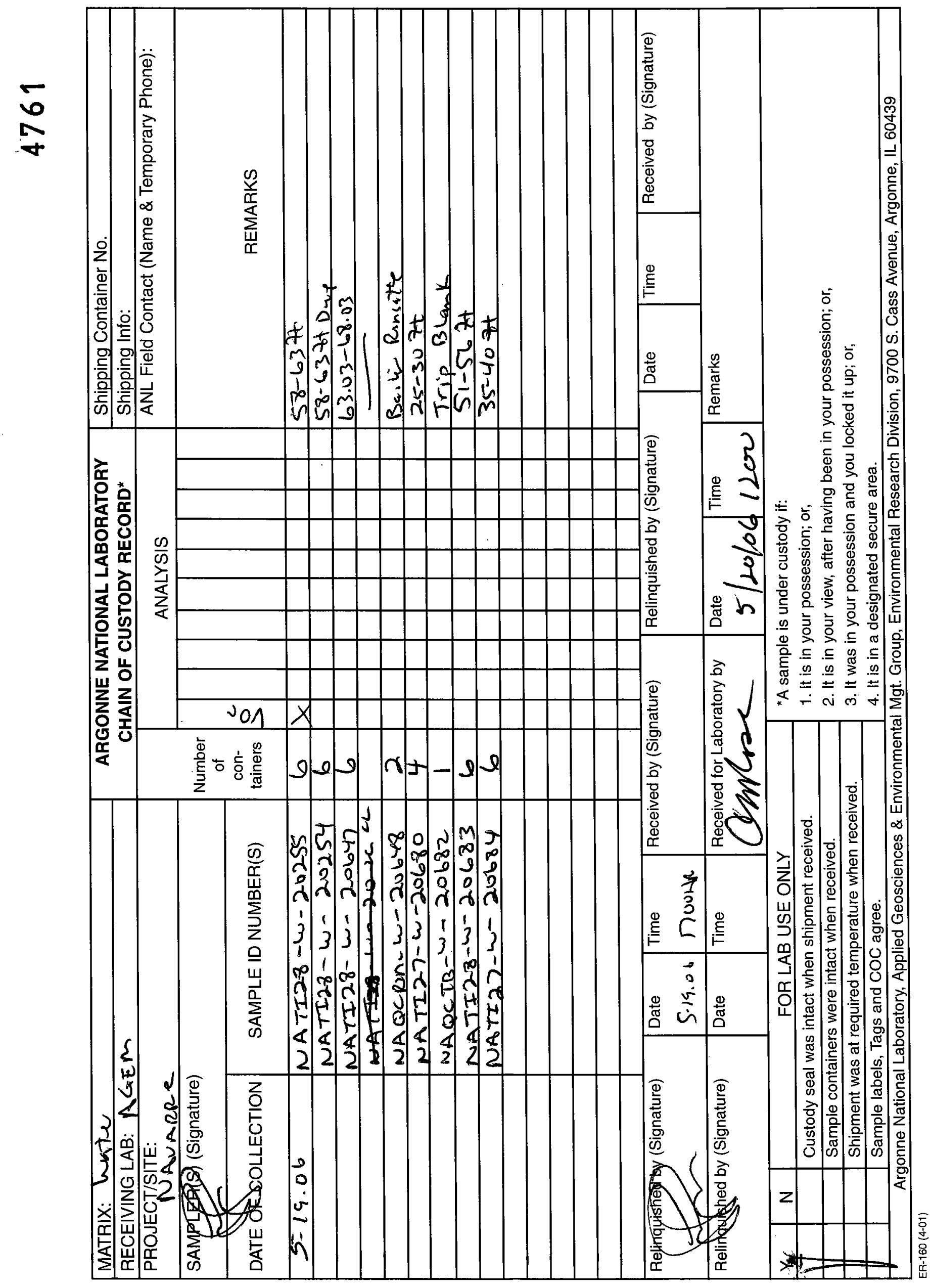




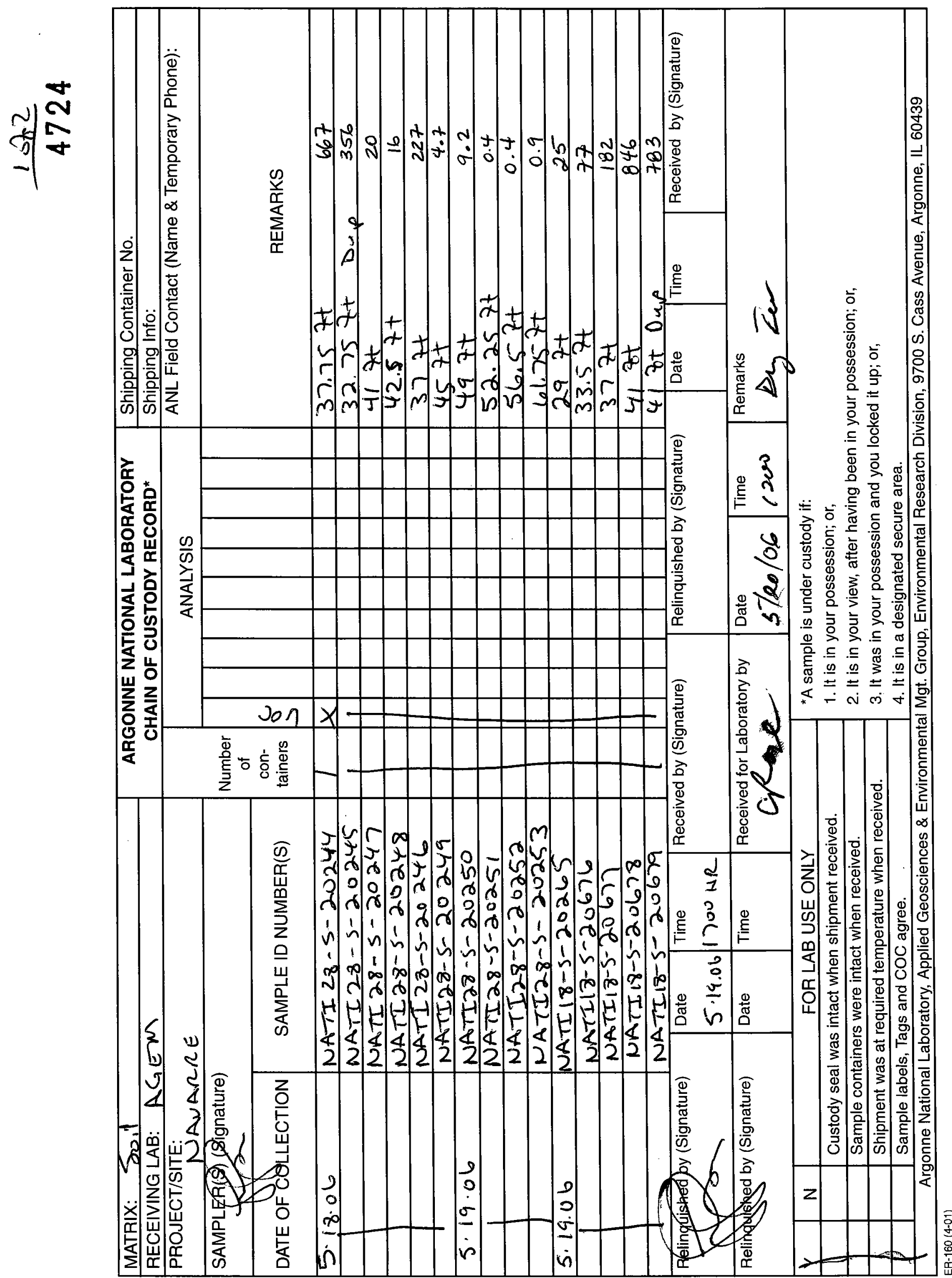




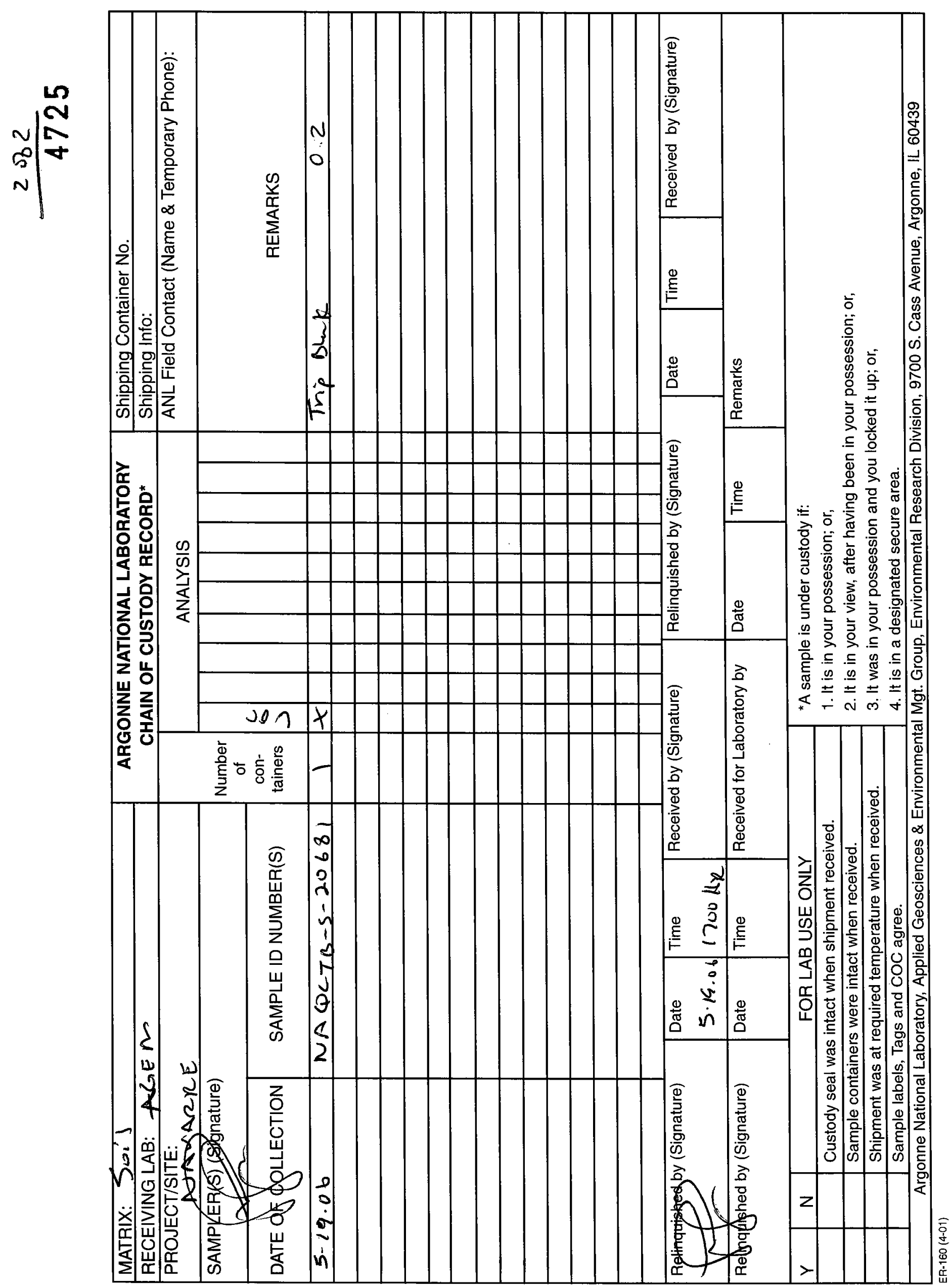




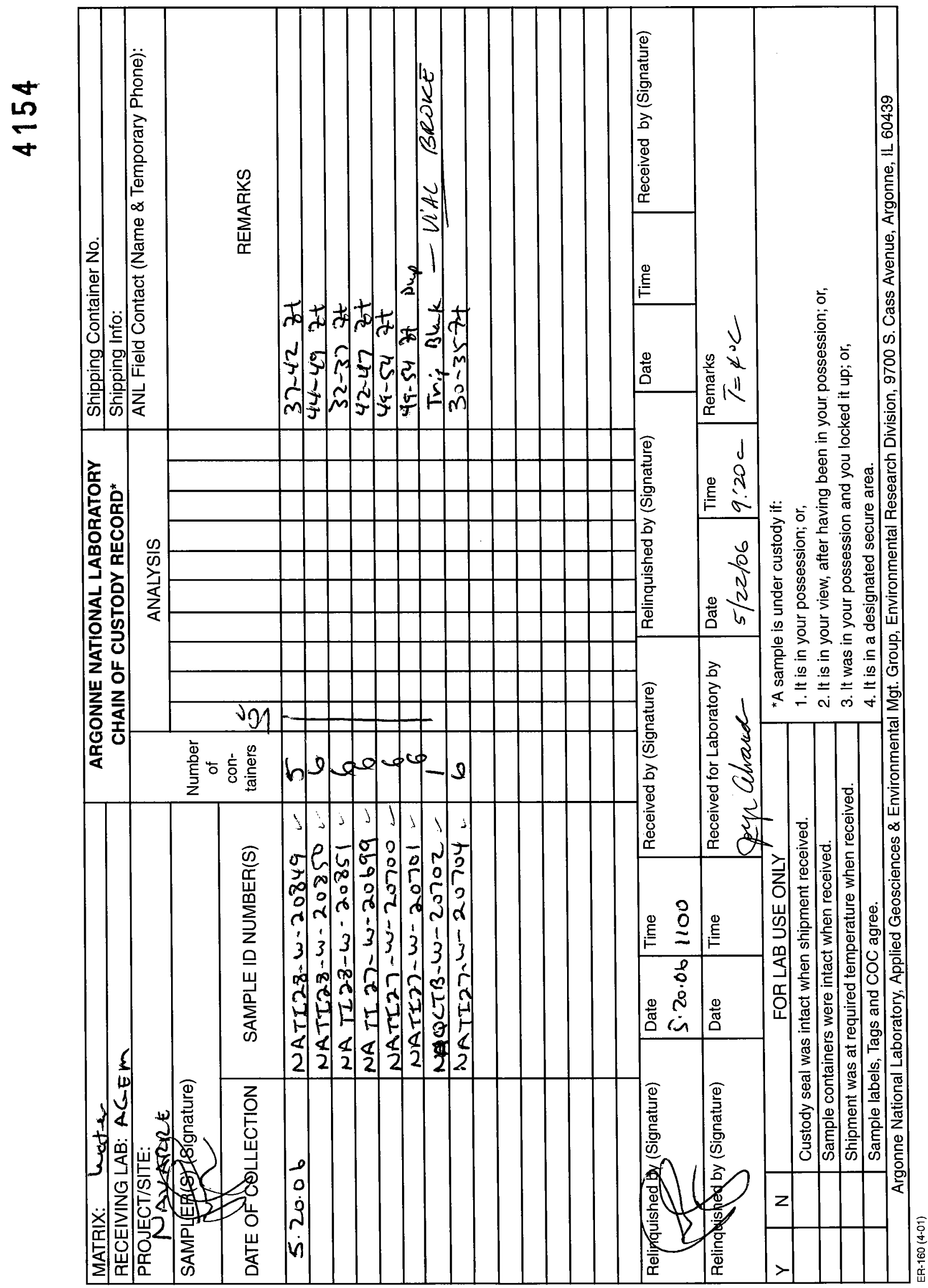




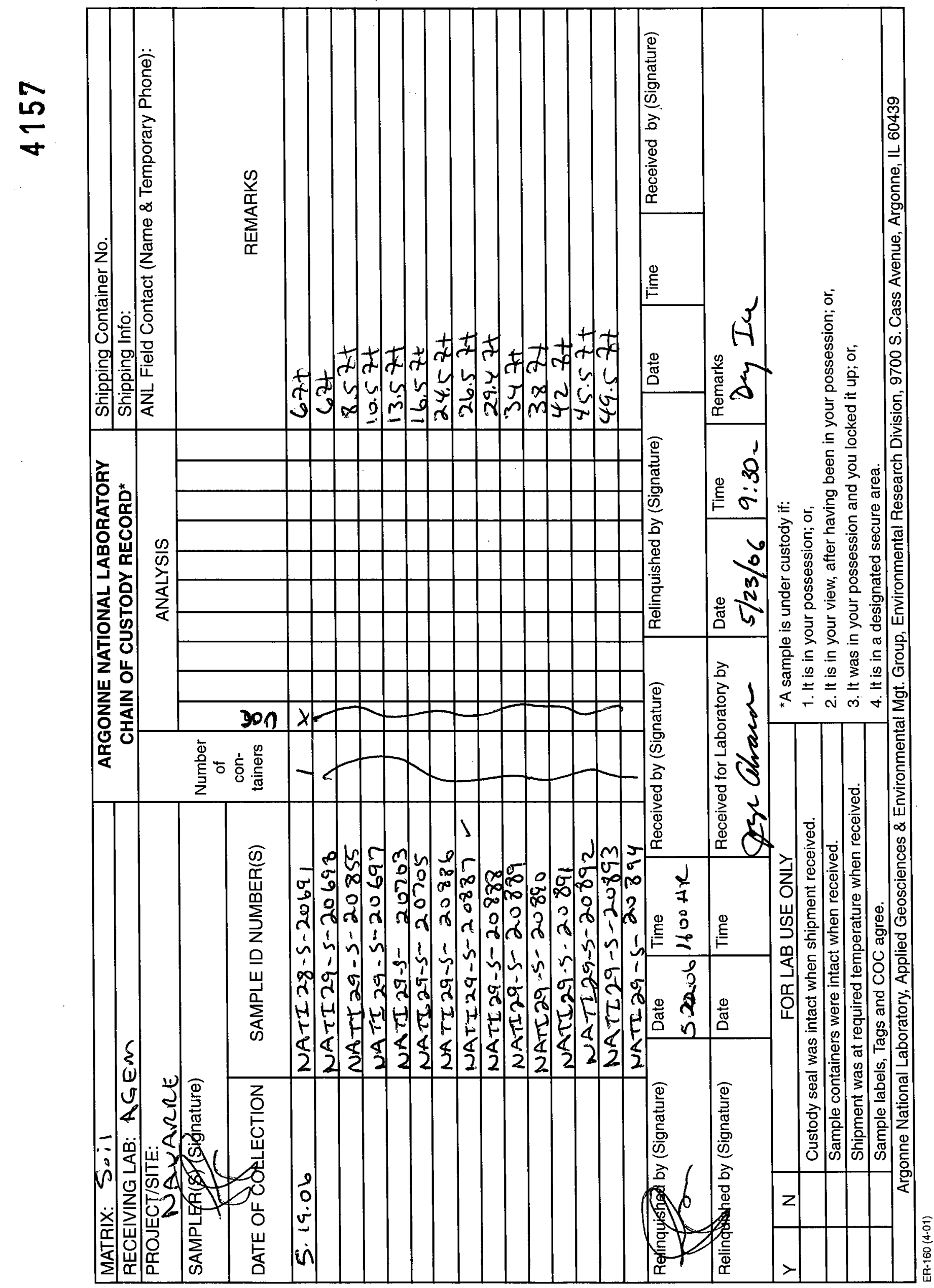




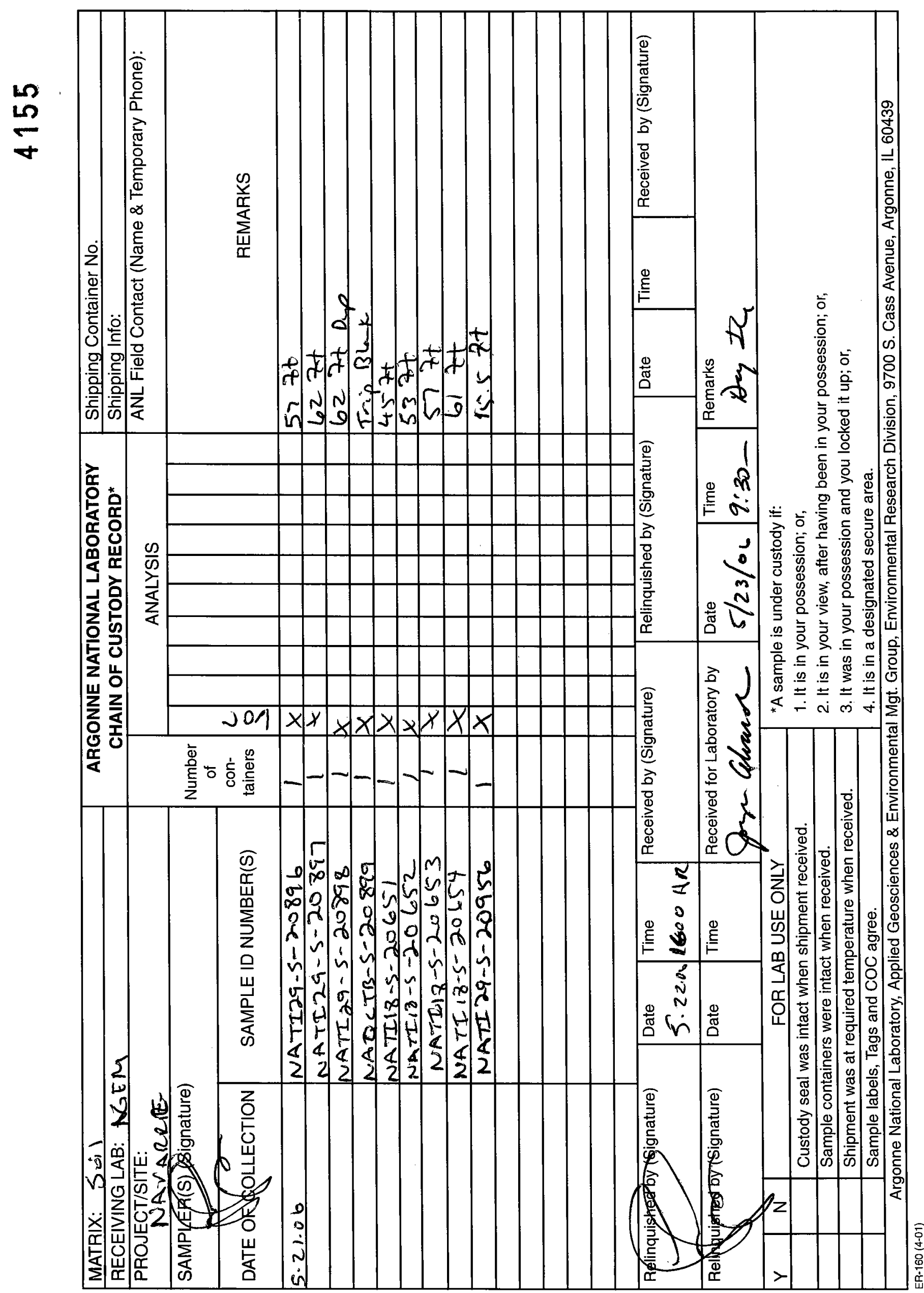




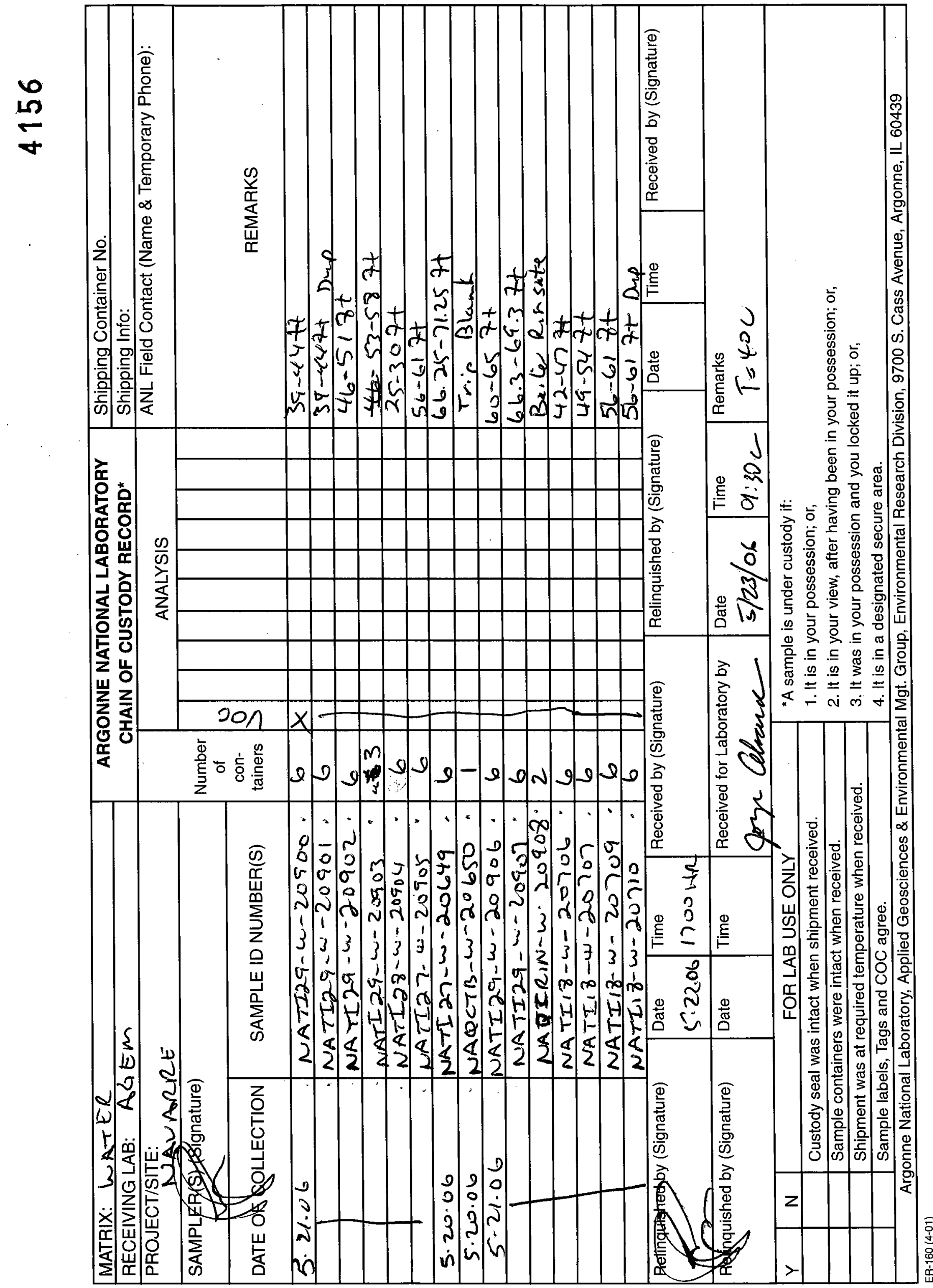




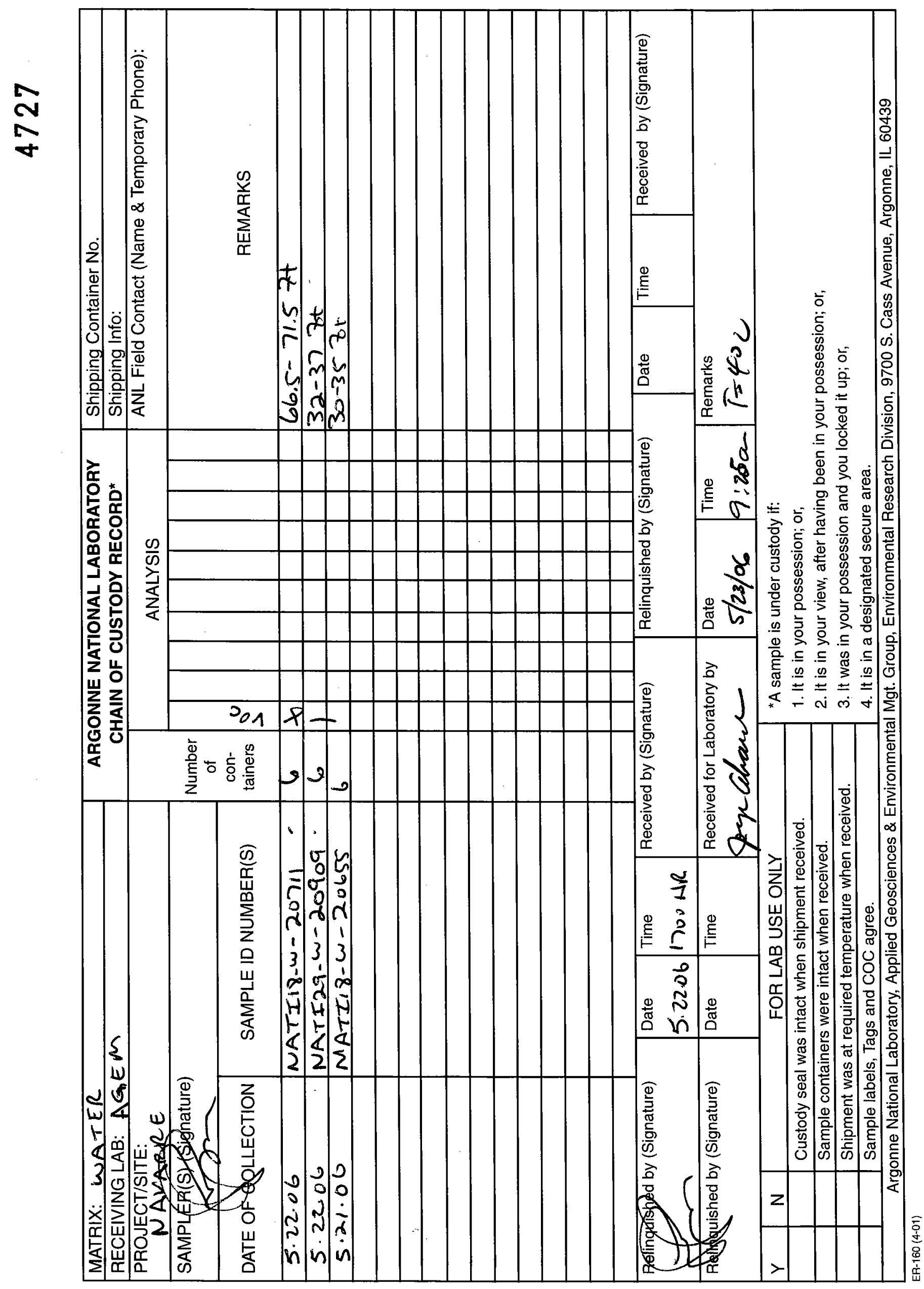




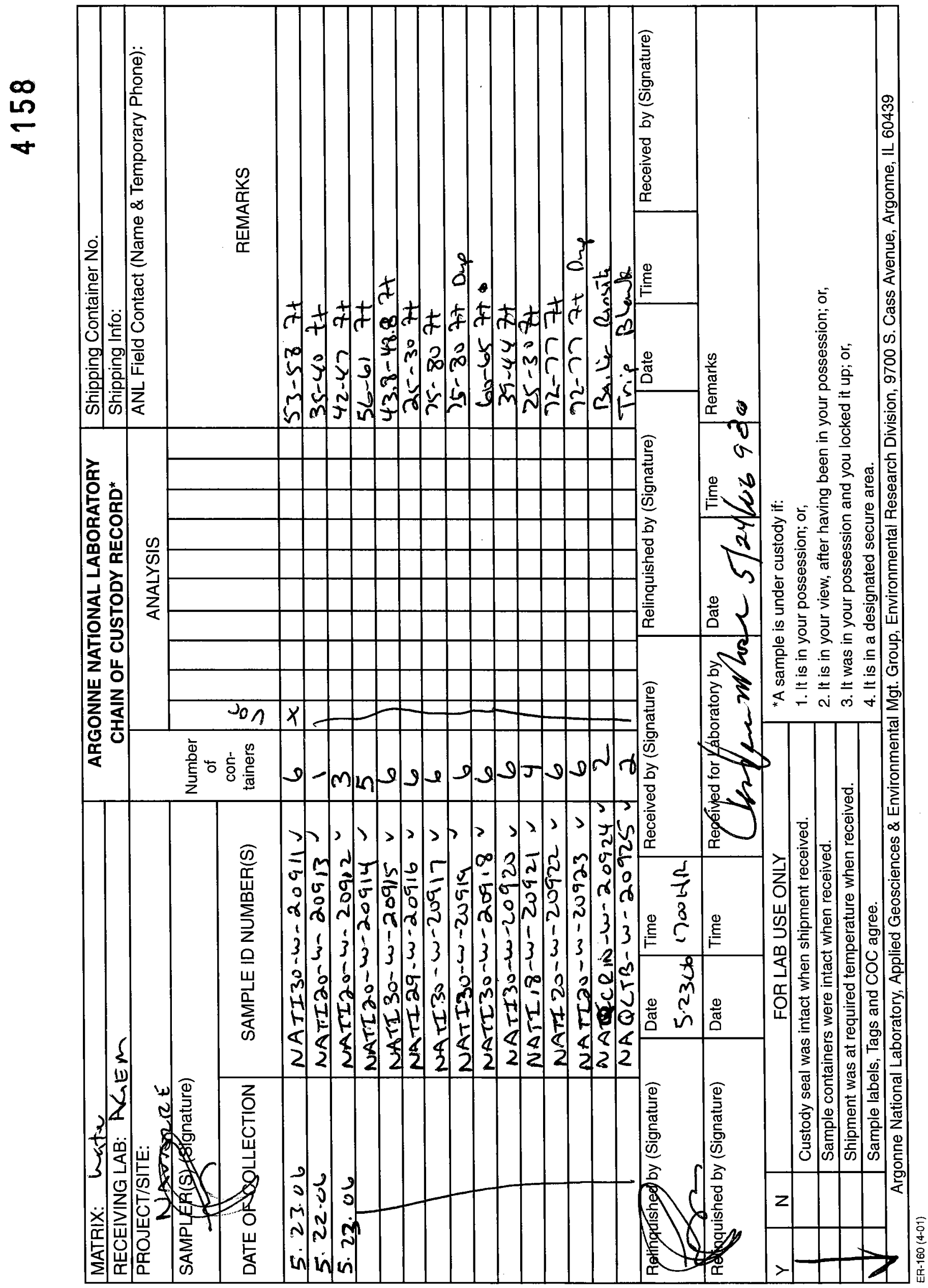




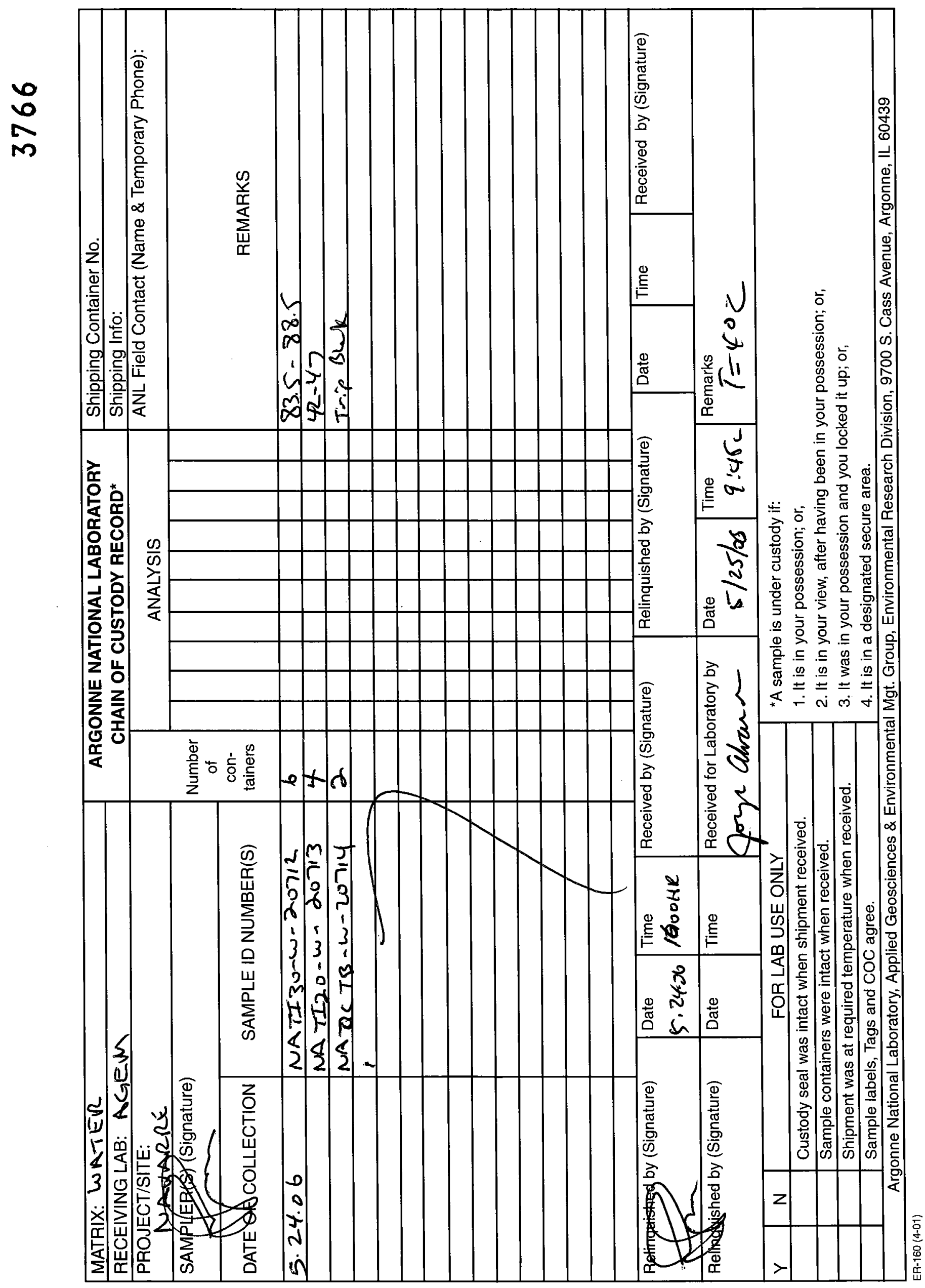




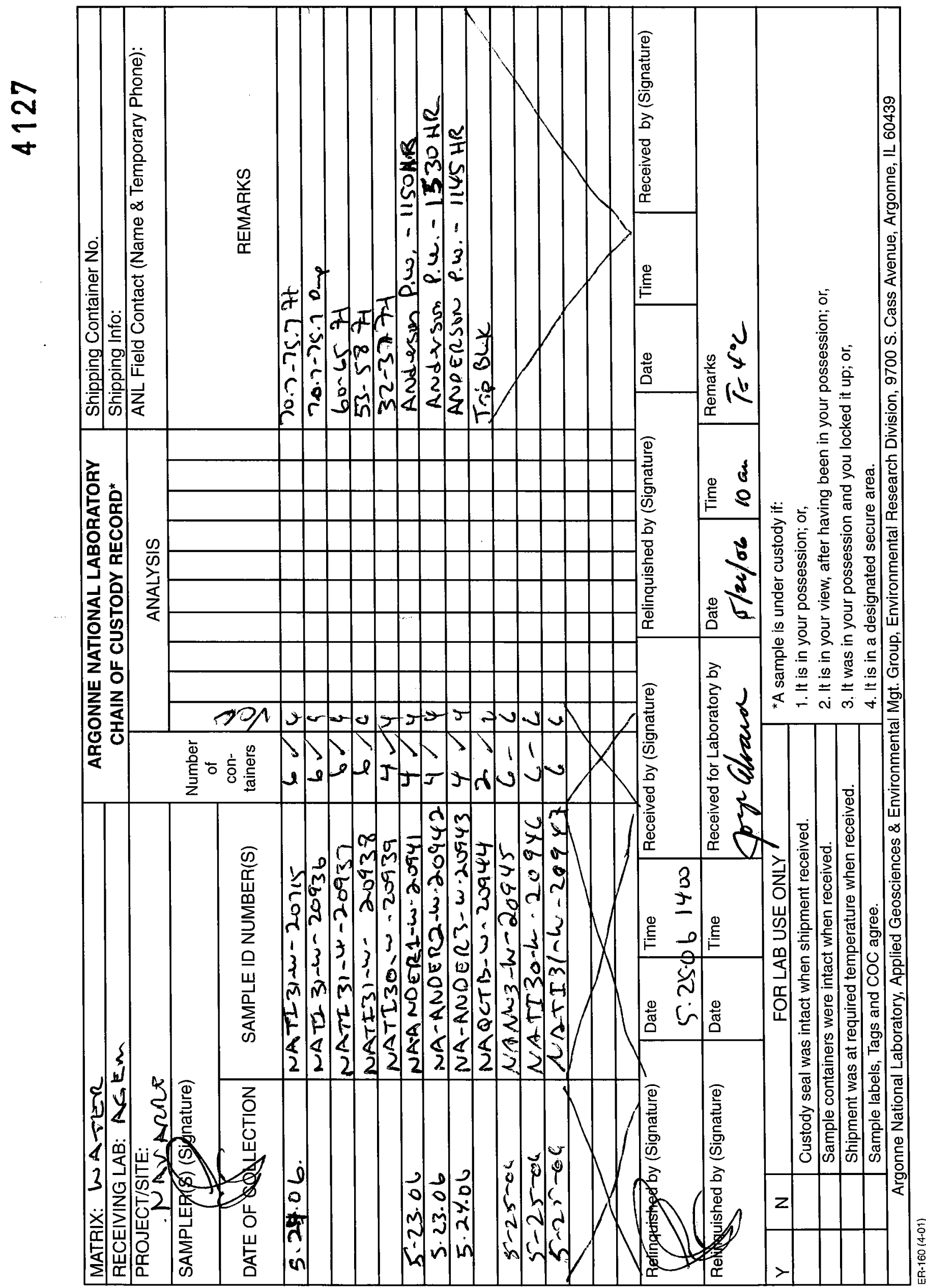




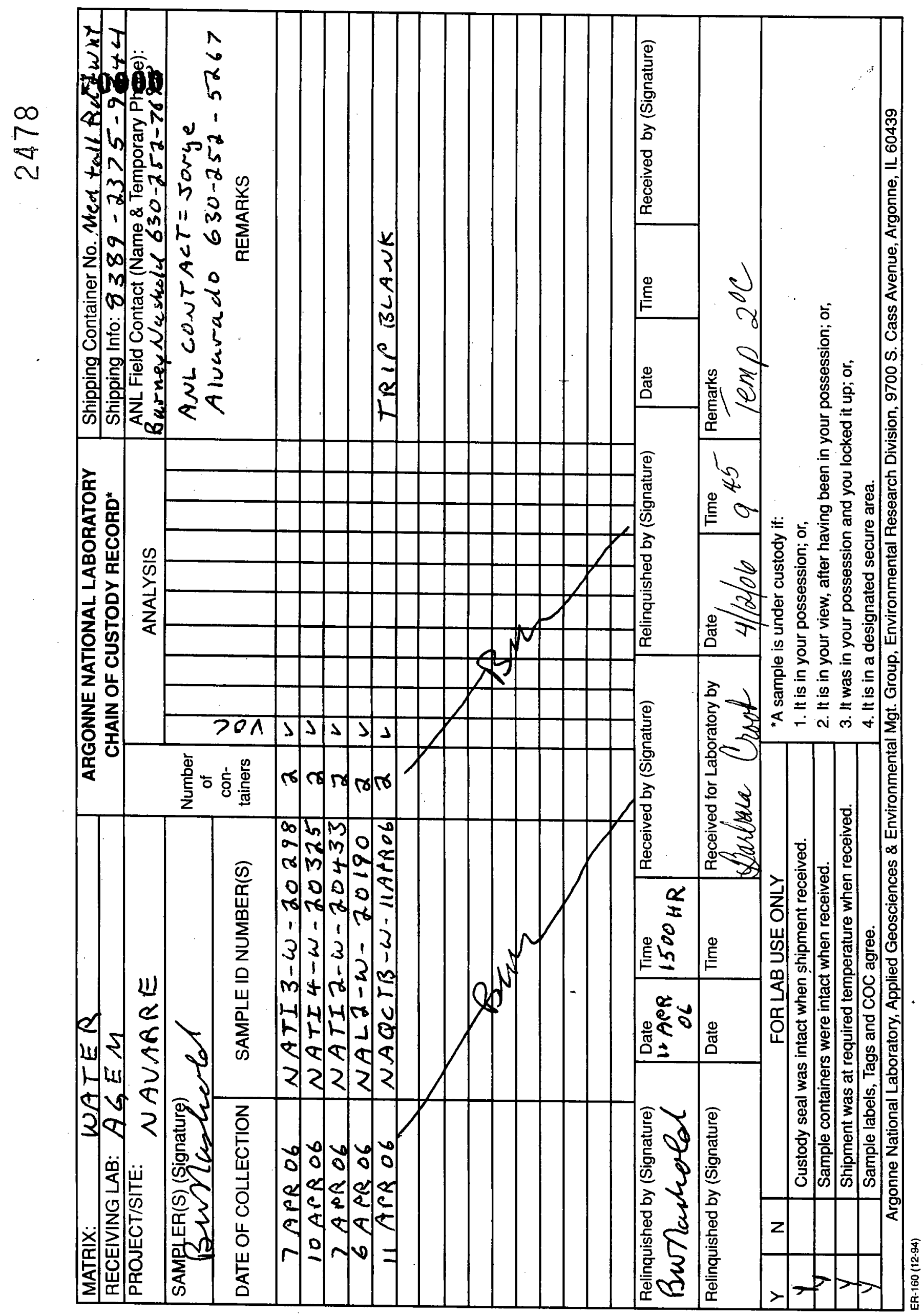




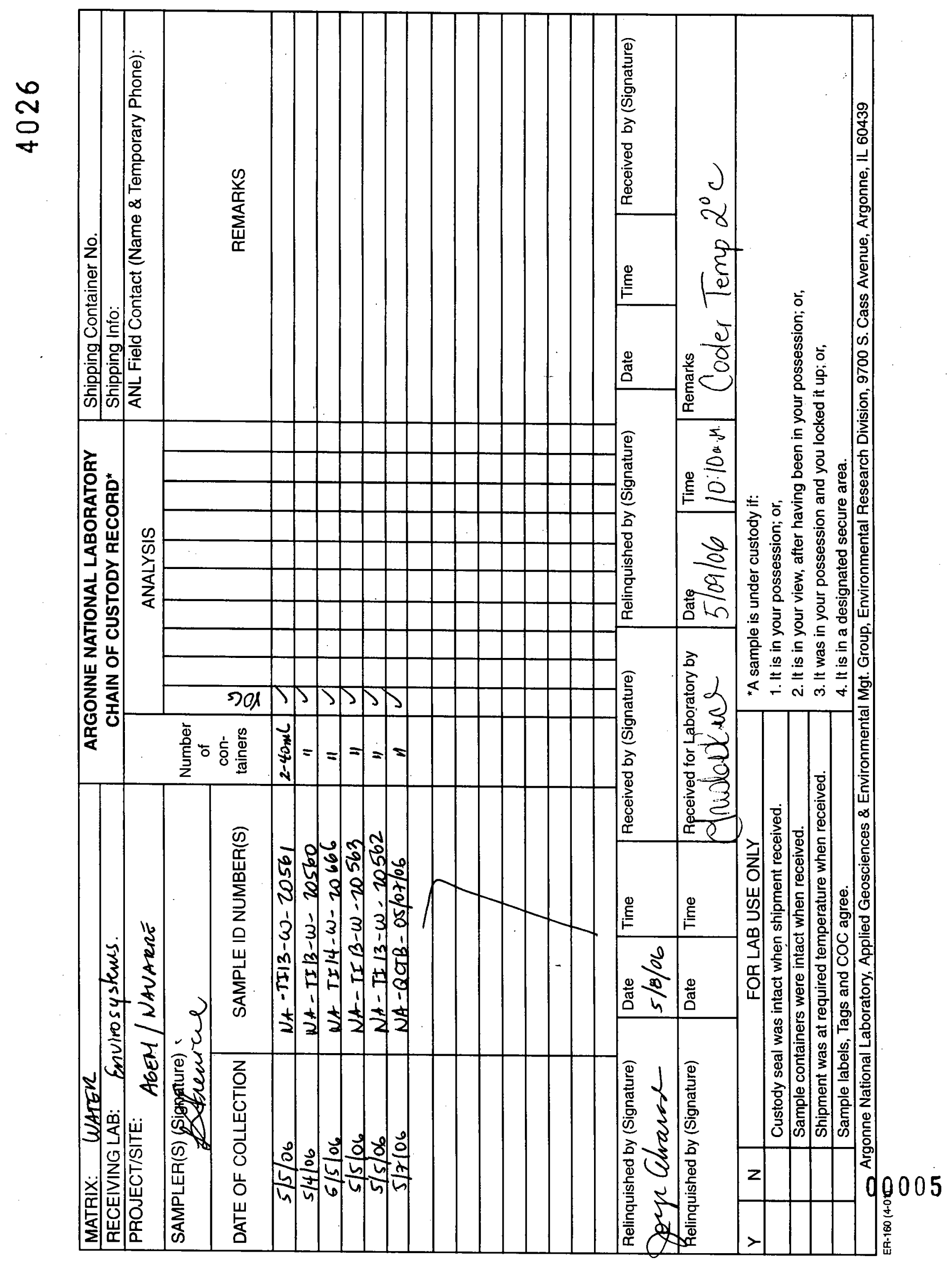




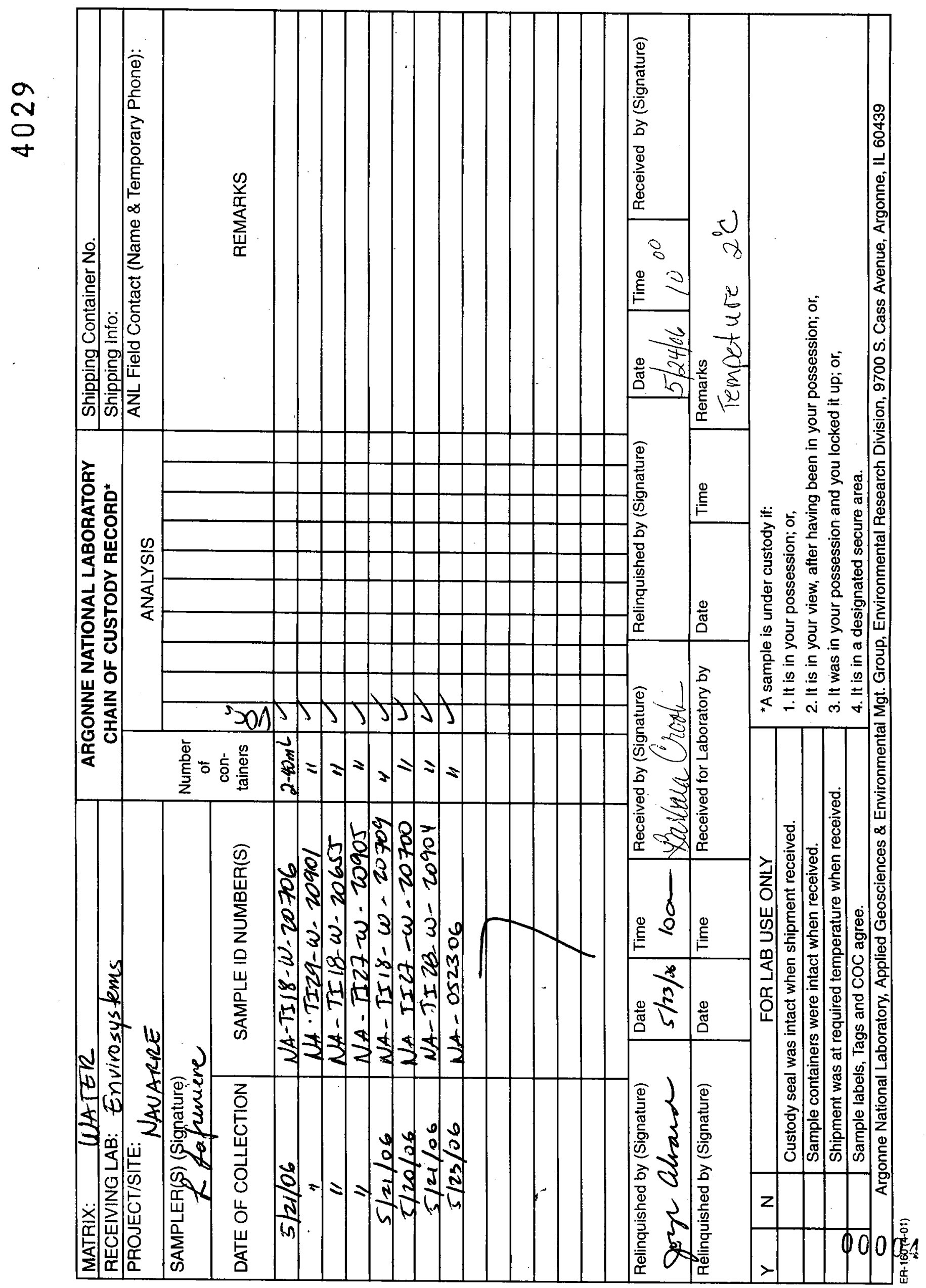




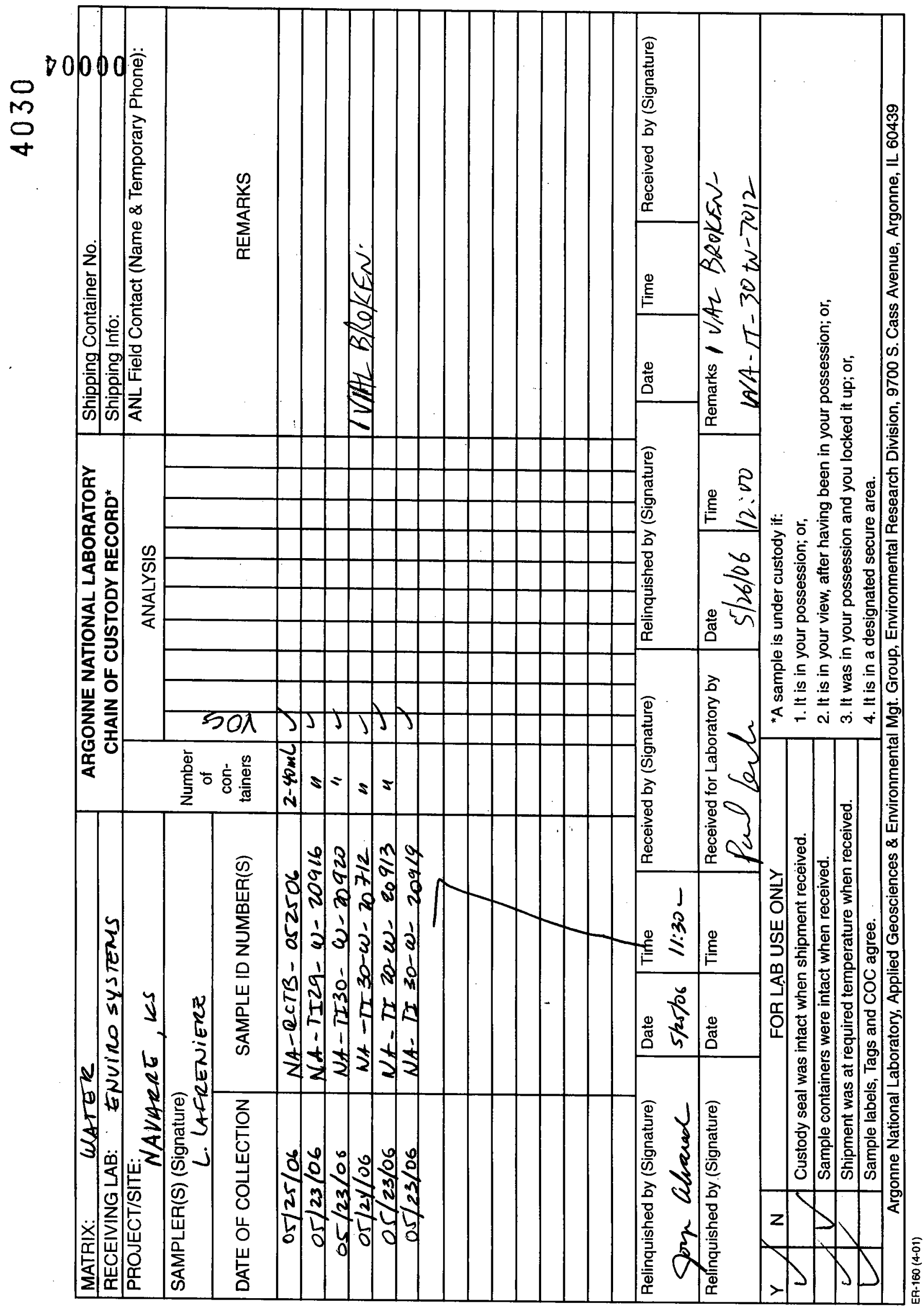




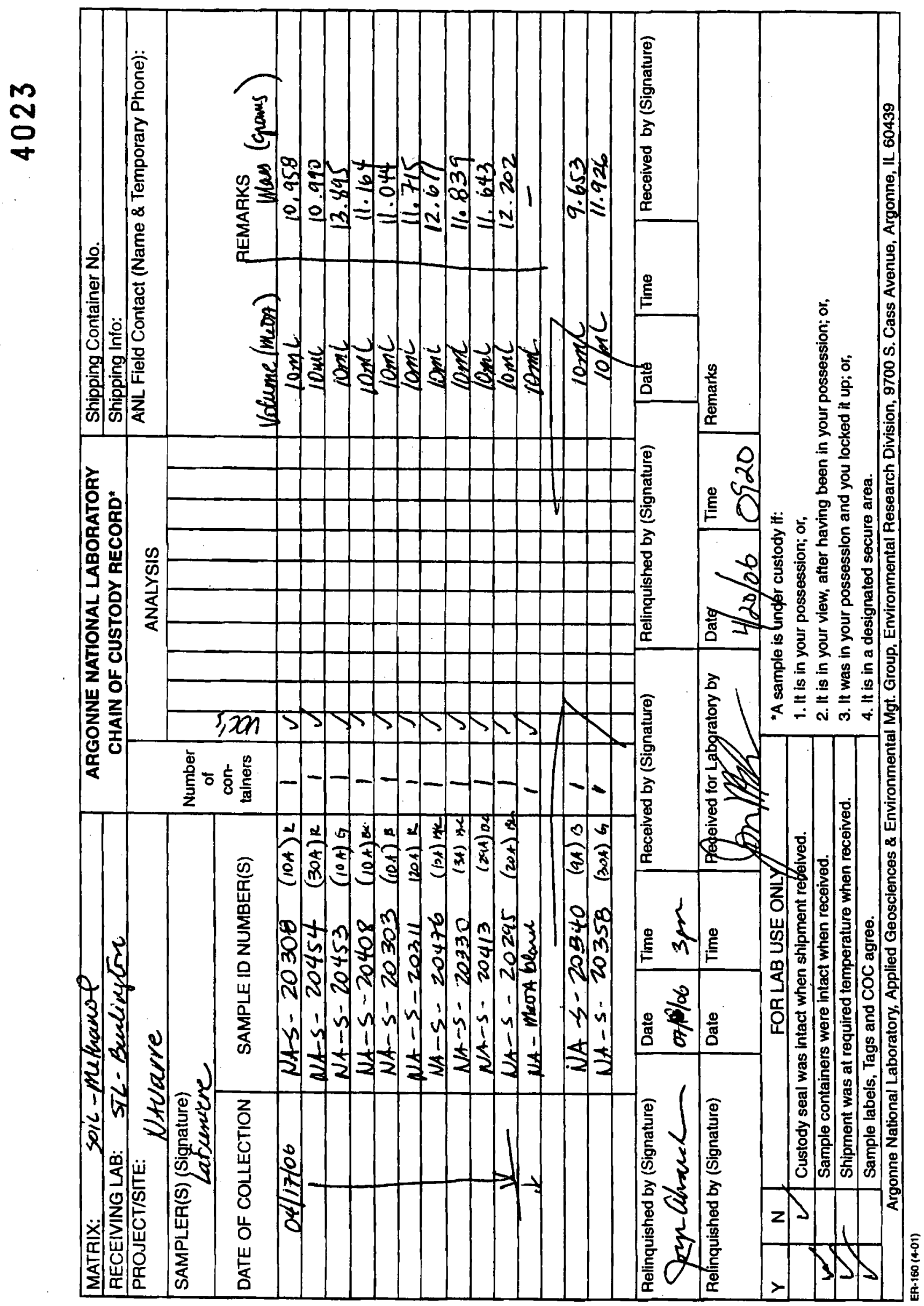




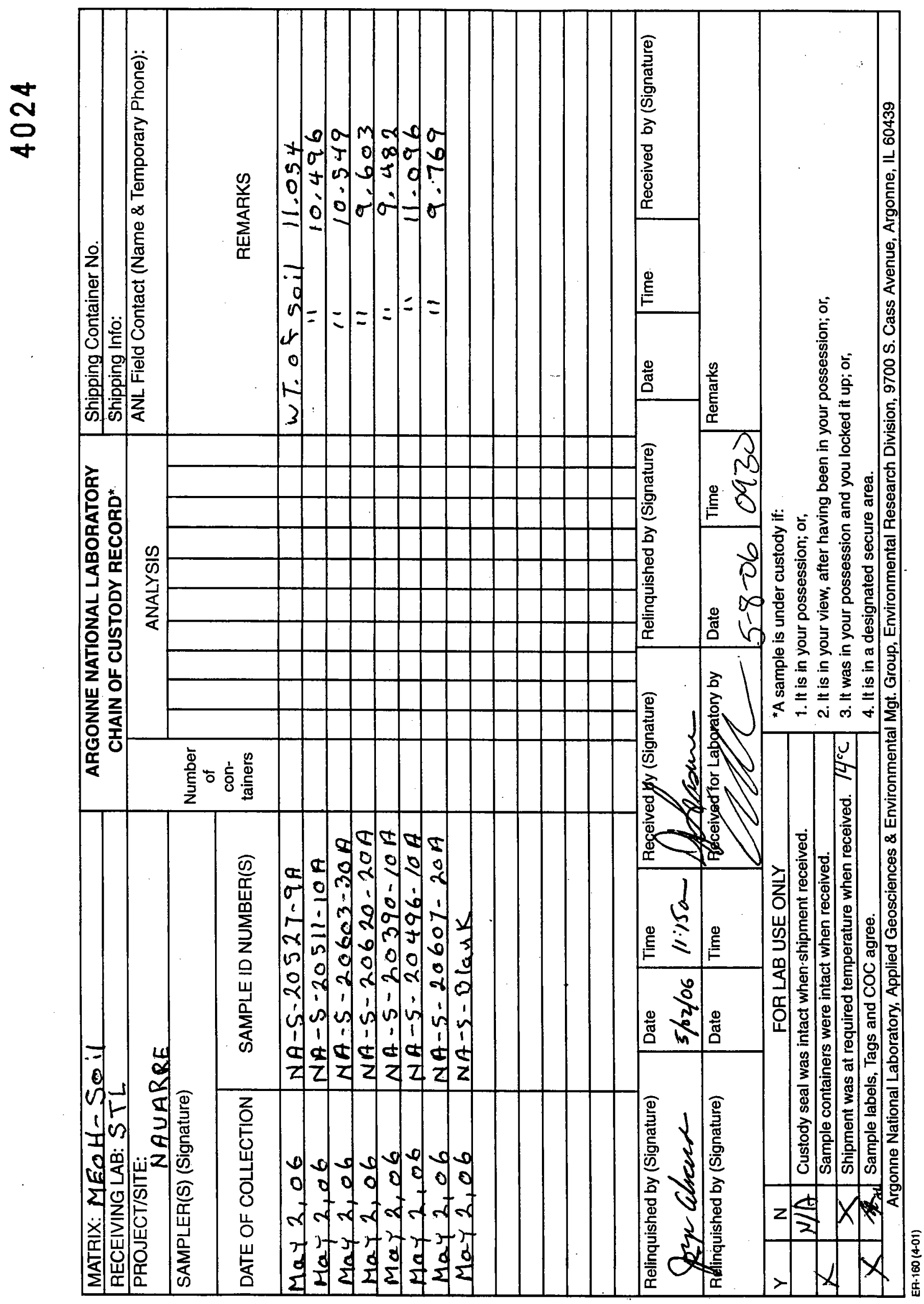




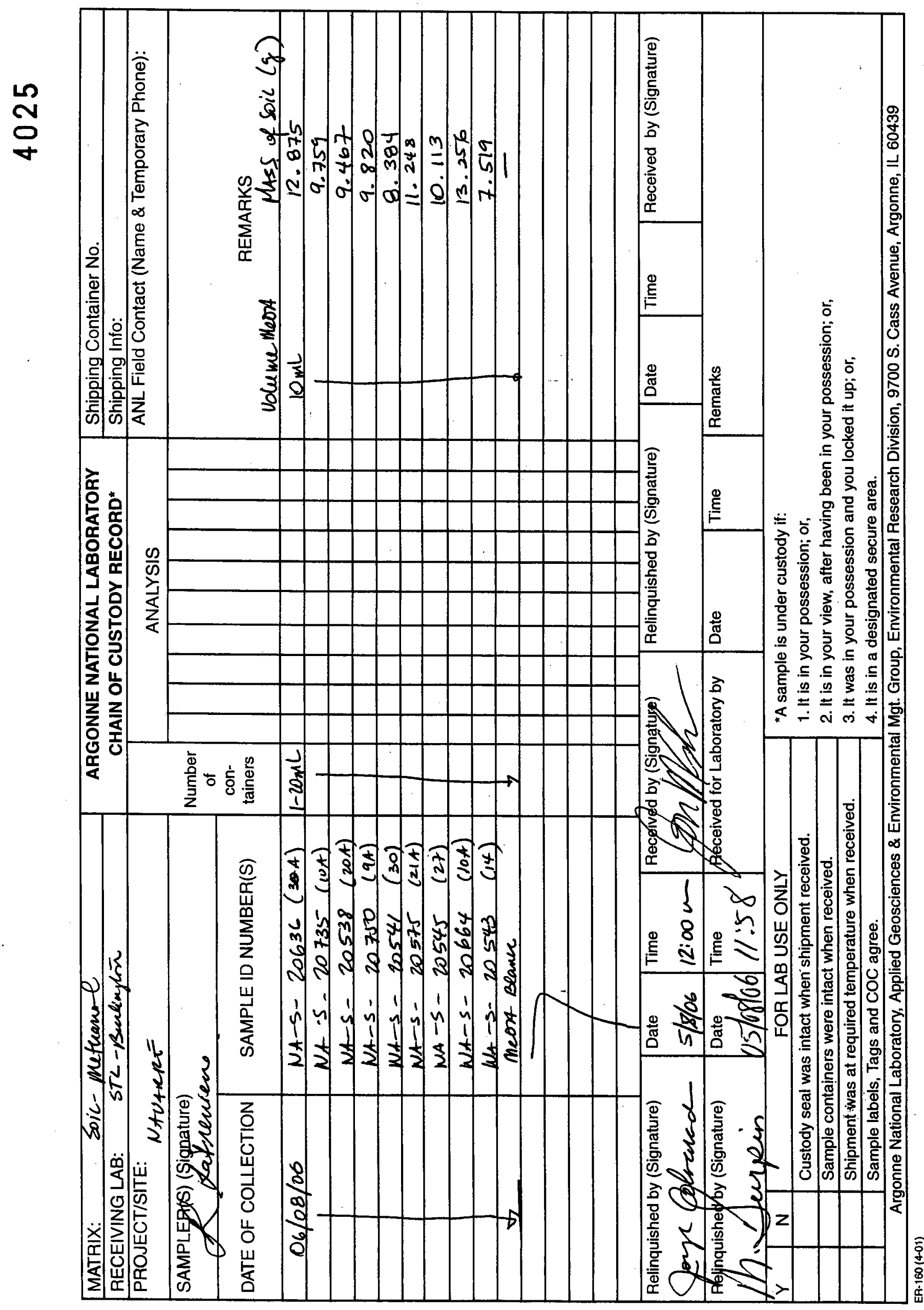




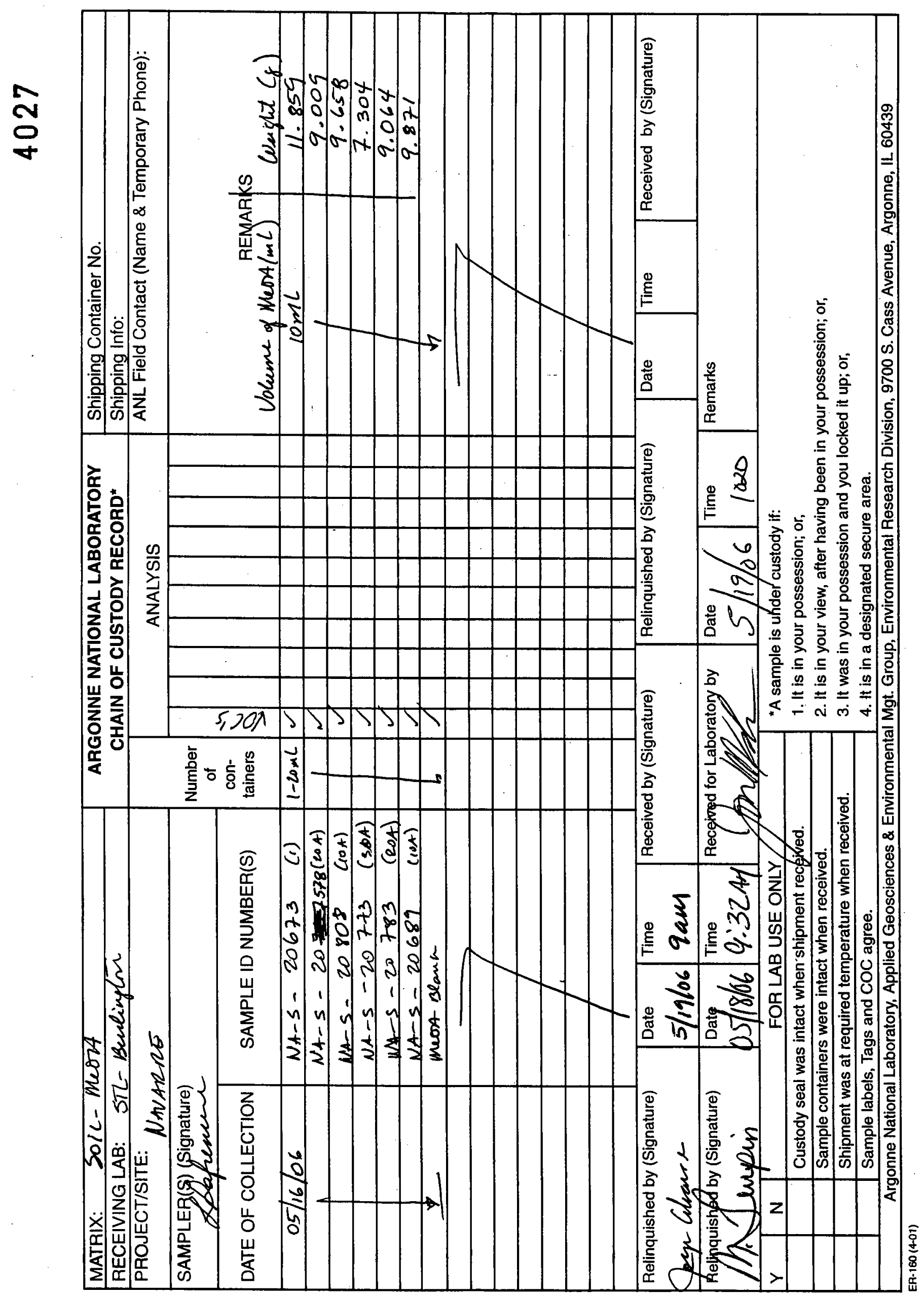




\section{ENVIROSYSTEMS, INC.}

9200 Rumsey Road • Suite B102 • Columbia, Maryland 21045-1934

Phone (410) 964-0330 - Fax (410) 740-9306

Email: info@envsystems.com - Webpage: www.envsystems.com/envsys

May 31, 2006

Jorge S. Alvarado, Ph.D

Argonne National Laboratory

Environmental Research Division

Applied Geoscience and Environmental

Management Section

9700 South Cass Avenue, ER-203

Argonne, Illinois 60439

\section{RE: ENVSY Report 0605051}

Dear Jorge:

Enclosed is the Analytical Data Package for the samples received on April 12th and $17^{\text {th }}, 2006$ for volatile organics analysis by US EPA CLP SOW OLM04.3

Please do not hesitate to call me if you have any questions, comments, or require additional information.

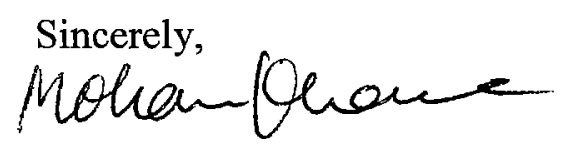

Mohan Khare, Ph.D

President/CEO

$\mathrm{MK} / \mathrm{sp}$ 
1. Narrative

00001 
LABORATORY NAME: ENVIROSYSTEMS, INC.

CLIENT: Argonne National Laboratory

DATA SAMPLES RECEIVED AT LABORATORY: April $12^{\text {th }}$ and $17^{\text {th }}, 2006$

SAMPLE ANALYSES INCLUDED IN THIS REPORT:

$\begin{array}{lllll}\text { CLIENT } \# & \text { LAB ID\# } & \text { ANALYSIS } & \text { MATRIX } & \text { VOA Ph } \\ \text { NATI3-W-20298 } & 0060407-01 & \text { VOA } & \text { WATER } & 7 \\ \text { NATI4-W-20325 } & 0060407-02 & \text { VOA } & \text { WATER } & 7 \\ \text { NATI2-W-20433 } & 0060407-03 & \text { VOA } & \text { WATER } & 7 \\ \text { NAL2-W-20190 } & 0060407-04 & \text { VOA } & \text { WATER } & 7 \\ \text { NAQCTB-W-11APR06 } & 0060407-05 & \text { VOA } & \text { WATER } & 7 \\ \text { WA-2-11-16891 } & 0060418-01 & \text { VOA } & \text { WATER } & 7 \\ \text { WA-5A-11-16892 } & 0060418-02 & \text { VOA } & \text { WATER } & 7 \\ \text { WA-5E-11-16893 } & 0060418-03 & \text { VOA } & \text { WATER } & 7 \\ \text { WA-9-16894 } & 0060418-04 & \text { VOA } & \text { WATER } & 7 \\ \text { WA-DUP-16885 } & 0060418-05 & \text { VOA } & \text { WATER } & 7 \\ \text { WA-10-16896 } & 0060418-06 & \text { VOA } & \text { WATER } & 7 \\ \text { WA-11-16897 } & 0060418-07 & \text { VOA } & \text { WATER } & 7 \\ \text { WA-12-16898 } & 0060418-08 & \text { VOA } & \text { WATER } & 7 \\ \text { WA-FB-0411064 } & 0060418-09 & \text { VOA } & \text { WATER } & 7\end{array}$

No Matrix spike/matrix spike duplicate analysis was performed for this case. Sample number WA-FB-0411064 was not spiked with the internal standard making this sample non-reportable.

Samples for this SDG are analyzed by EPA SOW OLMO4.3 for multi-media multi-concentration organics. Sample detection limits have been modified to meet client requirements.

The cooler temperature was measured to be 2 and 6 degrees $c$ for samples received on April $12^{\text {th }}$ and $17^{\text {th }}$ respectively.

The volatile analysis was performed on a Agilent 5975 GC/MS using a Restek RTX-624 20 meter column with an inner diameter of $0.18 \mathrm{~mm}$ and a 1 micron film thickness. The trap used with the autosampler is a $0.3 \mathrm{~cm}$ OD x 28.5 cm L ENCON Ambient Packed Trap.

Most of the samples have at least one surrogate recovery out of the quality control range. Also, one of the samples, WA-11-16897 had all three internal standards slightly lower than the quality control limits.

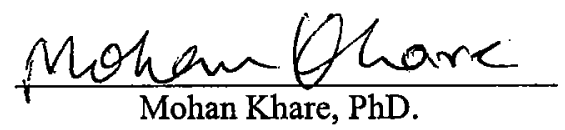

DATE: $\frac{5 / 30 / 86}{30 \operatorname{May} 30,2006}$ 
2. SGD Cover Sheet/Traffic Reports 


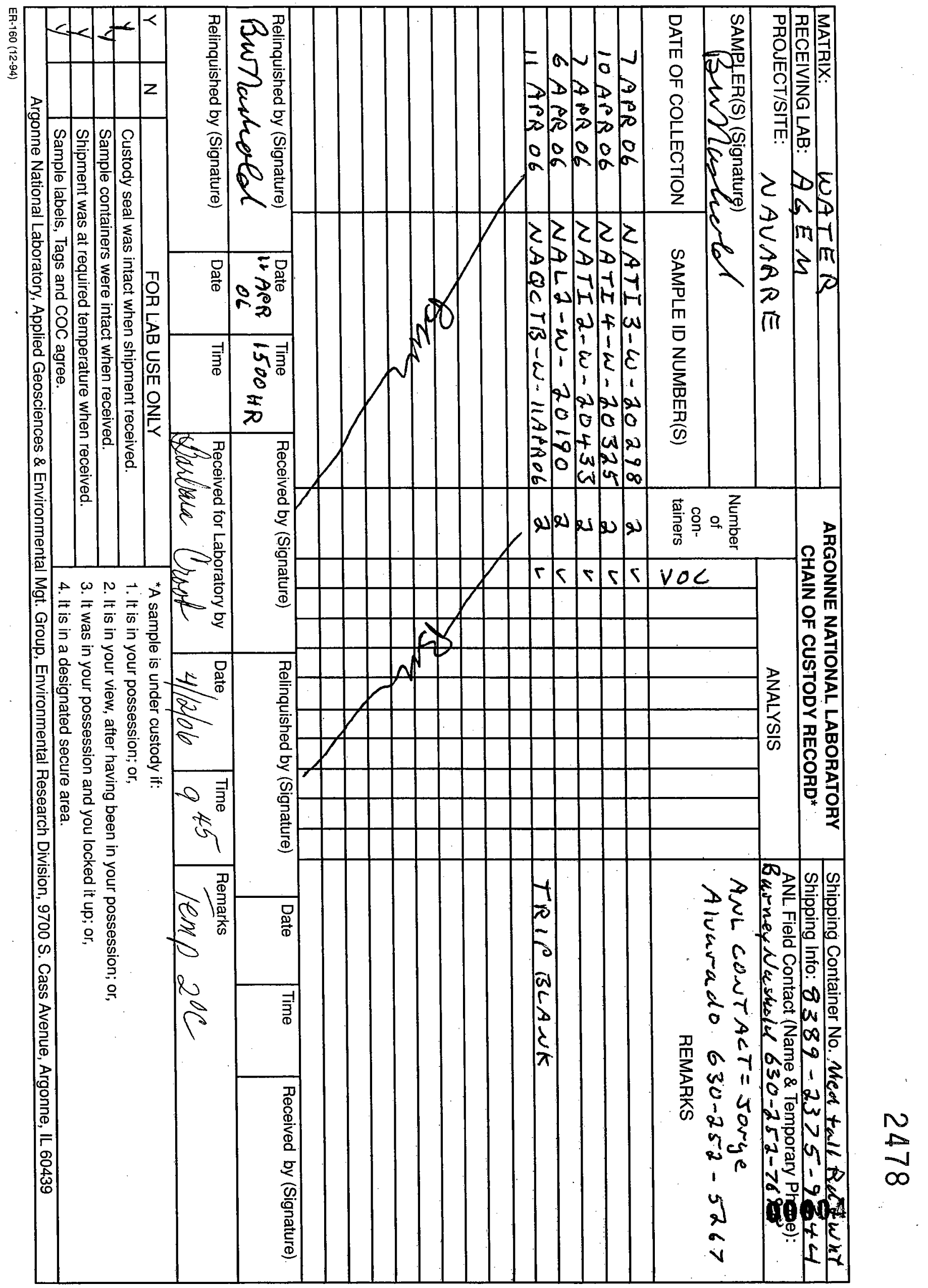


VOLATILE SAMPLE DATA

00012 
Lab Name: ENVIROSYSTEMS, INC.

Lab Code: ENVSYS Case No.: ARG0406 SAS No.:

SDG No.: ARG0406

Matrix: (soil/water) WATER

Sample wt/vol : $\quad 5.000(\mathrm{~g} / \mathrm{mL}) \mathrm{ML}$

Level: (low/med) LOW

\% Moisture: not dec.

GC Column: RTX-624 ID: 0.18 (mm)

Soil Extract Volume: (uL)
Lab Sample ID: 0060407-01

Lab File ID: 0000087_1

Date Received: 04/12/06

Date Analyzed: 04/20/06

Dilution Factor: 1.0

Soil Aliquot Volume: (uL)

CONCENTRATION UNITS:

CAS NO.

COMPOUND
UG/L Q

\begin{tabular}{|c|c|c|c|}
\hline $\begin{array}{r}75-71-8 \\
74-87-3 \\
75-01-4 \\
74-83-9 \\
75-00-3 \\
75-69-4 \\
75-35-4 \\
76-13-1 \\
67-64-1 \\
75-15-0 \\
79-20-9 \\
75-09-2 \\
156-60-5 \\
1634-04-4 \\
75-34-3 \\
156-59-2 \\
78-93-3 \\
67-66-3 \\
71-55-6 \\
110-82-7 \\
56-23-5 \\
71-43-2 \\
107-06-2\end{array}$ & $\begin{array}{l}\text { Dichlorodifluoromethane } \\
\text { Chloromethane } \\
\text { Vinyl Chloride } \\
\text { Bromomethane } \\
\text { Chloroethane } \\
\text { Trichlorofluoromethane } \\
\text { 1,1-Dichloroethene } \\
\text { 1,1,2-Trichloro-1,2,2-trifluoroethane } \\
\text { Acetone } \\
\text { Carbon Disulfide } \\
\text { Methyl Acetate } \\
\text { Methylene Chloride } \\
\text { trans-1,2-Dichloroethene } \\
\text { Methyl tert-Butyl Ether } \\
\text { 1,1-Dichloroethane } \\
\text { cis-1,2-Dichloroethene } \\
\text { 2-Butanone } \\
\text { Chloroform } \\
\text { 1,1,1-Trichloroethane } \\
\text { Cyclohexane } \\
\text { Carbon Tetrachloride } \\
\text { Benzene } \\
\text { 1,2-Dichloroethane }\end{array}$ & $\begin{array}{r}5.0 \\
5.0 \\
5.0 \\
5.0 \\
5.0 \\
5.0 \\
5.0 \\
5.0 \\
5.0 \\
5.0 \\
5.0 \\
1.6 \\
5.0 \\
5.0 \\
5.0 \\
5.0 \\
5.0 \\
11 \\
5.0 \\
5.0 \\
23 \\
5.0 \\
5.0\end{array}$ & $\begin{array}{l}\mathrm{U} \\
\mathrm{U} \\
\mathrm{U} \\
\mathrm{U} \\
\mathrm{U} \\
\mathrm{U} \\
\mathrm{U} \\
\mathrm{U} \\
\mathrm{U} \\
\mathrm{U} \\
\mathrm{U} \\
\mathrm{JB} \\
\mathrm{U} \\
\mathrm{U} \\
\mathrm{U} \\
\mathrm{U} \\
\mathrm{U} \\
\overline{\mathrm{U}} \\
\mathrm{U} \\
\overline{\mathrm{U}} . \\
\mathrm{U}\end{array}$ \\
\hline
\end{tabular}


Lab Name: ENVIROSYSTEMS, INC.

Contract: ARGONNE

Lab Code: ENVSYS Case No.: ARG0406 SAS No.:

SDG No.: ARG0406

Matrix: (soil/water) WATER

Sample wt/vol: $\quad 5.000(\mathrm{~g} / \mathrm{mL}) \mathrm{ML}$

Level: (low/med) LoW.

응 Moisture: not dec.

GC Column: RTX-624 ID: 0.18 (mm)

Soil Extract Volume: (uL)
Lab Sample ID: 0060407-01

Lab File ID: 0000087_1

Date Received: 04/12/06

Date Analyzed: 04/20/06

Dilution Factor: 1.0

Soil Aliquot Volume: (uL)

CONCENTRATION UNITS :

CAS NO.

COMPOUND

(ug/L or $\mathrm{ug} / \mathrm{Kg}$ )

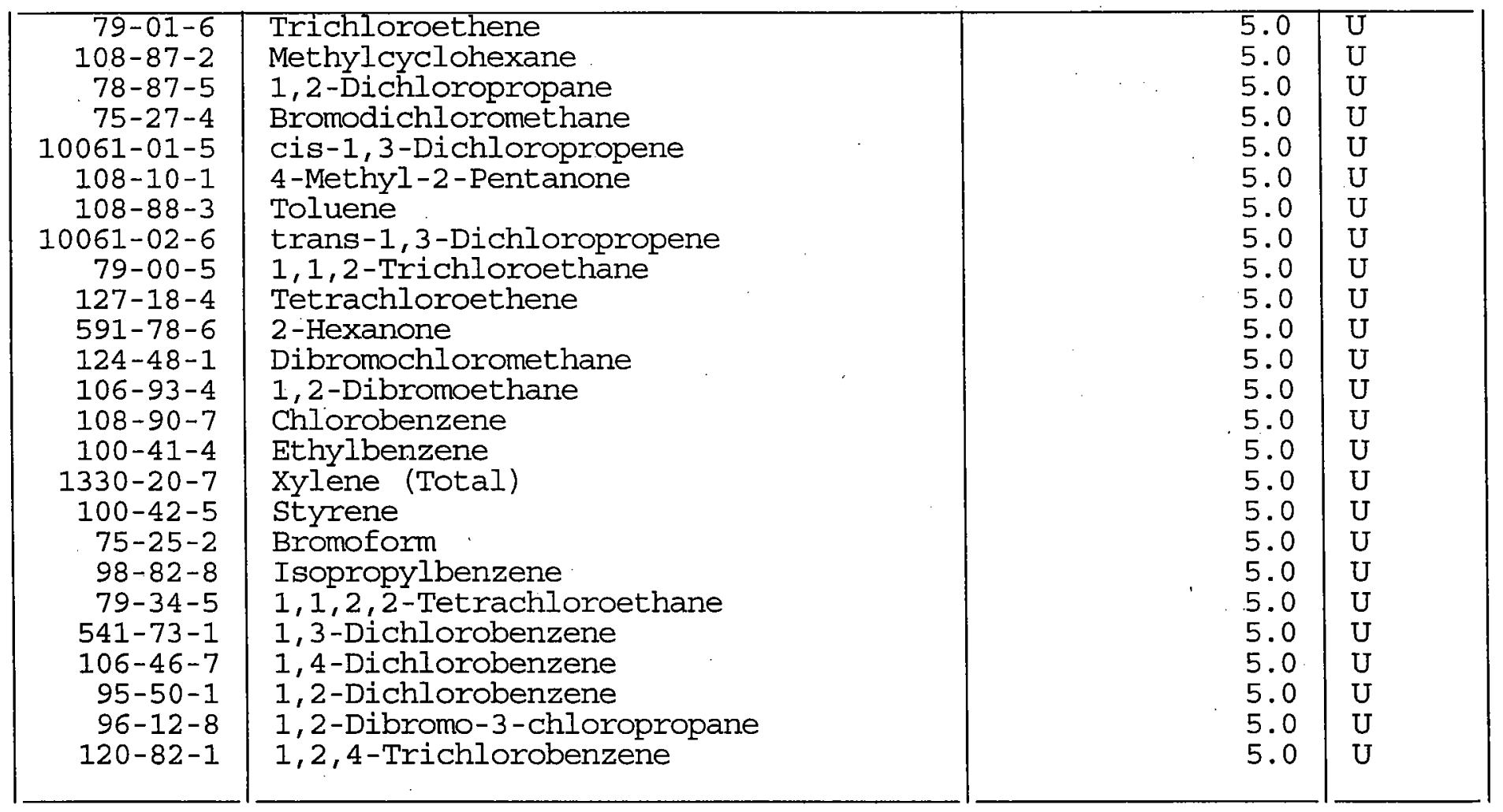


VOLATILE ORGANICS ANALYSIS DATA SHEET

TENTATIVELY IDENTIFIED COMPOUNDS

EPA SAMPLE NO.

NATI $3-W-20298$

Lab Name: ENVIROSYSTEMS, INC.

Contract: ARGONNE

Lab Code: ENVSYS Case No.: ARG0406 SAS No.:

SDG No. : ARG0406

Matrix: (soil/water) WATER

Sample wt/vol: $5.000(\mathrm{~g} / \mathrm{mL}) \mathrm{ML}$

Level: (low/med) LOW

\% Moisture: not dec.

GC Column: RTX-624 ID: 0.18 (mm)

Soil Extract Volume:

Number TICs found: 0
Lab Sample ID: 0060407-01

Lab File ID: 0000087 _

Date Received: 04/12/06

Date Analyzed: 04/20/06

Dilution Factor: 1.0

Soil Aliquot Volume: (uL)

CONCENTRATION UNITS :

(ug/L or $\mathrm{ug} / \mathrm{Kg}$ ) ug/L

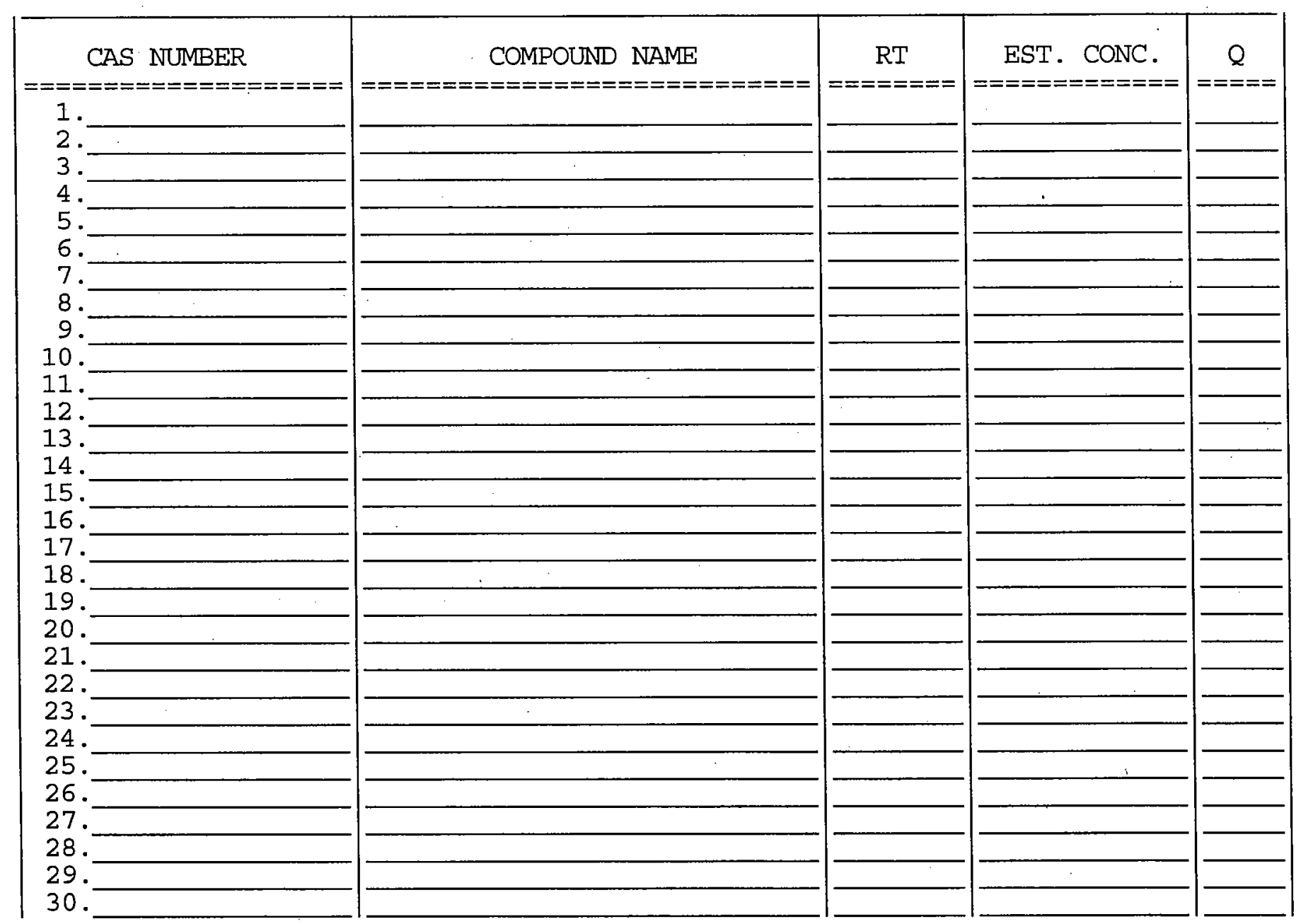


Lab Name: ENVIROSYSTEMS, INC.

Contract: ARGONNE

EPA SAMPLE NO.

NATI $4-W-20325$

Lab Code: ENVSYS Case No.: ARG0406 SAS No.:

SDG No. : ARG0406

Matrix: (soil/water) WATER

Lab Sample ID: 0060407-02

Sample wt/vol:

$5.000(\mathrm{~g} / \mathrm{mL}) \mathrm{ML}$

Lab File ID: 0000087_2

Level: (low/med) LOW

Date Received: 04/12/06

$\%$ Moisture: not dec.

Date Analyzed: 04/20/06

GC Column: RTX-624 ID: 0.18 (mm)

Dilution Factor: 1.0

Soil Extract Volume: (uL)

Soil Aliquot Volume: (uL)

CONCENTRATION UNITS:

CAS NO.

COMPOUND

(ug/L or $\mathrm{ug} / \mathrm{Kg}$ )

UG/L Q

\begin{tabular}{|c|c|c|c|}
\hline $\begin{array}{r}75-71-8 \\
74-87-3 \\
75-01-4 \\
74-83-9 \\
75-00-3 \\
75-69-4 \\
75-35-4 \\
76-13-1 \\
67-64-1 \\
75-15-0 \\
79-20-9 \\
75-09-2 \\
156-60-5 \\
1634-04-4 \\
75-34-3 \\
156-59-2 \\
78-93-3 \\
67-66-3 \\
71-55-6 \\
110-82-7 \\
56-23-5 \\
71-43-2 \\
107-06-2\end{array}$ & $\begin{array}{l}\text { Dichlorodifluoromethane } \\
\text { Chloromethane } \\
\text { Vinyl Chloride } \\
\text { Bromomethane } \\
\text { Chloroethane } \\
\text { Trichlorofluoromethane } \\
\text { 1,1-Dichloroethene } \\
\text { 1,1,2-Trichloro-1,2,2-trifluoroethane } \\
\text { Acetone } \\
\text { Carbon Disulfide } \\
\text { Methyl Acetate } \\
\text { Methylene Chloride } \\
\text { trans-1,2-Dichloroethene } \\
\text { Methyl tert-Butyl Ether } \\
\text { 1,1-Dichloroethane } \\
\text { cis-1,2-Dichloroethene } \\
\text { 2-Butanone } \\
\text { Chloroform } \\
\text { 1,1,1-Trichloroethane } \\
\text { Cyclohexane } \\
\text { Carbon Tetrachloride } \\
\text { Benzene } \\
\text { 1,2-Dichloroethane }\end{array}$ & $\begin{array}{r}5.0 \\
5.0 \\
5.0 \\
5.0 \\
5.0 \\
5.0 \\
5.0 \\
5.0 \\
5.0 \\
5.0 \\
5.0 \\
4.7 \\
5.0 \\
5.0 \\
5.0 \\
5.0 \\
5.0 \\
78 \\
5.0 \\
5.0 \\
95 \\
5.0 \\
5.0\end{array}$ & $\begin{array}{l}\mathrm{U} \\
\mathrm{U} \\
\mathrm{U} \\
\mathrm{U} \\
\mathrm{U} \\
\mathrm{U} \\
\mathrm{U} \\
\mathrm{U} \\
\mathrm{U} \\
\mathrm{U} \\
\mathrm{U} \\
\mathrm{J} \\
\mathrm{U} \\
\mathrm{U} \\
\mathrm{U} \\
\mathrm{U} \\
\mathrm{U}\end{array}$ \\
\hline
\end{tabular}


Lab Name: ENVIROSYSTEMS, INC.

Contract: ARGONNE

EPA SAMPLE NO.

Lab Code: ENVSYS Case No.: ARG0406 SAS No.:

NATI 4-W-20325

Matrix: (soil/water) WATER

Lab Sample ID: 0060407-02

Sample wt/vol:

$5.000(\mathrm{~g} / \mathrm{mL}) \mathrm{ML}$

Lab File ID: 0000087_2

Level : (low/med) LOW

Date Received: 04/12/06

\% Moisture: not dec.

GC Column: RTX-624 ID: 0.18 (mm)

Date Analyzed: 04/20/06

Soil Extract Volume: (uL)

Dilution Factor: 1.0

Soil Aliquot Volume: (uL)

CONCENTRATION UNITS :

CAS NO.

COMPOUND (ug/L or $\mathrm{ug} / \mathrm{Kg}$ )

UG/I

\begin{tabular}{|c|c|c|c|}
\hline $\begin{array}{r}79-01-6 \\
108-87-2 \\
78-87-5 \\
75-27-4 \\
10061-01-5 \\
108-10-1 \\
108-88-3 \\
10061-02-6 \\
79-00-5 \\
127-18-4 \\
591-78-6 \\
124-48-1 \\
106-93-4 \\
108-90-7 \\
100-41-4 \\
1330-20-7 \\
100-42-5 \\
75-25-2 \\
98-82-8 \\
79-34-5 \\
541-73-1 \\
106-46-7 \\
95-50-1 \\
96-12-8 \\
120-82-1\end{array}$ & $\begin{array}{l}\text { Trichloroethene } \\
\text { Methylcyclohexane } \\
\text { 1,2-Dichloropropane } \\
\text { Bromodichloromethane } \\
\text { cis-1,3-Dichloropropene } \\
\text { 4-Methyl-2-Pentanone } \\
\text { Toluene } \\
\text { trans-1,3-Dichloropropene } \\
\text { 1,1,2-Trichloroethane } \\
\text { Tetrachloroethene } \\
\text { 2-Hexanone } \\
\text { Dibromochloromethane } \\
\text { 1,2-Dibromoethane } \\
\text { Chlorobenzene } \\
\text { Ethylbenzene } \\
\text { Xylene (Total) } \\
\text { Styrene } \\
\text { Bromoform } \\
\text { Isopropylbenzene } \\
\text { 1,1,2,2-Tetrachloroethane } \\
\text { 1,3-Dichlorobenzene } \\
\text { 1,4-Dichlorobenzene } \\
\text { 1,2-Dichlorobenzene } \\
\text { 1,2-Dibromo-3-chloropropane } \\
\text { 1,2,4-Trichlorobenzene }\end{array}$ & $\begin{array}{l}5.0 \\
5.0 \\
5.0 \\
5.0 \\
5.0 \\
5.0 \\
5.0 \\
5.0 \\
5.0 \\
5.0 \\
5.0 \\
5.0 \\
5.0 \\
5.0 \\
5.0 \\
5.0 \\
5.0 \\
5.0 \\
5.0 \\
5.0 \\
5.0 \\
5.0 \\
5.0 \\
5.0 \\
5.0\end{array}$ & $\begin{array}{l}\mathrm{U} \\
\mathrm{U} \\
\mathrm{U} \\
\mathrm{U} \\
\mathrm{U} \\
\mathrm{U} \\
\mathrm{U} \\
\mathrm{U} \\
\mathrm{U} \\
\mathrm{U} \\
\mathrm{U} \\
\mathrm{U} \\
\mathrm{U} \\
\mathrm{U} \\
\mathrm{U} \\
\mathrm{U} \\
\mathrm{U} \\
\mathrm{U} \\
\mathrm{U} \\
\mathrm{U} \\
\mathrm{U} \\
\mathrm{U} \\
\mathrm{U} \\
\mathrm{U} \\
\mathrm{U}\end{array}$ \\
\hline
\end{tabular}


Lab Name: ENVIROSYSTEMS, INC.

Contract : ARGONNE

EPA SAMPLE NO.

NATI4 -W-20325

Lab Code: ENVSYS Case No.: ARG0406 SAS No.:

SDG No.: ARG0406

Matrix: (soil/water) WATER

Sample wt/vol: $5.000(\mathrm{~g} / \mathrm{mL}) \mathrm{ML}$

Level: (low/med) LOW

\% Moisture: not dec.

GC Column: RTX-624 ID: 0.18 (mm)

Soil Extract Volume:

Number TICs found: 0
Lab Sample ID: 0060407-02

Lab File ID: 0000087_2

Date Received: 04/12/06

Date Analyzed: 04/20/06

Dilution Factor: 1.0

Soil Aliquot Volume: (uL)

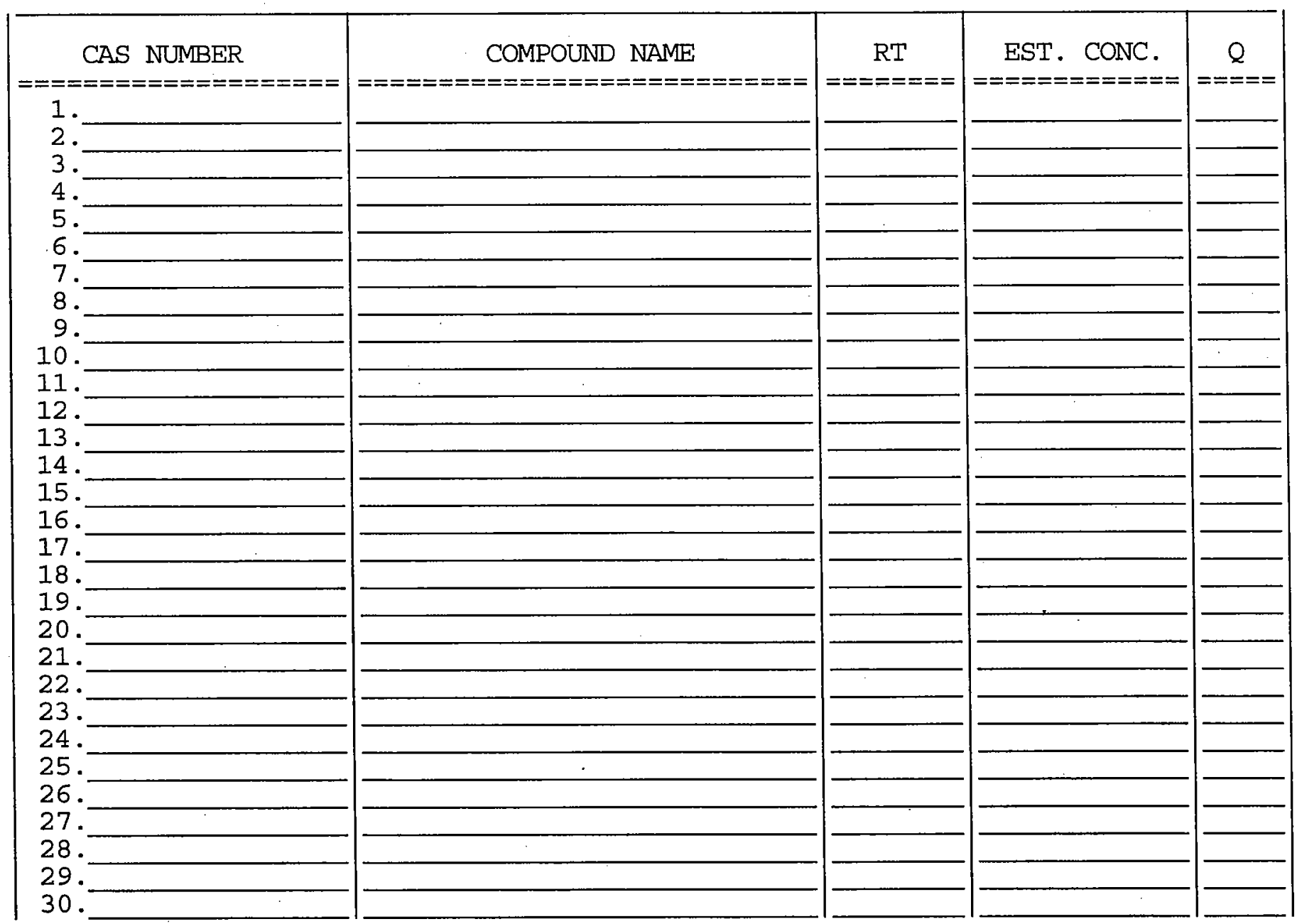


Lab Name: ENVIROSYSTEMS, INC.

Contract: ARGONNE

Lab Code: ENVSYS Case No.: ARG0406 SAS No.:

SDG No.: ARG0406

Matrix: (soil/water) WATER

Sample wt/vol: $\quad 5.000(\mathrm{~g} / \mathrm{mL}) \mathrm{ML}$

Level: (low/med) LOW

\% Moisture: not dec.

GC Column: RTX-624 ID: 0.18 (mm)

Soil Extract Volume: (uL)
Lab Sample ID: 0060407-03

Lab File ID: 0000087_3

Date Received: 04/12/06

Date Analyzed: 04/20/06

Dilution Factor: 1.0

Soil Aliquot Volume: (uL)

CONCENTRATION UNITS :

CAS NO.

COMPOUND

UG/L Q

\begin{tabular}{|c|c|c|c|}
\hline $\begin{array}{r}75-71-8 \\
74-87-3 \\
75-01-4 \\
74-83-9 \\
75-00-3 \\
75-69-4 \\
75-35-4 \\
76-13-1 \\
67-64-1 \\
75-15-0 \\
79-20-9 \\
75-09-2 \\
156-60-5 \\
1634-04-4 \\
75-34-3 \\
156-59-2 \\
78-93-3 \\
67-66-3 \\
71-55-6 \\
110-82-7 \\
56-23-5 \\
71-43-2 \\
107-06-2\end{array}$ & $\begin{array}{l}\text { Dichlorodifluoromethane } \\
\text { Chloromethane } \\
\text { Vinyl Chloride } \\
\text { Bromomethane } \\
\text { Chloroethane } \\
\text { Trichlorofluoromethane } \\
\text { 1,1-Dichloroethene } \\
\text { 1,1,2-Trichloro-1,2,2-trifluoroethane } \\
\text { Acetone } \\
\text { Carbon Disulfide } \\
\text { Methyl Acetate } \\
\text { Methylene Chloride } \\
\text { trans-1,2-Dichloroethene } \\
\text { Methyl tert-Butyl Ether } \\
\text { 1,1-Dichloroethane } \\
\text { cis-1,2-Dichloroethene } \\
\text { 2-Butanone } \\
\text { Chloroform } \\
\text { 1,1,1-Trichloroethane } \\
\text { Cyclohexane } \\
\text { Carbon Tetrachloride } \\
\text { Benzene } \\
\text { 1,2-Dichloroethane }\end{array}$ & $\begin{array}{l}5.0 \\
5.0 \\
5.0 \\
5.0 \\
5.0 \\
5.0 \\
5.0 \\
5.0 \\
4.1 \\
5.0 \\
5.0 \\
2.0 \\
5.0 \\
5.0 \\
5.0 \\
5.0 \\
5.0 \\
5.0 \\
5.0 \\
5.0 \\
5.0 \\
5.0 \\
5.0\end{array}$ & $\begin{array}{l}U \\
U \\
U \\
U \\
U \\
U \\
U \\
U \\
J \\
U \\
U \\
U \\
J B \\
U \\
U \\
U \\
U \\
U \\
U \\
U \\
U \\
U \\
U \\
U \\
U\end{array}$ \\
\hline
\end{tabular}


Lab Name: ENVIROSYSTEMS, INC.

Contract: ARGONNE

NATI2 $-W-20433$

Lab Code: ENVSYS Case No.: ARG0406 SAS No.:

SDG No.: ARG0406

Matrix: (soil/water) WATER

Sample wt/vol: $\quad 5.000(\mathrm{~g} / \mathrm{mL}) \mathrm{ML}$

Level: (low/med) LOW

\% Moisture: not dec.

GC Column: RTX-624 ID: 0.18 (mm)

Soil Extract Volume: (uL)
Lab Sample ID: 0060407-03

Lab File ID: 0000087 3

Date Received: 04/12/06

Date Analyzed: 04/20/06

Dilution Factor: 1.0

Soil Aliquot Volume:
CONCENTRATION UNITS :

$(\mathrm{ug} / \mathrm{L}$ or $\mathrm{ug} / \mathrm{Kg})$ UG/L Q

\begin{tabular}{|c|c|c|c|}
\hline $\begin{array}{r}79-01-6 \\
108-87-2 \\
78-87-5 \\
75-27-4 \\
10061-01-5 \\
108-10-1 \\
108-88-3 \\
10061-02-6 \\
79-00-5 \\
127-18-4 \\
591-78-6 \\
124-48-1 \\
106-93-4 \\
108-90-7 \\
100-41-4 \\
1330-20-7 \\
100-42-5 \\
75-25-2 \\
98-82-8 \\
79-34-5 \\
541-73-1 \\
106-46-7 \\
95-50-1 \\
96-12-8 \\
120-82-1\end{array}$ & $\begin{array}{l}\text { Trichloroethene } \\
\text { Methylcyclohexane } \\
\text { 1,2-Dichloropropane } \\
\text { Bromodichloromethane } \\
\text { cis-1,3-Dichloropropene } \\
\text { 4-Methyl-2-Pentanone } \\
\text { Toluene } \\
\text { trans-1,3-Dichloropropene } \\
\text { 1,1,2-Trichloroethane } \\
\text { Tetrachloroethene } \\
\text { 2-Hexanone } \\
\text { Dibromochloromethane } \\
\text { 1,2-Dibromoethane } \\
\text { Chlorobenzene } \\
\text { Ethylbenzene } \\
\text { Xylene (Total) } \\
\text { Styrene } \\
\text { Bromoform } \\
\text { Isopropylbenzene } \\
\text { 1,1,2,2-Tetrachloroethane } \\
\text { 1,3-Dichlorobenzene } \\
\text { 1,4-Dichlorobenzene } \\
\text { 1,2-Dichlorobenzene } \\
\text { 1,2-Dibromo-3-chloropropane } \\
\text { 1,2,4-Trichlorobenzene }\end{array}$ & $\begin{array}{l}5.0 \\
5.0 \\
5.0 \\
5.0 \\
5.0 \\
5.0 \\
5.0 \\
5.0 \\
5.0 \\
5.0 \\
5.0 \\
5.0 \\
5.0 \\
5.0 \\
5.0 \\
5.0 \\
5.0 \\
5.0 \\
5.0 \\
5.0 \\
5.0 \\
5.0 \\
5.0 \\
5.0 \\
5.0\end{array}$ & $\begin{array}{l}\mathrm{U} \\
\mathrm{U} \\
\mathrm{U} \\
\mathrm{U} \\
\mathrm{U} \\
\mathrm{U} \\
\mathrm{U} \\
\mathrm{U} \\
\mathrm{U} \\
\mathrm{U} \\
\mathrm{U} \\
\mathrm{U} \\
\mathrm{U} \\
\mathrm{U} \\
\mathrm{U} \\
\mathrm{U} \\
\mathrm{U} \\
\mathrm{U} \\
\mathrm{U} \\
\mathrm{U} \\
\mathrm{U} \\
\mathrm{U} \\
\mathrm{U} \\
\mathrm{U} \\
\mathrm{U}\end{array}$ \\
\hline
\end{tabular}


$1 \mathrm{~F}$

VOLATILE ORGANICS ANALYSIS DATA SHEET

TENTATIVELY IDENTIFIED COMPOUNDS
EPA SAMPLE NO.

NATI2 $-W-20433$

Lab Name: ENVIROSYSTEMS, INC.

Contract: ARGONNE

Lab Code: ENVSYS Case No.: ARG0406 SAS No.:

SDG No. : ARG0406

Matrix: (soil/water) WATER

Lab Sample ID: 0060407-03

Sample wt/vol: $5.000(\mathrm{~g} / \mathrm{mL}) \mathrm{ML}$

Lab File ID: 0000087 3

Level : (low/med) LOW

Date Received: 04/12/06

\% Moisture: not dec.

Date Analyzed: 04/20/06

GC Column: RTX-624 ID: 0.18 (mm)

Dilution Factor: 1.0

Soil Extract Volume:

Soil Aliquot Volume:

Number TICs found: 0

CONCENTRATION UNITS :

(ug/L or $\mathrm{ug} / \mathrm{Kg}) \mathrm{ug} / \mathrm{L}$

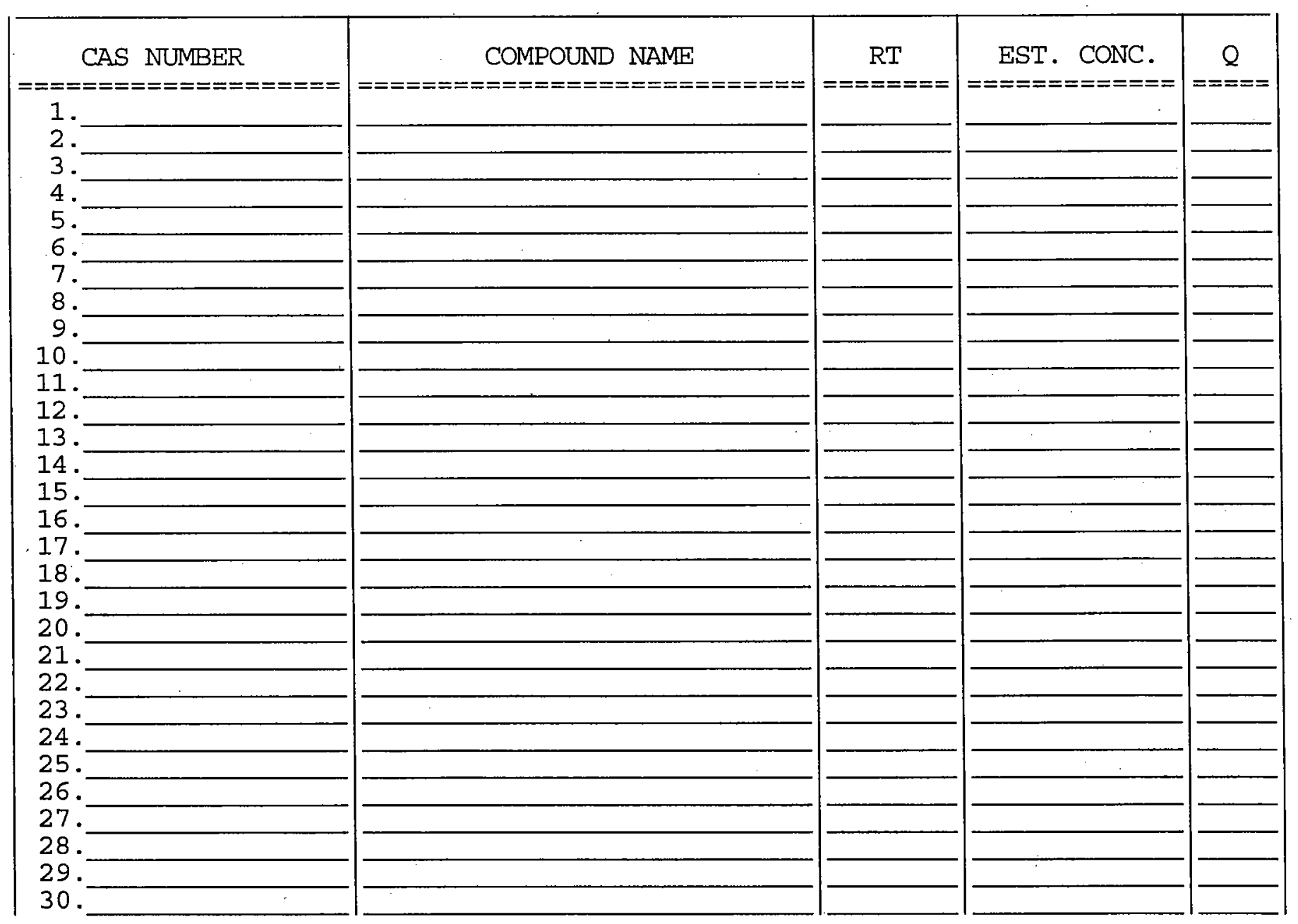

FORM I VOA-TIC 
Lab Name: ENVIROSYSTEMS, INC. Contract: ARGONNE EPA SAMPLE NO.

Lab Code: ENVSYS Case No.: ARG0406 SAS No.: NAL2 -W-20190

Matrix: (soil/water) WATER

Lab Sample ID: 0060407-04

Sample wt/vol:

$5.000(\mathrm{~g} / \mathrm{mL}) \mathrm{ML}$

Lab File ID: 000008744

Level : (low/med) LOW

Date Received: 04/12/06

$\%$ Moisture: not dec.

GC Column: RTX-624 ID: 0.18 (mm)

Date Analyzed: 04/20/06

Soil Extract Volume: (uL)

Dilution Factor: 1.0

Soil Aliquot Volume: (uI)

CONCENTRATION UNITS:

CAS NO.

COMPOUND

(ug/L or ug/Kg) UG/L Q

\begin{tabular}{|r|l|c|c|}
\hline $75-71-8$ & Dichlorodifluoromethane & 5.0 & $\mathrm{U}$ \\
$74-87-3$ & Chloromethane & 5.0 & $\mathrm{U}$ \\
$75-01-4$ & Vinyl Chloride & 5.0 & $\mathrm{U}$ \\
$74-83-9$ & Bromomethane & 5.0 & $\mathrm{U}$ \\
$75-00-3$ & Chloroethane & 5.0 & $\mathrm{U}$ \\
$75-69-4$ & Trichlorofluoromethane & 5.0 & $\mathrm{U}$ \\
$75-35-4$ & 1,1-Dichloroethene & 5.0 & $\mathrm{U}$ \\
$76-13-1$ & $1,1,2-$ Trichloro-1,2,2-trifluoroethane & 5.0 & $\mathrm{U}$ \\
$67-64-1$ & Acetone & 5.0 & $\mathrm{U}$ \\
$75-15-0$ & Carbon Disulfide & 5.0 & $\mathrm{U}$ \\
$79-20-9$ & Methyl Acetate & 5.0 & $\mathrm{U}$ \\
$75-09-2$ & Methylene Chloride & 1.3 & $\mathrm{JB}$ \\
$156-60-5$ & trans-1,2-Dichloroethene & 5.0 & $\mathrm{U}$ \\
$1634-04-4$ & Methyl tert-Butyl Ether & 5.0 & $\mathrm{U}$ \\
$75-34-3$ & $1,1-$ Dichloroethane & 5.0 & $\mathrm{U}$ \\
$156-59-2$ & Cis-1,2-Dichloroethene & 5.0 & $\mathrm{U}$ \\
$78-93-3$ & 2-Butanone & 5.0 & $\mathrm{U}$ \\
$67-66-3$ & Chloroform & 5.0 & $\mathrm{U}$ \\
$71-55-6$ & $1,1,1-$ Trichloroethane & 5.0 & $\mathrm{U}$ \\
$110-82-7$ & Cyclohexane & 5.0 & $\mathrm{U}$ \\
$56-23-5$ & Carbon Tetrachloride & 5.0 & $\mathrm{U}$ \\
$71-43-2$ & Benzene & 5.0 & $\mathrm{U}$ \\
$107-06-2$ & 1,2-Dichloroethane & 5.0 & $\mathrm{U}$ \\
\hline
\end{tabular}


Lab Name: ENVIROSYSTEMS, INC.

Contract: ARGONNE

NAL2 $-W-20190$

Lab Code: ENVSYS Case No.: ARG0406 SAS No.:

SDG No.: ARG0406

Matrix: (soil/water) WATER

Sample wt/vol:

$5.000(\mathrm{~g} / \mathrm{mL}) \mathrm{ML}$

Level: (low/med) LOW

\% Moisture: not dec.

GC Column: RTX-624 ID: 0.18 (mm)

Soil Extract Volume: (uL)
Lab Sample ID: 0060407-04

Lab File ID: 0000087_4

Date Received: 04/12/06

Date Analyzed: 04/20/06

Dilution Factor: 1.0

Soil Aliquot Volume: (uI)
CAS NO.

COMPOUND
CONCENTRATION UNITS : (ug/L or $\mathrm{ug} / \mathrm{Kg}$ ) UG/L Q

\begin{tabular}{|r|}
$79-01-6$ \\
$108-87-2$ \\
$78-87-5$ \\
$75-27-4$ \\
$10061-01-5$ \\
$108-10-1$ \\
$108-88-3$ \\
$10061-02-6$ \\
$79-00-5$ \\
$127-18-4$ \\
$591-78-6$ \\
$124-48-1$ \\
$106-93-4$ \\
$108-90-7$ \\
$100-41-4$ \\
$1330-20-7$ \\
$100-42-5$ \\
$75-25-2$ \\
$98-82-8$ \\
$79-34-5$ \\
$541-73-1$ \\
$106-46-7$ \\
$95-50-1$ \\
$96-12-8$ \\
$120-82-1$ \\
\hline
\end{tabular}

\begin{tabular}{l|} 
Trichloroethene \\
Methylcyclohexane \\
1,2-Dichloropropane \\
Bromodichloromethane \\
cis-1,3-Dichloropropene \\
4-Methyl-2-Pentanone \\
Toluene \\
trans-1,3-Dichloropropene \\
1,1,2-Trichloroethane \\
Tetrachloroethene \\
2-Hexanone \\
Dibromochloromethane \\
1,2-Dibromoethane \\
Chlorobenzene \\
Ethylbenzene \\
Xylene (Total) \\
Styrene \\
Bromoform \\
Isopropylbenzene \\
1,1,2,2-Tetrachloroethane \\
1,3-Dichlorobenzene \\
1,4-Dichlorobenzene \\
1,2-Dichlorobenzene \\
1,2-Dibromo-3-chloropropane \\
1,2,4-Trichlorobenzene
\end{tabular}

\begin{tabular}{|l|l|}
\hline 5.0 & $\mathrm{U}$ \\
5.0 & $\mathrm{U}$ \\
5.0 & $\mathrm{U}$ \\
5.0 & $\mathrm{U}$ \\
5.0 & $\mathrm{U}$ \\
5.0 & $\mathrm{U}$ \\
5.0 & $\mathrm{U}$ \\
5.0 & $\mathrm{U}$ \\
5.0 & $\mathrm{U}$ \\
5.0 & $\mathrm{U}$ \\
5.0 & $\mathrm{U}$ \\
5.0 & $\mathrm{U}$ \\
5.0 & $\mathrm{U}$ \\
5.0 & $\mathrm{U}$ \\
5.0 & $\mathrm{U}$ \\
5.0 & $\mathrm{U}$ \\
5.0 & $\mathrm{U}$ \\
5.0 & $\mathrm{U}$ \\
5.0 & $\mathrm{U}$ \\
5.0 & $\mathrm{U}$ \\
5.0 & $\mathrm{U}$ \\
5.0 & $\mathrm{U}$ \\
5.0 & $\mathrm{U}$ \\
5.0 & $\mathrm{U}$ \\
5.0 & $\mathrm{U}$ \\
\hline
\end{tabular}


$1 \mathrm{~F}$

VOLATILE ORGANICS ANALYSIS DATA SHEET

TENTATIVELY IDENTIFIED COMPOUNDS
EPA SAMPLE NO:

NAL2 $-W-20190$

Lab Name: ENVIROSYSTEMS, INC.

Contract: ARGONNE

Lab Code: ENVSYS Case No.: ARG0406 SAS No.:

SDG No.: ARG0406

Matrix: (soil/water) WATER

Lab Sample ID: 0060407-04

Sample wt/vol: $5.000(\mathrm{~g} / \mathrm{mL}) \mathrm{ML}$

Lab File ID: 0000087_4

Level: (low/med) LOW

Date Received: 04/12/06

\% Moisture: not dec.

Date Analyzed: 04/20/06

GC Column: RTX-624 ID: 0.18 (mm)

Dilution Factor: 1.0

Soil Extract Volume: (u山)

Soil Aliquot Volume:

Number TICs found: 0

CONCENTRATION UNITS :

(ug/L or $u g / \mathrm{Kg}$ ) ug/L

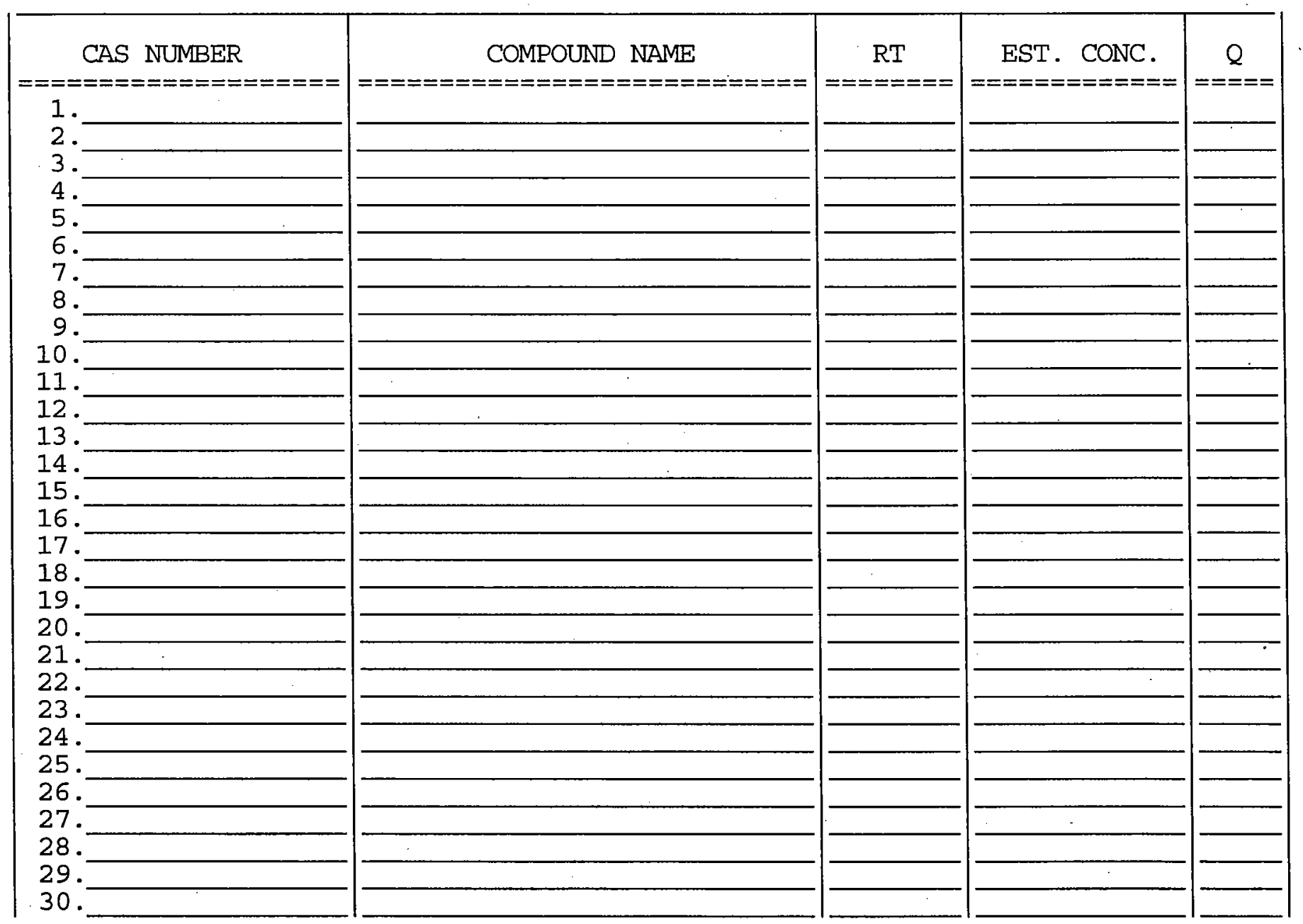


$1 \mathrm{~A}$

VOLATILE ORGANICS ANALYSIS DATA SHEET

Lab Name: ENVIROSYSTEMS, INC.

Contract: ARGONNE

Lab Code: ENVSYS Case No.: ARG0406 SAS No.:

SDG No.: ARG0406

Matrix: (soil/water) WATER

Lab Sample ID: 0060407-05

Sample wt/vol:

$5.000(\mathrm{~g} / \mathrm{mL}) \mathrm{ML}$

Level: (low/med) LOW

\% Moisture: not dec.

GC Column: RTX-624 ID: 0.18 (mm) (u广)

Lab File ID: 0000087_5

Date Received: 04/12/06

Date Analyzed: 04/20/06

Soil Extract Volume:

Dilution Factor: 1.0

Soil Aliquot Volume: (uL)

CONCENTRATION UNITS:

CAS NO.

COMPOUND

(ug/L or $\mathrm{ug} / \mathrm{Kg}$ )

UG/I Q

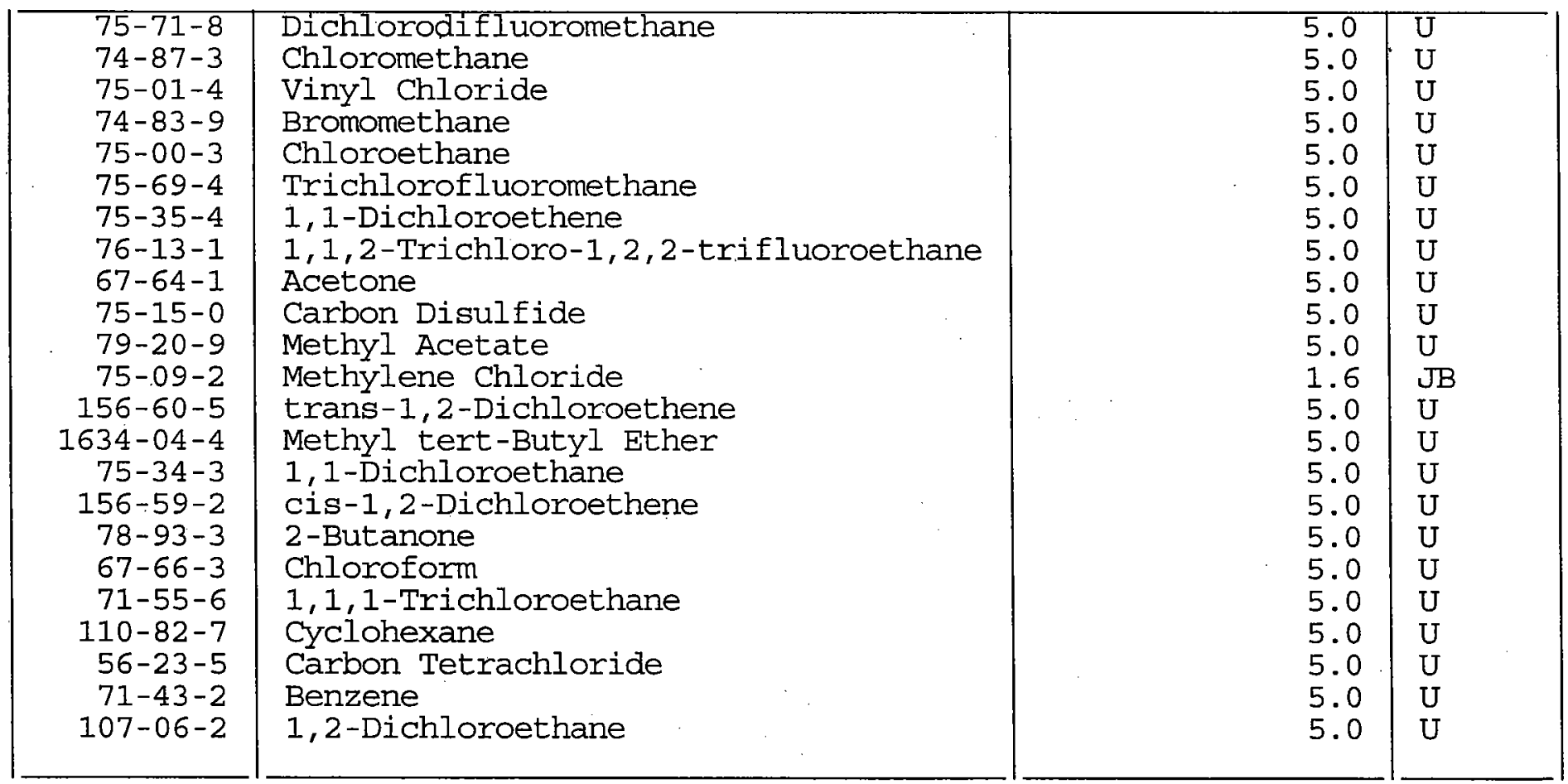


$1 \mathrm{~B}$

VOLATILE ORGANICS ANALYSIS DATA SHEET

Lab Name: ENVIROSYSTEMS, INC.

Contract: ARGONNE

EPA SAMPLE NO.

NAQCTB-W

-11APR06

Lab Code: ENVSYS Case No.: ARG0406 SAS No.:

SDG No.: ARG0406

Matrix: (soil/water) WATER

Sample wt/vol:

$5.000(\mathrm{~g} / \mathrm{mL}) \mathrm{ML}$

Level: (low/med) LoW

\% Moisture: not dec.

GC Column: RTX-624 ID: 0.18 (mm)

Soil Extract Volume: (uI)

COMPOUND
Lab Sample ID: 0060407-05

Lab File ID: 0000087 _5

Date Received: 04/12/06

Date Analyzed: 04/20/06

Dilution Factor: 1.0

Soil Aliquot Volume: (UI)
CAS NO.

CAS NO.

\begin{tabular}{|r|}
\hline $79-01-6$ \\
$108-87-2$ \\
$78-87-5$ \\
$75-27-4$ \\
$10061-01-5$ \\
$108-10-1$ \\
$108-88-3$ \\
$10061-02-6$ \\
$79-00-5$ \\
$127-18-4$ \\
$591-78-6$ \\
$124-48-1$ \\
$106-93-4$ \\
$108-90-7$ \\
$100-41-4$ \\
$1330-20-7$ \\
$100-42-5$ \\
$75-25-2$ \\
$98-82-8$ \\
$79-34-5$ \\
$541-73-1$ \\
$106-46-7$ \\
$95-50-1$ \\
$96-12-8$ \\
$120-82-1$ \\
\hline
\end{tabular}

CONCENTRATION UNITS: (ug/L or ug/Kg) UG/L Q 
$1 \mathrm{~F}$

VOLATILE ORGANICS ANALYSIS DATA SHEET

TENTATIVELY IDENTIFIED COMPOUNDS
EPA SAMPLE NO.

NAQCTB-W

-11APR06

Lab Name: ENVIROSYSTEMS, INC.

Contract : ARGONNE

Lab Code: ENVSYS Case No.: ARG0406 SAS No.:

SDG No.: ARG0406

Matrix: (soil/water) WATER

Sample wt/vol: $5.000(\mathrm{~g} / \mathrm{mL}) \mathrm{ML}$

Level: (low/med) LOW

\% Moisture: not dec.

GC Column: RTX-624 ID: 0.18 (mm)

Soil Extract Volume:

(uL)

Number TICs found: 0
Lab Sample ID: 0060407-05

Lab File ID: 0000087 _5

Date Received: 04/12/06

Date Analyzed: 04/20/06

Dilution Factor: 1.0

Soil Aliquot Volume: (u山)

CONCENTRATION UNITS :

(ug/L or $\mathrm{ug} / \mathrm{Kg}$ ) ug/L

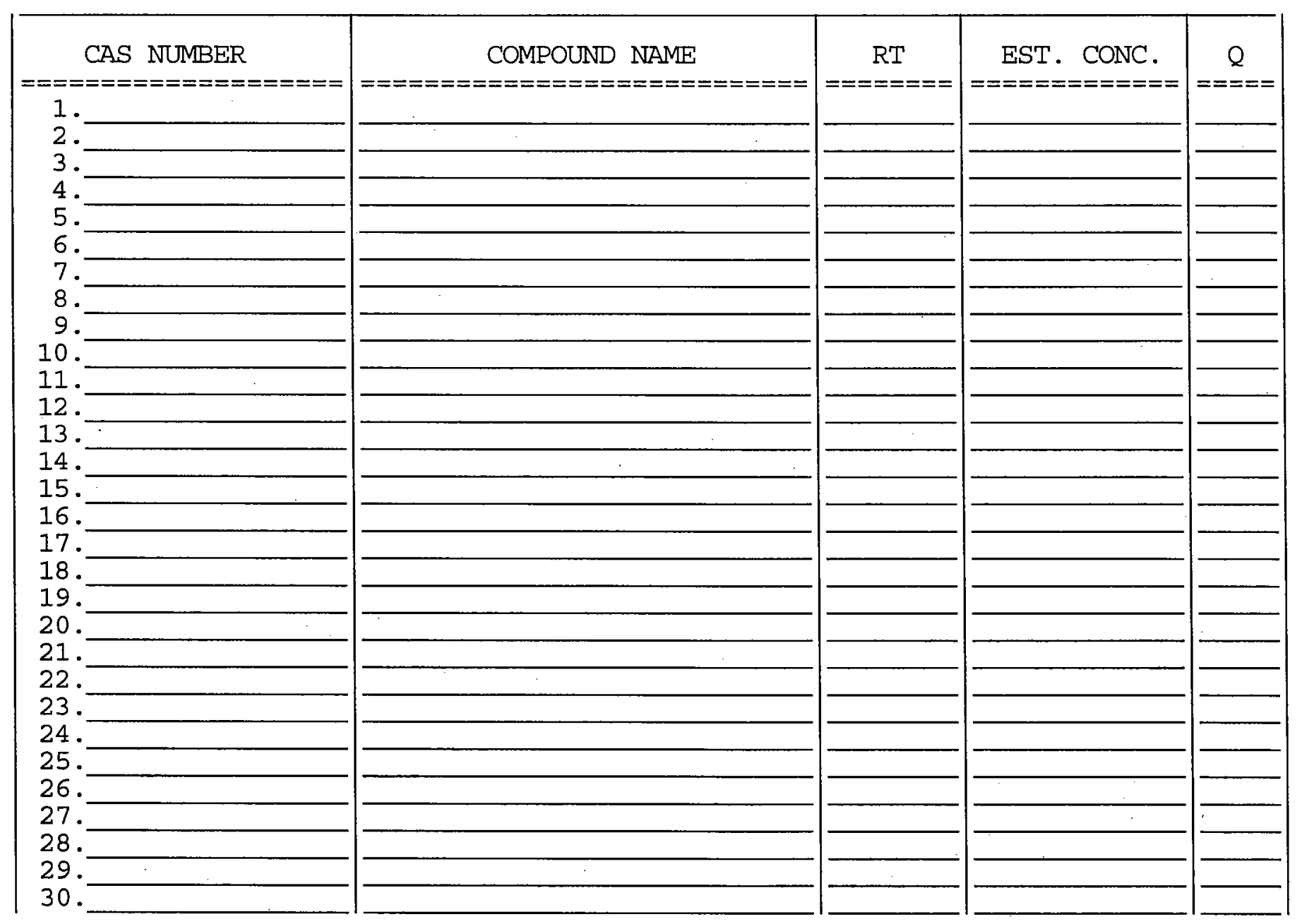




\section{ENVIROSYSTEMS, INC.}

9200 Rumsey Road • Suite B102 • Columbia, Maryland 21045-1934

Phone (410) 964-0330 - Fax (410) 740-9306

Email: info@envsystems.com • Webpage: www.envsystems.com/envsys

June 6, 2006

Jorge S. Alvarado, Ph.D

Argonne National Laboratory

Environmental Research Division

Applied Geoscience and Environmental

Management Section

9700 South Cass Avenue, ER-203

Argonne, Illinois 60439

\section{RE: ENVSYS Report 0606053}

Dear Jorge:

Enclosed is the Analytical Data Package for the samples received on May 24, 2006 for volatile organics analysis by US EPA CLP SOW OLM04.3

Please do not hesitate to call me if you have any questions, comments, or require additional information.

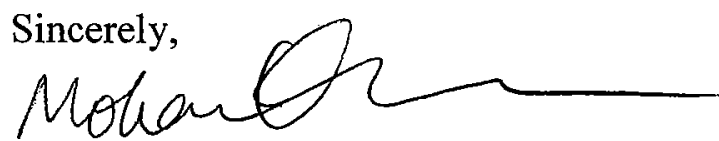

Mohan Khare, Ph.D

President/CEO

$\mathrm{MK} / \mathrm{sp}$ 
1. Narrative 
LABORATORY NAME: ENVIROSYSTEMS, INC.

CLIENT: Argonne National Laboratory RESORT \# 0606053

DATA SAMPLES RECEIVED AT LABORATORY: MAY 24TH, 2006

SAMPLE ANALYSES INCLUDED IN THIS REPORT:

\begin{tabular}{|c|c|c|c|c|}
\hline CLIENT \# & LAB ID\# & ANALYSIS & MATRIX & VOA Ph \\
\hline NA-TI18-W-20706 & $0060511-01$ & VOA & WATER & 7 \\
\hline NA-TI29-W-20901 & $0060511-02$ & VOA & WATER & 7 \\
\hline NA-TI18-W-20655 & $0060511-03$ & VOA & WATER & 7 \\
\hline NA-TI27-W-20905 & 0060511-04 & VOA & WATER & 7 \\
\hline NA-TI18-W-20709 & $0060511-05$ & VOA & WATER & 7 \\
\hline NA-TI27-W-20700 & 0060511-06 & VOA & WATER & 7 \\
\hline NA-TI28-W-20904 & $0060511-07$ & VOA & WATER & 7 \\
\hline NA-052306 & $0060511-08$ & VOA & WATER & 7 \\
\hline
\end{tabular}

Matrix spike/matrix spike duplicate analysis was not performed for this case.

The following samples had to be diluted due to concentrations exceeding the upper calibration range: NA-TI18-W20706, NA-TI29-W-20901, NA-TI18-W-20655, and NA-TI28-W-20904.

Samples for this SDG are analyzed by EPA SOW OLMO4.3 for multi-media multi-concentration organics. Sample detection limits have been modified to meet client requirements.

The cooler temperature was measured to be 2 degrees Celsius upon receipt

The volatile analysis was performed on an Agilent 5975 GC/MS using a Restek RTX-624 20 meter column with an inner diameter of $0.18 \mathrm{~mm}$ and a 1 micron film thickness. The trap used with the autosampler is a $0.3 \mathrm{~cm}$ OD x 28.5 cm L ENCON Ambient Packed Trap.

Three compounds did not meet the initial calibration criteria. Percent RSD for bromomethane and bromoform were greater than 20.5 and the response factor for 1,1,2,2 tetrachloroethane was below the minimum required. The continuing calibration VSTD050HW, VSTD050HD, and VSTD050HE had three compounds with percent D values greater than 25.0.

All other QC criteria were met for all samples included in this report.

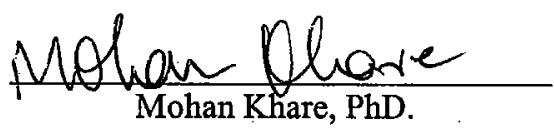

DATE: $\quad 6 / 5 / 06$ 
2. SGD Cover Sheet/Traffic Reports 


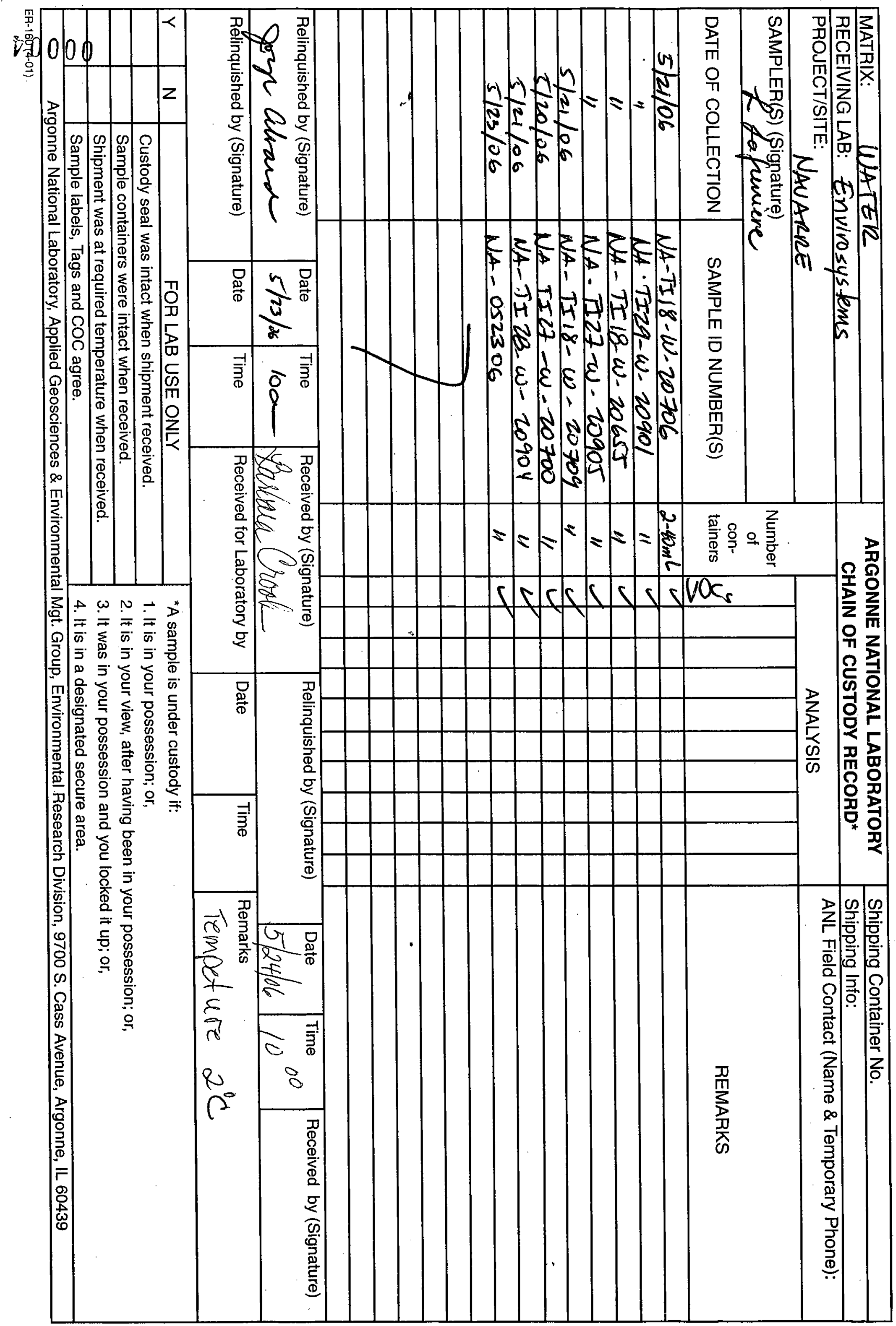


VOLATILE SAMPLE DATA 
Lab Name: ENVIROSYSTEMS, INC.

Contract: ARGONNE

NA-TI18-

$\mathrm{W}-20706 \mathrm{HW}$

Lab Code: ENVSYS Case No.: AR0511W SAS No.:

SDG NO.: AR0511W

Matrix: (soil/water) WATER

Lab Sample ID: 0060511-01

Sample wt/vol:

$5.000(\mathrm{~g} / \mathrm{mL}) \mathrm{ML}$

Lab File ID: AG75HC065

Level: (low/med) LOW

Date Received: 05/24/06

$\%$ Moisture: not dec.

GC Column: RTX-624 ID: 0.18 (mm)

Date Analyzed: 05/24/06

Soil Extract Volume:

(uL)

Dilution Factor: 1.0

Soil Aliquot Volume:

CAS NO.

COMPOUND

CONCENTRATION UNITS:

(ug/I or ug/Kg) UG/L Q

\begin{tabular}{|c|c|c|c|}
\hline $\begin{array}{r}75-71-8 \\
74-87-3 \\
75-01-4 \\
74-83-9 \\
75-00-3 \\
75-69-4 \\
75-35-4 \\
76-13-1 \\
67-64-1 \\
75-15-0 \\
79-20-9 \\
75-09-2 \\
156-60-5 \\
1634-04-4 \\
75-34-3 \\
156-59-2 \\
78-93-3 \\
67-66-3 \\
71-55-6 \\
110-82-7 \\
56-23-5 \\
71-43-2 \\
107-06-2\end{array}$ & $\begin{array}{l}\text { Dichlorodifluoromethane } \\
\text { Chloromethane } \\
\text { Vinyl Chloride } \\
\text { Bromomethane } \\
\text { Chloroethane } \\
\text { Trichlorofluoromethane } \\
\text { 1,1-Dichloroethene } \\
\text { 1,1,2-Trichloro-1,2,2-trifluoroethane } \\
\text { Acetone } \\
\text { Carbon Disulfide } \\
\text { Methyl Acetate } \\
\text { Methylene Chloride } \\
\text { trans-1,2-Dichloroethene } \\
\text { Methyl tert-Butyl Ether } \\
\text { 1,1-Dichloroethane } \\
\text { cis-1,2-Dichloroethene } \\
2-\text { Butanone } \\
\text { Chloroform } \\
\text { 1,1,1-Trichloroethane } \\
\text { Cyclohexane } \\
\text { Carbon Tetrachloride } \\
\text { Benzene } \\
\text { 1,2-Dichloroethane }\end{array}$ & $\begin{array}{r}5.0 \\
5.0 \\
5.0 \\
5.0 \\
5.0 \\
5.0 \\
5.0 \\
5.0 \\
5.0 \\
5.0 \\
5.0 \\
4.4 \\
5.0 \\
5.0 \\
5.0 \\
5.0 \\
5.0 \\
55 \\
5.0 \\
5.0 \\
240 \\
5.0 \\
5.0\end{array}$ & $\begin{array}{l}U \\
U \\
U \\
U \\
U \\
U \\
U \\
U \\
U \\
U \\
U \\
J B \\
U \\
U \\
U \\
U \\
U \\
U \\
U \\
E \\
U \\
U\end{array}$ \\
\hline
\end{tabular}


Lab Name: ENVIROSYSTEMS, INC.

Contract: ARGONNE

NA-TI18$\mathrm{W}-20706 \mathrm{HW}$

Lab Code: ENVSYS Case No.: AR0511W SAS No.:

SDG No.: AR0511W

Matrix: (soil/water) WATER

Sample wt/vol:

$5.000(\mathrm{~g} / \mathrm{mL}) \mathrm{ML}$

Level: (low/med) LOW

\% Moisture: not dec.

GC Column: RTX-624 ID: 0.18 (mm)

Soil Extract Volume: (uL)
Lab Sample ID: 0060511-01

Lab File ID: AG75HC065

Date Received: 05/24/06

Date Analyzed: 05/24/06

Dilution Factor: 1.0

Soil Aliquot Volume: (uL)

CONCENTRATION UNITS:

CAS NO.

COMPOUND

(ug/L or $\mathrm{ug} / \mathrm{Kg}$ )

UG/L

\begin{tabular}{|c|c|c|c|}
\hline $\begin{array}{r}79-01-6 \\
108-87-2 \\
78-87-5 \\
75-27-4 \\
10061-01-5 \\
108-10-1 \\
108-88-3 \\
10061-02-6 \\
79-00-5 \\
127-18-4 \\
591-78-6 \\
124-48-1 \\
106-93-4 \\
108-90-7 \\
100-41-4 \\
1330-20-7 \\
100-42-5 \\
75-25-2 \\
98-82-8 \\
79-34-5 \\
541-73-1 \\
106-46-7 \\
95-50-1 \\
96-12-8 \\
120-82-1\end{array}$ & $\begin{array}{l}\text { Trichloroethene } \\
\text { Methylcyclohexane } \\
\text { 1,2-Dichloropropane } \\
\text { Bromodichloromethane } \\
\text { cis-1,3-Dichloropropene } \\
\text { 4-Methyl-2-Pentanone } \\
\text { Toluene } \\
\text { trans-1,3-Dichloropropene } \\
\text { 1,1,2-Trichloroethane } \\
\text { Tetrachloroethene } \\
\text { 2-Hexanone } \\
\text { Dibromochloromethane } \\
\text { 1,2-Dibromoethane } \\
\text { Chlorobenzene } \\
\text { Ethylbenzene } \\
\text { Xylene (Total) } \\
\text { Styrene } \\
\text { Bromoform } \\
\text { Isopropylbenzene } \\
\text { 1,1,2,2-Tetrachloroethane } \\
\text { 1,3-Dichlorobenzene } \\
\text { 1,4-Dichlorobenzene } \\
\text { 1,2-Dichlorobenzene } \\
\text { 1,2-Dibromo-3-chloropropane } \\
1,2,4 \text {-Trichlorobenzene }\end{array}$ & $\begin{array}{l}5.0 \\
5.0 \\
5.0 \\
5.0 \\
5.0 \\
5.0 \\
5.0 \\
5.0 \\
5.0 \\
5.0 \\
5.0 \\
5.0 \\
5.0 \\
5.0 \\
5.0 \\
5.0 \\
5.0 \\
5.0 \\
5.0 \\
5.0 \\
5.0 \\
5.0 \\
5.0 \\
5.0 \\
5.0\end{array}$ & $\begin{array}{l}U \\
U \\
U \\
U \\
U \\
U \\
U \\
U \\
U \\
U \\
U \\
U \\
U \\
U \\
U \\
U \\
U \\
U \\
U \\
U \\
U \\
U \\
U \\
U \\
U\end{array}$ \\
\hline
\end{tabular}


Lab Name: ENVIROSYSTEMS, INC. Contract: ARGONNE

Lab Code: ENVSYS Case No.: AR0511W SAS No.:

SDG No. : AR0511W

Matrix: (soil/water) WATER

Sample $w t / \mathrm{Vol}: 5.000(\mathrm{~g} / \mathrm{mL}) \mathrm{ML}$

Level: (low/med) LOW

\% Moisture: not dec.

GC Column: RTX-624 ID: 0.18 (mm)

Soil Extract Volume: (uL)

Number TICs found: 1
Lab Sample ID: 0060511-01

Lab File ID: AG75HC065

Date Received: 05/24/06

Date Analyzed: 05/24/06

Dilution Factor: 1.0

Soil Aliquot Volume: (uL)

(ug/L or $u g / \mathrm{Kg}$ ) ug/L

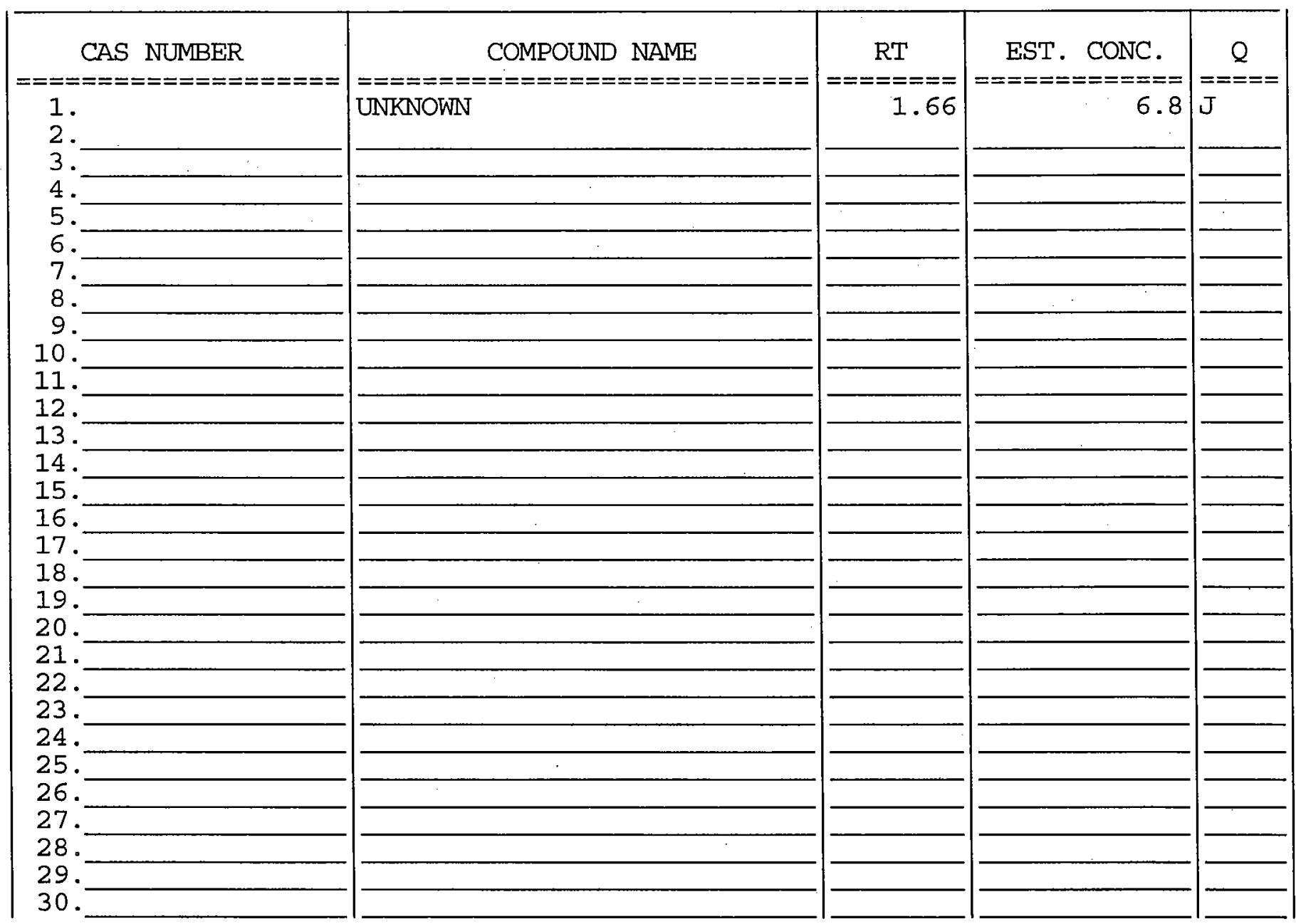


Lab Name: ENVIROSYSTEMS, INC.

Contract: ARGONNE

Lab Code: ENVSYS Case No.: AR0511W SAS No.:

SDG No. : AR0511W

Matrix: (soil/water) WATER

Sample wt/vol: $\quad 5.000(\mathrm{~g} / \mathrm{mL}) \mathrm{ML}$

Level: (low/med) LOW

$\%$ Moisture: not dec.

GC Column: RTX-624 ID: 0.18 (mm)

Soil Extract Volume: (uL)
Lab Sample ID: 0060511-01RE

Lab File ID: AG75HC113

Date Received: 05/24/06

Date Analyzed: 05/31/06

Dilution Factor: 2.0

Soil Aliquot Volume: (uJ)

CONCENTRATION UNITS :

CAS NO.

COMPOUND

(ug/L or $\mathrm{ug} / \mathrm{Kg}$ )

UG/L Q

\begin{tabular}{|c|c|c|c|}
\hline $\begin{array}{r}75-71-8 \\
74-87-3 \\
75-01-4 \\
74-83-9 \\
75-00-3 \\
75-69-4 \\
75-35-4 \\
76-13-1 \\
67-64-1 \\
75-15-0 \\
79-20-9 \\
75-09-2 \\
156-60-5 \\
1634-04-4 \\
75-34-3 \\
156-59-2 \\
78-93-3 \\
67-66-3 \\
71-55-6 \\
110-82-7 \\
56-23-5 \\
71-43-2 \\
107-06-2\end{array}$ & $\begin{array}{l}\text { Dichlorodifluoromethane } \\
\text { Chloromethane } \\
\text { Vinyl Chloride } \\
\text { Bromomethane } \\
\text { Chloroethane } \\
\text { Trichlorofluoromethane } \\
\text { 1,1-Dichloroethene } \\
\text { 1,1,2-Trichloro-1,2,2-trifluoroethane } \\
\text { Acetone } \\
\text { Carbon Disulfide } \\
\text { Methyl Acetate } \\
\text { Methylene Chloride } \\
\text { trans-1,2-Dichloroethene } \\
\text { Methyl tert-Butyl Ether } \\
\text { 1,1-Dichloroethane } \\
\text { cis-1,2-Dichloroethene } \\
\text { 2-Butanone } \\
\text { Chloroform } \\
\text { 1,1,1-Trichloroethane } \\
\text { Cyclohexane } \\
\text { Carbon Tetrachloride } \\
\text { Benzene } \\
\text { 1,2-Dichloroethane }\end{array}$ & $\begin{array}{r}10 \\
10 \\
10 \\
10 \\
10 \\
10 \\
10 \\
10 \\
10 \\
10 \\
10 \\
7.3 \\
10 \\
10 \\
10 \\
10 \\
10 \\
55 \\
10 \\
10 \\
270 \\
10 \\
10\end{array}$ & $\begin{array}{l}U \\
U \\
U \\
U \\
U \\
U \\
U \\
U \\
U \\
U \\
U \\
J B \\
U \\
U \\
U \\
U \\
U \\
\bar{U} \\
U\end{array}$ \\
\hline
\end{tabular}


Lab Name: ENVIROSYSTEMS, INC.

Contract: ARGONNE

Lab Code: ENVSYS Case No.: AR0511W SAS No.:

SDG No. : AR0511W

Matrix: (soil/water) WATER

Sample wt/vol: $\quad 5.000(\mathrm{~g} / \mathrm{mL}) \mathrm{ML}$

Level: (low/med) LOW

\% Moisture: not dec.

GC Column: RTX-624 ID: 0.18 (mm)

Soil Extract Volume: (uL)
Lab Sample ID: 0060511-01RE

Lab File ID: AG75HC113

Date Received: 05/24/06

Date Analyzed: 05/31/06

Dilution Factor: 2.0

Soil Aliquot Volume: (uL)

CONCENTRATION UNITS:

CAS NO.

COMPOUND
UG/L Q

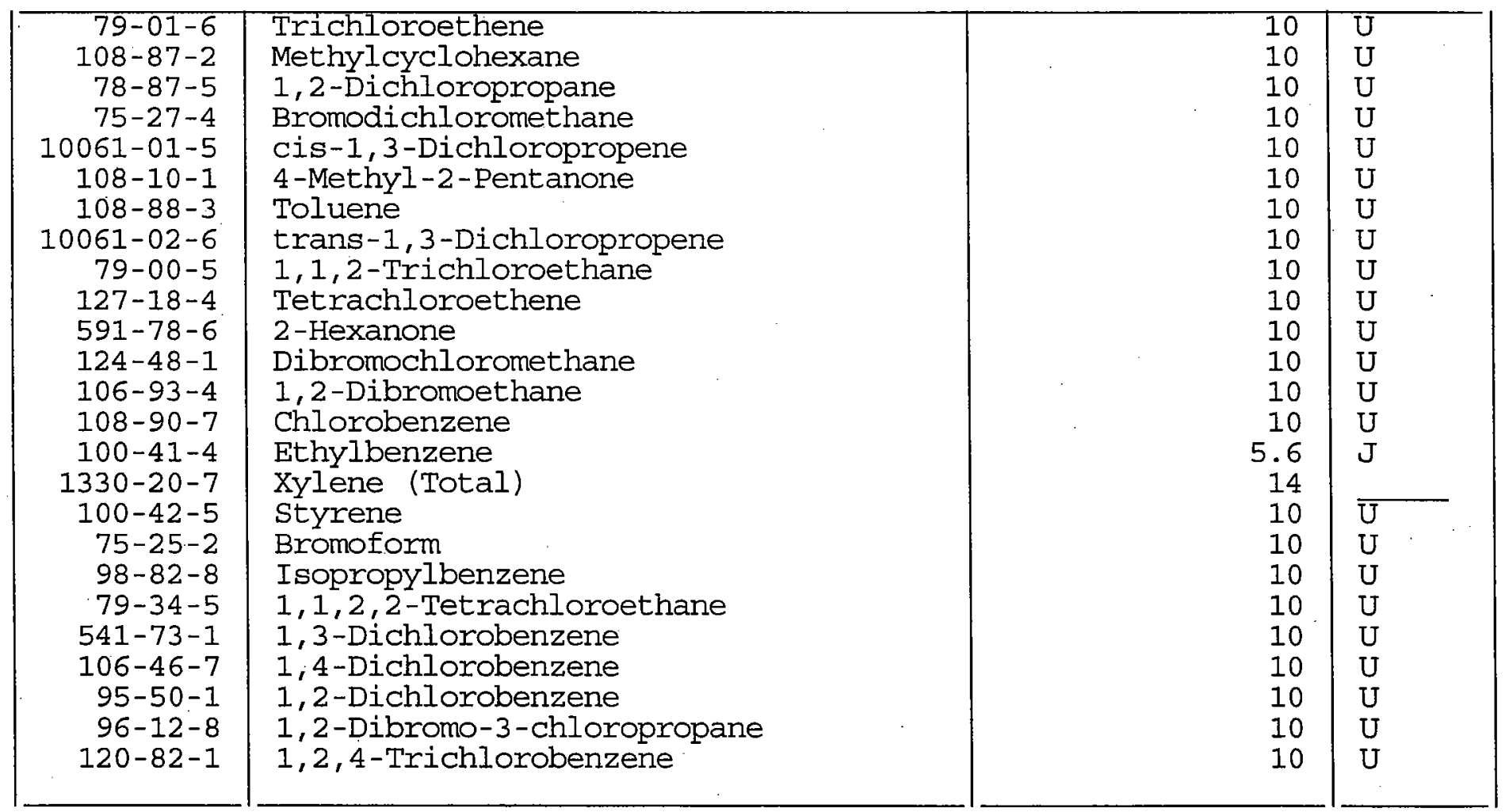


Lab Name: ENVIROSYSTEMS, INC.

Contract: ARGONNE

Lab Code: ENVSYS Case No.: AR0511W SAS No.:

SDG No.: AR0511W

Matrix: (soil/water) WATER

Sample wt/vol: $5.000(\mathrm{~g} / \mathrm{mL}) \mathrm{ML}$

Level: (low/med) LOW

\% Moisture: not dec.

GC Column: RTX-624 ID: 0.18 (mm)

Soil Extract Volume:

(uL)

Number TICs found: 2
Lab Sample ID: 0060511-01RE

Lab File ID: AG75HC113

Date Received: 05/24/06

Date Analyzed: 05/31/06

Dilution Factor: 2.0

Soil Aliquot Volume: (UL)

CONCENTRATION UNITS :

(ug/L or $u g / \mathrm{Kg}$ ) ug/L

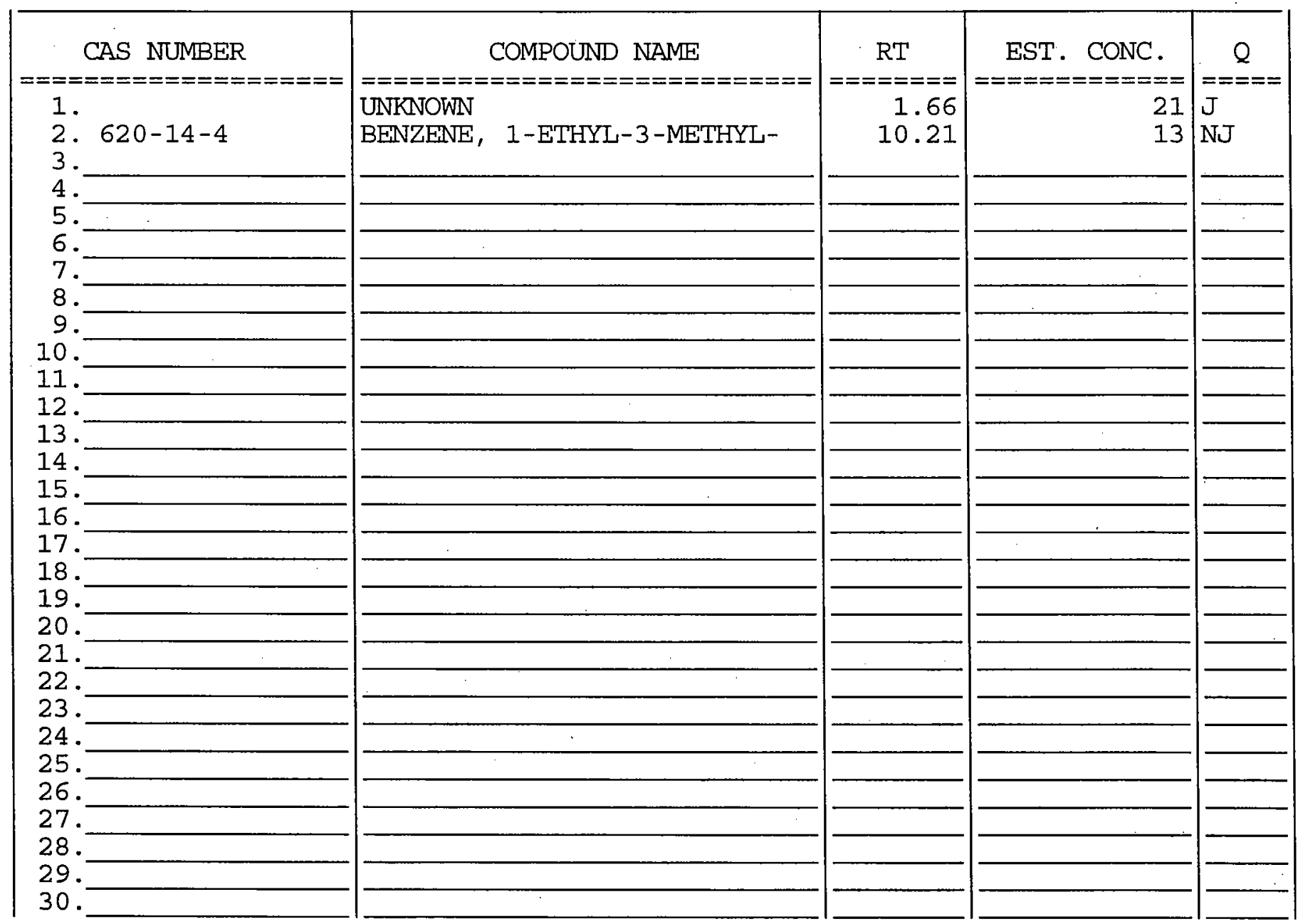


$1 A$

VOLATILE ORGANICS

Lab Name: ENVIROSYSTEMS, INC.

Contract: ARGONNE

Lab Code: ENVSYS Case No.: AR0511W SAS No.:

Lab Sample ID: 0060511-02

Matrix: (soil/water) WATER

Sample wt/vol: $\quad 5.000(\mathrm{~g} / \mathrm{mL}) \mathrm{ML}$

Level: (low/med) LOW

Lab File ID: AG75HC066

$\%$ Moisture: not dec.

GC Column: RTX-624 ID: 0.18 (mm)

Soil Extract Volume: (uL)

SDG NO. : AR0511W

Date Received: 05/24/06

Date Analyzed: 05/24/06

Dilution Factor: 1.0

Soil Aliquot Volume:
EPA SAMPLE NO.

NA-TI29-

W-20901HW (uL)

CONCENTRATION UNITS:

CAS NO.

COMPOUND

(ug/L or $\mathrm{ug} / \mathrm{Kg}$ )

UG/L Q

\begin{tabular}{|c|c|c|c|}
\hline $\begin{array}{r}75-71-8 \\
74-87-3 \\
75-01-4 \\
74-83-9 \\
75-00-3 \\
75-69-4 \\
75-35-4 \\
76-13-1 \\
67-64-1 \\
75-15-0 \\
79-20-9 \\
75-09-2 \\
156-60-5 \\
1634-04-4 \\
75-34-3 \\
156-59-2 \\
78-93-3 \\
67-66-3 \\
71-55-6 \\
110-82-7 \\
56-23-5 \\
71-43-2 \\
107-06-2\end{array}$ & $\begin{array}{l}\text { Dichlorodifluoromethane } \\
\text { Chloromethane } \\
\text { Vinyl Chloride } \\
\text { Bromomethane } \\
\text { Chloroethane } \\
\text { Trichlorofluoromethane } \\
\text { 1,1-Dichloroethene } \\
\text { 1,1,2-Trichloro-1,2,2-trifluoroethane } \\
\text { Acetone } \\
\text { Carbon Disulfide } \\
\text { Methyl Acetate } \\
\text { Methylene Chloride } \\
\text { trans-1,2-Dichloroethene } \\
\text { Methyl tert-Butyl Ether } \\
\text { 1,1-Dichloroethane } \\
\text { cis-1,2-Dichloroethene } \\
\text { 2-Butanone } \\
\text { Chloroform } \\
\text { 1,1,1-Trichloroethane } \\
\text { Cyclohexane } \\
\text { Carbon Tetrachloride } \\
\text { Benzene } \\
\text { 1,2-Dichloroethane }\end{array}$ & $\begin{array}{l}5.0 \\
5.0 \\
5.0 \\
5.0 \\
5.0 \\
5.0 \\
5.0 \\
5.0 \\
5.0 \\
5.0 \\
5.0 \\
5.8 \\
5.0 \\
5.0 \\
5.0 \\
5.0 \\
5.0 \\
75 \\
5.0 \\
5.0 \\
230 \\
5.0 \\
5.0\end{array}$ & $\begin{array}{l}\mathrm{U} \\
\mathrm{U} \\
\mathrm{U} \\
\mathrm{U} \\
\mathrm{U} \\
\mathrm{U} \\
\mathrm{U} \\
\mathrm{U} \\
\mathrm{U} \\
\mathrm{U} \\
\mathrm{U} \\
\mathrm{B} \\
\mathrm{U} \\
\mathrm{U} \\
\mathrm{U} \\
\mathrm{U} \\
\mathrm{U}\end{array}$ \\
\hline
\end{tabular}


Lab Name: ENVIROSYSTEMS, INC.

Contract : ARGONNE

NA-TI29-

W-20901HW

Lab Code: ENVSYS Case No.: AR0511W SAS No.:

SDG NO. : AR0511W

Matrix: (soil/water) WATER

Sample wt/vol: $\quad 5.000(\mathrm{~g} / \mathrm{mL}) \mathrm{ML}$

Level: (low/med) Low

\% Moisture: not dec.

GC Column: RTX-624 ID: 0.18 (mm)

Soil Extract Volume: (uL)
Lab Sample ID: 0060511-02

Lab File ID: AG75HC066

Date Received: 05/24/06

Date Analyzed: 05/24/06

Dilution Factor: 1.0

Soil Aliquot Volume: (uL)
CAS NO.

COMPOUND
CONCENTRATION UNITS:

(ug/L or $\mathrm{ug} / \mathrm{Kg}$ ) UG/L Q

\begin{tabular}{|c|c|c|c|}
\hline $\begin{array}{r}79-01-6 \\
108-87-2 \\
78-87-5 \\
75-27-4 \\
10061-01-5 \\
108-10-1 \\
108-88-3 \\
10061-02-6 \\
79-00-5 \\
127-18-4 \\
591-78-6 \\
124-48-1 \\
106-93-4 \\
108-90-7 \\
100-41-4 \\
1330-20-7 \\
100-42-5 \\
75-25-2 \\
98-82-8 \\
79-34-5 \\
541-73-1 \\
106-46-7 \\
95-50-1 \\
96-12-8 \\
120-82-1\end{array}$ & 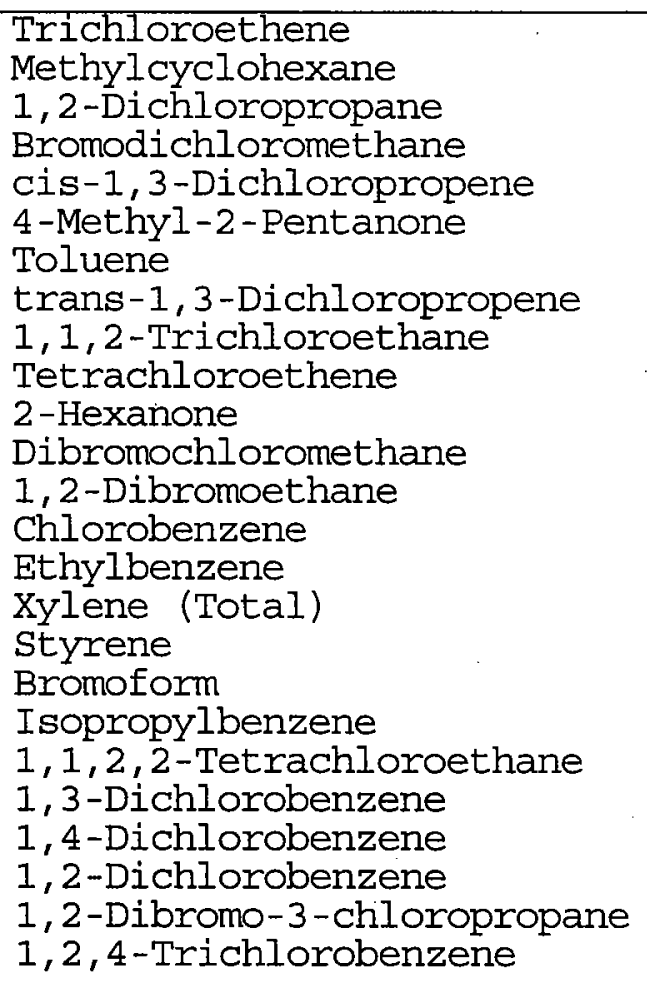 & $\begin{array}{l}5.0 \\
5.0 \\
5.0 \\
5.0 \\
5.0 \\
5.0 \\
5.0 \\
5.0 \\
5.0 \\
5.0 \\
5.0 \\
5.0 \\
5.0 \\
5.0 \\
5.0 \\
5.0 \\
5.0 \\
5.0 \\
5.0 \\
5.0 \\
5.0 \\
5.0 \\
5.0 \\
5.0 \\
5.0\end{array}$ & $\begin{array}{l} \\
U \\
U \\
U \\
U \\
U \\
U \\
U \\
U \\
U \\
U \\
U \\
U \\
U \\
U \\
U \\
U \\
U \\
U \\
U \\
U \\
U \\
U \\
U \\
U\end{array}$ \\
\hline
\end{tabular}


Lab Name: ENVIROSYSTEMS, INC.

Contract: ARGONNE

EPA SAMPLE NO.

NA-TI29W-20901HW

Lab Code: ENVSYS Case No.: AR0511W SAS No.:

SDG No.: AR0511W

Matrix: (soil/water) WATER

Sample wt/vol: $5.000(\mathrm{~g} / \mathrm{mL}) \mathrm{ML}$

Level : (low/med) LOW

\% Moisture: not dec.

GC Column: RTX-624 ID: 0.18 (mm)

Soil Extract Volume:

(uL)

Number TICs found: 0
Lab Sample ID: 0060511-02

Lab File ID: AG75HC066

Date Received: 05/24/06

Date Analyzed: 05/24/06

Dilution Factor: 1.0

Soil Aliquot Volume:

CONCENTRATION UNITS :

(ug/L or ug/Kg) ug/L

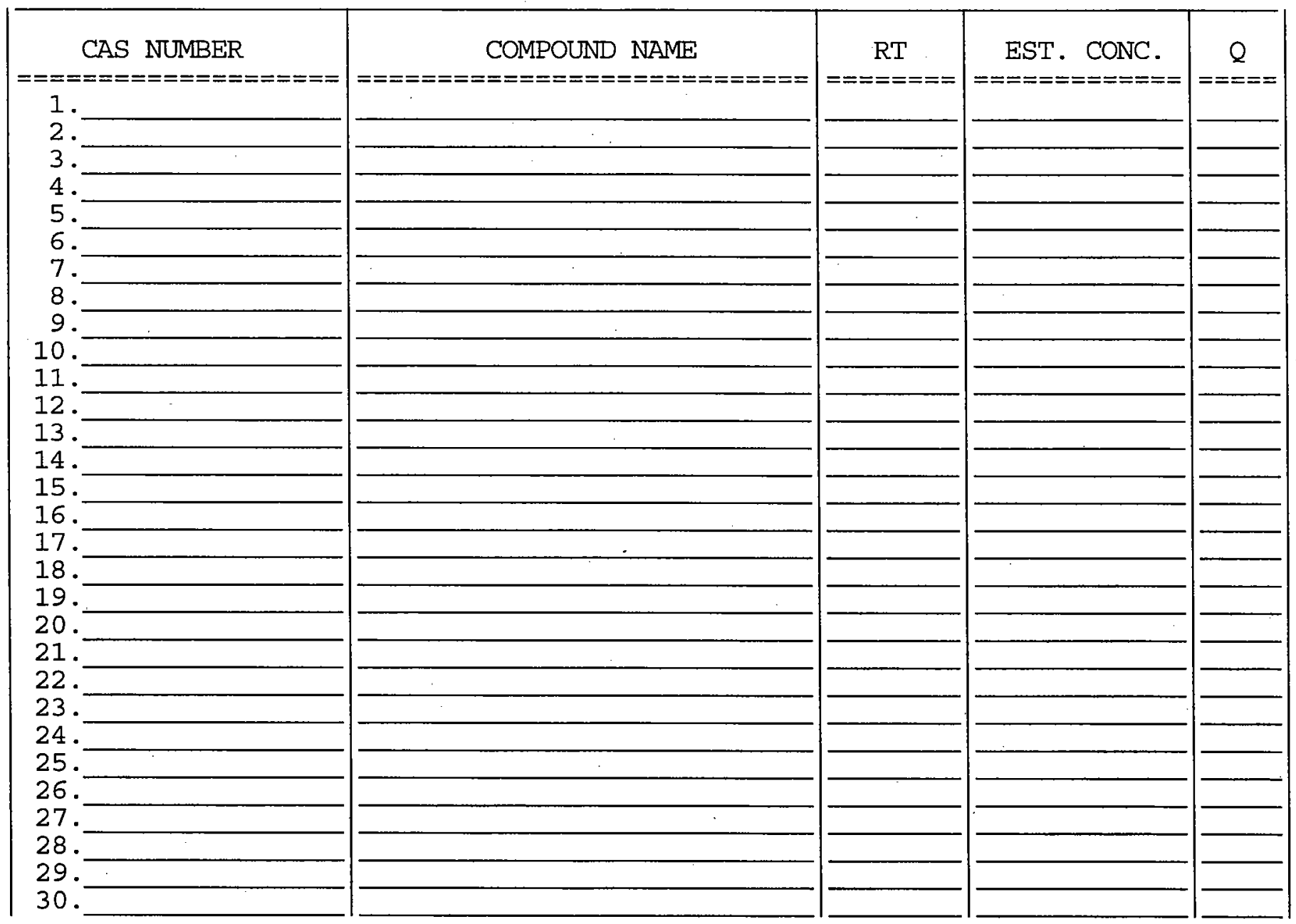


Lab Name: ENVIROSYSTEMS, INC.

Contract: ARGONNE

Lab Code: ENVSYS Case No.: AR0511W SAS No.:

SDG No.: AR0511W

Matrix: (soil/water) WATER

Sample wt/vol:

$5.000(\mathrm{~g} / \mathrm{mL}) \mathrm{ML}$

Level: (low/med) LOW

\% Moisture: not dec.

GC Column: RTX-624 ID: 0.18 (mm)

Soil Extract Volume: (uL)
Lab Sample ID: 0060511-02RE

Lab File ID: AG75HC114

Date Received: 05/24/06

Date Analyzed: 05/31/06

Dilution Factor: 2.0

Soil Aliquot Volume: (uL)

CAS NO.

COMPOUND

CONCENTRATION UNITS:

(ug/L or ug/Kg) UG/L Q

\begin{tabular}{|c|c|c|c|}
\hline $\begin{array}{r}75-71-8 \\
74-87-3 \\
75-01-4 \\
74-83-9 \\
75-00-3 \\
75-69-4 \\
75-35-4 \\
76-13-1 \\
67-64-1 \\
75-15-0 \\
79-20-9 \\
75-09-2 \\
156-60-5 \\
1634-04-4 \\
75-34-3 \\
156-59-2 \\
78-93-3 \\
67-66-3 \\
71-55-6 \\
110-82-7 \\
56-23-5 \\
71-43-2 \\
107-06-2\end{array}$ & $\begin{array}{l}\text { Dichlorodifluoromethane } \\
\text { Chloromethane } \\
\text { Vinyl Chloride } \\
\text { Bromomethane } \\
\text { Chloroethane } \\
\text { Trichlorofluoromethane } \\
\text { 1,1-Dichloroethene } \\
\text { 1,1,2-Trichloro-1,2,2-trifluoroethane } \\
\text { Acetone } \\
\text { Carbon Disulfide } \\
\text { Methyl Acetate } \\
\text { Methylene Chloride } \\
\text { trans-1,2-Dichloroethene } \\
\text { Methyl tert-Butyl Ether } \\
\text { 1,1-Dichloroethane } \\
\text { cis-1,2-Dichloroethene } \\
\text { 2-Butanone } \\
\text { Chloroform } \\
\text { 1,1,1-Trichloroethane } \\
\text { Cyclohexane } \\
\text { Carbon Tetrachloride } \\
\text { Benzene } \\
\text { 1,2-Dichloroethane }\end{array}$ & $\begin{array}{r}10 \\
10 \\
10 \\
10 \\
10 \\
10 \\
10 \\
10 \\
10 \\
10 \\
10 \\
8.6 \\
10 \\
10 \\
10 \\
10 \\
10 \\
77 \\
10 \\
10 \\
220 \\
10 \\
10\end{array}$ & $\begin{array}{l}U \\
U \\
U \\
U \\
U \\
U \\
U \\
U \\
U \\
U \\
U \\
J B \\
U \\
U \\
U \\
U \\
U \\
U \\
U \\
\bar{U} \\
U\end{array}$ \\
\hline
\end{tabular}


Lab Name: ENVIROSYSTEMS, INC.

Contract: ARGONNE EPA SAMPLE NO.

Lab Code: ENvSYS Case No.: AR0511W SAS No.:

SDG No.: AR0511W

Matrix: (soil/water) WATER

Sample wt/vol: $\quad 5.000(\mathrm{~g} / \mathrm{mL}) \mathrm{ML}$

Level: (low/med) LOW

\% Moisture: not dec.

GC Column: RTX-624 ID: 0.18 (mm)

Soil Extract Volume:

(uIs)
Lab Sample ID: 0060511-02RE

Lab File ID: AG75HC114

Date Received: 05/24/06

Date Analyzed: 05/31/06

Dilution Factor: 2.0

Soil Aliquot Volume:

CONCENTRATION UNITS:

CAS NO.

COMPOUND (ug/L or $\mathrm{ug} / \mathrm{Kg}$ )

UG/L Q

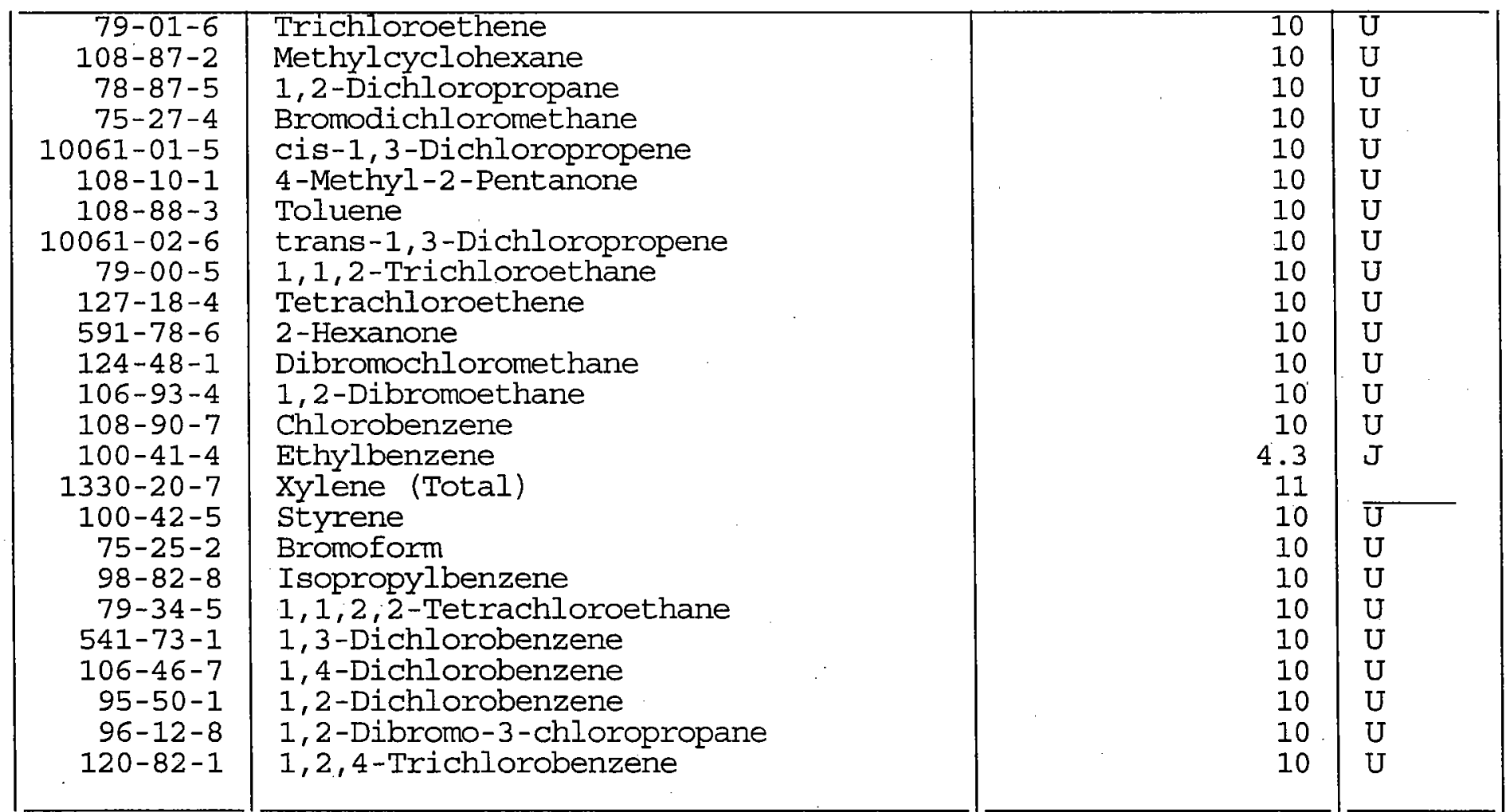


$1 \mathrm{~F}$

VOLATILE ORGANICS ANALYSIS DATA SHEET

TENTATIVELY IDENTIFIED COMPOUNDS
EPA SAMPLE NO.

NA-TI29-W-20901

Lab Name: ENVIROSYSTEMS, INC.

Contract : ARGONNE

Lab Code: ENVSYS Case No.: AR0511W SAS No.:

SDG NO.: AR0511W

Matrix: (soil/water) WATER

Sample wt/vol: $5.000(\mathrm{~g} / \mathrm{mL}) \mathrm{ML}$

Level: (low/med) LOW

\% Moisture: not dec.

GC Column: RTX-624 ID: 0.18 (mm)

Soil Extract Volume:

(uL)

Number TICs found: 1
Lab Sample ID: 0060511-02RE

Lab File ID: AG75HC114

Date Received: 05/24/06

Date Analyzed: 05/31/06

Dilution Factor: 2.0

Soil Aliquot Volume: (uL)

CONCENTRATION UNITS :

(ug/L or $\mathrm{ug} / \mathrm{Kg}$ ) ug/L

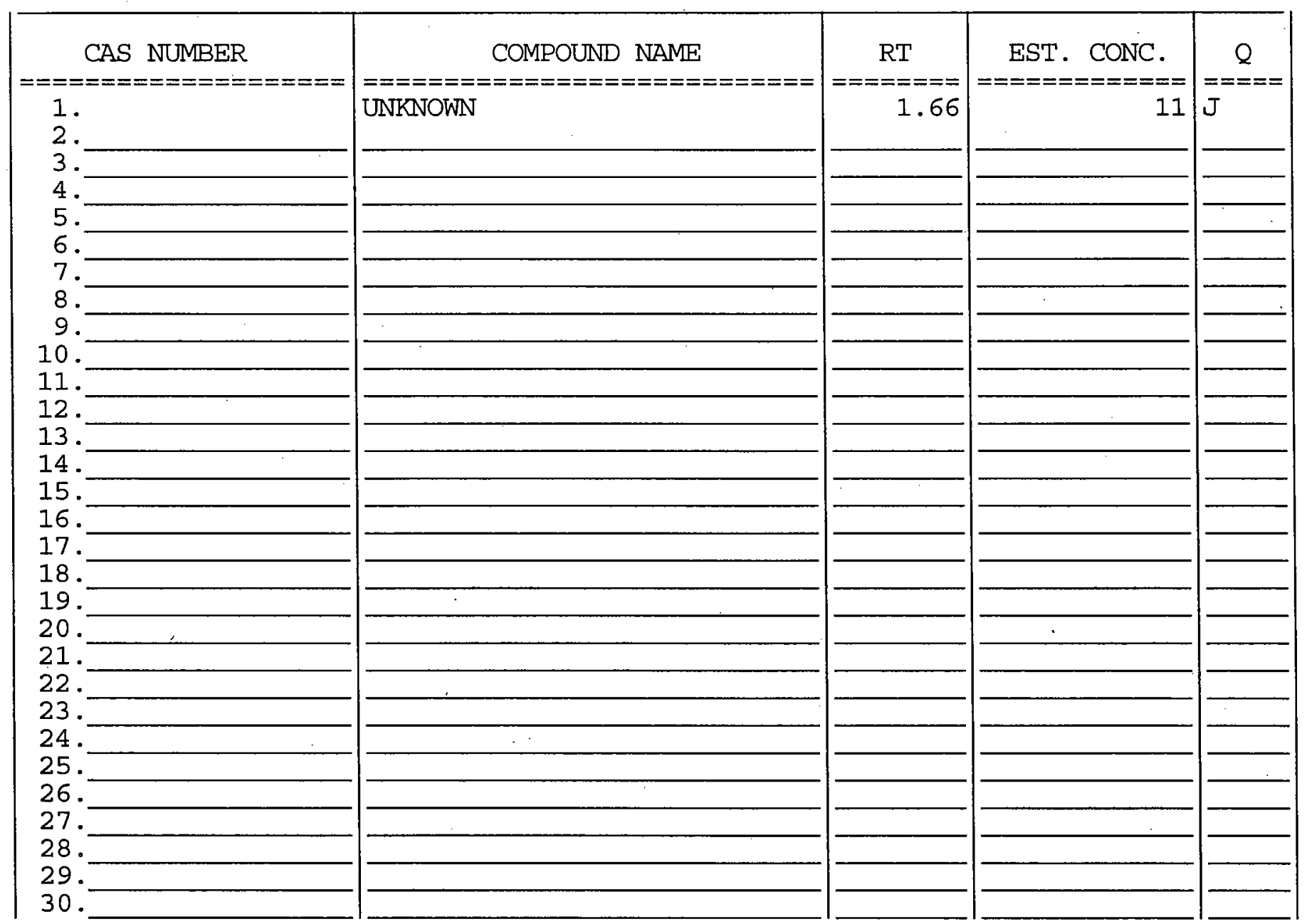


Lab Name: ENVIROSYSTEMS, INC.

Contract: ARGONNE

Lab Code: ENVSYS Case No.: AR0511W SAS No.:

SDG NO.: AR0511W

Matrix: (soil/water) WATER

Sample wt/vol:

$5.000(\mathrm{~g} / \mathrm{mL}) \mathrm{ML}$

Level: (low/med) LOW

\% Moisture: not dec.

GC Column: RTX-624 ID: 0.18 (mm)

Soil Extract Volume: (UI)
Lab Sample ID: 0060511-03

Lab File ID: AG75HC067

Date Received: 05/24/06

Date Analyzed: 05/24/06

Dilution Factor: 1.0

Soil Aliquot Volume: (uL)

CONCENTRATION UNITS:

CAS NO.

COMPOUND (ug/L or $\mathrm{ug} / \mathrm{Kg}$ )

UG/L Q

\begin{tabular}{|c|c|c|c|}
\hline $\begin{array}{r}75-71-8 \\
74-87-3 \\
75-01-4 \\
74-83-9 \\
75-00-3 \\
75-69-4 \\
75-35-4 \\
76-13-1 \\
67-64-1 \\
75-15-0 \\
79-20-9 \\
75-09-2 \\
156-60-5 \\
1634-04-4 \\
75-34-3 \\
156-59-2 \\
78-93-3 \\
67-66-3 \\
71-55-6 \\
110-82-7 \\
56-23-5 \\
71-43-2 \\
107-06-2\end{array}$ & $\begin{array}{l}\text { Dichlorodifluoromethane } \\
\text { Chloromethane } \\
\text { Vinyl Chloride } \\
\text { Bromomethane } \\
\text { Chloroethane } \\
\text { Trichlorofluoromethane } \\
\text { 1,1-Dichloroethene } \\
\text { 1,1,2-Trichloro-1,2,2-trifluoroethane } \\
\text { Acetone } \\
\text { Carbon Disulfide } \\
\text { Methyl Acetate } \\
\text { Methylene Chloride } \\
\text { trans-1,2-Dichloroethene } \\
\text { Methyl tert-Butyl Ether } \\
\text { 1,1-Dichloroethane } \\
\text { cis-1,2-Dichloroethene } \\
\text { 2-Butanone } \\
\text { Chloroform } \\
\text { 1,1,1-Trichloroethane } \\
\text { Cyclohexane } \\
\text { Carbon Tetrachloride } \\
\text { Benzene } \\
\text { 1,2-Dichloroethane }\end{array}$ & $\begin{array}{r}5.0 \\
5.0 \\
5.0 \\
5.0 \\
5.0 \\
5.0 \\
5.0 \\
5.0 \\
5.0 \\
5.0 \\
5.0 \\
4.1 \\
5.0 \\
5.0 \\
5.0 \\
5.0 \\
5.0 \\
39 \\
5.0 \\
5.0 \\
420 \\
5.0 \\
5.0\end{array}$ & $\begin{array}{l}U \\
U \\
U \\
U \\
U \\
U \\
U \\
U \\
U \\
U \\
U \\
J B \\
U \\
U \\
U \\
U \\
U \\
U \\
U \\
E \\
U \\
U\end{array}$ \\
\hline
\end{tabular}


Lab Name: ENVIROSYSTEMS, INC.

Contract: ARGONNE

Lab Code: ENVSYS Case No.: AR0511W SAS No.:

Matrix: (soil/water) WATER

Sample wt/vol: $\quad 5.000(\mathrm{~g} / \mathrm{mL}) \mathrm{ML}$

Level: (low/med) LOW

\% Moisture: not dec.

GC Column: RTX-624 ID: 0.18 (mm)

Soil Extract Volume: (UL)
Lab Sample ID: 0060511-03

Lab File ID: AG75HC067

Date Received: 05/24/06

Date Analyzed: 05/24/06

Dilution Factor: 1.0

Soil Aliquot Volume:

CONCENTRATION UNITS :

CAS NO.

COMPOUND

\begin{tabular}{|c|c|c|c|}
\hline $\begin{array}{r}79-01-6 \\
108-87-2 \\
78-87-5 \\
75-27-4 \\
10061-01-5 \\
108-10-1 \\
108-88-3 \\
10061-02-6 \\
79-00-5 \\
127-18-4 \\
591-78-6 \\
124-48-1 \\
106-93-4 \\
108-90-7 \\
100-41-4 \\
1330-20-7 \\
100-42-5 \\
75-25-2 \\
98-82-8 \\
79-34-5 \\
541-73-1 \\
106-46-7 \\
95-50-1 \\
96-12-8 \\
120-82-1\end{array}$ & $\begin{array}{l}\text { Trichloroethene } \\
\text { Methylcyclohexane } \\
\text { 1,2-Dichloropropane } \\
\text { Bromodichloromethane } \\
\text { cis-1,3-Dichloropropene } \\
\text { 4-Methyl-2-Pentanone } \\
\text { Toluene } \\
\text { trans-1,3-Dichloropropene } \\
\text { 1,1,2-Trichloroethane } \\
\text { Tetrachloroethene } \\
\text { 2-Hexanone } \\
\text { Dibromochloromethane } \\
\text { 1,2-Dibromoethane } \\
\text { Chlorobenzene } \\
\text { Ethylbenzene } \\
\text { XYlene (Total) } \\
\text { Styrene } \\
\text { Bromoform } \\
\text { Isopropylbenzene } \\
\text { 1,1,2,2-Tetrachloroethane } \\
\text { 1,3-Dichlorobenzene } \\
\text { 1,4-Dichlorobenzene } \\
\text { 1,2-Dichlorobenzene } \\
\text { 1,2-Dibromo-3-chloropropane } \\
\text { 1,2,4-Trichlorobenzene }\end{array}$ & $\begin{array}{l}5.0 \\
5.0 \\
5.0 \\
5.0 \\
5.0 \\
5.0 \\
5.0 \\
5.0 \\
5.0 \\
5.0 \\
5.0 \\
5.0 \\
5.0 \\
5.0 \\
5.0 \\
5.0 \\
5.0 \\
5.0 \\
5.0 \\
5.0 \\
5.0 \\
5.0 \\
5.0 \\
5.0 \\
5.0\end{array}$ & $\begin{array}{l}\mathrm{U} \\
\mathrm{U} \\
\mathrm{U} \\
\mathrm{U} \\
\mathrm{U} \\
\mathrm{U} \\
\mathrm{U} \\
\mathrm{U} \\
\mathrm{U} \\
\mathrm{U} \\
\mathrm{U} \\
\mathrm{U} \\
\mathrm{U} \\
\mathrm{U} \\
\mathrm{U} \\
\mathrm{U} \\
\mathrm{U} \\
\mathrm{U} \\
\mathrm{U} \\
\mathrm{U} \\
\mathrm{U} \\
\mathrm{U} \\
\mathrm{U} \\
\mathrm{U} \\
\mathrm{U}\end{array}$ \\
\hline
\end{tabular}


VOLATILE ORGANICS ANALYSIS DATA SHEET TENTATIVELY IDENTIFIED COMPOUNDS

EPA SAMPLE NO.

INC. Contract: ARGONNE

Lab Name: ENVIROSYSTEMS, INC.

SAS NO.:

SDG No. : AR0511W

Matrix: (soil/water) WATER

Sample wt/vol: $5.000(\mathrm{~g} / \mathrm{mL}) \mathrm{ML}$

Level : (low/med) LOW

\% Moisture: not dec.

GC Column: RTX-624 ID: 0.18 (mm)

Soil Extract Volume:

Number TICs found: 1
Lab Sample ID: 0060511-03

Lab File ID: AG75HC067

Date Received: 05/24/06

Date Analyzed: 05/24/06

Dilution Factor: 1.0

Soil Aliquot Volume: (uL)

(ug/L or $u g / \mathrm{Kg}$ ) ug/L

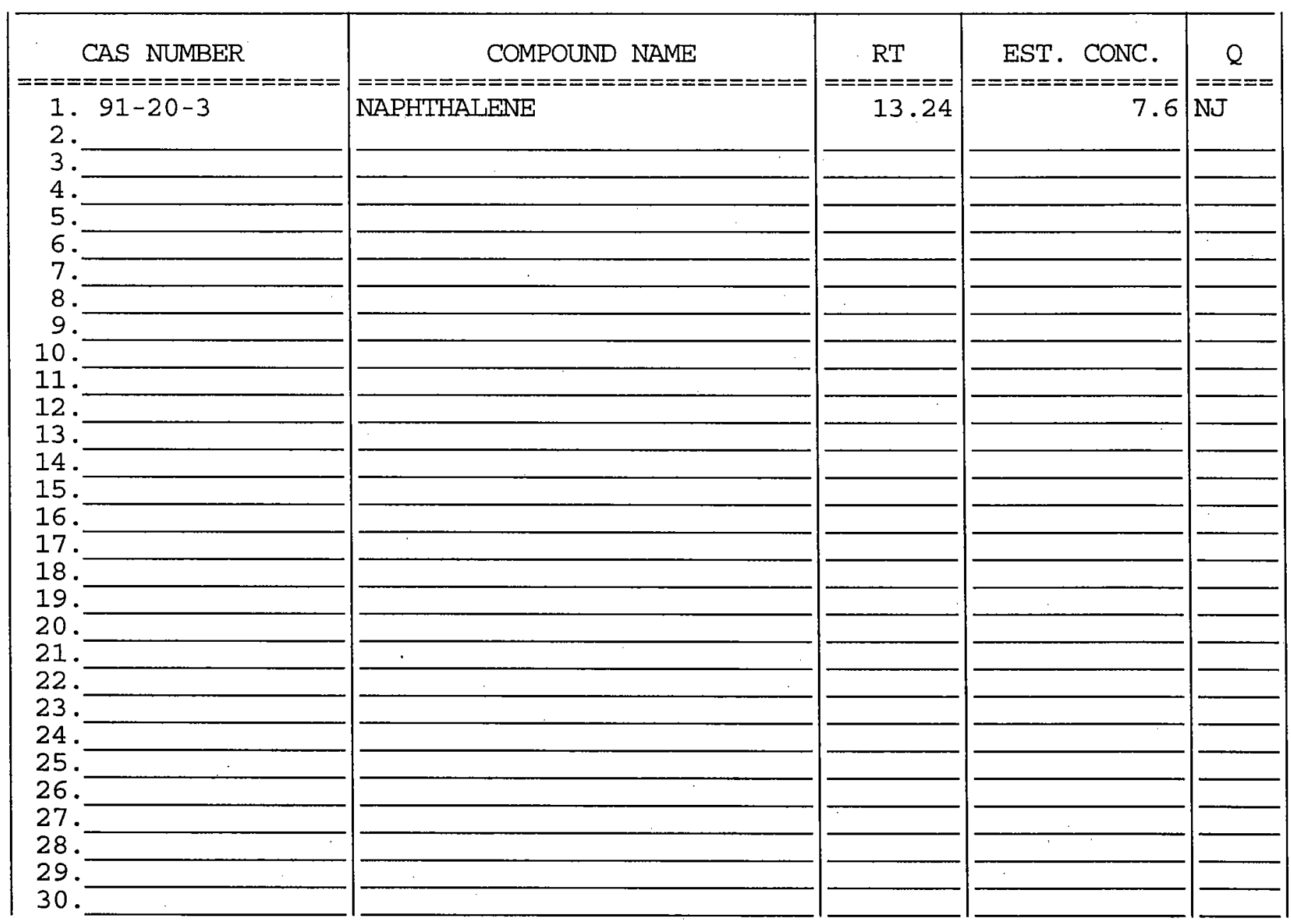


Lab Name: ENVIROSYSTEMS, INC.

Contract: ARGONNE NA-TI 18-W-20655

Lab Code: ENVSYS Case No.: AR0511W SAS No.:

SDG No.: AR0511W

Matrix: (soil/water) WATER

Sample wt/vol:

$5.000(\mathrm{~g} / \mathrm{mL}) \mathrm{ML}$

Level: (low/med) LOW

\% Moisture: not dec.

GC Column: RTX-624 ID: 0.18 (mm)

Soil Extract Volume: (uL)
Lab Sample ID: 0060511-03RE

Lab File ID: AG75HC115

Date Received: 05/24/06

Date Analyzed: 05/31/06

Dilution Factor: 5.0

Soil Aliquot Volume: (uL)
CAS NO.

COMPOUND
CONCENTRATION UNITS : (ug/L or $\mathrm{ug} / \mathrm{Kg}$ ) UG/L Q

\begin{tabular}{|c|c|c|c|}
\hline $\begin{array}{r}75-71-8 \\
74-87-3 \\
75-01-4 \\
74-83-9 \\
75-00-3 \\
75-69-4 \\
75-35-4 \\
76-13-1 \\
67-64-1 \\
75-15-0 \\
79-20-9 \\
75-09-2 \\
156-60-5 \\
1634-04-4 \\
75-34-3 \\
156-59-2 \\
78-93-3 \\
67-66-3 \\
71-55-6 \\
110-82-7 \\
56-23-5 \\
71-43-2 \\
107-06-2\end{array}$ & $\begin{array}{l}\text { Dichlorodifluoromethane } \\
\text { Chloromethane } \\
\text { Vinyl Chloride } \\
\text { Bromomethane } \\
\text { Chloroethane } \\
\text { Trichlorofluoromethane } \\
\text { 1,1-Dichloroethene } \\
\text { 1,1,2-Trichloro-1,2,2-trifluoroethane } \\
\text { Acetone } \\
\text { Carbon Disulfide } \\
\text { Methyl Acetate } \\
\text { Methylene Chloride } \\
\text { trans-1,2-Dichloroethene } \\
\text { Methyl tert-Butyl Ether } \\
\text { 1,1-Dichloroethane } \\
\text { cis-1,2-Dichloroethene } \\
\text { 2-Butanone } \\
\text { Chloroform } \\
\text { 1,1,1-Trichloroethane } \\
\text { Cyclohexane } \\
\text { Carbon Tetrachloride } \\
\text { Benzene } \\
\text { 1,2-Dichloroethane }\end{array}$ & $\begin{array}{r}25 \\
25 \\
25 \\
25 \\
25 \\
25 \\
25 \\
25 \\
25 \\
25 \\
25 \\
16 \\
25 \\
25 \\
25 \\
25 \\
25 \\
39 \\
25 \\
25 \\
390 \\
25 \\
25\end{array}$ & $\begin{array}{l}U \\
U \\
U \\
U \\
U \\
U \\
U \\
U \\
U \\
U \\
U \\
J B \\
U \\
U \\
U \\
U \\
U \\
\bar{U} \\
U \\
U \\
U\end{array}$ \\
\hline
\end{tabular}


Lab Name: ENVIROSYSTEMS, INC.

Contract: ARGONNE

Lab Code: ENVSYS Case No.: AR0511W SAS No.:

SDG No. : AR0511W

Matrix: (soil/water) WATER

Sample wt/vol:

$5.000(\mathrm{~g} / \mathrm{mL}) \mathrm{ML}$

Level: (low/med) LOW

\% Moisture: not dec.

GC Column: RTX-624 ID: 0.18 (mm) (UL)

Soil Extract Volume:
Lab Sample ID: 0060511-03RE

Lab File ID: AG75HC115

Date Received: 05/24/06

Date Analyzed: 05/31/06

Dilution Factor: 5.0

Soil Aliquot Volume: (uI)

CONCENTRATION UNITS:

CAS NO.

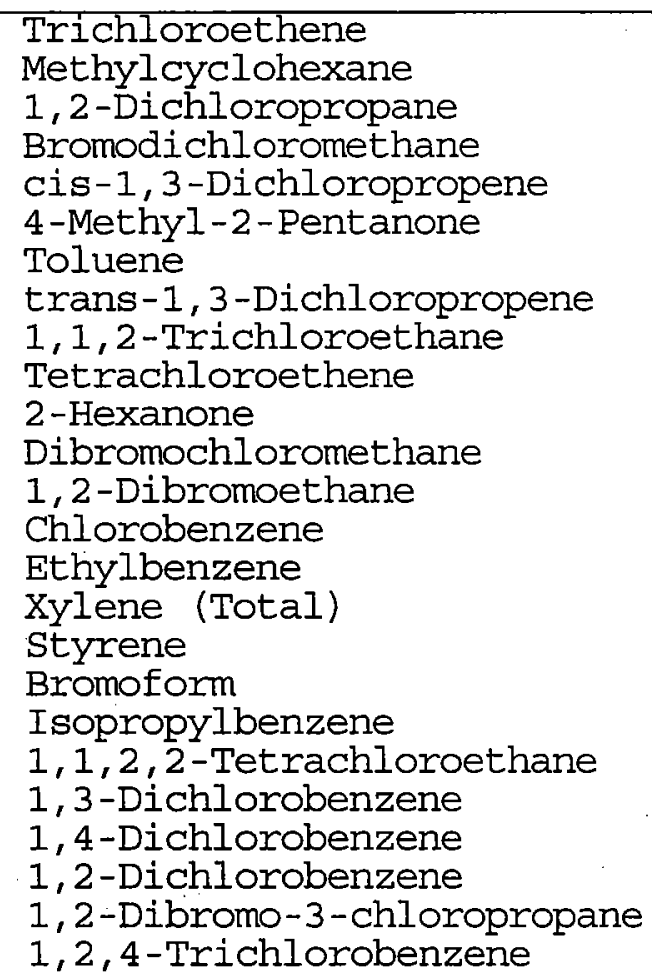

$108-90-7$

$100-41-4$

$1330-20-7$

$100-42-5$

$75-25-2$

$98-82-8$
$79-34-5$

$541-73-1$

$106-46-7$

$95-50-1$

$96-12-8$

120-82-1

(ug/L or $u g / \mathrm{kg}$ )

UG/L Q

\begin{tabular}{|l|l|l|}
\hline & $\mathrm{U}$ \\
25 & $\mathrm{U}$ \\
25 & $\mathrm{U}$ \\
25 & $\mathrm{U}$ \\
25 & $\mathrm{U}$ \\
25 & $\mathrm{U}$ \\
25 & $\mathrm{U}$ \\
25 & $\mathrm{U}$ \\
25 & $\mathrm{U}$ \\
25 & $\mathrm{U}$ \\
25 & $\mathrm{U}$ \\
25 & $\mathrm{U}$ \\
25 & $\mathrm{U}$ \\
25 & $\mathrm{U}$ \\
8.9 & $\mathrm{~J}$ \\
23 & $\mathrm{~J}$ \\
25 & $\mathrm{U}$ \\
25 & $\mathrm{U}$ \\
25 & $\mathrm{U}$ \\
25 & $\mathrm{U}$ \\
25 & $\mathrm{U}$ \\
25 & $\mathrm{U}$ \\
25 & $\mathrm{U}$ \\
25 & $\mathrm{U}$ \\
25 & $\mathrm{U}$ \\
& \\
\hline
\end{tabular}

FORM I VOA-2

OLM0 4.3 
$1 F$

VOLATILE ORGANICS ANALYSIS DATA SHEET

TENTATIVELY IDENTIFIED COMPOUNDS
EPA SAMPLE NO.

NA-TI18-W-20655

Lab Name: ENVIROSYSTEMS, INC.

Contract: ARGONNE

Lab Code: ENVSYS Case No.: AR0511W SAS No.:

SDG No.: AR0511W

Matrix: (soil/water) WATER

Sample wt/vol: $5.000(\mathrm{~g} / \mathrm{mL}) \mathrm{ML}$

Level: (low/med) LOW

\% Moisture: not dec.

GC Column: RTX-624 ID: 0.18 (mm)

Soil Extract Volume:

Number TICs found: 0

(uL)

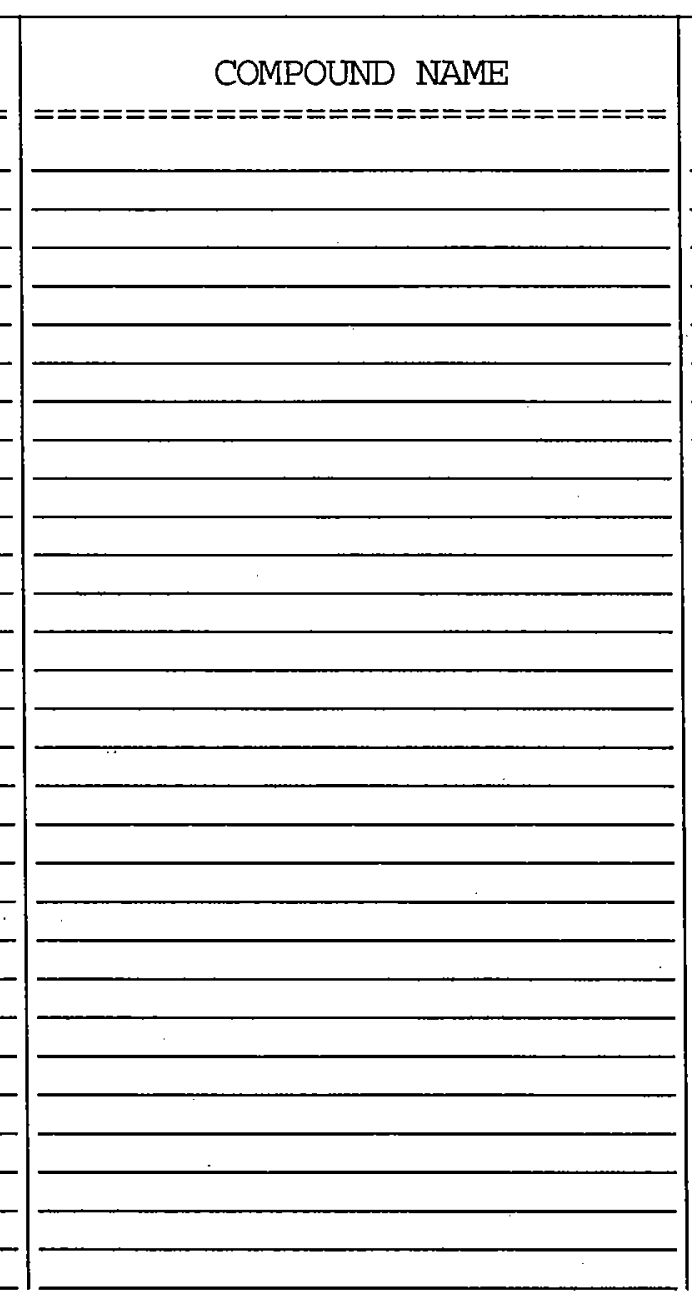

Lab Sample ID: 0060511-03RE

Lab File ID: AG75HC115

Date Received: 05/24/06

Date Analyzed: 05/31/06

Dilution Factor: 5.0

Soil Aliquot Volume: (uL)

CONCENTRATION UNITS:

(ug/L or $\mathrm{ug} / \mathrm{Kg}$ ) ug/L

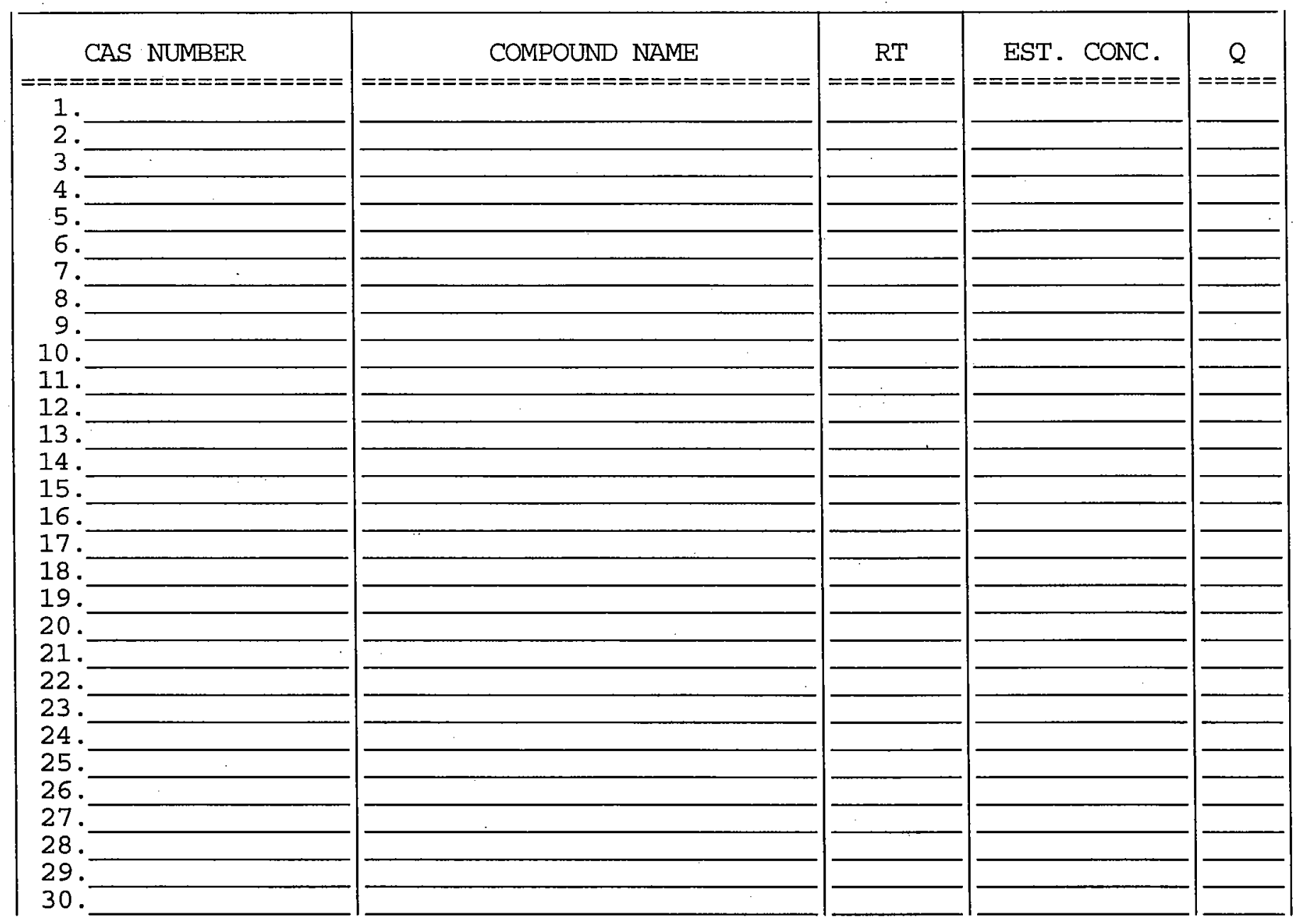


Lab Name: ENVIROSYSTEMS, INC.

Contract : ARGONNE

NA-TI27W-20905HW

Iab Code: ENVSYS Case No.: AR0511W SAS No.:

SDG NO. : AR0511W

Matrix: (soil/water) WATER

Sample wt/vol:

$5.000(\mathrm{~g} / \mathrm{mL}) \mathrm{ML}$

Level: (low/med) LOW

\% Moisture: not dec.

GC Column: RTX-624 ID: 0.18 (mm)

Soil Extract Volume: (uL)
CAS NO.

$75-71-8$

$74-87-3$

$75-01-4$

$74-83-9$

$75-00-3$

$75-69-4$

$75-35-4$

$76-13-1$

$67-64-1$

75-15-0

$79-20-9$

75-09-2

$156-60-5$

1634-04-4

$75-34-3$

156-59-2

$78-93-3$

$67-66-3$

$71-55-6$

$110-82-7$

$56-23-5$

$71-43-2$

107-06-2
COMPOUND

CONCENTRATION UNITS :

(ug/L or $\mathrm{ug} / \mathrm{Kg}$ ) UG/L Q

Dichlorodifluoromethane
Chloromethane
Vinyl Chloride
Bromomethane
Chloroethane
Trichlorofluoromethane
1,1-Dichloroethene
1,1,2-Trichloro-1,2,2-trifluoroethane
Acetone
Carbon Disulfide
Methyl Acetate
Methylene Chloride
trans-1,2-Dichloroethene
Methyl tert-Butyl Ether
1,1-Dichloroethane
cis-1,2-Dichloroethene
2-Butanone
Chloroform
1,1,1-Trichloroethane
Cyclohexane
Carbon Tetrachloride
Benzene
1,2-Dichloroethane

Dichlorodifluoromethane

loromethane

Trichlorofluoromethane

1,1-Dichloroethene

1,1,2-Trichloro-1,2,2-trifluoroethane

Methyl Acetate

Methylene Chloride

trans-1,2-Dichloroethene

Methyl tert-Butyl Ether

2 -Butanone

Chloroform

1,1,1-Trichloroethane

Cyclohexane

Benzene

1,2-Dichloroethane

\begin{tabular}{|l|l|l|}
\hline 5.0 & $\mathrm{U}$ \\
5.0 & $\mathrm{U}$ \\
5.0 & $\mathrm{U}$ \\
5.0 & $\mathrm{U}$ \\
5.0 & $\mathrm{U}$ \\
5.0 & $\mathrm{U}$ \\
5.0 & $\mathrm{U}$ \\
5.0 & $\mathrm{U}$ \\
5.0 & $\mathrm{U}$ \\
5.0 & $\mathrm{U}$ \\
5.0 & $\mathrm{U}$ \\
3.0 & $\mathrm{JB}$ \\
5.0 & $\mathrm{U}$ \\
5.0 & $\mathrm{U}$ \\
5.0 & $\mathrm{U}$ \\
5.0 & $\mathrm{U}$ \\
5.0 & $\mathrm{U}$ \\
5.0 & $\mathrm{U}$ \\
5.0 & $\mathrm{U}$ \\
5.0 & $\mathrm{U}$ \\
1.6 & $\mathrm{~J}$ \\
5.0 & $\mathrm{U}$ \\
5.0 & $\mathrm{U}$ \\
\hline
\end{tabular}


$1 B$

VOLATILE ORGANICS ANALYSIS DATA SHEET

Lab Name: ENVIROSYSTEMS, INC.

Contract : ARGONNE
EPA SAMPLE NO.

NA-TI27W-20905HW

Lab Code: ENVSYS Case No.: AR0511W SAS No.:

SDG No.: AR0511W

Matrix: (soil/water) WATER

Sample wt/vol:

$5.000(\mathrm{~g} / \mathrm{mL}) \mathrm{ML}$

Level: (low/med) LOW

\% Moisture: not dec.

GC Column: RTX-624 ID: 0.18 (mm).

Soil Extract Volume: (UI)
Lab Sample ID: 0060511-04

Lab File ID: AG75HC068

Date Received: 05/24/10

Date Analyzed: 05/24/06

Dilution Factor: 1.0

Soil Aliquot Volume: (uL)

CONCENTRATION UNITS :

CAS NO.

COMPOUND

UG/L Q

\begin{tabular}{|r|l|l|l|}
\hline $79-01-6$ & Trichloroethene & 5.0 & $\mathrm{U}$ \\
$108-87-2$ & Methylcyclohexane & 5.0 & $\mathrm{U}$ \\
$78-87-5$ & 1,2-Dichloropropane & 5.0 & $\mathrm{U}$ \\
$75-27-4$ & Bromodichloromethane & 5.0 & $\mathrm{U}$ \\
$10061-01-5$ & Cis-1,3-Dichloropropene & 5.0 & $\mathrm{U}$ \\
$108-10-1$ & $4-$ Methyl-2-Pentanone & 5.0 & $\mathrm{U}$ \\
$108-88-3$ & Toluene & 5.0 & $\mathrm{U}$ \\
$10061-02-6$ & trans-1,3-Dichloropropene & 5.0 & $\mathrm{U}$ \\
$79-00-5$ & $1,1,2-$ Trichloroethane & 5.0 & $\mathrm{U}$ \\
$127-18-4$ & Tetrachloroethene & 5.0 & $\mathrm{U}$ \\
$591-78-6$ & 2-Hexanone & 5.0 & $\mathrm{U}$ \\
$124-48-1$ & Dibromochloromethane & 5.0 & $\mathrm{U}$ \\
$106-93-4$ & 1,2-Dibromoethane & 5.0 & $\mathrm{U}$ \\
$108-90-7$ & Chlorobenzene & 5.0 & $\mathrm{U}$ \\
$100-41-4$ & Ethylbenzene & 5.0 & $\mathrm{U}$ \\
$1330-20-7$ & Xylene (Total) & 5.0 & $\mathrm{U}$ \\
$100-42-5$ & Styrene & 5.0 & $\mathrm{U}$ \\
$75-25-2$ & Bromoform & 5.0 & $\mathrm{U}$ \\
$98-82-8$ & Isopropylbenzene & 5.0 & $\mathrm{U}$ \\
$79-34-5$ & $1,1,2,2-T e t r a c h l o r o e t h a n e$ & 5.0 & $\mathrm{U}$ \\
$541-73-1$ & $1,3-$ Dichlorobenzene & 5.0 & $\mathrm{U}$ \\
$106-46-7$ & 1,4-Dichlorobenzene & 5.0 & $\mathrm{U}$ \\
$95-50-1$ & 1,2-Dichlorobenzene & 5.0 & $\mathrm{U}$ \\
$96-12-8$ & 1,2-Dibromo-3-chloropropane & 5.0 & $\mathrm{U}$ \\
$120-82-1$ & 1,2,4-Trichlorobenzene & 5.0 & $\mathrm{U}$ \\
\hline
\end{tabular}


VOLATILE ORGANICS ANALYSIS DATA SHEET TENTATIVELY IDENTIFIED COMPOUNDS

Lab Name: ENVIROSYSTEMS, INC.

Contract: ARGONNE

EPA SAMPLE NO.

Lab Code: ENVSYS Case No.: AR0511W SAS No.:

SDG No.: AR0511W

Matrix: (soil/water) WATER

Sample wt/vol: 5.000 (g/mL) ML

Level: (low/med) LOW

\% Moisture: not dec.

GC Column: RTX-624 ID: 0.18 (mm)

Soil Extract Volume:

(uI)

Number TICs found: 0
Lab Sample ID: 0060511-04

Lab File ID: AG75HC068

Date Received: 05/24/10

Date Analyzed: 05/24/06

Dilution Factor: 1.0

Soil Aliquot Volume:

(uL)

CONCENTRATION UNITS :

(ug/L or $\mathrm{ug} / \mathrm{Kg}$ ) ug/L

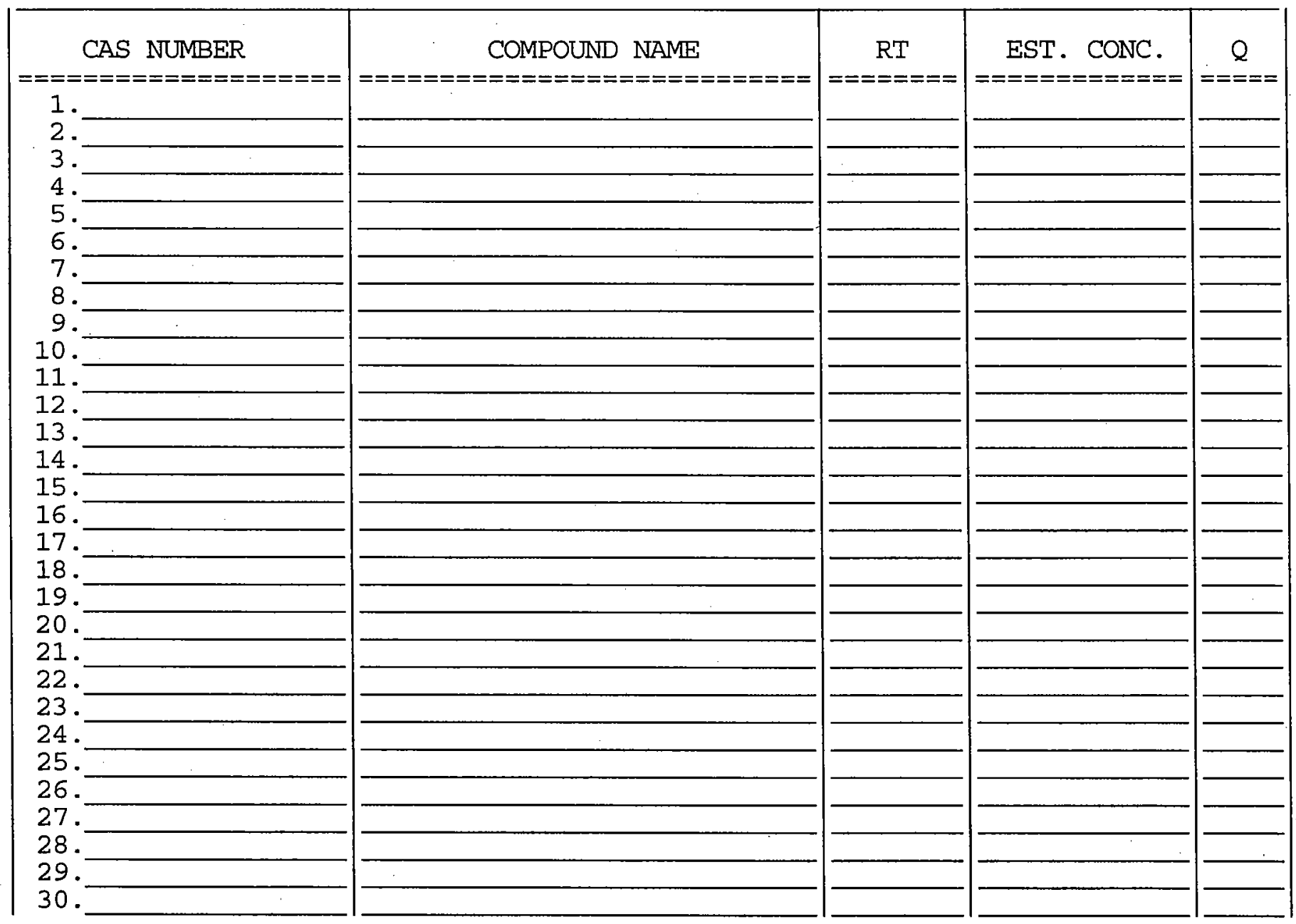


$1 A$

VOLATILE ORGANICS ANALYSIS DATA SHEET

Lab Name: ENVIROSYSTEMS, INC.

Lab Code: ENVSYS Case No.: AR0511W. SAS No.:

Contract: ARGONNE
EPA SAMPLE NO.

NA-TI18-

W-20709HW

SDG No.: AR0511W

Matrix: (soil/water) WATER

Lab Sample ID: 0060511-05-NA

Sample wt/vol:

$5.000(\mathrm{~g} / \mathrm{mL}) \mathrm{ML}$

Lab File ID: AG75HC069

Level: (low/med) LOW

Date Received: 05/24/06

\% Moisture: not dec.

GC Column: RTX-624 ID: 0.18 (mm)

Date Analyzed: 05/24/06

Soil Extract Volume: (UL)

Dilution Factor: 1.0

Soil Aliquot Volume: (UL)

CONCENTRATION UNITS :

CAS NO. COMPOUND $(u g / L$ or ug/ $\mathrm{Kg})$ UG/L Q

\begin{tabular}{|c|c|c|c|}
\hline $\begin{array}{r}75-71-8 \\
74-87-3 \\
75-01-4 \\
74-83-9 \\
75-00-3 \\
75-69-4 \\
75-35-4 \\
76-13-1 \\
67-64-1 \\
75-15-0 \\
79-20-9 \\
75-09-2 \\
156-60-5 \\
1634-04-4 \\
75-34-3 \\
156-59-2 \\
78-93-3 \\
67-66-3 \\
71-55-6 \\
110-82-7 \\
56-23-5 \\
71-43-2 \\
107-06-2\end{array}$ & $\begin{array}{l}\text { Dichlorodifluoromethane } \\
\text { Chloromethane } \\
\text { Vinyl Chloride } \\
\text { Bromomethane } \\
\text { Chloroethane } \\
\text { Trichlorofluoromethane } \\
\text { 1,1-Dichloroethene } \\
\text { 1,1,2-Trichloro-1,2,2-trifluoroethane } \\
\text { Acetone } \\
\text { Carbon Disulfide } \\
\text { Methyl Acetate } \\
\text { Methylene Chloride } \\
\text { trans-1,2-Dichloroethene } \\
\text { Methyl tert-Butyl Ether } \\
\text { 1,1-Dichloroethane } \\
\text { cis-1,2-Dichloroethene } \\
\text { 2-Butanone } \\
\text { Chloroform } \\
\text { 1,1,1-Trichloroethane } \\
\text { Cyclohexane } \\
\text { Carbon Tetrachloride } \\
\text { Benzene } \\
\text { 1,2-Dichloroethane }\end{array}$ & $\begin{array}{r}5.0 \\
5.0 \\
5.0 \\
5.0 \\
5.0 \\
5.0 \\
5.0 \\
5.0 \\
5.0 \\
5.0 \\
5.0 \\
4.0 \\
5.0 \\
5.0 \\
5.0 \\
5.0 \\
5.0 \\
44 \\
5.0 \\
5.0 \\
110 \\
5.0 \\
5.0\end{array}$ & $\begin{array}{l}U \\
U \\
U \\
U \\
U \\
U \\
U \\
U \\
U \\
U \\
U \\
J B \\
U \\
U \\
U \\
U \\
U \\
\bar{U} \\
U \\
U \\
U\end{array}$ \\
\hline
\end{tabular}


Lab Name: ENVIROSYSTEMS, INC.

Contract : ARGONNE

NA-TI18W-20709HW

Lab Code: ENVSYS Case No.: AR0511W SAS No.:

SDG No.: AR051IW

Matrix: (soil/water) WATER

Sample wt/vol:

$5.000(\mathrm{~g} / \mathrm{mL}) \mathrm{ML}$

Level : (low/med) LOW

\% Moisture: not dec.

GC Column: RTX-624 ID: 0.18 (mm)

Soil Extract Volume: (uL)

COMPOUND
CONCENTRATION UNITS :

(ug/L or $\mathrm{ug} / \mathrm{Kg}$ ) UG/L Q

\begin{tabular}{|c|c|c|c|}
\hline $\begin{array}{r}79-01-6 \\
108-87-2 \\
78-87-5 \\
75-27-4 \\
10061-01-5 \\
108-10-1 \\
108-88-3 \\
10061-02-6 \\
79-00-5 \\
127-18-4 \\
591-78-6 \\
124-48-1 \\
106-93-4 \\
108-90-7 \\
100-41-4 \\
1330-20-7 \\
100-42-5 \\
75-25-2 \\
98-82-8 \\
79-34-5 \\
541-73-1 \\
106-46-7 \\
95-50-1 \\
96-12-8 \\
120-82-1\end{array}$ & $\begin{array}{l}\text { Trichloroethene } \\
\text { Methylcyclohexane } \\
\text { 1,2-Dichloropropane } \\
\text { Bromodichloromethane } \\
\text { cis-1,3-Dichloropropene } \\
\text { 4-Methyl-2-Pentanone } \\
\text { Toluene } \\
\text { trans-1,3-Dichloropropene } \\
\text { 1,1,2-Trichloroethane } \\
\text { Tetrachloroethene } \\
\text { 2-Hexanone } \\
\text { Dibromochloromethane } \\
\text { 1,2-Dibromoethane } \\
\text { Chlorobenzene } \\
\text { Ethylbenzene } \\
\text { Xylene (Total) } \\
\text { Styrene } \\
\text { Bromoform } \\
\text { Isopropylbenzene } \\
\text { 1,1,2,2-Tetrachloroethane } \\
\text { 1,3-Dichlorobenzene } \\
\text { 1,4-Dichlorobenzene } \\
\text { 1,2-Dichlorobenzene } \\
\text { 1,2-Dibromo-3-chloropropane } \\
\text { 1,2,4-Trichlorobenzene }\end{array}$ & $\begin{array}{l}5.0 \\
5.0 \\
5.0 \\
5.0 \\
5.0 \\
5.0 \\
5.0 \\
5.0 \\
5.0 \\
5.0 \\
5.0 \\
5.0 \\
5.0 \\
5.0 \\
5.0 \\
5.0 \\
5.0 \\
5.0 \\
5.0 \\
5.0 \\
5.0 \\
5.0 \\
5.0 \\
5.0 \\
5.0\end{array}$ & $\begin{array}{l}\mathrm{U} \\
\mathrm{U} \\
\mathrm{U} \\
\mathrm{U} \\
\mathrm{U} \\
\mathrm{U} \\
\mathrm{U} \\
\mathrm{U} \\
\mathrm{U} \\
\mathrm{U} \\
\mathrm{U} \\
\mathrm{U} \\
\mathrm{U} \\
\mathrm{U} \\
\mathrm{U} \\
\mathrm{U} \\
\mathrm{U} \\
\mathrm{U} \\
\mathrm{U} \\
\mathrm{U} \\
\mathrm{U} \\
\mathrm{U} \\
\mathrm{U} \\
\mathrm{U} \\
\mathrm{U}\end{array}$ \\
\hline
\end{tabular}


$1 \mathrm{~F}$

VOLATILE ORGANICS ANALYSIS DATA SHEET TENTATIVELY IDENTIFIED COMPOUNDS

Lab Name: ENVIROSYSTEMS, INC.

Contract: ARGONNE

EPA SAMPLE NO.

NA-TII8W-20709HW

Lab Code: ENVSYS Case No.: AR0511W SAS No.:

SDG No.: AR0511W

Matrix: (soil/water) WATER

Sample wt/vol: $5.000(\mathrm{~g} / \mathrm{mL}) \mathrm{ML}$

Level: (low/med) LOW

\% Moisture: not dec.

GC Column: RTX-624 ID: 0.18 (mm)

Soil Extract Volume:

(uL)

Number TICs found: 1
Lab Sample ID: 0060511-05-NA

Lab File ID: AG75HC069

Date Received: 05/24/06

Date Analyzed: 05/24/06

Dilution Factor: 1.0

Soil Aliquot Volume:

CONCENTRATION UNITS:

(ug/L or $\mathrm{ug} / \mathrm{Kg}$ ) ug/L

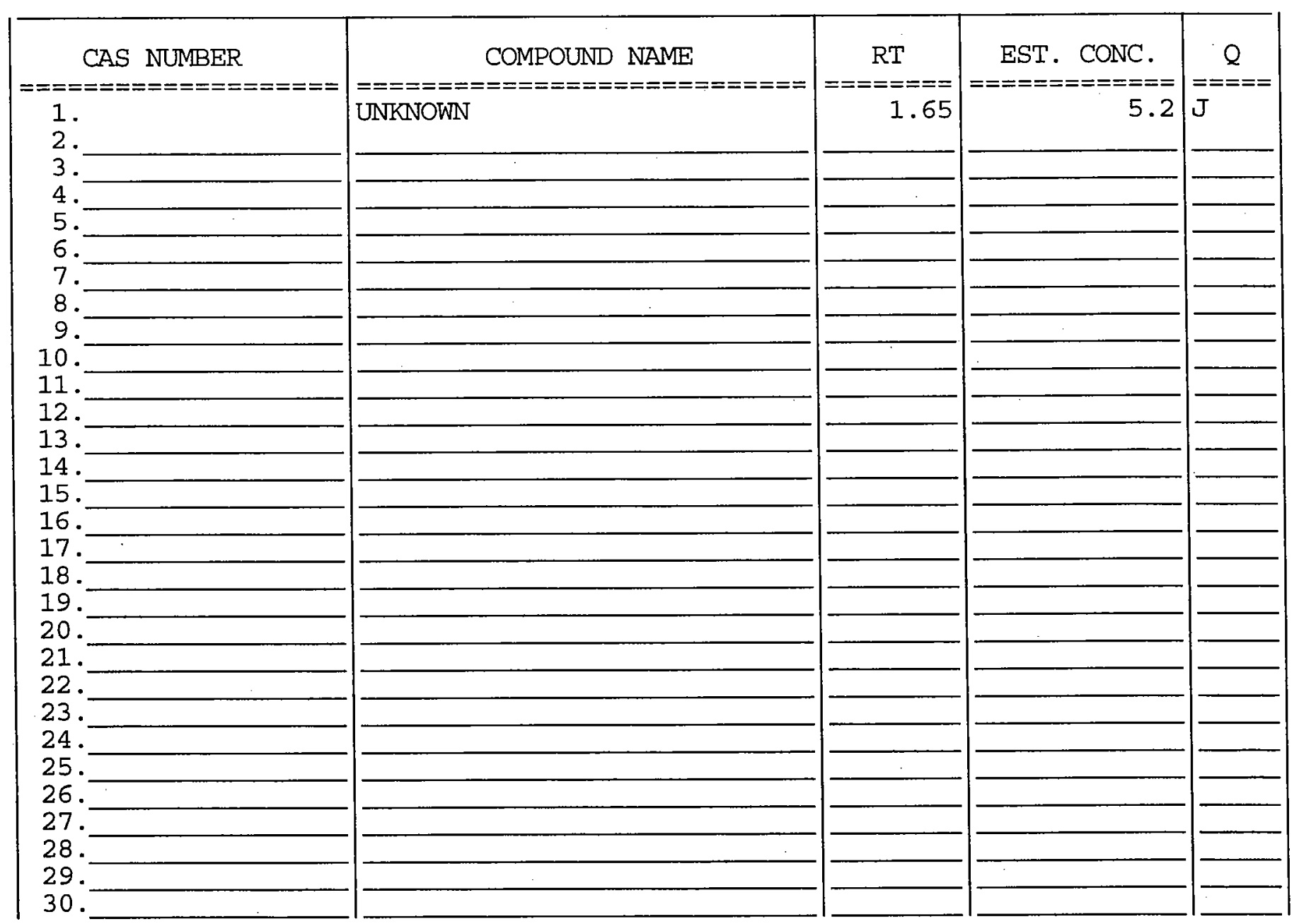

FORM I VOA-TIC 
$1 \mathrm{~A}$

VOLATILE ORGANICS ANALYSIS DATA SHEET

Lab Name: ENVIROSYSTEMS, INC.

Contract: ARGONNE
EPA SAMPLE NO.

NA-TI27W-20700HW

Lab Code: ENVSYS Case No.: AR0511W SAS No.:

SDG No.: AR0511W

Matrix: (soil/water) WATER

Sample wt/vol : $\quad 5.000(\mathrm{~g} / \mathrm{mL}) \mathrm{ML}$

Level: (low/med) LOW

\% Moisture: not dec.

GC Column: RTX-624 ID: 0.18 (mm)

Soil Extract Volume: (uL)
Lab Sample ID: 0060511-06

Lab File ID: AG75HC070

Date Received: 05/24/06

Date Analyzed: 05/24/06

Dilution Factor: 1.0

Soil Aliquot Volume:
CONCENTRATION UNITS :

(ug/L or $\mathrm{ug} / \mathrm{Kg}) \quad \mathrm{UG} / \mathrm{L} \quad \mathrm{Q}$
CAS NO.

COMPOUND
T

\begin{tabular}{|l|l|l|}
\hline 5.0 & $\mathrm{U}$ \\
5.0 & $\mathrm{U}$ \\
5.0 & $\mathrm{U}$ \\
5.0 & $\mathrm{U}$ \\
5.0 & $\mathrm{U}$ \\
5.0 & $\mathrm{U}$ \\
5.0 & $\mathrm{U}$ \\
5.0 & $\mathrm{U}$ \\
5.0 & $\mathrm{U}$ \\
5.0 & $\mathrm{U}$ \\
5.0 & $\mathrm{U}$ \\
2.5 & $\mathrm{JB}$ \\
5.0 & $\mathrm{U}$ \\
5.0 & $\mathrm{U}$ \\
5.0 & $\mathrm{U}$ \\
5.0 & $\mathrm{U}$ \\
5.0 & $\mathrm{U}$ \\
0.59 & $\mathrm{~J}$ \\
5.0 & $\mathrm{U}$ \\
5.0 & $\mathrm{U}$ \\
1.3 & $\mathrm{~J}$ \\
5.0 & $\mathrm{U}$ \\
5.0 & $\mathrm{U}$ \\
\hline
\end{tabular}

Dichlorodifluoromethane
Chloromethane
Vinyl Chloride
Bromomethane
Chloroethane
Trichlorofluoromethane
1,1-Dichloroethene
1,1,2-Trichloro-1,2,2-trifluoroethane
Acetone
Carbon Disulfide
Methyl Acetate
Methylene Chloride
trans-1,2-Dichloroethene
Methyl tert-Butyl Ether
1,1-Dichloroethane
cis-1,2-Dichloroethene
2-Butanone
Chloroform
1,1,1-Trichloroethane
Cyclohexane
Carbon Tetrachloride
Benzene
1,2-Dichloroethane

Dichlorodifluoromethane

$74-87-3$

$75-01-4$

$74-83-9$

$75-00-3$

$75-69-4$

$75-35-4$

$76-13-1$

67-64-1

75-15-0

$79-20-9$

75-09-2

156-60-5

1634-04-4

$75-34-3$

156-59-2

$78-93-3$

$67-66-3$

$71-55-6$

110-82-7

56-23-5

$71-43-2$

107-06-2 
Lab Name: ENVIROSYSTEMS, INC.

Contract: ARGONNE

Lab Code: ENVSYS Case No.: AR0511W SAS No.:

SDG NO.: AR0511W

Matrix: (soil/water) WATER

Sample wt/vol:

$5.000(\mathrm{~g} / \mathrm{mL}) \mathrm{ML}$

Level: (low/med) LOW

\% Moisture: not dec.

GC Column: RTX-624 ID: $0.18 \quad(\mathrm{~mm})$

Soil Extract Volume: (uL)
Lab Sample ID: 0060511-06

Lab File ID: AG75HC070

Date Received: 05/24/06

Date Analyzed: 05/24/06

Dilution Factor: 1.0

Soil Aliquot Volume:
CAS NO.

COMPOUND
(ug/L or ug/Kg).

\begin{tabular}{|r|l|l|l|}
\hline $79-01-6$ & Trichloroethene & $\mathrm{U}$ \\
$108-87-2$ & Methylcyclohexane & 5.0 & $\mathrm{U}$ \\
$78-87-5$ & 1,2-Dichloropropane & 5.0 & $\mathrm{U}$ \\
$75-27-4$ & Bromodichloromethane & 5.0 & $\mathrm{U}$ \\
$10061-01-5$ & Cis-1,3-Dichloropropene & 5.0 & $\mathrm{U}$ \\
$108-10-1$ & 4-Methyl-2-Pentanone & 5.0 & $\mathrm{U}$ \\
$108-88-3$ & Toluene & 5.0 & $\mathrm{U}$ \\
$10061-02-6$ & trans-1,3-Dichloropropene & 5.0 & $\mathrm{U}$ \\
$79-00-5$ & 1,1,2-Trichloroethane & 5.0 & $\mathrm{U}$ \\
$127-18-4$ & Tetrachloroethene & 5.0 & $\mathrm{U}$ \\
$591-78-6$ & 2-Hexanone & 5.0 & $\mathrm{U}$ \\
$124-48-1$ & Dibromochloromethane & 5.0 & $\mathrm{U}$ \\
$106-93-4$ & 1,2-Dibromoethane & 5.0 & $\mathrm{U}$ \\
$108-90-7$ & Chlorobenzene & 5.0 & $\mathrm{U}$ \\
$100-41-4$ & Ethylbenzene & 5.0 & $\mathrm{U}$ \\
$1330-20-7$ & Xylene (Total) & 5.0 & $\mathrm{U}$ \\
$100-42-5$ & Styrene & 5.0 & $\mathrm{U}$ \\
$75-25-2$ & Bromoform & 5.0 & $\mathrm{U}$ \\
$98-82-8$ & Isopropylbenzene & 5.0 & $\mathrm{U}$ \\
$79-34-5$ & 1,1,2,2-Tetrachloroethane & 5.0 & $\mathrm{U}$ \\
$541-73-1$ & $1,3-$ Dichlorobenzene & 5.0 & $\mathrm{U}$ \\
$106-46-7$ & $1,4-$ Dichlorobenzene & 5.0 & $\mathrm{U}$ \\
$95-50-1$ & 1,2-Dichlorobenzene & 5.0 & $\mathrm{U}$ \\
$96-12-8$ & 1,2-Dibromo-3-chloropropane & 5.0 & $\mathrm{U}$ \\
$120-82-1$ & 1,2,4-Trichlorobenzene & 5.0 & $\mathrm{U}$ \\
\hline
\end{tabular}


Lab Name: ENVIROSYSTEMS, INC.

Contract: ARGONNE

Lab Code: ENVSYS Case No.: AR0511W SAS No.:

SDG No.: AR0511W

Matrix: (soil/water) WATER

Sample wt/vol: $5.000(\mathrm{~g} / \mathrm{mL}) \mathrm{ML}$

Level : (low/med) LOW

․․ Moisture: not dec.

GC Column: RTX-624 ID: 0.18 (mm)

Soil Extract Volume: (uL)

Number TICs found: 0
Lab Sample ID: 0060511-06

Lab File ID: AG75HC070

Date Received: 05/24/06

Date Analyzed: 05/24/06

Dilution Factor: 1.0

Soil Aliquot Volume:

CONCENIRATION UNITS :

(ug/L or $u g / K g$ ) ug/L

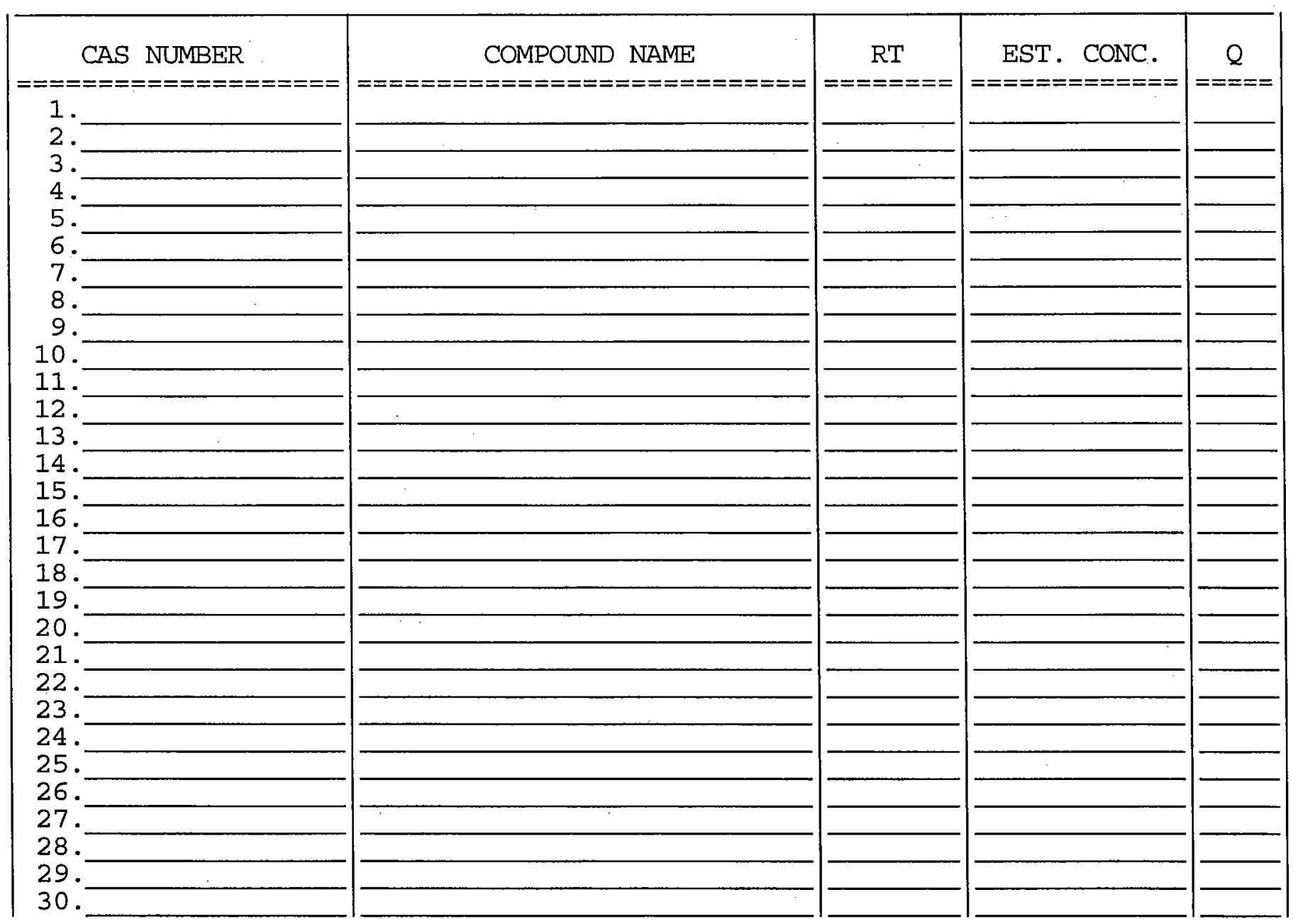


Lab Name: ENVIROSYSTEMS, INC.

Contract: ARGONNE

EPA SAMPLE NO.

Lab Code: ENVSYS Case No.: AR0511W SAS No.:

NA-TI28W-20904HW

Matrix: (soil/water) WATER

SDG NO.: AR0511W

Sample wt/vol: $\quad 5.000(\mathrm{~g} / \mathrm{mL}) \mathrm{ML}$

Lab Sample ID: 0060511-07

Level: (low/med) LOW

Lab File ID: AG75HC071

\% Moisture: not dec.

GC Column: RTX-624 ID: 0.18 (mm)

Date Received: 05/24/06

Soil Extract Volume:

Date Analyzed: 05/24/06

Dilution Factor: 1.0

Soil Aliquot Volume: (uL)

CAS NO.

COMPOUND

CONCENTRATION UNITS:

(ug/L or $\mathrm{ug} / \mathrm{Kg}$ ) UG/L Q

\begin{tabular}{|c|c|c|c|}
\hline $\begin{array}{r}75-71-8 \\
74-87-3 \\
75-01-4 \\
74-83-9 \\
75-00-3 \\
75-69-4 \\
75-35-4 \\
76-13-1 \\
67-64-1 \\
75-15-0 \\
79-20-9 \\
75-09-2 \\
156-60-5 \\
1634-04-4 \\
75-34-3 \\
156-59-2 \\
78-93-3 \\
67-66-3 \\
71-55-6 \\
110-82-7 \\
56-23-5 \\
71-43-2 \\
107-06-2\end{array}$ & $\begin{array}{l}\text { Dichlorodifluoromethane } \\
\text { Chloromethane } \\
\text { Vinyl Chloride } \\
\text { Bromomethane } \\
\text { Chloroethane } \\
\text { Trichlorofluoromethane } \\
\text { 1,1-Dichloroethene } \\
\text { 1,1,2-Trichloro-1,2,2-trifluoroethane } \\
\text { Acetone } \\
\text { Carbon Disulfide } \\
\text { Methyl Acetate } \\
\text { Methylene Chloride } \\
\text { trans-1,2-Dichloroethene } \\
\text { Methyl tert-Butyl Ether } \\
\text { 1,1-Dichloroethane } \\
\text { cis-1,2-Dichloroethene } \\
\text { 2-Butanone } \\
\text { Chloroform } \\
\text { 1,1,1-Trichloroethane } \\
\text { Cyclohexane } \\
\text { Carbon Tetrachloride } \\
\text { Benzene } \\
\text { 1,2-Dichloroethane }\end{array}$ & $\begin{array}{r}5.0 \\
5.0 \\
5.0 \\
5.0 \\
5.0 \\
5.0 \\
5.0 \\
5.0 \\
5.0 \\
5.0 \\
5.0 \\
4.2 \\
5.0 \\
5.0 \\
5.0 \\
5.0 \\
5.0 \\
210 \\
5.0 \\
5.0 \\
1800 \\
5.0 \\
5.0\end{array}$ & $\begin{array}{l}U \\
U \\
U \\
U \\
U \\
U \\
U \\
U \\
U \\
U \\
U \\
J B \\
U \\
U \\
U \\
U \\
U \\
E \\
U \\
U \\
E \\
U \\
U\end{array}$ \\
\hline
\end{tabular}


Lab Name: ENVIROSYSTEMS, INC.

Contract: ARGONNE

Lab Code: ENVSYS Case No.: AR0511W SAS No.:

SDG No.: AR0511W

Matrix: (soil/water) WATER

Sample wt/vol: $\quad 5.000(\mathrm{~g} / \mathrm{mL}) \mathrm{ML}$

Level: (low/med) LOW

\% Moisture: not dec.

GC Column: RTX-624 ID: 0.18 (mm)

Soil Extract Volume:

(uL)
Lab Sample ID: 0060511-07

Lab File ID: AG75HC071

Date Received: 05/24/06

Date Analyzed: 05/24/06

Dilution Factor: 1.0

Soil Aliquot Volume:

CONCENTRATION UNITS:

CAS NO.

COMPOUND (ug/L or $\mathrm{ug} / \mathrm{Kg}$ )

UG/L Q

\begin{tabular}{|c|c|c|c|}
\hline $\begin{array}{r}79-01-6 \\
108-87-2 \\
78-87-5 \\
75-27-4 \\
10061-01-5 \\
108-10-1 \\
108-88-3 \\
10061-02-6 \\
79-00-5 \\
127-18-4 \\
591-78-6 \\
124-48-1 \\
106-93-4 \\
108-90-7 \\
100-41-4 \\
1330-20-7 \\
100-42-5 \\
75-25-2 \\
98-82-8 \\
79-34-5 \\
541-73-1 \\
106-46-7 \\
95-50-1 \\
96-12-8 \\
120-82-1\end{array}$ & 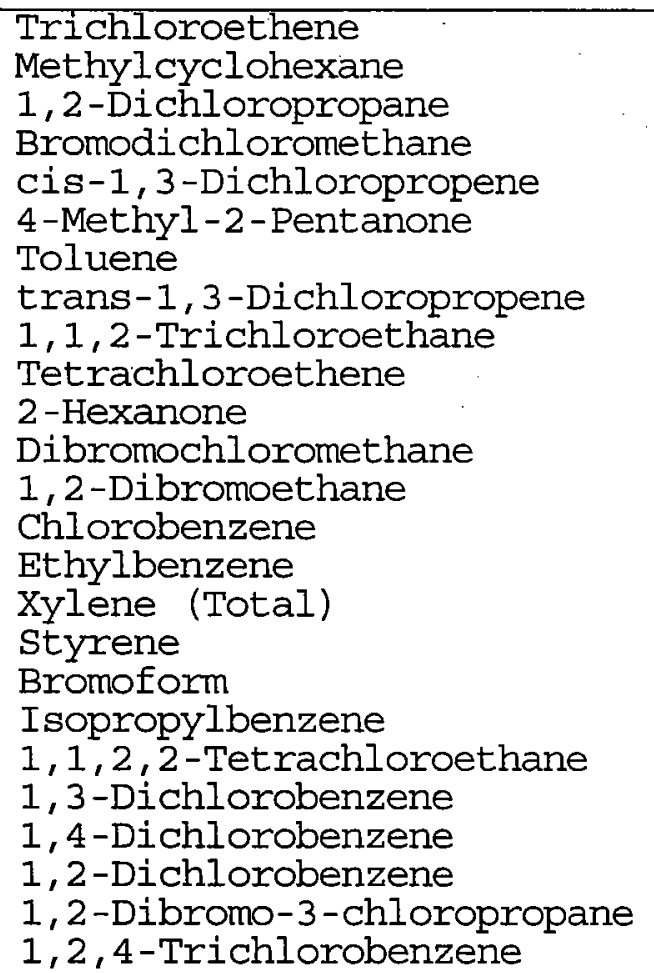 & $\begin{array}{l}5.0 \\
5.0 \\
5.0 \\
5.0 \\
5.0 \\
5.0 \\
5.0 \\
5.0 \\
5.0 \\
5.0 \\
5.0 \\
5.0 \\
5.0 \\
5.0 \\
5.0 \\
5.0 \\
5.0 \\
5.0 \\
5.0 \\
5.0 \\
5.0 \\
5.0 \\
5.0 \\
5.0 \\
5.0\end{array}$ & $\begin{array}{l}\mathrm{U} \\
\mathrm{U} \\
\mathrm{U} \\
\mathrm{U} \\
\mathrm{U} \\
\mathrm{U} \\
\mathrm{U} \\
\mathrm{U} \\
\mathrm{U} \\
\mathrm{U} \\
\mathrm{U} \\
\mathrm{U} \\
\mathrm{U} \\
\mathrm{U} \\
\mathrm{U} \\
\mathrm{U} \\
\mathrm{U} \\
\mathrm{U} \\
\mathrm{U} \\
\mathrm{U} \\
\mathrm{U} \\
\mathrm{U} \\
\mathrm{U} \\
\mathrm{U} \\
\mathrm{U}\end{array}$ \\
\hline
\end{tabular}


VOLATILE ORGANICS ANALYSIS DATA SHEET TENTATIVELY IDENTIFIED COMPOUNDS

Lab Name: ENVIROSYSTEMS, INC. Contract: ARGONNE
EPA SAMPLE NO.

NA-TI28W-20904HW

Lab Code: ENVSYS Case No.: AR0511W SAS No.:

SDG No.: AR0511W

Matrix: (soil/water) WATER

Sample wt/vol: $5.000(\mathrm{~g} / \mathrm{mL}) \mathrm{ML}$

Level : (low/med) LOW

$\%$ Moisture: not dec.

GC Column: RTX-624 ID: 0.18 (mm)

Soil Extract Volume:

(uL)

Number TICs found: 0
Lab Sample ID: 0060511-07

Lab File ID: AG75HC071

Date Received: 05/24/06

Date Analyzed: 05/24/06

Dilution Factor: 1.0

Soil Aliquot Volume: (uL)

CONCENTRATION UNITS :

$(\mathrm{ug} / \mathrm{L}$ or $\mathrm{ug} / \mathrm{Kg}) \mathrm{ug} / \mathrm{L}$

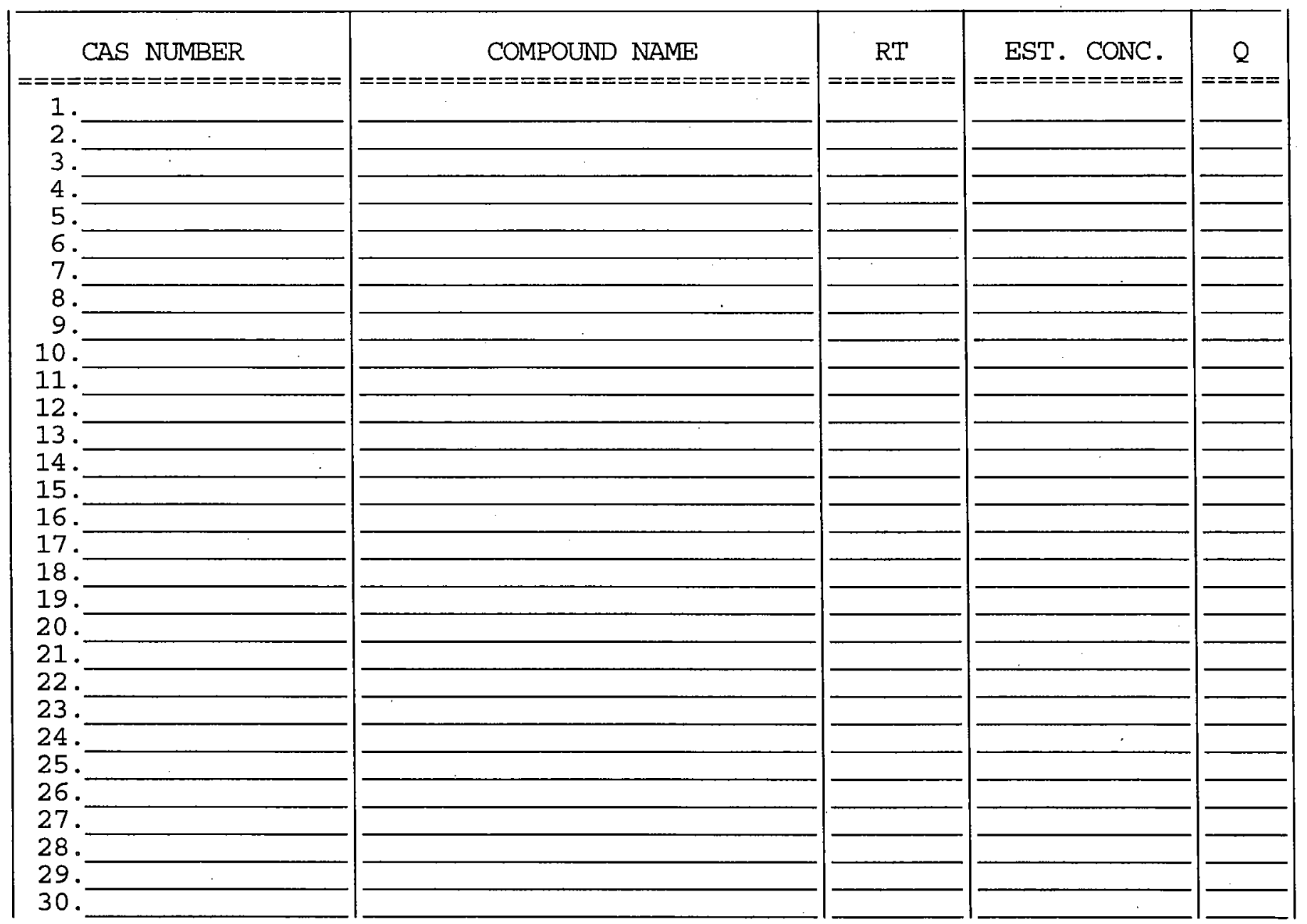


Lab Name: ENVIROSYSTEMS, INC.

Contract: ARGONNE

Lab Code: ENVSYS Case No.: AR0511W SAS No.:

SDG No.: AR0511W

Matrix: (soil/water) WATER

Lab Sample ID: 0060511-07RE2HE

Sample wt/vol: $\quad 5.000(\mathrm{~g} / \mathrm{mL}) \mathrm{ML}$

Lab File ID: AG75HC133

Level: (low/med) LOW

Date Received: 05/24/06

\% Moisture: not dec.

Date Analyzed: 06/01/06

GC Column: RTX-624 ID: $0.18,(\mathrm{~mm})$

Dilution Factor: 40.0

Soil Extract Volume: (uL)

Soil Aliquot Volume: (u山)

CAS NO.

COMPOUND

CONCENTRATION UNITS:

(ug/L or $\mathrm{ug} / \mathrm{Kg}) \quad$ UG/L Q

\begin{tabular}{|r|l|c|c|}
\hline $75-71-8$ & Dichlorodifluoromethane & 200 & $\mathrm{U}$ \\
$74-87-3$ & Chloromethane & 200 & $\mathrm{U}$ \\
$75-01-4$ & Vinyl Chloride & 200 & $\mathrm{U}$ \\
$74-83-9$ & Bromomethane & 200 & $\mathrm{U}$ \\
$75-00-3$ & Chloroethane & 200 & $\mathrm{U}$ \\
$75-69-4$ & Trichlorofluoromethane & 200 & $\mathrm{U}$ \\
$75-35-4$ & $1,1-$ Dichloroethene & 200 & $\mathrm{U}$ \\
$76-13-1$ & $1,1,2-$ Trichloro-1,2,2-trifluoroethane & 200 & $\mathrm{U}$ \\
$67-64-1$ & Acetone & 200 & $\mathrm{U}$ \\
$75-15-0$ & Carbon Disulfide & 200 & $\mathrm{U}$ \\
$79-20-9$ & Methyl Acetate & 200 & $\mathrm{U}$ \\
$75-09-2$ & Methylene Chloride & 150 & $\mathrm{JB}$ \\
$156-60-5$ & trans-1,2-Dichloroethene & 200 & $\mathrm{U}$ \\
$1634-04-4$ & Methyl tert-Butyl Ether & 200 & $\mathrm{U}$ \\
$75-34-3$ & $1,1-$ Dichloroethane & 200 & $\mathrm{U}$ \\
$156-59-2$ & Cis-1,2-Dichloroethene & 200 & $\mathrm{U}$ \\
$78-93-3$ & 2-Butanone & 200 & $\mathrm{U}$ \\
$77-66-3$ & Chloroform & 200 & \\
$71-55-6$ & 1,1,1-Trichloroethane & 200 & $\mathrm{U}$ \\
$110-82-7$ & Cyclohexane & 200 & $\mathrm{U}$ \\
$56-23-5$ & Carbon Tetrachloride & 3100 & \\
$71-43-2$ & Benzene & 200 & $\mathrm{U}$ \\
$107-06-2$ & 1,2-Dichloroethane & 200 & $\mathrm{U}$ \\
\hline
\end{tabular}


Lab Name: ENVIROSYSTEMS, INC.

Contract : ARGONNE

Lab Code: ENVSYS Case No.: AR0511W SAS No.:

SDG No.: AR0511W

Matrix: (soil/water) WATER

Sample wt/vol: $\quad 5.000(\mathrm{~g} / \mathrm{mL}) \mathrm{ML}$

Level : (low/med) LOW

$\%$ Moisture: not dec.

GC Column: RTX-624 ID: 0.18 (mm)

Soil Extract Volume: (uL)
Lab Sample ID: 0060511-07RE2HE

Lab File ID: AG75HC133

Date Received: 05/24/06

Date Analyzed: 06/01/06

Dilution Factor: 40.0

Soil Aliquot Volume: (uL)

CONCENTRATION UNITS:

CAS NO.

COMPOUND
UG/I Q

\begin{tabular}{|c|c|c|c|}
\hline $\begin{array}{r}79-01-6 \\
108-87-2 \\
78-87-5 \\
75-27-4 \\
10061-01-5 \\
108-10-1 \\
108-88-3 \\
10061-02-6 \\
79-00-5 \\
127-18-4 \\
591-78-6 \\
124-48-1 \\
106-93-4 \\
108-90-7 \\
100-41-4 \\
1330-20-7 \\
100-42-5 \\
75-25-2 \\
98-82-8 \\
79-34-5 \\
541-73-1 \\
106-46-7 \\
95-50-1 \\
96-12-8 \\
120-82-1\end{array}$ & $\begin{array}{l}\text { Trichloroethene } \\
\text { Methylcyclohexane } \\
\text { 1,2-Dichloropropane } \\
\text { Bromodichloromethane } \\
\text { cis-1,3-Dichloropropene } \\
\text { 4-Methyl-2-Pentanone } \\
\text { Toluene } \\
\text { trans-1,3-Dichloropropene } \\
\text { 1,1,2-Trichloroethane } \\
\text { Tetrachloroethene } \\
\text { 2-Hexanone } \\
\text { Dibromochloromethane } \\
\text { 1,2-Dibromoethane } \\
\text { Chlorobenzene } \\
\text { Ethylbenzene } \\
\text { Xylene (Total) } \\
\text { Styrene } \\
\text { Bromoform } \\
\text { Isopropylbenzene } \\
\text { 1,1,2,2-Tetrachloroethane } \\
\text { 1,3-Dichlorobenzene } \\
\text { 1,4-Dichlorobenzene } \\
\text { 1,2-Dichlorobenzene } \\
\text { 1,2-Dibromo-3-chloropropane } \\
\text { 1,2,4-Trichlorobenzene }\end{array}$ & $\begin{array}{l}200 \\
200 \\
200 \\
200 \\
200 \\
200 \\
200 \\
200 \\
200 \\
200 \\
200 \\
200 \\
200 \\
200 \\
200 \\
200 \\
200 \\
200 \\
200 \\
200 \\
200 \\
200 \\
200 \\
200 \\
200\end{array}$ & $\begin{array}{l}\mathrm{U} \\
\mathrm{U} \\
\mathrm{U} \\
\mathrm{U} \\
\mathrm{U} \\
\mathrm{U} \\
\mathrm{U} \\
\mathrm{U} \\
\mathrm{U} \\
\mathrm{U} \\
\mathrm{U} \\
\mathrm{U} \\
\mathrm{U} \\
\mathrm{U} \\
\mathrm{U} \\
\mathrm{U} \\
\mathrm{U} \\
\mathrm{U} \\
\mathrm{U} \\
\mathrm{U} \\
\mathrm{U} \\
\mathrm{U} \\
\mathrm{U} \\
\mathrm{U} \\
\mathrm{U}\end{array}$ \\
\hline
\end{tabular}


Lab Name: ENVIROSYSTEMS, INC.

Contract : ARGONNE

NA-TI28-W-20104

Lab Code: ENVSYS Case No.: AR0511W SAS No.:

SDG No.: AR0511W

Matrix: (soil/water) WATER

Sample wt/vol: $5.000(\mathrm{~g} / \mathrm{mL}) \mathrm{ML}$

Level : (low/med) LOW

$\%$ Moisture: not dec.

GC Column: RTX-624 ID: 0.18 (mm)

Soil Extract Volume:

(uL)

Number TICs found: 0

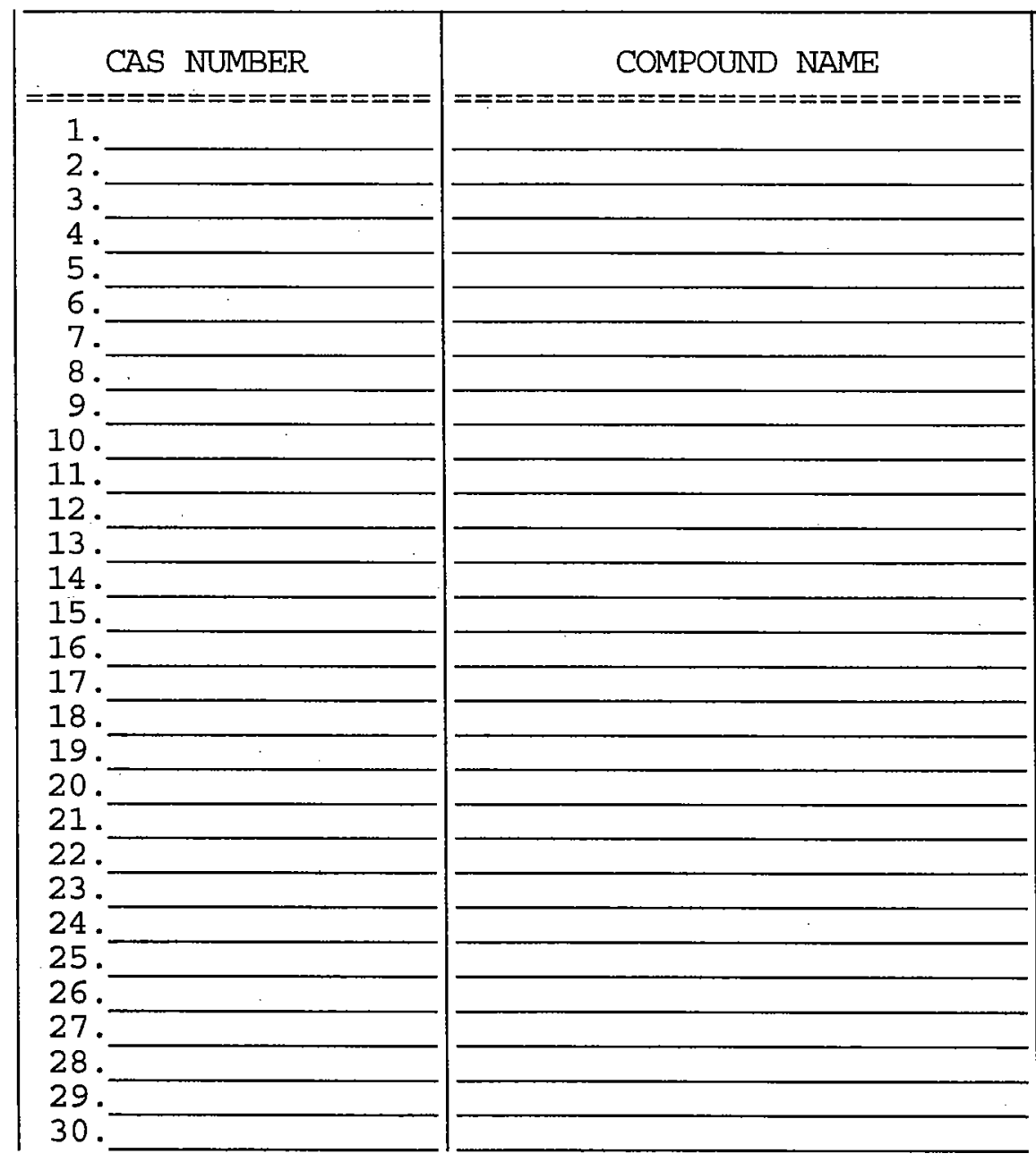

Lab Sample ID: 0060511-07RE2HE

Lab File ID: AG75HC133

Date Received: 05/24/06

Date Analyzed: 06/01/06

Dilution Factor: 40.0

Soil Aliquot Volume: (uL)

CONCENTRATION UNITS:

(ug/L or $u g / \mathrm{Kg}$ ) ug/L

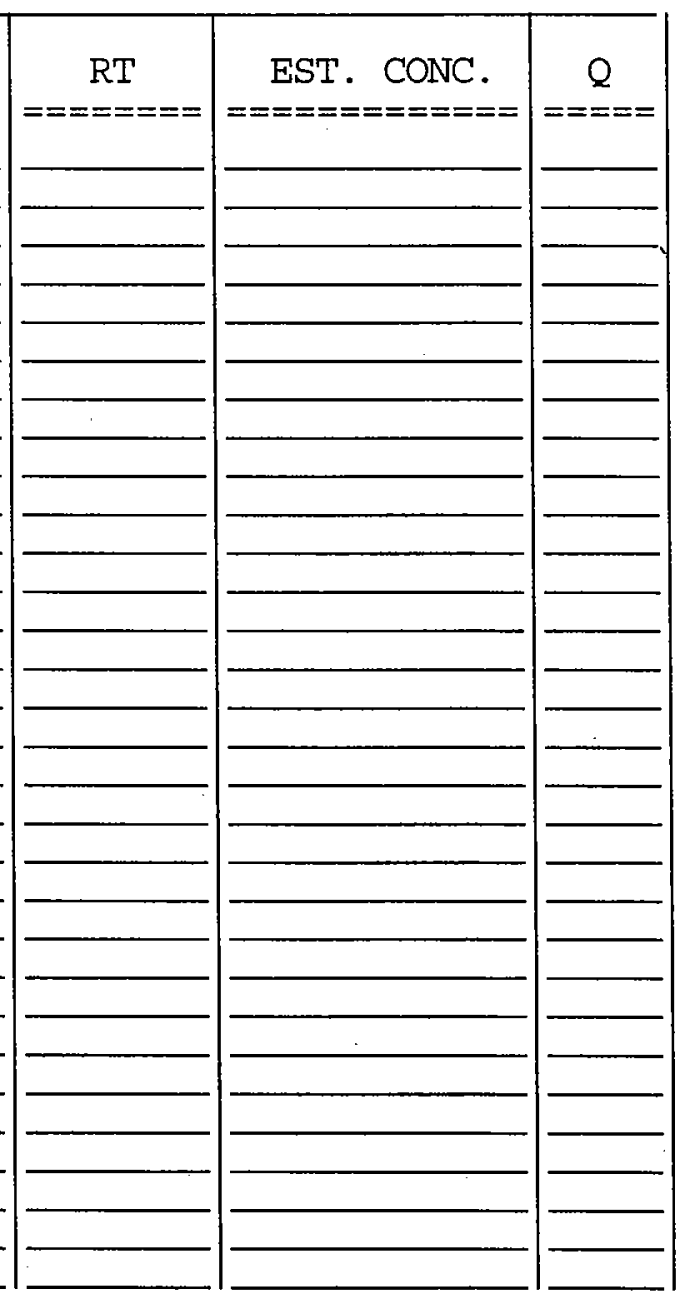


Lab Name: ENVIROSYSTEMS, INC. Contract: ARGONNE

NA-052306HW

Lab Code: ENVSYS Case No.: AR0511W SAS No.:

SDG No. : AR0511W

Matrix: (soil/water) WATER

Lab Sample ID: 0060511-08

Sample wt/vol:

$5.000(\mathrm{~g} / \mathrm{mL}) \mathrm{ML}$

Lab File ID: AG75HC072

Level: (low/med) LOW

Date Received: 05/24/06

$\%$ Moisture: not dec.

Date Analyzed: 05/24/06

GC Column: RTX-624 ID: 0.18 (mm)

Dilution Factor: 1.0

Soil Extract Volume: (uL)

Soil Aliquot Volume:

(uL)

CAS NO.

COMPOUND

CONCENTRATION UNITS:

(ug/L or $\mathrm{ug} / \mathrm{Kg}$ ) UG/L Q

\begin{tabular}{|c|c|c|c|}
\hline $\begin{array}{r}75-71-8 \\
74-87-3 \\
75-01-4 \\
74-83-9 \\
75-00-3 \\
75-69-4 \\
75-35-4 \\
76-13-1 \\
67-64-1 \\
75-15-0 \\
79-20-9 \\
75-09-2 \\
156-60-5 \\
1634-04-4 \\
75-34-3 \\
156-59-2 \\
78-93-3 \\
67-66-3 \\
71-55-6 \\
110-82-7 \\
56-23-5 \\
71-43-2 \\
107-06-2\end{array}$ & $\begin{array}{l}\text { Dichlorodifluoromethane } \\
\text { Chloromethane } \\
\text { Vinyl Chloride } \\
\text { Bromomethane } \\
\text { Chloroethane } \\
\text { Trichlorofluoromethane } \\
\text { 1,1-Dichloroethene } \\
\text { 1,1,2-Trichloro-1,2,2-trifluoroethane } \\
\text { Acetone } \\
\text { Carbon Disulfide } \\
\text { Methyl Acetate } \\
\text { Methylene Chloride } \\
\text { trans-1,2-Dichloroethene } \\
\text { Methyl tert-Butyl Ether } \\
\text { 1,1-Dichloroethane } \\
\text { cis-1,2-Dichloroethene } \\
\text { 2-Butanone } \\
\text { Chloroform } \\
\text { 1,1,1-Trichloroethane } \\
\text { Cyclohexane } \\
\text { Carbon Tetrachloride } \\
\text { Benzene } \\
\text { 1,2-Dichloroethane }\end{array}$ & $\begin{array}{l}5.0 \\
5.0 \\
5.0 \\
5.0 \\
5.0 \\
5.0 \\
5.0 \\
5.0 \\
5.0 \\
5.0 \\
5.0 \\
3.0 \\
5.0 \\
5.0 \\
5.0 \\
5.0 \\
5.0 \\
5.0 \\
5.0 \\
5.0 \\
1.5 \\
5.0 \\
5.0\end{array}$ & $\begin{array}{l}U \\
U \\
U \\
U \\
U \\
U \\
U \\
U \\
U \\
U \\
U \\
J B \\
U \\
U \\
U \\
U \\
U \\
U \\
U \\
U \\
J \\
U \\
U\end{array}$ \\
\hline
\end{tabular}


Lab Name: ENVIROSYSTEMS, INC.

Contract: ARGONNE

NA-052306HW

Lab Code: ENVSYS Case No.: AR0511W SAS No.:

SDG NO.: AR0511W

Matrix: (soil/water) WATER

Sample wt/vol: $\quad 5.000(\mathrm{~g} / \mathrm{mL}) \mathrm{ML}$

Level: (low/med) LOW

$\%$ Moisture: not dec.

GC Column: RTX-624 ID: 0.18 (mm)

Soil Extract Volume: (uL)
Lab Sample ID: 0060511-08

Lab File ID: AG75HC072

Date Received: 05/24/06

Date Analyzed: 05/24/06

Dilution Factor: 1.0

Soil Aliquot Volume:

(uL)

CONCENTRATION UNITS:

CAS NO.

COMPOUND

(ug/L or $u g / \mathrm{kg}) \quad \mathrm{UG} / \mathrm{L} \quad Q$

\begin{tabular}{|r|l|l|l|}
\hline $79-01-6$ & Trichloroethene & 5.0 & $\mathrm{U}$ \\
$108-87-2$ & Methylcyclohexane & 5.0 & $\mathrm{U}$ \\
$78-87-5$ & 1,2-Dichloropropane & 5.0 & $\mathrm{U}$ \\
$75-27-4$ & Bromodichloromethane & 5.0 & $\mathrm{U}$ \\
$10061-01-5$ & cis-1,3-Dichloropropene & 5.0 & $\mathrm{U}$ \\
$108-10-1$ & 4-Methyl-2-Pentanone & 5.0 & $\mathrm{U}$ \\
$108-88-3$ & Toluene & 5.0 & $\mathrm{U}$ \\
$10061-02-6$ & trans-1,3-Dichloropropene & 5.0 & $\mathrm{U}$ \\
$79-00-5$ & 1,1,2-Trichloroethane & 5.0 & $\mathrm{U}$ \\
$127-18-4$ & Tetrachloroethene & 5.0 & $\mathrm{U}$ \\
$591-78-6$ & 2-Hexanone & 5.0 & $\mathrm{U}$ \\
$124-48-1$ & Dibromochloromethane & 5.0 & $\mathrm{U}$ \\
$106-93-4$ & 1,2-Dibromoethane & 5.0 & $\mathrm{U}$ \\
$108-90-7$ & Chlorobenzene & 5.0 & $\mathrm{U}$ \\
$100-41-4$ & Ethylbenzene & 5.0 & $\mathrm{U}$ \\
$1330-20-7$ & Xylene (Total) & 5.0 & $\mathrm{U}$ \\
$100-42-5$ & Styrene & 5.0 & $\mathrm{U}$ \\
$75-25-2$ & Bromoform & 5.0 & $\mathrm{U}$ \\
$98-82-8$ & Isopropylbenzene & 5.0 & $\mathrm{U}$ \\
$79-34-5$ & 1,1,2,2-Tetrachloroethane & 5.0 & $\mathrm{U}$ \\
$541-73-1$ & 1,3-Dichlorobenzene & 5.0 & $\mathrm{U}$ \\
$106-46-7$ & 1,4-Dichlorobenzene & 5.0 & $\mathrm{U}$ \\
$95-50-1$ & 1,2-Dichlorobenzene & 5.0 & $\mathrm{U}$ \\
$96-12-8$ & 1,2 -Dibromo-3-chloropropane & 5.0 \\
$120-82-1$ & 1,2,4-Trichlorobenzene & $\mathrm{U}$ \\
\hline
\end{tabular}


VOLATILE ORGANICS ANALYSIS DATA SHEET TENTATIVELY IDENTIFIED COMPOUNDS

Lab Name: ENVIROSYSTEMS, INC.

Contract : ARGONNE

Lab Code: ENVSYS Case No.: AR0511W SAS No.:

SDG No. : AR0511W

Matrix: (soil/water) WATER

Sample wt/vol: $5.000(\mathrm{~g} / \mathrm{mL}) \mathrm{ML}$

Level: (low/med) LOW

$\%$ Moisture: not dec.

GC Column: RTX-624 ID: 0.18 (mm)

Soil Extract Volume:

(uL)

Number TICs found: 0
Lab Sample ID: 0060511-08

Lab File ID: AG75HCO72

Date Received: 05/24/06

Date Analyzed: 05/24/06

Dilution Factor: 1.0

Soil Aliquot Volume:

CONCENTRATION UNITS:

(ug/L or $\mathrm{ug} / \mathrm{Kg}$ ) ug/L

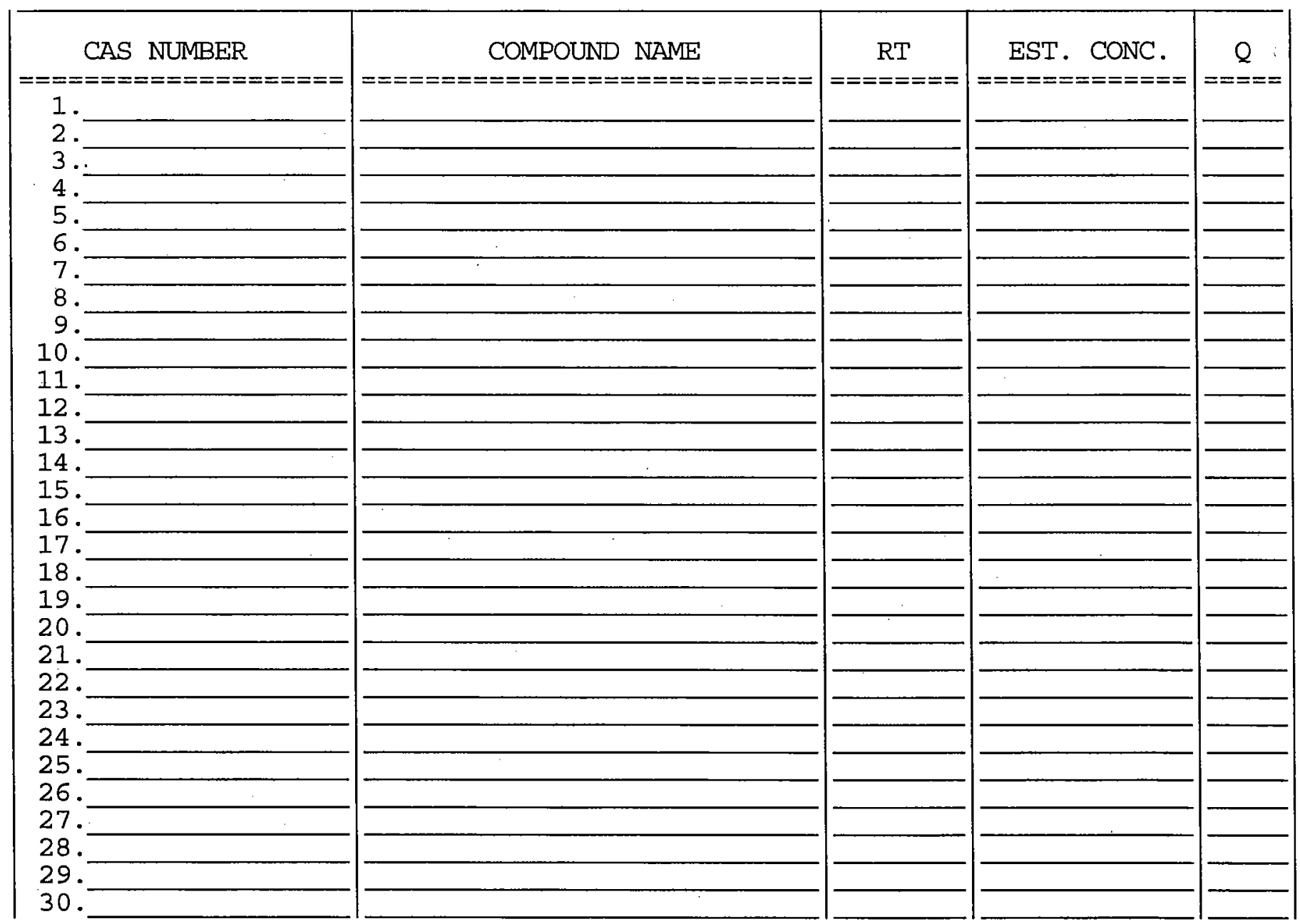




\section{ENVIROSYSTEMS, InC.}

9200 Rumsey Road • Suite B102 - Columbia, Maryland 21045-1934

Phone (410) 964-0330 - Fax (410) 740-9306

Email: info@envsystems.com -Webpage: www.envsystems.com/envsys

June 6, 2006

Jorge S. Alvarado, Ph.D

Argonne National Laboratory

Environmental Sciences Division

Applied Geoscience and Environmental

Management Section

9700 South Cass Avenue, EV-203-A137

Argonne, Illinois 60439

\section{RE: ENVSYS Report 0606054}

Dear Jorge:

Enclosed is the Analytical Data Package for the samples received on May 26, 2006 for volatile organics analysis by US EPA CLP SOW OLM04.3

Please do not hesitate to call me if you have any questions, comments, or require additional information.

Sincerely,<smiles>C=CC(C)CCCCC</smiles>

Mohan Khare, Ph.D

President/CEO

$\mathrm{MK} / \mathrm{sp}$ 
1. Narrative

00248

$$
001 \text { PL } 6 / 6106
$$




\section{SDG NARRATIVE}

LABORATORY NAME: ENVIROSYSTEMS, INC.

CLIENT: ARGONNE NATIONAL LABORATORY

DATA SAMPLES RECEIVED AT LABORATORY: MAY $26^{\text {th }}, 2006$

SAMPLE ANALYSES INCLUDED IN THIS REPORT:

$\begin{array}{lllll}\text { CLIENT \# } & \text { LAB ID\# } & \text { ANALYSIS } & \text { MATRIX } & \text { VOA pH } \\ \text { NA-QCTB-052506 } & 0060512-01 & \text { VOA } & \text { WATER } & 7 \\ \text { NA-TI29-W-20916 } & 0060512-02 & \text { VOA } & \text { WATER } & 7 \\ \text { NA-TI30-W-20920 } & 0060512-03 & \text { VOA } & \text { WATER } & 7 \\ \text { NA-TI30-W-20712 } & 0060512-04 & \text { VOA } & \text { WATER } & 7 \\ \text { NA-TI20-W-20913 } & 0060512-05 & \text { VOA } & \text { WATER } & 7 \\ \text { NA-TI30-W-20919 } & 0060512-06 & \text { VOA } & \text { WATER } & 7\end{array}$

Matrix spike/matrix spike duplicate analysis was not performed for this case.

Samples for this SDG are analyzed by EPA SOW OLMO4.3 for multi-media multi-concentration organics. Sample detection limits have meet client requirements.

The cooler temperature was measured to be the required temperture upon receipt. One vial NA-TI30-W-20712 was received broken at the laboratory. Sufficient sample was available to perform VOC analysis.

The volatile analysis was performed on an Agilent 5975 GC/MS using a Restek RTX-624 20 meter column with an inner diameter of $0.18 \mathrm{~mm}$ and a 1 micron film thickness. The trap used with the autosampler is a $0.3 \mathrm{~cm} \mathrm{OD} 28.5$ cm L ENCON Ambient Packed Trap.

Three compounds did not meet the initial calibration criteria. Percent RSD for bromomethane and bromoform were greater than 20.5 and the response factor for 1,1,2,2 tetrachloroethane was below the minimum required. Continuing calibration VSTD050HD had two compounds with percent $\mathrm{D}$ values greater than 25 and one compound not meeting the required RRF of 0.300 .

All other QC criteria were met for all samples included in this report.

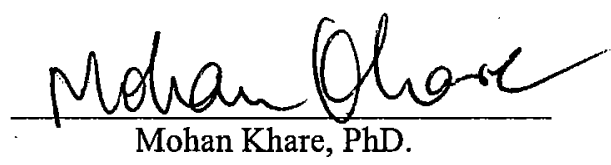

DATE: $\frac{6 / 6(06}{06^{\text {th }} \text { UNE } 2006}$ 
2. SGD Cover Sheet/Traffic Reports 


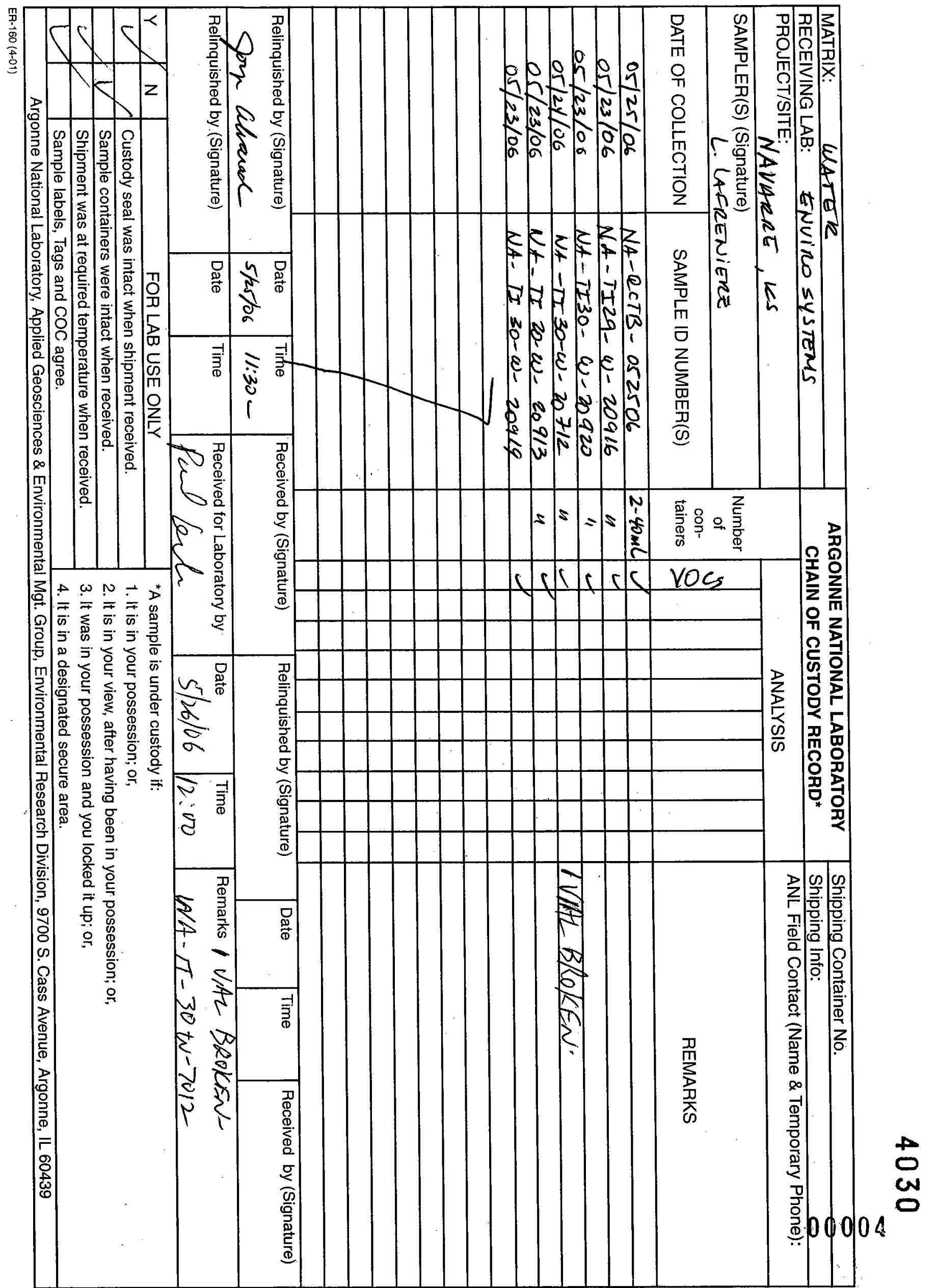


VOLATILE SAMPLE DATA

00012 
Lab Name: ENVIROSYSTEMS, INC.

Contract: ARGONNE

Lab Code: ENVSYS Case No.: AR0526 SAS No.:

SDG No. : AR0512W

Matrix: (soil/water) WATER

Lab Sample ID: 0060512-01

Sample wt/vol:

$5.000(\mathrm{~g} / \mathrm{mL}) \quad \mathrm{ML}$

Lab File ID: AG75HC120

Level : (low/med) LOW

Date Received: 05/26/06

\% Moisture: not dec.

GC Column: RTX-624 ID: 0.18 (mm)

Date Analyzed: 05/31/06

Soil Extract Volume: (uL)

Dilution Factor: 1.0

Soil Aliquot Volume:

CAS NO.

COMPOUND

CONCENTRATION UNITS:

(ug/L or ug/Kg) UG/L Q

$\begin{array}{r}75-71-8 \\ 74-87-3 \\ 75-01-4 \\ 74-83-9 \\ 75-00-3 \\ 75-69-4 \\ 75-35-4 \\ 76-13-1 \\ 67-64-1 \\ 75-15-0 \\ 79-20-9 \\ 75-09-2 \\ 156-60-5 \\ 1634-04-4 \\ 75-34-3 \\ 156-59-2 \\ 78-93-3 \\ 67-66-3 \\ 71-55-6 \\ 110-82-7 \\ 56-23-5 \\ 71-43-2 \\ 107-06-2 \\ \hline\end{array}$

Dichlorodifluoromethane
Chloromethane
Vinyl Chloride
Bromomethane
Chloroethane
Trichlorofluoromethane
1,1-Dichloroethene
1,1,2-Trichloro-1,2,2-trifluoroethane
Acetone
Carbon Disulfide
Methyl Acetate
Methylene Chloride
trans-1,2-Dichloroethene
Methyl tert-Butyl Ether
1,1-Dichloroethane
cis-1,2-Dichloroethene
2-Butanone
Chloroform
1,1,1-Trichloroethane
Cyclohexane
Carbon Tetrachloride
Benzene
1,2-Dichloroethane

(1)

\begin{tabular}{l|l|}
5.0 & $\mathrm{U}$ \\
5.0 & $\mathrm{U}$ \\
5.0 & $\mathrm{U}$ \\
5.0 & $\mathrm{U}$ \\
5.0 & $\mathrm{U}$ \\
5.0 & $\mathrm{U}$ \\
5.0 & $\mathrm{U}$ \\
5.0 & $\mathrm{U}$ \\
5.0 & $\mathrm{U}$ \\
5.0 & $\mathrm{U}$ \\
5.0 & $\mathrm{U}$ \\
3.1 & $\mathrm{~J}$ \\
5.0 & $\mathrm{U}$ \\
5.0 & $\mathrm{U}$ \\
5.0 & $\mathrm{U}$ \\
5.0 & $\mathrm{U}$ \\
5.0 & $\mathrm{U}$ \\
5.0 & $\mathrm{U}$ \\
5.0 & $\mathrm{U}$ \\
5.0 & $\mathrm{U}$ \\
5.0 & $\mathrm{U}$ \\
5.0 & $\mathrm{U}$ \\
5.0 & $\mathrm{U}$ \\
\hline
\end{tabular}


Lab Name: ENVIROSYSTEMS, INC.

Lab Code: ENVSYS Case No.:

Matrix: (soil/water) WATER

Sample wt/vol:

$5.000(\mathrm{~g} / \mathrm{mL}) \mathrm{ML}$

Level: (low/med) LOW

$\div$ Moisture: not dec.

GC Column: RTX-624 ID: 0.18 (mm)

Soil Extract Volume: (uL)
Contract: ARGONNE

SAS NO. :

SDG No. : AR0512W

Lab Sample ID: 0060512-01

Lab File ID: AG75HC120

Date Received: 05/26/06

Date Analyzed: 05/31/06

Dilution Factor: 1.0

Soil Aliquot Volume: (UL)

CONCENTRATION UNITS:

CAS NO.

COMPOUND

(ug/L or $u g / \mathrm{Kg}) \quad$ UG/L Q

\begin{tabular}{l|} 
Trichloroethene \\
Methylcyclohexane \\
1,2-Dichloropropane \\
Bromodichloromethane \\
cis-1,3-Dichloropropene \\
4-Methyl-2-Pentanone \\
Toluene \\
trans-1,3-Dichloropropene \\
1,1,2-Trichloroethane \\
Tetrachloroethene \\
2-Hexanone \\
Dibromochloromethane \\
1,2-Dibromoethane \\
Chlorobenzene \\
Ethylbenzene \\
Xylene (Total) \\
Styrene \\
Bromoform \\
Isopropylbenzene \\
$1,1,2,2-$ Tetrachloroethane \\
$1,3-$ ichlorobenzene \\
$1,4-D i c h l o r o b e n z e n e$ \\
$1,2-D i c h l o r o b e n z e n e$ \\
$1,2-D i b r o m o-3-c h l o r o p r o p a n e$ \\
$1,2,4-$ Trichlorobenzene
\end{tabular}

Trichloroethene

$108-87-2$

$78-87-5$

$75-27-4$

$10061-01-5$

$108-10-1$

$108-88-3$

$10061-02-6$

$79-00-5$

$127-18-4$

$591-78-6$

124-48-1

106-93-4

$108-90-7$

$100-41-4$

$1330-20-7$

$100-42-5$

$75-25-2$

$98-82-8$

$79-34-5$

$541-73-1$

$106-46-7$

$95-50-1$

96-12-8

120-82-1

\begin{tabular}{l|l|}
5.0 & $\mathrm{U}$ \\
5.0 & $\mathrm{U}$ \\
5.0 & $\mathrm{U}$ \\
5.0 & $\mathrm{U}$ \\
5.0 & $\mathrm{U}$ \\
5.0 & $\mathrm{U}$ \\
5.0 & $\mathrm{U}$ \\
5.0 & $\mathrm{U}$ \\
5.0 & $\mathrm{U}$ \\
5.0 & $\mathrm{U}$ \\
5.0 & $\mathrm{U}$ \\
5.0 & $\mathrm{U}$ \\
5.0 & $\mathrm{U}$ \\
5.0 & $\mathrm{U}$ \\
5.0 & $\mathrm{U}$ \\
5.0 & $\mathrm{U}$ \\
5.0 & $\mathrm{U}$ \\
5.0 & $\mathrm{U}$ \\
5.0 & $\mathrm{U}$ \\
5.0 & $\mathrm{U}$ \\
5.0 & $\mathrm{U}$ \\
5.0 & $\mathrm{U}$ \\
5.0 & $\mathrm{U}$ \\
5.0 & $\mathrm{U}$ \\
5.0 & $\mathrm{U}$ \\
\hline
\end{tabular}


Lab Name: ENVIROSYSTEMS, INC.

Lab Code: ENVSYS Case No.:

Matrix: (soil/water) WATER

Sample wt/vol: $5.000(\mathrm{~g} / \mathrm{mL}) \mathrm{ML}$

Level : (low/med) LOW

\% Moisture: not dec.

GC Column: RTX-624 ID: 0.18 (mm)

Soil Extract Volume:

(uL)

Number TICs found: 0
Contract: ARGONNE

SAS NO.:

SDG No.: AR0512W

Lab Sample ID: 0060512-01

Lab File ID: AG75HC120

Date Received: 05/26/06

Date Analyzed: 05/31/06

Dilution Factor: 1.0

Soil Aliquot Volume: (uW)

CONCENTRATION UNITS:

(ug/L or $\mathrm{ug} / \mathrm{Kg}$ ) ug/L

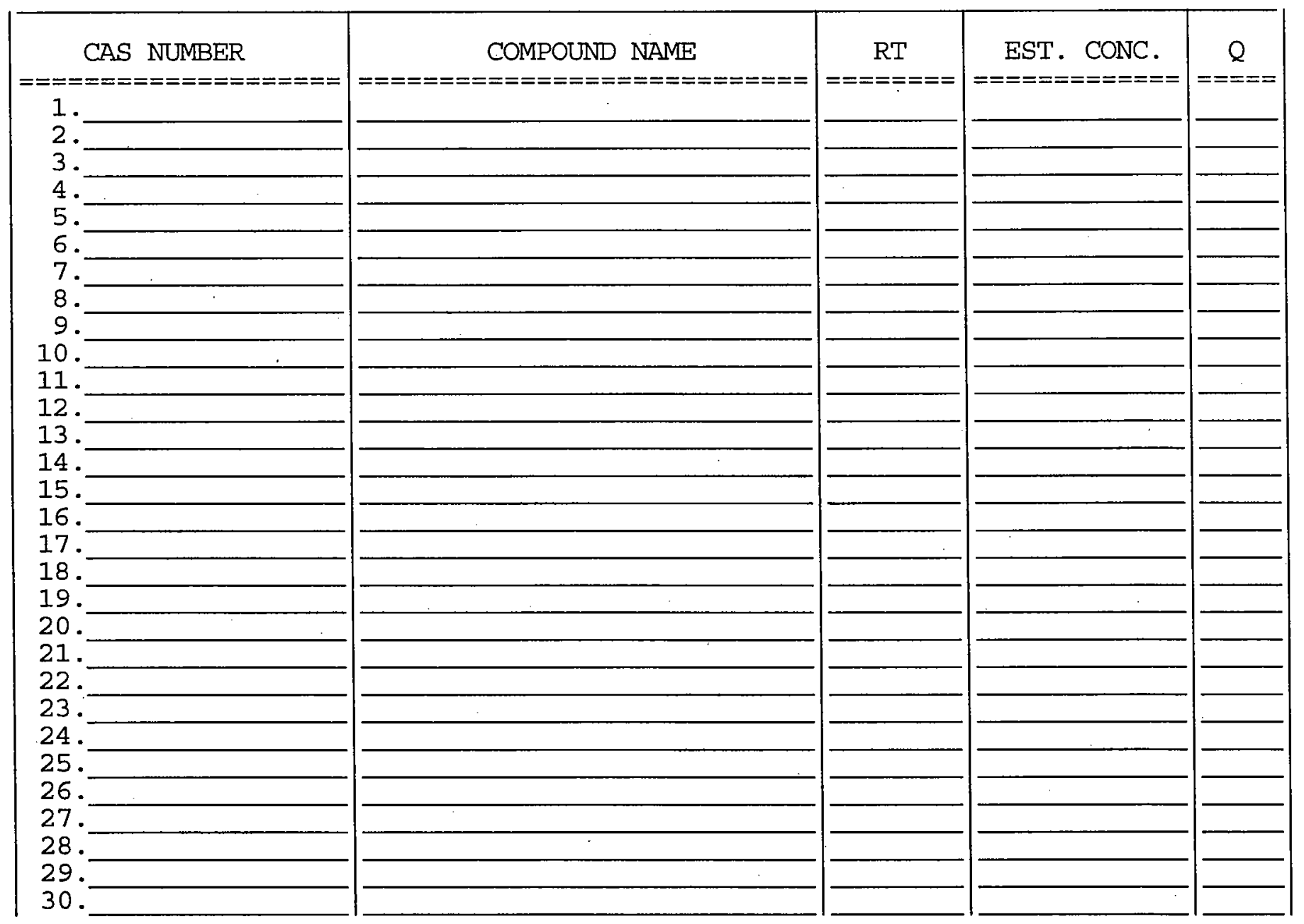


Lab Name: ENVIROSYSTEMS, INC.

Contract: ARGONNE

Lab Code: ENVSYS Case No.: AR0526 SAS No.:

SDG No. : AR0512W

Matrix: (soil/water) WATER

Lab Sample ID: 0060512-02

Sample wt/vol:

$5.000(\mathrm{~g} / \mathrm{mL}) \mathrm{ML}$

Lab File ID: AG75HC121

Level: (low/med) LOW

Date Received: 05/26/06

\% Moisture: not dec.

Date Analyzed: 05/31/06

GC Column: RTX-624 ID: 0.18 (mm)

Dilution Factor: 1.0

Soil Extract Volume: (uL)

Soil Aliquot Volume:

CAS NO.

COMPOUND

CONCENTRATION UNITS : (ug/L or $u g / \mathrm{Kg}$ ) UG/L Q

\begin{tabular}{|r|}
$75-71-8$ \\
$74-87-3$ \\
$75-01-4$ \\
$74-83-9$ \\
$75-00-3$ \\
$75-69-4$ \\
$75-35-4$ \\
$76-13-1$ \\
$67-64-1$ \\
$75-15-0$ \\
$79-20-9$ \\
$75-09-2$ \\
$156-60-5$ \\
$1634-04-4$ \\
$75-34-3$ \\
$156-59-2$ \\
$78-93-3$ \\
$67-66-3$ \\
$71-55-6$ \\
$110-82-7$ \\
$56-23-5$ \\
$71-43-2$ \\
$107-06-2$ \\
\hline
\end{tabular}

Dichlorodifluoromethane
Chloromethane
Vinyl Chloride
Bromomethane
Chloroethane
Trichlorofluoromethane
1,1-Dichloroethene
1,1,2-Trichloro-1,2,2-trifluoroethane
Acetone
Carbon Disulfide
Methyl Acetate
Methylene Chloride
trans-1,2-Dichloroethene
Methyl tert-Butyl Ether
1,1-Dichloroethane
cis-1,2-Dichloroethene
2-Butanone
Chloroform
1,1,1-Trichloroethane
Cyclohexane
Carbon Tetrachloride
Benzene
1,2-Dichloroethane

|

\begin{tabular}{|l|l|}
5.0 & $\mathrm{U}$ \\
5.0 & $\mathrm{U}$ \\
5.0 & $\mathrm{U}$ \\
5.0 & $\mathrm{U}$ \\
5.0 & $\mathrm{U}$ \\
5.0 & $\mathrm{U}$ \\
5.0 & $\mathrm{U}$ \\
5.0 & $\mathrm{U}$ \\
33 & \\
5.0 & $\mathrm{U}$ \\
5.0 & $\mathrm{U}$ \\
6.0 & $\mathrm{~B}$ \\
5.0 & $\mathrm{U}$ \\
5.0 & $\mathrm{U}$ \\
5.0 & $\mathrm{U}$ \\
5.0 & $\mathrm{U}$ \\
5.4 & \\
35 & \\
5.0 & $\mathrm{U}$ \\
5.0 & $\mathrm{U}$ \\
38 & \\
5.0 & $\mathrm{U}$ \\
5.0 & $\mathrm{U}$ \\
\hline
\end{tabular}


Lab Name: ENVIROSYSTEMS, INC.

Lab Code: ENVSYS Case No.:

Matrix: (soil/water) WATER

Sample wt/vol:

$5.000(\mathrm{~g} / \mathrm{mL}) \mathrm{ML}$

Level: (low/med) LOW

\% Moisture: not dec.

GC Column: RTX-624 ID: 0.18 (mm)

Soil Extract Volume: (uL)
Contract: ARGONNE

SAS NO.:

SDG No. : AR0512W

Lab Sample ID: 0060512-02

Lab File ID: AG75HC121

Date Received: 05/26/06

Date Analyzed: 05/31/06

Dilution Factor: 1.0

Soil Aliquot Volume: (uL)

CONCENTRATION UNITS :

CAS NO. COMPOUND

(ug/L or $\mathrm{ug} / \mathrm{Kg}$ ) UG/L Q

\begin{tabular}{|c|c|c|c|}
\hline $\begin{array}{r}79-01-6 \\
108-87-2 \\
78-87-5 \\
75-27-4 \\
10061-01-5 \\
108-10-1 \\
108-88-3 \\
10061-02-6 \\
79-00-5 \\
127-18-4 \\
591-78-6 \\
124-48-1 \\
106-93-4 \\
108-90-7 \\
100-41-4 \\
1330-20-7 \\
100-42-5 \\
75-25-2 \\
98-82-8 \\
79-34-5 \\
541-73-1 \\
106-46-7 \\
95-50-1 \\
96-12-8 \\
120-82-1\end{array}$ & $\begin{array}{l}\text { Trichloroethene } \\
\text { Methylcyclohexane } \\
\text { 1,2-Dichloropropane } \\
\text { Bromodichloromethane } \\
\text { cis-1,3-Dichloropropene } \\
\text { 4-Methyl-2-Pentanone } \\
\text { Toluene } \\
\text { trans-1,3-Dichloropropene } \\
\text { 1,1,2-Trichloroethane } \\
\text { Tetrachloroethene } \\
\text { 2-Hexanone } \\
\text { Dibromochloromethane } \\
\text { 1,2-Dibromoethane } \\
\text { Chlorobenzene } \\
\text { Ethylbenzene } \\
\text { Xylene (Total) } \\
\text { Styrene } \\
\text { Bromoform } \\
\text { Isopropylbenzene } \\
\text { 1,1,2,2-Tetrachloroethane } \\
\text { 1,3-Dichlorobenzene } \\
\text { 1,4-Dichlorobenzene } \\
\text { 1,2-Dichlorobenzene } \\
\text { 1,2-Dibromo-3-chloropropane } \\
\text { 1,2,4-Trichlorobenzene }\end{array}$ & $\begin{array}{l}5.0 \\
5.0 \\
5.0 \\
5.0 \\
5.0 \\
5.0 \\
5.0 \\
5.0 \\
5.0 \\
5.0 \\
5.0 \\
5.0 \\
5.0 \\
5.0 \\
5.0 \\
5.0 \\
5.0 \\
5.0 \\
5.0 \\
5.0 \\
5.0 \\
5.0 \\
5.0 \\
5.0 \\
5.0\end{array}$ & $\begin{array}{l}\mathrm{U} \\
\mathrm{U} \\
\mathrm{U} \\
\mathrm{U} \\
\mathrm{U} \\
\mathrm{U} \\
\mathrm{U} \\
\mathrm{U} \\
\mathrm{U} \\
\mathrm{U} \\
\mathrm{U} \\
\mathrm{U} \\
\mathrm{U} \\
\mathrm{U} \\
\mathrm{U} \\
\mathrm{U} \\
\mathrm{U} \\
\mathrm{U} \\
\mathrm{U} \\
\mathrm{U} \\
\mathrm{U} \\
\mathrm{U} \\
\mathrm{U} \\
\mathrm{U} \\
\mathrm{U}\end{array}$ \\
\hline
\end{tabular}


Lab Name: ENVIROSYSTEMS, INC.

Lab Code: ENVSYS Case No. :

Matrix: (soil/water) WATER

Sample wt/vol: $5.000(\mathrm{~g} / \mathrm{mL}) \mathrm{ML}$

Level: (low/med) LOW

\% Moisture: not dec.

GC Column: RTX-624 ID: 0.18 (mm)

Soil Extract Volume:

Number TICs found: 0
Contract: ARGONNE

SAS NO. :

SDG No.: AR0512W

Lab Sample ID: 0060512-02

Lab File ID: AG75HC121

Date Received: 05/26/06

Date Analyzed: 05/31/06

Dilution Factor: 1.0

Soil Aliquot Volume: (uL)

CONCENTRATION UNITS :

(ug/L or $u g / \mathrm{Kg}$ ) ug/L

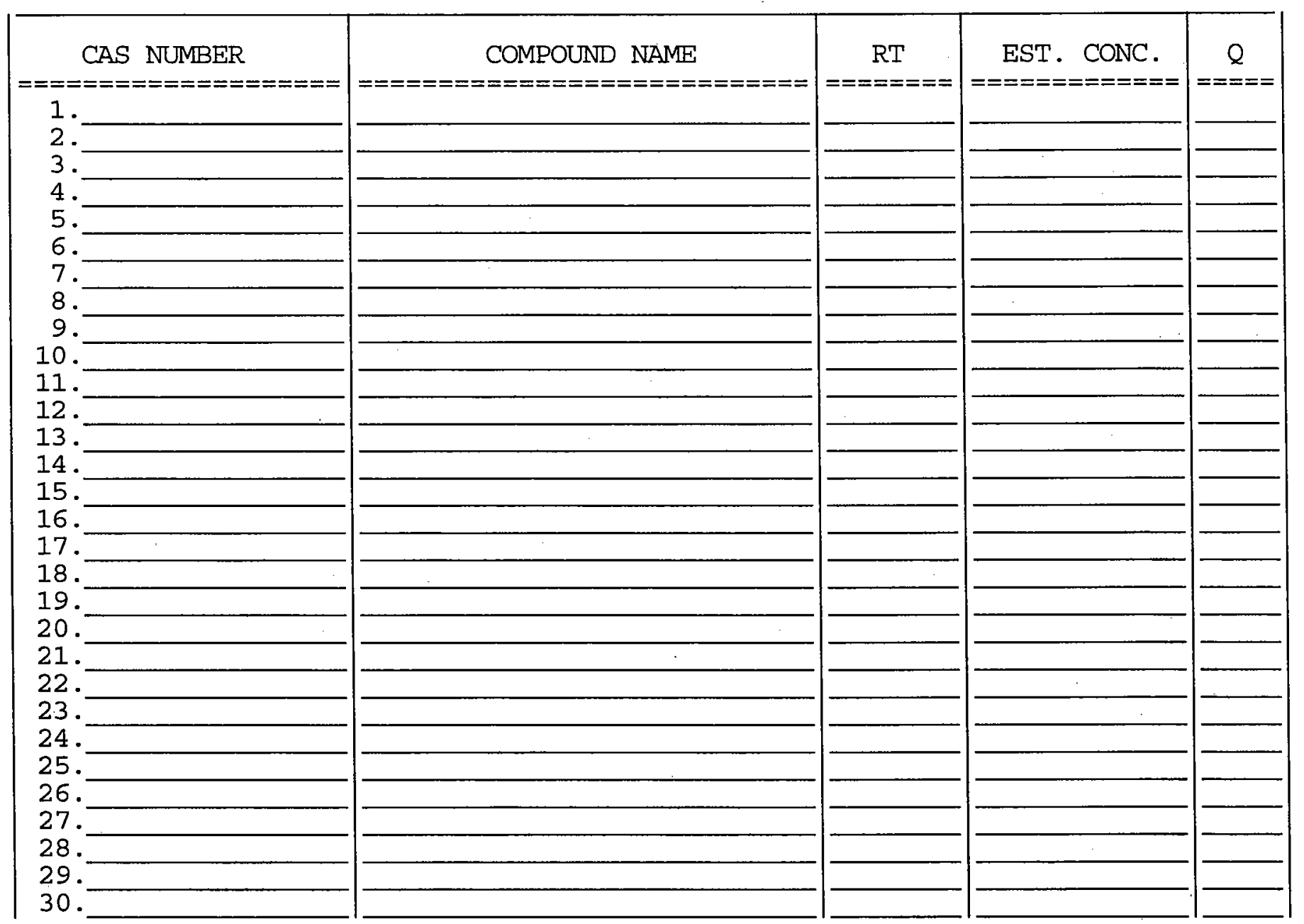


Lab Name: ENVIROSYSTEMS, INC.

Contract: ARGONNE

Lab Code: ENVSYS Case No.: AR0526 SAS No.:

SDG No.: AR0512W

Matrix: (soil/water) WATER

Lab Sample ID: 0060512-03

Sample wt/vol:

$5.000(\mathrm{~g} / \mathrm{mL}) \mathrm{ML}$

Lab File ID: AG75HC122

Level : (low/med) LOW

Date Received: 05/26/06

\% Moisture: not dec.

GC Column: RTX-624 ID: 0.18 (mm)

Date Analyzed: 05/31/06

Soil Extract Volume: (uL)

Dilution Factor: 1.0

Soil Aliquot Volume: (uL)

CONCENTRATION UNITS:

CAS NO.

COMPOUND

(ug/L or $\mathrm{ug} / \mathrm{Kg}$ ) UG/L

\begin{tabular}{|c|c|c|c|}
\hline $\begin{array}{r}75-71-8 \\
74-87-3 \\
75-01-4 \\
74-83-9 \\
75-00-3 \\
75-69-4 \\
75-35-4 \\
76-13-1 \\
67-64-1 \\
75-15-0 \\
79-20-9 \\
75-09-2 \\
156-60-5 \\
1634-04-4 \\
75-34-3 \\
156-59-2 \\
78-93-3 \\
67-66-3 \\
71-55-6 \\
110-82-7 \\
56-23-5 \\
71-43-2 \\
107-06-2\end{array}$ & $\begin{array}{l}\text { Dichlorodifluoromethane } \\
\text { Chloromethane } \\
\text { Vinyl Chloride } \\
\text { Bromomethane } \\
\text { Chloroethane } \\
\text { Trichlorofluoromethane } \\
\text { 1,1-Dichloroethene } \\
\text { 1,1,2-Trichloro-1,2,2-trifluoroethane } \\
\text { Acetone } \\
\text { Carbon Disulfide } \\
\text { Methyl Acetate } \\
\text { Methylene Chloride } \\
\text { trans-1,2-Dichloroethene } \\
\text { Methyl tert-Butyl Ether } \\
\text { 1,1-Dichloroethane } \\
\text { cis-1,2-Dichloroethene } \\
\text { 2-Butanone } \\
\text { Chloroform } \\
\text { 1,1,1-Trichloroethane } \\
\text { Cyclohexane } \\
\text { Carbon Tetrachloride } \\
\text { Benzene } \\
\text { 1,2-Dichloroethane }\end{array}$ & $\begin{array}{l}5.0 \\
5.0 \\
5.0 \\
5.0 \\
5.0 \\
5.0 \\
5.0 \\
5.0 \\
5.0 \\
5.0 \\
5.0 \\
3.3 \\
5.0 \\
5.0 \\
5.0 \\
5.0 \\
5.0 \\
5.0 \\
5.0 \\
5.0 \\
1.1 \\
5.0 \\
5.0\end{array}$ & $\begin{array}{l}U \\
U \\
U \\
U \\
U \\
U \\
U \\
U \\
U \\
U \\
U \\
J B \\
U \\
U \\
U \\
U \\
U \\
U \\
U \\
U \\
J \\
U \\
U\end{array}$ \\
\hline
\end{tabular}


Lab Name: ENVIROSYSTEMS, INC.

Lab Code: ENVSYS Case No.:

Matrix: (soil/water) WATER

Sample wt/vol: $\quad 5.000(\mathrm{~g} / \mathrm{mL}) \mathrm{ML}$

Level: (low/med) LOW

\% Moisture: not dec.

GC Column: RTX-624 ID: 0.18 (mm)

Soil Extract Volume: (uL)
Contract: ARGONNE

SAS No. :

SDG No. : AR0512

Lab Sample ID: 0060512-03

Lab File ID: AG75HC122

Date Received: 05/26/06

Date Analyzed: 05/31/06

Dilution Factor: 1.0

Soil Aliquot Volume: (uL)

CONCENTRATION UNITS:

CAS NO.

COMPOUND

(ug/L or $\mathrm{ug} / \mathrm{Kg}$ )

$\mathrm{UG} / \mathrm{L}$

$Q$

\begin{tabular}{|c|c|c|c|}
\hline $\begin{array}{r}79-01-6 \\
108-87-2 \\
78-87-5 \\
75-27-4 \\
10061-01-5 \\
108-10-1 \\
108-88-3 \\
10061-02-6 \\
79-00-5 \\
127-18-4 \\
591-78-6 \\
124-48-1 \\
106-93-4 \\
108-90-7 \\
100-41-4 \\
1330-20-7 \\
100-42-5 \\
75-25-2 \\
98-82-8 \\
79-34-5 \\
541-73-1 \\
106-46-7 \\
95-50-1 \\
96-12-8 \\
120-82-1\end{array}$ & $\begin{array}{l}\text { Trichloroethene } \\
\text { Methylcyclohexane } \\
\text { 1,2-Dichloropropane } \\
\text { Bromodichloromethane } \\
\text { cis-1,3-Dichloropropene } \\
\text { 4-Methyl-2-Pentanone } \\
\text { Toluene } \\
\text { trans-1,3-Dichloropropene } \\
\text { 1,1,2-Trichloroethane } \\
\text { Tetrachloroethene } \\
\text { 2-Hexanone } \\
\text { Dibromochloromethane } \\
\text { 1,2-Dibromoethane } \\
\text { Chlorobenzene } \\
\text { Ethylbenzene } \\
\text { Xylene (Total) } \\
\text { Styrene } \\
\text { Bromoform } \\
\text { Isopropylbenzene } \\
\text { 1,1,2,2-Tetrachloroethane } \\
\text { 1,3-Dichlorobenzene } \\
\text { 1,4-Dichlorobenzene } \\
\text { 1,2-Dichlorobenzene } \\
\text { 1,2-Dibromo-3-chloropropane } \\
1,2,4 \text {-Trichlorobenzene }\end{array}$ & $\begin{array}{l}5.0 \\
5.0 \\
5.0 \\
5.0 \\
5.0 \\
5.0 \\
5.0 \\
5.0 \\
5.0 \\
5.0 \\
5.0 \\
5.0 \\
5.0 \\
5.0 \\
5.0 \\
5.0 \\
5.0 \\
5.0 \\
5.0 \\
5.0 \\
5.0 \\
5.0 \\
5.0 \\
5.0 \\
5.0\end{array}$ & $\begin{array}{l}\mathrm{U} \\
\mathrm{U} \\
\mathrm{U} \\
\mathrm{U} \\
\mathrm{U} \\
\mathrm{U} \\
\mathrm{U} \\
\mathrm{U} \\
\mathrm{U} \\
\mathrm{U} \\
\mathrm{U} \\
\mathrm{U} \\
\mathrm{U} \\
\mathrm{U} \\
\mathrm{U} \\
\mathrm{U} \\
\mathrm{U} \\
\mathrm{U} \\
\mathrm{U} \\
\mathrm{U} \\
\mathrm{U} \\
\mathrm{U} \\
\mathrm{U} \\
\mathrm{U} \\
\mathrm{U}\end{array}$ \\
\hline
\end{tabular}


Lab Name: ENVIROSYSTEMS, INC.

Lab Code: ENVSYS Case No.:

Matrix: (soil/water) WATER

Sample wt/vol: $5.000(\mathrm{~g} / \mathrm{mL}) \mathrm{ML}$

Level: (low/med) LOW

\% Moisture: not dec.

GC Column: RTX-624 ID: 0.18 (mm)

Soil Extract Volume:

Number TICs found: 0
Contract: ARGONNE

SAS NO. :

SDG No.: AR0512W

Lab Sample ID: 0060512-03

Lab File ID: AG75HC122

Date Received: 05/26/06

Date Analyzed: 05/31/06

Dilution Factor: 1.0

Soil Aliquot Volume: (uT)

CONCENTRATION UNITS:

(ug/L or $\mathrm{ug} / \mathrm{Kg}$ ) ug/L

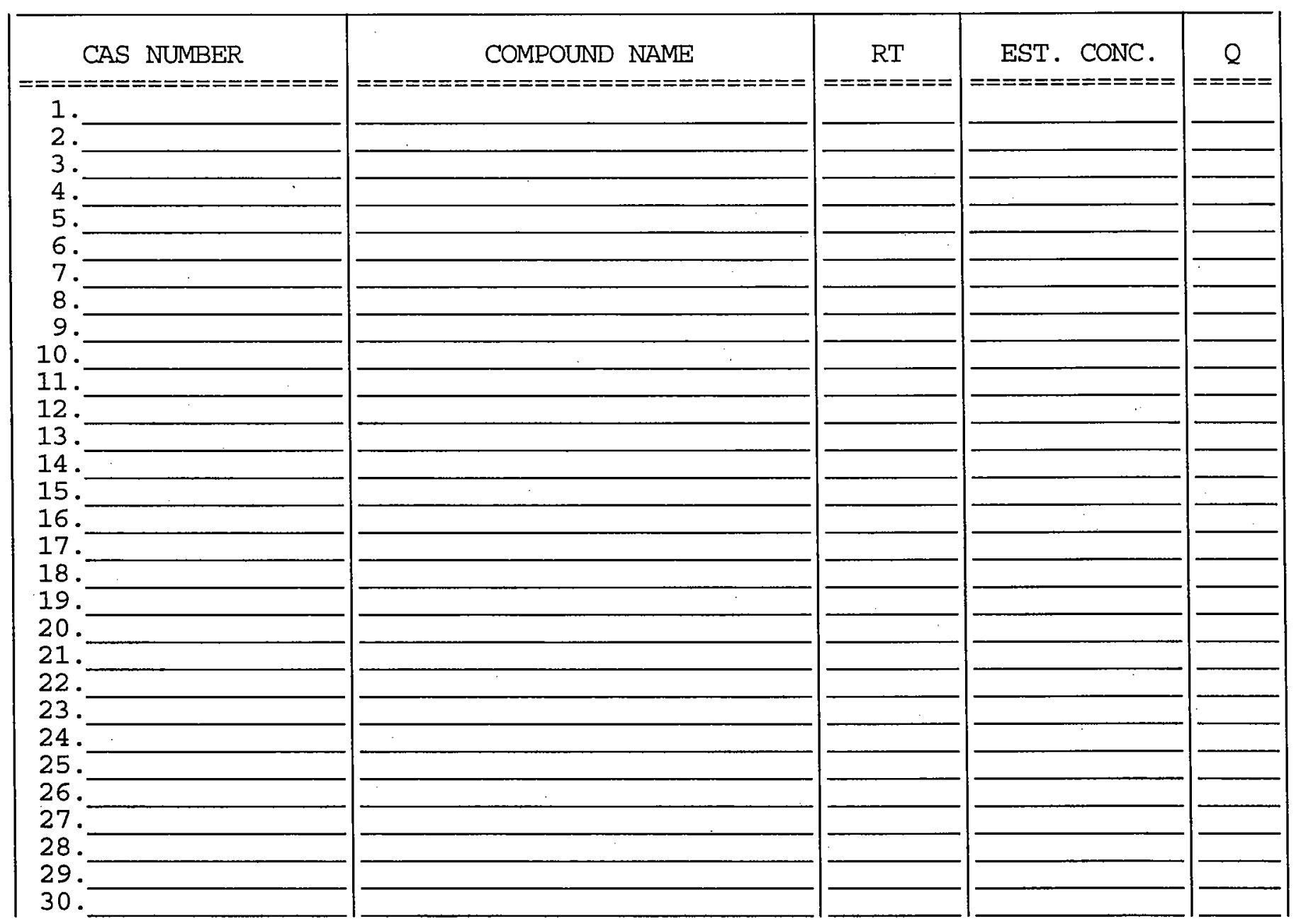


$1 \mathrm{~A}$

VOLATILE ORGANICS ANALYSIS DATA SHEET

Lab Name: ENVIROSYSTEMS, INC.

Lab Code: ENVSYS Case No.: AR0526 SAS No.:

Matrix: (soil/water) WATER

Sample wt/vol: $\quad 5.000(\mathrm{~g} / \mathrm{mL}) \mathrm{ML}$

Level: (low/med) LOW

\% Moisture: not dec.

GC Column: RTX-624 ID: 0.18 (mm) (uL)

Contract: ARGONNE

Soil Extract Volume:
EPA SAMPIEE NO.

NA-TI30-W-20712

Lab Sample ID: 0060512-04

Lab File ID: AG75HC123

Date Received: 05/26/06

Date Analyzed: 05/31/06

Dilution Factor: 1.0

Soil Aliquot Volume: (uL)

CONCENTRATION UNITS:

CAS NO.

COMPOUND

(ug/L or $\mathrm{ug} / \mathrm{Kg}) \quad \mathrm{UG} / \mathrm{L} \quad \mathrm{Q}$

\begin{tabular}{|c|c|c|c|}
\hline $\begin{array}{r}75-71-8 \\
74-87-3 \\
75-01-4 \\
74-83-9 \\
75-00-3 \\
75-69-4 \\
75-35-4 \\
76-13-1 \\
67-64-1 \\
75-15-0 \\
79-20-9 \\
75-09-2 \\
156-60-5 \\
1634-04-4 \\
75-34-3 \\
156-59-2 \\
78-93-3 \\
67-66-3 \\
71-55-6 \\
110-82-7 \\
56-23-5 \\
71-43-2 \\
107-06-2\end{array}$ & $\begin{array}{l}\text { Dichlorodifluoromethane } \\
\text { Chloromethane } \\
\text { Vinyl Chloride } \\
\text { Bromomethane } \\
\text { Chloroethane } \\
\text { Trichlorofluoromethane } \\
\text { 1,1-Dichloroethene } \\
\text { 1,1,2-Trichloro-1,2,2-trifluoroethane } \\
\text { Acetone } \\
\text { Carbon Disulfide } \\
\text { Methyl Acetate } \\
\text { Methylene Chloride } \\
\text { trans-1,2-Dichloroethene } \\
\text { Methyl tert-Butyl Ether } \\
\text { 1,1-Dichloroethane } \\
\text { cis-1,2-Dichloroethene } \\
\text { 2-Butanone } \\
\text { Chloroform } \\
\text { 1,1,1-Trichloroethane } \\
\text { Cyclohexane } \\
\text { Carbon Tetrachloride } \\
\text { Benzene } \\
\text { 1,2-Dichloroethane }\end{array}$ & $\begin{array}{r}5.0 \\
5.0 \\
5.0 \\
5.0 \\
5.0 \\
5.0 \\
5.0 \\
5.0 \\
26 \\
5.0 \\
5.0 \\
3.4 \\
5.0 \\
5.0 \\
5.0 \\
5.0 \\
5.0 \\
5.0 \\
5.0 \\
5.0 \\
5.0 \\
5.0 \\
5.0\end{array}$ & $\begin{array}{l}U \\
U \\
U \\
U \\
U \\
U \\
U \\
U \\
U \\
U \\
J B \\
U \\
U \\
U \\
U \\
U \\
U \\
U \\
U \\
U \\
U \\
U\end{array}$ \\
\hline
\end{tabular}


Lab Name: ENVIROSYSTEMS, INC.

Contract: ARGONNE

NA-TI30-W-20712

Lab Code: ENVSYS Case No.:

SAS NO. :

SDG No.: AR0512W

Matrix: (soil/water) WATER

Sample wt/vol : $\quad 5.000(\mathrm{~g} / \mathrm{mL}) \mathrm{ML}$

Level: (low/med) LOW

\% Moisture: not dec.

GC Column: RTX-624 ID: 0.18 (mm)

Soil Extract Volume: (uL)
Lab Sample ID: 0060512-04

Lab File ID: AG75HC123

Date Received: 05/26/06

Date Analyzed: 05/31/06

Dilution Factor: 1.0

Soil Aliquot Volume: (uL)

CONCENTRATION UNITS :

CAS NO.

COMPOUND (ug/L or $\mathrm{ug} / \mathrm{Kg}$ )

UG/L Q

\begin{tabular}{|c|c|c|c|}
\hline $\begin{array}{r}79-01-6 \\
108-87-2 \\
78-87-5 \\
75-27-4 \\
10061-01-5 \\
108-10-1 \\
108-88-3 \\
10061-02-6 \\
79-00-5 \\
127-18-4 \\
591-78-6 \\
124-48-1 \\
106-93-4 \\
108-90-7 \\
100-41-4 \\
1330-20-7 \\
100-42-5 \\
75-25-2 \\
98-82-8 \\
79-34-5 \\
541-73-1 \\
106-46-7 \\
95-50-1 \\
96-12-8 \\
120-82-1\end{array}$ & $\begin{array}{l}\text { Trichloroethene } \\
\text { Methylcyclohexane } \\
\text { 1,2-Dichloropropane } \\
\text { Bromodichloromethane } \\
\text { cis-1,3-Dichloropropene } \\
\text { 4-Methyl-2-Pentanone } \\
\text { Toluene } \\
\text { trans-1,3-Dichloropropene } \\
\text { 1,1,2-Trichloroethane } \\
\text { Tetrachloroethene } \\
\text { 2-Hexanone } \\
\text { Dibromochloromethane } \\
\text { 1,2-Dibromoethane } \\
\text { Chlorobenzene } \\
\text { Ethylbenzene } \\
\text { Xylene (Total) } \\
\text { Styrene } \\
\text { Bromoform } \\
\text { Isopropylbenzene } \\
\text { 1,1,2,2-Tetrachloroethane } \\
\text { 1,3-Dichlorobenzene } \\
\text { 1,4-Dichlorobenzene } \\
\text { 1,2-Dichlorobenzene } \\
\text { 1,2-Dibromo-3-chloropropane } \\
\text { 1,2,4-Trichlorobenzene }\end{array}$ & $\begin{array}{l}5.0 \\
5.0 \\
5.0 \\
5.0 \\
5.0 \\
5.0 \\
5.0 \\
5.0 \\
5.0 \\
5.0 \\
5.0 \\
5.0 \\
5.0 \\
5.0 \\
5.0 \\
5.0 \\
5.0 \\
5.0 \\
5.0 \\
5.0 \\
5.0 \\
5.0 \\
5.0 \\
5.0 \\
5.0\end{array}$ & $\begin{array}{l}\mathrm{U} \\
\mathrm{U} \\
\mathrm{U} \\
\mathrm{U} \\
\mathrm{U} \\
\mathrm{U} \\
\mathrm{U} \\
\mathrm{U} \\
\mathrm{U} \\
\mathrm{U} \\
\mathrm{U} \\
\mathrm{U} \\
\mathrm{U} \\
\mathrm{U} \\
\mathrm{U} \\
\mathrm{U} \\
\mathrm{U} \\
\mathrm{U} \\
\mathrm{U} \\
\mathrm{U} \\
\mathrm{U} \\
\mathrm{U} \\
\mathrm{U} \\
\mathrm{U} \\
\mathrm{U}\end{array}$ \\
\hline
\end{tabular}


IF

VOLATILE ORGANICS ANALYSIS DATA SHEET

TENTATIVELY IDENTIFIED COMPOUNDS
EPA SAMPLE NO.

NA-TI30-W-20712
Lab Name: ENVIROSYSTEMS, INC.

Lab Code: ENVSYS Case No.:

Matrix: (soil/water) WATER

Sample wt/vol: $5.000(\mathrm{~g} / \mathrm{mL}) \mathrm{ML}$

Level: (low/med) LOW

\% Moisture: not dec.

GC Column: RTX-624 ID: 0.18 (mm)

Soil Extract Volume:

Number TICs found: 8
Contract: ARGONNE

SAS NO. :

SDG No.: AR0512W

Lab Sample ID: 0060512-04

Lab File ID: AG75HC123

Date Received: 05/26/06

Date Analyzed: 05/31/06

Dilution Factor: 1.0

Soil Aliquot Volume: (uL)

CONCENTRATION UNITS :

(ug/L or $\mathrm{ug} / \mathrm{Kg}$ ) ug/L

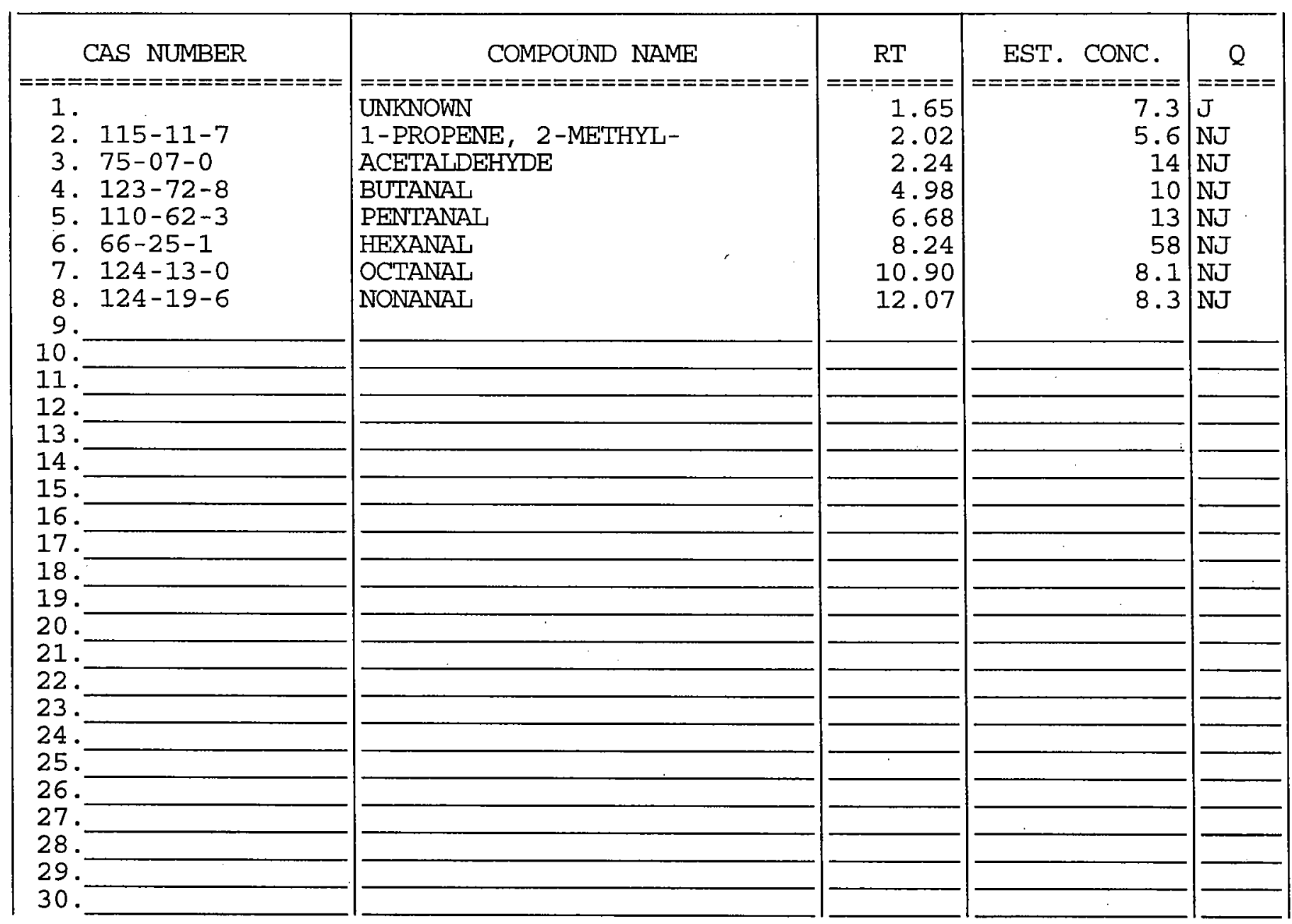


$1 \mathrm{~A}$

VOLATILE ORGANICS

ANALYSIS DATA SHEET

Lab Name: ENVIROSYSTEMS, INC.

Contract: ARGONNE

Lab Code: ENVSYS Case No.: AR0526 SAS No.:

Lab Sample ID: 0060512-05

Sample wt/vol:

$5.000(\mathrm{~g} / \mathrm{mL}) \mathrm{ML}$

Level: (low/med) LOW

Lab File ID: AG75HC124

\% Moisture: not dec.

GC Column: RTX-624 ID: 0.18 (mm)

Soil Extract Volume: (uL)

CONCENTRATION UNITS :

CAS NO.

COMPOUND
UG/L Q

\begin{tabular}{|c|c|c|c|}
\hline $\begin{array}{r}75-71-8 \\
74-87-3 \\
75-01-4 \\
74-83-9 \\
75-00-3 \\
75-69-4 \\
75-35-4 \\
76-13-1 \\
67-64-1 \\
75-15-0 \\
79-20-9 \\
75-09-2 \\
156-60-5 \\
1634-04-4 \\
75-34-3 \\
156-59-2 \\
78-93-3 \\
67-66-3 \\
71-55-6 \\
110-82-7 \\
56-23-5 \\
71-43-2 \\
107-06-2\end{array}$ & $\begin{array}{l}\text { Dichlorodifluoromethane } \\
\text { Chloromethane } \\
\text { Vinyl Chloride } \\
\text { Bromomethane } \\
\text { Chloroethane } \\
\text { Trichlorofluoromethane } \\
\text { 1,1-Dichloroethene } \\
\text { 1,1,2-Trichloro-1,2,2-trifluoroethane } \\
\text { Acetone } \\
\text { Carbon Disulfide } \\
\text { Methyl Acetate } \\
\text { Methylene Chloride } \\
\text { trans-1,2-Dichloroethene } \\
\text { Methyl tert-Butyl Ether } \\
\text { 1,1-Dichloroethane } \\
\text { cis-1,2-Dichloroethene } \\
\text { 2-Butanone } \\
\text { Chloroform } \\
\text { 1,1,1-Trichloroethane } \\
\text { Cyclohexane } \\
\text { Carbon Tetrachloride } \\
\text { Benzene } \\
\text { 1,2-Dichloroethane }\end{array}$ & $\begin{array}{r}5.0 \\
5.0 \\
5.0 \\
5.0 \\
5.0 \\
5.0 \\
5.0 \\
5.0 \\
5.0 \\
5.0 \\
5.0 \\
3.7 \\
5.0 \\
5.0 \\
5.0 \\
5.0 \\
5.0 \\
5.5 \\
5.0 \\
5.0 \\
15 \\
5.0 \\
5.0\end{array}$ & $\begin{array}{l}\mathrm{U} \\
\mathrm{U} \\
\mathrm{U} \\
\mathrm{U} \\
\mathrm{U} \\
\mathrm{U} \\
\mathrm{U} \\
\mathrm{U} \\
\mathrm{U} \\
\mathrm{U} \\
\mathrm{U} \\
\mathrm{JB} \\
\mathrm{U} \\
\mathrm{U} \\
\mathrm{U} \\
\mathrm{U} \\
\mathrm{U}\end{array}$ \\
\hline
\end{tabular}


Lab Name: ENVIROSYSTEMS, INC.

Lab Code: ENVSYS Case No.:

Matrix: (soil/water) WATER

Sample wt/vol:

$5.000(\mathrm{~g} / \mathrm{mL}) \mathrm{ML}$

Level: (low/med) LOW

\% Moisture: not dec.

GC Column: RTX-624 ID: 0.18 (nm)

Soil Extract Volume: (UL)
Contract: ARGONNE

SAS NO. :

SDG No.: AR0512W

Lab Sample ID: 0060512-05

Lab File ID: AG75HC124

Date Received: 05/26/06

Date Analyzed: 05/31/06

Dilution Factor: 1.0

Soil Aliquot Volume: (uL)

CONCENTRATION UNITS:

(ug/L or $\mathrm{ug} / \mathrm{Kg}$ ) UG/L Q

\begin{tabular}{|r|l|l|l|}
\hline $79-01-6$ & Trichloroethene & 5.0 & $\mathrm{U}$ \\
$108-87-2$ & Methylcyclohexane & 5.0 & $\mathrm{U}$ \\
$78-87-5$ & 1,2-Dichloropropane & 5.0 & $\mathrm{U}$ \\
$75-27-4$ & Bromodichloromethane & 5.0 & $\mathrm{U}$ \\
$10061-01-5$ & cis-1,3-Dichloropropene & 5.0 & $\mathrm{U}$ \\
$108-10-1$ & 4-Methyl-2-Pentanone & 5.0 & $\mathrm{U}$ \\
$108-88-3$ & Toluene & 5.0 & $\mathrm{U}$ \\
$10061-02-6$ & trans-1,3-Dichloropropene & 5.0 & $\mathrm{U}$ \\
$79-00-5$ & 1,1,2-Trichloroethane & 5.0 & $\mathrm{U}$ \\
$127-18-4$ & Tetrachloroethene & 5.0 & $\mathrm{U}$ \\
$591-78-6$ & 2-Hexanone & 5.0 & $\mathrm{U}$ \\
$124-48-1$ & Dibromochloromethane & 5.0 & $\mathrm{U}$ \\
$106-93-4$ & 1,2-Dibromoethane & 5.0 & $\mathrm{U}$ \\
$108-90-7$ & Chlorobenzene & 5.0 & $\mathrm{U}$ \\
$100-41-4$ & Ethylbenzene & 5.0 & $\mathrm{U}$ \\
$1330-20-7$ & Xylene (Total) & 5.0 & $\mathrm{U}$ \\
$100-42-5$ & Styrene & 5.0 & $\mathrm{U}$ \\
$75-25-2$ & Bromoform & 5.0 & $\mathrm{U}$ \\
$98-82-8$ & Isopropylbenzene & 5.0 & $\mathrm{U}$ \\
$79-34-5$ & 1, 1,2,-Tetrachloroethane & 5.0 & $\mathrm{U}$ \\
$541-73-1$ & 1,3-Dichlorobenzene & 5.0 & $\mathrm{U}$ \\
$106-46-7$ & 1,4-Dichlorobenzene & 5.0 & $\mathrm{U}$ \\
$95-50-1$ & 1,2-Dichlorobenzene & 5.0 & $\mathrm{U}$ \\
$96-12-8$ & 1,2-Dibromo-3-chloropropane & 5.0 & $\mathrm{U}$ \\
$120-82-1$ & 1,2,4-Trichlorobenzene & 5.0 & $\mathrm{U}$ \\
\hline
\end{tabular}


VOLATILE ORGANICS ANALYSIS DATA SHEET TENTATIVELY IDENTIFIED COMPOUNDS

Lab Name: ENVIROSYSTEMS, INC.

Lab Code: ENVSYS Case No.:

Matrix: (soil/water) WATER

Sample wt/vol: $5.000(\mathrm{~g} / \mathrm{mL}) \mathrm{ML}$

Level: (low/med) LOW

\% Moisture: not dec.

GC Column: RTX-624 ID: 0.18 (mm)

Soil Extract Volume:

Number TICs found: 0
Contract: ARGONNE

SAS NO. :
EPA SAMPLE NO.

NA-TI20-W-20913

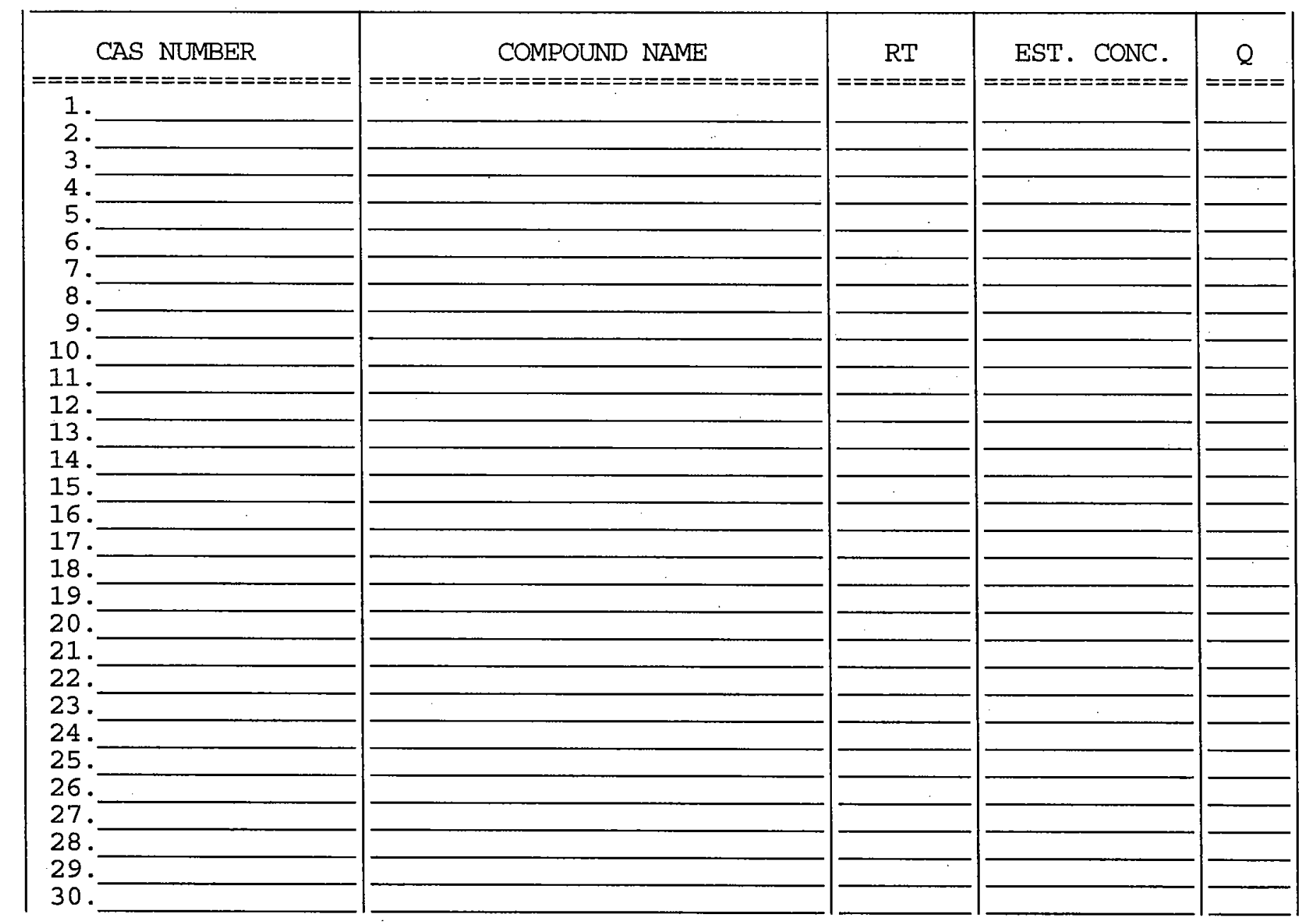

Lab Sample ID: 0060512-05

Lab File ID: AG75HC124

Date Received: 05/26/06

Date Analyzed: 05/31/06

Dilution Factor: 1.0

Soil Aliquot Volume: (uL) CONCENTRATION UNITS : (ug/L or ug/Kg) ug/L 
$1 \mathrm{~A}$

VOLATILE ORGANICS

ANALYSIS DATA SHEET

Lab Name: ENVIROSYSTEMS, INC.

Contract: ARGONNE

Lab Code: ENVSYS Case No.: AR0526 SAS No.:

Lab Sample ID: 0060512-06

Matrix: (soil/water) WATER

Sample wt/vol: $\quad 5.000(\mathrm{~g} / \mathrm{mL}) \mathrm{ML}$

Level: (low/med) Low

\% Moisture: not dec.

GC Column: RTX-624 ID: 0.18 (mm)

Soil Extract Volume: (uL)

Lab File ID: AG75HC125

Date Received: 05/26/06

Date Analyzed: 05/31/06

Dilution Factor: 1.0

Soil Aliquot Volume: (uL)

CONCENTRATION UNITS:

CAS NO.

COMPOUND

(ug/L or $\mathrm{ug} / \mathrm{Kg}$ )

UG/L Q

\begin{tabular}{|c|c|c|c|}
\hline $\begin{array}{r}75-71-8 \\
74-87-3 \\
75-01-4 \\
74-83-9 \\
75-00-3 \\
75-69-4 \\
75-35-4 \\
76-13-1 \\
67-64-1 \\
75-15-0 \\
79-20-9 \\
75-09-2 \\
156-60-5 \\
1634-04-4 \\
75-34-3 \\
156-59-2 \\
78-93-3 \\
67-66-3 \\
71-55-6 \\
110-82-7 \\
56-23-5 \\
71-43-2 \\
107-06-2\end{array}$ & $\begin{array}{l}\text { Dichlorodifluoromethane } \\
\text { Chloromethane } \\
\text { Vinyl Chloride } \\
\text { Bromomethane } \\
\text { Chloroethane } \\
\text { Trichlorofluoromethane } \\
\text { 1,1-Dichloroethene } \\
\text { 1,1,2-Trichloro-1,2,2-trifluoroethane } \\
\text { Acetone } \\
\text { Carbon Disulfide } \\
\text { Methyl Acetate } \\
\text { Methylene Chloride } \\
\text { trans-1,2-Dichloroethene } \\
\text { Methyl tert-Butyl Ether } \\
\text { 1,1-Dichloroethane } \\
\text { cis-1,2-Dichloroethene } \\
\text { 2-Butanone } \\
\text { Chloroform } \\
\text { 1,1,1-Trichloroethane } \\
\text { Cyclohexane } \\
\text { Carbon Tetrachloride } \\
\text { Benzene } \\
\text { 1,2-Dichloroethane }\end{array}$ & $\begin{array}{l}5.0 \\
5.0 \\
5.0 \\
5.0 \\
5.0 \\
5.0 \\
5.0 \\
5.0 \\
5.0 \\
5.0 \\
5.0 \\
3.9 \\
5.0 \\
5.0 \\
5.0 \\
5.0 \\
5.0 \\
5.0 \\
5.0 \\
5.0 \\
5.0 \\
5.0 \\
5.0\end{array}$ & $\begin{array}{l}U \\
U \\
U \\
U \\
U \\
U \\
U \\
U \\
U \\
U \\
U \\
J B \\
U \\
U \\
U \\
U \\
U \\
U \\
U \\
U \\
U \\
U \\
U\end{array}$ \\
\hline
\end{tabular}


Lab Name: ENVIROSYSTEMS, INC.

Lab Code: ENVSYS Case No.:

Matrix: (soil/water) WATER

Sample wt/vol :

$5.000(\mathrm{~g} / \mathrm{mL}) \mathrm{ML}$

Level: (low/med) LOW

\% Moisture: not dec.

GC Column: RTX-624 ID: 0.18 (mm) (uL)

Soil Extract Volume:

CAS NO.

COMPOUND
Contract: ARGONNE

SAS NO. :

SDG No.: AR0512W

Lab Sample ID: 0060512-06

Lab File ID: AG75HC125

Date Received: 05/26/06

Date Analyzed: 05/31/06

Dilution Factor: 1.0

Soil Aliquot Volume: (uU)

CONCENTRATION UNITS :

(ug/L or $u g / K g) \quad U G / L \quad Q$

\begin{tabular}{|c|c|c|c|}
\hline $\begin{array}{r}79-01-6 \\
108-87-2 \\
78-87-5 \\
75-27-4 \\
10061-01-5 \\
108-10-1 \\
108-88-3 \\
10061-02-6 \\
79-00-5 \\
127-18-4 \\
591-78-6 \\
124-48-1 \\
106-93-4 \\
108-90-7 \\
100-41-4 \\
1330-20-7 \\
100-42-5 \\
75-25-2 \\
98-82-8 \\
79-34-5 \\
541-73-1 \\
106-46-7 \\
95-50-1 \\
96-12-8 \\
120-82-1\end{array}$ & 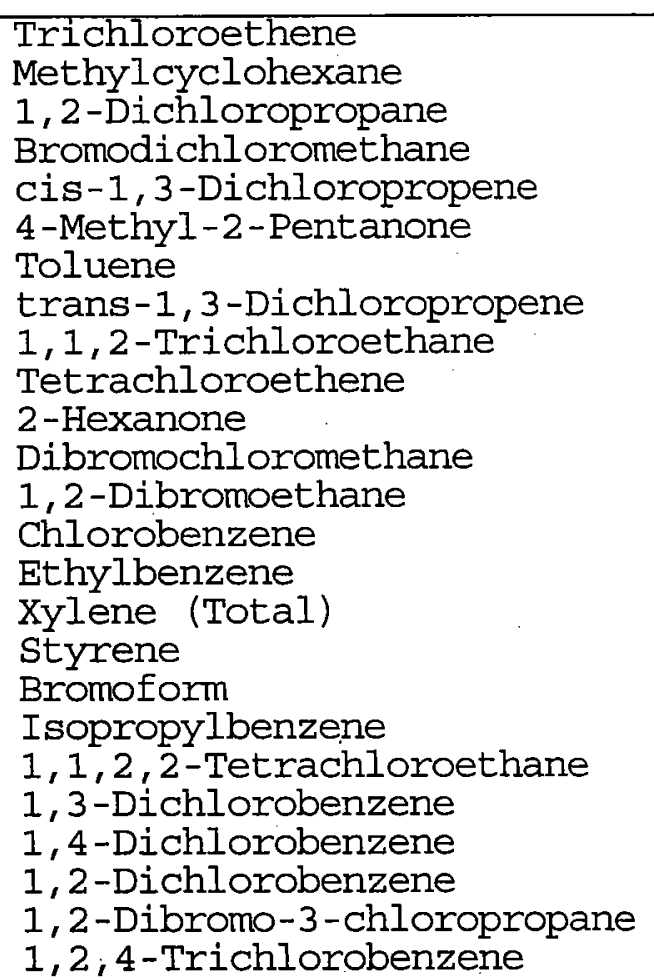 & $\begin{array}{l}5.0 \\
5.0 \\
5.0 \\
5.0 \\
5.0 \\
5.0 \\
5.0 \\
5.0 \\
5.0 \\
5.0 \\
5.0 \\
5.0 \\
5.0 \\
5.0 \\
5.0 \\
5.0 \\
5.0 \\
5.0 \\
5.0 \\
5.0 \\
5.0 \\
5.0 \\
5.0 \\
5.0 \\
5.0\end{array}$ & $\begin{array}{l}U \\
U \\
U \\
U \\
U \\
U \\
U \\
U \\
U \\
U \\
U \\
U \\
U \\
U \\
U \\
U \\
U \\
U \\
U \\
U \\
U \\
U \\
U \\
U \\
U\end{array}$ \\
\hline
\end{tabular}


Lab Name: ENVIROSYSTEMS, INC.

Lab Code: ENVSYS Case No.:

Matrix: (soil/water) WATER

Sample wt/vol: $5.000(\mathrm{~g} / \mathrm{mL}) \mathrm{ML}$

Level: (low/med) LOW

\% Moisture: not dec.

GC Column: RTX-624 ID: 0.18 (mm)

Soil Extract Volume: (uL)

Number TICs found: 0
Contract: ARGONNE

SAS NO.:

SDG NO.: AR0512W

Lab Sample ID: 0060512-06

Lab File ID: AG75HC125

Date Received: 05/26/06

Date Analyzed: 05/31/06

Dilution Factor: 1.0

Soil Aliquot Volume: (uL) CONCENTRATION UNITS: (ug/L or $\mathrm{ug} / \mathrm{Kg}$ ) ug/L

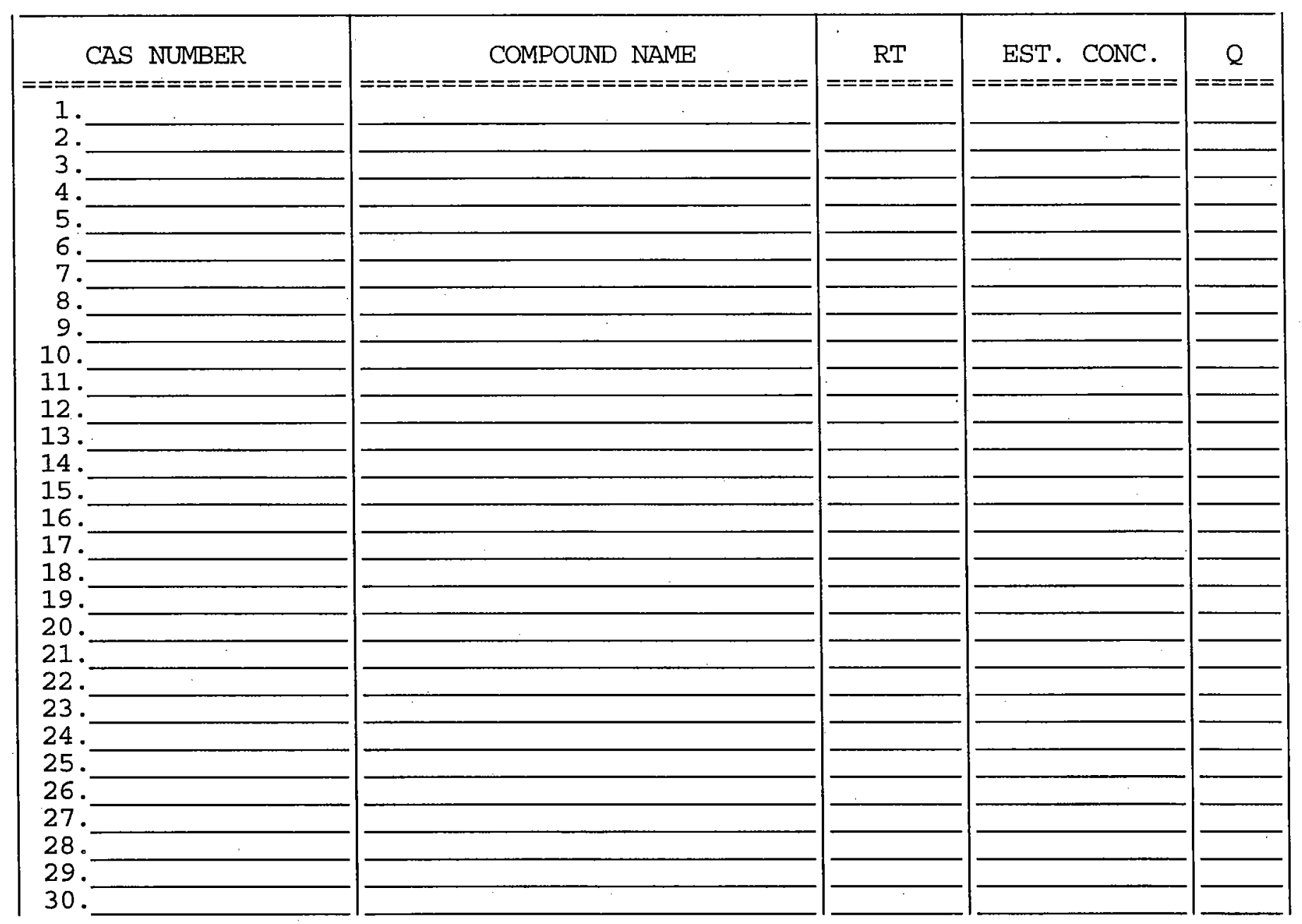




\section{ENVIROSYSTEMS, INC.}

9200 Rumsey Road • Suite B102 • Columbia, Maryland 21045-1934

Phone (410) 964-0330 - Fax (410) 740-9306

Email: info@envsystems.com - Webpage: www.envsystems.com/envsys

June 12, 2006

Jorge S. Alvarado, Ph.D

Argonne National Laboratory

Environmental Sciences Division

Applied Geoscience and Environmental

Management Section

9700 South Cass Avenue, EV-203-A137

Argonne, Illinois 60439

\section{RE: ENVSYS Report 0606057}

Dear Jorge:

Enclosed is the Analytical Data Package for the samples received on May 09, 2006 for volatile organics analysis by US EPA CLP SOW OLM04.3

Please do not hesitate to call me if you have any questions, comments, or require additional information.

Sincerely,

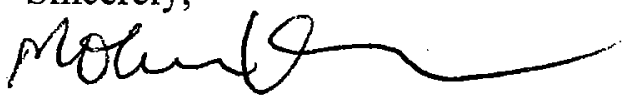

Mohan Khare, Ph.D

President/CEO

$\mathrm{MK} / \mathrm{pl}$ 
1. Narrative 


\section{SDG NARRATIVE}

LABORATORY NAME: ENVIROSYSTEMS, INC.

CLIENT: ARGONNE NATIONAL LABORATORY

DATA SAMPLES RECEIVED AT LABORATORY: MAY $09^{\text {th }}, 2006$

SAMPLE ANALYSES INCLUDED IN THIS REPORT:

CLIENT \#

NA-TI13-W-20561

NA-TI13-W-20560

NA-TI14-W-20666

NA-TI13-W-20563

NA-TI13-W-20562

NA-QCTB-05/07/06
LAB ID\#

0060504-01

0060504-02

$0060504-03$

0060504-04

0060504-05

0060504-06
ANALYSIS

VOA

VOA

VOA

VOA

VOA

VOA
MATRIX

VOA pH

WATER 7

WATER 7

WATER 7

WATER 7

WATER 7

WATER 7

Matrix spike/matrix spike duplicate analysis was not performed for this case.

Samples NA-TI14-W-2066 and NA-TI13-W-20563 had to be diluted due to a target compound(s) exceeding the maximum calibration standard. The surrogate spike Bromofluorobenzene did not meet the required recovery on the first run for any sample.

Samples for this SDG are analyzed by EPA SOW OLMO4.3 for multi-media multi-concentration organics. Sample detection limits have meet client requirements.

The cooler temperature was measured to be two degrees Celsius upon arrival.

The initial volatile analysis were performed on a HP5973 GC/MS using a Restek 62420 meter column with an inner diameter of $0.18 \mathrm{~mm}$ and a 1.0 micron film thickness. The trap used with the autosampler is a OI analytical trap packed with Tenax/silica gel $/ \mathrm{cms}$. The second analysis for dilution runs were performed on an Agilent 5975 GC/MS using a Restek RTX-624 20 meter column with an inner diameter of $0.18 \mathrm{~mm}$ and a 1.0 micron film thickness. The trap used with the autosampler is a $0.3 \mathrm{~cm}$ OD x $28.5 \mathrm{~cm} \mathrm{~L}$ ENCON Ambient Packed Trap.

Three compounds did not meet the first initial calibration criteria. Percent RSD for 1,2,4 Trichlorobenzen was greater than 20.5 and the response factor for 1,1,2,2 tetrachloroethane and 1,3-Dichlorobenzene was below the minimum required. Three compounds did not meet the second initial calibration criteria. Percent RSD for Bromomethane was greater than 20.5 and the response factor for Bromoform and for 1,1,2,2 tetrachloroethane were below the required criteria. Continuing calibration VSTD050FK had two compounds not meeting the required minimum RRF. Continuing calibration VSTD050HP had one compound that exceed the 25.0 percent $\mathrm{D}$ and one compound that was below the minimum required RRF of 0.300 . 
All other QC criteria were met for all samples included in this report.

moham Oh ave

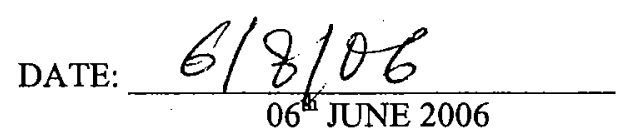

00003 
2. SGD Cover Sheet/Traffic Reports 


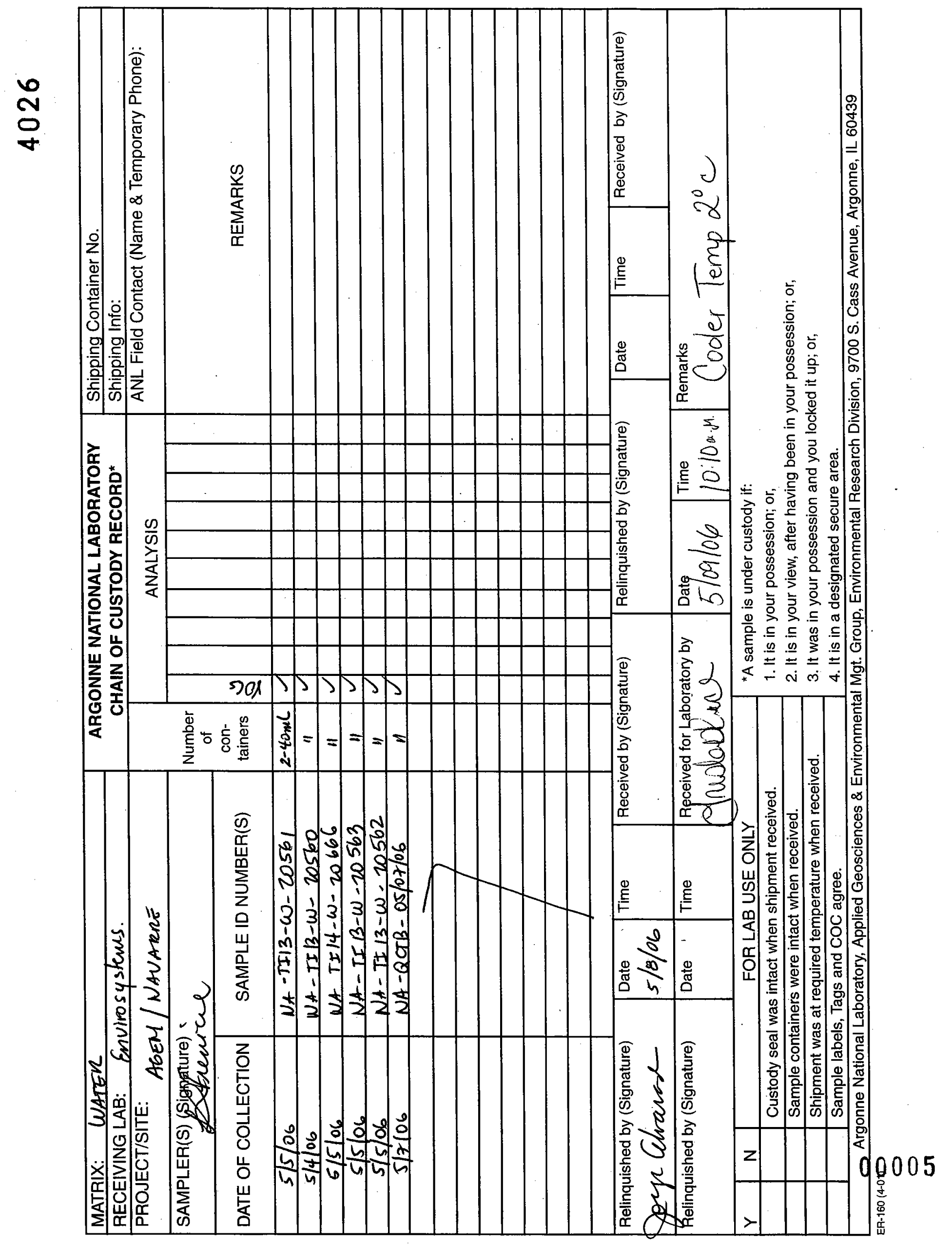


VOLATILE SAMPLE DATA

00017 
Lab Name: ENVIROSYSTEMS, INC.

Lab Code: ENVSYS Case No.: AR0504

Matrix: (soil/water) WATER

Sample wt/vol: $\quad 5.000(\mathrm{~g} / \mathrm{mL}) \mathrm{ML}$

Level: (low/med) LOW

\% Moisture: not dec.

GC Column: RTX-624 ID: 0.18 (mm)

Soil Extract Volume: (uL)
Contract: ARGONNE

SAS NO. :

SDG NO. : AR0504

Lab Sample ID: 0060504-01

Lab File ID: H73FF638

Date Received: 05/09/06

Date Analyzed: 05/13/06

Dilution Factor: 1.0

Soil Aliquot Volume:

CONCENTRATION UNITS:

CAS NO.

COMPOUND

$(u g / L$ or $u g / K g) \quad U G / L \quad Q$

\begin{tabular}{|r|l|}
\hline $75-71-8$ & Dichlorodifluoromethane \\
$74-87-3$ & Chloromethane \\
$75-01-4$ & Vinyl Chloride \\
$74-83-9$ & Bromomethane \\
$75-00-3$ & Chloroethane \\
$75-69-4$ & Trichlorofluoromethane \\
$75-35-4$ & $1,1-$ Dichloroethene \\
$76-13-1$ & $1,1,2-T r i c h l o r o-1,2,2-t r i f l u o r o e t h a n e$ \\
$67-64-1$ & Acetone \\
$75-15-0$ & Carbon Disulfide \\
$79-20-9$ & Methyl Acetate \\
$75-09-2$ & Methylene Chloride \\
$156-60-5$ & trans-1,2-Dichloroethene \\
$1634-04-4$ & Methyl tert-Butyl Ether \\
$75-34-3$ & $1,1-$ Dichloroethane \\
$156-59-2$ & Cis-1,2-Dichloroethene \\
$78-93-3$ & $2-$ Butanone \\
$67-66-3$ & Chloroform \\
$71-55-6$ & $1,1,1-T r i c h l o r o e t h a n e$ \\
$110-82-7$ & Cyclohexane \\
$56-23-5$ & Carbon Tetrachloride \\
$71-43-2$ & Benzene \\
$107-06-2$ & $1,2-$ Dichloroethane \\
&
\end{tabular}

\begin{tabular}{|l|l|}
5.0 & $\mathrm{U}$ \\
5.0 & $\mathrm{U}$ \\
5.0 & $\mathrm{U}$ \\
5.0 & $\mathrm{U}$ \\
5.0 & $\mathrm{U}$ \\
5.0 & $\mathrm{U}$ \\
5.0 & $\mathrm{U}$ \\
5.0 & $\mathrm{U}$ \\
5.0 & $\mathrm{U}$ \\
5.0 & $\mathrm{U}$ \\
5.0 & $\mathrm{U}$ \\
2.2 & $\mathrm{~J}$ \\
5.0 & $\mathrm{U}$ \\
5.0 & $\mathrm{U}$ \\
5.0 & $\mathrm{U}$ \\
5.0 & $\mathrm{U}$ \\
5.0 & $\mathrm{U}$ \\
5.6 & \\
5.0 & $\mathrm{U}$ \\
5.0 & $\mathrm{U}$ \\
18 & \\
5.0 & $\mathrm{U}$ \\
5.0 & $\mathrm{U}$ \\
\hline
\end{tabular}

FORM I VOA-1

OLM0 4.3 
Lab Name: ENVIROSYSTEMS, INC.

Lab Code: ENVSYS

Case No.: AR0504

Matrix: (soil/water) WATER

Sample wt/vol:

$5.000(\mathrm{~g} / \mathrm{mL}) \mathrm{ML}$

Level: (low/med) LOW

\% Moisture: not dec.

GC Column: RTX-624 ID: 0.18 (mm)

Soil Extract Volume: (uL)
Contract: ARGONNE

NA-TI13-W-20561

SAS NO. :

SDG No.: AR0504

Lab Sample ID: 0060504-01

Lab File ID: H73FF638

Date Received: 05/09/06

Date Analyzed: 05/13/06

Dilution Factor: 1.0

Soil Aliquot Volume: (uL)

CONCENTRATION UNITS :

CAS NO.

COMPOUND

(ug/I or $u g / K g) \quad U G / I$

\begin{tabular}{|c|c|c|c|}
\hline $\begin{array}{r}79-01-6 \\
108-87-2 \\
78-87-5 \\
75-27-4 \\
10061-01-5 \\
108-10-1 \\
108-88-3 \\
10061-02-6 \\
79-00-5 \\
127-18-4 \\
591-78-6 \\
124-48-1 \\
106-93-4 \\
108-90-7 \\
100-41-4 \\
1330-20-7 \\
100-42-5 \\
75-25-2 \\
98-82-8 \\
79-34-5 \\
541-73-1 \\
106-46-7 \\
95-50-1 \\
96-12-8 \\
120-82-1\end{array}$ & $\begin{array}{l}\text { Trichloroethene } \\
\text { Methylcyclohexane } \\
\text { 1,2-Dichloropropane } \\
\text { Bromodichloromethane } \\
\text { cis-1,3-Dichloropropene } \\
\text { 4-Methyl-2-Pentanone } \\
\text { Toluene } \\
\text { trans-1,3-Dichloropropene } \\
\text { 1,1,2-Trichloroethane } \\
\text { Tetrachloroethene } \\
\text { 2-Hexanone } \\
\text { Dibromochloromethane } \\
\text { 1,2-Dibromoethane } \\
\text { Chlorobenzene } \\
\text { Ethylbenzene } \\
\text { Xylene (Total) } \\
\text { Styrene } \\
\text { Bromoform } \\
\text { Isopropylbenzene } \\
\text { 1,1,2,2-Tetrachloroethane } \\
1,3 \text {-Dichlorobenzene } \\
\text { 1,4-Dichlorobenzene } \\
1,2-\text { Dichlorobenzene } \\
1,2-\text { Dibromo-3-chloropropane } \\
1,2,4 \text {-Trichlorobenzene }\end{array}$ & $\begin{array}{l}5.0 \\
5.0 \\
5.0 \\
5.0 \\
5.0 \\
5.0 \\
5.0 \\
5.0 \\
5.0 \\
5.0 \\
5.0 \\
5.0 \\
5.0 \\
5.0 \\
5.0 \\
5.0 \\
5.0 \\
5.0 \\
5.0 \\
5.0 \\
5.0 \\
5.0 \\
5.0 \\
5.0 \\
5.0\end{array}$ & $\begin{array}{l}\mathrm{U} \\
\mathrm{U} \\
\mathrm{U} \\
\mathrm{U} \\
\mathrm{U} \\
\mathrm{U} \\
\mathrm{U} \\
\mathrm{U} \\
\mathrm{U} \\
\mathrm{U} \\
\mathrm{U} \\
\mathrm{U} \\
\mathrm{U} \\
\mathrm{U} \\
\mathrm{U} \\
\mathrm{U} \\
\mathrm{U} \\
\mathrm{U} \\
\mathrm{U} \\
\mathrm{U} \\
\mathrm{U} \\
\mathrm{U} \\
\mathrm{U} \\
\mathrm{U} \\
\mathrm{U}\end{array}$ \\
\hline
\end{tabular}


$1 \mathrm{~F}$

VOLATILE ORGANICS ANALYSIS DATA SHEET

TENTATIVELY IDENTIFIED COMPOUNDS
EPA SAMPLE NO.

NA-TI13-W-20561

Lab Name: ENVIROSYSTEMS, INC.

Contract: ARGONNE

Lab Code: ENVSYS Case NQ.: AR0504 SAS No.:

SDG NO.: AR0504

Matrix: (soil/water) WATER

Sample wt/vol: $5.000(\mathrm{~g} / \mathrm{mL}) \mathrm{ML}$

Level: (low/med) LOW

号 Moisture: not dec.

GC Column: RTX-624 ID: 0.18 (mm)

Soil Extract Volume:

(uL)

Number TICs found: 0
Lab Sample ID: 0060504-01

Lab File ID: H73FF638

Date Received: 05/09/06

Date Analyzed: 05/13/06

Dilution Factor: 1.0

Soil Aliquot Volume: (uL)

CONCENTRATION UNITS :

(ug/L or $\mathrm{ug} / \mathrm{Kg}$ ) ug/L

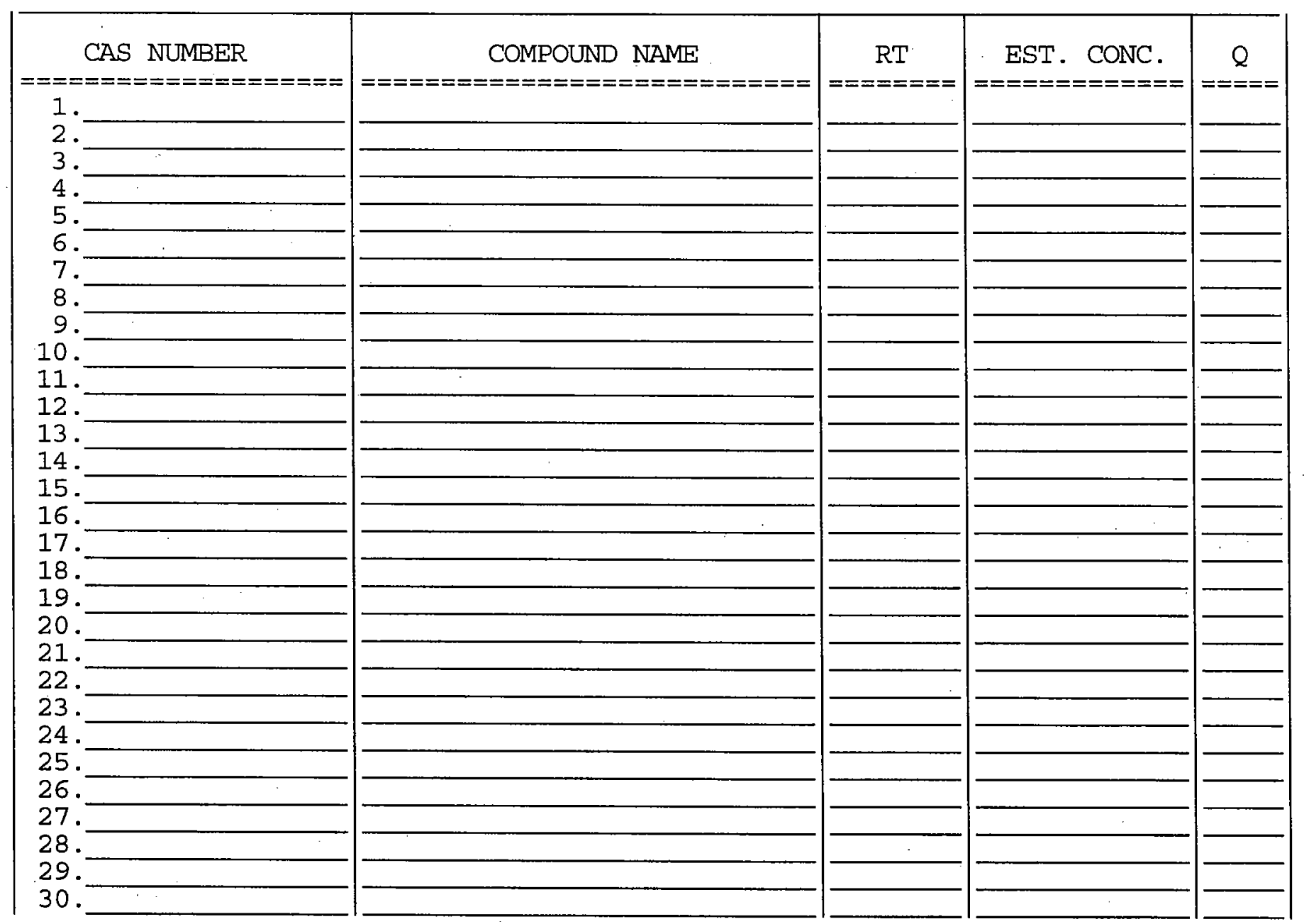


Lab Name: ENVIROSYSTEMS, INC.

Lab Code: ENVSYS Case No.: AR0504 SAS No.:

Matrix: (soil/water) WATER

Sample wt/vol:

$5.000(\mathrm{~g} / \mathrm{mL}) \mathrm{ML}$

Level: (low/med) LOW

\% Moisture: not dec.

GC Column: RTX-624 ID: 0.18 (mm)

Soil Extract Volume: (uL)
Contract: ARGONNE

SDG NO.: AR0504

Lab Sample ID: 0060504-02

Lab File ID: H73FF639

Date Received: 05/09/06

Date Analyzed: 05/13/06

Dilution Factor: 1.0

Soil Aliquot Volume:

CONCENTRATION UNITS :

CAS NO.

COMPOUND

(ug/L or ug/Kg) UG/L Q

\begin{tabular}{|c|c|c|c|}
\hline $\begin{array}{r}75-71-8 \\
74-87-3 \\
75-01-4 \\
74-83-9 \\
75-00-3 \\
75-69-4 \\
75-35-4 \\
76-13-1 \\
67-64-1 \\
75-15-0 \\
79-20-9 \\
75-09-2 \\
156-60-5 \\
1634-04-4 \\
75-34-3 \\
156-59-2 \\
78-93-3 \\
67-66-3 \\
71-55-6 \\
110-82-7 \\
56-23-5 \\
71-43-2 \\
107-06-2\end{array}$ & $\begin{array}{l}\text { Dichlorodifluoromethane } \\
\text { Chloromethane } \\
\text { Vinyl Chloride } \\
\text { Bromomethane } \\
\text { Chloroethane } \\
\text { Trichlorofluoromethane } \\
\text { 1,1-Dichloroethene } \\
\text { 1,1,2-Trichloro-1,2,2-trifluoroethane } \\
\text { Acetone } \\
\text { Carbon Disulfide } \\
\text { Methyl Acetate } \\
\text { Methylene Chloride } \\
\text { trans-1,2-Dichloroethene } \\
\text { Methyl tert-Butyl Ether } \\
\text { 1,1-Dichloroethane } \\
\text { cis-1,2-Dichloroethene } \\
\text { 2-Butanone } \\
\text { Chloroform } \\
\text { 1,1,1-Trichloroethane } \\
\text { Cyclohexane } \\
\text { Carbon Tetrachloride } \\
\text { Benzene } \\
\text { 1,2-Dichloroethane }\end{array}$ & $\begin{array}{r}5.0 \\
5.0 \\
5.0 \\
5.0 \\
5.0 \\
5.0 \\
5.0 \\
5.0 \\
5.0 \\
5.0 \\
5.0 \\
2.0 \\
5.0 \\
5.0 \\
5.0 \\
5.0 \\
5.0 \\
5.2 \\
5.0 \\
5.0 \\
16 \\
5.0 \\
5.0\end{array}$ & $\begin{array}{l}\mathrm{U} \\
\mathrm{U} \\
\mathrm{U} \\
\mathrm{U} \\
\mathrm{U} \\
\mathrm{U} \\
\mathrm{U} \\
\mathrm{U} \\
\mathrm{U} \\
\mathrm{U} \\
\mathrm{U} \\
\mathrm{J} \\
\mathrm{U} \\
\mathrm{U} \\
\mathrm{U} \\
\mathrm{U} \\
\mathrm{U}\end{array}$ \\
\hline
\end{tabular}


Lab Name: ENVIROSYSTEMS, INC.

Lab Code: ENVSYS

Case No.: AR0504

Matrix: (soil/water) WATER

Sample wt/vol:

$5.000(\mathrm{~g} / \mathrm{mL}) \mathrm{ML}$

Level: (low/med) LOW

$\%$ Moisture: not dec.

GC Column: RTX-624 ID: 0.18 (mm)

Soil Extract Volume: (uL)
Contract : ARGONNE

NA-TI13-W-20560
SAS NO. :

SDG No.: AR0504

Lab Sample ID: 0060504-02

Lab File ID: H73FF639

Date Received: 05/09/06

Date Analyzed: 05/13/06

Dilution Factor: 1.0

Soil Aliquot Volume: (uL)

CONCENTRATION UNITS:

CAS NO.

COMPOUND

(ug/L or $\mathrm{ug} / \mathrm{Kg}$ ) UG/L

\begin{tabular}{|r|l|l|l|}
\hline $79-01-6$ & Trichloroethene & $\mathrm{U}$ \\
$108-87-2$ & Methylcyclohexane & 5.0 & $\mathrm{U}$ \\
$78-87-5$ & 1,2-Dichloropropane & 5.0 & $\mathrm{U}$ \\
$75-27-4$ & Bromodichloromethane & 5.0 & $\mathrm{U}$ \\
$10061-01-5$ & Cis-1,3-Dichloropropene & 5.0 & $\mathrm{U}$ \\
$108-10-1$ & 4-Methyl-2-Pentanone & 5.0 & $\mathrm{U}$ \\
$108-88-3$ & Toluene & 5.0 & $\mathrm{U}$ \\
$10061-02-6$ & trans-1,3-Dichloropropene & 5.0 & $\mathrm{U}$ \\
$79-00-5$ & 1,1,2-Trichloroethane & 5.0 & $\mathrm{U}$ \\
$127-18-4$ & Tetrachloroethene & 5.0 & $\mathrm{U}$ \\
$591-78-6$ & 2-Hexanone & 5.0 & $\mathrm{U}$ \\
$124-48-1$ & Dibromochloromethane & 5.0 & $\mathrm{U}$ \\
$106-93-4$ & 1,2-Dibromoethane & 5.0 & $\mathrm{U}$ \\
$108-90-7$ & Chlorobenzene & 5.0 & $\mathrm{U}$ \\
$100-41-4$ & Ethylbenzene & 5.0 & $\mathrm{U}$ \\
$1330-20-7$ & Xylene (Total) & 5.0 & $\mathrm{U}$ \\
$100-42-5$ & Styrene & 5.0 & $\mathrm{U}$ \\
$75-25-2$ & Bromoform & 5.0 & $\mathrm{U}$ \\
$98-82-8$ & Isopropylbenzene & 5.0 & $\mathrm{U}$ \\
$79-34-5$ & 1, 1,2,2-Tetrachloroethane & 5.0 & $\mathrm{U}$ \\
$541-73-1$ & 1,3-Dichlorobenzene & & $\mathrm{U}$ \\
$106-46-7$ & 1,4-Dichlorobenzene & & $\mathrm{U}$ \\
$95-50-1$ & 1,2-Dichlorobenzene & & $\mathrm{U}$ \\
$96-12-8$ & 1,2-Dibromo-3-chloropropane & & $\mathrm{U}$ \\
$120-82-1$ & 1,2,4-Trichlorobenzene & & $\mathrm{U}$ \\
\hline
\end{tabular}


$1 F$

VOLATILE ORGANICS ANALYSIS DATA SHEET

TENTATIVELY IDENTIFIED COMPOUNDS

Lab Name: ENVIROSYSTEMS, INC.

Contract: ARGONNE

Lab Code: ENVSYS Case No.: AR0504 SAS No.:

SDG NO.: AR0504

Matrix: (soil/water) WATER

Sample wt/vol: $5.000(\mathrm{~g} / \mathrm{mL}) \mathrm{ML}$

Level: (low/med) LOW

\% Moisture: not dec.

GC Column: RTX-624 ID: 0.18 (mm)

Soil Extract Volume:

Number TICs found: 1
EPA SAMPLE NO.

NA-TI13-W-20560

\begin{tabular}{|c|c|c|c|c|}
\hline $\begin{array}{c}\text { CAS NUMBER } \\
==================\end{array}$ & 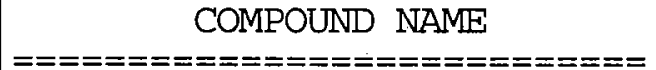 & $\begin{array}{c}\mathrm{RT} \\
========1\end{array}$ & $\begin{aligned} \text { EST. CONC. } \\
============\end{aligned}$ & 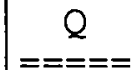 \\
\hline$\frac{1}{2}$ & UNKNOWN & 2.23 & 5.8 & \\
\hline 3.- & & & & \\
\hline $5 .-$ & & & & \\
\hline 6.7 & & & & \\
\hline 8. & & & & \\
\hline 9 & & & & \\
\hline 10. & & & & \\
\hline 11. & & & & \\
\hline 12. & & & & \\
\hline 13. & & & & \\
\hline 14. & & & & \\
\hline 16. & & & & \\
\hline 17. & & & & \\
\hline 18. & & & & \\
\hline 20 & 1 & - & 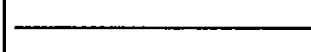 & \\
\hline 21 & & & & \\
\hline 22 . & & & & \\
\hline 23. & & & 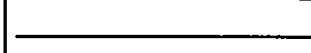 & \\
\hline 25. & 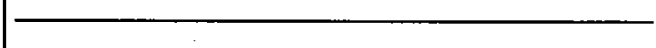 & & & \\
\hline 26. & & & & \\
\hline 27. & & & & \\
\hline 28. & 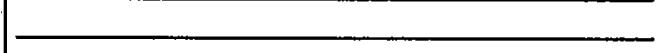 & & Z & \\
\hline 30. & & & & \\
\hline
\end{tabular}


Lab Name: ENVIROSYSTEMS, INC.

Lab Code: ENVSYS Case No.: AR0504 SAS No.:

Matrix: (soil/water) WATER

Sample wt/vol: $\quad 5.000(\mathrm{~g} / \mathrm{mL}) \mathrm{ML}$

Level: (low/med) LOW

\% Moisture: not dec.

GC Column: RTX-624 ID: 0.18 (mm)

Soil Extract Volume: (uL)

Contract: ARGONNE

SDG NO.: AR0504

Lab Sample ID: 0060504-03

Lab File ID: H73FF640

Date Received: 05/09/06

Date Analyzed: 05/13/06

Dilution Factor: 1.0

Soil Aliquot Volume: (uL)

CONCENTRATION UNITS:

CAS NO.

COMPOUND

(ug/L or $\mathrm{ug} / \mathrm{Kg}$ ) UG/L Q

\begin{tabular}{|r|}
$75-71-8$ \\
$74-87-3$ \\
$75-01-4$ \\
$74-83-9$ \\
$75-00-3$ \\
$75-69-4$ \\
$75-35-4$ \\
$76-13-1$ \\
$67-64-1$ \\
$75-15-0$ \\
$79-20-9$ \\
$75-09-2$ \\
$156-60-5$ \\
$1634-04-4$ \\
$75-34-3$ \\
$156-59-2$ \\
$78-93-3$ \\
$67-66-3$ \\
$71-55-6$ \\
$110-82-7$ \\
$56-23-5$ \\
$71-43-2$ \\
$107-06-2$ \\
\hline
\end{tabular}

Dichlorodifluoromethane
Chloromethane
Vinyl Chloride
Bromomethane
Chloroethane
Trichlorofluoromethane
1,1-Dichloroethene
1,1,2-Trichloro-1,2,2-trifluoroethane
Acetone
Carbon Disulfide
Methyl Acetate
Methylene Chloride
trans-1,2-Dichloroethene
Methyl tert-Butyl Ether
1,1-Dichloroethane
cis-1,2-Dichloroethene
2-Butanone
Chloroform
$1,1,1-T r i c h l o r o e t h a n e$
Cyclohexane
Carbon Tetrachloride
Benzene
1,2-Dichloroethane

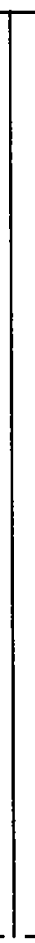

\begin{tabular}{l|l|}
5.0 & $\mathrm{U}$ \\
5.0 & $\mathrm{U}$ \\
5.0 & $\mathrm{U}$ \\
5.0 & $\mathrm{U}$ \\
5.0 & $\mathrm{U}$ \\
5.0 & $\mathrm{U}$ \\
5.0 & $\mathrm{U}$ \\
5.0 & $\mathrm{U}$ \\
5.0 & $\mathrm{U}$ \\
5.0 & $\mathrm{U}$ \\
5.0 & $\mathrm{U}$ \\
12 & \\
5.0 & $\mathrm{U}$ \\
5.0 & $\mathrm{U}$ \\
5.0 & $\mathrm{U}$ \\
5.0 & $\mathrm{U}$ \\
5.0 & $\mathrm{U}$ \\
240 & $\mathrm{E}$ \\
5.0 & $\mathrm{U}$ \\
5.0 & $\mathrm{U}$ \\
4.0 & $\mathrm{~J}$ \\
5.0 & $\mathrm{U}$ \\
5.0 & $\mathrm{U}$ \\
\hline
\end{tabular}


Lab Name: ENVIROSYSTEMS, INC.

Lab Code: ENVSYS Case No.: AR0504 SAS No.:

Matrix: (soil/water) WATER

Sample wt/vol:

$5.000(\mathrm{~g} / \mathrm{mL}) \mathrm{ML}$

Level: (low/med) LOW

\% Moisture: not dec.

GC Column: RTX-624 ID: 0.18 (mm)

Soil Extract Volume: (uI)
Contract: ARGONNE

SDG NO.: AR0504

Lab Sample ID: 0060504-03

Lab File ID: H73FF640

Date Received: 05/09/06

Date Analyzed: 05/13/06

Dilution Factor: 1.0

Soil Aliquot Volume: (uL)

CONCENTRATION UNITS :

CAS NO.

COMPOUND

(ug/L or $\mathrm{ug} / \mathrm{Kg}) \quad \mathrm{UG} / \mathrm{L} \quad \mathrm{Q}$

\begin{tabular}{|r|}
$79-01-6$ \\
$108-87-2$ \\
$78-87-5$ \\
$75-27-4$ \\
$10061-01-5$ \\
$108-10-1$ \\
$108-88-3$ \\
$10061-02-6$ \\
$79-00-5$ \\
$127-18-4$ \\
$591-78-6$ \\
$124-48-1$ \\
$106-93-4$ \\
$108-90-7$ \\
$100-41-4$ \\
$1330-20-7$ \\
$100-42-5$ \\
$75-25-2$ \\
$98-82-8$ \\
$79-34-5$ \\
$541-73-1$ \\
$106-46-7$ \\
$95-50-1$ \\
$96-12-8$ \\
$120-82-1$
\end{tabular}

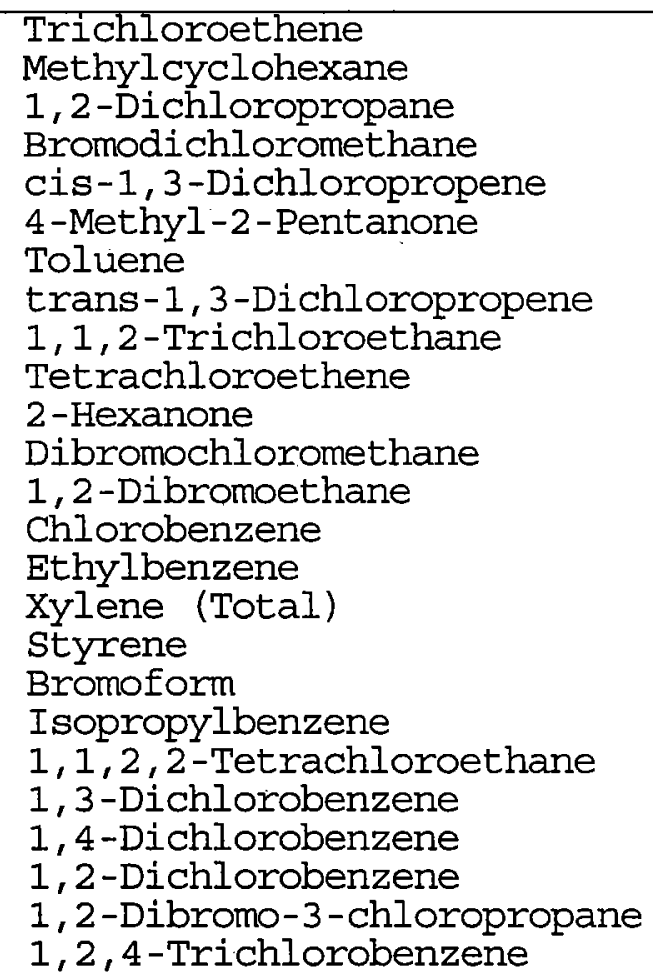

\begin{tabular}{|l|l|}
\hline 5.0 & $\mathrm{U}$ \\
5.0 & $\mathrm{U}$ \\
5.0 & $\mathrm{U}$ \\
5.0 & $\mathrm{U}$ \\
5.0 & $\mathrm{U}$ \\
5.0 & $\mathrm{U}$ \\
5.0 & $\mathrm{U}$ \\
5.0 & $\mathrm{U}$ \\
5.0 & $\mathrm{U}$ \\
5.0 & $\mathrm{U}$ \\
5.0 & $\mathrm{U}$ \\
5.0 & $\mathrm{U}$ \\
5.0 & $\mathrm{U}$ \\
5.0 & $\mathrm{U}$ \\
5.0 & $\mathrm{U}$ \\
5.0 & $\mathrm{U}$ \\
5.0 & $\mathrm{U}$ \\
5.0 & $\mathrm{U}$ \\
5.0 & $\mathrm{U}$ \\
5.0 & $\mathrm{U}$ \\
5.0 & $\mathrm{U}$ \\
5.0 & $\mathrm{U}$ \\
5.0 & $\mathrm{U}$ \\
5.0 & $\mathrm{U}$ \\
5.0 & $\mathrm{U}$ \\
\hline
\end{tabular}


$1 F$

VOLATILE ORGANICS ANALYSIS DATA SHEET

TENTATIVELY IDENTIFIED COMPOUNDS
EPA SAMPLE NO.

NA-TI14-W-20666

Lab Name: ENVIROSYSTEMS, INC.

Contract : ARGONNE

Lab Code: ENVSYS Case No.: AR0504 SAS No.:

SDG NO.: AR0504

Matrix: (soil/water) WATER

Sample wt/vol: $5.000(\mathrm{~g} / \mathrm{mL}) \mathrm{ML}$

Level: (low/med) LOW

\% Moisture: not dec.

GC Column: RTX-624 ID: 0.18 (mm)

Soil Extract Volume:

Number TICs found: 1
Lab Sample ID: 0060504-03

Lab File ID: H73FF640

Date Received: 05/09/06

Date Analyzed: 05/13/06

Dilution Factor: 1.0

Soil Aliquot Volume: (uL)

CONCENTRATION UNITS :

(ug/L or $\mathrm{ug} / \mathrm{Kg}$ ) ug/L

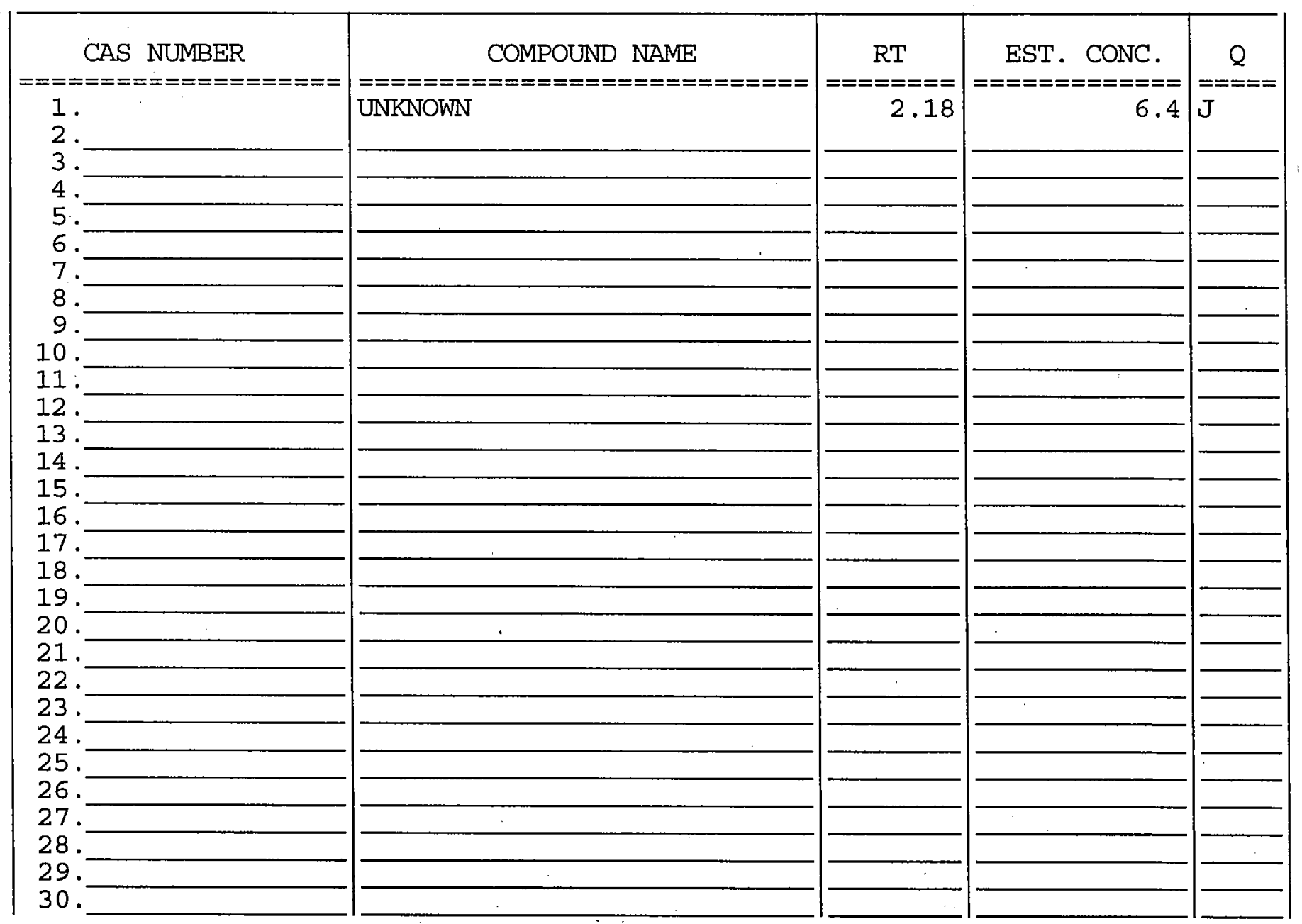


$1 \mathrm{~A}$

VOLATILE ORGANICS ANALYSIS DATA SHEET

Lab Name: ENVIROSYSTEMS, INC.

Lab Code: ENVSYS Case No.: KCI0504 SAS No.:

Matrix: (soil/water) WATER

Sample wt/vol: $\quad 5.000(\mathrm{~g} / \mathrm{mL}) \mathrm{ML}$

Level: (low/med) LOW

\% Moisture: not dec.

GC Column: RTX-624 ID: 0.18 (mm) (uL)

Soil Extract Volume:

COMPOUND

CAS NO.
EPA SAMPLE NO.

NA-TI19-

W-20666DL

SDG No. : AR0504

Lab Sample ID: 0060504-03DL

Lab File ID: AG75HD010

Date Received: 05/09/06

Date Analyzed: 05/17/06

Dilution Factor: 2.0

Soil Aliquot Volume: (uL)

CONCENTRATION UNITS :

Dichlorodifluoromethane

$74-87-3$

Chloromethane

Vinyl Chloride

$75-01-4$

Bromomethane

$75-00-3$

$75-69-4$

Chloroethane

Trichlorofluoromethane

$75-35-4$

$76-13-1$

$67-64-1$

$75-15-0$

$79-20-9$

$75-09-2$

$156-60-5$

$1634-04-4$

$75-34-3$

$156-59-2$

$78-93-3$

$67-66-3$

$71-55-6$

$110-82-7$

$56-23-5$

$71-43-2$

$107-06-2$

1,1-Dichloroethene

1, 1,2-Trichloro-1,2,2-trifluoroethane Acetone

Carbon Disulfide

Methyl Acetate

Methylene Chloride

trans-1,2-Dichloroethene

Methyl tert-Butyl Ether

1,1-Dichloroethane

cis-1,2-Dichloroethene

2-Butanone

Chloroform

1, 1, 1-Trichloroethane

Cyclohexane

Carbon Tetrachloride

Benzene

1,2-Dichloroethane

(ug/L or $\mathrm{ug} / \mathrm{Kg}$ ) UG/L Q 
Lab Name: ENVIROSYSTEMS, INC. Contract : KCI

NA-TI13W-20666DL

Lab Code: ENVSYS Case No.: KCI0504 SAS No.: SDG NO.: AR0504

Matrix: (soil/water) WATER

Sample wt/vol:

$5.000(\mathrm{~g} / \mathrm{mL}) \mathrm{ML}$

Level: (low/med) LOW

\% Moisture: not dec.

GC Column: RTX-624 ID: 0.18 (mm)

Soil Extract Volume: (uL)
Lab Sample ID: 0060504-03DL

Lab File ID: AG75HD010

Date Received: 05/09/06

Date Analyzed: 05/17/06

Dilution Factor: 2.0

Soil Aliquot Volume: (uL)

CONCENTRATION UNITS :

CAS NO.

COMPOUND

(ug/L or $\mathrm{ug} / \mathrm{Kg}$ ) UG/I

\begin{tabular}{|c|c|c|c|}
\hline $\begin{array}{r}79-01-6 \\
108-87-2 \\
78-87-5 \\
75-27-4 \\
10061-01-5 \\
108-10-1 \\
108-88-3 \\
10061-02-6 \\
79-00-5 \\
127-18-4 \\
591-78-6 \\
124-48-1 \\
106-93-4 \\
108-90-7 \\
100-41-4 \\
1330-20-7 \\
100-42-5 \\
75-25-2 \\
98-82-8 \\
79-34-5 \\
541-73-1 \\
106-46-7 \\
95-50-1 \\
96-12-8 \\
120-82-1\end{array}$ & $\begin{array}{l}\text { Trichloroethene } \\
\text { Methylcyclohexane } \\
\text { 1,2-Dichloropropane } \\
\text { Bromodichloromethane } \\
\text { cis-1,3-Dichloropropene } \\
\text { 4-Methyl-2-Pentanone } \\
\text { Toluene } \\
\text { trans-1,3-Dichloropropene } \\
\text { 1,1,2-Trichloroethane } \\
\text { Tetrachloroethene } \\
\text { 2-Hexanone } \\
\text { Dibromochloromethane } \\
\text { 1,2-Dibromoethane } \\
\text { Chlorobenzene } \\
\text { Ethylbenzene } \\
\text { Xylene (Total) } \\
\text { Styrene } \\
\text { Bromoform } \\
\text { Isopropylbenzene } \\
\text { 1,1,2,2-Tetrachloroethane } \\
\text { 1,3-Dichlorobenzene } \\
\text { 1,4-Dichlorobenzene } \\
\text { 1,2-Dichlorobenzene } \\
\text { 1,2-Dibromo -3-chloropropane } \\
\text { 1,2,4-Trichlorobenzene }\end{array}$ & $\begin{array}{l}10 \\
10 \\
10 \\
10 \\
10 \\
10 \\
10 \\
10 \\
10 \\
10 \\
10 \\
10 \\
10 \\
10 \\
10 \\
10 \\
10 \\
10 \\
10 \\
10 \\
10 \\
10 \\
10 \\
10 \\
10\end{array}$ & $\begin{array}{l}\mathrm{U} \\
\mathrm{U} \\
\mathrm{U} \\
\mathrm{U} \\
\mathrm{U} \\
\mathrm{U} \\
\mathrm{U} \\
\mathrm{U} \\
\mathrm{U} \\
\mathrm{U} \\
\mathrm{U} \\
\mathrm{U} \\
\mathrm{U} \\
\mathrm{U} \\
\mathrm{U} \\
\mathrm{U} \\
\mathrm{U} \\
\mathrm{U} \\
\mathrm{U} \\
\mathrm{U} \\
\mathrm{U} \\
\mathrm{U} \\
\mathrm{U} \\
\mathrm{U} \\
\mathrm{U}\end{array}$ \\
\hline
\end{tabular}


$1 \mathrm{~F}$

VOLATILE ORGANICS ANALYSIS DATA SHEET

TENTATIVELY IDENTIFIED COMPOUNDS
EPA SAMPLE NO.

Contract: $\mathrm{KCI}$
NA-TI13-

W-20666DL

Lab Name: ENVIROSYSTEMS, INC.

Lab Code: ENVSYS Case No.: KCI0504 SAS No.:

SDG NO.: AR0504

Lab Sample ID: 0060504-03DL

Sample wt/vol: $5.000(\mathrm{~g} / \mathrm{mL})$ ML

Lab File ID: AG75HD010

Level: (low/med) LOW

Date Received: 05/09/06

\% Moisture: not dec.

Date Analyzed: 05/17/06

GC Column: RTX-624 ID: 0.18 (mm)

Dilution Factor: 2.0

Soil Extract Volume:

(uL)

Soil Aliquot Volume:

Number TICs found: 0

CONCENTRATION UNITS :

(ug/L or $\mathrm{ug} / \mathrm{Kg}$ ) ug/L

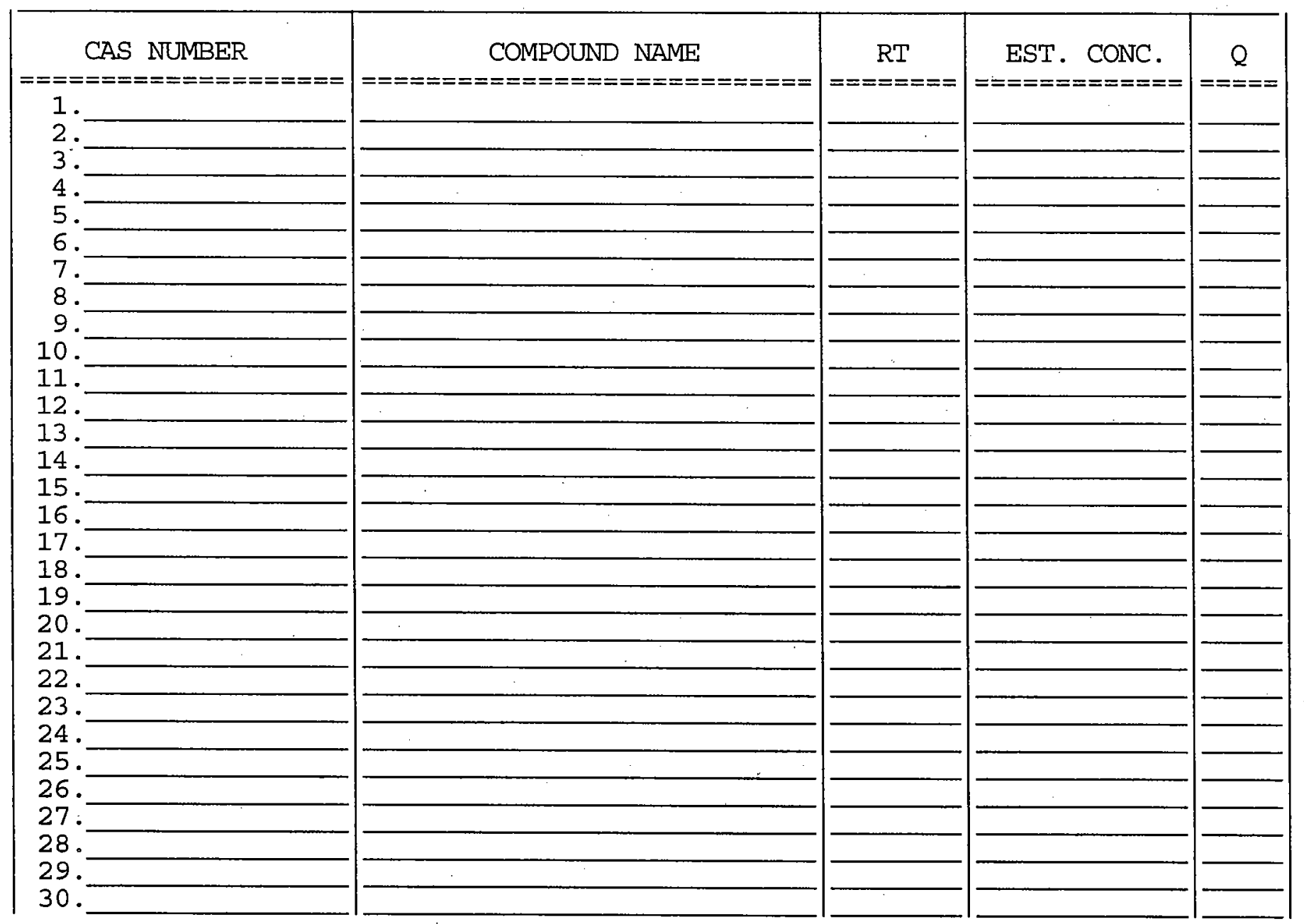


Lab Name: ENVIROSYSTEMS, INC.

Lab Code: ENVSYS Case No.: AR0504

Matrix: (soil/water) WATER

Sample wt/vol: $\quad 5.000(\mathrm{~g} / \mathrm{mL}) \mathrm{ML}$

Level: (low/med) LOW

\% Moisture: not dec.

GC Column: RTX-624 ID: 0.18 (mm)

Soil Extract Volume: (uL)

COMPOUND
Contract : ARGONNE

NA-TI13-W-20563
SAS NO. :

SDG No.: AR0504
Lab Sample ID: 0060504-04

Lab File ID: H73FF641

Date Received: 05/09/06

Date Analyzed: 05/13/06

Dilution Factor: 1.0

Soil Aliquot Volume: (uL)

\begin{tabular}{|c|c|c|c|}
\hline $\begin{array}{r}75-71-8 \\
74-87-3 \\
75-01-4 \\
74-83-9 \\
75-00-3 \\
75-69-4 \\
75-35-4 \\
76-13-1 \\
67-64-1 \\
75-15-0 \\
79-20-9 \\
75-09-2 \\
156-60-5 \\
1634-04-4 \\
75-34-3 \\
156-59-2 \\
78-93-3 \\
67-66-3 \\
71-55-6 \\
110-82-7 \\
56-23-5 \\
71-43-2 \\
107-06-2\end{array}$ & $\begin{array}{l}\text { Dichlorodifluoromethane } \\
\text { Chloromethane } \\
\text { Vinyl Chloride } \\
\text { Bromomethane } \\
\text { Chloroethane } \\
\text { Trichlorofluoromethane } \\
\text { 1,1-Dichloroethene } \\
\text { 1,1,2-Trichloro-1,2,2-trifluoroethane } \\
\text { Acetone } \\
\text { Carbon Disulfide } \\
\text { Methyl Acetate } \\
\text { Methylene Chloride } \\
\text { trans-1,2-Dichloroethene } \\
\text { Methyl tert-Butyl Ether } \\
\text { 1,1-Dichloroethane } \\
\text { cis-1,2-Dichloroethene } \\
\text { 2-Butanone } \\
\text { Chloroform } \\
\text { 1,1,1-Trichloroethane } \\
\text { Cyclohexane } \\
\text { Carbon Tetrachloride } \\
\text { Benzene } \\
\text { 1,2-Dichloroethane }\end{array}$ & $\begin{array}{l}5.0 \\
5.0 \\
5.0 \\
5.0 \\
5.0 \\
5.0 \\
5.0 \\
5.0 \\
5.0 \\
5.0 \\
5.0 \\
2.5 \\
5.0 \\
5.0 \\
5.0 \\
5.0 \\
5.0 \\
180 \\
5.0 \\
5.0 \\
330 \\
5.0 \\
5.0\end{array}$ & $\begin{array}{l}\mathrm{U} \\
\mathrm{U} \\
\mathrm{U} \\
\mathrm{U} \\
\mathrm{U} \\
\mathrm{U} \\
\mathrm{U} \\
\mathrm{U} \\
\mathrm{U} \\
\mathrm{U} \\
\mathrm{U} \\
\mathrm{J} \\
\mathrm{U} \\
\mathrm{U} \\
\mathrm{U} \\
\mathrm{U} \\
\mathrm{U}\end{array}$ \\
\hline
\end{tabular}


Lab Name: ENVIROSYSTEMS, INC.

Contract: ARGONNE

Lab Code: ENVSYS Case No.: AR0504 SAS No.:

SDG No.: AR0504

Matrix: (soil/water) WATER

Lab Sample ID: 0060504-04

Sample wt/vol:

$5.000(\mathrm{~g} / \mathrm{mL}) \mathrm{ML}$

Level: (low/med) LoW

Lab File ID: H73FF641

\% Moisture: not dec.

GC Column: RTX-624 ID: 0.18 (mm)

Date Received: 05/09/06

Soil Extract Volume: (uL)

Date Analyzed: 05/13/06

Dilution Factor: 1.0

Soil Aliquot Volume: (uL)

CONCENTRATION UNITS:

CAS NO. COMPOUND

(ug/L or $\mathrm{ug} / \mathrm{Kg})$ UG/L Q

\begin{tabular}{|r|l|r|l|}
\hline $79-01-6$ & Trichloroethene & 5.0 & $\mathrm{U}$ \\
$108-87-2$ & Methylcyclohexane & 5.0 & $\mathrm{U}$ \\
$78-87-5$ & 1,2-Dichloropropane & 5.0 & $\mathrm{U}$ \\
$75-27-4$ & Bromodichloromethane & 5.0 & $\mathrm{U}$ \\
$10061-01-5$ & Cis-1,3-Dichloropropene & 5.0 & $\mathrm{U}$ \\
$108-10-1$ & 4 -Methyl-2-Pentanone & 5.0 & $\mathrm{U}$ \\
$108-88-3$ & Toluene & 5.0 & $\mathrm{U}$ \\
$10061-02-6$ & trans-1,3-Dichloropropene & 5.0 & $\mathrm{U}$ \\
$79-00-5$ & $1,1,2$-Trichloroethane & 5.0 & $\mathrm{U}$ \\
$127-18-4$ & Tetrachloroethene & 5.0 & $\mathrm{U}$ \\
$591-78-6$ & 2-Hexanone & 5.0 & $\mathrm{U}$ \\
$124-48-1$ & Dibromochloromethane & 5.0 & $\mathrm{U}$ \\
$106-93-4$ & 1,2-Dibromoethane & 5.0 & $\mathrm{U}$ \\
$108-90-7$ & Chlorobenzene & 5.0 & $\mathrm{U}$ \\
$100-41-4$ & Ethylbenzene & 5.0 & $\mathrm{U}$ \\
$1330-20-7$ & XYlene (Total) & 5.0 & $\mathrm{U}$ \\
$100-42-5$ & Styrene & 5.0 & $\mathrm{U}$ \\
$75-25-2$ & Bromoform & 5.0 & $\mathrm{U}$ \\
$98-82-8$ & Isopropylbenzene & 5.0 & $\mathrm{U}$ \\
$79-34-5$ & 1,1,2,2-Tetrachloroethane & 5.0 & $\mathrm{U}$ \\
$541-73-1$ & $1,3-$ Dichlorobenzene & 5.0 & $\mathrm{U}$ \\
$106-46-7$ & 1,4 -Dichlorobenzene & 5.0 & $\mathrm{U}$ \\
$95-50-1$ & 1,2 -Dichlorobenzene & 5.0 & $\mathrm{U}$ \\
$96-12-8$ & 1,2-Dibromo-3-chloropropane & 5.0 & $\mathrm{U}$ \\
$120-82-1$ & 1,2,4-Trichlorobenzene & 5.0 & $\mathrm{U}$ \\
\hline
\end{tabular}


Lab Name: ENVIROSYSTEMS, INC.

Lab Code: ENVSYS Case No.: AR0504 SAS No.:

Matrix: (soil/water) WATER

Sample wt/vol: $5.000(\mathrm{~g} / \mathrm{mL}) \mathrm{ML}$

Level: (low/med) LOW.

\% Moisture: not dec.

GC Column: RTX-624 ID: 0.18 (mm)

Soil Extract Volume:

Number TICs found: 1
Contract: ARGONNE

SDG No.: AR0504
Lab Sample ID: 0060504-04

Lab File ID: H73FF641

Date Received: 05/09/06

Date Analyzed: 05/13/06

Dilution Factor: 1.0

Soil Aliquot Volume: (uL) CONCENTRATION UNITS: (ug/L or ug/Kg) ug/L

\begin{tabular}{|c|c|c|c|c|}
\hline $\begin{aligned} & \text { CAS NUMBER } \\
&================== \\
& \\
& 1 .\end{aligned}$ & $\begin{array}{l}\text { COMPOUND NAME } \\
========================== \\
\text { UNKNOWN }\end{array}$ & $\begin{array}{r}\mathrm{RT} \\
======= \\
2.22\end{array}$ & $\begin{array}{r}\text { EST. CONC. } \\
============ \\
6.2\end{array}$ & $\begin{array}{l}Q \\
==== \\
\mathrm{J}\end{array}$ \\
\hline & & & & \\
\hline${ }^{4}$. & & & & \\
\hline & & & & \\
\hline $8 .=$ & & & & \\
\hline & & & & \\
\hline 10. & & & & \\
\hline 12. & & & & \\
\hline & $\square$ & 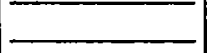 & & \\
\hline $\begin{array}{l}140^{\circ} \\
15\end{array}$ & & - & & \\
\hline 16. & & & & \\
\hline 17. & & & & \\
\hline $10^{\circ}$ & & & $\longrightarrow$ & \\
\hline 20. & & & $\bar{C}$ & \\
\hline 21. & -1 & & - & \\
\hline 23 & & & & \\
\hline 24. & & & & \\
\hline $\begin{array}{l}25 \\
26\end{array}$ & & & & \\
\hline 27. & & & & \\
\hline 28 & & & 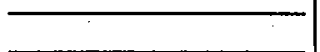 & \\
\hline & & & & \\
\hline & & & & \\
\hline
\end{tabular}


$1 A$

VOLATILE ORGANICS ANALYSIS DATA SHEET

Lab Name: ENVIROSYSTEMS, INC. Contract : KCI
EPA SAMPLE NO.

NA-TI13W-20563DL

Lab Code: ENVSYS Case No.: KCI0504 SAS No.:

SDG NO.: AR0504

Matrix: (soil/water) WATER

Sample wt/vol : $\quad 5.000(\mathrm{~g} / \mathrm{mL}) \mathrm{ML}$

Level: (low/med) Low

\% Moisture: not dec:

GC Column: RTX-624 ID: 0.18 (mm) (UL)

Soil Extract Volume:

COMPOUND

CONCENTRATION UNITS:

CAS NO.

Dichlorodifluoromethane
Chloromethane
Vinyl Chloride
Bromomethane
Chloroethane
Trichlorofluoromethane
1,1-Dichloroethene
1,1,2-Trichloro-1,2,2-trifluoroethane
Acetone
Carbon Disulfide
Methyl Acetate
Methylene Chloride
trans-1,2-Dichloroethene
Methyl tert-Butyl Ether
1,1-Dichloroethane
cis-1,2-Dichloroethene
2-Butanone
Chloroform
1,1,1-Trichloroethane
Cyclohexane
Carbon Tetrachloride
Benzene
1,2-Dichloroethane

Dichlorodifluoromethane

$74-87-3$

$75-01-4$

$74-83-9$

$75-00-3$

$75-69-4$

$75-35-4$

$76-13-1$

$67-64-1$

75-15-0

$79-20-9$

$75-09-2$

$156-60-5$

1634-04-4

$75-34-3$

156-59-2

$78-93-3$

$67-66-3$

$71-55-6$

$110-82-7$

$56-23-5$

$71-43-2$

107-06-2

Date Received: 05/09/06
Lab Sample ID: 0060504-04

Lab File ID: AG75HD011

Date Analyzed: 05/17/06

Dilution Factor: 2.0

Soil Aliquot Volume: (uL) 
Lab Name: ENVIROSYSTEMS, INC.

Contract: $\mathrm{KCI}$

EPA SAMPLE NO.

Lab Code: ENVSYS Case No.: KCI0504 SAS No.:

NA-TI13W-20563DL

Matrix: (soil/water) WATER

Sample wt/vol: $\quad 5.000(\mathrm{~g} / \mathrm{mL}) \mathrm{ML}$

Level: (low/med) LOW

$\%$ Moisture: not dec.

GC Column: RTX-624 ID: 0.18 (mm)

Soil Extract Volume: (uL)

Lab Sample ID: 0060504-04

Lab File ID: AG75HD011

Date Received: 05/09/06

Date Analyzed: 05/17/06

Dilution Factor: 2.0

Soil Aliquot Volume: (u山)

CONCENTRATION UNITS :

CAS NO.

COMPOUND

(ug/L or $\mathrm{ug} / \mathrm{Kg}$ )

$\mathrm{UG} / \mathrm{L} \quad \mathrm{Q}$

\begin{tabular}{|c|c|c|c|}
\hline $\begin{array}{r}79-01-6 \\
108-87-2 \\
78-87-5 \\
75-27-4 \\
10061-01-5 \\
108-10-1 \\
108-88-3 \\
10061-02-6 \\
79-00-5 \\
127-18-4 \\
591-78-6 \\
124-48-1 \\
106-93-4 \\
108-90-7 \\
100-41-4 \\
1330-20-7 \\
100-42-5 \\
75-25-2 \\
98-82-8 \\
79-34-5 \\
541-73-1 \\
106-46-7 \\
95-50-1 \\
96-12-8 \\
120-82-1\end{array}$ & $\begin{array}{l}\text { Trichloroethene } \\
\text { Methylcyclohexane } \\
\text { 1,2-Dichloropropane } \\
\text { Bromodichloromethane } \\
\text { cis-1,3-Dichloropropene } \\
\text { 4-Methyl-2-Pentanone } \\
\text { Toluene } \\
\text { trans-1,3-Dichloropropene } \\
\text { 1,1,2-Trichloroethane } \\
\text { Tetrachloroethene } \\
\text { 2-Hexanone } \\
\text { Dibromochloromethane } \\
\text { 1,2-Dibromoethane } \\
\text { Chlorobenzene } \\
\text { Ethylbenzene } \\
\text { Xylene (Total) } \\
\text { Styrene } \\
\text { Bromoform } \\
\text { Isopropylbenzene } \\
1,1,2,2-\text { Tetrachloroethane } \\
1,3-\text { Dichlorobenzene } \\
\text { 1,4-Dichlorobenzene } \\
\text { 1,2-Dichlorobenzene } \\
\text { 1,2-Dibromo-3-chloropropane } \\
\text { 1,2,4-Trichlorobenzene }\end{array}$ & $\begin{array}{l}10 \\
10 \\
10 \\
10 \\
10 \\
10 \\
10 \\
10 \\
10 \\
10 \\
10 \\
10 \\
10 \\
10 \\
10 \\
10 \\
10 \\
10 \\
10 \\
10 \\
10 \\
10 \\
10 \\
10 \\
10\end{array}$ & $\begin{array}{l}\bar{U} \\
U \\
U \\
U \\
U \\
U \\
U \\
U \\
U \\
U \\
U \\
U \\
U \\
U \\
U \\
U \\
U \\
U \\
U \\
U \\
U \\
U \\
U \\
U \\
U\end{array}$ \\
\hline
\end{tabular}


$1 F$

VOLATILE ORGANICS ANALYSIS DATA SHEET TENTATIVELY IDENTIFIED COMPOUNDS

Lab Name: ENVIROSYSTEMS, INC.

Contract: KCI

Lab Code: ENVSYS Case No.: KCI0504 SAS No.:

Lab Sample ID: 0060504-04

Sample wt/vol: $5.000(\mathrm{~g} / \mathrm{mL}) \mathrm{ML}$

Lab File ID: AG75HD011

Level: (low/med): LOW

\% Moisture: not dec.

GC Column: RTX-624 ID: $0.18 \quad(\mathrm{~mm})$

Soil Extract Volume:

(uI)

Number TICs found: 0

Date Received: 05/09/06

Date Analyzed: 05/17/06

Dilution Factor: 2.0

Soil Aliquot Volume:

CONCENTRATION UNITS:

$(u g / \mathrm{L}$ or $\mathrm{ug} / \mathrm{Kg}) \mathrm{ug} / \mathrm{L}$
EPA SAMPLE NO.

NA-TI13-

W-20563DL (uL)

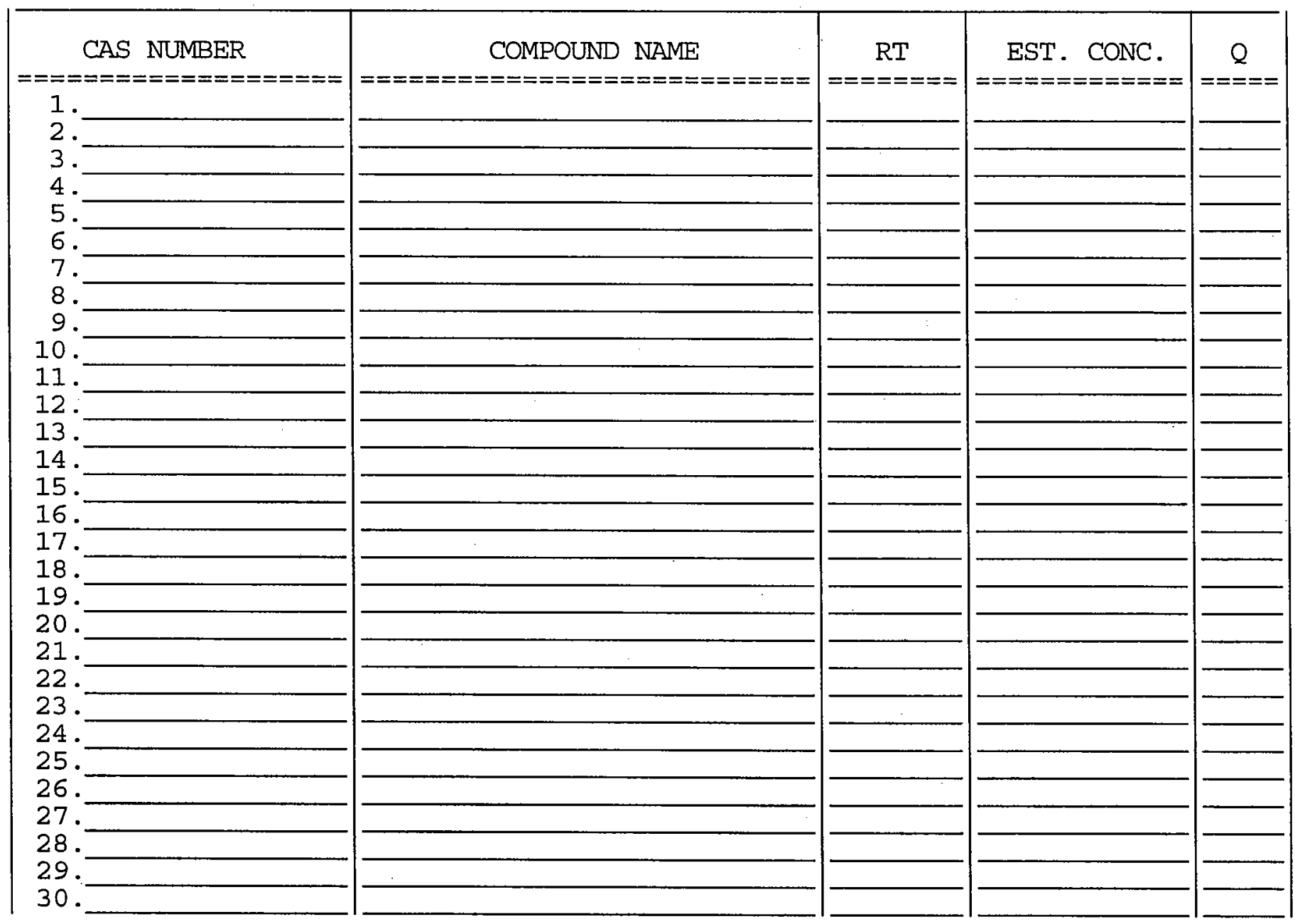


Lab Name: ENVIROSYSTEMS, INC.

Contract: ARGONNE

Lab Code: ENVSYS Case No.: AŔR0504 SAS No.:

SDG No.: AR0504

Matrix: (soil/water) WATER

Lab Sample ID: 0060504-05

Sample wt/vol:

$5.000(\mathrm{~g} / \mathrm{mL}) \mathrm{ML}$

Level: (low/med) LoW

Lab File ID: H73FF642

\% Moisture: not dec.

GC Column: RTX-624 ID: 0.18 (mm)

Date Received: 05/09/06

Soil Extract Volume: (uL)

Date Analyzed: 05/13/06

Dilution Factor: 1.0

Soil Aliquot Volume: (uL)

CAS NO. COMPOUND

CONCENTRATION UNITS:

(ug/L or ug/Kg) UG/L Q

\begin{tabular}{|r|l|}
\hline $75-71-8$ & Dichlorodifluoromethane \\
$74-87-3$ & Chloromethane \\
$75-01-4$ & Vinyl Chloride \\
$74-83-9$ & Bromomethane \\
$75-00-3$ & Chloroethane \\
$75-69-4$ & Trichlorofluoromethane \\
$75-35-4$ & $1,1-$ Dichloroethene \\
$76-13-1$ & $1,1,2-T r i c h l o r o-1,2,2-$ trifluoroethane \\
$67-64-1$ & Acetone \\
$75-15-0$ & Carbon Disulfide \\
$79-20-9$ & Methyl Acetate \\
$75-09-2$ & Methylene Chloride \\
$156-60-5$ & trans-1,2-Dichloroethene \\
$1634-04-4$ & Methyl tert-Butyl Ether \\
$75-34-3$ & $1,1-$ Dichloroethane \\
$156-59-2$ & cis-1,2-Dichloroethene \\
$78-93-3$ & $2-$-Butanone \\
$67-66-3$ & Chloroform \\
$71-55-6$ & $1,1,1-T r i c h l o r o e t h a n e$ \\
$110-82-7$ & Cyclohexane \\
$56-23-5$ & Carbon Tetrachloride \\
$71-43-2$ & Benzene \\
$107-06-2$ & $1,2-D i c h l o r o e t h a n e$ \\
& \\
\hline
\end{tabular}

\begin{tabular}{l|l|}
5.0 & $\mathrm{U}$ \\
5.0 & $\mathrm{U}$ \\
5.0 & $\mathrm{U}$ \\
5.0 & $\mathrm{U}$ \\
5.0 & $\mathrm{U}$ \\
5.0 & $\mathrm{U}$ \\
5.0 & $\mathrm{U}$ \\
5.0 & $\mathrm{U}$ \\
5.0 & $\mathrm{U}$ \\
5.0 & $\mathrm{U}$ \\
5.0 & $\mathrm{U}$ \\
2.9 & $\mathrm{~J}$ \\
5.0 & $\mathrm{U}$ \\
5.0 & $\mathrm{U}$ \\
5.0 & $\mathrm{U}$ \\
5.0 & $\mathrm{U}$ \\
5.0 & $\mathrm{U}$ \\
73 & \\
5.0 & $\mathrm{U}$ \\
5.0 & $\mathrm{U}$ \\
130 & \\
5.0 & $\mathrm{U}$ \\
5.0 & $\mathrm{U}$ \\
\hline
\end{tabular}


Lab Name: ENVIROSYSTEMS, INC.

Lab Code: ENVSYS Case No.: AR0504 SAS No.:

Matrix: (soil/water) WATER

Sample wt/vol:

$5.000(\mathrm{~g} / \mathrm{mL}) \mathrm{ML}$

Level: (low/med) LOW

\% Moisture: not dec.

GC Column: RTX-624 ID: 0.18 (mm)

Soil Extract Volume: (uL)

Contract: ARGONNE

SDG NO.: AR0504

Lab Sample ID: 0060504-05

Lab File ID: H73FF642

Date Received: 05/09/06

Date Analyzed: 05/13/06

Dilution Factor: 1.0

Soil Aliquot Volume: (uL)

CONCENTRATION UNITS :

CAS NO.

COMPOUND

(ug/L or $u g / K g) \quad U G / L \quad Q$

\begin{tabular}{|c|c|c|c|}
\hline $\begin{array}{r}79-01-6 \\
108-87-2 \\
78-87-5 \\
75-27-4 \\
10061-01-5 \\
108-10-1 \\
108-88-3 \\
10061-02-6 \\
79-00-5 \\
127-18-4 \\
591-78-6 \\
124-48-1 \\
106-93-4 \\
108-90-7 \\
100-41-4 \\
1330-20-7 \\
100-42-5 \\
75-25-2 \\
98-82-8 \\
79-34-5 \\
541-73-1 \\
106-46-7 \\
95-50-1 \\
96-12-8 \\
120-82-1\end{array}$ & $\begin{array}{l}\text { Trichloroethene } \\
\text { Methylcyclohexane } \\
\text { 1,2-Dichloropropane } \\
\text { Bromodichloromethane } \\
\text { cis-1,3-Dichloropropene } \\
\text { 4-Methyl-2-Pentanone } \\
\text { Toluene } \\
\text { trans-1,3-Dichloropropene } \\
\text { 1,1,2-Trichloroethane } \\
\text { Tetrachloroethene } \\
\text { 2-Hexanone } \\
\text { Dibromochloromethane } \\
\text { 1,2-Dibromoethane } \\
\text { Chlorobenzene } \\
\text { Ethylbenzene } \\
\text { Xylene (Total) } \\
\text { Styrene } \\
\text { Bromoform } \\
\text { Isopropylbenzene } \\
\text { 1,1,2,2-Tetrachloroethane } \\
\text { 1,3-Dichlorobenzene } \\
1,4-\text {-Dichlorobenzene } \\
1,2 \text {-Dichlorobenzene } \\
\text { 1,2-Dibromo-3-chloropropane } \\
1,2,4-\text { Trichlorobenzene }\end{array}$ & $\begin{array}{l}5.0 \\
5.0 \\
5.0 \\
5.0 \\
5.0 \\
5.0 \\
5.0 \\
5.0 \\
5.0 \\
5.0 \\
5.0 \\
5.0 \\
5.0 \\
5.0 \\
5.0 \\
5.0 \\
5.0 \\
5.0 \\
5.0 \\
5.0 \\
5.0 \\
5.0 \\
5.0 \\
5.0 \\
5.0\end{array}$ & $\begin{array}{l}\mathrm{U} \\
\mathrm{U} \\
\mathrm{U} \\
\mathrm{U} \\
\mathrm{U} \\
\mathrm{U} \\
\mathrm{U} \\
\mathrm{U} \\
\mathrm{U} \\
\mathrm{U} \\
\mathrm{U} \\
\mathrm{U} \\
\mathrm{U} \\
\mathrm{U} \\
\mathrm{U} \\
\mathrm{U} \\
\mathrm{U} \\
\mathrm{U} \\
\mathrm{U} \\
\mathrm{U} \\
\mathrm{U} \\
\mathrm{U} \\
\mathrm{U} \\
\mathrm{U} \\
\mathrm{U}\end{array}$ \\
\hline
\end{tabular}




\section{$1 \mathrm{~F}$}

VOLATILE ORGANICS ANALYSIS DATA SHEET

TENTATIVELY IDENTIFIED COMPOUNDS

Lab Name: ENVIROSYSTEMS, INC.

Contract : ARGONNE

Lab Code: ENVSYS Case No.: AR0504 SAS No.:

SDG No.: AR0504

Matrix: (soil/water) WATER

Sample wt/vol: $5.000(\mathrm{~g} / \mathrm{mL}) \mathrm{ML}$

Level: (low/med) LOW

$\because$ Moisture: not dec.

GC Column: RTX-624 ID: 0.18 (mm)

Soil Extract Volume:

Number TICs found: 0 .

Lab Sample ID: 0060504-05

Lab File ID: H73FF642

Date Received: 05/09/0.6

Date Analyzed: 05/13/06

Dilution Factor: 1.0

Soil Aliquot Volume:

CONCENTRATION UNITS:

(ug/L or ug/Kg) ug/L
EPA SAMPLE NO.

NA-TI13-W-20562

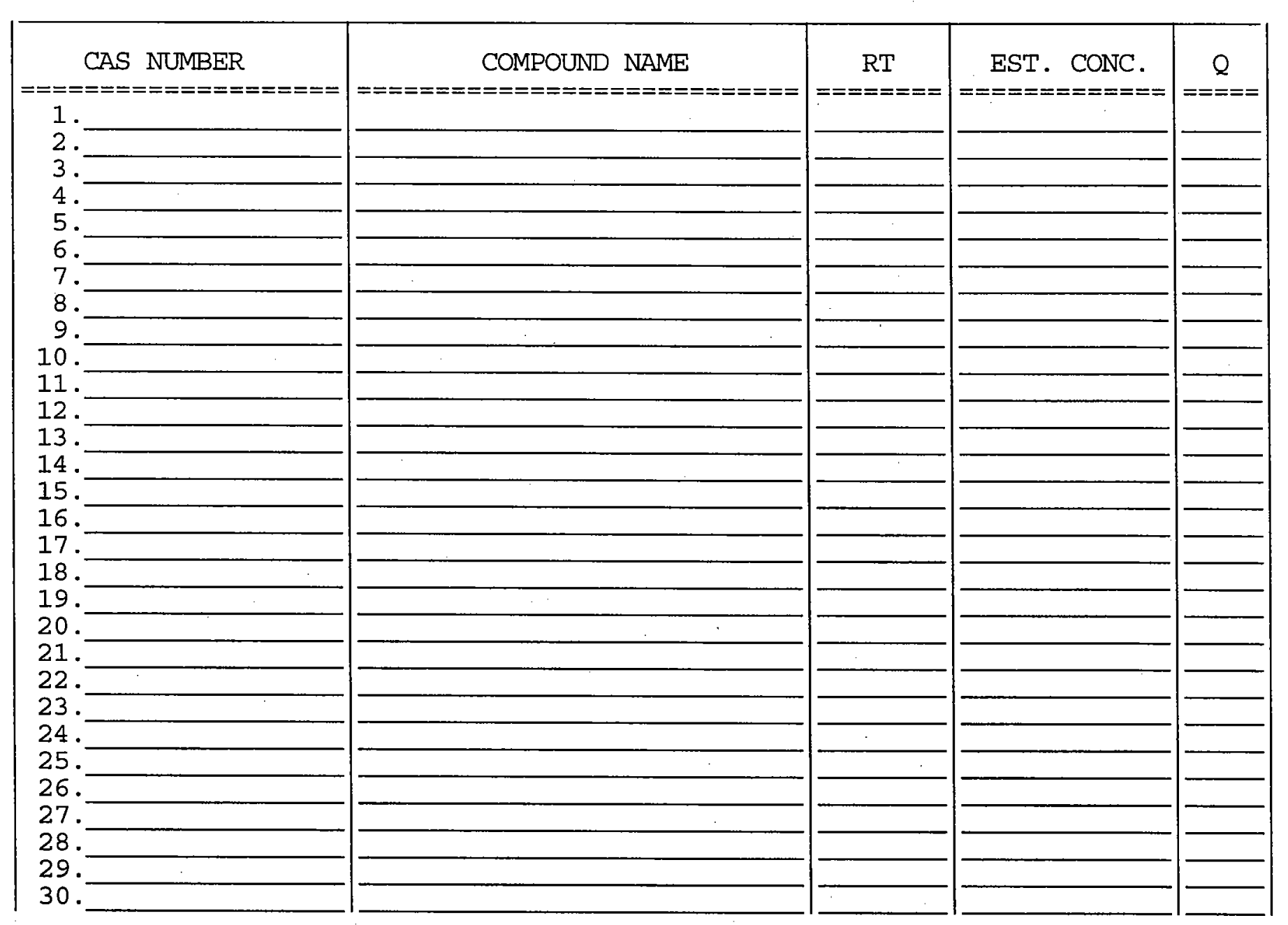


$1 \mathrm{~A}$

VOLATILE ORGANICS ANALYSIS DATA SHEET

Lab Name: ENVIROSYSTEMS, INC.

Contract: ARGONNE

EPA SAMPLE NO.

Lab Code: ENVSYS Case No.: AR0504 SAS No.:

Lab Sample ID: 0060504-06

Matrix: (soil/water) WATER

Sample wt/vol:

$5.000(\mathrm{~g} / \mathrm{mL}) \mathrm{ML}$

Level: (low/med) LOW

\% Moisture: not dec.

GC Column: RTX-624 ID: 0.18 (mm)

Soil Extract Volume: (uL)
Lab File ID: H73FF643

Date Received: 05/09/06

Date Analyzed: 05/13/06

Dilution Factor: 1.0

Soil Aliquot Volume: (uL)

CAS NO. COMPOUND

CONCENTRATION UNITS :

(ug/L or $u g / K g) \quad U G / L \quad Q$

\begin{tabular}{|c|c|c|c|}
\hline $\begin{array}{r}75-71-8 \\
74-87-3 \\
75-01-4 \\
74-83-9 \\
75-00-3 \\
75-69-4 \\
75-35-4 \\
76-13-1 \\
67-64-1 \\
75-15-0 \\
79-20-9 \\
75-09-2 \\
156-60-5 \\
1634-04-4 \\
75-34-3 \\
156-59-2 \\
78-93-3 \\
67-66-3 \\
71-55-6 \\
110-82-7 \\
56-23-5 \\
71-43-2 \\
107-06-2\end{array}$ & $\begin{array}{l}\text { Dichlorodifluoromethane } \\
\text { Chloromethane } \\
\text { Vinyl Chloride } \\
\text { Bromomethane } \\
\text { Chloroethane } \\
\text { Trichlorofluoromethane } \\
\text { 1,1-Dichloroethene } \\
\text { 1,1,2-Trichloro-1,2,2-trifluoroethane } \\
\text { Acetone } \\
\text { Carbon Disulfide } \\
\text { Methyl Acetate } \\
\text { Methylene Chloride } \\
\text { trans-1,2-Dichloroethene } \\
\text { Methyl tert-Butyl Ether } \\
\text { 1,1-Dichloroethane } \\
\text { cis-1,2-Dichloroethene } \\
\text { 2-Butanone } \\
\text { Chloroform } \\
\text { 1,1,1-Trichloroethane } \\
\text { Cyclohexane } \\
\text { Carbon Tetrachloride } \\
\text { Benzene } \\
\text { 1,2-Dichloroethane }\end{array}$ & $\begin{array}{l}5.0 \\
5.0 \\
5.0 \\
5.0 \\
5.0 \\
5.0 \\
5.0 \\
5.0 \\
5.0 \\
5.0 \\
5.0 \\
2.2 \\
5.0 \\
5.0 \\
5.0 \\
5.0 \\
5.0 \\
5.0 \\
5.0 \\
5.0 \\
5.0 \\
5.0 \\
5.0\end{array}$ & $\begin{array}{l}U \\
U \\
U \\
U \\
U \\
U \\
U \\
U \\
U \\
U \\
U \\
J \\
U \\
U \\
U \\
U \\
U \\
U \\
U \\
U \\
U \\
U \\
U\end{array}$ \\
\hline
\end{tabular}


Lab Name: ENVIROSYSTEMS, INC.

Contract : ARGONNE

NA-QCTB$05 / 07 / 06$

Lab Code: ENVSYS Case No.: AR0504 SAS No.:

SDG No.: AR0504

Matrix: (soil/water) WATER

Lab Sample ID: 0060504-06

Sample wt/vol:

$5.000(\mathrm{~g} / \mathrm{mL}) \mathrm{ML}$

Lab File ID: H73FF643

Level : (low/med) LOW

Date Received: 05/09/06

\% Moisture: not dec.

GC Column: RTX-624 ID: 0.18 (mm)

Date Analyzed: 05/13/06

Soil Extract Volume: (UL)

Dilution Factor: 1.0

Soil Aliquot Volume: (uI)

CONCENTRATION UNITS:

CAS NO.

COMPOUND

(ug/L or $u g / K g) \quad U G / L \quad Q$

\begin{tabular}{|c|c|c|c|}
\hline $\begin{array}{r}79-01-6 \\
108-87-2 \\
78-87-5 \\
75-27-4 \\
10061-01-5 \\
108-10-1 \\
108-88-3 \\
10061-02-6 \\
79-00-5 \\
127-18-4 \\
591-78-6 \\
124-48-1 \\
106-93-4 \\
108-90-7 \\
100-41-4 \\
1330-20-7 \\
100-42-5 \\
75-25-2 \\
98-82-8 \\
79-34-5 \\
541-73-1 \\
106-46-7 \\
95-50-1 \\
96-12-8 \\
120-82-1\end{array}$ & $\begin{array}{l}\text { Trichloroethene } \\
\text { Methylcyclohexane } \\
\text { 1,2-Dichloropropane } \\
\text { Bromodichloromethane } \\
\text { cis-1,3-Dichloropropene } \\
\text { 4-Methyl-2-Pentanone } \\
\text { Toluene } \\
\text { trans-1,3-Dichloropropene } \\
\text { 1,1,2-Trichloroethane } \\
\text { Tetrachloroethene } \\
\text { 2-Hexanone } \\
\text { Dibromochloromethane } \\
\text { 1,2-Dibromoethane } \\
\text { Chlorobenzene } \\
\text { Ethylbenzene } \\
\text { Xylene (Total) } \\
\text { Styrene } \\
\text { Bromoform } \\
\text { Isopropylbenzene } \\
\text { 1,1,2,2-Tetrachloroethane } \\
\text { 1,3-Dichlorobenzene } \\
\text { 1,4-Dichlorobenzene } \\
\text { 1,2-Dichlorobenzene } \\
\text { 1,2-Dibromo-3-chloropropane } \\
\text { 1,2,4-Trichlorobenzene }\end{array}$ & $\begin{array}{l}5.0 \\
5.0 \\
5.0 \\
5.0 \\
5.0 \\
5.0 \\
5.0 \\
5.0 \\
5.0 \\
5.0 \\
5.0 \\
5.0 \\
5.0 \\
5.0 \\
5.0 \\
5.0 \\
5.0 \\
5.0 \\
5.0 \\
5.0 \\
5.0 \\
5.0 \\
5.0 \\
5.0 \\
5.0\end{array}$ & $\begin{array}{l}\mathrm{U} \\
\mathrm{U} \\
\mathrm{U} \\
\mathrm{U} \\
\mathrm{U} \\
\mathrm{U} \\
\mathrm{U} \\
\mathrm{U} \\
\mathrm{U} \\
\mathrm{U} \\
\mathrm{U} \\
\mathrm{U} \\
\mathrm{U} \\
\mathrm{U} \\
\mathrm{U} \\
\mathrm{U} \\
\mathrm{U} \\
\mathrm{U} \\
\mathrm{U} \\
\mathrm{U} \\
\mathrm{U} \\
\mathrm{U} \\
\mathrm{U} \\
\mathrm{U}\end{array}$ \\
\hline
\end{tabular}


$1 \mathrm{~F}$

VOLATILE ORGANICS ANALYSIS DATA SHEET TENTATIVELY IDENTIFIED COMPOUNDS
EPA SAMPLE NO.

NA-QCTB05/07/06

Lab Name: ENVIROSYSTEMS, INC.

Contract: ARGONNE

Lab Code: ENVSYS Case No.: AR0504 SAS No.:

SDG No.: AR0504

Matrix: (soil/water) WATER

Sample wt/vol: $5.000(\mathrm{~g} / \mathrm{mL}) \mathrm{ML}$

Level: (low/med) LOW

\% Moisture: not dec.

GC Column: RTX-624 ID: 0.18 (mm)

Soil Extract Volume: (uL)

Number TICs found: 1
Lab Sample ID: 0060504-06

Lab File ID: H73FF643

Date Received: 05/09/06

Date Analyzed: 05/13/06

Dilution Factor: 1.0

Soil Aliquot Volume:

CONCENTRATION UNITS:

(ug/L or $\mathrm{ug} / \mathrm{Kg}$ ) ug/L

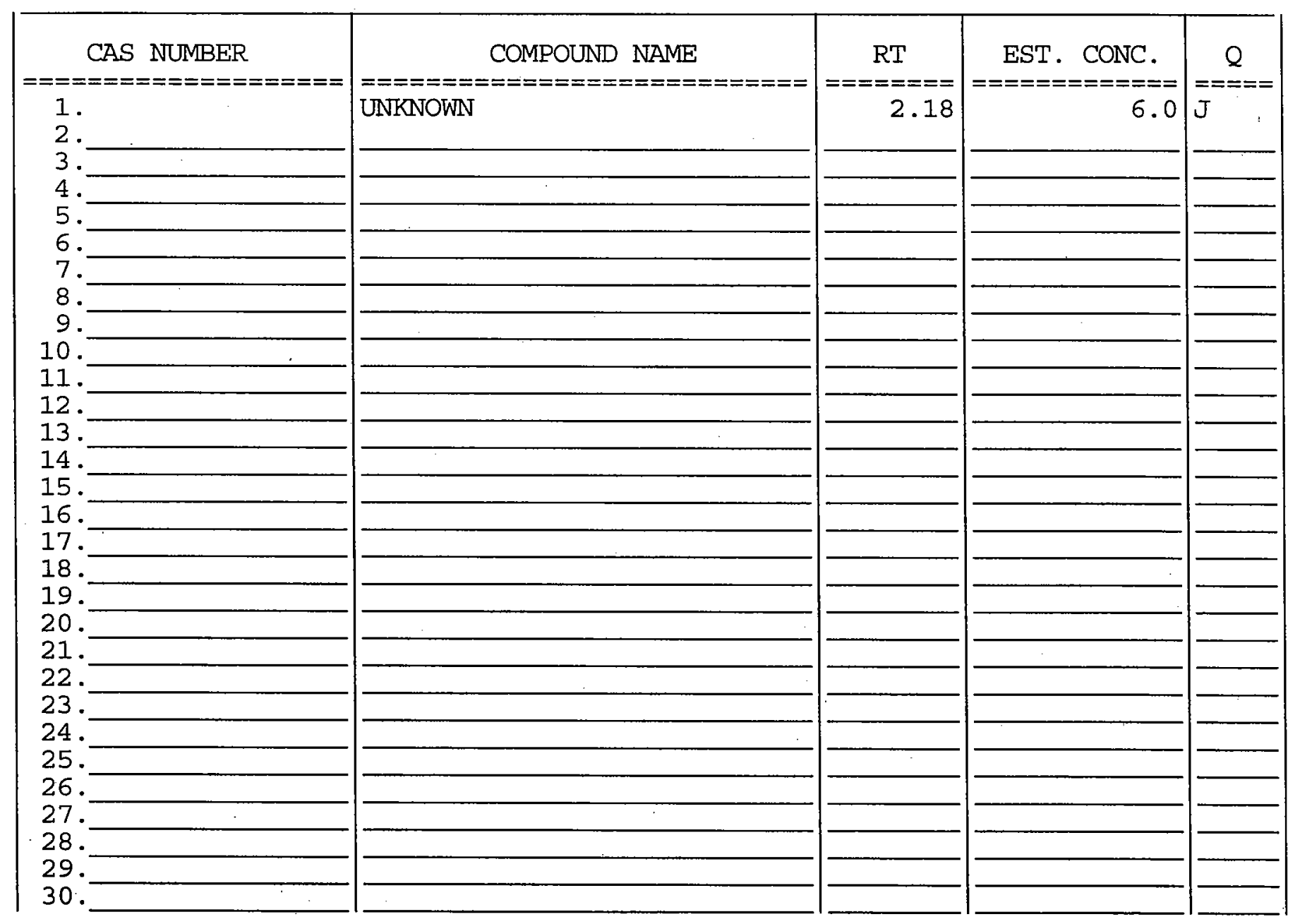




\section{S E V E R N}

\section{T R E N T}

May 3, 2006

Mr. Clyde Dennis

Argonne National Laboratory

9700 S. Cass Avenue

Building 203, Office B149

Argonne, IL 60439
STL Burlington

208 South Park Drive, Suite 1

Colchester, VT 05446

Tel: 8026551203 Fax: 8026551248

www.stl-inc.com

Re: Laboratory Project No. 21005

Case: NAVARRE; SDG: 113901

Dear Mr. Dennis:

Enclosed are the analytical results for the samples that were received by STL Burlington on April $20^{\text {th }}, 2006$. Laboratory identification numbers were assigned, and designated as follows:

$\begin{array}{llll}\text { Lab ID } & \begin{array}{l}\text { Client } \\ \text { Sample ID }\end{array} & \begin{array}{l}\text { Sample } \\ \text { Date }\end{array} & \begin{array}{l}\text { Sample } \\ \text { Matrix }\end{array} \\ & \text { Received: 04/20/06 ETR No: } 113901 & \\ 666620 & \text { NA-S-20308 } & 04 / 17 / 06 & \text { MEOH } \\ 666621 & \text { NA-S-20454 } & 04 / 17 / 06 & \text { MEOH } \\ 666622 & \text { NA-S-20453 } & 04 / 17 / 06 & \text { MEOH } \\ 666623 & \text { NA-S-20408 } & 04 / 17 / 06 & \text { MEOH } \\ 666624 & \text { NA-S-20303 } & 04 / 17 / 06 & \text { MEOH } \\ 666625 & \text { NA-S-20311 } & 04 / 17 / 06 & \text { MEOH } \\ 666626 & \text { NA-S-20476 } & 04 / 17 / 06 & \text { MEOH } \\ 666627 & \text { NA-S-20330 } & 04 / 17 / 06 & \text { MEOH } \\ 666628 & \text { NA-S-20413 } & 04 / 17 / 06 & \text { MEOH } \\ 666629 & \text { NA-S-20295 } & 04 / 17 / 06 & \text { MEOH } \\ 666630 & \text { NA-MEOH BLANK } & 04 / 17 / 06 & \text { MEOH } \\ 666631 & \text { NA-S-20340 } & & \text { MEOH } \\ 666632 & \text { NA-S-20358 } & & \text { MEOH }\end{array}$

Documentation of the condition of the samples at the time of their receipt and any exceptions to the laboratory's Sample Acceptance Policy is included in the Sample Handling section of this submittal.

The samples were analyzed by Method $8260 \mathrm{~B}$, using a low-level calibration. In performing the analytical work, 500 microliters of the methanol extract were added to the 5 milliliter purge volume. In each analysis having 500 microliters of methanol, the recovery of one of the surrogate controls, 1,2-dichlorobenzene- $d_{4}$, was low. The recovery values generally ranged between 60 and 70 percent. Additionally, the recovery of bromofluorobenzene was elevated in certain of the analyses. There was good internal standard stability in each of the analyses associated with the sample set. Two types of laboratory control sample analyses were performed as part of the analytical sequence. One was performed to evaluate method performance, and one was 
performed with 500 microliters of methanol added to the purge volume in order to characterize the affect on the analytical process. The target analytes were recovered well in each of the laboratory control sample analyses that defined the method performance. In the laboratory control sample analysis with methanol, several of the earlier eluting compounds did exhibit lower recoveries, as did several of the later eluting compounds. Most profoundly affected was the performance of 1,2-dibromo-3-chloropropane, 1,2,4-trichlorobenzene, naphthalene, and 1,2,3-trichlorobenzene, for which the recovery values were below 10 percent. Chloroform and carbon tetrachloride were recovered well in each of the laboratory control sample analyses. Matrix spike and matrix spike duplicate analyses were not performed on samples in this sample set. The analysis of the instrument blank that was analyzed in association with the samples was free of contamination. The laboratory did provide for the analysis of a method blank with the addition of 500 microliters of methanol, however the methanol that was used was not from the same lot as that was used in the extraction of the samples.

If there are any questions regarding this submittal, please contact me at (802) 655-1203.

The analytical results associated with the samples presented in this test report were generated under a quality system that adheres to the requirements specified in the NELAC standard. Release of the data contained in this test report and any associated electronic deliverables is authorized by the Laboratory Director's designee as verified by the following signature.

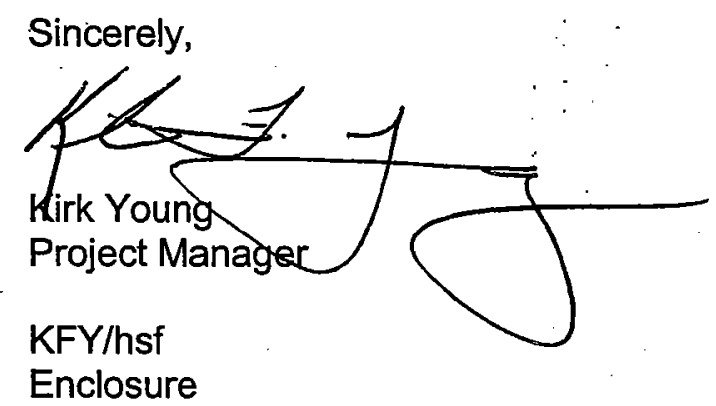




\section{STL Burlington Data Qualifier Definitions}

\section{Organic}

U: Compound analyzed but not detected at a concentration above the reporting limit.

J: $\quad$ Estimated value.

$\mathrm{N}$ : Indicates presumptive evidence of a compound. This flag is used only for tentatively identified compounds (TICs) where the identification of a compound is based on a mass spectral library search.

P: $\quad$ SW-846: Greater than $40 \%$ difference for detected concentrations between two GC columns. Unless otherwise specified the higher of the two values is reported on the Form I.

CLP SOW: Greater than $25 \%$ difference for detected concentrations between two GC columns. Unless otherwise specified the lower of the two values is reported on the Form I.

C: Pesticide result whose identification has been confirmed by GC/MS.

B: Analyte is found in the sample and the associated method blank. The flag is used for tentatively identified compounds as well as positively identified compounds.

E: Compounds whose concentrations exceed the upper limit of the calibration range of the instrument for that specific analysis.

D: Concentrations identified from analysis of the sample at a secondary dilution.

A: Tentatively identified compound is a suspected aldol condensation product.

$X, Y, Z$ : Laboratory defined flags that may be used alone or combined, as needed. If used, the description of the flag is defined in the project narrative.

\section{Inorganic/Metals}

E: Reported value is estimated due to the presence of interference.

N: Matrix spike sample recovery is not within control limits.

* Duplicate sample analysis is not within control limits.

B: The result reported is less than the reporting limit but greater than the instrument detection limit.

U: Analyte was analyzed for but not detected above the reporting limit.

\section{Method Codes:}

P ICP-AES

MS ICP-MS

CV Cold Vapor AA

AS Semi-Automated Spectrophotometric 


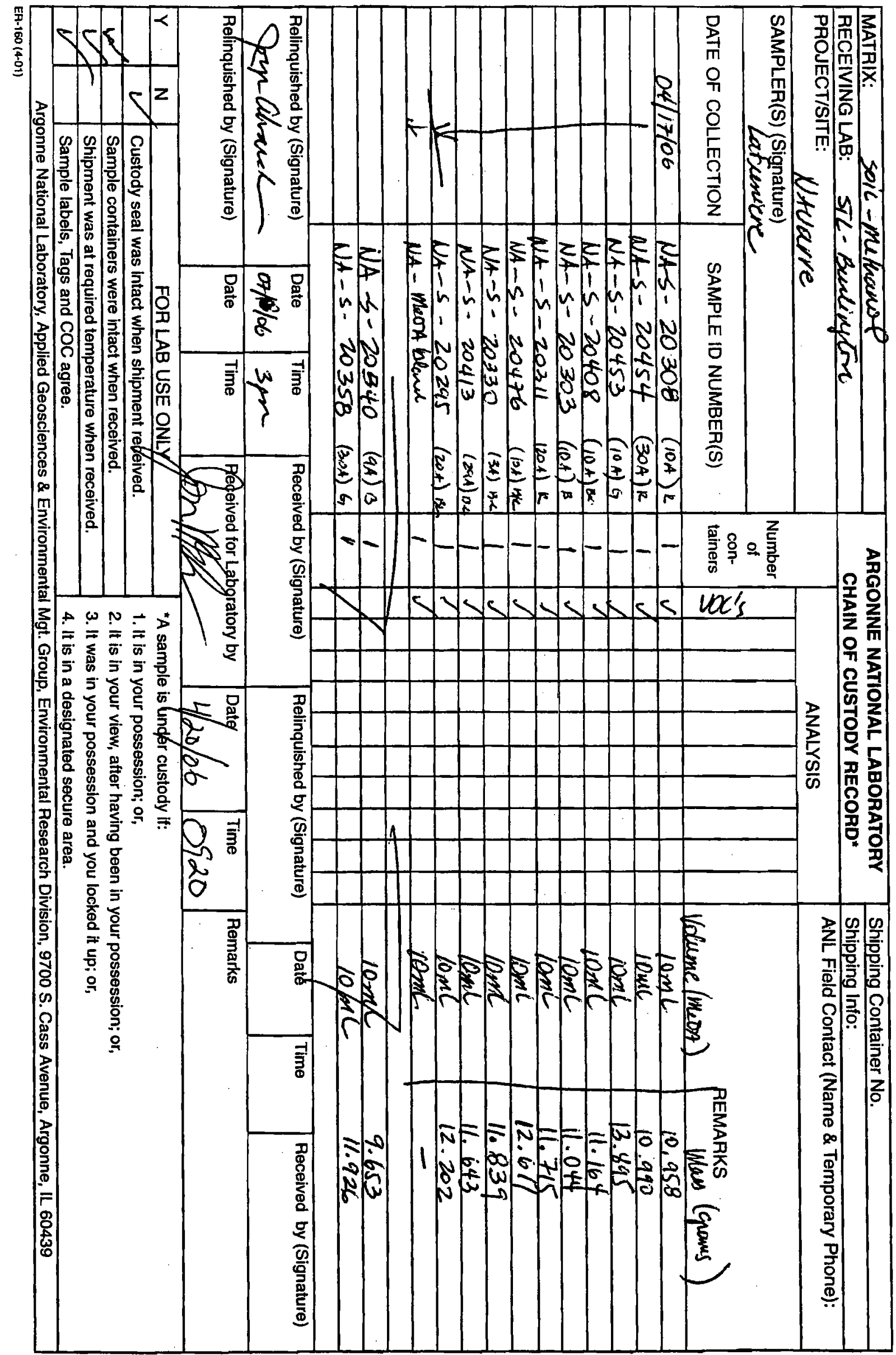




\section{$\frac{\text { SEVERN }}{\text { TRENT }}$ STL}

\section{METHOD 8260B}

\section{VOLATILE ORGANIC ANALYSIS}

SAMPLE DATA SUMMARY PACKAGE 
FORM 1

VOLATILE ORGANICS ANALYSIS DATA SHEET

Lab Name: STL BURLINGTON

Contract : 21005

Lab Code: STLVT Case NO.: NAVARRE SAS No.:

Matrix: (soil/water) SoIL

Sample wt/vol:

$10.0(\mathrm{~g} / \mathrm{mL}) \mathrm{G}$

Level: (low/med) MED

\%oisture: not dec.

GC Colum: CAP ID: 0.53 (mm)

Soil Extract Volume: 10000 (UU)
ARGLAB SAMPLE NO.

NA-MEOH BLANK

SDG No.: 113901

Lab Sample ID: 666630

Lab File ID: $\quad 666630$

Date Received: 04/20/06

Date Analyzed: 04/29/06

Dilution Factor: 1.0

Soil Aliquot Volume: $\quad 500$ (UU)

CONCENTRATION UNITS:

(ug/L or $\mathrm{ug} / \mathrm{Kg}$ ) UG/KG

Q 
FORM 1

VOIATILE ORGANICS ANALYSIS DATA SHEET

Lab Name: STL BURLINGTON

Contract: 21005

Lab Code: STLVT Case No.: NAVARRE SAS No.:

ARGLAB SAMPLE NO.

NA-MEOH BLANK
Matrix: (soil/water) SOIL

Sample wt/vol:

$10.0(\mathrm{~g} / \mathrm{mL}) \mathrm{G}$

Level: (low/med) MED

\% Moisture: not dec.

GC Column: CAP

Soil Extract Volume: 10000 (uL)
Lab Sample ID: 666630

Iab File ID: 666630

Date Received: 04/20/06

Date Analyzed: 04/29/06

Dilution Factor: 1.0

Soil Aliquot Volume:

500 (UL)

CAS NO.

COMPOUND

CONCENIRATION UNITS:

(ug/L or $\mathrm{ug} / \mathrm{Kg}$ ) UG/KG

$Q$

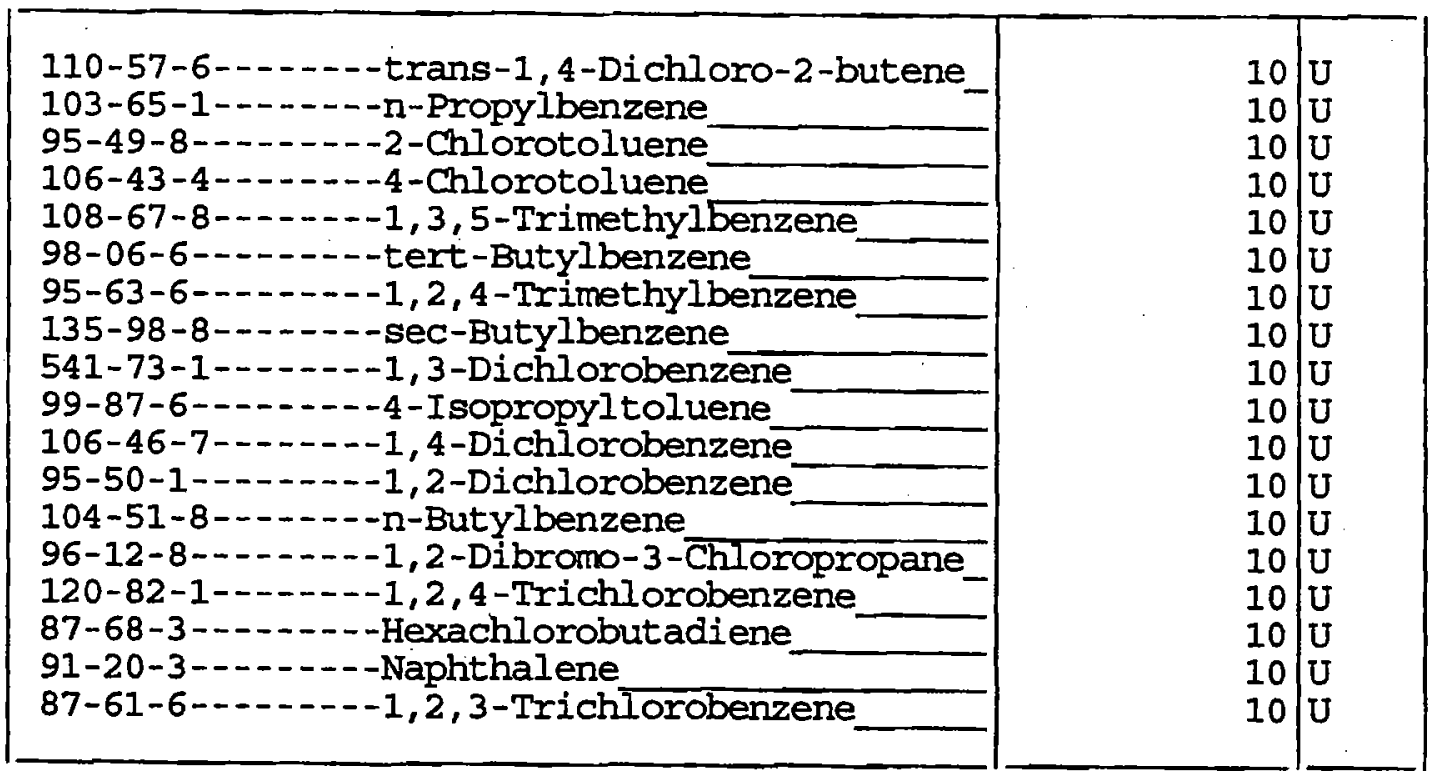


FORM 1

VOLATILE ORGANICS ANAIYSIS DATA SHEET

- Lab Name: STL BURLINGTON

Contract: 21005

Lab Code: STIJTT Case No.: NAVARRE SAS No.:

Lab Sample ID: 666629

Matrix: (soil/water) SOIL

Lab File ID: 666629

Sample wt/vol:

$12.0(\mathrm{~g} / \mathrm{mL}) \mathrm{G}$

Level: (low/med) MED

\& Moisture: not dec.

GC Column: CAP ID: 0.53 (mm)

Soil Extract Volume: 10000 (UU)
ARGLAB SAMPLE NO.

NA $-S-20295$

SDG No.: 113901
Date Received: 04/20/06

Date Analyzed: 04/29/06

Dilution Factor: 1.0

Soil Aliquot Volume: $\quad 500$ (uI)

CONCENTRATION UNITS:
(ug/L or $\mathrm{ug} / \mathrm{Kg}$ ) UG/KG

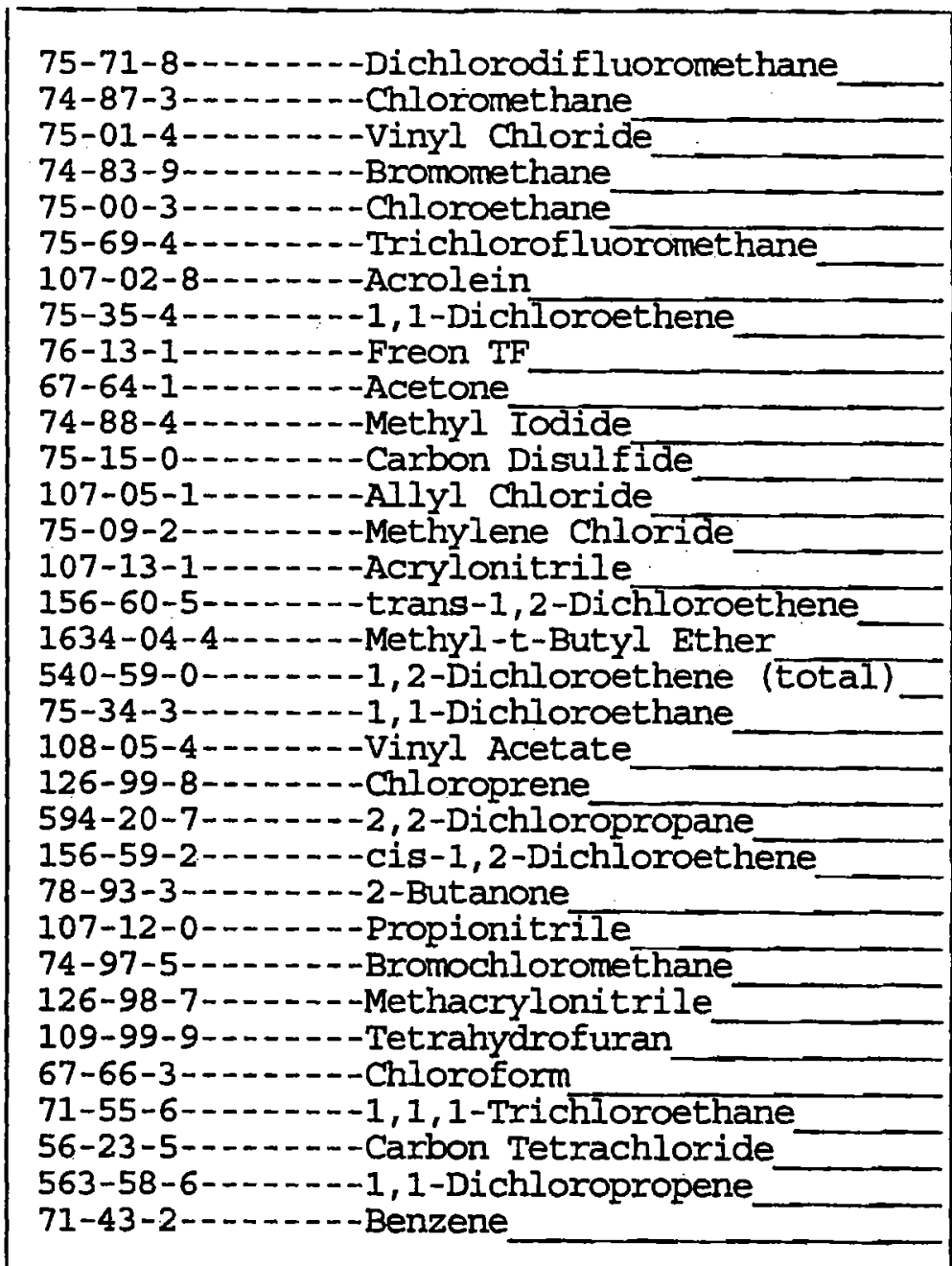

\begin{tabular}{|r|r|}
8.3 & $U$ \\
8.3 & $U$ \\
8.3 & $U$ \\
3.5 & $U$ \\
8.3 & $U$ \\
8.3 & $U$ \\
41 & $U$ \\
8.3 & $U$ \\
8.3 & $U$ \\
41 & $U$ \\
2.9 & $U$ \\
8.3 & $U$ \\
8.3 & $U$ \\
8.3 & $U$ \\
8.3 & $U$ \\
8.3 & $U$ \\
8.3 & $U$ \\
8.3 & $U$ \\
8.3 & $U$ \\
8.3 & $U$ \\
8.3 & $U$ \\
8.3 & $U$ \\
8.3 & $U$ \\
41 & $U$ \\
33 & $U$ \\
8.3 & $U$ \\
8.3 & $U$ \\
120 & $U$ \\
8.3 & $U$ \\
8.3 & $U$ \\
8.3 & $U$ \\
8.3 & $U$ \\
8.3 & $U$ \\
& \\
\hline
\end{tabular}


FORM 1

VOLATILE ORGANICS ANALYSIS DATA SHEET

Lab Name: STL BURLINGTON

Contract : 21005

Lab code: STLVT Case No.: NAVARRE SAS No.:
ARGLAB SAMPLE NO.

NA-S-20295
Matrix: (soil/water) SOIL

Sample wt/vol:

$12.0(\mathrm{~g} / \mathrm{mL}) \mathrm{G}$

Level: (low/med) MED

\& Moisture: not dec.

GC Column: CAP ID: 0.53 (mm)

Soil Extract Volume: 10000 (uf)
Lab Sample ID: 666629

Lab File ID: $\quad 666629$

Date Received: 04/20/06

Date Analyzed: 04/29/06

Dilution Factor: 1.0

Soil Aliquot volume: $\quad 500$ (uI)

CAS NO.

COMPOUND

CONCENIRATION UNITS:

(ug/L or ug/Kg) UG/KG

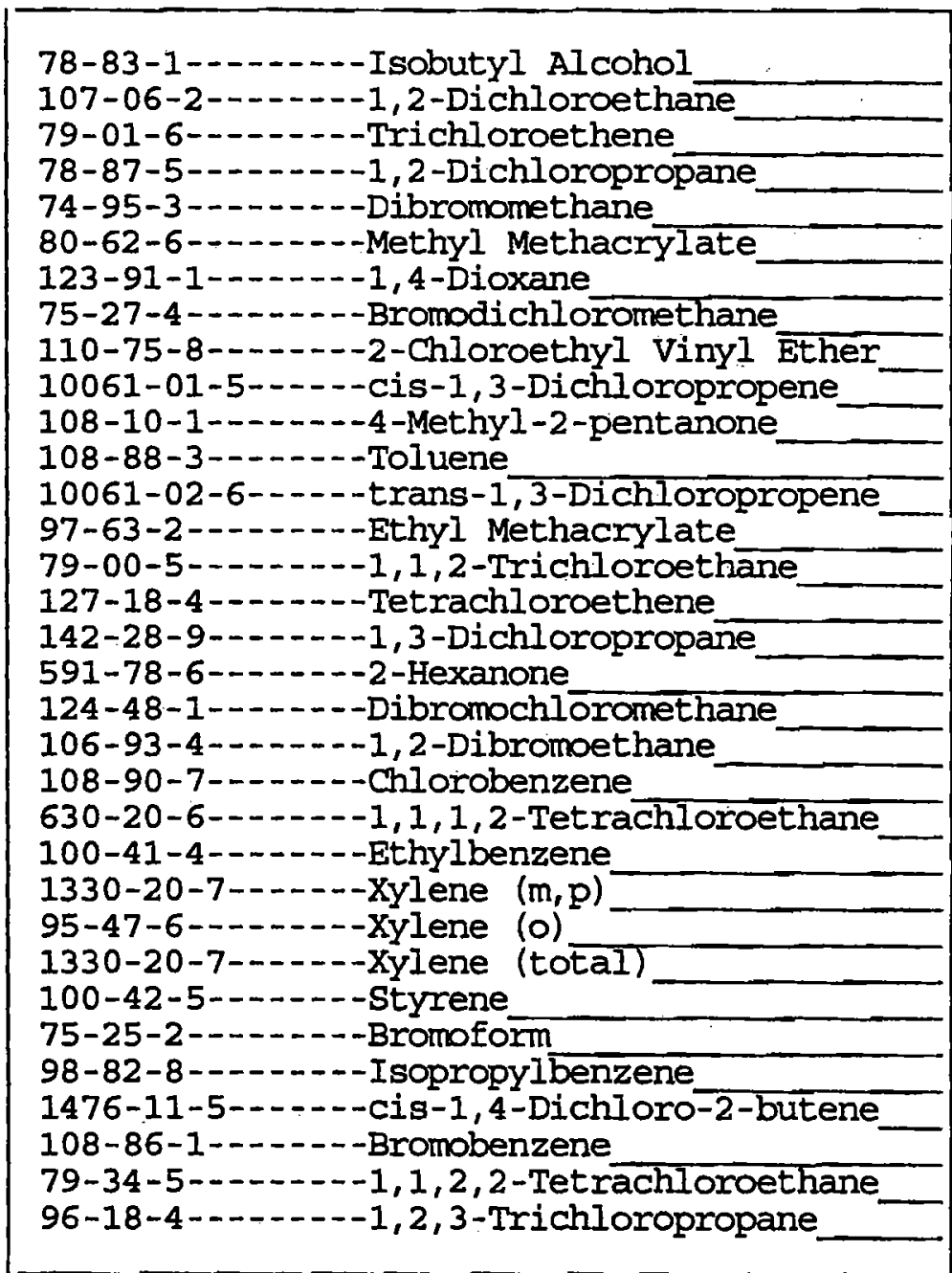

\begin{tabular}{|l|l|}
\hline 420 & $\mathrm{U}$ \\
8.3 & $\mathrm{U}$ \\
8.3 & $\mathrm{U}$ \\
8.3 & $\mathrm{U}$ \\
8.3 & $\mathrm{U}$ \\
8.3 & $\mathrm{U}$ \\
420 & $\mathrm{U}$ \\
8.3 & $\mathrm{U}$ \\
8.3 & $\mathrm{U}$ \\
8.3 & $\mathrm{U}$ \\
41 & $\mathrm{U}$ \\
8.3 & $\mathrm{U}$ \\
8.3 & $\mathrm{U}$ \\
8.3 & $\mathrm{U}$ \\
8.3 & $\mathrm{U}$ \\
8.3 & $\mathrm{U}$ \\
8.3 & $\mathrm{U}$ \\
41 & $\mathrm{U}$ \\
8.3 & $\mathrm{U}$ \\
8.3 & $\mathrm{U}$ \\
8.3 & $\mathrm{U}$ \\
8.3 & $\mathrm{U}$ \\
8.3 & $\mathrm{U}$ \\
8.3 & $\mathrm{U}$ \\
8.3 & $\mathrm{U}$ \\
8.3 & $\mathrm{U}$ \\
8.3 & $\mathrm{U}$ \\
8.3 & $\mathrm{U}$ \\
8.3 & $\mathrm{U}$ \\
8.3 & $\mathrm{U}$ \\
8.3 & $\mathrm{U}$ \\
8.3 & $\mathrm{U}$ \\
8.3 & $\mathrm{U}$ \\
& \\
\hline & \\
\hline
\end{tabular}


FORM 1

VOLATILE ORGANICS ANALYSIS DATA SHEET

Lab Name: STL BURLINGTON

Contract: 21005

Lab Code: STLVT Case No.: NAVARRE SAS No.:

Lab Sample ID: 666629

Sample wt/vol:

$12.0(\mathrm{~g} / \mathrm{mL}) \mathrm{G}$

Lab File ID: $\quad 666629$

Level: (low/med) MED

Date Received: 04/20/06

\% Moisture: not dec.

GC Colum: CAP

ID: 0.53 (mm)

Date Analyzed: 04/29/06

Dilution Factor: 1.0

Soil Extract Volume: 10000 (uL)

Soil Aliquot Volume:

500 (UL)

CONCENTRATION UNITS:

CAS NO.

COMPOUND

$Q$
$8.3 \mathrm{U}$

$8.3 \mathrm{U}$

$8.3 \mathrm{U}$

$8.3 \mathrm{U}$

$8.3 \mathrm{U}$

$8.3 \mathrm{U}$

$8.3 \mathrm{U}$

$8.3 \mathrm{U}$

$8.3 \mathrm{U}$

$8.3 \mathrm{U}$

$8.3 \mathrm{U}$

$8.3 \mathrm{U}$

$8.3 \mathrm{U}$

$8.3 \mathrm{U}$

$8.3 \mathrm{U}$

$8.3 \mathrm{U}$

$8.3 \mathrm{U}$

$8.3 \mathrm{U}$ 
FORM 1

VOLATILE ORGANICS ANALYSIS DATA SHEET

Lab Name: STL BURIINGTON

Contract : 21005

Lab Code: STLVT Case No.: NAVARRE SAS No.:

Lab Sample ID: 666624

Matrix: (soil/water) SOIL

Lab File ID: $\quad 666624$

Sample wt/vol:

$11.0(\mathrm{~g} / \mathrm{mL}) \mathrm{G}$

Level: (low/med) MED

Date Received: 04/20/06

\% Moisture: not dec.

GC Column: CAP

ID: $0.53 \quad(\mathrm{~mm})$

Soil Extract Volume: 10000 (UL)

CAS NO.

COMPOUND CONCENTRATION UNITS: (ug/L or $\mathrm{ug} / \mathrm{Kg}$ ) UG/KG

Q
ARGLAB SAMPLE NO.

NA-S-20303

SDG No.: 113901
Date Analyzed: 04/29/06

Dilution Factor: 1.0

Soil Aliquot Volume:

500 (uL)
$9.1 \mathrm{U}$

$2.0 \mathrm{~J}$

$9.1 \mathrm{U}$

$5.2 \mathrm{~J}$

$9.1 \mathrm{U}$

$9.1 \mathrm{U}$

$45 \mathrm{U}$

$9.1 \mathrm{U}$

$9.1 \mathrm{U}$

$45 \mathrm{U}$

$4.7 \mathrm{~J}$

$9.1 \mathrm{U}$

$9.1 \mathrm{U}$

$9.1 \mathrm{U}$

$9.1 \mathrm{U}$

$9.1 \mathrm{U}$

$9.1 \mathrm{U}$

$9.1 \mathrm{U}$

$9.1 \mathrm{U}$

9.1 U

$9.1 \mathrm{U}$

$9.1 \mathrm{U}$

$9.1 \mathrm{U}$

$25 \mathrm{~J}$

$36 \mathrm{U}$

$9.1 \mathrm{U}$

$9.1 \mathrm{U}$

$130 \mathrm{U}$

$9.1 \mathrm{U}$

$9.1 \mathrm{U}$

$9.1 \mathrm{U}$

$9.1 \mathrm{U}$

$9.1 \mathrm{U}$ 
FORM 1

VOLATILE ORGANICS ANALYSIS DATA SHEET

Lab Name: STL BURLINGTON

Contract: 21005

Lab code: STLVT Case No.: NAVARRE SAS No.:

Lab Sample ID: 666624

Matrix: (soil/water) SOIL

Lab File ID: $\quad 666624$

Sample wt/vol: $\quad 11.0(\mathrm{~g} / \mathrm{mL}) \mathrm{G}$

Level: (low/med) MED

\% Moisture: not dec.

GC Column: CAP

ID: $0.53(\mathrm{~mm})$

Soil Extract Volume: 10000 (U山)

Date Received: 04/20/06

Date Analyzed: 04/29/06

Dilution Factor: 1.0
ARGLAB SAMPLE NO.

NA-S-20303

Soil Aliquot Volume: $\quad 500$ (u山)

CONCENTRATION UNITS:

CAS NO. COMPOUND $(u g / L$ or $u g / K g) ~ U G / K G \quad Q$

\begin{tabular}{|c|c|c|}
\hline 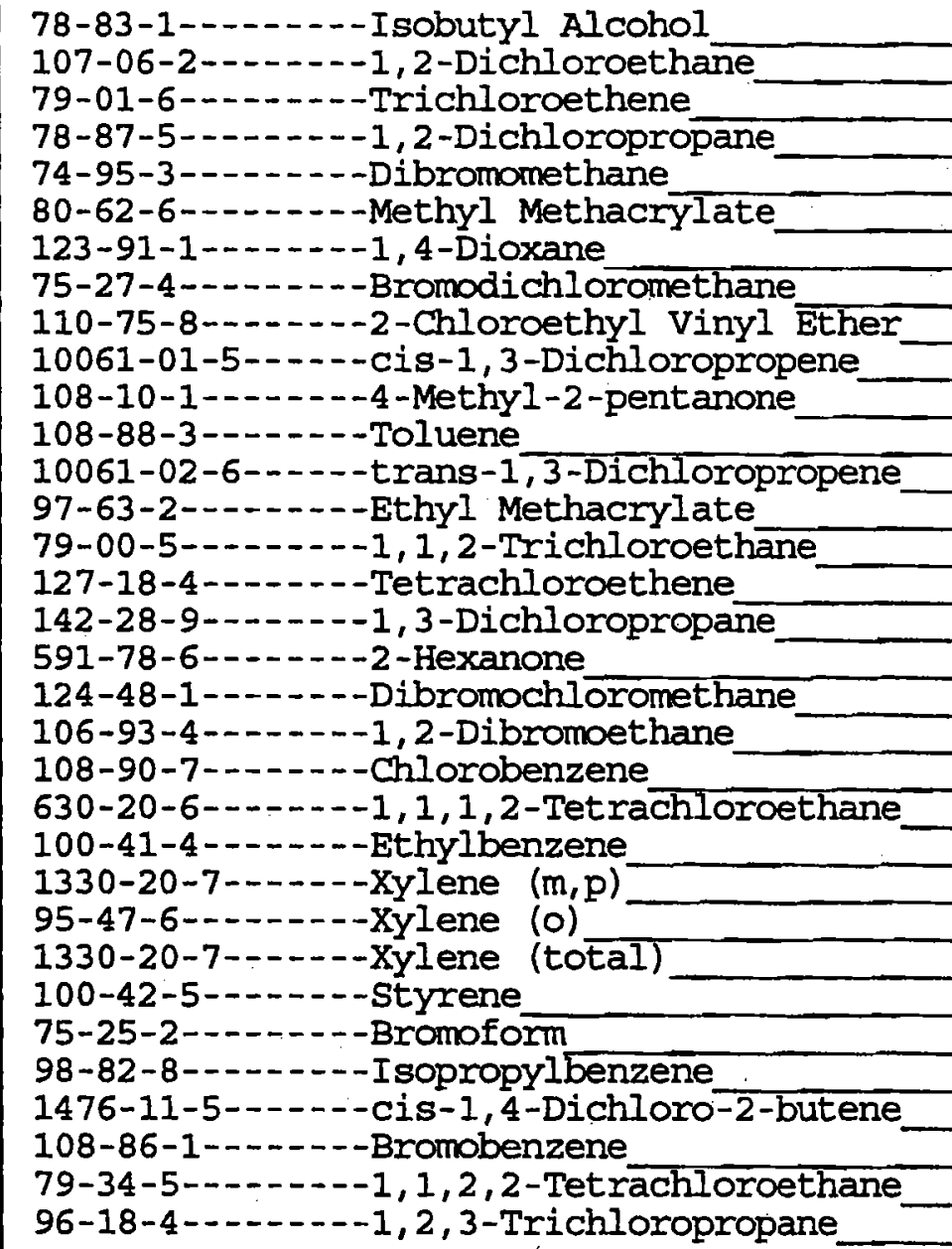 & $\begin{array}{l}450 \\
9.1 \\
9.1 \\
9.1 \\
9.1 \\
9.1 \\
450 \\
9.1 \\
9.1 \\
9.1 \\
45 \\
9.1 \\
9.1 \\
9.1 \\
9.1 \\
9.1 \\
9.1 \\
45 \\
9.1 \\
9.1 \\
9.1 \\
9.1 \\
9.1 \\
9.1 \\
9.1 \\
9.1 \\
9.1 \\
9.1 \\
9.1 \\
9.1 \\
9.1 \\
9.1 \\
9.1\end{array}$ & $\begin{array}{l}U \\
U \\
U \\
U \\
U \\
U \\
U \\
U \\
U \\
U \\
U \\
U \\
U \\
U \\
U \\
U \\
U \\
U \\
U \\
U \\
U \\
U \\
U \\
U \\
U \\
U \\
U \\
U \\
U \\
U \\
U \\
U \\
U\end{array}$ \\
\hline
\end{tabular}


FORM 1

VOLATILE ORGANICS ANALYSIS DATA SHEET

Lab Name: STL BURLINGTON

Contract: 21005

Lab code: STLVT Case No.: NAVARRE SAS No.:

Matrix: (soil/water) SOIL

Sample wt/vol:

$11.0(\mathrm{~g} / \mathrm{mL}) \mathrm{G}$

Level: (low/med) MED

\%oisture: not dec.

GC Column: CAP ID: 0.53 (mm)

Soil Extract Volume: 10000 (uL)

CAS NO.

COMPOUND
ARGLAB SAMPLE NO.

NA-S-20303

SDG No.: 113901
Lab Sample ID: 666624

Lab File ID: 666624

Date Received: 04/20/06

Date Analyzed: 04/29/06

Dilution Factor: 1.0

Soil Aliquot Volume:

500 (UL)

CONCENTRATION UNITS:

(ug/L or $\mathrm{ug} / \mathrm{Kg}$ ) UG/KG

Q

$9.1 \mathrm{U}$

$9.1 \mathrm{U}$

$9.1 \mathrm{U}$

$9.1 \mathrm{U}$

$9.1 \mathrm{U}$

$9.1 \mathrm{U}$

$9.1 \mathrm{U}$

$9.1 \mathrm{U}$

$9.1 \mathrm{U}$

$9.1 \mathrm{U}$

$9.1 \mathrm{U}$

$9.1 \mathrm{U}$

$9.1 \mathrm{U}$

$9.1 \mathrm{U}$

$9.1 \mathrm{U}$

$9.1 \mathrm{U}$

$9.1 \mathrm{U}$

$9.1 \mathrm{U}$ 
FORM 1

VOLATIIE ORGANICS ANALYSIS DATA SHEET

Lab Name: STL BURLINGTON

Contract: 21005

Lab code: STLVT Case No.: NAVARRE SAS No.:

Lab Sample ID: 666620

Matrix: (soil/water) SOIL

Lab File ID: $\quad 666620$

Sample wt/vol:

$11.0(\mathrm{~g} / \mathrm{mL}) \mathrm{G}$

Level: (low/med) MED

Date Received: 04/20/06

q Moisture: not dec.

GC Column: CAP

ID: $0.53 \quad(\mathrm{~mm})$

Soil Extract Volume: 10000 (ut)

CAS NO.

COMPOUND

Q

CONCENTRATION UNITS:

(ug/L or $u g / \mathrm{Kg}$ ) UG/KG
ARGLAB SAMPLE NO.

NA-S-20308

SDG No.: 113901
Date Analyzed: 04/29/06

Dilution Factor: 1.0

Soil Aliquot Volume:

500 (UT)

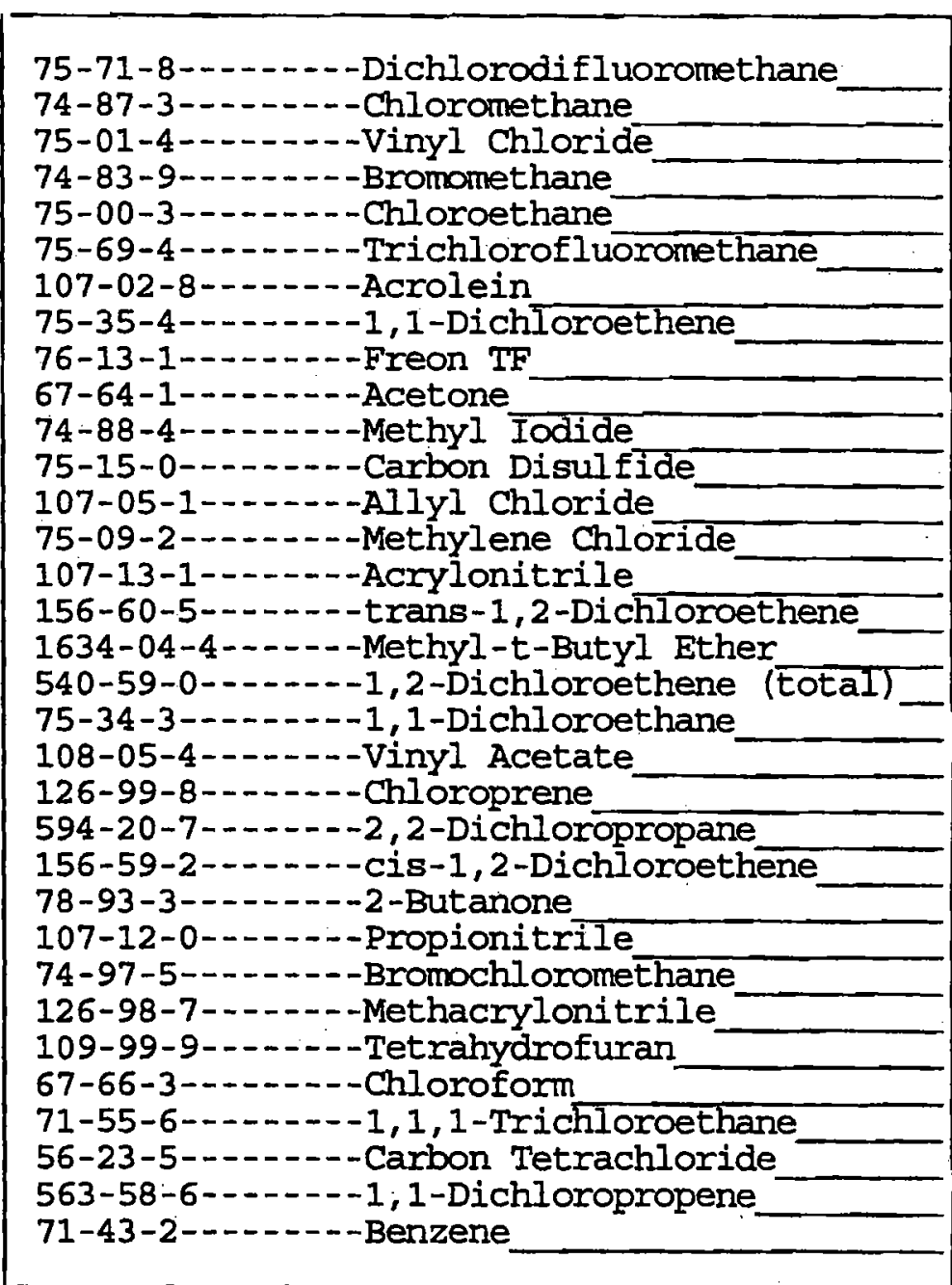

\begin{tabular}{l|l}
9.1 & $\mathrm{U}$ \\
3.1 & $\mathrm{~J}$ \\
9.1 & $\mathrm{U}$ \\
9.1 & $\mathrm{U}$ \\
9.1 & $\mathrm{U}$ \\
9.1 & $\mathrm{U}$ \\
45 & $\mathrm{U}$ \\
9.1 & $\mathrm{U}$ \\
9.1 & $\mathrm{U}$ \\
45 & $\mathrm{U}$ \\
9.4 & \\
9.1 & $\mathrm{U}$ \\
9.1 & $\mathrm{U}$ \\
9.1 & $\mathrm{U}$ \\
9.1 & $\mathrm{U}$ \\
9.1 & $\mathrm{U}$ \\
9.1 & $\mathrm{U}$ \\
9.1 & $\mathrm{U}$ \\
9.1 & $\mathrm{U}$ \\
9.1 & $\mathrm{U}$ \\
9.1 & $\mathrm{U}$ \\
9.1 & $\mathrm{U}$ \\
9.1 & $\mathrm{U}$ \\
40 & $\mathrm{~J}$ \\
36 & $\mathrm{U}$ \\
9.1 & $\mathrm{U}$ \\
9.1 & $\mathrm{U}$ \\
130 & $\mathrm{U}$ \\
9.1 & $\mathrm{U}$ \\
9.1 & $\mathrm{U}$ \\
9.1 & $\mathrm{U}$ \\
9.1 & $\mathrm{U}$ \\
9.1 & $\mathrm{U}$ \\
\hline & \\
\hline
\end{tabular}


FORM 1

VOLATIIE ORGANICS ANALYSIS DATA SHEET

Lab Name: STL BURLINGTON

Contract: 21005

Lab Code: SILVT Case No.: NAVARRE SAS No.:

Lab Sample ID: 666620

Matrix: (soil/water) soIL

Sample wt/vol:

$11.0(\mathrm{~g} / \mathrm{mL}) \mathrm{G}$

Level : (low/med) MED

\& Moisture: not dec.

GC Column: CAP ID: 0.53 (mm)

Soil Extract Volume: 10000 (UL)

Lab File ID: $\quad 666620$

Date Received: 04/20/06

Date Analyzed: 04/29/06

Dilution Factor: 1.0

Soil Aliquot Volume:

COMPOUND

500 (UL)

CAS NO.

CONCENTRATION UNITS:

(ug/L or $u g / K g) ~ U G / K G$

Q

\begin{tabular}{|c|c|c|}
\hline 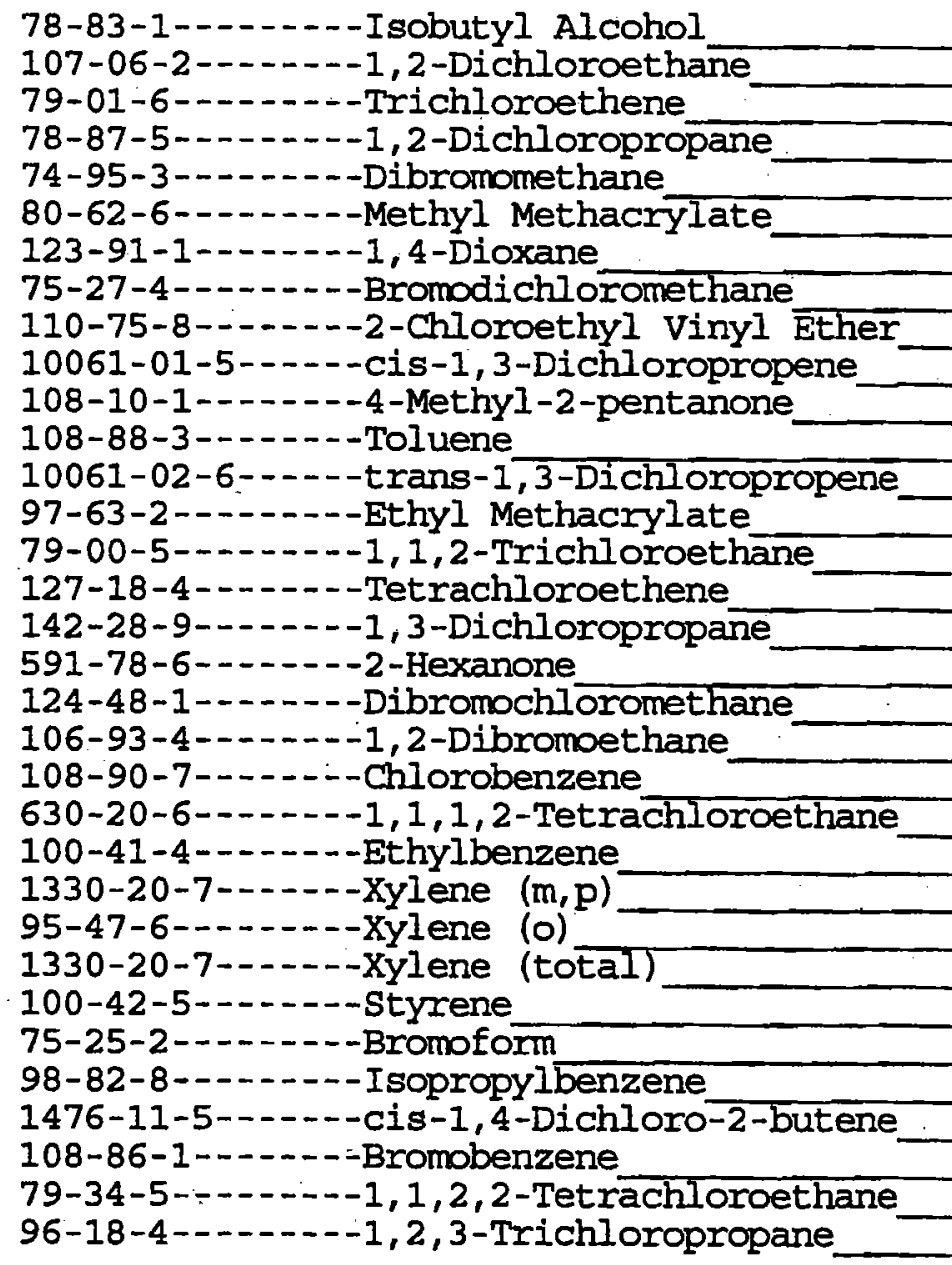 & $\begin{array}{l}460 \\
9.1 \\
9.1 \\
9.1 \\
9.1 \\
9.1 \\
460 \\
9.1 \\
9.1 \\
9.1 \\
45 \\
9.1 \\
9.1 \\
9.1 \\
9.1 \\
9.1 \\
9.1 \\
45 \\
9.1 \\
9.1 \\
9.1 \\
9.1 \\
9.1 \\
9.1 \\
9.1 \\
9.1 \\
9.1 \\
9.1 \\
9.1 \\
9.1 \\
9.1 \\
9.1 \\
9.1\end{array}$ & $\begin{array}{l}U \\
U \\
U \\
U \\
U \\
U \\
U \\
U \\
U \\
U \\
U \\
U \\
U \\
U \\
U \\
U \\
U \\
U \\
U \\
U \\
U \\
U \\
U \\
U \\
U \\
U \\
U \\
U \\
U \\
U \\
U \\
U \\
U \\
U \\
U \\
U \\
U \\
U \\
U\end{array}$ \\
\hline
\end{tabular}


FORM 1

VOLATILE ORGANICS ANALYSIS DATA SHEET

Lab Name: STL BURLINGTON

Contract: 21005
ARGLAB SAMPLE NO.

NA-S -20308

Lab code: STLVT Case No.: NAVARRE SAS No.:

SDG NO.: 113901

Matrix: (soil/water) SOIL

Sample wt/vol:

$11.0(\mathrm{~g} / \mathrm{mL}) \mathrm{G}$

Level: (low/med) MED

\% Moisture: not dec.

GC Column: CAP

ID: $0.53(\mathrm{~mm})$

Soil Extract Volume: 10000 (u)
Iab Sample ID: 666620

Lab File ID: $\quad 666620$

Date Received: 04/20/06

Date Analyzed: 04/29/06

Dilution Factor: 1.0

Soil Aliquot Volume:

500 (uL)

CAS NO.

COMPOUND

CONCENTRATION UNITS:

(ug/L or $u g / K g$ ) UG/KG

Q

$9.1 \mathrm{U}$

$9.1 \mathrm{U}$

$9.1 \mathrm{U}$

$9.1 \mathrm{U}$

$9.1 \mathrm{U}$

$9.1 \mathrm{U}$

$9.1 \mathrm{U}$

$9.1 \mathrm{U}$

$9.1 \mathrm{U}$

$9.1 \mathrm{U}$

$9.1 \mathrm{U}$

$9.1 \mathrm{U}$

$9.1 \mathrm{U}$

$9.1 \mathrm{U}$

$9.1 \mathrm{U}$

$9.1 \mathrm{U}$

$9.1 \mathrm{U}$

9.1 U 
FORM 1

VOLATILE ORGANICS ANALYSIS DATA SHEET

Lab Name: STL BURLINGTON

Contract : 21005

Lab Code: STUVT Case No.: NAVARRE SAS No.:

Lab Sample ID: 666625

Matrix: (soil/water) SOIL

Lab File ID: $\quad 666625$

Sample wt/vol: $\quad 11.7(\mathrm{~g} / \mathrm{mL}) \mathrm{G}$

Date Received: 04/20/06

Level: (low/med) MED

Date Analyzed: 04/29/06

GC Column: CAP

ID: $0.53(\mathrm{~mm})$

Dilution Factor: 1.0

Soil Extract Volume: 10000 (uL)

500 (uL)

CONCENTRATION UNITS:

CAS NO.

COMPOUND

(ug/L or $\mathrm{ug} / \mathrm{Kg}$ ) UG/KG

Q

\begin{tabular}{|c|c|c|}
\hline 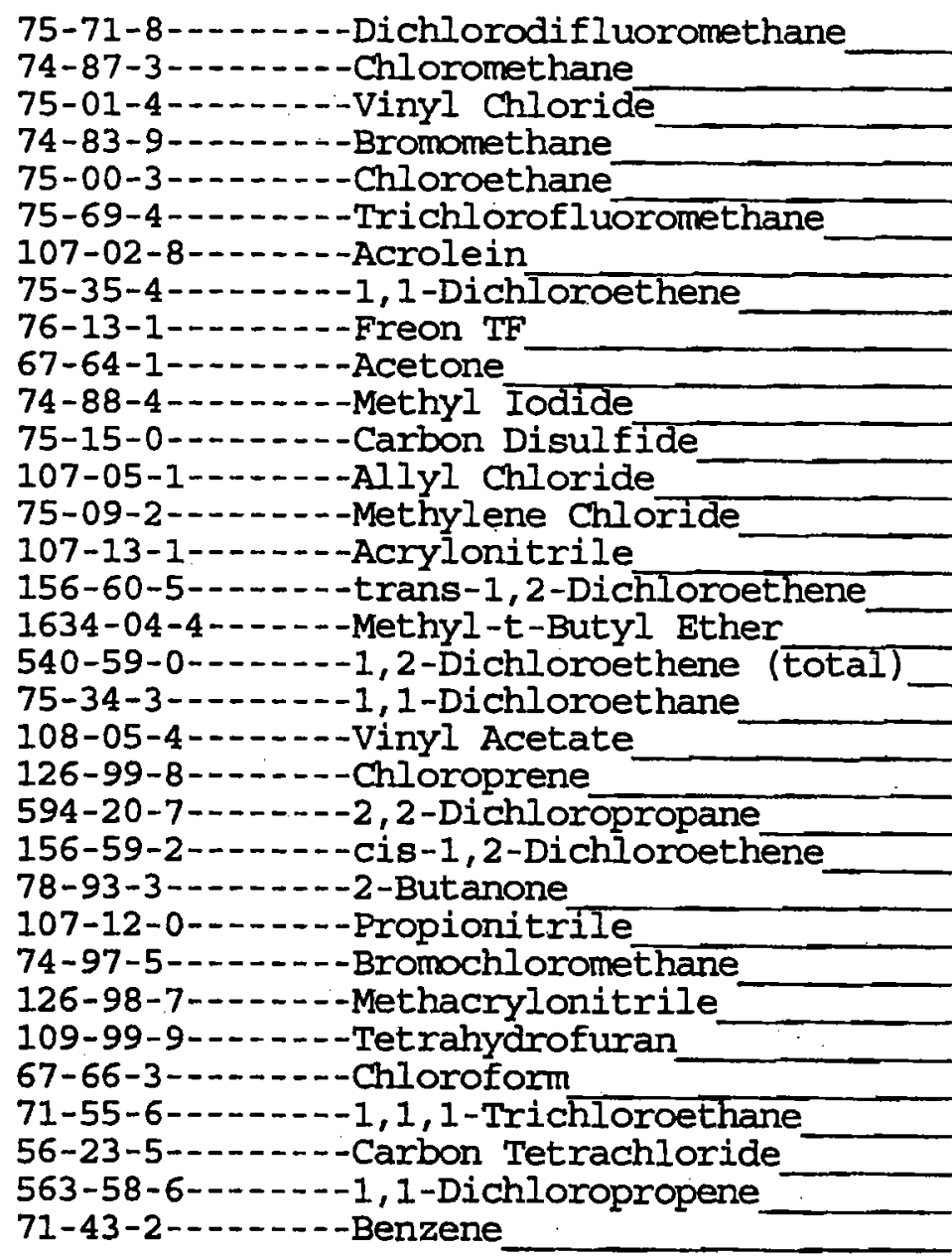 & $\begin{array}{r}8.5 \\
8.5 \\
8.5 \\
6.7 \\
8.5 \\
8.5 \\
42 \\
8.5 \\
8.5 \\
42 \\
5.1 \\
8.5 \\
8.5 \\
8.5 \\
8.5 \\
8.5 \\
8.5 \\
8.5 \\
8.5 \\
8.5 \\
8.5 \\
8.5 \\
8.5 \\
42 \\
34 \\
8.5 \\
8.5 \\
120 \\
8.5 \\
8.5 \\
8.5 \\
8.5 \\
8.5\end{array}$ & $\begin{array}{l}U \\
U \\
U \\
J \\
U \\
U \\
U \\
U \\
U \\
U \\
U \\
U \\
U \\
U \\
U \\
U \\
U \\
U \\
U \\
U \\
U \\
U \\
U \\
U \\
U \\
U \\
U \\
U \\
U \\
U \\
U \\
U \\
U\end{array}$ \\
\hline
\end{tabular}


FORM 1

VOLATILE ORGANICS ANALYSIS DATA SHEET

Lab Name: STL BURLINGTON

Contract: 21005

Lab code: STLVT Case No.: NAVARRE SAS No.:
Matrix: (soil/water) SOIL

Sample wt/vol:

$11.7(\mathrm{~g} / \mathrm{mL}) \mathrm{G}$

Level : (low/med) MED

\% Moisture: not dec.

GC Colunn: CAP ID: 0.53 (mm)

Soil Extract Volume: 10000 (uL)
ARGLAB SAMPLE NO.

NA-S-20311

SDG No.: 113901

Lab Sample ID: 666625

Lab File ID: $\quad 666625$

Date Received: 04/20/06

Date Analyzed: 04/29/06

Dilution Factor: 1.0

Soil Aliquot volume:

500 (U)

CONCENTRATION UNITS:

CAS NO. COMPOUND $\quad(u g / L$ or $u g / \mathrm{Kg})$ UG/KG

Q

\begin{tabular}{|c|c|c|}
\hline 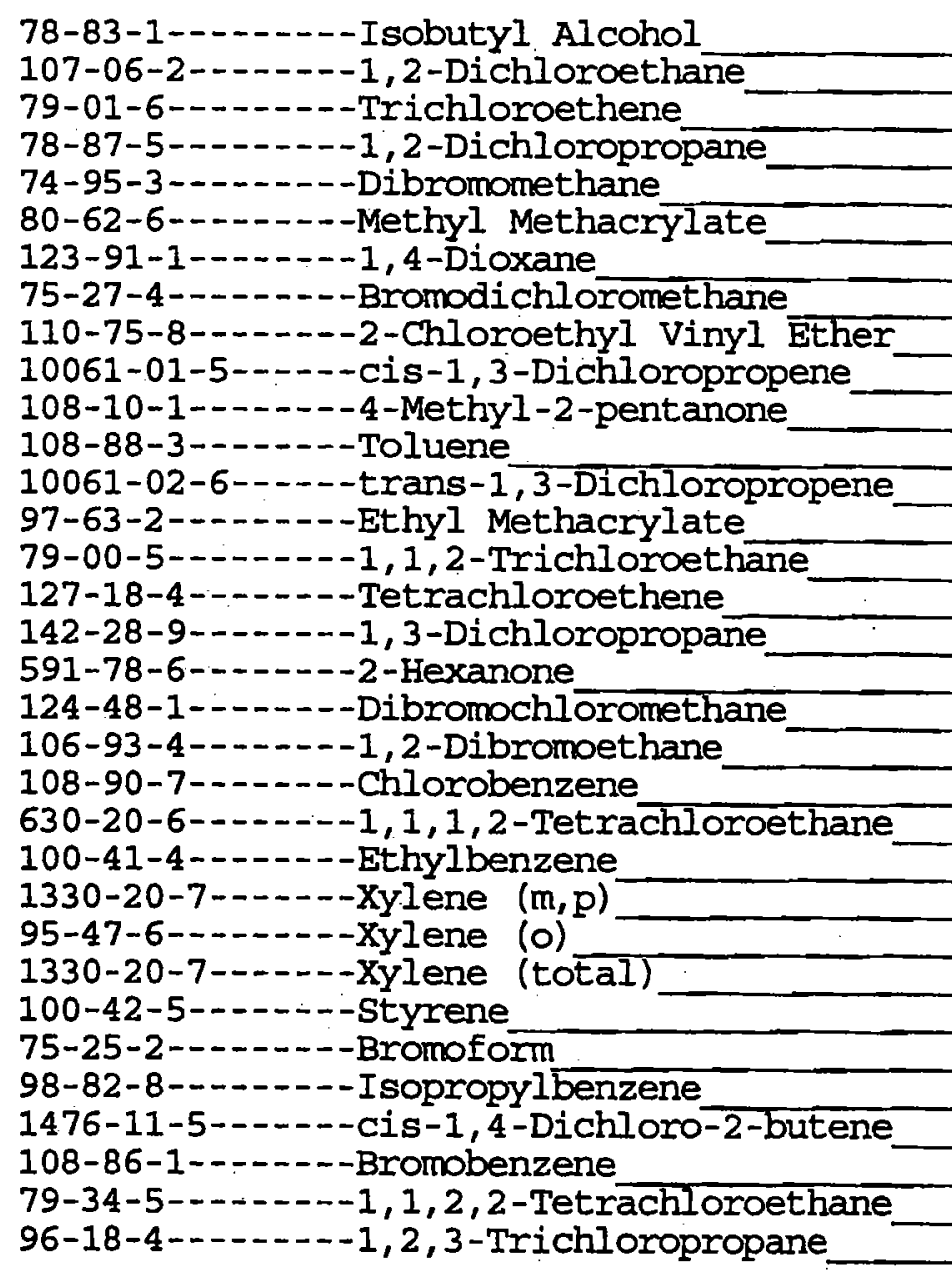 & $\begin{array}{r}430 \\
8.5 \\
8.5 \\
8.5 \\
8.5 \\
8.5 \\
430 \\
8.5 \\
8.5 \\
8.5 \\
422 \\
8.5 \\
8.5 \\
8.5 \\
8.5 \\
8.5 \\
8.5 \\
42 \\
8.5 \\
8.5 \\
8.5 \\
8.5 \\
8.5 \\
8.5 \\
8.5 \\
8.5 \\
8.5 \\
8.5 \\
8.5 \\
8.5 \\
8.5 \\
8.5 \\
8.5\end{array}$ & \begin{tabular}{|l}
$U$ \\
$U$ \\
$U$ \\
$U$ \\
$U$ \\
$U$ \\
$U$ \\
$U$ \\
$U$ \\
$U$ \\
$U$ \\
$U$ \\
$U$ \\
$U$ \\
$U$ \\
$U$ \\
$U$ \\
$U$ \\
$U$ \\
$U$ \\
$U$ \\
$U$ \\
$U$ \\
$U$ \\
$U$ \\
$U$ \\
$U$ \\
$U$ \\
$U$ \\
$U$ \\
$U$ \\
$U$ \\
$U$
\end{tabular} \\
\hline
\end{tabular}


FORM 1

VOLATILE ORGANICS ANALYSIS DATA SHEET

Lab Name: STL BURLINGTON

Contract: 21005

Lab Code: STLVT Case No.: NAVARRE SAS No.:

Lab Sample ID: 666625

Matrix: (soil/water) soIL

$11.7(\mathrm{~g} / \mathrm{mL}) \mathrm{G}$

Lab File ID: 666625

Level: (low/med) MED

\& Moisture: not dec.

GC Column: CAP ID: 0.53 (mm)

Soil Extract Volume: 10000 (W)

CAS NO.
ARGLAB SAMPLE NO.

NA-S-20311

SDG No.: 113901

\begin{tabular}{|c|c|c|}
\hline 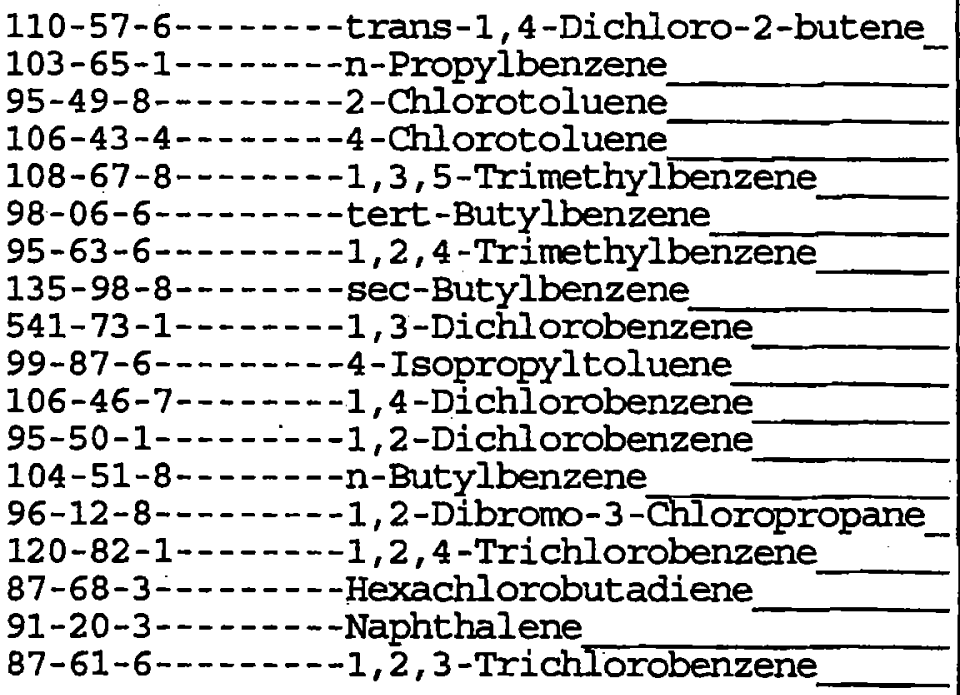 & $\begin{array}{l}8.5 \\
8.5 \\
8.5 \\
8.5 \\
8.5 \\
8.5 \\
8.5 \\
8.5 \\
8.5 \\
8.5 \\
8.5 \\
8.5 \\
8.5 \\
8.5 \\
8.5 \\
8.5 \\
8.5 \\
8.5\end{array}$ & $\begin{array}{l}U \\
U \\
U \\
U \\
U \\
U \\
U \\
U \\
U \\
U \\
U \\
U \\
U \\
U \\
U \\
U \\
U \\
U\end{array}$ \\
\hline
\end{tabular}


FORM 1

VOLATILE ORGANICS ANALYSIS DATA SHEET

Lab Name: STL BURIJINGTON

Contract : 21005

Lab code: STLVT Case No.: NAVARRE SAS No.:

Lab Sample ID: 666627

Matrix: (soil/water) sOIL

Lab File ID: $\quad 666627$

Sample wt/vol: $\quad 11.8(\mathrm{~g} / \mathrm{mL}) \mathrm{G}$

Date Received: 04/20/06

Level: (low/med) MED

Date Analyzed: 04/29/06

\% Moisture: not dec.

GC Column: CAP

ID : $0.53(\mathrm{~mm})$

Soil Extract Volume: 10000 (u)
ARGLAB SAMPLE NO.

$N A-S-20330$

SDG No.: 113901
Dilution Factor: 1.0

Soil Aliquot Volume:

500 (ut)

CAS NO.

COMPOUND

CONCENTRATION UNITS:

(ug/L or $u g / K g$ ) UG/KG

Q

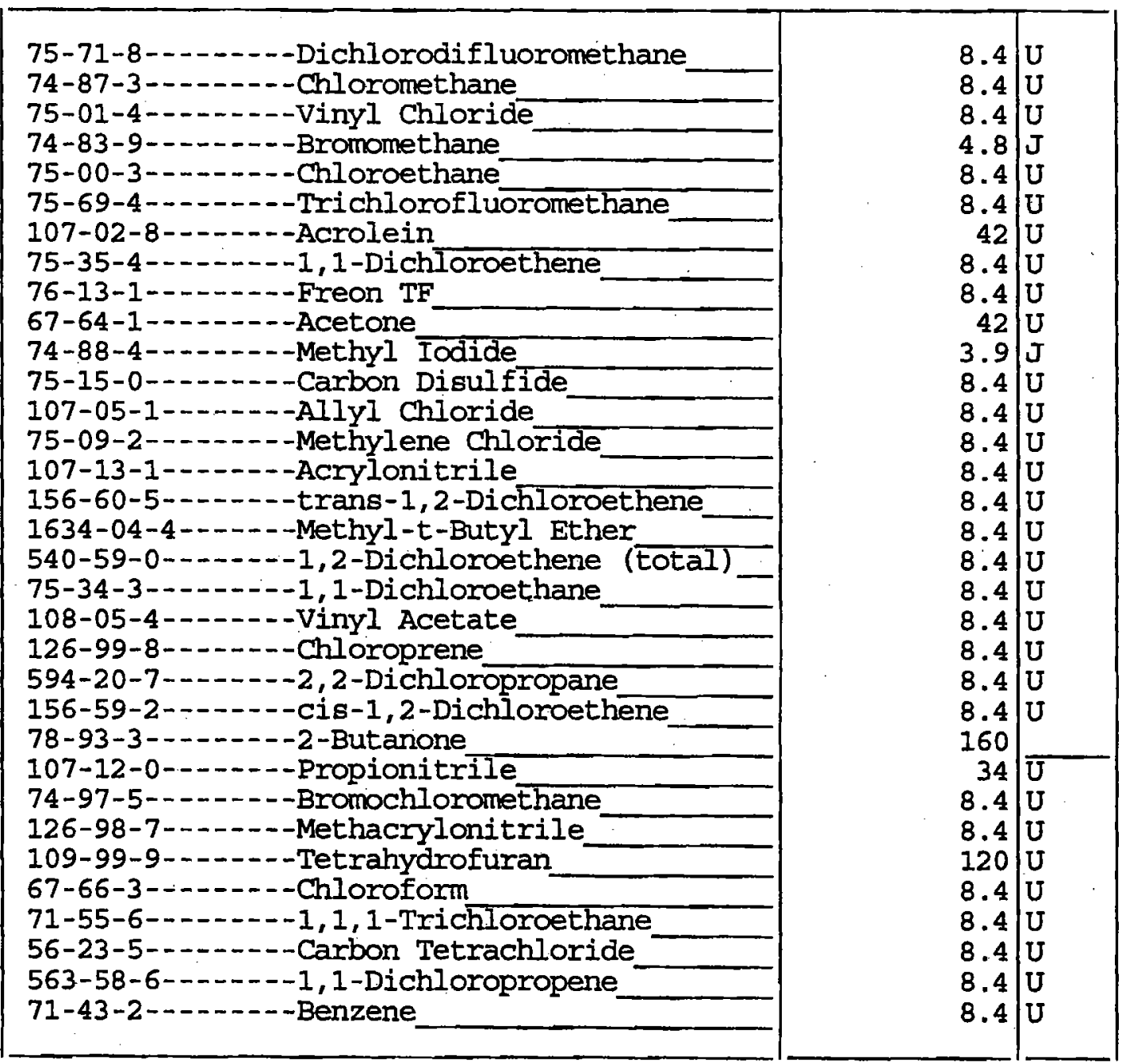


FORM 1

VOLATILE ORGANICS ANALYSIS DATA SHEET

Lab Name: STL BURLINGTON

Contract: 21005
ARGLAB SAMPLE NO.

NA-S -20330

Lab Code: STLVT Case No.: NAVARRE SAS No.:

SDG No.: 113901

Matrix: (soil/water) SOIL

Sample wt/vol: $\quad 11.8(\mathrm{~g} / \mathrm{mL}) \mathrm{G}$

Level: (low/med) MED

\& Moisture: not dec.

GC Colunn: CAP

ID: $0.53 \quad(\mathrm{~mm})$

Soil Extract Volume: 10000 (uL)
Lab Sample ID: 666627

Lab File ID: $\quad 666627$

Date Received: 04/20/06

Date Analyzed: 04/29/06

Dilution Factor: 1.0

Soil Aliquot Volume:

500 (UL)

CAS NO.

COMPOUND

CONCENTRATION UNITS:

(ug/L or $\mathrm{ug} / \mathrm{Kg}$ ) UG/KG

Q

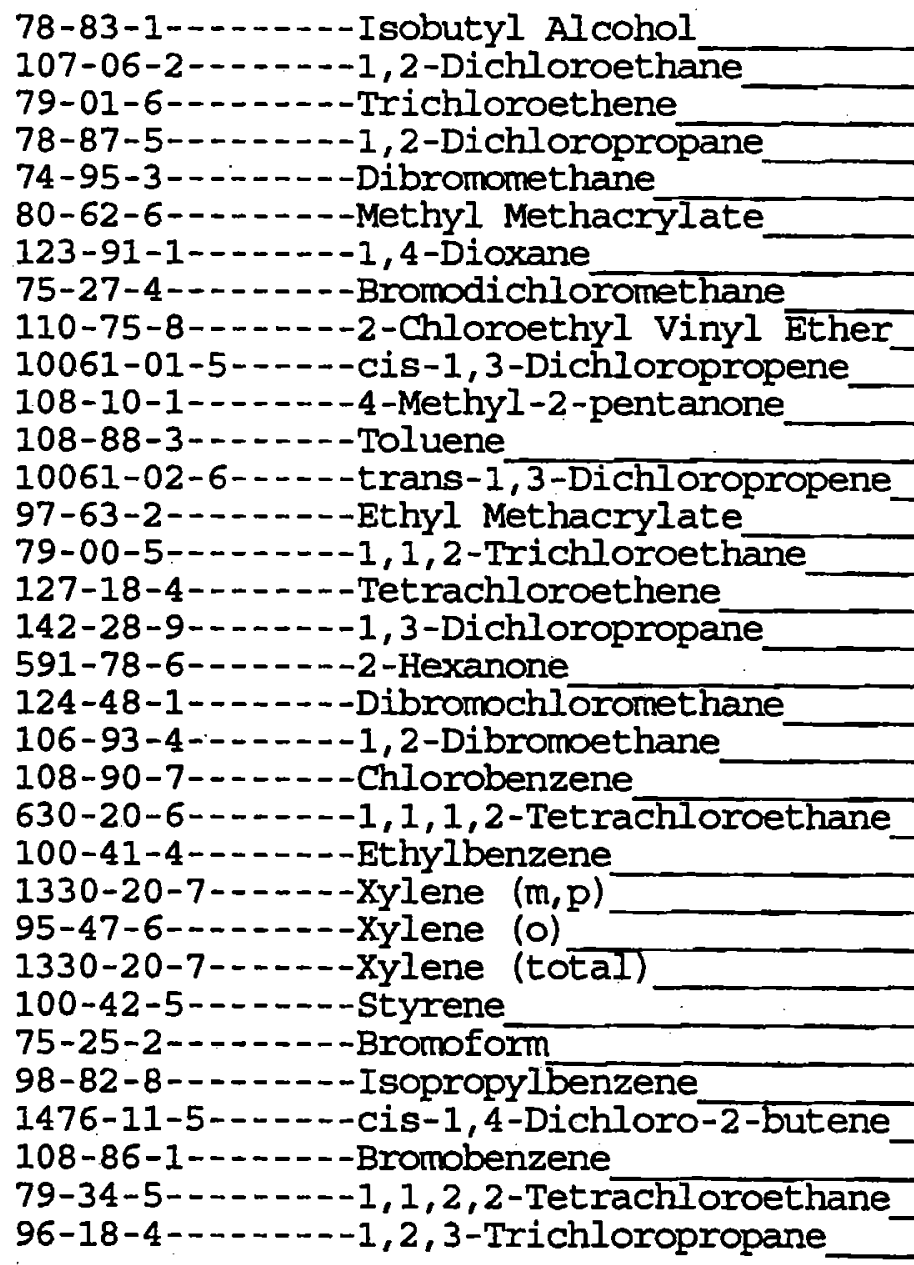

\begin{tabular}{l|l|}
420 & $\mathrm{U}$ \\
8.4 & $\mathrm{U}$ \\
8.4 & $\mathrm{U}$ \\
8.4 & $\mathrm{U}$ \\
8.4 & $\mathrm{U}$ \\
8.4 & $\mathrm{U}$ \\
420 & $\mathrm{U}$ \\
8.4 & $\mathrm{U}$ \\
8.4 & $\mathrm{U}$ \\
8.4 & $\mathrm{U}$ \\
42 & $\mathrm{U}$ \\
8.4 & $\mathrm{U}$ \\
8.4 & $\mathrm{U}$ \\
8.4 & $\mathrm{U}$ \\
8.4 & $\mathrm{U}$ \\
8.4 & $\mathrm{U}$ \\
8.4 & $\mathrm{U}$ \\
42 & $\mathrm{U}$ \\
8.4 & $\mathrm{U}$ \\
8.4 & $\mathrm{U}$ \\
8.4 & $\mathrm{U}$ \\
8.4 & $\mathrm{U}$ \\
8.4 & $\mathrm{U}$ \\
2.2 & $\mathrm{~J}$ \\
8.4 & $\mathrm{U}$ \\
2.3 & $\mathrm{~J}$ \\
8.4 & $\mathrm{U}$ \\
8.4 & $\mathrm{U}$ \\
8.4 & $\mathrm{U}$ \\
8.4 & $\mathrm{U}$ \\
8.4 & $\mathrm{U}$ \\
8.4 & $\mathrm{U}$ \\
8.4 & $\mathrm{U}$ \\
\hline & \\
\hline &
\end{tabular}


FORM 1

VOLATILE ORGANICS ANALYSIS DATA SHEET

Lab Name: STL BURLINGTON

Contract : 21005

Lab Code: STLVT Case No.: NAVARRE SAS No.:

ARGLAB SAMPLE NO.

Matrix: (soil/water) SOIL

Lab Sample ID: 666627

Sample wt/vol:

$11.8(\mathrm{~g} / \mathrm{mL}) \mathrm{G}$

Lab File ID: $\quad 666627$

Level : (low/med) MED

Date Received: 04/20/06

\& Moisture: not dec.

GC Column: CAP ID: 0.53 (mm)

Date Analyzed: 04/29/06

Soil Extract Volume: 10000 (uس)

Dilution Factor: 1.0

Soil Aliquot Volume:

500 (U工)

CONCENTRATION UNITS:

CAS NO.

COMPOUND

Q

\begin{tabular}{|c|c|c|}
\hline 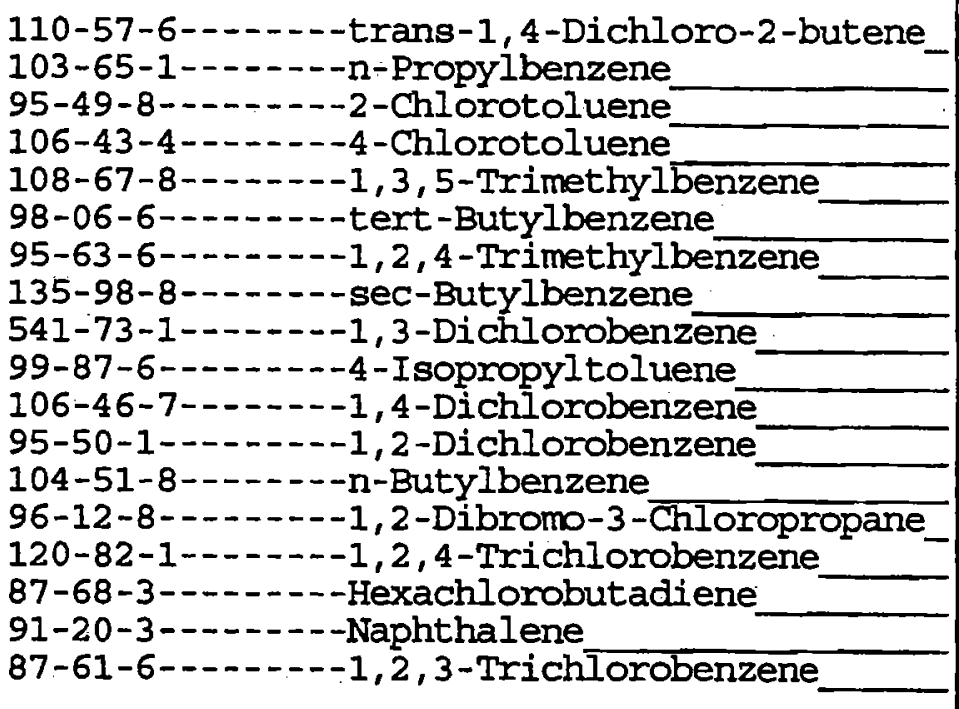 & $\begin{array}{l}8.4 \\
8.4 \\
8.4 \\
8.4 \\
8.4 \\
8.4 \\
8.4 \\
8.4 \\
8.4 \\
8.4 \\
8.4 \\
8.4 \\
8.4 \\
8.4 \\
8.4 \\
8.4 \\
8.4 \\
8.4\end{array}$ & $\begin{array}{l}U \\
U \\
U \\
U \\
U \\
U \\
U \\
U \\
U \\
U \\
U \\
U \\
U \\
U \\
U \\
U \\
U \\
U \\
U\end{array}$ \\
\hline
\end{tabular}


FORM 1

VOLATILE ORGANICS ANALYSIS DATA SHEET

Lab Name: STL BURLINGTON

Contract: 21005

Lab Code: STLVT Case No.: NAVARRE SAS No.:

Lab Sample ID: 666631

Matrix: (soil/water) SOIL

Sample wt/vol:

$9.7(\mathrm{~g} / \mathrm{mL}) \mathrm{G}$

Lab File ID: 666631

Level: (low/med) MED

Date Received: 04/20/06

\% Moisture: not dec.

GC Colum: CAP ID: 0.53 (mm)

Soil Extract Volume: 10000 (uس)
ARGLAB SAMPLE NO.

NA-S-20340

SDG No.: 113901
Date Analyzed: 04/29/06

Dilution Factor: 1.0

Soil Aliquot Volume:

500 (UL)

CAS NO.

CONCENTRATION UNITS:

(ug/L or $\mathrm{ug} / \mathrm{Kg}$ ) UG/KG

Q

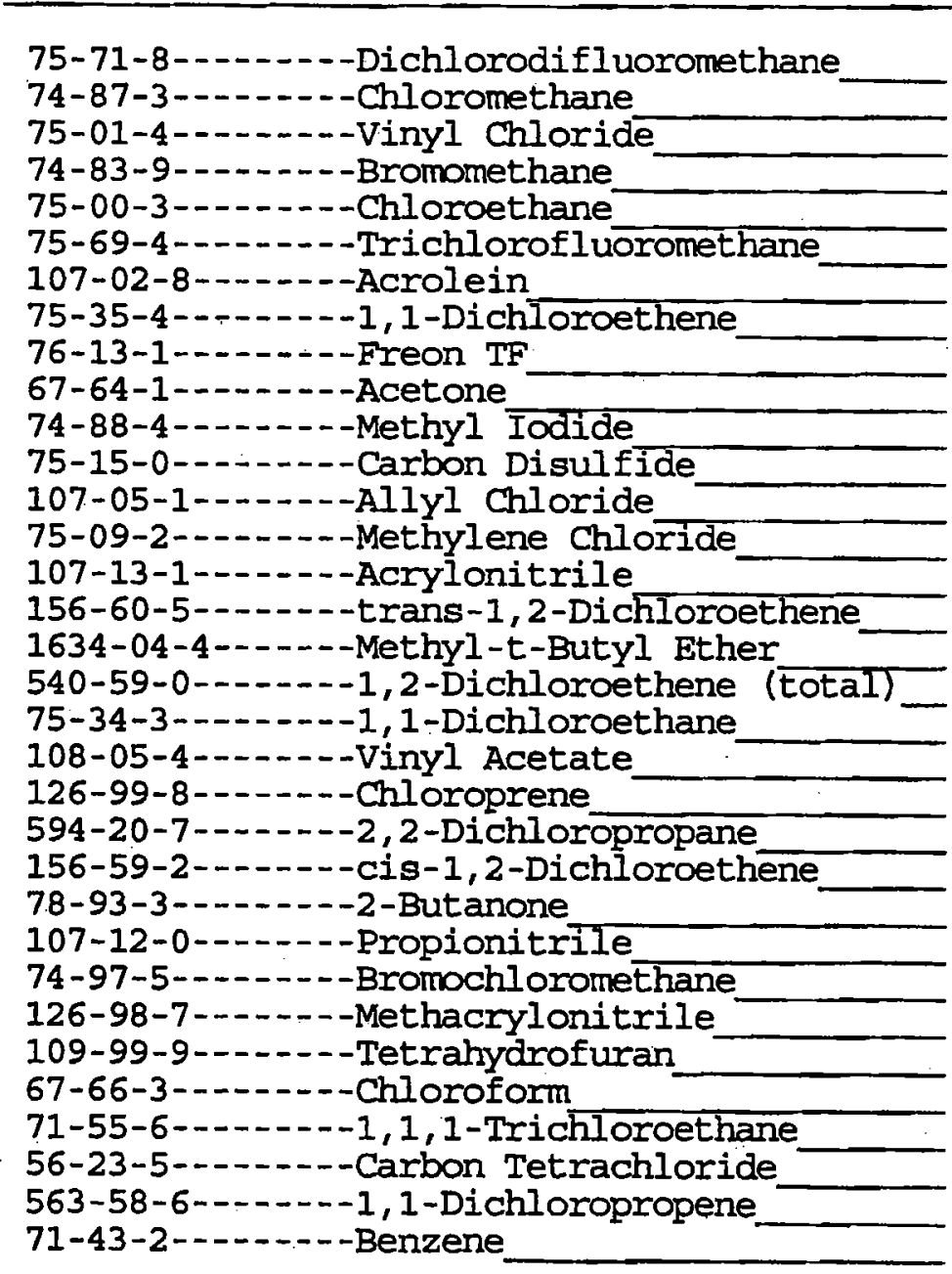

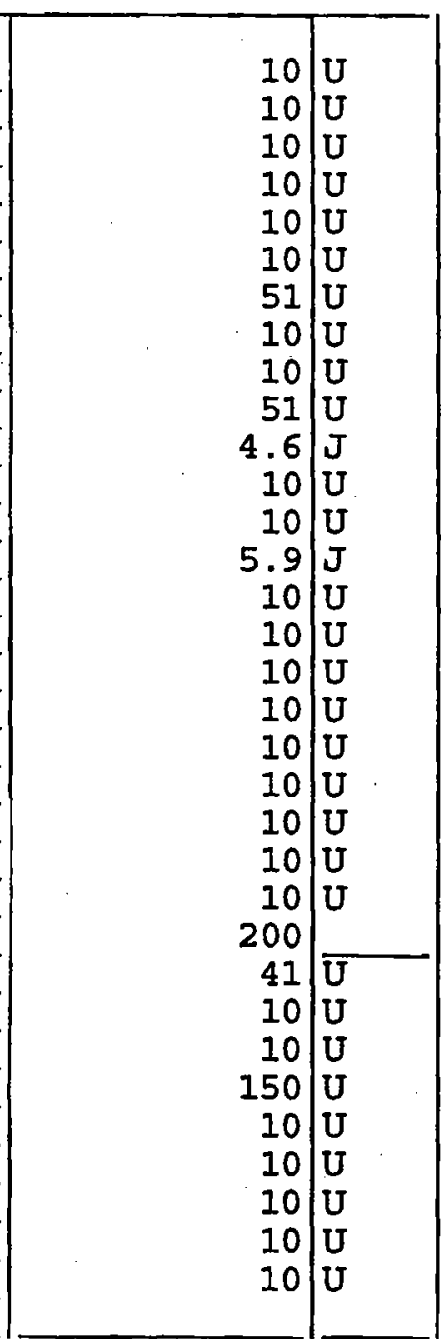


FORM 1

VOLATILE ORGANICS ANALYSIS DATA SHEET

Lab Name: STL BURLINGTON

Contract: 21005
ARGLAB SAMPLE NO.

NA-S-20340

SDG No.: 113901
Matrix: (soil/water) SOIL

Sample wt/vol: $\quad 9.7(\mathrm{~g} / \mathrm{mL})$ G

Level: (low/med) MED

\& Moisture: not dec.

GC Colunn: CAP ID: 0.53 (mm)

Soil Extract Volume: 10000 (uJ)
Lab Sample ID: 666631

Lab File ID: 666631

Date Received: 04/20/06

Date Analyzed: 04/29/06

Dilution Factor: 1.0

Soil Aliquot Volume:

500 (UL)

CAS NO.

COMPOUND

CONCENTRATION UNITS:

(ug/L or ug/Kg) UG/KG . Q

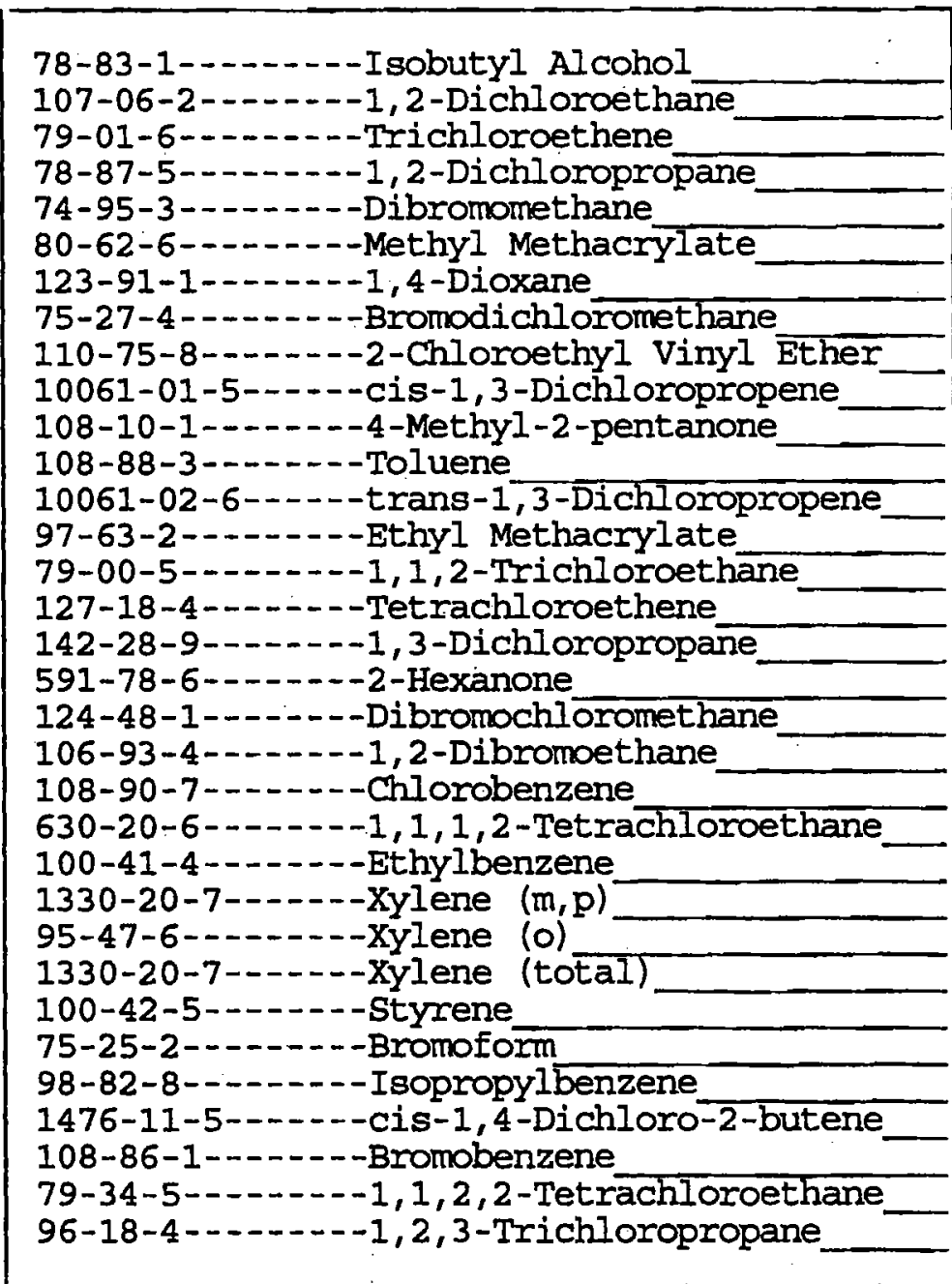


FORM 1

VOLATILE ORGANICS ANALYSIS DATA SHEET

Lab Name: STL BURLINGTON

Contract : 21005

Iab Code: STLVT Case No.: NAVARRE SAS No.:

Lab Sample ID: 666631

Matrix: (soil/water) SOIL

Sample wt/vol:

$9.7(\mathrm{~g} / \mathrm{mL}) \mathrm{G}$

Lab File ID: 666631

Level: (low/med) MED

Date Received: 04/20/06

\% Moisture: not dec.

GC Colunn: CAP ID: 0.53 (mm)

Soil Extract Volume: 10000 (uس)
ARGIAB SAMPLE NO.

NA-S -20340
Date Analyzed: 04/29/06

Dilution Factor: 1.0

Soil Aliquot Volume:

500 (UL)

CAS NO.

COMPOUND

CONCENTRATION UNITS :

(ug/L or $\mathrm{ug} / \mathrm{Kg}$ ) UG/KG

Q

\begin{tabular}{|c|c|}
\hline 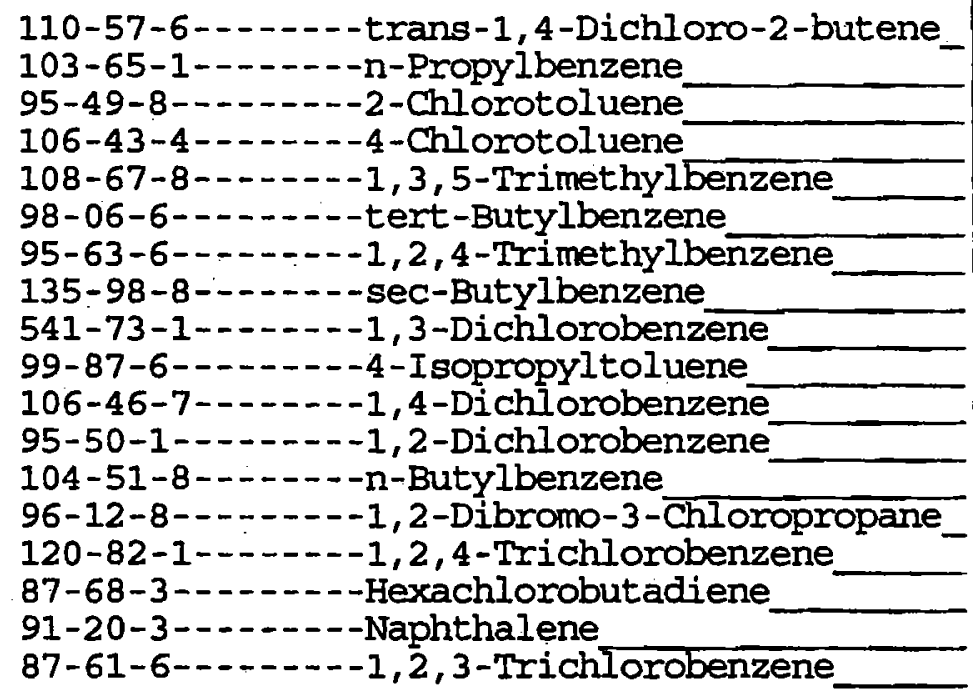 & \begin{tabular}{l|l}
10 & $U$ \\
10 & $U$ \\
10 & $U$ \\
10 & $U$ \\
10 & $U$ \\
10 & $U$ \\
10 & $U$ \\
10 & $U$ \\
10 & $U$ \\
10 & $U$ \\
10 & $U$ \\
10 & $U$ \\
10 & $U$ \\
10 & $U$ \\
10 & $U$ \\
10 & $U$ \\
10 & $U$ \\
10 & $U$
\end{tabular} \\
\hline
\end{tabular}


FORM 1

VOLATILE ORGANICS ANALYSIS DATA SHEET

Lab Name: STL BURLINGTON

Contract : 21005

Lab Code: SILVT Case No.: NAVARRE SAS No.:

Lab Sample ID: 666632

Matrix: (soil/water) SOIL

Lab File ID: 666632

Sample wt/vol: $\quad 11.9(\mathrm{~g} / \mathrm{mL}) \mathrm{G}$

Date Received: 04/20/06

Level: (low/med) MED

\% Moisture: not dec.

GC Column: CAP

ID: $0.53(\mathrm{~mm})$

Soil Extract Volume: 10000 (UI)
ARGLAB SAMPLE NO.

NA-S-20358

SDG No.: 113901
Date Analyzed: 04/29/06

Dilution Factor: 1.0

Soil Aliquot Volume:

500 (UI)

\begin{tabular}{|c|c|c|}
\hline 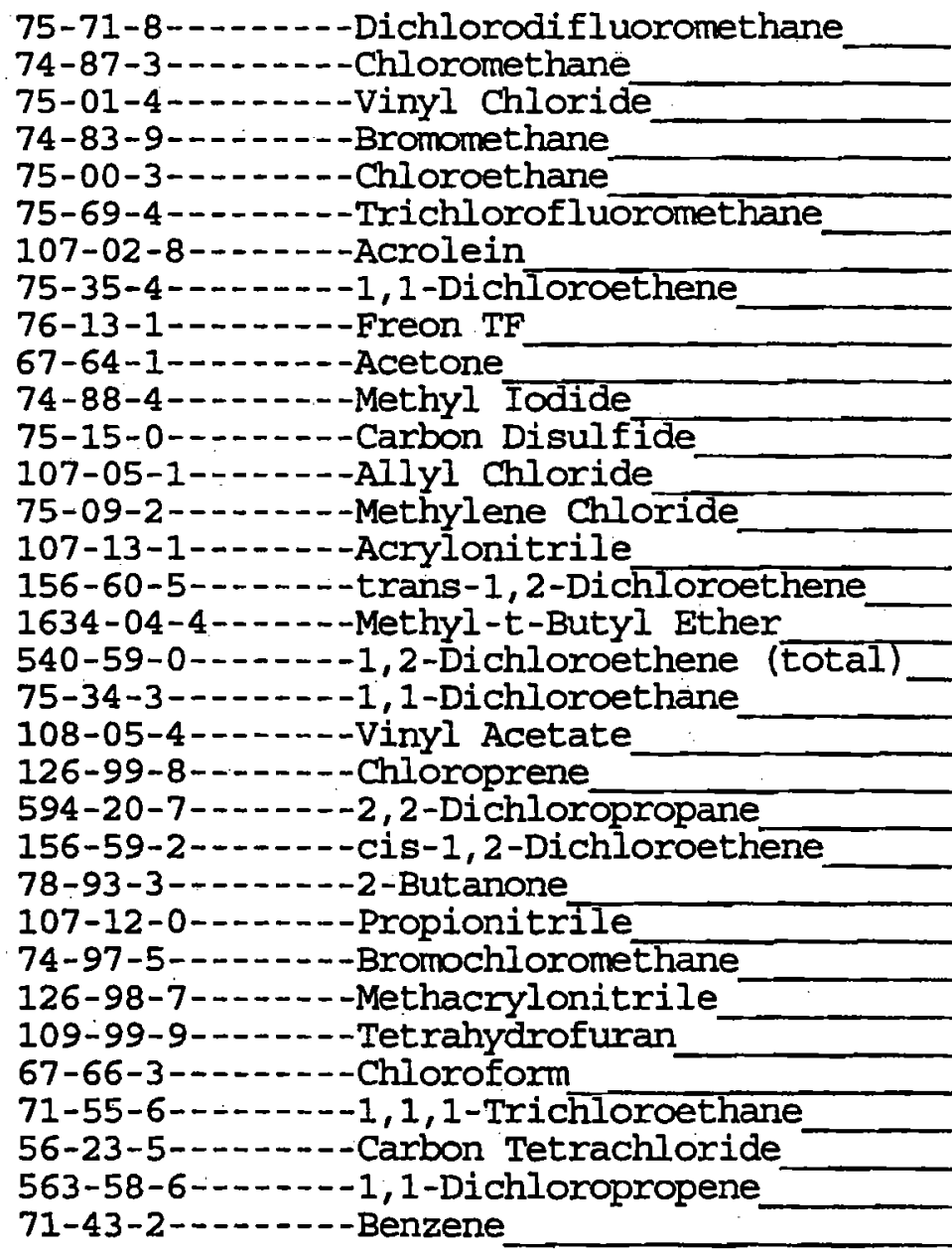 & $\begin{array}{r}8.4 \\
8.4 \\
8.4 \\
2.5 \\
8.4 \\
8.4 \\
42 \\
8.4 \\
8.4 \\
42 \\
2.5 \\
8.4 \\
8.4 \\
7.9 \\
8.4 \\
8.4 \\
8.4 \\
8.4 \\
8.4 \\
8.4 \\
8.4 \\
8.4 \\
8.4 \\
160 \\
344 \\
8.4 \\
8.4 \\
120 \\
8.4 \\
8.4 \\
8.4 \\
8.4 \\
8.4\end{array}$ & $\begin{array}{l}U \\
U \\
U \\
J \\
U \\
U \\
U \\
U \\
U \\
U \\
J \\
U \\
U \\
J \\
U \\
U \\
U \\
U \\
U \\
U \\
U \\
U \\
U \\
U \\
U \\
U \\
U \\
U \\
U \\
U \\
U \\
U \\
U\end{array}$ \\
\hline
\end{tabular}


FORM 1

VOLATILE ORGANICS ANALYSIS DATA SHEET

Lab Name: STL BURLINGTON

Contract : 21005
ARGLAB SAMPLE NO.

NA-S-20358

Lab code: STLVT Case No.: NAVARRE SAS No.:

SDG No.: 113901

Matrix: (soil/water) SOIL

Sample wt/vol: $\quad 11.9(\mathrm{~g} / \mathrm{mL}) \mathrm{G}$

Level: (low/med) MED

\%oisture: not dec.

GC Column: CAP ID: 0.53 (mm)

Soil Extract Volume: 10000 (uL)
Lab Sample ID: 666632

Lab File ID: $\quad 666632$

Date Received: 04/20/06

Date Analyzed: 04/29/06

Dilution Factor: 1.0

Soil Aliquot Volume: 500 (uL)

CAS NO.

COMPOUND

CONCENTRATION UNITS:

\begin{tabular}{|c|}
\hline 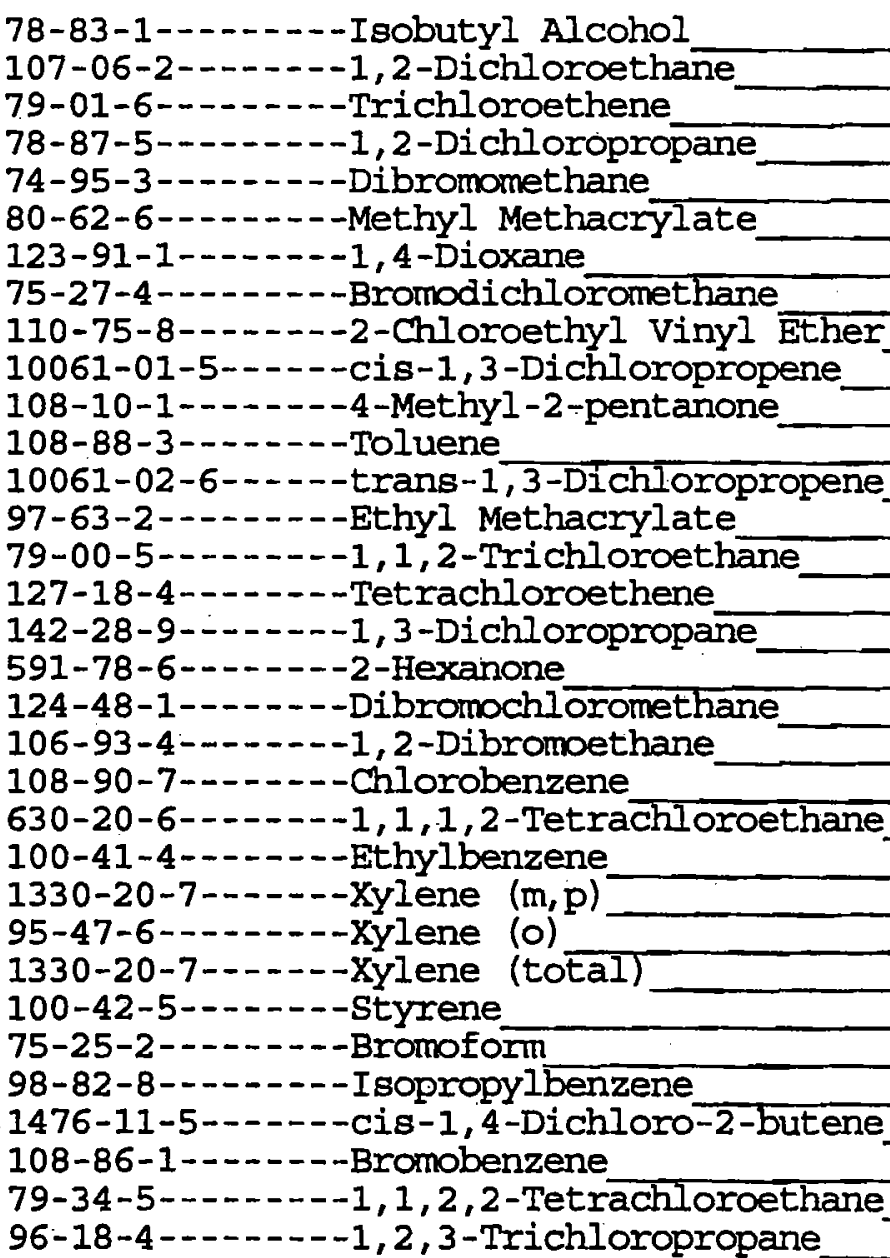 \\
\hline
\end{tabular}

(ug/L or $\mathrm{ug} / \mathrm{Kg}$ ) UG/KG

Q 
FORM 1

VOLATIIE ORGANICS ANALYSIS DATA SHEET

Lab Name: STL BURLINGTON

Contract: 21005
ARGLAB SAMPLE NO.

NA-S-20358

Lab Code: STLVT Case No. : NAVARRE SAS No.:

SDG No.: 113901

Matrix: (soil/water) SoIL

Sample wt/vol:

$11.9(\mathrm{~g} / \mathrm{mW}) \mathrm{G}$

Level: (low/med) MED

\% Moisture: not dec.

GC Column: CAP

ID: $0.53 \quad(\mathrm{~mm})$

Soil Extract volume: 10000 (UI)
Lab Sample ID: 666632

Lab File ID: 666632

Date Received: 04/20/06

Date Analyzed: 04/29/06

Dilution Factor: 1.0

Soil Aliquot volume:

500 (uL)

CAS NO.

COMPOUND

CONCENTRATION UNITS:

(ug/L or $\mathrm{ug} / \mathrm{Kg}$ ) UG/KG

Q

$8.4 \mathrm{U}$

$8.4 \mathrm{U}$

$8.4 \mathrm{U}$

$8.4 \mathrm{U}$

$8.4 \mathrm{U}$

$8.4 \mathrm{U}$

$8.4 \mathrm{U}$

$8.4 \mathrm{U}$

$8.4 \mathrm{U}$

$8.4 \mathrm{U}$

$8.4 \mathrm{U}$

$8.4 \mathrm{U}$

$8.4 \mathrm{U}$

$8.4 \mathrm{U}$

$8.4 \mathrm{U}$

$8.4 \mathrm{U}$

$8.4 \mathrm{U}$

$8.4 \mathrm{U}$ 
FORM 1

VOLATILE ORGANICS ANALYSIS DATA SHEET

Lab Name: STL BURLINGTON

Contract: 21005

Lab Code: STLVT Case No.: NAVARRE SAS No.:

Lab Sample ID: 666623

Matrix: (soil/water) SOIL

Lab File ID: 666623

Sample wt/vol: $\quad 11.2(\mathrm{~g} / \mathrm{mL}) \mathrm{G}$

Date Received: 04/20/06

Level: (low/med) MED

Date Analyzed: 04/29/06

GC Column: CAP

ID: $0.53(\mathrm{~mm})$

Soil Extract Volume: 10000 (uL)

COMPOUND

CAS NO.

Dilution Factor: 1.0

Soil Aliquot Volume:

CONCENTRATION UNITS:
ARGLAB SAMPLE NO.

NA-S-20408

SDG No.: 113901

500 (UI)

\begin{tabular}{|c|c|c|}
\hline 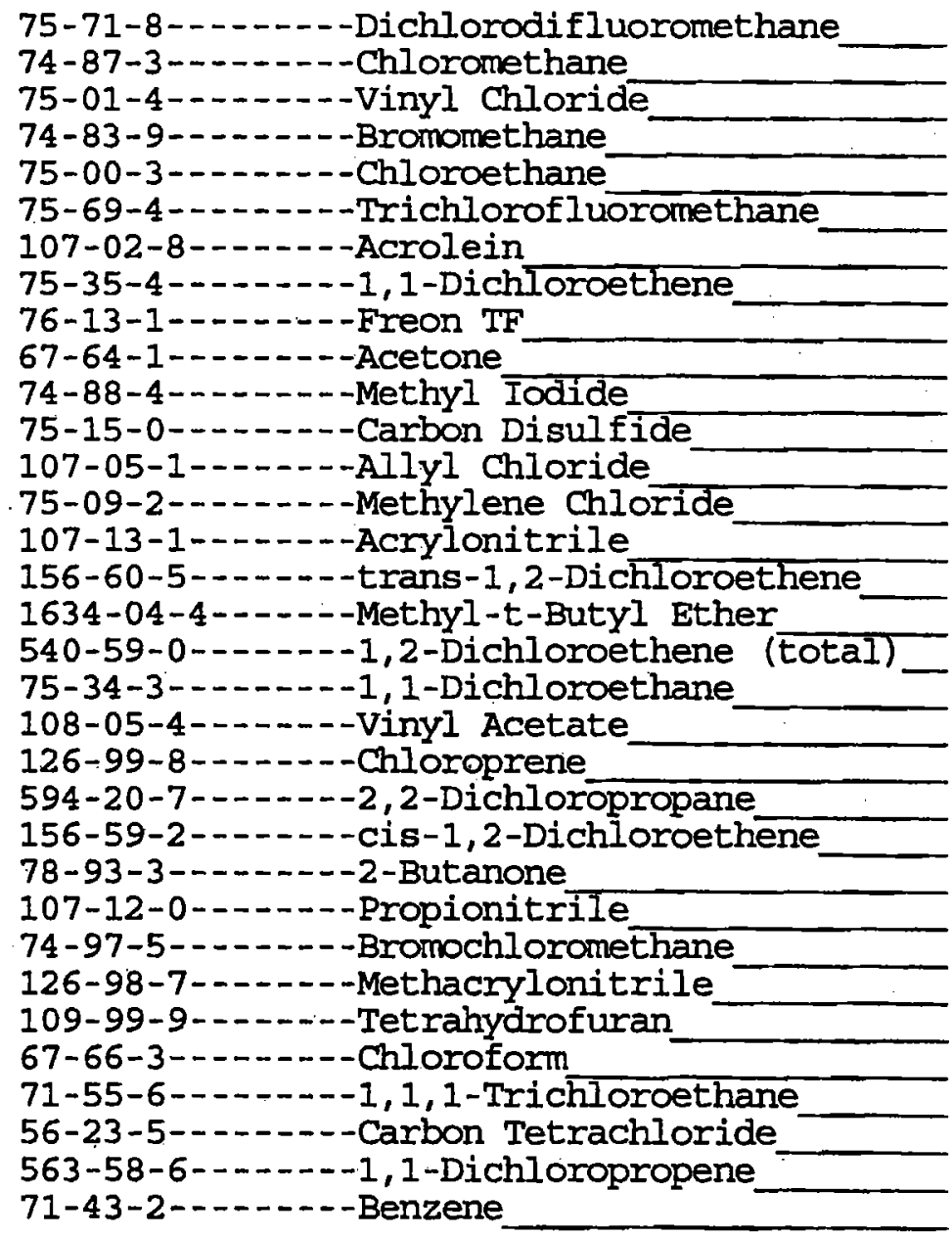 & $\begin{array}{r}9.0 \\
2.1 \\
9.0 \\
7.3 \\
9.0 \\
9.0 \\
44 \\
9.0 \\
9.0 \\
44 \\
5.5 \\
9.0 \\
9.0 \\
9.0 \\
9.0 \\
9.0 \\
9.0 \\
9.0 \\
9.0 \\
9.0 \\
9.0 \\
9.0 \\
9.0 \\
46 \\
36 \\
9.0 \\
9.0 \\
130 \\
9.0 \\
9.0 \\
9.0 \\
9.0 \\
9.0\end{array}$ & $\begin{array}{l}\mathrm{U} \\
\mathrm{J} \\
\mathrm{U} \\
\mathrm{J} \\
\mathrm{U} \\
\mathrm{U} \\
\mathrm{U} \\
\mathrm{U} \\
\mathrm{U} \\
\mathrm{U} \\
\mathrm{J} \\
\mathrm{U} \\
\mathrm{U} \\
\mathrm{U} \\
\mathrm{U} \\
\mathrm{U} \\
\mathrm{U} \\
\mathrm{U} \\
\mathrm{U} \\
\mathrm{U} \\
\mathrm{U} \\
\mathrm{U} \\
\mathrm{U} \\
\mathrm{U} \\
\mathrm{U} \\
\mathrm{U} \\
\mathrm{U} \\
\mathrm{U} \\
\mathrm{U} \\
\mathrm{U} \\
\mathrm{U} \\
\mathrm{U}\end{array}$ \\
\hline
\end{tabular}


FORM 1

VOLATILE ORGANICS ANALYSIS DATA SHEET

Lab Name: STL BURLINGTON

Contract: 21005

Lab Code: STLVT Case No.: NAVARRE SAS No.:

Lab Sample ID: 666623

Matrix: (soil/water) SOIL

Sample wt/vol:

$11.2(\mathrm{~g} / \mathrm{mL}) \mathrm{G}$

Level : (low/med) MED

\% Moisture: not dec.

GC Column: CAP ID: 0.53 (mm)

Soil Extract Volume: 10000 (uI)

Soil Aliquot Volume:
ARGLAB SAMPLE NO.

NA-S-20408

SDG NO.: 113901

Date Received: 04/20/06

Date Analyzed: 04/29/06

Dilution Factor: 1.0

500 (uL)

CAS NO.

COMPOUND

CONCENTRATION UNITS:

(ug/L or ug/Kg) UG/KG

Q

\begin{tabular}{|c|}
\hline 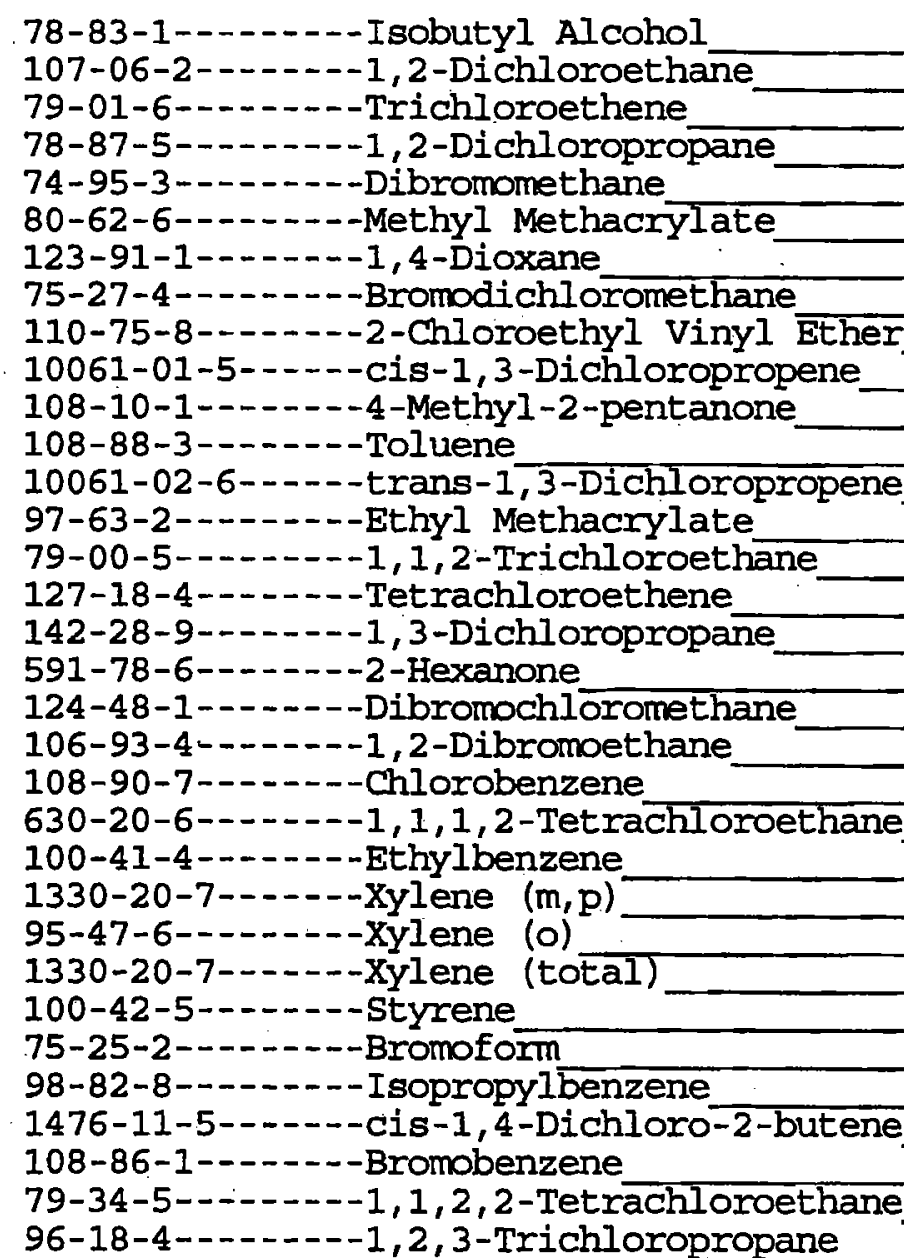 \\
\hline
\end{tabular}

$450 \mathrm{U}$

$9.0 \mathrm{U}$

$9.0 \mathrm{U}$

$9.0 \mathrm{U}$

$9.0 \mathrm{U}$

$9.0 \mathrm{U}$

$450 \mathrm{U}$

$9.0 \mathrm{U}$

$9.0 \mathrm{U}$

$9.0 \mathrm{U}$

$44 \mathrm{U}$

$9.0 \mathrm{U}$

$9.0 \mathrm{U}$

$9.0 \mathrm{U}$

$9.0 \mathrm{U}$

$9.0 \mathrm{U}$

$9.0 \mathrm{U}$

$44 \mathrm{U}$

$9.0 \mathrm{U}$

$9.0 \mathrm{U}$

$9.0 \mathrm{U}$

$9.0 \mathrm{U}$

$9.0 \mathrm{U}$

$9.0 \mathrm{U}$

$9.0 \mathrm{U}$

$9.0 \mathrm{U}$

$9.0 \mathrm{U}$

$9.0 \mathrm{U}$

$9.0 \mathrm{U}$

$9.0 \mathrm{U}$

$9.0 \mathrm{U}$

$9.0 \mathrm{U}$

$9.0 \mathrm{U}$ 
FORM 1

VOLATILE ORGANICS ANALYSIS DATA SHEET

Lab Name: STL BURLINGTON

Contract : 21005
ARGLAB SAMPLE NO.

NA-S-20408

Lab Code: STLVT Case No.: NAVARRE SAS No.:

SDG No.: 113901

Matrix: (soil/water) SOIL

Sample wt/vol:

$11.2(\mathrm{~g} / \mathrm{mL}) \mathrm{G}$

Level: (low/med) MED

\% Moisture: not dec.

GC Column: CAP

ID: $0.53(\mathrm{~mm})$

Soil Extract Volume: 10000 (UL)
Lab Sample ID: 666623

Lab File ID: $\quad 666623$

Date Received: 04/20/06

Date Analyzed: 04/29/06

Dilution Factor: 1.0

Soil Aliquot Volume:

$500(\mathrm{uL})$

CAS NO.

COMPOUND

CONCENTRATION UNITS:

(ug/L or $u g / K g)$ UG/KG

Q

$9.0 \mathrm{U}$

$9.0 \mathrm{U}$

$9.0 \mathrm{U}$

$9.0 \mathrm{U}$

$9.0 \mathrm{U}$

$9.0 \mathrm{U}$

$9.0 \mathrm{U}$

$9.0 \mathrm{U}$

$9.0 \mathrm{U}$

$9.0 \mathrm{U}$

$9.0 \mathrm{U}$

$9.0 \mathrm{U}$

$9.0 \mathrm{U}$

$9.0 \mathrm{U}$

$9.0 \mathrm{U}$

$9.0 \mathrm{U}$

$9.0 \mathrm{U}$

$9.0 \mathrm{U}$ 
FORM 1

VOLATILE ORGANICS ANALYSIS DATA SHEET

Lab Name: STL BURLINGTON

Contract : 21005

Lab Code: STLVT Case No.: NAVARRE SAS No.:

ARGLAB SAMPLE NO.

NA-S-20413

SDG No.: 113901
Matrix: (soil/water) SOIL

Sarmple wt/vol:

$11.6(\mathrm{~g} / \mathrm{mL}) \mathrm{G}$

Level: (low/med) MED

\% Moisture: not dec.

GC Colunn: CAP

Soil Extract Volume: 10000 (uI)
Lab Sample ID: 666628

Lab File ID: $\quad 666628$

Date Received: 04/20/06

Date Analyzed: 04/29/06

Dilution Factor: 1.0

Soil Aliquot Volume:

$500\left(u_{s}\right)$

CAS NO.

COMPOUND

CONCENTRATION UNITS:

(ug/L or $\mathrm{ug} / \mathrm{Kg}$ ) UG/KG

Q

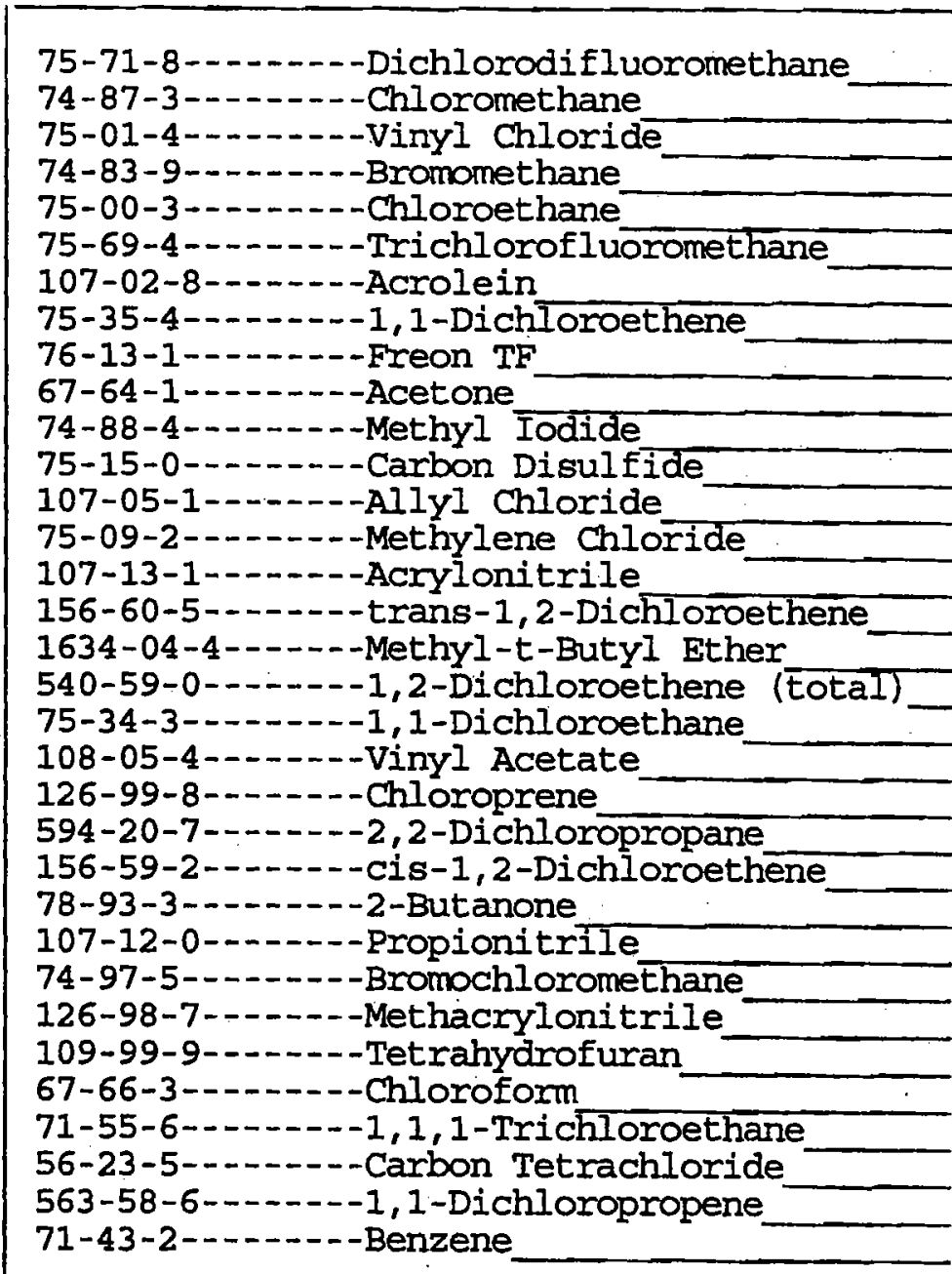

$8.6 \mathrm{U}$

$2.2 \mathrm{~J}$

$8.6 \mathrm{U}$

$2.3 \mathrm{~J}$

$8.6 \mathrm{U}$

$8.6 \mathrm{U}$

$43 \mathrm{U}$

$8.6 \mathrm{U}$

$8.6 \mathrm{U}$

$43 \mathrm{U}$

$3.8 \mathrm{~J}$

$8.6 \mathrm{U}$

$8.6 \mathrm{U}$

$8.6 \mathrm{U}$

$8.6 \mathrm{U}$

$8.6 \mathrm{U}$

$8.6 \mathrm{U}$

$8.6 \mathrm{U}$

$8.6 \mathrm{U}$

$8.6 \mathrm{U}$

$8.6 \mathrm{U}$

$8.6 \mathrm{U}$

$8.6 \mathrm{U}$

$43 \mathrm{U}$

$34 \mathrm{U}$

$8.6 \mathrm{U}$

$8.6 \mathrm{U}$

$120 \mathrm{U}$

$8.6 \mathrm{U}$

$8.6 \mathrm{U}$

$8.6 \mathrm{U}$

$8.6 \mathrm{U}$

$8.6 \mathrm{U}$ 
FORM 1

VOLATIIE ORGANICS ANALYSIS DATA SHEET

Lab Name: STL BURLINGTON

Contract: 21005

Iab Code: STLVT Case No.: NAVARRE SAS No.:

ARGLAB SAMPLE NO.

NA-S-20413
Matrix: (soil/water) soIL

Sample wt/vol: $\quad 11.6(\mathrm{~g} / \mathrm{mL}) \mathrm{G}$

Level: (low/med) MED

\& Moisture: not dec.

GC Colum: CAP. ID: 0.53 (mm)

Soil Extract Volume: 10000 (ur)
Lab Sample ID: 666628

Lab File ID: $\quad 666628$

Date Received: 04/20/06

Date Analyzed: 04/29/06

Dilution Factor: 1.0

Soil Aliquot volume:

500 (uL)

CAS NO.

COMPOUND

CONCENTRATION UNITS:

(ug/L or $\mathrm{ug} / \mathrm{Kg}$ ) UG/KG

$Q$

\begin{tabular}{|c|c|c|}
\hline 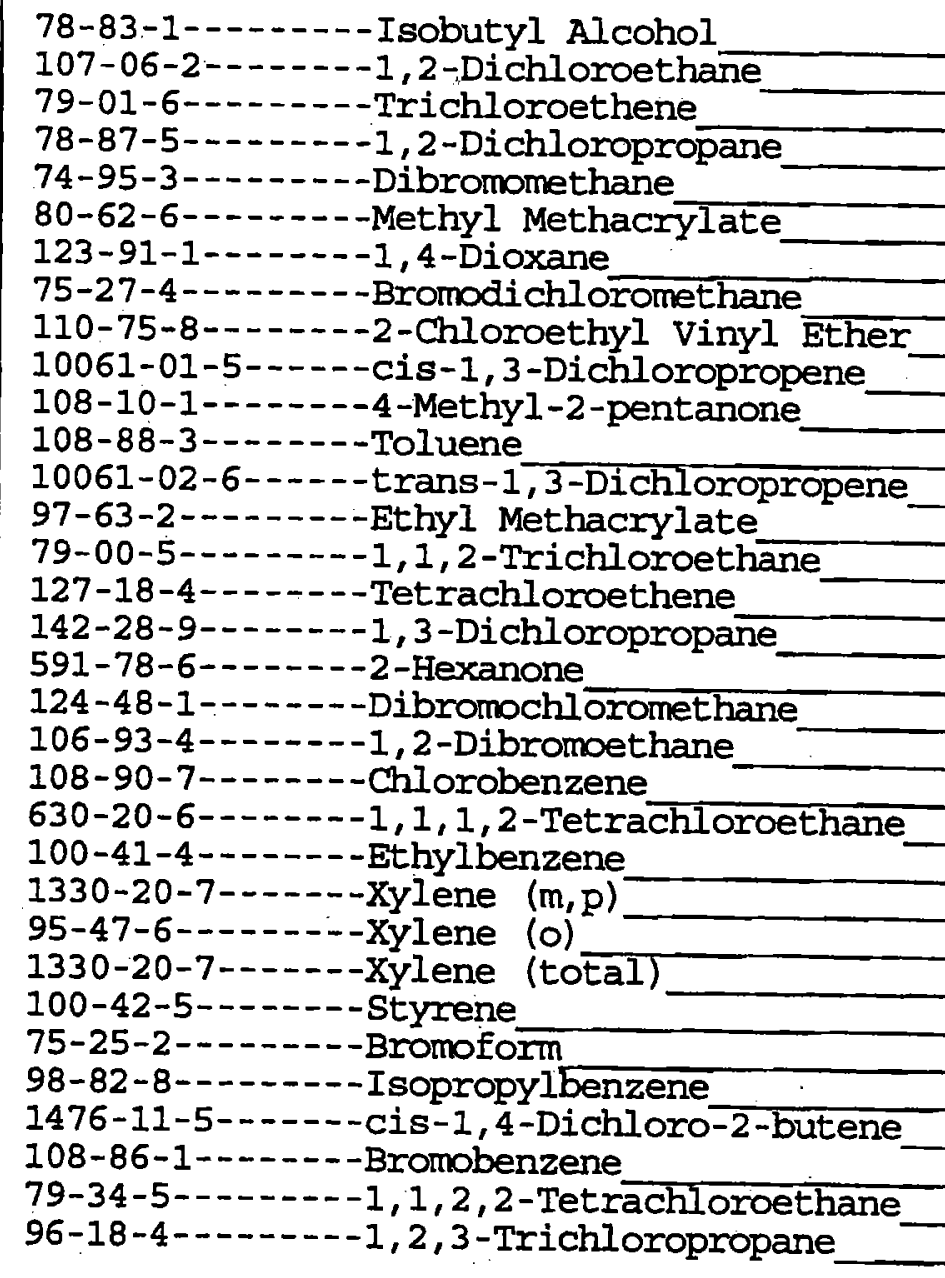 & $\begin{array}{r}430 \\
8.6 \\
8.6 \\
8.6 \\
8.6 \\
13 \\
430 \\
8.6 \\
8.6 \\
8.6 \\
43 \\
8.6 \\
8.6 \\
8.6 \\
8.6 \\
8.6 \\
8.6 \\
43 \\
8.6 \\
8.6 \\
8.6 \\
8.6 \\
8.6 \\
8.6 \\
8.6 \\
8.6 \\
8.6 \\
8.6 \\
8.6 \\
8.6 \\
8.6 \\
8.6 \\
8.6\end{array}$ & $\begin{array}{l}U \\
U \\
U \\
U \\
U \\
U \\
U \\
U \\
U \\
U \\
U \\
U \\
U \\
U \\
U \\
U \\
U \\
U \\
U \\
U \\
U \\
U \\
U \\
U \\
U \\
U \\
U \\
U \\
U \\
U \\
U \\
U\end{array}$ \\
\hline
\end{tabular}


FORM 1

VOLATILE ORGANICS ANALYSIS DATA SHEET

Lab Name: STL BURLINGTON

Contract: 21005
ARGLAB SAMPLE NO.

NA-S-20413

Lab Code: STLVT Case No.: NAVARRE SAS No.:

SDG No.: 113901

Matrix: (soil/water) SOIL

Sample wt/vol:

$11.6(\mathrm{~g} / \mathrm{mL}) \mathrm{G}$

Level: (low/med) MED

\% Moisture: not dec.

GC Column: CAP

Soil Extract Volume: 10000 (uL)
Lab Sample ID: 666628

Lab File ID: 666628

Date Received: 04/20/06

Date Analyzed: 04/29/06

Dilution Factor: 1.0

Soil Aliquot Volume:

500 (UL)

CAS NO.

COMPOUND

CONCENTRATION UNITS:

(ug/L or $\mathrm{ug} / \mathrm{Kg}$ ) UG/KG

Q

$8.6 \mathrm{U}$

$8.6 \mathrm{U}$

$8.6 \mathrm{U}$

$8.6 \mathrm{U}$

$8.6 \mathrm{U}$

$8.6 \mathrm{U}$

$8.6 \mathrm{U}$

$8.6 \mathrm{U}$

$8.6 \mathrm{U}$

$8.6 \mathrm{U}$

$8.6 \mathrm{U}$

$8.6 \mathrm{U}$

$8.6 \mathrm{U}$

$8.6 \mathrm{U}$

$8.6 \mathrm{U}$

$8.6 \mathrm{U}$

$8.6 \mathrm{U}$

$8.6 \mathrm{U}$ 
FORM 1

VOLATILE ORGANICS ANALYSIS DATA SHEET

Lab Name: STL BURLINGTON

Contract: 21005
ARGLAB SAMPLE NO.

NA-S-20453

Lab Code: STLVT Case No.: NAVARRE SAS No.:

SDG NO.: 113901

Matrix: (soil/water) soIL

Iab Sample ID: 666622

Sample wt/vol:

$13.9(\mathrm{~g} / \mathrm{mL}) \mathrm{G}$

Lab File ID: $\quad 666622$

Level: (low/med) MED

Date Received: 04/20/06

\% Moisture: not dec.

GC Colurn: CAP ID: 0.53 (mm)

Date Analyzed: 04/29/06

Soil Extract Volume: 10000 (UL)

Dilution Factor: 1.0

Soil Aliquot Volume:

500 (UL)

CONCENTRATION UNITS:

CAS NO.

COMPOUND

(ug/L or ug/Kg) UG/KG

Q

\begin{tabular}{|c|c|c|}
\hline 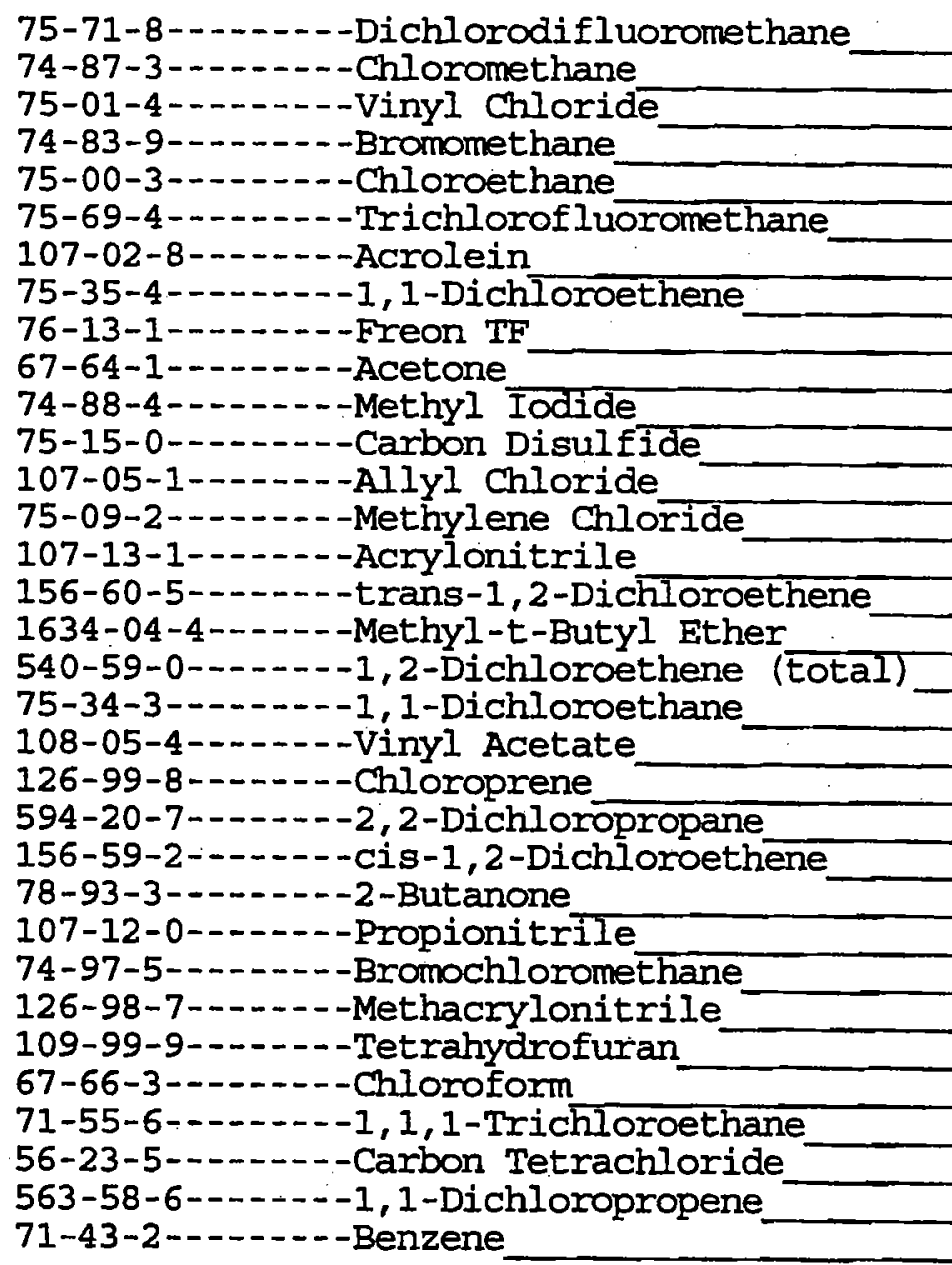 & $\begin{array}{r}7.2 \\
1.7 \\
7.2 \\
4.0 \\
7.2 \\
7.2 \\
36 \\
7.2 \\
7.2 \\
36 \\
5.2 \\
7.2 \\
7.2 \\
7.2 \\
7.2 \\
7.2 \\
7.2 \\
7.2 \\
7.2 \\
7.2 \\
7.2 \\
7.2 \\
7.2 \\
33 \\
2.9 \\
7.2 \\
7.2 \\
100 \\
7.2 \\
7.2 \\
7.2 \\
7.2 \\
7.2\end{array}$ & $\begin{array}{l}U \\
U \\
U \\
J \\
U \\
U \\
U \\
U \\
U \\
U \\
U \\
J \\
U \\
U \\
U \\
U \\
U \\
U \\
U \\
U \\
U \\
U \\
U \\
U \\
U \\
J \\
U \\
U \\
U \\
U \\
U \\
U \\
U \\
U \\
U\end{array}$ \\
\hline
\end{tabular}


FORM 1

VOLATIIE ORGANICS ANALYSIS DATA SHEET

Lab Name: STL BURLINGTON

Contract : 21005
ARGLAB SAMPLE NO.

NA-S-20453

Lab Code: STLVT Case No.: NAVARRE SAS No.:

SDG No.: 113901

Matrix: (soil/water) soIL

Sample wt/vol:

$13.9(\mathrm{~g} / \mathrm{mL}) \mathrm{G}$

Level : (low/med) MED

\% Moisture: not dec.

GC Column: CAP

ID $: 0.53(\mathrm{~mm})$

Soil Extract Volume: 10000 (UI)
Lab Sample ID: 666622

Lab File ID: 666622

Date Received: 04/20/06

Date Analyzed: 04/29/06

Dilution Factor: 1.0

Soil Aliquot Volume:

500 (u山)

CAS NO.

COMPOUND

CONCENTRATION UNITS:

$(\mathrm{ug} / \mathrm{L}$ or $\mathrm{ug} / \mathrm{Kg}$ ) UG/KG

Q

$7.2 \mathrm{U}$

$7.2 \mathrm{U}$

$7.2 \mathrm{U}$

$7.2 \mathrm{U}$

$360 \mathrm{U}$

$7.2 \mathrm{U}$

$7.2 \mathrm{U}$

$7.2 \mathrm{U}$

$36 \mathrm{U}$

$7.2 \mathrm{U}$

$7.2 \mathrm{U}$

$7.2 \mathrm{U}$

$7.2 \mathrm{U}$

$7.2 \mathrm{U}$

$7.2 \mathrm{U}$

$36 \mathrm{U}$

$7.2 \mathrm{U}$

$7.2 \mathrm{U}$

$7.2 \mathrm{U}$

7.2 U

$7.2 \mathrm{U}$

$7.2 \mathrm{U}$

$7.2 \mathrm{U}$

7.2 U

$7.2 \mathrm{U}$

$7.2 \mathrm{U}$

$7.2 \mathrm{U}$

$7.2 \mathrm{U}$

$7.2 \mathrm{U}$

$7.2 \mathrm{U}$

$7.2 \mathrm{U}$ 
FORM 1

VOLATILE ORGANICS ANALYSIS DATA SHEET

Lab Name: STL BURLINGTON

Contract : 21005
ARGLAB SAMPLE NO.

NA-S-20453

Lab Code: STLVT Case No.: NAVARRE SAS No.:

SDG No.: 113901

Matrix: (soil/water) soIL

Sample wt/vol: $\quad 13.9(\mathrm{~g} / \mathrm{mL})$ G

Level: (low/med) MED

\% Moisture: not dec.

GC Colunn: CAP

ID: 0.53 (mm)

Soil Extract Volume: 10000 (uL)
Lab Sample ID: 666622

Lab File ID: $\quad 666622$

Date Received: 04/20/06

Date Analyzed: 04/29/06

Dilution Factor: 1.0

Soil Aliquot volume:

500 (UL)

CAS NO.

COMPOUND CONCENTRATION UNITS: (ug/L or $u g / \mathrm{Kg}$ ) UG/KG

Q

$7.2 \mathrm{U}$

$7.2 \mathrm{U}$

$7.2 \mathrm{U}$

$7.2 \mathrm{U}$

$7.2 \mathrm{U}$

$7.2 \mathrm{U}$

$7.2 \mathrm{U}$

$7.2 \mathrm{U}$

$7.2 \mathrm{U}$

$7.2 \mathrm{U}$

$7.2 \mathrm{U}$

$7.2 \mathrm{U}$

$7.2 \mathrm{U}$

$7.2 \mathrm{U}$

$7.2 \mathrm{U}$

$7.2 \mathrm{U}$

$7.2 \mathrm{U}$

$7.2 \mathrm{U}$ 
FORM 1

VOLATILE ORGANICS ANALYSIS DATA SHEET

Lab Name: STL BURLINGTON

Contract: 21005

Lab Code: STLVT Case No.: NAVARRE SAS No.:

Lab Sample ID: 666621

Matrix: (soil/water) SOIL

Lab File ID: 666621

Sample wt/vol:

$11.0(\mathrm{~g} / \mathrm{mL}) \mathrm{G}$

Level: (low/med) MED

Date Received: 04/20/06

\% Moisture: not dec.

GC Column: CAP

ID: 0.53 (mm)

Soil Extract Volume: 10000 (UL)

SDG No.: 113901

Dilution Factor: 1.0

Soil Aliquot Volume:
ARGLAB SAMPLE NO.

NA-S-20454

500 (uU)

CONCENTRATION UNITS:

CAS NO. COMPOUND (ug/L or $\mathrm{ug} / \mathrm{Kg}$ ) UG/KG

Q

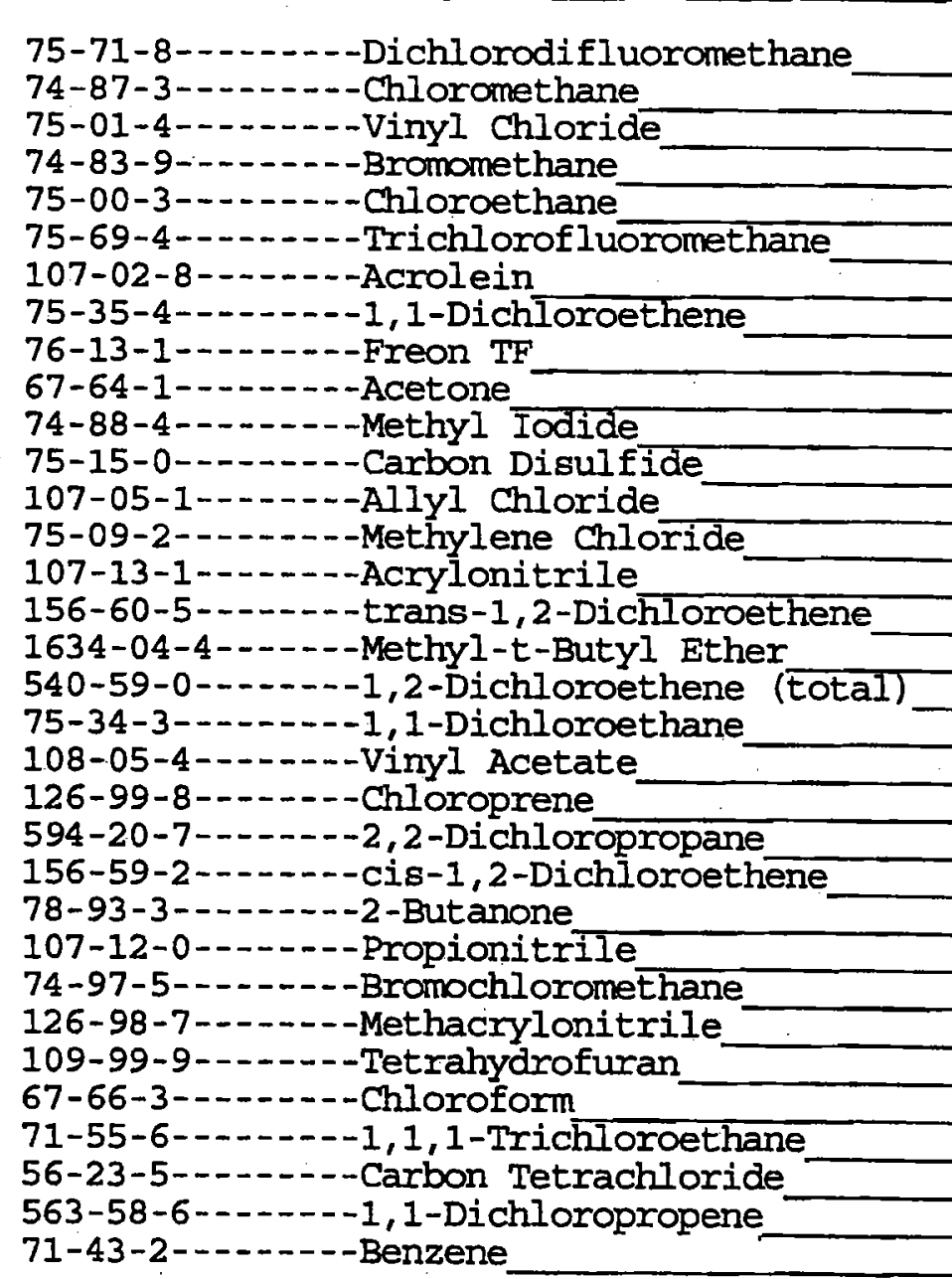

$9.1 \mathrm{U}$

$2.8 \mathrm{~J}$

$9.1 \mathrm{U}$

$8.9 \mathrm{~J}$

$9.1 \mathrm{U}$

$9.1 \mathrm{U}$

$45 \mathrm{U}$

$9.1 \mathrm{U}$

$9.1 \mathrm{U}$

$45 \mathrm{U}$

$7.7 \mathrm{~J}$

$9.1 \mathrm{U}$

$9.1 \mathrm{U}$

9.1 U

$9.1 \mathrm{U}$

$9.1 \mathrm{U}$

$9.1 \mathrm{U}$

$9.1 U$

$9.1 \mathrm{U}$

$9.1 \mathrm{U}$

$9.1 \mathrm{U}$

$9.1 \mathrm{U}$

$9.1 \mathrm{U}$

$31 \mathrm{~J}$

$36 \mathrm{U}$

$9.1 \mathrm{U}$

$9.1 \mathrm{U}$

$130 \mathrm{U}$

$9.1 \mathrm{U}$

$9.1 \mathrm{U}$

$9.1 \mathrm{U}$

$9.1 \mathrm{U}$

$9.1 \mathrm{U}$ 
FORM 1

VOLATILE ORGANICS ANALYSIS DATA SHEET

Lab Name: STL BURLINGTON

Contract : 21005

Lab Code: STLVT Case No.: NAVARRE SAS No.:

Lab Sample ID: 666621

Matrix: (soil/water) SoIL

Lab File ID: 666621

Sample wt/vol:

$11.0(\mathrm{~g} / \mathrm{mL}) \mathrm{G}$

Level: (low/med) MED

of Moisture: not dec.

GC Column: CAP . ID: 0.53 (mm)

Soil Extract Volume: 10000 (uL)
ARGLAB SAMPLE NO.

NA-S-20454

SDG No.: 113901
Date Received: 04/20/06

Date Analyzed: 04/29/06

Dilution Factor: 1.0

Soil Aliquot Volume: $\quad 500$ (uL)

CAS NO. COMPOUND (ug/L or ug/ $\mathrm{Kg}$ ) UG/KG Q

\begin{tabular}{|c|c|c|}
\hline 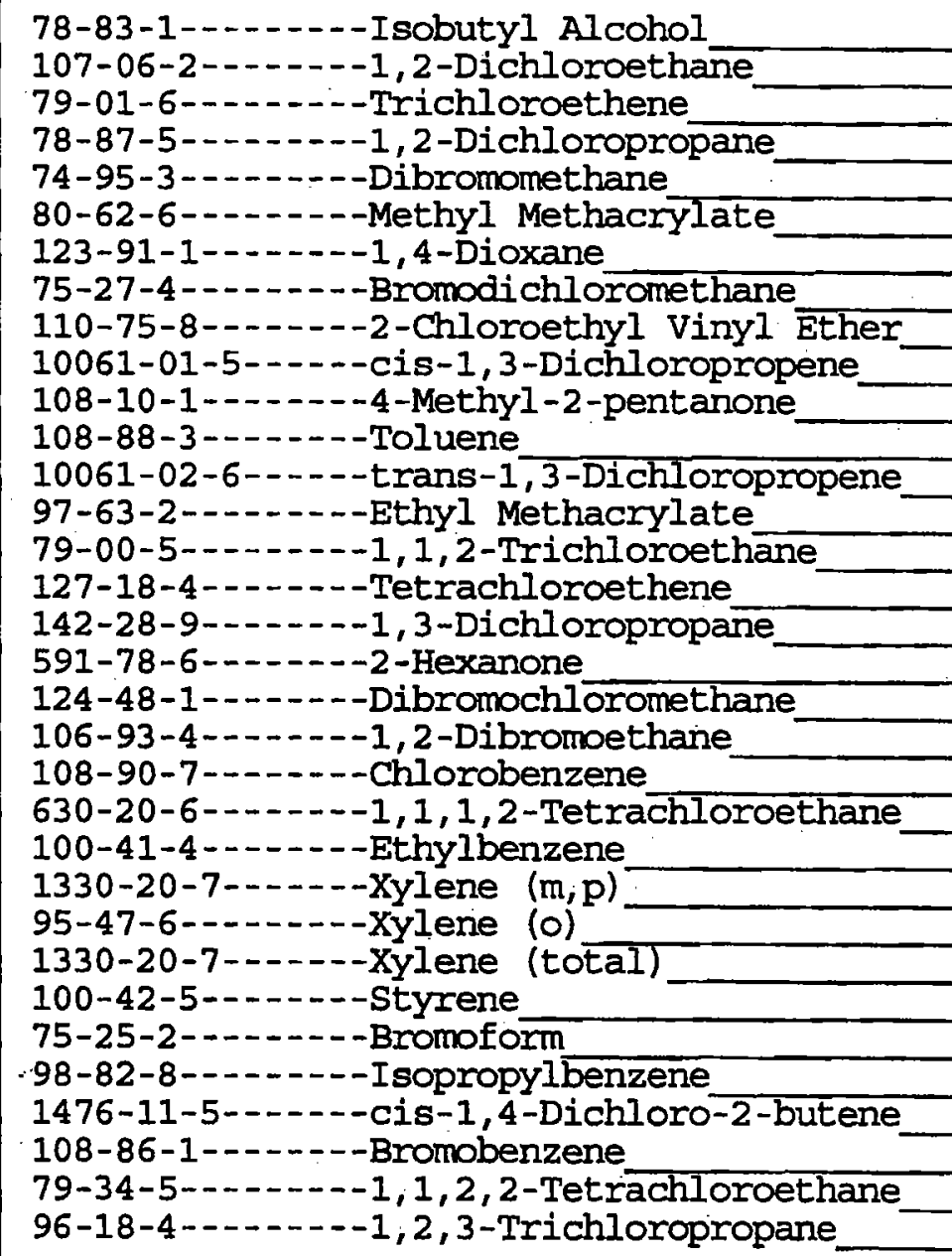 & $\begin{array}{r}450 \\
9.1 \\
9.1 \\
9.1 \\
9.1 \\
9.1 \\
450 \\
9.1 \\
9.1 \\
9.1 \\
45 \\
9.1 \\
9.1 \\
9.1 \\
9.1 \\
9.1 \\
9.1 \\
45 \\
9.1 \\
9.1 \\
9.1 \\
9.1 \\
9.1 \\
9.1 \\
9.1 \\
9.1 \\
9.1 \\
9.1 \\
9.1 \\
9.1 \\
9.1 \\
9.1 \\
9.1\end{array}$ & $\begin{array}{l}\mathrm{U} \\
\mathrm{U} \\
\mathrm{U} \\
\mathrm{U} \\
\mathrm{U} \\
\mathrm{U} \\
\mathrm{U} \\
\mathrm{U} \\
\mathrm{U} \\
\mathrm{U} \\
\mathrm{U} \\
\mathrm{U} \\
\mathrm{U} \\
\mathrm{U} \\
\mathrm{U} \\
\mathrm{U} \\
\mathrm{U} \\
\mathrm{U} \\
\mathrm{U} \\
\mathrm{U} \\
\mathrm{U} \\
\mathrm{U} \\
\mathrm{U} \\
\mathrm{U} \\
\mathrm{U} \\
\mathrm{U} \\
\mathrm{U} \\
\mathrm{U} \\
\mathrm{U} \\
\mathrm{U} \\
\mathrm{U} \\
\mathrm{U} \\
\mathrm{U}\end{array}$ \\
\hline
\end{tabular}


FORM 1

VOLATILE ORGANICS ANALYSIS DATA SHEET

Lab Name: STI BURLINGTON

Contract: 21005

Lab Code: STLVT Case No.: NAVARRE SAS No.:

Lab Sample ID: 666621

Matrix: (soil/water) SoIL

Lab File ID: 666621

Sample wt/vol: $\quad 11.0(\mathrm{~g} / \mathrm{mL}) \mathrm{G}$

Level: (low/med) MED

Date Received: 04/20/06

\& Moisture: not dec.

GC Column: CAP

ID: $0.53(\mathrm{~mm})$

Date Analyzed: 04/29/06

Dilution Factor: 1.0

Soil Extract Volume: 10000 (uL)

Soil Aliquot volume:

500 (uL)

COMPOUND (ug/L or $\mathrm{ug} / \mathrm{Kg}$ ) UG/KG Q

$9.1 \mathrm{U}$

$9.1 \mathrm{U}$

$9.1 \mathrm{U}$

$9.1 \mathrm{U}$

$9.1 \mathrm{U}$

$9.1 \mathrm{U}$

$9.1 \mathrm{U}$

$9.1 \mathrm{U}$

$9.1 \mathrm{U}$

$9.1 \mathrm{U}$

$9.1 \mathrm{U}$

$9.1 \mathrm{U}$

$9.1 \mathrm{U}$

$9.1 U$

$9.1 \mathrm{U}$

$9.1 \mathrm{U}$

$9.1 \mathrm{U}$

$9.1 \mathrm{U}$ 
FORM 1

VOLATILE ORGANICS ANALYSIS DATA SHEET

Iab Name: STL BURLINGTON

Contract: 21005

Iab Code: STLVT Case No.: NAVARRE SAS No.:

Lab Sample ID: 666626

Matrix: (soil/water) soIL

Lab File ID: 666626

Sarmle wt/vol: $\quad 12.6(\mathrm{~g} / \mathrm{mL})$ G

Date Received: 04/20/06

Level : (low/med) MED

Date Analyzed: 04/29/06

GC Column: CAP

ID: $0.53(\mathrm{~mm})$

Dilution Factor: 1.0

Soil Extract Volume: 10000 (UI)

Soil Aliquot Volume:

500 (uL)

CONCENTRATION UNITS:

CAS NO.

COMPOUND

(ug/L or $\mathrm{ug} / \mathrm{Kg}$ ) UG/KG

Q

\begin{tabular}{|c|c|c|}
\hline 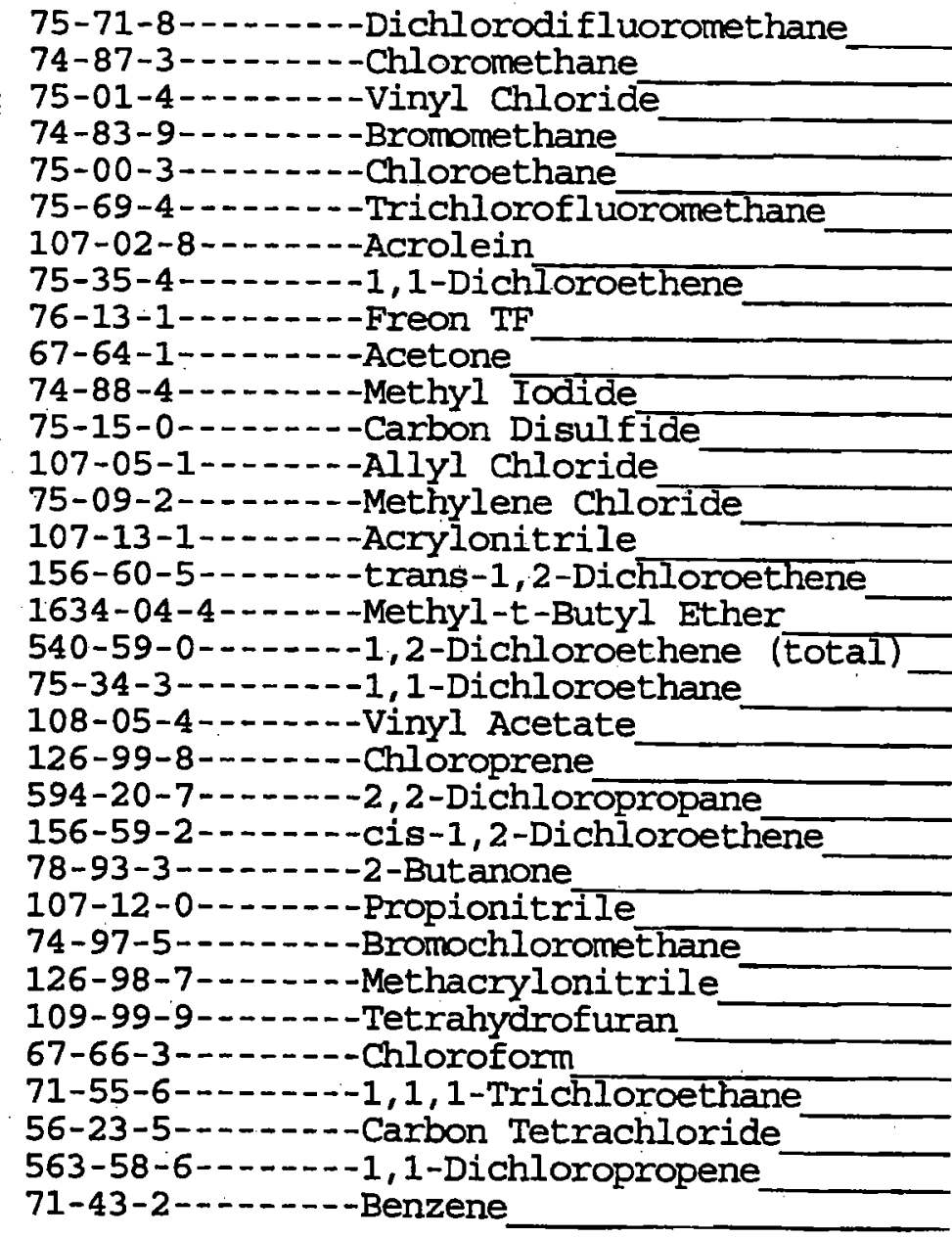 & $\begin{array}{r}7.9 \\
7.9 \\
7.9 \\
4.8 \\
7.9 \\
7.9 \\
39 \\
7.9 \\
7.9 \\
39 \\
4.8 \\
7.9 \\
7.9 \\
7.7 \\
7.9 \\
7.9 \\
7.9 \\
7.9 \\
7.9 \\
7.9 \\
7.9 \\
7.9 \\
7.9 \\
120 \\
32 \\
7.9 \\
7.9 \\
110 \\
11 \\
7.9 \\
29 \\
7.9 \\
7.9\end{array}$ & $\begin{array}{l}\mathrm{U} \\
\mathrm{U} \\
\mathrm{U} \\
\mathrm{U} \\
\mathrm{U} \\
\mathrm{U} \\
\mathrm{U} \\
\mathrm{U} \\
\mathrm{U} \\
\mathrm{U} \\
\mathrm{U} \\
\mathrm{U} \\
\mathrm{U} \\
\mathrm{U} \\
\mathrm{U} \\
\mathrm{U} \\
\mathrm{U} \\
\mathrm{U} \\
\mathrm{U} \\
\mathrm{U}\end{array}$ \\
\hline
\end{tabular}


FORM 1

VOLATILE ORGANICS ANALYSIS DATA SHEET

Lab Name: STL BURLINGTON

Contract: 21005
ARGLAB SAMPLE NO.

NA-S-20476

Lab Code: STLVT Case No.: NAVARRE SAS No.:

SDG No.: 113901

Matrix: (soil/water) SOIL

Sample wt/vol: $\quad 12.6(\mathrm{~g} / \mathrm{mL}) \mathrm{G}$

Level: (low/med) MED

o Moisture: not dec.

GC Colunm: CAP

ID: $0.53(\mathrm{~mm})$

Soil Extract Volume: 10000 (UU)
Lab Sample ID: 666626

Lab File ID: 666626

Date Received: 04/20/06

Date Analyzed: 04/29/06

Dilution Factor: 1.0

Soil Aliquot Volume:

500 (u山)

CAS NO.

COMPOUND

CONCENTRATION UNITS :

$(\mathrm{ug} / \mathrm{L}$ or $\mathrm{ug} / \mathrm{Kg}$ ) UG/KG

Q

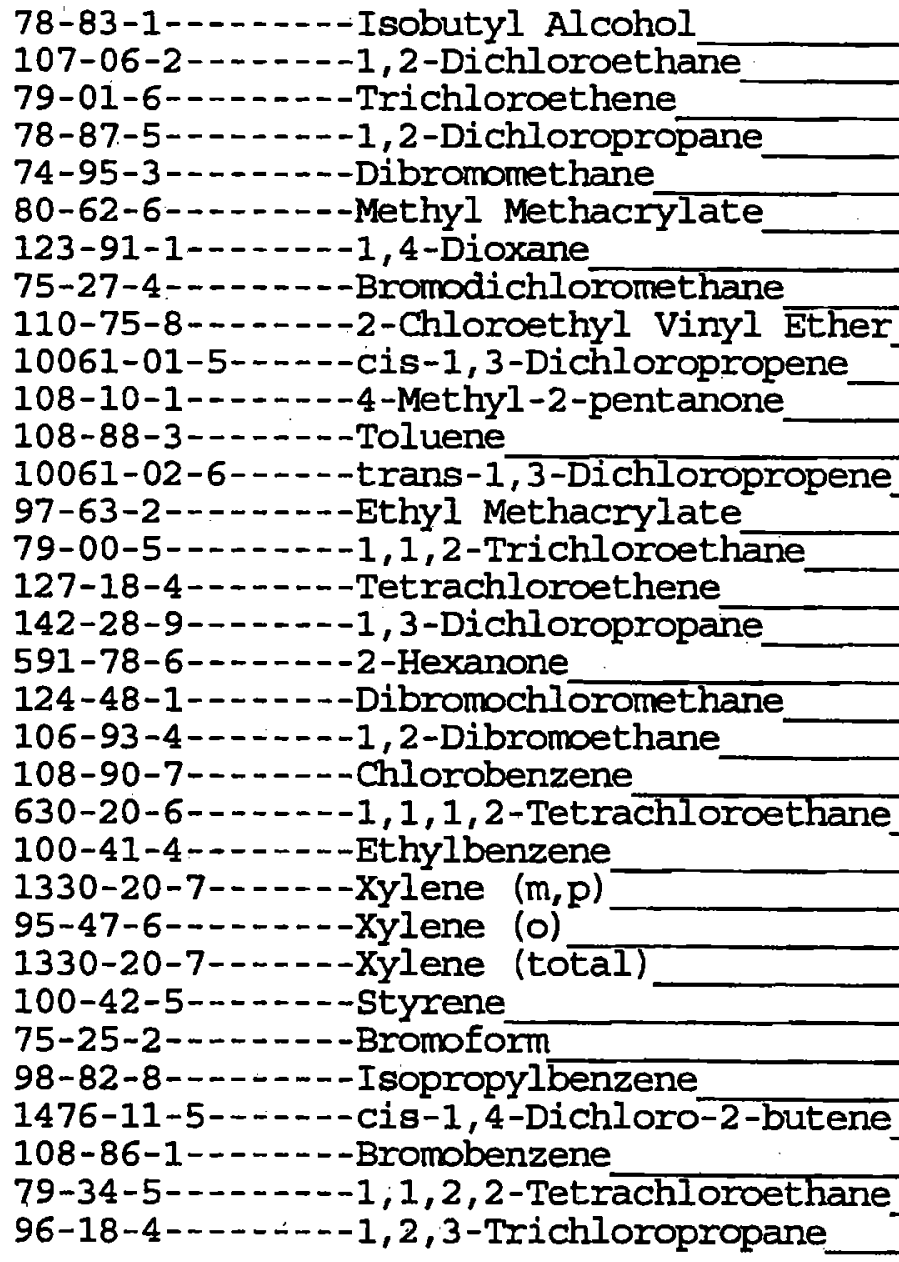

$7.9 \mathrm{U}$

$39 \mathrm{U}$

$7.9 \mathrm{U}$

$7.9 \mathrm{U}$

$7.9 \mathrm{U}$

$7.9 \mathrm{U}$

7.9 U

$7.9 \mathrm{U}$

$39 \mathrm{U}$

$7.9 \mathrm{U}$

$7.9 \mathrm{U}$

$7.9 \mathrm{U}$

$7.9 \mathrm{U}$

$7.9 \mathrm{U}$

$2.0 \mathrm{~J}$

$7.9 \mathrm{U}$

$2.2 \mathrm{~J}$

$7.9 \mathrm{U}$

$7.9 \mathrm{U}$

$7.9 \mathrm{U}$

$7.9 \mathrm{U}$

$7.9 \mathrm{U}$

$7.9 \mathrm{U}$

$7.9 \mathrm{U}$ 
FORM 1

VOLATIIE ORGANICS ANALYSIS DATA SHEET

Lab Name: STL BURLINGTON

Contract: 21005
ARGLAB SAMPLE NO.

NA-S-20476

Lab Code: STLVT Case No.: NAVARRE SAS No.:

SDG No.: 113901

Matrix: (soil/water) SOIL

Lab Sample ID: 666626

Sample wt/vol:

$12.6(\mathrm{~g} / \mathrm{mL}) \mathrm{G}$

Lab File ID: 666626

Level: (low/med) MED

Date Received: 04/20/06

\& Moisture: not dec.

GC Colurm: CAP

ID: 0.53 (mm)

Date Analyzed: 04/29/06

Dilution Factor: 1.0

Soil Extract Volume: 10000 (uL)

Soil Aliquot Volume:

500 (uL)

CONCENTRATION UNITS:

CAS NO.

COMPOUND

(ug/L or ug/Kg) UG/KG

Q

$7.9 \mathrm{U}$

$7.9 \mathrm{U}$

$7.9 \mathrm{U}$

$7.9 \mathrm{U}$

$7.9 \mathrm{U}$

$7.9 \mathrm{U}$

$7.9 \mathrm{U}$

$7.9 \mathrm{U}$

$7.9 \mathrm{U}$

$7.9 \mathrm{U}$

$7.9 \mathrm{U}$

$7.9 \mathrm{U}$

$7.9 \mathrm{U}$

$7.9 \mathrm{U}$

$7.9 \mathrm{U}$

$7.9 \mathrm{U}$

$7.9 \mathrm{U}$

$7.9 \mathrm{U}$ 


\section{S E V E R N}

T R E N T
May 19, 2006

Mr. Clyde Dennis

Argonne National Laboratory

9700 S. Cass Avenue

Building 203, Office 149

Argonne, IL 60439
STL Burlington

208 South Park Drive, Suite 1

Colchester, VT 05446

Tel: 8026551203 Fax: 8026551248

www.stl-inc.com

Re: Laboratory Project No. 21005

Case: NAVARRE; SDG: 114211

Dear Mr. Dennis:

Enclosed are analytical results for samples that were received by STL Burlington on May $8^{\text {th }}, 2006$. Laboratory identification numbers were assigned, and designated as follows:

$\begin{array}{llll}\underline{\text { Lab ID }} & \begin{array}{l}\text { Client } \\ \text { Sample ID }\end{array} & \begin{array}{l}\text { Sample } \\ \text { Date }\end{array} & \begin{array}{l}\text { Sample } \\ \text { Matrix }\end{array} \\ & \text { Received: 05/08/06 ETR No: } 114211 & \\ 668542 & \text { NA-S-20527-9A } & 05 / 02 / 06 & \text { Liquid } \\ 668543 & \text { NA-S-20511-10A } & 05 / 02 / 06 & \text { Liquid } \\ 668544 & \text { NA-S-20603-30A } & 05 / 02 / 06 & \text { Liquid } \\ 668545 & \text { NA-S-20620-20A } & 05 / 02 / 06 & \text { Liquid } \\ 668546 & \text { NA-S-20390-10A } & 05 / 02 / 06 & \text { Liquid } \\ 668547 & \text { NA-S-20496-10A } & 05 / 02 / 06 & \text { Liquid } \\ 668548 & \text { NA-S-20607-20A } & 05 / 02 / 06 & \text { Liquid } \\ 668549 & \text { NA-S-BLANK } & 05 / 02 / 06 & \text { Liquid }\end{array}$

Documentation of the condition of the samples at the time of their receipt and any exception to the laboratory's Sample Acceptance Policy is documented in the Sample Handling section of this submittal. It should be noted that at the time that they were received, the sample volumes were at near ambient temperature.

The samples were analyzed by Method $8260 \mathrm{~B}$, using a low-level calibration. In performing the analytical work, 500 microliters of the methanol extract were added to the 5 milliliter purge volume. The surrogate controls were recovered well in each of the analyses associated with the sample set, and each analysis exhibited good internal standard stability. Two types of laboratory control sample analyses were performed as part of the analytical sequence. One was performed to evaluate method performance, and one was performed with $\mathbf{5 0 0}$ microliters of methanol added to the purge volume in order to characterize the affect on the analytical process. The target analytes were recovered well in the laboratory control sample analysis that defined the method performance. In the laboratory control sample analysis with methanol, several of the earlier eluting compounds did exhibit lower recoveries, as did several of the later eluting compounds. Most profoundly affected was the performance of chloroethane, naphthalene, and 1,2,3-trichlorobenzene, for which the recovery values were below 10 percent. Chloroform and 
carbon tetrachloride were recovered well in each of the laboratory control sample analyses. Matrix spike and matrix spike duplicate analyses were not performed on samples in this sample set. The analysis of the instrument blank that was analyzed in association with the samples was free of contamination. The laboratory did provide for the analysis of a method blank with the addition of 500 microliters of methanol, however the methanol that was used was not from the same lot as that used in the extraction of the samples.

If there are any questions regarding this submittal, please contact me at (802) 655-1203.

The analytical results associated with the samples presented in this test report were generated under a quality system that adheres to requirements specified in the NELAC standard. Release of the data in this test report and any associated electronic deliverables is authorized by the Laboratory Director's designee as verified by the following signature.

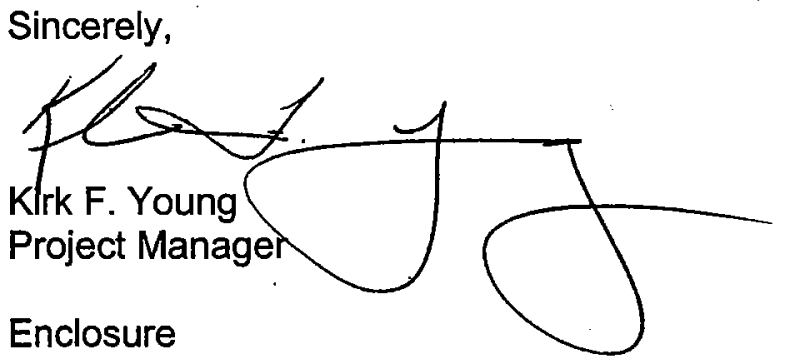




\section{STL Burlington Data Qualifier Definitions}

\section{Organic}

$\mathrm{U}$ : Compound analyzed but not detected at a concentration above the reporting limit.

J: Estimated value.

$\mathrm{N}$ : Indicates presumptive evidence of a compound. This flag is used only for tentatively identified compounds (TICs) where the identification of a compound is based on a mass spectral library search.

P: SW-846: Greater than $40 \%$ difference for detected concentrations between two $\mathrm{GC}$ columns. Unless otherwise specified the higher of the two values is reported on the Form I.

CLP SOW: Greater than 25\% difference for detected concentrations between two $\mathrm{GC}$ columns. Unless otherwise specified the lower of the two values is reported on the Form 1 .

C: $\quad$ Pesticide result whose identification has been confirmed by GC/MS.

B: Analyte is found in the sample and the associated method blank. The flag is used for tentatively identified compounds as well as positively identified compounds.

E: Compounds whose concentrations exceed the upper limit of the calibration range of the instrument for that speciific analysis.

D: Concentrations identified from analysis of the sample at a secondary dilution.

A: Tentatively identified compound is a suspected aldol condensation product.

$X, Y, Z$ : Laboratory defined flags that may be used alone or combined, as needed. If used, the description of the flag is defined in the project narrative.

\section{Inorganic/Metals}

E: Reported value is estimated due to the presence of interference.

$\mathrm{N}$ : Matrix spike sample recovery is not within control limits.

* Duplicate sample analysis is not within control limits.

B: The result reported is less than the reporting limit but greater than the instrument detection limit.

$\mathrm{U}: \quad$ Analyte was analyzed for but not detected above the reporting limit.

Method Codes:

P ICP-AES

MS ICP-MS

CV Cold Vapor AA

AS Semi-Automated Spectrophotometric 


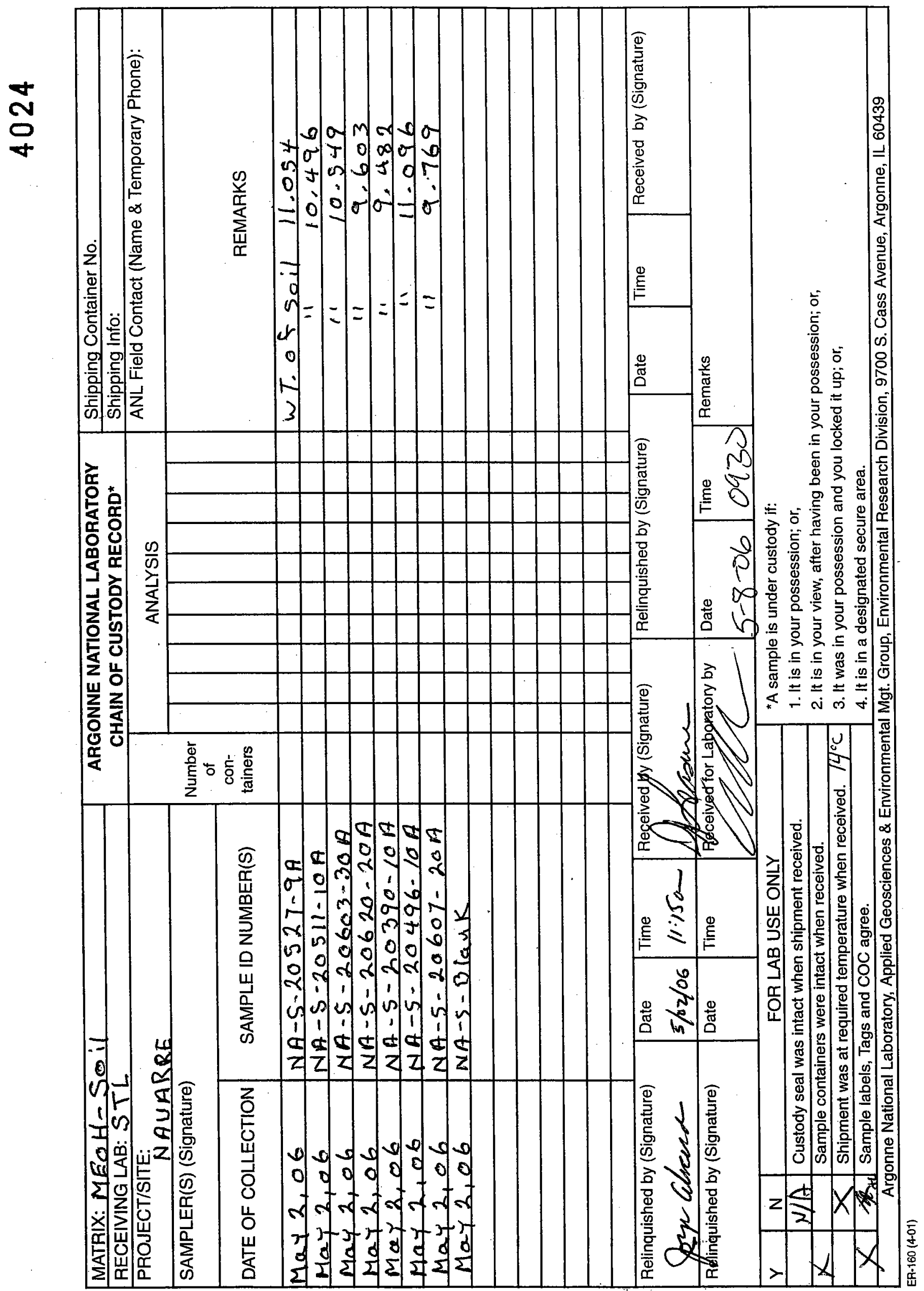




\section{SEVER N \\ TRENT $\boldsymbol{O} \boldsymbol{L}$}

METHOD 8260B

\section{VOLATILE ORGANIC ANALYSIS}

\section{SAMPLE DATA SUMMARY PACKAGE}


FORM 1

VOLATILE ORGANICS ANALYSIS DATA SHEET
Lab Name: STL BURLINGTON

Contract: 21005
ARGLAB SAMPLE NO.

NA-S-BLANK

Lab Code: STLVT Case No.: NAVARRE SAS No.:

SDG No.: 114211

Matrix: (soil/water) SOIL

Sample wt/vol: $\quad 10.0(\mathrm{~g} / \mathrm{mL}) \mathrm{G}$

Level: (low/med) MED

\% Moisture: not dec.

GC Column: CAP ID: 0.53 (mm)

Soil Extract Volume: 10000 (UL)
Lab Sample ID: 668549

Lab File ID: 668549

Date Received: 05/08/06

Date Analyzed: 05/10/06

Dilution Factor: 1.0

Soil Aliquot Volume:

$500(u L)$

CAS NO. COMPOUND $\quad(u g / L$ or $\mathrm{ug} / \mathrm{Kg})$ UG/KG Q

\begin{tabular}{|c|c|c|}
\hline 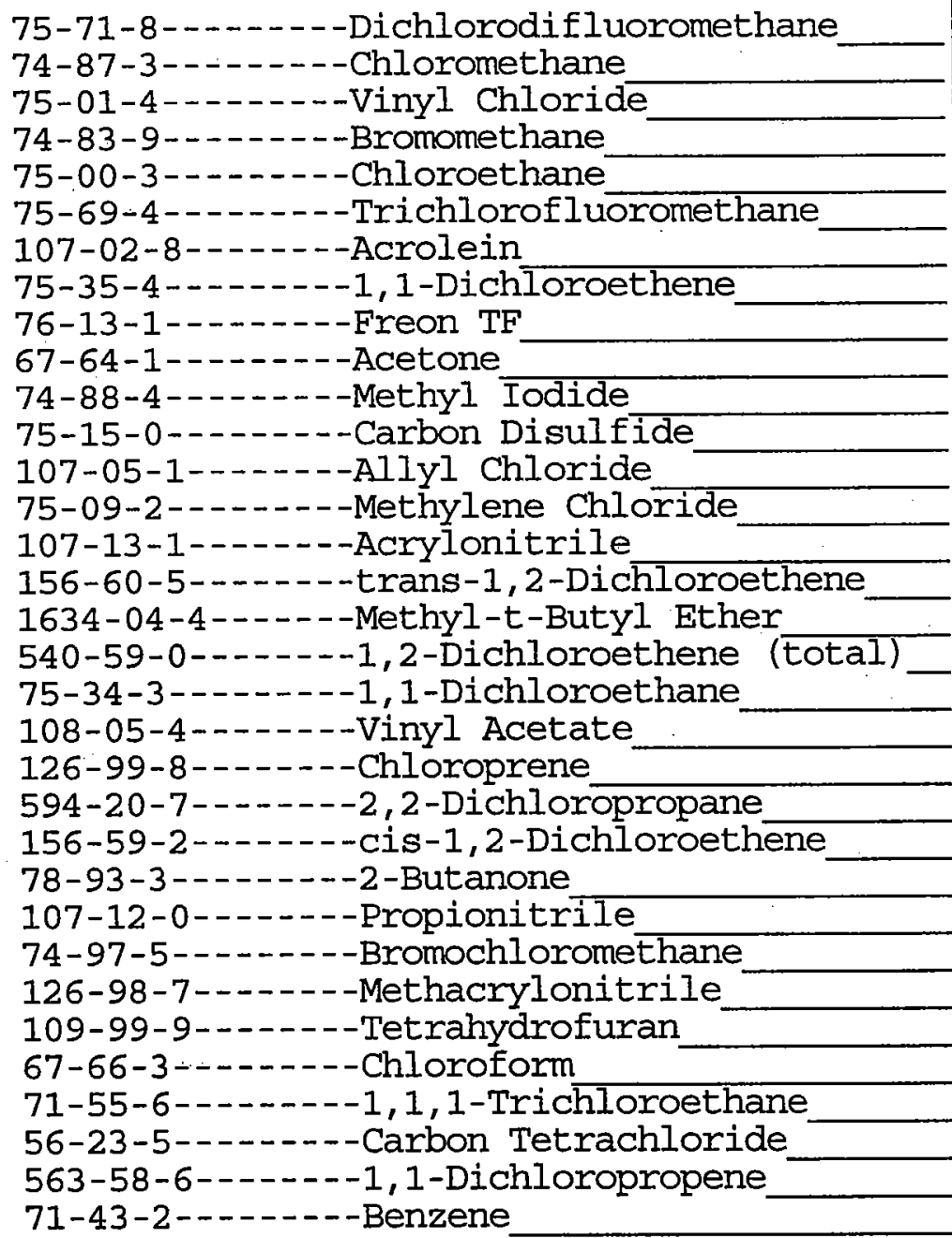 & $\begin{array}{r}10 \\
4.8 \\
10 \\
8.3 \\
10 \\
10 \\
50 \\
10 \\
10 \\
50 \\
6.0 \\
10 \\
10 \\
3.5 \\
10 \\
10 \\
10 \\
10 \\
10 \\
10 \\
10 \\
10 \\
10 \\
430 \\
40 \\
10 \\
10 \\
140 \\
10 \\
10 \\
10 \\
10 \\
10\end{array}$ & $\begin{array}{l}\mathrm{U} \\
\mathrm{JB} \\
\mathrm{U} \\
\mathrm{JB} \\
\mathrm{U} \\
\mathrm{U} \\
\mathrm{U} \\
\mathrm{U} \\
\mathrm{U} \\
\mathrm{U} \\
\mathrm{JB} \\
\mathrm{U} \\
\mathrm{U} \\
\mathrm{J} \\
\mathrm{U} \\
\mathrm{U} \\
\mathrm{U} \\
\mathrm{U} \\
\mathrm{U} \\
\mathrm{U} \\
\mathrm{U} \\
\mathrm{U} \\
\mathrm{U} \\
\mathrm{B} \\
\mathrm{U} \\
\mathrm{U} \\
\mathrm{U} \\
\mathrm{U} \\
\mathrm{U} \\
\mathrm{U} \\
\mathrm{U} \\
\mathrm{U} \\
\mathrm{U}\end{array}$ \\
\hline
\end{tabular}


FORM 1

VOLATILE ORGANICS ANALYSIS DATA SHEET
Lab Name: STL BURLINGTON

Contract: 21005
ARGLAB SAMPLE NO.

NA-S-BLANK

Lab Code: STLVT Case No.: NAVARRE SAS No.:

SDG No.: 114211

Matrix: (soil/water) SOIL

Sample wt/vol: $\quad 10.0(\mathrm{~g} / \mathrm{mL}) \mathrm{G}$

Level : (low/med) MED

\% Moisture: not dec.

GC Column: CAP

Soil Extract Volume: 10000 (uL)
Lab Sample ID: 668549

Lab File ID: 668549

Date Received: 05/08/06

Date Analyzed: 05/10/06

Dilution Factor: 1.0

Soil Aliquot Volume:

500 (uL)

CAS NO.

COMPOUND

CONCENTRATION UNITS :

(ug/L or $\mathrm{ug} / \mathrm{Kg}$ ) UG/KG

\begin{tabular}{|c|c|c|}
\hline 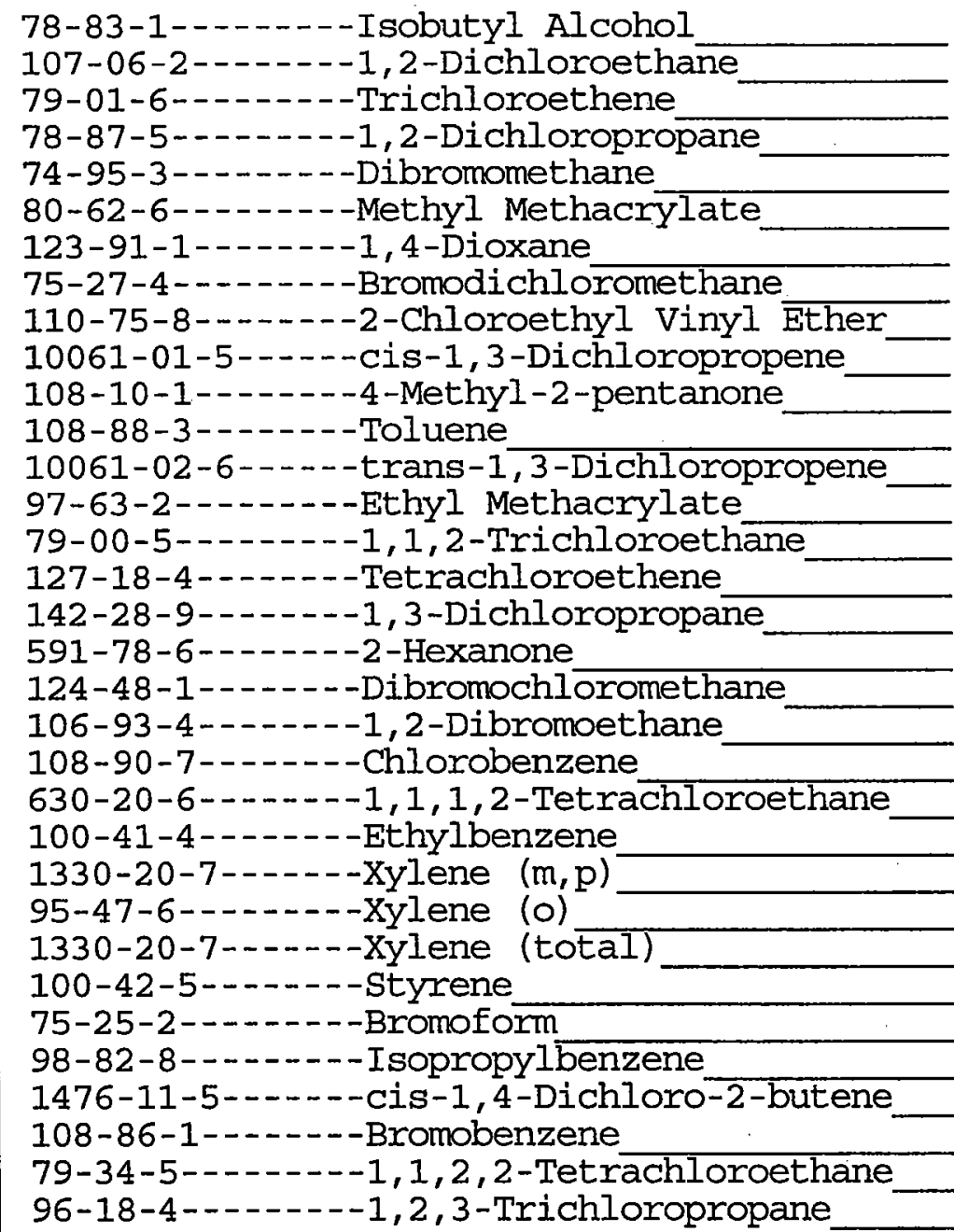 & $\begin{array}{r}500 \\
10 \\
10 \\
10 \\
10 \\
10 \\
500 \\
10 \\
10 \\
10 \\
50 \\
10 \\
10 \\
10 \\
10 \\
10 \\
10 \\
50 \\
10 \\
10 \\
10 \\
10 \\
10 \\
3.1 \\
10 \\
3.2 \\
10 \\
10 \\
10 \\
10 \\
10 \\
10 \\
10\end{array}$ & 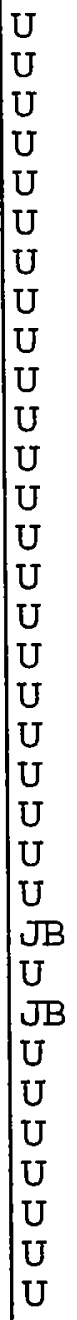 \\
\hline
\end{tabular}


FORM 1

VOLATILE ORGANICS ANALYSIS DATA SHEET

Lab Name: STL BURLINGTON

Contract: 21005

Lab Code: STLVT Case No.: NAVARRE SAS No.:

Lab Sample ID: 668549

Matrix: (soil/water) SOIL

Lab File ID: 668549

Sample wt/vol: $\quad 10.0(\mathrm{~g} / \mathrm{mL}) \mathrm{G}$

Date Received: 05/08/06

Level : (low/med) MED

\% Moisture: not dec.

GC Column: CAP

ID: $0.53 \quad(\mathrm{~mm})$

Soil Extract Volume: 10000 (uL)
ARGLAB SAMPLE NO.

NA-S-BLANK
Date Analyzed: 05/10/06

Dilution Factor: 1.0

Soil Aliquot Volume:

500 (uL)

CAS NO. CONCENTRATION UNITS :

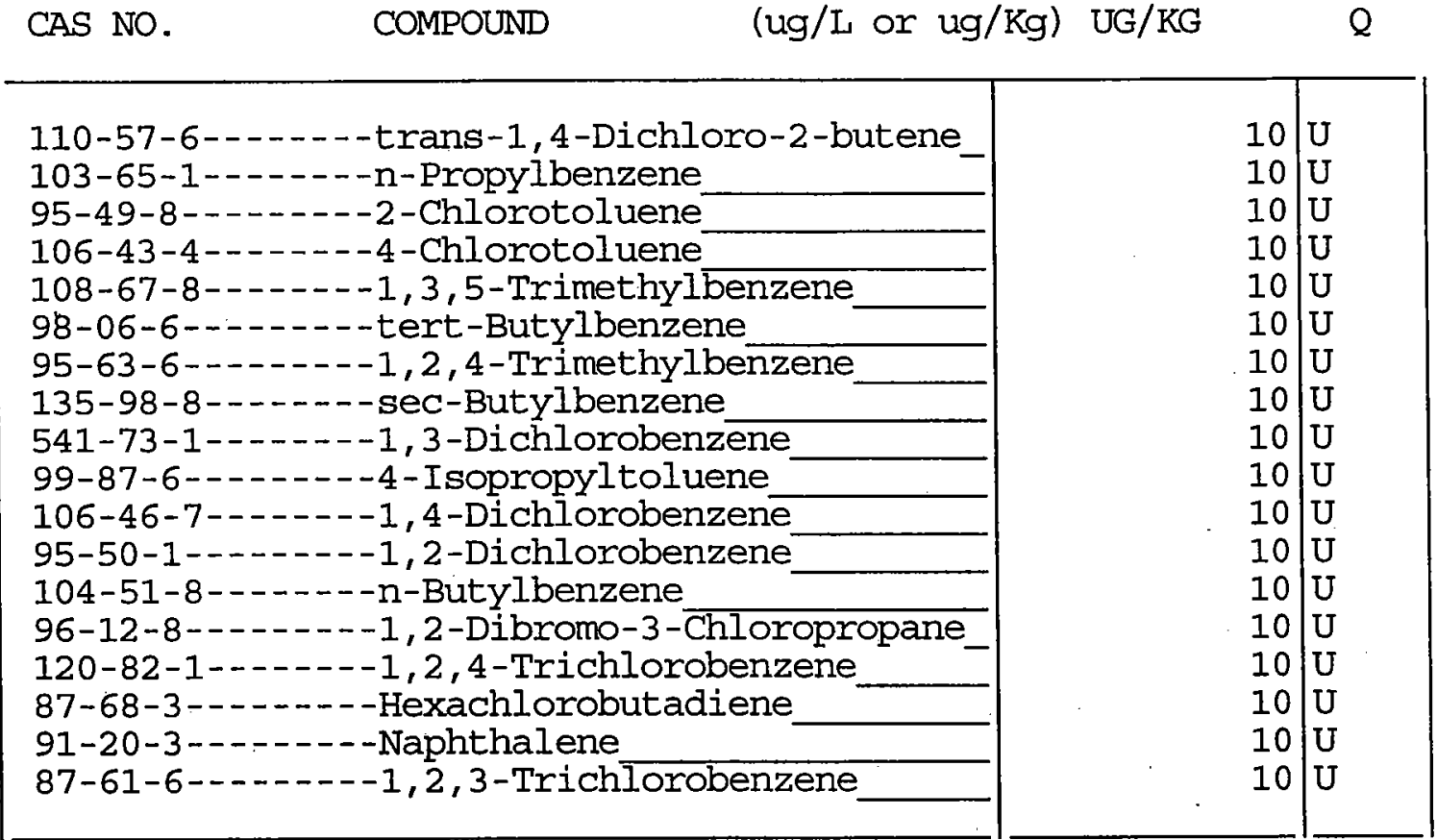


FORM 1

VOLATILE ORGANICS ANALYSIS DATA SHEET

Lab Name: STL BURLINGTON

Contract: 21005
ARGLAB SAMPLE NO.

NAS20390-10A

Lab Code: STLVT Case No.: NAVARRE SAS No.:

SDG No. : 114211

Matrix: (soil/water) SOIL

Sample wt/vol: $\quad 9.5(\mathrm{~g} / \mathrm{mL})$ G

Level: (low/med) MED

\% Moisture: not dec.

GC Column: CAP ID: 0.53 (mm)

Soil Extract Volume: 10000 (uL)
Lab Sample ID: 668546

Lab File ID: 668546

Date Received: 05/08/06

Date Analyzed: 05/10/06

Dilution Factor: 1.0

Soil Aliquot Volume:

500 (uL)

CAS NO. CONCENTRATION UNITS:

CAS NO. COMPOUND (ug/L or $\mathrm{ug} / \mathrm{Kg})$ UG/KG Q

\begin{tabular}{|c|c|c|}
\hline 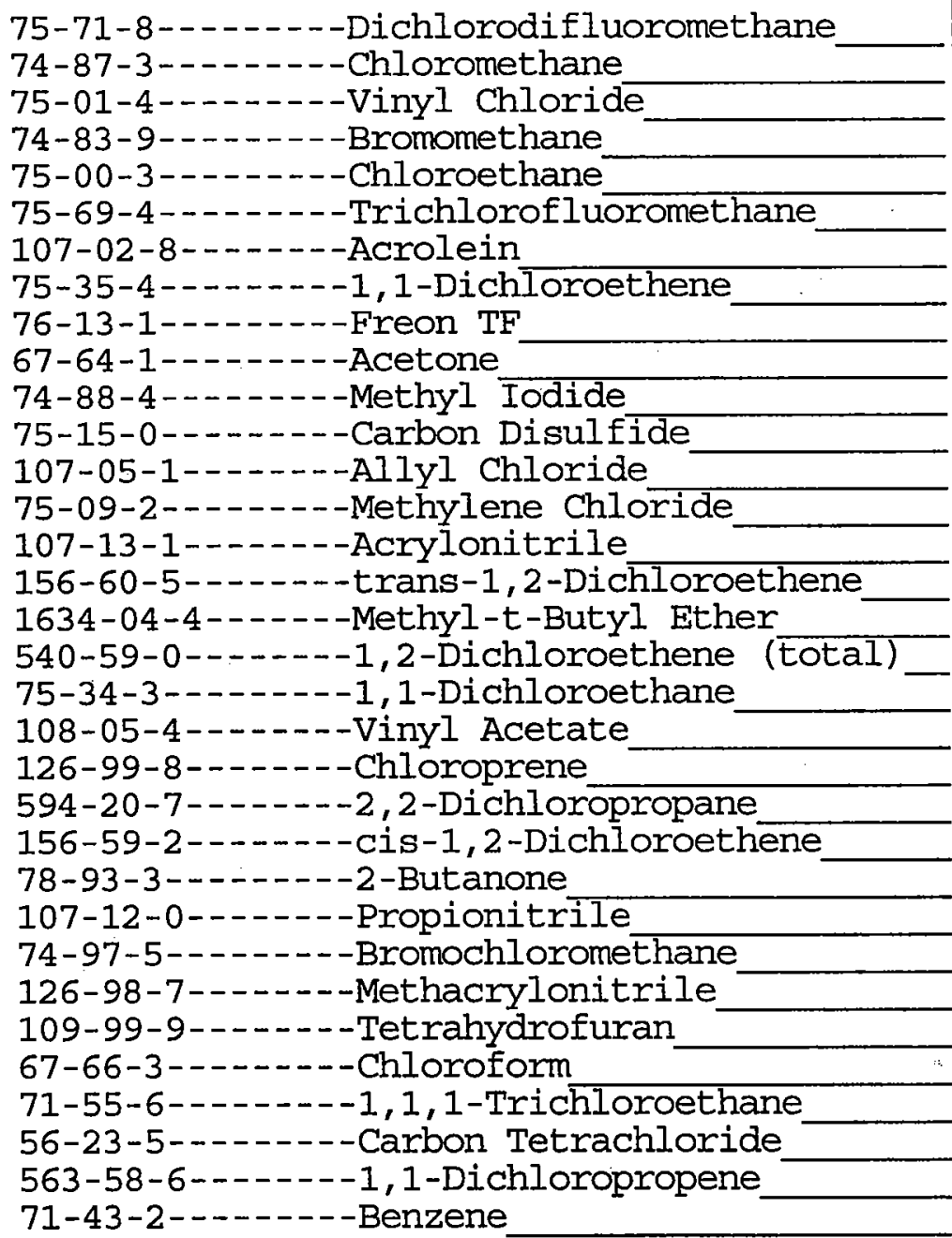 & $\begin{array}{r}11 \\
5.6 \\
11 \\
8.4 \\
11 \\
11 \\
52 \\
11 \\
11 \\
52 \\
7.6 \\
11 \\
11 \\
5.7 \\
11 \\
11 \\
11 \\
11 \\
11 \\
11 \\
11 \\
11 \\
11 \\
250 \\
42 \\
11 \\
11 \\
150 \\
11 \\
11 \\
11 \\
11 \\
11\end{array}$ & $\begin{array}{l}U \\
\mathrm{JB} \\
\mathrm{U} \\
\mathrm{JB} \\
\mathrm{U} \\
\mathrm{U} \\
\mathrm{U} \\
\mathrm{U} \\
\mathrm{U} \\
\mathrm{U} \\
\mathrm{JB} \\
\mathrm{U} \\
\mathrm{U} \\
\mathrm{J} \\
\mathrm{U} \\
\mathrm{U} \\
\mathrm{U} \\
\mathrm{U} \\
\mathrm{U} \\
\mathrm{U} \\
\mathrm{U} \\
\mathrm{U} \\
\mathrm{U} \\
\mathrm{B} \\
\mathrm{U} \\
\mathrm{U} \\
\mathrm{U} \\
\mathrm{U} \\
\mathrm{U} \\
\mathrm{U} \\
\mathrm{U} \\
\mathrm{U} \\
\mathrm{U}\end{array}$ \\
\hline
\end{tabular}


FORM 1

VOLATILE ORGANICS ANALYSIS DATA SHEET
Lab Name: STL BURLINGTON

Contract : 21005
ARGLAB SAMPLE NO.

NAS20390-10A

Lab Code: STLVT Case No.: NAVARRE SAS No.:

SDG No.: 114211

Matrix: (soil/water) soIL

Sample wt/vol:

$9.5(\mathrm{~g} / \mathrm{mL}) \mathrm{G}$

Level: (low/med) MED

$\%$ Moisture: not dec.

GC Column: CAP

ID: 0.53 (mm)

Soil Extract Volume: 10000 (uL)
Lab Sample ID: 668546

Lab File ID: $\quad 668546$

Date Received: 05/08/06

Date Analyzed: 05/10/06

Dilution Factor: 1.0

Soil Aliquot Volume:

$500(\mathrm{uL})$

CONCENTRATION UNITS:
$(u g / L$ or $\mathrm{ug} / \mathrm{Kg})$ UG/KG

$\begin{array}{ll} & \text { CONCENTRATION UNITS: } \\ \text { CAS NO. } & \text { COMPOUND }\end{array}$

\begin{tabular}{|c|c|c|}
\hline 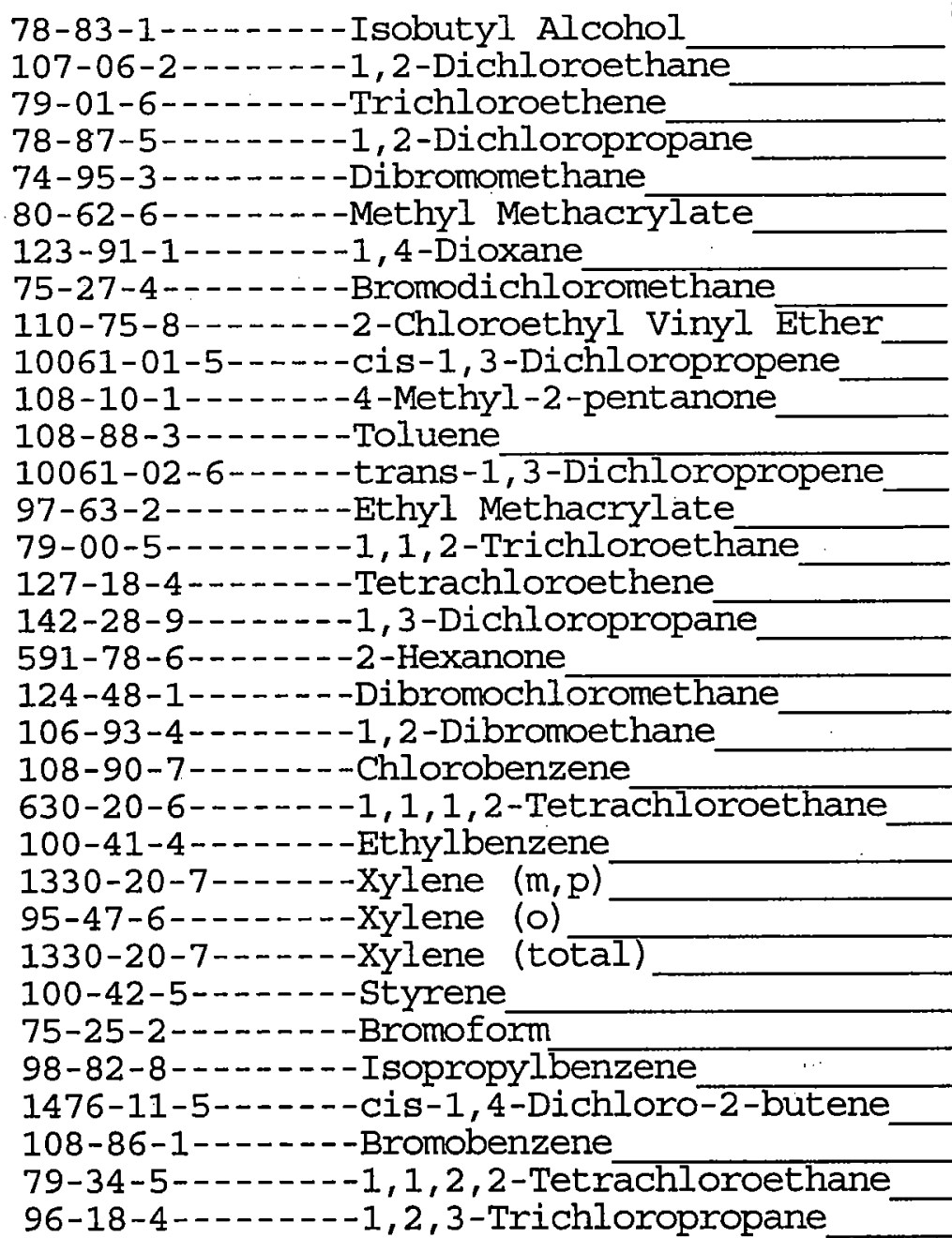 & $\begin{array}{r}530 \\
11 \\
11 \\
11 \\
11 \\
4.4 \\
530 \\
11 \\
11 \\
11 \\
52 \\
11 \\
11 \\
11 \\
11 \\
11 \\
11 \\
52 \\
11 \\
11 \\
11 \\
11 \\
11 \\
3.0 \\
11 \\
3.2 \\
11 \\
11 \\
11 \\
11 \\
11 \\
11 \\
11\end{array}$ & $\begin{array}{l}U \\
U \\
U \\
U \\
U \\
U \\
U \\
U \\
U \\
U \\
U \\
U \\
U \\
U \\
U \\
U \\
U \\
U \\
U \\
U \\
U \\
U \\
U \\
U \\
U \\
J B \\
U \\
U \\
U B \\
U \\
U \\
U \\
U \\
U \\
U \\
U \\
U\end{array}$ \\
\hline
\end{tabular}


FORM 1

VOLATILE ORGANICS ANALYSIS DATA SHEET
Lab Name: STL BURLINGTON

Contract : 21005
ARGLAB SAMPLE NO.

$$
\text { NAS20390-10A }
$$

Lab Code: STLVT Case No.: NAVARRE SAS No.:

SDG No. : 114211

Matrix: (soil/water) SOIL

Sample wt/vol: $\quad 9.5(\mathrm{~g} / \mathrm{mL}) \mathrm{G}$

Level : (low/med) MED

\% Moisture: not dec.

GC Column: CAP ID: $0.53 \quad(\mathrm{~mm})$

Soil Extract Volume: 10000 (uL)
Lab Sample ID: 668546

Lab File ID: 668546

Date Received: 05/08/06

Date Analyzed: 05/10/06

Dilution Factor: 1.0

Soil Aliquot Volume:

500 (uL)

CAS NO. CONCENTRATION UNITS:

CAS NO. COMPOUND $\quad(u g / L$ or $u g / K g) ~ U G / K G$

Q

$11 \mathrm{U}$

$11 \mathrm{U}$

$11 \mathrm{U}$

$11 \mathrm{U}$

$11 \mathrm{U}$

$11 \mathrm{U}$

$11 \mathrm{U}$

$11 \mathrm{U}$

$11 \mathrm{U}$

$11 \mathrm{U}$

$11 \mathrm{U}$

$11 \mathrm{U}$

$11 \mathrm{U}$

$11 \mathrm{U}$

$11 \mathrm{U}$

$11 \mathrm{U}$

$11 \mathrm{U}$

$11 \mathrm{U}$ 
FORM 1

VOLATILE ORGANICS ANALYSIS DATA SHEET

Lab Name: STL BURLINGTON

Contract : 21005
ARGLAB SAMPLE NO.

NAS2 0496-10A

Lab Code: STLVT Case No.: NAVARRE SAS No.:

SDG No.: 114211

Matrix: (soil/water) SOIL

Sample wt/vol: $\quad 11.1(\mathrm{~g} / \mathrm{mL}) \mathrm{G}$

Level: (low/med) MED

\% Moisture: not dec.

GC Column: CAP ID: 0.53 (mm)

Soil Extract Volume: 10000 (uL)
Lab Sample ID: 668547

Lab File ID: 668547

Date Received: 05/08/06

Date Analyzed: 05/10/06

Dilution Factor: 1.0

Soil Aliquot Volume:

500 (uL)
CAS NO. COMPOUND $\quad(u g / L$ or $\mathrm{ug} / \mathrm{Kg})$ UG/KG

$Q$

\begin{tabular}{|c|c|c|}
\hline 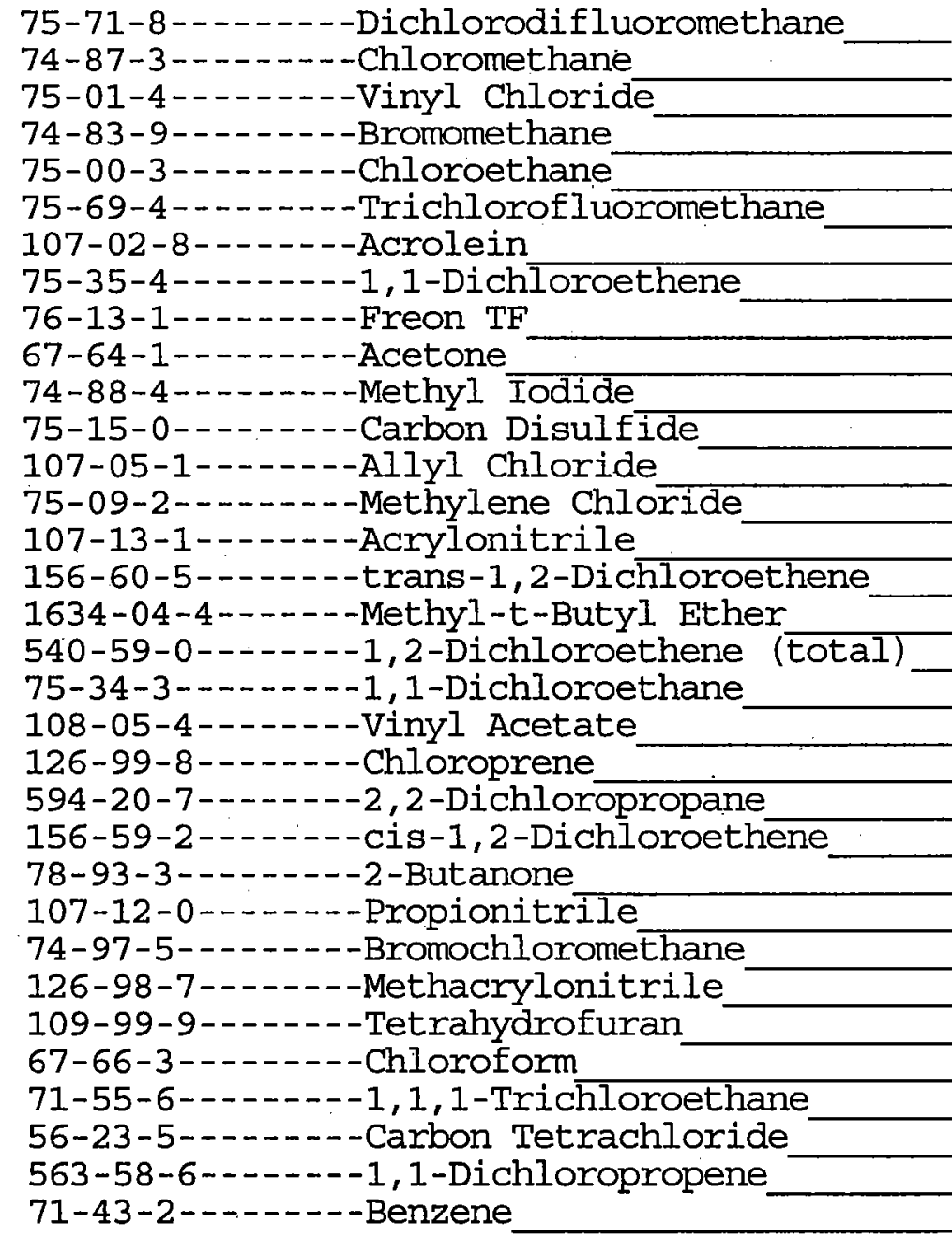 & $\begin{array}{r}9.0 \\
4.9 \\
9.0 \\
7.0 \\
9.0 \\
9.0 \\
45 \\
9.0 \\
9.0 \\
45 \\
6.8 \\
9.0 \\
9.0 \\
5.1 \\
9.0 \\
9.0 \\
9.0 \\
9.0 \\
9.0 \\
9.0 \\
9.0 \\
9.0 \\
9.0 \\
170 \\
36 \\
9.0 \\
9.0 \\
130 \\
9.0 \\
9.0 \\
9.0 \\
9.0 \\
9.0\end{array}$ & $\begin{array}{l}\text { U } \\
J B \\
U \\
J B \\
U \\
U \\
U \\
U \\
U \\
U \\
U \\
U B \\
U \\
U \\
J \\
U \\
U \\
U \\
U \\
U \\
U \\
U \\
U \\
U \\
U \\
B \\
U \\
U \\
U \\
U \\
U \\
U \\
U \\
U \\
U \\
U\end{array}$ \\
\hline
\end{tabular}


FORM 1

VOLATILE ORGANICS ANALYSIS DATA SHEET

Lab Name: STL BURLINGTON

Contract : 21005

ARGLAB SAMPLE NO.

Lab Code: STLVT Case No.: NAVARRE SAS No.:

NAS20496-10A

Matrix: (soil/water) SOIL

Lab Sample ID: 668547

Sample wt/vol:

$11.1(\mathrm{~g} / \mathrm{mL}) \mathrm{G}$

Lab File ID: 668547

Level: (low/med) MED

\% Moisture: not dec.

GC Column: CAP ID: 0.53 (mm)

Date Received: 05/08/06

Soil Extract Volume: 10000 (uL)

Date Analyzed: 05/10/06

Dilution Factor: 1.0

Soil Aliquot Volume:

500 (uL)

CONCENTRATION UNITS:

CAS NO. COMPOUND (ug/L or ug/ $\mathrm{kg}$ ) UG/KG

Q

\begin{tabular}{|c|c|c|}
\hline 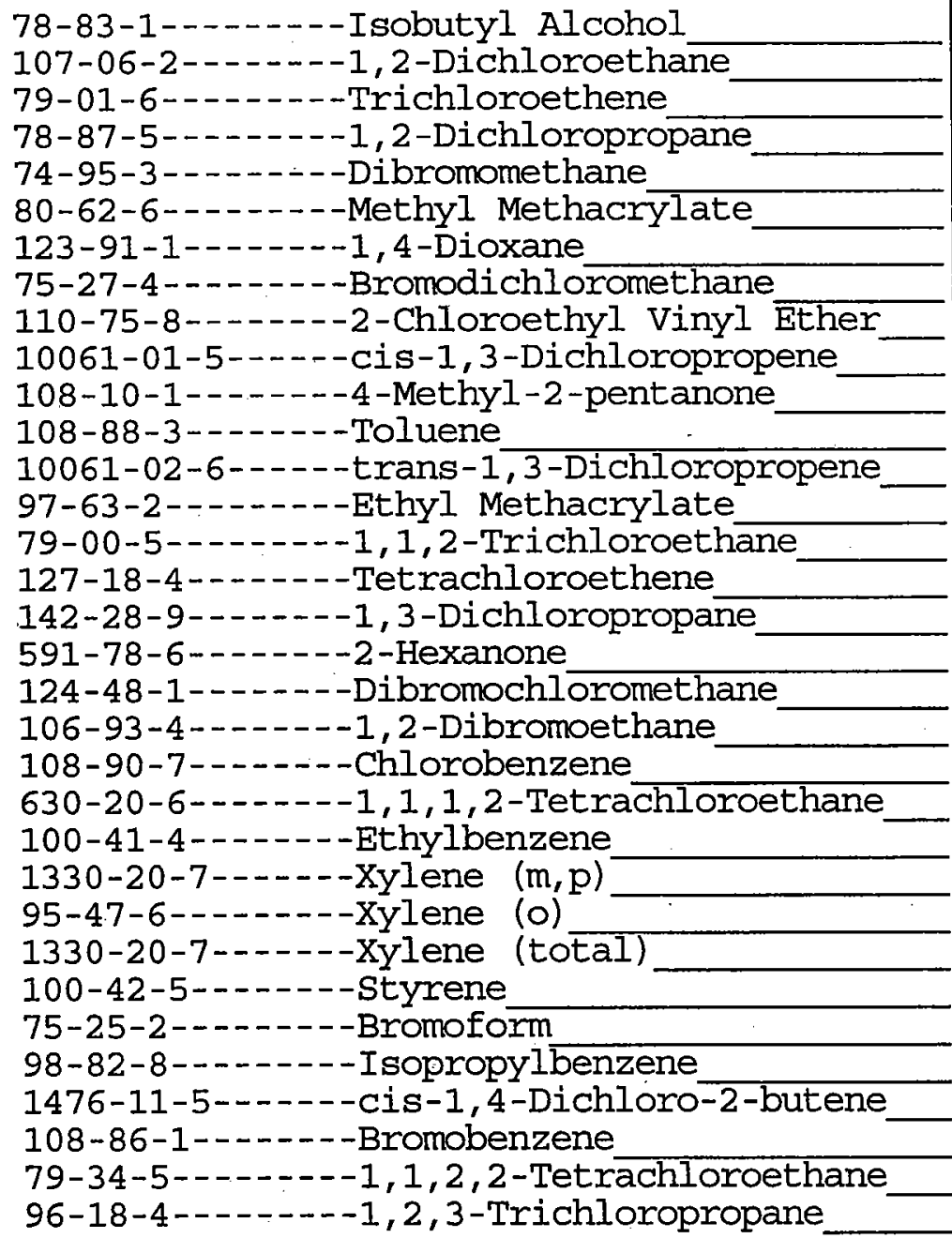 & $\begin{array}{r}450 \\
9.0 \\
9.0 \\
9.0 \\
9.0 \\
3.4 \\
450 \\
9.0 \\
9.0 \\
9.0 \\
45 \\
9.0 \\
9.0 \\
9.0 \\
9.0 \\
9.0 \\
9.0 \\
45 \\
9.0 \\
9.0 \\
9.0 \\
9.0 \\
9.0 \\
2.5 \\
9.0 \\
2.7 \\
9.0 \\
9.0 \\
9.0 \\
9.0 \\
9.0 \\
9.0 \\
9.0\end{array}$ & $\begin{array}{l}U \\
U \\
U \\
U \\
U \\
U \\
U \\
U \\
U \\
U \\
U \\
U \\
U \\
U \\
U \\
U \\
U \\
U \\
U \\
U \\
U \\
U \\
U \\
U \\
J B \\
U \\
J B \\
U \\
U \\
U \\
U \\
U \\
U \\
U \\
U\end{array}$ \\
\hline
\end{tabular}


FORM 1

VOLATILE ORGANICS ANALYSIS DATA SHEET

Lab Name: STL BURLINGTON

Contract : 21005
ARGLAB SAMPLE NO.

NAS20496-10A

Lab Code: STLVT Case No.: NAVARRE SAS No.:

SDG No. : 114211

Matrix: (soil/water) SOIL

Lab Sample ID: 668547

Sample wt/vol:

$11.1(\mathrm{~g} / \mathrm{mL}) \mathrm{G}$

Lab File ID: $\quad 668547$

Level: (low/med) MED

Date Received: 05/08/06

\% Moisture: not dec.

GC Column: CAP

ID: $0.53(\mathrm{~mm})$

Date Analyzed: 05/10/06

Soil Extract Volume: 10000 (UL)

Dilution Factor: 1.0

Soil Aliquot Volume:

500 (uس)

CAS NO.

COMPOUND

CONCENTRATION UNITS:

(ug/L or $\mathrm{ug} / \mathrm{Kg}$ ) UG/KG

$Q$

\begin{tabular}{|c|c|c|}
\hline 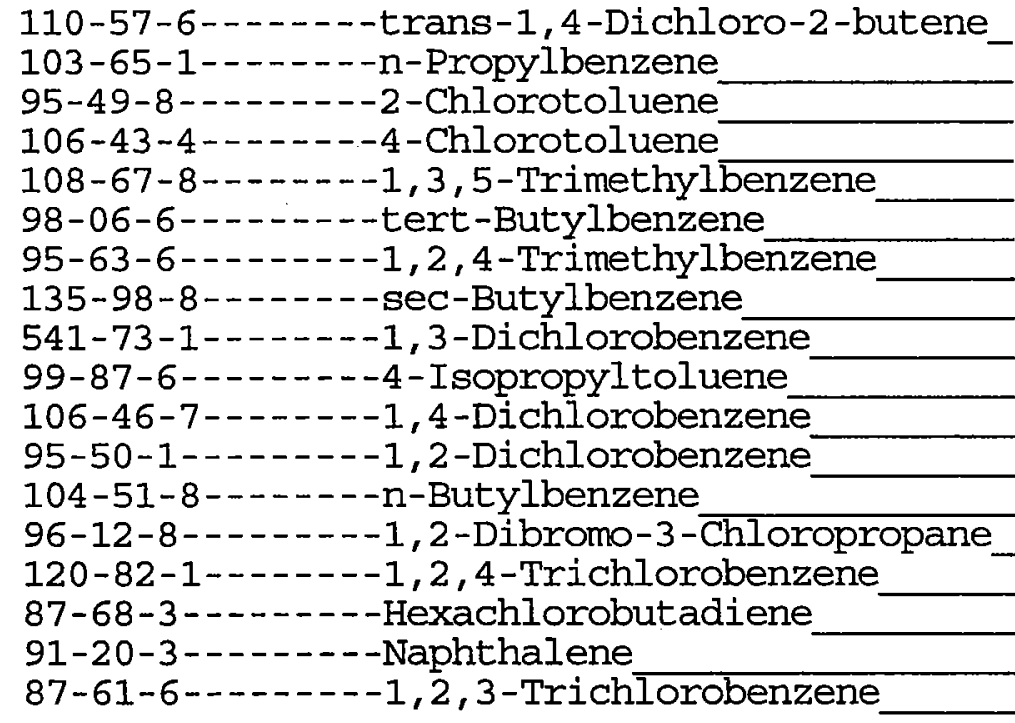 & $\begin{array}{l}9.0 \\
9.0 \\
9.0 \\
9.0 \\
9.0 \\
9.0 \\
9.0 \\
9.0 \\
9.0 \\
9.0 \\
9.0 \\
9.0 \\
9.0 \\
9.0 \\
9.0 \\
9.0 \\
9.0 \\
9.0\end{array}$ & \begin{tabular}{|l}
$\mathrm{U}$ \\
$\mathrm{U}$ \\
$\mathrm{U}$ \\
$\mathrm{U}$ \\
$\mathrm{U}$ \\
$\mathrm{U}$ \\
$\mathrm{U}$ \\
$\mathrm{U}$ \\
$\mathrm{U}$ \\
$\mathrm{U}$ \\
$\mathrm{U}$ \\
$\mathrm{U}$ \\
$\mathrm{U}$ \\
$\mathrm{U}$ \\
$\mathrm{U}$ \\
$\mathrm{U}$ \\
$\mathrm{U}$ \\
$\mathrm{U}$
\end{tabular} \\
\hline
\end{tabular}


FORM 1

VOLATILE ORGANICS ANALYSIS DATA SHEET
Lab Name: STL BURLINGTON

Contract: 21005
ARGLLAB SAMPLE NO.

NAS20511-10A

Lab Code: STLVT Case No.: NAVARRE SAS No.:

SDG No.: 114211

Matrix: (soil/water) SOIL

Sample wt/vol:

$10.5(\mathrm{~g} / \mathrm{mL}) \mathrm{G}$

Level: (low/med) MED

$\because$ Moisture: not dec.

GC Column: CAP

ID $: 0.53(\mathrm{~mm})$

Soil Extract Volume: 10000 (u山)
Lab Sample ID: 668543

Lab File ID: $\quad 668543$

Date Received: 05/08/06

Date Analyzed: 05/10/06

Dilution Factor: 1.0

Soil Aliquot Volume:

500 (uL)

CAS NO.

COMPOUND

CONCENTRATION UNITS :

(ug/L or $u g / \mathrm{Kg}$ ) UG/KG

Q

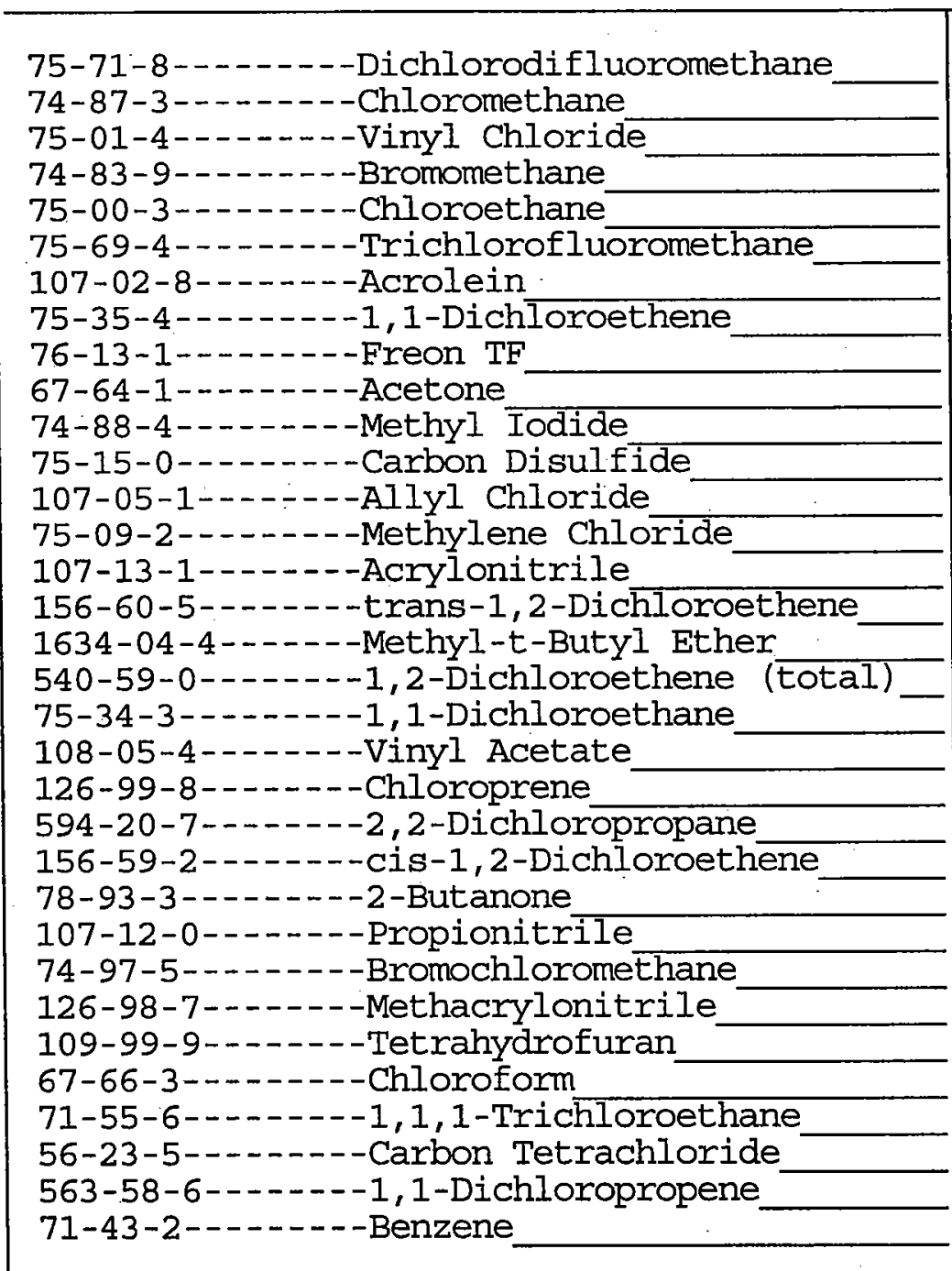

$9.5 \mathrm{U}$

$8.4 \mathrm{JB}$

$9.5 \mathrm{U}$

$13 \mathrm{~B}$

$9.5 \mathrm{U}$

$9.5 \mathrm{U}$

$47 \mathrm{U}$

$9.5 \mathrm{U}$

$9.5 \mathrm{U}$

$47 \mathrm{U}$

$12 \mathrm{~B}$

$9.5 \mathrm{U}$

$9.5 \mathrm{U}$

$4.6 \mathrm{~J}$

$9.5 \mathrm{U}$

$9.5 \mathrm{U}$

$9.5 \mathrm{U}$

$9.5 \mathrm{U}$

$9.5 \mathrm{U}$

$9.5 \mathrm{U}$

$9.5 \mathrm{U}$

$9.5 \mathrm{U}$

$9.5 \mathrm{U}$

200 B

$38 \mathrm{U}$

$9.5 \mathrm{U}$

$9.5 \mathrm{U}$

$130 \mathrm{U}$

$9.5 \mathrm{U}$

$9.5 \mathrm{U}$

$9.5 \mathrm{U}$

$9.5 \mathrm{U}$

$9.5 \mathrm{U}$ 
FORM 1

VOLATILE ORGANICS ANALYSIS DATA SHEET
Lab Name: STL BURLINGTON

Contract: 21005
ARGLAB SAMPLE NO.

NAS20511-10A

Lab Code: STLVT Case No.: NAVARRE SAS No.:

SDG No. : 114211

Matrix: (soil/water) SOIL

Sample wt/vol: $\quad 10.5(\mathrm{~g} / \mathrm{mL}) \mathrm{G}$

Level: (low/med) MED

$\%$ Moisture: not dec.

GC Column: CAP ID: 0.53 (mm)

Soil Extract Volume: 10000 (uL)
Lab Sample ID: 668543

Lab File ID: 668543

Date Received: 05/08/06

Date Analyzed: 05/10/06

Dilution Factor: 1.0

Soil Aliquot Volume:

500 (ur)

CAS NO. COMPOUND (ug/L or ug/Kg) UG/KG $Q$

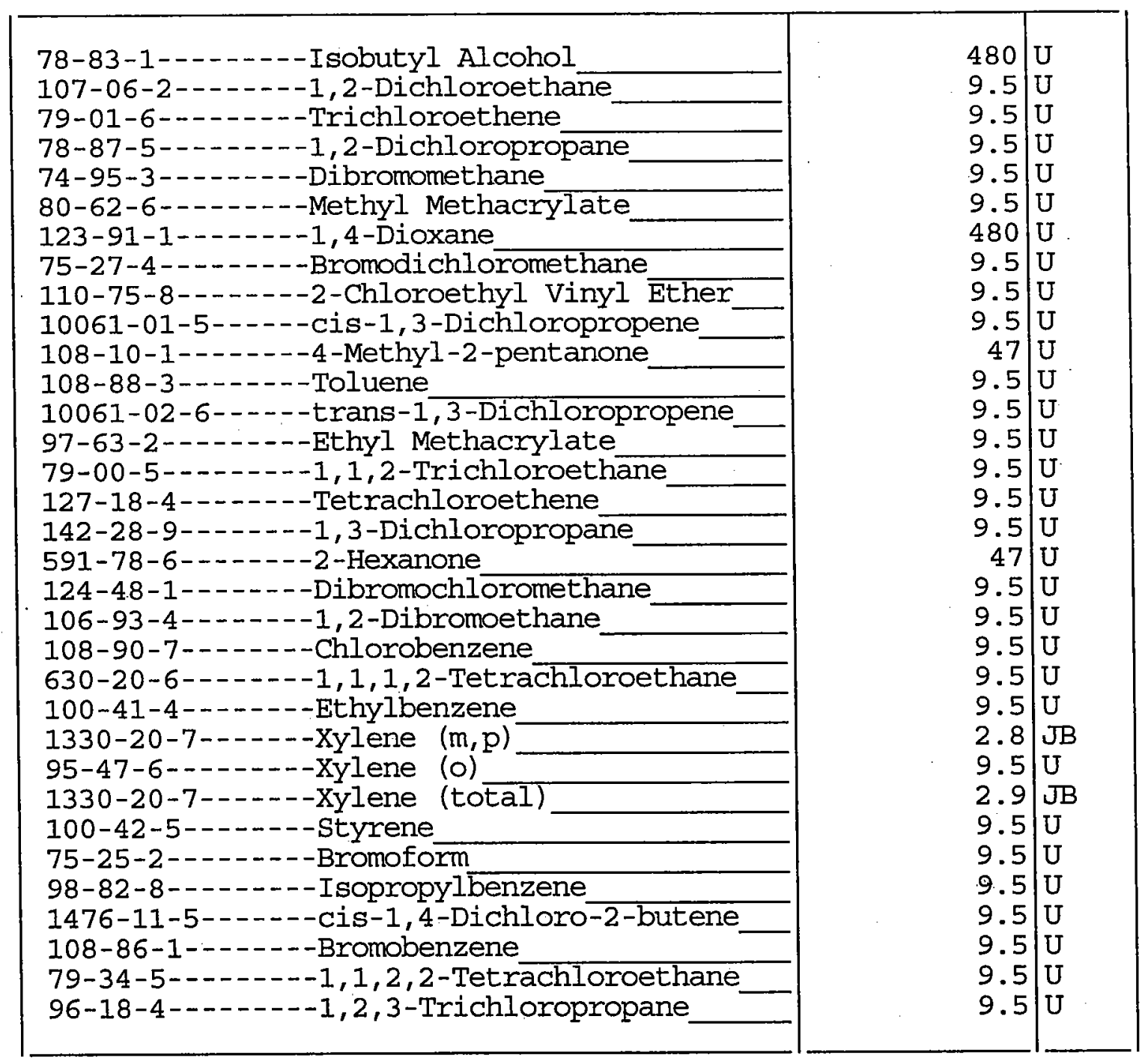


FORM 1

VOLATILE ORGANICS ANALYSIS DATA SHEET
Lab Name: STL BURLINGTON

Contract: 21005
ARGLAB SAMPLE NO.

$$
\text { NAS20511-10A }
$$

Lab Code: STLVT Case No.: NAVARRE SAS No.:

SDG No. : 114211

Matrix: (soil/water) SOIL

Sample wt/vol: $\quad 10.5(\mathrm{~g} / \mathrm{mL})$ G

Level: (low/med) MED

\% Moisture: not dec.

GC Column: CAP ID: 0.53 (mm)

Soil Extract Volume: 10000 (uL)
Lab Sample ID: 668543

Lab File ID: 668543

Date Received: 05/08/06

Date Analyzed: 05/10/06

Dilution Factor: 1.0

Soil Aliquot Volume:

500 (uL)

CAS NO. CONCENTRATION UNITS:

CAS NO. COMPOUND (ug/L or $\mathrm{ug} / \mathrm{Kg})$ UG/KG

Q

$9.5 \mathrm{U}$

$9.5 \mathrm{U}$

$9.5 \mathrm{U}$

$9.5 \mathrm{U}$

$9.5 \mathrm{U}$

$9.5 \mathrm{U}$

$9.5 \mathrm{U}$

$9.5 \mathrm{U}$

$9.5 \mathrm{U}$

$9.5 \mathrm{U}$

$9.5 \mathrm{U}$

$9.5 \mathrm{U}$

$9.5 \mathrm{U}$

$9.5 \mathrm{U}$

$9.5 \mathrm{U}$

$9.5 \mathrm{U}$

$9.5 \mathrm{U}$

$9.5 \mathrm{U}$ 
FORM 1

VOLATILE ORGANICS ANALYSIS DATA SHEET
Lab Name: STL BURLINGTON

Contract : 21005
ARGLAB SAMPLE NO.

NAS20527-9A

Lab Code: STLVT Case No.: NAVARRE SAS No.:

SDG No.: 114211

Matrix: (soil/water) SOIL

Sample wt/vol: $\quad 11.1(\mathrm{~g} / \mathrm{mL}) \mathrm{G}$

Level: (low/med) MED

$\%$ Moisture: not dec.

GC Column: CAP

Soil Extract Volume: 10000 (uL)
Lab Sample ID: 668542

Lab File ID: $\quad 668542$

Date Received: 05/08/06

Date Analyzed: 05/10/06

Dilution Factor: 1.0

Soil Aliquot Volume:

500 (uL)

(ug/L or ug/Kg) UG/KG Q

\begin{tabular}{|c|c|c|}
\hline 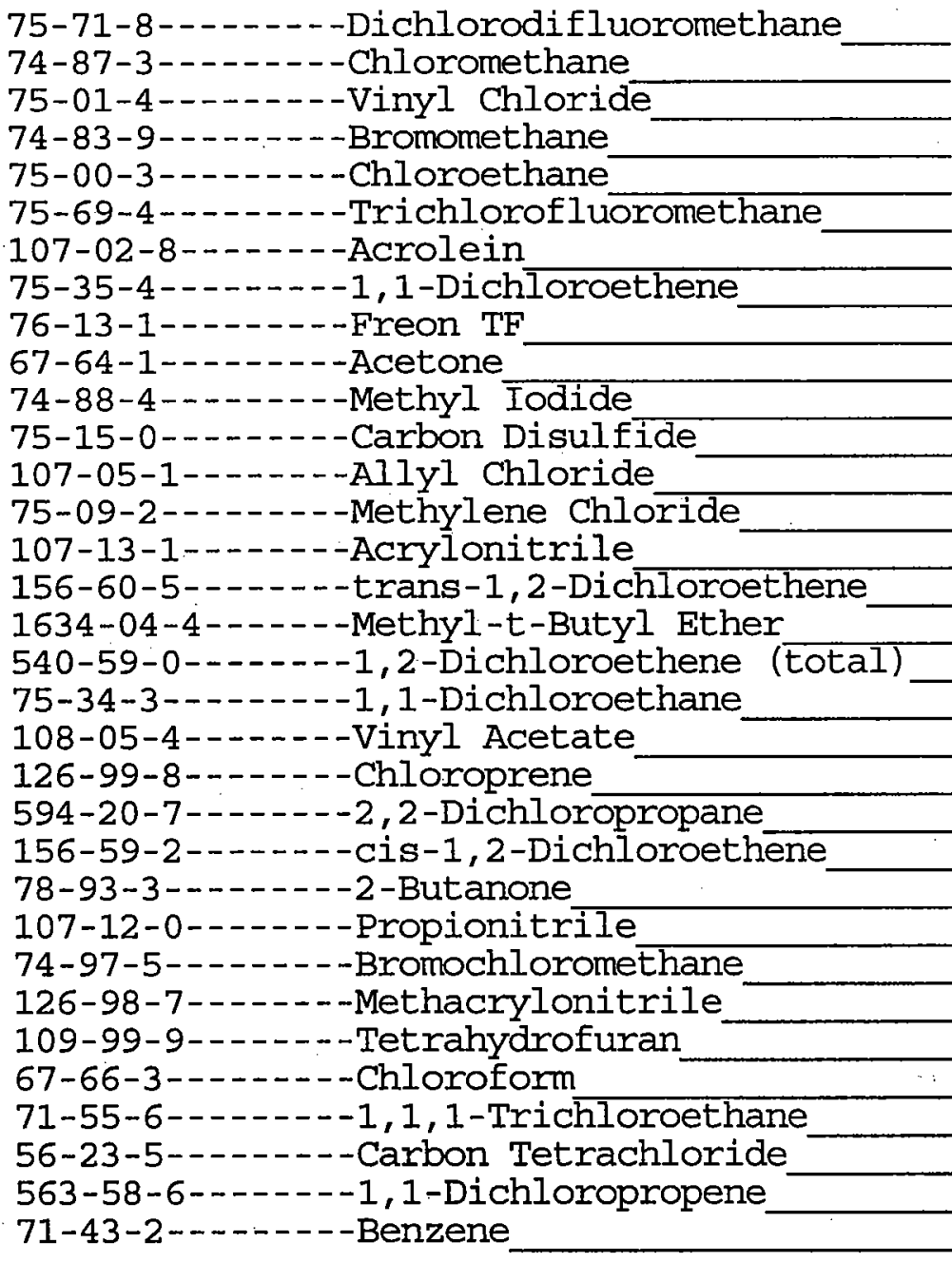 & $\begin{array}{r}9.0 \\
13 \\
9.0 \\
16 \\
9.0 \\
9.0 \\
45 \\
9.0 \\
9.0 \\
45 \\
16 \\
9.0 \\
9.0 \\
3.6 \\
9.0 \\
9.0 \\
9.0 \\
9.0 \\
9.0 \\
9.0 \\
9.0 \\
9.0 \\
9.0 \\
220 \\
36 \\
9.0 \\
9.0 \\
130 \\
9.0 \\
9.0 \\
9.0 \\
9.0 \\
9.0\end{array}$ & $\begin{array}{l}\mathrm{U} \\
\mathrm{B} \\
\mathrm{U} \\
\mathrm{B} \\
\mathrm{U} \\
\mathrm{U} \\
\mathrm{U} \\
\mathrm{U} \\
\mathrm{U} \\
\mathrm{U} \\
\mathrm{B} \\
\mathrm{U} \\
\mathrm{U} \\
\mathrm{J} \\
\mathrm{U} \\
\mathrm{U} \\
\mathrm{U} \\
\mathrm{U} \\
\mathrm{U} \\
\mathrm{U} \\
\mathrm{U} \\
\mathrm{U} \\
\mathrm{U} \\
\mathrm{B} \\
\mathrm{U} \\
\mathrm{U} \\
\mathrm{U} \\
\mathrm{U} \\
\mathrm{U} \\
\mathrm{U} \\
\mathrm{U} \\
\mathrm{U} \\
\mathrm{U}\end{array}$ \\
\hline
\end{tabular}


FORM 1

VOLATILE ORGANICS ANALYSIS DATA SHEET

Lab Name: STL BURLINGTON

Contract: 21005
ARGLAB SAMPLE NO.

NAS20527-9A

Lab Code: STLVT Case No.: NAVARRE SAS No.:

SDG No. : 114211

Matrix: (soil/water) SOIL

Sample wt/vol: $\quad 11.1(\mathrm{~g} / \mathrm{mL}) \mathrm{G}$

Level: (low/med) MED

\% Moisture: not dec.

GC Column: CAP ID: 0.53 (mm)

Soil Extract Volume: 10000 (uL)
Lab Sample ID: 668542

Lab File ID: 668542

Date Received: 05/08/06

Date Analyzed: 05/10/06

Dilution Factor: 1.0

Soil Aliquot Volume:

500 (UL)

CAS NO. COMPOUND $\quad(u g / L$ or $\mathrm{ug} / \mathrm{Kg}) \quad \mathrm{UG} / \mathrm{KG}$

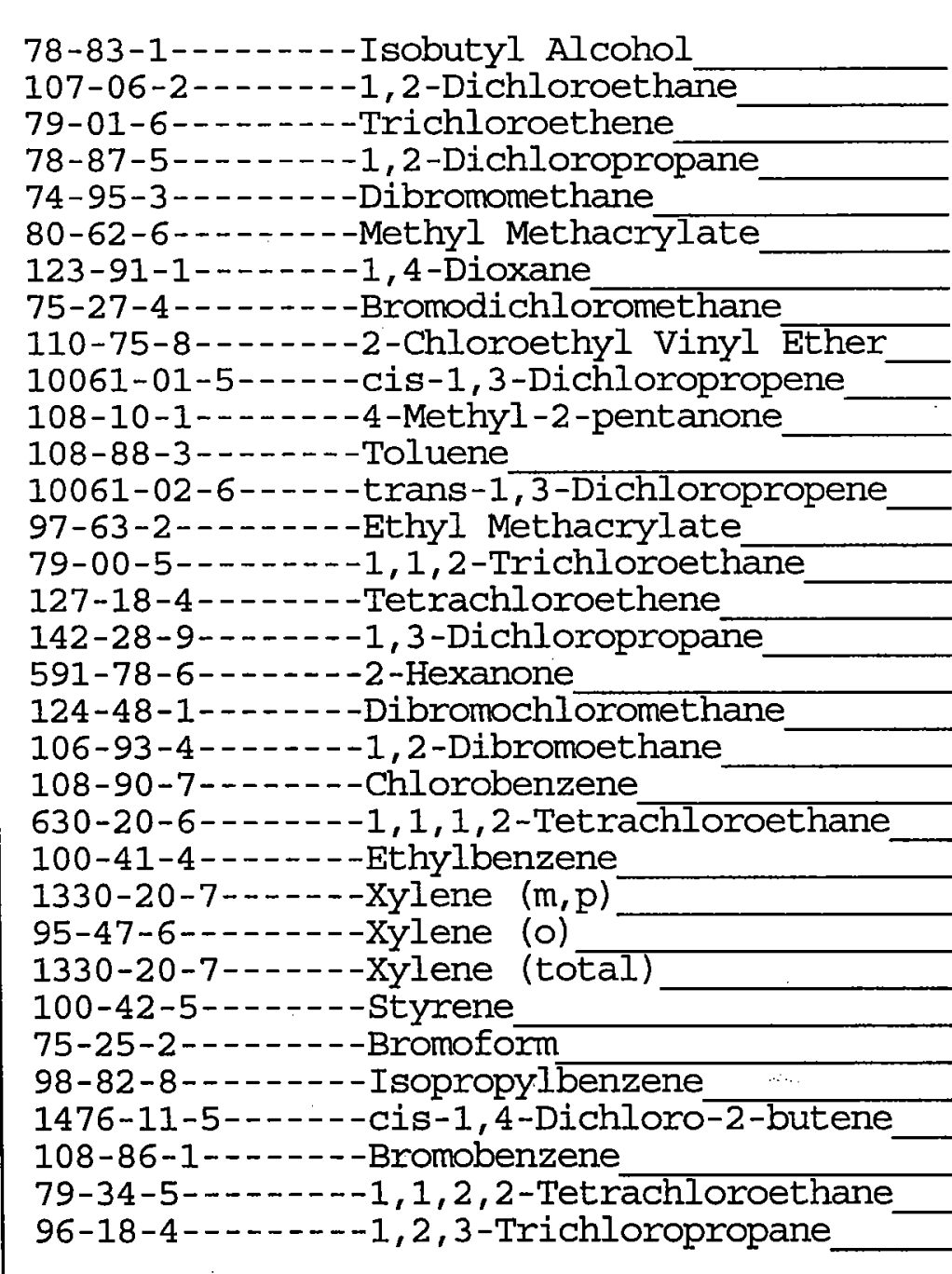


FORM 1

VOLATILE ORGANICS ANALYSIS DATA SHEET

Lab Name: STL BURLINGTON

Contract: 21005

ARGLAB SAMPLE NO.

Lab Code: STLVT Case No.: NAVARRE SAS No.:

SDG No.: 114211

Matrix: (soil/water) SOIL

Lab Sample ID: 668542

Sample wt/vol:

$11.1(\mathrm{~g} / \mathrm{mL}) \quad \mathrm{G}$

Lab File ID: 668542

Level: (low/med) MED

Date Received: 05/08/06

\% Moisture: not dec.

Date Analyzed: 05/10/06

GC Column: CAP

ID: $0.53 \quad(\mathrm{~mm})$

Dilution Factor: 1.0

Soil Extract Volume: 10000 (uL)

Soil Aliquot Volume:

500 (uL)

CONCENTRATION UNITS :

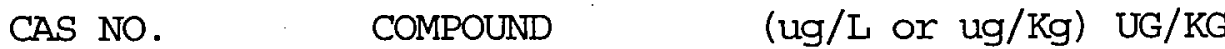

Q

$9.0 \mathrm{U}$

$9.0 \mathrm{U}$

$9.0 \mathrm{U}$

9.0 U

$9.0 \mathrm{U}$

$9.0 \mathrm{U}$

$9.0 \mathrm{U}$

$9.0 \mathrm{U}$

$9.0 \mathrm{U}$

$9.0 \mathrm{U}$

$9.0 \mathrm{U}$

$9.0 \mathrm{U}$

$9.0 \mathrm{U}$

$9.0 \mathrm{U}$

$3.6 \mathrm{~J}$

$9.0 \mathrm{U}$

$3.2 \mathrm{~J}$

$3.4 \mathrm{~J}$ 
FORM 1

VOLATILE ORGANICS ANALYSIS DATA SHEET

Lab Name: STL BURLINGTON

Contract: 21005
ARGLAB SAMPLE NO.

NAS2 $0603-30 A$

Lab Code: STLVT Case No.: NAVARRE SAS No.:

SDG No.: 114211

Matrix: (soil/water) SOIL

Sample wt/vol: $\quad 10.6(\mathrm{~g} / \mathrm{mL}) \mathrm{G}$

Level: (low/med) MED

\% Moisture: not dec.

GC Column: CAP ID: 0.53 (mm)

Soil Extract Volume: 10000 (uL)
Lab Sample ID: 668544

Lab File ID: 668544

Date Received: 05/08/06

Date Analyzed: 05/10/06

Dilution Factor: 1.0

Soil Aliquot Volume:

500 (uس)

CAS NO

CAS NO. COMPOUND (ug/L or $\mathrm{ug} / \mathrm{Kg})$ UG/KG Q

\begin{tabular}{|c|c|c|}
\hline 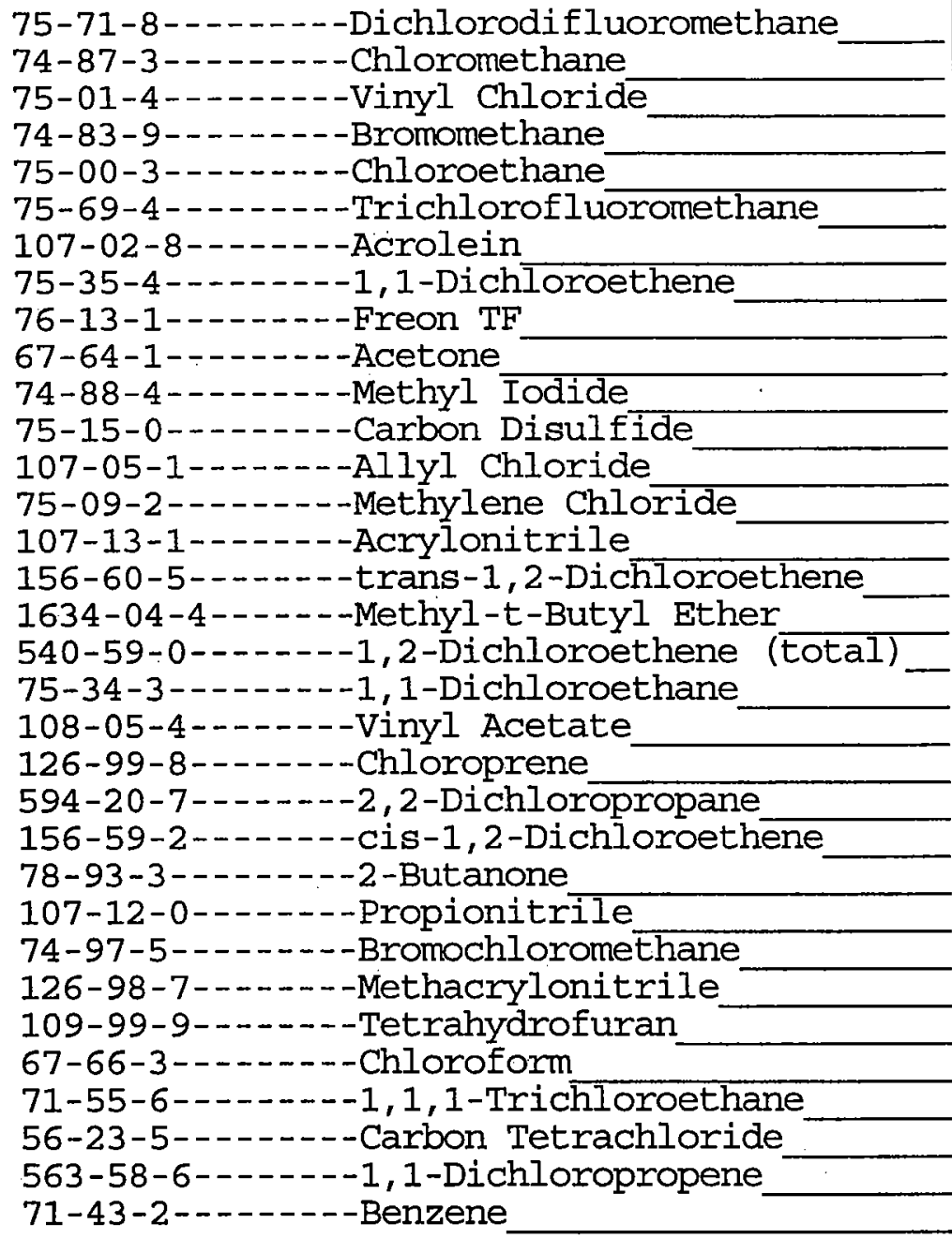 & $\begin{array}{r}9.5 \\
5.6 \\
9.5 \\
11 \\
9.5 \\
9.5 \\
47 \\
9.5 \\
9.5 \\
47 \\
11 \\
9.5 \\
9.5 \\
4.3 \\
9.5 \\
9.5 \\
9.5 \\
9.5 \\
9.5 \\
9.5 \\
9.5 \\
9.5 \\
9.5 \\
180 \\
38 \\
9.5 \\
9.5 \\
130 \\
9.5 \\
9.5 \\
3.5 \\
9.5 \\
9.5\end{array}$ & $\begin{array}{l}U \\
J B \\
U \\
B \\
U \\
U \\
U \\
U \\
U \\
U \\
B \\
U \\
U \\
J \\
U \\
U \\
U \\
U \\
U \\
U \\
U \\
U \\
U \\
B \\
U \\
U \\
U \\
U \\
U \\
U \\
U \\
U \\
U \\
U\end{array}$ \\
\hline
\end{tabular}


FORM 1

VOLATILE ORGANICS ANALYSIS DATA SHEET

Lab Name: STL BURLINGTON

Contract : 21005
ARGLAB SAMPLE NO.

NAS20603-30A

Lab Code: STLVT Case No.: NAVARRE SAS No.:

SDG No.: 114211

Matrix: (soil/water) sOIL

Sample wt/vol : $\quad 10.6(\mathrm{~g} / \mathrm{mL}) \mathrm{G}$

Level : (low/med) MED

\% Moisture: not dec.

GC Column: CAP ID: 0.53 (mm)

Soil Extract Volume: 10000 (UL)
Lab Sample ID: 668544

Lab File ID: $\quad 668544$

Date Received: 05/08/06

Date Analyzed: 05/10/06

Dilution Factor: 1.0

Soil Aliquot Volume: 500 (UL)

CAS NO.

COMPOUND

CONCENTRATION UNITS:

(ug/L or $u g / \mathrm{Kg}$ ) UG/KG

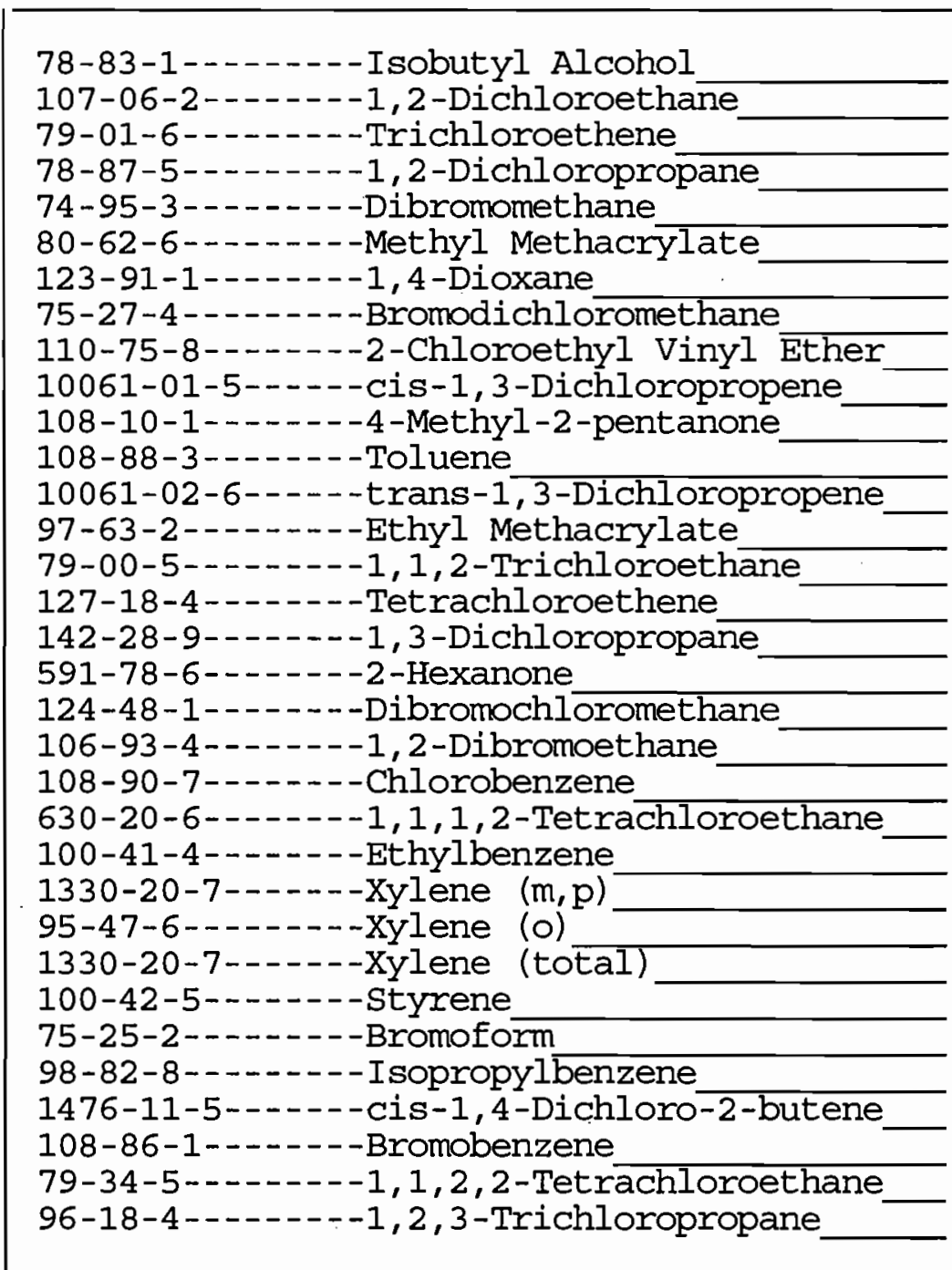

$470 \mathrm{U}$

$9.5 \mathrm{U}$

$9.5 \mathrm{U}$

$9.5 \mathrm{U}$

$9.5 \mathrm{U}$

$9.5 \mathrm{U}$

$470 \mathrm{U}$

$9.5 \mathrm{U}$

$9.5 \mathrm{U}$

$9.5 \mathrm{U}$

$47 \mathrm{U}$

$9.5 \mathrm{U}$

$9.5 \mathrm{U}$

$9.5 \mathrm{U}$

$9.5 \mathrm{U}$

$9.5 \mathrm{U}$

$9.5 \mathrm{U}$

$47 \mathrm{U}$

$9.5 \mathrm{U}$

$9.5 \mathrm{U}$

$9.5 \mathrm{U}$

$9.5 \mathrm{U}$

$9.5 \mathrm{U}$

2.9 JB

$9.5 \mathrm{U}$

3.1 JB

$9.5 \mathrm{U}$

$9.5 \mathrm{U}$

$9.5 \mathrm{U}$

$9.5 \mathrm{U}$

$9.5 \mathrm{U}$

$9.5 \mathrm{U}$

$9.5 \mathrm{U}$ 
FORM 1

VOLATILE ORGANICS ANALYSIS DATA SHEET

Lab Name: STL BURLINGTON

Contract : 21005

ARGLAB SAMPLE NO.

Lab Code: STLVT Case No.: NAVARRE SAS No.:

NAS20603-30A

Matrix: (soil/water) SOIL

Lab Sample ID: 668544

Sample wt/vol:

$10.6(\mathrm{~g} / \mathrm{mL}) \mathrm{G}$

Lab File ID: 668544

Level : (low/med) MED

Date Received: 05/08/06

$\%$ Moisture: not dec.

GC Column: CAP. ID: 0.53 (mm)

Date Analyzed: 05/10/06

Soil Extract Volume: 10000 (uL)

Dilution Factor: 1.0

Soil Aliquot Volume:

500 (uL)

CONCENTRATION UNITS :

CAS NO. COMPOUND (ug/L or ug/Kg) UG/KG Q

\begin{tabular}{|c|c|c|}
\hline 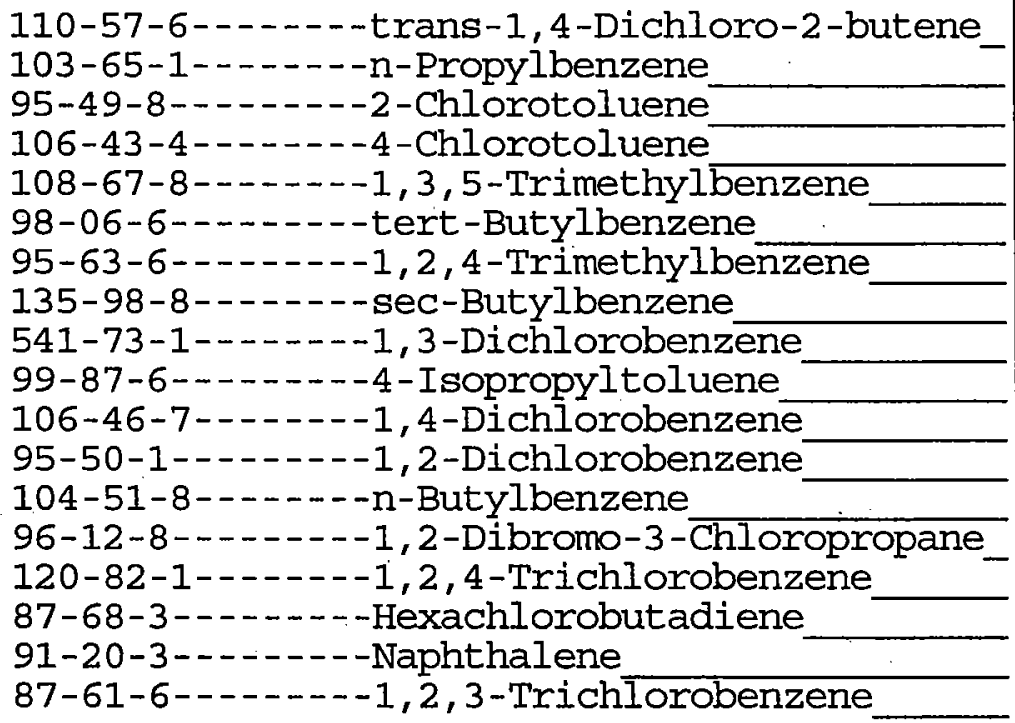 & $\begin{array}{l}9.5 \\
9 \cdot 5 \\
9.5 \\
9.5 \\
9.5 \\
9.5 \\
9.5 \\
9.5 \\
9.5 \\
9.5 \\
9.5 \\
9.5 \\
9.5 \\
9.5 \\
9.5 \\
9.5 \\
9.5 \\
9.5\end{array}$ & \begin{tabular}{|l}
$\mathrm{U}$ \\
$\mathrm{U}$ \\
$\mathrm{U}$ \\
$\mathrm{U}$ \\
$\mathrm{U}$ \\
$\mathrm{U}$ \\
$\mathrm{U}$ \\
$\mathrm{U}$ \\
$\mathrm{U}$ \\
$\mathrm{U}$ \\
$\mathrm{U}$ \\
$\mathrm{U}$ \\
$\mathrm{U}$ \\
$\mathrm{U}$ \\
$\mathrm{U}$ \\
$\mathrm{U}$ \\
$\mathrm{U}$ \\
$\mathrm{U}$
\end{tabular} \\
\hline
\end{tabular}


FORM 1

VOLATILE ORGANICS ANALYSIS DATA SHEET

Lab Name: STL BURLINGTON

Contract: 21005
ARGLAB SAMPLE NO.

NAS20607-20A

Lab Code: STLVT Case No.: NAVARRE SAS No.:

SDG No. : 114211

Matrix: (soil/water) SOIL

Sample wt/vol: $\quad 9.8(\mathrm{~g} / \mathrm{mL}) \mathrm{G}$

Level: $\quad($ low $/ \mathrm{med}) \quad$ MED

$\%$ Moisture: not dec.

GC Column: CAP ID: 0.53 (mm)

Soil Extract Volume: 10000 (u山)
Lab Sample ID: 668548

Lab File ID: 668548

Date Received: 05/08/06

Date Analyzed: 05/10/06

Dilution Factor: 1.0

Soil Aliquot Volume:

$500(u L)$

CAS NO.

COMPOUND

(ug/L or $u g / \mathrm{Kg}$ ) UG/KG

$Q$

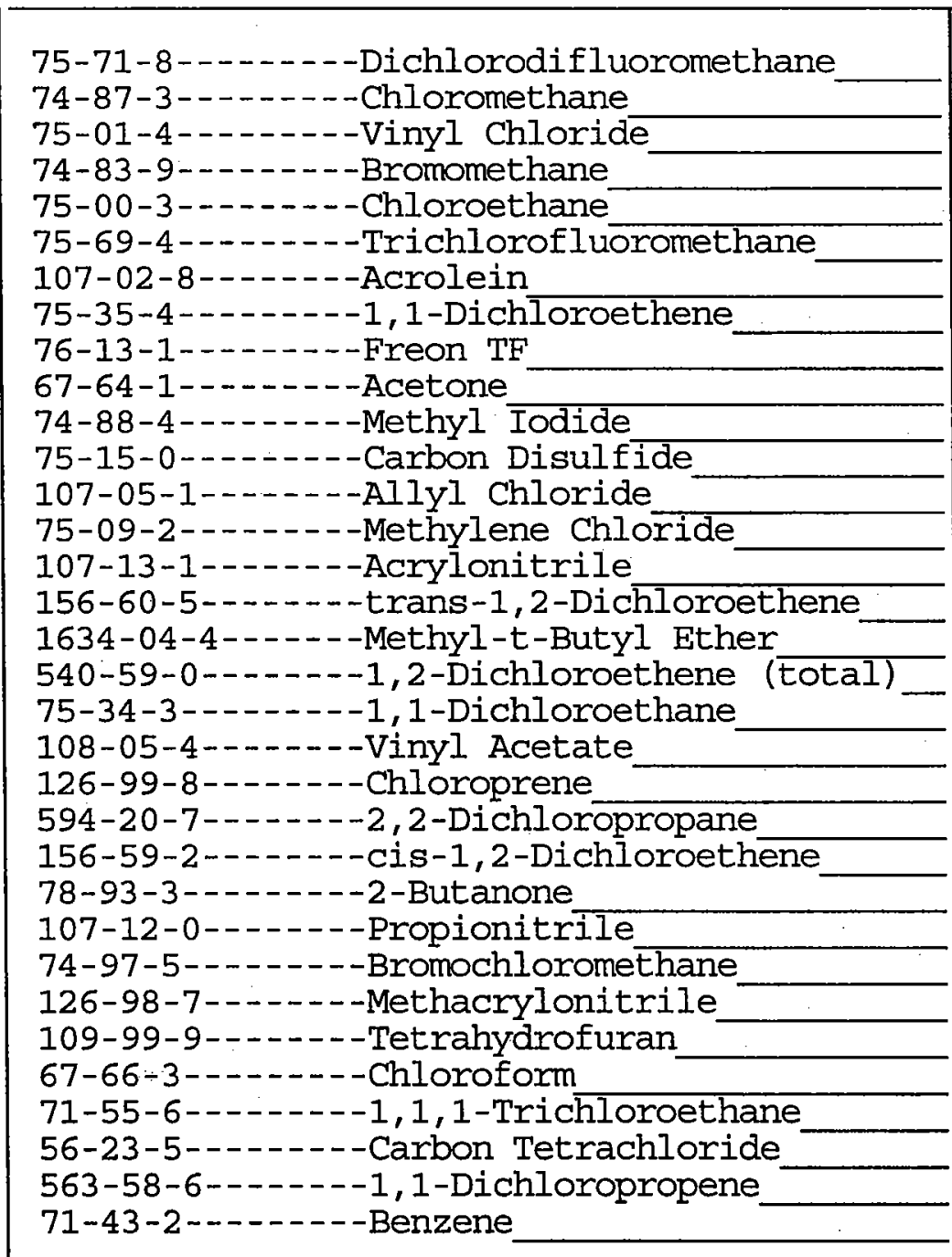

$10 \mathrm{U}$

$5.1 \mathrm{JB}$

$10 \mathrm{U}$

$5.6 \mathrm{JB}$

$10 \mathrm{U}$

$10 \mathrm{U}$

$51 \mathrm{U}$

$10 \mathrm{U}$

$10 \mathrm{U}$

$51 \mathrm{U}$

$5.9 \mathrm{JB}$

$10 \mathrm{U}$

$10 \mathrm{U}$

$6.4 \mathrm{~J}$

$10 \mathrm{U}$

$10 \mathrm{U}$

$10 \mathrm{U}$

$10 \mathrm{U}$

$10 \mathrm{U}$

$10 \mathrm{U}$

$10 \mathrm{U}$

$10 \mathrm{U}$

$10 \mathrm{U}$

$210 \mathrm{~B}$

$41 \mathrm{U}$

$10 \mathrm{U}$

$10 \mathrm{U}$

$140 \mathrm{U}$

$10 \mathrm{U}$

$10 \mathrm{U}$

$10 \mathrm{U}$

$10 \mathrm{U}$

$10 \mathrm{U}$ 
FORM 1

VOLATILE ORGANICS ANALYSIS DATA SHEET

Lab Name: STL BURLINGTON

Contract: 21005
ARGLAB SAMPLE NO.

NAS2 0607-20A

Lab Code: STLVT Case No.: NAVARRE SAS No.:

SDG No.: 114211

Matrix: (soil/water) SOIL

Sample wt/vol:

$9.8(\mathrm{~g} / \mathrm{mL}) \mathrm{G}$

Level : (low/med) MED

$\%$ Moisture: not dec.

GC Column: CAP

ID: $0.53(\mathrm{~mm})$

Soil Extract Volume: 10000 (uL)
Lab Sample ID: 668548

Lab File ID: 668548

Date Received: 05/08/06

Date Analyzed: 05/10/06

Dilution Factor: 1.0

Soil Aliquot Volume:

500 (uL)

CAS NO.

COMPOUND

CONCENTRATION UNITS :

(ug/L or $u g / \mathrm{Kg}$ ) UG/KG

$Q$

\begin{tabular}{|c|}
\hline 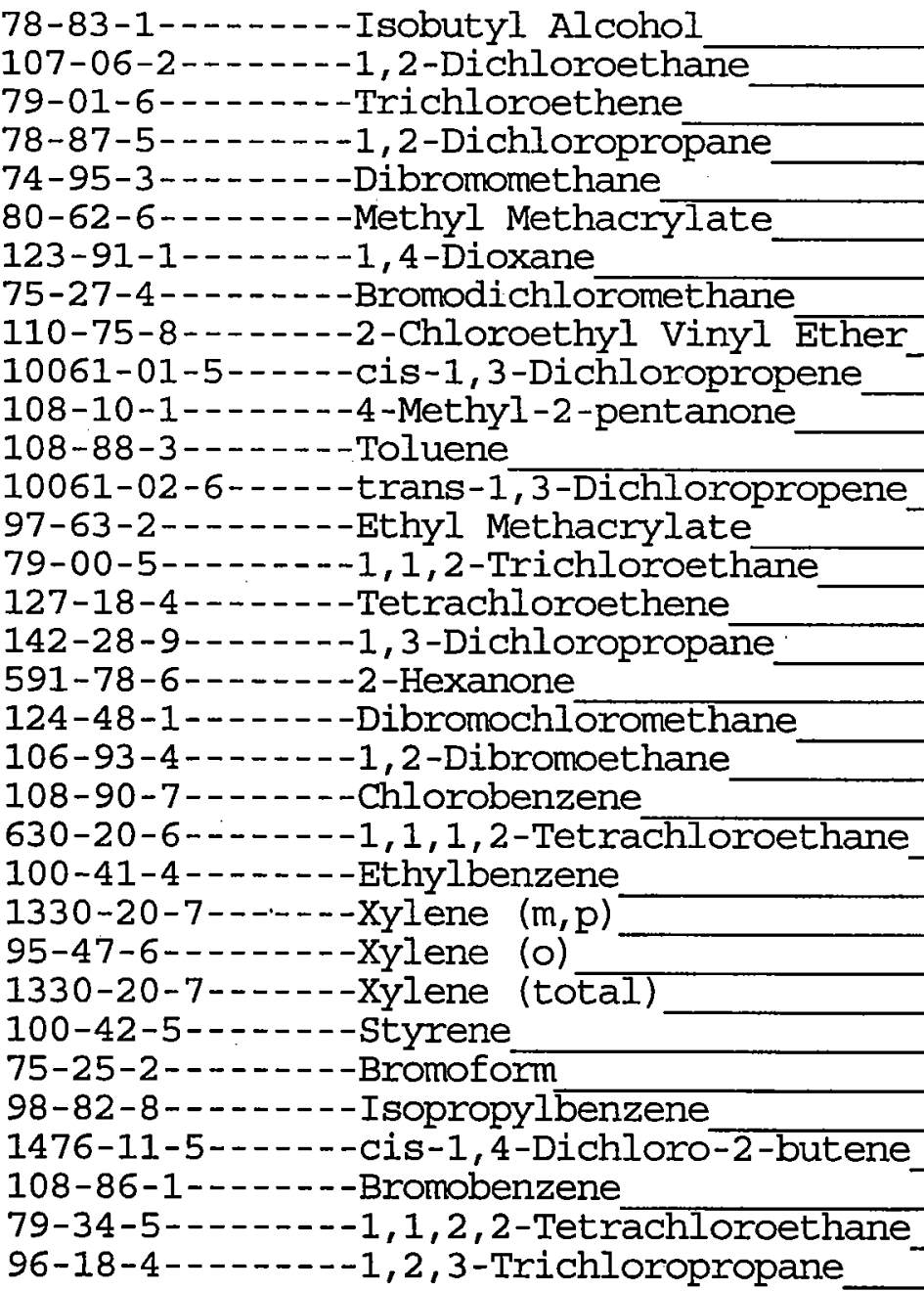 \\
\hline
\end{tabular}

\begin{tabular}{r|r|l|}
510 & $\mathrm{U}$ \\
10 & $\mathrm{U}$ \\
10 & $\mathrm{U}$ \\
10 & $\mathrm{U}$ \\
10 & $\mathrm{U}$ \\
10 & $\mathrm{U}$ \\
510 & $\mathrm{U}$ \\
10 & $\mathrm{U}$ \\
10 & $\mathrm{U}$ \\
10 & $\mathrm{U}$ \\
51 & $\mathrm{U}$ \\
10 & $\mathrm{U}$ \\
10 & $\mathrm{U}$ \\
10 & $\mathrm{U}$ \\
10 & $\mathrm{U}$ \\
10 & $\mathrm{U}$ \\
10 & $\mathrm{U}$ \\
51 & $\mathrm{U}$ \\
10 & $\mathrm{U}$ \\
10 & $\mathrm{U}$ \\
10 & $\mathrm{U}$ \\
10 & $\mathrm{U}$ \\
10 & $\mathrm{U}$ \\
2.9 & $\mathrm{JB}$ \\
10 & $\mathrm{U}$ \\
3.0 & $\mathrm{JB}$ \\
10 & $\mathrm{U}$ \\
10 & $\mathrm{U}$ \\
10 & $\mathrm{U}$ \\
10 & $\mathrm{U}$ \\
10 & $\mathrm{U}$ \\
10 & $\mathrm{U}$ \\
10 & $\mathrm{U}$ \\
& \\
\hline & \\
\hline$\cdots$ &
\end{tabular}


FORM 1

VOLATILE ORGANICS ANALYSIS DATA SHEET

Lab Name: STL BURLINGTON

Contract : 21005

Lab Code: STLVT Case No.: NAVARRE SAS No.:

Lab Sample ID: 668548

Matrix: (soil/water) SOIL

Lab File ID: $\quad 668548$

Sample wt/vol:

$9.8(\mathrm{~g} / \mathrm{mL}) \mathrm{G}$

Level : (low/med) MED

Date Received: 05/08/06

\% Moisture: not dec.

GC Column: CAP

ID $: 0.53(\mathrm{~mm})$

Date Analyzed: 05/10/06

Dilution Factor: 1.0

Soil Extract Volume: 10000 (uL)

Soil Aliquot Volume:

500 (UL)

CAS NO.

COMPOUND

CONCENTRATION UNITS:

(ug/L or $\mathrm{ug} / \mathrm{Kg}$ ) UG/KG Q

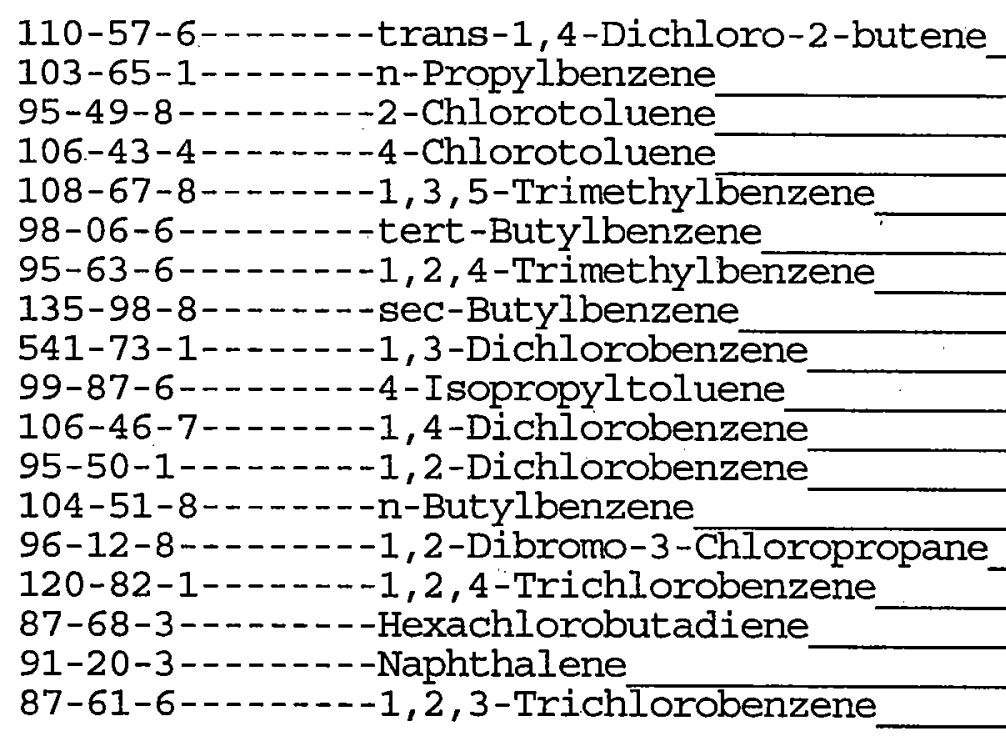


FORM 1

VOLATILE ORGANICS ANALYSIS DATA SHEET

Lab Name: STL BURLINGTON

Contract: 21005
ARGLAB SAMPLE NO.

NAS2 0620-20A

Lab Code: STLVT Case No.: NAVARRE SAS No.:

SDG No.: 114211

Matrix: (soil/water) SOIL

Sample wt/vol:

$9.6(\mathrm{~g} / \mathrm{mL}) \mathrm{G}$

Level: (low/med) MED

\% Moisture: not dec.

GC Column: CAP

ID $: 0.53(\mathrm{~mm})$

Soil Extract Volume: 10000 (uL)
Lab Sample ID: 668545

Lab File ID: 668545

Date Received: 05/08/06

Date Analyzed: 05/10/06

Dilution Factor: 1.0

Soil Aliquot Volume:

500 (uL)

CAS NO.

COMPOUND

CONCENTRATION UNITS:

(ug/L or $\mathrm{ug} / \mathrm{Kg}$ ) UG/KG

Q

\begin{tabular}{|c|c|c|}
\hline 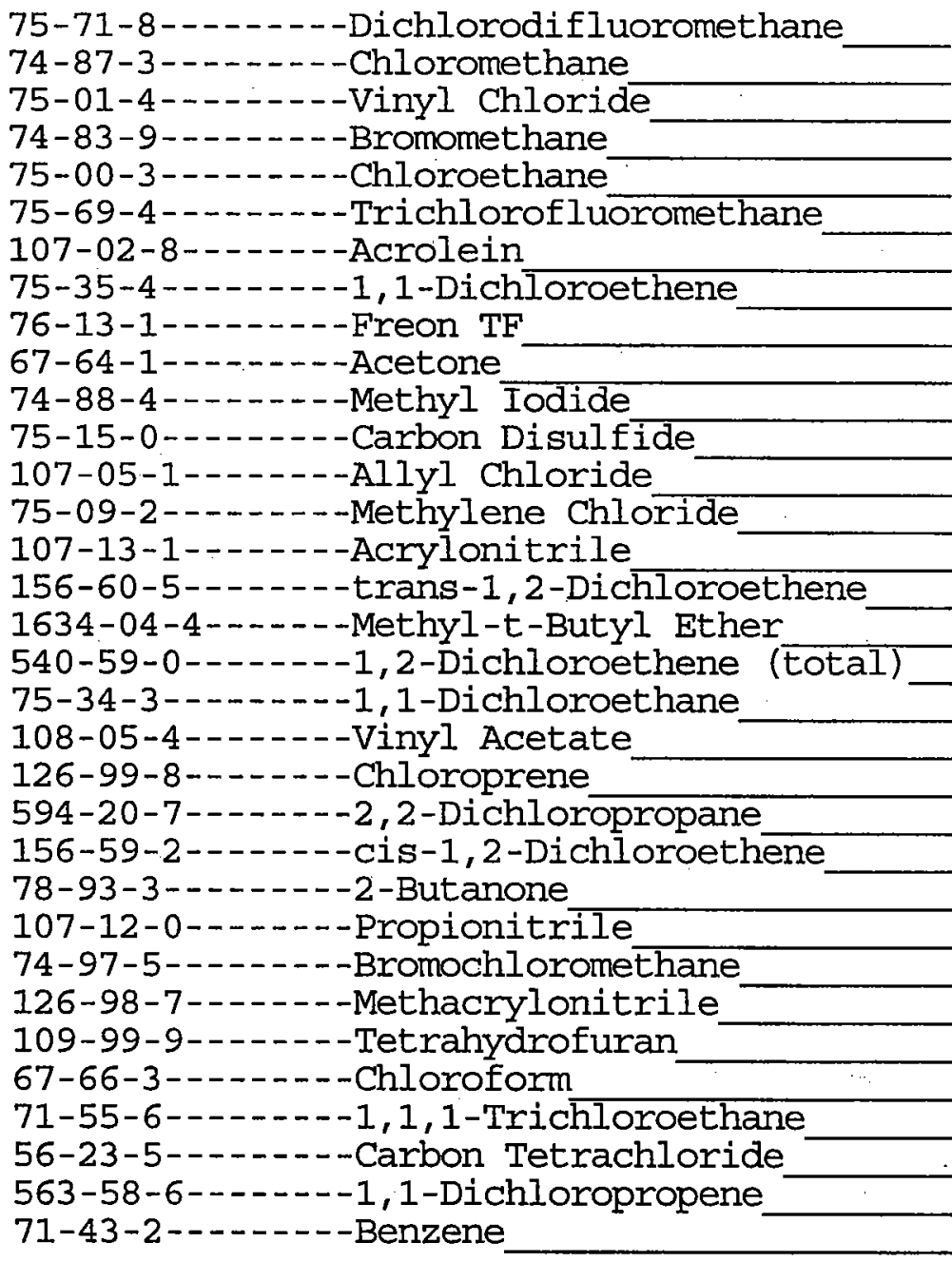 & $\begin{array}{r}10 \\
5.9 \\
10 \\
6.9 \\
10 \\
10 \\
52 \\
10 \\
10 \\
52 \\
9.2 \\
10 \\
10 \\
4.4 \\
10 \\
10 \\
10 \\
10 \\
10 \\
10 \\
10 \\
10 \\
10 \\
250 \\
42 \\
10 \\
10 \\
150 \\
10 \\
10 \\
10 \\
10 \\
10\end{array}$ & $\begin{array}{l}U \\
J B \\
U \\
J B \\
U \\
U \\
U \\
U \\
U \\
U \\
J B \\
U \\
U \\
U \\
J \\
U \\
U \\
U \\
U \\
U \\
U \\
U \\
U \\
U \\
B \\
U \\
U \\
U \\
U \\
U \\
U \\
U \\
U \\
U \\
U \\
U\end{array}$ \\
\hline
\end{tabular}


FORM 1

VOLATILE ORGANICS ANALYSIS DATA SHEET

Lab Name: STL BURLINGTON

Contract: 21005

Lab Code: STLVT Case No.: NAVARRE SAS No.:

Lab Sample ID: 668545

Matrix: (soil/water) SOIL

Lab File ID: 668545

Sample wt/vol: $\quad 9.6(\mathrm{~g} / \mathrm{mL}) \mathrm{G}$

Level : (low/med) MED

Date Received: 05/08/06

\% Moisture: not dec.

GC Column: CAP

ID: $0.53 \quad(\mathrm{~mm})$

Date Analyzed: 05/10/06

Dilution Factor: 1.0

Soil Extract Volume: 10000 (uL)

Soil Aliquot Volume:

500 (u山)

CAS NO.

COMPOUND

CONCENTRATION UNITS :

(ug/L or $u g / K g$ ) UG/KG

Q

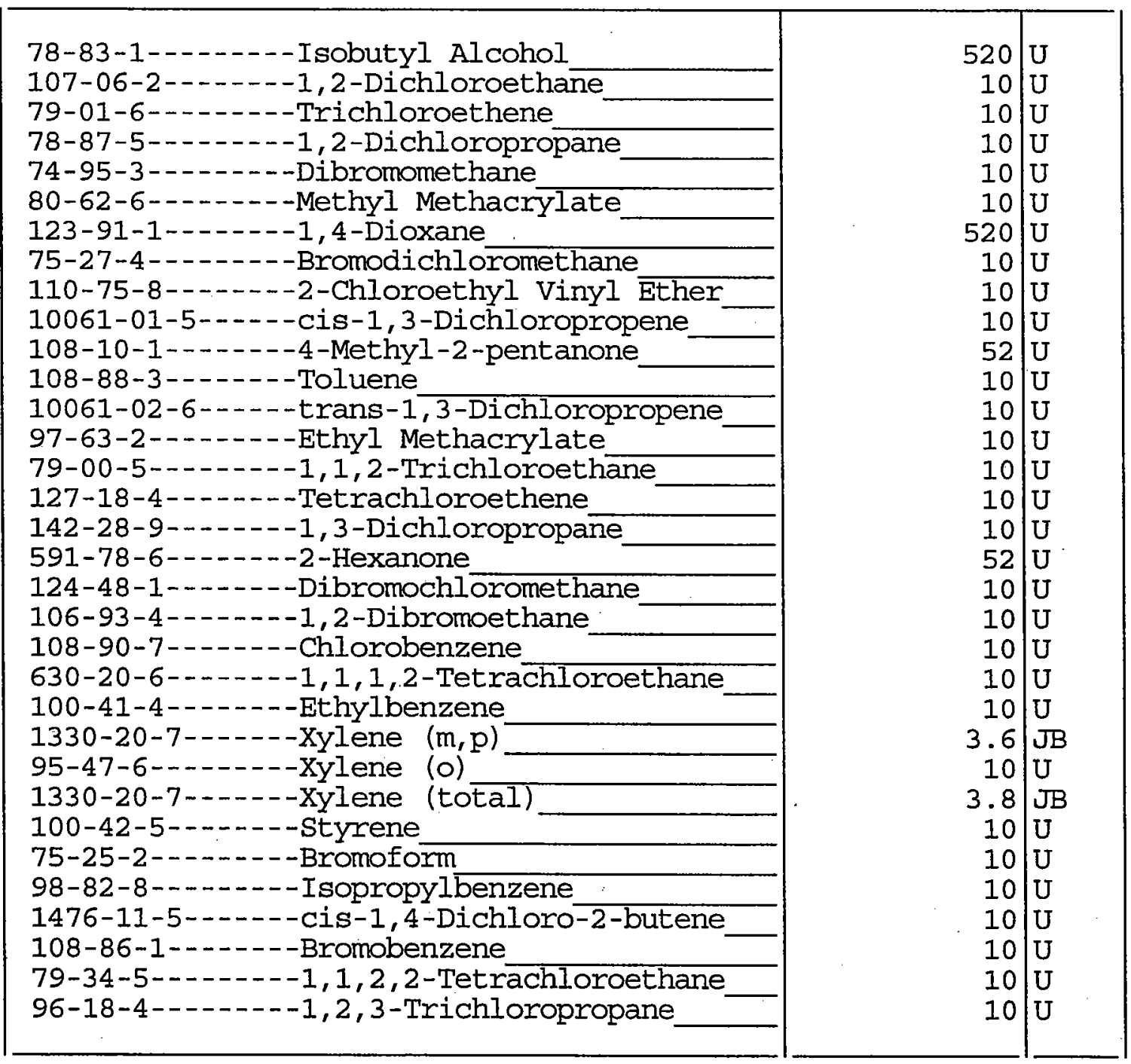


FORM 1

VOLATILE ORGANICS ANALYSIS DATA SHEET

Lab Name: STL BURLINGTON

Contract: 21005

ARGLAB SAMPLE NO.

Lab Code: STLVT Case No.: NAVARRE SAS No.:

NAS2 0620-20A

Matrix: (soil/water) SOIL

Lab Sample ID: 668545

Sample wt/vol:

$9.6(\mathrm{~g} / \mathrm{mL}) \mathrm{G}$

Lab File ID: 668545

Level : (low/med) MED

Date Received: 05/08/06

$\%$ Moisture: not dec.

GC Column: CAP

ID: $0.53 \quad(\mathrm{~mm})$

Date Analyzed: 05/10/06

Soil Extract Volume: 10000 (ut)

Dilution Factor: 1.0

Soil Aliquot Volume:

500 (U工)

CONCENTRATION UNITS:

CAS NO.

COMPOUND

(ug/L or $u g / K g$ ) UG/KG

Q

\begin{tabular}{|c|c|c|}
\hline 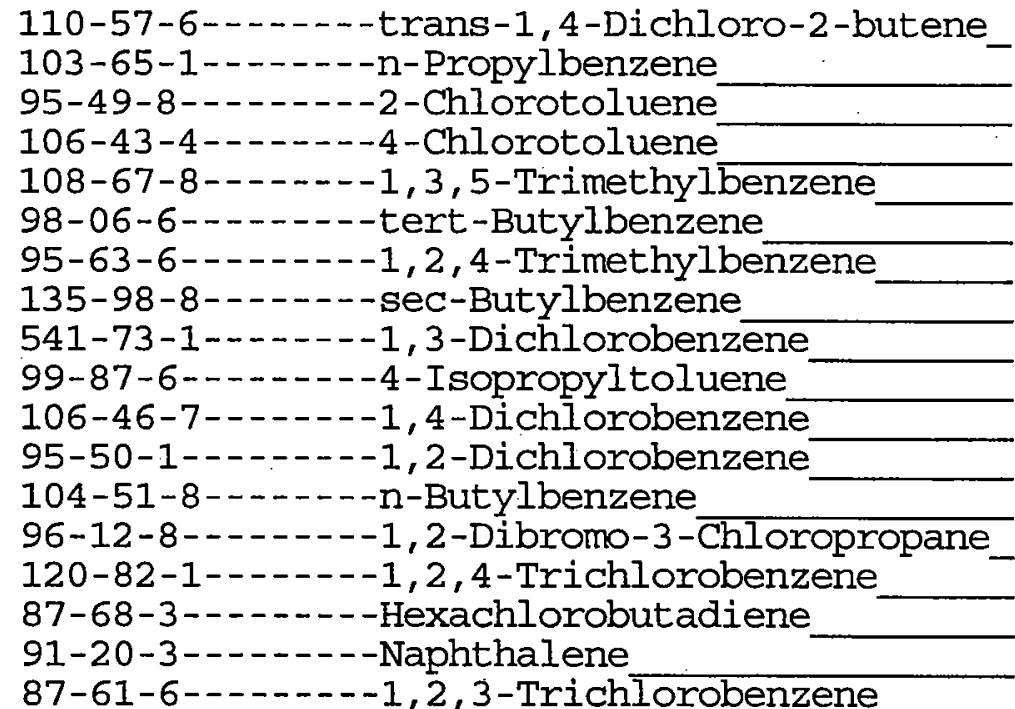 & $\begin{array}{l}10 \\
10 \\
10 \\
10 \\
10 \\
10 \\
10 \\
10 \\
10 \\
10 \\
10 \\
10 \\
10 \\
10 \\
10 \\
10 \\
10 \\
10\end{array}$ & $\begin{array}{l}\mathrm{U} \\
\mathrm{U} \\
\mathrm{U} \\
\mathrm{U} \\
\mathrm{U} \\
\mathrm{U} \\
\mathrm{U} \\
\mathrm{U} \\
\mathrm{U} \\
\mathrm{U} \\
\mathrm{U} \\
\mathrm{U} \\
\mathrm{U} \\
\mathrm{U} \\
\mathrm{U} \\
\mathrm{U} \\
\mathrm{U} \\
\mathrm{U}\end{array}$ \\
\hline
\end{tabular}


May 25, 2006

\section{S E V E R N}

T R E N T

Mr. Clyde Dennis

Argonne National Laboratory

9700 S. Cass Avenue

Building 203, Office 149

Argonne, IL 60439
STL Burlington

208 South Park Drive, Suite 1

Colchester, VT 05446

Tel: 8026551203 Fax: 8026551248

www.stl-inc.com

Re: Laboratory Project No. 21005

Case: NAVARRE; SDG: 114309

Dear Mr. Dennis:

Enclosed are analytical results for samples that were received by STL Burlington on May $12^{\text {th }}, 2006$. Laboratory identification numbers were assigned, and designated as follows:

$\begin{array}{llll}\text { Lab ID } & \begin{array}{l}\text { Client } \\ \text { Sample ID }\end{array} & \begin{array}{l}\text { Sample } \\ \text { Date }\end{array} & \begin{array}{l}\text { Sample } \\ \text { Matrix }\end{array} \\ & \text { Received: 05/12/06 ETR No: } 114309 & \\ 669174 & \text { NA-S-20636 } & 05 / 08 / 06 & \text { Liquid } \\ 669175 & \text { NA-S-20735 } & 05 / 08 / 06 & \text { Liquid } \\ 669176 & \text { NA-S-20538 } & 05 / 08 / 06 & \text { Liquid } \\ 669177 & \text { NA-S-20750 } & 05 / 08 / 06 & \text { Liquid } \\ 669178 & \text { NA-S-20541 } & 05 / 08 / 06 & \text { Liquid } \\ 669179 & \text { NA-S-20575 } & 05 / 08 / 06 & \text { Liquid } \\ 669180 & \text { NA-S-20545 } & 05 / 08 / 06 & \text { Liquid } \\ 669181 & \text { NA-S-20664 } & 05 / 08 / 06 & \text { Liquid } \\ 669182 & \text { NA-S-20543 } & 05 / 08 / 06 & \text { Liquid } \\ 669183 & \text { MEOH BLANK } & 05 / 08 / 06 & \text { Liquid }\end{array}$

Documentation of the condition of the samples at the time of their receipt and any exception to the laboratory's Sample Acceptance Policy is documented in the Sample Handling section of this submittal. It should be noted that at the time that they were received, the sample volumes were at near ambient temperature.

The samples were analyzed by Method $8260 \mathrm{~B}$, using a low-level calibration. In performing the analytical work, 500 microliters of the methanol extract were added to the 5 milliliter purge volume. The surrogate controls were recovered well in each of the analyses associated with the sample set, and each analysis exhibited good internal standard stability. Two types of laboratory control sample analyses were performed as part of the analytical sequence. One was performed to evaluate method performance, and one was performed with 500 microliters of methanol added to the purge volume in order to characterize the affect on the analytical process. With the exception of that for dichlorodifluoromethane, for which the recovery was 74 percent, the recovery of each target analyte was within the established control range in the laboratory control sample analysis that defined the method performance. In the laboratory control sample analysis with methanol, several of the earlier eluting compounds did exhibit lower recoveries, as did isobutyl 
alcohol and 1,4-dioxane. Chloroform and carbon tetrachloride were recovered well in each of the laboratory control sample analyses. Matrix spike and matrix spike duplicate analyses were not performed on samples in this sample set. The analysis of the instrument blank that was analyzed in association with the samples was free of contamination. The laboratory did associate the analysis of sample MEOH BLANK with each of the other field sample analyses in order to reference the blank association, and accordingly qualify the reported results.

If there are any questions regarding this submittal, please contact me at (802) 655-1203.

The analytical results associated with the samples presented in this test report were generated under a quality system that adheres to requirements specified in the NELAC standard. Release of the data in this test report and any associated electronic deliverables is authorized by the Laboratory Director's designee as verified by the following signature.

Sincerely,

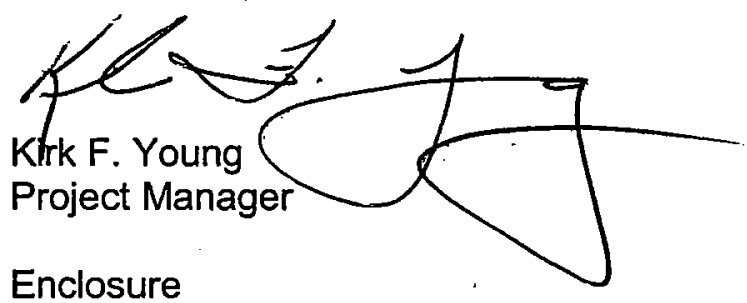




\section{STL Burlington Data Qualifier Definitions}

\section{Organic}

$\mathrm{U}$ : Compound analyzed but not detected at a concentration above the reporting limit.

J: $\quad$ Estimated value.

$\mathrm{N}$ : Indicates presumptive evidence of a compound. This flag is used only for tentatively identified compounds (TICs) where the identification of a compound is based on a mass spectral library search.

P: SW-846: Greater than $40 \%$ difference for detected concentrations between two GC columns. Unless otherwise specified the higher of the two values is reported on the Form I.

CLP SOW: Greater than $25 \%$ difference for detected concentrations between two GC columns. Uniess otherwise specified the lower of the two values is reported on the Form I.

C: Pesticide result whose identification has been confirmed by GC/MS.

B: $\quad$ Analyte is found in the sample and the associated method blank. The flag is used for tentatively identified compounds as well as positively identified compounds.

E: Compounds whose concentrations exceed the upper limit of the calibration range of the instrument for that specific analysis.

D: Concentrations identified from analysis of the sample at a secondary dilution.

A: Tentatively identified compound is a suspected aldol condensation product.

$X, Y, Z$ : Laboratory defined flags that may be used alone or combined, as needed. If used, the description of the flag is defined in the project narrative.

\section{Inorganic/Metals}

E: Reported value is estimated due to the presence of interference.

N: Matrix spike sample recovery is not within control limits.

* Duplicate sample analysis is not within control limits.

B: The result reported is less than the reporting limit but greater than the instrument detection limit.

U: $\quad$ Analyte was analyzed for but not detected above the reporting limit.

\section{Method Codes:}

$P \quad$ ICP-AES

MS ICP-MS

CV Cold Vapor AA

AS Semi-Automated Spectrophotometric 


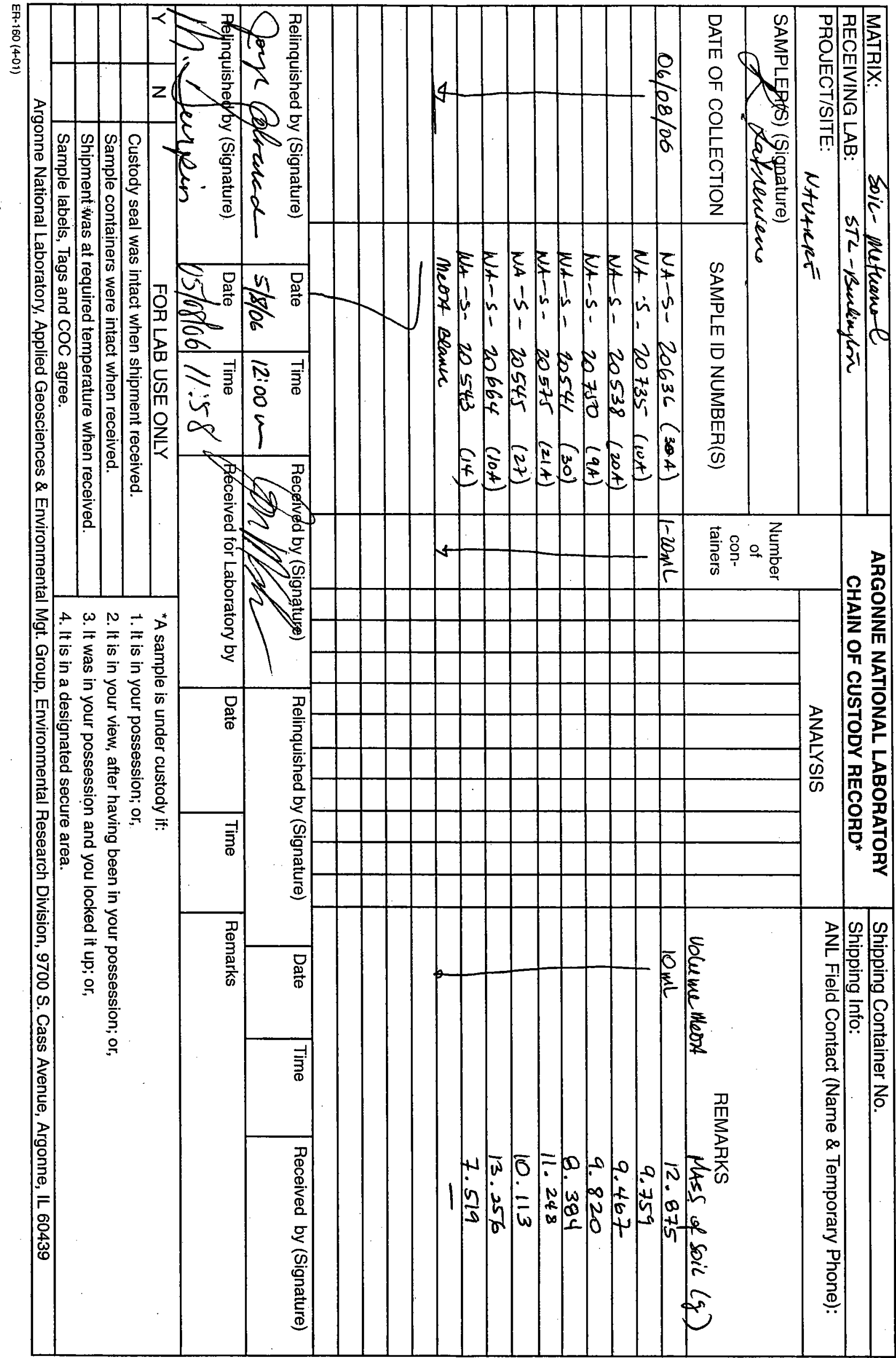




\section{SEVERN \\ T REN T \\ STL}

\section{METHOD 8260B}

\section{VOLATILE ORGANIC ANALYSIS}

SAMPLE DATA SUMMARY PACKAGE 
FORM 1

VOLATILE ORGANICS ANALYSIS DATA SHEET

Lab Name: STL BURLINGTON

Contract: 21005
ARGLAB SAMPLE NO.

NA-S-20538

Lab Code: STLVT Case No.: NAVARRE SAS No.:

SDG No.: 114309

Matrix: (soil/water) SOIL

Sample wt/vol:

$9.5(\mathrm{~g} / \mathrm{mL}) \mathrm{G}$

Level: (low/med) MED

음 Moisture: not dec.

GC Column: CAP ID: 0.53 (mm)

Soil Extract Volume: 10000 (UL)
Lab Sample ID: 669176

Lab File ID: $\quad 669176 \mathrm{E}$

Date Received: 05/12/06

Date Analyzed: 05/19/06

Dilution Factor: 1.0

Soil Aliquot Volume:

500 (uL)

(ug/L or $\mathrm{ug} / \mathrm{Kg})$ UG/KG Q

\begin{tabular}{|c|c|c|}
\hline 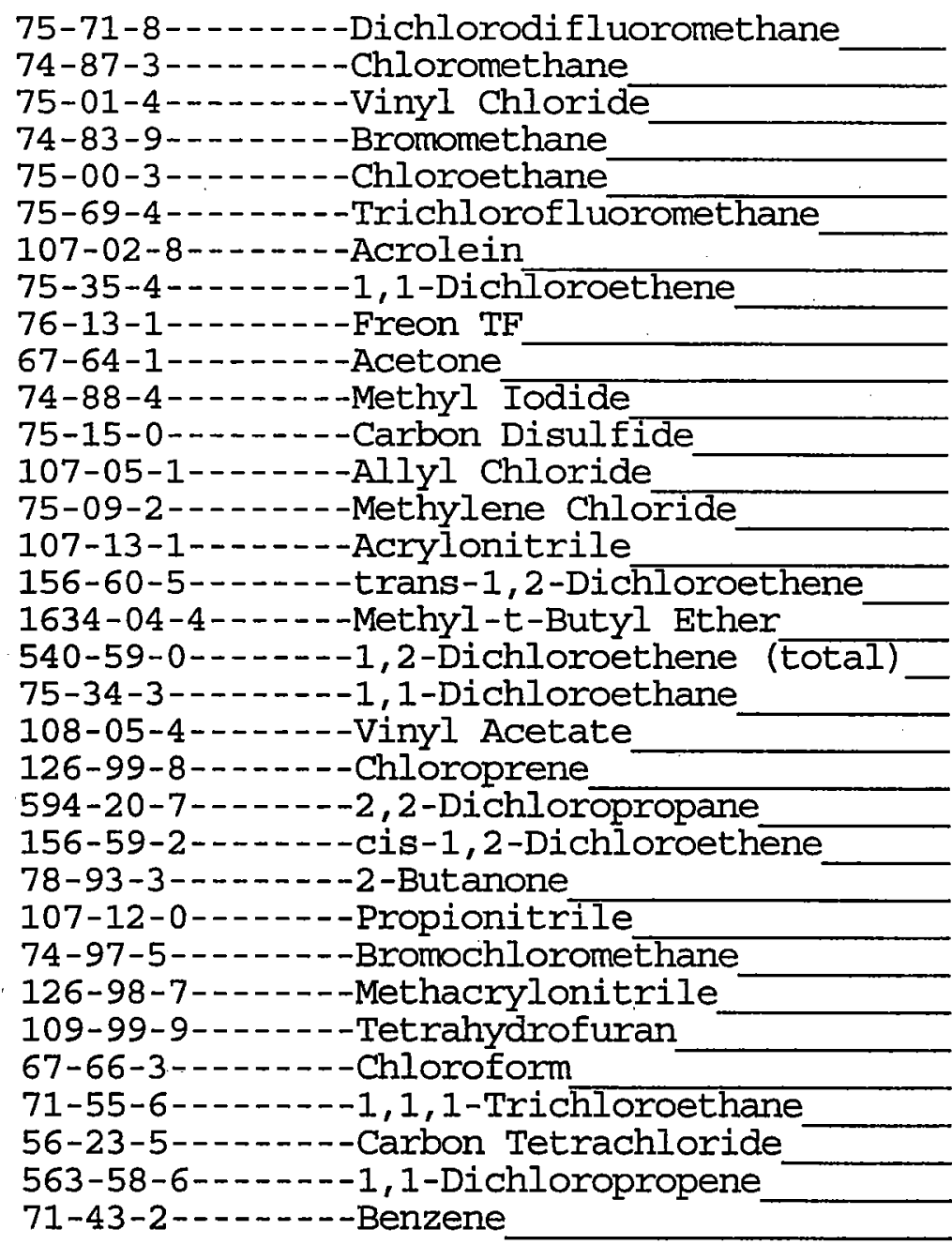 & $\begin{array}{r}11 \\
5.3 \\
11 \\
12 \\
11 \\
11 \\
52 \\
11 \\
11 \\
52 \\
9.0 \\
11 \\
11 \\
4.5 \\
11 \\
11 \\
11 \\
11 \\
11 \\
11 \\
11 \\
11 \\
11 \\
200 \\
42 \\
11 \\
11 \\
150 \\
11 \\
11 \\
11 \\
11 \\
11\end{array}$ & $\begin{array}{l}U \\
J \\
U \\
B \\
U \\
U \\
U \\
U \\
U \\
U \\
J B \\
U \\
U \\
J B \\
U \\
U \\
U \\
U \\
U \\
U \\
U \\
U \\
U \\
U \\
B \\
U \\
U \\
U \\
U \\
U \\
U \\
U \\
U \\
U \\
U \\
U\end{array}$ \\
\hline
\end{tabular}


FORM 1

VOLATILE ORGANICS ANALYSIS DATA SHEET

Lab Name: STL BURLINGTON

Contract: 21005

Lab Code: STLVT Case No.: NAVARRE SAS No.:

Lab Sample ID: 669176

Matrix: (soil/water) SOIL

Lab File ID: $669176 \mathrm{E}$

Sample wt/vol:

$9.5(\mathrm{~g} / \mathrm{mL}) \mathrm{G}$

Level: (low/med) MED

$\%$ Moisture: not dec.

GC Column: CAP ID: 0.53 (mm)

Soil Extract Volume: 10000 (uL)

Date Received: 05/12/06

Date Analyzed: 05/19/06

Dilution Factor: 1.0

Soil Aliquot Volume:
ARGLAB SAMPLE NO.

NA-S-20538

SDG No. : 114309

500 (uL)

\section{CONCENTRATION UNITS:}

CAS NO. COMPOUND (ug/L or ug/Kg) UG/KG Q

\begin{tabular}{|c|}
\hline 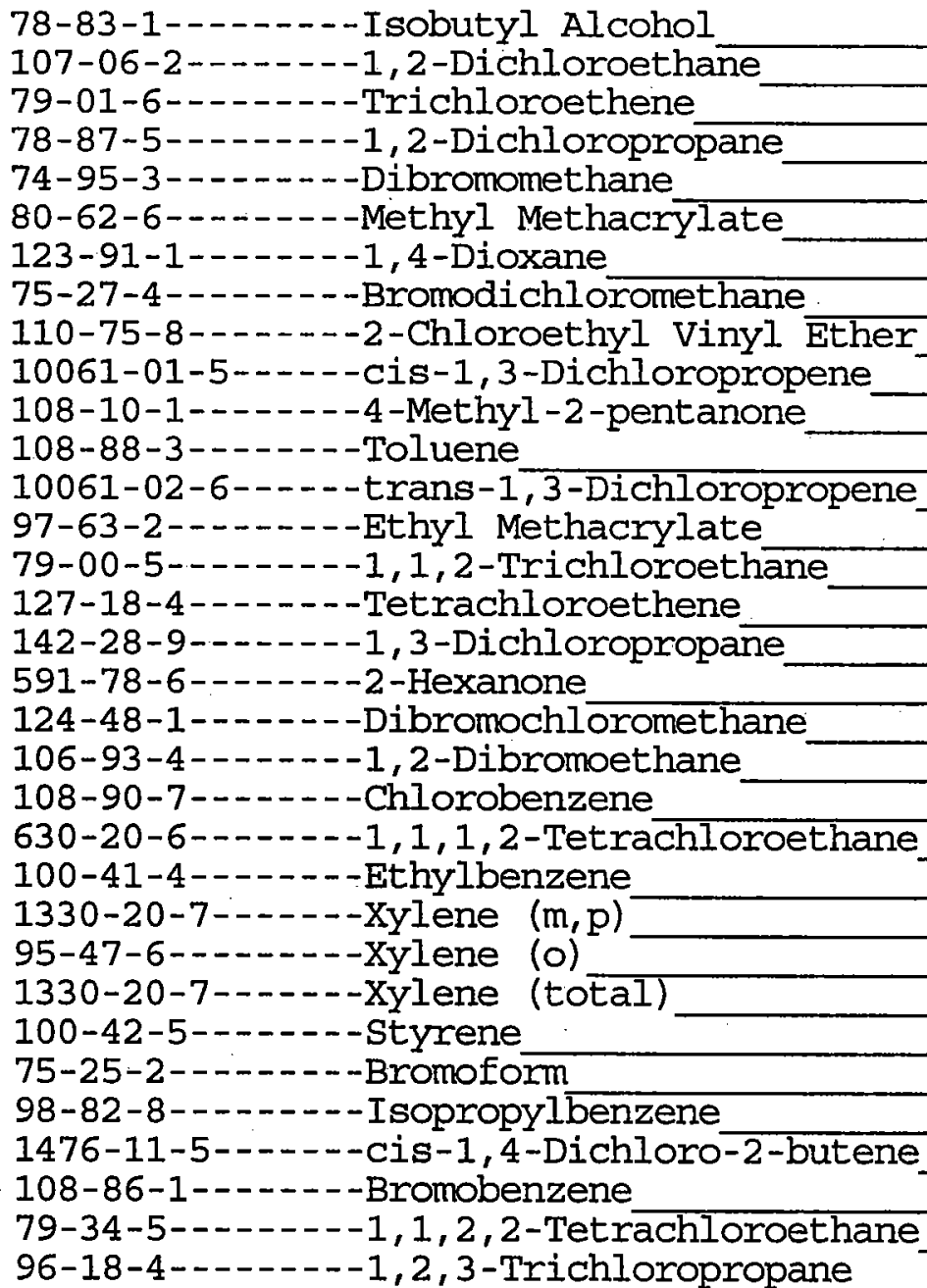 \\
\hline
\end{tabular}

$530 \mathrm{U}$

$11 \mathrm{U}$

$11 \mathrm{U}$

$11 \mathrm{U}$

$11 \mathrm{U}$

$11 \mathrm{U}$

$530 \mathrm{U}$

$11 \mathrm{U}$

$11 \mathrm{U}$

$11 \mathrm{U}$

$52 \mathrm{U}$

11 U

$11 \mathrm{U}$

$11 \mathrm{U}$

$11 \mathrm{U}$

$11 \mathrm{U}$

$11 \mathrm{U}$

$52 \mathrm{U}$

$11 \mathrm{U}$

$11 \mathrm{U}$

$11 \mathrm{U}$

11 U

$11 \mathrm{U}$

$3.2 \mathrm{~J}$

$11 \mathrm{U}$

$3.3 \mathrm{~J}$

$11 \mathrm{U}$

$11 \mathrm{U}$

$11 \mathrm{U}$

$11 \mathrm{U}$

$11 \mathrm{U}$

$11 \mathrm{U}$

$11 \mathrm{U}$ 
FORM 1

VOLATILE ORGANICS ANALYSIS DATA SHEET

Lab Name: STL BURIINGTON

Contract : 21005

Lab Code: STLVT Case No.: NAVARRE SAS No.:

Lab Sample ID: 669176

Matrix: (soil/water) SOIL

Lab File ID: $669176 \mathrm{E}$

Sample wt/vol:

$9.5(\mathrm{~g} / \mathrm{mL}) \mathrm{G}$

Le'vel: (low/med) MED

Date Received: 05/12/06

\% Moisture: not dec.

GC Column: CAP

ID : $0.53 \quad(\mathrm{~mm})$

Date Analyzed: 05/19/06

Dilution Factor: 1.0

Soil Extract Volume: 10000 (uL)

Soil Aliquot Volume:

500 (u山)

CONCENTRATION UNITS:

CAS NO. COMPOUND (ug/L or $\mathrm{ug} / \mathrm{Kg}$ ) UG/KG

Q

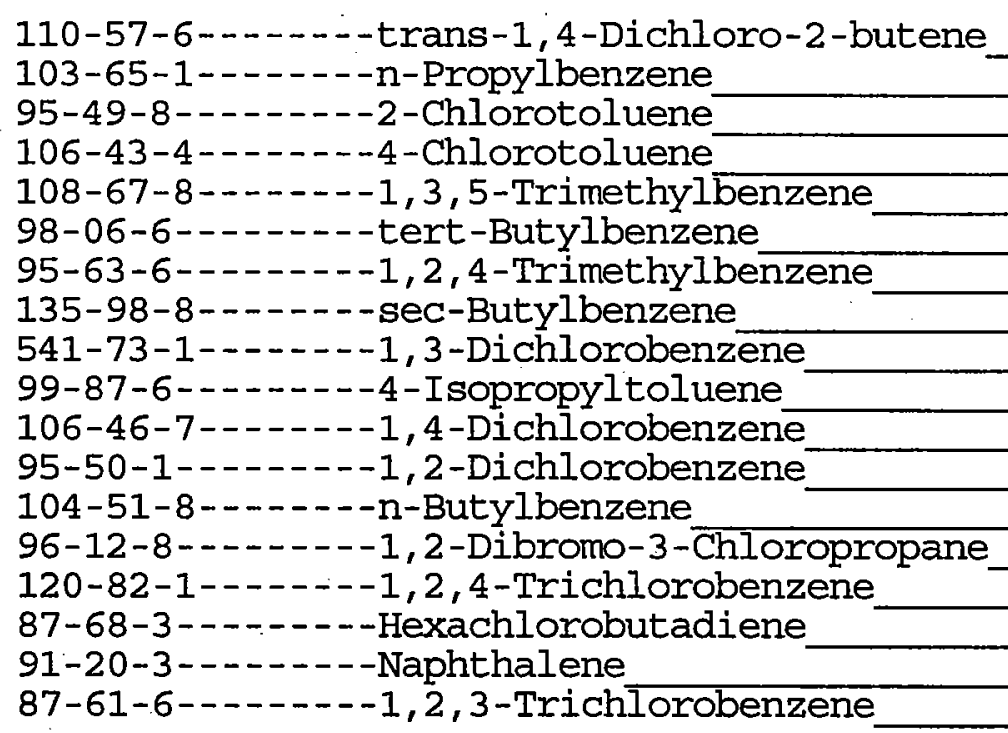

\begin{tabular}{|l|l|l|}
11 & $\mathrm{U}$ \\
11 & $\mathrm{U}$ \\
11 & $\mathrm{U}$ \\
11 & $\mathrm{U}$ \\
11 & $\mathrm{U}$ \\
11 & $\mathrm{U}$ \\
11 & $\mathrm{U}$ \\
11 & $\mathrm{U}$ \\
11 & $\mathrm{U}$ \\
11 & $\mathrm{U}$ \\
11 & $\mathrm{U}$ \\
11 & $\mathrm{U}$ \\
11 & $\mathrm{U}$ \\
11 & $\mathrm{U}$ \\
11 & $\mathrm{U}$ \\
11 & $\mathrm{U}$ \\
11 & $\mathrm{U}$ \\
11 & $\mathrm{U}$ \\
\hline
\end{tabular}


FORM 1

VOLATILE ORGANICS ANALYSIS DATA SHEET

Lab Name: STL BURLINGTON

Contract: 21005
ARGLAB SAMPLE NO.

NA-S-20541

Lab Code: STLVT Case No.: NAVARRE SAS No.:

SDG No.: 114309

Matrix: (soil/water) SOIL

Sample wt/vol:

$8.4(\mathrm{~g} / \mathrm{mL}) \mathrm{G}$

Level : (low/med) MED

$\%$ Moisture: not dec.

GC Column: CAP

ID $: 0.53(\mathrm{~mm})$

Soil Extract Volume: 10000 (UU)
Lab Sample ID: 669178

Lab File ID: $\quad 669178 \mathrm{E}$

Date Received: 05/12/06

Date Analyzed: 05/19/06

Dilution Factor: 1.0

Soil Aliquot Volume:

500 (uL)

CAS NO.

COMPOUND

CONCENTRATION UNITS:

(ug/L or ug/Kg) UG/KG

Q

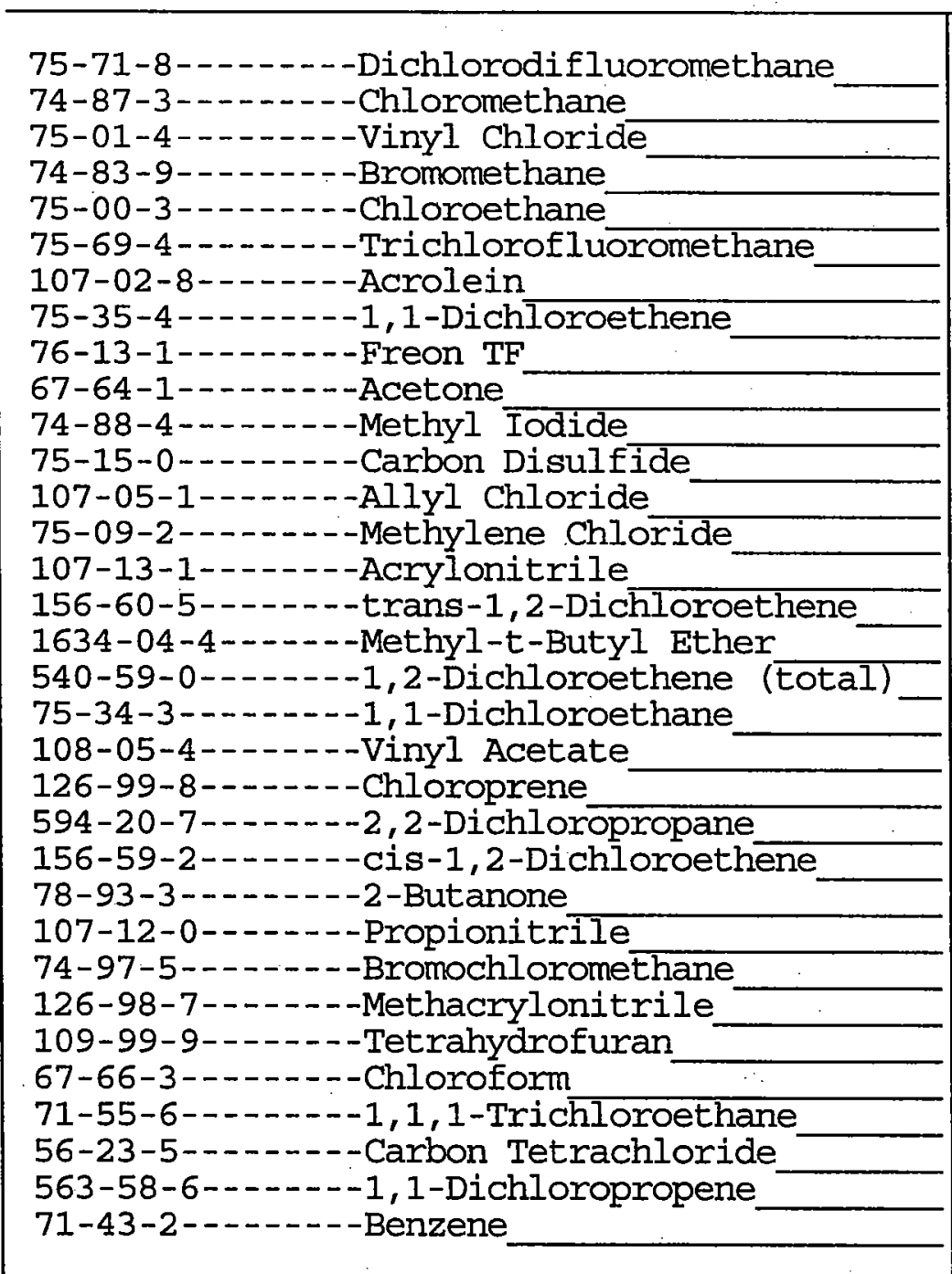

12

$2.7 \mathrm{JB}$

$12 \mathrm{U}$

$10 \mathrm{JB}$

$12 \mathrm{U}$

$12 \mathrm{U}$

$59 \mathrm{U}$

$12 \mathrm{U}$

$12 \mathrm{U}$

$59 \mathrm{U}$

$3.7 \mathrm{JB}$

$12 \mathrm{U}$

$12 \mathrm{U}$

$5.0 \mathrm{JB}$

$12 \mathrm{U}$

$12 \mathrm{U}$

$12 \mathrm{U}$

$12 \mathrm{U}$

$12 \mathrm{U}$

$12 \mathrm{U}$

$12 \mathrm{U}$

$12 \mathrm{U}$

$12 \mathrm{U}$

$310 \mathrm{~B}$

$48 \mathrm{U}$

$12 \mathrm{U}$

$12 \mathrm{U}$

$170 \mathrm{U}$

34

12 U

140

$12 \overline{\mathrm{U}}$

$12 \mathrm{U}$ 
FORM 1

VOLATILE ORGANICS ANALYSIS DATA SHEET

Lab Name: STL BURLINGTON

Contract: 21005
ARGLAB SAMPLE NO.

NA-S-20541

Lab Code: STLVT Case No.: NAVARRE SAS No.:

SDG No.: 114309

Matrix: (soil/water) SOIL

Sample wt/vol:

$8.4(\mathrm{~g} / \mathrm{mL}) \mathrm{G}$

Level: (low/med) MED

음 Moisture: not dec.

GC Column: CAP

ID: $0.53(\mathrm{~mm})$

Soil Extract Volume: 10000 (uL)
Lab Sample ID: 669178

Lab File ID: $\quad 669178 \mathrm{E}$

Date Received: 05/12/06

Date Analyzed: 05/19/06

Dilution Factor: 1.0

Soil Aliquot Volume:

500 (u山)

CAS NO.

COMPOUND

CONCENTRATION UNITS:

(ug/L or $\mathrm{ug} / \mathrm{Kg}$ ) UG/KG

Q

\begin{tabular}{|c|}
\hline 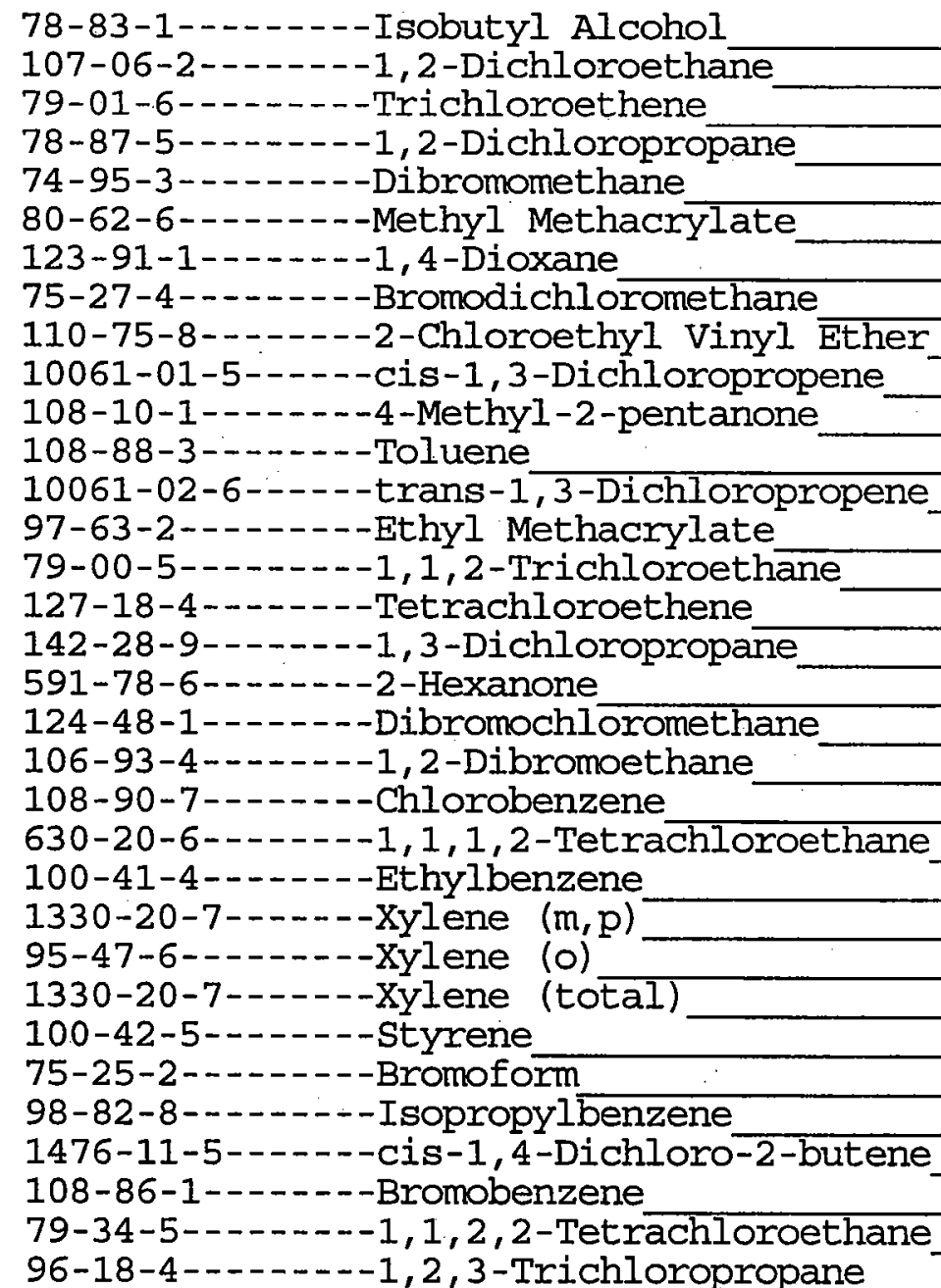 \\
\hline
\end{tabular}


FORM 1

VOLATILE ORGANICS ANALYSIS DATA SHEET

Lab Name: STL BURLINGTON

Contract: 21005
ARGLAB SAMPLE NO.

NA-S-20541

Lab Code: STLVT Case No.: NAVARRE SAS No.:

SDG No.: 114309

Matrix: (soil/water) SOIL

Lab Sample ID: 669178

Sample wt/vol:

$8.4(\mathrm{~g} / \mathrm{mL}) \mathrm{G}$

Lab File ID: $669178 \mathrm{E}$

Level: (low/med) MED

Date Received: 05/12/06

\% Moisture: not dec.

Date Analyzed: 05/19/06

GC Column: CAP

ID : $0.53(\mathrm{~mm})$

Dilution Factor: 1.0

Soil Extract Volume: 10000 (uL)

Soil Aliquot Volume:

500 (u山)

CAS NO.

COMPOUND

CONCENTRATION UNITS:

(ug/L or ug/Kg) UG/KG

Q

110-57-6--.-.-trans-1, 4-Dichloro-2-butene

103-65-1------n-Propylbenzene

95-49-8--1---2-Chlorotoluene

106-43-4-.-.--4-Chlorotoluene

108-67-8-....-1, 3, 5-Trimethyl benzene

98-06-6-------tert-Butylbenzene

95-63-6-------1, 2,4-Trimethylbenzene

135-98-8------sec-Butylbenzene

541-73-1-....-1, 3-Dichlorobenzene

99-87-6------4-Isopropyltoluene

106-46-7------1, 4-Dichlorobenzene

95-50-1----.--1, 2-Dichlorobenzene

104-51-8-.---n-Butylbenzene

96-12-8--.--1, 2-Dibromo-3-Chloropropane

120-82-1--.---1, 2,4-Trichlorobenzene

87-68-3-...-.-Hexachlorobutadiene

91-20-3-...---Naphthalene

87-61-6-...--1, 2, 3-Trichlorobenzene

\begin{tabular}{|l|l|l}
12 & $\mathrm{U}$ \\
12 & $\mathrm{U}$ \\
12 & $\mathrm{U}$ \\
12 & $\mathrm{U}$ \\
12 & $\mathrm{U}$ \\
12 & $\mathrm{U}$ \\
12 & $\mathrm{U}$ \\
12 & $\mathrm{U}$ \\
12 & $\mathrm{U}$ \\
12 & $\mathrm{U}$ \\
12 & $\mathrm{U}$ \\
12 & $\mathrm{U}$ \\
12 & $\mathrm{U}$ \\
12 & $\mathrm{U}$ \\
12 & $\mathrm{U}$ \\
12 & $\mathrm{U}$ \\
12 & $\mathrm{U}$ \\
12 & $\mathrm{U}$ \\
\hline
\end{tabular}


FORM 1

VOLATILE ORGANICS ANALYSIS DATA SHEET

Lab Name: STL BURLINGTON

Contract: 21005
ARGLAB SAMPLE NO.

NA-S-20543

Lab Code: STLVT Case No.: NAVARRE SAS No.:

SDG No.: 114309

Matrix: (soil/water) SOIL

Sample wt/vol : $\quad 7.5(\mathrm{~g} / \mathrm{mL})$ G

Level : (low/med) MED

$\%$ Moisture: not dec.

GC Column: CAP ID: 0.53 (mm)

Soil Extract Volume: 10000 (uJ)
Lab Sample ID: 669182

Lab File ID: $\quad$ 669182E

Date Received: 05/12/06

Date Analyzed: 05/19/06

Dilution Factor: 1.0

Soil Aliquot Volume:

500 (U山)

CAS NO.

COMPOUND

CONCENTRATION UNITS:

(ug/L or $\mathrm{ug} / \mathrm{Kg}$ ) UG/KG

Q

\begin{tabular}{|c|c|c|}
\hline 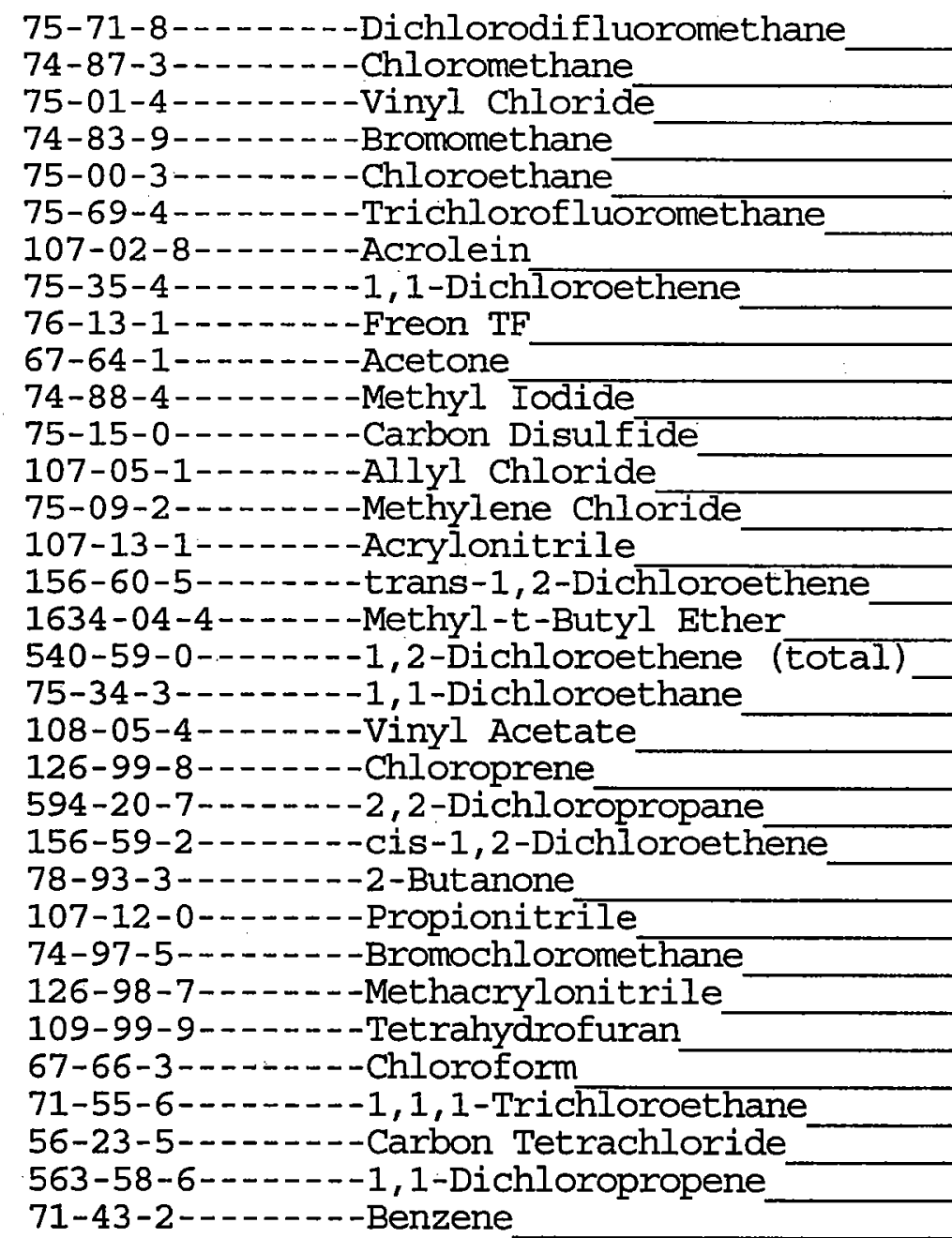 & $\begin{array}{r}13 \\
13 \\
13 \\
8.3 \\
13 \\
13 \\
66 \\
13 \\
13 \\
66 \\
6.0 \\
13 \\
13 \\
5.5 \\
13 \\
13 \\
13 \\
13 \\
13 \\
13 \\
13 \\
13 \\
13 \\
290 \\
53 \\
13 \\
13 \\
190 \\
5.4 \\
13 \\
15 \\
13 \\
13\end{array}$ & \begin{tabular}{|l}
$\mathrm{U}$ \\
$\mathrm{U}$ \\
$\mathrm{U}$ \\
$\mathrm{JB}$ \\
$\mathrm{U}$ \\
$\mathrm{U}$ \\
$\mathrm{U}$ \\
$\mathrm{U}$ \\
$\mathrm{U}$ \\
$\mathrm{U}$ \\
$\mathrm{JB}$ \\
$\mathrm{U}$ \\
$\mathrm{U}$ \\
$\mathrm{JB}$ \\
$\mathrm{U}$ \\
$\mathrm{U}$ \\
$\mathrm{U}$ \\
$\mathrm{U}$ \\
$\mathrm{U}$ \\
$\mathrm{U}$ \\
$\mathrm{U}$ \\
$\mathrm{U}$ \\
$\mathrm{U}$ \\
$\mathrm{B}$ \\
$\mathrm{U}$ \\
$\mathrm{U}$ \\
$\mathrm{U}$ \\
$\mathrm{U}$ \\
$\mathrm{J}$ \\
$\mathrm{U}$ \\
$\mathrm{U}$ \\
$\mathrm{U}$
\end{tabular} \\
\hline
\end{tabular}


FORM 1

VOLATILE ORGANICS ANALYSIS DATA SHEET

Lab Name: STL BURLINGTON

Contract : 21005
ARGLAB SAMPLE NO.

NA-S-20543

Lab Code: STLVT Case No.: NAVARRE SAS No.:

SDG No.: 114309

Matrix: (soil/water) ȘOIL

Sample wt/vol: $\quad 7.5(\mathrm{~g} / \mathrm{mL}) \mathrm{G}$

Level: (low/med) MED

\% Moisture: not dec.

GC Column: CAP

Soil Extract Volume: 10000 (UU)
Lab Sample ID: 669182

Lab File ID: $669182 \mathrm{E}$

Date Received: 05/12/06

Date Analyzed: 05/19/06

Dilution Factor: 1.0

Soil Aliquot Volume: $500(u L)$

CAS NO.

COMPOUND

CONCENTRATION UNITS:

(ug/L or $\mathrm{ug} / \mathrm{Kg}$ ) UG/KG

\begin{tabular}{|c|c|c|}
\hline 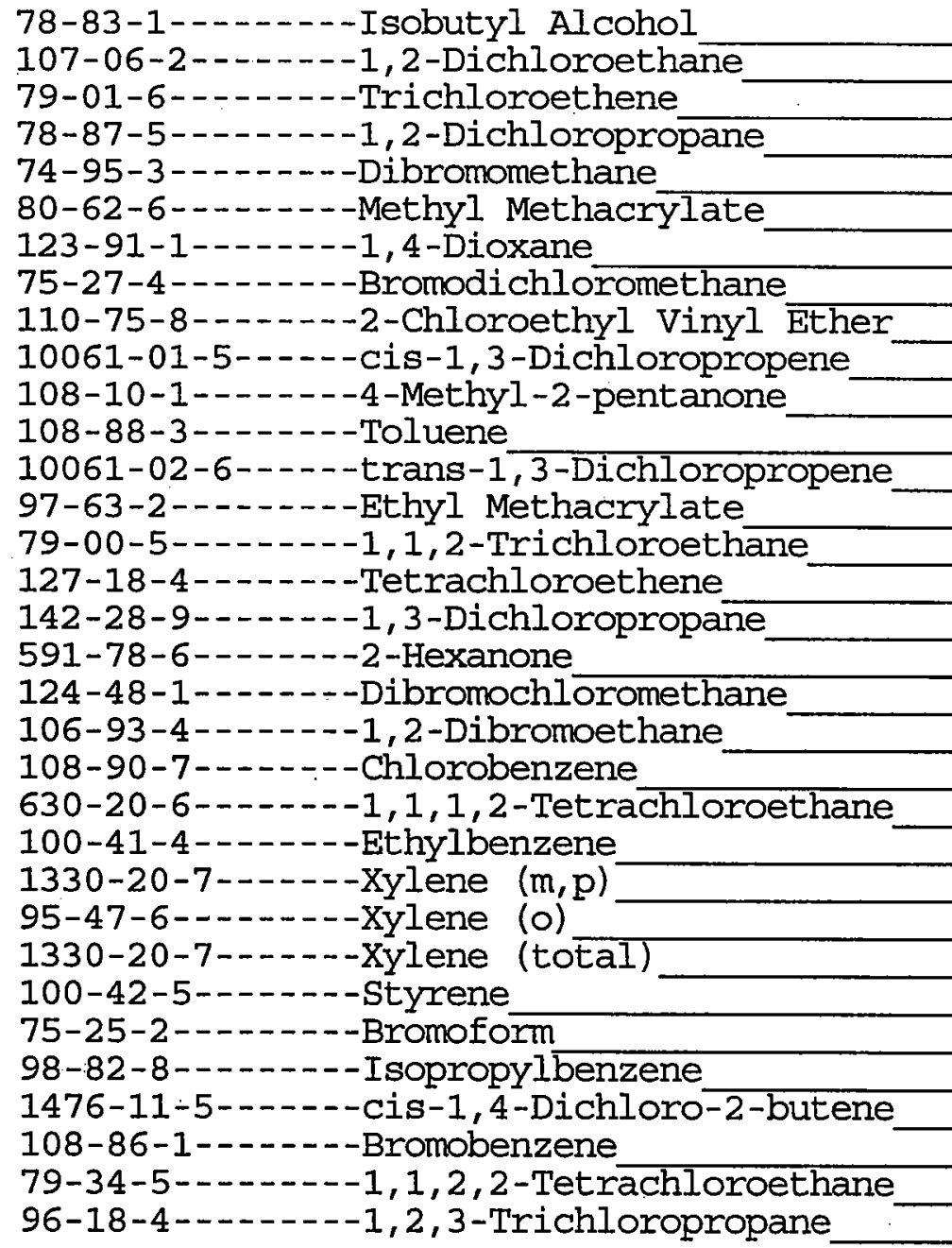 & $\begin{array}{r}660 \\
13 \\
13 \\
13 \\
13 \\
13 \\
660 \\
13 \\
13 \\
13 \\
66 \\
13 \\
13 \\
13 \\
13 \\
13 \\
13 \\
66 \\
13 \\
13 \\
13 \\
13 \\
13 \\
3.8 \\
13 \\
4.0 \\
13 \\
13 \\
13 \\
13 \\
13 \\
13 \\
13\end{array}$ & 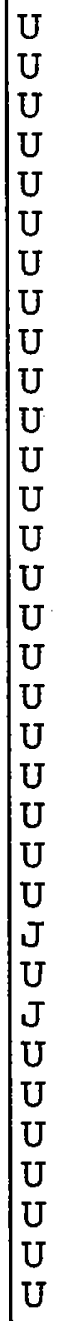 \\
\hline
\end{tabular}


FORM 1

VOLATILE ORGANICS ANALYSIS DATA SHEET

Lab Name: STL BURLINGTON

Contract : 21005
ARGLAB SAMPLE NO.

NA-S-20543

Lab Code: STLVT Case No.: NAVARRE SAS No.:

SDG No.: 114309

Matrix: (soil/water) SOIL

Lab Sample ID: 669182

Sample wt/vol:

$7.5(\mathrm{~g} / \mathrm{mL}) \mathrm{G}$

Lab File ID: $\quad 669182 \mathrm{E}$

Level: (low/med) MED

Date Received: 05/12/06

\% Moisture: not dec.

GC Column: CAP

ID: $0.53 \quad(\mathrm{~mm})$

Date Analyzed: 05/19/06

Soil Extract Volume: 10000 (uL)

Dilution Factor: 1.0

Soil Aliquot Volume:

500 (UL)

CONCENTRATION UNITS :

CAS NO.

COMPOUND

(ug/L or $u g / \mathrm{Kg}$ ) UG/KG

Q

110-57-6-------trans-1, 4-Dichloro-2-butene 103-65-1------n-Propylbenzene 95-49-8-2...-2-Chlorotoluene 106-43-4-n---4-Chlorotoluene 108-67-8------1, 3, 5-Trimethylbenzene 98-06-6-------tert-Butylbenzene 95-63-6--.-.--1, 2, 4-Trimethylbenzene 135-98-8-------sec-Butylbenzene 541-73-1------1, 3-Dichlorobenzene 99-87-6-------4-Isopropyltoluene 106-46-7------1, 4-Dichlorobenzene 95-50-1-.....-1, 2-Dichlorobenzene 104-51-8------n-Butylbenzene 96-12-8------1, 2-Dibromo-3-Chloropropane 120-82-1-.....-1, 2,4-Trichlorobenzene 87-68-3--.-----Hexachlorobutadiene 91-20-3--.-.-Naphthalene 87-61-6-...--1,2,3-Trichlorobenzene 
FORM 1

VOLATILE ORGANICS ANALYSIS DATA SHEET

Lab Name: STL BURLINGTON

Contract : 21005
ARGLAB SAMPLE NO.

NA-S-20545

Lab Code: STLVT Case No.: NAVARRE SAS No.:

SDG No.: 114309

Matrix: (soil/water) soIL

Sample wt/vol:

$10.1(\mathrm{~g} / \mathrm{mL}) \mathrm{G}$

Level: (low/med) MED

$\%$ Moisture: not dec.

GC Column: CAP

ID: $0.53 \quad(\mathrm{~mm})$

Soil Extract Volume: 10000 (uL)
Lab Sample ID: 669180

Lab File ID: $\quad 669180 \mathrm{E}$

Date Received: 05/12/06

Date Analyzed: 05/19/06

Dilution Factor: 1.0

Soil Aliquot Volume:

$500(\mathrm{uL})$

CAS NO.

COMPOUND

CONCENTRATION UNITS :

(ug/L or $\mathrm{ug} / \mathrm{Kg}$ ) UG/KG

Q

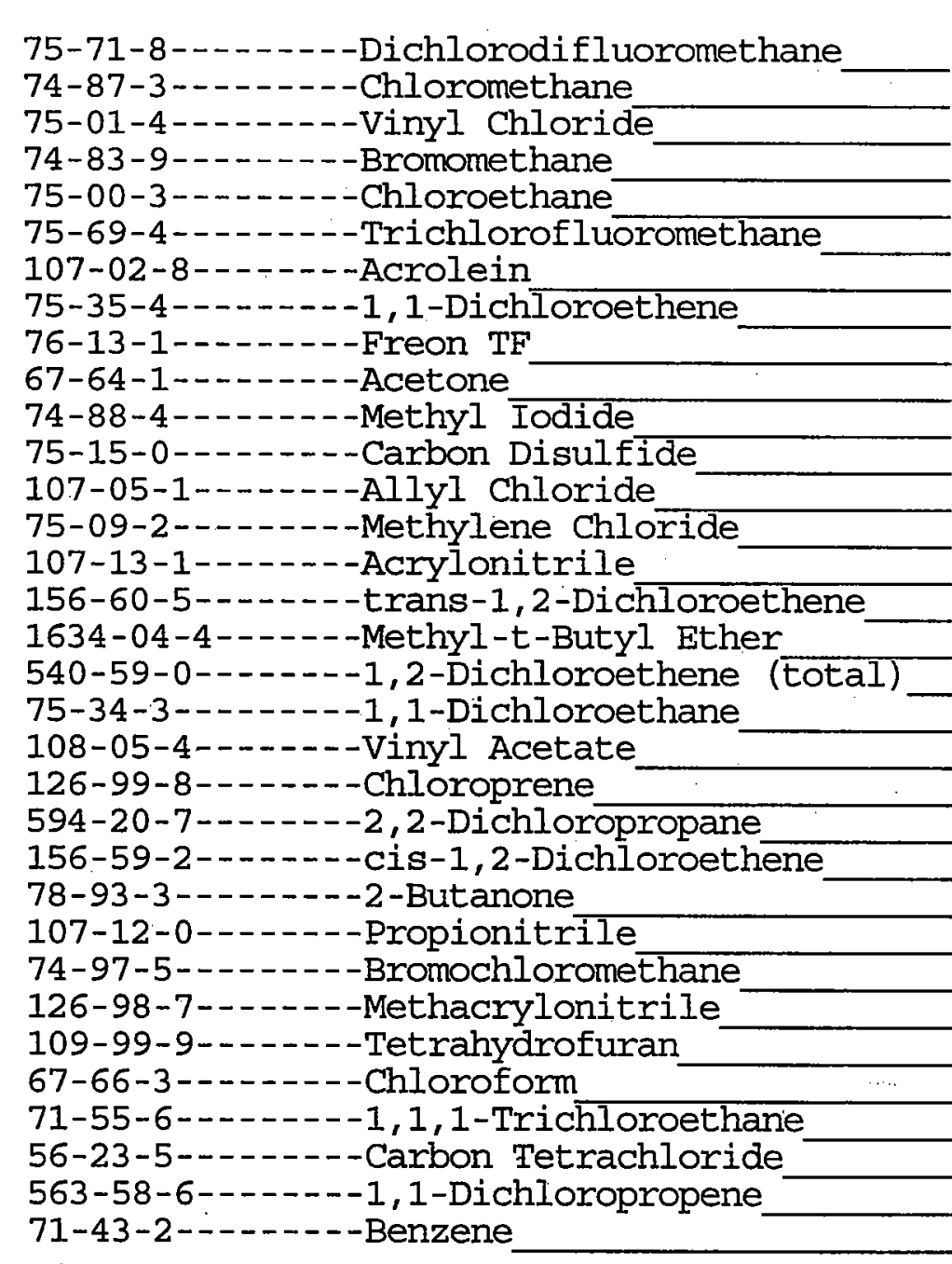

$9.9 \mathrm{U}$

$2.1 \mathrm{JB}$

$9.9 \mathrm{U}$

$7.2 \mathrm{JB}$

$9.9 \mathrm{U}$

$9.9 \mathrm{U}$

$49 \mathrm{U}$

$9.9 \mathrm{U}$

$9.9 \mathrm{U}$

$49 \mathrm{U}$

$3.0 \mathrm{JB}$

$9.9 \mathrm{U}$

$9.9 \mathrm{U}$

$4.0 \mathrm{JB}$

$9.9 \mathrm{U}$

$9.9 \mathrm{U}$

$9.9 \mathrm{U}$

$9.9 \mathrm{U}$

$9.9 \mathrm{U}$

$9.9 \mathrm{U}$

$9.9 \mathrm{U}$

$9.9 \mathrm{U}$

$9.9 \mathrm{U}$

210 B

$40 \mathrm{U}$

$9.9 \mathrm{U}$

$9.9 \mathrm{U}$

$140 \mathrm{U}$

29

9.9

35

$9.9 \overline{\mathrm{U}}$

$9.9 \mathrm{U}$ 
FORM 1

VOLATILE ORGANICS ANALYSIS DATA SHEET

Lab Name: STL BURLINGTON

Contract: 21005

Lab Code: STLVT Case No.: NAVARRE SAS No.:

Lab Sample ID: 669180

Sample wt/vol: $\quad 10.1(\mathrm{~g} / \mathrm{mL}) \mathrm{G}$

Lab File ID: $\quad 669180 E$

ID: $0.53(\mathrm{~mm})$

Soil Extract Volume: 10000 (uL)
ARGLAB SAMPLE NO.

NA-S-20545
Level: (low/med) MED

\% Moisture: not dec.

GC Column: CAP

Matrix: (soil/water) SOIL

CAS NO.
COMPOUND
Date Received: 05/12/06

Date Analyzed: 05/19/06

Dilution Factor: 1.0

Soil Aliquot Volume:

500 (uL)
CONCENTRATION UNITS :

(ug/L or $u g / \mathrm{Kg}$ ) UG/KG
$490 \mathrm{U}$

$9.9 \mathrm{U}$

$9.9 \mathrm{U}$

$9.9 \mathrm{U}$

$9.9 \mathrm{U}$

$9.9 \mathrm{U}$

$490 \mathrm{U}$

$9.9 \mathrm{U}$

$9.9 \mathrm{U}$

$9.9 \mathrm{U}$

$49 \mathrm{U}$

$9.9 \mathrm{U}$

$9.9 \mathrm{U}$

$9.9 \mathrm{U}$

$9.9 \mathrm{U}$

$9.9 \mathrm{U}$

$9.9 \mathrm{U}$

$49 \mathrm{U}$

$9.9 \mathrm{U}$

$9.9 \mathrm{U}$

$9.9 \mathrm{U}$

$9.9 \mathrm{U}$

$9.9 \mathrm{U}$

$2.8 \mathrm{~J}$

$9.9 \mathrm{U}$

$3.0 \mathrm{~J}$

$9.9 \mathrm{U}$

$9.9 \mathrm{U}$

$9.9 \mathrm{U}$

$9.9 \mathrm{U}$

$9.9 \mathrm{U}$

$9.9 \mathrm{U}$

$9.9 \mathrm{U}$ 
FORM 1

VOLATILE ORGANICS ANALYSIS DATA SHEET

Lab Name: STL BURLINGTON

Contract: 21005

Lab Code: STLVT Case No.: NAVARRE SAS No.:

ARGLAB SAMPLE NO.

NA-S-20545

SDG NO.: 114309
Matrix: (soil/water) SOIL

Sample wt/vol:

$10.1(\mathrm{~g} / \mathrm{mL}) \mathrm{G}$

Level: (low/med) MED

\% Moisture: not dec.

GC Column: CAP

ID $: 0.53 \quad(\mathrm{~mm})$

Soil Extract Volume: 10000 (uL)
Lab Sample ID: 669180

Lab File ID: $669180 \mathrm{E}$

Date Received: 05/12/06

Date Analyzed: 05/19/06

Dilution Factor: 1.0

Soil Aliquot Volume:

$500(u L)$

(ug/L or ug/Kg) UG/KG Q

$9.9 \mathrm{U}$

$9.9 \mathrm{U}$

$9.9 \mathrm{U}$

$9.9 \mathrm{U}$

$9.9 \mathrm{U}$

$9.9 \mathrm{U}$

$9.9 \mathrm{U}$

$9.9 \mathrm{U}$

$9.9 \mathrm{U}$

$9.9 \mathrm{U}$

$9.9 \mathrm{U}$

$9.9 \mathrm{U}$

$9.9 \mathrm{U}$

$9.9 \mathrm{U}$

$9.9 \mathrm{U}$

$9.9 \mathrm{U}$

$9.9 \mathrm{U}$

$9.9 \mathrm{U}$ 
FORM 1

VOLATILE ORGANICS ANALYSIS DATA SHEET

Lab Name: STL BURLINGTON

Contract: 21005
ARGLAB SAMPLE NO.

NA-S-20575

Lab Code: STLVT Case No.: NAVARRE SAS No.:

SDG No. : 114309

Matrix: (soil/water) SOIL

Sample wt/vol:

$11.2(\mathrm{~g} / \mathrm{mL}) \mathrm{G}$

Level: (low/med) MED

\% Moisture: not dec.

GC Column: CAP

ID : $0.53 \quad(\mathrm{~mm})$

Soil Extract Volume: 10000 (uL)
Lab Sample ID: 669179

Lab File ID: $\quad 669179 \mathrm{E}$

Date Received: 05/12/06

Date Analyzed: 05/19/06

Dilution Factor: 1.0

Soil Aliquot Volume:

500 (uL)

CAS NO.

COMPOUND

CONCENTRATION UNITS :

(ug/L or $\mathrm{ug} / \mathrm{Kg}$ ) UG/KG

Q

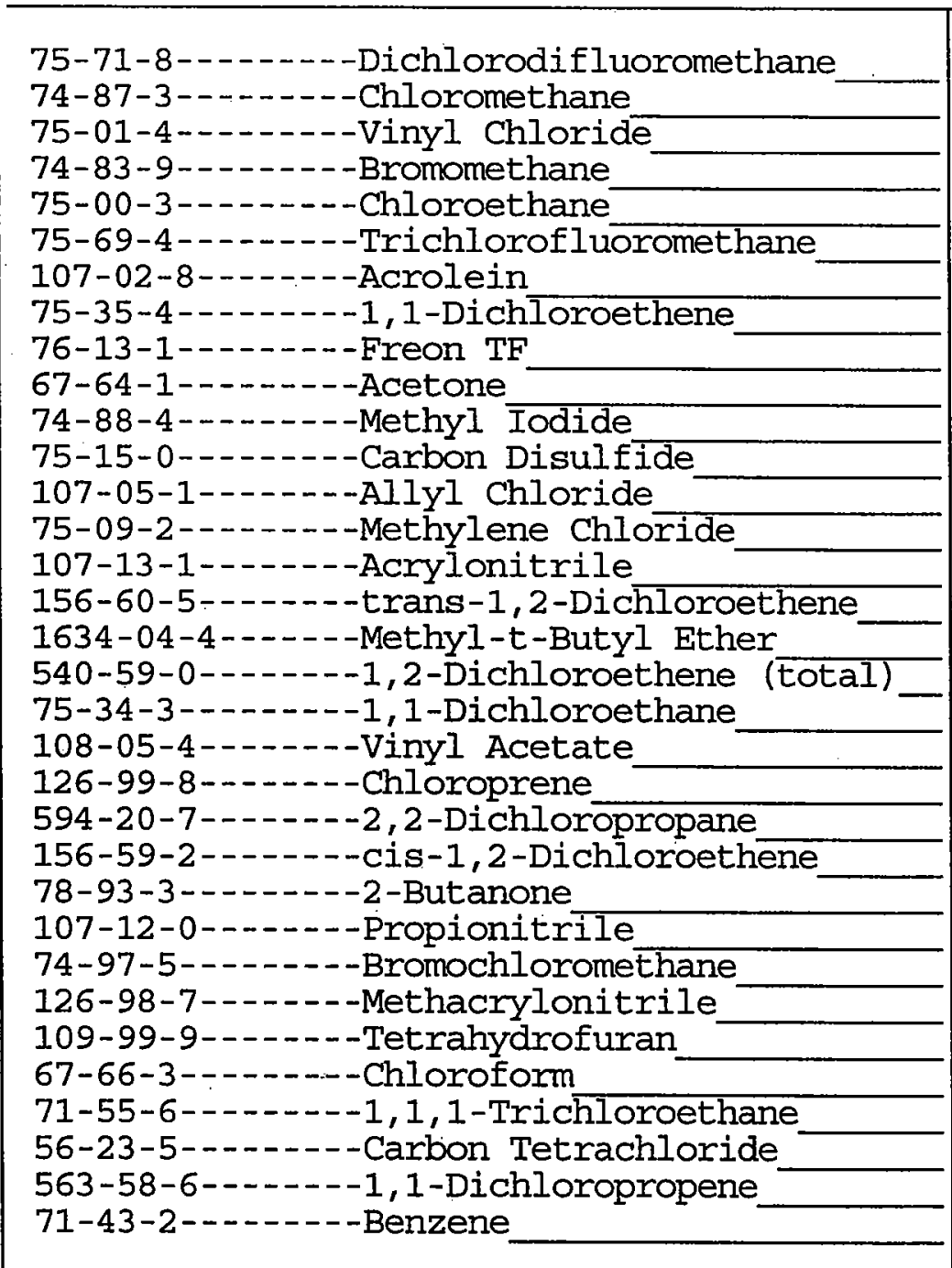

$8.9 \mathrm{U}$

$2.1 \mathrm{JB}$

$8.9 \mathrm{U}$

$8.9 \mathrm{~B}$

$8.9 \mathrm{U}$

$8.9 \mathrm{U}$

$44 \mathrm{U}$

$8.9 \mathrm{U}$

$8.9 \mathrm{U}$

$44 \mathrm{U}$

$5.0 \mathrm{JB}$

$8.9 \mathrm{U}$

$8.9 \mathrm{U}$

$6.5 \mathrm{JB}$

$8.9 \mathrm{U}$

$8.9 \mathrm{U}$

$8.9 \mathrm{U}$

$8.9 \mathrm{U}$

$8.9 \mathrm{U}$

$8.9 \mathrm{U}$

8.9 U

$8.9 \mathrm{U}$

$8.9 \mathrm{U}$

$44 \mathrm{JB}$

$36 \mathrm{U}$

$8.9 \mathrm{U}$

$8.9 \mathrm{U}$

$120 \mathrm{U}$

$2.7 \mathrm{~J}$

$8.9 \mathrm{U}$

77

$8.9 \mathrm{U}$

$8.9 \mathrm{U}$ 
FORM 1

VOLATILE ORGANICS ANALYSIS DATA SHEET

Lab Name: STL BURLINGTON

Contract: 21005

Lab Code: STLVT Càse No.: NAVARRE SAS No.:

Matrix: (soil/water) SOIL

Lab Sample ID: 669179

Sample wt/vol:

$11.2(\mathrm{~g} / \mathrm{mL}) \mathrm{G}$

Level: (low/med) MED

\% Moisture: not dec.

GC Column: CAP

ID: 0.53 (mm)

Soil Extract Volume: 10000 (uL)
ARGLAB SAMPLE NO.

NA-S-20575
Lab File ID: $\quad 669179 \mathrm{E}$

Date Received: 05/12/06

Date Analyzed: 05/19/06

Dilution Factor: 1.0

Soil Aliquot Volume:

500 (uL)

CAS NO.

COMPOUND

CONCENTRATION UNITS:

(ug/I or $\mathrm{ug} / \mathrm{Kg}$ ) UG/KG

\begin{tabular}{|c|c|c|}
\hline 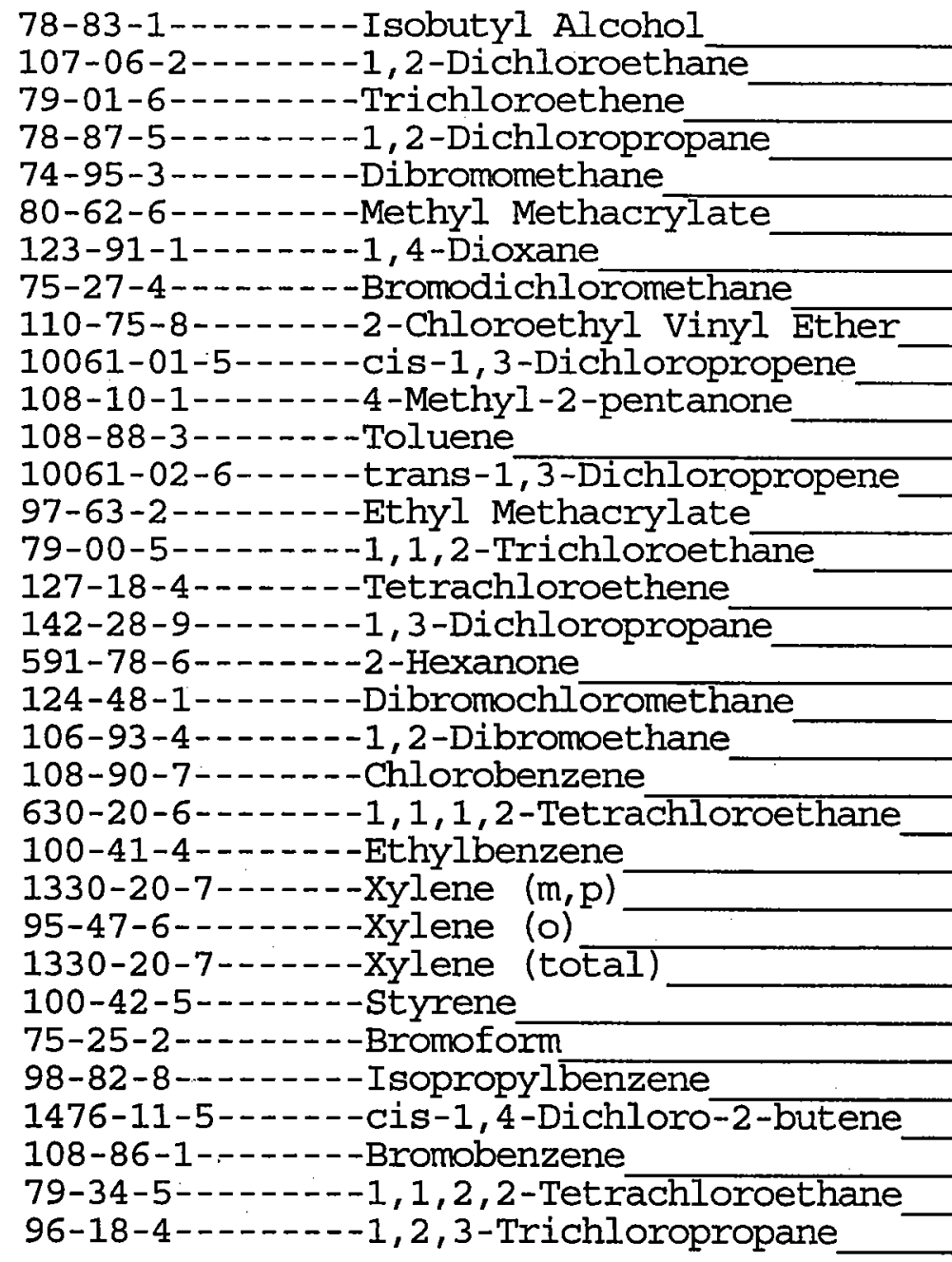 & $\begin{array}{r}440 \\
8.9 \\
8.9 \\
8.9 \\
8.9 \\
3.3 \\
440 \\
8.9 \\
8.9 \\
8.9 \\
44 \\
2.2 \\
8.9 \\
8.9 \\
8.9 \\
8.9 \\
8.9 \\
44 \\
8.9 \\
8.9 \\
8.9 \\
8.9 \\
8.9 \\
8.9 \\
8.9 \\
8.9 \\
8.9 \\
8.9 \\
8.9 \\
8.9 \\
8.9 \\
8.9 \\
8.9\end{array}$ & \begin{tabular}{|l}
$\mathrm{U}$ \\
$\mathrm{U}$ \\
$\mathrm{U}$ \\
$\mathrm{U}$ \\
$\mathrm{U}$ \\
$\mathrm{J}$ \\
$\mathrm{U}$ \\
$\mathrm{U}$ \\
$\mathrm{U}$ \\
$\mathrm{U}$ \\
$\mathrm{U}$ \\
$\mathrm{JB}$ \\
$\mathrm{U}$ \\
$\mathrm{U}$ \\
$\mathrm{U}$ \\
$\mathrm{U}$ \\
$\mathrm{U}$ \\
$\mathrm{U}$ \\
$\mathrm{U}$ \\
$\mathrm{U}$ \\
$\mathrm{U}$ \\
$\mathrm{U}$ \\
$\mathrm{U}$ \\
$\mathrm{U}$ \\
$\mathrm{U}$ \\
$\mathrm{U}$ \\
$\mathrm{U}$ \\
$\mathrm{U}$ \\
$\mathrm{U}$ \\
$\mathrm{U}$ \\
$\mathrm{U}$ \\
$\mathrm{U}$ \\
$\mathrm{U}$ \\
\end{tabular} \\
\hline
\end{tabular}


FORM 1

VOLATILE ORGANICS ANALYSIS DATA SHEET

Lab Name: STL BURLINGTON

Contract : 21005

Lab Code: STLVT Case No.: NAVARRE SAS No.:

ARGLAB SAMPLE NO.

$\mathrm{NA}-\mathrm{S}-20575$

SDG No.: 114309
Matrix: (soil/water) SOIL

Sample wt/vol:

$11.2(\mathrm{~g} / \mathrm{mL}) \mathrm{G}$

Level: (low/med) MED

\% Moisture: not dec.

GC Column: CAP

Soil Extract Volume: 10000 (uL)
Lab Sample ID: 669179

Lab File ID: $\quad 669179 \mathrm{E}$

Date Received: 05/12/06

Date Analyzed: 05/19/06

Dilution Factor: 1.0

Soil Aliquot Volume:

500 (uس)

CAS NO.

COMPOUND

CONCENTRATION UNITS:

(ug/L or $\mathrm{ug} / \mathrm{Kg}$ ) UG/KG

Q

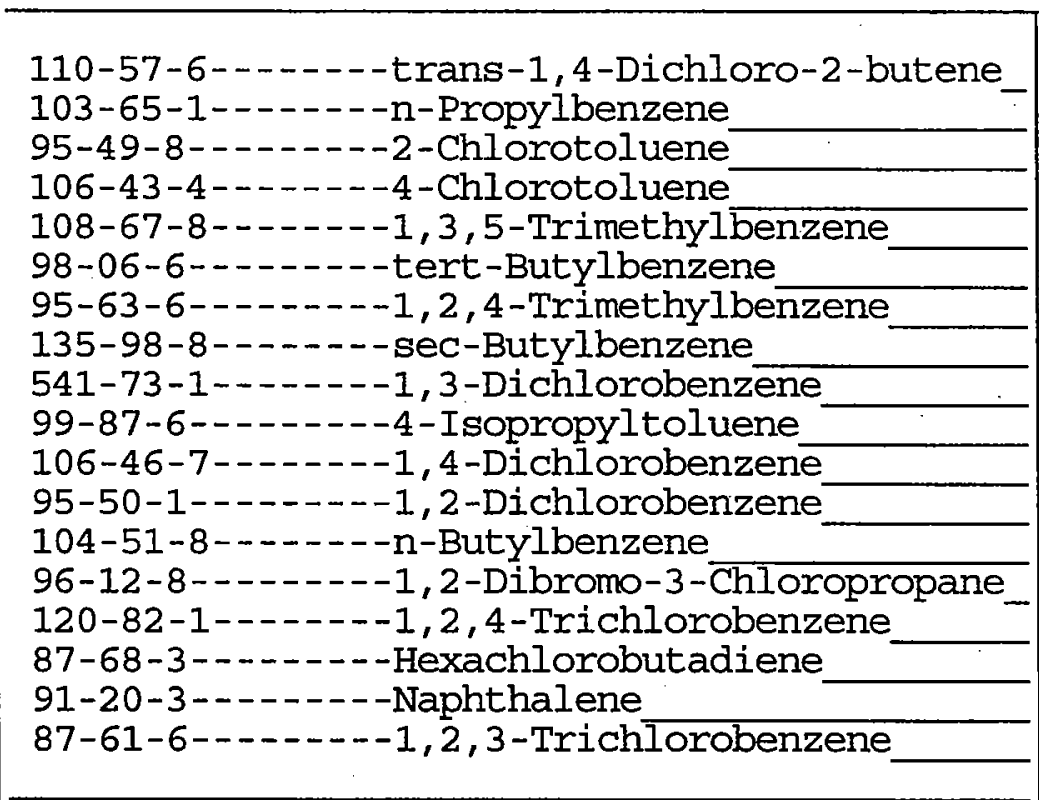

$8.9 \mathrm{U}$

$8.9 \mathrm{U}$

$8.9 \mathrm{U}$

$8.9 \mathrm{U}$

$8.9 \mathrm{U}$

$8.9 \mathrm{U}$

$8.9 \mathrm{U}$

$8.9 \mathrm{U}$

$8.9 \mathrm{U}$

$8.9 \mathrm{U}$

$8.9 \mathrm{U}$

$8.9 \mathrm{U}$

$8.9 \mathrm{U}$

$8.9 \mathrm{U}$

$8.9 \mathrm{U}$

$8.9 \mathrm{U}$

$8.9 \mathrm{U}$

$8.9 \mathrm{U}$ 
FORM 1

VOLATILE ORGANICS ANALYSIS DATA SHEET

Lab Name: STL BURLINGTON

Contract: 21005

ARGLAB SAMPLE NO.

Lab Code: STLVT Case No.: NAVARRE SAS No.:

NA-S-20636

Matrix: (soil/water) SOIL

Sample wt/vol: $\quad 12.9(\mathrm{~g} / \mathrm{mL}) \mathrm{G}$

Level: (low/med) MED

\% Moisture: not dec.

GC Column: CAP ID: $0.53 \quad$ (mm)

Soil Extract Volume: 10000 (uL)
Lab Sample ID: 669174

Lab File ID: $\quad 669174 E$

Date Received: 05/12/06

Date Analyzed: 05/19/06

Dilution Factor: 1.0

Soil Aliquot Volume:

500 (UL)

\begin{abstract}
CAS NO.
\end{abstract}
CONCENTRATION UNITS:

(ug/L or $\mathrm{ug} / \mathrm{Kg}$ ) UG/KG

$Q$

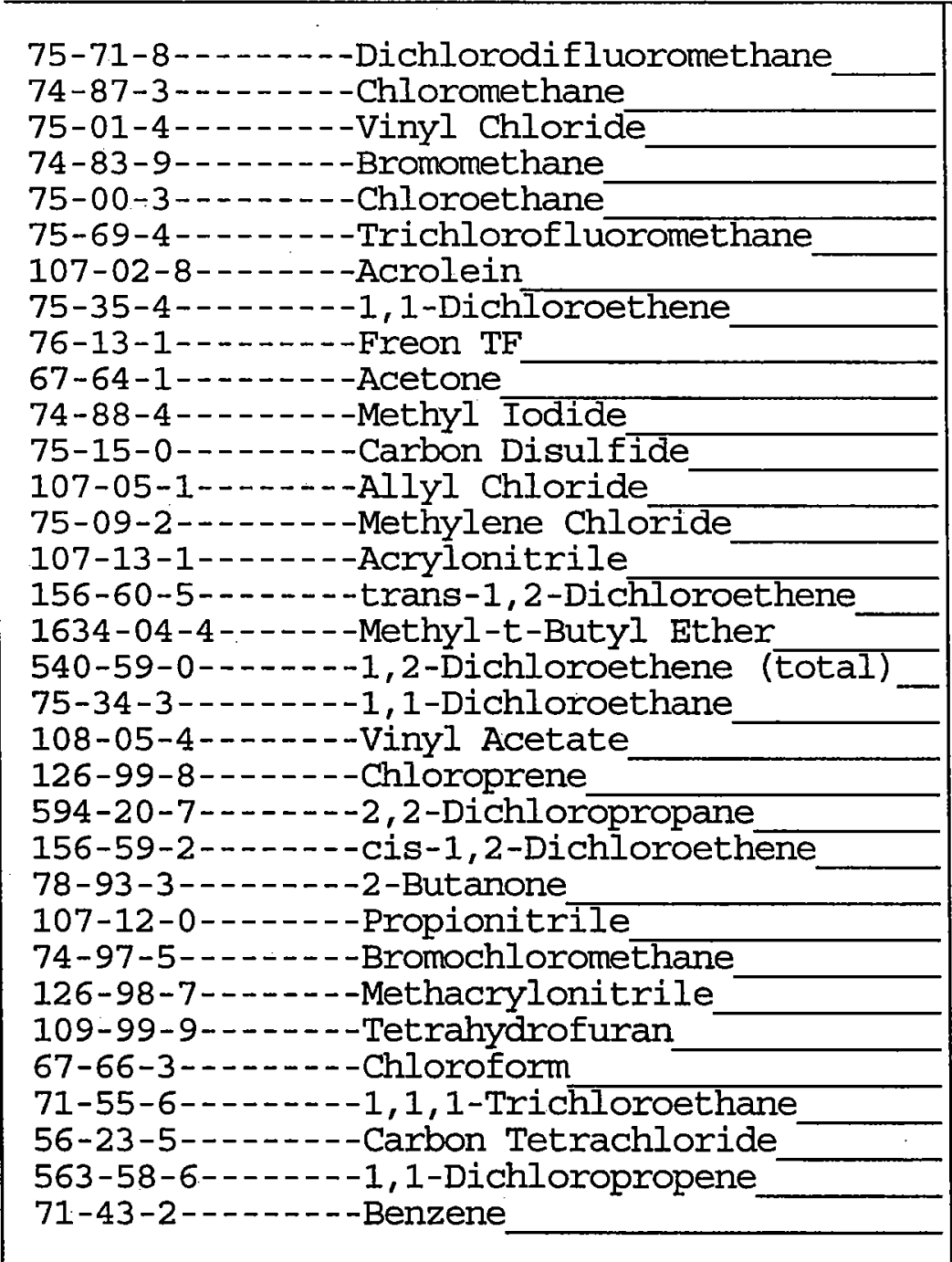

$7.8 \mathrm{U}$

$3.7 \mathrm{JB}$

$7.8 \mathrm{U}$

$13 \mathrm{~B}$

$7.8 \mathrm{U}$

$7.8 \mathrm{U}$

$39 \mathrm{U}$

$7.8 \mathrm{U}$

$7.8 \mathrm{U}$

$39 \mathrm{U}$

$8.7 \mathrm{~B}$

$7.8 \mathrm{U}$

$7.8 \mathrm{U}$

$18 \mathrm{~B}$

$7.8 \mathrm{U}$

$7.8 \mathrm{U}$

$7.8 \mathrm{U}$

$7.8 \mathrm{U}$

$7.8 \mathrm{U}$

$7.8 \mathrm{U}$

$7.8 \mathrm{U}$

$7.8 \mathrm{U}$

$7.8 \mathrm{U}$

$180 \mathrm{~B}$

$31 \mathrm{U}$

$7.8 \mathrm{U}$

$7.8 \mathrm{U}$

$110 \mathrm{U}$

$7.8 \mathrm{U}$

$7.8 \mathrm{U}$

$7.8 \mathrm{U}$

$7.8 \mathrm{U}$

$7.8 \mathrm{U}$ 
FORM 1

VOLATILE ORGANICS ANALYSIS DATA SHEET

Lab Name: STL BURLINGTON

Contract: 21005
ARGLAB SAMPLE NO.

NA-S-20636

Lab Code: STLVT Case No.: NAVARRE SAS No.:

SDG No. : 114309

Matrix: (soil/water) SOIL

Sample wt/vol:

$12.9(\mathrm{~g} / \mathrm{mL}) \mathrm{G}$

Level: (low/med) MED

\% Moisture: not dec.

GC Column: CAP

ID : $0.53 \quad(\mathrm{~mm})$

Soil Extract Volume: 10000 (uL)
Lab Sample ID: 669174

Lab File ID: $\quad$ 669174E

Date Received: 05/12/06

Date Analyzed: 05/19/06

Dilution Factor: 1.0

Soil Aliquot Volume:

500 (u山)

CAS NO.

COMPOUND

CONCENTRATION UNITS:

(ug/L or $u g / \mathrm{Kg}$ ) UG/KG

$Q$

$390 \mathrm{U}$

$7.8 \mathrm{U}$

$7.8 \mathrm{U}$

$7.8 \mathrm{U}$

$7.8 \mathrm{U}$

$6.5 \mathrm{~J}$

$390 \mathrm{U}$

$7.8 \mathrm{U}$

$7.8 \mathrm{U}$

$7.8 \mathrm{U}$

$39 \mathrm{U}$

$7.8 \mathrm{U}$

$7.8 \mathrm{U}$

$7.8 \mathrm{U}$

$7.8 \mathrm{U}$

$7.8 \mathrm{U}$

$7.8 \mathrm{U}$

$39 \mathrm{U}$

$7.8 \mathrm{U}$

$7.8 \mathrm{U}$

$7.8 \mathrm{U}$

$7.8 \mathrm{U}$

$7.8 \mathrm{U}$

$2.6 \mathrm{~J}$

$7.8 \mathrm{U}$

$2.7 \mathrm{~J}$

$7.8 \mathrm{U}$

$7.8 \mathrm{U}$

$7.8 \mathrm{U}$

$7.8 \mathrm{U}$

$7.8 \mathrm{U}$

$7.8 \mathrm{U}$

$7.8 \mathrm{U}$ 
FORM 1

VOLATILE ORGANICS ANALYSIS DATA SHEET

Lab Name: STL BURLINGTON

Contract : 21005

Lab Code: STLVT Case No.: NAVARRE SAS No.:

Lab Sample ID: 669174

Sample wt/vol: $\quad 12.9(\mathrm{~g} / \mathrm{mL}) \mathrm{G}$

Lab File ID: $\quad 669174 \mathrm{E}$

Level: (low/med) MED

Date Received: 05/12/06

$\%$ Moisture: not dec.

GC Column: CAP ID: 0.53 (mm)

Date Analyzed: 05/19/06

Dilution Factor: 1.0

Soil Extract Volume: 10000 (uL)

Soil Aliquot Volume:

$500(\mathrm{uL})$

CONCENTRATION UNITS:

CAS NO. COMPOUND (ug/L or $\mathrm{ug} / \mathrm{Kg})$ UG/KG

Q

\begin{tabular}{|c|c|c|}
\hline 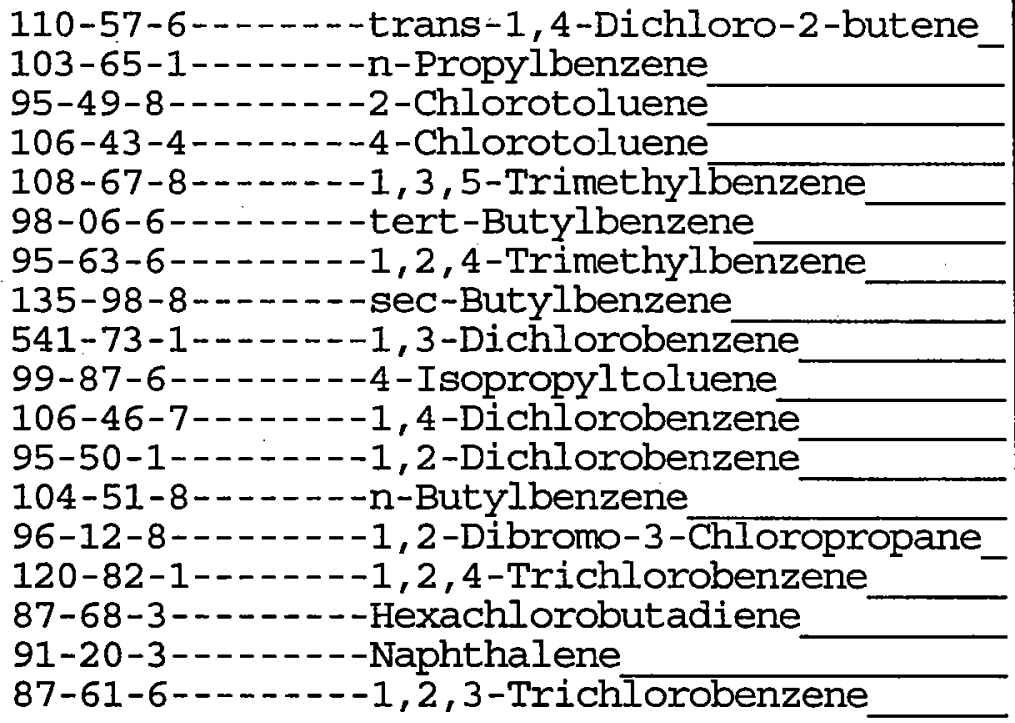 & $\begin{array}{l}7.8 \\
7.8 \\
7.8 \\
7.8 \\
7.8 \\
7.8 \\
7.8 \\
7.8 \\
7.8 \\
7.8 \\
7.8 \\
7.8 \\
7.8 \\
7.8 \\
7.8 \\
7.8 \\
7.8 \\
7.8\end{array}$ & $\begin{array}{l}\mathrm{U} \\
\mathrm{U} \\
\mathrm{U} \\
\mathrm{U} \\
\mathrm{U} \\
\mathrm{U} \\
\mathrm{U} \\
\mathrm{U} \\
\mathrm{U} \\
\mathrm{U} \\
\mathrm{U} \\
\mathrm{U} \\
\mathrm{U} \\
\mathrm{U} \\
\mathrm{U} \\
\mathrm{U} \\
\mathrm{U} \\
\mathrm{U}\end{array}$ \\
\hline
\end{tabular}


FORM 1

VOLATILE ORGANICS ANALYSIS DATA SHEET

Lab Name: STL BURLINGTON

Contract : 21005

ARGLAB SAMPLE NO.

NA-S-20664

Lab Code: STLVT Case No.: NAVARRE SAS No.:

SDG No.: 114309

Matrix: (soil/water) SOIL

Sample wt/vol: $\quad 13.3(\mathrm{~g} / \mathrm{mL}) \mathrm{G}$

Level: (low/med) MED

\% Moisture: not dec.

GC Column: CAP ID: $0.53 \quad(\mathrm{~mm})$

Soil Extract Volume: 10000 (uL)
Lab Sample ID: 669181

Lab File ID: $669181 \mathrm{E}$

Date Received: 05/12/06

Date Analyzed: 05/19/06

Dilution Factor: 1.0

Soil Aliquot Volume: $\quad 500$ (uس)

CONCENTRATION UNITS:

CAS NO. COMPOUND $\quad(u g / L$ or $u g / \mathrm{Kg}) \mathrm{UG} / \mathrm{KG}$

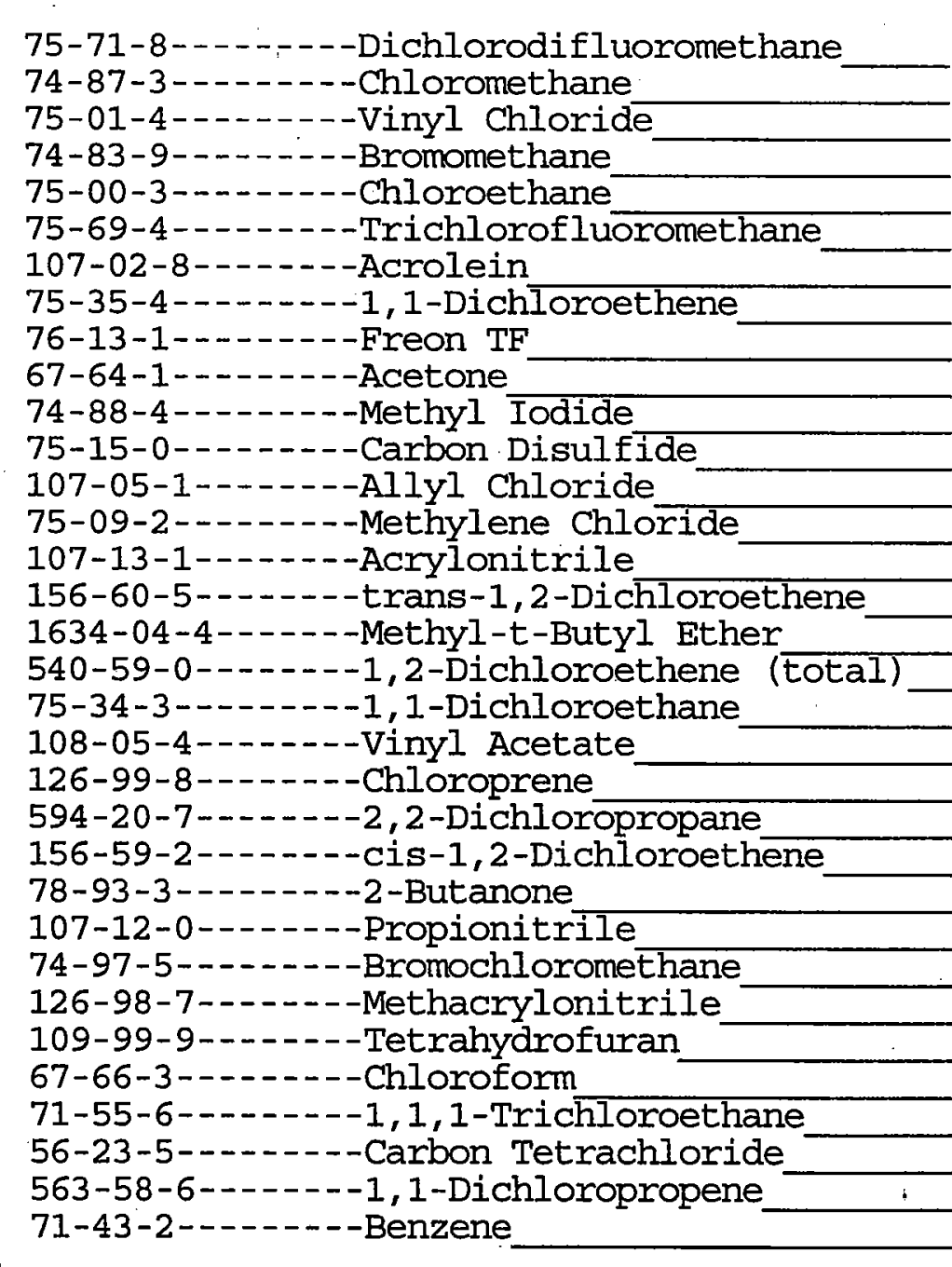

$7.5 \mathrm{U}$

3.3 JB

$7.5 \mathrm{U}$

$5.6 \mathrm{JB}$

$7.5 \mathrm{U}$

$7.5 \mathrm{U}$

$37 \mathrm{U}$

$7.5 \mathrm{U}$

$7.5 \mathrm{U}$

$37 \mathrm{U}$

$3.5 \mathrm{JB}$

$7.5 \mathrm{U}$

$7.5 \mathrm{U}$

$5.7 \mathrm{JB}$

$7.5 \mathrm{U}$

$7.5 \mathrm{U}$

$7.5 \mathrm{U}$

$7.5 \mathrm{U}$

$7.5 \mathrm{U}$

$7.5 \mathrm{U}$

7.5 U

$7.5 \mathrm{U}$

$7.5 \mathrm{U}$

$39 \mathrm{~B}$

$30 \mathrm{U}$

$7.5 \mathrm{U}$

$7.5 \mathrm{U}$

$110 \mathrm{U}$

$7.5 \mathrm{U}$

$7.5 \mathrm{U}$

$7.5 \mathrm{U}$

$7.5 \mathrm{U}$

$7.5 \mathrm{U}$ 
FORM 1

VOLATILE ORGANICS ANALYSIS DATA SHEET

Lab Name: STL BURLINGTON

Contract: 21005

Lab Code: STLVT Case No.: NAVARRE SAS No.:

ARGLAB SAMPLE NO.

NA-S-20664

SDG No.: 114309
Matrix: (soil/water) SoIL

Sample wt/vol : $\quad 13.3(\mathrm{~g} / \mathrm{mL}) \mathrm{G}$

Level : (low/med) MED

\% Moisture: not dec.

GC Column: CAP ID: 0.53 (mm)

Soil Extract Volume: 10000 (uL)
Lab Sample ID: 669181

Lab File ID: $669181 \mathrm{E}$

Date Received: 05/12/06

Date Analyzed: 05/19/06

Dilution Factor: 1.0

Soil Aliquot Volume: $\quad 500(u L)$

CAS NO. COMPOUND (ug/L or ug/Kg) UG/KG

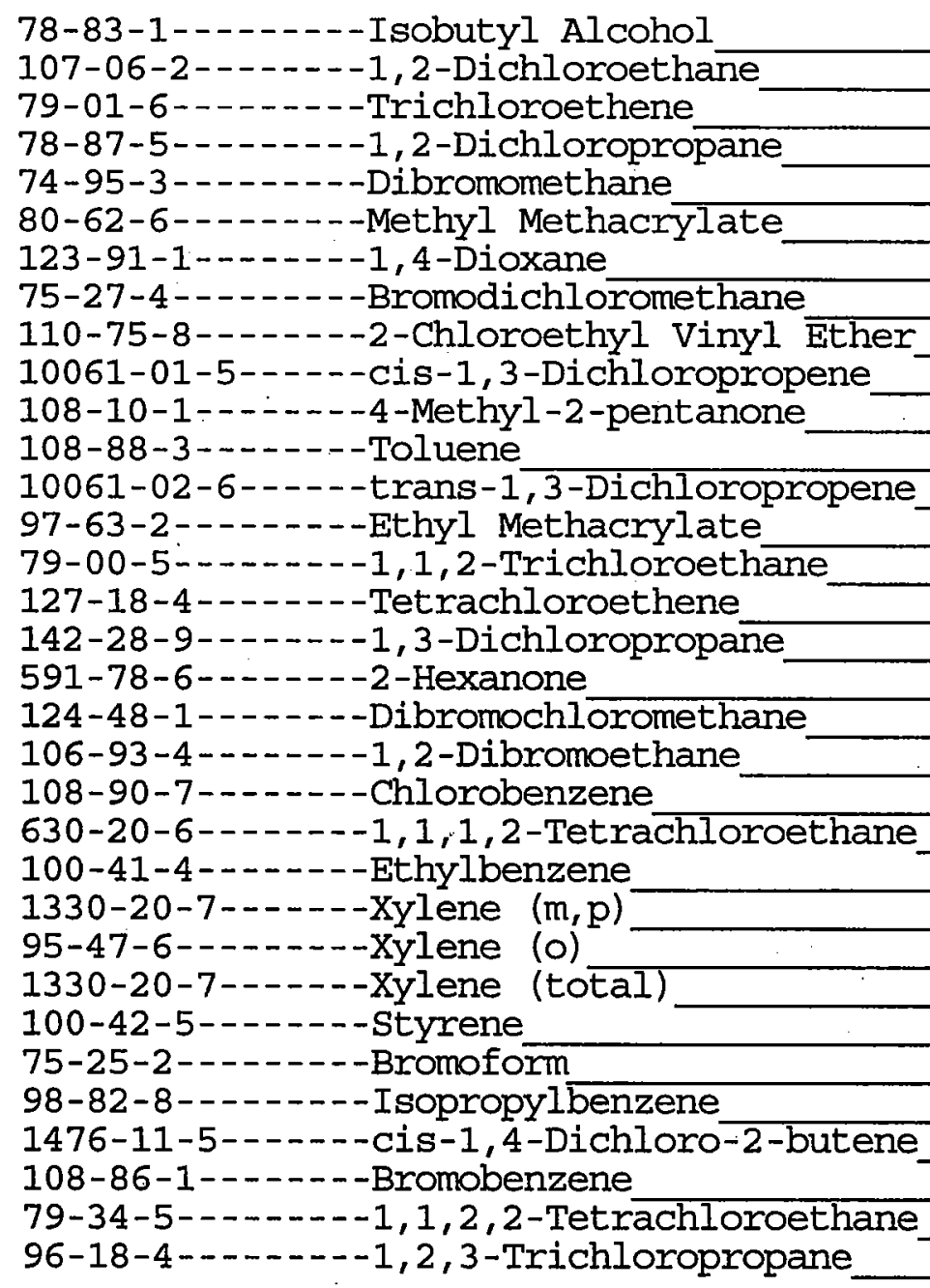

\begin{tabular}{l|l}
380 & $\mathrm{U}$ \\
7.5 & $\mathrm{U}$ \\
7.5 & $\mathrm{U}$ \\
7.5 & $\mathrm{U}$ \\
7.5 & $\mathrm{U}$ \\
7.5 & $\mathrm{U}$ \\
380 & $\mathrm{U}$ \\
7.5 & $\mathrm{U}$ \\
7.5 & $\mathrm{U}$ \\
7.5 & $\mathrm{U}$ \\
37 & $\mathrm{U}$ \\
1.9 & $\mathrm{JB}$ \\
7.5 & $\mathrm{U}$ \\
7.5 & $\mathrm{U}$ \\
7.5 & $\mathrm{U}$ \\
7.5 & $\mathrm{U}$ \\
7.5 & $\mathrm{U}$ \\
37 & $\mathrm{U}$ \\
7.5 & $\mathrm{U}$ \\
7.5 & $\mathrm{U}$ \\
7.5 & $\mathrm{U}$ \\
7.5 & $\mathrm{U}$ \\
7.5 & $\mathrm{U}$ \\
7.5 & $\mathrm{U}$ \\
7.5 & $\mathrm{U}$ \\
7.5 & $\mathrm{U}$ \\
7.5 & $\mathrm{U}$ \\
7.5 & $\mathrm{U}$ \\
7.5 & $\mathrm{U}$ \\
7.5 & $\mathrm{U}$ \\
7.5 & $\mathrm{U}$ \\
7.5 & $\mathrm{U}$ \\
7.5 & $\mathrm{U}$ \\
& \\
\hline & \\
\hline
\end{tabular}


FORM 1

VOLATILE ORGANICS ANALYSIS DATA SHEET

Lab Name: STL BURLINGTON

Contract: 21005
ARGLAB SAMPLE NO.

NA-S-20664

Lab Code: STLVT Case No. : NAVARRE SAS No.:

SDG No. : 114309

Matrix: (soil/water) SOIL

Sample wt/vol: $\quad 13.3(\mathrm{~g} / \mathrm{mL}) \mathrm{G}$

Level: (low/med) MED

号 Moisture: not dec.

GC Column: CAP ID: 0.53 (mm)

Soil Extract Volume: 10000 (UL)
Lab Sample ID: 669181

Lab File ID: $669181 \mathrm{E}$

Date Received: 05/12/06

Date Analyzed: 05/19/06

Dilution Factor: 1.0

Soil Aliquot Volume:

500 (u山)

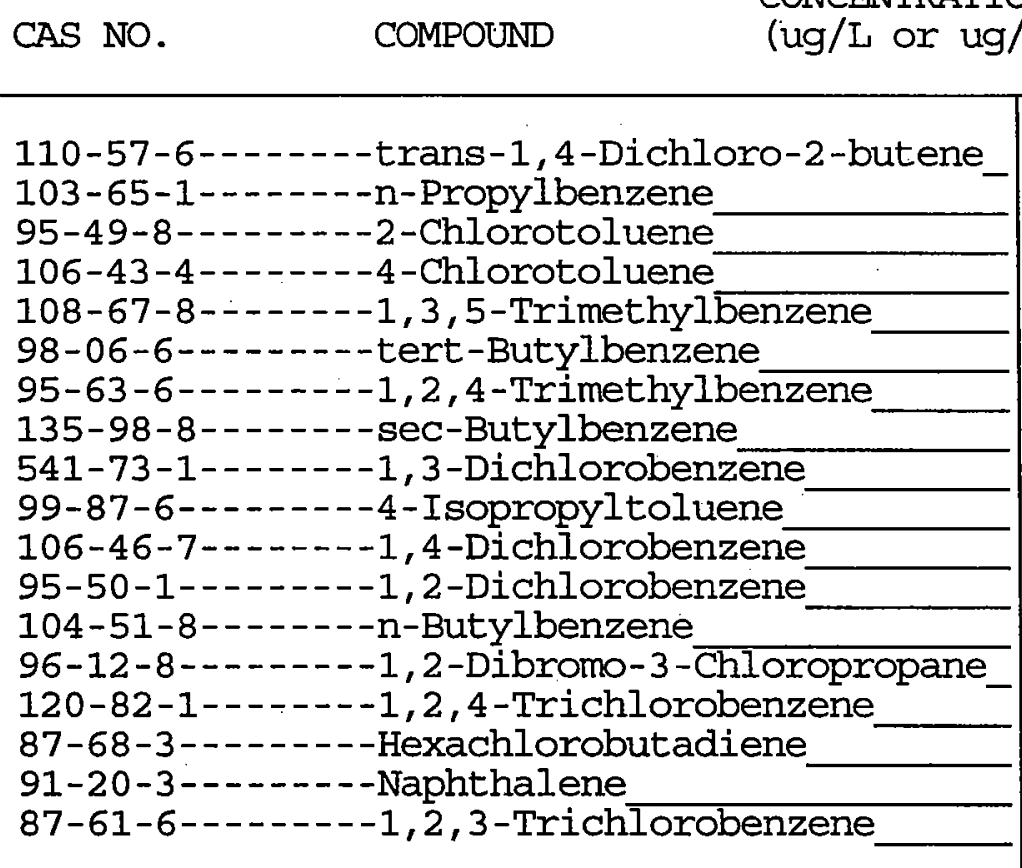

CONCENTRATION UNITS :
(ug/L or ug/Kg) UG/KG

Q

$7.5 \mathrm{U}$

$7.5 \mathrm{U}$

$7.5 \mathrm{U}$

$7.5 \mathrm{U}$

$7.5 \mathrm{U}$

$7.5 \mathrm{U}$

$7.5 \mathrm{U}$

$7.5 \mathrm{U}$

$7.5 \mathrm{U}$

$7.5 \mathrm{U}$

$7.5 \mathrm{U}$

$7.5 \mathrm{U}$

$7.5 \mathrm{U}$

$7.5 \mathrm{U}$

$7.5 \mathrm{U}$

$7.5 \mathrm{U}$

$7.5 \mathrm{U}$

$7.5 \mathrm{U}$ 
FORM 1

VOLATILE ORGANICS ANALYSIS DATA SHEET
Lab Name: STL BURLINGTON

Contract : 21005
ARGLAB SAMPLE NO.

NA-S-20735

Lab Code: STLVT Case No.: NAVARRE SAS No.:

SDG No.: 114309

Matrix: (soil/water) SOIL

Sample wt/vol: $\quad 9.8(\mathrm{~g} / \mathrm{mL}) \mathrm{G}$

Level: (low/med) MED

\% Moisture: not dec.

GC Column: CAP ID: 0.53 (mm)

Soil Extract Volume: 10000 (uL)
Lab Sample ID: 669175

Lab File ID: $\quad$ 669175E

Date Received: 05/12/06

Date Analyzed: 05/19/06

Dilution Factor: 1.0

Soil Aliquot Volume:

500 (UL)

$\begin{array}{llll}\text { CAS NO. COMPOUND } & (\mathrm{ug} / \mathrm{L} \text { or } \mathrm{ug} / \mathrm{Kg}) & \mathrm{UG} / \mathrm{KG} & \mathrm{K}\end{array}$

\begin{tabular}{|c|c|c|}
\hline 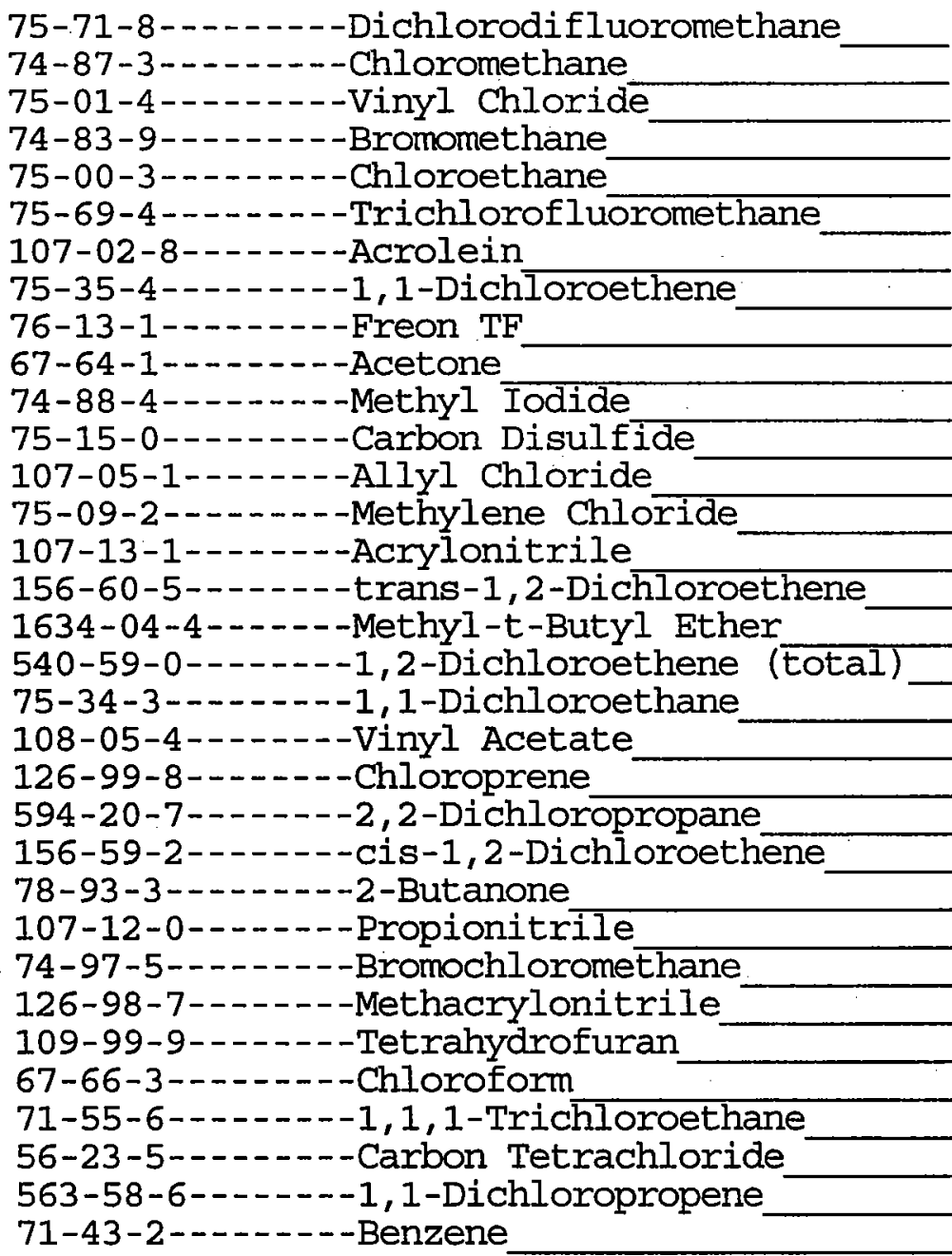 & $\begin{array}{r}10 \\
4.1 \\
10 \\
18 \\
10 \\
10 \\
51 \\
10 \\
10 \\
51 \\
9.9 \\
10 \\
10 \\
4.2 \\
10 \\
10 \\
10 \\
10 \\
10 \\
10 \\
10 \\
10 \\
10 \\
230 \\
41 \\
10 \\
10 \\
140 \\
10 \\
10 \\
10 \\
10 \\
10\end{array}$ & \begin{tabular}{|l}
$U$ \\
$J B$ \\
$U$ \\
$\mathrm{~B}$ \\
$\mathrm{U}$ \\
$\mathrm{U}$ \\
$\mathrm{U}$ \\
$\mathrm{U}$ \\
$\mathrm{U}$ \\
$\mathrm{U}$ \\
$\mathrm{JB}$ \\
$\mathrm{U}$ \\
$\mathrm{U}$ \\
$\mathrm{JB}$ \\
$\mathrm{U}$ \\
$\mathrm{U}$ \\
$\mathrm{U}$ \\
$\mathrm{U}$ \\
$\mathrm{U}$ \\
$\mathrm{U}$ \\
$\mathrm{U}$ \\
$\mathrm{U}$ \\
$\mathrm{U}$ \\
$\mathrm{B}$ \\
$\mathrm{U}$ \\
$\mathrm{U}$ \\
$\mathrm{U}$
\end{tabular} \\
\hline
\end{tabular}


FORM 1

VOLATILE ORGANICS ANALYSIS DATA SHEET
Lab Name: STL BURLINGTON

Contract : 21005
ARGLAB SAMPLE NO.

NA-S-20735

Lab Code: STLVT Case No.: NAVARRE SAS No.:

SDG No.: 114309

Matrix: (soil/water) SOIL

Sample wt/vol: $\quad 9.8(\mathrm{~g} / \mathrm{mL}) \mathrm{G}$

Level : (low/med) MED

\% Moisture: not dec.

GC Column: CAP ID: 0.53 (mm)

Soil Extract Volume: 10000 (uL)
Lab Sample ID: 669175

Lab File ID: $669175 \mathrm{E}$

Date Received: 05/12/06

Date Analyzed: 05/19/06

Dilution Factor: 1.0

Soil Aliquot Volume:

500 (UL)

CONCENTRATION UNITS:
(ug/L or $\mathrm{ug} / \mathrm{Kg}$ ) UG/KG

Q

\begin{tabular}{|c|}
\hline 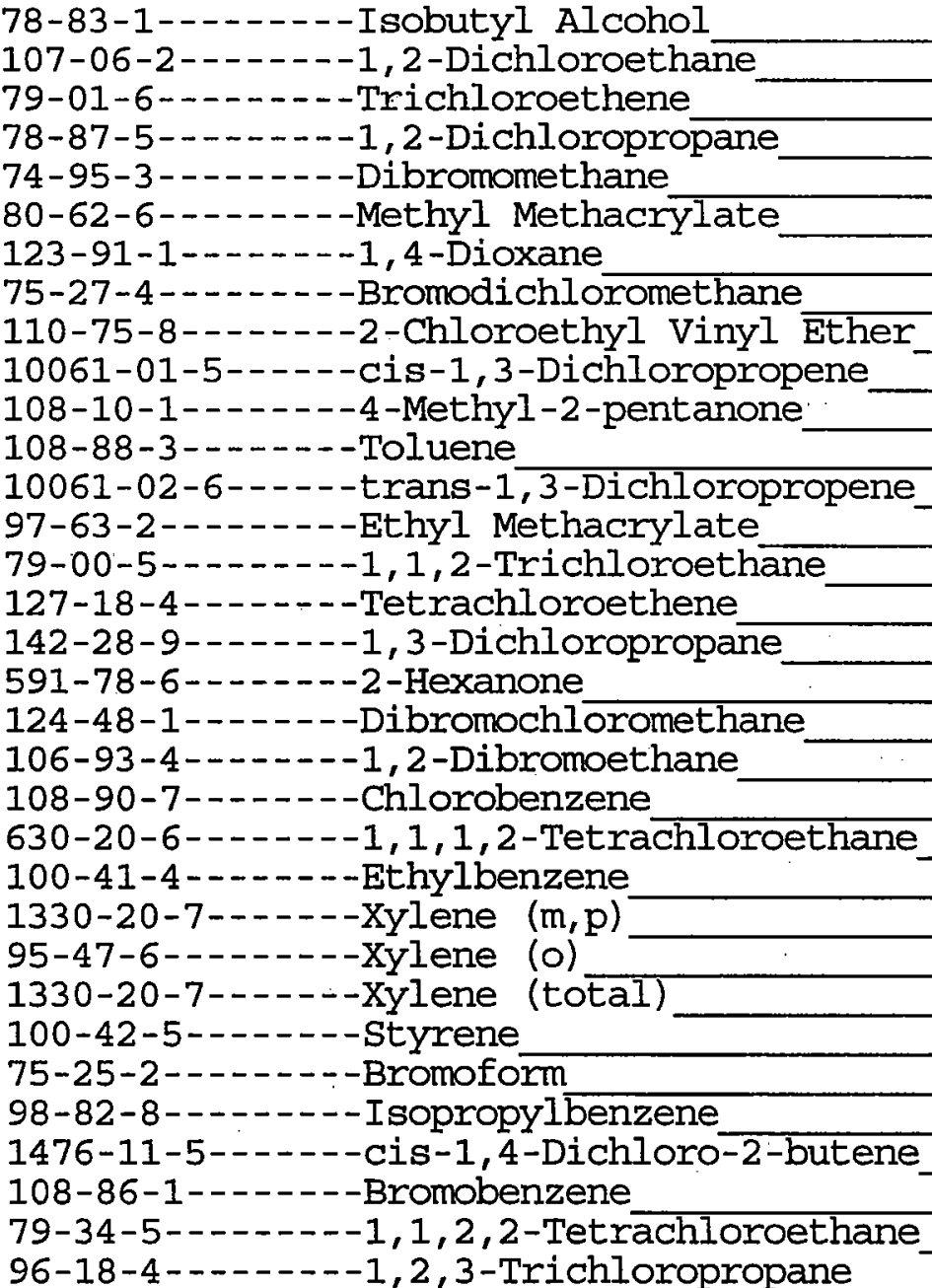 \\
\hline
\end{tabular}

\begin{tabular}{|r|r|r|}
510 & $\mathrm{U}$ \\
10 & $\mathrm{U}$ \\
10 & $\mathrm{U}$ \\
10 & $\mathrm{U}$ \\
10 & $\mathrm{U}$ \\
10 & $\mathrm{U}$ \\
510 & $\mathrm{U}$ \\
10 & $\mathrm{U}$ \\
10 & $\mathrm{U}$ \\
10 & $\mathrm{U}$ \\
51 & $\mathrm{U}$ \\
10 & $\mathrm{U}$ \\
10 & $\mathrm{U}$ \\
10 & $\mathrm{U}$ \\
10 & $\mathrm{U}$ \\
10 & $\mathrm{U}$ \\
10 & $\mathrm{U}$ \\
51 & $\mathrm{U}$ \\
10 & $\mathrm{U}$ \\
10 & $\mathrm{U}$ \\
10 & $\mathrm{U}$ \\
10 & $\mathrm{U}$ \\
10 & $\mathrm{U}$ \\
3.1 & $\mathrm{~J}$ \\
10 & $\mathrm{U}$ \\
3.3 & $\mathrm{~J}$ \\
10 & $\mathrm{U}$ \\
10 & $\mathrm{U}$ \\
10 & $\mathrm{U}$ \\
10 & $\mathrm{U}$ \\
10 & $\mathrm{U}$ \\
10 & $\mathrm{U}$ \\
10 & $\mathrm{U}$ \\
& \\
\hline
\end{tabular}


FORM 1

VOLATILE ORGANICS ANALYSIS DATA SHEET

Lab Name: STL BURLINGTON

Contract: 21005

Lab Code: STLVT Case No.: NAVARRE SAS No.:

ARGLAB SAMPLE NO.

NA-S-20735

SDG No.: 114309
Matrix: (soil/water) SOIL

Sample wt/vol:

$9.8(\mathrm{~g} / \mathrm{mL}) \mathrm{G}$

Level: (low/med) MED

\% Moisture: not dec.

GC Column: CAP

Soil Extract Volume: 10000 (uL)
Lab Sample ID: 669175

Lab File ID: 669175E

Date Received: 05/12/06

Date Analyzed: 05/19/06

Dilution Factor: 1.0

Soil Aliquot Volume:

500 (uL)

\begin{tabular}{|c|c|c|}
\hline 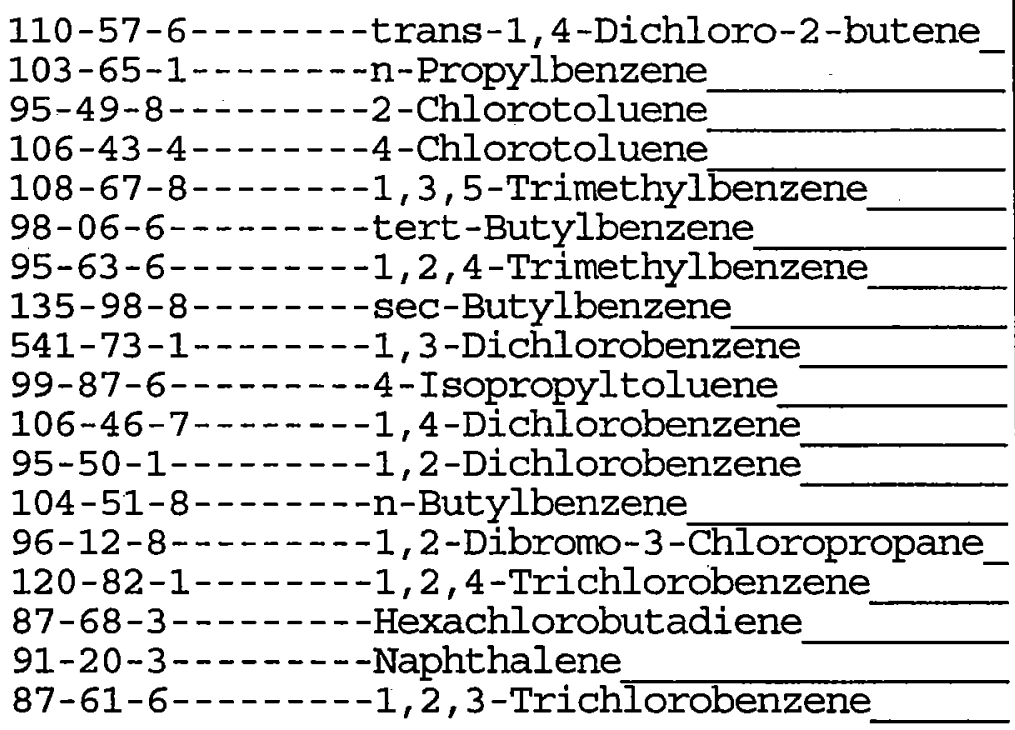 & $\begin{array}{l}10 \\
10 \\
10 \\
10 \\
10 \\
10 \\
10 \\
10 \\
10 \\
10 \\
10 \\
10 \\
10 \\
10 \\
10 \\
10 \\
10 \\
10\end{array}$ & $\begin{array}{l}U \\
U \\
U \\
U \\
U \\
U \\
U \\
U \\
U \\
U \\
U \\
U \\
U \\
U \\
U \\
U \\
U \\
U\end{array}$ \\
\hline
\end{tabular}


FORM 1

VOLATILE ORGANICS ANALYSIS DATA SHEET
Lab Name: STL BURLINGTON

Contract: 21005

Lab Code: STLVT Case No.: NAVARRE SAS No.:

Matrix: (soil/water) soIL

Sample wt/vol:

$9.8(\mathrm{~g} / \mathrm{mL}) \mathrm{G}$

Level: (low/med) MED

\% Moisture: not dec.

GC Column: CAP

ID: $0.53(\mathrm{~mm})$

Soil Extract Volume: 10000 (uL)
ARGLAB SAMPLE NO.

NA-S-20750

SDG No.: 114309

Lab Sample ID: 669177

Lab File ID: $\quad$ 669177E

Date Received: 05/12/06

Date Analyzed: 05/19/06

Dilution Factor: 1.0

Soil Aliquot Volume: $\quad 500$ (uL)

CONCENTRATION UNITS :

CAS NO.

COMPOUND

(ug/L or ug/Kg) UG/KG

\section{Q}

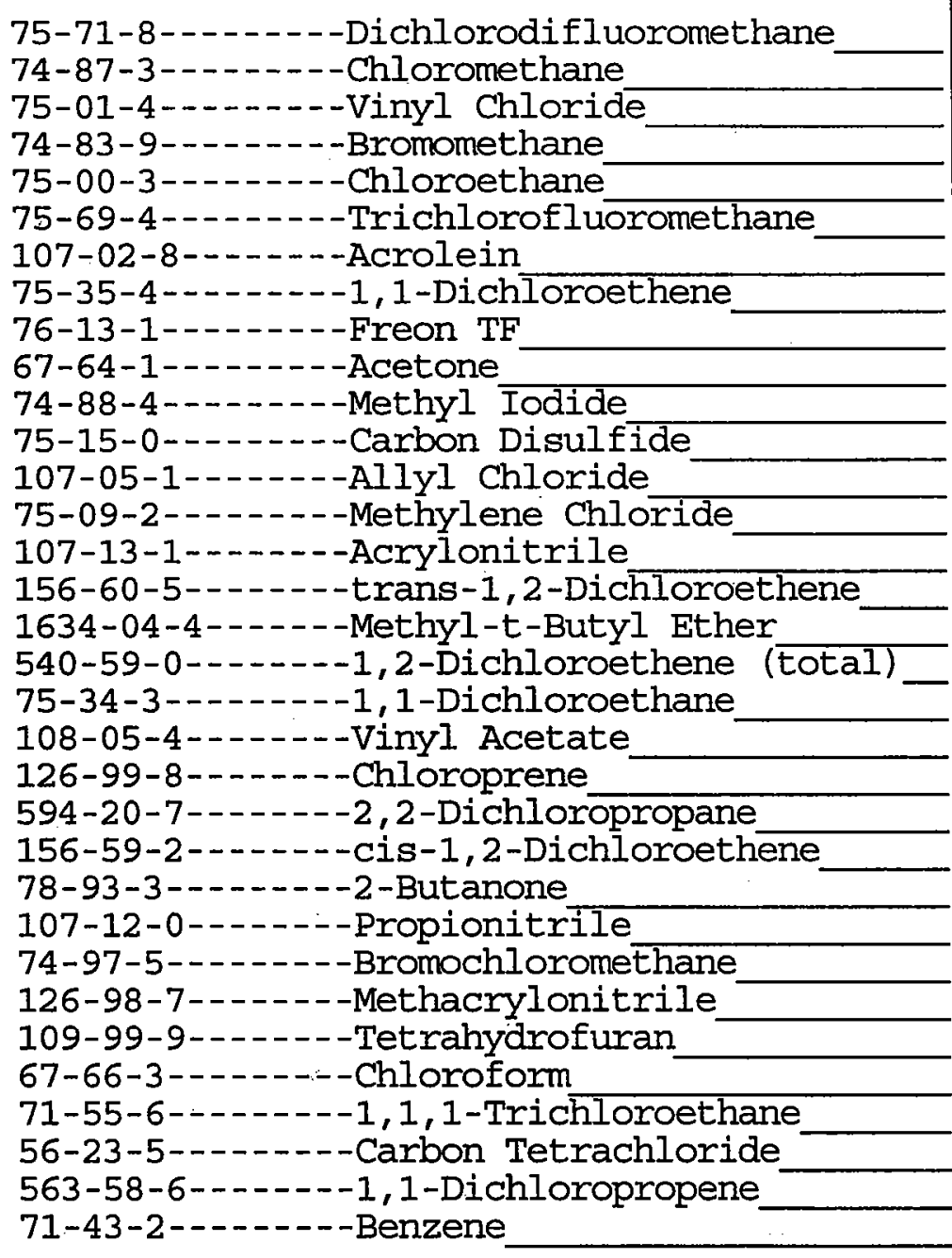

$10 \mathrm{U}$

$2.9 \mathrm{JB}$

$10 \mathrm{U}$

$6.0 \mathrm{JB}$

$10 \mathrm{U}$

$10 \mathrm{U}$

$51 \mathrm{U}$

$10 \mathrm{U}$

$10 \mathrm{U}$

$51 \mathrm{U}$

$7.5 \mathrm{JB}$

$10 \mathrm{U}$

$10 \mathrm{U}$

$3.2 \mathrm{JB}$

$10 \mathrm{U}$

$10 \mathrm{U}$

$10 \mathrm{U}$

$10 \mathrm{U}$

$10 \mathrm{U}$

$10 \mathrm{U}$

$10 \mathrm{U}$

$10 \mathrm{U}$

$10 \mathrm{U}$

52 B

$41 \mathrm{U}$

$10 \mathrm{U}$

$10 \mathrm{U}$

$140 \mathrm{U}$

$10 \mathrm{U}$

$10 \mathrm{U}$

$10 \mathrm{U}$

$10 \mathrm{U}$

$10 \mathrm{U}$ 
FORM 1

VOLATILE ORGANICS ANALYSIS DATA SHEET

Lab Name: STL BURLINGTON

Contract : 21005

Lab Code: STLVT Case No.: NAVARRE SAS No.:

Lab Sample ID: 669177

Matrix: (soil/water) SOIL

Sample wt/vol: $\quad 9.8(\mathrm{~g} / \mathrm{mL}) \mathrm{G}$

Level: (low/med) MED

Lab File ID: $\quad 669177 \mathrm{E}$

\% Moisture: not dec.

GC Column: CAP ID: 0.53 (mm)

Soil Extract Volume: 10000 (uL)
ARGLAB SAMPLE NO.

NA-S-20750

SDG No.: 114309
Date Received: 05/12/06

Date Analyzed: 05/19/06

Dilution Factor: 1.0

Soil Aliquot Volume:

500 (uL)

CAS NO.

COMPOUND

CONCENTRATION UNITS:

(ug/L or $\mathrm{ug} / \mathrm{Kg}$ ) UG/KG

$Q$

\begin{tabular}{|c|c|c|}
\hline 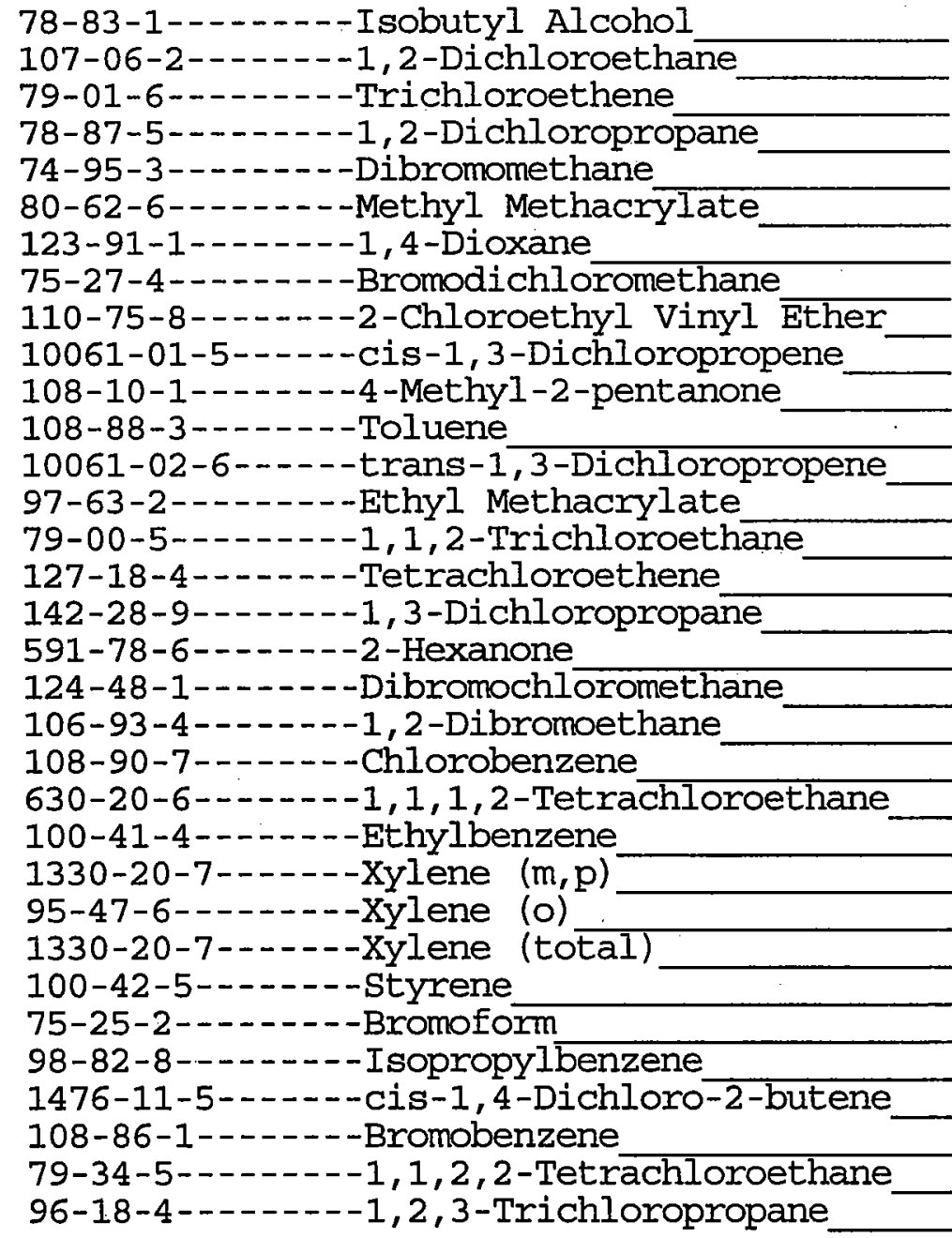 & $\begin{array}{r}510 \\
10 \\
10 \\
10 \\
10 \\
10 \\
510 \\
10 \\
10 \\
10 \\
51 \\
10 \\
10 \\
10 \\
10 \\
10 \\
10 \\
51 \\
10 \\
10 \\
10 \\
10 \\
10 \\
10 \\
10 \\
10 \\
10 \\
10 \\
10 \\
10 \\
10 \\
10 \\
10\end{array}$ & $\begin{array}{l}U \\
U \\
U \\
U \\
U \\
U \\
U \\
U \\
U \\
U \\
U \\
U \\
U \\
U \\
U \\
U \\
U \\
U \\
U \\
U \\
U \\
U \\
U \\
U \\
U \\
U \\
U \\
U \\
U \\
U \\
U \\
U \\
U \\
U\end{array}$ \\
\hline
\end{tabular}


FORM 1

VOLATILE ORGANICS ANALYSIS DATA SHEET

Lab Name: STL BURLINGTON

Contract: 21005

Lab Code: STIUT Case No.: NAVARRE SAS No.:

Lab Sample ID: 669177

Matrix: (soil/water) SOIL

Sample wt/vol:

$9.8(\mathrm{~g} / \mathrm{mL}) \mathrm{G}$

Level: (low/med) MED

\% Moisture: not dec.

GC Column: CAP

ID: $0.53 \quad(\mathrm{~mm})$

Soil Extract Volume: 10000 (uL)

CAS NO.

COMPOUND
ARGLAB SAMPLE NO.

NA-S-20750

SDG No.: 114309
Lab File ID: $669177 \mathrm{E}$

Date Received: 05/12/06

Date Analyzed: 05/19/06

Dilution Factor: 1.0

Soil Aliquot Volume:

500 (uL)

CONCENTRATION UNITS:

(ug/L or $\mathrm{ug} / \mathrm{Kg}$ ) UG/KG

Q

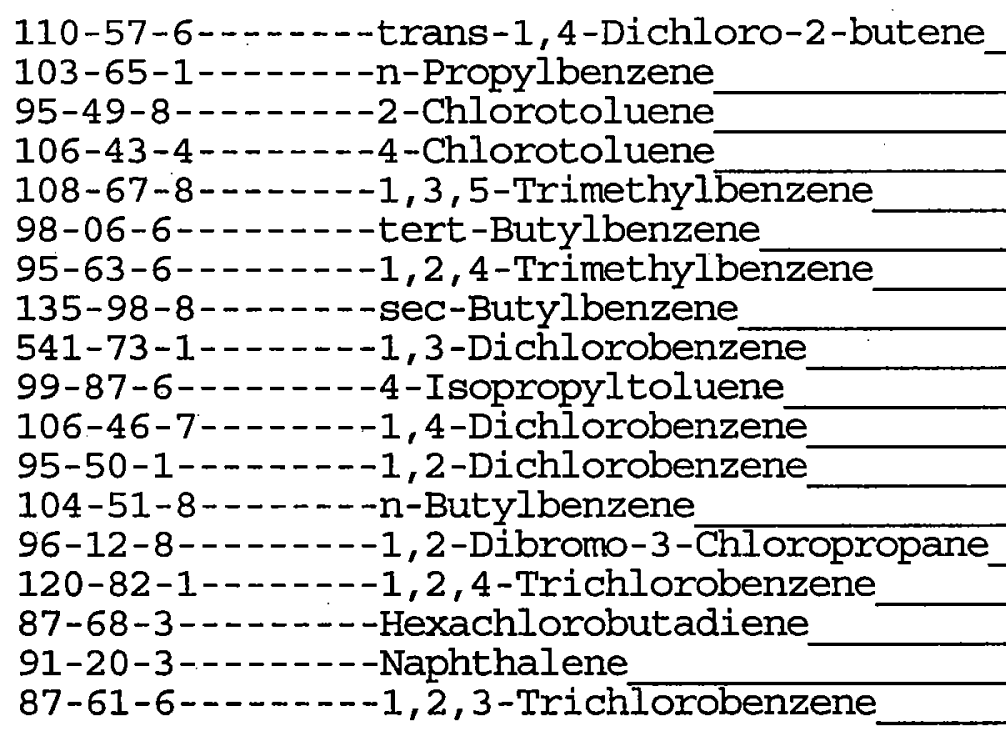

\begin{tabular}{|l|l|l|}
10 & $\mathrm{U}$ \\
10 & $\mathrm{U}$ \\
10 & $\mathrm{U}$ \\
10 & $\mathrm{U}$ \\
10 & $\mathrm{U}$ \\
10 & $\mathrm{U}$ \\
10 & $\mathrm{U}$ \\
10 & $\mathrm{U}$ \\
10 & $\mathrm{U}$ \\
10 & $\mathrm{U}$ \\
10 & $\mathrm{U}$ \\
10 & $\mathrm{U}$ \\
10 & $\mathrm{U}$ \\
10 & $\mathrm{U}$ \\
10 & $\mathrm{U}$ \\
10 & $\mathrm{U}$ \\
10 & $\mathrm{U}$ \\
10 & $\mathrm{U}$ \\
\hline
\end{tabular}


FORM 1

VOLATILE ORGANICS ANALYSIS DATA SHEET

Lab Name: STL BURLINGTON

Contract: 21005

Lab Code: STLVT Case No.: NAVARRE SAS No.:

Lab Sample ID: 669183

Matrix: (soil/water) SOIL

Sample wt/vol: $\quad 10.0(\mathrm{~g} / \mathrm{mL})$ G

Level: (low/med) MED

\% Moisture: not dec.

GC Column: CAP ID: 0.53 (mm)

Soil Extract Volume: 10000 (uL)
ARGLAB SAMPLE NO.

MEOH BLANK
Lab File ID: 669183E

Date Received: 05/12/06

Date Analyzed: 05/19/06

Dilution Factor: 1.0

Soil Aliquot Volume:

500 (uL)

CAS NO.

COMPOUND

CONCENTRATION UNITS:

(ug/L or $\mathrm{ug} / \mathrm{Kg}$ ) UG/KG

Q

\begin{tabular}{|c|c|c|}
\hline 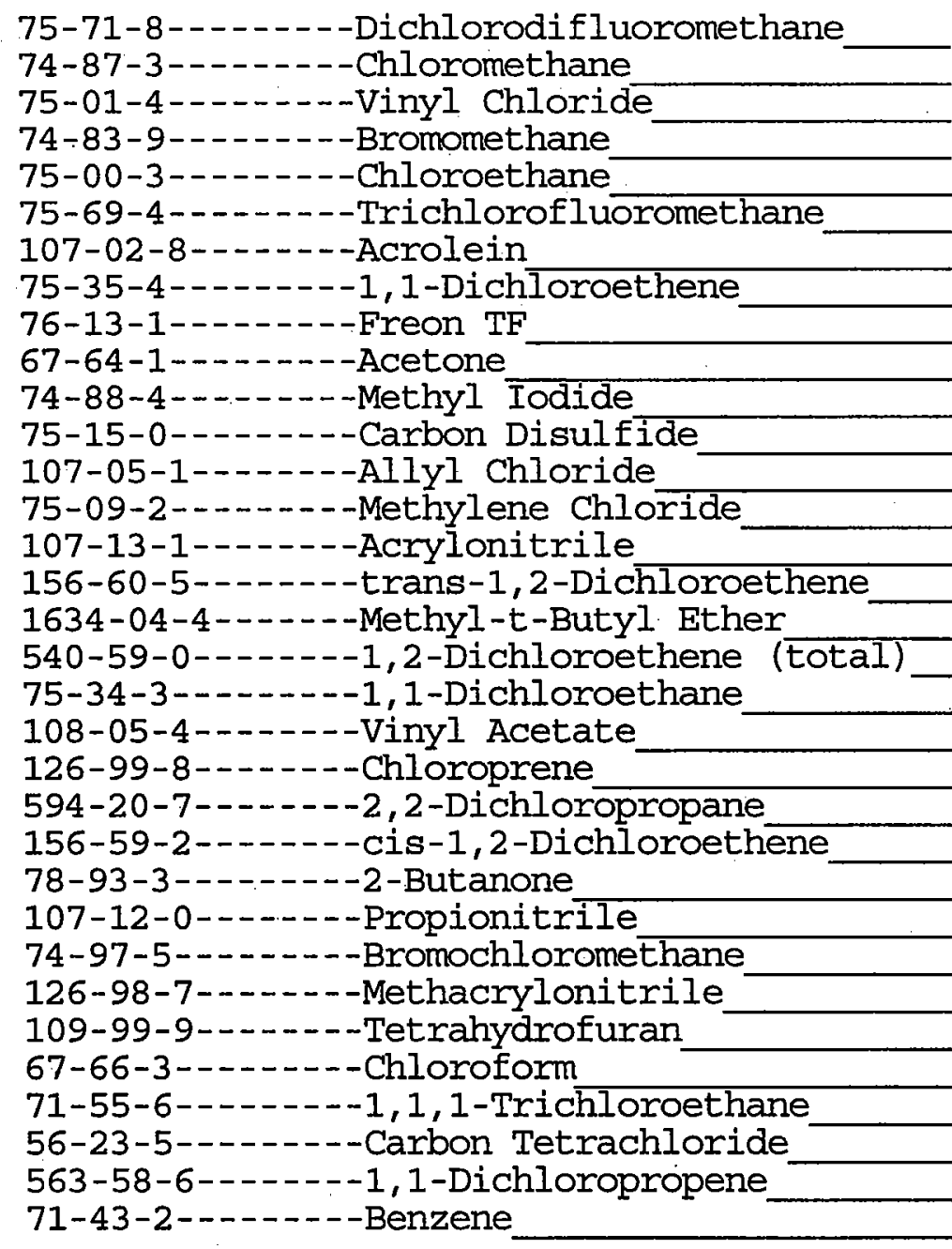 & $\begin{array}{r}10 \\
5.7 \\
10 \\
14 \\
10 \\
10 \\
50 \\
10 \\
10 \\
50 \\
13 \\
10 \\
10 \\
9.1 \\
10 \\
10 \\
10 \\
10 \\
10 \\
10 \\
10 \\
10 \\
10 \\
69 \\
40 \\
10 \\
10 \\
140 \\
-10 \\
10 \\
10 \\
10 \\
2.2\end{array}$ & $\begin{array}{l}\mathrm{U} \\
\mathrm{J} \\
\mathrm{U} \\
\mathrm{U} \\
\mathrm{U} \\
\mathrm{U} \\
\mathrm{U} \\
\mathrm{U} \\
\mathrm{U} \\
\mathrm{U} \\
\mathrm{U} \\
\mathrm{J} \\
\mathrm{U} \\
\mathrm{U} \\
\mathrm{U} \\
\mathrm{U} \\
\mathrm{U} \\
\mathrm{U} \\
\mathrm{U} \\
\mathrm{U} \\
\mathrm{U} \\
\mathrm{U} \\
\mathrm{U} \\
\mathrm{U} \\
\mathrm{U} \\
\mathrm{U} \\
\mathrm{U} \\
\mathrm{U} \\
\mathrm{U} \\
\mathrm{J}\end{array}$ \\
\hline
\end{tabular}


FORM 1

VOLATILE ORGANICS ANALYSIS DATA SHEET

Lab Name: STL BURLINGTON

Lab code: STLVT Case No.: NAVARRE SAS No.:

MEOH BL_ANK

Matrix: (soil/water) SOIL

Sample wt/vol: $\quad 10.0(\mathrm{~g} / \mathrm{mL}) \mathrm{G}$

Level: (low/med) MED

$\%$ Moisture: not dec.

GC Column: CAP ID: 0.53 (mm)

Soil Extract Volume: 10000 (ut)
Lab Sample ID: 669183

Lab File ID: $669183 \mathrm{E}$

Date Received: 05/12/06

Date Analyzed: 05/19/06

Dilution Factor: 1.0

Soil Aliquot Volume:

500 (UL)
CONCENTRATION UNITS:

(ug/L or $\mathrm{ug} / \mathrm{Kg}$ ) UG/KG

Q

$500 \mathrm{U}$

$10 \mathrm{U}$

$10 \mathrm{U}$

$10 \mathrm{U}$

$10 \mathrm{U}$

$10 \mathrm{U}$

$500 \mathrm{U}$

$10 \mathrm{U}$

$10 \mathrm{U}$

$10 \mathrm{U}$

$50 \mathrm{U}$

$3.0 \mathrm{~J}$

$10 \mathrm{U}$

$10 \mathrm{U}$

$10 \mathrm{U}$

$10 \mathrm{U}$

$10 \mathrm{U}$

$50 \mathrm{U}$

$10 \mathrm{U}$

$10 \mathrm{U}$

$10 \mathrm{U}$

$10 \mathrm{U}$

$10 \mathrm{U}$

$10 \mathrm{U}$

$10 \mathrm{U}$

$10 \mathrm{U}$

$10 \mathrm{U}$

$10 \mathrm{U}$

$10 \mathrm{U}$

$10 \mathrm{U}$

$10 \mathrm{U}$

$10 \mathrm{U}$

$10 \mathrm{U}$ 
FORM 1

VOLATILE ORGANICS ANALYSIS DATA SHEET

Lab Name: STL BURLINGTON

Contract : 21005

ARGLAB SAMPLE NO.

Lab Code: STLVT Case No.: NAVARRE SAS No.:

MEOH BLANK

Matrix: (soil/water) SOIL

Lab Sample ID: 669183

Sample wt/vol:

$10.0(\mathrm{~g} / \mathrm{mL}) \mathrm{G}$

Lab File ID: $669183 \mathrm{E}$

Level: (low/med) MED

Date Received: 05/12/06

\% Moisture: not dec.

GC Column: CAP ID: 0.53 (mm)

Date Analyzed: 05/19/06

Soil Extract Volume: 10000 (uL)

Dilution Factor: 1.0

Soil Aliquot Volume:

500 (UL)

CONCENTRATION UNITS:

CAS NO. COMPOUND (ug/L or ug/Kg) UG/KG

Q 
May 31,2006

\title{
S E V E R N
}

T R E N T

\author{
. \\ Mr. Clyde Dennis \\ Argonne National Laboratory \\ 9700 S. Cass Avenue \\ Building 203, Office 149 \\ Argonne, IL 60439 \\ $\begin{array}{ll}\text { Re: } & \text { Laboratory Project No. } 21005 \\ & \text { Case: NAVARRE; SDG: } 114417\end{array}$
}

STL Burlington

208 South Park Drive, Suite 1

Colchester, VT 05446

Tel: 8026551203 Fax: 8026551248

www.stl-inc.com

Dear Mr. Dennis:

Enclosed are analytical results for samples that were received by STL Burlington on May $19^{\text {th }}, 2006$. Laboratory identification numbers were assigned, and designated as follows:

$\begin{array}{llll}\text { Lab ID } & \begin{array}{l}\text { Client } \\ \text { Sample ID }\end{array} & \begin{array}{l}\text { Sample } \\ \text { Date }\end{array} & \begin{array}{l}\text { Sample } \\ \text { Matrix }\end{array} \\ & \text { Received: 05/19/06 ETR No: } & 114417 & \\ 669921 & \text { NA-S-20673 } & 05 / 16 / 06 & \text { Liquid } \\ 669922 & \text { NA-S-20578 } & 05 / 16 / 06 & \text { Liquid } \\ 669923 & \text { NA-S-20808 } & 05 / 16 / 06 & \text { Liquid } \\ 669924 & \text { NA-S-20773 } & 05 / 16 / 06 & \text { Liquid } \\ 669925 & \text { NA-S-20783 } & 05 / 16 / 06 & \text { Liquid } \\ 669926 & \text { NA-S-20689 } & 05 / 16 / 06 & \text { Liquid } \\ 669927 & \text { MEOH BLANK } & 05 / 16 / 06 & \text { Liquid }\end{array}$

Documentation of the condition of the samples at the time of their receipt and any exception to the laboratory's Sample Acceptance Policy is documented in the Sample Handling section of this submittal. It should be noted that at the time that they were received, the sample volumes were at near ambient temperature.

The samples were analyzed by Method $8260 \mathrm{~B}$, using a low-level calibration. In performing the analytical work, 500 microliters of the methanol extract were added to the 5 milliliter purge volume. The recovery of the surrogate controls were generally elevated in the analysis of sample NA-S-20673 (approximating 135 percent). Additionally, the analysis of samples MEOH BLANK and NA-S-20808 did yield a high recovery of toluene-d $d_{8}$ (approximating 119 percent). Each of the analyses associated with the sample set exhibited good internal standard stability. Two types of laboratory control sample analyses were performed as part of the analytical sequence. One was performed to evaluate method performance, and one was performed with 500 microliters of methanol added to the purge volume in order to characterize the affect on the analytical process. With the exception of that for dichlorodifluoromethane (69 percent) and methyl-t-butyl ether (77 percent), the recovery of each target analyte was within the established control range in the laboratory control sample analysis that defined the method performance. In the laboratory control sample analysis with methanol, several of the earlier eluting compounds did exhibit lower recoveries, as did isobutyl alcohol,1,4-dioxane, and certain of the other later eluting compounds. Most profoundly affected was the performance of isobutyl alcohol and 1,4-dioxane, for which the 
recovery values were below 20 percent. The recoveries of chloroform and carbon tetrachloride were within the control range in each of the laboratory control sample analyses. It should be noted, however, that the derived recovery value for carbon tetrachloride was at the lower control limit of 75 percent in the laboratory control sample analysis with 500 milliiters of methanol. Matrix spike and matrix spike duplicate analyses were not performed on samples in this sample set. The analysis of the instrument blank that was analyzed in association with the samples was free of contamination. The laboratory did associate the analysis of sample MEOH BLANK with each of the other field sample analyses in order to reference the blank association, and accordingly qualify the reported results.

If there are any questions regarding this submittal, please contact me at (802) 655-1203.

The analytical results associated with the samples presented in this test report were generated under a quality system that adheres to requirements specified in the NELAC standard. Release of the data in this test report and any associated electronic deliverables is authorized by the Laboratory Director's designee as verified by the following signature.

Sincerely,

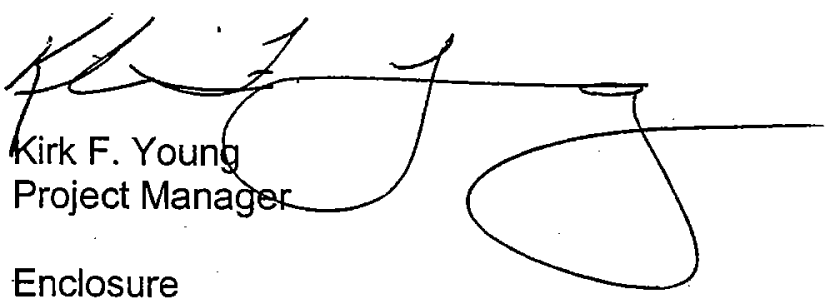




\section{STL Burlington Data Qualifier Definitions}

\section{Organic}

U: Compound analyzed but not detected at a concentration above the reporting limit.

$\mathrm{J}: \quad$ Estimated value.

$\mathrm{N}$ : Indicates presumptive evidence of a compound. This flag is used only for tentatively identified compounds (TICs) where the identification of a compound is based on a mass spectral library search.

P: $\quad$ SW-846: Greater than $40 \%$ difference for detected concentrations between two $\mathrm{GC}$ columns. Unless otherwise specified the higher of the two values is reported on the Form 1.

CLP SOW: Greater than 25\% difference for detected concentrations between two GC columns. Unless otherwise specified the lower of the two values is reported on the Form I.

C: Pesticide result whose identification has been confirmed by GC/MS.

B: $\quad$ Analyte is found in the sample and the associated method blank. The flag is used for tentatively identified compounds as well as positively identified compounds.

E: Compounds whose concentrations exceed the upper limit of the calibration range of the instrument for that speciric analysis.

D: Concentrations identified from analysis of the sample at a secondary dilution.

A: Tentatively identified compound is a suspected aldol condensation product.

$X, Y, Z$ : Laboratory defined flags that may be used alone or combined, as needed. If used, the description of the flag is defined in the project narrative.

\section{Inorganic/Metals}

E: Reported value is estimated due to the presence of interference.

$\mathrm{N}$ : Matrix spike sample recovery is not within control limits.

* Duplicate sample analysis is not within control limits.

B: The result reported is less than the reporting limit but greater than the instrument detection limit.

U: $\quad$ Analyte was analyzed for but not detected above the reporting limit.

Method Codes:

$P \quad$ ICP-AES

MS ICP-MS

CV Cold Vapor AA

AS Semi-Automated Spectrophotometric 


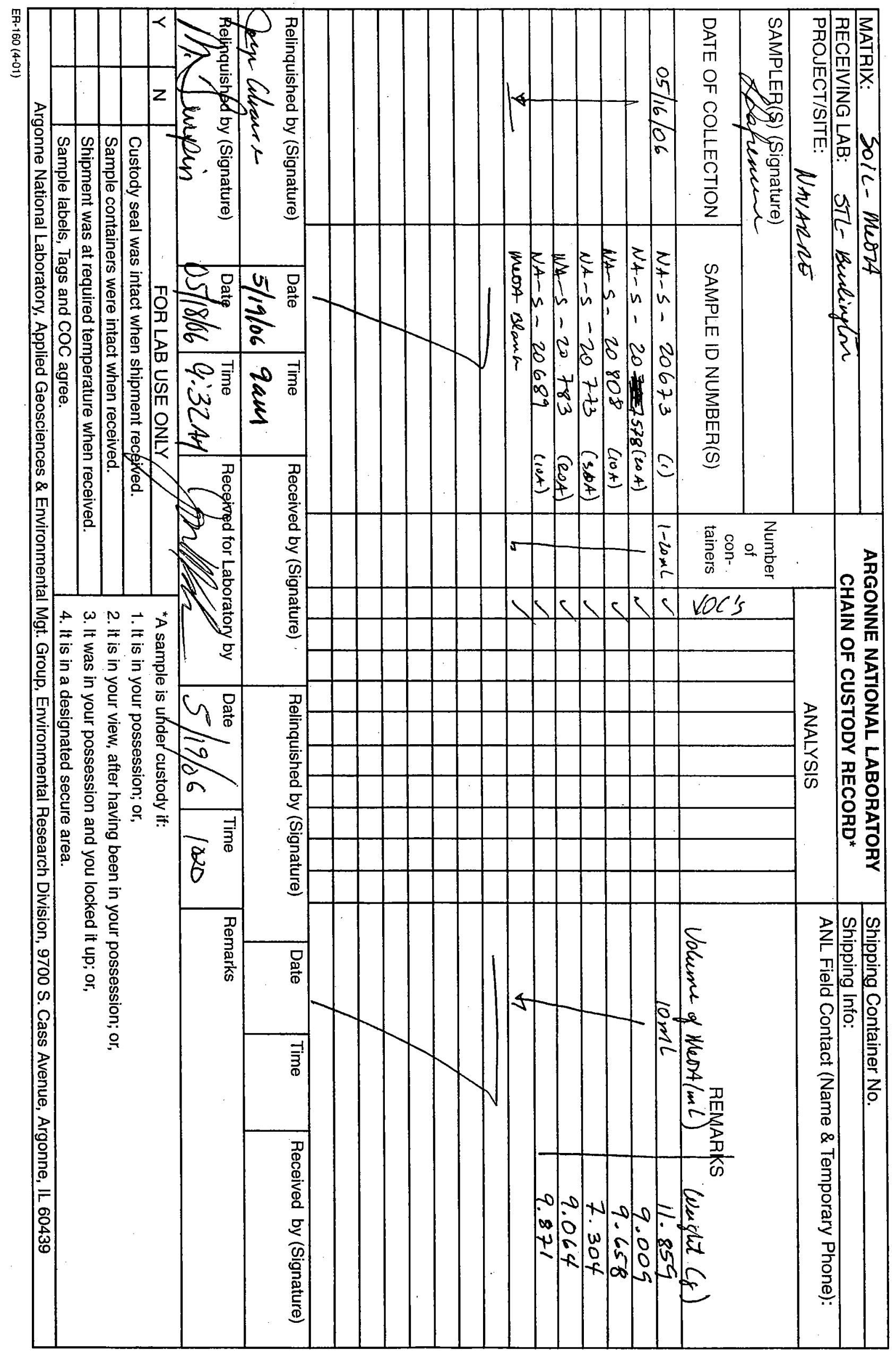




\section{SEVER N \\ T R E N T \\ STL}

\section{METHOD 8260B}

\section{VOLATILE ORGANIC ANALYSIS}

SAMPLE DATA SUMMARY PACKAGE 
FORM 1

VOLATILE ORGANICS ANALYSIS DATA SHEET

Lab Name: STL BURLINGTON

Contract: 21005

Lab Code: STLVT Case No.: NAVARRE SAS No.:

Lab Sample ID: 669922

Matrix: (soil/water) SOIL

Sample wt/vol:

$9.0(\mathrm{~g} / \mathrm{mL}) \mathrm{G}$

Level : (low/med) MED

\% Moisture: not dec.

GC Column: CAP

ID: $0.53(\mathrm{~mm})$

Soil Extract Volume: 10000 (ut)

Lab File ID: $\quad 669922$

Date Received: 05/19/06

Date Analyzed: 05/24/06

Dilution Factor: 1.0

Soil Aliquot Volume:
ARGLAB SAMPLE NO.

NA-S-20578

500 (uL)

CONCENTRATION UNITS :

CAS NO.

COMPOUND

(ug/L or $u g / \mathrm{Kg}$ ) UG/KG

$Q$

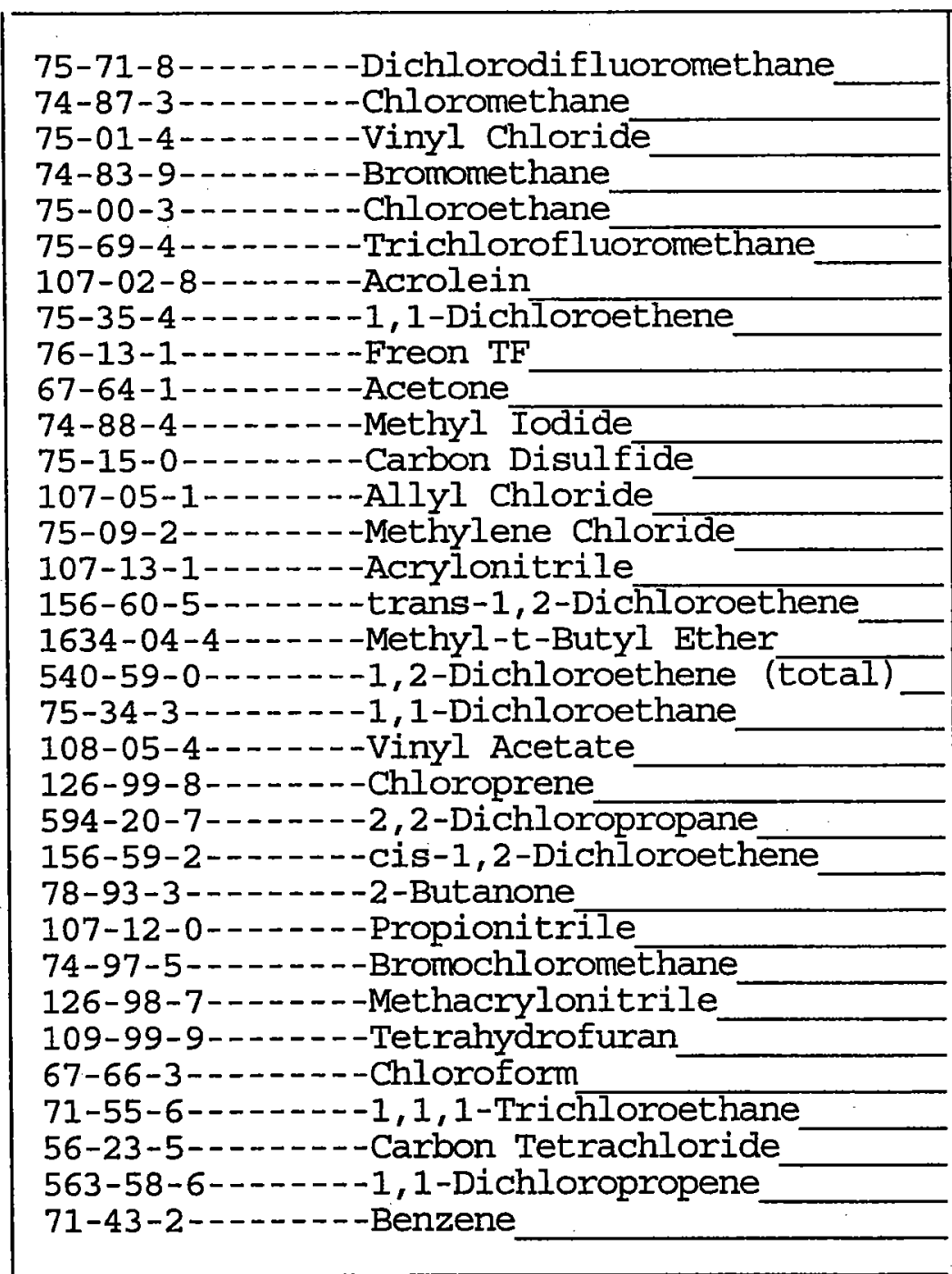

$11 \mathrm{U}$

$2.7 \mathrm{JB}$

$11 \mathrm{U}$

$8.7 \mathrm{JB}$

$11 \mathrm{U}$

$11 \mathrm{U}$

$55 \mathrm{U}$

$11 \mathrm{U}$

$11 \mathrm{U}$

$55 \mathrm{U}$

$7.4 \mathrm{JB}$

$11 \mathrm{U}$

$11 \mathrm{U}$

$44 \mathrm{~B}$

$11 \mathrm{U}$

$11 \mathrm{U}$

$11 \mathrm{U}$

$11 \mathrm{U}$

$11 \mathrm{U}$

$11 \mathrm{U}$

$11 \mathrm{U}$

$11 \mathrm{U}$

$11 \mathrm{U}$

$55 \mathrm{U}$

$44 \mathrm{U}$

$11 \mathrm{U}$

$11 \mathrm{U}$

$160 \mathrm{U}$

$11 \mathrm{U}$

$3.3 \mathrm{JB}$

$5.9 \mathrm{~J}$

$11 \mathrm{U}$

$11 \mathrm{U}$ 
FORM 1

VOLATILE ORGANICS ANALYSIS DATA SHEET

Lab Name: STL BURLINGTON

Contract: 21005

ARGLAB SAMPIE NO.

Lab code: STLVT Case No.: NAVARRE SAS No.:

NA-S-20578

SDG No. : 114417

Matrix: (soil/water) SOIL

Lab Sample ID: 669922

Sample wt/vol: $\quad 9.0(\mathrm{~g} / \mathrm{mL}) \mathrm{G}$

Lab File ID: 669922

Level : (low/med) MED

Date Received: 05/19/06

\% Moisture: not dec.

GC Column: CAP ID: 0.53 (mm)

Date Analyzed: 05/24/06

Soil Extract Volume: 10000 (uL)

Dilution Factor: 1.0

Soil Aliquot Volume:

500 (UL)

CONCENTRATION UNITS:

CAS NO.

COMPOUND

(ug/L or $\mathrm{ug} / \mathrm{Kg}$ ) UG/KG

Q

\begin{tabular}{|r|l|l|}
550 & $\mathrm{U}$ \\
11 & $\mathrm{U}$ \\
11 & $\mathrm{U}$ \\
11 & $\mathrm{U}$ \\
11 & $\mathrm{U}$ \\
11 & $\mathrm{U}$ \\
550 & $\mathrm{U}$ \\
11 & $\mathrm{U}$ \\
11 & $\mathrm{U}$ \\
11 & $\mathrm{U}$ \\
55 & $\mathrm{U}$ \\
11 & $\mathrm{U}$ \\
11 & $\mathrm{U}$ \\
11 & $\mathrm{U}$ \\
11 & $\mathrm{U}$ \\
11 & $\mathrm{U}$ \\
11 & $\mathrm{U}$ \\
55 & $\mathrm{U}$ \\
11 & $\mathrm{U}$ \\
11 & $\mathrm{U}$ \\
11 & $\mathrm{U}$ \\
11 & $\mathrm{U}$ \\
11 & $\mathrm{U}$ \\
11 & $\mathrm{U}$ \\
11 & $\mathrm{U}$ \\
11 & $\mathrm{U}$ \\
11 & $\mathrm{U}$ \\
11 & $\mathrm{U}$ \\
11 & $\mathrm{U}$ \\
11 & $\mathrm{U}$ \\
11 & $\mathrm{U}$ \\
11 & $\mathrm{U}$ \\
11 & $\mathrm{U}$ \\
& \\
\hline & \\
\hline
\end{tabular}


FORM 1

VOLATILE ORGANICS ANALYSIS DATA SHEET

Lab Name: STL BURIINGTON

Contract : 21005

Lab Code: STLVT Case No.: NAVARRE SAS No::

Lab Sample ID: 669922

Matrix: (soil/water) SOIL

Lab File ID: 669922

Sample wt/vol:

$9.0(\mathrm{~g} / \mathrm{mL}) \mathrm{G}$

Level: (low/med) MED

Date Received: 05/19/06

음 Moisture: not dec.

GC Column: CAP

ID: $0.53 \quad(\mathrm{~mm})$

Soil Extract Volume: 10000 (uL)
Date Analyzed: 05/24/06

Dilution Factor: 1.0
ARGLAB SAMPLE NO.

NA-S-20578

CONCENTRATION UNITS :

CAS NO.

COMPOUND

110-57-6-------trans-1,4-Dichloro-2-butene 103-65-1------n-Propylbenzene

95-49-8--.---2-Chlorotoluene 106-43-4-..---4-Chlorotoluene 108-67-8--.---1, 3,5-Trimethylbenzene 98-06-6-------tert-Butylbenzene 95-63-6-...--1, 2, 4-Trimethylbenzene 135-98-8--.----sec-Butylbenzene 541-73-1------1, 3-Dichlorobenzene 99-87-6-------4-Isopropyltoluene 106-46-7-----1, 4-Dichlorobenzene 95-50-1-2.--1,2-Dichlorobenzene 104-51-8--.---n-Butylbenzene 96-12-8---- --1, 2-Dibromo-3-Chloropropane 120-82-1--.---1,2,4-Trichlorobenzene 87-68-3-...--Hexachlorobutadiene 91-20-3-..---Naphthalene 87-61-6-...--1,2,3-Trichlorobenzene 
FORM 1

VOLATILE ORGANICS ANALYSIS DATA SHEET
Lab Name: STL BURLINGTON

Contract: 21005
ARGLAB SAMPLE NO.

NA-S-20673

Iab Code: STLVT Case No.: NAVARRE SAS No.:

SDG No.: 114417

Matrix: (soil/water) SOIL

Sample wt/vol: $\quad 11.9(\mathrm{~g} / \mathrm{mL})$ G

Level: (low/med) MED

웅 Moisture: not dec.

GC Column: CAP

Soil Extract Volume: 10000 (uI)
Lab Sample ID: 669921

Lab File ID: 669921

Date Received: 05/19/06

Date Analyzed: 05/24/06

Dilution Factor: 1.0

Soil Aliquot Volume: $\quad 500$ (uL)

CAS NO.

COMPOUND

CONCENTRATION UNITS:

(ug/L or ug/Kg) UG/KG

Q

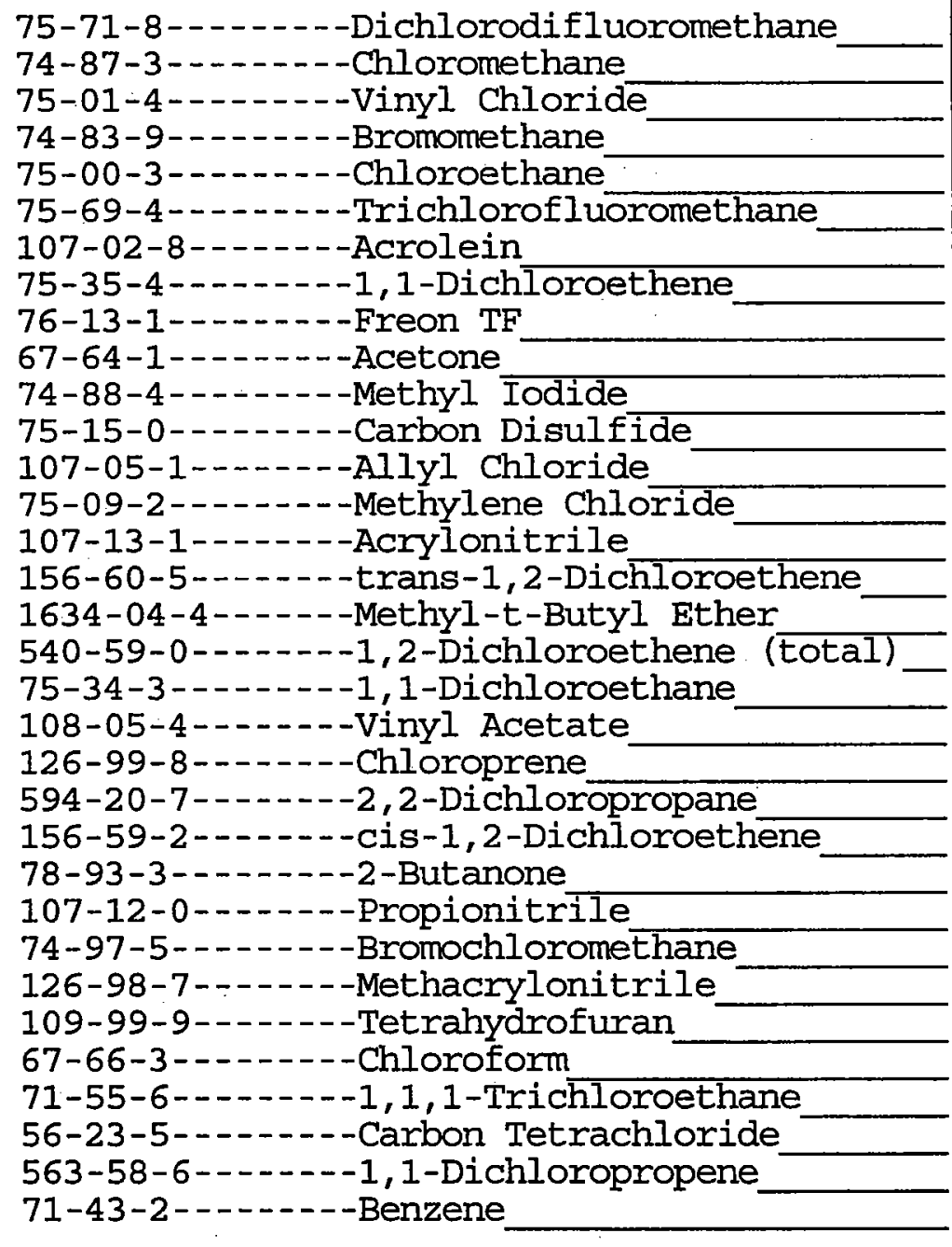

$8.4 \mathrm{U}$

2.2 JB

$8.4 \mathrm{U}$

$8.8 \mathrm{~B}$

$8.4 \mathrm{U}$

$8.4 \mathrm{U}$

$42 \mathrm{U}$

$8.4 \mathrm{U}$

$8.4 \mathrm{U}$

$42 \mathrm{U}$

$6.0 \mathrm{JB}$

$8.4 \mathrm{U}$

$8.4 \mathrm{U}$

$7.0 \mathrm{JB}$

$8.4 \mathrm{U}$

$8.4 \mathrm{U}$

$8.4 \mathrm{U}$

$8.4 \mathrm{U}$

$8.4 \mathrm{U}$

$8.4 \mathrm{U}$

$8.4 \mathrm{U}$

$8.4 U$

$8.4 \mathrm{U}$

$20 \mathrm{~J}$

$34 \mathrm{U}$

$8.4 \mathrm{U}$

$8.4 \mathrm{U}$

$120 \mathrm{U}$

$11 \mathrm{~B}$

$8.4 \mathrm{U}$

110

$8.4 \overline{\mathrm{U}}$

$8.4 \mathrm{U}$ 
FORM 1

VOLATILE ORGANICS ANALYSIS DATA SHEET

Lab Name: STL BURLINGTON

Contract: 21005

Lab Code: STLVT Case No.: NAVARRE SAS No.:

Lab Sample ID: 669921

Matrix: (soil/water) sOIL

Sample wt/vol:

$11.9(\mathrm{~g} / \mathrm{mL}) \mathrm{G}$

Level: (low/med) MED

\% Moisture: not dec.

GC Column: CAP

ID: $0.53 \quad(\mathrm{~mm})$

Soil Extract Volume: 10000 (uL)

Lab File ID: 669921

Date Received: 05/19/06

Date Analyzed: 05/24/06

Dilution Factor: 1.0

Soil Aliquot Volume:
ARGLAB SAMPLE NO.

NA-S-20673

SDG No.: 114417

500 (uL)

CONCENTRATION UNITS:

CAS NO.

COMPOUND

(ug/L or $\mathrm{ug} / \mathrm{Kg}$ ) UG/KG

Q

$420 \mathrm{U}$

$8.4 \mathrm{U}$

$8.4 \mathrm{U}$

$8.4 \mathrm{U}$

$8.4 \mathrm{U}$

$8.4 \mathrm{U}$

$420 \mathrm{U}$

$8.4 \mathrm{U}$

$8.4 \mathrm{U}$

$8.4 \mathrm{U}$

$42 \mathrm{U}$

$8.4 \mathrm{U}$

$8.4 \mathrm{U}$

$8.4 \mathrm{U}$

$8.4 \mathrm{U}$

$8.4 \mathrm{U}$

$8.4 \mathrm{U}$

$42 \mathrm{U}$

$8.4 \mathrm{U}$

$8.4 \mathrm{U}$

$8.4 \mathrm{U}$

$8.4 \mathrm{U}$

$8.4 \mathrm{U}$

$8.4 \mathrm{U}$

$8.4 \mathrm{U}$

$8.4 \mathrm{U}$

$8.4 \mathrm{U}$

$8.4 \mathrm{U}$

$8.4 \mathrm{U}$

$8.4 \mathrm{U}$

$8.4 \mathrm{U}$

$8.4 \mathrm{U}$

$8.4 \mathrm{U}$ 
FORM 1

VOLATILE ORGANICS ANALYSIS DATA SHEET

Lab Name: STL BURIINGTON

Contract: 21005
ARGLAB SAMPLE NO.

NA-S-20673

Lab Code: STLVT Case No.: NAVARRE SAS No.:

SDG No.: 114417

Matrix: (soil/water) SOIL

Sample wt/vol:

$11.9(\mathrm{~g} / \mathrm{mL}) \mathrm{G}$

Level: (low/med) MED

\% Moisture: not dec.

GC Column: CAP

ID: $0.53 \quad(\mathrm{~mm})$

Soil Extract Volume: 10000 (uL)
Lab Sample ID: 669921

Lab File ID: 669921

Date Received: 05/19/06

Date Analyzed: 05/24/06

Dilution Factor: 1.0

Soil Aliquot Volume:

500 (uL)

CAS NO.

COMPOUND

(ug/L or $\mathrm{ug} / \mathrm{Kg}$ ) UG/KG

Q

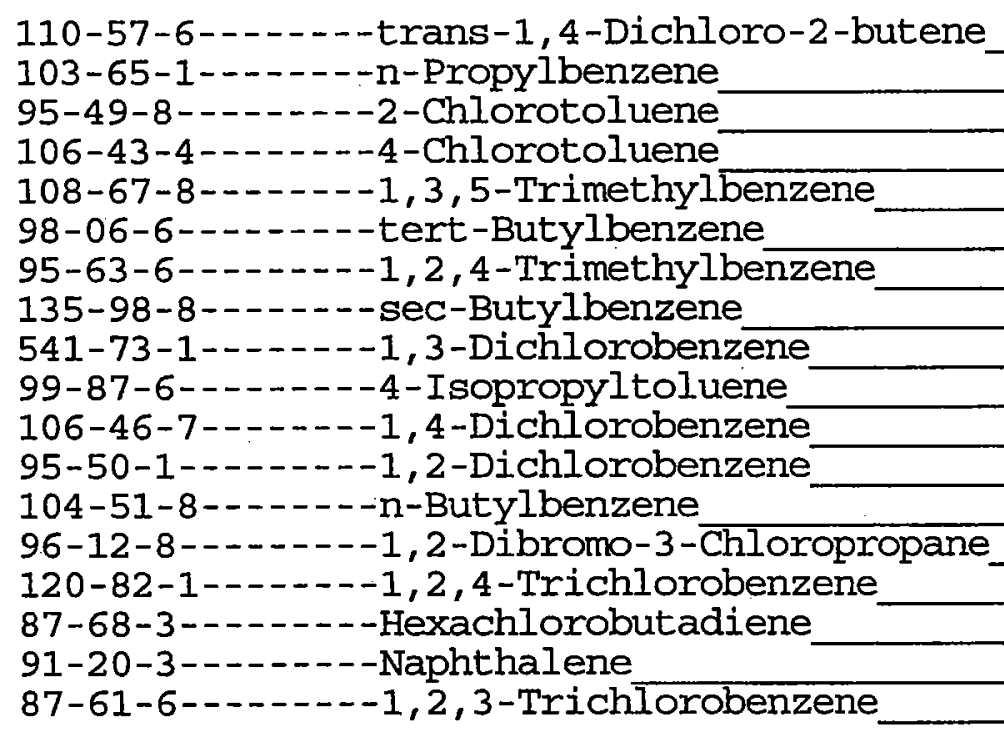


FORM 1

VOLATILE ORGANICS ANALYSIS DATA SHEET

Lab Name: STL BURLINGTON

Contract: 21005

ARGLAB SAMPIE NO.

Lab Code: STLVT Case No.: NAVARRE SAS No.:

NA-S-20689

Matrix: (soil/water) soIL

SDG No.: 114417

Sample wt/vol: $\quad 9.9(\mathrm{~g} / \mathrm{mL}) \mathrm{G}$

Lab Sample ID: 669926

Level: (low/med) MED

Lab File ID: $\quad 669926$

Date Received: 05/19/06

\% Moisture: not dec.

GC Column: CAP ID: $0.53 \cdot(\mathrm{mm})$

Date Analyzed: 05/24/06

Soil Extract Volume: 10000 (uL)

Dilution Factor: 1.0

Soil Aliquot Volume:

$500(\mathrm{uL})$

CAS NO.

COMPOUND

CONCENTRATION UNITS:

(ug/L or $\mathrm{ug} / \mathrm{Kg}$ ) UG/KG

\begin{tabular}{|c|}
\hline 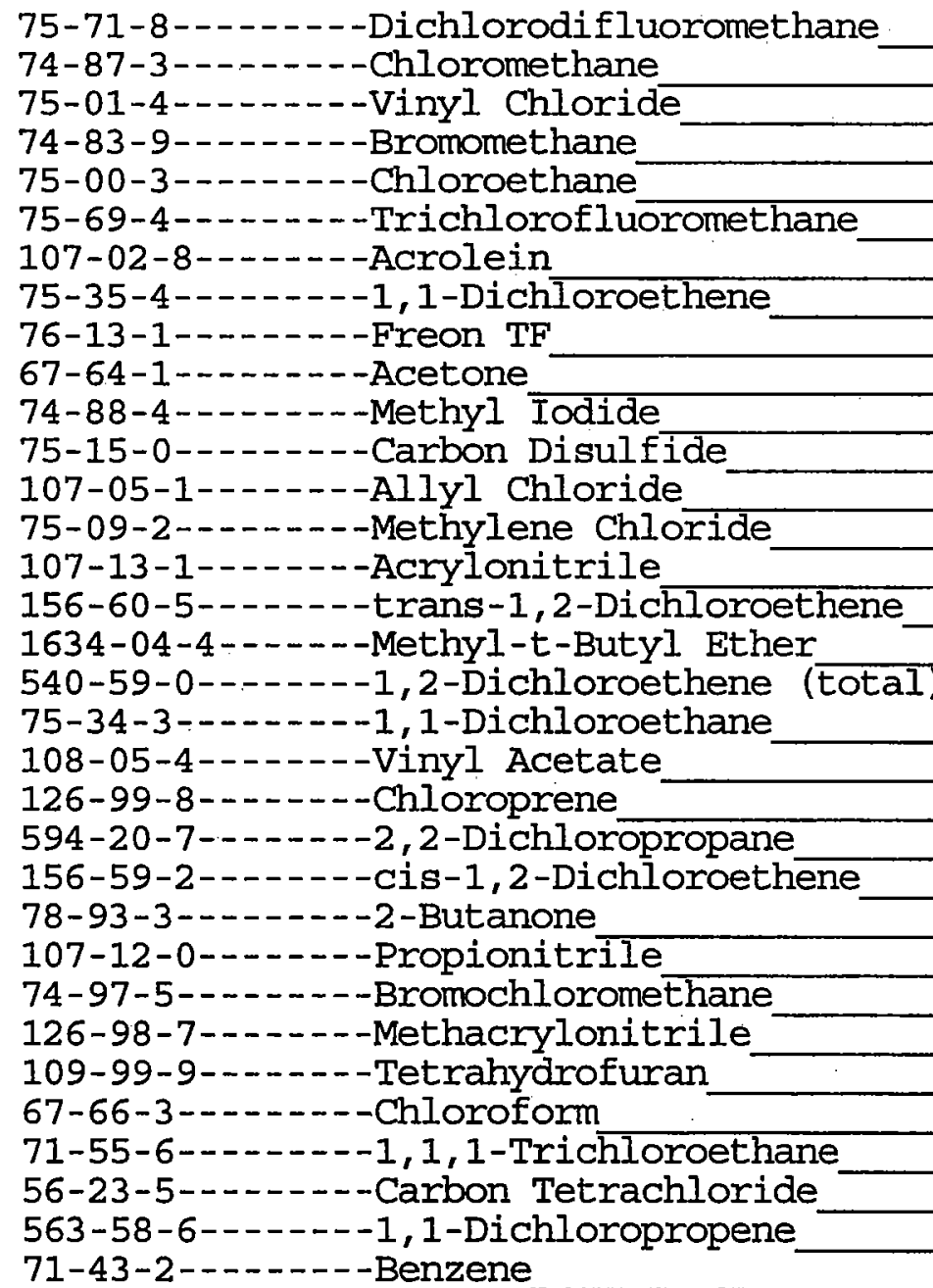 \\
\hline
\end{tabular}

\begin{tabular}{|r|l|l|}
10 & $\mathrm{U}$ \\
10 & $\mathrm{U}$ \\
10 & $\mathrm{U}$ \\
4.3 & $\mathrm{JB}$ \\
10 & $\mathrm{U}$ \\
10 & $\mathrm{U}$ \\
50 & $\mathrm{U}$ \\
10 & $\mathrm{U}$ \\
10 & $\mathrm{U}$ \\
50 & $\mathrm{U}$ \\
3.3 & $\mathrm{JB}$ \\
10 & $\mathrm{U}$ \\
10 & $\mathrm{U}$ \\
6.1 & $\mathrm{JB}$ \\
10 & $\mathrm{U}$ \\
10 & $\mathrm{U}$ \\
10 & $\mathrm{U}$ \\
10 & $\mathrm{U}$ \\
10 & $\mathrm{U}$ \\
10 & $\mathrm{U}$ \\
10 & $\mathrm{U}$ \\
10 & $\mathrm{U}$ \\
10 & $\mathrm{U}$ \\
27 & $\mathrm{~J}$ \\
41 & $\mathrm{U}$ \\
10 & $\mathrm{U}$ \\
10 & $\mathrm{U}$ \\
140 & $\mathrm{U}$ \\
10 & $\mathrm{U}$ \\
10 & $\mathrm{U}$ \\
16 & \\
10 & $\mathrm{U}$ \\
10 & $\mathrm{U}$ \\
& & \\
\hline
\end{tabular}


FORM 1

VOLATILE ORGANICS ANALYSIS DATA SHEET

Lab Name: STL BURLINGTON

Contract: 21005

Lab Code: STLVT Case No.: NAVARRE SAS No.:

ARGLAB SAMPLE NO.

NA-S-20689

SDG No. : 114417
Matrix: (soil/water) SOIL

Sample wt/vol: $\quad 9.9(\mathrm{~g} / \mathrm{mL}) \mathrm{G}$

Level: (low/med) MED

음 Moisture: not dec.

GC Column: CAP ID: $0.53 \quad(\mathrm{~mm})$

Soil Extract Volume: 10000 (uL)
Lab Sample ID: 669926

Lab File ID: 669926

Date Received: 05/19/06

Date Analyzed: 05/24/06

Dilution Factor: 1.0

Soil Aliquot Volume: 500 (uL)

CAS NO.

COMPOUND

CONCENTRATION UNITS:

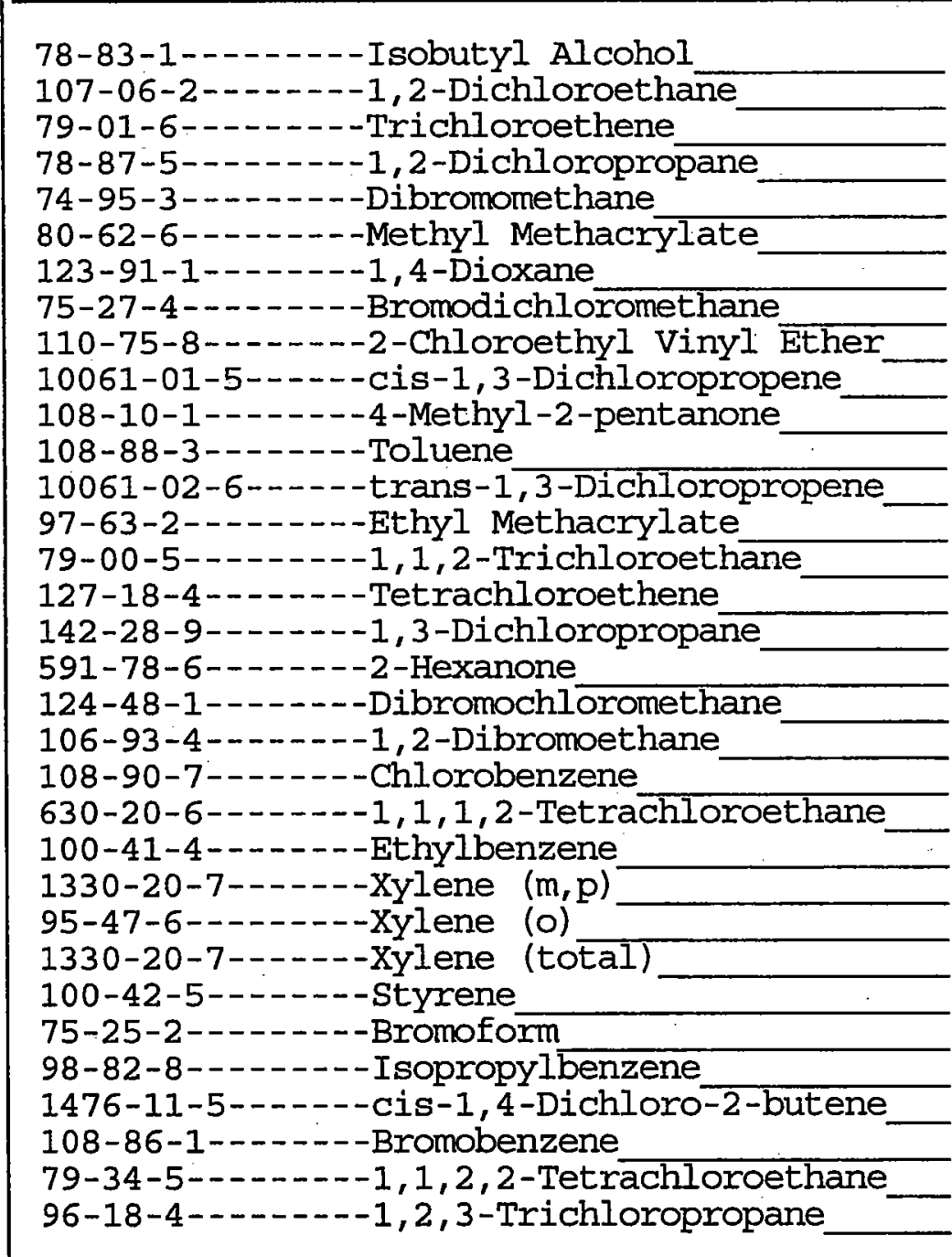


FORM 1

VOLATILE ORGANICS ANALYSIS DATA SHEET

Lab Name: STL BURLINGTON

Contract: 21005

Lab code: STLVT Case No.: NAVARRE SAS No.:

ARGLAB SAMPLE NO.

Matrix: (soil/water) SOII

Sample wt/vol: $\quad 9.9(\mathrm{~g} / \mathrm{mL}) \mathrm{G}$

Lab Sample ID: 669926

Level : (low/med) MED

Lab File ID: 669926

\% Moisture: not dec.

GC Column: CAP ID: 0.53 (mm)

Soil Extract Volume: 10000 (uس)
NA-S-20689

SDG No.: 114417
COMPOUND
CAS NO.
(ug/L or $\mathrm{ug} / \mathrm{Kg}$ ) UG/KG
$Q$

Date Analyzed: 05/24/06

Dilution Factor: 1.0

Soil Aliquot Volume:

500 (uس)
Date Received: 05/19/06
CONCENTRATION UNITS:$$
\text { 110-57-6-------trans-1, 4-Dichloro-2-butene }
$$$$
\text { 103-65-1------n-Propylbenzene. }
$$$$
\text { 95-49-8--.---2-Chlorotoluene- }
$$$$
\text { 106-43-4-----4-Chlorotoluene }
$$$$
\text { 108-67-8-...-1, 3, 5-Trimethylbenzene }
$$$$
\text { 98-06-6---.---tert-Butylbenzene }
$$$$
\text { 95-63-6 -...--1,2,4-Trimethylbenzene }
$$$$
\text { 135-98-8------sec-Butylbenzene }
$$$$
\text { 541-73-1-..--1, 3-Dichlorobenzene }
$$$$
\text { 99-87-6-------4-Isopropyltoluene }
$$$$
\text { 106-46-7--.----1,4-Dichlorobenzene }
$$$$
\text { 95-50-1--..--1, 2-Dichlorobenzene }
$$$$
\text { 104-51-8-...-n-Butylbenzene }
$$$$
\text { 96-12-8-...--1, 2-Dibromo-3-Chloropropane }
$$$$
\text { 120-82-1-...--1,2,4-Trichlorobenzene }
$$$$
\text { 87-68-3-...-.-Hexachlorobutadiene }
$$$$
\text { 91-20-3-...--Naphthalene }
$$$$
\text { 87-61-6-..---1,2,3-Trichlorobenzene }
$$

\begin{tabular}{l|l|}
10 & $\mathrm{U}$ \\
10 & $\mathrm{U}$ \\
10 & $\mathrm{U}$ \\
10 & $\mathrm{U}$ \\
10 & $\mathrm{U}$ \\
10 & $\mathrm{U}$ \\
10 & $\mathrm{U}$ \\
10 & $\mathrm{U}$ \\
10 & $\mathrm{U}$ \\
10 & $\mathrm{U}$ \\
10 & $\mathrm{U}$ \\
10 & $\mathrm{U}$ \\
10 & $\mathrm{U}$ \\
10 & $\mathrm{U}$ \\
10 & $\mathrm{U}$ \\
10 & $\mathrm{U}$ \\
10 & $\mathrm{U}$ \\
10 & $\mathrm{U}$ \\
\hline
\end{tabular}


FORM 1

VOLATILE ORGANICS ANALYSIS DATA SHEET

Lab Name: STL BURLINGTON

Contract: 21005

Lab Code: STLVT Case No.: NAVARRE SAS No.:

ARGLAB SAMPLE NO.

Matrix: (soil/water) SOIL

Lab Sample ID: 669924

Sample wt/vol: $\quad 7.3(\mathrm{~g} / \mathrm{mL}) \mathrm{G}$

Lab File ID: $\quad 669924$

Level: (low/med) MED

Date Received: 05/19/06

\% Moisture: not dec.

GC Column: CAP ID: 0.53 (mm)

Soil Extract Volume: 10000 (U山)

CAS NO.
COMPOUND
NA-S-20773
Date Analyzed: 05/24/06

Dilution Factor: 1.0

Soil Aliquot Volume: $\quad 500(u \mathrm{u})$

\begin{tabular}{|c|c|c|}
\hline 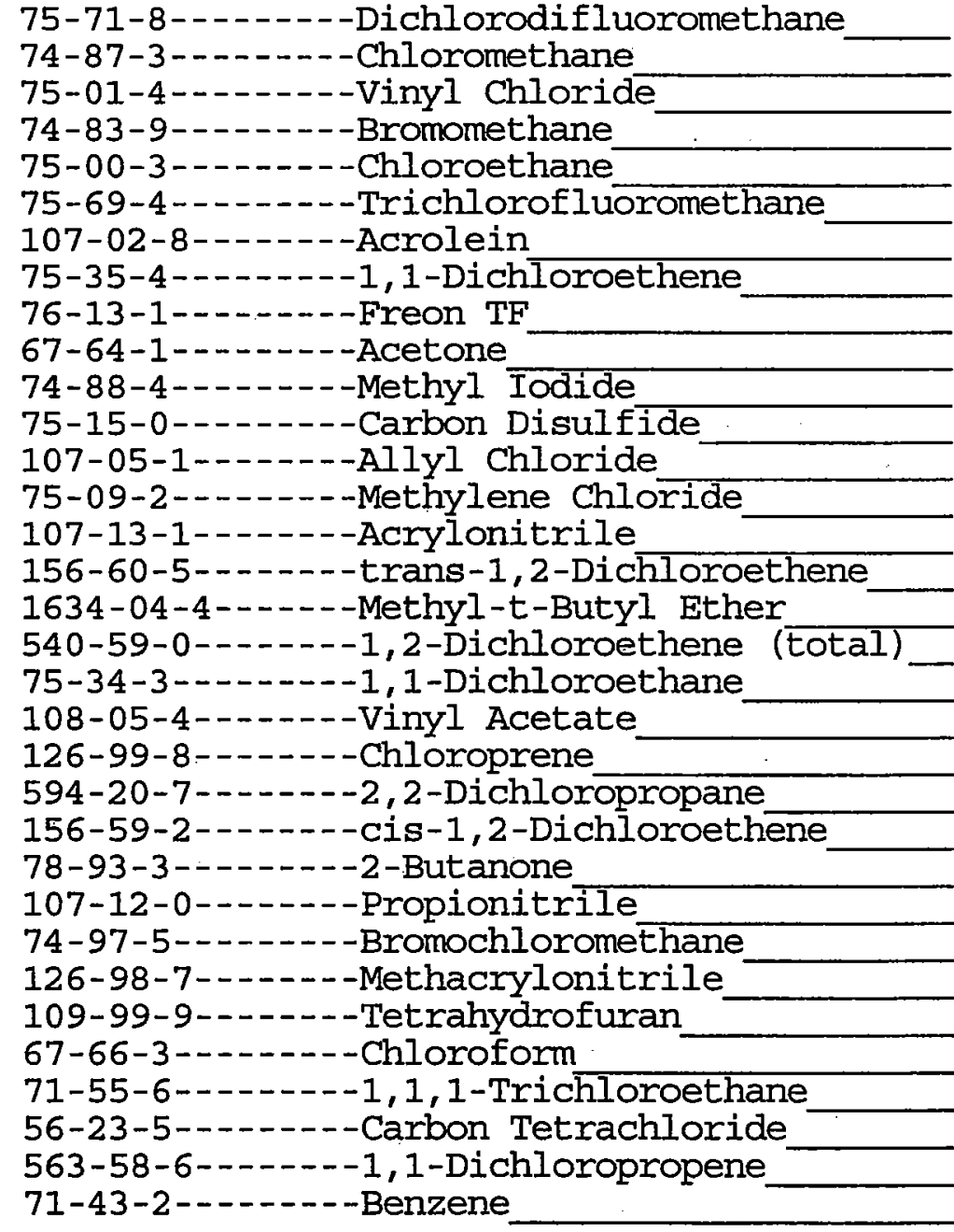 & $\begin{array}{r}14 \\
14 \\
14 \\
8.2 \\
14 \\
14 \\
68 \\
14 \\
14 \\
68 \\
14 \\
14 \\
14 \\
8.9 \\
14 \\
14 \\
14 \\
14 \\
14 \\
14 \\
14 \\
14 \\
14 \\
33 \\
55 \\
14 \\
14 \\
190 \\
14 \\
14 \\
14 \\
14 \\
14\end{array}$ & \begin{tabular}{|l}
$U$ \\
$U$ \\
$U$ \\
$J B$ \\
$U$ \\
$U$ \\
$U$ \\
$U$ \\
$U$ \\
$U$ \\
$U$ \\
$U$ \\
$U$ \\
$U$ \\
$U$ \\
$U$ \\
$J B$ \\
$U$ \\
$U$ \\
$U$ \\
$U$ \\
$U$ \\
$U$ \\
$U$ \\
$U$ \\
$U$ \\
$U$ \\
$U$ \\
$U$ \\
$U$ \\
$U$ \\
$U$ \\
$U$ \\
$U$ \\
$U$ \\
$U$ \\
$U$ \\
$U$ \\
$U$ \\
$U$ \\
$U$ \\
$U$
\end{tabular} \\
\hline
\end{tabular}


FORM 1

VOLATILE ORGANICS ANALYSIS DATA SHEET

Lab Name: STL BURLINGTON

Contract : 21005

Lab code: STLVT Case No.: NAVARRE SAS No.:

Lab Sample ID: 669924

Matrix: (soil/water) SOIL

Sample wt/vol: $\quad 7.3(\mathrm{~g} / \mathrm{mL})$ G

Level: (low/med) MED

Lab File ID: 669924

$\%$ Moisture: not dec.

GC Column: CAP ID: 0.53 (mm)

Soil Extract Volume: 10000 (UL)
ARGLAB SAMPLE NO.

NA-S-20773

SDG No.: 114417
Date Received: 05/19/06

Date Analyzed: 05/24/06

Dilution Factor: 1.0

Soil Aliquot Volume:

500 (ut)

CAS NO. COMPOUND (ug/L or ug/Kg) UG/KG

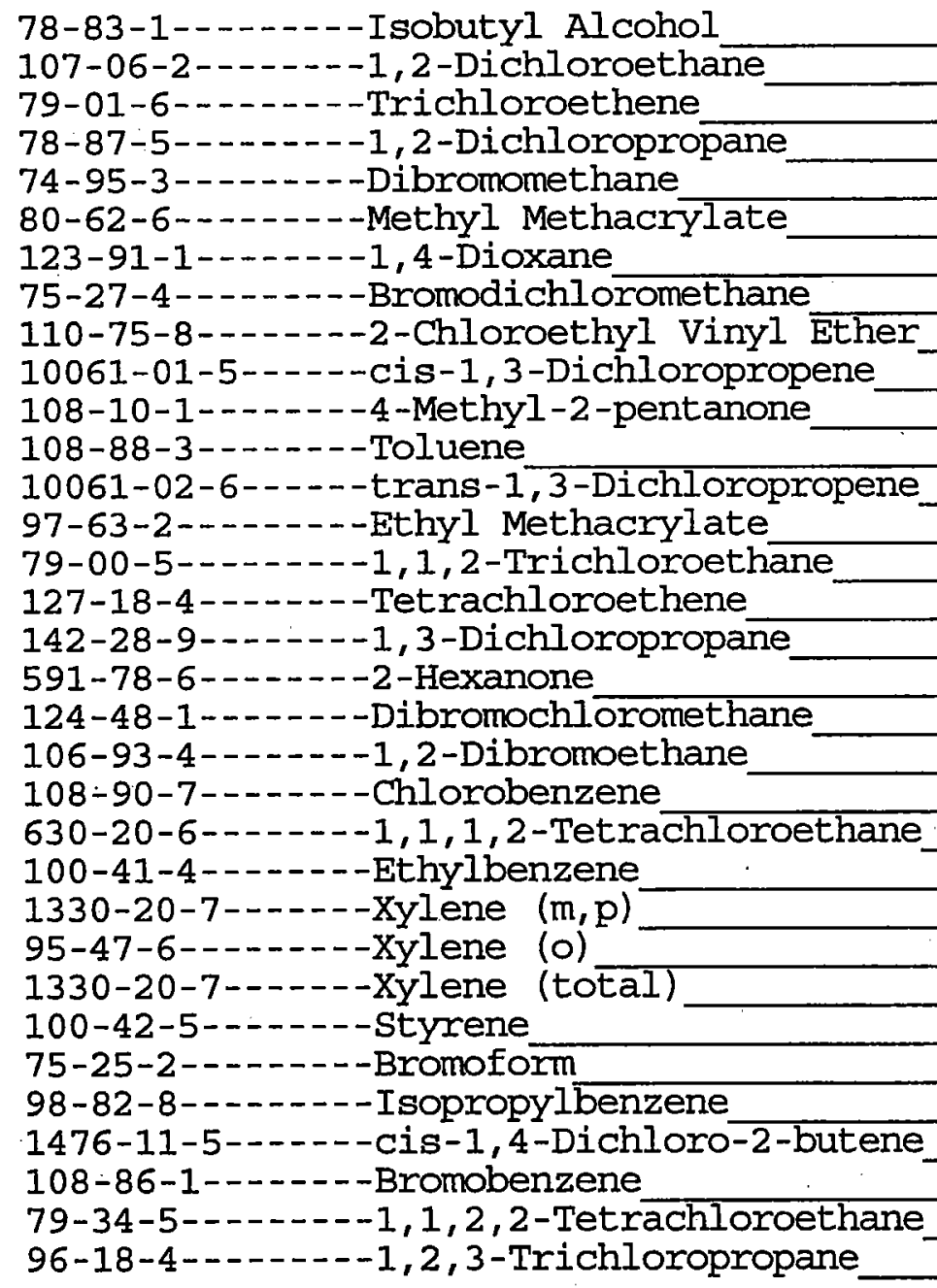

\begin{tabular}{r|l}
680 & $\mathrm{U}$ \\
14 & $\mathrm{U}$ \\
14 & $\mathrm{U}$ \\
14 & $\mathrm{U}$ \\
14 & $\mathrm{U}$ \\
14 & $\mathrm{U}$ \\
680 & $\mathrm{U}$ \\
14 & $\mathrm{U}$ \\
14 & $\mathrm{U}$ \\
14 & $\mathrm{U}$ \\
68 & $\mathrm{U}$ \\
14 & $\mathrm{U}$ \\
14 & $\mathrm{U}$ \\
14 & $\mathrm{U}$ \\
14 & $\mathrm{U}$ \\
14 & $\mathrm{U}$ \\
14 & $\mathrm{U}$ \\
68 & $\mathrm{U}$ \\
14 & $\mathrm{U}$ \\
14 & $\mathrm{U}$ \\
14 & $\mathrm{U}$ \\
14 & $\mathrm{U}$ \\
14 & $\mathrm{U}$ \\
14 & $\mathrm{U}$ \\
14 & $\mathrm{U}$ \\
14 & $\mathrm{U}$ \\
14 & $\mathrm{U}$ \\
14 & $\mathrm{U}$ \\
14 & $\mathrm{U}$ \\
14 & $\mathrm{U}$ \\
14 & $\mathrm{U}$ \\
14 & $\mathrm{U}$ \\
14 & $\mathrm{U}$ \\
& \\
\hline &
\end{tabular}


FORM 1

VOLATILE ORGANICS ANALYSIS DATA SHEET

Lab Name: STL BURLINGTON

Contract: 21005

ARGLAB SAMPLE NO.

$\mathrm{NA}-\mathrm{S}-20773$

Lab Code: STLVT Case No.: NAVARRE SAS No.:

SDG No.: 114417

Matrix: (soil/water) SOIL

Lab Sample ID: 669924

Sample wt/vol:

$7.3(\mathrm{~g} / \mathrm{mL}) \mathrm{G}$

Lab File ID: $\quad 669924$

Level: (low/med) MED

Date Received: 05/19/06

\% Moisture: not dec.

Date Analyzed: 05/24/06

GC Column: CAP ID: 0.53 (mm)

Dilution Factor: 1.0

Soil Extract Volume: 10000 (u山)

Soil Aliquot Volume:

500 (uL)

CONCENTRATION UNITS:

CAS NO.

COMPOUND

(ug/L or $u g / \mathrm{Kg}$ ) UG/KG

Q

$14 \mathrm{U}$

$14 \mathrm{U}$

$14 \mathrm{U}$

$14 \mathrm{U}$

$14 \mathrm{U}$

$14 \mathrm{U}$

$14 \mathrm{U}$

$14 \mathrm{U}$

$14 \mathrm{U}$

$14 \mathrm{U}$

$14 \mathrm{U}$

$14 \mathrm{U}$

$14 \mathrm{U}$

$14 \mathrm{U}$

$14 \mathrm{U}$

$14 \mathrm{U}$

$14 \mathrm{U}$

$14 \mathrm{U}$ 
FORM 1

VOLATILE ORGANICS ANALYSIS DATA SHEET

Lab Name: STL BURLINGTON

Contract: 21005

Lab Code: STLVT Case No.: NAVARRE SAS No.:

Lab Sample ID: 669925

Matrix: (soil/water) SOIL

Lab File ID: 669925

Sample wt/vol:

$9.1(\mathrm{~g} / \mathrm{mL}) \mathrm{G}$

Level: (low/med) MED

Date Received: 05/19/06

\% Moisture: not dec.

GC Column: CAP

ID: $0.53 \quad(\mathrm{~mm})$

Date Analyzed: 05/24/06

Soil Extract Volume: 10000 (UL)
NA-S-20783

SDG No. : 114417
Dilution Factor: 1.0

Soil Aliquot Volume:

CAS NO.

Dichlorodifluoromethane

74-87-3----.--Chloromethane

75-01-4-...-.--Vinyl Chloride

74-83-9-1-.---Bromomethane

75-00-3-------Chloroethane

75-69-4-..-- - Trichlorofluoromethane

107-02-8-...--Acrolein

75-35-4-- - - 1, 1-Dichloroethene

76-13-1--.----Freon TF

67-64-1--.-.---Acetone

74-88-4-...-.--Methyl Iodide

75-15-0-.....-Carbon Disulf $\overline{i d e}$

107-05-1--.--Allyl Chloride

75-09-2 -...--Methylene Chloride

107-13-1.....-Acrylonitrile

156-60-5------trans-1, 2-Dichloroethene

1634-04-4------Methyl-t-Butyl Ether

CONCENTRATION UNITS:

500 (uL)

540-59-0-..--1,2-Dichloroethene (total)

75-34-3-..---1,1-Dichloroethane

108-05-4------Vinyl Acetate

126-99-8-.-.---Chloroprene

594-20-7---.--2, 2-Dichloropropane

156-59-2------cis-1,2-Dichloroethene

78-93-3--.----2-Butanone

107-12-0-...---Propionitrile

74-97-5-...--Bromochloromethane

126-98-7------Methacrylonitrile

109-99-9------Tetrahydrofuran

67-66-3--.---Chloroform

71-55-6-...-1, 1,1-Trichloroethane

56-23-5-.....-.-Carbon Tetrachloride

563-58-6-----1, 1-Dichloropropene

71-43-2-.-.-.--Benzene

\begin{tabular}{r|l}
11 & $\mathrm{U}$ \\
11 & $\mathrm{U}$ \\
11 & $\mathrm{U}$ \\
2.9 & $\mathrm{JB}$ \\
11 & $\mathrm{U}$ \\
11 & $\mathrm{U}$ \\
55 & $\mathrm{U}$ \\
11 & $\mathrm{U}$ \\
11 & $\mathrm{U}$ \\
55 & $\mathrm{U}$ \\
3.9 & $\mathrm{BB}$ \\
11 & $\mathrm{U}$ \\
11 & $\mathrm{U}$ \\
6.4 & $\mathrm{BB}$ \\
11 & $\mathrm{U}$ \\
11 & $\mathrm{U}$ \\
11 & $\mathrm{U}$ \\
11 & $\mathrm{U}$ \\
11 & $\mathrm{U}$ \\
11 & $\mathrm{U}$ \\
11 & $\mathrm{U}$ \\
11 & $\mathrm{U}$ \\
11 & $\mathrm{U}$ \\
55 & $\mathrm{U}$ \\
44 & $\mathrm{U}$ \\
11 & $\mathrm{U}$ \\
11 & $\mathrm{U}$ \\
150 & $\mathrm{U}$ \\
3.6 & $\mathrm{JB}$ \\
11 & $\mathrm{U}$ \\
13 & \\
11 & $\mathrm{U}$ \\
11 & $\mathrm{U}$ \\
& \\
\hline & \\
\hline
\end{tabular}


FORM 1

VOLATIIE ORGANICS ANALYSIS DATA SHEET

Lab Name: STL BURLINGTON

Contract: 21005

Lab Code: STLVT Case No.: NAVARRE SAS No.:

ARGLAB SAMPLE NO.

NA-S-20783

SDG No.: 114417
Matrix: (soil/water) SOIL

Sample wt/vol:

$9.1(\mathrm{~g} / \mathrm{mL}) \mathrm{G}$

Level: (low/med) MED

\% Moisture: not dec.

GC Column: CAP

ID : $0.53(\mathrm{~mm})$

Soil Extract Volume: 10000 (uL)
Lab Sample ID: 669925

Lab File ID: $\quad 669925$

Date Received: 05/19/06

Date Analyzed: 05/24/06

Dilution Factor: 1.0

Soil Aliquot Volume: $\quad 500(u L)$

CAS NO.

COMPOUND

CONCENTRATION UNITS:

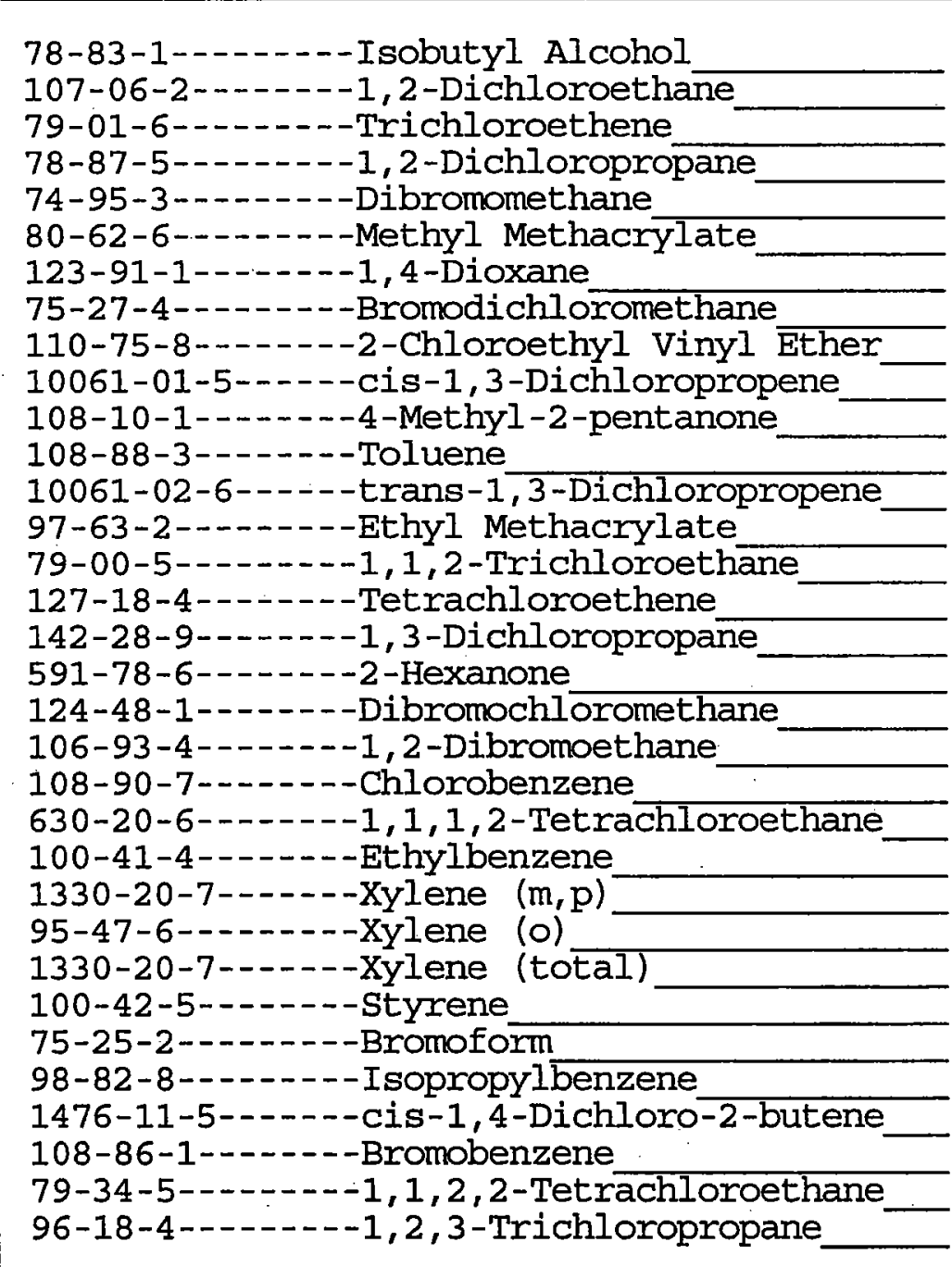

(ug/L or $\mathrm{ug} / \mathrm{Kg}$ ) UG/KG

$Q$ 
FORM 1

VOLATILE ORGANICS ANALYSIS DATA SHEET

Lab Name: STL BURLINGTON

Contract: 21005

Lab Code: STLVT Case No.: NAVARRE SAS No.:

Lab Sample ID: 669925

Matrix: (soil/water) SOIL

Sample wt/vol:

$9.1(\mathrm{~g} / \mathrm{mL}) \mathrm{G}$

Level : (low/med) MED

: Moisture: not dec.

GC Colum: CAP ID: 0.53 (mm)

Soil Extract Volume: 10000 (uL)

Lab File ID: 669925

Date Received: 05/19/06

Date Analyzed: 05/24/06

Dilution Factor: 1.0

Soil Aliquot Volume:
ARGLAB SAMPLE NO.

NA-S-20783

SDG No. : 114417

500 (uL)

CONCENTRATION UNITS:

CAS NO. COMPOUND (ug/I or ug/Kg) UG/KG

Q

$11 \mathrm{U}$

$11 \mathrm{U}$

$11 \mathrm{U}$

$11 \mathrm{U}$

$11 \mathrm{U}$

$11 \mathrm{U}$

$11 \mathrm{U}$

$11 \mathrm{U}$

$11 \mathrm{U}$

$11 \mathrm{U}$

$11 \mathrm{U}$

$11 \mathrm{U}$

$11 \mathrm{U}$

$11 \mathrm{U}$

$11 \mathrm{U}$

$11 \mathrm{U}$

$11 \mathrm{U}$

$11 \mathrm{U}$ 
FORM 1

VOLATILE ORGANICS ANALYSIS DATA SHEET

Lab Name: STL BURLINGTON

Contract: 21005

Lab Code: STLVT Case No.: NAVARRE SAS No.:

Matrix: (soil/water) SOIL

Sample wt/vol:

$9.7(\mathrm{~g} / \mathrm{mL}) \mathrm{G}$

Level : (low/med) MED

\% Moisture: not dec.

GC Column: CAP ID: $0.53 \quad(\mathrm{~mm})$

Soil Extract Volume: 10000 (uL)
ARGLAB SAMPLE NO.

NA-S-20808

SDG No.: 114417

Lab Sample ID: 669923

Lab File ID: 669923

Date Received: 05/19/06

Date Analyzed: 05/24/06

Dilution Factor: 1.0

Soil Aliquot Volume:

500 (UL)

CONCENT'RATION UNITS :

CAS NO. COMPOUND (ug/L or $\mathrm{ug} / \mathrm{Kg})$ UG/KG

Q

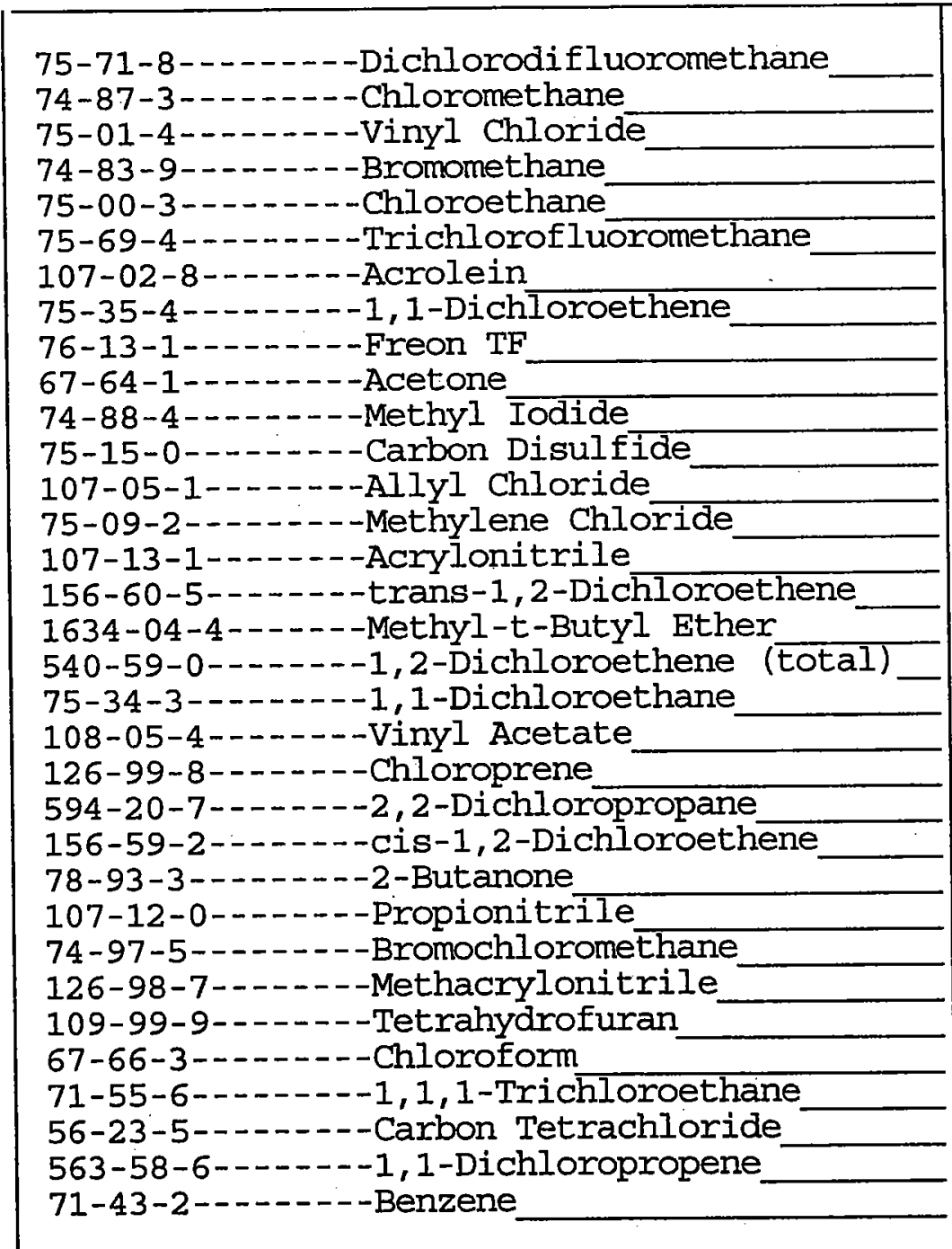

\begin{tabular}{r|l|l}
10 & $\mathrm{U}$ \\
10 & $\mathrm{U}$ \\
10 & $\mathrm{U}$ \\
7.7 & $\mathrm{JB}$ \\
10 & $\mathrm{U}$ \\
10 & $\mathrm{U}$ \\
51 & $\mathrm{U}$ \\
10 & $\mathrm{U}$ \\
10 & $\mathrm{U}$ \\
51 & $\mathrm{U}$ \\
4.4 & $\mathrm{JB}$ \\
10 & $\mathrm{U}$ \\
10 & $\mathrm{U}$ \\
6.7 & $\mathrm{JB}$ \\
10 & $\mathrm{U}$ \\
10 & $\mathrm{U}$ \\
10 & $\mathrm{U}$ \\
10 & $\mathrm{U}$ \\
10 & $\mathrm{U}$ \\
10 & $\mathrm{U}$ \\
10 & $\mathrm{U}$ \\
10 & $\mathrm{U}$ \\
10 & $\mathrm{U}$ \\
26 & $\mathrm{~J}$ \\
41 & $\mathrm{U}$ \\
10 & $\mathrm{U}$ \\
10 & $\mathrm{U}$ \\
140 & $\mathrm{U}$ \\
10 & $\mathrm{U}$ \\
10 & $\mathrm{U}$ \\
3.2 & $\mathrm{~J}$ \\
10 & $\mathrm{U}$ \\
10 & $\mathrm{U}$ \\
& \\
\hline & \\
\hline
\end{tabular}


FORM 1

VOLATILE ORGANICS ANALYSIS DATA SHEET

Lab Name: STL BURLINGTON

Lab Code: STLVT Case No.: NAVARRE SAS No.:
ARGLAB SAMPLE NO.

NA-S-20808

SDG No. : 114417

Matrix: (soil/water) SOIL

Sample wt/vol: $\quad 9.7(\mathrm{~g} / \mathrm{mL}) \mathrm{G}$

Level: (low/med) MED

․ㅡㅁ Moisture: not dec.

GC Column: CAP $\quad$ ID: 0.53 (mm)

Soil Extract Volume: 10000 (ut)
Lab Sample ID: 669923

Lab File ID: 669923

Date Received: 05/19/06

Date Analyzed: 05/24/06

Dilution Factor: 1.0

Soil Aliquot Volume:

500 (uL)

CAS NO.

COMPOUND

CONCENTRATION UNITS :

(ug/L or $u g / \mathrm{Kg}$ ) UG/KG

78-83-1-...---Isobutyl Alcohol

107-06-2------1,2-Dichloroethane

79-01-6--.----Trichloroethene

78-87-5-1---1,2-Dichloropropane

74-95-3--.----Dibromomethane

80-62-6-...--Methyl Methacrylate

123-91-1-..---1,4-Dioxane

75-27-4--1----Bromodichloromethane

110-75-8-..--2-Chloroethyl Vinyl Ether

10061-01-5--.--cis-1,3-Dichloropropene

108-10-1-..---4-Methyl-2-pentanone

108-88-3--.-.--Toluene

10061-02-6-----trans-1, 3-Dichloropropene

97-63-2-1.----Ethyl Methacrylate

79-00-5-...--1, 1,2-Trichloroethane

127-18-4------Tetrachloroethene

142-28-9-..--1, 3-Dichloropropane

591-78-6--.---2-Hexanone

124-48-1------Dibromochloromethane

106-93-4-----1, 2-Dibromoethane

108-90-7---.----Chlorobenzene

630-20-6-...-1, 1, 1, 2-Tetrachloroethane

100-41-4-...-.-Ethylbenzene

1330-20-7-..--Xylene $(\mathrm{m}, \mathrm{p})$

95-47-6--.---Xylene (o)

1330-20-7-..---Xylene (total)

100-42-5---.---styrene

75-25-2 - . - - -Bromoform

98-82-8--.----Isopropyl benzene

1476-11-5-..---cis-1, 4-Dichloro-2-butene

108-86-1-..---Bromobenzene

79-34-5------1, 1,2,2-Tetrachloroethane

96-18-4--.--1, 2,3-Trichloropropane

\begin{tabular}{r|l}
520 & $\mathrm{U}$ \\
10 & $\mathrm{U}$ \\
10 & $\mathrm{U}$ \\
10 & $\mathrm{U}$ \\
10 & $\mathrm{U}$ \\
10 & $\mathrm{U}$ \\
520 & $\mathrm{U}$ \\
10 & $\mathrm{U}$ \\
10 & $\mathrm{U}$ \\
10 & $\mathrm{U}$ \\
51 & $\mathrm{U}$ \\
10 & $\mathrm{U}$ \\
10 & $\mathrm{U}$ \\
10 & $\mathrm{U}$ \\
10 & $\mathrm{U}$ \\
10 & $\mathrm{U}$ \\
10 & $\mathrm{U}$ \\
51 & $\mathrm{U}$ \\
10 & $\mathrm{U}$ \\
10 & $\mathrm{U}$ \\
10 & $\mathrm{U}$ \\
10 & $\mathrm{U}$ \\
10 & $\mathrm{U}$ \\
10 & $\mathrm{U}$ \\
10 & $\mathrm{U}$ \\
10 & $\mathrm{U}$ \\
10 & $\mathrm{U}$ \\
10 & $\mathrm{U}$ \\
10 & $\mathrm{U}$ \\
10 & $\mathrm{U}$ \\
10 & $\mathrm{U}$ \\
10 & $\mathrm{U}$ \\
10 & $\mathrm{U}$ \\
& \\
&
\end{tabular}


FORM 1

VOLATILE ORGANICS ANALYSIS DATA SHEET

Lab Name: STL BURLINGTON

Contract: 21005
ARGLABB SAMPLE NO.

NA-S-20808

Lab Code: STLVT Case No.: NAVARRE SAS No.:

SDG No. : 114417

Matrix: (soil/water) soIL

Lab Sample ID: 669923

Sample wt/vol: $\quad 9.7(\mathrm{~g} / \mathrm{mL}) \mathrm{G}$

Lab File ID: 669923

Level: (low/med) MED

Date Received: 05/19/06

응 Moisture: not dec.

GC Column: CAP ID: 0.53 (mm)

Date Analyzed: 05/24/06

Soil Extract Volume: 10000 (uL)

Dilution Factor: 1.0

Soil Aliquot Volume:

500 (UT)

CONCENIRATION UNITS:

CAS NO. COMPOUND (ug/L or ug/Kg) UG/KG

Q

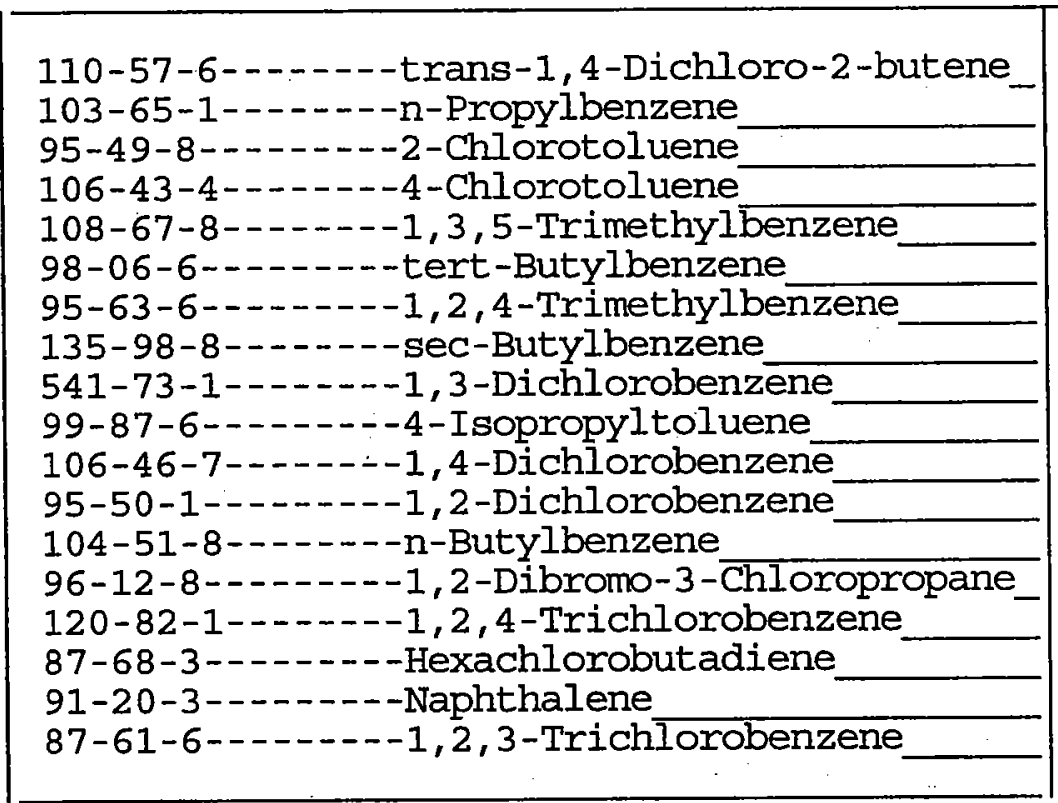

\begin{tabular}{|l|l|l|}
10 & $\mathrm{U}$ \\
10 & $\mathrm{U}$ \\
10 & $\mathrm{U}$ \\
10 & $\mathrm{U}$ \\
10 & $\mathrm{U}$ \\
10 & $\mathrm{U}$ \\
10 & $\mathrm{U}$ \\
10 & $\mathrm{U}$ \\
10 & $\mathrm{U}$ \\
10 & $\mathrm{U}$ \\
10 & $\mathrm{U}$ \\
10 & $\mathrm{U}$ \\
10 & $\mathrm{U}$ \\
10 & $\mathrm{U}$ \\
10 & $\mathrm{U}$ \\
10 & $\mathrm{U}$ \\
10 & $\mathrm{U}$ \\
10 & $\mathrm{U}$ \\
\hline
\end{tabular}


FORM 1

VOLATILE ORGANICS ANALYSIS DATA SHEET

Lab Name: STL BURLINGTON

Contract: 21005
ARGLAB SAMPLE NO.

MEOH BLANK

Lab Code: STLVT Case No.: NAVARRE SAS No.:

SDG No.: 114417

Matrix: (soil/water) SoIL

Lab Sample ID: 669927

Sample wt/vol:

$10.0(\mathrm{~g} / \mathrm{mL}) \mathrm{G}$

Lab File ID: 669927

Level: (low/med) MED

Date Received: 05/19/06

\% Moisture: not dec.

GC Column: CAP ID: 0.53 (mm)

Date Analyzed: 05/24/06

Soil Extract Volume: 10000 (uL)

Dilution Factor: 1.0

Soil Aliquot Volume:

500 (uL)

CAS NO.

COMPOUND

CONCENTRATION UNITS:

$(u g / L$ or $u g / K g) ~ U G / K G$

Q

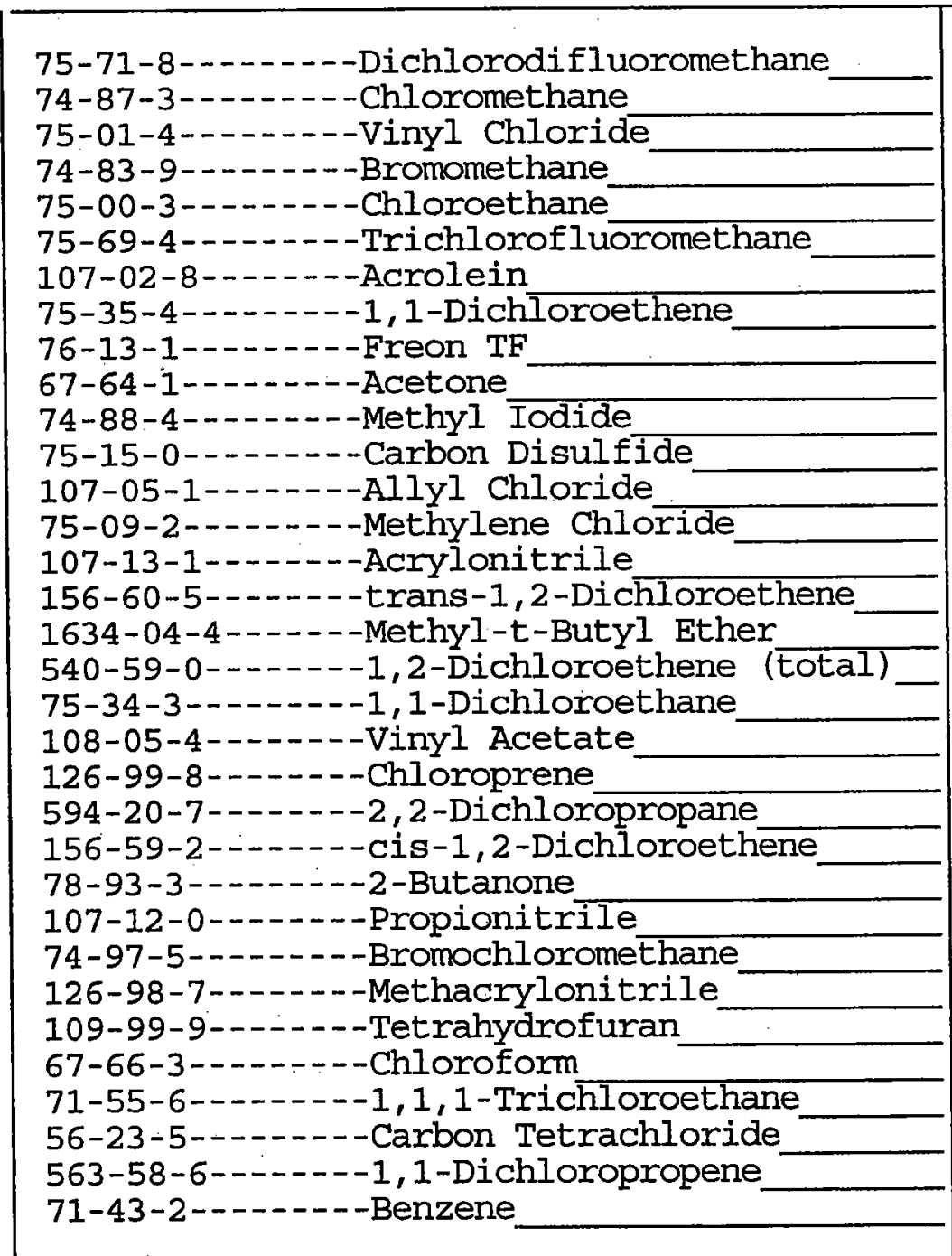

\begin{tabular}{r|l|}
10 & $\mathrm{U}$ \\
3.5 & $\mathrm{~J}$ \\
10 & $\mathrm{U}$ \\
13 & \\
10 & $\mathrm{U}$ \\
10 & $\mathrm{U}$ \\
50 & $\mathrm{U}$ \\
10 & $\mathrm{U}$ \\
10 & $\mathrm{U}$ \\
50 & $\mathrm{U}$ \\
9.5 & $\mathrm{~J}$ \\
10 & $\mathrm{U}$ \\
10 & $\mathrm{U}$ \\
200 & \\
10 & $\mathrm{U}$ \\
10 & $\mathrm{U}$ \\
10 & $\mathrm{U}$ \\
10 & $\mathrm{U}$ \\
10 & $\mathrm{U}$ \\
10 & $\mathrm{U}$ \\
10 & $\mathrm{U}$ \\
10 & $\mathrm{U}$ \\
10 & $\mathrm{U}$ \\
50 & $\mathrm{U}$ \\
40 & $\mathrm{U}$ \\
10 & $\mathrm{U}$ \\
10 & $\mathrm{U}$ \\
140 & $\mathrm{U}$ \\
3.2 & $\mathrm{~J}$ \\
55 & \\
10 & $\mathrm{U}$ \\
10 & $\mathrm{U}$ \\
10 & $\mathrm{U}$ \\
& \\
\hline
\end{tabular}


FORM 1

VOLATIIE ORGANICS ANAIYSIS DATA SHEET

Lab Name: STL BURIINGTON

Contract: 21005

Lab Code: STLVT Case No.: NAVARRE SAS No.:

Lab Sample ID: 669927

Matrix: (soil/water) SOIL

Lab File ID: $\quad 669927$

Sample wt/vol:

$10.0(\mathrm{~g} / \mathrm{mL}) \mathrm{G}$

Level: (low/med) MED

\% Moisture: not dec.

GC Column: CAP ID: 0.53 (mm)

Soil Extract Volume: 10000 (uL)
Date Analyzed: 05/24/06

Dilution Factor: 1.0

Soil Aliquot Volume:
ARGLAB SAMPLE NO.

MEOH BLANK

SDG No.: 114417

CONCENTRATION UNITS :

CAS NO.

COMPOUND

(ug/L or $u g / \mathrm{Kg}$ ) UG/KG

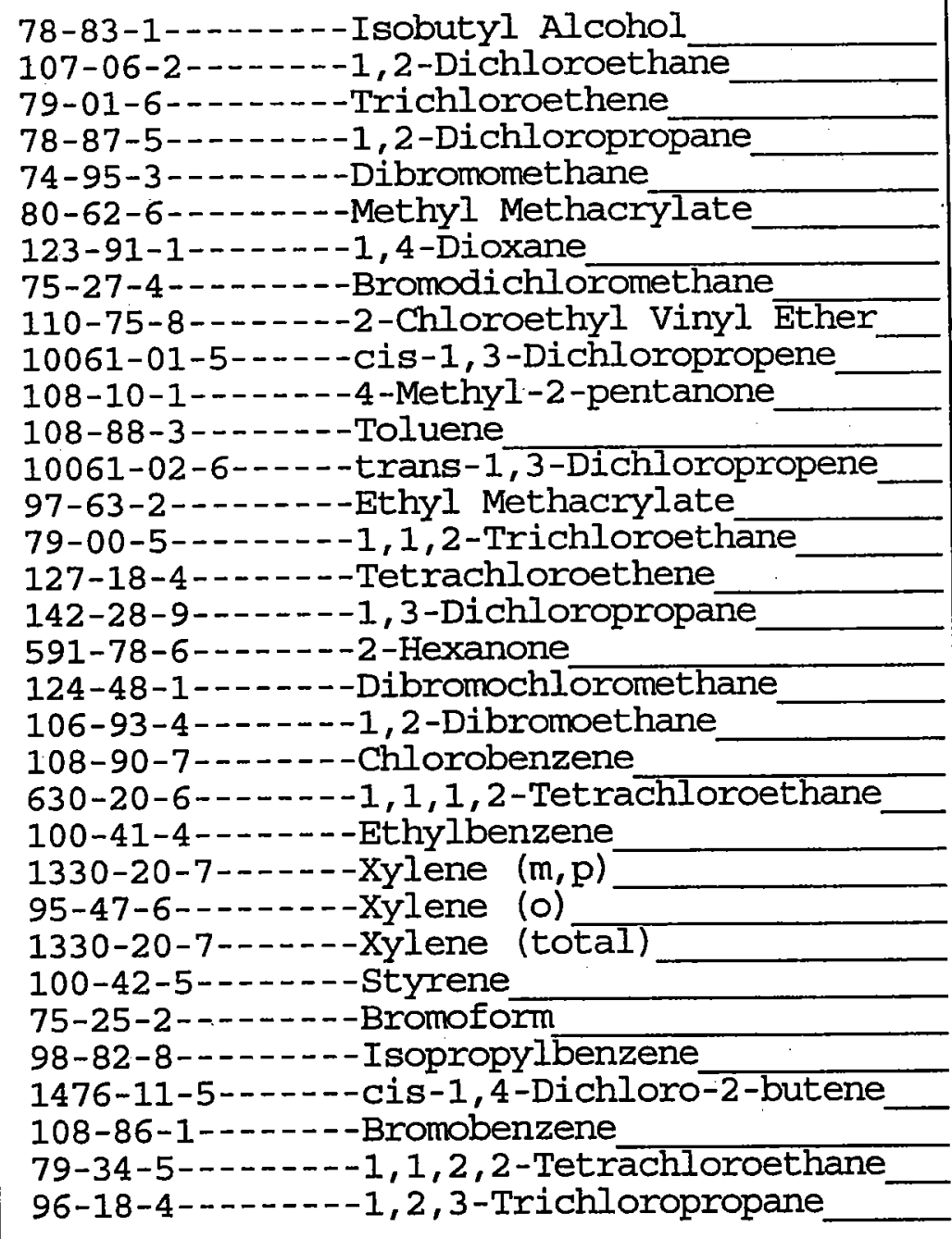


FORM 1

VOLATILE ORGANICS ANALYSIS DATA SHEET

Lab Name: STL BURLINGTON

Contract : 21005

Lab Code: STLVT Case No.: NAVARRE SAS No.:

Lab Sample ID: 669927

Matrix: (soil/water) soIL

Lab File ID: 669927

Sample wt/vol:

$10.0(\mathrm{~g} / \mathrm{mL}) \mathrm{G}$

Level: (low/med) MED

\% Moisture: not dec.

GC Column: CAP

ID: $0.53 \quad(\mathrm{~mm})$

Soil Extract Volume: 10000 (uI)
ARGLAB SAMPLE NO.

MEOH BLANK

SDG No.: 114417
Date Received: 05/19/06

Date Analyzed: 05/24/06

Dilution Factor: 1.0

Soil Aliquot Volume:

500 (UL)

CAS NO.

CONCENTRATION UNITS :

(ug/L or $\mathrm{ug} / \mathrm{Kg}$ ) UG/KG

$Q$

$10 \mathrm{U}$

$10 \mathrm{U}$

$10 \mathrm{U}$

$10 \mathrm{U}$

$10 \mathrm{U}$

$10 \mathrm{U}$

$10 \mathrm{U}$

$10 \mathrm{U}$

$10 \mathrm{U}$

$10 \mathrm{U}$

$10 \mathrm{U}$

$10 \mathrm{U}$

$10 \mathrm{U}$

$10 \mathrm{U}$

$10 \mathrm{U}$

$10 \mathrm{U}$

$10 \mathrm{U}$

$10 \mathrm{U}$

87-61-6-..-- - 1, 2, 3-Trich lorobenzene 


\section{Supplement 6:}

Wastewater Characterization Data 
M.D. Chemical and Testing, Inc.

P.O. Box 19321, Forbes Field, Bldg 281. Topeka, KS 66619

Kansas Certification No. E-10135 (785)862-3500 fax(785)862-5132

Sample Collected By: T.G.

Received In lab: 6/15/2006

Date Reported: 6/19/2006

Project Name: Centralia/Morrill/Everest

Project Number:
Lab Number: 1062574

Client: Larsen \& Associates 913 Rhode lsland P.O. Box 1447

Lawrence, KS 66044

ATTN: Lisa Larsen

\begin{tabular}{|c|c|c|c|c|c|c|}
\hline Analysis & Method & Result & $\begin{array}{l}\text { Detection } \\
\text { Limit }\end{array}$ & Units & $\begin{array}{c}\text { Date } \\
\text { Analyzed }\end{array}$ & Analyst \\
\hline \multicolumn{7}{|c|}{ Sample ID: NV-CM-2-20220 } \\
\hline \multicolumn{7}{|c|}{ Sampled: $6 / 12 / 2006$} \\
\hline \multicolumn{7}{|c|}{ VOLATILE ORGANICS - METHOD } \\
\hline 2-Chloroethyl vinyl ether & EPA $624 / 8260$ & Not Detected & {$[0.5]$} & $\mu g / L(p p b)$ & $6 / 15 / 2006$ & TPJ \\
\hline Vinyl Chloride & EPA $624 / 8260$ & Not Detected & {$[0.5]$} & $\mu \mathrm{g} / \mathrm{L}(\mathrm{ppb})$ & & \\
\hline Trichlorofluoromethane & EPA $624 / 8260$ & Not Detected & {$[0.5]$} & $\mu \mathrm{g} / \mathrm{L}(\mathrm{ppb})$ & & \\
\hline 1,1-Dichloroethylene & $\mathrm{EPA} 624 / 8260$ & Not Detected & {$[0.5]$} & $\mu \mathrm{g} / \mathrm{L}(\mathrm{ppb})$ & & \\
\hline Dichloromethane & EPA $624 / 8260$ & Not Detected & {$[0.5]$} & $\mu \mathrm{g} / \mathrm{L}(\mathrm{ppb})$ & & . \\
\hline Trans 1,2-Dichloroethylene & EPA $624 / 8260$ & Not Detected & {$[0.5]$} & $\mu \mathrm{g} / \mathrm{L}(\mathrm{ppb})$ & & \\
\hline 1,1-Dichloroethane & EPA $624 / 8260$ & Not Detected & {$[0.5]$} & $\mu \mathrm{g} / \mathrm{L}(\mathrm{ppb})$ & & \\
\hline Trichloromethane & EPA $624 / 8260$ & Not Detected & {$[0.5]$} & $\mu \mathrm{g} / \mathrm{L}(\mathrm{ppb})$ & : & \\
\hline 1,1,1-Trichloroethane & EPA $624 / 8260$ & Not Detected & {$[0.5]$} & $\mu \mathrm{g} / \mathrm{L}(\mathrm{ppb})$ & & \\
\hline Tetrachloromethane & EPA $624 / 8260$ & Not Detected & {$[0.5]$} & $\mu \mathrm{g} / \mathrm{L}(\mathrm{ppb})$ & & \\
\hline Benzene & EPA $624 / 8260$ & Not Detected & {$[0.5]$} & $\mu \mathrm{g} / \mathrm{L}(\mathrm{ppb})$ & & \\
\hline 1,2-Dichloroethane & EPA $624 / 8260$ & Not Detected & {$[0.5]$} & $\mu \mathrm{g} / \mathrm{L}(\mathrm{ppb})$ & & \\
\hline Trichloroethylene & EPA $624 / 8260$ & Not Detected & {$[0.5]$} & $\mu \mathrm{g} / \mathrm{L}(\mathrm{ppb})$ & & \\
\hline 1,2-Dichloropropane & EPA $624 / 8260$ & Not Detected & {$[0.5]$} & $\mu \mathrm{g} / \mathrm{L}(\mathrm{ppb})$ & & \\
\hline Bromodichloromiethane & EPA $624 / 8260$ & Not Detected & {$[0.5]$} & $\mu \mathrm{g} / \mathrm{L}(\mathrm{ppb})$ & & \\
\hline Trans 1,3-Dichloropropene & EPA $624 / 8260$ & Not Detected & {$[0.5]$} & $\mu g / L(p p b)$ & & \\
\hline Toluene & EPA $624 / 8260$ & Not Detected & {$[0.5]$} & $\mu \mathrm{g} / \mathrm{L}(\mathrm{ppb})$ & & \\
\hline Cis-1,3-Dichloropropene & EPA $624 / 8260$ & Not Detected & {$[0.5]$} & $\mu \mathrm{g} / \mathrm{L}(\mathrm{ppb})$ & & \\
\hline 1,1,2-Trichloroethane & EPA $624 / 8260$ & Not Detected & {$[0.5]$} & $\mu \mathrm{g} / \mathrm{L}(\mathrm{ppb})$ & & \\
\hline Tetrachloroethylene & EPA $624 / 8260$ & Not Detected & {$[0.5]$} & $\mu \mathrm{g} / \mathrm{L}(\mathrm{ppb})$ & & \\
\hline Dibromochloromethane & EPA $624 / 8260$ & Not Detected & {$[0.5]$} & $\mu \mathrm{g} / \mathrm{L}(\mathrm{ppb})$ & & \\
\hline Chiorobenzene & EPA $624 / 8260$ & Not Detected & {$[0.5]$} & $\mu \mathrm{g} / \mathrm{L}(\mathrm{ppb})$ & & \\
\hline Ethylbenzene & EPA $624 / 8260$ & Not Detected & {$[0.5]$} & $\mu \mathrm{g} / \mathrm{L}(\mathrm{ppb})$ & & \\
\hline Bromoform & EPA $624 / 8260$ & Not Detected & {$[0.5]$} & $\mu \mathrm{g} / \mathrm{L}(\mathrm{ppb})$ & & \\
\hline $1,1,2,2$-Tetrachloroethane & EPA $624 / 8260$ & Not Detected & {$[0.5]$} & $\mu \mathrm{g} / \mathrm{L}(\mathrm{ppb})$ & & \\
\hline $1,3 / 1,4-X y l e n e(m / p)$ & EPA $624 / 8260$ & Not Detected & {$[0.5]$} & $\mu \mathrm{g} / \mathrm{L}(\mathrm{ppb})$ & & \\
\hline 1,2-Xylene(o) & EPA $624 / 8260$ & Not Detected & {$[0.5]$} & $\mu \mathrm{g} / \mathrm{L}(\mathrm{ppb})$ & & \\
\hline 1,3-Dichlorobenzene(m) & EPA $624 / 8260$ & Not Detected & {$[0.5]$} & $\mu \mathrm{g} / \mathrm{L}(\mathrm{ppb})$ & & \\
\hline 1,4-Dichlorobenzene(p) & EPA $624 / 8260$ & Not Detected & {$[0.5]$} & $\mu \mathrm{g} / \mathrm{L}(\mathrm{ppb})$ & & \\
\hline 1,2-Dichlorobenzene(o) & EPA $624 / 8260$ & Not Detected & {$[0.5]$} & $\mu \mathrm{g} / \mathrm{L}(\mathrm{ppb})$ & & \\
\hline
\end{tabular}




\section{Supplement 7:}

\section{Property Documentation}




\section{Supplement 7:}

\section{Property Documentation}

Argonne's description of property boundaries for the former CCC/USDA grain storage facility at Navarre (Figure S7.1) ${ }^{\mathrm{a}}$ is based on the following three levels of documentation:

- Aerial Photographs. A series of nine historical aerial photographs taken in 19572002 provides a visual record of the changing grain storage operations over time and their positions relative to fixed landmarks.

- Lease Records. Lease records obtained from the Atchison, Topeka, and Santa Fe Railroad (ATSF) by the U.S. Environmental Protection Agency in 1992 — and provided to the CCC/USDA and Argonne in 2000 - identify the location of the former CCC/USDA facility and record the expansion of the Navarre Co-op over time. A timeline related to property records is in Table S7.1. ${ }^{a}$

- Survey Data. Survey coordinates of sampling locations and selected structures during the 2006 investigation fix these positions accurately.

\section{S7.1 Aerial Photographs}

The following descriptions refer to some features that are labeled in Figure S7.1 but not in the individual historical photographs.

\section{S7.1.1 July 13, 1957, Photograph}

The 1957 photograph (Figure S7.2a, left) shows the former CCC/USDA grain storage facility at the height of its operation. An extension of Strole Street (refer to Figure S7.1) provides access for the former CCC/USDA facility and the former stockyard $50 \mathrm{ft}$ to the north. Pastureland (the site of the future ball field) is to the northwest of the former CCC/USDA

\footnotetext{
a Figures and a table follow the introductory text. These are followed by copies of the property documents on file at Argonne.
} 
facility, and the former railway depot is to the east. The concrete elevator to the north has been recently added (as of 1957) by the Co-op at the location of a northern stockyard identified in lease documents (Section S7.2).

\section{S7.1.2 August 12, 1965, Photograph}

The 1965 photograph (Figure S7.2a, right) shows that eastern silos (refer to Figure S7.1) with an underground tunnel and overhead walk have been added by the Co-op near the concrete elevator. The flat storage building has been constructed, and drums and other aboveground storage containers lie immediately to its south. Remnants of the stockyard are still visible in the original photograph $50 \mathrm{ft}$ north of the former CCC/USDA facility. The Strole Street extension has been removed, and the area northwest of the former CCC/USDA facility is still pastureland. A gravel or dirt roadway has been extended along the western edge of the flat storage building to provide access to the former CCC/USDA facility. Shadows show that only five bins remain within the former CCC/USDA property, but all of the bin bases are still present. The depot is still visible east of the former CCC/USDA facility.

\section{S7.1.3 June 28, 1971, Photograph}

The 1971 photograph (Figure S7.2b, left) shows that the Co-op has expanded southward and has constructed the dry fertilizer storage building (refer to Figure S7.1) inside the northern border of the former CCC/USDA property. Bulk liquid storage is visible just north of the dry storage building. Remnants of the stockyard $50 \mathrm{ft}$ north of the former CCC/USDA property and the depot to the east are no longer apparent. Hard-packed gravel now covers the northern portion of the former CCC/USDA facility, but the southern portion has been returned to cropland. A ball park is visible northwest of the former CCC/USDA facility.

\section{S7.1.4 October 31, 1976, Photograph}

The 1976 photograph (Figure S7.2b, right) shows that the Co-op has grown across the entire former CCC/USDA facility, and an office and scale have been constructed in the northern portion of the Co-op. Liquid fertilizer storage tanks have been added north of the dry fertilizer building. Anhydrous ammonia tanks in an "L" configuration are visible south of the dry fertilizer 
storage building. Hard-packed gravel extends throughout the Co-op facility. The baseball field seen in the 1971 photograph is no longer present.

\section{S7.1.5 June 14, 1980, Photograph}

The 1980 photograph (Figure S7.2c, left) shows that a fuel tank area has been added south of the anhydrous ammonia tanks ("L" configuration), near the southern extent of the former CCC/USDA facility.

\section{S7.1.6 September 27, 1981, Photograph}

The 1981 photograph (Figure S7.2c, right) shows that the Co-op has expanded south of the former CCC/USDA facility, adding molasses tanks south of the anhydrous ammonia tank storage area. The shadows of the molasses tanks are visible.

\section{S7.1.7 May 25, 1985, Photograph}

In the 1985 photograph (Figure S7.2d, left), a large excavated pit is visible south of the molasses tanks, south of the former CCC/USDA facility. Deposition \#89 by Daryl Anderson (Co-op manager) states the following: "In approximately 1987, the Co-op constructed a shop area in the northern portion of the Co-op. Prior to construction, fill dirt was taken from the southernmost end of the Co-op property leaving an indentation approximately $10 \mathrm{ft}$ by $30 \mathrm{ft}$ in size. The area was subsequently used as a burn pit and general waste disposal area."

\section{S7.1.8 October 1, 1991, Photograph}

Container storage is visible in the 1991 photograph (Figure S7.2d, right) throughout the former ball field northwest of the former CCC/USDA property; a surface drainage pathway appears to bisect that storage area. The molasses tanks and the portable anhydrous ammonia storage tank area (south of the fuel tank area) are visible at the southern end of the Co-op area. The large pit to the south (seen in the 1985 photograph) has been filled in. 


\section{S7.1.9 2002 Photograph}

The 2002 aerial photograph (Figure S7.2e) shows the Co-op site basically as it exists at present. A concrete-bermed area for storage of bulk liquid fertilizer lies just south of the flat storage building (north of the former bulk storage area seen in the 1991 photograph), at the location of the former stockyard.

\section{S7.2 Lease Records}

Lease records and associated property documents specify the location of the former CCC/USDA grain storage facility and provide information about its operations, as well as the locations of expanding operations at the Navarre Co-op. Significant lease records and property documents are summarized below.

\section{S7.2.1 Lease Records for the Former CCC/USDA Grain Storage Facility}

A lease agreement was entered into on June 21, 1954, between the ATSF and the CCC/USDA. The lease was terminated on January 17, 1966 (per an agreement dated February 2, 1966). The 1965 aerial photo (Figure S7.2a, right) shows that by August 1965 only five bins remained at the facility; according to the Johnson deposition, Volume 1, page 102, the CCC/USDA grain bins were being removed as early as 1963. The location of the former CCC/USDA facility, as shown in Figure S7.3 (which accompanied the lease agreement), is west of the depot and $50 \mathrm{ft}$ south of the railroad stockyard (".35 stockyard"). The dimensions and location of the former CCC/USDA property are determined by mile markers MP44 + $2365 \mathrm{ft}$ and MP44 + $1981 \mathrm{ft}$ (toward the bottom of Figure S7.3). The area of the former CCC/USDA property is calculated as follows:

$$
\text { Length }=2,365 \mathrm{ft}-1,981 \mathrm{ft}=384 \mathrm{ft}
$$

Width (difficult to read in Figure S7.3) $=98 \mathrm{ft}$

$$
\text { Area }=384 \mathrm{ft} \times 98 \mathrm{ft}=37,632 \mathrm{ft}^{2}
$$


The 1954 CCC/USDA lease appears overlaid on the 2002 aerial photograph in Figure S7.1.

\section{S7.2.2 Lease Records for the Navarre Co-op}

The Navarre Farmers Union Cooperative Association was incorporated on April 18, 1919. Numerous lease documents on file (or referenced in subsequent lease documents) show the southward expansion of the Co-op operations from the northern extent of the current property, toward and beyond the former CCC/USDA grain storage facility. Figures accompanying eight of the lease documents (discussed below) are significant, because they fix locations and dimensions for not only the Co-op property in general but also for the flat storage building north of the former CCC/USDA facility and the dry fertilizer storage building just inside the northern boundary of the former CCC/USDA facility.

\section{S7.2.2.1 May 1, 1916, Lease (Figure S7.4)}

The earliest lease on file at Argonne for the Navarre Co-op property is between the ATSF and the Fullington Lumber Company. This 1916 lease describes a 3,776- $\mathrm{ft}^{2}$ parcel (32 $\mathrm{ft}$ by $118 \mathrm{ft}$ ) in the northern portion of the current Co-op property. This 1916 lease is cited in a subsequent (1921) lease between ATSF and the Navarre Co-op, transferring control of the property from the lumber company to the Co-op. The figure accompanying the 1916 lease (Figure S7.4) shows the location of the 3,776- $\mathrm{ft}^{2}$ parcel between mile markers MP44 $+3059 \mathrm{ft}$ and MP44 + $2941 \mathrm{ft}$. Just south of the leased parcel are the Hoffman Elevator Company office and a (now demolished) wooden elevator. Farther to the south is the northern stockyard, the future location of the Navarre Co-op concrete elevator.

\section{S7.2.2.2 July 6, 1916, Lease (Not on File)}

Not on file at Argonne is a Co-op lease dated July 6, 1916, that is cited in the June 1, 1921, lease (Section S7.2.2.4). The property described lies between mile markers MP44 + 3059 and MP44 + 2941, which are visible in Figure S7.4. 


\section{S7.2.2.3 May 6, 1920, Lease (Not on File)}

Not on file at Argonne is a Co-op lease dated May 6, 1920, that is cited in the June 5, 1934, lease (Section S7.2.2.5). The 1920 lease concerns a coal bin and elevator, apparently those shown in Figure S7.4 (and also without labels in Figure S7.5).

\section{S7.2.2.4 June 1, 1921, Lease (No Figure)}

The 1921 lease document transfers property held by the Fullington Lumber Company to the Navarre Co-op. No lease figure accompanies Argonne's copy of this lease.

\section{S7.2.2.5 June 5, 1934, Lease (Figure S7.5)}

The 1934 lease between the ATSF and the Navarre Co-op assigns property to be used for an elevator, coal bins, bulk oil station, and other buildings. The accompanying lease figure (Figure S7.5) shows the property to be $27,476 \mathrm{ft}^{2}$, centered on mile marker MP44 $+2941 \mathrm{ft}$ and extending to the former northern stockyard.

\section{S7.2.2.6 April 13, 1949, Lease (Figure S7.6)}

The 1949 lease between ATSF and the Navarre Co-op increases the Co-op-controlled property to $48,276 \mathrm{ft}^{2}$. Noticeable on the accompanying figure (Figure S7.6) is the assignment of the former northern stockyard as the location of a new concrete elevator near mile marker MP44 $+2680 \mathrm{ft}$.

\section{S7.2.2.7 October 8, 1952, Lease (Figure S7.7)}

The 1952 lease with accompanying figure (Figure S7.7) appears to decrease the Co-opcontrolled property to $35,166 \mathrm{ft}^{2}$, eliminating property east of the railroad. 


\section{S7.2.2.8 August 4, 1958, Lease (Figure S7.8)}

The 1958 lease states (on the cover page) that the leased property is to be used for an elevator, coal bins, bulk oil station, a "warehouse," and other buildings. The addition of "warehouse" to the earlier descriptions for property use refers to the construction of the flat storage building. A figure accompanying the lease (Figure S7.8) shows the flat storage building between mile markers MP44 + $2618 \mathrm{ft}$ and MP44 + $2497 \mathrm{ft}$. The 1958 Co-op lease is overlaid on the 2002 aerial photograph in Figure S7.1, along with the 1954 CCC/USDA lease.

\section{S7.2.2.9 January 22, 1962, Lease (No Figure)}

The 1962 lease is for the addition of the tunnel, conveyor, and overhead walk at the location of the elevators and silos in the northern portion of the Co-op property. Diagrams with the lease show the construction details of the new additions.

\section{S7.2.2.10 August 5, 1966, Lease (Figure S7.9)}

The 1966 lease itself is not on file at Argonne. The date of the lease is known from the 1969 lease (Section S7.2.2.11), which states as item \#23 that Contract No. 123081 is terminated. The 1969 lease extends the boundary of the property leased in 1966. The property leased in 1966 under contract No. 123081 is identified in a figure on file (Figure S7.9) as the parcel north of the former CCC/USDA facility location, between it and the former stockyard (approximately an inch above the bottom of the image).

\section{S7.2.2.11 November 21, 1969, Lease (Figure S7.10)}

The 1969 lease concerns additional land south of the flat storage building (in the northern portion of the former CCC/USDA grain storage facility), to be used for the construction of the dry fertilizer storage building. The use of the leased parcel is identified as handling liquid and bulk fertilizer, plus storing of fertilizer equipment and a portable loader. The accompanying lease figure (Figure S7.10) shows the position of the property between mile markers MP44 + $2405 \mathrm{ft}$ and MP44 + $2165 \mathrm{ft}$. The former CCC/USDA facility was located between mile markers MP44+ $2365 \mathrm{ft}$ and MP44 $+1981 \mathrm{ft}$. Therefore, this new parcel is approximately the northern half of the 
former CCC/USDA facility, plus property extending northward almost to the former stockyard shown in the CCC/USDA lease (Figure S7.3).

\section{S7.2.2.12 December 10, 1971, Lease (No Figure)}

The 1971 lease is for the construction of an additional rail line to "serve a grain, coal and bulk oil handling facility."

\section{S7.2.2.13 April 25, 1975, Lease (Figure S7.11)}

The 1975 lease between ATSF and the Co-op expands the property under Co-op control to $194,663 \mathrm{ft}^{2}$. The figure accompanying the lease (Figure S7.11a,b) shows the Co-op property extending $216 \mathrm{ft}$ south of the former CCC/USDA grain storage facility, to mile marker MP44+ $1765 \mathrm{ft}$. (The former CCC/USDA facility extended south to MP44 + $1981 \mathrm{ft}$; Figure S7.3.)

\section{S7.2.2.14 January 30, 1976, Lease (No Figure)}

The 1976 lease between ATSF and Farmland Industries and the Co-op allowed the installation of the 30,000-gallon anhydrous ammonia storage tank north of the dry fertilizer building. (Bulk liquid fertilizer storage was subsequently moved to the south of the flat storage building, within the concrete-bermed area.)

\section{S7.3 Survey Data}

At the conclusion of the CCC/USDA-Argonne 2006 targeted investigation, the sampling locations and selected building corners were surveyed to fix their locations accurately. Northing and easting positions were determined for each point (Figure S7.12a,b). The comparison below of the northing coordinates shows the relative positions of some points of interest.

\begin{tabular}{lc}
\multicolumn{1}{c}{ Location } & Northing $(\mathrm{ft})$ \\
\hline Flat Storage Building (SW corner) & 169831.7 \\
Concrete Berm (NW corner) & 169810.4 \\
Concrete Berm (SE corner) & 169782.7 \\
Dry Fertilizer Building (NE corner) & 169682.0
\end{tabular}




\section{S7.4 Summary}

Consideration of all of the levels of property documentation described here indicates that the north edge of the present dry fertilizer building marks the northern extent of the former CCC/USDA property at Navarre (Figure S7.1).

\section{S7.5 Sources of Photographs}

NAIP, 2002, aerial photograph of Navarre, Kansas, National Agricultural Imagery Program, U.S. Department of Agriculture, http://www.apfo.usda.gov/NAIP.html.

USDA, 1957, aerial photograph AYH-2T-127, U.S. Department of Agriculture, July 13.

USDA, 1965, aerial photograph AYH-4FF-101, U.S. Department of Agriculture, August 12.

USDA, 1971, aerial photograph AYH-3MM-147, U.S. Department of Agriculture, June 28.

USDA, 1976, aerial photograph 20041176 11L, U.S. Department of Agriculture, October 31.

USDA, 1980, aerial photograph 20041180 71A, U.S. Department of Agriculture, June 14.

USDA, 1981, aerial photograph HAP81 171 183L, U.S. Department of Agriculture, September 27.

USDA, 1985, aerial photograph NHAP2 21 46L, U.S. Department of Agriculture, May 25.

USGS, 1991, aerial photograph of Navarre, Kansas, U.S. Geological Survey, October 1. 


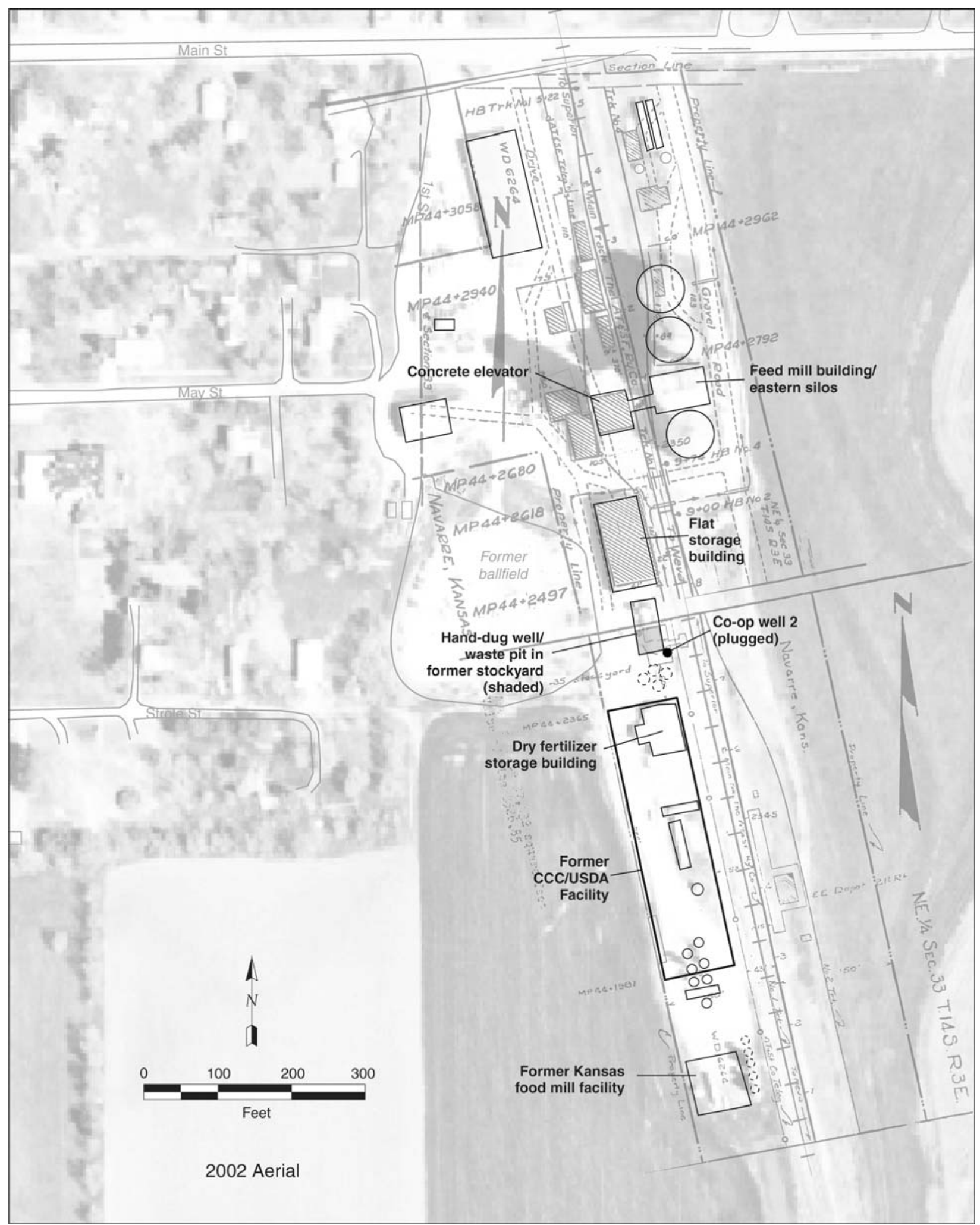

FIGURE S7.1 2002 aerial photo of Navarre overlain with historic lease data (1958, green; 1954, purple). Source of photograph: NAIP (2002). 

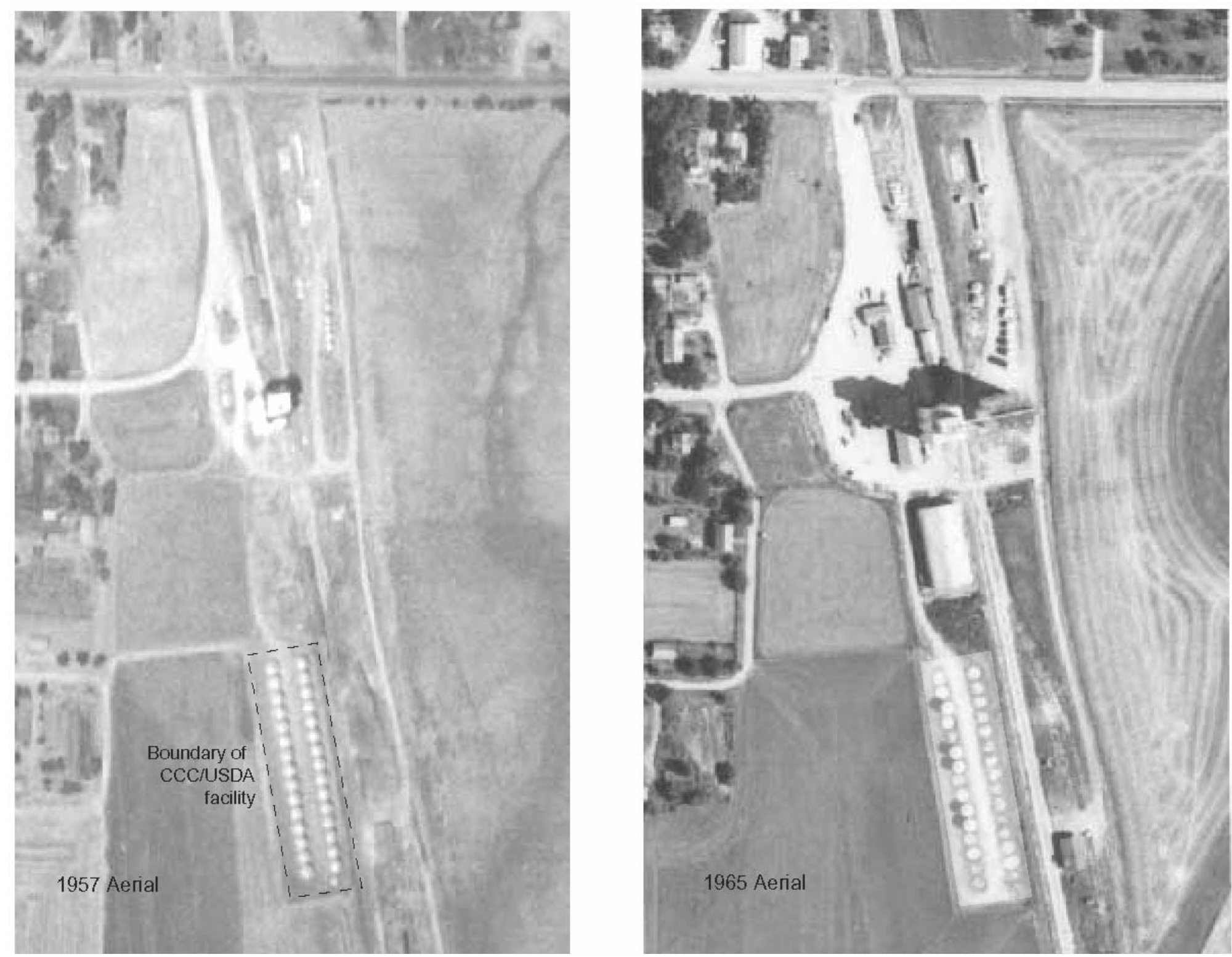

FIGURE S7.2a Navarre, 1957 and 1965. Sources of photographs: USDA $(1957,1965)$. 

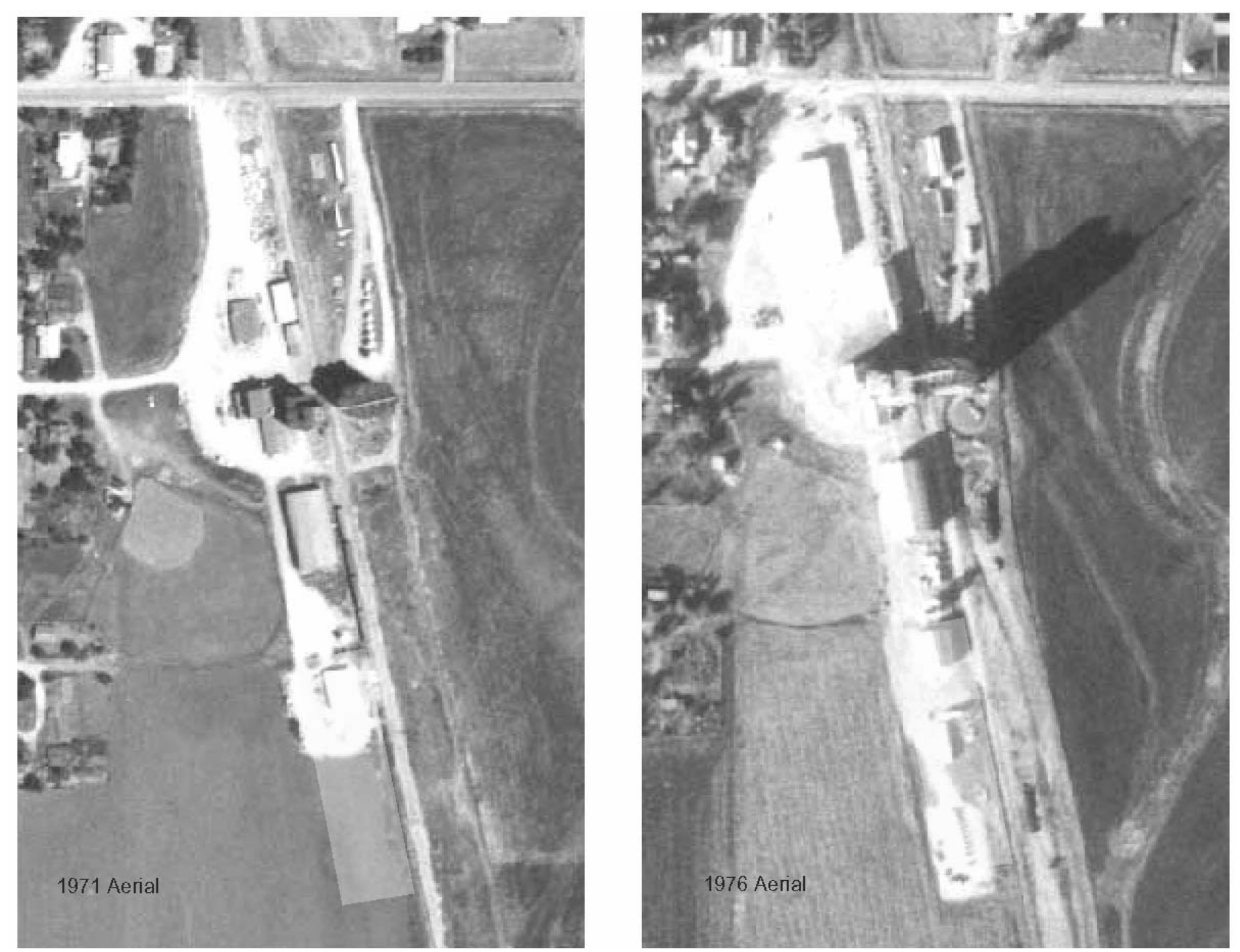

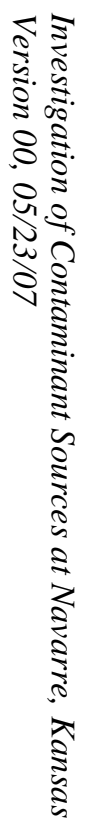

FIGURE S7.2b Navarre, 1971 and 1976. Sources of photographs: USDA $(1971,1976)$. 

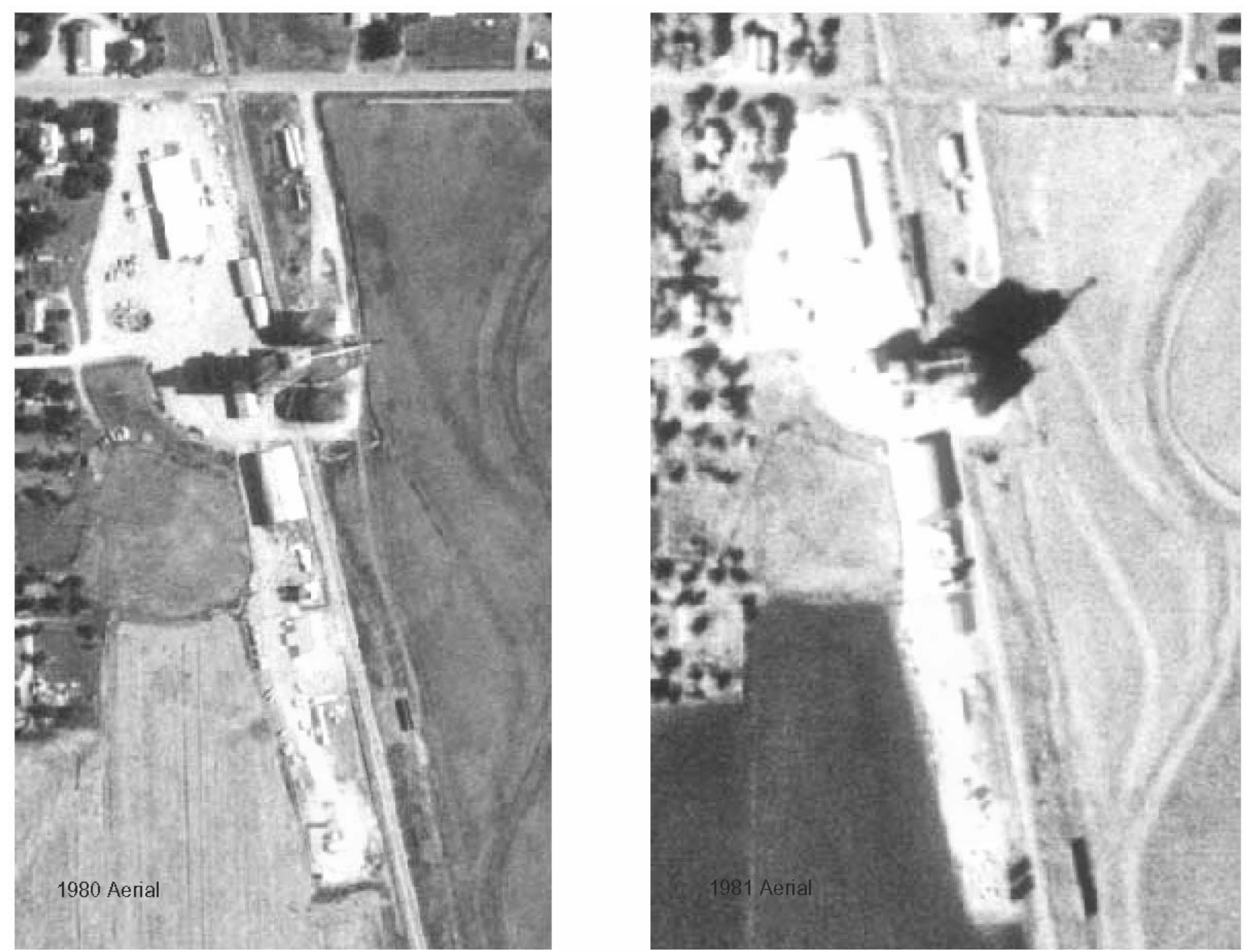

FIGURE S7.2c Navarre, 1980 and 1981. Sources of photographs: USDA (1980, 1981). 

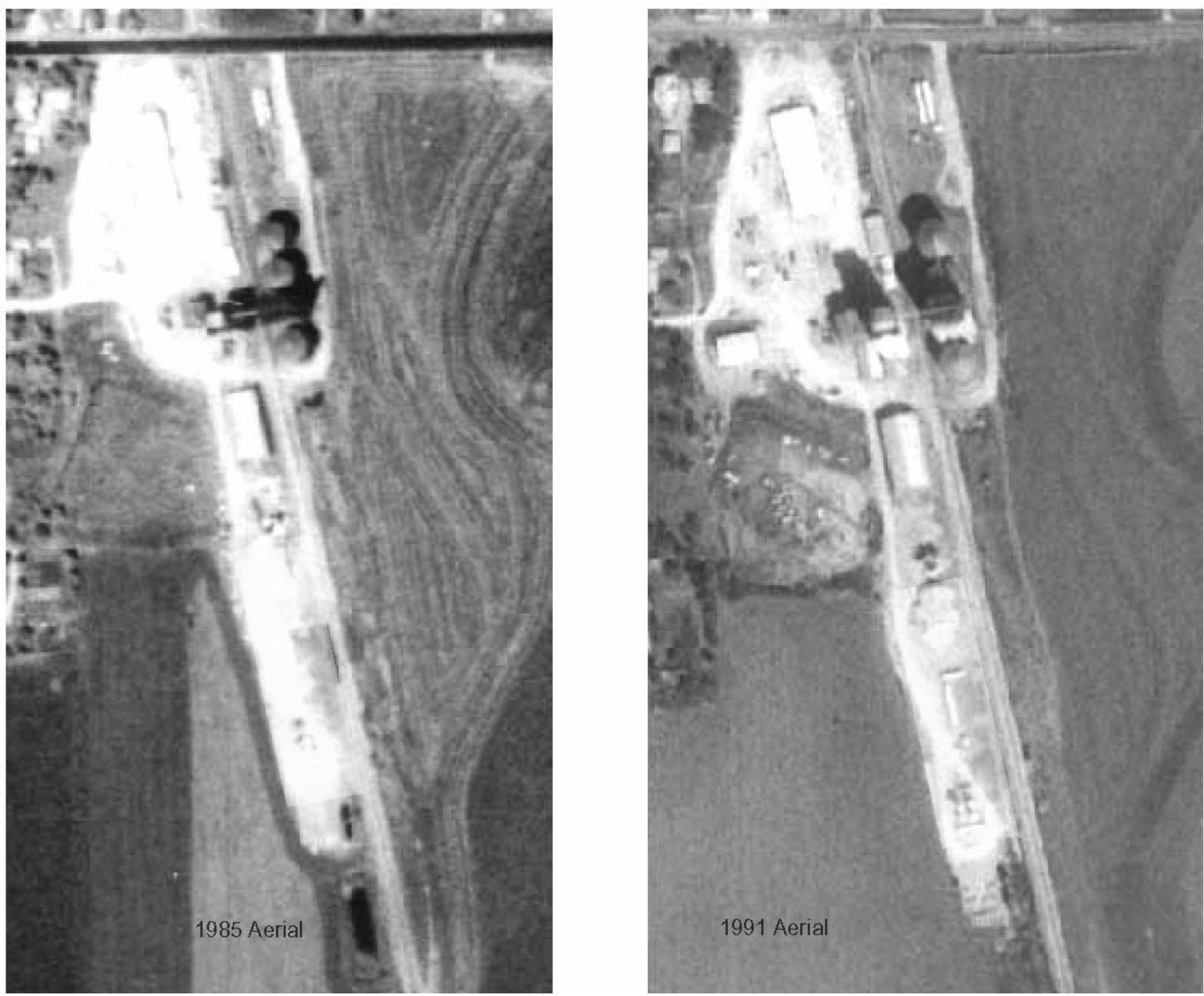

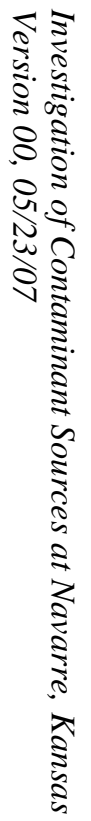

FIGURE S7.2d Navarre, 1985 and 1991. Sources of photographs: USDA (1985) and USGS (1991). 

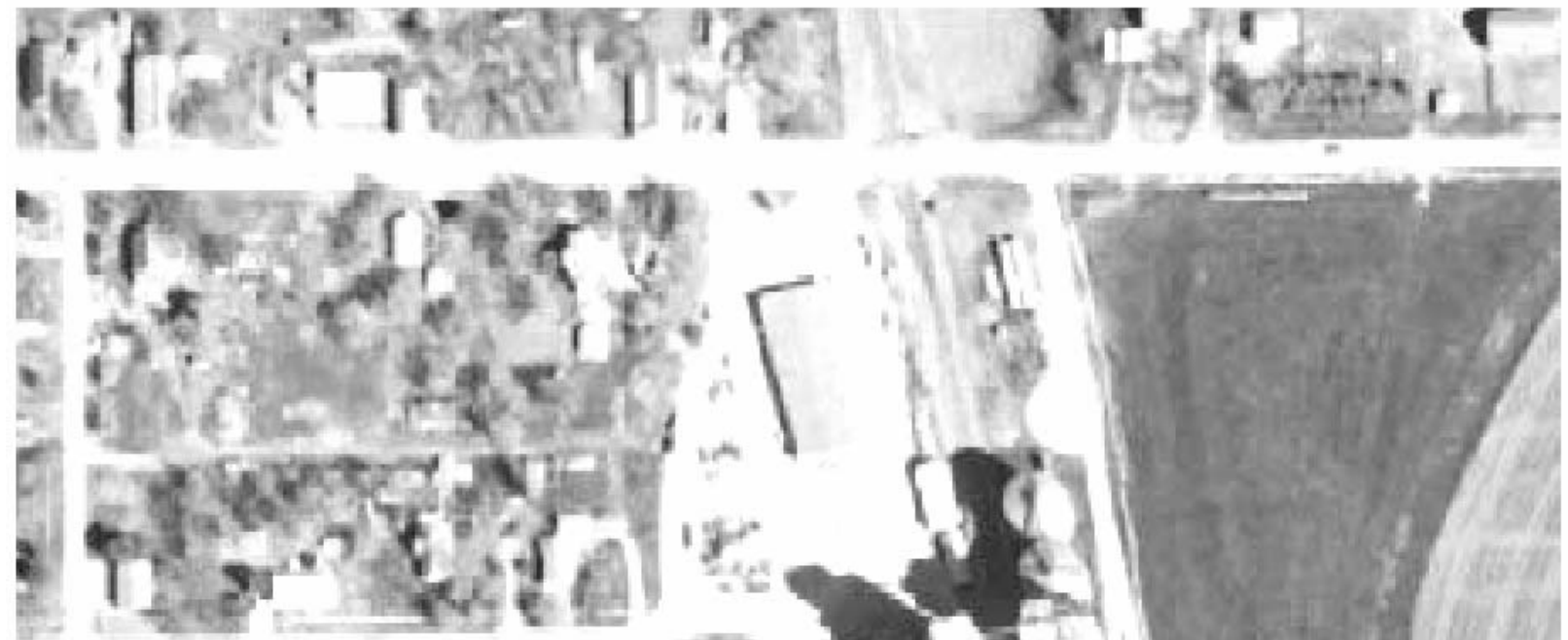
.

1. 4 . 74
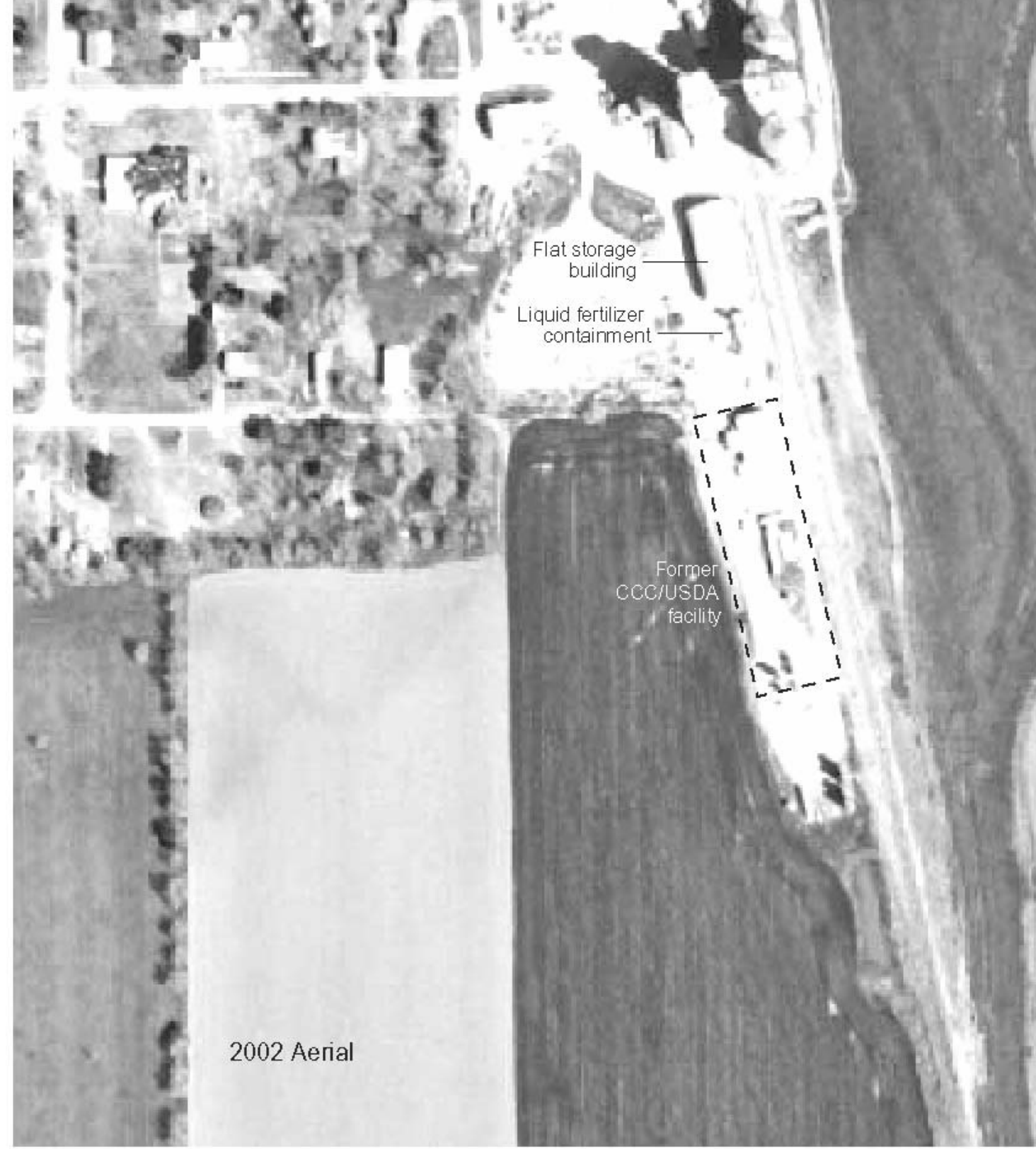

FIGURE S7.2e Investigation area at Navarre, 2002. Source of photograph: NAIP (2002). 


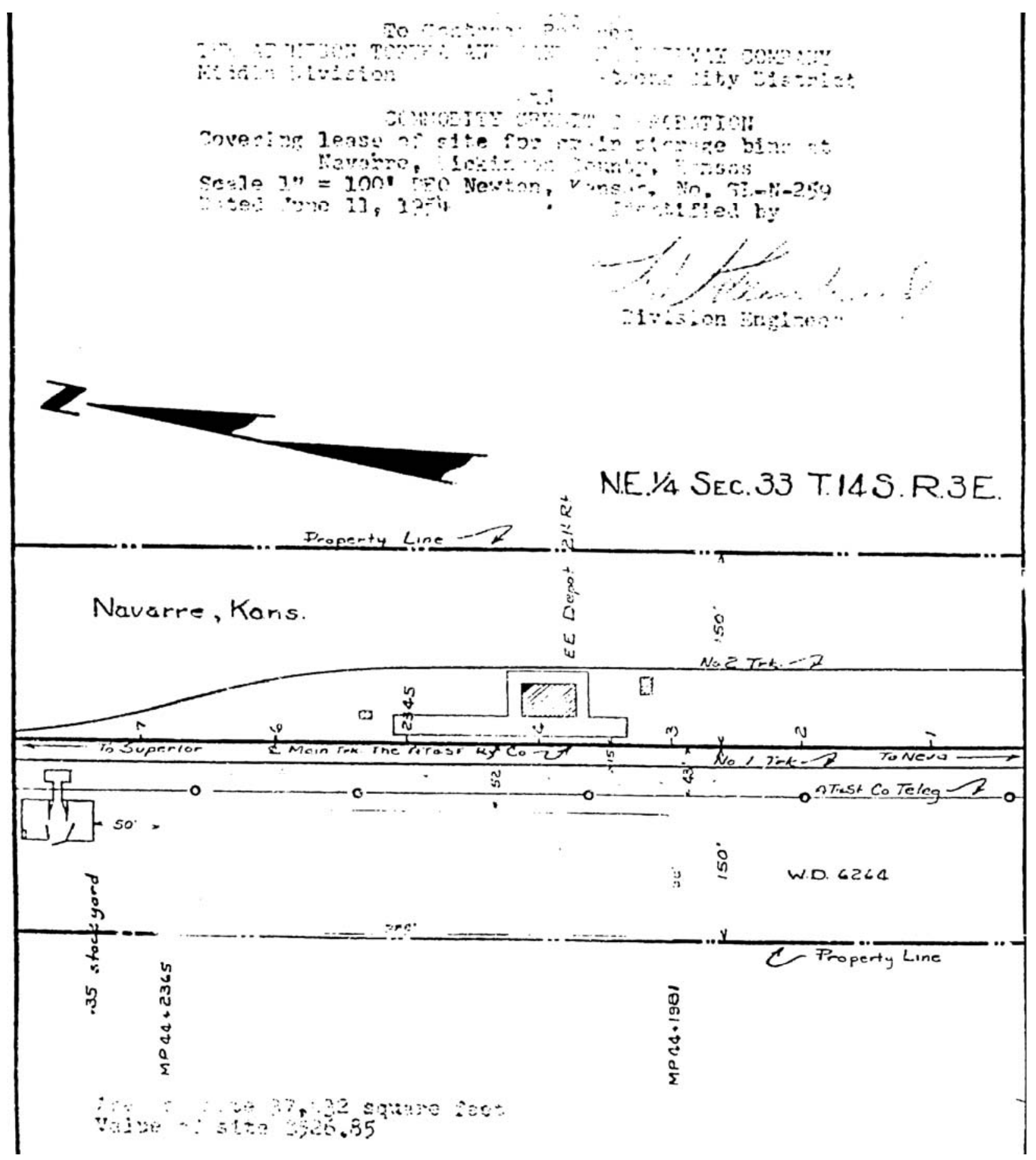

FIGURE S7.3 1954 CCC/USDA lease illustration. 


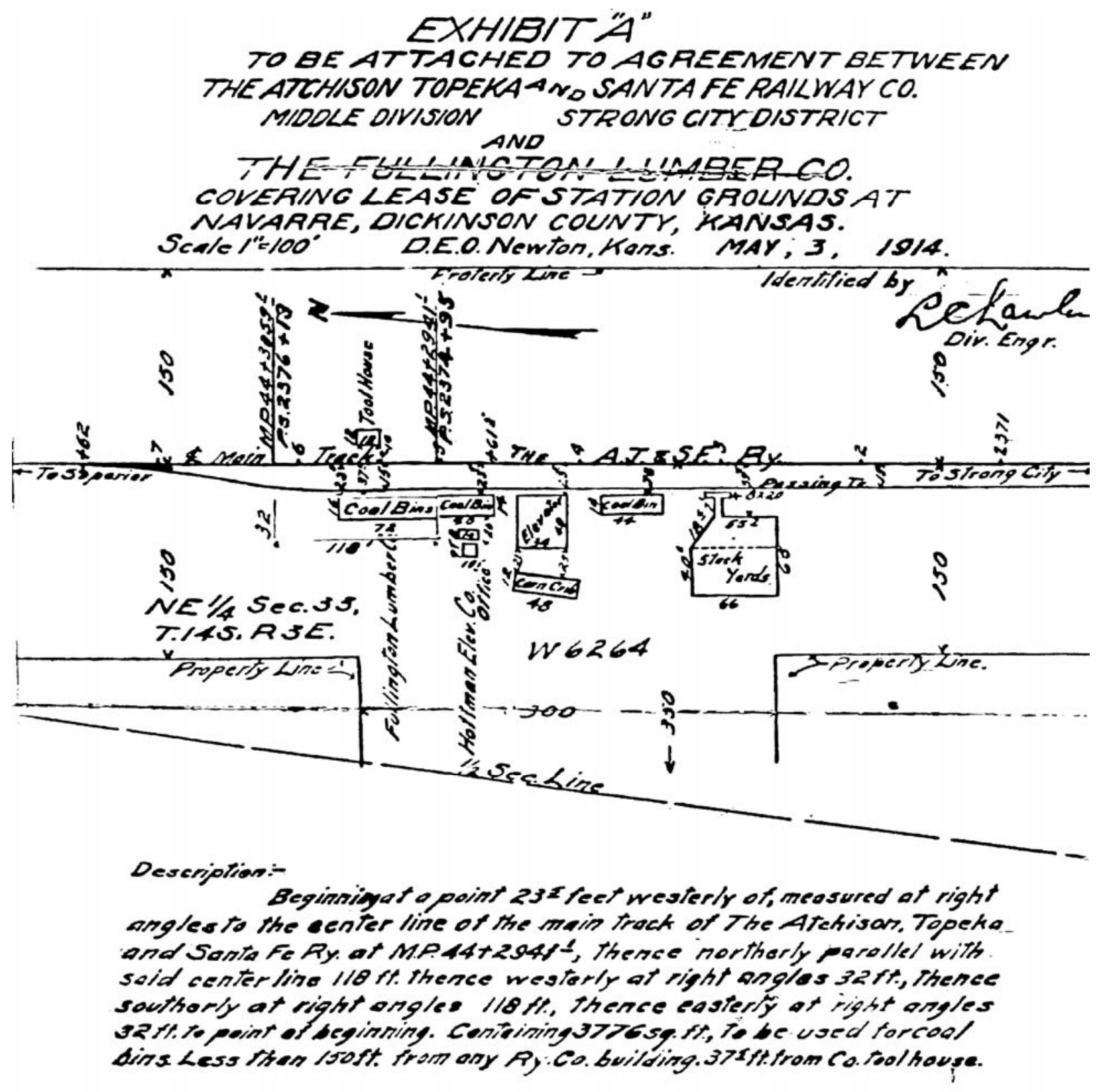

FIGURE S7.4 1916 Co-op lease illustration. 


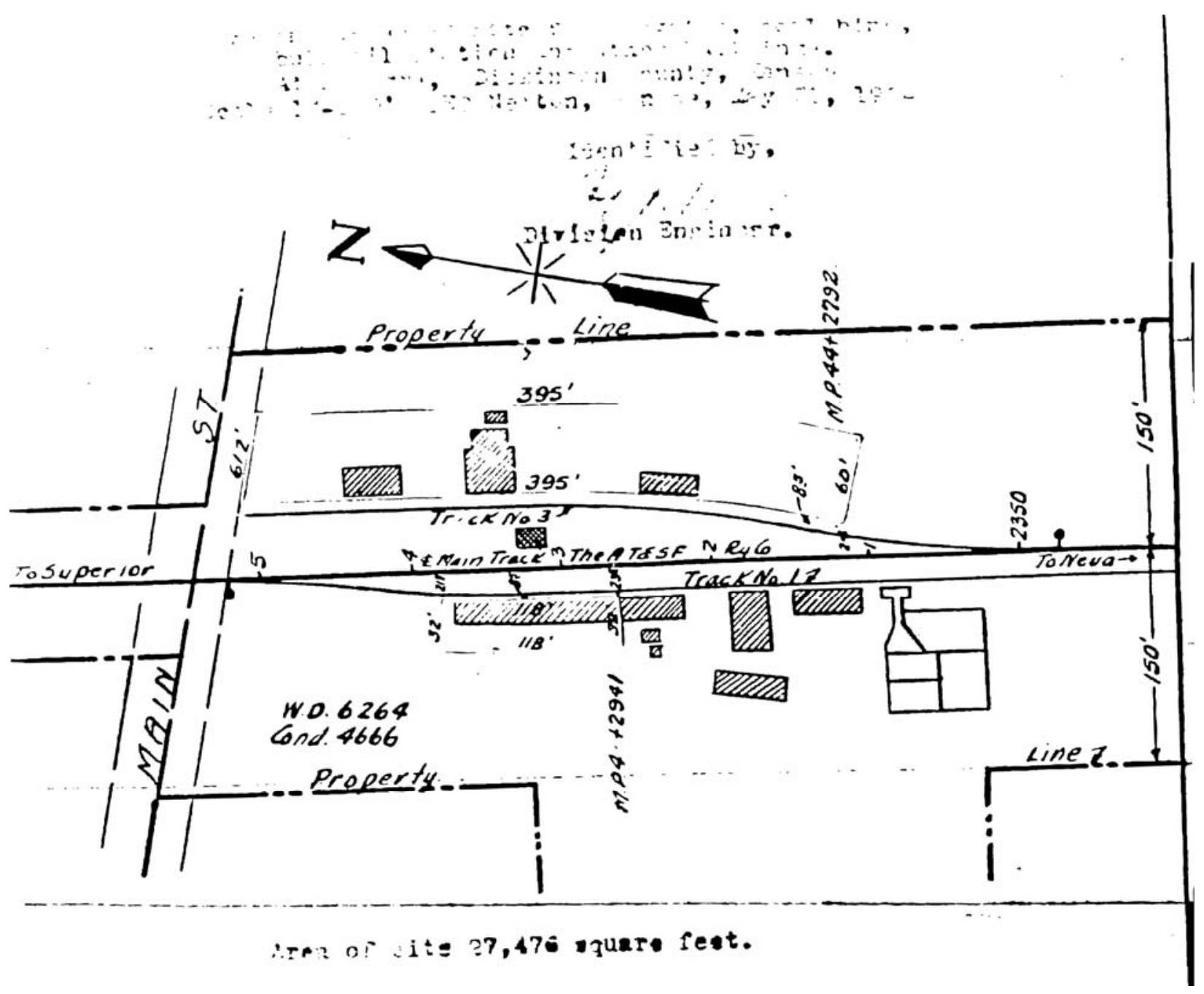

FIGURE S7.5 1934 Co-op lease illustration. 


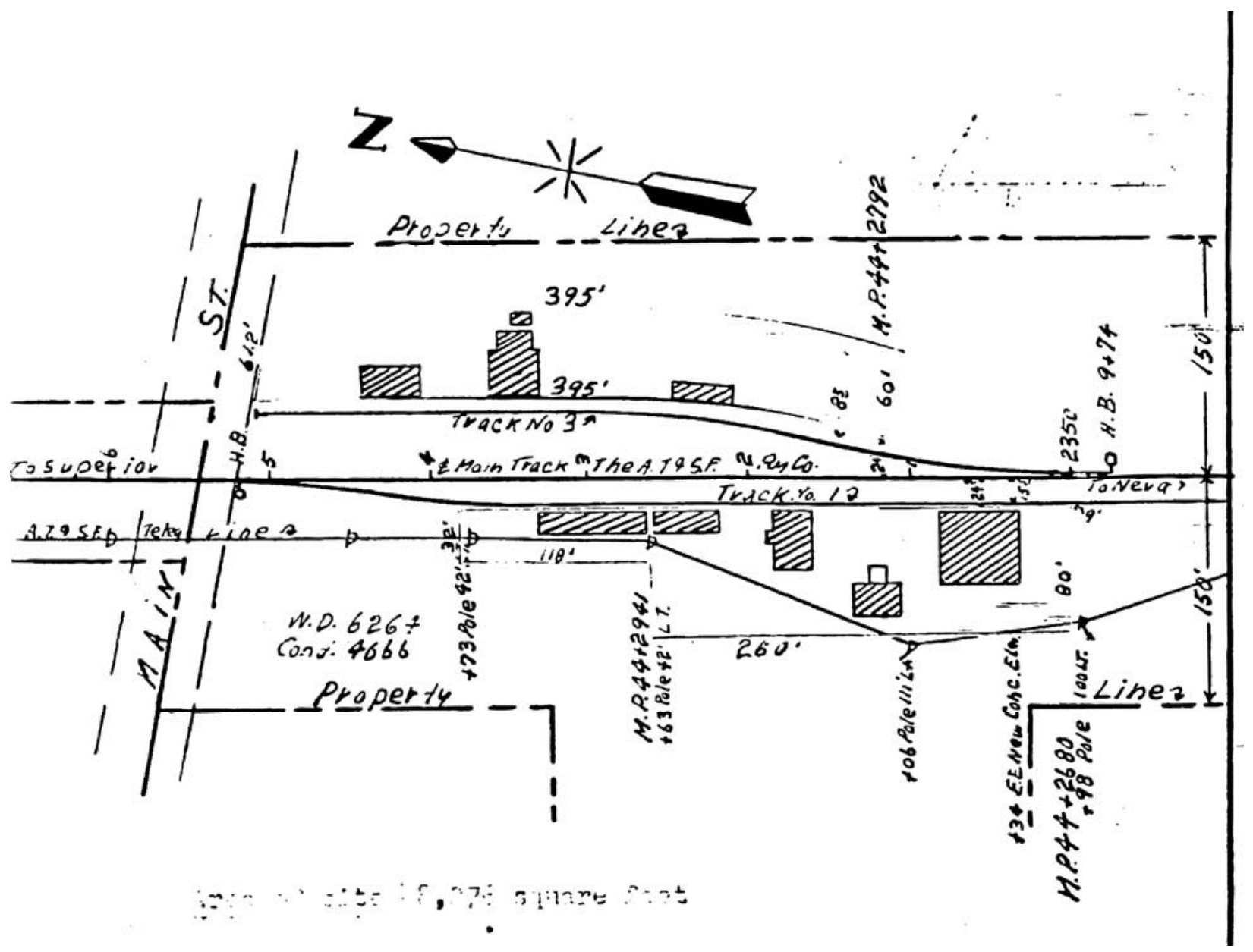

FIGURE S7.6 1949 Co-op lease illustration. 


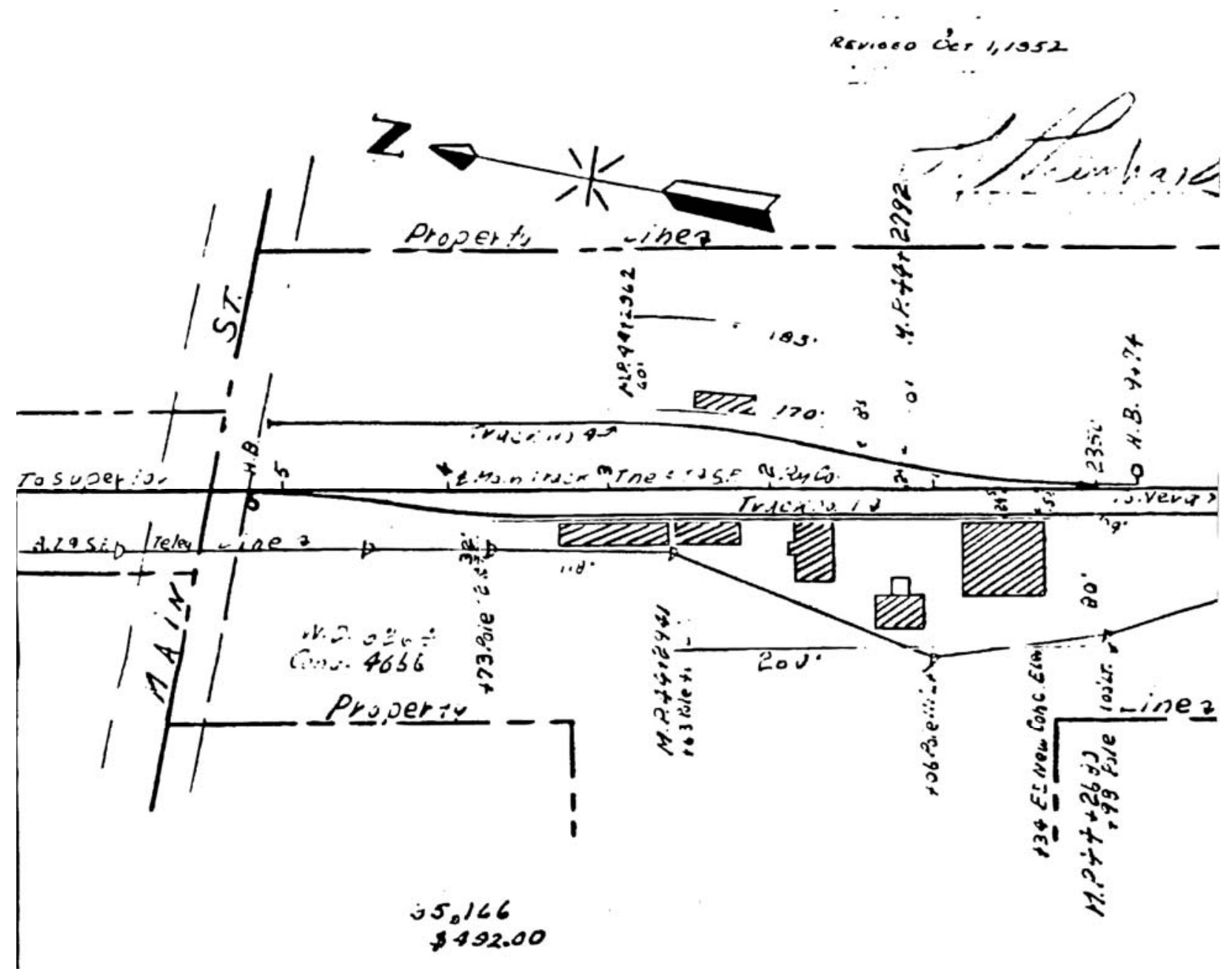

FIGURE S7.7 1952 Co-op lease illustration. 


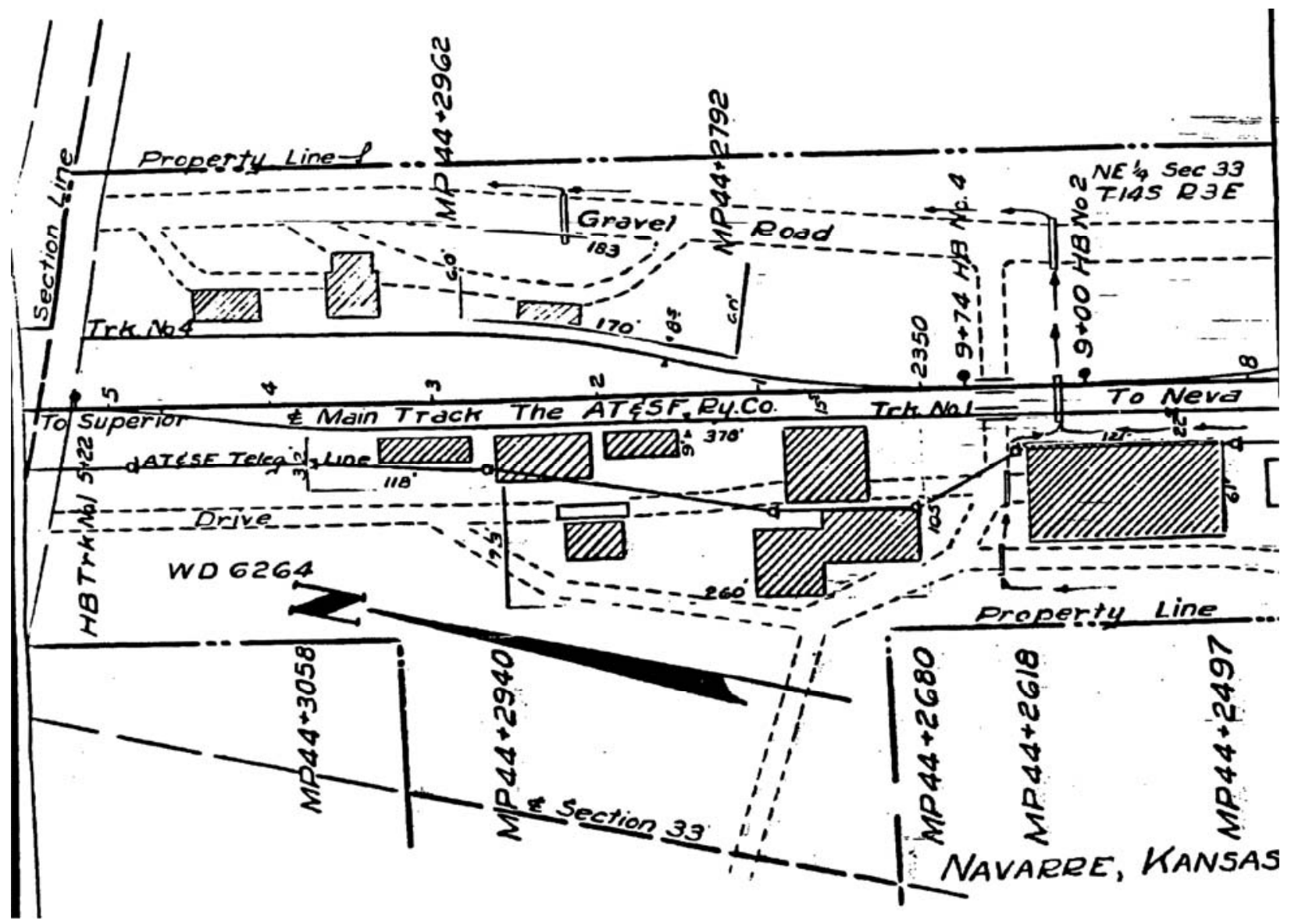

FIGURE S7.8 1958 Co-op lease illustration. 


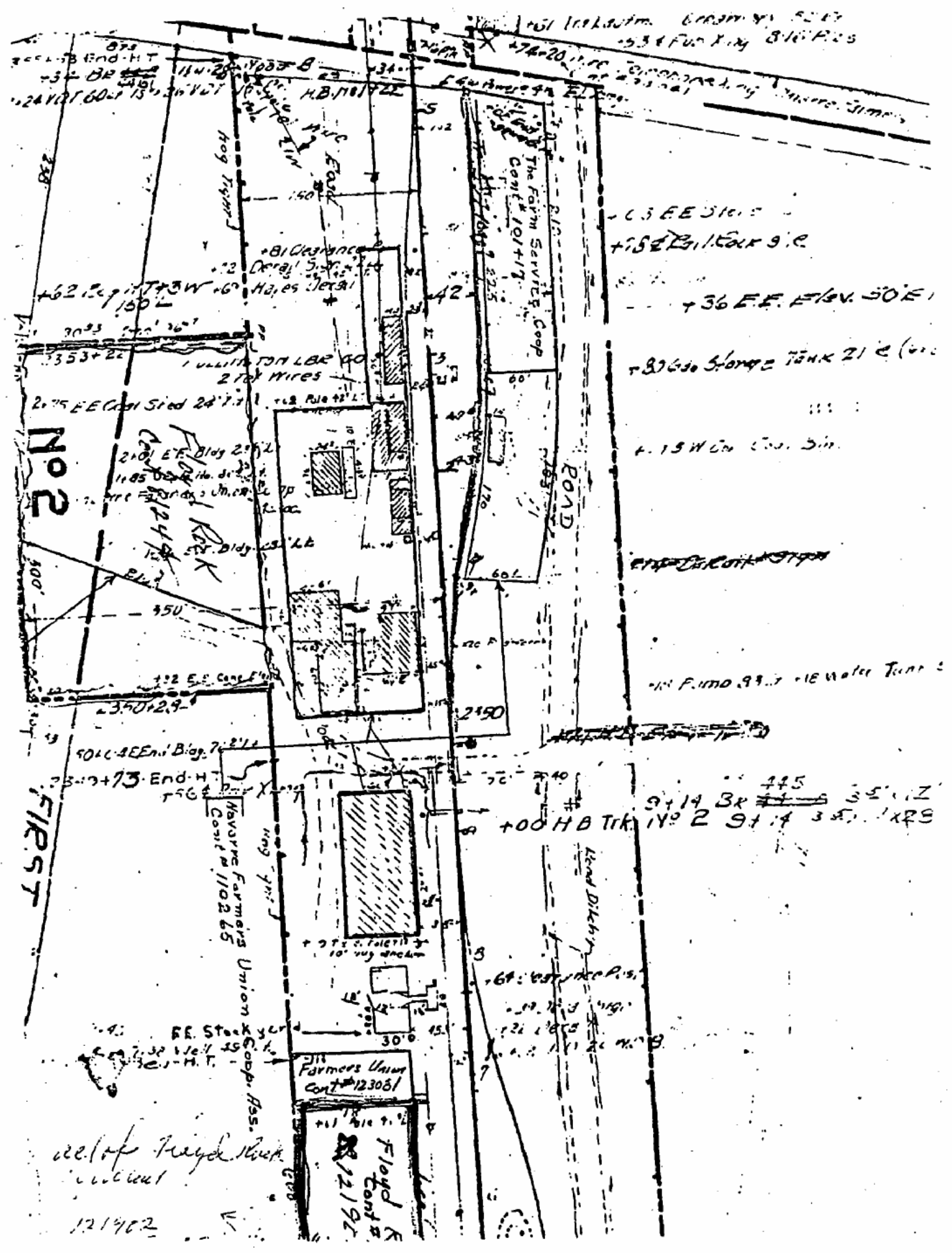

FIGURE S7.9 1966 Co-op lease illustration. 


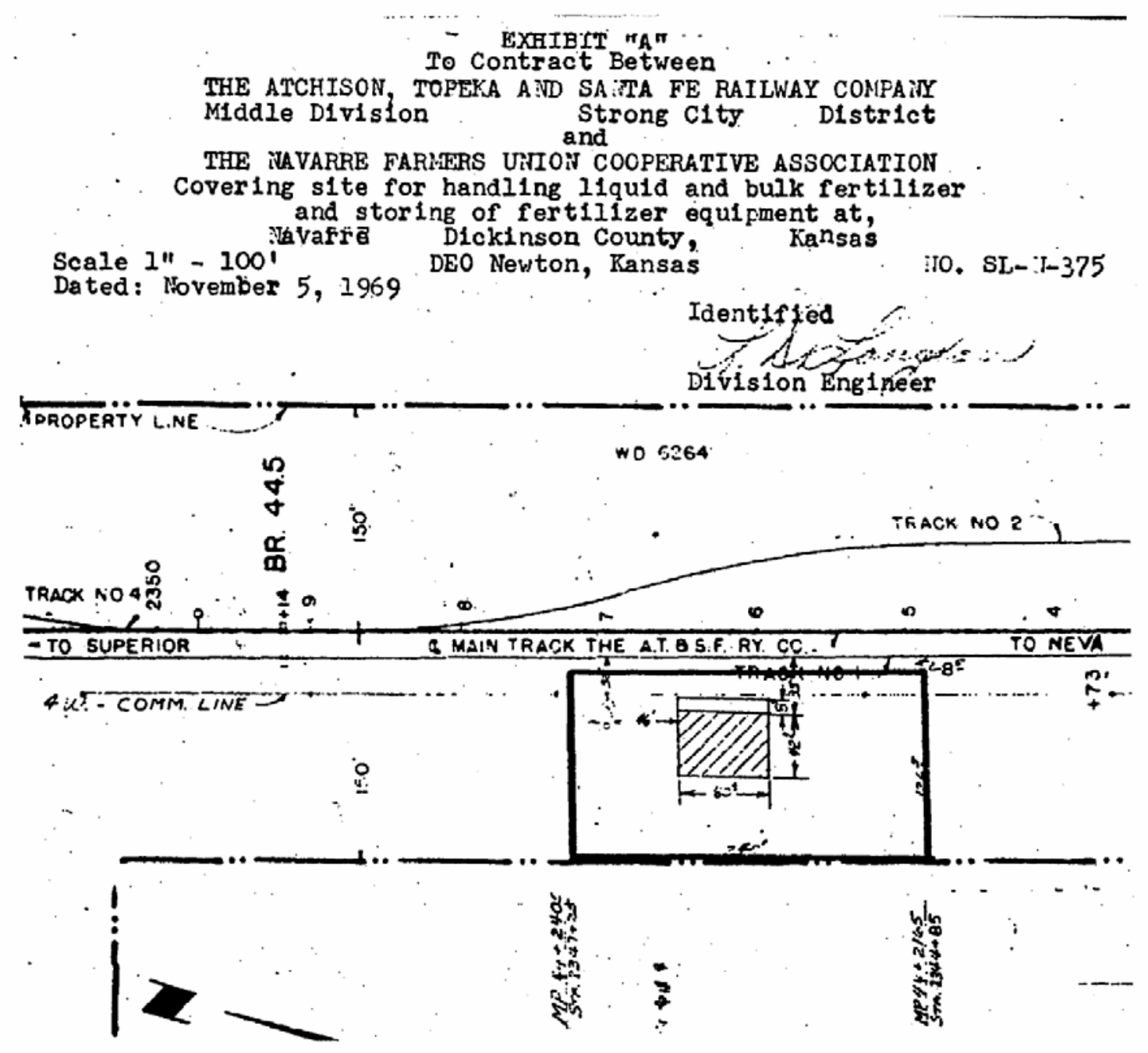

FIGURE S7.10 1969 Co-op lease illustration. 


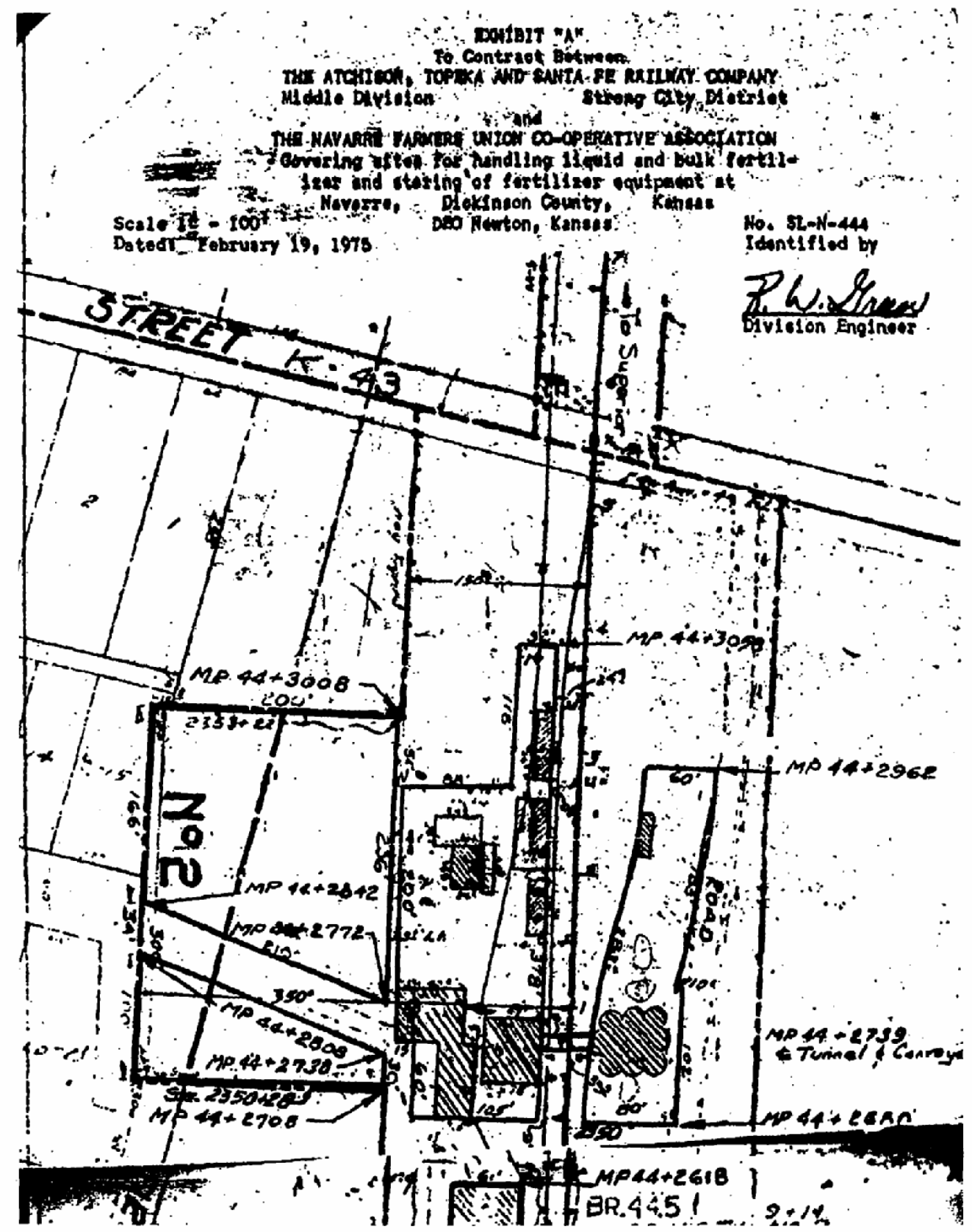

FIGURE S7.11a 1975 Co-op lease illustration (top). 


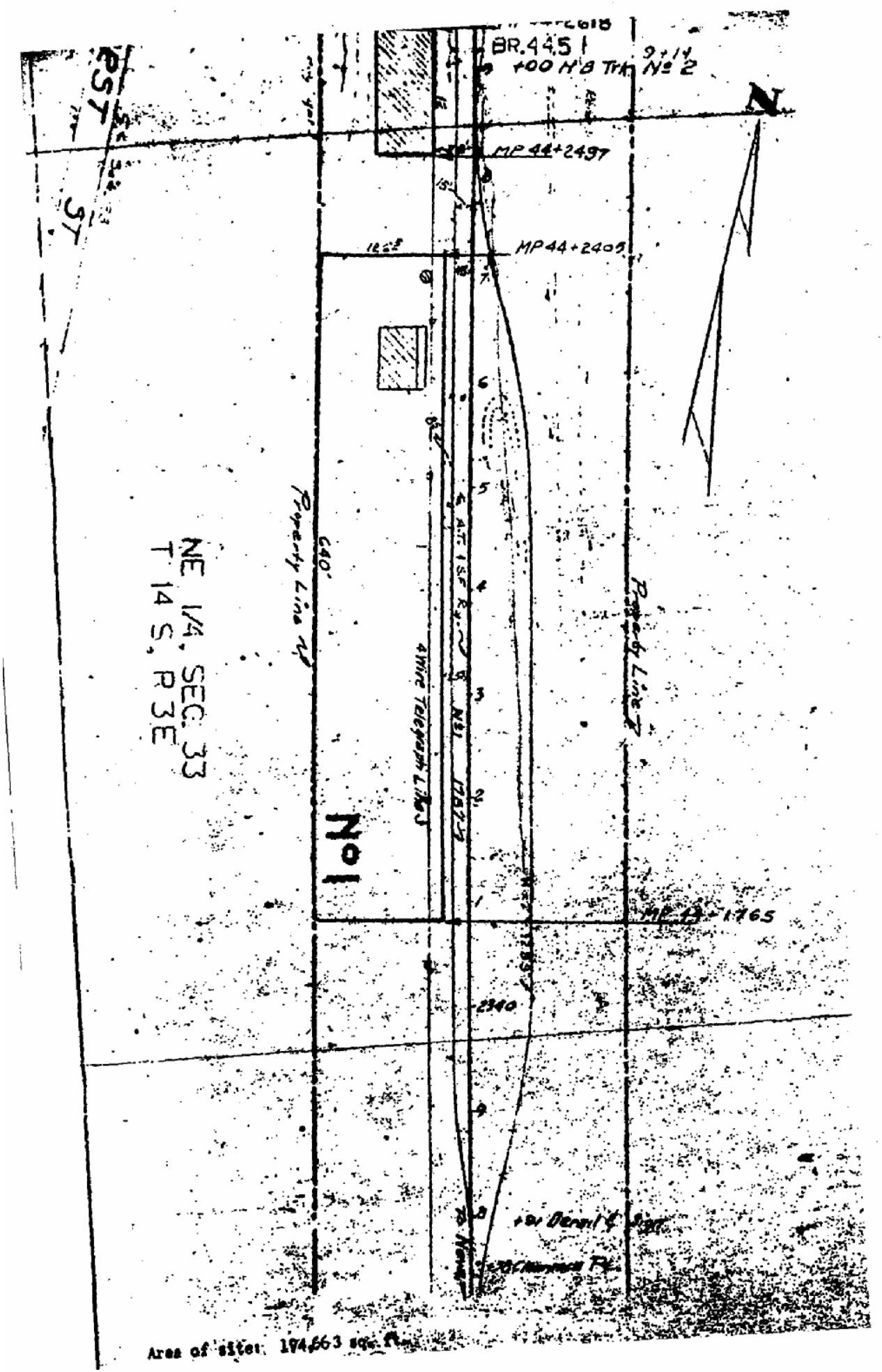

FIGURE S7.11b 1975 Co-op lease illustration (bottom). 


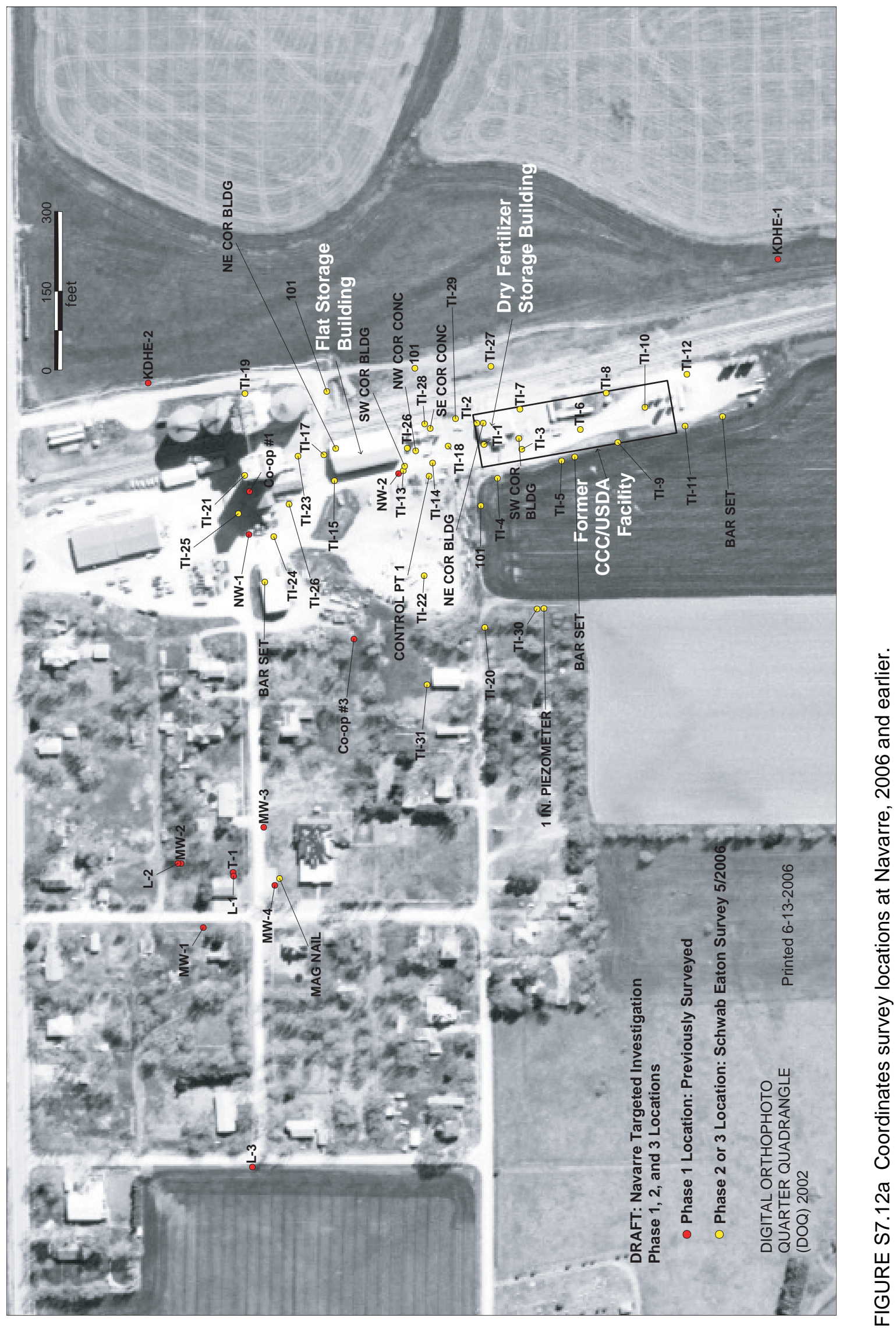




\begin{tabular}{|c|c|c|c|c|c|}
\hline POINT & NORTH & EAST & ELEVATION & \multicolumn{2}{|l|}{ DESCRIPTION } \\
\hline 1 & 232955.482 & 1573378.100 & \multicolumn{3}{|c|}{ 1223.74 SMOKY } \\
\hline 2 & 230446.105 & 1575646.109 & \multicolumn{3}{|c|}{ 1210.21 STATION CHECK } \\
\hline 3 & 197216.254 & 1567080.425 & 1248.33 & & 101 \\
\hline 5 & 170077.270 & 1567436.047 & \multicolumn{3}{|c|}{ 1351.09 MAG NAIL } \\
\hline 6 & 170099.532 & 1567997.486 & \multicolumn{3}{|c|}{ 1351.73 BAR SET } \\
\hline 7 & 169228.424 & 1568301.808 & \multicolumn{3}{|c|}{ 1351.90 BAR SET } \\
\hline 8 & 169509.233 & 1568228.333 & \multicolumn{3}{|c|}{ 1352.21 BAR SET } \\
\hline 9 & 169977.756 & 1568357.387 & 1349.76 & & 101 \\
\hline 10 & 169810.261 & 1568399.934 & 1351.91 & & 101 \\
\hline 11 & 169687.916 & 1568137.482 & 1352.58 & & 101 \\
\hline 50 & 169299.401 & 1568284.386 & \multicolumn{3}{|c|}{ 1352.07 TI-11 } \\
\hline 51 & 169295.189 & 1568382.529 & \multicolumn{3}{|c|}{$1351.87 \mathrm{TI}-12$} \\
\hline 52 & 169375.023 & 1568321.065 & \multicolumn{3}{|c|}{$1353.21 \mathrm{TI}-10$} \\
\hline 53 & 169427.001 & 1568254.976 & \multicolumn{3}{|c|}{$1351.55 \mathrm{TI}-9$} \\
\hline 54 & 169498.115 & 1568280.322 & \multicolumn{3}{|c|}{$1352.73 \mathrm{TI}-6$} \\
\hline 55 & 169448.449 & 1568348.940 & \multicolumn{3}{|c|}{$1351.21 \mathrm{TI}-8$} \\
\hline 56 & 169534.213 & 1568221.318 & \multicolumn{3}{|c|}{$1351.96 \mathrm{TI}-5$} \\
\hline 57 & 169609.492 & 1568244.016 & \multicolumn{3}{|c|}{$1352.92 \mathrm{TI}-3$} \\
\hline 58 & 169612.025 & 1568320.129 & \multicolumn{3}{|c|}{$1351.14 \mathrm{TI}-7$} \\
\hline 59 & 169693.739 & 1568294.251 & \multicolumn{3}{|c|}{$1351.64 \mathrm{TI}-2$} \\
\hline 60 & 169680.825 & 1568253.983 & \multicolumn{3}{|c|}{$1352.62 \mathrm{TI}-1$} \\
\hline 61 & 169656.098 & 1568189.406 & \multicolumn{3}{|c|}{$1351.63 \mathrm{TI}-4$} \\
\hline 62 & 169748.891 & 1568251.468 & \multicolumn{3}{|c|}{$1353.02 \mathrm{Tl}-18$} \\
\hline 63 & 169778.675 & 1568219.992 & \multicolumn{3}{|c|}{$1352.68 \mathrm{TI}-14$} \\
\hline 64 & 169785.756 & 1568194.611 & 1352.29 & CONTROL PT 1 & \\
\hline 65 & 169834.283 & 1568205.747 & 1351.73 & $\mathrm{TI}-13$ & \\
\hline 66 & 169792.658 & 1568294.183 & 1348.85 & TI-28 & \\
\hline 67 & 169734.532 & 1568303.312 & 1349.53 & TI-29 & \\
\hline 68 & 169666.384 & 1568401.793 & 1350.43 & TI-27 & \\
\hline 69 & 169965.491 & 1568188.481 & 1351.48 & TI-15 & \\
\hline 70 & 169984.611 & 1568237.475 & 1351.54 & TI-17 & \\
\hline 71 & 170051.693 & 1568144.665 & 1352.67 & TI-26 & \\
\hline 72 & 170081.286 & 1568083.370 & 1352.64 & TI-24 & \\
\hline 73 & 170135.159 & 1568200.213 & 1352.16 & TI-21 & \\
\hline 74 & 170147.822 & 1568127.860 & 1352.37 & TI-25 & \\
\hline 75 & 169796.837 & 1568006.400 & 1352.68 & TI-22 & \\
\hline 76 & 169793.896 & 1567799.797 & 1353.68 & TI-31 & \\
\hline 77 & 169583.697 & 1567941.394 & 1353.77 & TI-30 & \\
\hline 78 & 169570.588 & 1567942.246 & 1353.65 & 1 IN. PIEZOMETE & \\
\hline 79 & 169682.042 & 1568294.320 & 1352.02 & NE COR BLDG & \\
\hline 80 & 169782.763 & 1568285.590 & 1354.07 & SE COR CONC & \\
\hline 81 & 169961.942 & 1568249.252 & 1351.65 & NE COR BLDG & \\
\hline 82 & 170033.385 & 1568235.733 & 1351.79 & TI-23 & \\
\hline 83 & 170132.606 & 1568355.171 & 1349.12 & TI-19 & \\
\hline 84 & 169614.770 & 1568264.631 & 1352.98 & SW COR BLDG & \\
\hline 85 & 169810.451 & 1568243.282 & 1354.10 & NW COR CONC & \\
\hline 86 & 169826.265 & 1568248.293 & 1352.19 & TI-16 & \\
\hline 87 & 169831.725 & 1568214.414 & 1351.71 & SW COR BLDG & \\
\hline 88 & 169682.829 & 1567907.391 & 1353.45 & TI-20 & \\
\hline
\end{tabular}

FIGURE S7.12b Coordinates survey data for Navarre locations, May 2006. 
TABLE S7.1 Timeline related to property records for the location of the former CCC/USDA grain storage facility at Navarre, Kansas.

\begin{tabular}{|c|c|c|c|c|c|}
\hline $\begin{array}{l}\text { Document } \\
\text { Date }\end{array}$ & Title & $\begin{array}{l}\text { No. of } \\
\text { Pages }\end{array}$ & Contract No. & Document Description & Relevance to Property Boundaries \\
\hline 2/7/2000 & Transmittal letter & 11 & - & $\begin{array}{l}\text { Transmittal of Navarre property documents to } \\
\text { Argonne (J. Burton) from CCC/USDA } \\
\text { (G. Fremerman). }\end{array}$ & \\
\hline $5 / 1 / 1916$ & $\begin{array}{l}\text { Right-of-way lease } \\
\text { between Atchison, } \\
\text { Topeka, and Santa } \\
\text { Fe Railroad (ATSF) } \\
\text { and Fullington } \\
\text { Lumber Co. }\end{array}$ & 4 & 36189 & $\begin{array}{l}\text { Lease of } 3776 \mathrm{ft}^{2} \text { to Fullington Lumber Co. to be } \\
\text { used for coal bins. Leased property is located } \\
\text { between mile markers MP44 }+3059 \mathrm{ft} \text { and } \\
\text { MP44 }+2941 \mathrm{ft} \text {. }\end{array}$ & $\begin{array}{l}\text { Future Co-op property in the vicinity of the } \\
\text { current elevator. Lease figure shows } \\
\text { locations of coal bins, elevator, corn crib, and } \\
\text { stockyard west of tracks, plus toolshed east } \\
\text { of tracks. The old wooden elevator described } \\
\text { no longer exists; it was north of current } \\
\text { elevator location. }\end{array}$ \\
\hline 7/6/1916 & $\begin{array}{l}\text { Assignment by } \\
\text { Fullington Lumber } \\
\text { Co. to Navarre } \\
\text { Co-op }\end{array}$ & 0 & 36189 & $\begin{array}{l}\text { This lease assignment document is not on file at } \\
\text { Argonne, but the lease, its date, and the } \\
\text { contract number are referenced in the } \\
6 / 1 / 1921 \text { lease. }\end{array}$ & $\begin{array}{l}\text { Property assigned to the Fullington Lumber Co. } \\
\text { under contract } 36189 \text { is transferred by } \\
\text { Fullington to the Navarre Co-op. Leased } \\
\text { property is located between mile markers } \\
\text { MP44 + } 3059 \mathrm{ft} \text { and MP44 + } 2941 \mathrm{ft} \text {. }\end{array}$ \\
\hline $4 / 18 / 1919$ & $\begin{array}{l}\text { Articles of } \\
\text { incorporation }\end{array}$ & 5 & - & $\begin{array}{l}\text { Charter for the Navarre Farmers Union } \\
\text { Cooperative Association. }\end{array}$ & Establishes incorporation date of Co-op. \\
\hline $5 / 6 / 1920$ & $\begin{array}{l}\text { Lease between ATSF } \\
\text { and Navarre Co-op }\end{array}$ & 0 & 43928 & $\begin{array}{l}\text { This lease is not on file at Argonne. Lease date } \\
\text { and contract number are identified in the } \\
6 / 5 / 1934 \text { lease. Property is leased by ATSF to } \\
\text { the Co-op as a site for a coal bin and elevator } \\
\text { under contract } 43928 \text {. }\end{array}$ & $\begin{array}{l}\text { Establishes the date of grain storage operations } \\
\text { by the Navarre Co-op as May } 1920 .\end{array}$ \\
\hline $6 / 1 / 1921$ & $\begin{array}{l}\text { Lease from Fullington } \\
\text { Lumber Co. to } \\
\text { Navarre Co-op }\end{array}$ & 3 & 36189 & $\begin{array}{l}\text { Lease held by Lumber Co. under contract } 36189 \\
\text { (dated } 7 / 6 / 1916) \text {, for a coal bin location, is } \\
\text { transferred in whole to the Co-op. }\end{array}$ & $\begin{array}{l}\text { Initial lease on file at Argonne specifically citing } \\
\text { the Navarre Farmers Union Cooperative } \\
\text { Association. }\end{array}$ \\
\hline $6 / 5 / 1934$ & $\begin{array}{l}\text { Right-of-way lease } \\
\text { between ATSF and } \\
\text { Navarre Co-op }\end{array}$ & 5 & 74624 & $\begin{array}{l}\text { Expansion of Co-op east of tracks. Contracts } \\
36189 \text { (dated } 7 / 16 / 1916 \text { [sic]) and } 43928 \\
\text { (dated 5/6/1920) are terminated, combining } \\
\text { both parcels under contract } 74624 \text {. }\end{array}$ & $\begin{array}{l}\text { Co-op controls } 27,476 \mathrm{ft}^{2} \text { of the ATSF right-of- } \\
\text { way. Figure shows the current (as of 1934) } \\
\text { extent of the Co-op property, centered on } \\
\text { mile marker MP44 }+2941 \mathrm{ft} \text {. }\end{array}$ \\
\hline
\end{tabular}


TABLE S7.1 (Cont.)

\begin{tabular}{ccc}
\hline $\begin{array}{l}\text { Document } \\
\text { Date }\end{array}$ & No. of & \\
& Title & Pages Contract No. Document Description
\end{tabular}

\begin{tabular}{|c|c|c|c|c|}
\hline $3 / 27 / 1940$ & First National Bank & 1 & - & $\begin{array}{l}\text { Resolution passed by Farmers Union } \\
\text { Cooperative establishing the Navarre Elevator } \\
\text { as the registered office of the corporation and } \\
\text { D.S. Strole as the registered agent. }\end{array}$ \\
\hline 4/13/1949 & $\begin{array}{l}\text { Lease from ATSF to } \\
\text { Navarre Co-op }\end{array}$ & 3 & 95727 & $\begin{array}{l}\text { Argonne's copy is incomplete, missing page } 2 \\
\text { and signature page. Lease of additional right- } \\
\text { of-way for elevator, coal bins, bulk oil station, } \\
\text { and other buildings. (Under this lease the } \\
\text { Co-op will construct a new concrete elevator.) }\end{array}$ \\
\hline
\end{tabular}
Lease from ATSF to
Navarre Co-op

5

\section{6/21/1954 Lease from ATSF to Commodity Credit Corporation}

Unknown Lease from ATSF to

$$
\text { Navarre Co-op }
$$

8/4/1958 Lease from ATSF to Navarre Co-op
101411 After construction of concrete elevator, Co-op vacates land east of the tracks and to the north by terminating contract 74624 (dated 6/5/1934).

CCC/USDA leases property from railroad under contract 104166 (identified in the lease termination agreement dated 2/2/1966). CCC/USDA controls $37,632 \mathrm{ft}^{2}(98 \mathrm{ft} \times 384 \mathrm{ft})$. Mile marker locations MP44 $+2365 \mathrm{ft}$ and MP44 + $1981 \mathrm{ft}$ determine the position and the $384-\mathrm{ft}$ length of the property.

The first page is missing from Argonne's copy. Contract 101411 (dated 10/8/1952) is terminated. Contract number is identified in figure associated with 8/5/1966 lease.
Corporate office of the Co-op moved to Navarre, Kansas.

Lease figure shows Co-op expanding southward, taking over the former northern stockyard area, identified in the figure as a new concrete elevator. Co-op controls $48,276 \mathrm{ft}^{2}$. Southern extent of Co-op property is at mile marker MP44 $+2680 \mathrm{ft}$.

Co-op controls $35,166 \mathrm{ft}^{2}$. Southern extent of Co-op property is still at mile marker MP44 + $2680 \mathrm{ft}$.

Lease figure shows the area leased by the CCC/USDA. Fifty feet north of the CCC/USDA area is a former railroad stockyard. East of the CCC/USDA leasehold are railroad track 1 (the passing track), the main track, the former depot, and track 2.

The stockyard is believed to be the location of the former hand-dug well.

Co-op resumes control of the area east of the railroad tracks.

Co-op property extends southward, toward the former CCC/USDA facility. The lease figure shows the southern extent of the flat storage building at mile marker MP44 + $2497 \mathrm{ft}$. The southern boundary of the parcel available for Co-op operations is not specified on the figure. description of the property leased by th (i.e., the flat storage building), which was not present in the 1957 aerial photo but is in the 1965 photo. Contract 95727 (dated 4/13/1949) is terminated. 
TABLE S7.1 (Cont.)

\begin{tabular}{lll}
$\begin{array}{c}\text { Document } \\
\text { Date }\end{array}$ & No. of \\
& Pages Contract No. Document Description & \\
\hline
\end{tabular}

\begin{tabular}{|c|c|c|c|c|}
\hline 10/19/1959 & $\begin{array}{l}\text { Certificate of } \\
\text { amendment }\end{array}$ & 2 & - & $\begin{array}{l}\text { Certificate of amendment to articles of } \\
\text { incorporation, establishing amount of Co-op's } \\
\text { capital stock at } \$ 100,000 \text {. }\end{array}$ \\
\hline $9 / 20 / 1960$ & $\begin{array}{l}\text { Lease from ATSF to } \\
\text { Navarre Co-op }\end{array}$ & 0 & 113081 & $\begin{array}{l}\text { This lease is not on file at Argonne. Lease date } \\
\text { and contract number are known from } \\
1 / 22 / 1962 \text { lease, which adds land for the } \\
\text { tunnel conveyor and states that contract } \\
113081 \text { (dated } 9 / 20 / 1960 \text { ) is terminated. }\end{array}$ \\
\hline
\end{tabular}

Registered office of Co-op is in Navarre,

Kansas.

The leased land specific to this contract is unknown; it could include the land between the flat storage building (first cited in the $8 / 4 / 1958$ lease) and the parcel just north of the former CCC/USDA facility (identified under contract 123081 in the 8/5/1966 lease).

$3 / 27 / 196$

Change of location of registered office

$4 / 12 / 1961$

\section{Supplemental} agreement between ATSF and CCC/USDA

1/22/1962 Lease from ATSF to Navarre Co-op

2/18/1963 Change of resident agent

1/22/1965 Change of resident agent

8/16/1965 Amendment to articles of incorporation
$-$

Resident agent of Co-op corporation is changed from D.S. Strole to Ralph Funston.

104166

Supplemental agreement between railroad and CCC/USDA to increase annual rental payment for property defined under contract 104166.

Lease of land for tunnel conveyor and overhead walk is added by the Co-op. Contract 113081 (dated 9/20/1960) is terminated.

Resident agent for corporation is changed from Ralph Funston to Marvin Foos.

Resident agent for corporation is changed from Marvin Foos to Duane Rufenor.

- $\quad$ Defines the nature and character of business for which the Navarre Farmers Union Cooperative Association was organized and limits the number of corporate directors.

104166
The 6/21/1954 lease between ATSF and the CCC/USDA for the property defined under contract 104166 is terminated as of $1 / 17 / 1966$.
Registered office of Co-op remains in Navarre Kansas.

CCC/USDA property boundary is that cited in the $6 / 21 / 1954$ lease.

Area of Co-op site is stated as $60,503 \mathrm{ft}^{2}$.

Registered office of Co-op remains in Navarre Kansas.

Registered office of Co-op remains in Navarre, Kansas.

Registered office of Co-op remains in Navarre, Kansas.

CCC/USDA property boundary is that cited in the $6 / 21 / 1954$ lease. 
TABLE S7.1 (Cont.)

\begin{tabular}{|c|c|c|c|c|c|}
\hline $\begin{array}{l}\text { Document } \\
\text { Date }\end{array}$ & Title & $\begin{array}{l}\text { No. of } \\
\text { Pages }\end{array}$ & Contract No. & Document Description & Relevance to Property Boundaries \\
\hline 8/5/1966 & $\begin{array}{l}\text { Lease between ATSF } \\
\text { and Navarre Co-op }\end{array}$ & 1 & 123081 & $\begin{array}{l}\text { This lease is not on file at Argonne, but a figure } \\
\text { showing the location of the land specified } \\
\text { under contract } 123081 \text { is on file. Lease date } \\
\text { and contract } 128081 \text { are cited in the } \\
11 / 21 / 1969 \text { lease. }\end{array}$ & $\begin{array}{l}\text { Co-op moves farther south, onto land just north } \\
\text { of the former CCC/USDA facility and south of } \\
\text { the stockyard. }\end{array}$ \\
\hline 2/24/1969 & $\begin{array}{l}\text { Certificate of } \\
\text { reinstatement, } \\
\text { restoration, or } \\
\text { renewal }\end{array}$ & 2 & - & $\begin{array}{l}\text { The Navarre Farmers Union Cooperative } \\
\text { Association, organized on } 4 / 18 / 1919 \text { for a } \\
\text { period of } 50 \text { years, until } 4 / 18 / 1969 \text {, is } \\
\text { extended for a period of } 50 \text { years. }\end{array}$ & $\begin{array}{l}\text { Registered office of Co-op remains in Navarre, } \\
\text { Kansas. }\end{array}$ \\
\hline 11/21/1969 & $\begin{array}{l}\text { Lease between ATSF } \\
\text { and Navarre Co-op }\end{array}$ & 5 & 133460 & $\begin{array}{l}\text { Co-op leases additional land for handling of } \\
\text { liquid and bulk fertilizer and for storing } \\
\text { fertilizer equipment and a portable loader. } \\
\text { Area covered includes the portion under } \\
\text { contract } 123081 \text { (just north of former } \\
\text { CCC/USDA facility) and additional area } \\
\text { extending south into the former CCC/USDA } \\
\text { facility, for a total area of } 30,360 \mathrm{ft}^{2} \text {. Contract } \\
123081 \text { (dated } 8 / 5 / 1966 \text { ) is terminated. }\end{array}$ & $\begin{array}{l}\text { The lease figure indicates that at this point the } \\
\text { Co-op controls about half of the former } \\
\text { CCC/USDA facility (its northern portion). The } \\
\text { lease figure shows the southern extent of the } \\
\text { Co-op property at mile marker MP } 44+ \\
2165 \mathrm{ft} \text {. (The former CCC/USDA facility was } \\
\text { located between mile markers MP44 + } \\
2365 \mathrm{ft} \text { and MP44 + } 1981 \mathrm{ft} \text {.) }\end{array}$ \\
\hline 12/10/1971 & $\begin{array}{l}\text { Contract for industry } \\
\text { track }\end{array}$ & 5 & & $\begin{array}{l}\text { Contract between Co-op and railroad for } \\
\text { construction of northern track by the railroad. }\end{array}$ & - \\
\hline $1 / 14 / 1974$ & ATSF letter to Co-op & 4 & - & $\begin{array}{l}\text { Letter from railroad to Co-op regarding request } \\
\text { by Co-op to take over northern land presently } \\
\text { controlled by Floyd Rock for construction of a } \\
\text { new office and scale. }\end{array}$ & - \\
\hline 1/9/1975 & $\begin{array}{l}\text { Certificate of } \\
\text { amendment }\end{array}$ & 2 & - & $\begin{array}{l}\text { Amendment to Co-op articles of incorporation } \\
\text { establishing the amount of capital stock of the } \\
\text { corporation at } \$ 500,000 \text {. }\end{array}$ & $\begin{array}{l}\text { Registered office of Co-op remains in Navarre, } \\
\text { Kansas. }\end{array}$ \\
\hline
\end{tabular}


TABLE S7.1 (Cont.)

\begin{tabular}{|c|c|c|c|c|c|}
\hline $\begin{array}{l}\text { Document } \\
\text { Date }\end{array}$ & Title & $\begin{array}{l}\text { No. of } \\
\text { Pages }\end{array}$ & Contract No. & Document Description & Relevance to Property Boundaries \\
\hline $4 / 25 / 1975$ & $\begin{array}{l}\text { Lease from ATSF to } \\
\text { Navarre Co-op }\end{array}$ & 7 & 147780 & $\begin{array}{l}\text { Expansion of Co-op to } 194,663 \mathrm{ft}^{2} \text {, terminating } \\
\text { two previous contracts and combining them } \\
\text { into one lease. Contracts } 114732 \text { (dated } \\
1 / 22 / 1962 \text { ) and } 133460 \text { (dated } 11 / 21 / 1969 \text { ) } \\
\text { are terminated. At this point the Co-op } \\
\text { controls all of the former CCC/USDA property. }\end{array}$ & $\begin{array}{l}\text { Co-op property expands south of the former } \\
\text { CCC/USDA grain storage facility. Southern } \\
\text { extent of Co-op property is shown on lease } \\
\text { figure as mile marker MP44 }+1765 \mathrm{ft} \text {. (The } \\
\text { former CCC/USDA facility was located } \\
\text { between mile markers MP44 }+2365 \mathrm{ft} \text { and } \\
\text { MP44 + 1981 ft.) }\end{array}$ \\
\hline $1 / 30 / 1976$ & Agreement & 2 & - & $\begin{array}{l}\text { Farmland Industries subleases land from Co-op } \\
\text { to install a } 30,000 \text {-gal fertilizer tank. No figure } \\
\text { is on file with the lease at Argonne. }\end{array}$ & $\begin{array}{l}\text { Installation of bulk liquid fertilizer storage tank } \\
\text { just north of the dry fertilizer storage building. }\end{array}$ \\
\hline $3 / 14 / 1978$ & $\begin{array}{l}\text { Supplemental } \\
\text { agreement }\end{array}$ & 3 & 147780 & $\begin{array}{l}\text { Supplemental agreement to increase annual } \\
\text { payment for property leased under contract } \\
147780 \text { (dated 4/25/1975). }\end{array}$ & $\begin{array}{l}\text { Co-op continues at } 194,663 \mathrm{ft}^{2} \text { specified under } \\
4 / 25 / 1975 \text { lease. }\end{array}$ \\
\hline $4 / 9 / 1981$ & $\begin{array}{l}\text { Supplemental } \\
\text { agreement }\end{array}$ & 3 & 147780 & $\begin{array}{l}\text { Supplemental agreement to increase annual } \\
\text { payment for property leased under contract } \\
147780 \text { (dated } 4 / 25 / 1975 \text { ). }\end{array}$ & $\begin{array}{l}\text { Co-op continues at } 194,663 \mathrm{ft}^{2} \text { specified under } \\
4 / 25 / 1975 \text { lease. }\end{array}$ \\
\hline 10/31/1991 & $\begin{array}{l}\text { Corporate annual } \\
\text { report }\end{array}$ & 3 & - & $\begin{array}{l}\text { Corporate annual report for Navarre Farmers } \\
\text { Union Cooperative Association, listing } \\
\text { liabilities and assets as of tax closing date } \\
10 / 31 / 1991 .\end{array}$ & $\begin{array}{l}\text { Registered office of Co-op remains in Navarre, } \\
\text { Kansas. }\end{array}$ \\
\hline 3/18/1992 & Title search certificate & 6 & - & $\begin{array}{l}\text { Statement of record for a title search by the } \\
\text { Dickinson County Title Company. }\end{array}$ & $\begin{array}{l}\text { Title search record includes the } 6 / 21 / 1954 \\
\text { lease between the ATSF and the } \\
\text { CCC/USDA as an attachment, noting that } \\
\text { the lease was recorded in the Dickinson } \\
\text { County Register of Deeds Office, Record } \\
\text { Book 172, page } 416 \text {. }\end{array}$ \\
\hline
\end{tabular}


D.C.

20250-1400
$C K, 3$

$\mathrm{Ci}$

February 7, 2000

\section{VIA OVERNIGHT MAIL}

Jacqueline C. Burton, Ph.D.

Argonne National Laboratory

9700 South Cass Avenue

Building 203, Room B121

Argonne, Illinois 60439

\section{PRIVILEGED \& CONFIDENTIAL;} ATTORNEY WORK PRODUCT

\section{Re: $\quad$ Navarre, KS Lawsuit}

Dear Jackie:

Following up on our recent discussion, please find enclosed various lease agreements between the Navarre Coop and the Atchison, Topeka and Santa Fe Railway Company at the Navarre, KS site (and some other related materials) that we just received from EPA. Hopefully, these lease agreements will help us "nail down" the Coop's responsibility for the carbon tetrachloride contamination at the site.

Please let me know how these lease documents affect your analysis of this matter at your earliest convenience.

Sincerely,

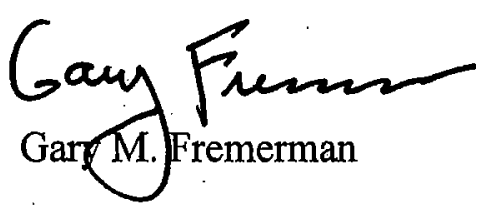

GMF:

Enclosure 


\section{UNITED STATES ENVIRONMENTAL PROTECTION AGENCY \\ REGION VII \\ 901 NORTH 5THSTREET \\ KANSAS CITY, KANSAS 66101}

February 2, 2000

Gary Fremerman, Attorney

U.S. Department of Agriculture

Office of General Counsel, Pollution Control Team

4624 South Building

1400 Independence Avenue, S.W.

Washington, D.C. 20250

\section{Re: Documents from EPA Region VII file on the Navarre, Kansas groundwater contamination site}

\section{Dear Gary:}

Enclosed is a copy of a 1992 investigative report for the Navarre, Kansas groundwater site, with some attached agreements between the Santa Fe Railway and the Navarre Co-op, as you requested.

If you have questions or need further information, please call me at (913) 551-7252.

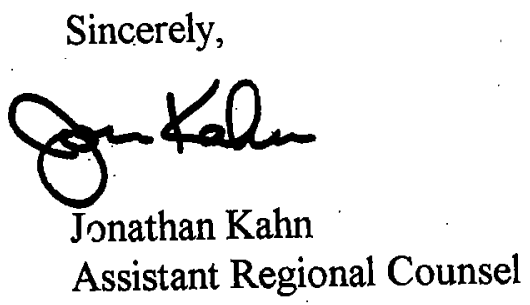

Enclosure 


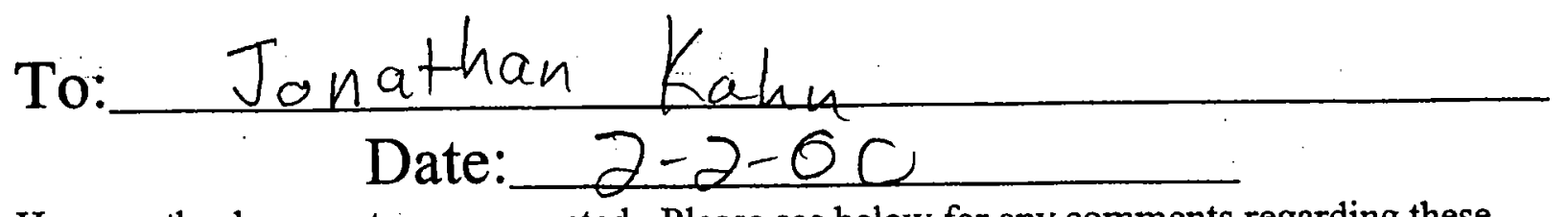

Here are the documents you requested. Please see below for any comments regarding these documents.

\begin{tabular}{ll} 
Mary Calloway & 7994 \\
Melissa Cox & 7443 \\
Frances Gingles & 7113 \\
Marleyne Marsh & 7134 \\
Tricia Mobley & 7427 \\
Odessa Murphy & 7095 \\
Linda Norris & 7827 \\
Sharon Robertson & 7634 \\
Trebreh Samuels & 7186 \\
\hline LaVerne Weber & 7447 \\
\hline Melissa Yocum & 7476 \\
\hline Monique Yocum & 7504 \\
\hline
\end{tabular}


2 copies

March 26, 1992

Mr. Gene Gunn

U.S. EPA, Region 7

726 Minnesota Avenue

Kansas City, Kansas 66101

Re: EPA Contract No. 68-W9-0006

Work Assignment No. C07023-009

Investigative Report for Navarre, Kansas
PRC Environmental Management, Inc. 650 Minnesola Avenue Kansas City, KS 66101 913-281-2277

Fax 913-281-5383

\section{DRE}

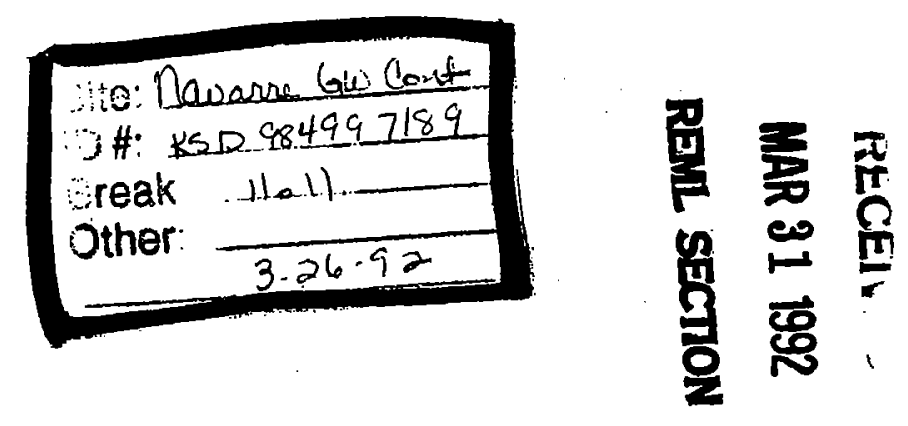

Dear Mr. Caron:

PRC Environmental Management, Inc., is pleased to submit two copies of the investigative report for the Navarre, Kansas, groundwater site prepared by Clarence M. Kelley and Associates (Clarence Kelley). Mr. Dave Linder, the attorney representing the Navarre Co-op, did not allow Clarence Kelley to interview Mr. Rufener, the Co-op's manager, regarding the use of 80/20. He stated that he would respond to written questions from an attorney representing EPA. Clarence Kelley has advised EPA's attorney, Ms. Pat Miller, of this request.

If you have any questions or comments on this submittal, please do not hesitate to call me at (913) 281-2277.

Sincerely,

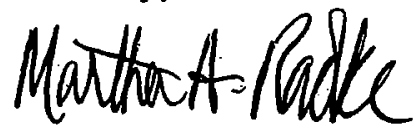

Martha A. Radke

Project Manager

cc: Norman Caron, U.S. EPA-WAM

Pat Miller, U.S. EPA

Maureen Hunt, U.S. EPA-RPO

Kay Mesia, PRC EMI 
RE: Navarre Groundwater Contamination Site
G. GuNN

RECEIY. -

MAR 311992

REML SECTION
REPORT FOR:

REPORT BY:

REPORT DATE:
Ms. Martha Radke

PRC Environmental Management, Inc.

650 Minnesota Avenue

Kansas City, Kansas 66101

Clarence M. Kelley \& Associates

4041 Central Street

Kansas City, Missouri 64111

March 20, 1992

Kansas City, Kansas 66101

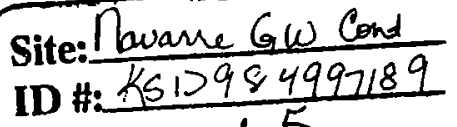

Break:

Other:

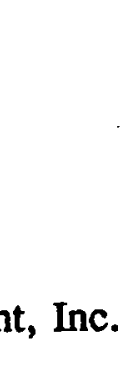

\section{CONEIDENTIAL}

CMK \#896-8 


\section{TABLE OF CONTENTS}

\section{PAGE}

Predication $\ldots \ldots \ldots \ldots \ldots \ldots \ldots \ldots \ldots \ldots \ldots \ldots$

Synopsis $\ldots \ldots \ldots \ldots \ldots \ldots \ldots \ldots \ldots \ldots \ldots \ldots \ldots \ldots$

Interviews with Duane Rufener and Orville Robson $\ldots \ldots \ldots \ldots . \ldots .4$

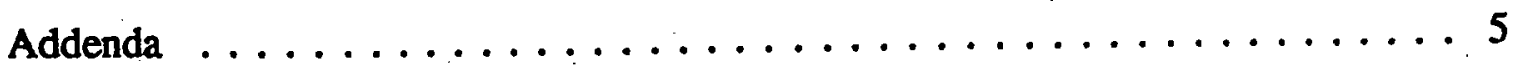




\section{PREDICATION}

This investigation predicated upon a request from the EPA to conduct a title search on the property along the east edge of the town at the current location of the Navarre CO-OP and south of that location in the area of the former USDA grain storage operation. Also conduct interviews of Duane Rufener and Orville W. Robson regarding their knowledge of the use of 80/20 grain fumigant at the elevator.

\section{SYNOPSIS}

David C. Linder, attorney for the Navarre Farmers Union Cooperative Association, declined to allow a verbal interview with Duane Rufener regarding the use of $80 / 20$ and its application at Navarre. Linder said he will respond to a written request from an attorney representing EPA. Orville Robson said he never worked for Navarre CO-OP but did help apply 80/20 there as an employee of the local ASC office. 


\section{INTERVIEWS}

Duane Rufener

Manager, Navarre CO-OP

Box 78, Hope, Kansas

Telephone: (913) 479-2221 (office)

(913 949-2427 (home)

On March 16, 1992, Mr. Duane Rufener was contacted at his place of employment as agreed per my telephone conversation with him on March 14, 1992. Present in Mr. Rufener's office was Mr. David C. Linder, Attorney at Law with the firm of Spencer, Fane, Britt and Browne, 1400 Commerce Bank Building, 1000 Walnut Street, Kansas City, Missouri 64106, telephone (816) 474-8100.

Mr. Rufener advised he has been manager here since 1962. He has located lease agreements for the property in question and has furnished them to Mr. Linder to photo copy for the investigator.

With regard to the questions provided to the investigator for answer by Rufener, Mr. Linder said that these questions should be submitted by the appropriate EPA attorney to him in writing. Linder declined to answer the questions in an interview type format.

With regard to rodent control around the elevator, Rufener said he has always used a professional exterminator whom he identifies as follows: Larry Rakowsky, ECO-LAB, Manhattan, Kansas. 
Mr. Orville W. Robson

Route 3, Box 251

Abilene, Kansas 67410

Telephone: (913) 479-5990

Mr. Robson was contacted at his residence on March 16, 1992, and advised as follows:

Mr. Robson is a retired farmer, age 81 , and still lives on his farm which he now rents to his neighbor.

Dobson said he became involved with helping to apply 80/20 in the early 1950's while employed on a part-time basis by the local Agricultural Stabilization and Conservation Office (ASC). He worked part-time to bring in extra cash while continuing to farm. His supervisor at ASC was Merl Hill, who did the actual spraying. Hill is now deceased. Robson stopped working for ASC in about 1959. He never worked for the Navarre CO-OP.

Robson said that one of the places they sprayed was the Commodity Credit Corporation storage site at Navarre. The bins themselves were located on the railroad right of way and the bin sites were owned by the government, to his knowledge.

Robson said he remembered loading two or three 50 gallon barrels of $80 / 20$ on the back of his pickup along with a small gas powered engine which was used to pump the 80/20 out of the barrels. His job was to operate the engine while Hill did the spraying. Hill never entered any of the grain bins to spray but did wear a mask.

With regard to the construction of the grain bins during that time period, Robson recalled that a cylindrical pit was dug about one foot in depth. The bin was then erected starting in the pit and proceeding upward. After completed with a roof, the floor of the bin would be covered with sand over which aluminum panels would be cut and laid. He recalled that there were about 18 - 
20 such bins at Navarre which were all about 16 feet high. They were at least 3,000 bushel capacity each.

Spraying was usually done in the fall and then sealed for the winter. ASC would require that the grain be checked periodically for insects. If insects were found, it would be necessary to spray again. Robson said that all the grain treated with $80 / 20$ at Navarre was stored there by the government.

Robson could not recall any incidents of spillage of $80 / 20$ nor any use of $80 / 20$ to pour down rat holes or pumped into the ground to kill termites, ants, or poured down prairie dog.holes. He was certain that neither he or Merl Hill ever used $80 / 20$ for any purpose other than treating grain. 


\section{ADDENDA}

1. Farmer Union Cooperative Association Railroad Leases.

2. Articles of Incorporation.

3. Title Search. 


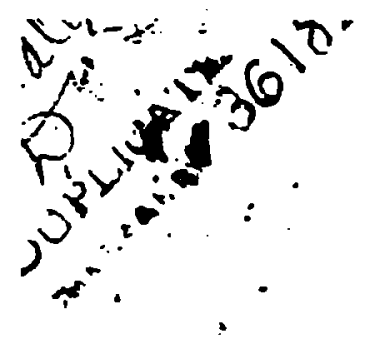

Form 1616 Stendard

(Approved by Generad Bolleitor.)

RIGHT OF WAY LEASE.

Chis $\mathfrak{I n s t r u m e n t}$, Executed in duplicate between

TEE ATCHI SOR TOPREA ATD SARTA TE RAILTAY COLPAR.

(Write or atamp in full aame of Companyi

a.

Kengens ..corporation (hereinafter called the "Lessor"), party of the first part, and

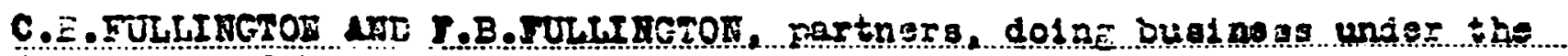

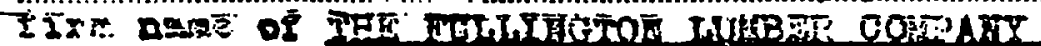

(hereinafter called the "Lessee"), part.20.6 of the second part,

Witnesseth, That for and in consideration of the payment of the rental hereinafter specified, and of the performance by the Lessee of the covenants hereinafter set forth to be performed by the Lessee, the Lessor hereby leases to the Lessee a part of the right of way or station grounds now in the possession of the Lessor, situated at or near.

Hipise station, in the County of.

Diokiason.

and State of

$\operatorname{Rang} 8$ described as follows, to wit:

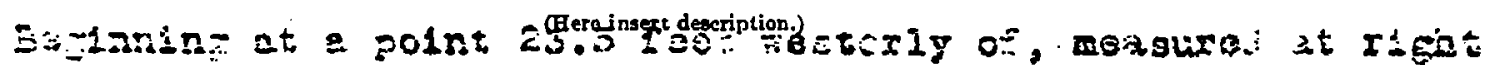
…1. E:

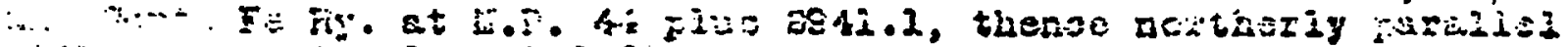

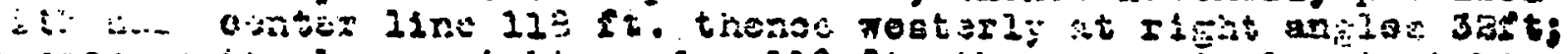

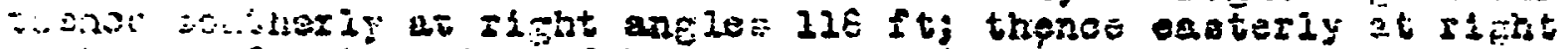

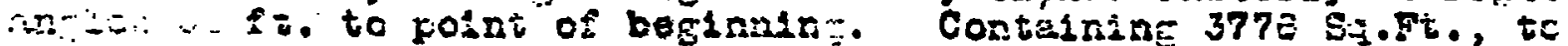

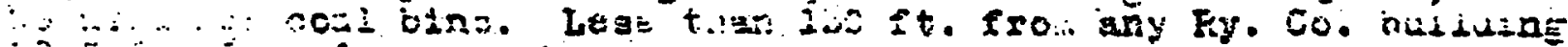

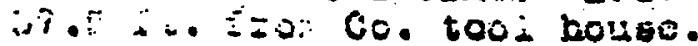

the location and dimensions of said premises being more definitely shomn on the print hereunto annexed, designated "Exhilitit $A$ " and made a part hereof.

To Fave and to Fold the same from Mar..... 20 t. 19.18 , until such time as this contract shall be terminated, as hereinafter provided.

In Consideration of the aforesaid Lease, the Lessee covenants and agrees to and with the Lessor as follows:

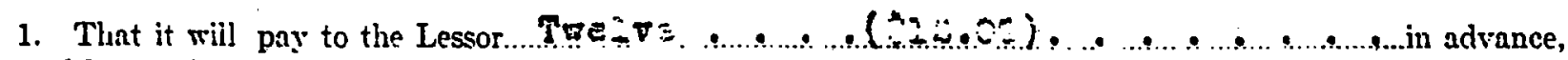
as rentsl for the demised premises, a sum which shall represent interest at the ratc of six per cent $(6 \%)$ per annum on the fair ralue of said premises. but in no case shall said rental he less than Twelve Doillars (S12.00) per year. Such fair value shall be increased from time to time hy the amount of any charge or assessment iexcept general property taxes; which the Lessor shall be required to par on account of or in respect to said nremises for paring. curbing, sidewalks, sewers, benefit districts and the like. For the purposes of this lease, the fair value of the demised premises at

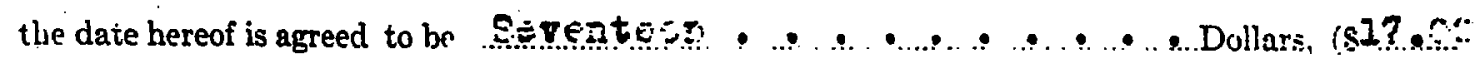

2. That it will pay before the same become delinquent all taxes, charges, rates and assessments which may, -during the term of this lease, be levied upon or assessed against or he equitably chargeable to or assessed in respect to any buildings and improvements which may be placed upon the demised promises br the Lessee; and where any such tax, rate, charge or assessment may be embraced in the general amount of taxes charged upon the demised premises separately or in cornection with other property of the Lessor and the Lessor shall pay all of said taxes, then the Lessee will promptly repay or refund to the Lessor the amount or part of the tax. charge, rate or assessment equitably or fairly apportionable to any buildings or improvements placed on the demised premises by the Lessee.

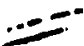




\section{EXHIEIT \#"}

TO GE ATT ACAED TO AGFEENENT BETWEEN THE ATCHHSON TOPEWA $A$ NO SANTA FE PAILWAY $C O$. MIOOLE DWISHOW STRONG CITYDISTRICT

\section{$\rightarrow N O$}

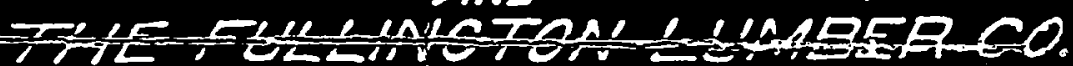

COVEFING LEASE OFSTATION GFOUNDS AT

NAWAFAE, DICHINSON COUNTY, HANSAS.

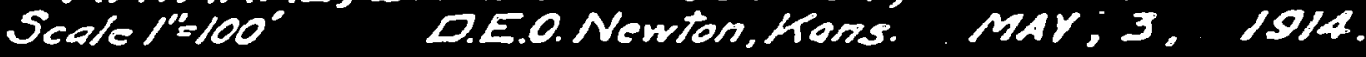

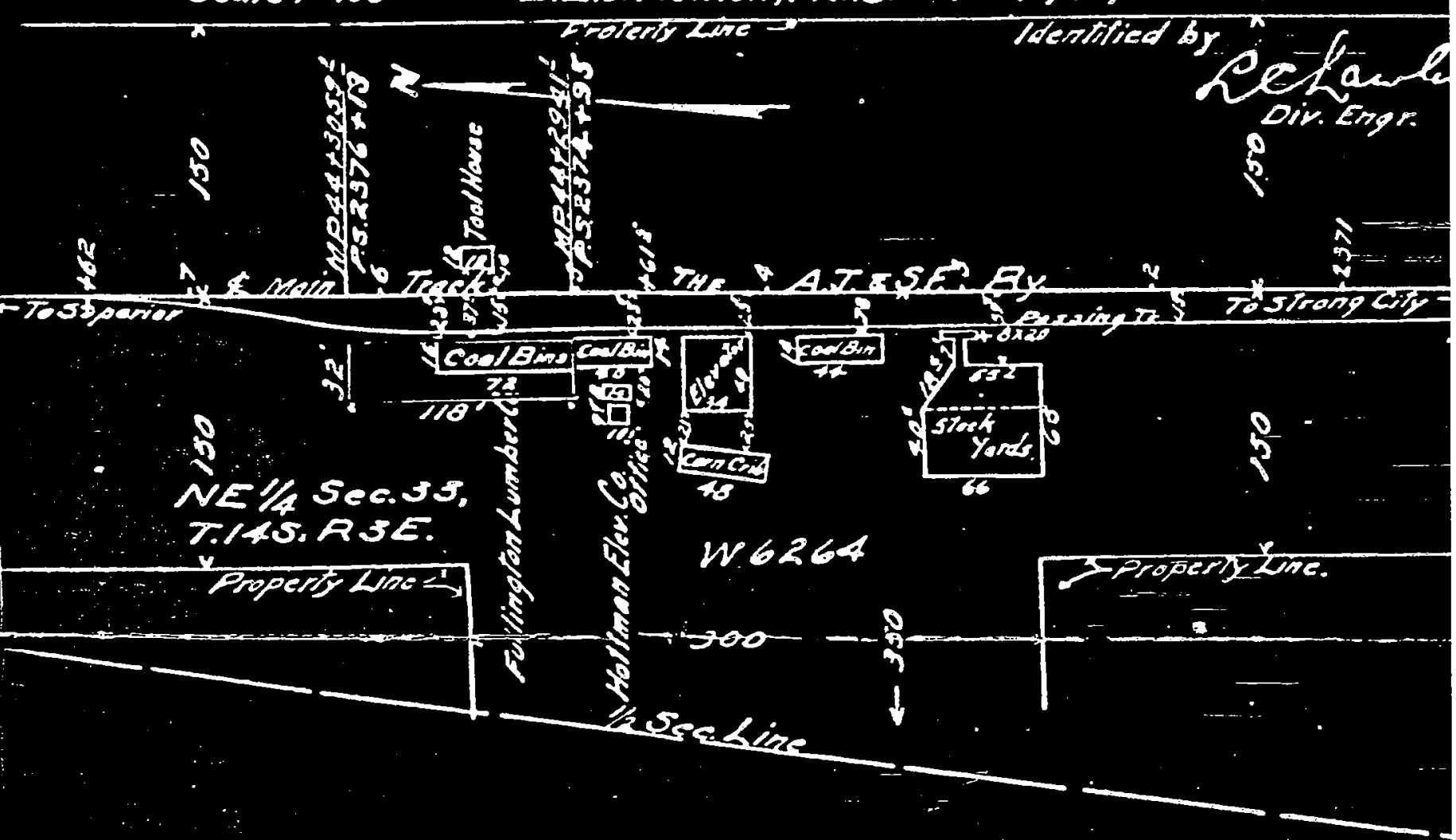

Descriotion:

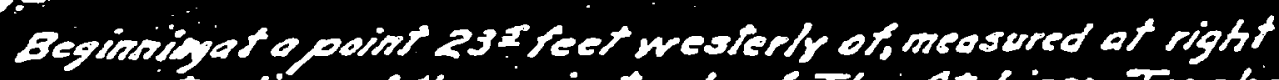

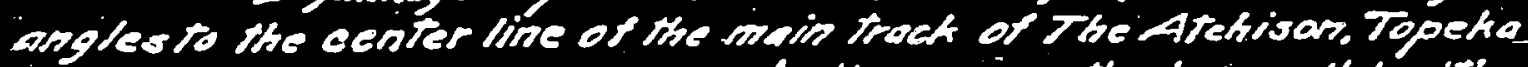

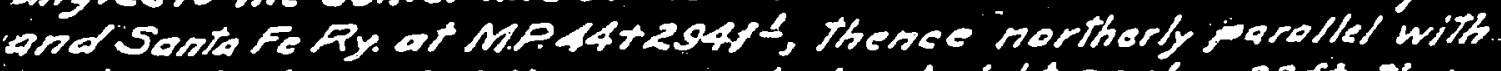

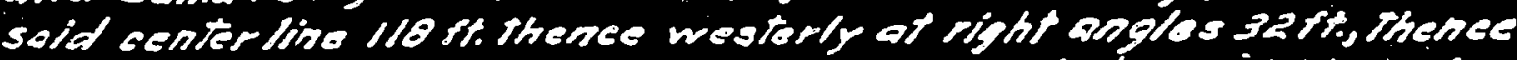

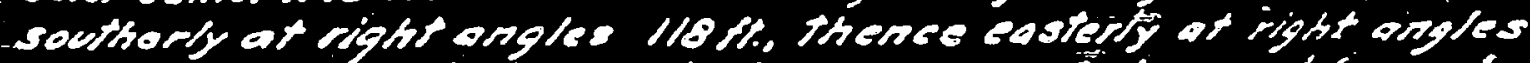

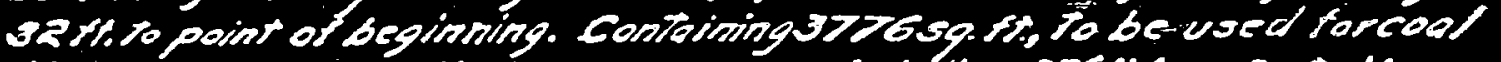

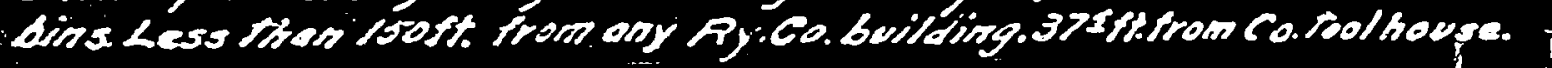




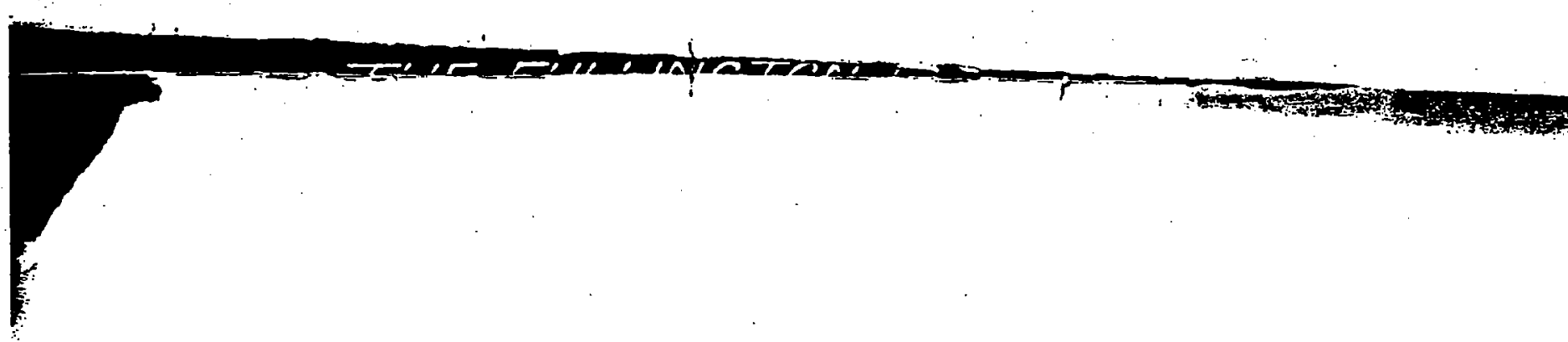

CONSENT OF LESSTOR TO ASSIGNMENT.

The Lessor named in the foregoing lease hereby consents to an assignment of said lease by the Lessee in it named to. provided

the assignee named shall agree in writing to assume and be bound by all the obligations of the Lesgee thereunder.

Dated this. day of. 19.

Lessor.

$$
\text { By }
$$

Its

\section{ASSIGNMENT.}

For value received, the Lessee named in the foregoing lease hereby assigns and transfers this lease and all of the rights.conferred by it to.

and guarantees that said. will keep and perform all the covenants and agreements of the Lessee thereunder.

Dated this day of .19

\section{ACCEPTANCE.}

The undersigned accepts the foregoing assignment of the lease mentioned therein and agrees to discharge all of the obligations imposed by said lease upon the Lessee named therein, and to use and occupy the premises described in said lease upon and subject to its terms and conditions.

Dated this day of .19 
$4+2$
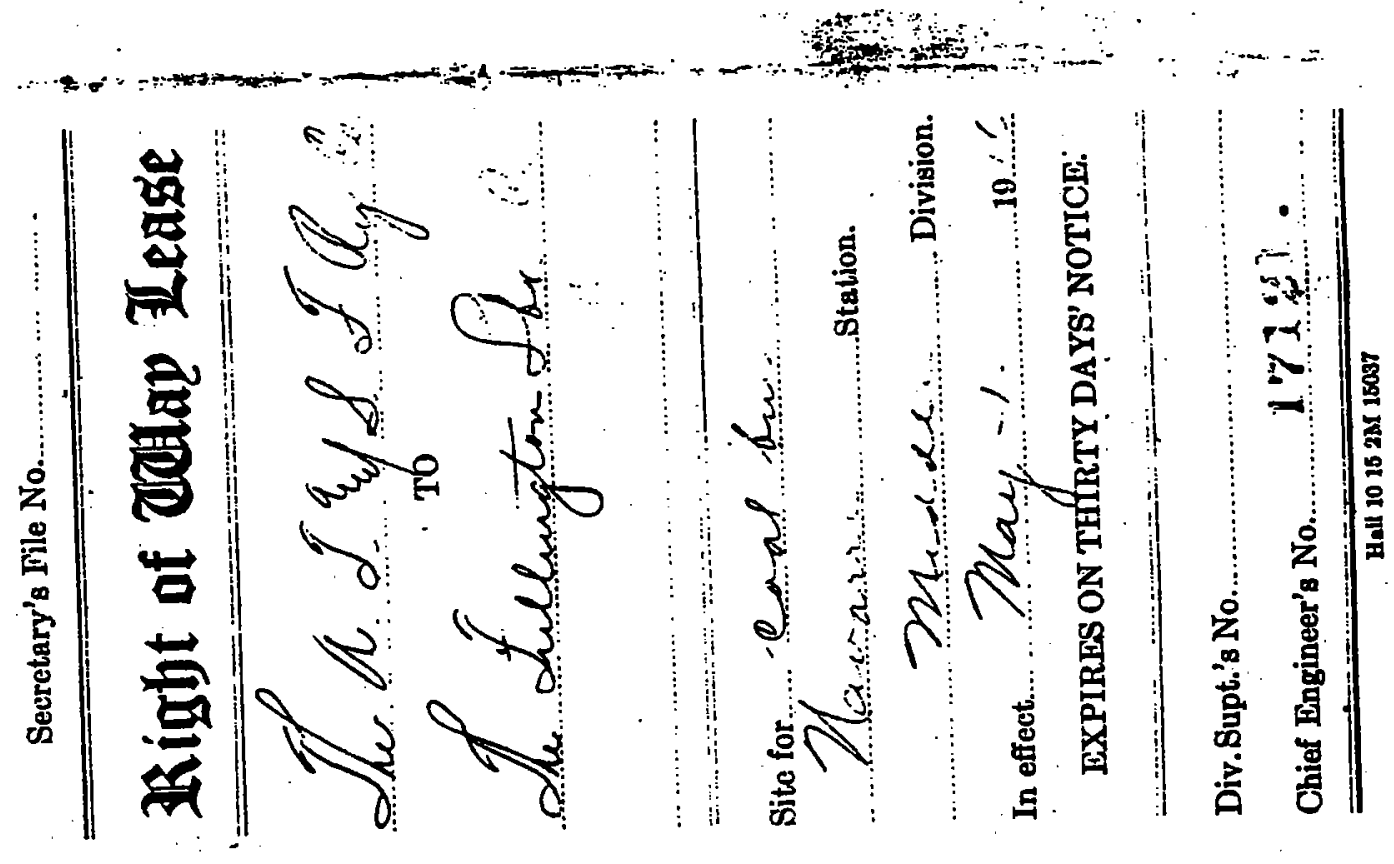

-

$\cdots$
$\because 10$
$\because 3$
$\cdots$
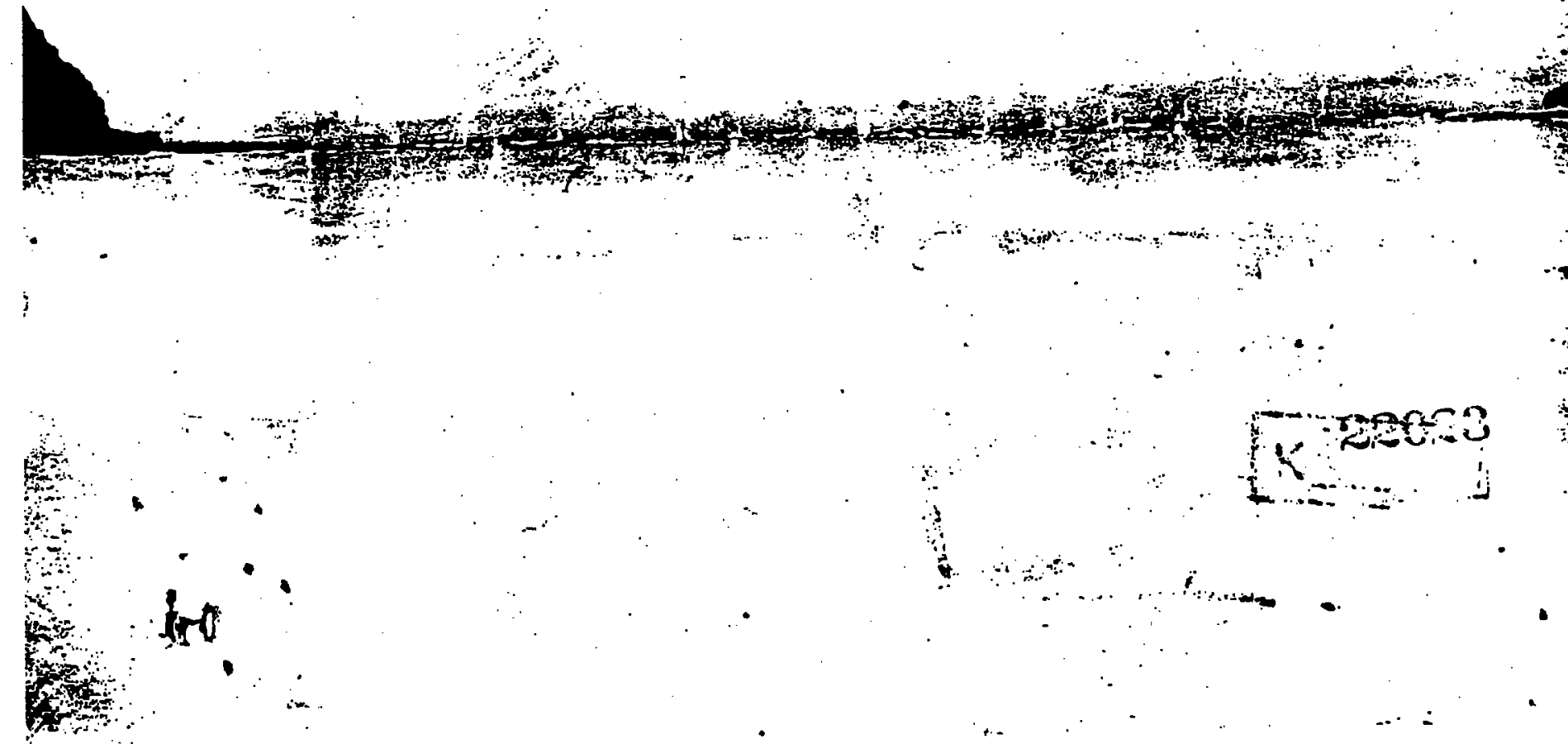


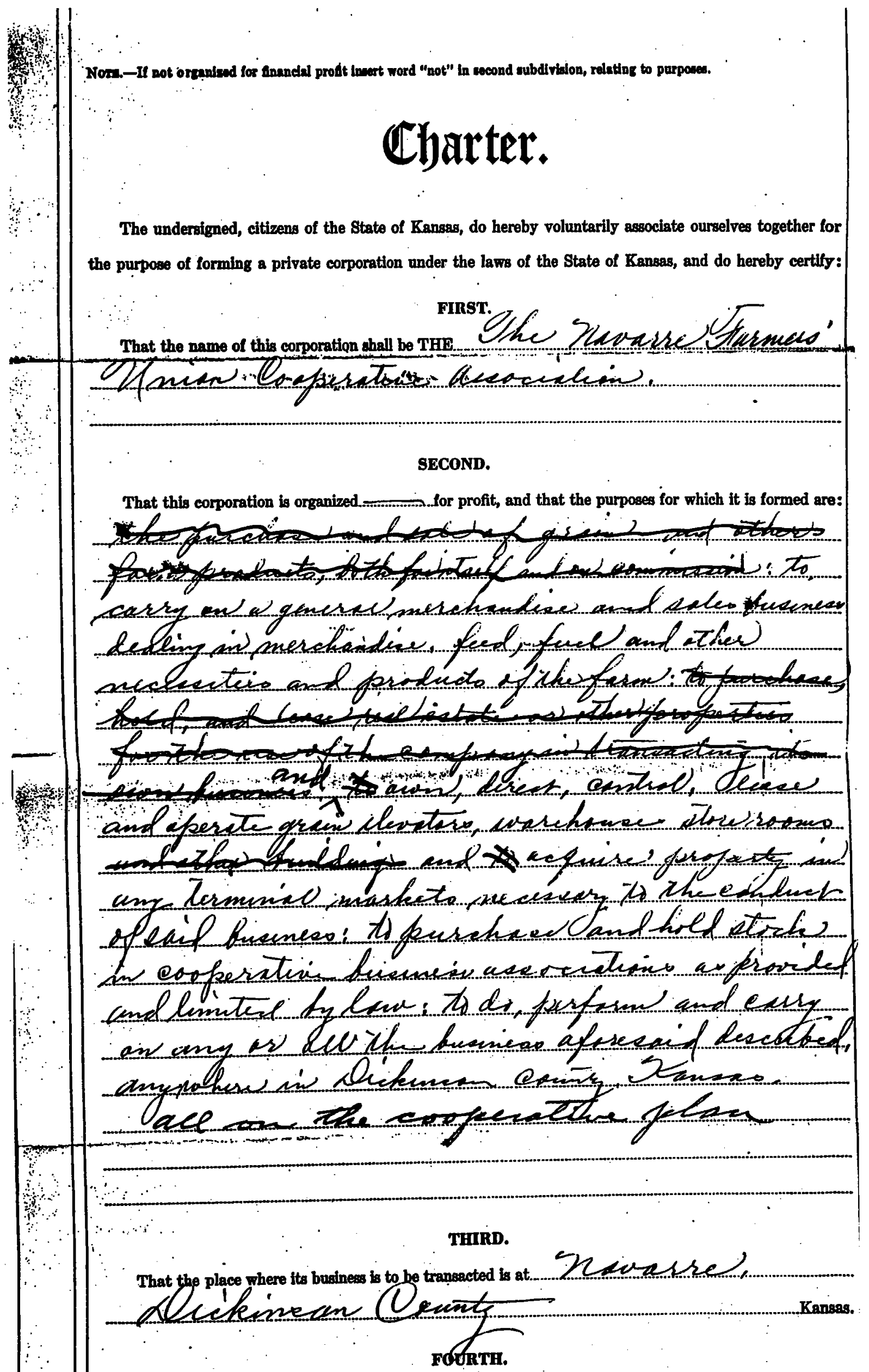

That the term for which this corporation is to exist is FUTYY Y TARS. 


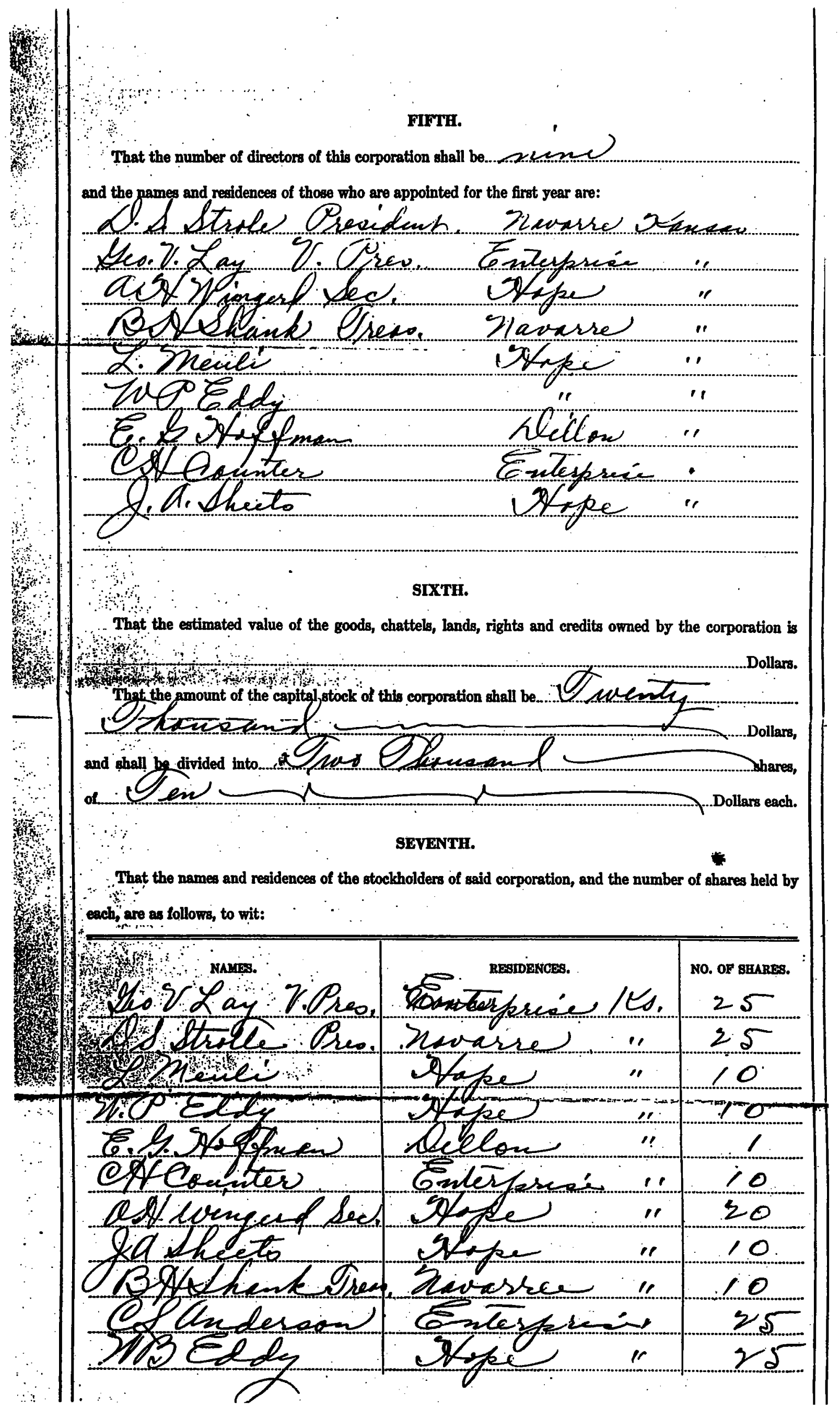




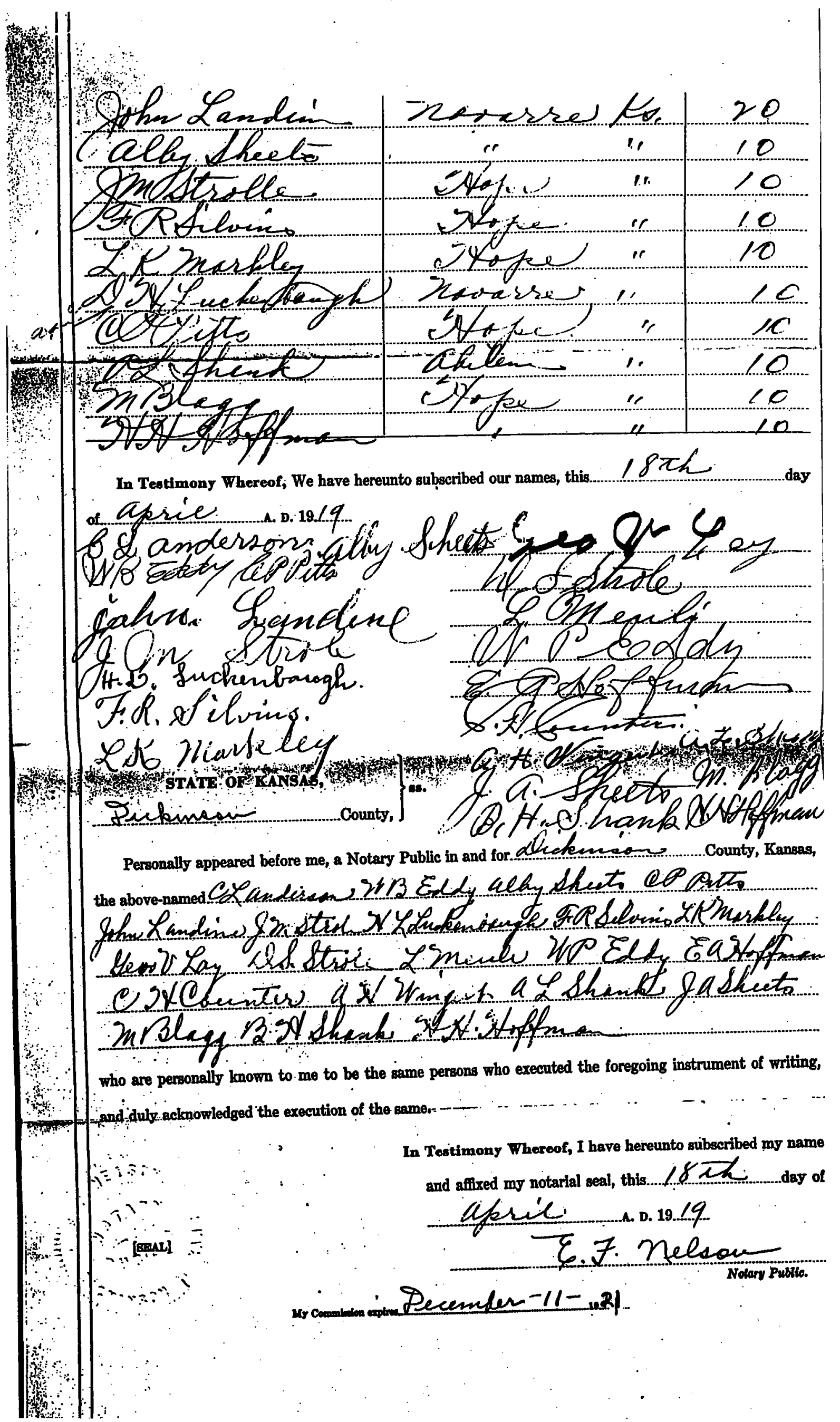


THE DICKINSON COUNTY FARMERS

EDUCATIONAL ÁND CO-OPERATIVE UNNION NO. 58

abdene tano $4 / 18$ ig1

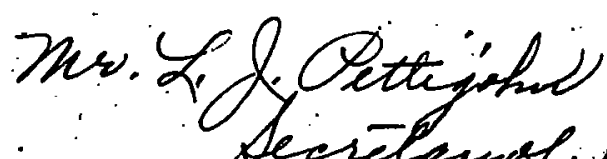

decretangs ifiate

spena Jtaino.

Llear Lis: Endoed herewith jawnielfint Application fochastw togither with chick for fier.

appeicituin fac \$s $\$ 25$

Recordiniy

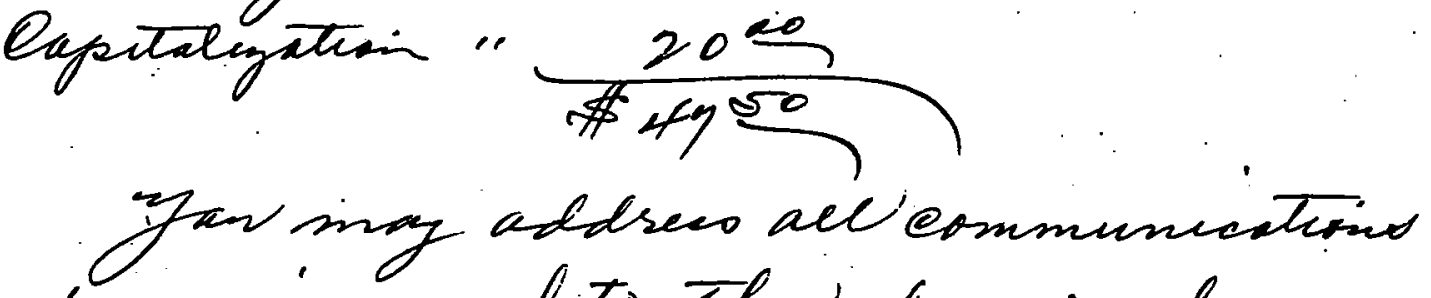
to mo inregand to the above and reng. mmak alliju.

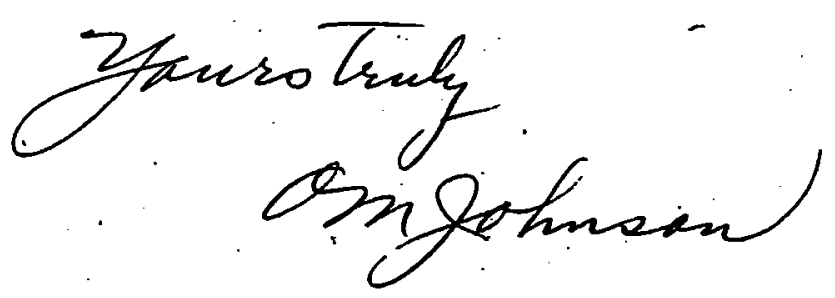




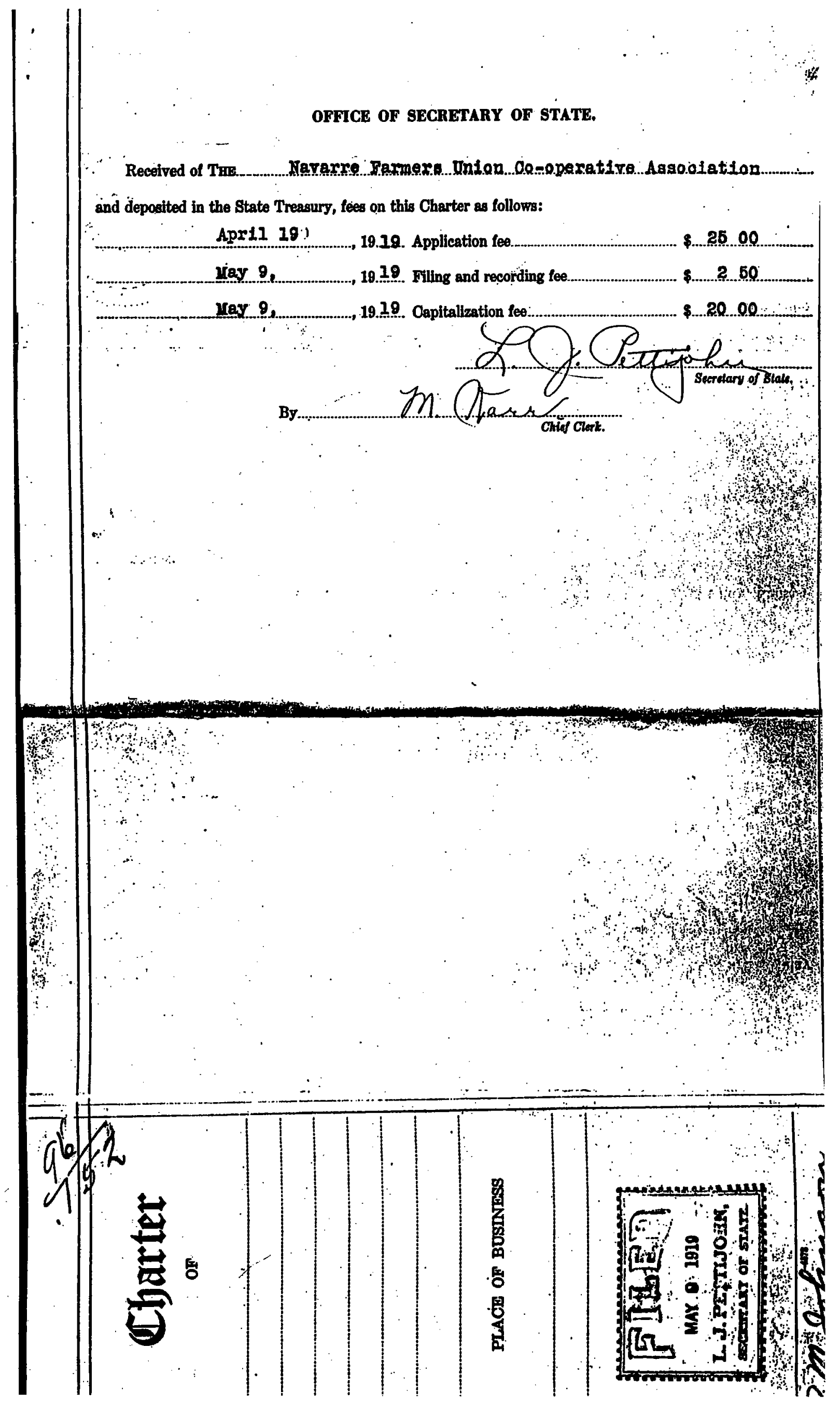



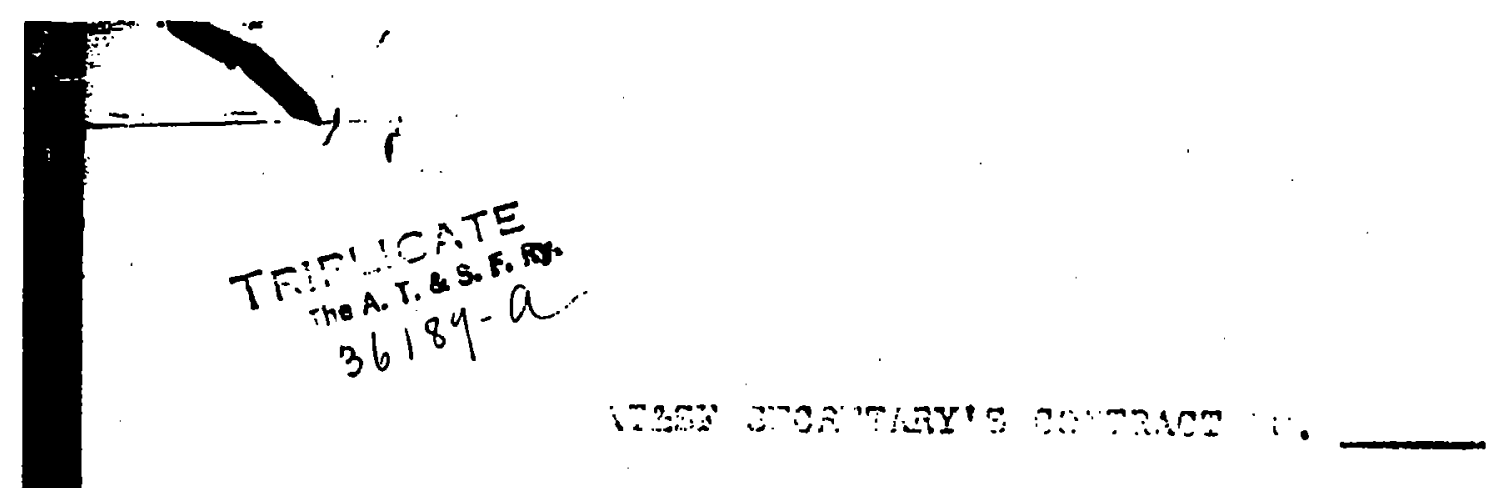

$-\infty-\infty \mathrm{OU} 0-\infty-\infty$

jorisist je

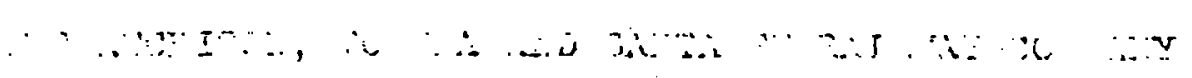

io in $30 \pi i$ ingent by

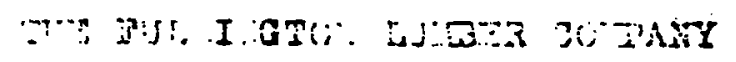

to

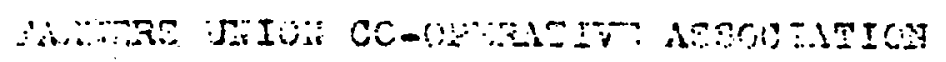

of

ATsis jeoretary'a Jontract

io. 3G139, Jatad July 6th.

1916, selitinis io 2 coal

bin location ri Yararre,

Fansas.

$--0-000-00$ 
AgFringrem, Yade tris B Rirst day of June

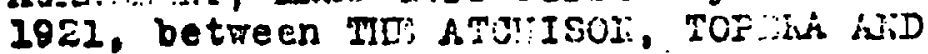

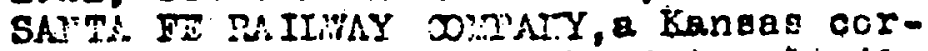
poretion, hereinafter oilied the "ii:il-

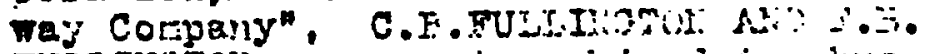

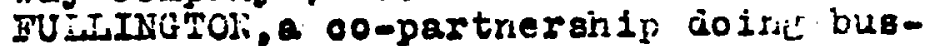
inese under firm mame The Fuliniton Lumber Company, herolnafter collective-

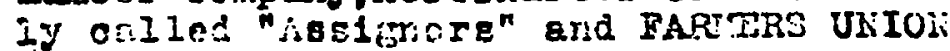
CC-OFEUATIV ASSOCIATION, a KangaB COTyoratiou, rereinistor ociled the

"Assignee".

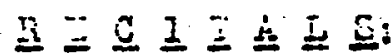

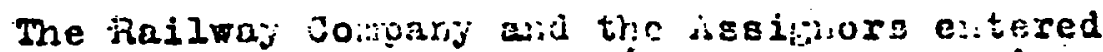

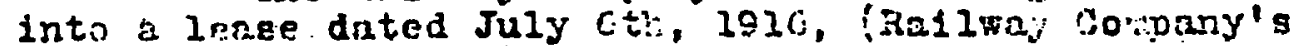

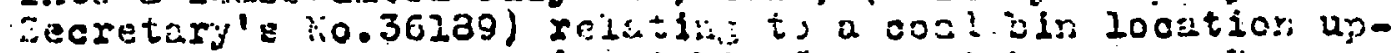
on the iallwa; Company's ririt of why at savarre, isaneas.

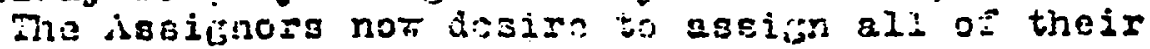

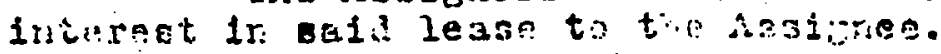

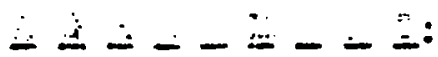

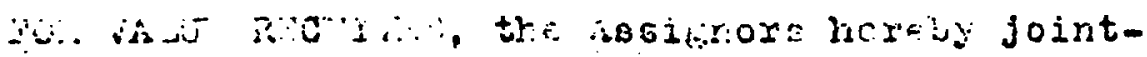

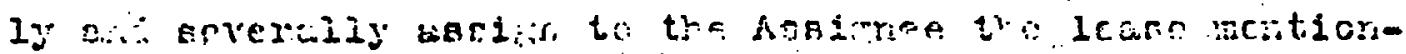

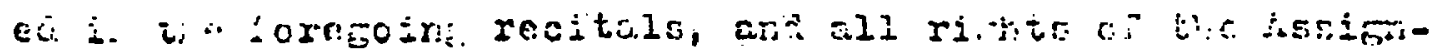

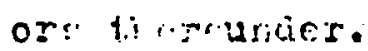

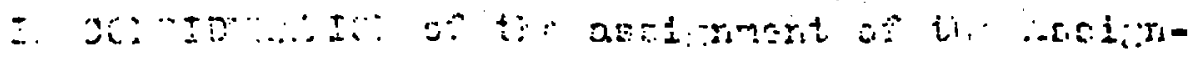

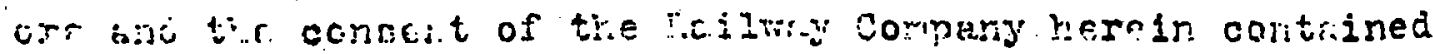

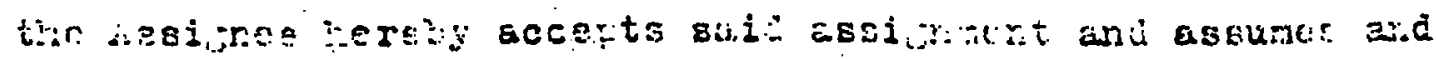
ayese to obgerve ard digcharme ull of the conditiore and ouligatione in the eforegaiz lease wlici ars by the terme thereof to be observed an: iegt by the assignor, and the 
Asalgnee further agrees not to aosien cald lease or any right or interest thexnix, and net to oublet the demised premises or any part thereof without the written corsert. of the Rallaky Companj in each Instarce.

IF CONSIDYRSEION of the promides ard of the covenante of the Aerigree rernin cortained, and tres filthful performerce of the orme, tie rislins. Jompeng colssente to tref aseirntent by the Aeglignors to tre hesignee of the ebeve rertiared Irage.

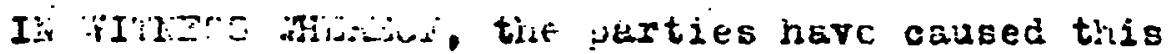

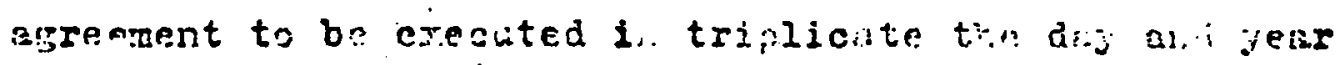
Firet Bbove vritter.

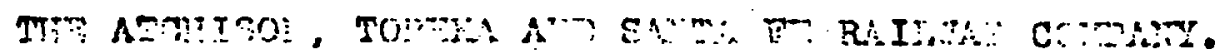

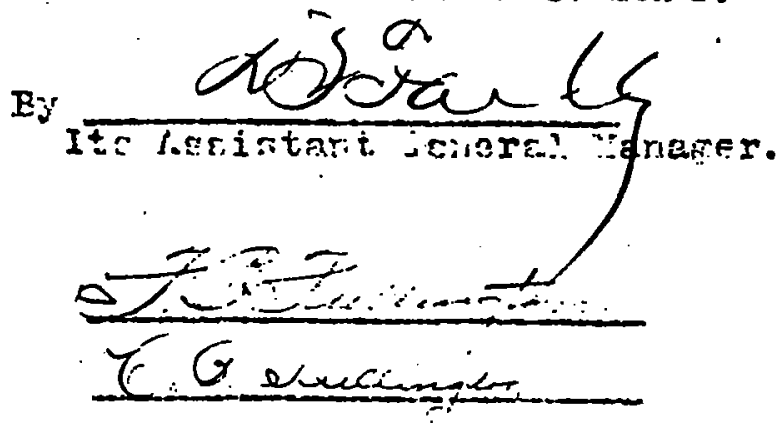

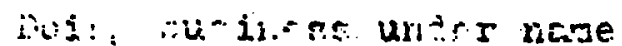
Mis : ul.lintor sumber Company.

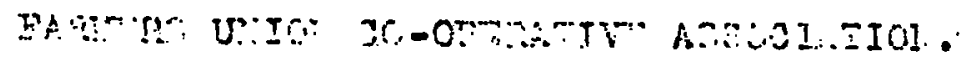

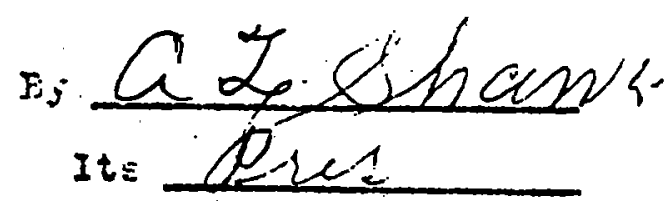

644075 


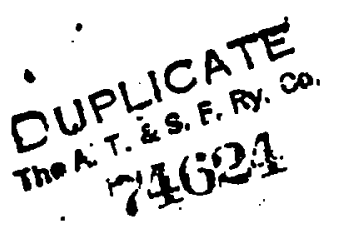

Form 1616 Standard

(Appooved by Coavel sollettor)

RIGHT OF WAY LEASE

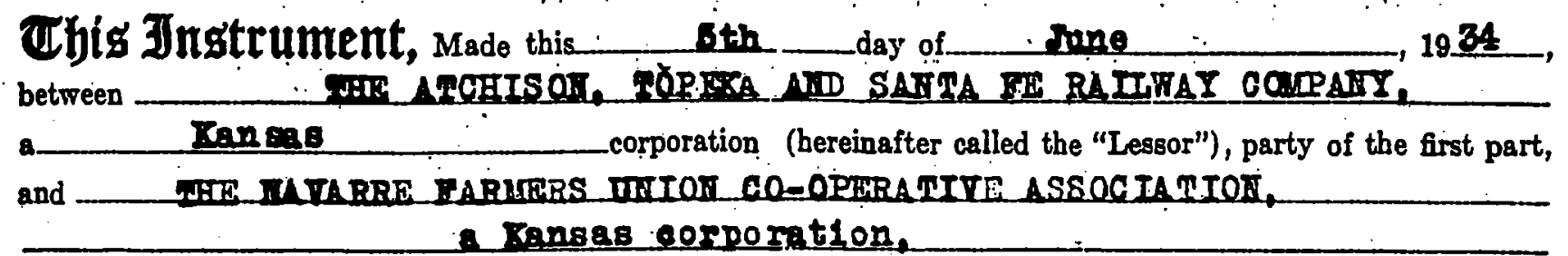

hereinafter (whether one or more persons or corporations) called the "Lessee," party of the second part.

Witneacth, That for and in consideration of the payment of the rental hereinafter epecified, and of the performance by the Leasee of the covenants hereinafter set forth to, be performed by the Iessee, the Lessor hereby leases to the Iessee that part of the right of way or station grounds now in the possession of the Lessor, situated at or near_ Iararee station, in the County of Diovelnson and State of Kansas______ outlined in red coloring on the print hereto attached,

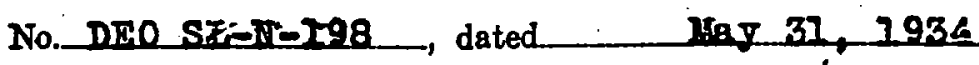
, marked "Exhibit A", and made a part hereof.

Provided, however, the Lessor hereby reserves unto itself and/or its licensees, the right to operate, maintain and renew such pipe lines, electric transmission lines, telephone lines, telegraph lines, signal lines, and other facilities of like character as may exist upon, under, or over the demised premises as of the date of this lease, it being agreed that this lesse is subject and subordinate to any and all rights granted by the Lessor for any such existing lines and facilities; and the Lessee releases the Railway Company from any and all loss or damage which the Iessee may sustain on account thereof.

To Have and to Hold the same from Lay 11, this lease shall be terminated as hereinafter provided. 19 34, until such time as

In Consideration of the aforesaid lease, the Lessee covenants and agrees to and with the Lessor as follows:

1. That it will pay to the Lessor annually in advance, as rental for the demised premises, a sum which shall represent interest at the rate of six per cent $(6 \%)$ per annum on the fair rental value of said premises, but in no case shall said rental be less than Mreire and $\mathrm{No} / 100$

Dollars $(\$ .22 .00 \ldots \ldots$ ) per year. Such value shall be increased from time to time by the amount of any charge or assessment (except general property taxes) payable on account of or in respect to said premises for paving, curbing, sidewalks, sewers, benefit districts and the like, and shall also be subject to revision at least once every five (5) years during which this lease may continue in effect. For the purposes of this lease, the fair rental value of the demised premises at the date hereof is agreed to be

-. Three Hundred If ehty-itire and $\pi 0 / 100^{\circ}$ and the initial rental shall be_menty-three and $10 / 100$ $(\$ 23.10 \quad$ per annum.

2. That it will pay before the same become delinquent all taxes, charges, rates and. assessments which may, during the term of this lease, be levied upon or assessed against or be equitably chargeable to or assessed in respect to any buildings and improvements which may be placed upon the demised premises by the Lessee; and where any such tax, rate, charge or assessment may be embraced in the general amount of taxes charged upon the demised premises separately or in connection with other property of the Lessor and the Lessor shall pay all of said taxes, then the Lessee will promptly repay or refund to the Lessor the amount or part of the tax, charge, rate or assegsment equitably or fairly apportionable to any buildings or improvements placed on the demised premises by the Lessee.

3. That it will use the demised premises exclusively as a site for elerator, ooz bins, buli- o11 station and other builalngs

4. That it will keep the demised premises and the buildings and structures thereon in a condition satisfactory to the Lessor, and will from time to time, as may be required by the Lessor, paint all such buildings and structurea with paints of a color approved by the Lessor. Should the Lessee fail or refuse within fifteen (15) days to comply with any request made by the Lessor to place the premises or any building or gtructure thereon in proper condition, or to paint any such building or structure, the Lessor may, at its option, perform such work, and in such event the Iessee shall promptly reimburse the Lessor for the cost so incurred.

5. That it will perform, observe and comply with all federal and state laws, orders or regulations and municipal ordinances or regulations regarding inspection, sanitation, safety devices, fires and other matters connected with the maintenance and use of gaid premises, and in the event the demised premises shall be used for the loading, unloading, st:anes or otherwise fling of any petroleum products, that it 
all the regulations, anct dations from time to time published by

can Railway Association, $\rightarrow$ aus successor agency, and that it will indemnify Bureau of Explosives of the Ameriall fines, penalties, claims, demands and suits, at law or in equity, and loss or damso harmless the Lessor against manner out of the non-observance by the Lessee of any federal or state law, order, or to property, arising in any nance or regulation, or Bureau of Explosives regulation or recommendation as aforessid, or oun, municipal ordiLessee of the demised premises or the buildings or improvements erected thereon or out of the of the use by the acts, omissions or negligence of the Lessee or of the servants or agents of the Lessee in on sole or contributing premises in the use thereof by the Lessee, and will promptly pay to the Lessor the amount of about the demised to property sustained by the Lessor and the amount of any fine, penalty or judgment which may be laid or damage ered against the Lessor on account of any matter or thing againgt which the Lessor is indemnified as in or recovgraph 5 provided.

6. That it will at all times keep a space of six (6) feet from the nearest rail of any railroad track entirely clear of structures, material and obstructions of every sort but, nevertheless, the Lessee may erect loading platforms which shall not be higher than three (3) feet and six (6) inches above the top of the rails and which at no point shall be nearer than four (4) feet to the nearest rail of such track; provided, however, if by statute or order of competent public authority different. clearances shall be required than those provided for in this Section 6 , then - the Lessee shall strictly comply with such statute or order. In case of breach of these obligations, or any of them, the Lessee assumes and agrees to indemnify the Lessor against all liability for loss, damage, injury and death arising therefrom and to reimburse the Lessor for any sums which the Lessor may have been required to pay in the way of damages, fines, penalties or other expense resulting from the violation by the Lessee of any
statute or order as aforesaid.

7. That it will indemnify and hold harmless the Lessor from all loss or damage, by fire, to all buildings structures ur improvements at any time upon the demised premises, and all property of any sort belonging to the Lessee or others in said buildings and structures or upon the demised premises, whether such fire shall be caused by the negligence of the Lessor or its employes, or otherwise.

8. That it will waive all claims which it might or could have for injury to stock or animals pasturing, working or being on the demised premises, whether the same arise from such animals or stock taking fright or other-
wise.

9. That neither the Leasee, its legal representatives, successors or assigns, nor any subsequent assignee, shall underlease or sublet the demised premises or the buildings or improvements erected thereon, or uny part thereof, nor assign this lease or any interest herein, without the written consent and approval in each instance of the Lessor, and that, at the option of the Lessor, this lease shall be forfeited by any such voluntary sublease or assignment
or by any assignment thereof by operation of law.

10. That in case of eviction of the Lessee by anyone owning or claiming title to the said land, the Lessor shall not be liable to the Lessee for any damage of any nature whatsoever, or to refund any rental paid bereunder.

11. That if any rent hereunder shall be due and unpaid, or if default shall be made in any of the covenants of the Lessee herein contained, then it shall be lawful for the Lessor to re-enter the demised premises and to re-
move all persons therefrom.

12. That this lease may be terminated at any time by either party upon thirty (30) days' notice in writing to be served upon the other party, stating therein the date that such termination shall take place and upon the expiration of the time specified in such notice this lease and all rights of the Lessee hereunder shall absolutely cease and determine; but upon any such termination the Lessee shall be entitled to have refunded by the Lessor a proportionate part of any rentals paid in advance.

13. Any notice to be given by the Lessor to the Lessee hereunder shall be deemed to be properly served if the same be delipered to the Lessee, or if left with any of the agents, servants or employes of the Lessee on the leased premises, or if posted on the leased premises, or if deposited in the postofice, postpaid, addressed to the Lessee at Hararre: Kansas.

14. That upon the expiration or termination of this lease in any manner herein provided, the Lessee upor demand of the-Lessor, without further notice, shall deliver up to the Lessor the possession of the demised premiset and shall remove all the improvements placed thereon by the Lossee and restore the demised premises to substantially their former atste, and in case the Lessee shall fail within thirty (30) days after the date of expiration on termination of this lease to make such removal or restoration, then the Lessor may, at its election, either remove said improvements and restore said premises for the account and at the sole cost of the Lessee, or may take and hold the said improvements as its sole property.

15. No termination or cancellation hereof shall release the Lessee from any liability or obligation (whethes of indemnity or otherwise) which may have attached or accrued previous to or which may be accruing at the time of such termination or cancellation.

16. In the event that the Lessee herein embraces two or more persons or corporations, all the covenants anc agreements of the Lessee herein shall be the joint and several covenants and agreements of auch persons or cor
porations.

17. That all the covenants and provisions of this lease shall be binding upon the Lessee and the executors administrators, successors and assigns of the Lessee, and shall inure to the benefit of the successors and assigns 0
the Lessor.

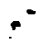


$\mathrm{By}$

(Lessor.)

(Lessee.)
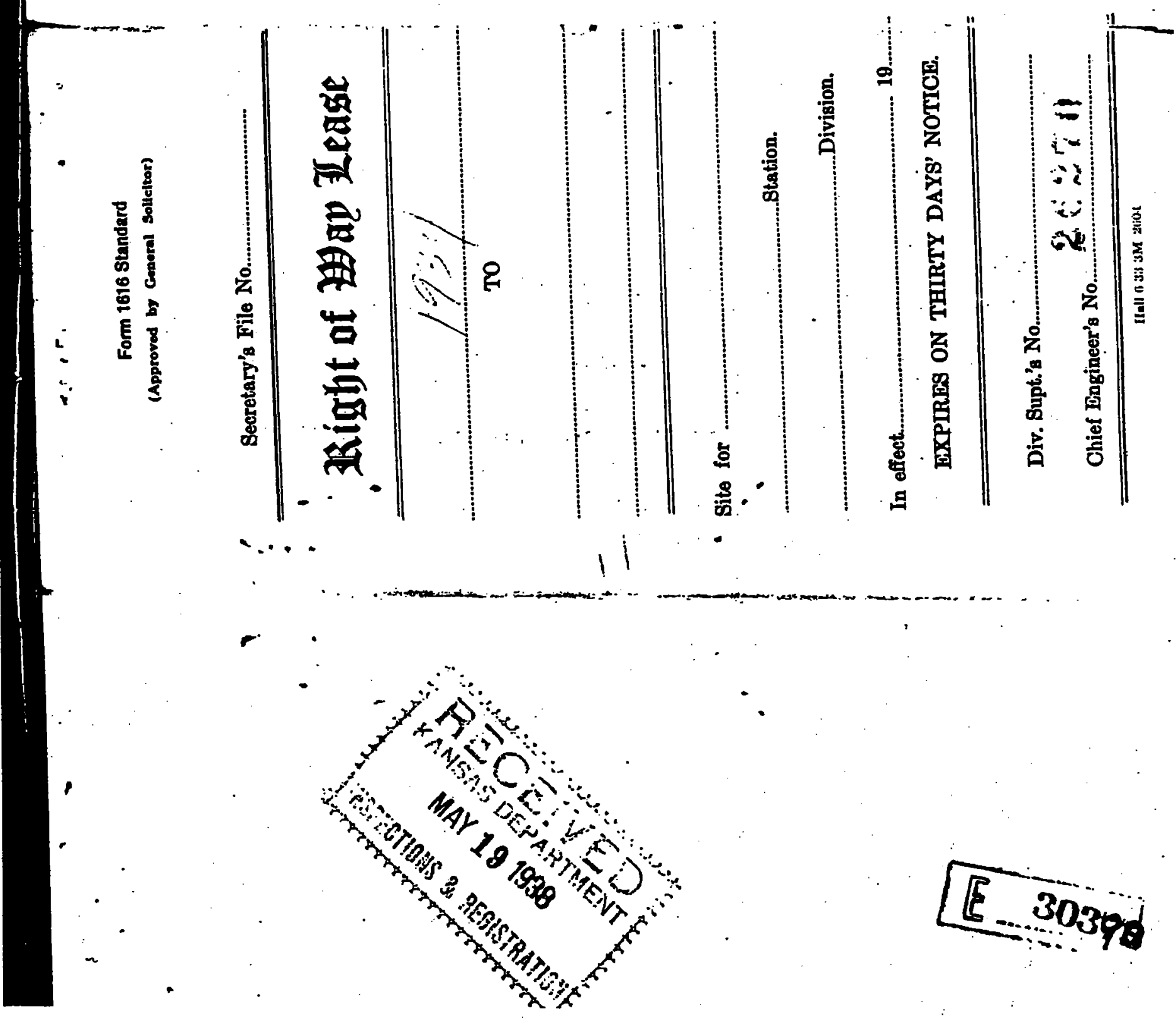

CUPLICATE

thet ing 6324 
It 18 motrolly agreod that the certain agroemont dated ros 6 ,

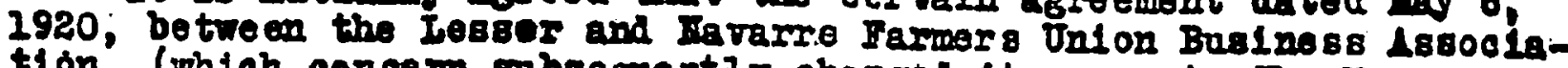
t1on, (whioh oonoem sobsequentis changed its name to ine Iavarys Tarmers Jn1on 00-Oporatiro Assoolation). Iossor's Soerotarg's Contract II0. 43928, relatine to the use of a portion of Leseor'B proporty at Iavamo, Jansas, as a site for coal bln and olovetor, shall be and the same is heroby terminsted as of the effeotive deite hereof.

It 15 further motually agreed that the certaln agroement dated

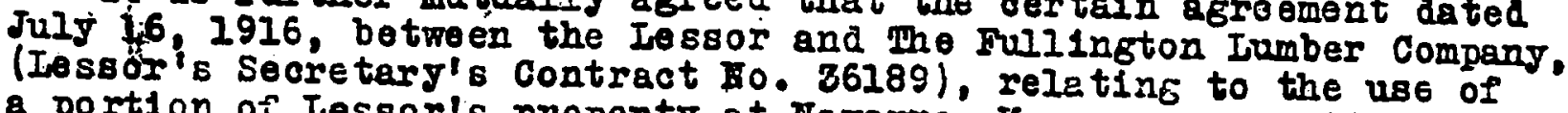
a portion of Lessor's property at Havarre, Kansas, as a eite for coal bin as as8i Gnea under date of June 1, 1921, to the Farmers Union Co-operative Ascociation, (to all of whose rights and obligations in caid agreement the Iessee has suoceded, shall be and the same is hereby. terminated as of the effective date hereof.

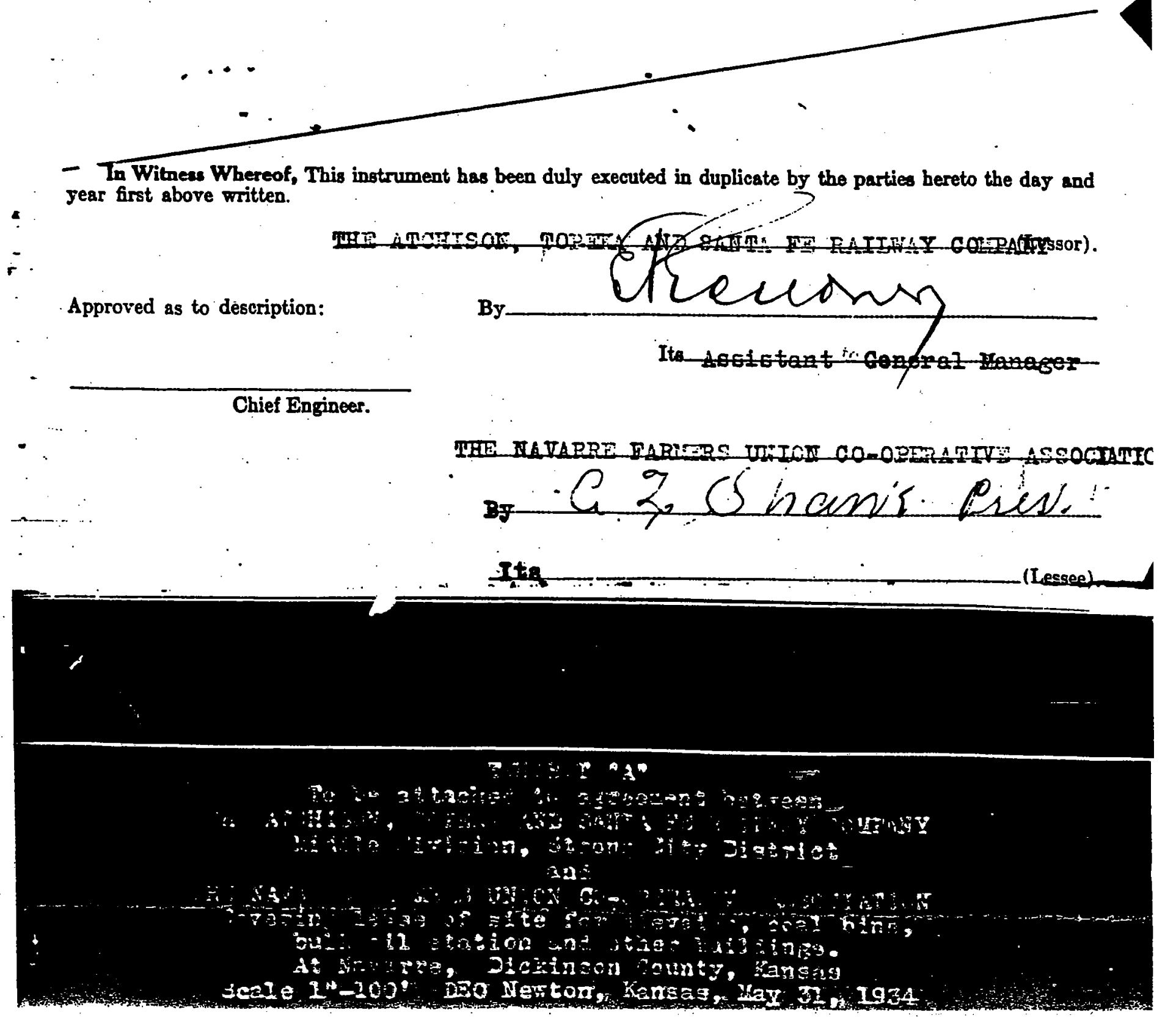




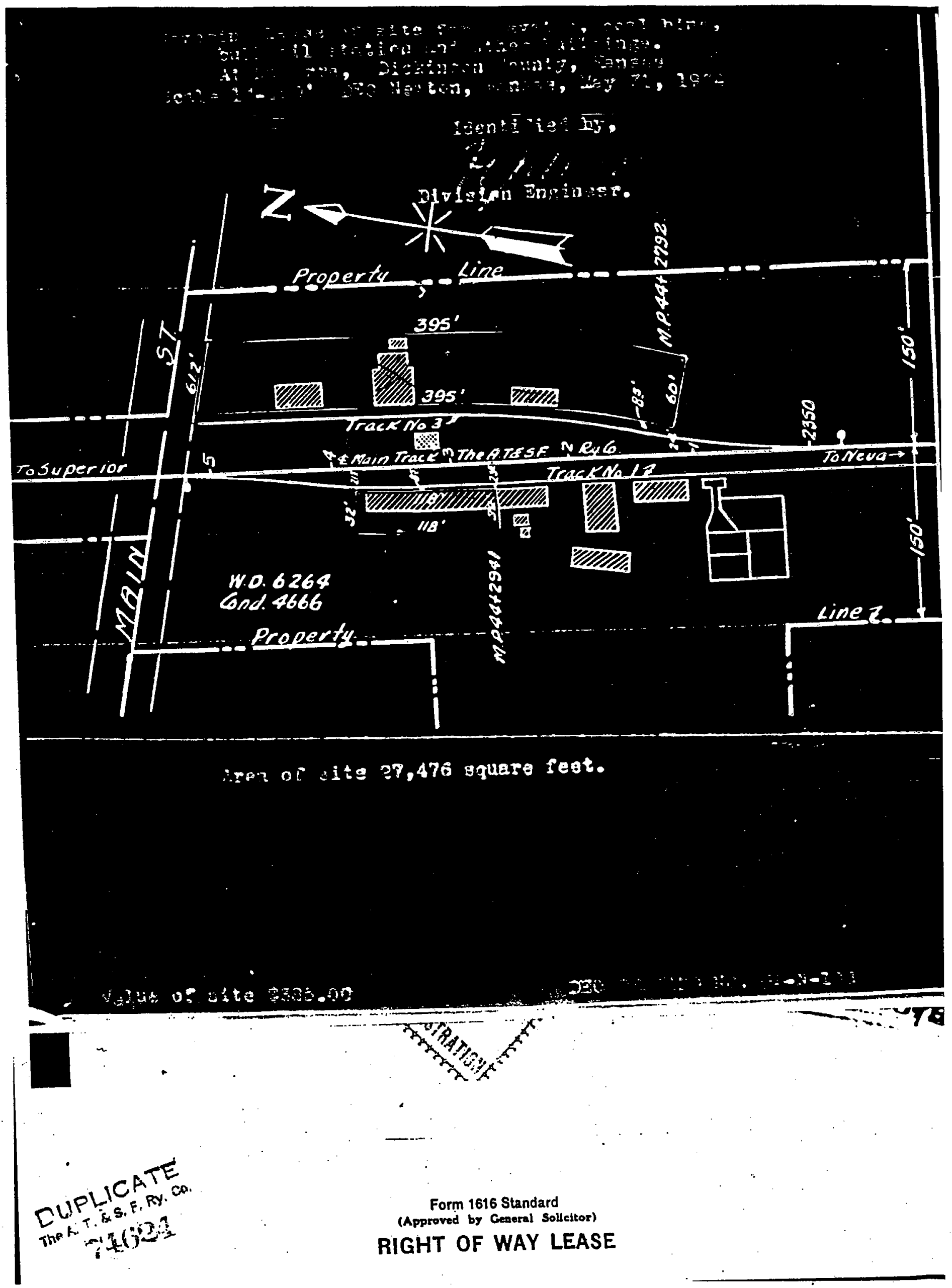




\section{The First Nationar Bank 23}

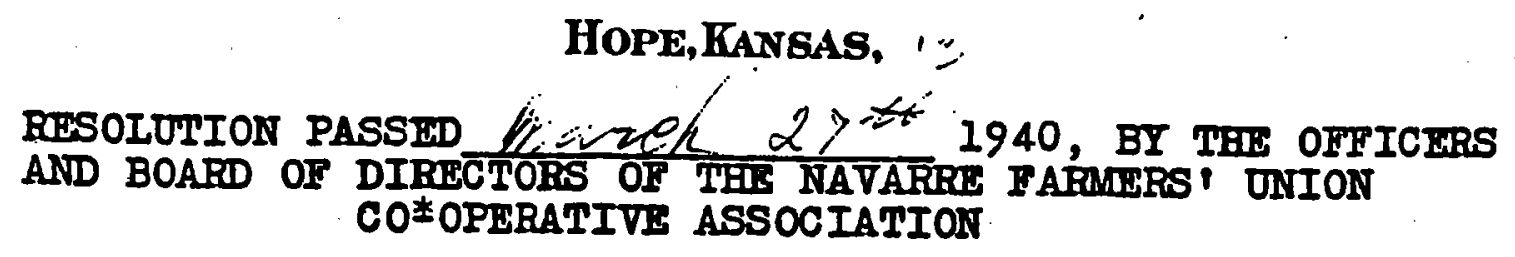

WHFPEAS, it is required by Section 142 of Chapter 152, of the 1939 Session Laws of the state of Kansas, that every corporation shall maintain a Registered Office and Resident Agent in this State, it is therefore now and hereby

RESOLVED and ORDERED, by

the Officers and Directors of the NAVARRE FARMRRS' URION CO-

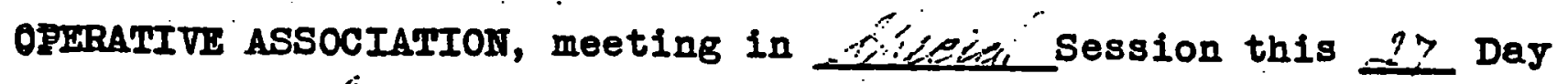
of Lilezeri A. D. 1940, that the REGISTEERED OFTICE of this corporation shall be at Navarre, Kansas, at the office of the Elevator owned and operated by this corporation at said place; and that $\because \frac{1}{2}$ shall be hereby designated as the RESIDENT AGENT of this Corporation, his address being laterelile Kansas.

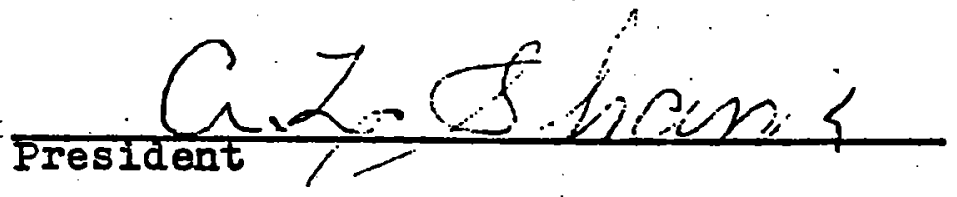

으르므

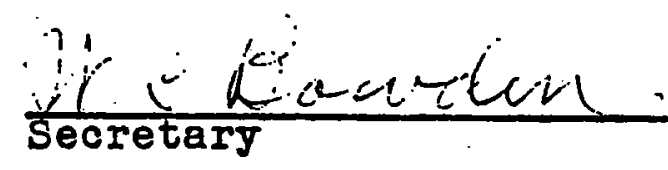




\section{Form 1616 Standard \\ (Approved by Generel Soliodtor) \\ LEASE OF LAND (Short Term)}

TEIS LEASE, Made as of the

13th

day of.......pril

$19 . .49$

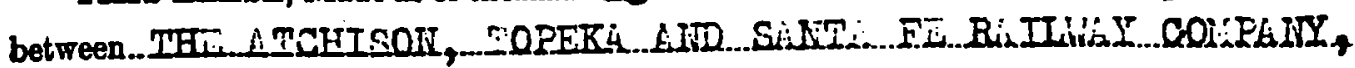

a.

a Kansas corporation (hereinafter called "Lessor"),

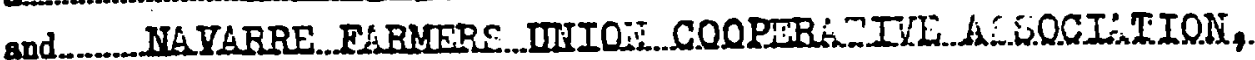

a Kansas cosporation,

(hereinafter, whether one party or more, called "Lessee").

WIINZSSITE, That the parties hereto for the considerations hereinafter expressed covenant and agree as follows:

1. Lessor bereby leases to Lessee, subject to the rights and easements hereinafter excepted and reserved, and upon the terms and conditions hereinafter set forth, the land (hereinafter called "Premises") situated at or near

$17=$ yarre County of

Dialsinson.

State of..K:ins:se , outlined in red coloring on the print bereto attached,

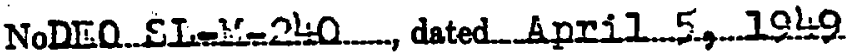

marked "Exhibit A" and made a part hereof, for a term beginning on.

and ending when this lease shall be terminated as hereinafter provided.

2. Lessor hereby excepts and reserves the right, to be exercised by Lessor and by any others who have obtained or may obtain permission or authority from Lessor so to do, (a) to operate, maintain, renew and relocate any and all existing pipe, power, and communication lines and appurtenances and other facilities of like character upon, over or under the surface of the Premises; and (b) from time to time to construct, operate, maintain, rener and relocate such additional facilities of the same character as will not unreasonably interfere with the use of the Premises by Lessee for the purpose specified in paragraph 6 hereof.

3. Lessee shall pay to Lessor on or before the first day of each period of one year during the continuance of this lease as rental for the use of the Premises for such period, a sum equal to six per cent $(6 \%)$ of the fair rental value of

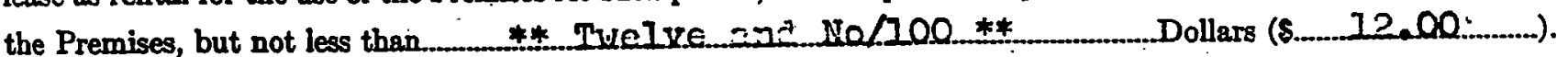
For the purposes of this lease the fair rental.value of the Premises at the effective date hereof is agreed to be. * six Eurared seventy-six.and jo/100.** Dollars (8...676́.00........) and the initial rental shall be * Forty Enc...50/100.**

Dollars ( $8 \ldots$ 4.... $56 \ldots$.....) per annum. Such fair rental value shall be increased from time to time by the amount of any governmental charge or assessment (except general property taxes) payable on account of or in respect to the Premises for the construction of public improvements.

4. Lessee covenants and warrants that Lessee either oivns, or has obtained from the owner or owners thereof the right to use, any improvements now on the Premises show? or described on said Exhibit A as "I Lessee's Existing Improvements." Such improvements, if any, together with any other improvements hereafter placed upon the Premises by or for account of Lessee are hereinsfter called "Improvements."

5. Lessee shall pay before the same become delinquent all taxes, charges, rates, and assessments which may, during the term of this lease, be levied upon, or assessed against, or be equitably chargeable to or assessed in respect of the Improvements; and where any such tax, rate, charge, or assessment may be embraced in the general amount of tarse ohargad upon the Premises nenarately or in connection with other property of Lessor and Lessor shall pay all of said texes, then Lessee shall promptly repay or refund to Lessor the amount or part of the tax, charge, rate or assessment equitably or fairly apportionable to the Improvements.

6. Lessee shall use the Premises exclusively as a site for elexzator....coe 1...bins,...bulk.

olistation an other...bulidings

7. Lessee shall keep and maintain the Premises and Improvements in such safe, sanitary, and sightly condition as shall be satisfactory to Lessor, and, if required by Lessor, shall paint the Improvements with paints of a color approved by Lessor; and if Lessee fails or refuses within fifteen (15) daps after receipt of any request by Lessor so to do, Lessor may, at its option, perform such work, and in such event Lessee shall within thirty (30) days after the reddition of bill therefor reimburse Lessor for the cost so incurred. 
。

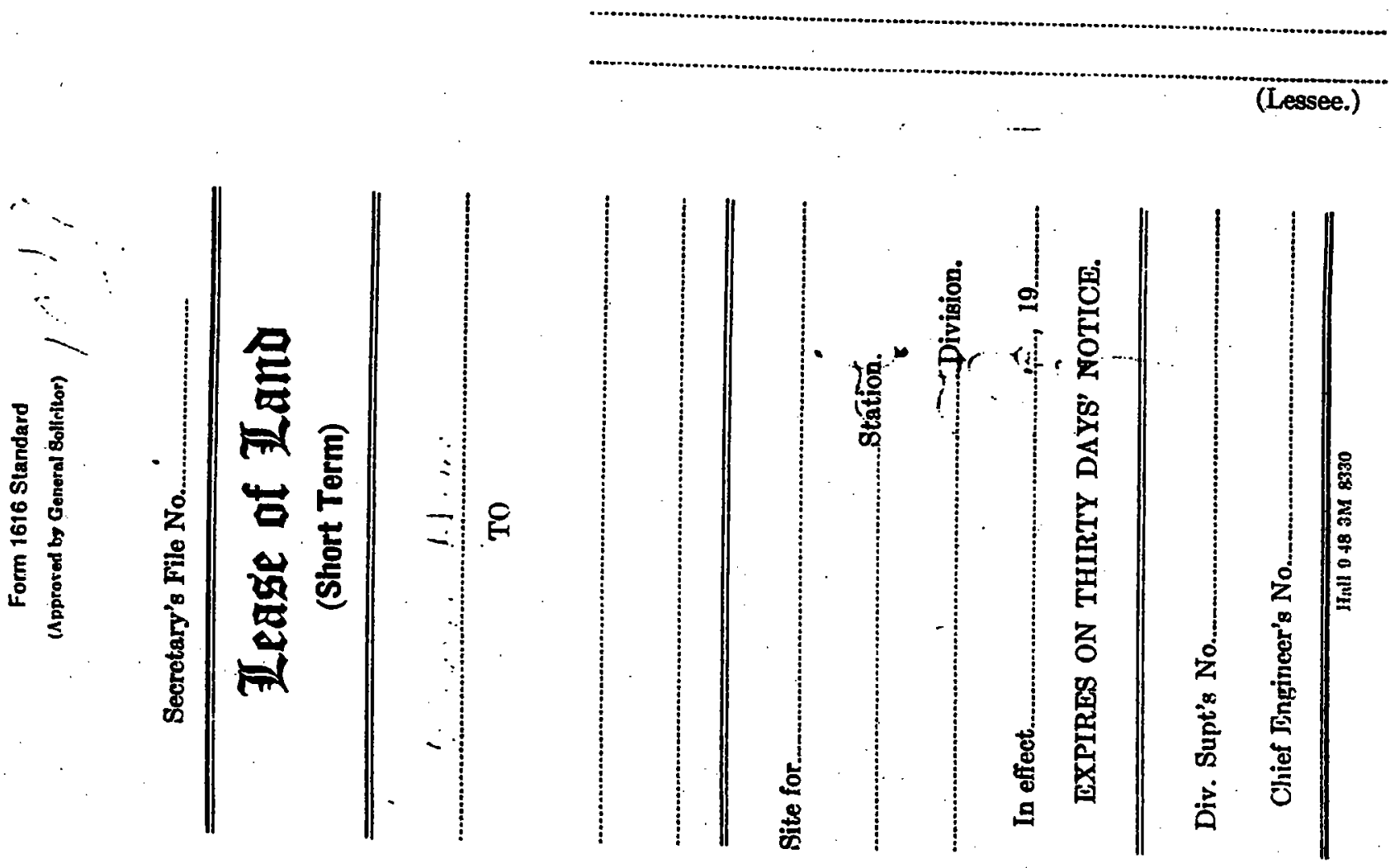




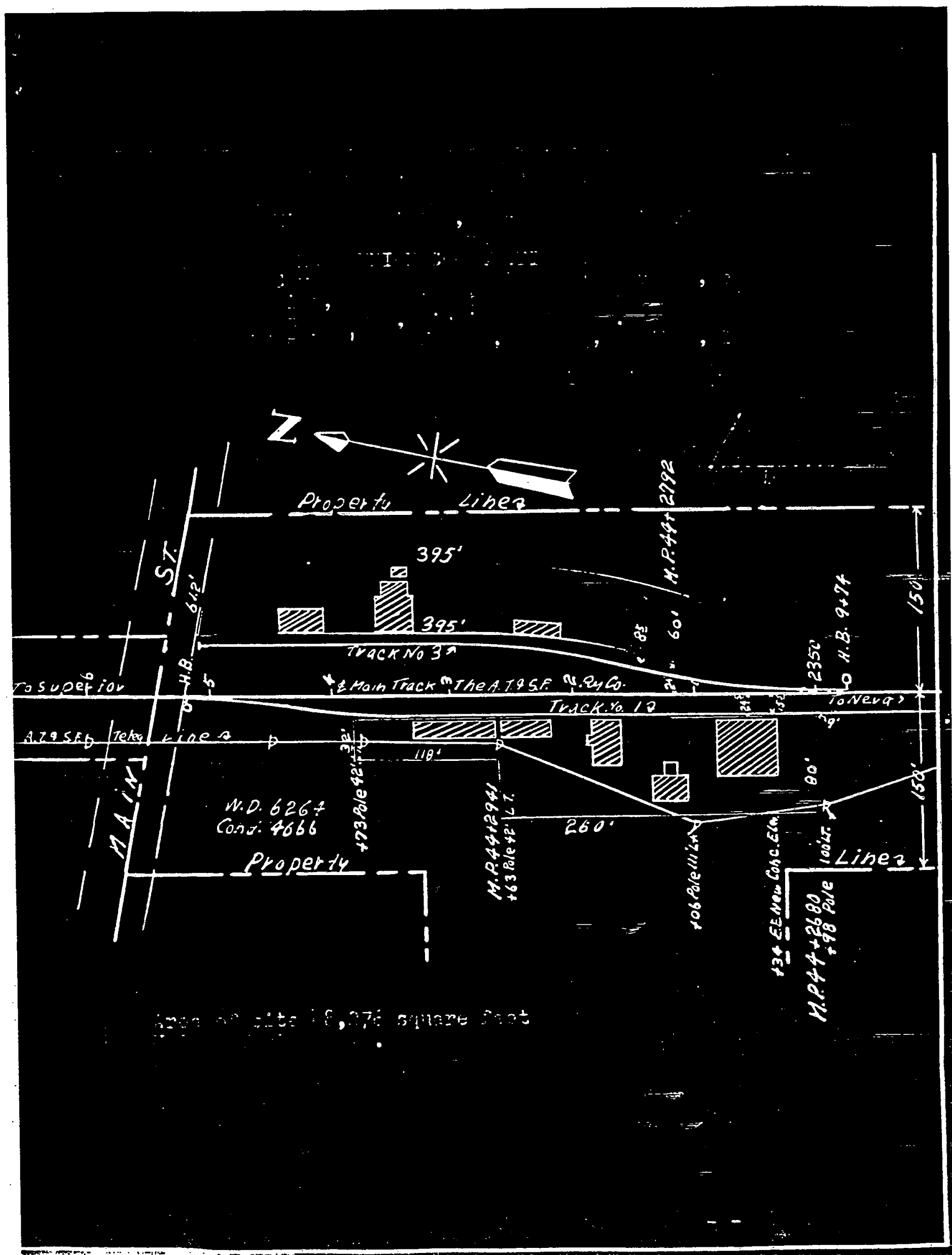




\section{Form 1616 Standand

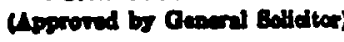 \\ LEASE OF LAND (Short Term)}

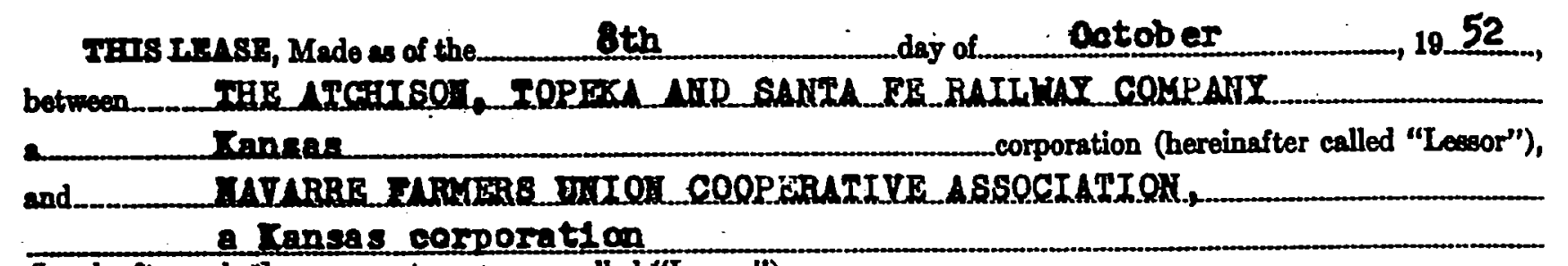

(hereinafter, whether one party or more, called "Lessee"). follows:

FIIInssivTH, That the parties hereto for the considerations hereinafter expressed covenant and agree as

1. Lewor hereby leases to Lessee, subject to the rights and easements hereinafter excepted and reserved, and upan the terms and conditions hereinafter set forth, the land (hereinafter called "Premises") situated at or near Tavare Dickinson

State of Tansas._._._outlined in red coloring on the print hereto attached,

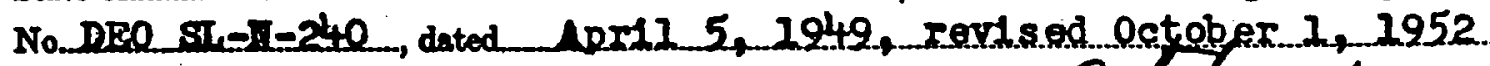

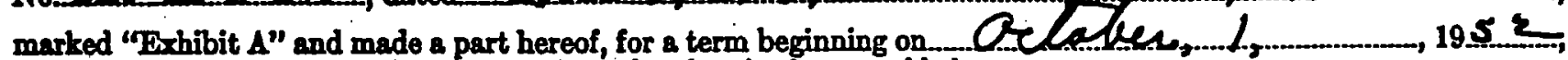
and ending when this lease shall be terminated as hereinafter provided.

2. Leasor hereby excepts and reserves the right, to be exercised by Lessor and by any others who have obtained or may obtain permission or authority from Leasor so to do, (a) to operate, maintain, renew and relocate any and all existing pipe, power, and communication lines and appurtenances and other facilities of like character upon, over or under the surface of the Premises; and (b) from time to time to construct, operate, maintain, renew and relocate wuch additional facilities of the same character as will not unreasonably interfere with the use of the Premises by Leosee for the purpose specified in paragraph 6 hereof.

3. I essee shall pay to Lessor on or before the first day of each period of one year during the continuance of this lease as rental for the use of the Premises for such period, a sum equal to six per cent $(6 \%)$ of the fair rental value of the Premises, but not less than Twa7 Ie and Do/200 Dollars (\$..... 12.00 ........).

For the purposes of this lease the fair rental value of the Premises at the effective date hereof is agreed to be Four Fundred rinaty $=$ tiwo and $\mathrm{H} 0 / 100$ Dollars (\$. 1492.00 and the initial rental shall be__nenty $=$ nine and $52 / 100$

Dollars (\$29.52 j) per annum. Such fair rental value shall be increased from time to time by the amount of any governmental charge or assessment (except general property taxes) payable on account of or in respect to the Premises for the construction of public improvements.

4. Lessee covenants and warrants that Leasee either owns, or has obtained from the owner or owners thereof the right to use, any improvements now on the Premises shown or described on 8aid Exhibit A as "Lessee's Existing Improvements." Buch improvements, if any, together with any other improvements hereafter placed upon the Premises by or for account of Lessee are hereinafter called "Improvements."

5. Lessee shall pay before the same become delinquent all taxes, charges, rates, and assessments which may, during the term of this lease, be levied upon, or assessed against, or be equitably chargeable to or assessed in respect of the Improvements; and where any such tax, rate, charge, or assessment may be embraced in the general amount of taxes charged upon the Premises separately or in connection with other property of Lessor and Lessor shall pay all of said taxes, then Lessee shall promptly repay or refund to Lessor the amount or part of the tax, charge, rate or assessment equitably or fairly apportionable to the Improvements.

6. Lessee shall use the Premises exclusively as a site for_elerator,..coal fbins, ...bulk.all station and other:bullatngs

7. Lessee shall keep and maintain the Premises and Improvements in such safe, sanitary, and sightly condition as shall be satisfactory to Lessor, and, if required by Lessor, shall paint the Improvements with paints of a color approved by Lessor; and if Lessee fails or refuses within fifteen (15) days after receipt of any request by Lessoir $0_{0}$ to do, Leasor may, at its option, perform such work, and in such event Lessee shall within thirty (30) days after the rendition of bill therefor reimburse Lessor for the cost 80 incurred. 
8. In using the Premises, and in constructing, maintaining, operating and using the Improvements thereon Lessee shall comply with any and all requirements imposed by federal or state statutes, or by ordinances, orders or regulations of any governmental body having jurisdiction thereover. In the event the Premises or Improve ments shall be used for the loading, unloading, storing, or otherwise handling of any petroleum products, Lesse shall comply with all applicable regulations and recommendations from time to time promulgated by the Bureau o Explosives of the Association of American Railroads, or any successor agency. All artificial lighting in pump houses warehouses, or other enclosures upon the Premises, where oil or other inflammable fluid supplies are handled o stored by Lessee, except in unbroken original containers, shall be by electricity, and such electrical installation an any other electrical installation upon the Premises shall at all times conform to and be maintained in accordanc with the provisions of the then current edition of the National Electrical Code with respect to Class I hazardou locations. Lessee shall promptly pay and discharge any and all liens arising out of any construction, alteration repair work done, or suffered or permitted to be done, by Lessee on the Premises, and Lessor is hereby authorize to post any notices or take any other action upon or with respect to the Premises that is or may be permitted by las to prevent the attachment of any such liens to the Premises; provided, however, that the tailure of Lessor to take an such action shall not relieve Lessee of any obligation or liability under this or any other paragraph hereot.

9. Lessee shall at all times keep a space of six (6) feet from the nearest rail of any railroad track entiraly clea of structures, material and obstructions of every sort and shall observe an overhead clearance of not less than tirenty five (25) feet above the top of rail; but, nevertheless, for convenience in handling freight to and from cars on an railroad track serving the Premises, the Lessee may install, use and maintain (a) loading or unloading cranes $c$ other devices not nearer than six (6) feet from the nearest rail of such track and no part of which shall at any iim project or extend in the direction of such track except when crane or device is being used for loading or unloadin freight and (b) platforms which shall be not more than three (3) feet and six (6) inches higher than the top of th rails, and which at no point shall be nearer than four ( $t$ ) feet to the nearest side of the head of the nearest rail c such track; provided, however, if by statute or order of competent public authority different clearances shall be re quired, then Lessee shall strictly comply with such statute or order.

10. Lessee agrees to indemnify and save harmless Lessor against all loss, damage or expense which Lessor ma sustain, incur or become liable for, including loss of or damage to property or injury to or death of persons and fin or penalties imposed upon or assessed against Lessor, arising in any manner out of (a) the use of the Premises c Improvements by Lessee, (b) any breach by Lessee of the terms, covenants or conditions in this instrument containec or (c) the sole or contributing acts or omissions of Lessee or the employes, agents, patrons or invitees of Lessee in, o or about the Premises or Improvements, except that if Lessor shall participate in any such contributing acts or omi sions, then the loss, damage or expense arising therefrom shall be borne by the parties hereto equally; provide however, that Lessee hereby assumes the risk of, and agrees to indemnify Lessor against liability for, loss of or damas to the property of Lessee or of others upon the Premises (except any rolling stock or shipments in the course of tran portation and except any property of Lessor or others placed or kept on the Premises pursuant to paragraph 2 hereo due to fire communicated from locomotives while being operated by Lessor upon any tracks within or in the vicinit of the Premises, regardless of Lessor's negligence, if any.

11. Neither Lessee, nor the heirs, legal representatives, successors or assigns of Lessee, nor any subseque] assignee, shall underlease or sublet the Premises or the Improvements, or any part thereof, nor assign or transf this lease or any interest herein, without the written consent and approval in each instance of Lessor.

12. In case of the èviction of Lessee by anyone owning or claiming title to or any interest in the Premise Lessor shall not be liable to Lessee for any damage of any nature whatsoever, or to refund any rental paid hereund $\epsilon$ except the proportionate part of any rental paid in advance.

13. If any rental hereunder shall be due and unpaid, or if default shall be made in any of the covenants or agre ments of Lessee herein contained, or in case of any assignment or transfer of this lease by operation of law, Less may, at its option, terminate this lease by serving five (5) days' notice in writing upon Lessee; but any waiver Lessor of any default or defaults shall not constitute a waiver of the right to terminate this lease for any subseque default or defaults.

14. This lease may be terminated at any time by either party by serving thirty (30) days' written notice termination upon the other party, stating therein the date that such termination shall take place, and upon the piration of the time specified in such notice this lease and all rights of Lessee hereunder shall absolutely cease a determine; but upon any such termination Lessee shall be entitled to have refunded by Lessor a proportionate $\mathrm{p}$ of any rentals paid in advance.

15. Any notice to be given by Lessor to Lessee hereunder shall be deemed to be properly served if the same delivered to Lessee, or if left with any of the agents, servants or emplayes of Lessee, of if posted on the Premises, if deposited in the Post Office, postpaid, addressed to Lessee at.....Navar.ro..... Kansa. 8

16. Upon the termination of this lease in any manner herein provided, Lessee shall forthwith surrender to Les the possession of the Premises and shall remove the Improvements and restore the Premises to substantially state in which they were prior to the construction of the Improvements, and in case Lessee shall fail within thirty ( days after the date of such termination to make such removal or restoration, then Lessor may, at its election to exercised within thirty (30) days thereafter, either remove the Improvements and restore the Premises for the acco of Lessee, and in such event Lessee shall within thirty (30) days after the rendition of bill therefor reimburse Le: for the cost so incurred, or may take and bold the Improvements as its sole property. 
of Lessee, and in such event Lessee shall within thirty (30) days after the oun s cowse uue r remises ror tne accol or the cost so incurred, or may take and hold the Improvements as its solodition of bill therefor reimburse Les the Improvements as its sole property.

17. If Lessee fails to surrender to Lessor the Premises, upon any- termination of this lease, all the liabilitie and obligations of Lessee hereunder shall continue in effect until the Premises are surrendered; and no terminatio hereof shall release Lessee from any liability or obligation hereunder, whether of indemnity or otherwise, resultin ments are removed and the Premises restored or Lessor elects to termination or the date, if later, when the Improve as hereinabove in paragraph 16 provided.

18. In the event that Lessee consists of two or more parties, all the covenants and agreements of Lessee hereir contained shall be the joint and several covenants and agreements of such parties.

19. All the covenants and agreements of Lessee herein contained shall be binding upon the heirs, legal representatives, successors and assigns of Lessee, and shall inure to the benefit of the successors and assigns of Lessor.

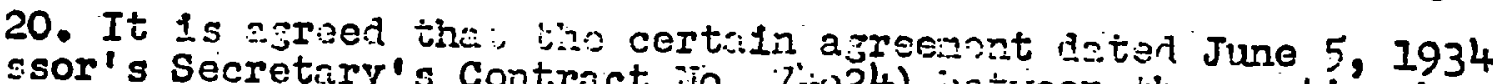
(Lessor's Secretary's Contract io. 7 ':024) jetreen 'he parties hereto, Ielatinj to tile use of $a$ portion ch Lecsor's property at Jlevarre, oll st.:. as of the dete hereof. $\ldots$ ming, is hereby timinated effecive

Approved as to description:

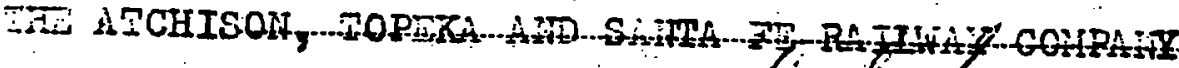
(Lessc and year first above written.

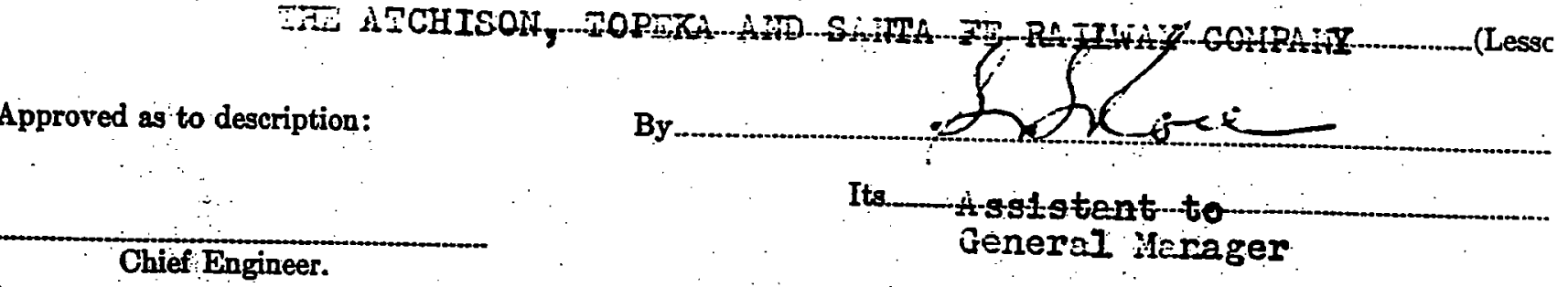

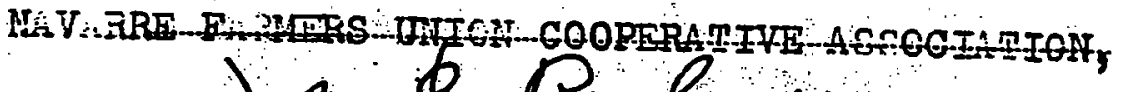

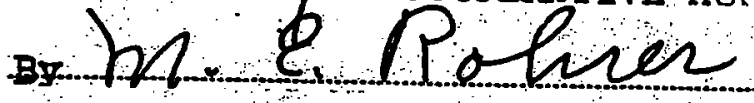




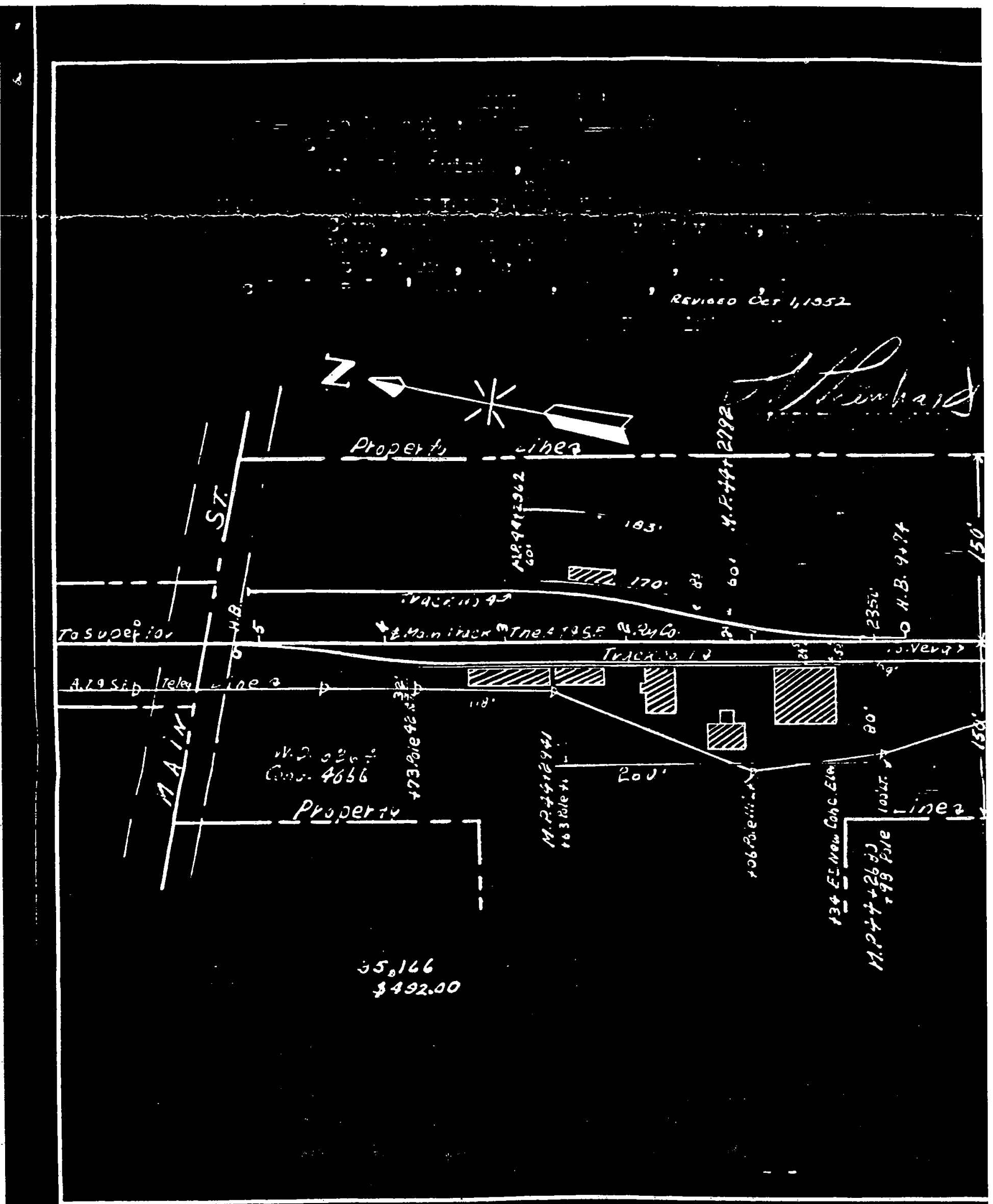


CATOETHATOK AND 8OBBHNDBR

The parties mutually agree that the foregoing lease is hereby cancelled as of the. das of 19............, and the premises therein described surrendered to the Lessor.

Dated this. dey of 19

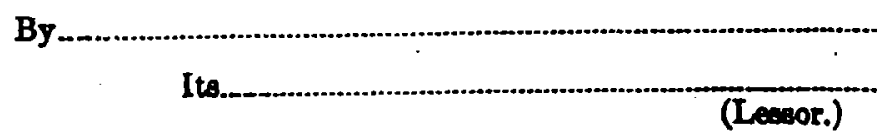

(Lesser.)
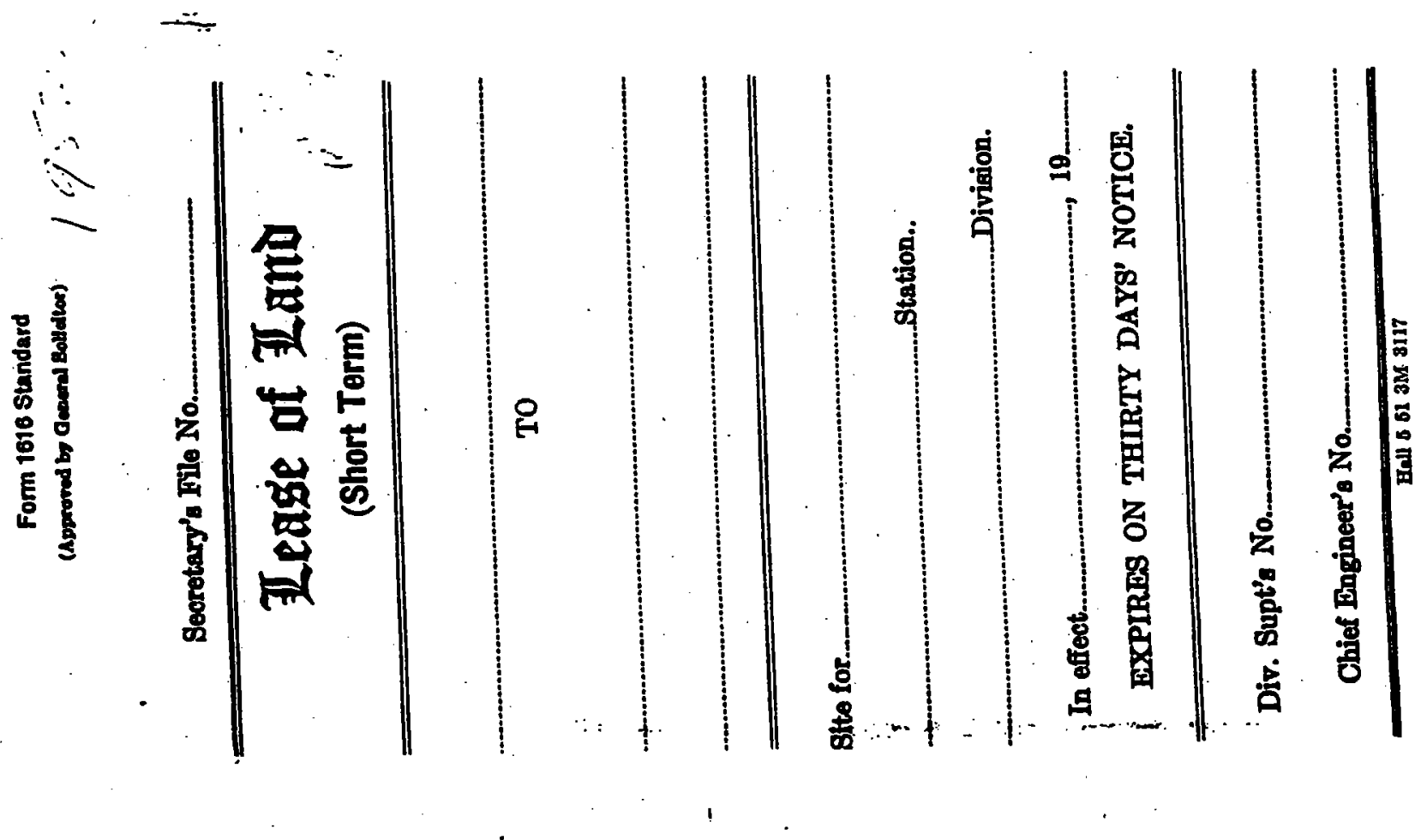


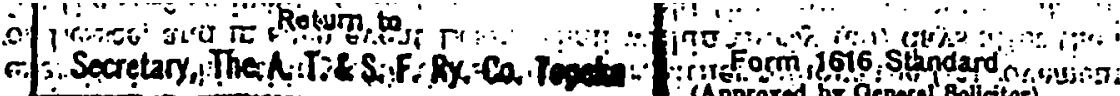

i

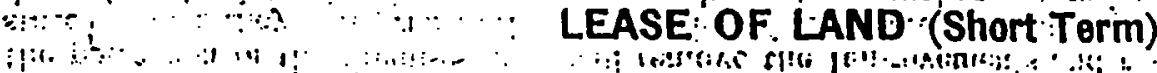

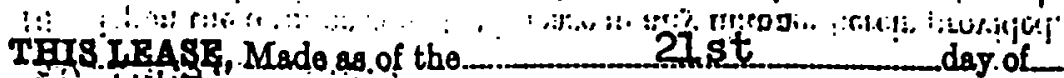
day. of June

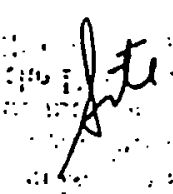

1. ND SANPA FE RAILWAY COMPANY Kansas

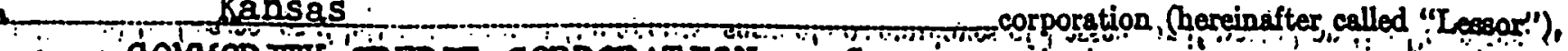

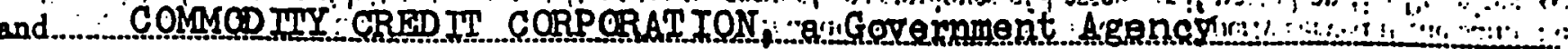
Chereinafter; whether one party or more; called "Lespes") WITKESSETE; That the parties hereto for the considerations bereinafter expreseed covenant and agree as

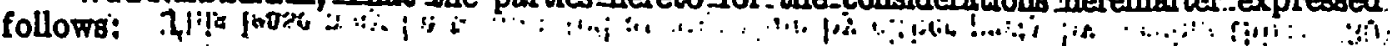

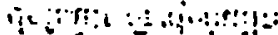

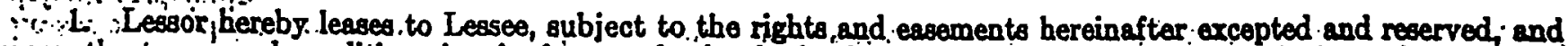
upon the-terms and conditions hereinafter set. forth, the iland (hereinafter called "'Premises") situated at :or near Navarre

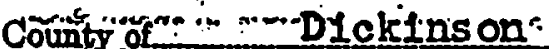
Stato of Känges outlined in red coloring on the print hereto attached,

NODEO:ST-I-259 dated

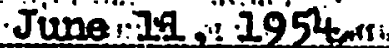

Tin tiates of dated. marked "Fxhibit Al" and made a part bereof, for a term beginning of

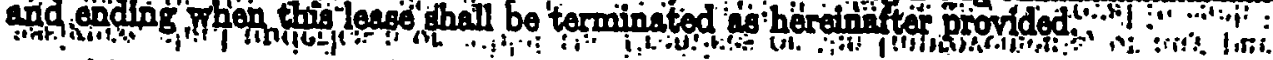

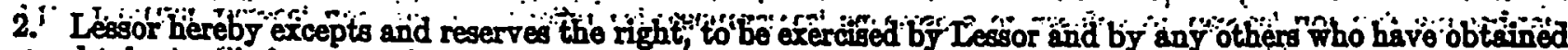

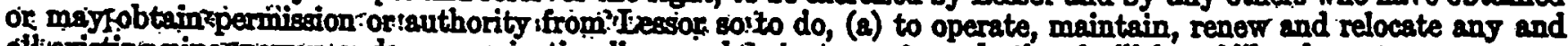

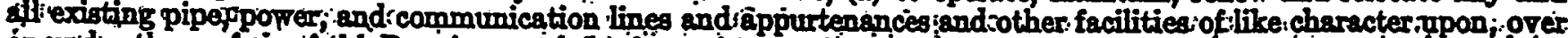

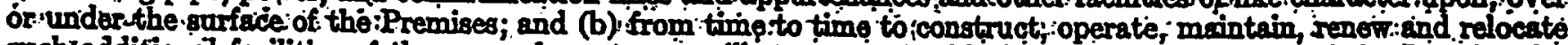
such additional facilities of the same character as will pot unreasonably. interfere with the use of the Premises by

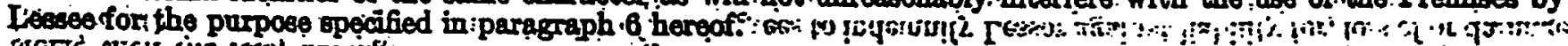

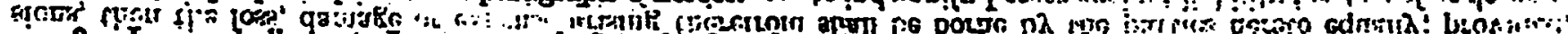

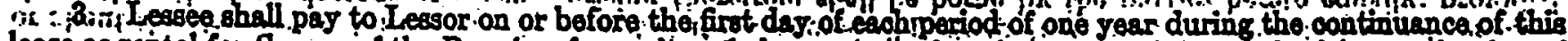

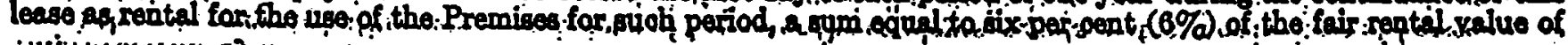

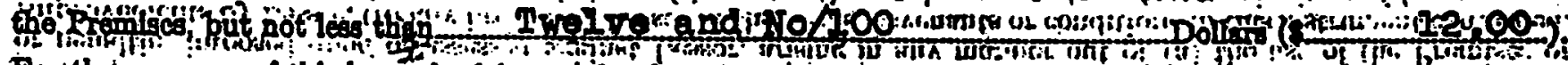

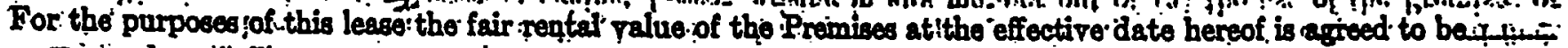

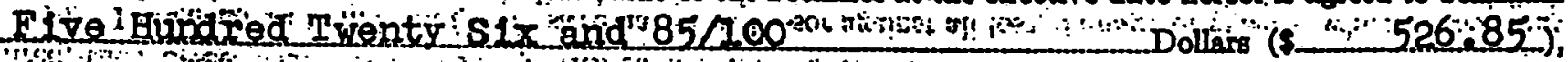
and theinitial rental eball be Thirty one and $61 / 100$

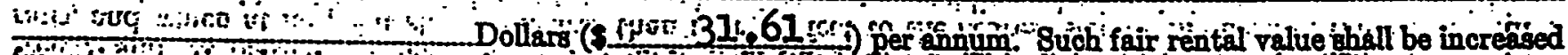

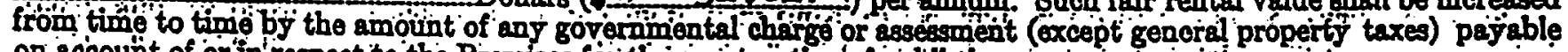
on accoupt of or in reopeot to tho Premises for the corigtriotion of publio improviomonts.

13!4'4 Lesseescovenants and warrants that Lesses either owns; or has obtained from the owner or owners thereof

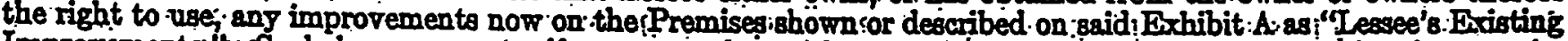
Improvements: ; i Such :improvements; if iang; together : with any. other improvements :hereafter placed :upon the

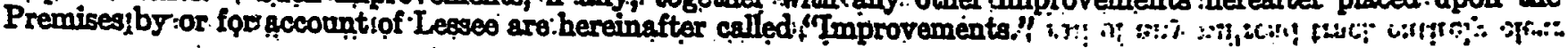

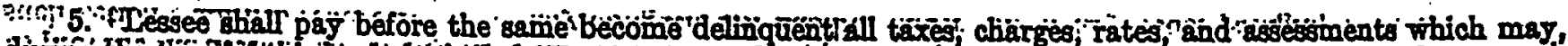

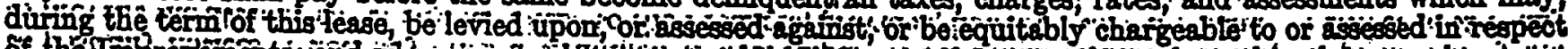

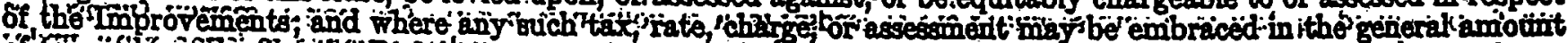

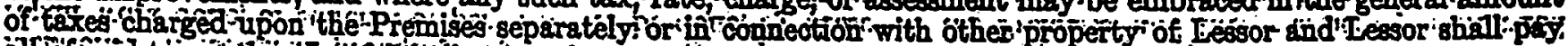

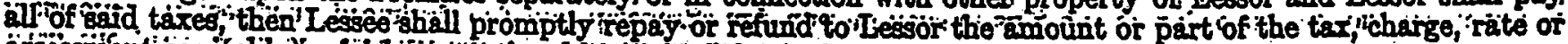

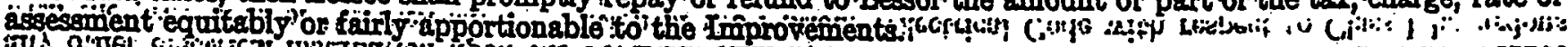

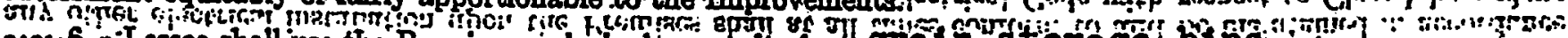

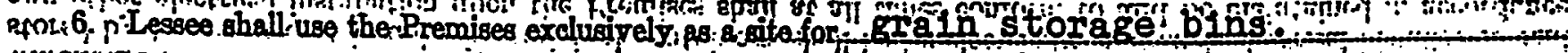

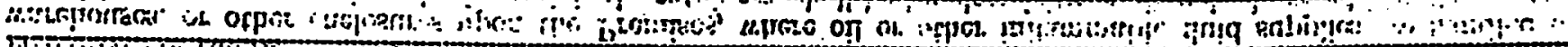

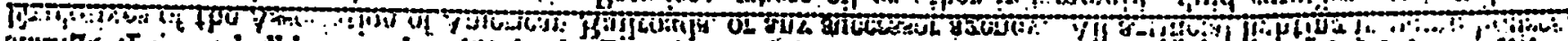

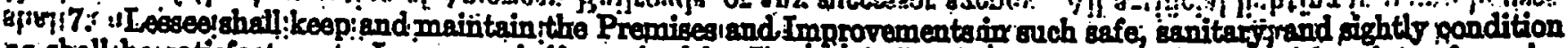

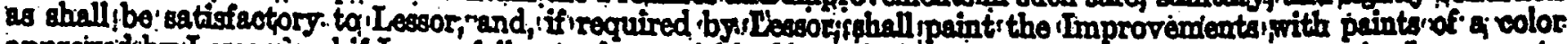

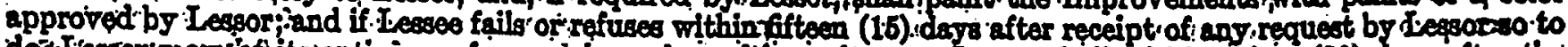

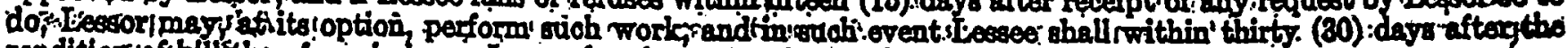

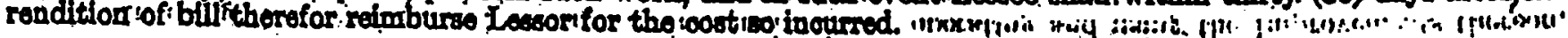




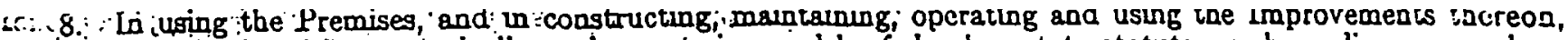
Lesseo ahall tcomply! with: any and sill. requirements imposed by federal or state statutes, or: by ordinances, orders, or:iregulationg of- any governmental body -having jurisdiction thereover. In the event the:Premises or Improve-

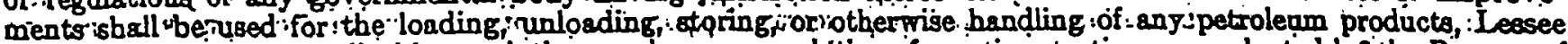
shall comply:with:all applicable regulations and recommendations from: time to time promulgated by the. Bureau of Explosives of the Association of American Railrogds, or any successor agency. All artificial lighting in pump houses, warehouses, or other oncloyures upon the Premises, where oil or othor infammable fluid supplies are handled or

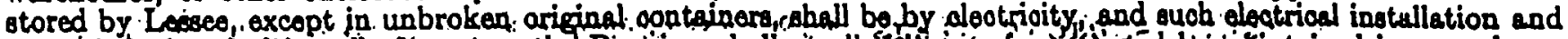
any other electrical installation upon tho Preraises shall at all tifíes conform to ańd be trikintained in accordance

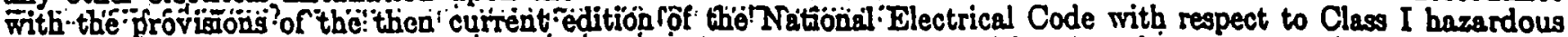

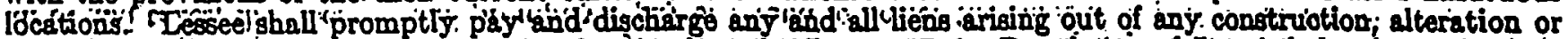

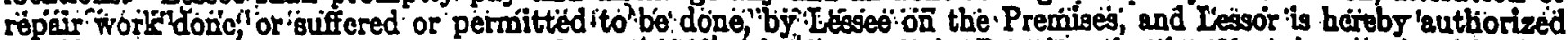

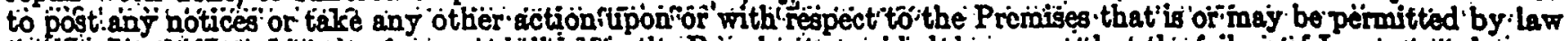

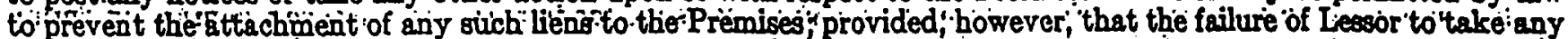

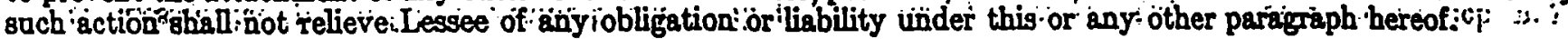

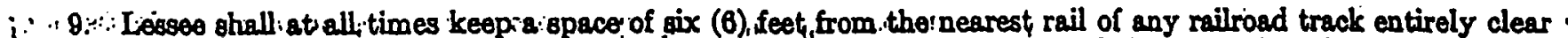
of structures; material rand obstructions of every sort and shall observe an overhoad olearance of:not leas.than twenty-

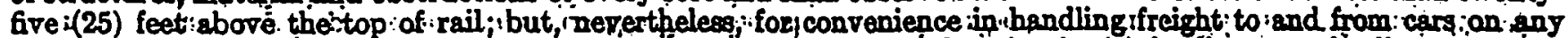

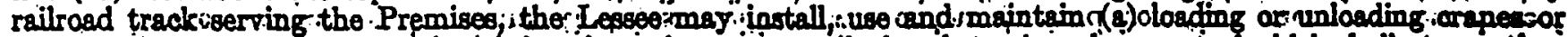
other devjos not nearer than six (6) feet from the nearest rail of euch track and no part of which ahall-at any timo project or extend in the direction of such track except when crane ö device is being used for loading or unloading

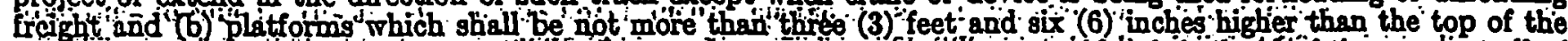

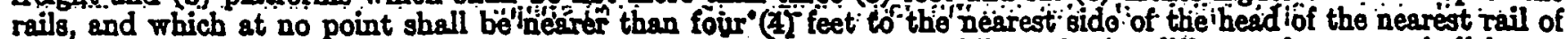

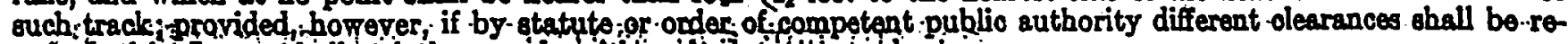
quired, then Lessee shall strictly comply with' zud 'otatutb'or order.

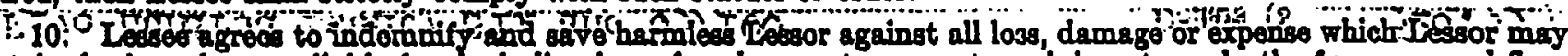

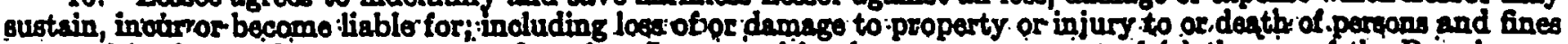
or penalties jmposed upon or essessed against Lessor, grising in any manner out of (a) the use of the Premises or

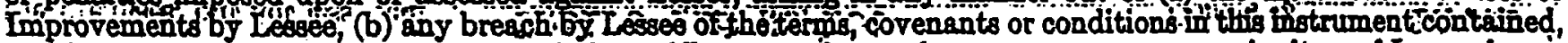

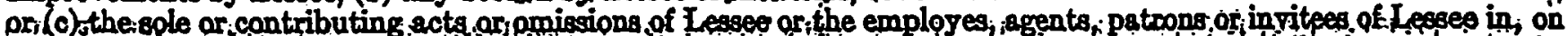

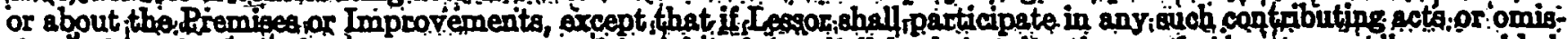
sions, then the lose, damage or expense arising therefrom shill be borne by the parties hereto equally; provided, however; that Lessee hereby. assumes thë:riak of, and agrees to indemnify Lessor against liability for, loss of or damage to the property of Tessee or of others upon:the:Premises:(excepteang. rolling stock: or ghipments:in the coumse: of tranis-

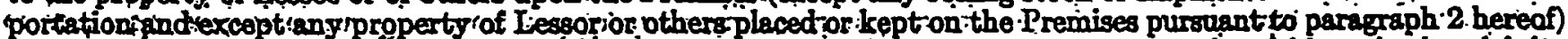

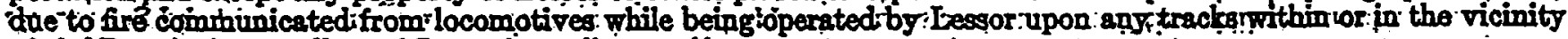

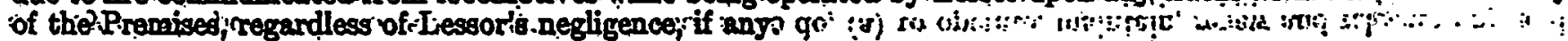

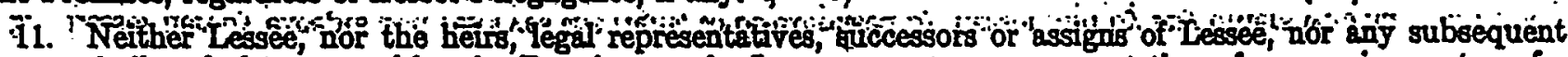
asgignee, shall underlease or sublet the Premises or the Improvements, or any part thereof, nor assign or transfer

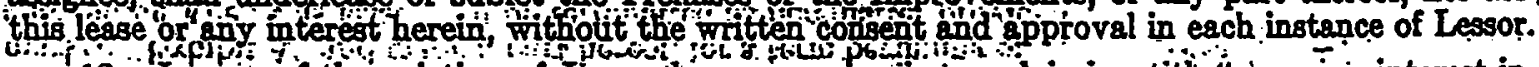

12. In case of the eviction of Iessee by anyone owning or claiming title to or siny interest in the Premises,

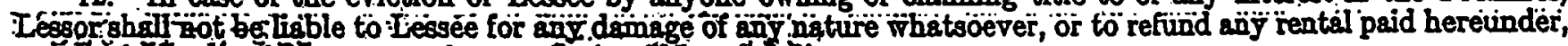
except the.proportionate part of any rental paid in advance.

13. If 5 . ments of Lessec berem-contained, or in case of any-assienment or transfer iof thisilease by operation of law, Lessor

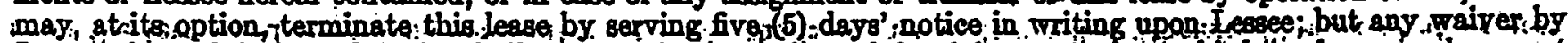

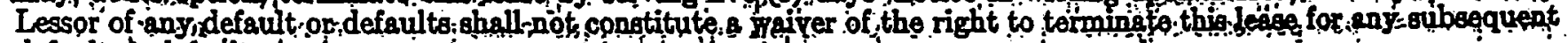
default or defailts.

ir. 14: This lease may be terminated at any time by either party by serving thirty (30) days' written notice of termination' ipon'the other party, 'stating therein' the date that ouch termination shall take place, and upon the expiration of the time specified in such notice this lease and all rights of Lessee bereunder sball absolutely cease and

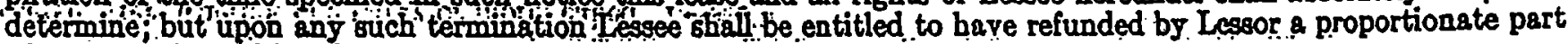
of any rentals paid in advance.

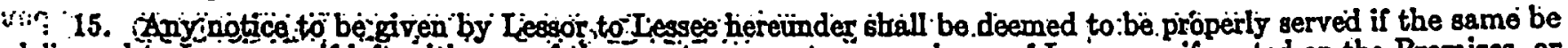
:delivered to.Lesses or if left with any of the.agents, iervants or employes of Lessee, or if posted on the Premises, or jif depogited in. the. Post. Office, postpaid addressed to lesseo at
Abit

16. Upon the termination of this lease in ah manner herein provided, Lessee iball forthwith surrender to the possession of the Premises and eball remove the Improvements and restore the Premises to substantially the state in which they were prior to the conjiguctiog of thie'fipprovenfeints, aridiin case Lessee shall fail within thirty (30) days after the dato of such, termination to:make such remoral or restoration, then Lessor may, at its election to be exercised rithinathirty: (80) days thereafter, either remors. the Improviements and restore the Premises for the account of Lessce, and in euch event Lessee shall within thijty (30) days after the rendition of bill therefor reimburse Lessor fof the coot 80 incurred, of may take and hold the Improvements as its sole property.

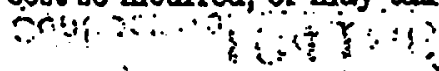

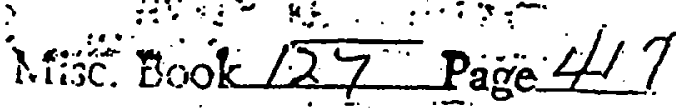




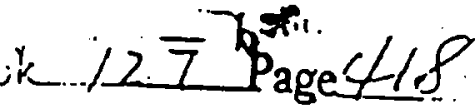

17. If Lesses fails to surrender to Lessor the Premises, upon än. termination of this lease, all the lisbilities and obligations of Lessee hereunder shall continue in effect until the Piemises are surrevidered; and no termination hereof shall release Lessec from any liability or obligation hereunder, whether of indemnity or otberwise, rejulting from any acta, omisaions or events happening prior to the date of termination or the date, if later; when the Improvements are remored and the Premises restoredor Lessor elects to take and hold the Improvemento as its sole property

18. In the event that Lessee consists of tiro or more parties, all tho covenants and agreements of Lessee herein contained shall bo the joint and several covenants and agreements of such.parties.

19. All the covenants and agreements of Lessee herein contained shäl be binding upoị the heirs, legal representatives, succesiors and assigns of Lessee, and shall inure to the benchit of the successors and assigns of Lessor.

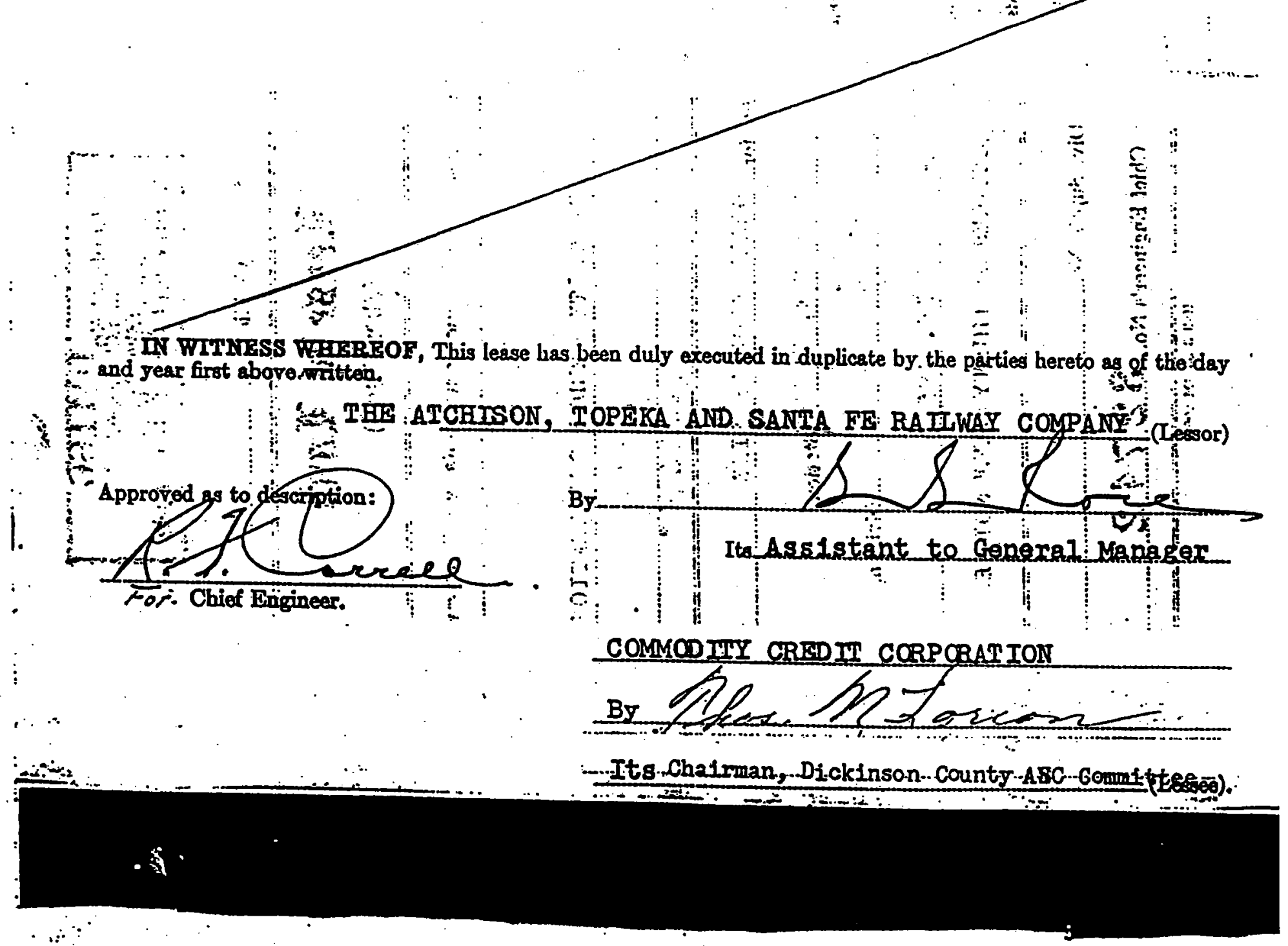

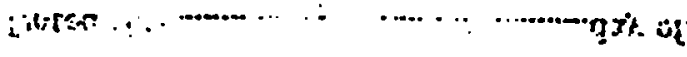

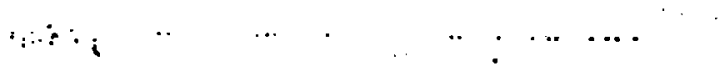

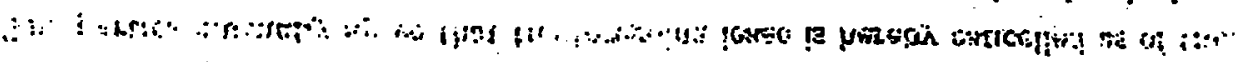

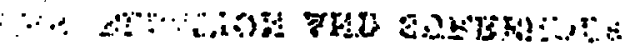

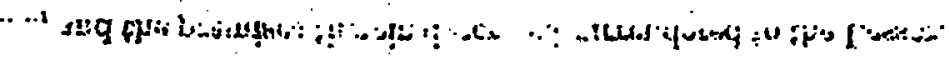




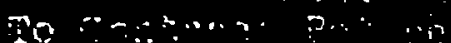

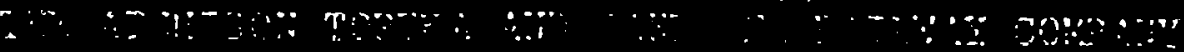

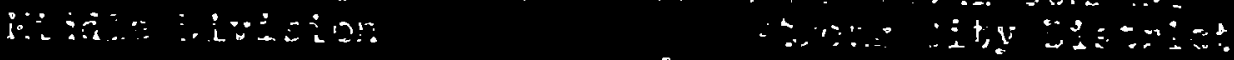

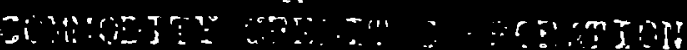

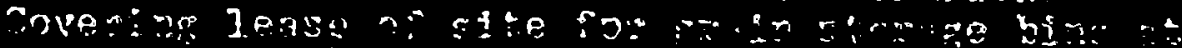

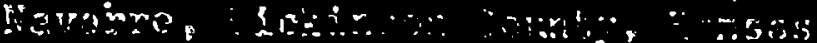

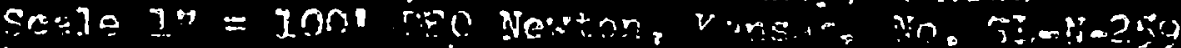

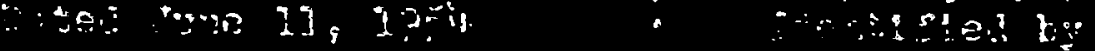

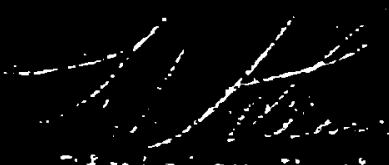

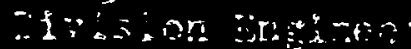
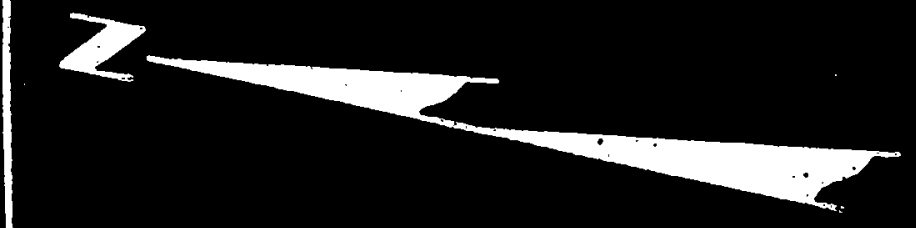

NE.1/4 SEC. 33 T.14S.R.3E.

Navarre, Kans.

E
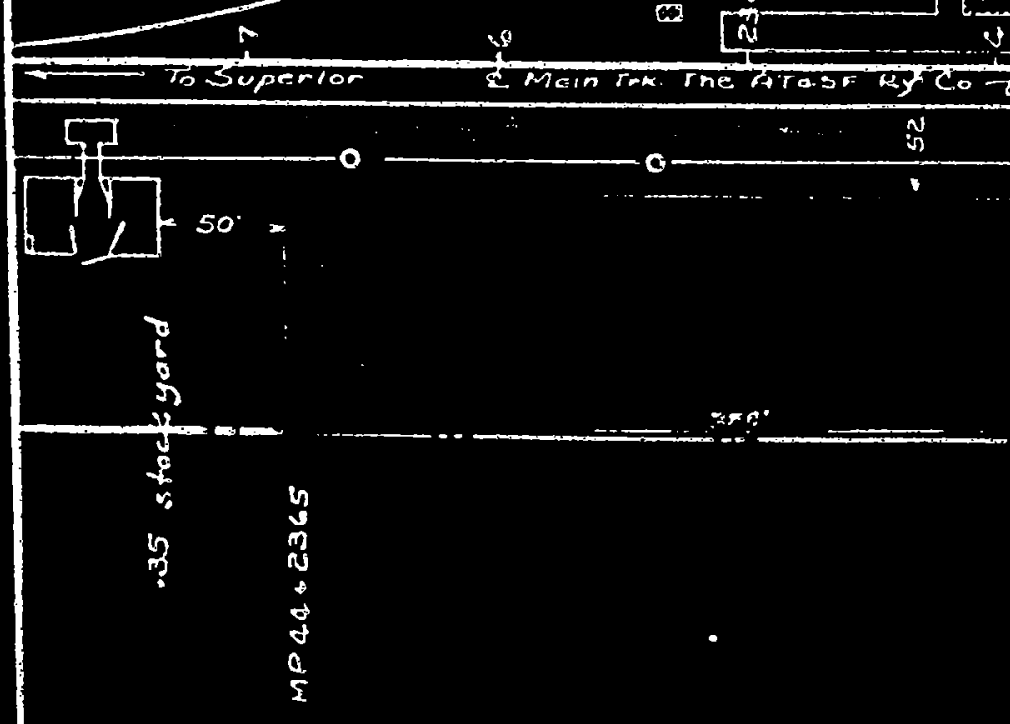
$\approx \times p^{\circ}$

$y$
$y$
in
0
0
0
$c$

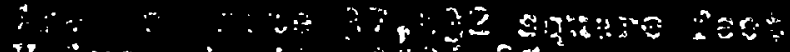

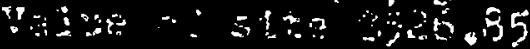


IN WITNESS WHERFOF, the parties hereto have executed this supplemental Agreement in duplicate the day and jear first above written.

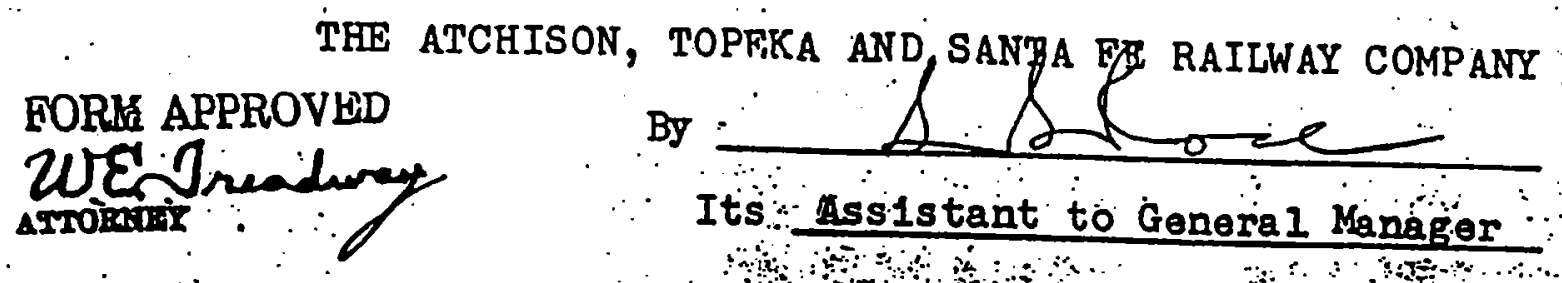

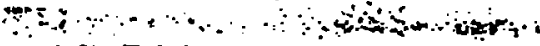

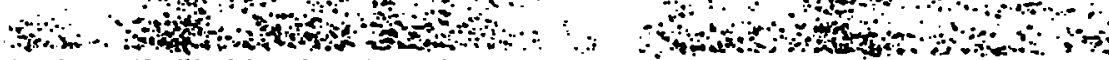

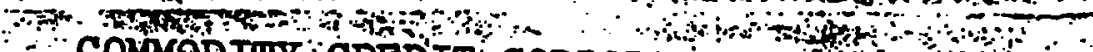
COMMODITY CREDIT CORPORATION AA

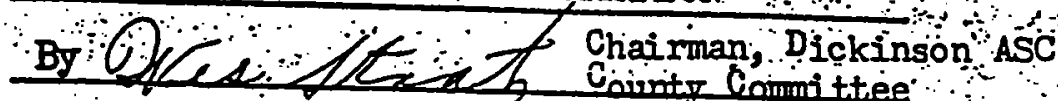

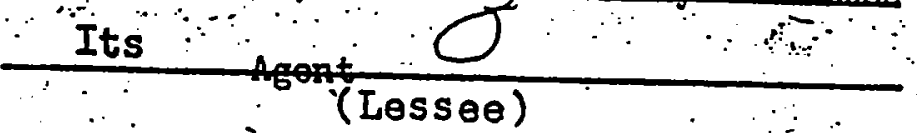
40

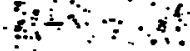

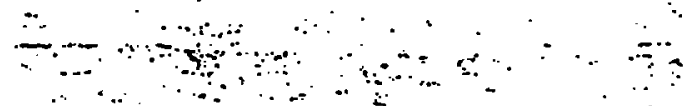

$\sin \sin \theta$

$\therefore$

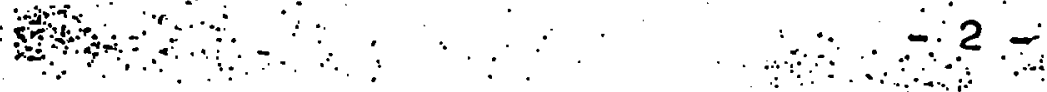


8. In using the Premises, and in constructing, maintaining, operating and using the Improvements thereo Iessee shall comply with any and all requirements imposed by federal or state atatutes, or by ordinances, orde or regulations of any governmental body having jurisdiction thereover. In the event the Premises or Improv ments shall be used for the loading, unloading, storing, or otherwise handling of any petroleum products, Improv shall comply with all applicable regulations and recommendations from time to time promulgated by the Bureau Explosives of the Association of American Railroads, or any successor agency. All artificial lighting in pump hau warehouses, or other enclosures upon the Premises, where oil or other inflammable fluid supplies are handled stored by Lessee, except in unbroken original containers, shall be by electricity, and such electrical installation any other electrical installation upon the Premises shall at all times conform to and be maintained in accordan with the provisions of the then current edition of the National Electrical Code with respect to Class I hazardo locations. Lessee shall promptly pay and discharge any and all liens arising out of any construction, alteration repair work done, or suffered or permitted to be done, by Lessee on the Premises, and Lessor is hereby authoriz to post any notices or take any other action upon or with respect to the Premises that is or may be permitted by ls to prevent the attachment of any such liens to the Premises; provided, however, that the failure of Lessor to take a such action shall not relieve Lessee of any obligation or liability under this or any other paragraph hereof.

9. Lessee shall at all times keep a space of six (6) feet from the nearest rail of any railroad track entirely cle of structures, material and obstructions of every sort and shall observe an overhead clearance of not less than twent five (25) feet above the top of rail; but, nevertheless, for convenience in handling freight to and from cars on a railroad track serving the Premises, the Leseee may install, use and maintain (a) loading or unloading cranes other devices not nearer than six (6) feet from the nearest rail of such track and no part of which shall at any ti project or extend in the direction of such track except when crane or device is being used for loading or unloadi freight and (b) platforms which shall be not more than three (3) feet and six (6) inches higher than the top of $t$ rails, and which at no point shall be nearer then" four (4) feet to the nearest side of the head of the nearest rail wuch track; provided, however, if by atatute or order of competent public authority different clearances shall be quired, then Lessee shall strictly comply with such statute or order.

10. Lessee agrees to indemnify and save harmless Lessor against all lose, damage or expense which Lessor $\mathrm{m}$ surtain, incur or become liable for, including loss of or damage to property or injury to or death of persons and fir or penalties imposed upon or assessed against Lessor, arising in any manner out of (a) the use nf the Premises Improvements by Lessee, (b) any breach by Lessee of the terms, covenants or conditions in this instrument contain or (c) the sole or contributing acts or omissions of Lessee or the employes, agents, patrons or invitees of Lessee in, or about the Premises or Improvements, except that if Lessor shall participate in any such contributing acts or om gions, then the los, damage or expense arising therefrom shall be borne by the parties hereto equally; provide however, that Lessee hereby assumes the risk of, and agrees to indemnify Lessor against liability for, loss of or dama to the property of Lessee or of others upon the Premises (except any rolling stock or shipments in the course of tra portation and except any property of Lessor or others placed or kept on the Premises pursuant to paragraph 2 here due to fire communicated from locomotives while being operated by Lessor upon any tracks within or in the vicin of the Premises, regardlese of Lessor's negligence, if any.

11. Neither Lessee, nor the heirs, legal representatives, successors or assigns of Lessee, nor any subseque assignee, shall underlesse or aublet the Premises or the Improvements, or any part thereof, nor assign or trans this lease or any interest herein, without the written consent and approval in each instance of Leasor.

12. In case of the eviction of Lessee by anyone owning or claiming title to or any interest in the Premis Lessor shall not be liable to Lessee for any damage of any nature whatsoever, or to refund any rental paid hereund except the proportionate part of any. rental paid in adyance

13. If any rental hereunder shall bë due and unpaid, or if default shall be made in any of the covenants or ag ments of Lessee'herein contained, or in case of any asgignment or transfer of this lease by operation of law, Les may, at its option, terminate this lease by serving five. (5) day' notice in writing upon Lessee; but. any waiver Lessor of any default or defaults shall not constitute a waiver of the right to terminate this lease for any subsequ default or defaults.

14. This lease may be terminated at any time by either party by serving thirty (30) days' written notice termination upon the other party, stating therein the date that such termination shall take place, and upon the piration of the time specified in such notice this lease and all rights of Lessee hereunder shall absolutely cease determine; but upon any such termination Lessee ahall be entitled to have refunded by Lessor a proportionate $\mathrm{p}$ of any rentals paid in advance.

15. Any notice to be given by Lessor to Lessee hereunder shall be deemed to be properly served if the esme delivered to Lessee, or if left with any of the agents, servants or employes of Lesees, or if posted on the Premises

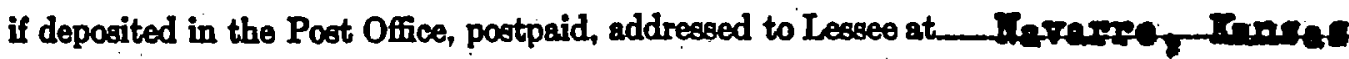

16. Upon the termination of this lease in any manner herein provided, Lessee shall forthwith surrender to Ler the possession of the Premises and shall remove the Improvements and restore the Premises to substantially state in which they were prior to the construction of the Improvements, and in case Lessee shall fail within thirty days aftor the date of such termination to make such remoral or restoration, then Lessor mas, at its election $t$ exercised within thirty (30) days thereafter, either remove the Improvements and restore the Premises for the acc of Lessee, and in such event Lessee shall within thirty. (30) days after the rendition of bill therefor reimburse Le for the cost 80 incurred, or may take and bold the Improvements as its sole property. 


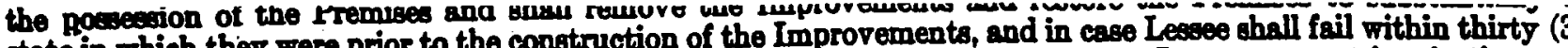
state in which they were prior to the construction of the Improvements, andin case Leser may, at its election to dayt aftor the date of such terminstion to make such removal or restorstion, then lasor ereroised within thirty (30) days thereafter, either remove the Improvements and restore the of Lessee, and in such event Leseee shall within thirty (30) days after itse rendition of

17. If Leesee fails to surrender to Leseor the Premises, upon any termination of this lease, all the liabilit and obligations of Lesse hereunder shall continue in effect until the Premises are surrendered; and no terminati hereof shall release Lessee from any liability or obligation hereunder, whether of indemnity or otherwise, result from any acta, omissions or events happening prior to the date of termination or the date, if later, when the Impro ments are removed and the Premises restored or Lessor elects to take and hold the Improvements as its sole prope as herejoabove in paragraph 16 provided.

18. In the event that Lesgee consists of two or more parties, all the covenants and agreements of Leasee her contained shall be the joint and several covenants and agreements of such parties.

19. All the covenants and agreements of Lessee herein contained shall be binding upon the heirs, legal rep sentatives, successors and assigns of Lesee, and shall inure to the benefit of the successors and assigns of Less

20. That in the use of the Premises, Iossee shall exercise utmost a extraordinary diligonce to the ond that no damage shall occur to Iossc communication Iine located upon the Premlsos, and Losseo heroby agrees to pay Inssor within twonty (20) days after fendition of bill therefor the entire cost of repaling any damage to sald communcetion ilne reeulting in any manner from or In connection with lassee's use of the Premises.

21. It 1s agreed that the agreement dated Ootober 8, 1952, (Lossor's socretary's Contraot No. 1014II) between the part1es hereto, relating the use or a portion of Inssor's property at Navarre, Dicidinson County rangs, as a site for elevator, cosi bins, bulk o1I station and other butldings is hereby terminated as of the effeotive date horeof.

If WTIRESS WHEREOF, This lease has been duly executed in duplicate by the parties hereto as of the $d$ and year first above written.

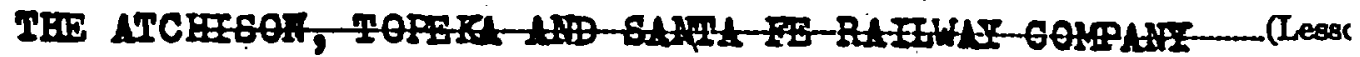

Approved as to description:

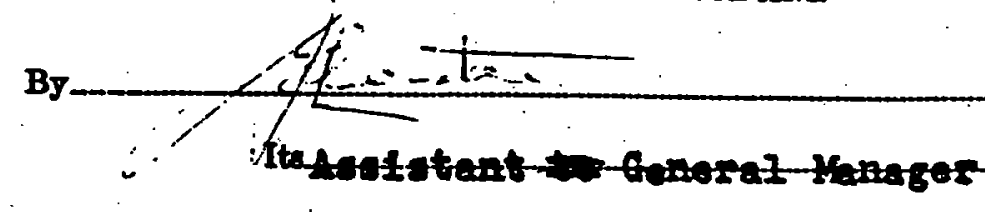

Chief Engineer.

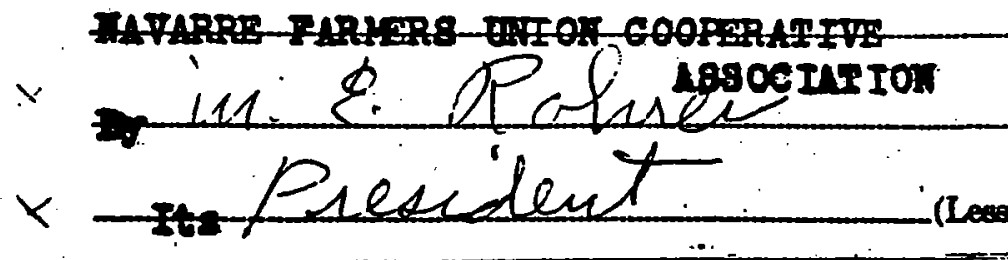




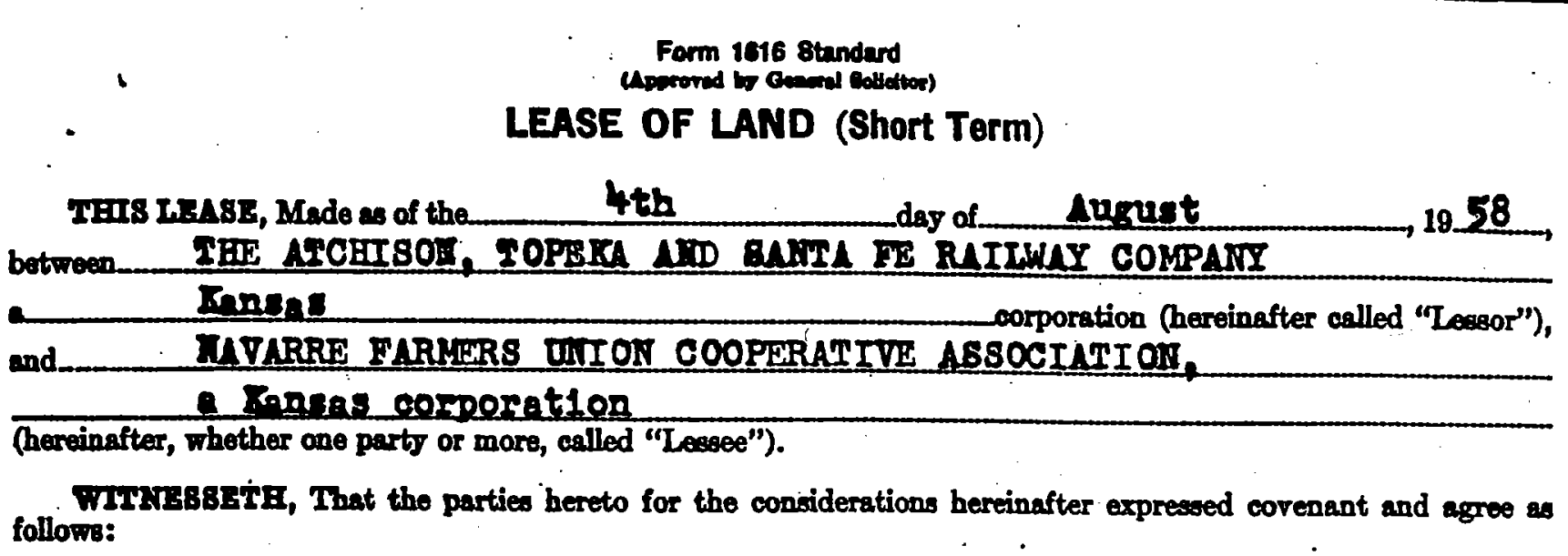

1. Inesor bereby leages to Leasee, eubject to the rights and easements hereinsfter excepted and reserved, and upon the terms and conditions hereinafter set forth, the land (hereinafter called "Premiges") situated at or near Eevarse County of Diokinson

State of Insge outlined in red coloring on the print hereto attached,

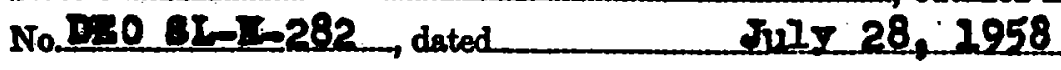

marked "Exhibit A" and made a part hereof, for a term beginning on Lovember $18,10.52$, and ending when this lease shall be terminated as hereinafter provided.

2. Leasor hereby excepts and reserves the right, to be exercised by Leasor and by any others who have obtained or msy obtain permission or suthority from Lessor 80 to do, (a) to operate, maintain, renew and relocate any and Il existing pipe, power, and communication lines and appurtenances and other facilities of like character upon, over or under the surface of the Premises; and (b) from time to time to construct, operate, maintain, renew and relocate guch idditional facilities of the game character as will not unreasonably interfere with the use of the Premises by Lessee for the purpose specified in paragraph 6 hereof.

8. Lessee shell pay to Lessor on or before the first day of each period of one year during the continuance of this lease is rental for the use of the Premises for such period, s sum equal to six per cent (6\%) of the fair rental value of the Premises, but not less than Irenty-I Ive and I0/100 Dollars ( 825.00

For the purposes of this lease the fair rental value of the Premises at the effective date hereof is agreed to be

Bit Bundred Eightr-51x and $10 / 200$ and the initial rental shall be Forty-one and $16 / 100$ Dollars $(\$ 686.00$ Dollars ( $\$ 41.16$ Dollars ( $\$ 41.16 \quad$ from time to time by the amount of any governmental charge or assessment (except general property taxes) payable on account of or in respect to the Premises for the construction of public improvements.

4. Lessee corenants and warrants that Lessee either owns, or has obtained from the owner or owners thereof the right to use, any improvements now on the Premises shown or described on said Exhibit A as "Lessee's Existing Improvements." . Such improvements, if any, together with any other improvements hereafter placed upon the Premises by or for account of Lessee are hereinafter called "Improvements."

5. Lesee shall pay before the same become delinquent all taxes, charges, rates, and assessments which may, during the term of this lease, be levied upon, or assessed against; or be equitably chargeable to or assessed in respect of taxes charged upon the Premises such tax, rate, charge, or assessment may be embraced in the general amount all of said taxes, then Lessee shall preparately or in connection with other property of Lessor and Lessor shall pay assessment equitably or fairly apportionsty repay or refund to Lessor the amount or part of the tax, charge, rate or assessment equitably or fairly apportionable to the Improvements.

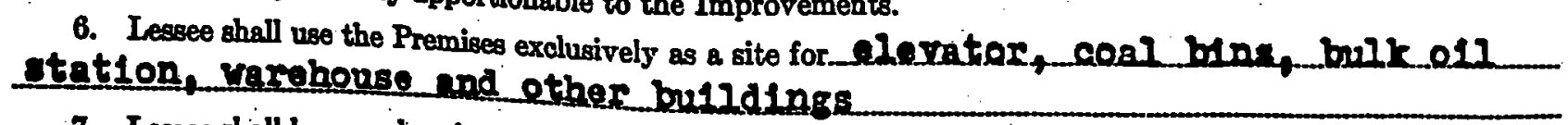

7. Lessee shall keep and maintain the Premises and Improvements in such safe, sanitary, and sightly condition as thall be satisfactory to Lessor, and, if required by Lessor, shall paint the Improvements with paints of a color do, Lessor may, at its option, perform such work, readition of bill therefor reimburse Leasor for the oost in such incuent Lessee shall within thirty (30) days after the 
8. In using the Premises, and in constructing, maintaining, operating and using the Improvements thereon, Lessee shall comply with any and all requirements imposed by federal or state statutes, or by ordinances, orders, or regulations of any governmental body having jurisdiction thereover. In the event the Premises or Improvements shall be used for the loading, unloading, storing, or otherwise handling of any petroleum products, Lessee shall comply with all applicable regulations and recommendations from time to time promulgated by the Bureau of Explosives of the Association of American Railroads, or any successor agency. All artificial lighting in pump houses, warehouses, or other enclosures upon the Premises, where oil or other inflammable fluid supplies are handled or stored by Lessee, except in unbroken original containers, shall be by electricity, and such electrical installation and any other electrical installation upon the Premises shall at all times conform to and be maintained in accordance with the provisions of the then current edition of the National Electrical Code with respect to Class I hazardous locations. Lessee shall promptly pay and discharge any and all liens arising out of any construction, alteration or repair work done, or suffered or permitted to be done, by Lessee on the Premises, and Lessor is hereby authorized to post any notices or take any other action upon or with respect to the Premises that is or may be permitted by law to prevent the attachment of any such liens to the Premises; provided, however, that the failure of Lessor to take any such action shall not relieve Lessee of any obligation or liability under this or any other paragraph hereof.

9. Lessee shall at all times keep a space of six (6) feet from the nearest rail of any railroad track entirely clear of structures, material and obstructions of every sort and shall observe an overhead clearance of not less than twentyGive (25) feet above the top of rail; but, nevertheless, for convenience in handling freight to and from cars on any railroad track serving the Premises, the Lessee may install, use and maintain (a) loading or unloading cranes or other devices not nearer than six (6) feet from the nearest rail of such track and no part of which shall at any time project or extend in the direction of such track except when crane or device is being used for loading or unloading freight and (b) platforms which shall be not more than three (3) feet and six (6) inches higher than the top of the rails, and which at no point shall be nearer than four (4) feet to the nearest side of the head of the nearest rail of such track; provided, however, if by statute or order of competent public authority different clearances shall be required, then Lessee shall strictly comply with such statute or order.

10. Lessee agrees to indemnify and save harmless Lessor against all loss, damage or expense which Lessor may sustain, incur or become liable for, including loss of or damage to property or injury to or death of persons and fines or penalties imposed upon or assessed against Lessor, arising in any manner out of (a) the use of the Premises or Improvements by Lessee, (b) any breach by Lessee of the terms, covenants or conditions in this instrument contained, or (c) the sole or contributing acts or omissions of Lessee or the employes, agents, patrons or invitees of Lessee in, on or about the Premises or Improvements, except that if Lessor shall participate in any such contributing acts or omissions, then the loss, damage or expense arising therefrom shall be borne by the parties hereto equally; provided, howerer, that Lessee hereby assumes the risk of, and agrees to indemnify Lessor against liability for, loss of or damage to the property of Lessee or of others upon the Premises (except any rolling stock or shipments in the course of transportation and except any property of Lessor or others placed or kept on the Premises pursuant to paragraph 2 hereof) due to fire communicated from locomotives while being operated by -Lessor upon any tracks within or in the vicinity of the Premises, regardless of Lessor's negligence, if any.

11. Neither Lessee, nor the heirs, legal representatives, successors or assigns of Lessee, nor any subsequent assignee, shall underlesse or sublet the Premises or the Improvements, or any part thereof, nor assign or transfer this lease or any interest herein, without the written consent and approval in each instance of Lessor.

12. In case of the eviction of Lessee by anyone owning or claiming title to or any interest in the Premises, Lessor shall not be liable to Lessee for any damage of any nature whatsoever, or to refund any rental paid hereunder, except the proportionate part of any rental paid in advance.

13. If any rental hereunder shall be due and unpaid, or if default shall be made in any of the covenants or agreements of Lessee herein contained, or in case of any assignment or transfer of this lease by operation of law, Lessor may, at its option, terminate this lease by serving five (5) days' notice in writing upon Lessee; but any waiver by may, at its option, terminate this lease by servitu te waiver of the right to terminate this lease for any subsequen! default or defaults.

14. This lease may be terminated at any time by either party by serving thirty (30) days' written notice o termination upon the other party, stating therein the date that such termination shall take place, and upon the ex piration of the time specified in such notice this lease and all rights of Lessee hereunder shall absolutely cease anc determine; but upon any such termination Lessee shall be entitled to have refunded by Lessor a proportionate par of any rentals paid in adrance.

15. Any notice to be given by Lessor to Lessee hereunder shall be deemed to be properly served if the same $b_{1}$ delivered to Lessee, or if left with any of the agents, servants or emplojes of Lessee, or if posted on the Premises, o if deposited in the Post Office, postpaid, addressed to Lessee at_Lavarre, Kansas

16. Upon the termination of this lease in any manner herein provided, Lessee shall forthwith surrender to Lesso the possession of the Premises and shall remove the Improvements and restore the Premises to substantially th gtate in which they were prior to the construction of the Improvements, and in case Lessee shall fail within thirty (3C days after the date of such termination to make such removal or restoration, then Lessor may, at its election to b exercised within thirty (30) days thereafter, either remove the Improvements and restore the Premises for the accour of Lessee, and in such event Lessee shall within thirty (30) days after the rendition of bill therefor reimburse Lessc for the cost so incurred, or may take and hold the Improvements as its sole property. 
17. If Lessee fails to surrender to Lessor the Premises, upon any termination of this lease, all the liabilities and obligations of Lessee hereunder shall continue in effect until the Premises are surrendered; and no termination 1 hereof shall release Leesee from any liability or obligation hereunder, whether of indemnity or otherwise, resulting from any acts, omissions or events happening prior to the date of termination or the date, if later, when the Improvements are removed and the Premises restored or Lessor elects to take and bold the Improvements as its sole property as hereinabove in paragraph 16 provided.

18. In the event that Lessee consists of two or more parties, all the covenants and agreements of Lessee herein contained shall be the joint and several covenants and agreements of such parties.

19. All the covenants and agreements of Lessee herein contained shall be binding upon the heirs, legal represontatives, successors and assigns of Lessee, and shall inure to the benefit of the successors and assigns of Lessor.

20. It 1s agreed that the agreenent dated Apr11 13, 1949, (Iessor's Secretary's Contract No. 95727 ) between the parties hereto, relating to the use of a portion of Lessor's property at Navarre, Dickinson County, Kansas, as a site for elevator, coal bins, bulk oil station and other buildings $1 \mathrm{~s}$ hereby terminated as of the effective date hereof.

IN WITNESS WHEREOF, This lease has been duly executed in duplicate by the parties hereto as of the day and year first above written.

IHE ATGHSON, TOPEKA BUDD SAITA FE FAILWAY GOMEANY(Lessor)

Approved as to description:

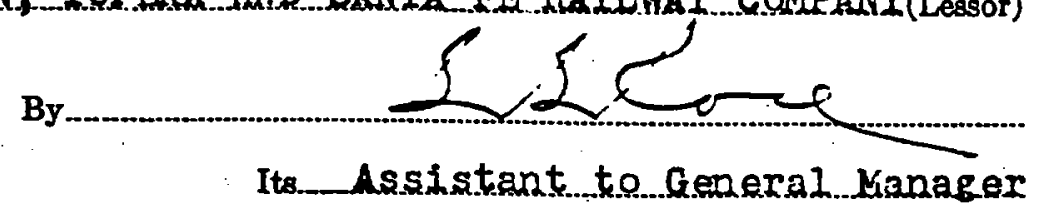

Chief Engineer.

MAVARRB EARMEBS. ONIOLX COOE WRATIYE...SSSOCIATION

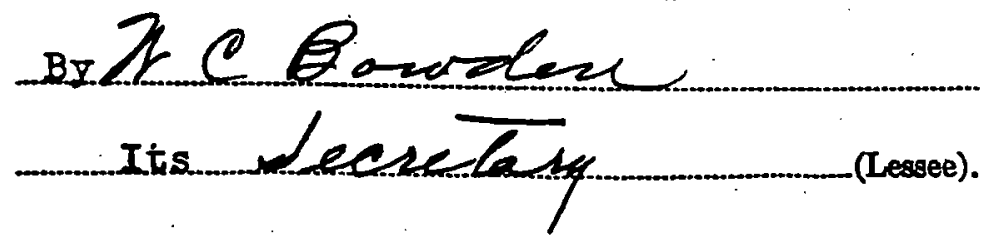

(Attach print here)

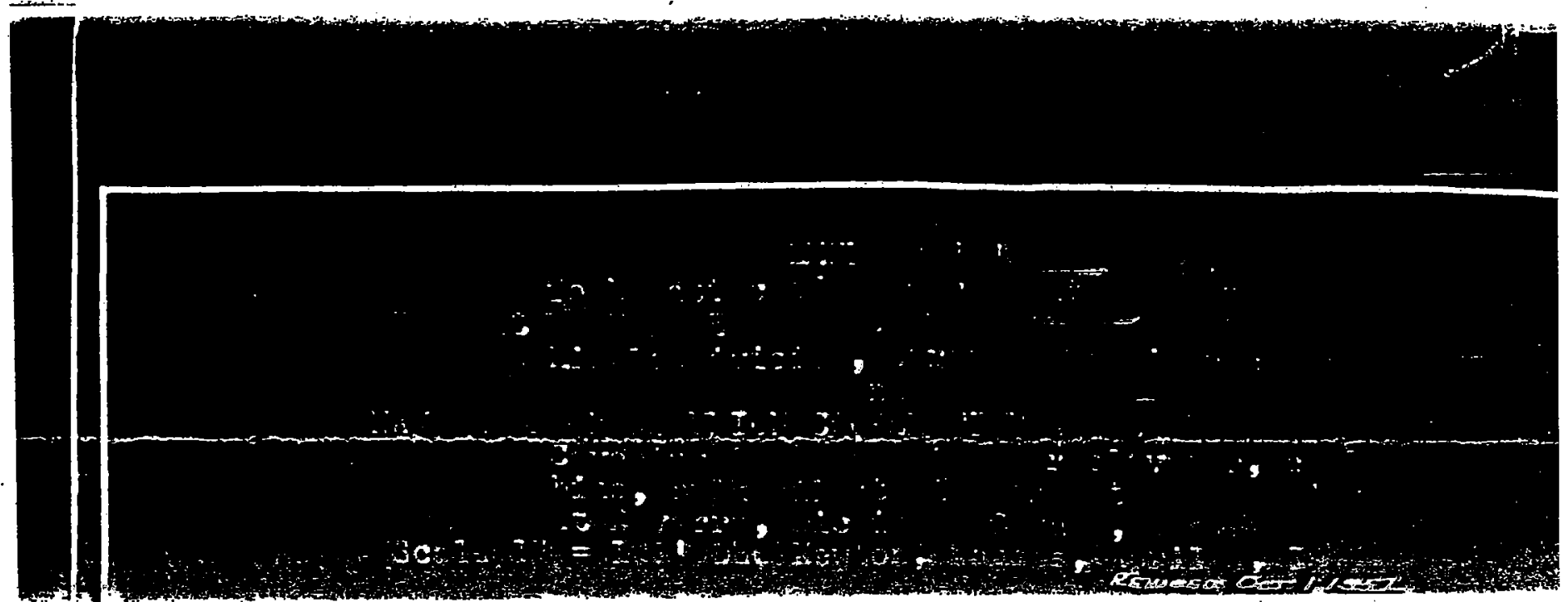




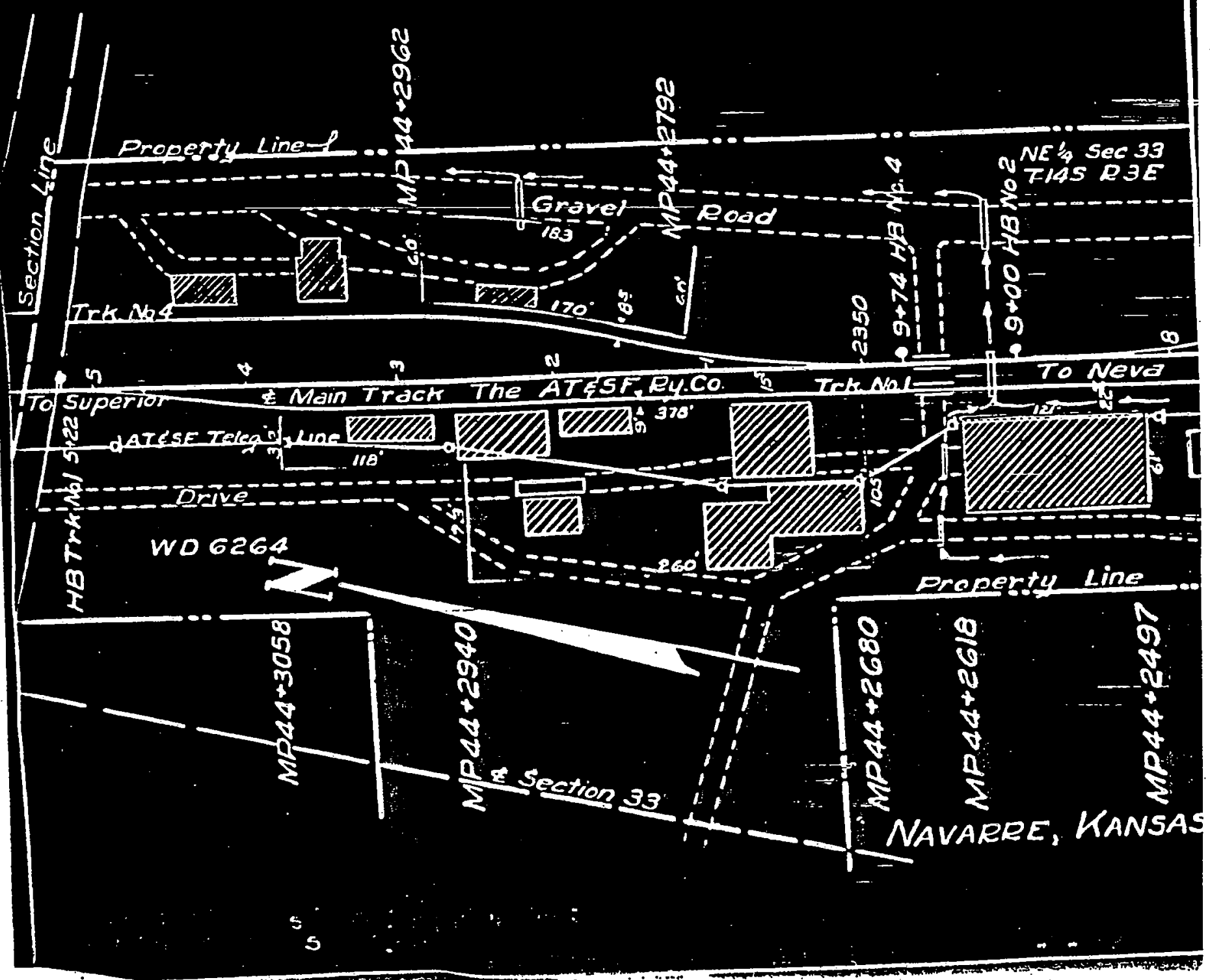

(

i

I.

NE\& Sec 33 Whas 
8. In using the Premises, and in constructing, maintaining, operating and using the Improvements thi Lessee shall comply with any and all requirements imposed by federal or state statutes, or by ordinances, o or regulations of any governmental body having jurisdiction thereover. In the event the Premises or Imf ments shall be used for the loading, unloading, storing, or otherwise handling of any petroleum products, I shall comply with all applicable regulations and recommendations from time to time promulgated by the Burc Explosives of the Association of American Railroads, or any successor agency. All artificial lighting in pump $h$ warehouses, or other enclosures upon the Premises, where oil or other inflammable fluid supplies are hand! stored by Lessee, except in unbroken original containers, shall be by electricity, and such electrical installatio any other electrical installation upon the Premises shall at all times conform to and be maintained in accor with the provisions of the then current edition of the National Electrical Code with respect to Class I baza locations. Lessee shall promptly pay and discharge any and all liens arising out of any construction, alterati repair work done, or suffered or permitted to be done, by Lessee on the Premises, and Lessor is hereby authc to post any notices or take any other action upon or mith respect to the Premises that is or may be permititeri to prevent the attachment of any such liens to the Premises; provided, however, that the failure of Lessor to tak

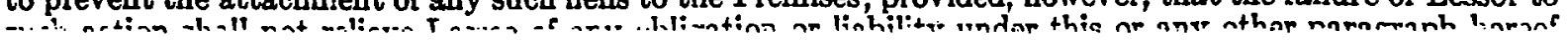
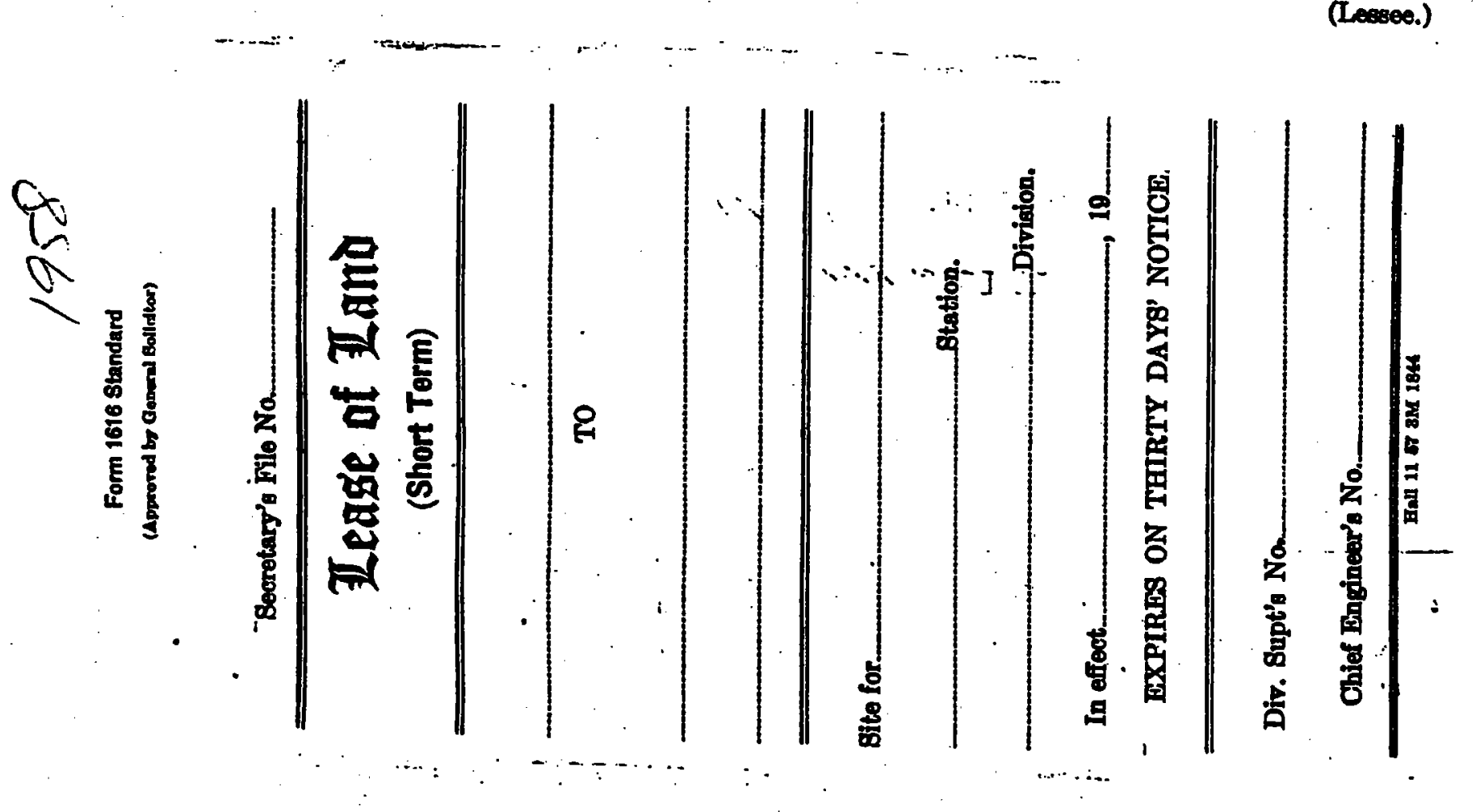

(Lessee.) 
THE MAYARRR. PARMERS UTIOA COLOPRAATTYR ASSOGTATION

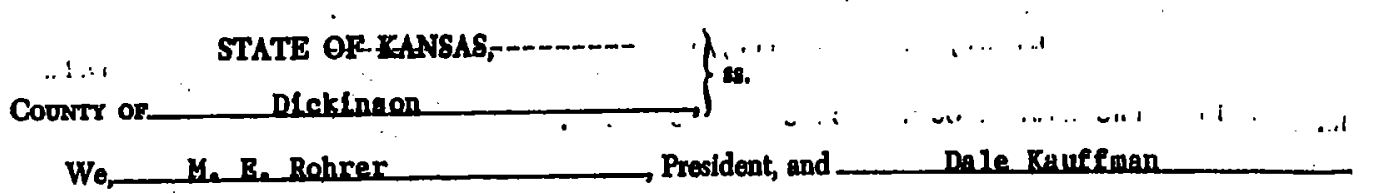

Secretary of The Navarre Barmere Uni on Co-operative Aseoclat ton

a corporation organized and existing under the laws of the State of Kansas, and whose registered office is

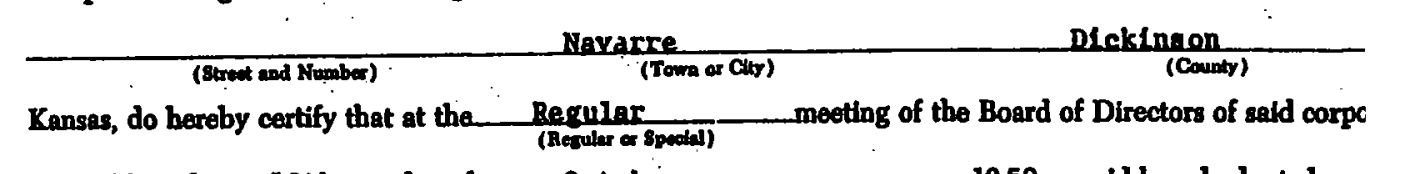
tion held on the 19th___day of___actober _ 19.59, sald board adopted a re lution setting forth the following amendment to the Articles of Incorporation and declared its advisability, to v (Article 6-Capltal stock) That the amount of capltal stock of this cor=poration shall be one Hundred Thousand Dollara $(\$ 100,000)$ conglating of Ten Thousand (10,000) ahares of Ten Dollars $(\$ 10.00)$ per share.

That thereafter, pursuant to said resolution and in accordance with the by-laws and the laws of the State Kansas, said directors called a meeting of stockholders for the consideration of said amendment, and thereal pursuant to said notice and in accordance with the statutes of the State of Kansas, on the lat

Pebrivary proposed amendment.

That at said meeting the stockholders entitled to vote did vote upon said amendment, and two judges $i$ appointed for the purpose conducted sald vote deciding upon the qualification of voters and declared that the jority of voting stockholders of the corporation had voted for the proposed amendment certifying that the $v$

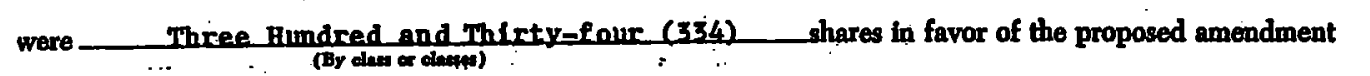
Thirteen (13) shares' against the amendment.

That the total number of nembers reflected by the bogka and records of aigd co That said amendment was duly adopted in accordance with the provisions of Chapter 17, Article 62 , Gen Statutes of Kansas, 1949, and amendments thereto.

That the capital of eaid corporation will not be reduced under or by reason of sald amendment. : :! 1... . i $\because$. ... IN Wriviss Wrisneor we have hereunto set our hands and affired the ser

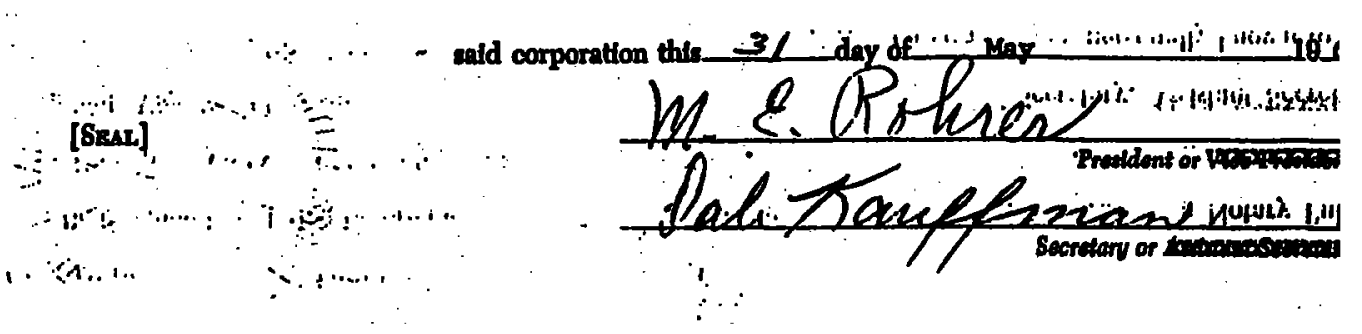




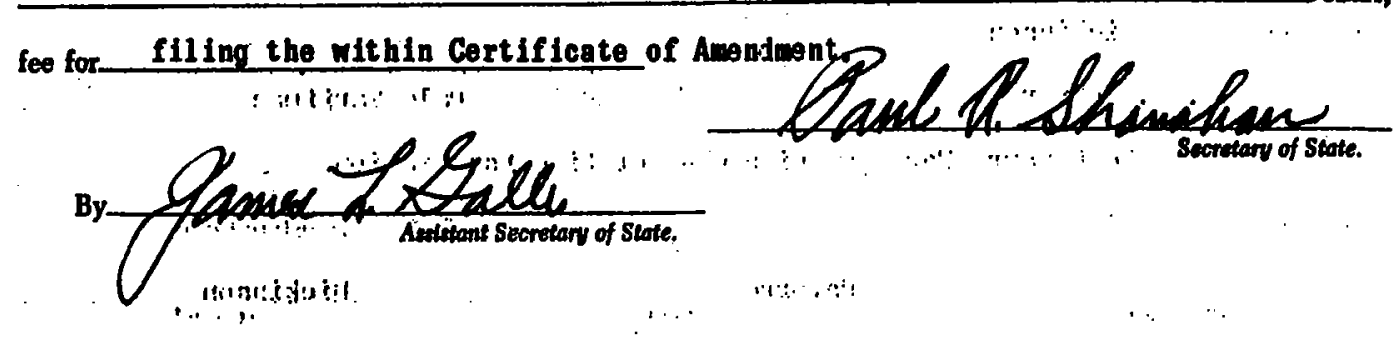

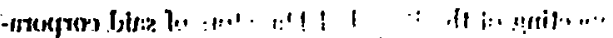

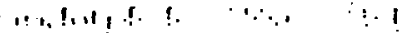

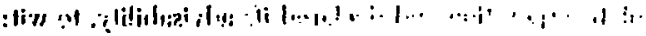

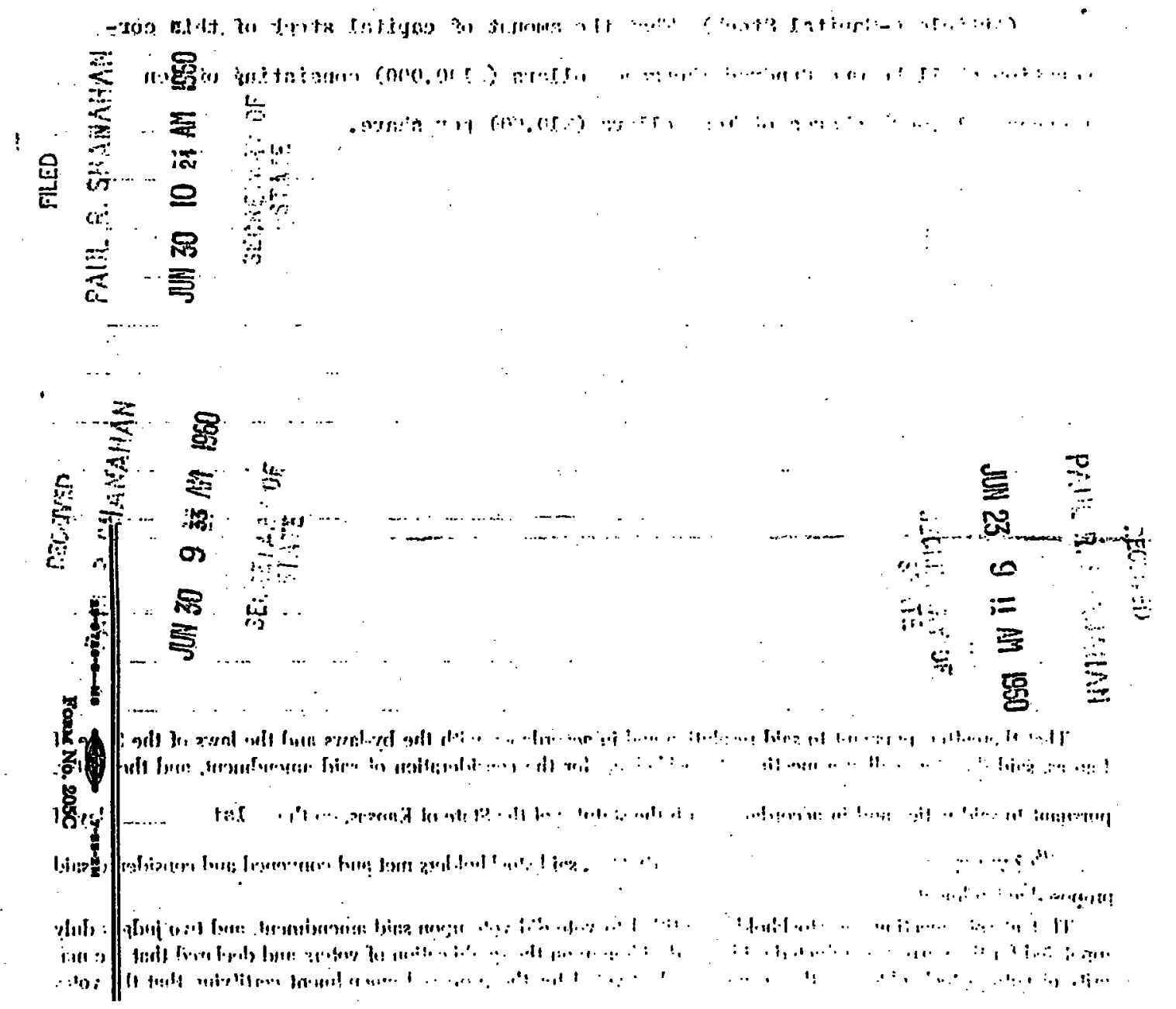

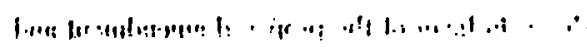

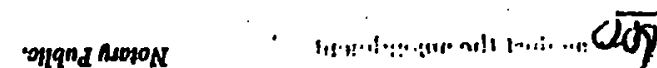

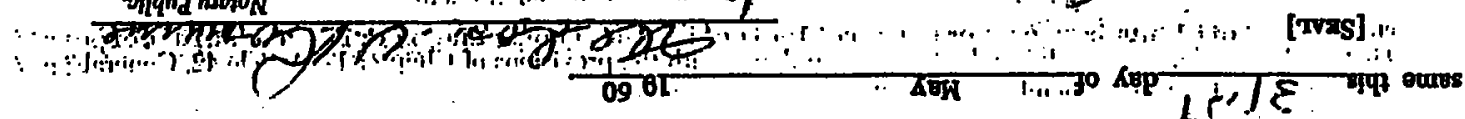

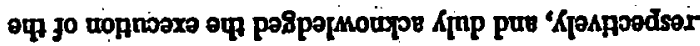

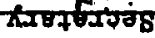
pue

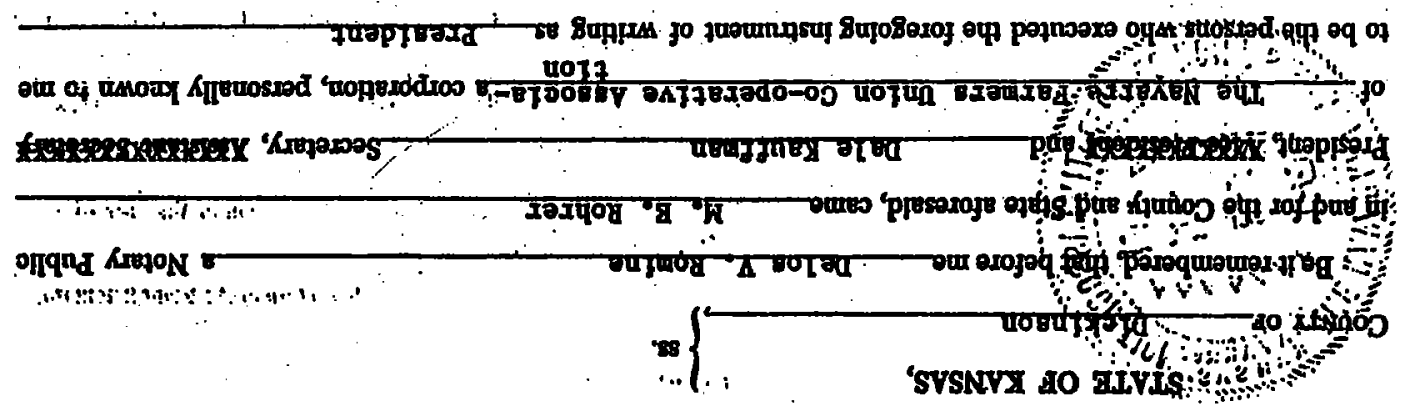




\section{CHANGE OF LOCATION OF REGISTERED OFFICE AND/OR}

CHANGE OF RESIDENT AGENT

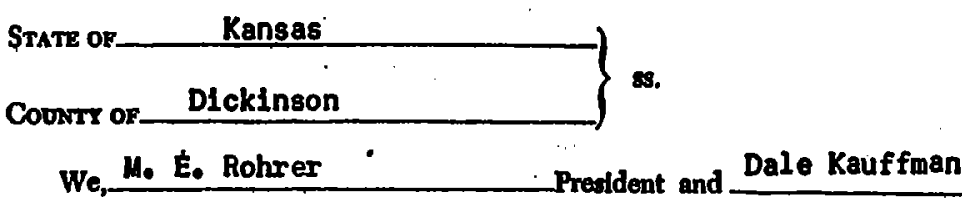

\section{Navarre Farmers Union Cooperative Association}

a corporation organized and existing under and by virtue of the laws of the State of Kan $8 a s$.

whose Registered Office in the State of Kansas is

Navarre, Kansas

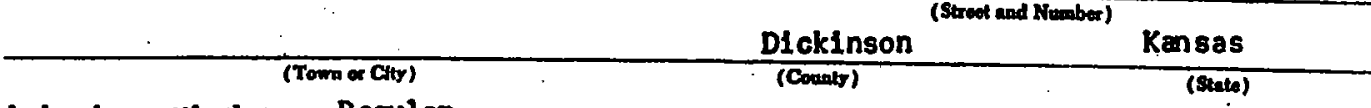

do hereby certify that a Reqular

(Aopuler or Speolal)

on the_ 27 _day of March meeting of the Board of Directors of said corporation hel

Be it resolved that the Registered Office in the State of Kansas of sald corporation be changed from

(Struct and Number)

(Towp ex City)

(County)

(8tate)

the same being of record in the office of the Secretary of State of Kansas to

(Stret and Number)

(Towa ox Chy) (Combly)

(state)

Be it further resolved that the Resldent Agent of ald corporation in the State of Kansas be changed from D. 5. Strole

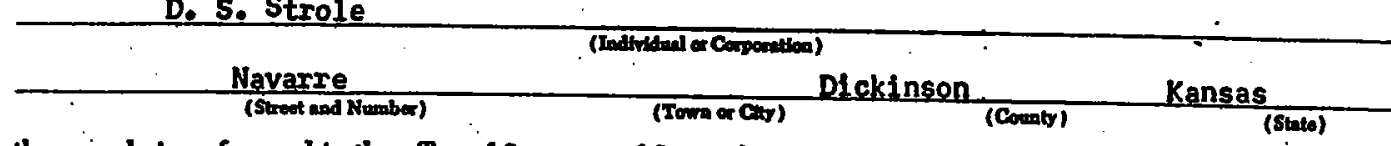

the same being of record in the office of Secretary of State of Kansas to

Ralph Funston

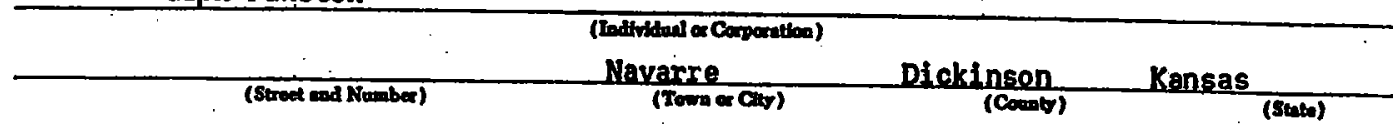

The President and Secretary are hereby authorked to fle and record the same in the manner as required by law.

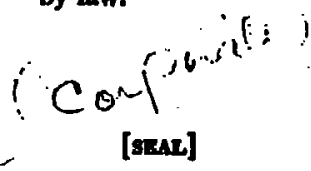

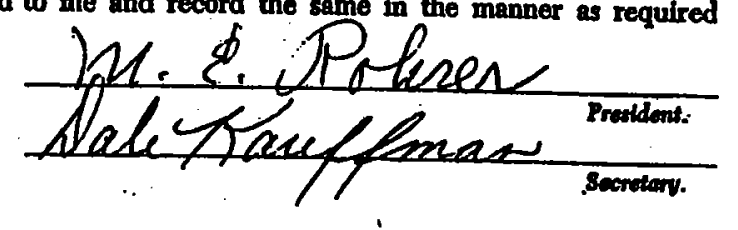

Fonu iva, sose 
Rro.ives or

THE NAVARRE FARUERS" UNION COOPERATIVE ASSOCIATION

Five and no/100 Dollars,

fee for Change of Location of REGISTERED OFFICE and/or CHANCE OF RESIDENT AGENT.

Dated this_-29th_day of

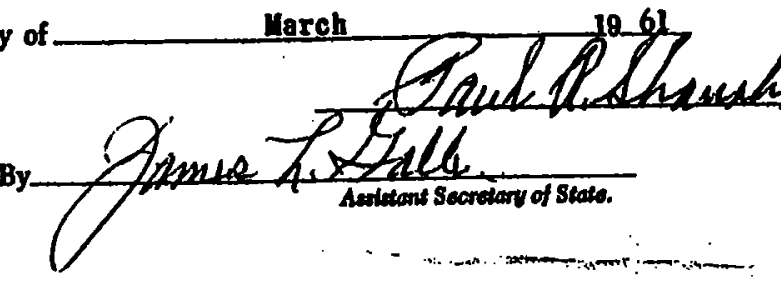

\section{seonetardo}

secretary of Scate.

B

- $-\ldots$

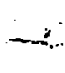

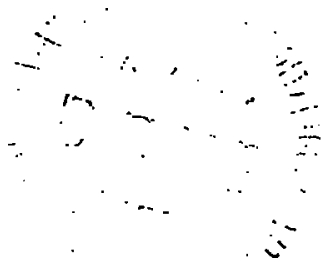

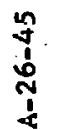
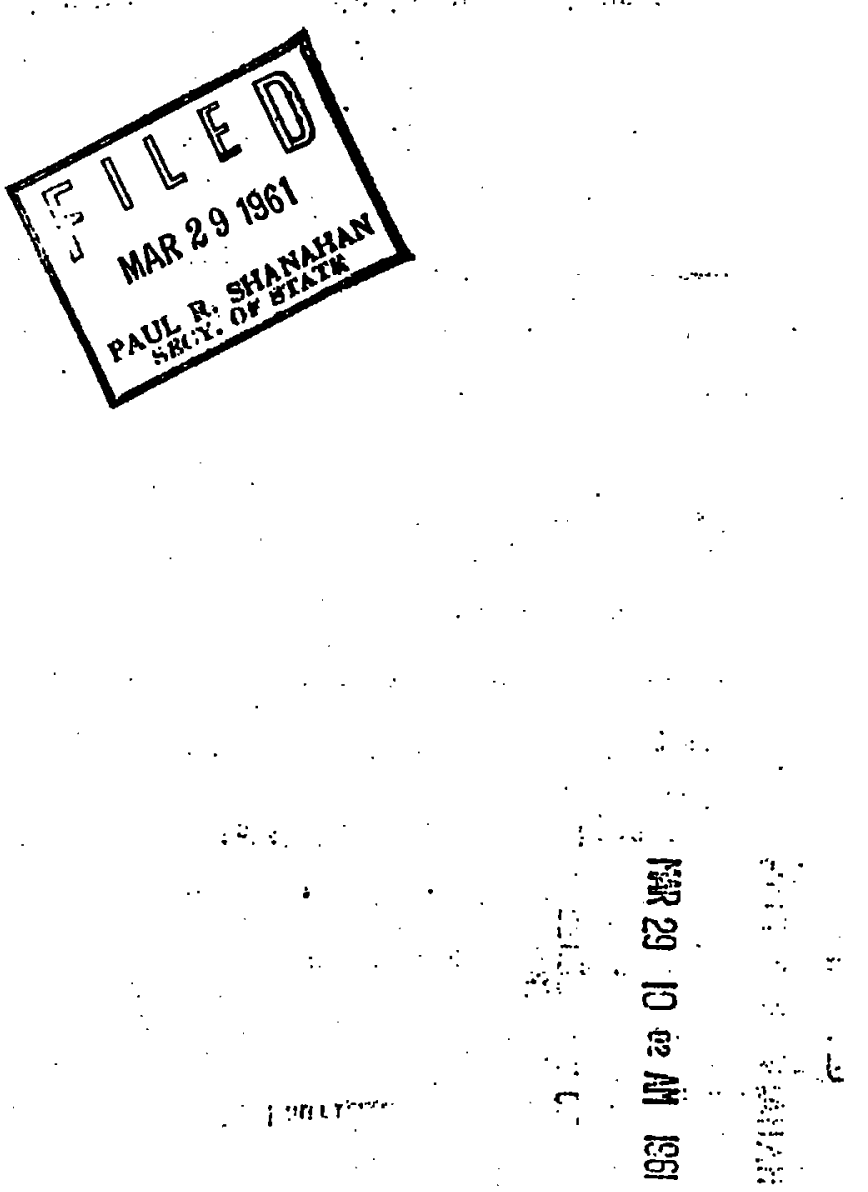


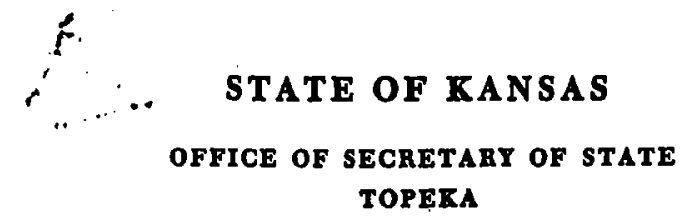

TOPEKA
RAUL R. SHANAHAN BRCMETART OF STATE

Narch 7, 1961

The Navarre Farmers Union Cooperative Assoctation Navarre, Kansas

Gentlemen:

IN RE: 1960 Annual Report

An examination of the charter records filed with this office for the subject corporation reveals that the resident agent, is:

D. S. Strole, Navarre, Kansas

and the location of the registersd office is: Navarre, Kansas

As shown by the last annual report, the resident agent is: Ralph Funst on, Navarre, Kansas

and the location of the registered office is

Navarre, Kansas

If there has been a change, as shown by the annual report, the enclosed blanks should be completed, in duplicate, and returned to this office, together with filing foe of $\$ 5.00$. It is requested that the address of the resident agent and the address of the registered office colncide in order to conform with our interpretation of Chapter 17, Article 44, G.S. 1959 S upp.

Your cooperation in this matter will be appreciated. If you have any questions, please do not hesitate to call or write.
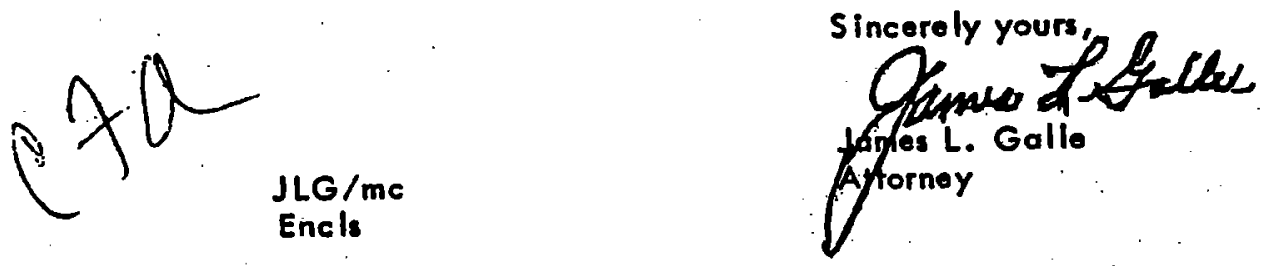
SUPPLFMFNTAL AGRFFMFINT, Made this $\frac{12 \text { th }}{\text { between THE ATCHISON TOPEKA AND }}$ SANTA EE' RAILWAY COMPANY, a Kansas corporation, hereinafter referred to as "Lessor", and COMMODITY CREDIT CORPORATION, a Government Agency,

hereinafter referred to as "Lessee".

R $\underline{\text { E }} \underline{\mathrm{I}} \underline{\mathrm{T}} \underline{\mathrm{A}} \underline{\mathrm{L}} \underline{\mathrm{S}}$

Lessor and Lessee are now parties to a lease dated June 21, 1954 104166 Lessor's Secretary's Contract No. underingl Lease"), under Which Lessee pays Lessor a rental of \$31.61 year for use of a portion of Lessor's property. at Navarre Dickinson County, Kansas, as a site for grain storage bins.

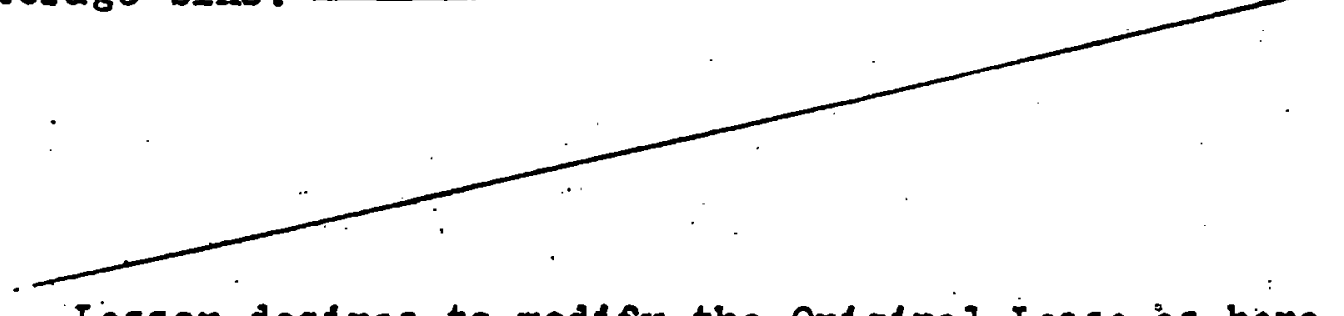

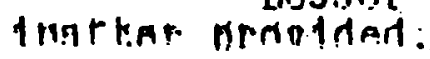

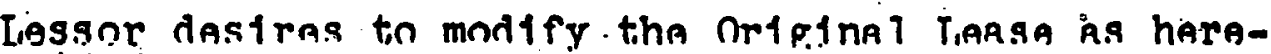
E $\underline{\text { E }}$ 最

It is mutually agreed that effective May 15, 1961

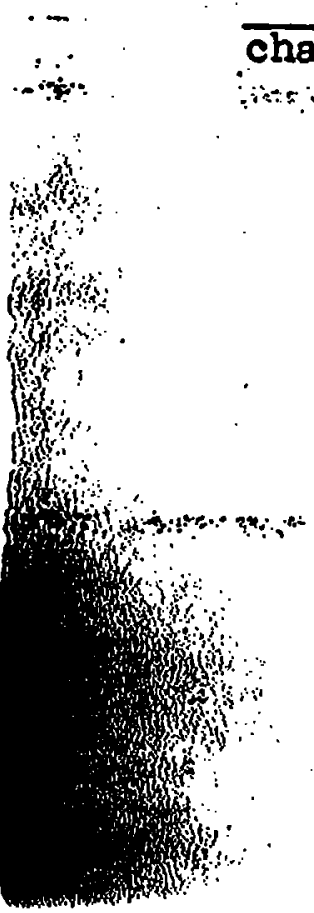
Section 3. of the Original Lease is hereby changed to read, as follows:

"Lessee shall pay to Lessor on or before the first day of each period of one year during the continuance of this lease as rental for the use of the Premises for such period, the sum of Thirty-six and 88/100 Dollars ( $\$ 36.88$ ) per annum. Such rental shall be subject to increase if rom time to time on eccount. of any. governmental charge or -assessment (except gen-... eral property taxes) pajable in respect to the Premis

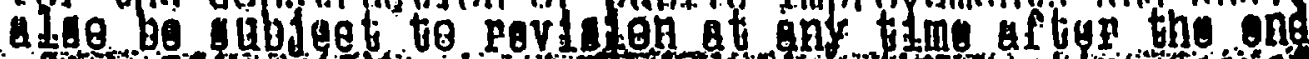

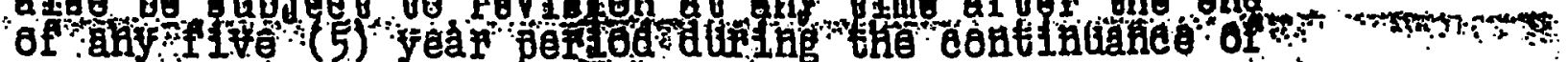
this lease."

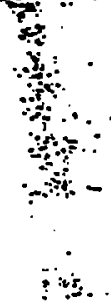


THIS LFA8E, Made as of the 22nd day of

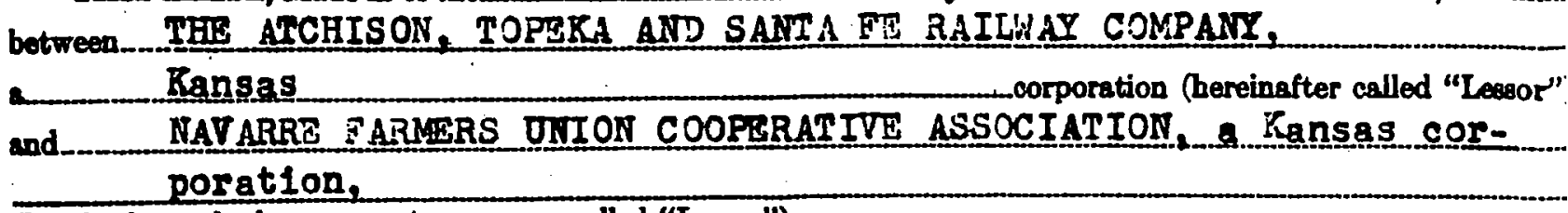

(hereinafter, whether one party or more, called "Lessee"). follows:

WITRKSSETE, That the parties hereto for the considerations hereinafter expressed covenant and agree :

1. Leesor bereby leases to Leesee, oubject to the rights and easements hereinafter excepted and reserved, an upon the termo and conditions hereinafter set forth, the land (hereinafter called "Premises") situated at or nea Narares County of Dickinson

State of Ransas outlined in red coloring on the print hereto attache No. DEO 8I-N-314, datod January 2, 1962

marked "Erhibit A" and made a part hereof, for a term beginning on and ending when this lease shall be terminated as hereinatter provided. 18

2. Lessor hereby excepts and reserves the right, to be exercised by Lessor and by any others who have obtaine or may obtain permission or authority from Lessor so to do, (a) to operate, maintain, renew and relocate any an all existing pipe, power, and communication lines and appurtenances and other facilities of like character upon, or or under the surface of the Premises; and (b) from time to time to construct, operate, maintain, renew and reloca ouch additional facilities of the same character as will not unreasonebly interfere with the use of the Premises b Lessee for the purpose opecified in paragraph 6 hereof.

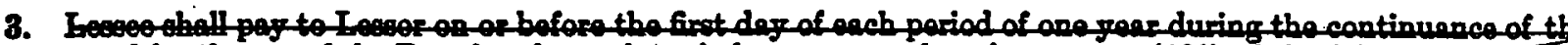
lease as rental for the use of the Premises for such period, a sum equal to aix per cent (6\%) of the fair renter ralue the Premises, but not lees than Dollents (5.

For the purposes of this lease the fair rental value of the Premises at the effective date hereof is agreed to be

and the initial rental shall be Dollars (2.

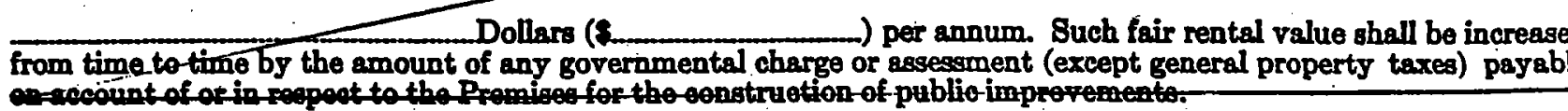

4. Lessee covenants and werrants that Lessee either owns, or has obtained from the owner or owners there the right to use, any improvements now on the Premises shown or described on said Exhibit A as "Lessee's Existi Improvements." Buch improvements, if any, together with any other improvements hereafter placed upon $t$ Premises by or for account of Lessee are hereinafter called "Improvements."

5. Lessee shall pay before the game become delinquent all taxes, charges, rates, and assesaments which ma during the term of this lease, be levied upon, or assessed against, or be equitably chargeable to or assessed in respe of the Improvements; and where any such tax, rate, charge, or assessment may be embraced in the general amou of tares charged upon the Premises separately or in connection with other property of Lessor and Lessor shall p1 all of said tares, then Lessee shall promptly repay or refund to Lessor the amount or part of the tax, charge, rate assessment equitably or fairly apportionable to the Improvements.

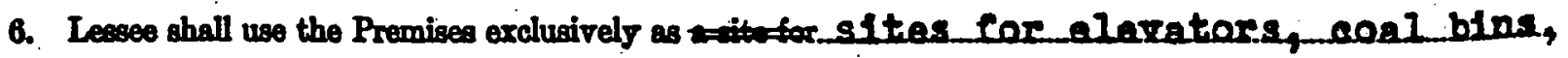

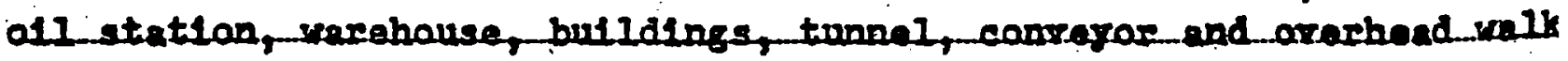


8. In using the Premisea, and in construoting, maintaining, operattng and using the Improvements thereon, Leesee shall comply with any and all requirements impoeed by federal or etate statutes, of by ordinances, arders, or regulations of any governmental body having jurisdiotion thereover. In the event the Premises or Improve. ments shall be used for the loading, unloading, staring, ar otherwise bandlings of any petroleum products, Leeseo shall comply with all applicable regulations and recommendations from time to time promulgated by the Bureau of Explosives of the Association of American Railroeds, or any successor agency. All artificial lighting in pump houses, warehouses, or other enclosures upon the Premises, where oil or other inflammable fluid supplice are haodled or stored by Lessee, except in unbroken original containers, shall be by electricity, and such electrical installation and any other electrial installation upon the Premises shall at all times conform to and be maintained in accondance with the provisions of the then current edition of the National Electrical Code with respect to Class I hasardous locations. Lessee shall promptly pay and discharge any and all liens arising out of any construction, alteration or repair work done, or euffered or permitted to be done, by Lessee on the Premises, and Lessor is hereby authorized to post any notices or take any other action upon or with respect to the Premises that is or may be permitted by law to prevent the attachment of any such liens to the Premises; provided, however, that the failure of Lessor to take any such action shall not relieve Lesee of any obligation or liability under this or any other paragraph hereof.

9. Lessee shall at all times keep a space of six (6) feet from the neareat rail of any railroad track entirely olear of structures, material and obstructions of every sort and ahall observe an overhesd olearance of not less than twentyfive (25) feet above the top of rail; but, nevertheless, for convenience in handling freight to and from cars on any railroad track serving the Premises, the Lessee may install, use and maintain (a) loading or unloading oranes or other devices not nearer than six (6) feet from the nearest rail of auch track and no part of which ahall at any time project or extend in the direction of such track except when crane or dexice in being used for loading or unloading

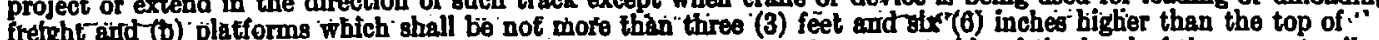
rails, and which at no point shall be nearer than four (4) feet to the nearest side of the head of the nearest rail c rails, and which at no point shall be nearer than or or ompetent public authority different clearances shall be required, then Lessee shall strictly comply with euch statute or order.

10. Leasee agrees to indemnify and save harmless Lessor againat all Isea, damage or expense which Lessor may suetain, theur or become liable for, including loss of or damage to property or injury to or death of persong and fines or penalties imposed upon or asseesed against Lcesor, arising in any manner out of (a) the use of the Premises or or penases (b) any breach by Lesses of the terms, covenants or conditions in this instrument contained, Improvements or contributing acts or omiseions of Lezsee or the employes, agents, patrons or invitees of Lessee in, on or sbout the Premises or. Improvements, except that if I oesot thall participato in any outeb-contributing acts or omisor about the the lose, damage or expense ariang therefrom shall be borne by the parties hereto equally; provided, gions, then the loss, damage or expe the riak of, and agrees to indemnify Leesor againist liability for, loss of or damage

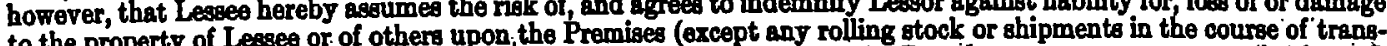
to the property of Lesses or of others upon; the Pramises (oxcept any ron the Premiges pursuant to paragraph 2 hereof) portation and except ant fro fre communicated from locomotives while being operated by Leser upon any tracks within or in the vicinity of the Premises, regardiess of Lessor's negligence, if any.

11. Neither Lessee, nor the heirs, legal representativee, successors or aseigns of Lessee, nor any subsequent assignee, ehall underlease or sublet the Premises or the Improvemente, or any part thereof, nor assign or transfer this lease or any interest herein, without the written consent and approval in eaoh instance of Lessor.

12. In case of the eviction of Lesee by anyone owning or claiming title to or any interest in the Premisea, I except the proportionate part of any rental paid in advance.

13. If any rental hereunder ahall be due and unpaid, or if default shall be made in any of the covenants or agreements of Lessee herein contained, or in case of any assignment or trangfer of this lease by operation of law, Leasor ments or iteseetion, terminate this lease by serving five (5) dajg' notice in writing upon Lessee; but any waiver by mey, at its opten, term defaults shall not constitute a waiver of the right to terminate this lease for any subsequent default or defaults.

14. This lease may be terminated at any time by either party by eorving thirty (30) dayg'. written notice of termin piration of the time opecified in such notice this lease and all rights of Leseo hereunder shall absolutely cease and piration of the timo opecifed in such notion Lesee shall bo entitled to bave refunded by Leseor a proportionate part of any rentals paddin advance.

18

15. Any notice to be given by Lessor to Lessee hereunder ehall bo deemed to be properly. served if the same be delivered to Lesee, or if lefit with any of the agents, Bervants or employes of Lessee, or if posted on the Premises, or if deposited in the Post Office, postpaid, addiesed to Lesseo at Navarre. Kanses.

16. Upan the termination of this lease in any manner herein provided, Lesee shall forthwith surrender to Lessor the posesesion of the Premises and shall remove the Improvements and restore the Premises to substantially the otate in which they were prior to the construction of the Improvements, and in case Lessee shall fail within thirty (30) date days after the date of such terms thereafter, either remove the Improvemente and restore the Premises for the account exercised within thirty (30) days thereatter, either remove (30) dajs after the rendition of bill therefor reimburse Lessor for the cost so incurred, or may take and hold the Improvements as its sole property.

17. If Iease fails to gurrender to Lesor the Premiises, upon any termination of this lease, all the liabilities

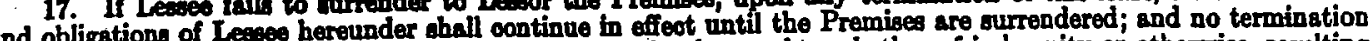
and obligation, of Lesee hereunder shablity or obligation hereunder, whother of indemnity or otherwise, resulting bereof shall release Lesses from any lisbility or obligation atrounder, whotion or the date, if later, when the Improvefrom any acts, omiscions or events happening prior to the date of termination or the date, if ater, when the Improve as hereinabove in paragraph 16 provided.

18. In the event that Lessee consists of two or more parties, all the covenants and agreements of Lessee herein contained shall be tho joint and several covenants and agreements of such parties.

19. All the covenants and agreements of Leasee herein contained shall be binding upon the heirs, legal repre-

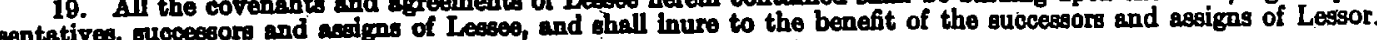

20. It 1s mutually agreed that RIder " $A$ " hereto attached, Identified by the signeture of $:$. $S$. Rose, 1 hereby made a part hereof. 


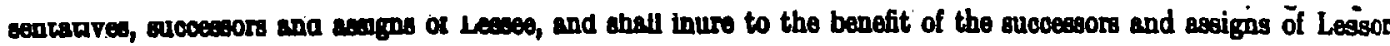

20. It 13 mutually agreed that Rider " $A$ " hereto attached, 1dentifle by the signeture of $:$. S. Tose, 19 hargby made a part hereof.

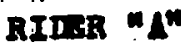 \\ To Iorice inted Jabunry 22, 1962 \\ Botwoon

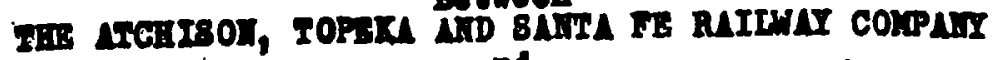 \\ and

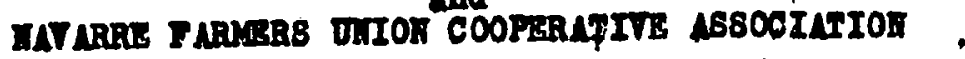

21. Inswee shall pay to Inseor on or beffore the f1rst day of each poriod of one joar during the continuanes of this loase as rontal fos the we of the Pronises for speh pariod the sm of 81xty-one and 41/100

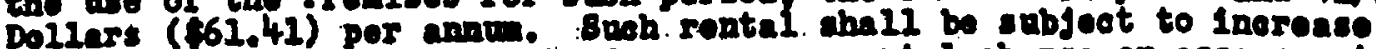
from tine to tim on aooount of anj. corermontal oherge. or apsessents (ercopt cenoral property taxes) payeble in respoot to the pronlees for the oonstruetion of publie inprorenents and shall aluo bo subjoot to rerislon at any time after the ond of any five (5) joar perlod during the continuanoe of this Iase.

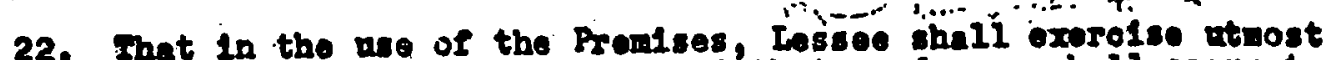
and extraordinary diligence to the ond that no fanage shall oocur to Iessor's comnuteation IIn located upon the Promlses, and Lesee horeby agrees to pay Iassor within twenty (20) days after rondtion of b111 therefor, the ontir cost of ropalring any danage to seid commication

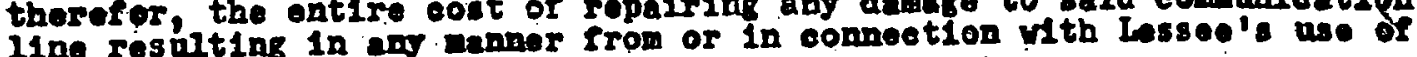
the Premises.

23. Notulthstanding arthing containod in Beetion 9 horoof to the contrary, Iossee nay, at its sole cost and exponse and in anner cot1sfactory to Lessor, construct, Install, use and maintain an orerhead serew oonveyor and walk, and an underground tunnel and apptartenances orer waer and adjacent to Ioseor's tracks in the lootion indioated and In accordance wth tho general design sown on sald Ixhibit "A" In such anner and of such waterlal as wil not at anj time be courco of denger to or Interference with or the saf operation of Lessor ': rallroad. Diring oonstruetion. Installation, use or repairing of sald twanel and/or orerbed serow conveyor and wall, and appurtenanoes, Iassee shell exercise utwost and extraordinary d111 conee to prevent Lassee shall exerclse utasor or infury to 1ts agonts or aplores. If at an time during the term hereof, Iessor shall desire to nake ang use of 1ts property with whioh sald tumel and/or okerhead sorew copveyor, ralk and appurtenanoes will in any way Interfore, Iessee shs Il, at rase 18 sole oost, within thirty (30) days after recelving y ritten not1ce fron Iescor to una offoct, walce such changes in said tunnel not and and appurtenances as in the judgment of Issor may be necessary to avold interference wth the proposed use

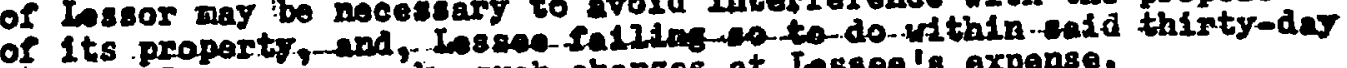
perlod, Lessor tey make suoh changes at Iossoo 's expense.

24. Jotwithstanding enthing oontained in Section 10 hereor to the contrary, Lesseo agrees that it will at il tiess Indernify and save hermless Lessor against all elaims, demands, netions or causes of actions. arising or growing out of 1058 of or damege to property inoluding said tunnel and/or overhead serew convoyor, waik and appurtenanoes or Injurs to or death of persons, Ineluaing onployes of Iossor, rosulting in any manner from the construction, Instaliation, rintenanoe, use; state of repalr or presence of sald tunnel and/or overhead sorew conveyor, walk and appurtenances under, over or adjacent to sald tracks whether such loss, damage, injary or death be capsed or contributed to by that it wil gence of Lessor, Its agonts or omployes, or otherwise, and that it wh prouptis pay to Iossor the fuil amount of any loss or di gus whion Lessor tessor may sustain, ineur or becoms in settlement of ang cleims on account may pay or

thereor.

25. It is mutualiy agroed that the agreement dated September 20, 1960,

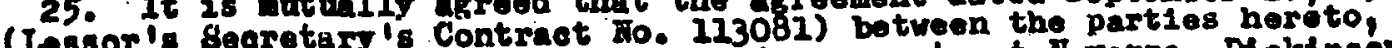
rolating to use of a portion of Lassor's property at Hevarre, Dickinson Count, crnses, as sites for elevator, coal bins, bulk oll station, varehouse and other buildings, is horbu termineted as of the offeetive date hereor. 
IN WITNEss WERBEOF, This lease has been duly executed in duplicate by the partie hereto as of the day and year first above written.

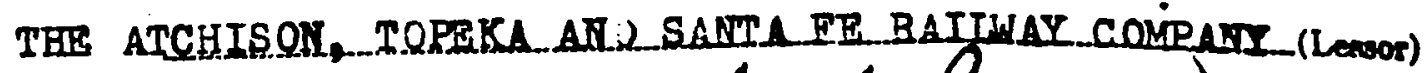

Approved as to description:

By

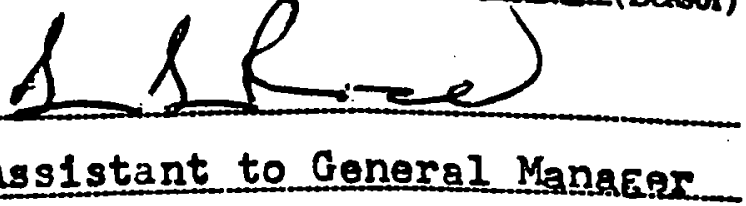

Chied Engineer.

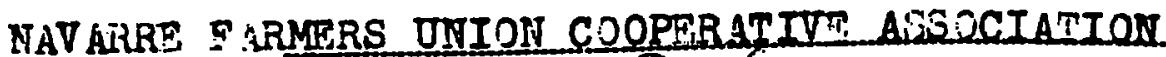

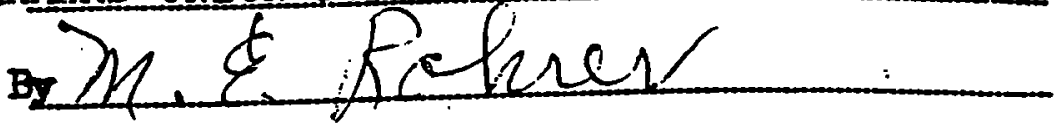

Its

Presidente (Leneer).

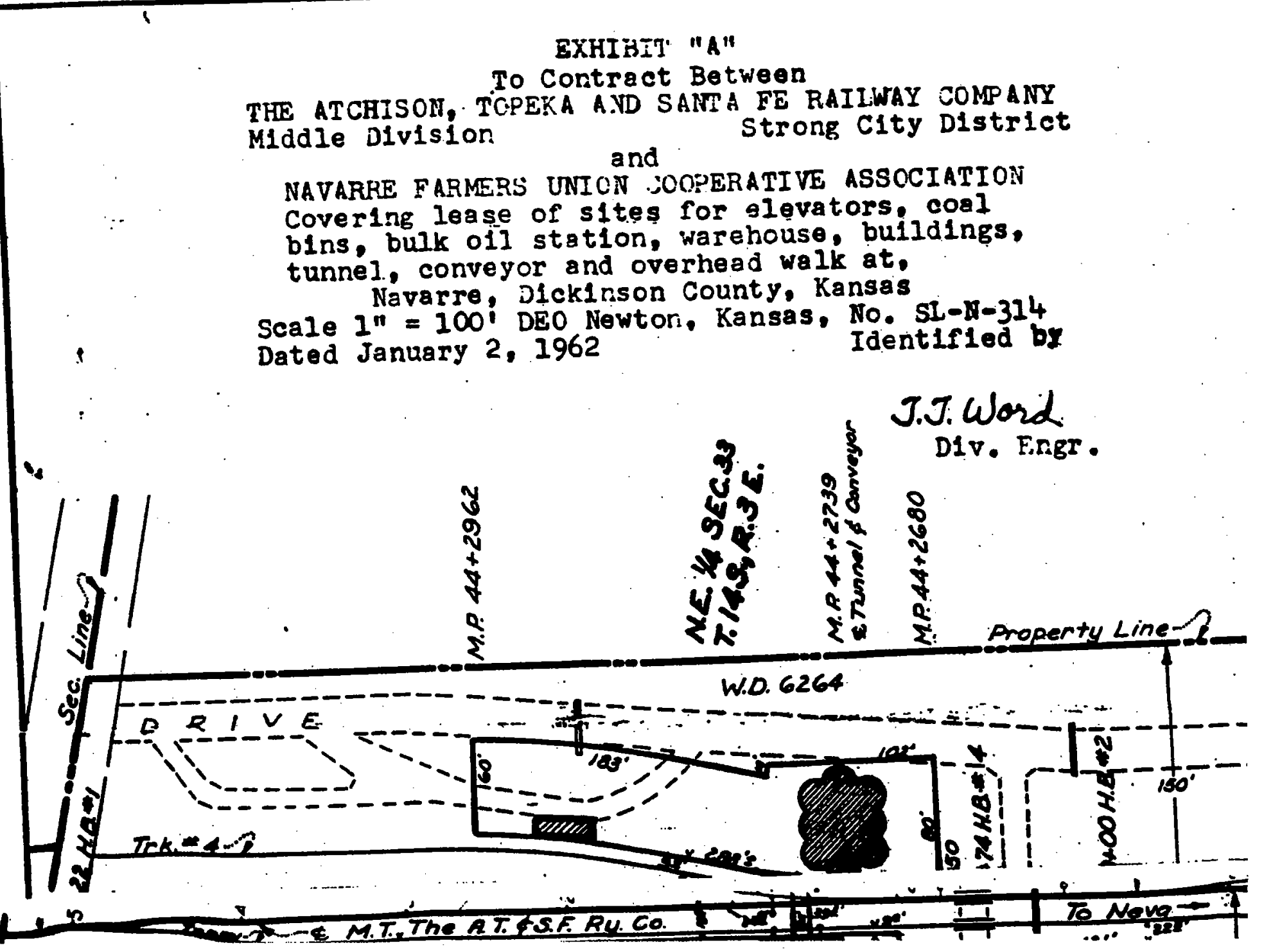




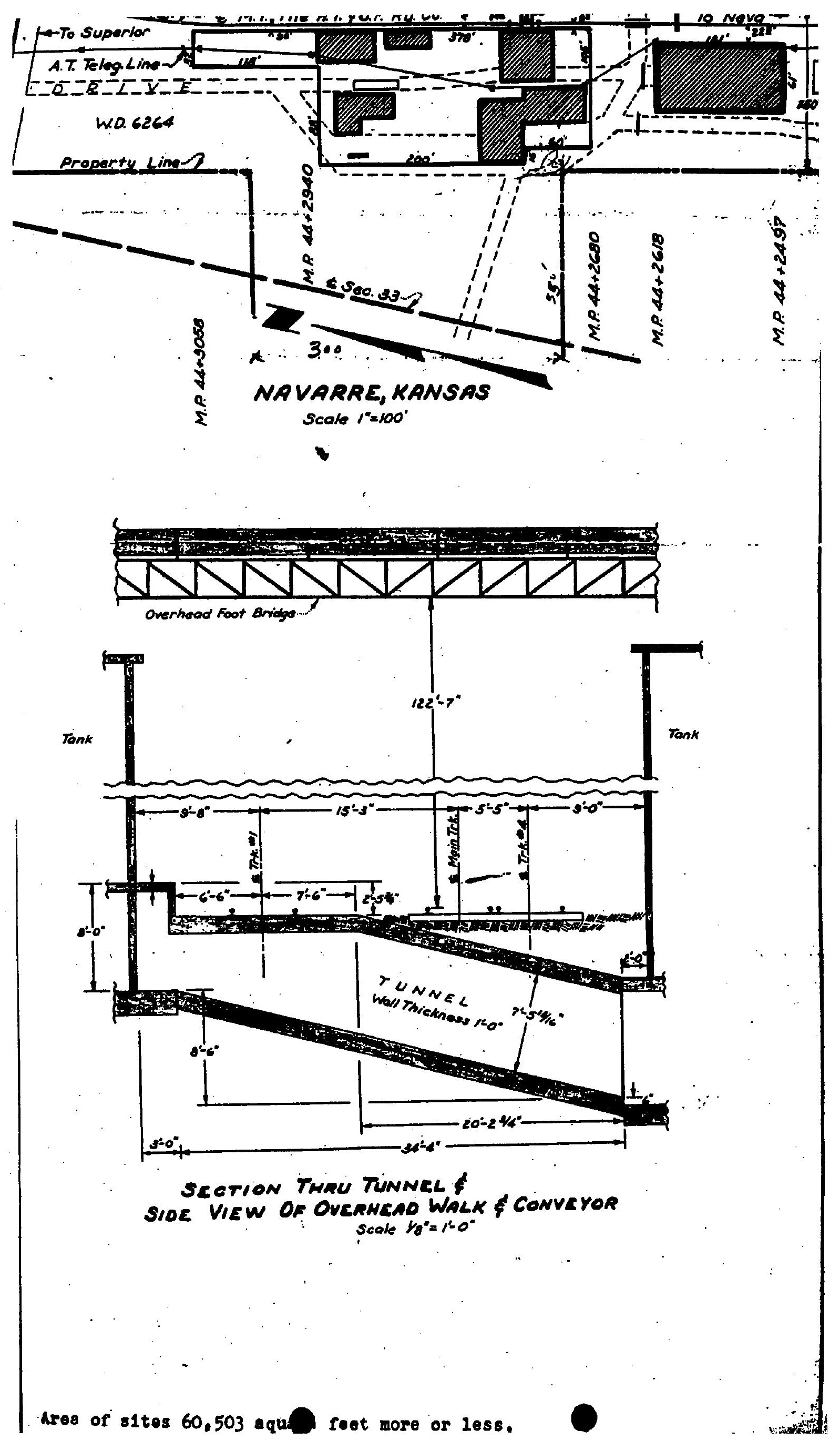




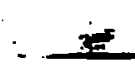

4

\section{GANCELLATION AND SURRENDRR}

villy apree thet the foregoing lease is hereby cancelled as of the 19. day of. 18
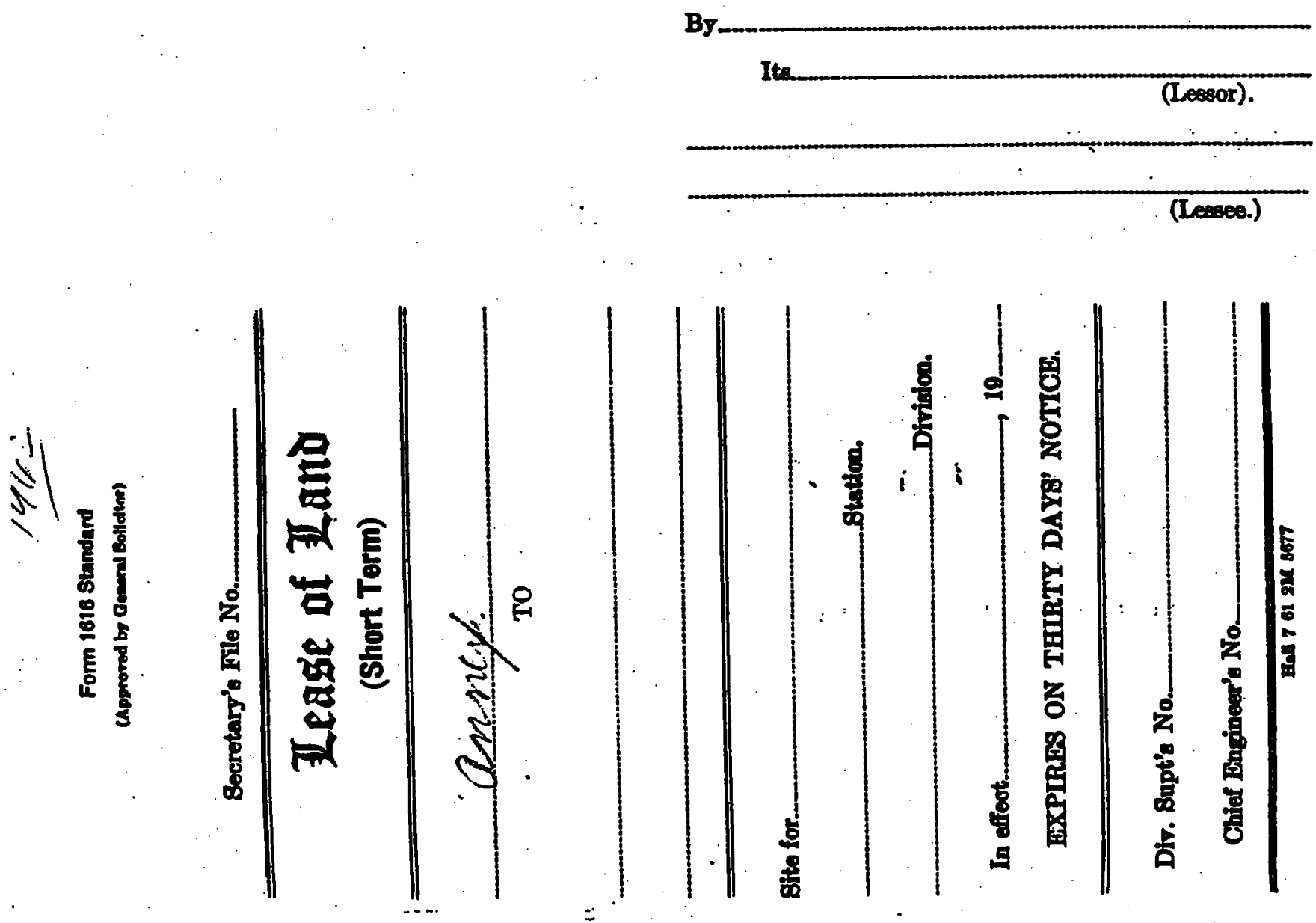


Strate of_Kansais

We, $\frac{\text { Dale Kauffman }}{\text { Navarre Farmers Union Conperative Association }}$ Secretary of

a corporation organized and existing under and by virtue of the laws of the State of

Kansas

whose Registered Office in the State of Kansas is

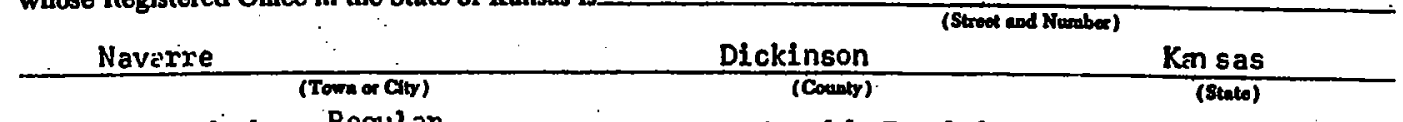
do hereby certify that a Regular on the 18 day of February meeting of the Board of Directors of said corporation held $19 \quad 63$, the following resolution was duly adopted.

Be it resolved that the Registered Office in the State of Kansas of said corporation be changed from

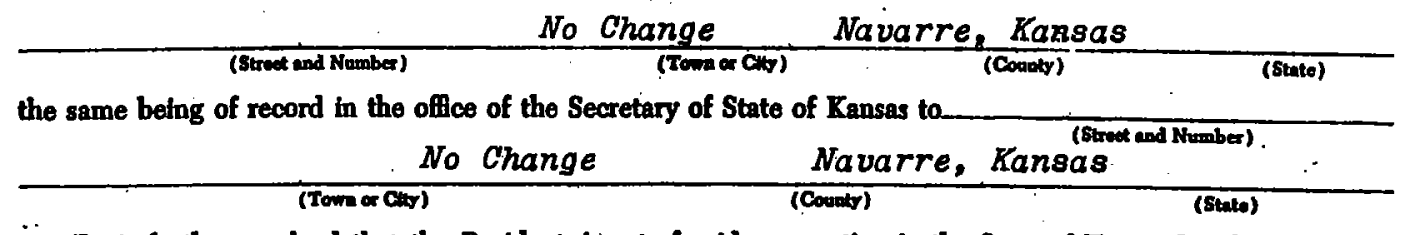

Be it further rosolved that the Resident Agent of eald corporation in the State of Kansas be changed from

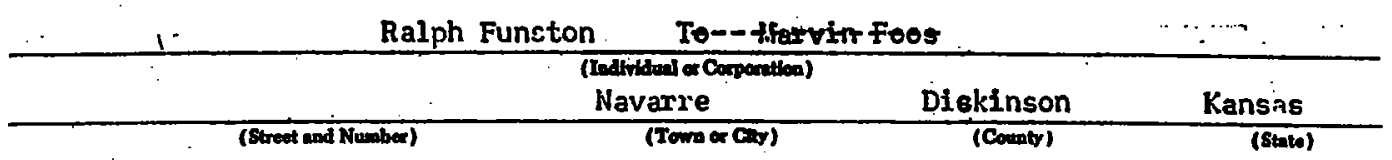
the same being of record in the offlce of Secretary of State of Kansas to

Marvin Foos

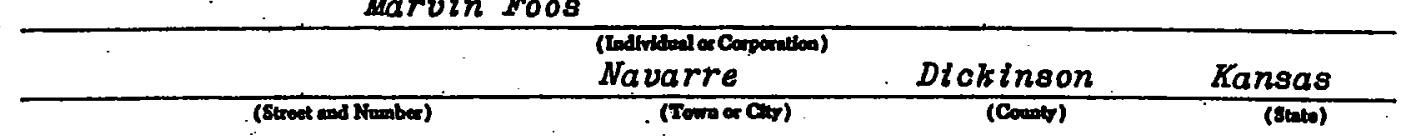

The President and Secretary are hereby authorized to fle, gnd record the same in the manner as required by law.

[seax]

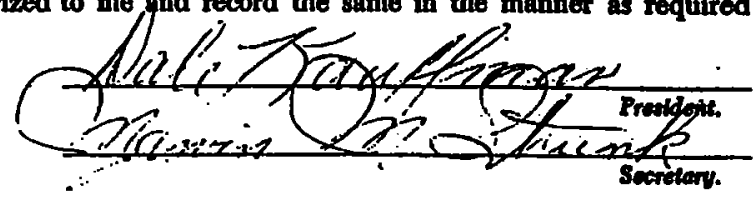

Toain Na. souc 


\section{CHANGE OF LOCATION OF REGISTERED OFFICE \\ AND/OR}

CHANGE OF RESIDENT AGENT

STATE or-KANSAS
Coovrs or OICKINSON

we, AbKIN SANdawe President and CLAReNeC GATCh Secretary of NAVAvhe Farmer's lunien coop Ass'x

a corparation arganized and existing under and by virtue of the laws of the state of / $1 / 1545$

whose Registered Office in the State of Kansas is

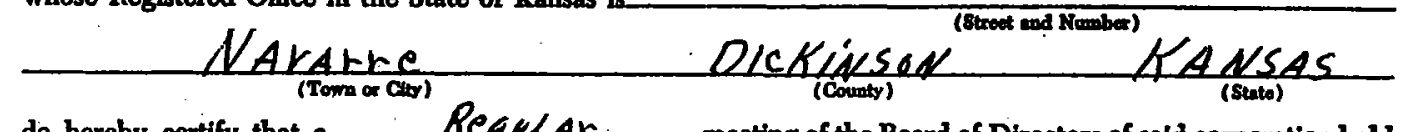
do hereby certify that a $\quad$ RequLAR on the 32 day of SANunAry meeting of the Board of Directors of sald corporation held

Be It resolved that the Registered Office in the State of Kansas of said corporation be changed from No ChANee

(Town of Clb)

1065 , the following resolution was duly adopted.

the same being of record in the office of the Secretary of State of Kansas to

(County)

(Stato)

(Strout and Nomber)

(Town ox C(y) (Comb)

(Stato)

Be it further resolved that the Resident Agent of said corporation in the State of Kansas be changed from MArrin Foos

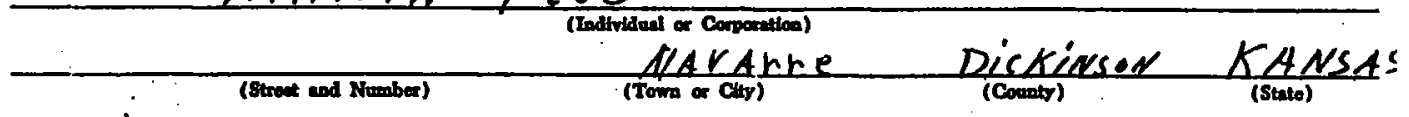

the same being of record in the office of Secretary of State of Kansas to

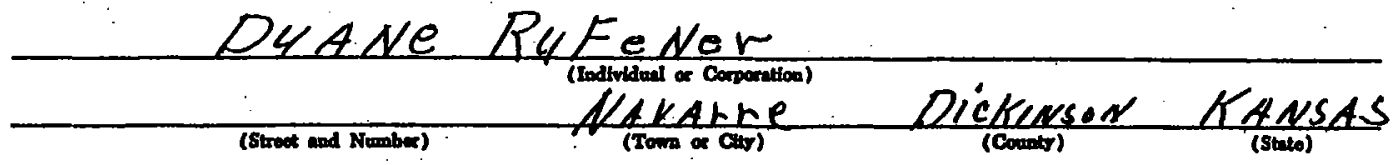

The President and Secretary are hereby authartzed to flle and recond the same in the manner as required by law.

SEAL.

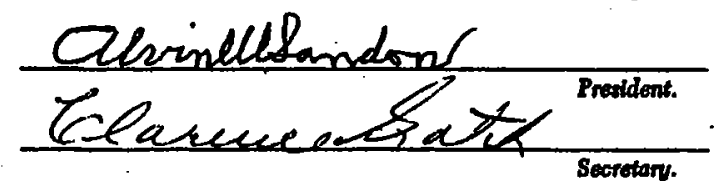

NOTE: Complete each ftem on form.

Address of Realdent Agent and Registered Ofico, as set forth above, must bo tho samo. (Ch. 17, Art, 14, 1859 Supp.)

OFFICE OF SECRETARY OF STATE

Topelae, Fonias

Rucerves or_the NAVARRE FARIERS UNION COOPERATIVE ASSOCIATION

FIVE and No/100

$(\$ 5.00)$ -Dollars,

fee for Change of Location of REGISTERED OFFICE and/g CIANGE OF RESIDENT AGENT.

Dated this 27th day of

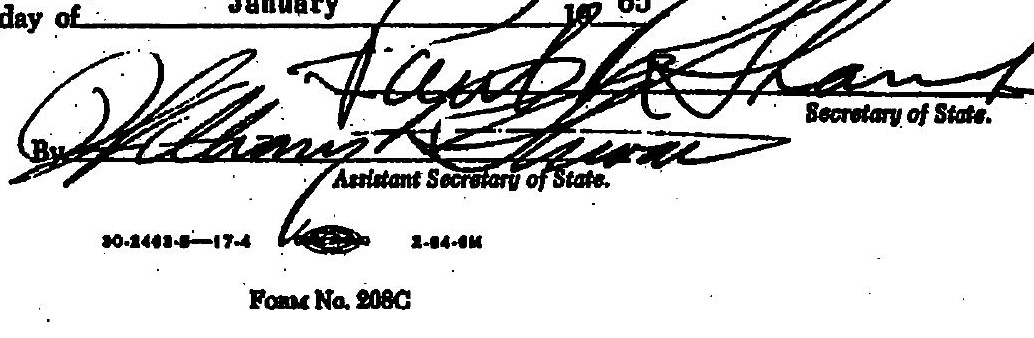


Navarre Farmers Union Co-operative Association

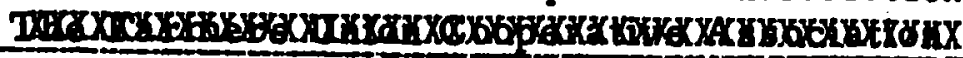

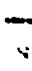

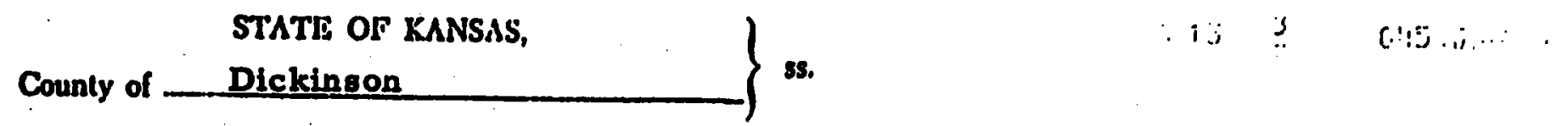
Wo, Jehn Murphy Presidgnt, and Lynn Rock Navarre Farmers Union Co-operative Association

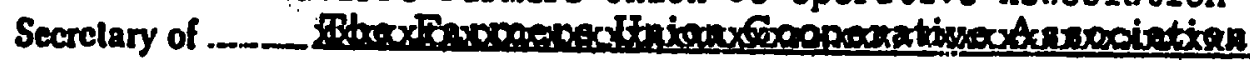

a corporation organized and existing uncler the laws of the State of Kansas, and whose registered office is

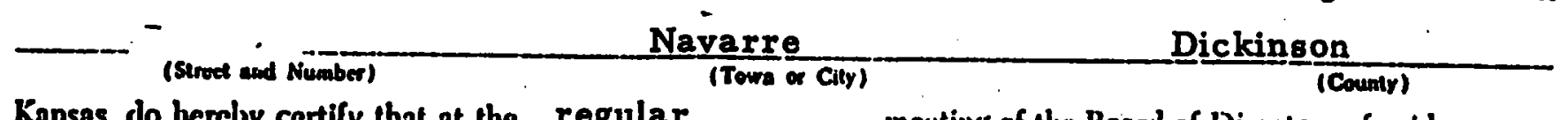
Kansas, do hereby certify that at the regular. meeting of (lie Board of Directors of said corporntion held on the 16th_ day of August 1965 said board aclopted a reso. Jution setting forth the following amendment to the Articles of Incorporation and declared its advisability, to wit: PIRST

That the name of this Corparre Farmers Union Cooperative Association.

\section{SECOND}

This Corporation is organized as a non-profit farmers' cooperative association, and the full nature and character of the business in which it proposes to engage is (1) to engage in any activity in connection with marketing or seliing of the agricultural products. of its members or with the harvesting, threshing, milling, preserving, ,drying, processing, canning, packing, storing, handing, shipping or utilization thereof, or the manufacturing or marketing of the by-products thereof; (2) to manufacture, sell or supply to lts members, machinery, equipment, and supplies, and to engage in any activity in connection therewith; (3) to do a public warehousing pusiness and store agricultural products in interstate commerce; (4) to engage in the financing of the above-stated activities; and (5) to handle the products of and rende any of the foregoing services to non-members; provided, that this Association shall not market, handle, process, store, or deal in che products of non-members in and further that it in value than such as is transacted by it with members; and provided further that it shall not engage in the business of banking. THIRD

That the place where its buisiness is to be transacted is at Navarre, Dickinson County, Kansas.

\section{FOURTH}

That the term for which this Corporation is to exist is fifty years. PIFIH

That the number of directors of this Corporation shall be not less than five (5) nor more than twenty-five (25) as may be provided in the bylaws of the Association. 
IT IS MUTUALLY AGREED that the instrument(s) described as follows.

Agreement dated June 21, 1954, Santa Fe's Secretary's Contract No. 104166, between the parties hereto, relating to use of a portion of Senta Fe's property at Navarre, Dickinson County, Kansas, as a site for grain storage bins, as modified by Supplemental Agreement dated April 12, 1961, increasing the rental from $\$ 31.61$ to $\$ 36.88$ per annum, effective May 15 , 1961,

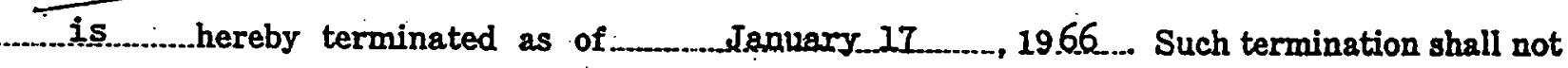
release any party hereto from any liability or obligation under the instrument(s) hereby terminated, whether of indemnity or otherwise, resulting from any acts, omissions or events happening prior to the date of termination or thereafter in case by the terms of said instrument $(8)$ it is provided that anything shall or may be done after termination thereof.

IN WITNESS WHEREOF the parties hereto have executed this agreement in duplicate the day and year first above written. 


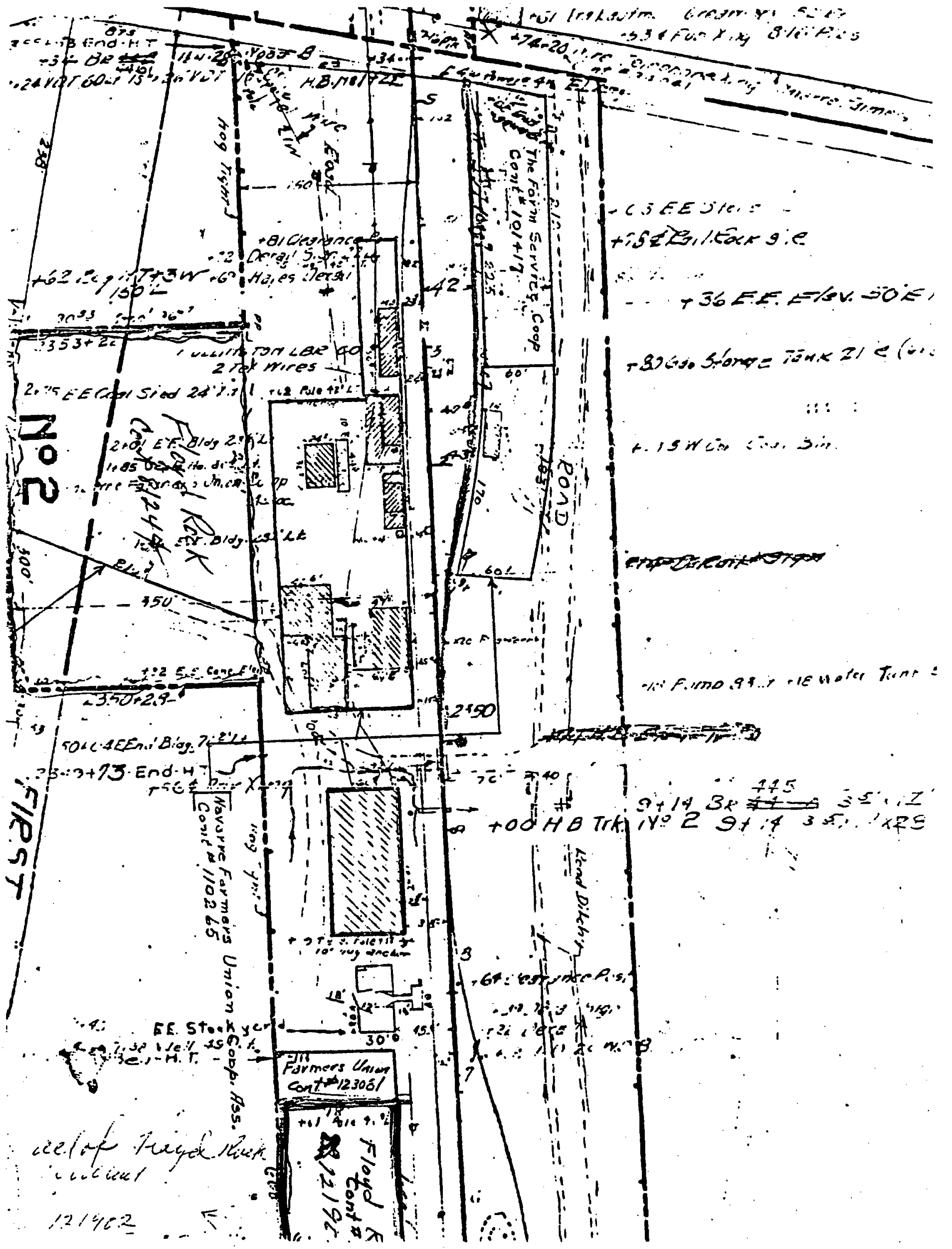




\section{CERTIFICATE OF REINSTATEMENT, RESTORATION, OR RENEWAL}

State or_ RANSAS

We, Alvin Sandow being the last acting President, Secretary, or Treasurer of The Navarre Farmers Uninn Cotop Ass'n provided by law, herewith file in behalf of said corporation this certificate for relnstatement, renewal, revival, res.. . : ... other officers elected as toration, and extension of its corporate existence or authority to engage in business in the State of Kansas:

(A) The correct name of the corporation is Navarre Farmers Union Cooperative Assaciation.

(B) The location of the corporate registered office in the State of Kansas is.

Box 85 Navarre (Town or Chy) Diakinson (Streot and Namber)

\section{(Town or Cus)}

(Coonty)

(C) The name and address of the corporate resident agent in the State of Kansas is

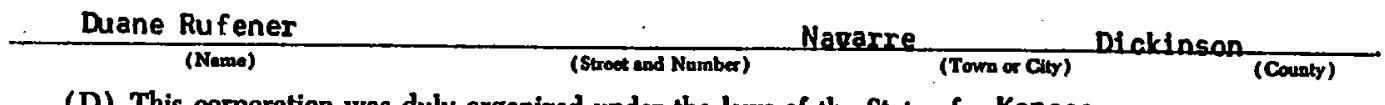

(D) This corporation was duly organized under the laws of the State of Kansas on the_18 day of Apri1 19

(E) The corporate existence, or authority to engage in business in the State of Kansas:

(1) Has expired, or will expire, by reason of time on thell___day of_Apr11 1969 and said corporate eristence, or authority to engage in business, is hereby extended for a period of fifty years from the 18 . day of April $19^{69}$

(2). Has been declared canceled by the Charter Board of the State of Kansas because said corporation failed to file its annual report and pay its annual fee provided by law for the year-

(3) Has been renewed, but through failure to comply strictly with the provisions of law, the validity of the renewal has been brought into question; hence, the corporate existence, or authority, is hereby extended for fifty years from the day of

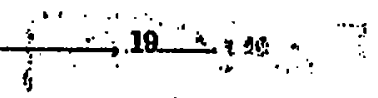

(F) This certificate is filed by authority of the duly elected directors or managers of the corparation in compliance with the provisions of Chapter 17, Article 48, G. S. of Kansas, 1949 and all amendments thereto.

IN TESTIMONY WHEREOF, we have hercunto set our hands and affixed the seal of the corporation this 24 day of February $10-69$

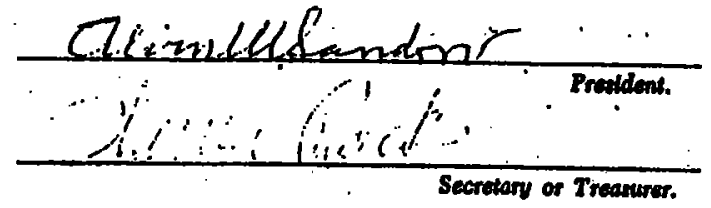

( SEAL) 


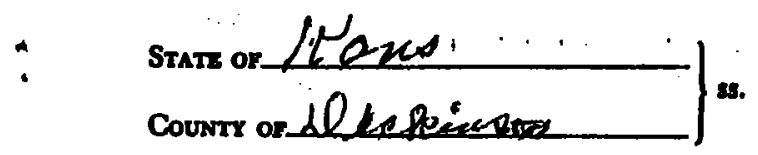

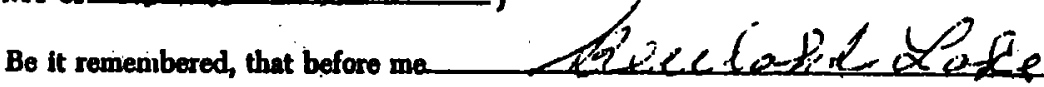
a Notary Publi

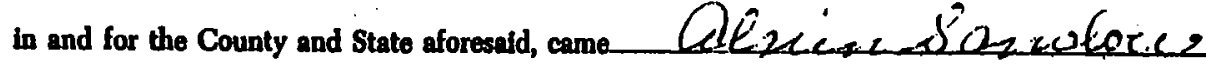
and 2 virsen $1 P$ se

personally known to me to be the persons who executed the foregoing instrument of writing, and duly acknowledged the execution of the same this__a day of Lep. 1869

(Sen)

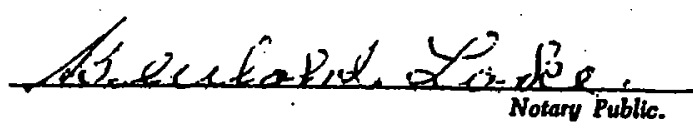

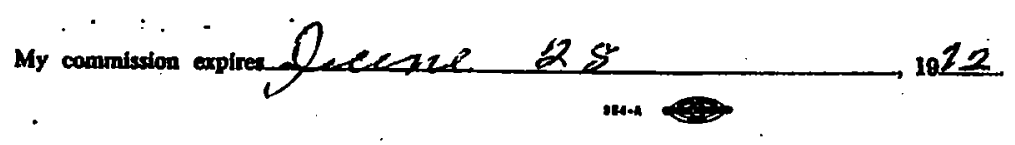

Foar No. 207C
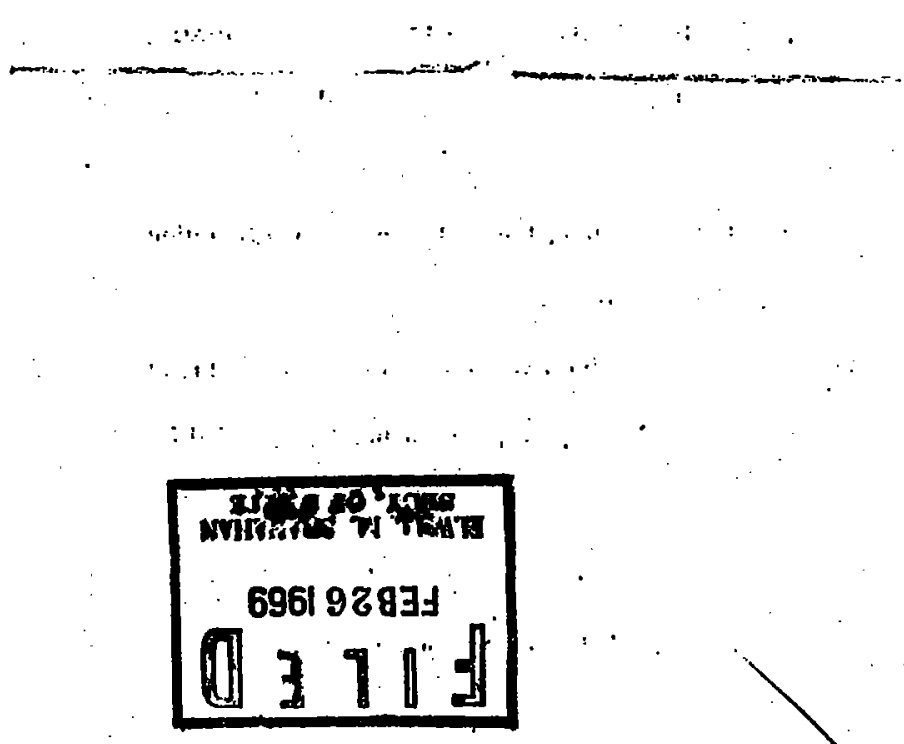

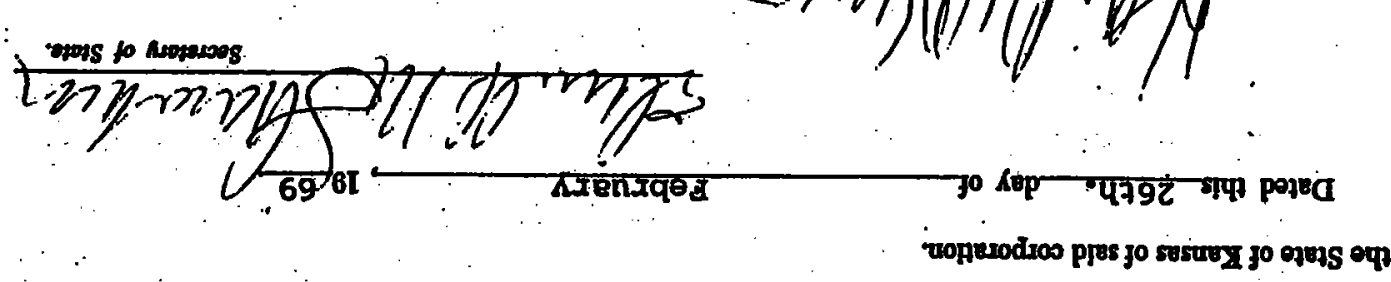

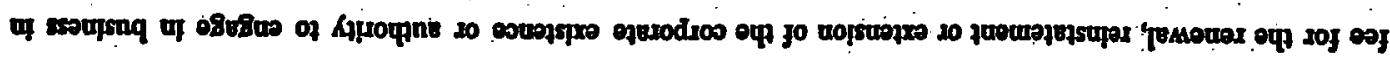


THIS LEAST, Made as of the 21 st day of Noratiber 1069 between THE ATCHSON, TOPEKA AND SANTA FE BATLWAY COKPANY, Kansar corporation (hereinafter callod "Lessor"),

and THE NAYARRE FARUERS UNTON COOPERATIYE ASSOCIATTOH,

a Kansar conporation

(hereinafter, whether one party or more, called "Lessee").

WTTNESSETH, That the parties hereto for the considerations hereinafter expressed covenant and agree as follows:

1. Lessor hereby leases to Lessee, subject to the rights and easements hereinafter excepted and reserved, and upon the terms and conditions hereinafter set forth, the land (hereinafter called "Premises") situated at or near. Navarea County of niakinan

State of Kansan , outlined in red coloring on the print hereto attached, No._SI_Ll-375 dated Norrembar 5,1969 marked "Exhibit A" and made a part hereof, for a term beginning on and ending when this lease shall be terminated as hereinafter provided.

2. Lessor hereby excepts and reserves the right, to be exercised by Lessor and by any others who have obtained or may obtain permission or authority from Lessor so to do, (a) to operate, maintain, renew and relocate any and all existing pipe, power, and communication lines and appurtenances and other facilities of like character upon, over or under the surface of the Premises; and (b) from time to time to construct, operate, maintain, renew and relocate such additional facilities of the same character as will

tha nas of the Promiase ho $T$ saxpa for the vurbose specified in paragraph

3. Lessee shall pay to Lessor as rental for the use of the Premises the sum of Eighty-fly and $\mathrm{Ho} / 100$ Dollars (\$_85.0e ) per. year. payable in advance. vals.

4. Lessee covenants and warrants that Lessee either owns, or has obtained from the owner or owners thereof the right to use, any improvements now on the Premises shown or described on said Exhibit A as "Lessee's Existing Improvements." Such improvements, if any, together with any other improvements hereafter placed upon the Premises by or for account of Lessee are hereinafter called "Im-
provements."

5. Lessee shall pay before the same became delinquent all taxes, charges, rates, and assessments which may, during the term of this lease, be levied upon, or assessed against, or be equitably chargeable to or assessed in respect of the Improvements ; and where any such tax, rate, charge, or assessment may be embraced in the general amount of taxes charged upon the Premises separately or in connection with other property of Lessor and Lessor shall pay all of said taxes, then Lessee shall promptly repay. or re. fund to Lessor the amount or part of the tax, charge, rate or assessment equitably or fairly apportion-

6. Lessee shall use the Premises exclusively as a site for handling Hquid and bulk fact ilize

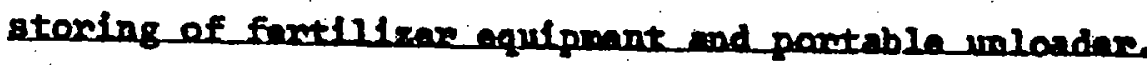

7. Lessee shall keep and maintain the Premises and Improvements in such safe, sanitary, and sightly condition as shall be satisfactory to Lessor, and, if required by Lessor, shall paint the Improvoments with paints of a color approved by Lessor; and if Lessee fails or refuses within fifteen (15) days after receipt of any request by Lessor so to do, Lessor may, at its option, perform such work, and in such event Lessee shall within thirty (80) deys after the rendition of bill therefor reimburse Lessor for the

8. In using the Premises, and in constructing, maintaining, operating and using the Improvements thereon, Lessee shall comply with any and all requirements imposed by federal oring the Improvements
ordinances, orders, or regulations of any governmental body having jurisdiction thereover. In th. Or by 
8. In using the Premises, and in constructing, msintaining, operating and using the Improvements thereon, Lessee shall comply with any and all requirements imposed by federal or state statutes, or by the Premises or Improvements shall be used for the loading, unloading, storing, or otherwise the event any petroleum products, Lessee shall comply with all applicable regulations and recommendations of time to time promulgated by the Bureau of Explosives of the Association of American Railroads from successor agency. All artificial lighting in pump houses, warehouses, or other enclosures upon the Prany ises, where oil or other inflammable fluid supplies are handled or stored by Lessee, except in unbroken original containers, shall be by electricity, and such electrical installation and any other electrical installation upon the Premises shall at all times conform to and be maintained in accordance with the provisions of the then current edition of the National Electrical Code with respect to Class I hazardous locations. Lessee shall pramptly pay and discharge any and all liens arising out of any construction, alteration or repair work done, or suffered or permitted to be done, by Lessee on the Premises, and Lessor is hereby authorized to post any notices or take any other action upon or with respect to the Premises that is or may be permitted by law to prevent the attachment of any such liens to the Premises; provided, however, that the failure of Lessor to take any such action shall not relieve Lessee of any obligation or liability under this or any other paragraph hereof.

9. Lessee agrees to indemnify and save harmless Lessor against all loss, damage or expense which Lessor may sustain, incur or become liable for, including loss of or damage to property or injury to or death of persons and fines or penalties imposed upon or assessed against Lessor, arising in any manner out of (a) the use of the Premises or Improvements by Lessee, (b) any breach by Lessee of the terms, covenants or conditions in this instrument contained, or (c) the sole or contributing acts or omissions of Lessee or the employes, agents, patrons or invitees of Lessee in, on or about the Premises or Improve. ments, except that if Lessor shall participate in any such contributing acts or omissions, then the loss, damage or expense arising therefrom shall be borne by the parties hereto equally.

10. Lessee shall at all times keep a space of six (6) feet from the nearest rail of any railroad track entirely clear of structures, material and obstructions of every sort and shall observe an overhead clearance of not less than twenty-five (25) feet above the top of rail; but, nevertheless, for convenience in handling freight to and from cars on any railroad track serving the Premises, the Lessee may install, use and maintain (a) loading or unlaading cranes or other devices not nearer than six (6) feet from the mearest rall of such track and no part of which shall at any time project or extend in the direction of such track except when crane or device is being used for loading or unloading freight and (b) platforms which shall be not more than three (3) feet and six. (6) inches higher than the top of the rails, and which at no point shall be nearer than four (4) feet to the nearest side of the head of the nearest rail of such track; provided, however, if by statute or order of competent public authority different clearances shall be required, then Lessee shall strictly comply with such statute or order. Irrespective of anything in Section 9 hereof contained, in case of a breach of the obligations contained in this Section 10, or of any of them, Lessee assumes and agrees to indemnify Lessor against all liability for loss, damage, injury and death resulting therefrom, and to reimburse Lessor for any sums which Lessor may have been required to pay in the way of damages, fines, penalties or other expense resulting, in whole or in part, from the failure of Lessee to comply with any of the provisions hereinabore in this Section 10 contained.

11. Neither Lessee, nor the heirs, legal representatives, successors or assigns of Lessee, nor any subsequent assignee, shall underlease or sublet the Premises or the Improvements, or any part thereof, nor assign or transfer this lease or any interest herein, without the written consent and approval in each instance of Lessor.

12. In case of the eviction of Lessee by anjone owning or claiming title to or any interest in the Premises, Lessor shall not be liable to Lessee for any damage of any nature whatsoever, or to refund any rental paid hereunder, except the proportionate part of any rental paid in advance.

13. If any rental hereunder shall be due and unpaid, or if default shall be made in any of the covenants or agreements of Lessee herein contained, or in case of any assignment or transfer of this lease by operation of law, Lessor may, at its option, terminate this lease by serving five (5) days' notice in writing upon Lessee; but any waiver by Lessor of any default or defaults shall not constitute a waiver of the right to terminate this lease for any subsequent default or defaults.

14. This lease may be terminated at any time by either party by serving thirty (80) days' written notice of termlnation upon the other party, stating therein the date that such termination shall take place, and upon the expiration of the time specified in such notice this lease and all rights of Lessee hereunder shall absolutely cease and determine; but upon any such termination Lessee shall be entitled to have refunded by Lessor a proportionate part of any rentals paid in advance. 
15. Any notice hereunder to be given by Lessor to Lessee shall be deemed to be properly served If it be deposited In the United States mail, postage prepaid, addressed to Lessee at

Naxuse, Kanere

Any notice to be given hereunder by Lessee to Lessor shall be deemed to be properly served if the same be deposited in the United States mail, postage prepaid, addressed to Lessor's

supanintandent at 201-Eat sixth-stpot,

Narton, Kansan 67114

16. Upon the termination of this lease in any manner herein provided, Lessee shall forthwith surrender to Lessor the possession of the Premises and shall remove the Improvements and restore the Premises to substantially the state in which they were prior to the construction of the Improvements, and in case Lessee shall fail within thirty (30) days after the date of such termination to make such removal or restoration, then Lessor may, at its election to be exercised within thirty (30) days thereafter, either remove the Improvements and restore the Premises for the account of Lessee, and in such event Lessee shall within thirty (30) days after the rendition of bill therefor reimburse Lessor for the cost so incurred, or may take and hold the Improvements as its sole property.

17. If Lessee fails to surrender to Lessor the Premises, upon any termination of this lease, all the liabilities and obligations of Lessee hereunder shall continue in effect until the Premises are surrendered; and no termination hereof shall release Lessee from any liability or obligation bereunder, whether of indemnity or otherwise, resulting from any acts, omissions or events happening prior to the date of termination or the date, if later, when the Improvements are removed and the Premises restored or Lessor elects to take and hold the. Improvements as its sole property as hereinabove in paragraph 16 provided.

18. In the event that Lessee consists of two or more parties, all the covenants and agreements of Lessee herein contained shall be the joint and several covenants and agreements of such parties.

19. All the covenants and agreements of Lessee herein contained shall be binding upon the heirs, legai representatives, successors and assigns of Lessee, and shall inure to the benefit of the successors and assigns of Lessor.

20. It is mutuslly agreed that Rider 'An hereto attachad, Identified by the olgotwo of D. E. Barnes, is hereby made a part hereof.

\author{
RIDER "A" \\ Attached to Lease Dated November 21, 1969 \\ Between \\ THE ATCHISON, TOPEKA AND SAMTA FE RAILHAY COMPANT \\ and \\ THE NAYARRE FARMERS UNIOH COOPERATIVE ASSOCIATIOH
}

21. Lessee agrees that it will at. all times, except when in actual use, keep sald portable unloader or any portion or appurtenances thereof a distance of not less than six (6) feet frow the nearest rail of Lessor's Track No. 1.

22. Notwithetanding anything contained in Section 9 hereof to the contrany, wessee agwes that it will at all times indernify and save hamless lossor against ell claims, dexunds, actions or causes of action, arialng or growing out of lose of or dange to property, Including but not limited to sold portablo unloader and appurtenancer, and injury to on death of persons, including but not 1 lmited to om or presence of said portIns in any manner fron the maintenance, use, atate of repald to sald Track Ho. 1, whother such able unlouder upon said prenises or upon or contributed to by the negligence of lessor, Its agents or enployes, or otherwiee, and that It will prontly pay to lessor the full or damage which lessor way sustaln, inciur or becom 11 able for and all oum which lessor way pay or be compelled to pay in iettlement of any clains on account thareat.

23. It Is mutually agred that the agrenment dated August 5, 1966, (Santa Fe's Secr tary's contrect 10,123081 ) between the parties bereto relating to use of a portion of

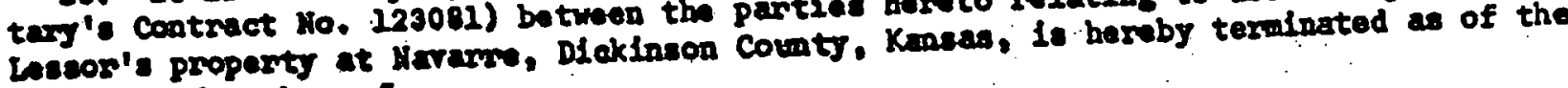
effective date bareol. 
(

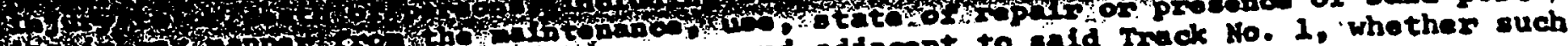

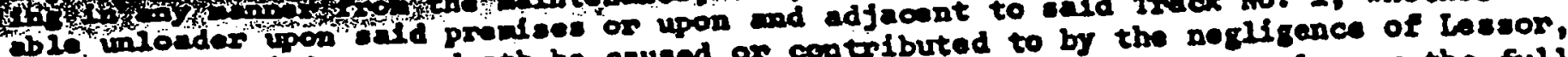

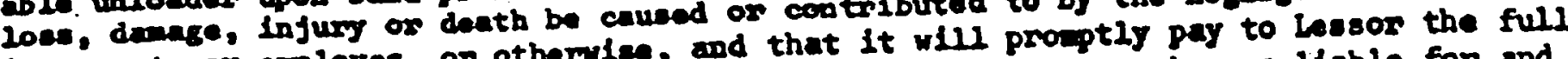

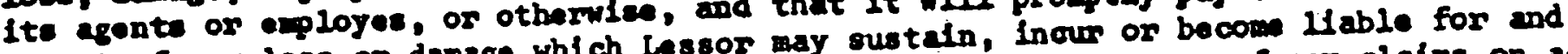

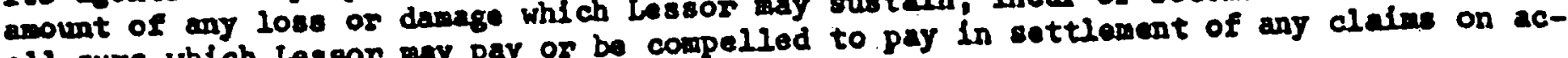
al wing which

23. It Is mutually agreed that the agmement dated August 5, 1966, (Santa Fe's Secre taxy's Contract No. 223081) between the parties bereto rolating to use of a portion of Lessor'a property at Mararre, Dlakinson County. Kansas, is hereby terminated as of the effective date barnof.
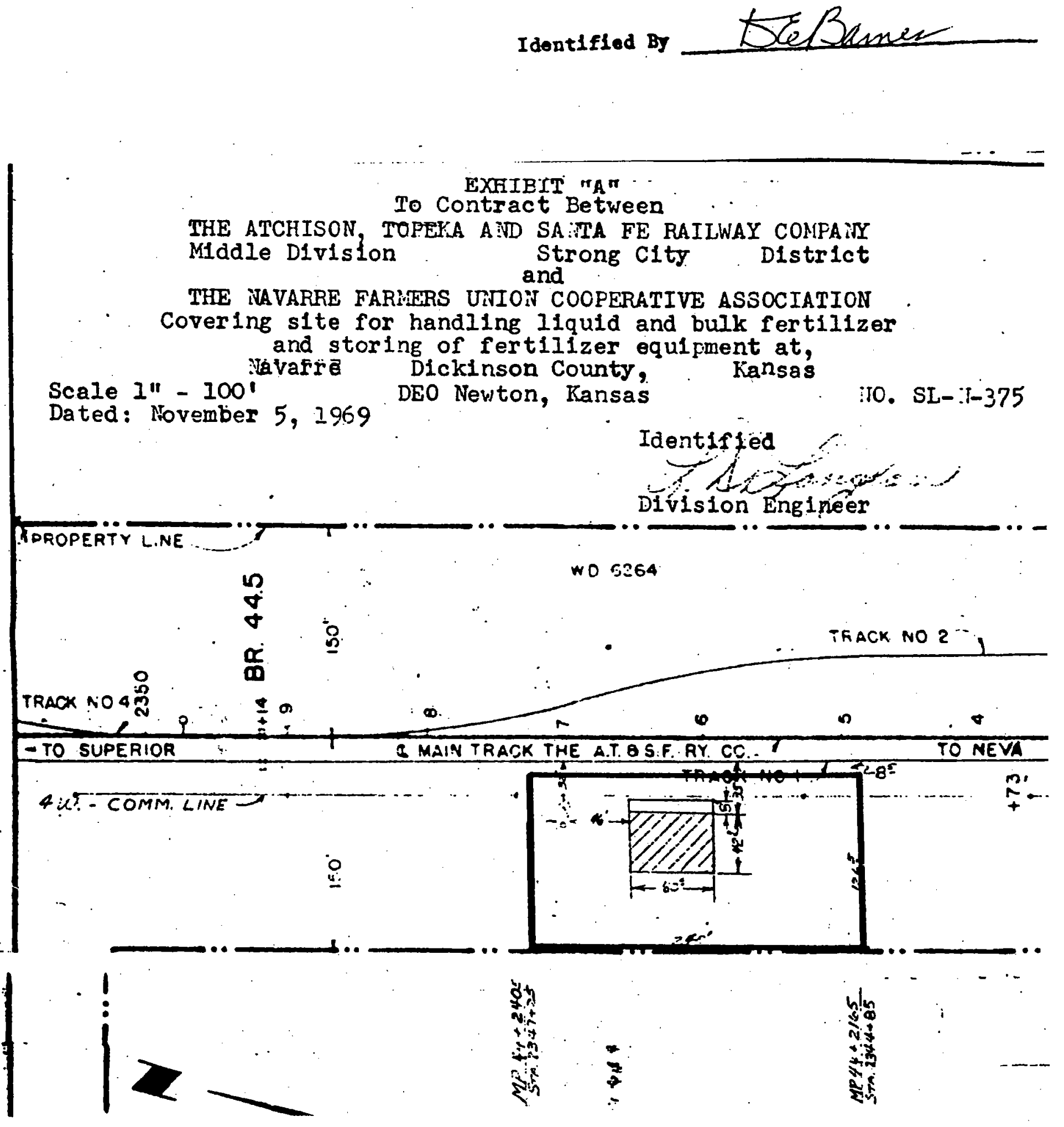


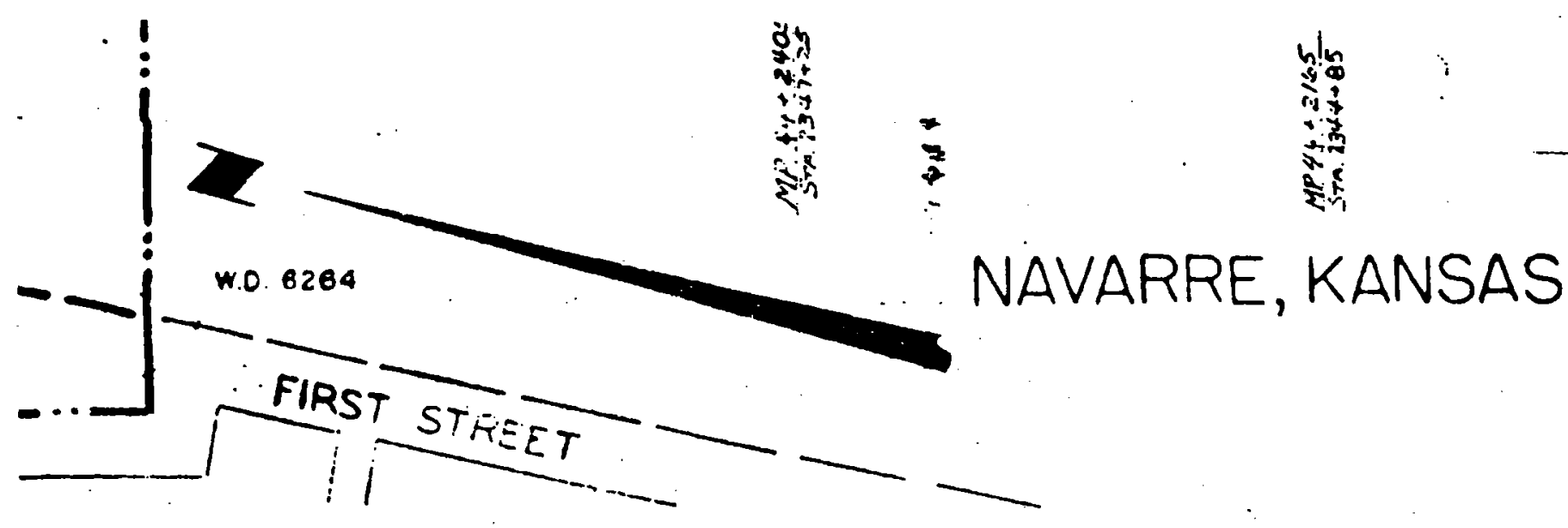

$\checkmark$

Area of site: 30,360 sc. ft.

DEO Drawing Number SL-..-375.

Corresfonderce File Itumber 66-34219
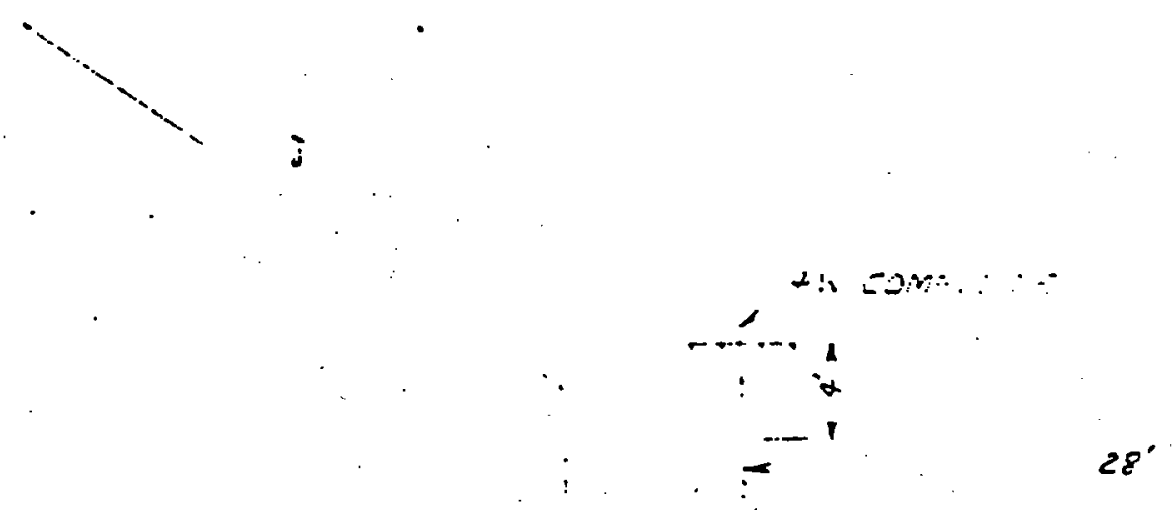

E-icis

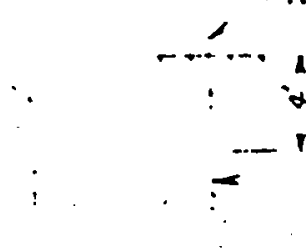

$16^{\circ}$

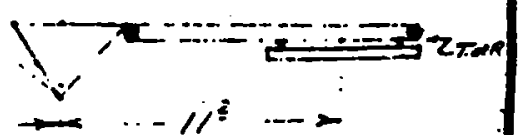




\section{CONTRACT FOR INDUSTRY TRACK}

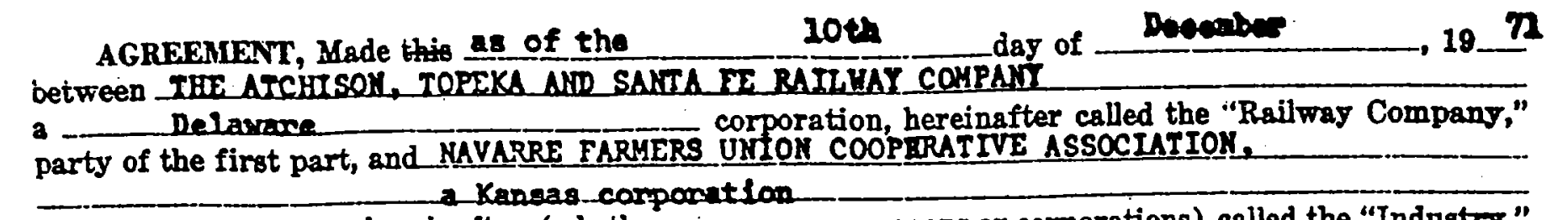

party.... of the second part.

hereinafter (whether one or more persons or corporations) called the "Industry,"

RECITALS:-The Industry has requested the Railway Company to operate and maintain a track or track extension, as the case may be, about 378. feet in length, hereinafter in its entirety referred to as "The Track," at or near the station of Maramp, DLokinson County, Kansas to serve a grain, coal and bulk oll handing facllity

- (hereinafter called the "Plant"), to be operated by the Industry. The Track is shown by red coloring upon the print hereto attached, marked "Exhibit $A$ " and made a part hereof.

\section{AGRDEMINT: $\quad$ ARTICLE $I$.}

In consideration of the covenants of the Railway Company, the Industry agrees as follows:

1. That it will and hereby does grant to the Railway Company for the term of this agreement, free of cost, a right of way, eight and one-half $(81 / 2)$ feet on each side of the center line thereof, for all that portion, if any, of The Track located on property belonging to the Industry, with the right to maintain and operate The Track thereon, and where any part of The Track lies on a public street or alley, or upon property belonging to third persons or corporations, that it will secure to the Railwas Company by ordinance or grant, as the case may be, in form satisfactory to the Railway Company's counsel, and without cost to the Railway Company, the right to maintain and operate The Track thereon, together with the right to remove the same; in the case of a grant, the right of way shall be eight and one-half $(81 / 2)$ feet on each side of the center line of The Track.

2. That it will pay to the Railway Company, in advance, the estimated cost of

$$
\text { Ho cost to be bosene by the Industry under this section. }
$$

the cost 80 to be paid by the Industry

being bereby estimated at. . If the actual cost shall be more or less than such estimated cost, the difference shall be promptly paid by the Industry or repaid by the Railway Company, as the case may be.

3. That it will pay to the Railway Company, from time to time, within twenty (20) days after bills are rendered therefor, the entire cost (or an equitable share thereof in case other industries shall be served by The Track or a part thereof, or in case the Railway Company shall make any other use of The Track in the exercise of the right given in Article III, Section 1) of maintaining and renewing that portion of The Track beyond the clearance point, the Railway Company to be the judge of the necessity for and character of maintenance required on The Track. It is understood that the expense of maintaining and renewing The Track shall include any expense to which the Railway Company may be put in the way of paving, sewers, crossing protection or other work, because of the existence of The Track.

4. That it will operate the Plant during the term hereof. 
5. The Industry agrees to indemnify and hold harmless the Rallway Company for lose, damage or injury from any act or omission of the Industry, its employes or agents, to the person or property of the parties hereto and their emplojes, and to the person or property of any other person or corporation, while on or about The Track; and if any claim or liability shall arise from the joint or concurring negliBence of both parties hereto, it ahall be borne by them equally.

6. That it will at all times keep a space of six (6) feet from the nearest rail of any railroad track entirely clear of structures, material and obstructions of every sort and will observe an overhead clearance of not less than twenty-five (25) feet above the top of rail; but, nevertheless, the Industry may erect loading platforms which shall not be higher than three (3) feet and six (6) inches above the top of the rails and which at no point shall be nearer than four (4) feet to the nearest rail of such track; provided, however, if by statute or order of competent public authority different clearances shall be required than those provided for in this Section 6, then the Industry shall strictly comply with such statute or order. In case of a breach of these obligations, or any of them, the Industry assumes and agrees to indemnify the Railway Company against all liability for 1058 , damage, injury and death arising therefrom and to reimburse the Railway Company for any sums which the Rallway Company may have been required to pay in the way of damages, fines, penalties or other expense resulting from the violation by the Induotry of any statute or order as aforesaid.

\section{ARTICLE II.}

In consideration of the covenants of the Industry, and the faithful performance thereof, the Railway Company subject to the provisions of Sections 2 and 3 of Article I hereof, agrees to maintain The Track during the term of this agreement, unless its obligation 80 to maintain it may be sooner terminated as herein provided, and to transfer cars, loaded or empty, thereover to and from the Plant subject to any lawful charges that may be imposed by the Railway Company for suck transfer.

\section{ARTICLE III.}

The parties mutually agree as follows:

1. The title to The Track and to all property furmished in the maintenance thereof shall be in the Railway Company. The Railway Company shall have the right to use The Track for other than the express purpose of serving the Plant.

2. If the Industry shall fail for a period of_alx (6)___ months in ang period of twelve monthis to operate the Plant, or shall fail or refuse to comply with or carry out any of the covenants or agreements herein contained, the Railway Company may, at its option, expressed in writing, terminate this agreement; but no termination shall release the Industry from any liability or obligation under this agreement (whether of indemnity or otherwise) resulting from any acts, omissions or events happening prior to the date of termination.

thint d if Unless earlier terminated as herein provided, this agreement shall be in force for the term of \&

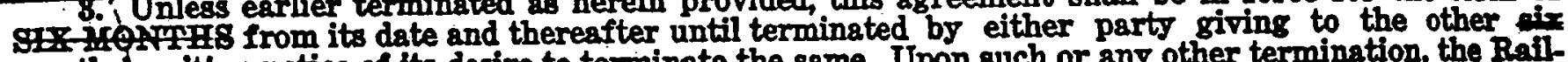
months written notice of its desire to terminate the same. Upon such or any other termination, the Rail way Company shall have the right to remove The Track and every part thereof. This agreement shall also terminate forthwith in the event the Railway Company shall be dispossessed of the right to operate over any privately owned track of which The Track or any part thereof is an extension.

4. Any notlce to be given by the Railway Company to the Industry hereunder shall be deemed to be properly served if the same be delivered to the Industry, or if left at the Plant with any of the agents, servants or employes of the Industry, or if posted on the Plant, or if deposited in the postoffice, postpaid, addressed to the Industry at Naraxe. Cans.

5. In the event that the Industry embraces two or more persons or corporations, all the covenants and agreements of the Industry herein shall be the joint and several covenants and agreements of such persons or corporations.

6. All the covenants and provisions of this agreement shall be binding upon the successors, legal representatives and assigns of the Industry to the same extent and effect as the same are binding upon the Industry, and each and every covenant herein shall inure in favor of and run to the successors and assigns of the Rallway Company and to each and every person, firm or corporation which may hereafter own or be in possession of or operate the railroad of the Railway Company to the same extent and as fully as though such person, firm or corporation were specifically named in this agreement in the place and stead of the Railway Company; provided, however, no assignment hereof by the Industry, its successors, legal representatives or asigns, or ans subsequent assignee, shall be binding upon the Railway Company without the written consent of a Vice-President or a General Manager of the Railway Company in each inistance. 
IN WITNESS WHEREOF, the parties have executed this agreement in duplicaterthe day and year first above written.

TEE ATCHISOY, TOPEKA NDP SAITA FE RAIIWAY COMPANY

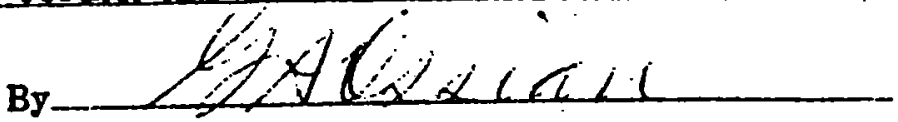

Approved:

Its_Aselatant to Canaral Manager.

As to Form

Genoral Attorney.

As to Description

MAVARRE TARUER UNCOK COORERATTYE ASSOCTATION

Chiet Encincer.

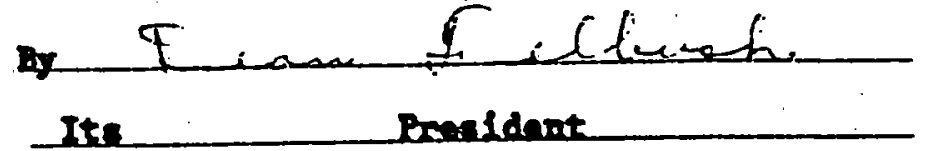

(Attach print here.)

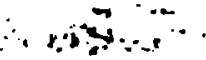




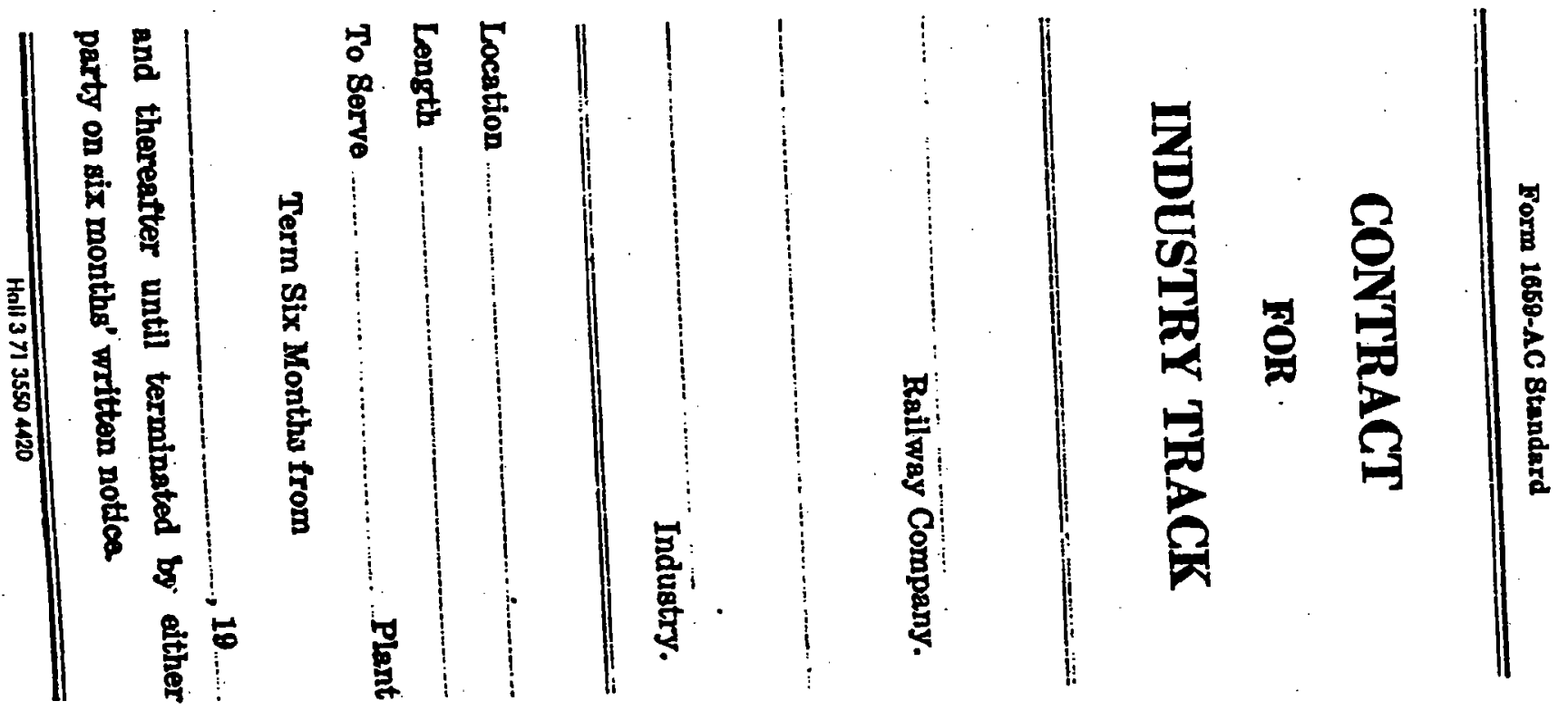




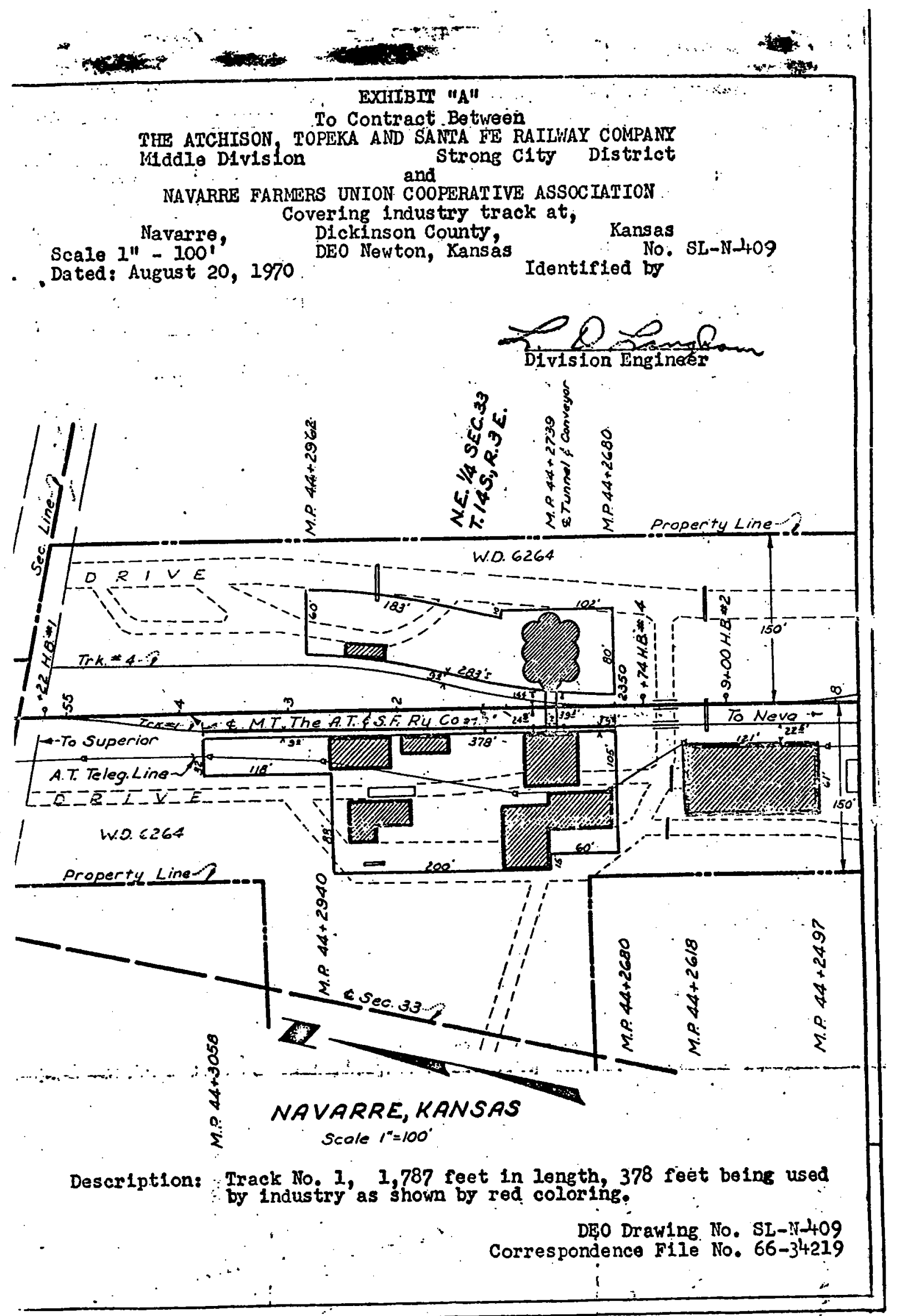




\section{The Atchison, Topeka and Santa Fe Rallway Company}

201 East Sixth Street

Newton, Kansas 67114

January 14,1974

$66-34219$

Mr. Duane Rufener, Manager

Navarre Farmers Union Cooperative Association

Navarre, Kansas 67469

Dear Sir:

This has reference to your telephone conversation with my Contract Clerk, Mr. Danby, concerning the possibility of leasing a portion of our right of way at Navarre, presentiy under lease to bir. Floyd Rock, for expansion of your facilities for construction of a new office and scale.

Upon checking our records, we find that we have three contracts with Mr. Floyd Rock at Navarre; in view of which, I am not just sure of which property you are referring to; however, am attaching a print from portion of our station grounds. Which I believe includes the area in which you are interested.

Ifill you please indicate on the sketch the area desired by Navarre Farmers Union Cooperative and return the sketch for further consideration.

Yours truly,

E. I) \&itimerc

E. Gillmore

Superintendent

thri jorensos 


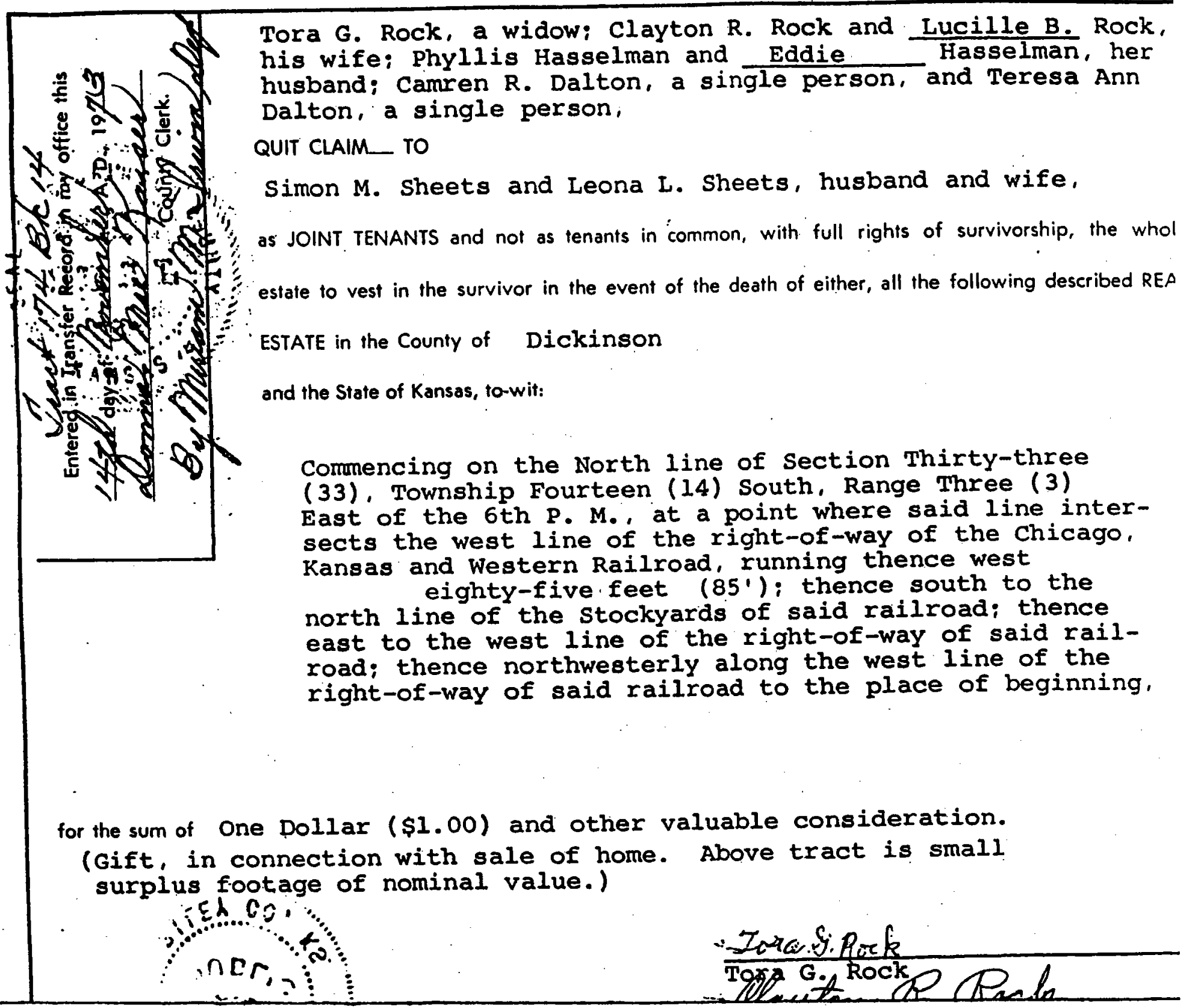




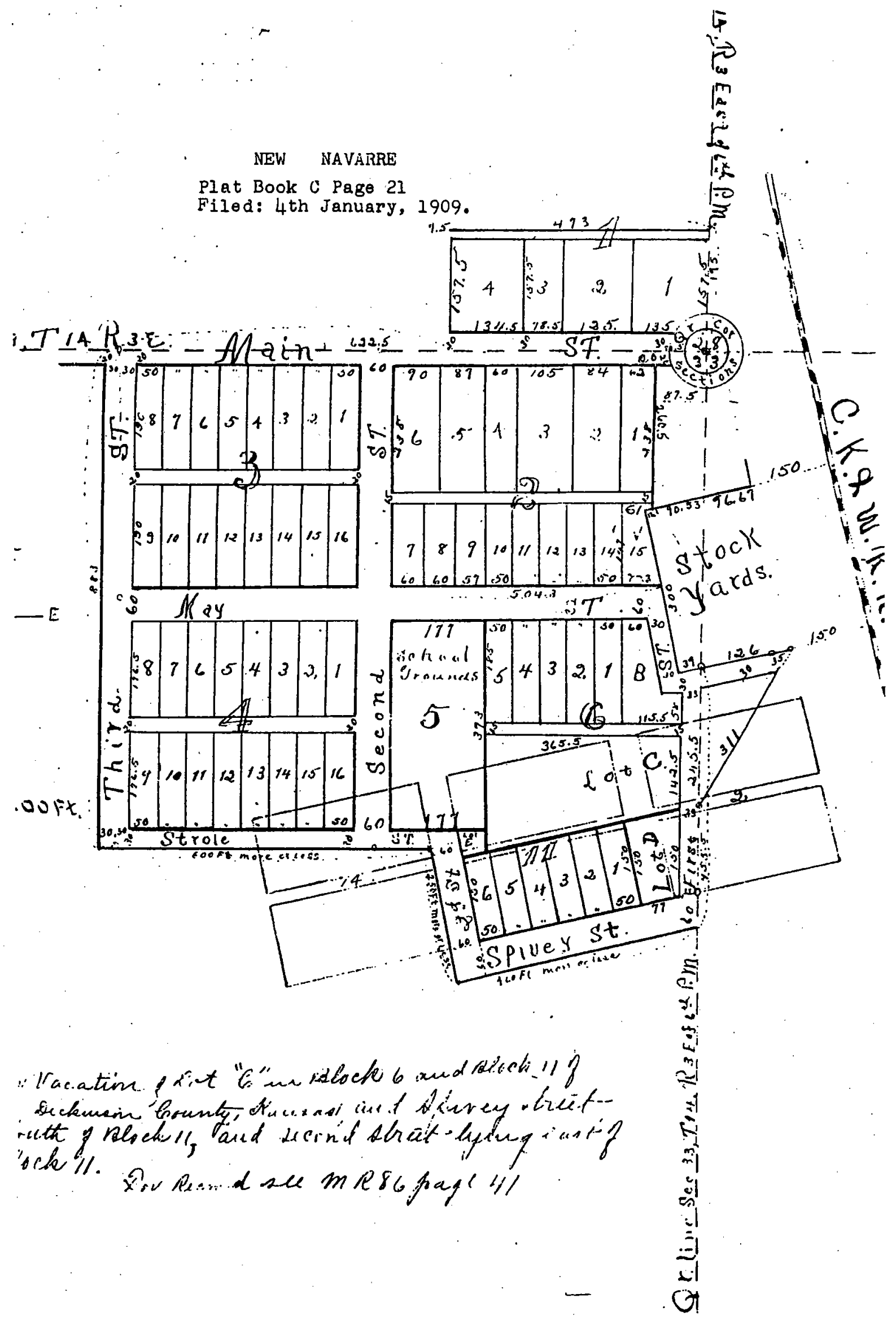




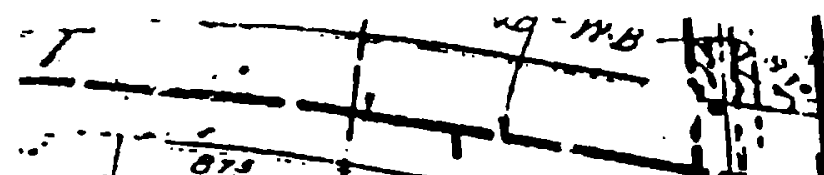

13

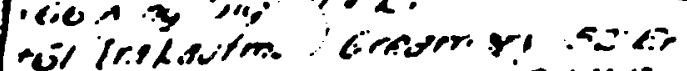

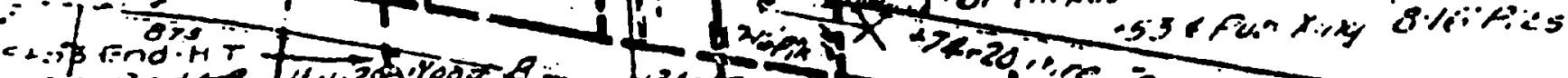

-jf be the fo.

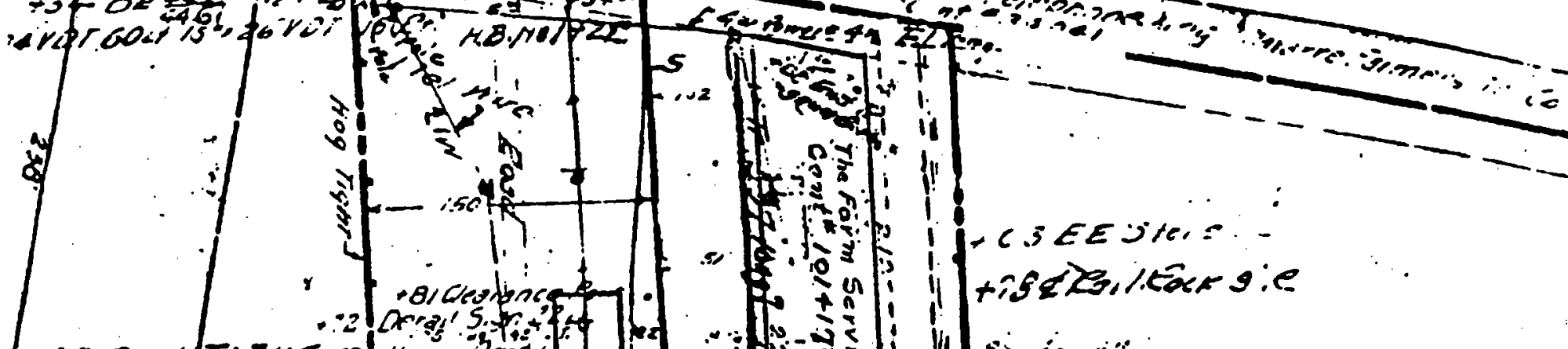

toz acy isp?

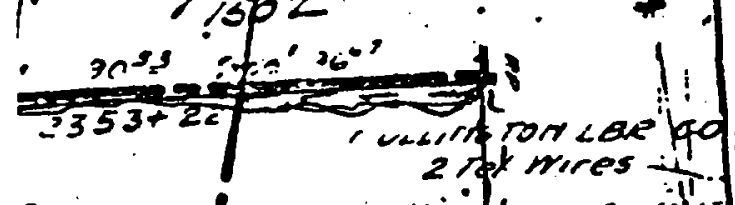

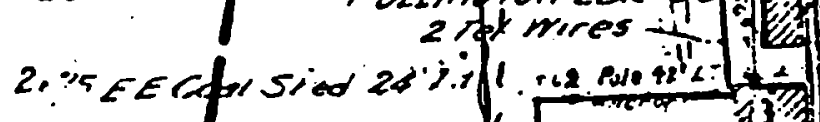

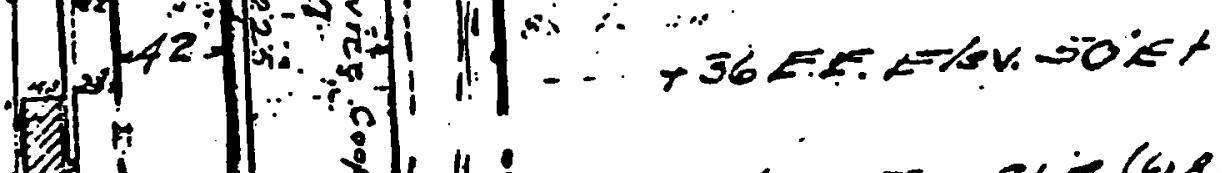

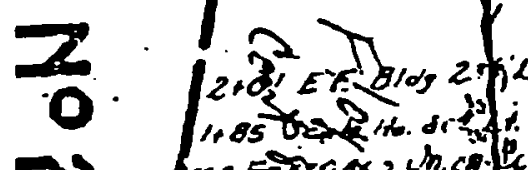

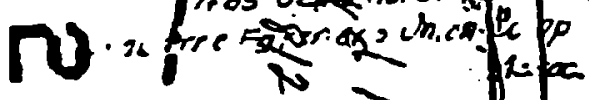

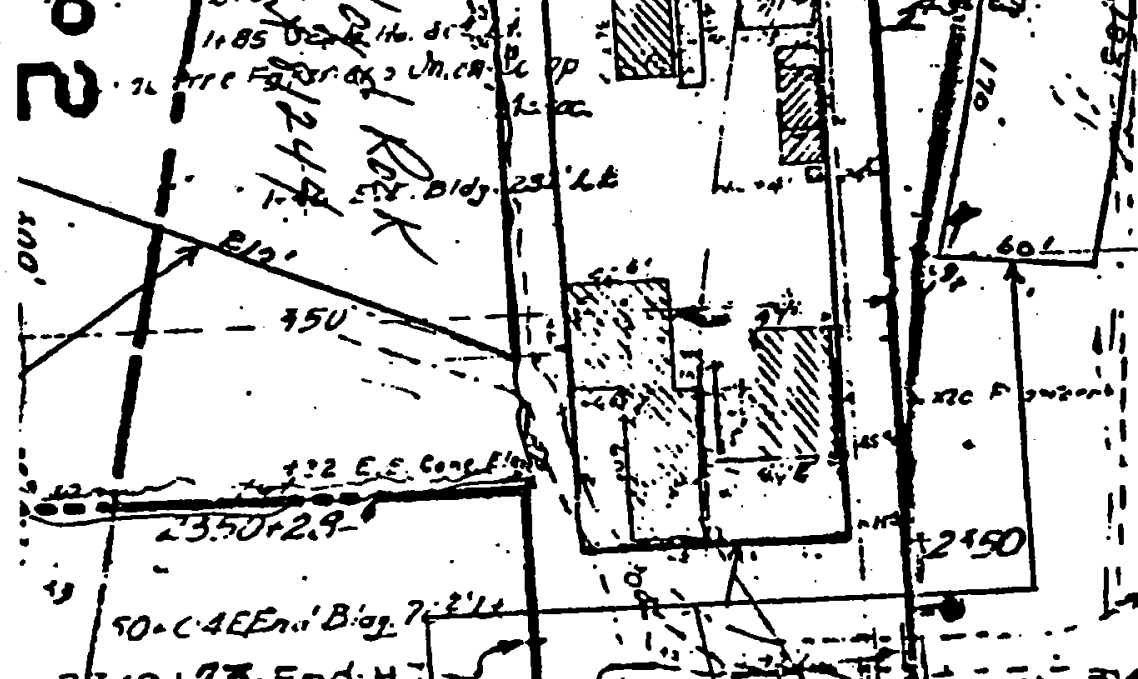

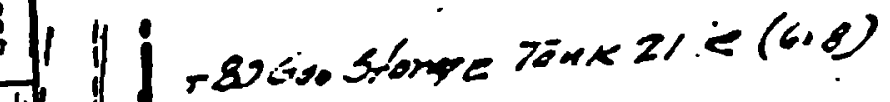

tising

\section{.}

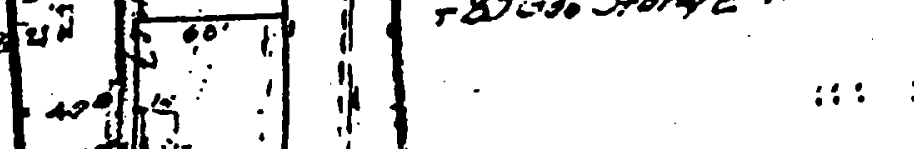




\section{CERTIFICATE OF AMENDMENT TO ARTICLES OF INCORPORATION \\ OF}

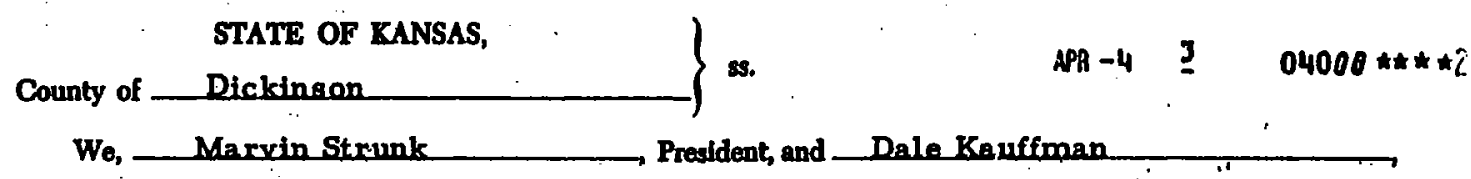

Secretary of Navarre Farmerg Union Cooperative Asepciation $\quad \cdot \cdots \cdot$

a corporation organized and existing under the laws of the State of Kansas, and whose reghted, officis

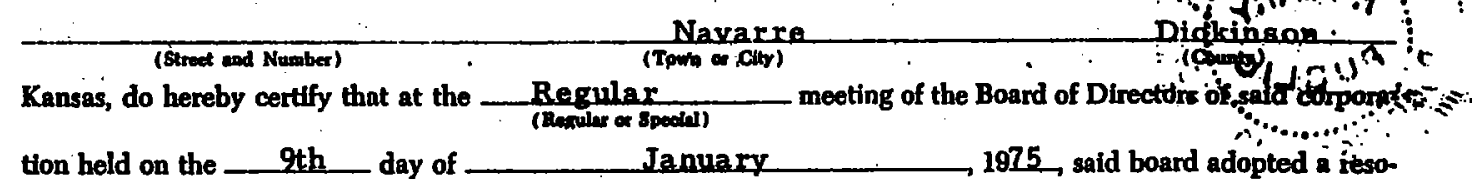

lution setting forth the following amendment to the Articles of Incorporation and declared its advisability, to wit:

RESOLVED, that Article VI of the Articles of Incorporation be changed to read as follows:

\section{SIXTH}

That the amount of the capital stock of this corporation shall be five hundred thousand dollars $(\$ 500,000)$ and shall be divided into fifty thousand $(50,000)$ shares of common stock of the par value of $\$ 10$ per share.

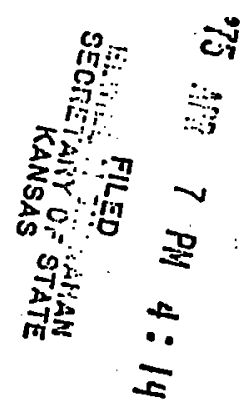

That thereafter, pursuant to said resolution and in accordance with the by-laws and the laws of the State of Kansas, said directors called a meeting of stockholders for the consideration of said amendment, and thereafter, pursuant to sald notice and in accordance with the statutes of the State of Kansas, on the 27th

January. 10.75 said stockholders met and convened and considered said proposed amendinent.

That at said meeting the stockholders entitled to vote did vote upon said amendment, and the majority of votting stockholders of the corporation had voted for the proposed amendment certifying that the votes were

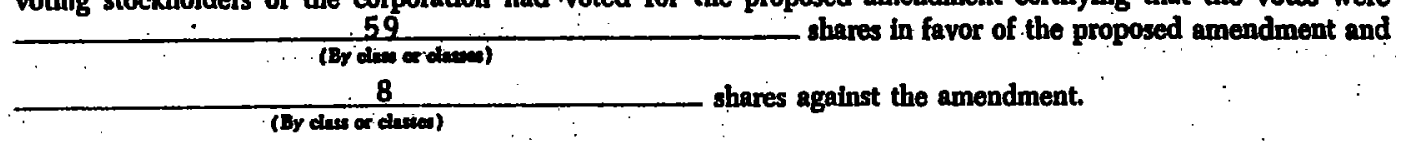

That said amendment was duly adopted in accordance with the provisions of K.S. A. 1972 Supp. 17-6602.

That the capital of said corporation will not be reduced under or by reason of said amendment. Is Wrizess Warpror we have hereunto set our hands and affixed the seal of sald corporation this

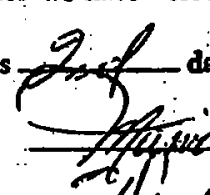

day of Cueseit 


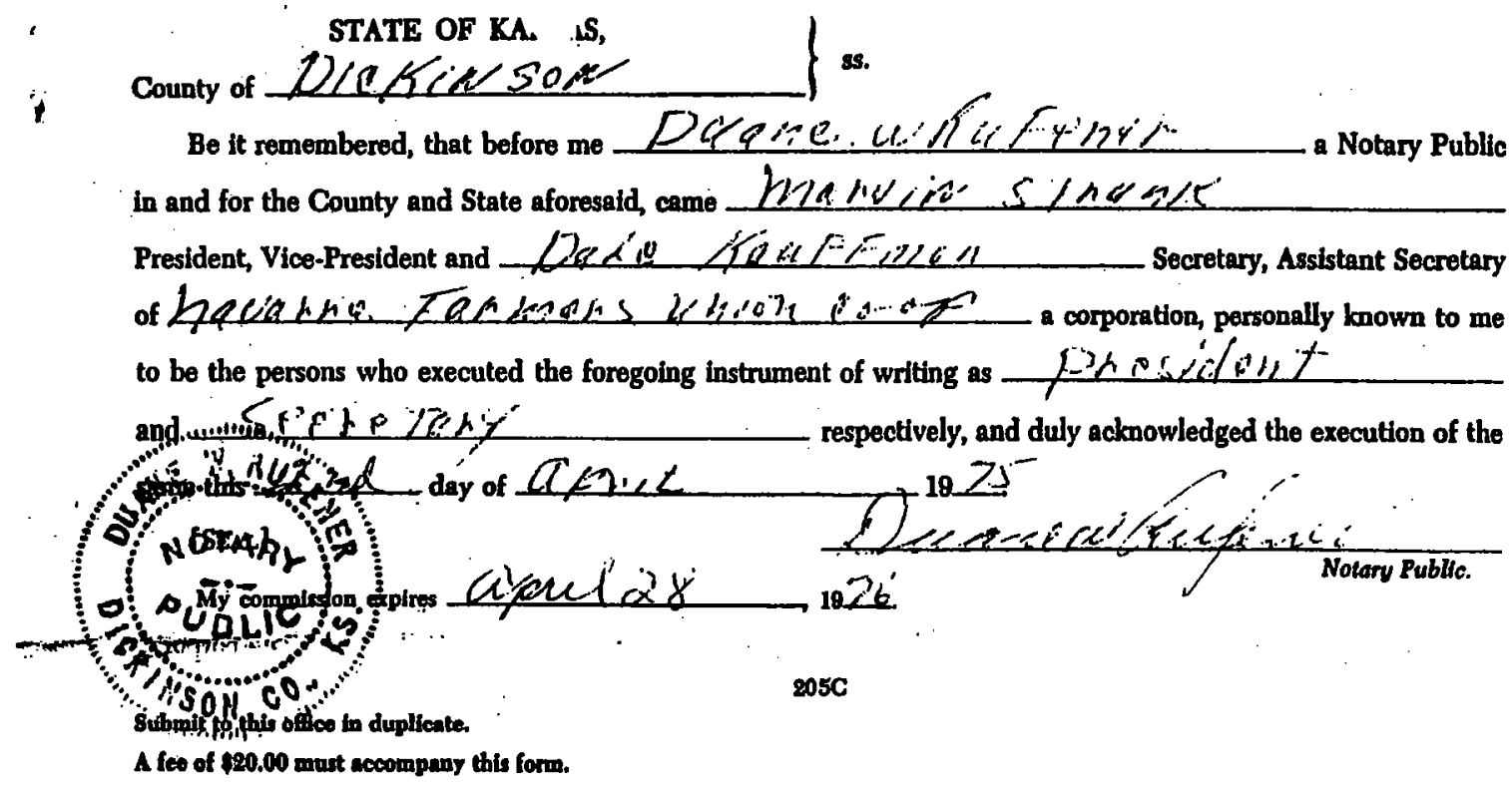


하두

Fon 1816 mandor

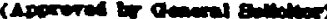

LEASE OF LAND (Short Term)

THIS LaAgI, Yade as of the

25th

des of

Apr11

10.7

betweon TIE ATChISOX, TOPERA AID SAITA FE RAILHAY COHPANY

2. Delaware

corporation (herelnafter callod "Temer"),

and _. IYR HAVARRE FARMEBS INIOH CO-OPRRATIVE ASSOCTATION.

a Kansas corporation

(hereinafter, whether one party or more, called "Lawbe").

WTrNEssETH, That the parties hereto for the consideratlonis hereinafter expressed corenant and acteo as follow:

1. Lessor hereby leases to Leasea, subject to the rights and easements hereinafter ercepted and re cerved, and upon the terms and conditions hereinafter and situated at or near Nararre County of

State of Rana outlined in red coloring on the print hercto attached, No._St-N-444_, dated_Eebruacy 12, 1975

marked "Exhibit A" and made a part hereol, lor a term berinning on" and ending when this lease thall be terminated as hereinnfter propided.

Nay 1 1075

2. Leesor bareby excopts and reserves the right to be exercisod by Leasor and by any othere who

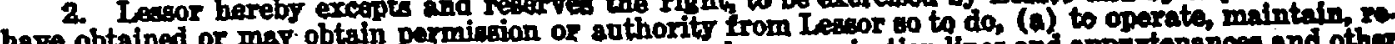
and

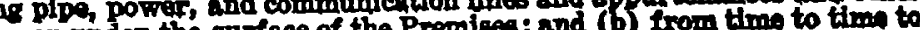

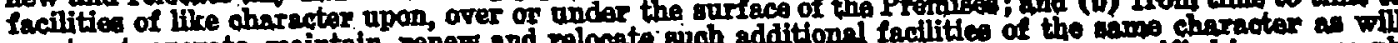

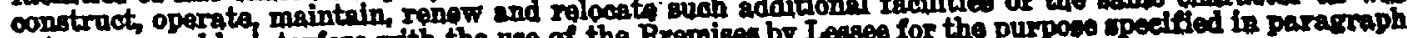
not unreasc 6 bareof.

3. Leage chall pay to Iassar as rental for the use of the Pramiges the wum of Blye Hundred Porty and No/100

Dollars (\$ 540.00 per___par payable in adrance

Said rental shall bo subject to revision at fro-(5) vals. three (3) 4. Ieesee covenants and warrants that Iessee aither opns, or has obtained from the owper or owners thereaf the sight to voe, any improvements now on the Premises any, together with any othat im hibit A as "Iessee's Haisting Improvements" Such improvements propements here provementa

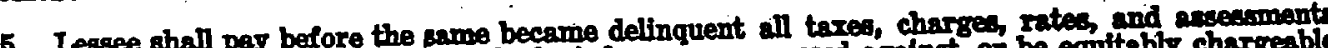
lor

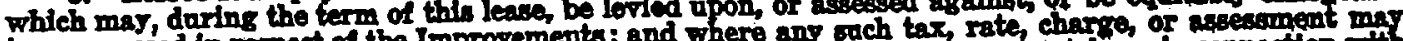
to or assessed in respect of the Improvements; and where ang ouch tax, rete, eratet of in connectlon with

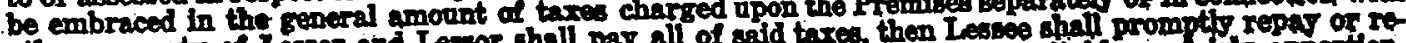

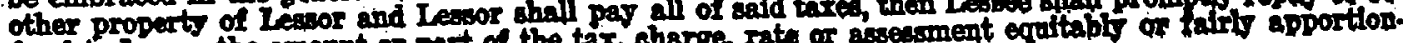
fund to Iessor the amonnt on able to the Improvementa.

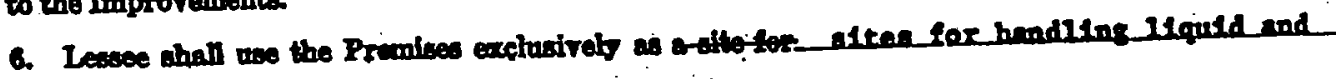

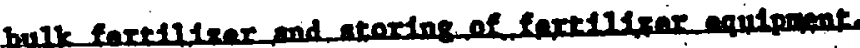

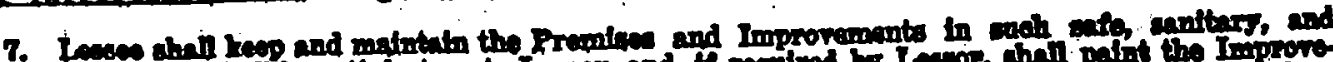

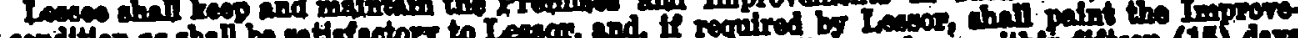

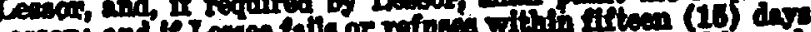

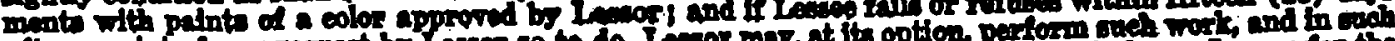

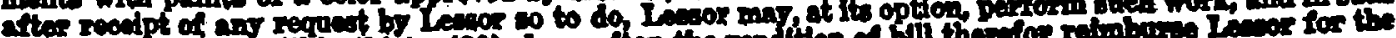

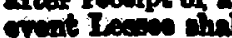
cost no inenreil 
15. Any notice hereunder to be given by Lessor to Leasee thall be deemed to be properby served If It be deposited in the United States mall, postage prepaid, addressed to Leseee at

Navarre, Kangan.

Anj notice to be given herennder by Leasee to Leasor ghall be deened to be properly served if the samo be deposited in tho United States mail, postage prepaid, addreased to Lesoor's

Superintendent.

at Neston, Rensas 67114

16. Upon the termination of this lease in any manner herein provided, Lessee shall forthwith wurrender to Leasor the possession of the Premises and shall remove the Improrement and reatore the Premises to substantially the tate in which they pere prior to the construetion of the Improvements, and in case Lessee shall fail within thirty (30) days after the date of such termination to make wach removal or restoration, then Lessor my, at its election to be exercised within thirty (30) day therearter, either remope the Improvements and restore the Premises for the account of Leasee, and in wuch event Lessee ahall within thilty (80) days after the rendition of bill therefor reimburve Leseor for the cost so incurred, or may take and hold the Improvement as its solo property.

17. If Leasee fails to gurrender to Lessor the Premises, upon any termination of this lease, all the Hablities and obligations of Lesiee hereunder ahall continue in effect until the Premisea are surrendered; and no termination hereof shall release Lesees trom any liabillty or obligation hereunder, whether of indemnity or otherwise resulting from ans acts, ormissions or eventi happening prior to the date of terminstion or the date if later, when the Improvements are remoped and the Premises restored or Lessor elects to take and hold the Improvements a its sole property as hereinabovo in paragraph 16 provided.

18. In the event that Lessee connists of two or more parties, all the coreinants and agreements of Leasee herein contained shall be the joint and several covenants and agreements of such parties.

19. All the covenants and agreements of Iowee berein contained shall be binding apon the heirs, legal representatives, successors and antigas of Iessee, and shall inure to the beneftt of the successors and acsigns of loasor.

20. It is mutually agreed that Rider " $A$ " hereto attached, Identified by the signature of $D$. $\Sigma$. Barnes, is hereby made a part hereof.

RIDER "A"

To Lease Dated April 25, 1975

Between

THE ATCHISON, TOPEKA AND SANTA FE RAILHAT COMPANY

and

THE MAYARRE FARYERS UHIOH CO-OPERATIVE ASSOCIATION

21. That In the use of the Premises, Lessee shall exercise utmost and extraordinary diligen 21. That In the use the ead that ao dames to pay Lessor uithin twenty (20) days after rendition of b111 therefor nd lessee hereby agrees to pay Lesson within twenty (20) days after resulting in any manner from he entire cost of repaining any damage to said commulcation line resulting in any manmer from $r$ in connectlon with Lessee's use of the Premlees.

22. Notwithatanding anything contained in Section 9 beroof to the contrany, Lessee may, at ts sole cost and expense and in manner satisfactory to Lessor, construct, Install, use and lafutain an overhead screw conveyor and ralk, portable unlosder, and an undargound tunnel and ppurtenances over, under and adjacent to Leseor's tracks in the location Indicated and In acoxdance with the goneral desim shown on said Exhlbit "A" In such a manner and of such materis 8 will jot at any time be a source of danger to or Interference with or the safe operation of es8or's rallroad. Durlng construction, Installation, use or repalring of eald tunnel and/or verhead screw conveyor and walk, portable unloader and appurtenances, Lessee shall exerclse itmost and extraondinary diligence to prevent damage to property of lessor or infury to its age iv employes. If at any tive during the term hereof, Lessor shall desire to make any use of its sroperty with which sald tunnel and/or overhead sarew conveyor, walk, portable unloader and (3C) 


$$
\begin{aligned}
& \text { RIDER "A" } \\
& \text { TO Lease Dated APr11 25, } 1975 \\
& \text { Between } \\
& \text { THE ATCHISON, TOREKA AND SANTA FE RAILHAY CONPAKY } \\
& \text { and } \\
& \text { THE NAVARRE FARIERS UHION CO-OPERATIVE ASSOCIATION }
\end{aligned}
$$

of the Premlses, Lessee shall exercise utmost and extraordinary diligence in the use of the Premises to Lessor's communication Iine located upon the Premlses, Lessee hereby agrees to pay Lessor within twenty (20) days after rendition of blll therefor, entire cost of repairing any damage to said commulcation line resulting in any manner from in connection with Lessee's use of the Prenlses.

22. Notilthstanding anything contained in Section 9 hereof to the contrary, Lessee may, at 80le orerhead screw conveyor and walk, portable unloader, and an underground tunnel and in adjacent to Lessor's tracks in the location Indicated and in ac-

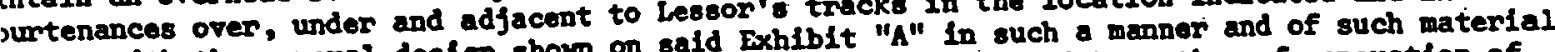
dance with the general dosign shown on sald to or interference with or the safe operation of will not at any time be a source of dantallation, use or repairing of said tunnel and/or s8or's railroad. During constmuction, Installation, lo or repaices, Iessee shall exerclse erhead screw conveyor and walk, portable unloader and appurtenan of Lessor or injury to Its agent most and extraordinary diligence to prevent dareof, Lessor shall desire to make any use of Its employes. If at any time during the term hereos, Lessor shall desin, portable unloader and operty with which sald tunnel and/or overhead sorj; at lessed's sole cost, within thirty (30) purtenances. will in any way Interfere, Lessee shall, at Lesses's sole cost, wanges in said tunnel ys after receiving written notice from Lessor to such and appurtenances as in the judgment id/or overhead screw conveyor, walk, portablo unloade andosed use of its property, and, Lessor may be necessary to avold interforence wlth the proposay make such changes at Lessee's ssee falling 80 to do within sald thirty Pense:

contained in Section 10 hereof to the contrary, Lessee agrees

23. Notwithstanding anything contained in section lossor against all claims, demands, action hat it will at all times Indemify and save haxmles lo on damage to pxoperty including aaid c causes of action, arlsing or groring out of loss of orloader and appirtenances or infury to or unnel and/or overhead screw conveyor, walk, portable unloader and appin from the construction, eath of persons, including employes of lessor, resulting in any manner from the constring or overhead nstallation, maintenance, use, state of repair or presence or said tur or adjacent to sald creu conveyor, walk, portable unloader and appurtenances under, ontributed to by the negligence racks whether such 2088 , damage, Injury or death be caul it will promptly pay to Lessor the if Lessor, 1ts agenti or employes, or otherwise, and that it incur or become liable for and all ill anount of any $108 s$ or damage which lessor may sustain, lncur or becolims on account thereof suis which leasor may pay or be compelled to pay in settlenent of any clains on account theres. 1

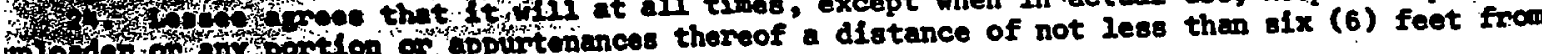

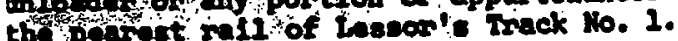

(n)

25. It Is mutually agreed that the asreement dated Jenuany 22, 1962, (Lessor's Secretary's Contract No. i14732) between the partles hereto, relating to use of a portion of Lessor's propenty at Navarre, Dickinson County, Kansas, as sites for elevators, coal bins, bulk oll station rachouse, busldinge tuminel, conveyor and overhead walk, as modified by Supplemental Agreement dated November 29,1971 increasing the compensation to $\$ 145.00$ par year, is hereby terminated of the effective date hereof.

26. It is muturily acreed that the agreement dated November. 21, 1969, (Lessor's Secretary Contract No. 133460) between the parties hereto, relating to use of a portion of Lessor's propenty at Havarre, Dickinson County, Kansas, a alte for handling 11 auld and bulk fertilizer, stong of fertilirer equipsont and portable unloader, is heraby terminated as of the effectiv date hereof. 
the ding and rear fint above wilteen.

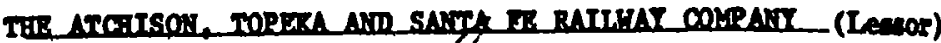

Approved as to deceription:

B $\mathbf{y}$

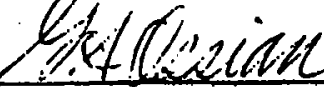

It Asoivtant to General Manager

Chief Engineer.

THE NATARRE RARYERS UNTOI CO-OPERATIVE ASSOCIATIOA

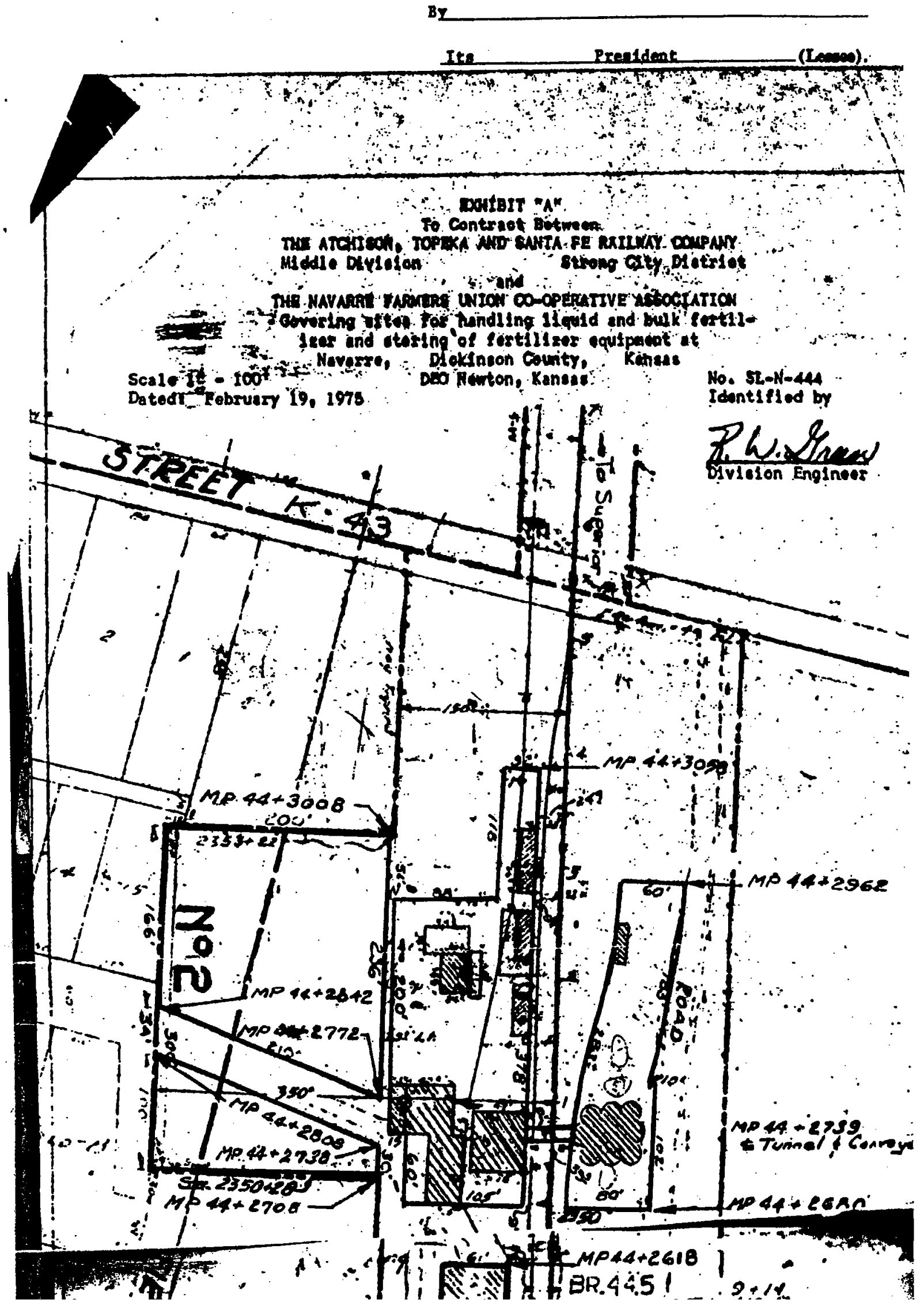




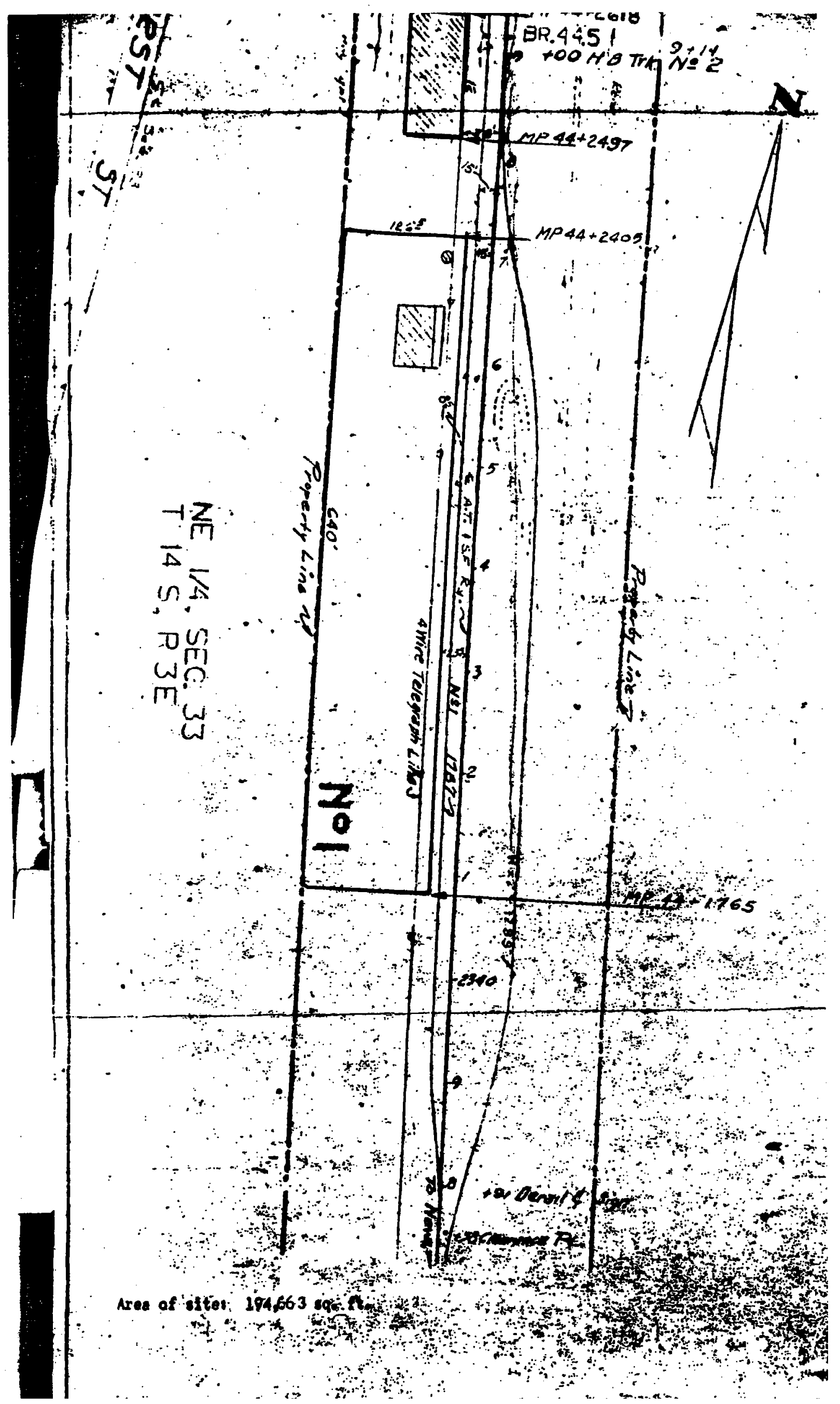




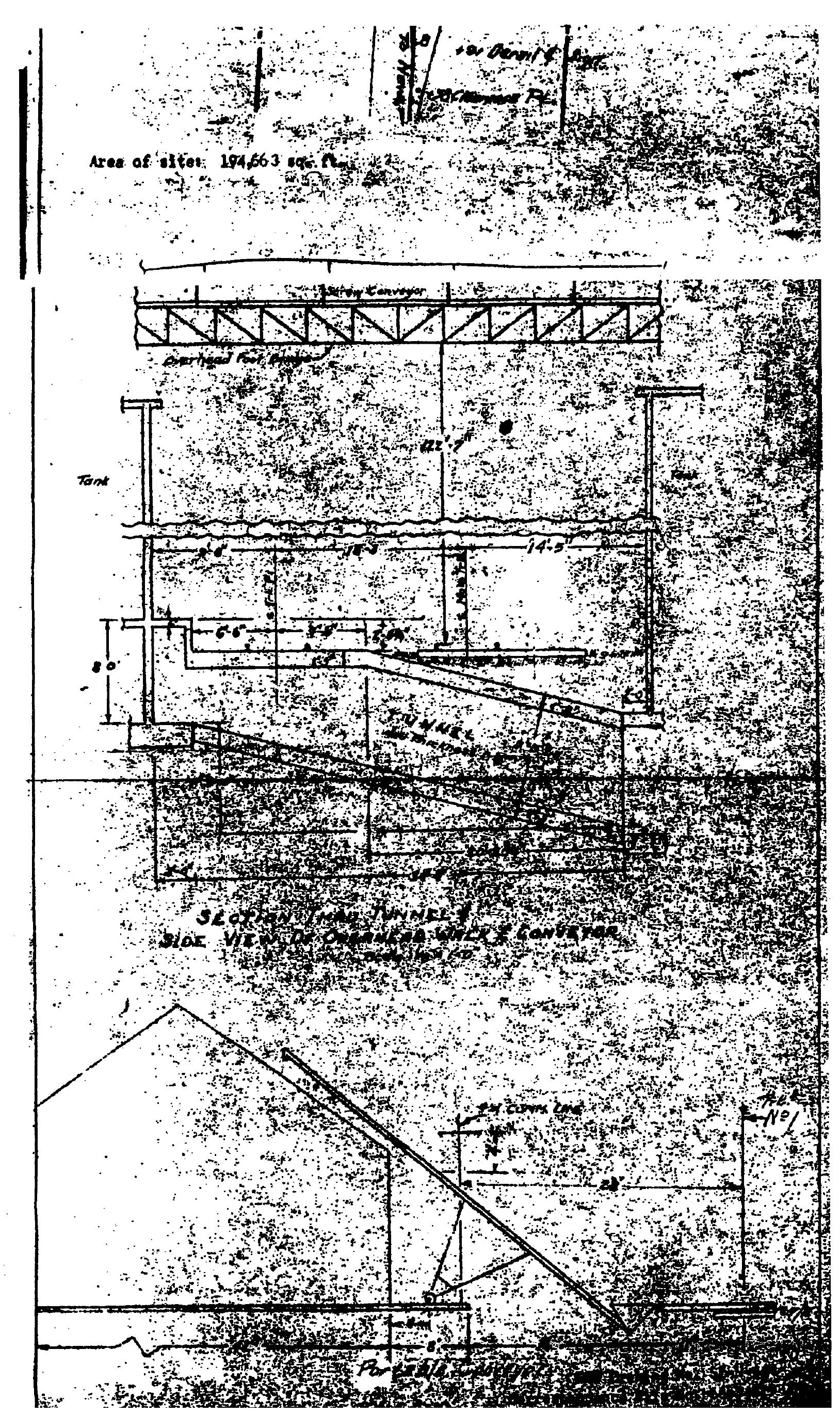




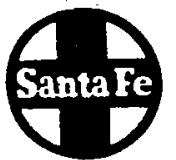

\section{The Atchison, Topeka and Santa Fe Rallway Company}

P.O. Box 1738, 900 Jackson Street, Topeka, Kansas 66628

Telephone 913/235-0041

Ext. 4224

July 15, 1975

$$
E-44391-F
$$

The Navarre Farmers Union Co-Operat1ve Association Navarre, Ransas

Gentlemen:

This has reference to your request to revise your lease area at Navarre, Ransas, as covered by our Secretary's Contract Nos. 114732 at Navarre, Ransas, as 133460 , and Include additional area, making a revised total square footage of 194,663 square feet of space.

As previousiy advised, we axe agreeable to leasing the 194,663 square feet of space to you at a rental of $\$ 540.00$. per year, effective May 1, 1975, and enclosed, In duplicate, is our Lease of Land, Form 1616, to cover, which agreement also provides for wutual termination of contract Nos. 114732 and 133460.

Please have the document executed by the President or a Vlce President of copy for your records and returning the copy marked "Santa Fe Original" to this office.

We are notifying our Audit offlce to revise billing under Contract Nos. 114732 and 133460 , prorated to the date of the ner lease agreemint, which you should recelve shortly.

Yours truly,

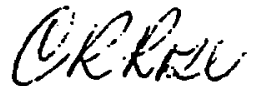

C. R. Rose

Assistant General Maneger 
THIS. AGREEAENT, Made and entered Into in triplicate as of the 30th day of January , 1976 , by and between THE ATCHISON, TOPEKA AND SANTA FE RAILWAT CORPANY, a Delaware corporation with offices at Topeka, Kansas, herelnafter called "Rallroad"; FARLIAND INDUSTRIES, INC, a Ransas corporation with offices In Ransas C1ty, Missour1, herelnafter called "Parmland"; and THE MAVARRF FARMFRS UNION CO-OPERATIVE ASSOCIATION; a Raneas corporation wth offlces at Navarre, Kansas,

herelnafter called "Coop" WITNESSETH THAT:

WHEREAS, Railroad permitted Coop___ to use certain premises at Navarre, Mickinson Co., Ks., under the terms of an agreement dated Apri1 25, 1975 , Identifled in the records of Rallroad as Secretary's No. 147780 , and WHEREAS, Farmland has entered into an agreement with Coop for the Installation and rental on sald Premises of one 30,000 gallon anhydrous amaonia storage $\operatorname{tank}(8)$.

NOW THEREFORE, in consideration of the premises, the parties hereto agree as follows:

1. Farmland may instali the aforesald tank(8) on sald premises. 2. Ownership of guch $\operatorname{tank}(8)$ shall be and remain in Farmland at all times as personal property regardless of how attached to the 1 and.

3. Rallroad assumes no 11ability in connection with the Instailation, malntenance, operat lon or presence of the tank(B) upon said premises and shall not be responsible for any damage thereto regardleas of the cause of ouch damage. Nothing contalned in thlo agreenent shall be construed to release Coop from any of Its obligations, Including but not IImlted to liablifty under provisions of sald agreenent dated Apr11.25. 1975

4. Farmland way remore said $\operatorname{tank}(8)$ at any time prior to the termination of sald agreement dated 1 pril 25, 1975, and In the event of termination thereof, agrees to rewove said tank(s) frow sald prentses within thirty days after belng given written notice of such teratation. In event 
Farmland fa1ls to remove the tank(s) within thirty days after notice of termination, then Rallroad may, at its option, remove the tank(s) from sald premises at cost of Farmland or may take and hold the tank(s) as its sole property. In such event, shall remain liable to the Rallroad for any damages to the premises or for fallure to restore said premises to its original condition.

5. Nelther Farmland nor Coop - nor their legal representatives, successors or assigns shall assign or transfer this agreement or any right or interest therein, w1thout the written consent and approval of Rallroad in each instance.

IN WITNESS WHEREOF, the partles hereto have duly executed this agreement in triplicate as of the day and year first above written.

THE ATCHISON, TOPEKA AND,SANTA FE RAILWAY COMPANY
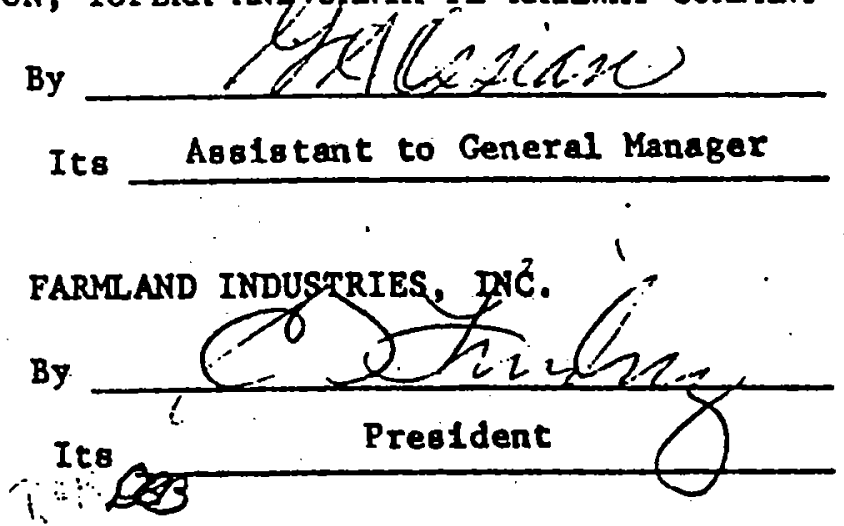

THE NAVARRE FARMERS UNION CO-ORERATIVE ASSOCIA:!

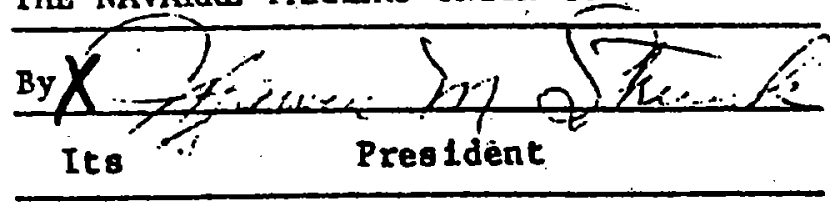


SUPPLadirtal AGREEYTNT, ade as of th1 $\frac{14 \text { th }}{\text { between THE ATCHISON, TOPEX }}$ 19 $\frac{78}{\text { NND SANT: }}$ FE RAILHAY COHPAN, - Delmare corporation, hereinafter referred to as "Sante Pe"), and THE NAVARRE FARMERS UNION CO-OPERATIVE ASSOCIATION, a Kansas corporation

hereinafter, whether one party or nore, referred to as "Second Party".

$\underline{R} \underline{\mathbf{C}} \underline{\mathrm{I}} \underline{\mathrm{A}} \underline{\mathrm{s}} \mathrm{s}$

Santa Fe and Second Party are now parties to a contract dated April 25, 1975 - Santa Pe'a Secretary's Contract No. 147780 torether with anv and all oodifleatlons, oupplewents and amendwents thereto, belnk hereinafter referred to as "Orlpinal Contract". under wich Second Party paye Santa Fe a compensation of $\$ 540.00$ per year for the use of - portion of Santa Fe property at or near Navarre, Dickinson County, Kansas a- - for sites for handling liquid and bulk fertilizer and storing of fertilizer equipment. provided.

The partieo desire to modify the Original Contract as hereinifter

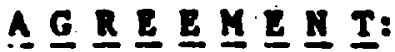

IN CONSIDERATION of the our of $\$ 1.00$, and other conolderation, the recelpt and oufficlency of which are hereby acknouledged by Second Party, it 1. wutully agreed that effective May 1,1978 - the compencation section of the OrIginal Contract $1 \overline{0}$ hereby changed to read, ce follows:

"Second Party ahall pay to Sanca Pe on or before the firat day of each period of one year during the continuance of this contract as compeneation for the use of the Prentece for such period the oun of $\$ 1,000.00$ - Sente Pe way revloe the amount of ouch yearly cowpensation after the end of each three (3) year period during which this contract way renain in effect, and without affecting the right of elther party hereto to terulnate this contract at any tine as any be provided elsewhere herein."

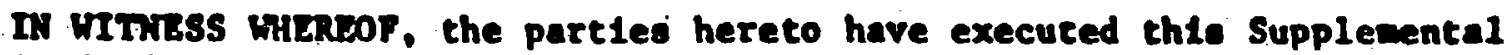
Agreement In duplicate as of the day and rear firet above written.

THE ATCAISON, TOPEKA AND SANTA FE RAILAAT COMPANY

IT Is MIIUALIT AOREED that as of the effective date hereof, the provialons of RIder " $A$ " attached hereto, Ideatlfled by the elgnature of D. $\mathrm{E}$. Barneo, are heroby ade a part of the Orsingel contract.

D)

Ite Assistant to General Manager

THE NAVARRE FARMERS UNION CO-OPERATIVE ASSOCIATIC

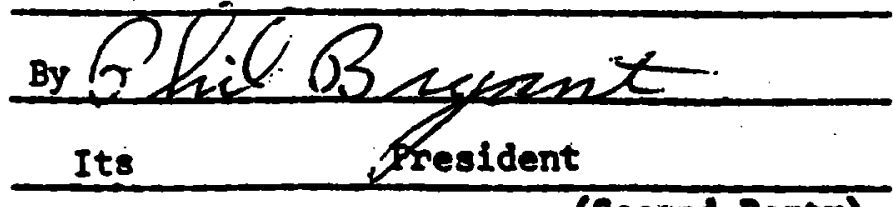




\author{
RIDER "A" \\ At tached to agreewent dated March 14, 1978 \\ Between \\ THE ATCHISON, TOPEKA AND SANTA FE RAILWAY COMPANY
}

and

THE NAVARRE FARMERS UNION CO OPERATIVE ASSOCIATTON

27. Notulthstanding any other provision of this lease, Lessee shall comply wth all environmental statutes, ordinances, rules, regulations, orders and declsions (herelnafter referred to as "Standards"), Issued by any federal, state or local governwental body or agency established thereby (hereinafter referred to as "Authority"), relating to Lessee's use of the Prem1ses. Lessee shall malntain the Premises In full compliance with all Standards set by any Authority, Including, but not limited to, Standards concerning alr quality, water quality and nolse. In the event Lessee falls to maintaln the Premises according to the Standards set by any Authority, Lessor may, after giving reasonable notice of the fallure to Lessee, take whatever action is necessary to bring the Premises into compliance. Lessee shall reimburse Lessor for all costs (Including, but not limited to, consultIng, engineering and legal costs) Incurred by Lessor in bringing the Premises Into compliance with such Standards, and also all such costs Incurred by Lessor in abating a violation of such Standards, protecting against a threatened violation of such Standarda, defending any claim of rlolation of such Standards in any proceeding before any Authority or court, and paying any fines or penalties lmposed for such violations. Lessee shall further indemify and save harmless Lessor from any clain of a violation of such Standards regardless of the nature thereof or the Authority or person asserting such claim, which results from Lessee's use of the Premises, whether such claim arises in whole or in part from the negligence of the lessor or otherwise. Lessee at its cost, shall assume the defense of all such clalms regardless of whether they are asserted agatnst l.essee or Lessor. 


\section{The Atchison, Topeka and Santa Fe Rallway Company}

March 21, 1978

$E-44391-F$

The Navarre Farmers Union

Co-Operative Association

Navarre, Kansas 67469

Gentlemen:

You are a party to an agreement dated April 25, 1975 with The Atchison, Topeka and Santa Fe Railway Company, and identified in Santa Fe's records as Contract No. 147780 , covering your use of a portion of our property at Navarre, Kansas as a site for handling liquid and bulk fertilizer and storing of fertilizer equipment.

We have made review of this contract and now find it necessary to revise the compensation, and are attaching, in duplicate, supplemental Agreement to the above contract, which revises the compensation to $\$ 1,000,00$ per year effective May 1, 1978.

Please have the document executed by the President or a vicePresident of your firm, clearly indicating correct spelling of that officer's name, retuming the copy marked "Santa Fe Original" to this office.

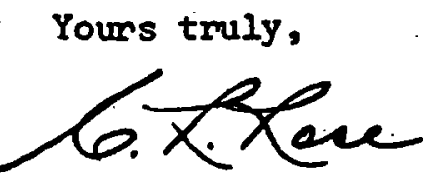

C. R. Rose Assistant General Manager

DJH: Ir 
SUPPLEMPSTAL AGPPEMRNT, made as of this 9 th day of Apr11, 1981 hetwen ThR ATChISON, TOPRKA AND SANTA PR RAILWAY COMPANT, a Delavare corporation (heretnafter referred to as "Santa $\mathrm{Pe}^{\circ)}$, and THE IAVARRE FARMERS INION COMPRRATIVP. ASSOCATION, a Kangas corporation, (hereinafter, whether one narty or more, referred to as "Second Party").

$\underline{R} \underline{\mathbb{R}} \underline{\mathrm{C}} \underline{\mathrm{I}} \underline{\mathrm{A}} \underline{\mathrm{L}} \underline{\mathrm{S}}:$

Santa Fe and Second Paxty are now narties to a contract dated Apr11 25, 1975 , Santa Pe's Secretary's Contract ito. 147780 together wth any and all modiflcations, supplements and anendments thereto,' helng herelnafter referred to as "nriglnal Contract", under hich Second Party pays Santa Fe a compensation of $\$ 1,000.00$ per year for the use of a portion of Santa Pe property at or near davarre Jickinson County, Ransas , sites for handilng liquid and bulk fert1lizer and storing of fert1lizer equipant. provided.

The parties desire to modify the neiginal Contract as herelnafter A G $\underline{\text { R }} \underline{\mathrm{E}} \underline{\mathrm{M}} \underline{\mathrm{E}} \underline{\mathrm{Y}} \underline{\mathrm{T}}$

IN GONSINRRATION of the sum of $\$ .0 n$, and other consideration, the recelpt and sufflclencr of which are hereby acknowledged by Second.Party, 1t is mutually agreed that effective May 1, 1981 the Origlnal Contract 1s hereby changed to read, as follows: Section 3 of

" 3 - Second Party shall pay to Santa Pe on or before the sirst day of each period of one year during the continuance of this contract as compensation for the use of the Premises for euch perlod the aum of $\$ 1,355.00$. - Santa Fe may revise the amount of such yearly compensation after the end of each three (3) year period during wich this contract may remain in effect, and without affecting the right of elther party hereto to terminate this contract at any time as may be provided elsewhere herein."

Section 14 of the Original Contract is hereby deleted and the following oubotituted therefor:

"14. Th1. contract may be terminated at any time by elther party upon thitty (30) daya' notice in writing to be served upon the other party, stating thereln the date that such termination shall take place, and upon the explration of the time opecifled in such notice this contract and all rights of Second Party hereunder shall absolutely cease and determine; but upon any termination of thie contract, Santa Fe shall retain as a minimu charge for the use 
of Santa Pe' t property a sum of $\$ 240.00$

and any rental in excese of eald sur shall be apportloned between the partes, and Santa Pe hereby agrees to refund to Second Party a proportionate part of any such excess amount pald in advance."

IN UITNESS WTRREOF, the part1es hereto have executed this Supplemental Apreement in duplicate as of the day and rear firat sbove witten.

TIE ATCHISON, TOPEKA AND SANTA FE RAILWAY COMPANT

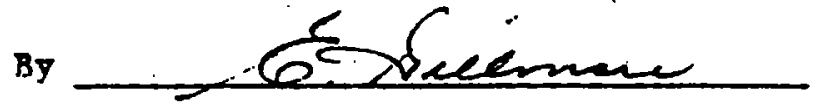

Its Assistant to General Manager

THE NAVARRE FARMERS ONION CO-OPERATIVE ASSOCIATION

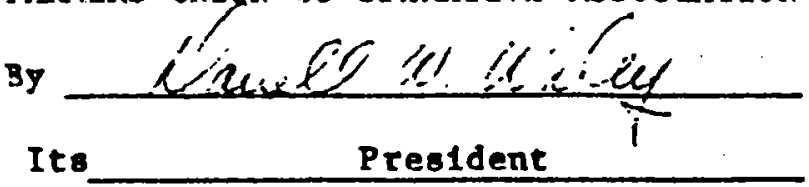




\section{The Atchison, Topeka and Santa Fe Rallway Company}

P.O. Box 1738, 900 Jackson Sireet, Topeka, Kansas 66628 Telephone 913/235-0041

Ext. 4826

Apri1 21, 1981

$\mathbf{E}-44391-\mathrm{F}$

The Navarre Farmers Unton Co-Operative Association

Navarre, Kansas 67469

Gentlemen :

You are a party to an agreement dated Apr11 25, 1975 w1th The Atchison, Topeka and Santa Fe Rallway Company, and Identified in Santa Fe's records as Contract No. 147780, covering sites for handling liquid and bulk fert111zer and storing of fertilizer equipment at Navarre, Dickinson County, Kansas.

We have made review of this contract and now find it necessary to revise the compensation, and are attaching, in duplicate, Supplemental Agreement to the ai ve contract, which revises the compensation to $\$ 1,355.00$ per year, effective May 1, 1981 .

Please have the document executed by the President or a Vice President of your firm, cleariy ind lcating correct speliling of that officer's name, returning the copy marked "Santa Fe Original" to this office.

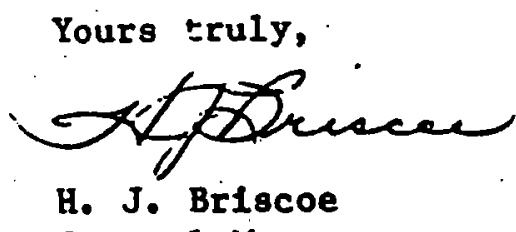

General Manager

LMR bjt 169 
mam

\section{CorporateAn}

in this box enter the exact coportion neme and pivilln

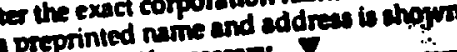
address. If e preprinied numect if necenery.

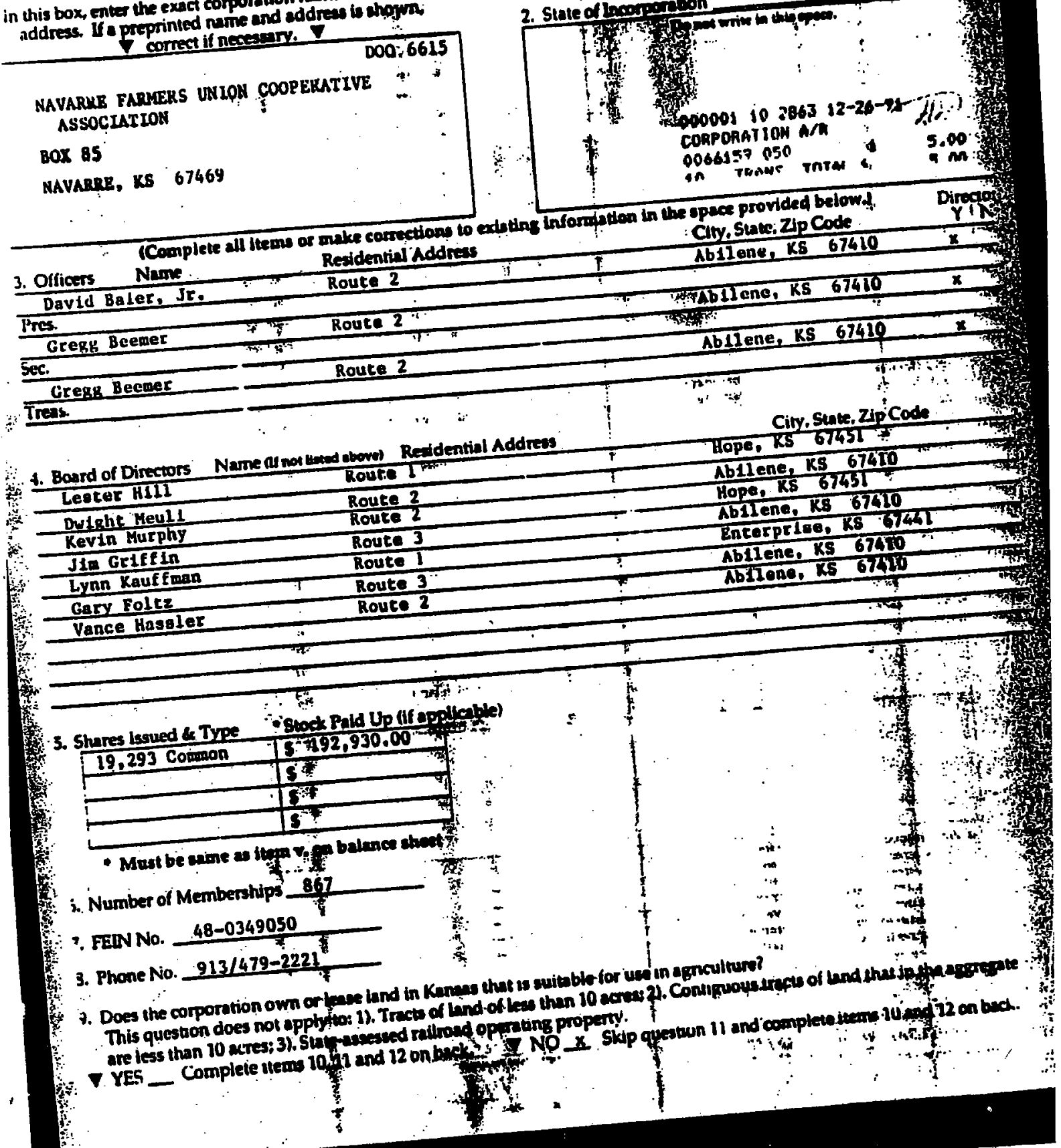




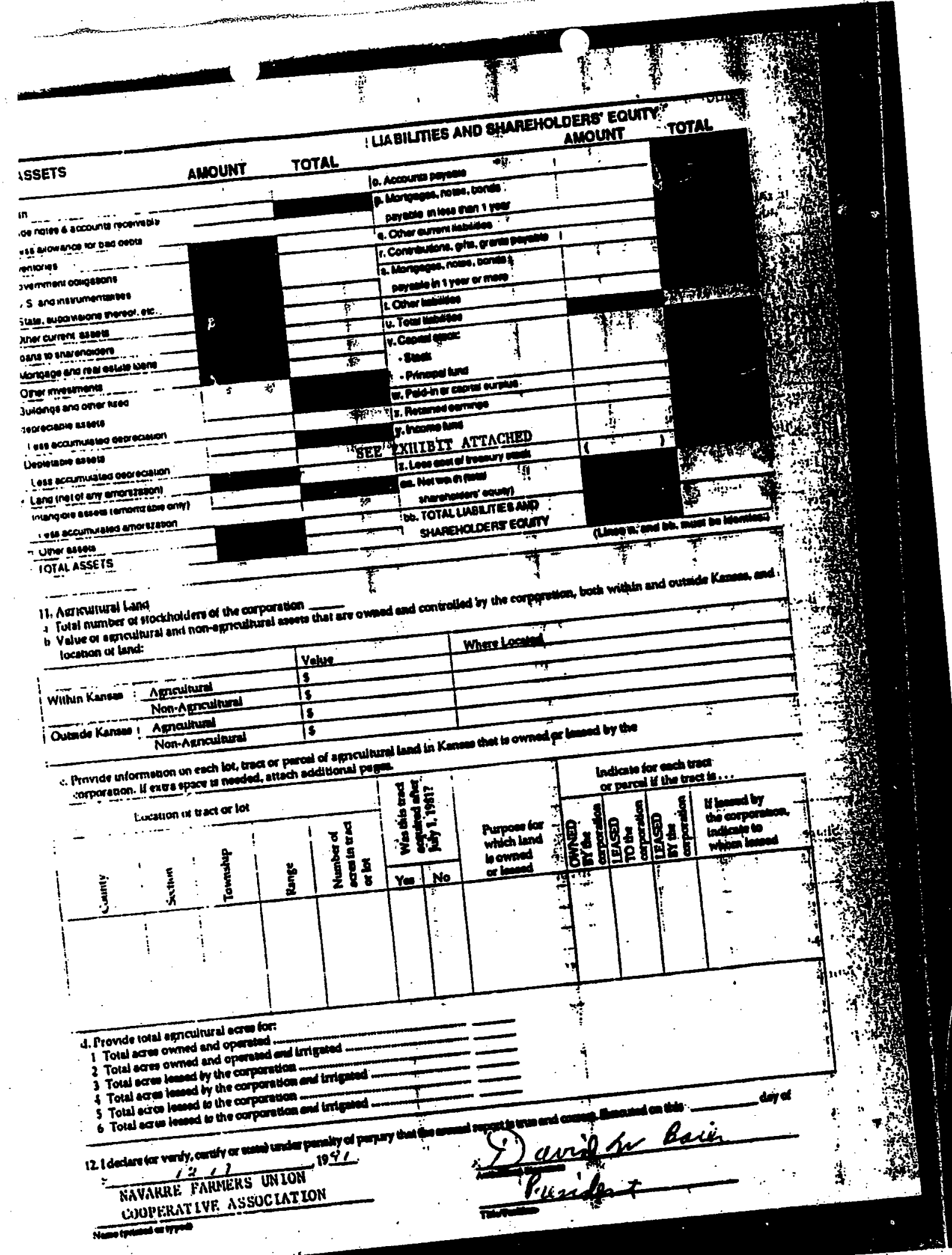




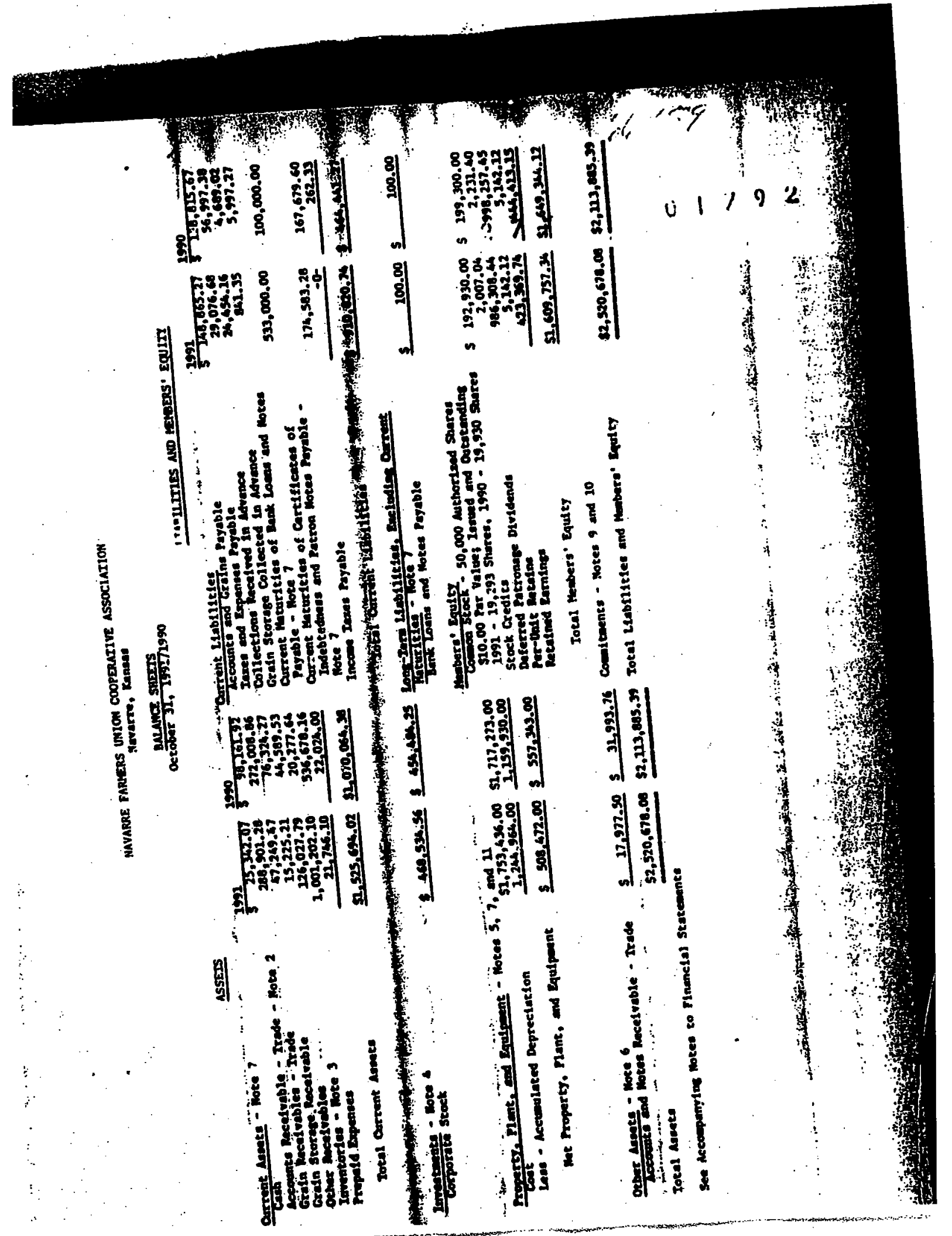




\title{
CER TIFICA T E
}

\author{
STATE OF KANSAS ) \\ DICKINSON COUNTY, \} \\ ss:
}

This is to certify. that the undersigned and made a thorough and complete check of the records of the Reglster of Deeds office of said county and state relating to the following described lands, subsequent to January 1, 1900.

All that part of the North half of Section 33, Township is South, Range 3 East bounded and described as follows:

Commencing at the intersection of the center line of Main track of the Chicago, Kansas and Hestern Rail Road (Strong City Extension) with the South llne of said hald Section, thence East on said South line to a point 150 feet distant at right angles from said center line; thence Northerly on a line 150 feet from and parallel to said center line to the North line of said Section 33, thence west on said North Line to a point West of and 150 feet distant at right angles from said center. line, thence southerly on a line 150 feet from and parallel to said center line to a point 300 feet Northerly from the North line of May Street in the Town of South Navarre, Dickinson County. Kansas according to the recorded plat of said Town; thence Westerly on a line 300 feet from and parallel to said North line of May street to a polnt 350 feet distant at right angles from said center line; thence southerly 350 feet from and parallel to said center line to the North line of May Street; thence Easterly along the North line of May Street 200 feet; thence Southerly on a line 150 feet from and parallel to said center line to the South line of said half Section; thence East on said South lline to the place of commencement.

Record Owner: The Atchison, Topeka and Santa Fe Rallway Company.

and

A tract of land beginning at a point 30 feet South of the STOCK YARDS PLAT on the one-half Section line, running thence South : on said line 240 feet, running thence directly east to the K.C. $\varepsilon$ R. R. right-of-way, running thence North along said rightof-way, to a point 40 feet south of the STOCK YARDS PLAT, running thence West to the point of beginning, said tract being located in the North East Quarter of Section 33, in Township 14 South, Range 3 East of the d6th. P.M.. Dickinson County; Kansas.

Record Owner: Irene E. Larsen

and

Commencing on the North lirie of Section 33, Township Commencing on the Sixth P.M., at a point where said line intersects the West line of the right where of the Chicago, Kansas and Western Raliroad, runing thence West 85 feet; thence South to the North line of the Stockyards of said rallraod; thence Ea'st of the West line of the right of way of said rallraod; thence Northwesterly along the West line of the right of way of said rallroad to the place of beginning. 
Recurd Owner: Navarre Farmers Union Cooperative Assoclation.

That the only lease of record as to any portion of said lands lying west of the center line of the railroad right of way is a certain Lease dated June 21, 1954, from The Atchison. Topeka and Santa Fe Railway Company and Commodity Credit Corporation, filed July 22, 1954 and recorded in Misc. Record Book 172 Page 416. (copy attached).

Dated at Abilene, Kansas this 18th day of March, A.D. 1992.

THE DICKINSON COUNTY TITLE CO.

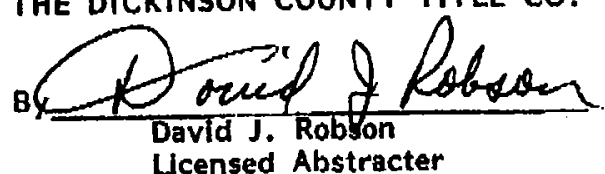




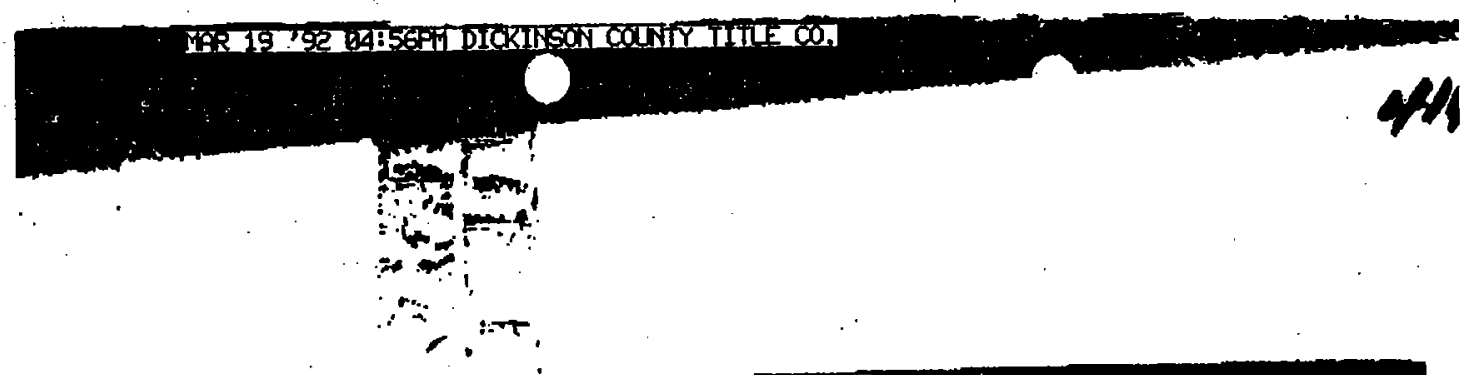

Nis: Buok Page - $-\cdots$ AAT'A GR TRIGINA

Farmit 1010 standaro

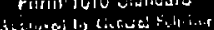

LEASE OF LAND (SHort Terni)

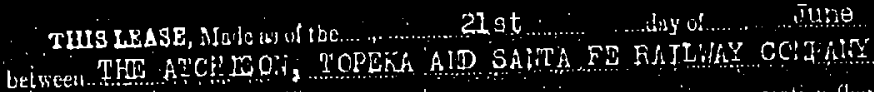

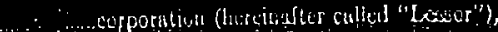

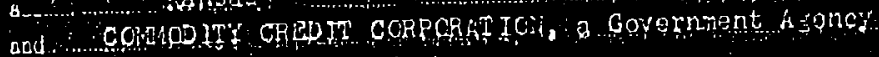

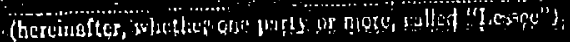

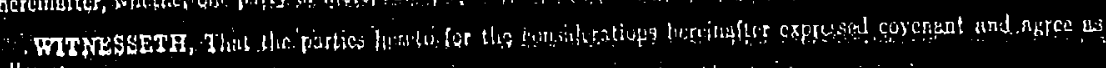
Pollawi:

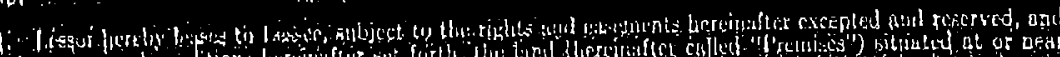

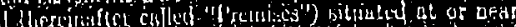

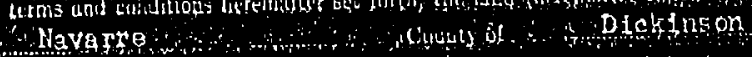

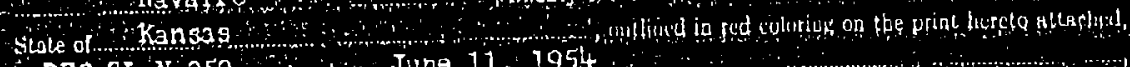

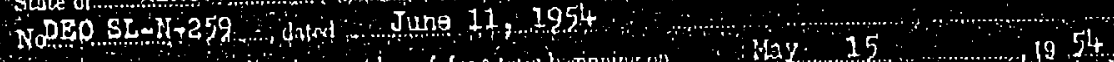

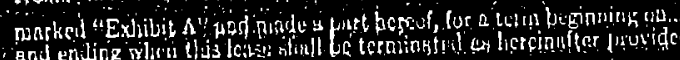

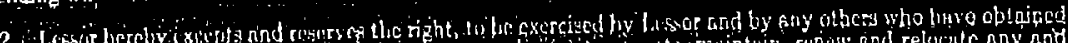
2. ot ber firilitite of tike chastus: fur upod, oyer

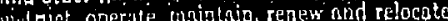

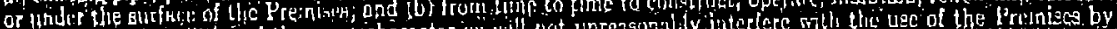

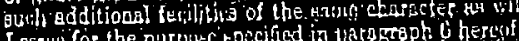

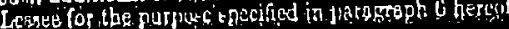

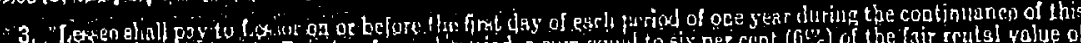

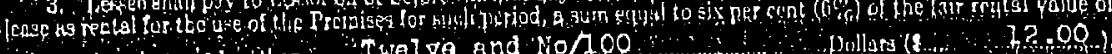

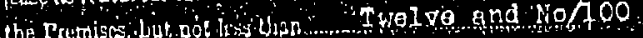

poullers (9

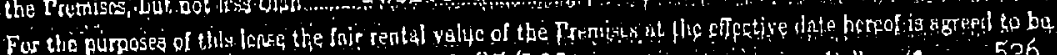
Five Hund red wenty $\$ \mathrm{~A} x$ and $85 / 1 \mathrm{co}$ Dollore if

$526.85\}$

\section{are ono and $61 / 100$}

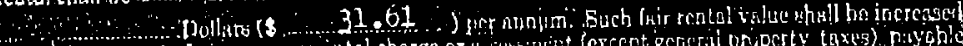

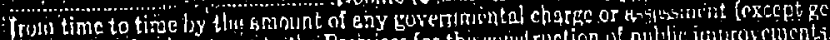

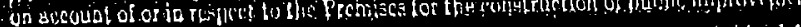

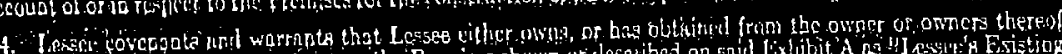

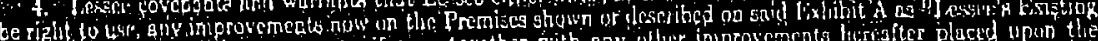

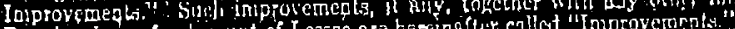

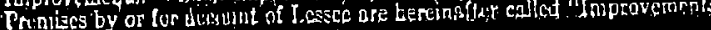

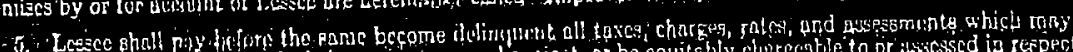

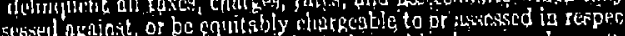

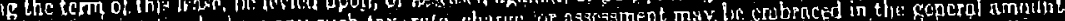

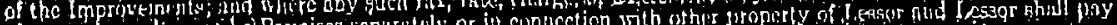

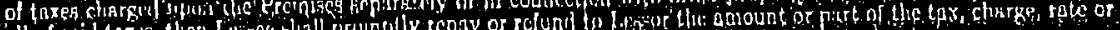

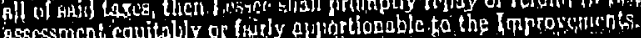

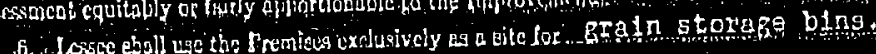

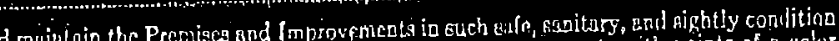

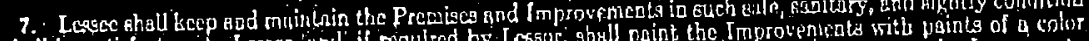

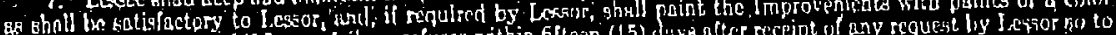

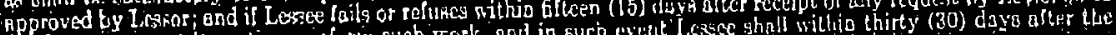

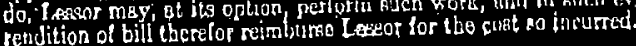


MAR 19 '92 64:5TRM DICKINSON COUNTY TITLE CO.

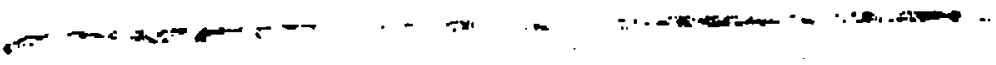

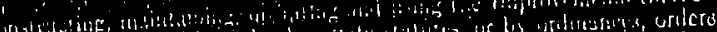

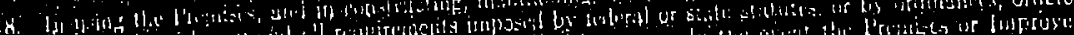

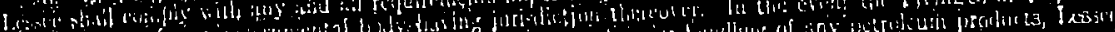

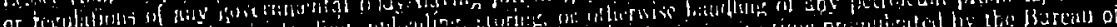
mont :

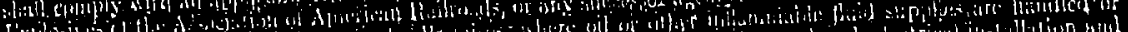
L.y.

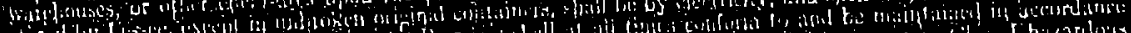

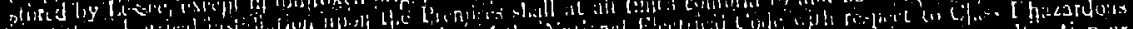

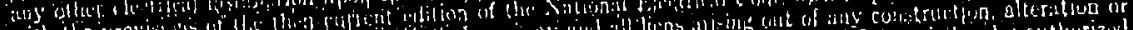

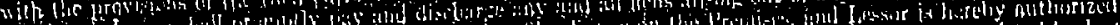

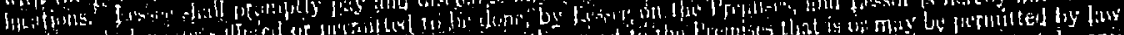

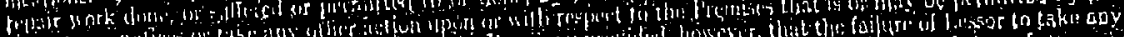

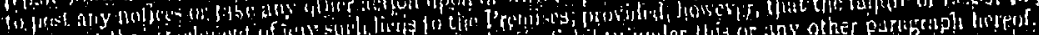

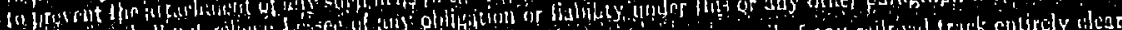

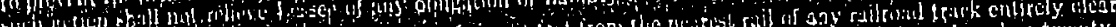

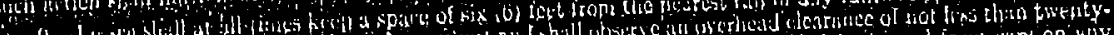

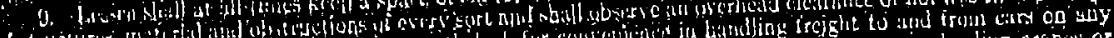

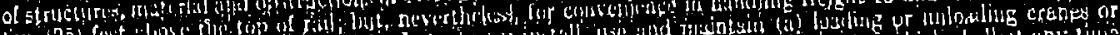

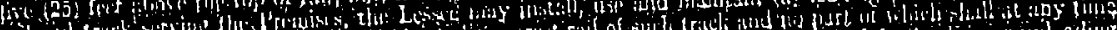

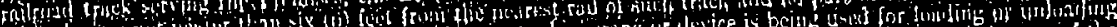

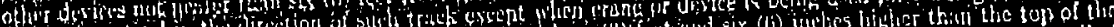

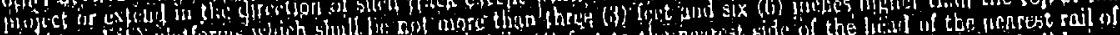

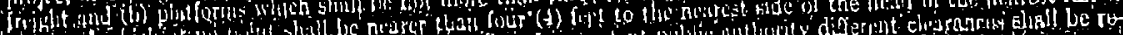

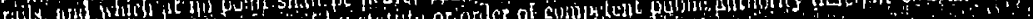

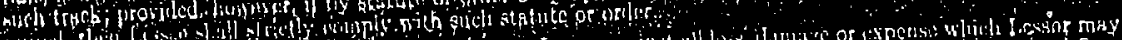

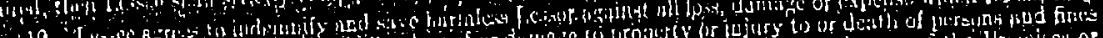

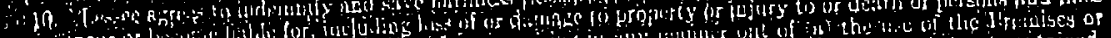

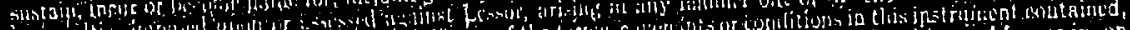

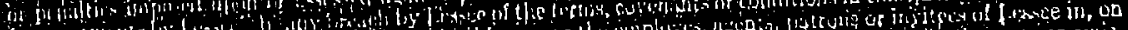

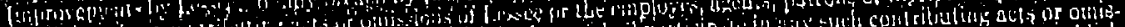

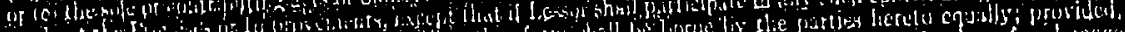

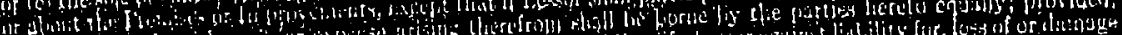

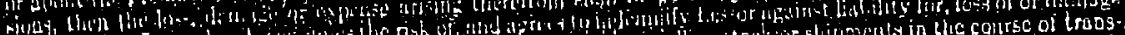

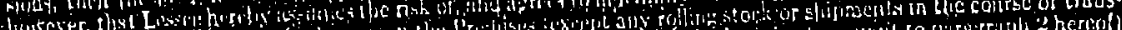

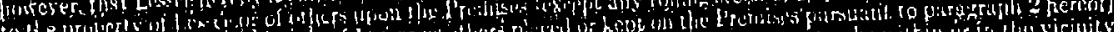

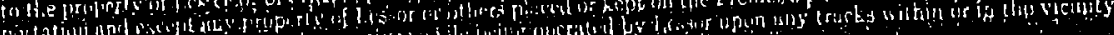

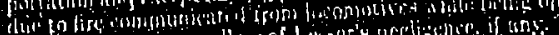

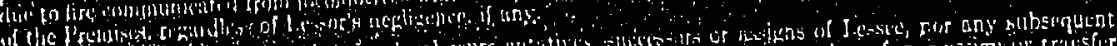

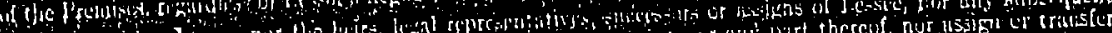

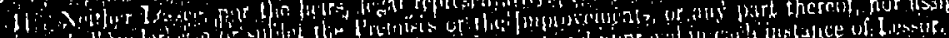

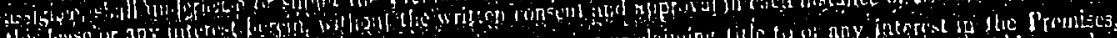

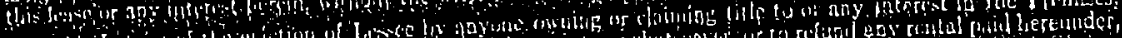

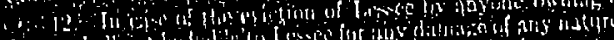

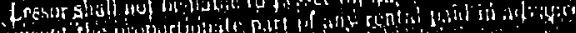

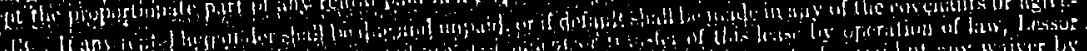

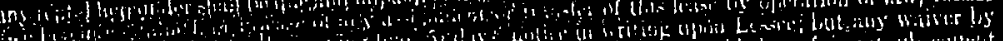

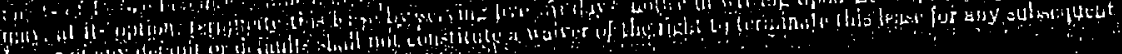

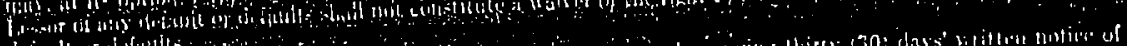

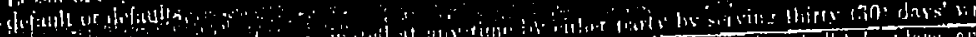

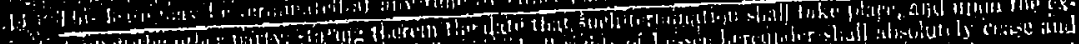

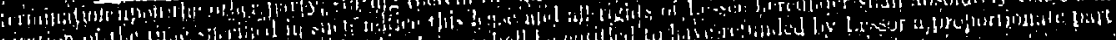

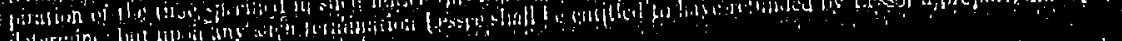

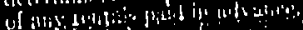

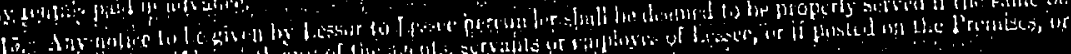

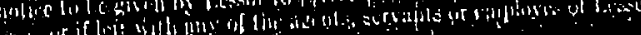

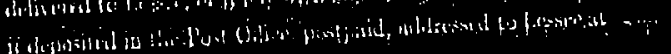

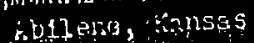

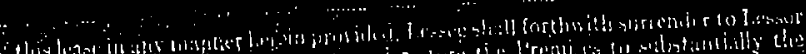

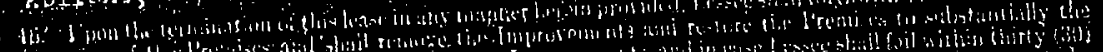

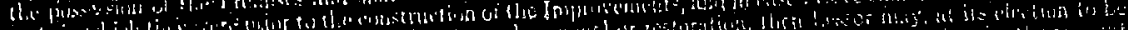

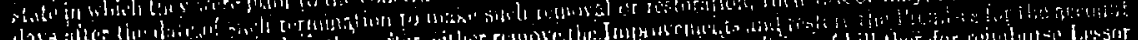
(a)

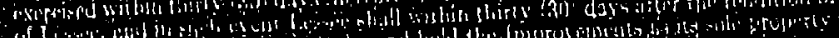

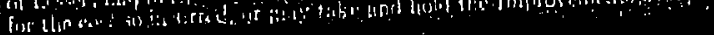




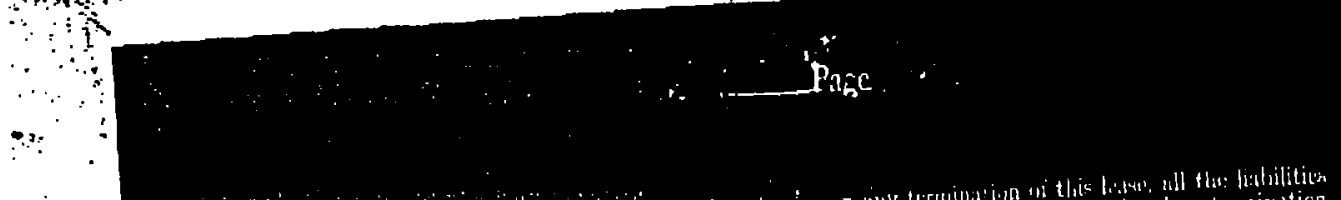

$=i^{\prime}$ a

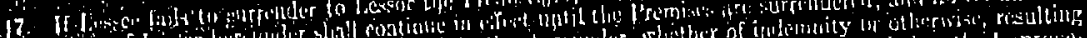

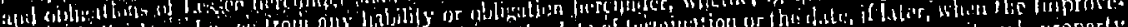

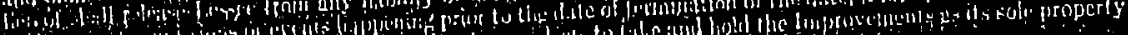

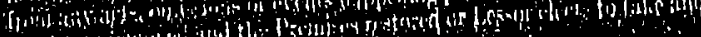

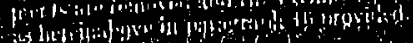

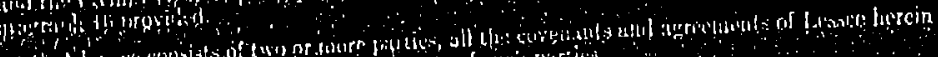

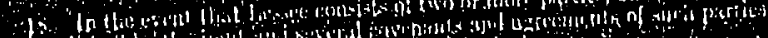

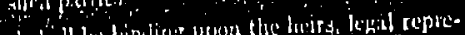

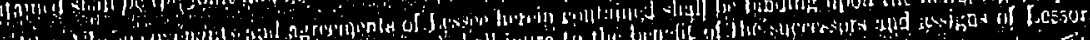

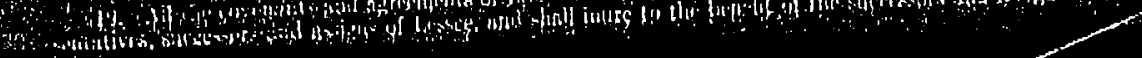

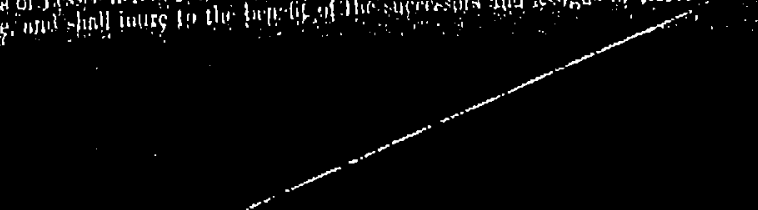

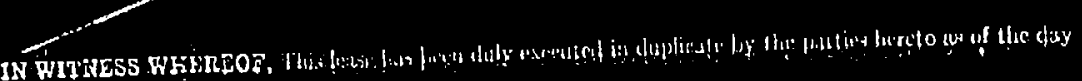

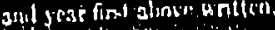

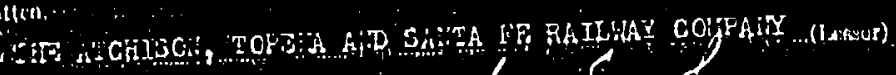

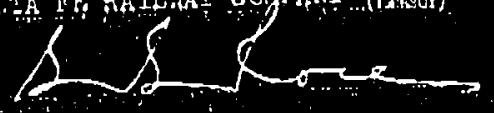

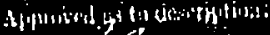

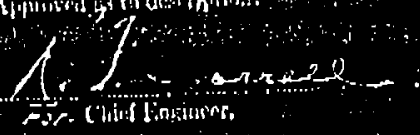

Hy

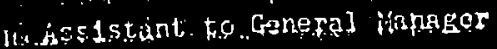

CEY OOFL CFETI CORPGUTTCN

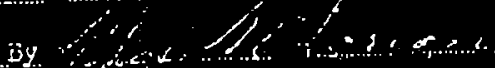

EXHIBIT " $\Lambda^{4}$

To Centirnet Ret.apun THE ATCHTSOR TOPEXA AND 3 ART Misde vivision und 5i. SHUCITY CREOTS gF PORATION coverine 1 ase of 1 te for graln jtor"se bins at Vausre, fickinson Jounty. Kansas

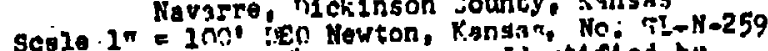
Dated June 11, 1954 . Inentifled by

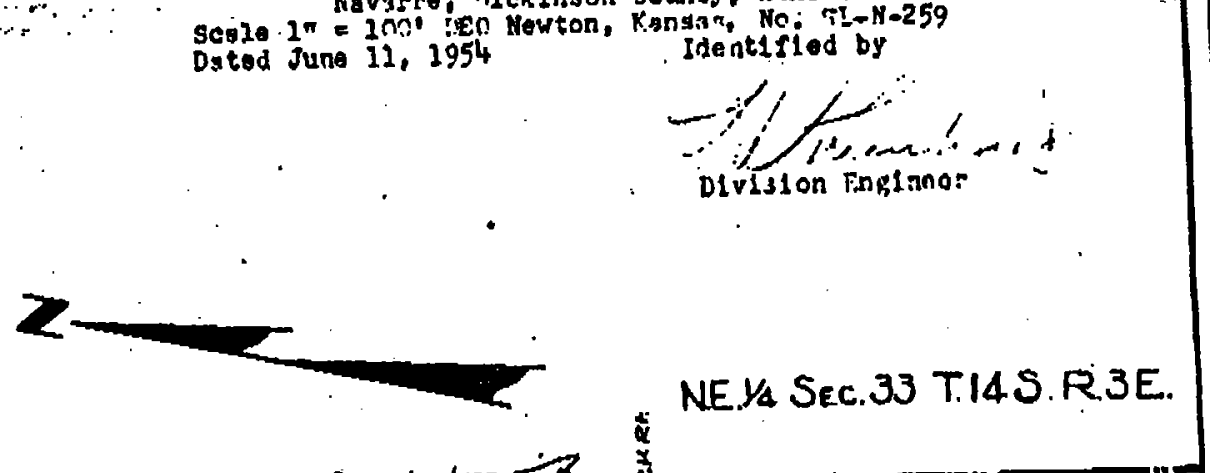

Anpaty fine .

NE. Y/4 SEC. 33 T.14S.R.3E. 

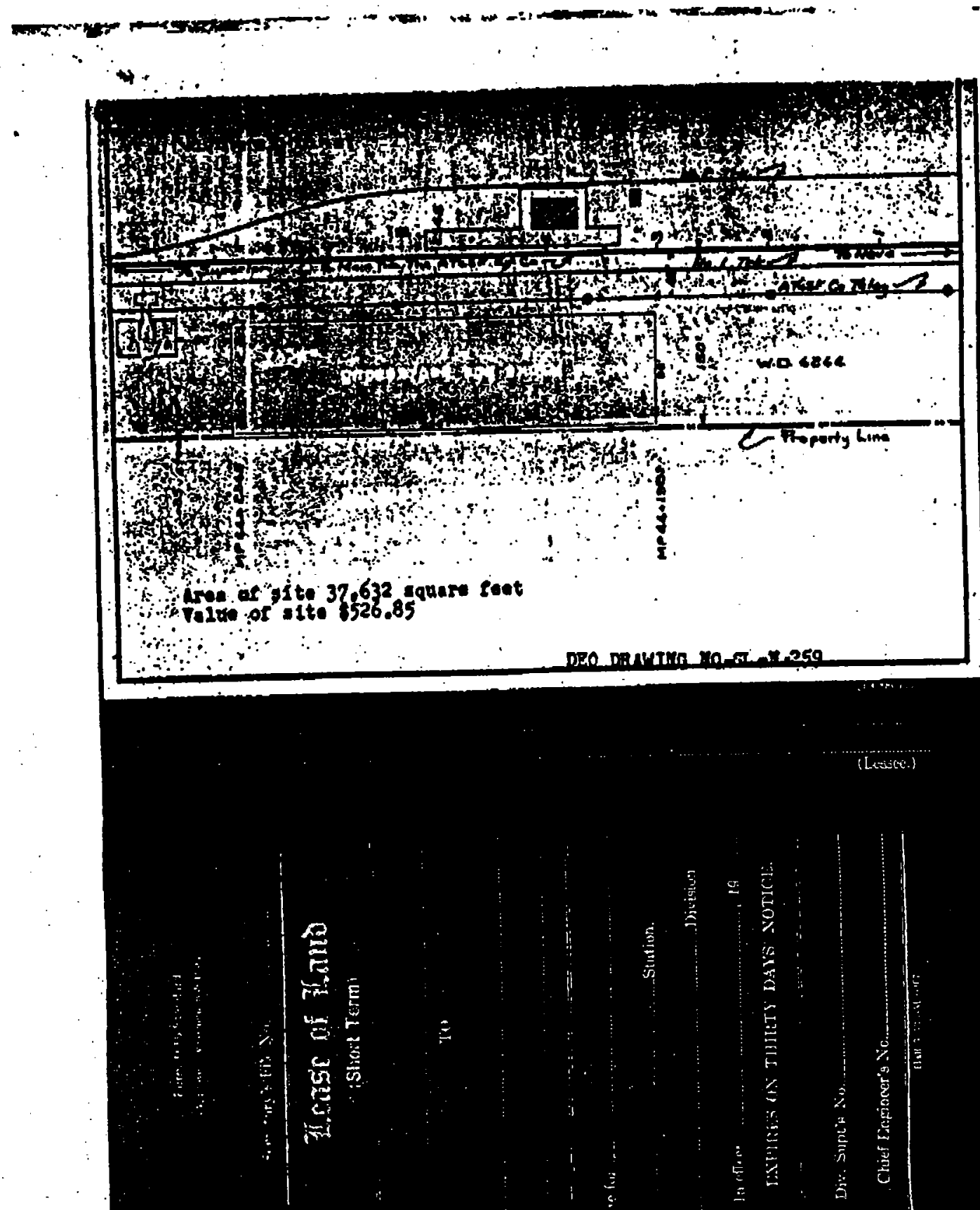
Argonne

Environmental Science Division

Argonne National Laboratory

9700 South Cass Avenue, Bldg. 203

Argonne, IL 60439-4843

www.anl.gov

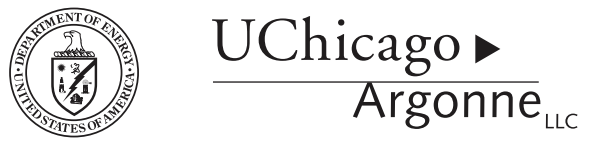

A U.S. Department of Energy laboratory

managed by UChicago Argonne, LLC 